Portland State University

PDXScholar

Environmental Science and Management

Professional Master's Project Reports

Winter 2022

\title{
Identifying and Prioritizing Urban and Commercial Stormwater Concerns: City of Grants Pass, Oregon
}

Amie Nicole Siedlecki

Portland State University

Follow this and additional works at: https://pdxscholar.library.pdx.edu/mem_gradprojects

Part of the Environmental Indicators and Impact Assessment Commons, Environmental Monitoring Commons, and the Water Resource Management Commons Let us know how access to this document benefits you.

\section{Recommended Citation}

Siedlecki, Amie Nicole, "Identifying and Prioritizing Urban and Commercial Stormwater Concerns: City of Grants Pass, Oregon" (2022). Environmental Science and Management Professional Master's Project Reports. 72.

https://pdxscholar.library.pdx.edu/mem_gradprojects/72

https://doi.org/10.15760/mem.75

This Project is brought to you for free and open access. It has been accepted for inclusion in Environmental Science and Management Professional Master's Project Reports by an authorized administrator of PDXScholar. Please contact us if we can make this document more accessible: pdxscholar@pdx.edu. 
Identifying and Prioritizing Urban and Commercial Stormwater Concerns: City of Grants Pass, Oregon

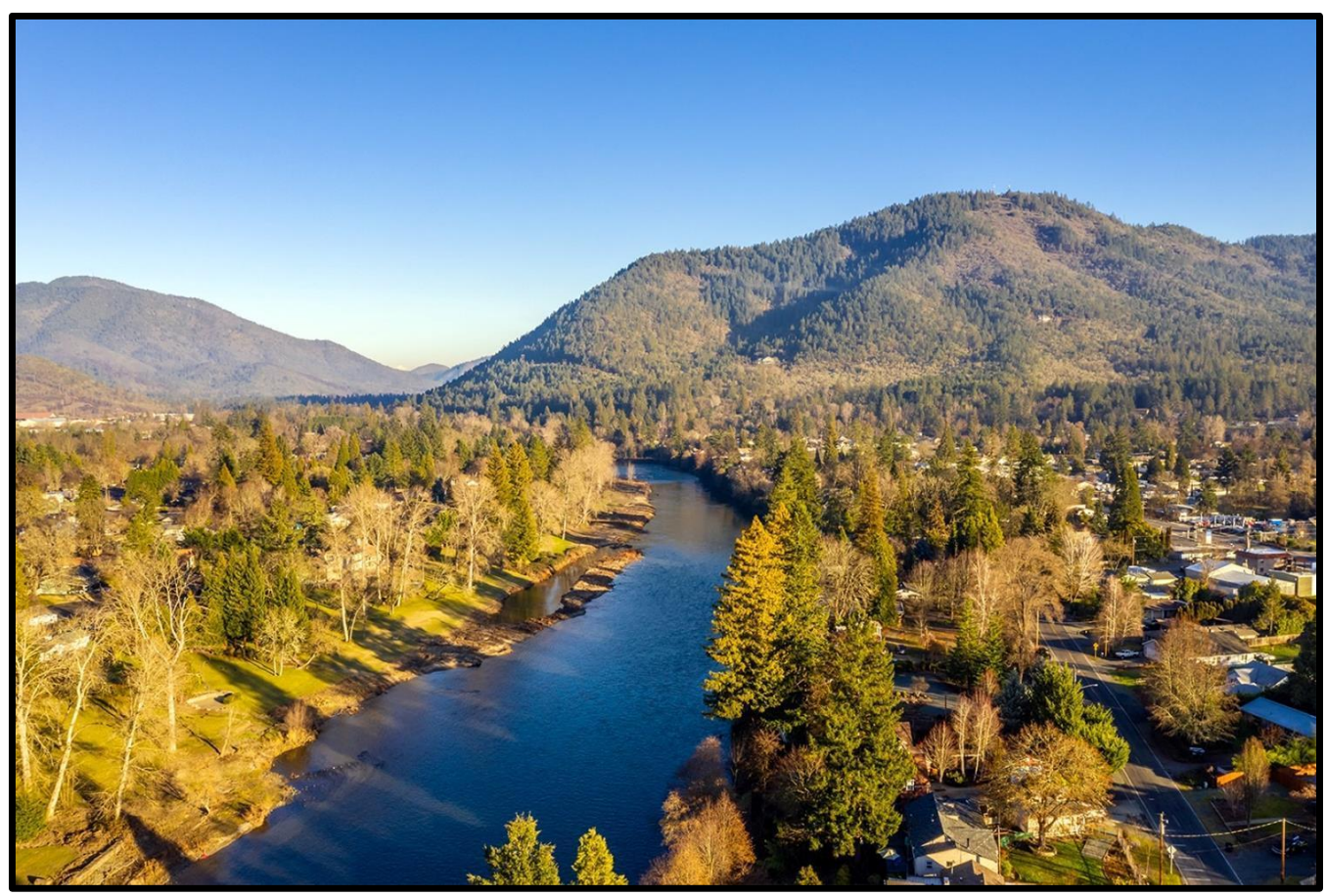

The Rogue River Watershed Council (RRWC), the City of Grants Pass, and the Medford Water Commission

Amie Nicole Siedlecki, Masters of Environmental Management (MEM) Candidate, Portland State University (PSU)

Winter 2022

Eugene Foster and Yangdong Pan, Advisors, PSU 


\begin{abstract}
For many communities, drinking water comes from surface water sources, or source water, such as rivers and creeks. Within the city of Grants Pass, Oregon, this is the case. The Rogue River, which spans 215 miles, beginning near Crater Lake and emptying into the ocean at Gold Beach, is Grants Pass' drinking water source. While the capacity of the Rogue River, in relation to drinking water, is rarely an issue for the City of Grants Pass' Public Works Department, the potential contaminant sources (PCS) from the urban, commercial, and industrial geographical areas of Grants Pass is a concern. In order to deploy treatment processes that are capable of targeting these PCS, it is important to have an idea of where and how these PCS are reaching the storm drains, creeks, and eventually the Rogue River. The purpose of this study was to identify area-specific risk components and how those components spatially aligned with PCS and their locations. Geographic Information System (GIS) analysis and a risk matrix were used to rank the PCS according to risk in relation to Grants Pass' source water intake. PCS ranked as high priority, or exuding the highest risk to drinking water quality, were followed up with onthe-ground surveys. After surveying the high priority PCS, best management practices (BMP) recommendations were made to the City of Grants Pass to better protect the drinking water quality. Branching off of this initial project work came similar studies in many other Rogue Basin communities. With this continued work, improvements were made to streamline the processes, such as recording survey observations. Overall, this project work has led to many discoveries regarding threats to drinking water quality and how to best respond to certain types of threats.
\end{abstract}




\section{ACKNOWLEDGEMENTS}

When one of my graduate advisors, Eugene Foster approached me with information about a group focused on drinking water quality in the Rogue Basin back in December 2017, I certainly was intrigued, but was unsure of what I could offer to the group. My interests were in stormwater quality and macroinvertebrate studies. How could this knowledge be of use within the drinking water realm? Despite my concerns, this was an opportunity I could not pass up. To earn my Master's degree through the completion of a project within my hometown of Grants Pass, Oregon, now that is a dream come true.

I quickly learned, after my first meeting with the newly-formed Rogue Drinking Water Partnership (RDWP), that stormwater and drinking water are absolutely related, especially when stormwater issues directly impact the amount of treatment required to supply clean drinking water to residents. Through the guidance of the Rogue River Watershed Council (RRWC), the City of Grants Pass, and the Medford Water Commission (MWC), a project focused on identifying and prioritizing urban and commercial stormwater concerns was not only created, but successfully completed.

The pilot project was so successful that, to my surprise, the RDWP advocated that the risk matrix, windshield survey, and best management practices (BMP) recommendation processes be applied within other Rogue Basin communities. Not only was I able to be a part of the process to help my hometown and the City of Grants Pass identify PCS, I was also involved in this process for the cities of Cave Junction, Eagle Point, Gold Hill, Rogue River, Shady Cove, and White City, as well as a private drinking water provider (DWP), Country View Mobile Home Estates in Trail. 
As a graduate student, I feel fulfilled and content with the work that I have been able to accomplish, which I could not have done without guidance from my advisors, Eugene Foster and Yangdong Pan, John Speece of the RRWC, Jason Canady and Wade Elliott of the City of Grants Pass, Craig Harper of the MWC, Jacquie Fern of the Department of Environmental Quality (DEQ), Greg Stabach of the Rogue Valley Council of Governments (RVCOG), my stepfather, who drove me around for the windshield surveys, my mother-in-law and father for encouraging me, sometimes sternly, to finish my Master's degree, and Eric Siedlecki, my husband and biggest supporter, who provided quality screenshots to use as figures in this report and gave me the intellectual space that I needed on Sunday mornings. Thank you all so much for encouraging me to be the best that I can be! 


\section{TABLE OF CONTENTS}

$\begin{array}{lll}\text { ABSTRACT } & \text { i }\end{array}$

ACKNOWLEDGEMENTS ii-iii

LIST OF FIGURES vi

LIST OF TABLES vii

GLOSSARY OF ACRONYMS viii

1.0 INTRODUCTION $\quad 1-15$

1.1 Overview: Source Water Protection $\quad 1-6$

1.2 National Drinking Water Issues $\quad$ 6-8

1.3 Local Drinking Water Concerns: Almeda and South Obenchain Fires 8-10

1.4 Statement of the Problem: City of Grants Pass 10-13

1.5 Rogue Drinking Water Partnership 13-14

1.6 Goals, Objectives, and Tasks and Deliverables 14-15

$\begin{array}{llr}2.0 & \text { METHODS } & 16-29\end{array}$

$2.1 \quad$ Study Area $\quad 16-20$

2.2 Study Approach $\quad$ 20-21

2.3 Total Number of PCS within the Source Water Area 21

$\begin{array}{lll}2.4 & \text { Risk Matrix } & 22-24\end{array}$

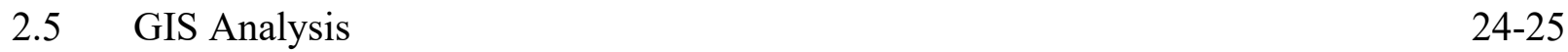

$2.6 \quad$ Windshield Surveys $25-27$

2.7 Public Water Supply Intake Risk 27-28

2.8 BMP Database 28-29

$\begin{array}{llr}3.0 & \text { RESULTS } & 30-35\end{array}$

3.1 Applying the Risk Matrix to GIS 30-31

3.2 Windshield Survey Findings $\quad$ 31-32

$\begin{array}{lll}3.3 & \text { Using the BMP Database } & 32-35\end{array}$

3.4 Communication of Results to the City of Grants Pass 35

$\begin{array}{llr}4.0 & \text { DISCUSSION } & 36-39\end{array}$

5.0 CONCLUSIONS AND RECOMMENDATIONS 40

6.0 REFERENCES $\quad 41-42$ 
7.0 APPENDICES

APPENDIX A. Updated Source Water Assessment: City of Grants Pass

APPENDIX B. Memorandum of Agreement - Rogue Drinking Water Partnership

APPENDIX C. Conceptual Model - Risk Matrix

APPENDIX D. Directions - Applying the Risk Matrix to GIS

APPENDIX E. Windshield Survey Sites

APPENDIX F. Source Water Protection Inspection Worksheet

APPENDIX G. BMP Database

APPENDIX H. USWA Excel File with PCS Information

APPENDIX I. Windshield Survey Database 


\section{LIST OF FIGURES}

Figure 1a. Rogue Basin with Surface Water Intakes and 8-Hour TOT 2

Figure 1b. City of Grants Pass Source Water Area, 8-Hour TOT, and PCS 3

Figure 1c. Legend of Map Symbols from the Updated SWA $\quad 4$

Figure 2. City of Grants Pass: DWPA 16

Figure 3. City of Grants Pass: 8-Hour TOT 17

Figure 4. City of Grants Pass: Stormwater Basins and UGB 18

Figure 5. City of Grants Pass: High Priority Stormwater Basins and PCS 19

Figure 6. Flow Chart of Study Design $\quad 21$

Figure 7. First Two Pages of the Inspection Worksheet 26

$\begin{array}{ll}\text { Figure 8a. Closed Business } & 27\end{array}$

$\begin{array}{ll}\text { Figure 8b. New Construction } & 27\end{array}$

$\begin{array}{ll}\text { Figure 8c. Wood Manufacturing } & 27\end{array}$

$\begin{array}{ll}\text { Figure 8d. General Contractor } & 27\end{array}$

$\begin{array}{ll}\text { Figure 9a. Brakes Plus } & 35\end{array}$

Figure 9b. Brakes Plus 35 


\section{LIST OF TABLES}

Table 1. City of Grants Pass: Climate Information in Comparison to United States Average

Table 2. EWEB's Scoring System for Magnitude Criteria

Table 3. Stormwater Basin Risk Component, Scoring, and Descriptions

Table 4. PCS Types in Grants Pass with a High SW Risk

Table 5. Subset of High Priority PCS Sites Surveyed

Table 6. Subset of Entries and Survey Responses Included in the Windshield Survey Database

Table 7. BMPs Associated with PCS Code C04

Table 8. BMPs Associated with PCS Code C07 


\section{GLOSSARY OF ACRONYMS}

ACWA: $\quad$ Association of Clean Water Administrators

BLM: $\quad$ Bureau of Land Management

BMP: $\quad$ Best Management Practices

CRWP: $\quad$ Clackamas River Water Providers

DEQ: $\quad$ Department of Environmental Quality

DSLR: $\quad$ Digital Single-Lens Reflex (type of camera)

DWP: $\quad$ Drinking Water Providers

DWPA: $\quad$ Drinking Water Protection Area

DWSA: $\quad$ Drinking Water Source Area

EPA: $\quad$ Environmental Protection Agency

ESRI: Environmental Systems Research Institute

EWEB: $\quad$ Eugene Water and Electric Board

FEMA: Federal Emergency Management Agency

GIS: $\quad$ Geographic Information System

JSWCD: Jackson Soil and Water Conservation District

MOA: $\quad$ Memorandum of Agreement

MWC: $\quad$ Medford Water Commission

NRCS: Natural Resources Conservation Service

NRDC: $\quad$ Natural Resources Defense Council

NWQI: National Water Quality Initiative

OHA: Oregon Health Authority

OWEB: $\quad$ Oregon Watershed Enhancement Board

PAH: $\quad$ Polycyclic Aromatic Hydrocarbon

PCS: $\quad$ Potential Contaminant Source

PFAS: $\quad$ Per- and Polyfluoroalkyl Substances

PPB: $\quad$ Parts Per Billion

PWS: $\quad$ Public Water Supplier

RDWP: $\quad$ Rogue Drinking Water Partnership

RRWC: $\quad$ Rogue River Watershed Council

RVCOG: Rogue Valley Council of Governments

RVSS: $\quad$ Rogue Valley Sewer Services

SVOC: $\quad$ Semi-Volatile Organic Compounds

SW: $\quad$ Surface Water

SWA: $\quad$ Source Water Assessment

SWP: $\quad$ Source Water Protection

TOC: $\quad$ Total Organic Carbon

TOT: Time-Of-Travel

TP: $\quad$ Total Phosphorus

UGB: $\quad$ Urban Growth Boundary

UST: $\quad$ Underground Storage Tank

USWA: Updated Source Water Assessment

VOC: $\quad$ Volatile Organic Compounds 


\subsection{INTRODUCTION}

\subsection{Overview: Source Water Protection}

Amongst those in the profession of drinking water treatment, source water is surface and/or groundwater that serves as a source of drinking water. Drinking water providers, either public or private and in the form of water utilities, community governments, or a combination of both, utilize source water assessments (SWA) to help identify threats to drinking water quality. SWAs are provided by the Oregon Department of Environmental Quality (DEQ) and the Oregon Health Authority (OHA) for the state of Oregon, and include important information tailored to each drinking water provider, or public water supplier (PWS), and its drinking water protection area (DWPA), or source area. Figure 1a. shows the Rogue Basin boundary, many of the cities within the basin, the river and stream corridors, the 8-hour time-of-travel (TOT), or the distance that potential contaminant sources (PCS) can travel within 8 hours in relation to surface water intakes (indicated with green circles), and a submap with the location of Grants Pass in relation to Portland. The studies and reports included within the SWAs pertain to PCS from certain activities, such as concentrated animal feeding operations, logging and mining sites, and industrial processing, as well as automobile repair shops, fueling stations, high-density housing, shopping malls, heavily-used roads, and others, which appear more densely-populated within urban centers (such as in Grants Pass, Figure 1b., with symbols described in Figure 1c.). These PCS and others have been identified as potential threats to drinking water treatment processes and the health and safety of residents within the DWPA, not to mention the impacts to aquatic and riparian organisms. 


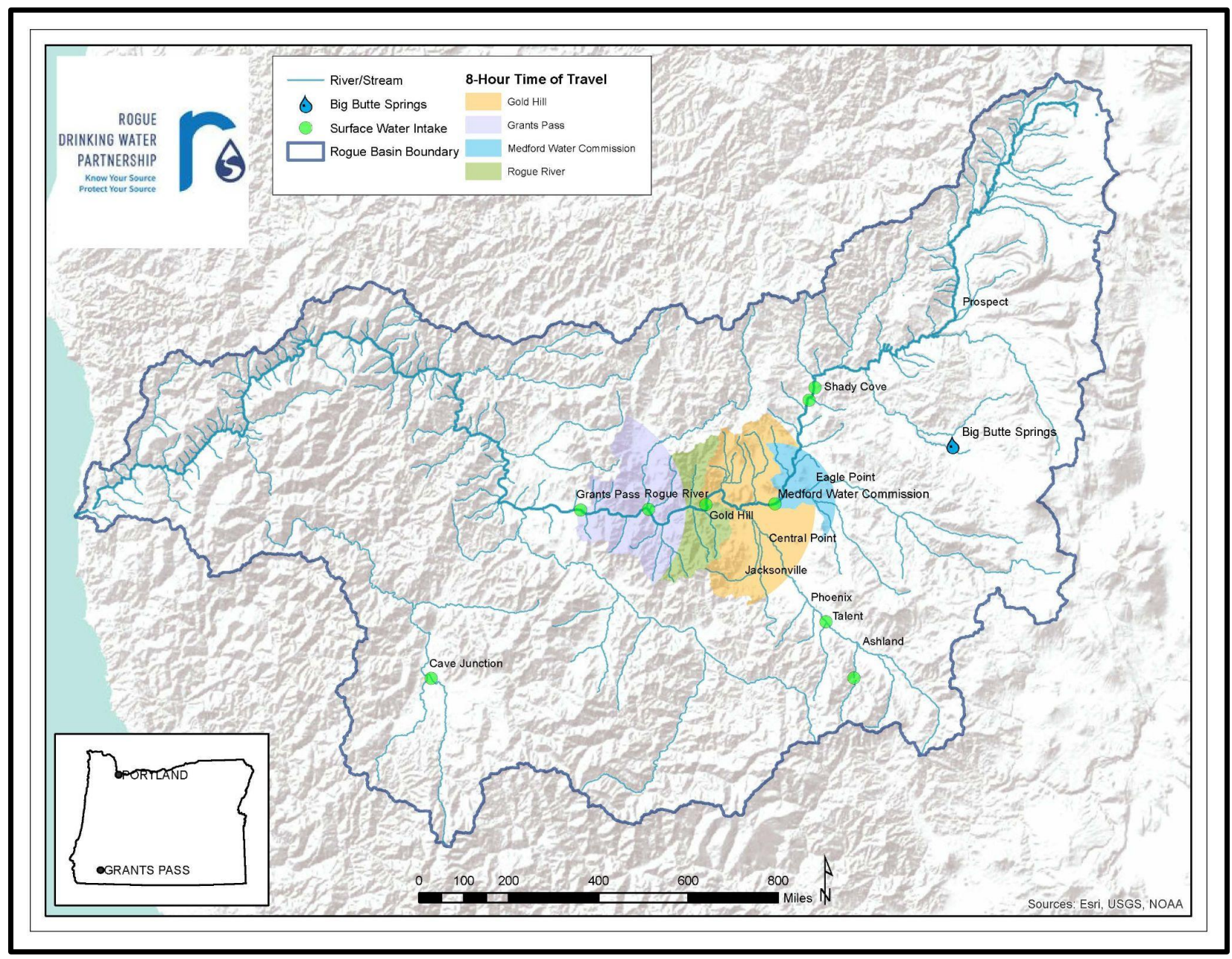

Figure 1a. Rogue Basin with Surface Water Intakes and 8-Hour TOT 


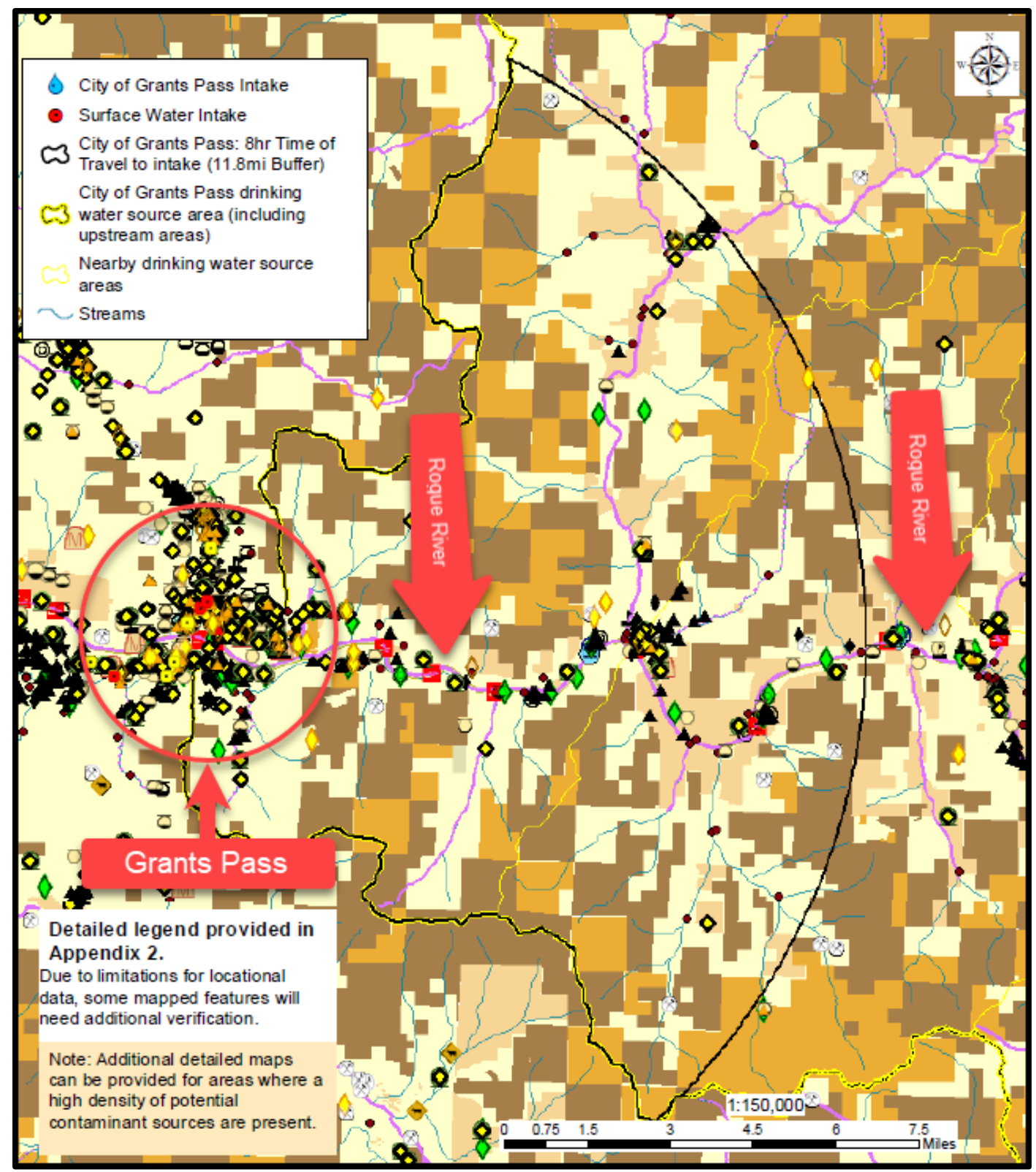

Figure 1b. City of Grants Pass Source Water Area, 8-Hour TOT, and PCS 


\begin{tabular}{|c|c|}
\hline $\begin{array}{l}\text { Urban Growth Boundary } \\
\text { County Boundary } \\
\text { Erosion Potential: } \\
\text { Streams near soils with significant erosion potential. } \\
\text { Erosion control measures (BMPs) may be necessary for } \\
\text { land management activites that disturb or leave bare } \\
\text { soils in these areas. } \\
\text { Streams (NHD) with significant erosion potential from } \\
\text { intensive (>75\%) soil surface disturbance (i.e.tilled or bare } \\
\text { soils) (NRCS-RUSLE2/ODA-EVl; see Note 4a). } \\
\text { Streams (NHD) with significant erosion potential from } \\
\text { substantial (50-75\%) soil surface disturbance (NRCS off- } \\
\text { road/off-trail ratings; see Note 4b). } \\
\text { Streams (NHD) with significant erosion potential (slope>30\% } \\
\text { using USFS SRI data, NRCS SSURGO data not avaliable; } \\
\text { see Note 4C). } \\
\text { Landslide Information } \\
\text { Landslide Deposits (non-rock material, includes } \\
\text { earth and debris slides, flows, slumps, falls and } \\
\text { complex) (DOGAMI SLIDO3.2) } \\
\text { Scarp Flanks (DOGAMI SLIDO-3.2) } \\
\text { Scarps (DOGAMI SLIDO-3.2) } \\
\text { Land Ownership/Use: } \\
\text { Private Urban Lands (within city limits) } \\
\text { Private Rural Lands (private non-industrial outside } \\
\text { city limits) } \\
\text { Agriculture (Ag Zoning (BLM) and NASS 2013) }\end{array}$ & 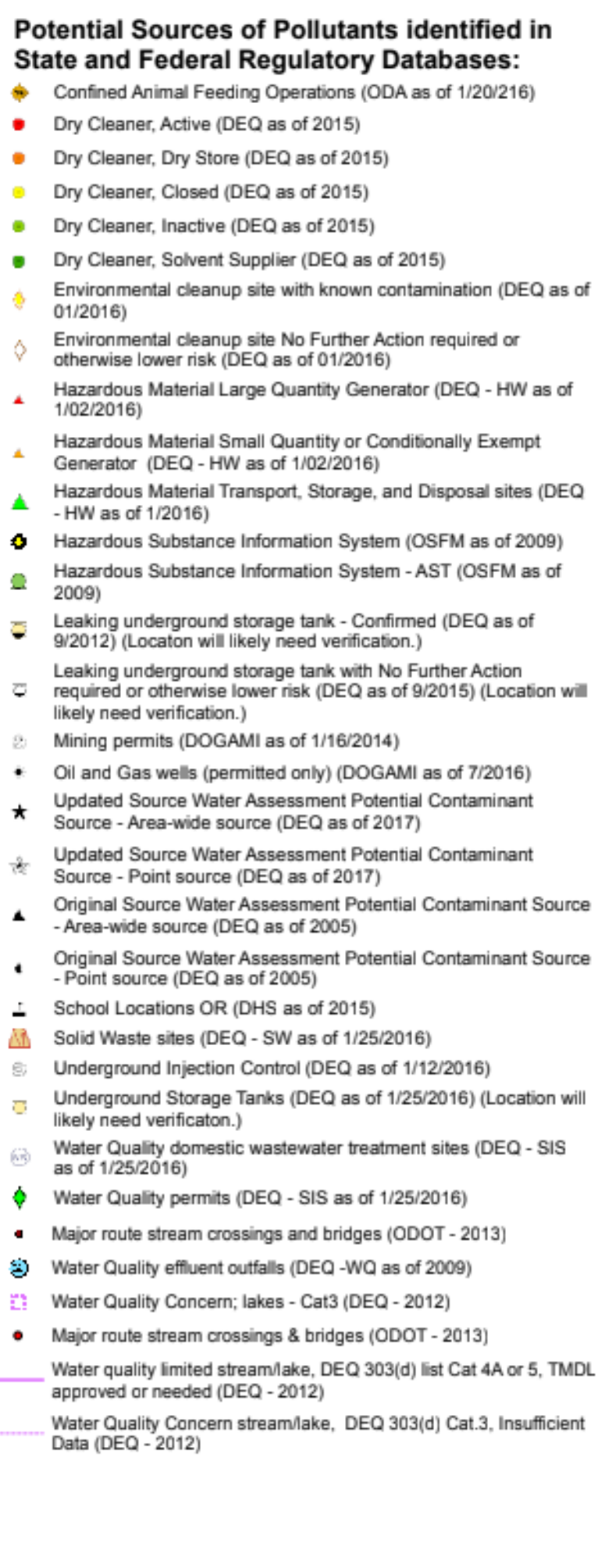 \\
\hline
\end{tabular}

Figure 1c. Legend of Map Symbols from the Updated SWA 
The updated SWA for the City of Grants Pass (March 2018; Appendix A.), begins with an opening letter from DEQ and OHA, followed by maps, and text, tables, and spreadsheets detailing the DWPA itself and PCS identified within the DWPA. Maps are included that display the following data: the delineated area of the entire Rogue Basin and the locations of surface water intakes (where the source water is collected for treatment), and erosion and soil surface disturbance potential, landslide hazards, landslide ownership and use related to anthropogenic sources and transportation corridors, all of which include the 8-hour TOT. The text, tables, and spreadsheets provide a DWPA summary (land use/ownership percentages, potential pollutants, water quality monitoring data, and treatment methods), additional resources for drinking water providers, and detailed information for each identified PCS.

The Clackamas River Water Providers (CRWP), which is a coalition of municipal water providers that source their drinking water from the Clackamas River, located just outside of the Portland metropolitan area, have utilized the information from their SWAs to develop a drinking water protection plan aimed at providing long-term source water protection strategies. The primary goals of this 2010 plan include identifying, preventing, minimizing, and mitigating the impacts of PCS and promoting public awareness and stewardship of a healthy Clackamas River watershed. Within their drinking water protection plan, the CRWP includes resources on BMPs that private citizens can adopt to help protect drinking water quality. These BMPs are relatively easy to put into practice and include keeping leaves, grass clippings, dirt, pet waste, and litter out of storm drains, planting ground cover, such as shrubs, on bare ground to hold soil in place and prevent erosion, and using non-toxic or less toxic pesticides and herbicides.

More recently, the City of Yachats, located on the central Oregon coast, presented a draft drinking water protection plan at a public meeting in April 2021. The draft plan includes 
information from the SWAs for Yachats regarding risks to drinking water quality and PCS. According to the meeting notes and presentation, the City of Yachats planned to submit a final drinking water protection plan to DEQ and OHA in June 2021.

The SWAs provide drinking water providers an opportunity to establish or build upon source water protection (SWP). Generally, SWP addresses the concerns associated with PCS by implementing behavioral and/or structural BMPs to protect drinking water quality. For example, the Eugene Water \& Electric Board (EWEB) has implemented several BMPs to protect source water, such as continuous monitoring equipment and wetland enhancements to treat stormwater runoff. In relation to both SWP and the aftermath of the Holiday Farm Fire of September 2020, EWEB also rallied collaborative erosion control efforts by Pure Water Partners, a program that encourages stewardship of healthy riparian forest areas and provides incentives to landowners to protect and/or restore riparian forests, and landowners, including hydroseeding, check dams, wattles, silt fencing, jute mats, and mulching. These BMPs have proven to aid in SWP.

Efforts to implement SWP measures and BMPs were identified as highly important within Grants Pass. This was due to the upstream locations of several industrial PCS in relation to the surface water intake, which will be discussed in detail within later sections.

\subsection{National Drinking Water Issues}

According to the Natural Resources Defense Council (NRDC), more than 100 million Americans are being exposed to per- and polyfluoroalkyl substances (PFAS) in their drinking water. PFAS are a class of "forever" chemicals which have been found at levels above the U.S. Environmental Protection Agency's (EPA) "health advisory" (2019). In order to better protect the health and safety of, first and foremost, our most vulnerable populations of people (i.e. pregnant and nursing mothers, infants, and children), major revisions to the EPA's Safe Drinking 
Water Act are required, with special attention to setting enforceable drinking water standards for PFAS.

Additionally, there are approximately 10.5 million lead service lines delivering drinking water into peoples' homes (2019). Lead contamination of drinking water has predominantly been displayed in Flint, Michigan. During the mid-20th century, Flint experienced great economic success, attributed to the automotive industry. However, the 1980s saw rising oil prices and an increase in imported cars. The economy in Flint began to plummet, as shown by $45 \%$ of its population living below the poverty line. Flint fell under state control once a \$25 million deficit was reached, and officials felt the pressure to cut costs to make up for the deficit.

In 2013, City of Flint officials decided to switch water providers from the Detroit Water and Sewerage Department, which utilizes Detroit River as its source, in favor of building a pipeline that would connect to the Karegnondi Water Authority and Lake Huron water, which would save the region $\$ 200$ million over 25 years (NPR, 2016). However, until the pipeline could be finished, the City of Flint decided to pipe treated Flint River water to its residents. Over the years, the Flint River has served as a waste disposal and raw sewage dumping site, and also receives agricultural and urban runoff and toxics leaching from landfills. As a highly corrosive drinking water source, lead began to leach away from the piping system and enter residents' homes.

Residents protested that the water was unsafe, which sparked Virginia Tech to conduct a study on the water. According to the study, nearly $17 \%$ of the samples collected by residents registered above the federal "action level" of 15 parts per billion (ppb) of lead, the level at which corrective action should be taken. Additionally, $40 \%$ of samples tested at $5 \mathrm{ppb}$ of lead, which indicates a very serious problem (NRDC, 2018). Lead contamination is especially harmful to 
infants and children, who were exposed for 18 months.

Lead exposure wasn't the only outcome of the switch in water sources. The third-largest outbreak of Legionnaires' disease, which is a severe form of pneumonia, in the U.S. also occurred. City workers found elevated levels of fecal coliform bacteria, and overcorrected that issue by using more chlorine as a disinfectant. However, too much chlorine led to elevated levels of total trihalomethanes, cancer-causing chemicals that are a byproduct of the chlorination of water.

After much court time, city and state officials were ordered to provide bottled water for its residents, as well as replace the lead and galvanized steel piping throughout Flint. As of October 2018, a little over 7,500 pipes have been upgraded, and lead levels within the water remain below the federal action level (NRDC, 2018). While this is good news, the pace at which pipes are being replaced is very slow, which has given the NRDC the opportunity to work with Flint residents to ensure a faster timeline in achieving safe, clean drinking water.

\subsection{Local Drinking Water Concerns: Almeda and South Obenchain Fires}

On September 8th, 2020, two fires were ignited within the Rogue Valley: the humancaused Almeda Fire, and the South Obenchain Wildfire. The Almeda Fire started in a dry brush field in Ashland along Almeda Drive behind the City of Ashland's Wastewater Treatment Plant, and the South Obenchain Wildfire broke out five miles east of Eagle Point. Due to high winds of more than 40 miles per hour, dry and hot weather conditions, and an abundance of fuel, specifically large colonies of Himalayan Blackberry, the Almeda Fire spread quickly within the Bear Creek Corridor, burning an estimated 3,000 acres and destroying approximately 2,800 structures. From the onset of the South Obenchain Wildfire until the end of September, 32,671 acres were burned. 
Potential drinking water quality concerns related to these local fires are elevated levels of aluminum (Al), perfluorinated compounds (PFAS; used for fire suppression), total phosphorus (TP), total organic carbon (TOC), turbidity, and volatile and semi-volatile organic compounds (VOC and SVOC). In the absence of healthy root systems to keep soils in place, these contaminants wash into waterways adjacent to burn areas. It is likely that $\mathrm{Al}$, phosphorus, and TOC are linked to turbidity, in that these materials are bonded and are adhered to soil particles. While natural sources and levels of $\mathrm{Al}$, phosphorus, and TOC exist in soils, current water samples indicate concentrations that have the potential to lead to major losses of macroinvertebrates and fish, as well as harmful algal blooms. As far as human impacts are concerned, the presence of these contaminants requires drinking water treatment facilities to increase their use of coagulants, chlorine, and/or chloramine.

Additional constituents of concern that have been identified following other wildfires, such as the 2015 Butte and Valley Wildfires, the 2017 Tubbs Fire, and the 2018 Camp Fire in Central and Northern California, include: bacteria (E. coli), ammonium and nitrates, metals (antimony, arsenic, cadmium, copper, lead, nickel, mercury, and zinc), pesticides and herbicides, polycyclic aromatic hydrocarbons (PAH; dioxins and furans), asbestos, polychlorinated biphenyls (PCB), and disinfection by-products, which are formed when water treatments, like chlorination, react with dissolved organic matter (EOS, 2020). It is important to note that harmful pollutants can also arise within drinking water distribution networks, rather than the source water itself, following urban fire events. For example, following the Tubbs Fire and the Camp Fire, benzene, a known carcinogen, was found in the distribution network, caused by the burning of plastic pipes and other plastics used in urban areas (EOS, 2020). 
Following these two fires, many organizations and groups within the Rogue Valley, such as the City of Grants Pass, Jackson County Parks, Jackson Soil and Water Conservation District (JSWCD), the Medford Water Commission (MWC), the Rogue River Watershed Council (RRWC), Rogue Riverkeeper, Rogue Valley Council of Governments (RVCOG), Rogue Valley Sewer Services (RVSS), and many others mobilized to protect water quality. To address erosion and sediment concerns, community volunteers and students were solicited to help these organizations spread seed mixes, including clover and barley, and straw within the riparian zones of the burn areas. Additionally, both in-stream and stormwater outfall water quality monitoring within the burn areas was carried out during rain events. As a result of the successful, collaborative work of these organizations, the Oregon Watershed Enhancement Board (OWEB) announced its support of a funding application to continue post-fire water quality monitoring within the burn scar of the Almeda Fire. With an increased capacity to monitor water quality within Bear Creek, which flows into the Rogue River, drinking water, especially for the communities of Gold Hill, Rogue River, and Grants Pass, may be better protected for its water users.

\subsection{Statement of the Problem: City of Grants Pass}

Since 1888, the primary drinking water source for residents within Grants Pass, Oregon has been surface water drawn from the Rogue River. The Rogue River also provides drinking water for other communities within the Rogue Valley, habitat for fish and wildlife, and recreational opportunities for thousands of people. The Rogue River spans 215 miles, beginning near Crater Lake and emptying into the ocean at Gold Beach. As such, the Rogue River traverses through varying landscapes, both "wild" (i.e. Western Cascades, Klamath Mountains, and the Kalmiopsis Wilderness) and "domesticated" (i.e. residential, urban, commercial, industrial, and 
agricultural). In regard to the "wild" landscapes, Bureau of Land Management (BLM) forest lands, State forests, and other State lands account for $34 \%$ of the drinking water source area (DWSA). As far as the "domesticated" landscapes are concerned, private rural and private urban areas account for $41 \%$, private industrial forests amount to $16 \%$, and agricultural lands make up 9\% of the DWSA. As the Rogue River travels downstream through forested lands, the water quality is most often described as pristine. Once the Rogue River begins to wind through farmlands, towns and cities, and commercial and industrial areas, the water quality is impacted by PCS and is no longer described as a pristine drinking water source.

For information related to climate, see Table 1. Climate, specifically precipitation events (rainfall and snowfall), can lead to a more rapid movement of PCS to storm drains, creeks, and the Rogue River.

Table 1. City of Grants Pass: Climate Information in Comparison to United States Average *Information from BestPlaces.net*

\begin{tabular}{|l|l|l|}
\hline Geographic Area & Grants Pass, Oregon & United States (Average) \\
\hline Rainfall (inches/centimeters) & $29.8 / 75.692$ & $38.1 / 96.774$ \\
\hline Snowfall (inches/centimeters) & $2.6 / 6.604$ & $27.8 / 70.612$ \\
\hline Precipitation (days) & 104.4 & 106.2 \\
\hline Sunny (days) & 196 & 205 \\
\hline $\begin{array}{l}\text { Average July High } \\
\text { Temperature. }\left({ }^{\circ} \mathrm{F} / \mathrm{C}\right)\end{array}$ & $90.7 / 32.61111$ & $85.8 / 29.88889$ \\
\hline $\begin{array}{l}\text { Average January Low } \\
\text { Temperature }\left({ }^{\circ} \mathrm{F} / \mathrm{C}\right)\end{array}$ & $33.9 / 1.055556$ & $21.7 /-5.722222$ \\
\hline Elevation (feet/meters) & $942 / 287.122$ & $2443 / 744.6264$ \\
\hline
\end{tabular}

Of concern within the urban, commercial, and industrial landscapes of Grants Pass are PCS. The major urban, commercial, and industrial centers of Grants Pass are located in the Fruitdale and Skunk Creek stormwater basins (along Highway 99 for Fruitdale Creek and south 
of Highway 199 for Skunk Creek; Figure 5.). PCS found within these parts of the city include junk, scrap, and salvage yards, metal plating, finishing, and fabrication shops, wood, paper, and pulp processing facilities, and others capable of negatively impacting the City of Grants Pass' public drinking water system and drinking water quality. These impacts come in the form of increased maintenance and operations costs (i.e., clogged filters in need of frequent filter backwashes and/or replacement, expanded uses of chlorine and/or chloramine for treatment, and additional staff time allocated for these procedures), and a higher rate of dissatisfaction among customers associated with the elevated concentrations of disinfectants (i.e., taste and smell).

For example, the City of Grants Pass occasionally receives complaints during certain periods of the year when additional disinfectants need to be used to treat the drinking water (i.e., high turbidity during heavy rainfall events, irrigation season, drought and low water levels, etc.). Some PCS may even pass through filters untreated, posing immediate health threats through consumption of the drinking water. Pairing these issues with that of a water treatment plant "well past its designed life cycle... becoming increasingly difficult and expensive to maintain" (City of Grants Pass: Public Works, 2021), addressing concerns with PCS before they reach the plant is of utmost importance for the City of Grants Pass. To paint a clearer picture of the water treatment plant and its challenges, the City of Grants Pass continues to operate within a plant originally built in 1931. To meet the needs of the continuously-growing city, the plant was expanded in the 50s, 60s, and 80s. Infrastructure failure and subsequent repairs have plagued the plant over the decades. However, in 2017, the Grant Pass City Council identified the replacement of the plant as their number one priority, with construction expected to begin in 2023.

To address the specific issue surrounding PCS, RRWC partnered with the City of Grants Pass and MWC under the Rogue Drinking Water Partnership (RDWP) to expand source water 
protection efforts to other PWS areas, as well as to grow the partnership, which will be discussed further in the following section.

\subsection{Rogue Drinking Water Partnership}

The Rogue Drinking Water Partnership (RDWP) is a collaborative partnership of municipal and private Rogue Basin drinking water providers (DWP) and other organizations, such as RRWC, that seeks to preserve, protect, and enhance drinking water quality from both surface and groundwater sources. The work of the RDWP, funded largely by Drinking Water Source Protection grants from OHA, looks to leverage the interests of many stakeholders to maximize the effectiveness of SWP and to support clean water throughout the Rogue Basin. Actions that prevent, minimize, and mitigate activities that may cause harmful impacts to drinking water quality include implementing BMPs, promoting public awareness and stewardship of drinking water sources, and developing resources and financial support to put in place an action plan, which is the current work of the RDWP. Presently, the RDWP meets quarterly to share project outcomes, improvements, and further actions that need to occur on the drinking water front. For additional information on the RDWP and its Memorandum of Agreement (MOA), refer to Appendix B.

Beginning in 2017, the RDWP initiated multiple efforts to protect drinking water quality and combat activities that may harm it, such as PCS associated with urban, commercial, and industrial stormwater, agricultural areas, and forested lands. First, the partnership requested SWAs from DEQ to help identify potential problem areas within the Rogue Basin. Starting in 2018, the RDWP received updated SWAs for the following Rogue Basin communities: the cities of Ashland, Cave Junction, Gold Hill, Grants Pass, and Rogue River, and the municipal and private DWPs, including Anglers Cove/Shady Cove Heights Water Company (Shady Cove, 
Oregon), Country View Mobile Home Estates (Trail, Oregon), Hiland Water Company (Shady

Cove, Oregon), and MWC (Medford, Oregon and neighboring communities). For its relevance to this report, refer to Appendix A. for the City of Grants Pass' updated SWA.

As one of the main applicants for the grants from OHA, the City of Grants Pass, with guidance from RRWC, was responsible for developing a pilot project to move the overall efforts of the RDWP forward. Due to my interests in urban, commercial, and industrial stormwater, paired with source water quality concerns related to Grants Pass' industrial area and its location directly upstream of the surface water intake, the following goals, objectives, and tasks and deliverables were developed for the surveys that began in December 2018.

\subsection{Goals, Objectives, and Tasks and Deliverables}

\section{Goals}

1. Identify and prioritize the risks of PCS within the urban, commercial, and industrial geographic areas of the DWPA for the City of Grants Pass.

2. Determine the site-specific BMPs that can be implemented to potentially reduce those risks, such as secondary containment, catch basin media inserts, emergency spillways, check dams, wattles, and silt fencing, among several others.

\section{Objectives}

1. Address and limit the impacts of chemicals and other pollutants on drinking water quality.

2. Establish protocols for identifying and ranking PCS to be used by municipalities, DWPs, and conservation organizations, utilizing readily-available resources.

3. Create a list of BMPs associated with specific PCS.

\section{Tasks and Deliverables}

1. Produce a ranking system using available data, Geographic Information System (GIS) analysis, local recommendations, and the updated SWAs to determine the risks (low, moderate, and high) of each PCS.

2. Establish a replicable field strategy for locating and surveying the impacts of urban and commercial PCS that threaten DWPs and treatment processes. 
3. Create an interactive database of implementable BMPs addressing erosion and pollution prevention, and runoff and sediment controls, to protect drinking water quality.

4. Enhance the protocols (field strategy, ranking system, and BMP database) developed for the City of Grants Pass, and apply them within other Rogue Basin communities. 


\subsection{METHODS}

\subsection{Study Area}

As mentioned in the overview, each DWP is responsible for maintaining the health and safety of its DWPA. For the City of Grants Pass, this area extends in a northeastern direction, or upstream, past both the city limits and the urban growth boundary (UGB; Figure 2.). In addition to this DWPA, the City of Grants Pass must also take into account the 8-hour TOT, or the distance that PCS can travel within 8 hours in relation to surface water intakes (Figure 3.) in order to respond quickly to spills and other emergencies related to drinking water quality.

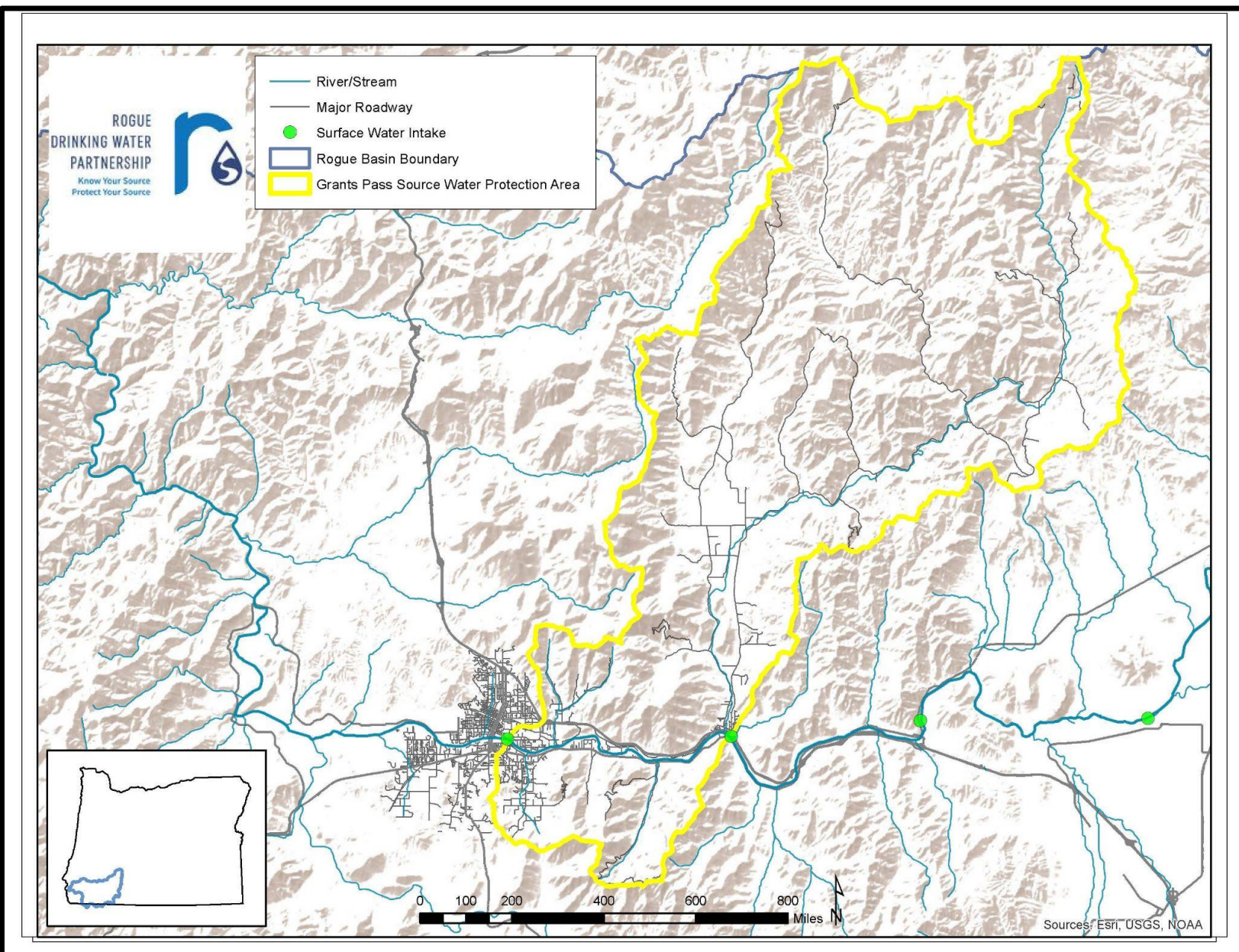

Figure 2. City of Grants Pass: DWPA 


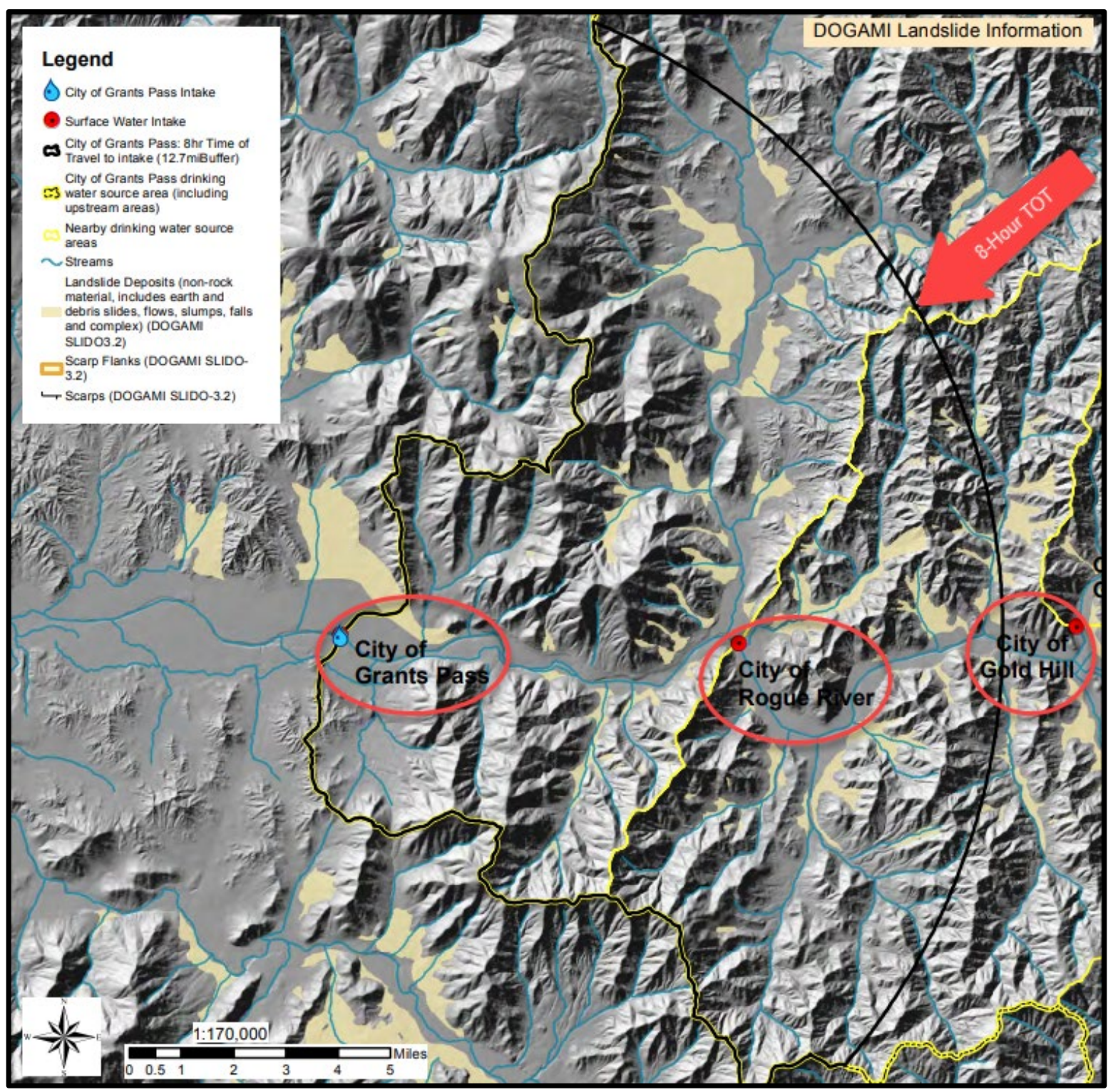

Figure 3. City of Grants Pass: 8-Hour TOT

With its stormwater utility in mind, which manages runoff to avoid flooding, water

pollution, and streambank erosion, the City of Grants Pass has organized its stormwater

infrastructure (i.e. systems of drains, pipes, culverts, ravines, and creeks) into stormwater basins.

There are seven stormwater basins within or surrounding the UGB (shown as a red line; Figure

4.). Runoff that enters a storm drain within the Gilbert Creek stormwater basin, for example, will empty into Gilbert Creek, and eventually the mainstem Rogue River. 


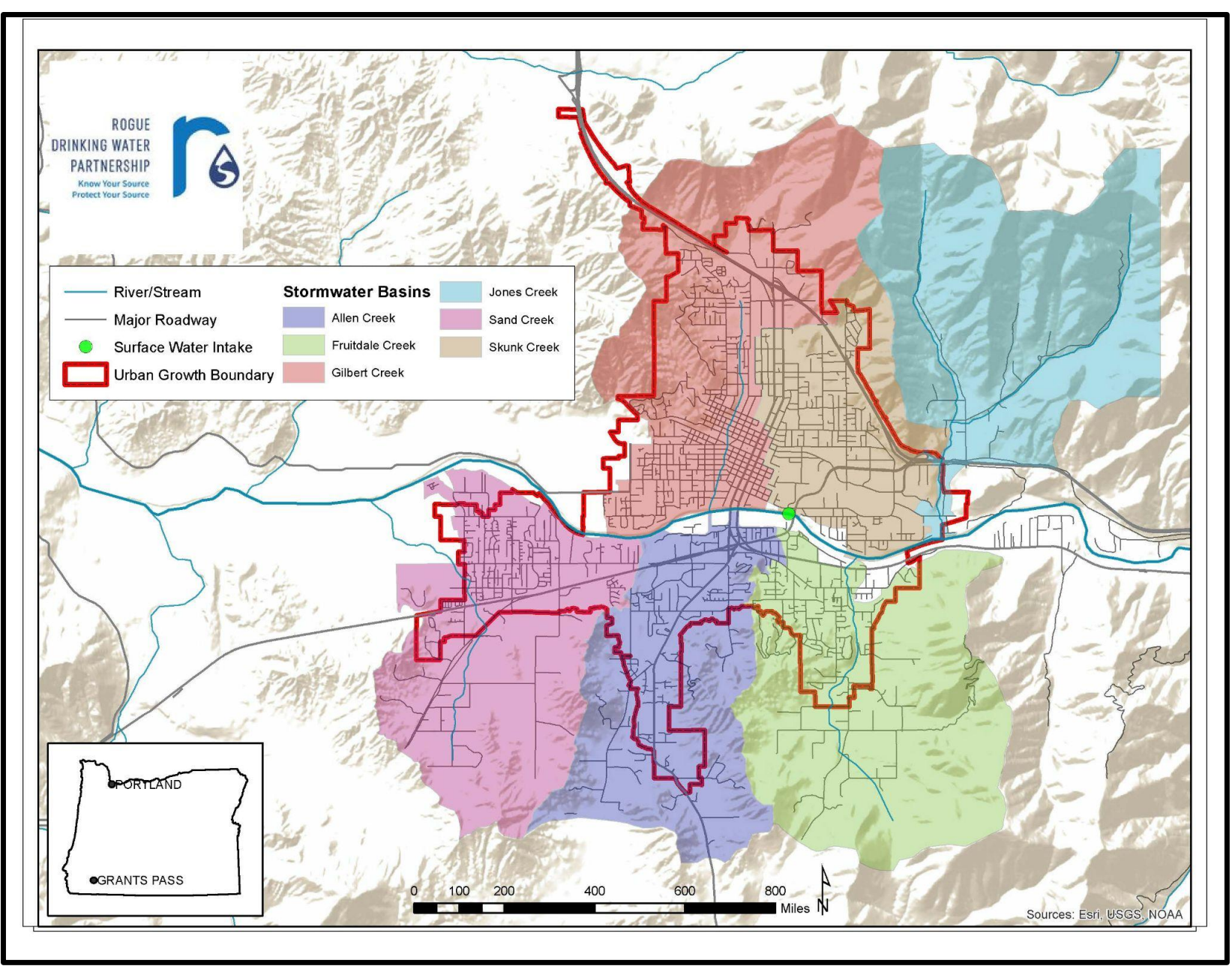

Figure 4. City of Grants Pass: Stormwater Basins and UGB

The Fruitdale, Jones, and Skunk Creek stormwater basins were prioritized for PCS

identification, and encompassed the study area due to their proximity and locations directly upstream of the drinking water intake, which is shown as the green circle (Figure 5.). Within the study area, commercial and industrial PCS were identified and evaluated to determine the immediate risks to drinking water quality. 


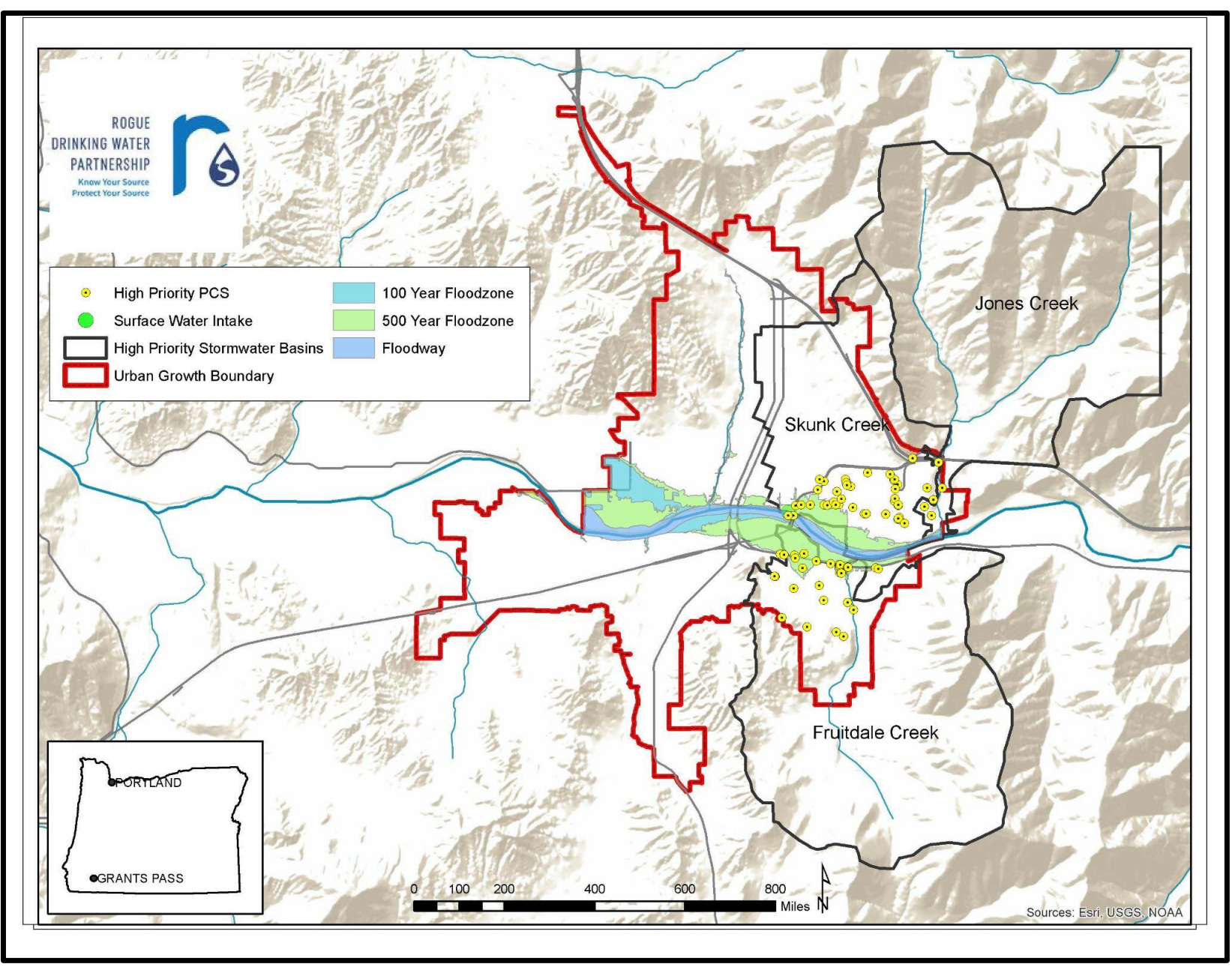

Figure 5. City of Grants Pass: High Priority Stormwater Basins and PCS

Expanding on the prioritization process, if a PCS (fuel, gasoline, or diesel from a major spill, for example) enters one of these three stormwater basins and the drinking water treatment plant is notified, plant operators and other responders may shut down the surface water intake and utilize emergency response strategies to protect the source water. Understanding the close proximity of the three creek inlets in relation to the intake, those responding to a spill or other pollutants must act in haste to avoid drawing in contaminated source water. As for creek inlets farther upstream and/or downstream of the intake, the threat is not as immediate, although response is still required to protect the source water. This is an emerging topic within the Rogue Valley and one that will be discussed later in this report. 
The highest priority PCS (yellow circles) are all located in the Fruitdale, Jones, and Skunk Creek stormwater basins and within the UGB (red line; Figure 5.). These high priority PCS were determined through the development of a risk matrix containing multiple components and associated scoring, which will be discussed in detail within the following section.

\subsection{Study Approach}

The framework for this study was developed from both the available information in the SWAs and the updated source water assessments (USWA; January 2018) and recommendations from the City of Grants Pass. The framework aimed to identify the total number of PCS within Grants Pass through the creation of a risk scoring matrix. The risk matrix helped to identify highest priority urban and commercial PCS after being applied within a pre-screen and prioritization of sites using GIS. Following the results of the GIS analyses, windshield surveys to verify priority PCS occurrence and locations were conducted. Conducting windshield surveys, or visual surveys, is an easily utilized strategy. These surveys do not necessarily require the surveyor to leave the seat of their field vehicle to record information. Instead, the only information that is documented is what they can determine when viewing that area from inside the vehicle, or outside from a distance. Additionally, pollutant risks to PWS intake were calculated and BMPs and actions to address those risks were identified (to be discussed in detail within the following paragraphs). All of this information was communicated to the City of Grants Pass following the completion of this pilot project (Figure 6.). 
Total number of PCS within the Grants Pass source water area (from USWA).
Develop risk matrix scoring; identify and verify highest

priority urban and commercial PCS.
Recommendations to the City of Grants Pass.
BMPs and actions to address the most significant risks to PWS intake.
Pre-screen and prioritize sites for windshield surveys using GIS

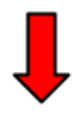

Windshield surveys; verify highest priority PCS.

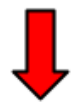

Calculate pollutant risk to PWS intake.

Figure 6. Flow Chart of Study Design

\subsection{Total Number of PCS within the Source Water Area}

Utilizing and narrowing down the information from the USWA and a supplementary Excel file with all PCS for several cities within Oregon provided by DEQ, it was determined that a total of 214 PCS can be found within Grants Pass. However, it is important to note that one location can be categorized as having multiple PCS. For example, Fruitdale Elementary School is categorized as 1. C20 - Parking Lots/Malls >50 Spaces, 2. M28 - Underground Storage Tank (UST) - Status Unknown, and, of course, 3. R15 - Schools. To simplify later procedures, each location was noted for having multiple PCS types and duplicate locations were deleted from the windshield survey list, which is discussed in detail in the Windshield Surveys section of this report. 


\subsection{Risk Matrix}

As mentioned above, using both the available information in the USWA and recommendations from the City of Grants Pass, a risk matrix was developed for numeric ranking of priority PCS within the City of Grants Pass' source water area. The following sections share details related to the inspiration for and the development of the risk matrix.

In May of 2018, the RDWP invited Karl Morgenstern from EWEB to discuss risk assessment and contingency planning during a quarterly meeting. Morgenstern presented on EWEB's “scoring system for magnitude criteria” from a 1995 Environmental Risk Assessment of the McKenzie Watershed, which aimed at identifying risks to Eugene's sole source of drinking water. This scoring system provides a ranking, general description, and an example for each category from a series of five, as seen in Table 2. We used this scoring system as an example to develop a risk matrix to apply within Grants Pass. This risk matrix included components known to pose risks to drinking water quality, along with associated scoring, to help pinpoint high priority PCS. 
Table 2. EWEB's Scoring System for Magnitude Criteria

\section{SCORING SYSTEM FOR MAGNITUDE CRITERIA}

\begin{tabular}{|c|l|l|}
\hline Ranking & \multicolumn{1}{|c|}{ Gene ral Description } & \multicolumn{1}{|c|}{ Examples } \\
\hline 1 & $\begin{array}{l}\text { Inactive sites or small volumes and/or } \\
\text { low toxicities }\end{array}$ & $\begin{array}{l}\text { Markets, motels, docomissioned service stations, } \\
\text { masonary business }\end{array}$ \\
\hline 2 & $\begin{array}{l}\text { Small to moderate volumes and/or } \\
\text { moderate toxicities }\end{array}$ & $\begin{array}{l}\text { Schools, animal clinics, feed stores, welding, small } \\
\text { auto repair, small manufacturing, logging and } \\
\text { cosntruction companies }\end{array}$ \\
\hline 3 & $\begin{array}{l}\text { Moderate to large volumes and/or } \\
\text { toxicities }\end{array}$ & $\begin{array}{l}\text { Auto repair, medium sized manufacturing, active } \\
\text { service stations, powerhouses }\end{array}$ \\
\hline 4 & $\begin{array}{l}\text { Large volumes and/or moderate high } \\
\text { toxicities }\end{array}$ & Storm sewer outfalls, cleaners, forestry activities \\
\hline 5 & $\begin{array}{l}\text { Large to very large volumes and/or } \\
\text { high toxicities }\end{array}$ & $\begin{array}{l}\text { Hazmat truck accidents, large manufacturing plants, } \\
\text { fish hatcheries }\end{array}$ \\
\hline
\end{tabular}

Using data from the City Utility Systems and Area Hazards interactive maps from the City of Grants Pass, recommendations from the Public Works Department, and the updated SWA from DEQ, the following risk components were included in the risk matrix (Appendix C.) to identify the highest priority PCS to address:

1. Stormwater basin (City Utility Systems),

2. Proximity to waterway* (recommendations from the Public Works Department),

3. Surface water risk (updated SWA),

4. Flood hazard (Area Hazards), and

5. Jurisdiction (City Utility Systems).

*With the assumption that PCS located closer to waterways pose a higher risk than those located farther from waterways, "proximity to waterway" was included in the risk matrix.

Each risk component is associated with a set of scoring and descriptions (seen in Appendix C.). For example, Table 3. depicts the stormwater basin risk component. To receive a score of 0, the PCS must have been located within the Allen, Gilbert, or Sand Creek stormwater basins. PCS within the Allen, Gilbert, and Sand Creek stormwater basins are downstream of the drinking water intake and do not pose a threat to the City of Grants Pass' drinking water quality. 
On the other hand, PCS within the Fruitdale and Skunk Creek stormwater basins are directly upstream of the drinking water intake, and therefore pose a greater risk (score of 4).

Table 3. Stormwater Basin Risk Component, Scoring, and Descriptions

\section{Stormwater Basin:}

\begin{tabular}{|l|l|}
\hline \multicolumn{1}{|c|}{ Score: } & \multicolumn{1}{c|}{ General Description: } \\
\hline 0 & $\begin{array}{l}\text { Gilbert/Allen/Sand creeks are downstream of the } \\
\text { drinking water intake }\end{array}$ \\
\hline 1 & $\begin{array}{l}\text { "Needs Basin ID” is upstream of the drinking water } \\
\text { intake, but does not cover any urban or industrial } \\
\text { areas }\end{array}$ \\
\hline 2 & $\begin{array}{l}\text { Jones Creek is farther upstream from the city center, } \\
\text { but does cover industrial areas }\end{array}$ \\
\hline 3 & $\begin{array}{l}\text { Skunk/Fruitdale creeks cover the city center, } \\
\text { industrial areas, and are both mostly upstream of the } \\
\text { drinking water intake }\end{array}$ \\
\hline
\end{tabular}

Stormwater Basin Data from the Interactive Maps, City of Grants Pass.

Applying the five risk matrix components and associated scoring, as well as the PCS location data from the updated SWA to GIS, each PCS received a total score. The overall risk matrix scoring ranges for low $(\mathrm{L})$, moderate $(\mathrm{M})$, and high $(\mathrm{H})$ risk PCS are 1-4, 5-8, and 9-13, respectively.

\subsection{GIS Analysis}

GIS analyses were used to pre-screen and prioritize sites for the windshield surveys. Available data and the appropriate GIS analysis was used to reduce the PCS count to complete the surveys in the timeframe for this project. To complete the analyses, data, including the USWA Excel file with PCS information and GIS layers, was downloaded and added to ArcMap: 
- Stormwater basin layer from the City of Grants Pass,

- Ditches and waterlines layer,

- USWA Excel file with PCS information, including surface water (SW) risk ratings (H, $\mathrm{M}$, and $\mathrm{L}$ ),

- Federal Emergency Management Agency (FEMA) floodplain layer (including the categories "floodway," "100-year flood," "500-year flood," and beyond), and

- UGB layer.

An "area of interest" polygon was created using the Editor tool. This area of interest captured both the city limits and the UGB for Grants Pass. Data was clipped to this area and scores/rankings were added for each of the data files for use in the prioritization.

For example, a high $(\mathrm{H})$ risk PCS within 0 - 500' of a waterway, in the 100-year flood zone, and inside of the UGB would have scores 2, 3, 2, and 2, respectively. Associated scores were manually added to the attribute table, which was then exported to Excel to calculate total scores. The example PCS above receives a score of 9, which categorizes it as a high risk PCS. All high risk PCS sites were visited in Grants Pass, unless they were located outside of the UGB. Full instructions for applying the risk matrices to GIS are available in Appendix D.

\subsection{Windshield Surveys}

The windshield surveys were conducted to verify, in the field, the highest priority PCS. Windshield survey site visits were identified based on the scoring results of the PCS using the risk matrix. As mentioned in the previous section, PCS that received an overall score of between 9 and 13, except those that landed outside of the UGB of Grants Pass, were surveyed. For example, a location with a score of 9, but lying outside of the UGB, was not surveyed. This decision was made based on the City of Grants Pass' position to offer BMP suggestions to property owners and/or businesses, as well as their authority to issue violation notices in the case of illicit discharges or improper behaviors in relation to PCS following several warnings. 
With the paired down list of PCS locations (highest priority PCS within the UGB), these locations were then listed in a geographical order or route that created a logical starting point and ending point (Appendix E.). This document guided the windshield surveys and allowed for notetaking (e.g. business is closed, subdivision is developed/no longer requires attention, etc.). In addition to this document, a 5-page inspection worksheet (Figure 7.), adapted from a collaborative RVSS and MWC document, was filled out for each location. The full inspection worksheet is available to view in Appendix F.

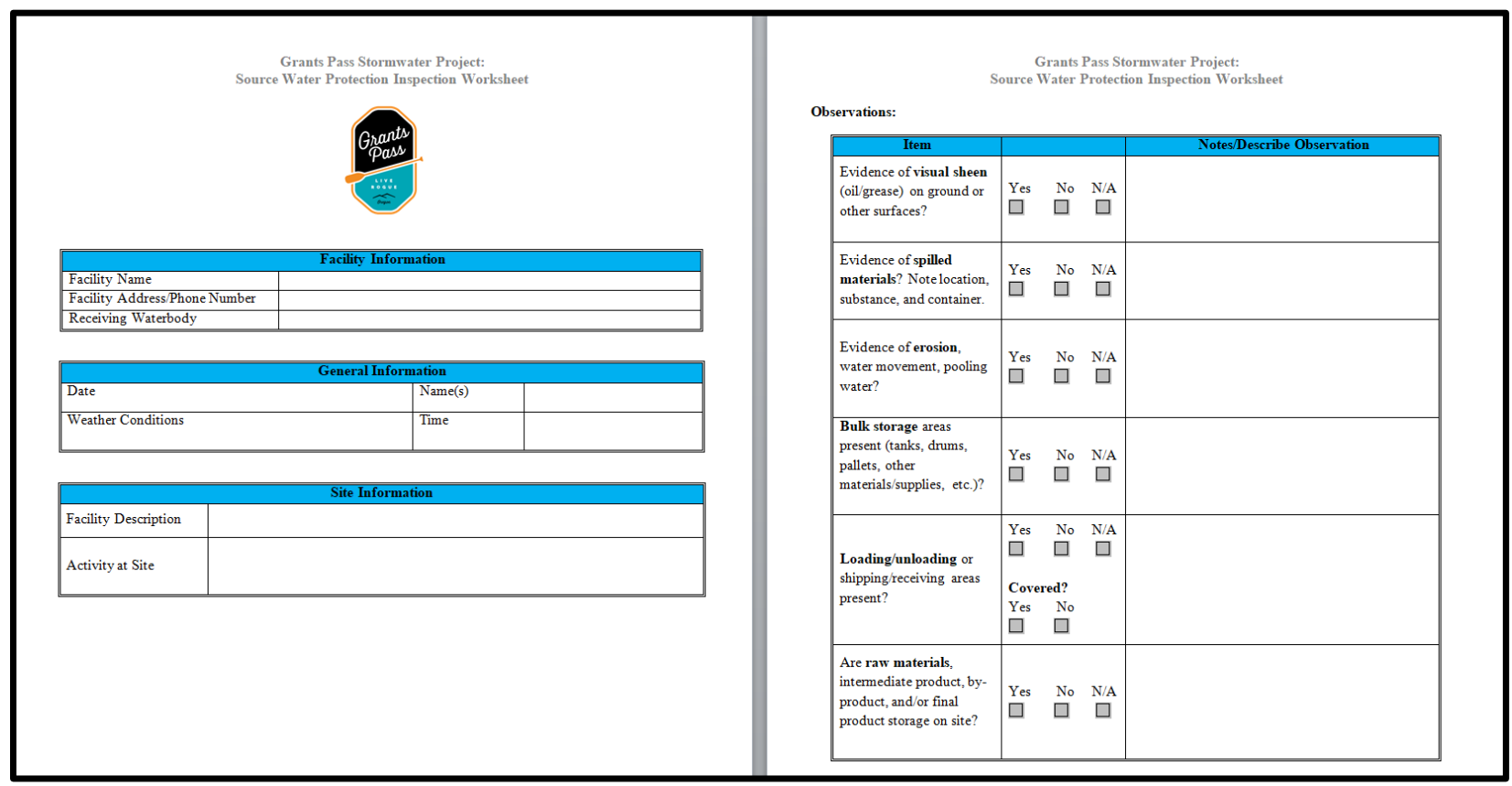

Figure 7. First Two Pages of the Inspection Worksheet

Site information collected included the name, address, and phone number of the business, date and time of inspection, name of inspector, stormwater basin (Fruitdale Creek, Jones Creek, or Skunk Creek), and activity at the site. Questions pertaining to visual sheen, ground disturbance, oil, gas, and chemical storage, erosion prevention and sediment control, stormwater BMPs, and others were asked throughout the worksheet regarding the appearance at each of the sites. Information obtained through completing the worksheets was transferred to a windshield 
survey database (Excel spreadsheet) for ease of viewing, sorting, and filtering the data. Overall, reviewing the inspection worksheet data and the pictures that were captured at each location using a digital single-lens reflex (DSLR) camera (examples below in Figures 8a., 8b., 8c., and 8d.), paired with the information included in the BMP Database (to be discussed in the following section), allowed for the recommendation of site-specific BMPs to protect drinking water quality.

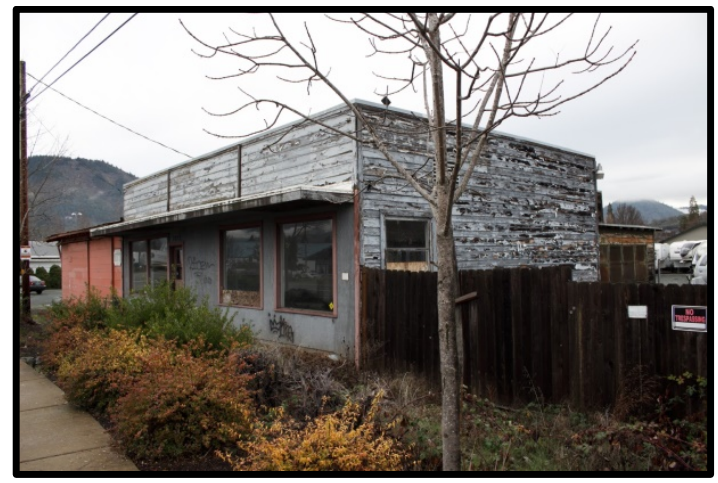

Figure 8a. Closed Business

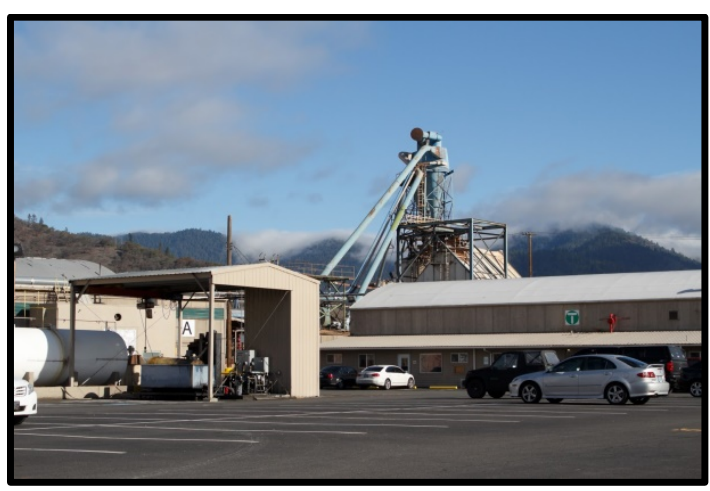

Figure 8c. Wood Manufacturing

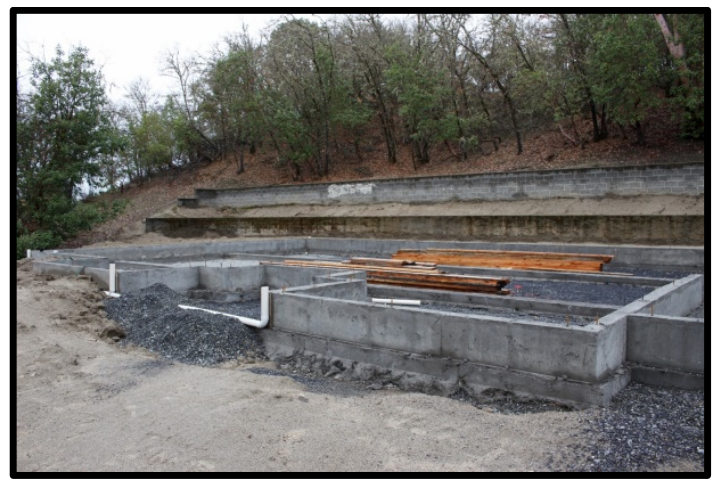

Figure 8b. New Construction

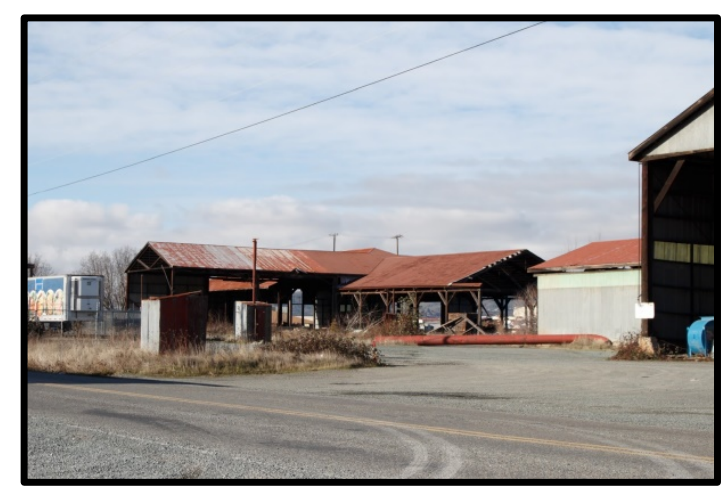

Figure 8d. General Contractor

\subsection{Public Water Supply Intake Risk}

To best protect source water, PWS are usually most concerned with PCS that are categorized as posing the highest risk to surface water, which is information found within the USWA. Table 4. lists some of the more common PCS types found within Grants Pass that pose 
the most concern in relation to surface water quality, and usually require immediate BMP implementation to protect source water.

Table 4. PCS Types in Grants Pass with a High SW Risk

\begin{tabular}{|c|l|c|}
\hline PCS Code & \multicolumn{1}{|c|}{ PCS Type } & $\begin{array}{c}\text { Surface Water } \\
\text { Risk }\end{array}$ \\
\hline C07 & Chemical/Petroleum Processing & $\mathrm{H}$ \\
\hline C14 & Junk/Scrap/Salvage Yards & $\mathrm{H}$ \\
\hline C15 & Machine Shops & $\mathrm{H}$ \\
\hline C20 & Parking Lots/Malls >50 Spaces & $\mathrm{H}$ \\
\hline C26 & Wood/Pulp/Paper Processing and Mills & $\mathrm{H}$ \\
\hline M06 & Auto - Gas Stations - Historic & $\mathrm{H}$ \\
\hline M32 & Construction/Demolition Area & $\mathrm{H}$ \\
\hline
\end{tabular}

\subsection{BMP Database}

\section{Description}

A BMP Database was created to assist DWPs and others with determining site-specific BMPs (Appendix G.). The BMP Database includes a variety of management strategies (e.g., proper fluids handling) and/or products (e.g., hydrodynamic separators) that can be implemented to protect drinking water quality. The BMP Database is organized in three tabs within an Excel spreadsheet:

1. BMP Inventory,

2. Assigned BMP, and

3. Master Lists.

- Within the BMP Inventory tab, the user will find designated BMP codes, names, descriptions, types, and sources for each BMP. The codes are only applicable within the BMP Database and do not have any secondary meaning. BMPs are categorized under the following types: erosion prevention, pollution prevention, runoff controls, and sediment controls. Sources, such as the Association of Clean Water Administrators (ACWA), are included to allow the user to investigate BMPs further. 
- The Assigned BMP tab includes PCS codes, "Activity at Site" descriptions, the surface water risk rating (low, L; moderate, M; high, $\mathrm{H}$ ), and designated BMP codes and names. Each PCS code and "Activity at Site" may have multiple BMP options. For example, Wells - Abandoned (M61) is associated with multiple potential BMPs to implement, such as: Equipment Maintenance (BMP34), Secondary Containment (BMP49), and Site Stabilization (BMP52). DWPs and other organizations may choose to implement just one of the BMPs or all three, depending on the costs linked to each of the BMPs.

- Lastly, the Master Lists tab includes all of the "Activity at Site" descriptions for PCS found within the City of Grants Pass, according to the SWA. Also, the BMP types are listed here to allow for a drop-down option under "Type" in the BMP Inventory tab.

\section{Application}

In order to use the BMP Database, DWPs and other organizations will need to have the

PCS codes for their locations of interest, or the USWA Excel file with PCS information

(Appendix H.) that was utilized for GIS mapping and analyses. This spreadsheet includes PCS information for several cities within Oregon. To determine the PCS codes associated with a particular location:

- Hold down Ctrl $+\mathrm{F}$ and enter the business name and/or address. Depending on the location, there may be multiple entries with the same information, but each entry may have a different PCS code.

- Record the relevant PCS codes, close the Excel spreadsheet, and open the BMP Database.

- Once the BMP Database is open, navigate to the Assigned BMP tab and either scroll down to find the PCS code associated with the location of interest, or hold down Ctrl + F to search for the PCS code. Depending on the PCS code, there may be several potential BMPs to implement at that particular location. Additional information for the BMPs can be found on the BMP Inventory tab. DWPs and other organizations may choose to investigate BMPs further by entering the BMP source in a search engine. 


\subsection{RESULTS}

\subsection{Applying the Risk Matrix to GIS}

The risk matrix components (stormwater basin, proximity to waterway, surface water risk, flood hazard, and jurisdiction) and scoring (Appendix C.) were analyzed in ArcMap to prioritize and target the highest priority PCS among the 214 total PCS recorded within the SWA for Grants Pass (Figure 5.). Detailed procedures for the application of the risk matrix and PCS information to GIS are provided in Appendix D. The attribute table from this initial narrowing down of the PCS was exported to an Excel spreadsheet and then examined for duplicate PCS, of which there were several. Duplicate entries from multiple SWAs (from 2005, 2008, 2013, 2015, 2016, 2017, and 2018) were combined and PCS known to no longer exist, based on information from Google Maps and local knowledge, were removed.

Further narrowing down of the PCS list using GIS analyses brought the total number of locations to survey to nearly 50 PCS. Within the final list of 50 PCS sites, 22 were categorized by PCS code and those that posed the highest risk for surface water include, but are not limited to, the following:

- C04 - Auto/Repair Shops (4/22 PCS, 18\%; gasoline, oil, coolant, and other automotive chemicals),

- C07 - Chemica1/Petroleum Processing/Storage (10/22 PCS, 45\%; variety of chemicals),

- C14 - Junk/Scrap/Salvage Yards (1/22 PCS, 5\%; gasoline, oil, coolant, and other automotive chemicals),

- C15 - Machine Shops (1/22 PCS, 5\%; variety of chemicals),

- C20 - Parking Lots/Malls $>50$ Spaces (1/22 PCS, 5\%; stormwater),

- C26 - Wood/Pulp/Paper Processing and Mills (2/22 PCS, 9\%; variety of chemicals), and

- M26 - Underground Storage Tank (UST) - Confirmed Leaking but listed NFA (3/22 PCS, $\sim 14 \%$; heating oil, gasoline, and other types of stored chemicals).

The final list included 39 high priority PCS, 22 of which were categorized by PCS code, to survey within Grants Pass (a subset of high priority PCS and scores are included in Table 5., 
Figure 5. shows the locations of the high priority PCS, and a complete list of the PCS surveyed is available in Appendix E.).

Table 5. Subset of High Priority PCS Sites Surveyed

\begin{tabular}{|c|c|c|c|c|c|c|c|}
\hline Common Name & Address & Basin & $\begin{array}{c}\text { Prox2Wate } \\
r\end{array}$ & $\begin{array}{l}\text { SW } \\
\text { Risk }\end{array}$ & $\begin{array}{c}\text { Flood } \\
\mathrm{Hz}\end{array}$ & Jurisd & Total \\
\hline $\begin{array}{l}\text { Clovercrest Service } \\
\text { Center }\end{array}$ & Cloverlawn Dr. and Crestview Loop & 3 & 3 & 1 & 0 & 2 & 9 \\
\hline $\begin{array}{l}\text { Construction - } \\
\text { Panoramic View } \\
\text { Estates }\end{array}$ & Panoramic Loop & 3 & 3 & 2 & 0 & 2 & 10 \\
\hline City of Grants Pass & 2202 SE HAVILAND DR & 3 & 3 & 2 & 0 & 2 & 10 \\
\hline $\begin{array}{l}\text { Willow Development } \\
\text { Consultants, LLC }\end{array}$ & 2221 HAVILAND DR & 3 & 2 & 2 & 0 & 2 & 9 \\
\hline $\begin{array}{l}\text { High Density Housing } \\
\text { - South of School }\end{array}$ & $\begin{array}{l}\text { SOUTH OF SCHOOL ON WEST SIDE OF } \\
\text { HAMILTON LANE }\end{array}$ & 3 & 3 & 1 & 0 & 2 & 9 \\
\hline Parkdale Subdivision & $\begin{array}{l}\text { AT SOUTH END OF PARKDALE/WELL } \\
\text { ON WEST SIDE OF ROAD }\end{array}$ & 3 & 3 & 1 & 0 & 2 & 9 \\
\hline \begin{tabular}{|l|} 
High Density Housing \\
- East of School
\end{tabular} & $\begin{array}{l}\text { EAST OF SCHOOL/EAST SIDE OF } \\
\text { HAMILTON LANE }\end{array}$ & 3 & 3 & 1 & 0 & 2 & 9 \\
\hline $\begin{array}{l}\text { Fruitdale Elementary } \\
\text { School }\end{array}$ & 1560 HAMILTON LANE & 3 & 3 & 2 & 0 & 2 & 10 \\
\hline Heating Oil Tank & 1560 HAMILTON LN & 3 & 3 & 0 & 1 & 2 & 9 \\
\hline
\end{tabular}

\subsection{Windshield Survey Findings}

After recording the inspection worksheet findings into the windshield survey database (a subset shown in Table 6. and the complete database in Appendix I.) and revisiting the survey data and photos, it was determined that 22 of the 39 high priority PCS within Grants Pass required follow-up in the form of BMP implementation, whether behavioral or structural. 
Table 6. Subset of Entries and Survey Responses Included in the Windshield Survey Database

\begin{tabular}{|l|l|l|l|l|}
\hline \multicolumn{1}{|c|}{ Facility Name } & BMPs & Functioning? & \multicolumn{1}{|c|}{ Stormwater Controls Observed } & \multicolumn{1}{c|}{ Photo \# } \\
\hline $\begin{array}{l}\text { Automania Car Sales } \\
\text { (Moved) }\end{array}$ & No & N/A & N/A & 4488 \\
\hline $\begin{array}{l}\text { Big Sam's Auto Repair } \\
\text { (Closed) }\end{array}$ & N/A & N/A & N/A & N/A \\
\hline Brakes Plus & No & N/A & $\begin{array}{l}\text { Catch Basin and Dumpster/Equipment } \\
\text { Cover }\end{array}$ & 4489,4490 \\
\hline Caveman RV & No & N/A & N/A & $4505,4506,4507$ \\
\hline $\begin{array}{l}\text { Clovercrest Service } \\
\text { Center (now NAPA Auto) }\end{array}$ & No & N/A & $\begin{array}{l}\text { Dumpster/Equipment Cover and } \\
\text { Drum/Container Containment }\end{array}$ & 4492,4493 \\
\hline $\begin{array}{l}\text { Fruitdale Elementary } \\
\text { School }\end{array}$ & & & $\begin{array}{l}\text { Oil/Water Separator, } \\
\text { Dumpster/Equipment Cover, and } \\
\text { Vegetated Buffer Zone }\end{array}$ & \\
\hline $\begin{array}{l}\text { Grants Pass Irrigation } \\
\text { District (GPID) Easement }\end{array}$ & No & Yes & N/A & Vegetated Buffer Zone \\
\hline Heating Oil Tank & N/A & N/A & N/A & 4491 \\
\hline
\end{tabular}

\subsection{Using the BMP Database}

Utilizing information from the USWA Excel file (Appendix H.), specifically PCS codes associated with "Activity at Site" descriptors, each PCS was assigned site-specific BMPs with the goal of protecting drinking water quality. For example, Brakes Plus (Figures 9a. and 9b.), an auto repair shop in the Fruitdale Creek stormwater basin, was assigned several BMPs. These BMPs are prescribed for PCS codes C04 (Auto - Repair Shops) and C07 (Chemical/Petroleum Processing/Storage) and include, but are not limited to, the following runoff controls and pollution prevention practices seen in Table 7. and Table 8. 
Table 7. BMPs Associated with PCS Code C04

\begin{tabular}{|l|l|l|l|l|}
\hline \multicolumn{1}{|c|}{ PCS } & \multicolumn{1}{c|}{ Activity at Site } & \multicolumn{1}{c|}{$\begin{array}{c}\text { SW } \\
\text { Risk }\end{array}$} & $\begin{array}{c}\text { BMP } \\
\text { Code }\end{array}$ & \multicolumn{1}{c|}{ BMP Name } \\
\hline C04 & Auto - Repair Shops & M & BMP30 & Spill and Leak Prevention \\
\hline C04 & Auto - Repair Shops & M & BMP31 & Spill Clean-Up \\
\hline C04 & Auto - Repair Shops & M & BMP32 & Proper Fluids Handling \\
\hline C04 & Auto - Repair Shops & M & BMP33 & Drain, Cover, and Contain Oily Parts Stored Outside \\
\hline C04 & Auto - Repair Shops & M & BMP34 & Equipment Maintenance \\
\hline C04 & Auto - Repair Shops & M & BMP36 & Housekeeping \\
\hline C04 & Auto - Repair Shops & M & BMP38 & Vehicle Owner Education \\
\hline C04 & Auto - Repair Shops & M & BMP39 & Proper Grease and Oil Disposal \\
\hline C04 & Auto - Repair Shops & M & BMP40 & Dumpster Maintenance \\
\hline C04 & Auto - Repair Shops & M & BMP49 & Secondary Containment \\
\hline C04 & Auto - Repair Shops & M & BMP51 & Materials Safety Data Sheets (MSDS) On-Hand \\
\hline C04 & Auto - Repair Shops & M & BMP13 & Emergency Spillway \\
\hline C04 & Auto - Repair Shops & M & BMP20 & Bio-Retention Practices \\
\hline C04 & Auto - Repair Shops & M & BMP22 & Hydrodynamic Separators \\
\hline C04 & Auto - Repair Shops & M & BMP23 & Catch Basin Media Inserts \\
\hline
\end{tabular}


Table 8. BMPs Associated with PCS Code C07

\begin{tabular}{|c|c|c|c|c|}
\hline $\begin{array}{l}\text { PCS } \\
\text { Code }\end{array}$ & Activity at Site & $\begin{array}{l}\text { SW } \\
\text { Risk }\end{array}$ & $\begin{array}{l}\text { BMP } \\
\text { Code }\end{array}$ & BMP Name \\
\hline $\mathrm{CO7}$ & \begin{tabular}{|l|} 
Chemical/Petroleum \\
Processing/Storage
\end{tabular} & $\mathrm{H}$ & BMP12 & Stockpile and Materials Management \\
\hline $\mathrm{CO}$ & \begin{tabular}{|l|} 
Chemical/Petroleum \\
Processing/Storage
\end{tabular} & $\mathrm{H}$ & BMP13 & Emergency Spillway \\
\hline $\mathrm{CO7}$ & $\begin{array}{l}\text { Chemical/Petroleum } \\
\text { Processing/Storage }\end{array}$ & $\mathrm{H}$ & BMP22 & Hydrodynamic Separators \\
\hline $\mathrm{CO7}$ & \begin{tabular}{|l|} 
Chemical/Petroleum \\
Processing/Storage
\end{tabular} & $\mathrm{H}$ & BMP23 & Catch Basin Media Inserts \\
\hline $\mathrm{CO}$ & $\begin{array}{l}\text { Chemical/Petroleum } \\
\text { Processing/Storage }\end{array}$ & $\mathrm{H}$ & BMP30 & Spill and Leak Prevention \\
\hline $\mathrm{CO7}$ & $\begin{array}{l}\text { Chemical/Petroleum } \\
\text { Processing/Storage }\end{array}$ & $\mathrm{H}$ & BMP31 & Spill Clean-Up \\
\hline $\mathrm{CO}$ & \begin{tabular}{|l|} 
Chemical/Petroleum \\
Processing/Storage
\end{tabular} & $\mathrm{H}$ & BMP32 & Proper Fluids Handling \\
\hline $\mathrm{CO}$ & $\begin{array}{l}\text { Chemical/Petroleum } \\
\text { Processing/Storage }\end{array}$ & $\mathrm{H}$ & BMP34 & Equipment Maintenance \\
\hline $\mathrm{C} 07$ & $\begin{array}{l}\text { Chemical/Petroleum } \\
\text { Processing/Storage }\end{array}$ & $\mathrm{H}$ & BMP36 & Housekeeping \\
\hline $\mathrm{CO7}$ & \begin{tabular}{|l} 
Chemical/Petroleum \\
Processing/Storage
\end{tabular} & $\mathrm{H}$ & BMP37 & Proper Wash Water Disposal \\
\hline $\mathrm{CO}$ & \begin{tabular}{|l|} 
Chemical/Petroleum \\
Processing/Storage
\end{tabular} & $\mathrm{H}$ & BMP40 & Dumpster Maintenance \\
\hline $\mathrm{CO}$ & \begin{tabular}{|l|} 
Chemical/Petroleum \\
Processing/Storage
\end{tabular} & $\mathrm{H}$ & BMP41 & Minimize Chlorinated Cleaners and Solvents \\
\hline $\mathrm{CO7}$ & \begin{tabular}{|l|} 
Chemical/Petroleum \\
Processing/Storage
\end{tabular} & $\mathrm{H}$ & BMP49 & Secondary Containment \\
\hline $\mathrm{CO}$ & $\begin{array}{l}\text { Chemical/Petroleum } \\
\text { Processing/Storage }\end{array}$ & $\mathrm{H}$ & BMP50 & Proper Hazardous Waste Disposal \\
\hline $\mathrm{CO}$ & $\begin{array}{l}\text { Chemical/Petroleum } \\
\text { Processing/Storage }\end{array}$ & $\mathrm{H}$ & BMP51 & Materials Safety Data Sheets (MSDS) On-Hand \\
\hline
\end{tabular}

These BMPs were assigned based on observations of an overabundance of materials onsite (specifically tires), oil sheens from leaking vehicles and/or storage containers, and an overfilled dumpster with trash overflowing onto the site. In addition to Brakes Plus, the 
remainder of the 21 high priority PCS sites were also assigned site-specific BMPs, and these BMP recommendations were forwarded to the City of Grants Pass' Public Works Department.
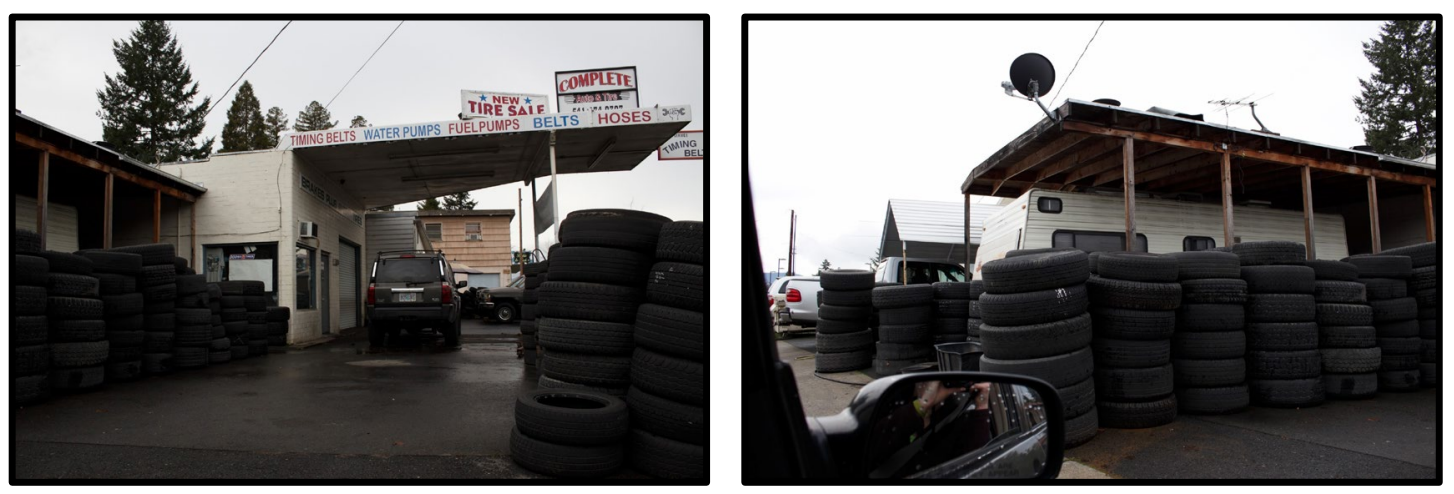

Figures 9a. and 9b. Brakes Plus

\subsection{Communication of Results to the City of Grants Pass}

Results of the project were shared with the City of Grants Pass. The risk matrix, the inspection worksheet, the results of the surveys, methodology for the surveys, and two maps showing both the elements of the risk matrix and the high priority PCS in Grants Pass were shared. These results were presented at an RDWP meeting. Lastly, the BMP Database and "howto" guide were shared with the City of Grants Pass. Overall, as a result of these deliverables, the impacts of chemicals and other pollutants on drinking water quality can be addressed and limited, resulting in the initial SWP efforts that the City of Grants Pass had hoped to achieve. 


\subsection{DISCUSSION}

In an ideal situation, all 22 of the high priority PCS sites would be addressed by followup surveys and, potentially, discussions between business and property owners and the City of Grants Pass. However, considering the amount of time required and the expense of visiting these 22 PCS sites, in addition to daily departmental tasks, it is likely that Public Works employees reviewed the windshield survey database (Appendix I.) and narrowed down the list of sites to include only those that they felt most concerned with and needed to follow up on. Further, Public Works is also well-aware of many of these PCS sites, some of which have a history of causing concern related to source water. Having these 22 PCS sites flagged is the first step in long-term monitoring to ensure that source water for residents within Grants Pass can be protected.

As expected, these 22 high priority sites house chemicals on-site, both known and unknown, provide storage of items with the potential of leakage, such as USTs, and can contribute large quantities of stormwater runoff during high rain events. Understanding where these PCS are located in relation to the drinking water intake, surveying the sites, and determining site-specific BMPs to protect the drinking water were all tasks accomplished within Grants Pass. As with EWEB's environmental risk assessment of the McKenzie Watershed and actions to take to protect that drinking water source, the City of Grants Pass now has a solid foundation for protecting the drinking water source for its residents. The momentum initiated by EWEB and continued by the City of Grants Pass has resulted in further work within the Rogue Basin.

While this process for identifying and responding to high priority PCS is replicable, there are limitations that may exclude DWPs, municipalities, and conservation organizations from using it. For example, in order to apply the risk matrix to GIS, the user will need to have access 
to and knowledge of ArcMap or a similar GIS program. Unfortunately, licenses for ArcGIS begin at $\$ 800 /$ year for the Desktop Basic program, and although many organizations can afford that expense, some smaller, private DWPs may not be able to justify the cost. Not only can the cost be a barrier, but the DWP will also need to know how to use the many tools within GIS programming. With online resources, training, courses, and peer-to-peer instruction, these programs can be learned. However, in order to have an in-depth understanding of the programs, much time will need to be allocated to this learning.

In addition to time allocated for learning GIS programs, depending on the size of the DWPA, a large amount of time may be required to complete the windshield surveys. This time includes compiling the list of PCS sites, placing them in geographical order, visiting each PCS and completing an inspection worksheet, and recording the survey data into the windshield survey database. For DWPs with a small staff, this amount of time may impact their routine tasks that need to be accomplished, and these DWPs may instead choose to contract out this type of work.

Lastly, although the current BMP Database includes over 50 recommended BMPs categorized as erosion prevention, pollution prevention, runoff controls, and/or sediment controls, there are likely many more BMPs that have not yet been identified and listed within the database. In the following section, resolutions to these limitations that were implemented within the Rogue Basin will be discussed, as well as other future plans.

While not all of the above limitations can be easily resolved, the incompleteness of the current BMP Database can be addressed through an adaptation into an open-source document. By simply moving away from a traditional Excel spreadsheet to a format as simple as a Google Sheets document shared between DWPs, municipalities, and conservation organizations, the 
BMP Database can be added to, edited, and shared with others as a living document, or one that is constantly being updated and improved upon.

Upon finding the December 2018 pilot project within the City of Grants Pass to be successful, the RDWP felt that applying this process within other Rogue Basin communities would be significantly helpful in identifying high priority PCS. Beginning in the summer of 2019, the following communities were surveyed:

- Shady Cove, Anglers Cove/Hiland Water Company/Shady Cove Heights Water Company - July 26th, 2019,

- City of Rogue River - August 1st, 2019,

- City of Gold Hill - August 7th, 2019,

- Trail, Country View Mobile Home Estates - August 15th, 2019,

- City of Cave Junction - August 21st, 2019,

- Eagle Point, MWC - January 14th and 15th, 2020, and

- White City, MWC - late February to late March, 2020.

Further, to simplify the survey process, the hard-copy inspection worksheet was scrapped and instead modified for the ArcGIS Survey 1-2-3 application. Rather than completing these surveys with paper and pencil, the Survey 1-2-3 application allowed for real-time data to be entered into an online form using a smartphone. Once the data is uploaded, it is compiled into an online database that shows the results for each question or prompt from all of the surveys. Utilizing this tool eliminated the need to scan physical paper copy inspection worksheets and record the data into the inspection worksheet database, which saves a great amount of time. Additionally, each survey entry is georeferenced and shows up as a point in an ArcGIS data layer. Overall, moving from paper copies to the Survey 1-2-3 application proved to save time and add a new technological element to the project.

Not only have many high priority PCS been identified within many of the Rogue Basin communities using a more technologically-advanced process, but other opportunities have surfaced from this initial work. Currently, reporting for a National Water Quality Initiative 
(NWQI) program funded by the Natural Resources Conservation Service (NRCS) targeting Upper and Middle Rogue subwatersheds is underway. This NWQI reporting will provide the NRCS and other conservation organizations a basis for approaching private, agricultural landowners with solutions and, potentially, funding for implementing BMPs to protect water quality.

Other projects that are underway include a spill response planning effort, a multijurisdictional post-fire monitoring project, and one that has grown from the initial PCS survey work completed within Eagle Point and White City, a Source Water Patrol project by RVCOG and funded by the MWC. Full details for this project are still being determined, but the overall goals are to conduct visual surveys of high priority PCS within the White City industrial area. Upon finding seemingly undesirable water quality conditions adjacent to these PCS, MWC has requested the use of Survey 1-2-3 to record and data, and if deemed necessary, collect water samples for chemical analyses.

In the following section, drinking water quality studies as a surrogate for stormwater quality and other environmental studies, as well as recommendations for continued work within the Rogue Basin, will be discussed. 


\subsection{CONCLUSIONS AND RECOMMENDATIONS}

As the drinking water source for residents of Grants Pass and other communities downstream, the Rogue River requires heightened attention in regard to PCS. Through the guidance of the RDWP, the risk matrix, windshield survey, and BMP recommendation process will likely continue to evolve and be applied within additional communities, perhaps even outside of the Rogue Basin, to protect drinking water.

However, to make the best use of these processes, I will share a few recommendations:

- Keep the risk matrix as concise as possible and do not include risk components that will add too much complexity during the transition to ArcGIS. For example, most of the risk matrices following the pilot project only included these risk components: surface water risk, proximity to waterway, flood hazard, and jurisdiction. The only risk matrix that included additional factors was the one created for White City, and these additions were specifically requested by the MWC,

- If the DWPs, municipalities, and conservation organizations are able to obtain an Environmental Systems Research Institute (ESRI) license, utilizing ArcGIS Online and Survey 1-2-3 will speed up the windshield survey process,

- Examine the BMP Database and add information that is currently missing, and

- Document the process, record changes, and share outcomes with others. 


\subsection{REFERENCES}

BestPlaces (2021). Climate in Grants Pass, Oregon. https://www.bestplaces.net/climate/city/oregon/grants_pass

City of Grants Pass: GIS Division (2020). Gallery for City of Grants Pass (AGOL). https://grantspassoregon.maps.arcgis.com/home/gallery.html?view=grid\&sortOrder=desc $\underline{\text { \&sortField }=\text { numviews }}$

City of Grants Pass: Public Works - Streets and Drainage Division (2020). City of Grants Pass Stormwater Utility. https://www.grantspassoregon.gov/1188/Stormwater-Utility

City of Grants Pass: Public Works - Streets and Drainage Division (2020). Grants Pass Stormwater Utility. https://www.grantspassoregon.gov/DocumentCenter/View/14509/ $\underline{\text { Stormwater-Utility-Brochure }}$

City of Grants Pass: Public Works - Water Treatment (2021).

Water Treatment Plant History. https://www.grantspassoregon.gov/444/Water-Treatment-Plant-History

Clackamas River Water Providers (2021). About Us. https://www.clackamasproviders.org/about-us/

Clackamas River Water Providers (2021). Drinking Water Protection Plan. https://www.clackamasproviders.org/drinking-water-protection-plan/

Clackamas River Water Providers (2021). What Can You Do To Prevent Water Pollution? https://www.clackamasproviders.org/wpcontent/uploads/2014/09/what_can_you_do to prevent_water_pollution.doc

EOS (2020). Wildfires Are Threatening Municipal Water Supplies. https://eos.org/science-updates/wildfires-are-threatening-municipal-water-supplies

Eugene Water \& Electric Board (2000). Drinking Water Protection Plan: Environmental Setting \& Risk Assessment. https://www.deq.state.or.us/wq/dwp/docs/swareports/00287EugeneSWA.pdf

Eugene Water \& Electric Board (2021). Pure Water Partners. http://www.eweb.org/community-and-environment/mckenzie-watershedprotection/landowner-assistance-programs/pure-water-partners

Eugene Water \& Electric Board (2021). State of the McKenzie Watershed Report. http://www.eweb.org/community-and-environment/mckenzie-watershedprotection/drinking-water-source-protection-plan 
National Public Radio (2016). Lead-Laced Water In Flint: A Step-By-Step Look At The Makings Of A Crisis.

https://www.npr.org/sections/thetwo-way/2016/04/20/465545378/lead-laced-water-inflint-a-step-by-step-look-at-the-makings-of-a-crisis

Natural Resources Defense Council (2018). Flint Water Crisis: Everything You Need to Know. https://www.nrdc.org/stories/flint-water-crisis-everything-you-need-know\#sec-summary

Natural Resources Defense Council (2019). The Broken Safe Drinking Water Act Won't Fix the PFAS Crisis. https://www.nrdc.org/experts/erik-d-olson/broken-safe-drinking-water-act$\underline{\text { wont-fix-pfas-crisis }}$

Natural Resources Defense Council (2019). What Can We Do to Fix the Drinking Water Problem in America?

https://www.nrdc.org/stories/what-can-we-do-fix-drinking-water-problem-america

Oregon Department of Environmental Quality (2018). Updated Source Water Assessment - City of Grants Pass. https://www.deq.state.or.us/wq/dwp/docs/uswareports/USWA_00342GrantsPass.pdf

Yachats, Oregon (2021). Drinking Water Protection Plan. https://www.yachatsoregon.org/372/Drinking-Water-Protection-Plan 
7.0 APPENDICES 
APPENDIX A. Updated Source Water Assessment: City of Grants Pass 


\section{Updated \\ Source Water Assessment}

\section{City of Grants Pass}

PWS \#4100342

March 2018

Prepared for:

City of Grants Pass

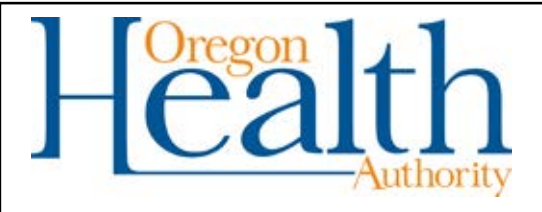

Prepared by:

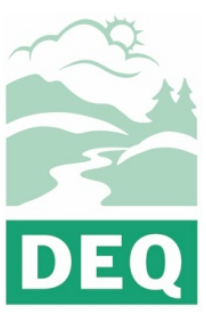

State of Oregon Department of Environmental Quality 


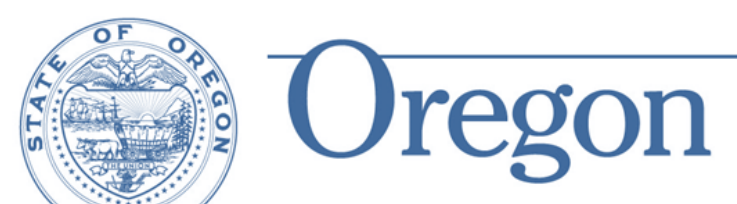

Kate Brown, Governor
Department of Environmental Quality

Agency Headquarters

700 NE Multnomah Street, Suite 600

Portland, OR 97232

(503) 229-5696

FAX (503) 229-6124

TTY 711

March 20, 2018

City of Grants Pass

Jason Canady, Public Works Director

Craig Kuhnert, Water Treatment Plant Supervisor

101 NW A Street

Grants Pass, OR 97526

\section{Re: Updated Source Water Assessment for PWS \# 4100342}

Dear Mr. Canady and Mr. Kuhnert,

On behalf of the Oregon Health Authority (OHA), the Oregon Department of Environmental Quality (DEQ) is pleased to provide your community with important information in this Updated Source Water

Assessment. The updated assessment is intended to provide information and resources to assist you and your community to implement local drinking water protection efforts. Since the first source water assessments were completed in 2005 , state agencies have significantly expanded analytical capabilities, including more detailed data for analyzing natural characteristics and potential pollutant sources. DEQ is currently completing the updated assessments for surface water systems and $\mathrm{OHA}$ is updating the groundwater system assessments.

As you know, assuring safe drinking water depends on public water suppliers implementing multiple successful practices. First, protect the drinking water source. Second, practice effective water treatment. Third, conduct regular monitoring for contaminants to assure safety. Fourth, protect the distribution system piping and finished water storage from recontamination. Finally, practice competent water system operation, maintenance, and construction. These practices are collectively called "multiple barrier public health protection". Source water protection is an important first step because starting with the best possible quality source water helps assure that water treatment can be effective at all times.

Source water protection is accomplished by effective state public health programs, environmental protection, land use policies, pro-active land stewardship, and by implementation of local drinking water protection efforts. The susceptibility of the public drinking water system source depends on both the natural conditions in the watershed as well as the anthropogenic activities in the watershed.

This letter, with attached figures and technical information, constitutes your Updated Source Water Assessment. It supplements your original Source Water Assessment (link here:

http://www.deq.state.or.us/wq/dwp/swrpts.asp ). One of the most important assets a public water system can have is accurate source water area mapping and visual resources to share with the community citizens and officials. The figures include a new regional map view of your watershed, topographic or aerial photograph basemap with the source area delineated, and maps with natural characteristics, anthropogenic land uses, potential sources of pollutants, 8-hour time-of-travel zone for the surface water intake, and historic landslides. Information on anthropogenic land uses in a drinking 
water source area is important for evaluating potential pollutant sources and working with stakeholders upstream. Tables are provided that include a summary of the types of potential pollutant sources present in your drinking water source area.

There are also a variety of resources included in this document to assist you with drinking water source protection efforts. Appendix \#1 provides a summary of how to use the information provided in the assessment to move forward to develop and implement source water protection. Appendix \#3 lists websites and resources available to public water systems and community members seeking technical assistance for work on watershed protection. Appendix \#4 provides brief descriptions and contact information for grants and loans to fund both drinking water infrastructure and source protection projects. Appendix \#5 contains details on potential sources of pollution identified in readily accessible state and federal databases and GIS layers.

For watersheds with more than one intake such as the Rogue Subbasin, all protection areas for intakes upstream of the water system's intake are included in their drinking water source area. Activities and impacts in upstream drinking water protection area also have the potential to impact downstream water users. We encourage public water suppliers to work with other water providers within the vicinity or subbasin as they develop protection strategies. State agency resources are also available to help you with mapping and information needs. Larger sizes of the source area maps and more details of landslide potential and other natural characteristics are available for you upon request (contact Julie Harvey at 503-229-5664). A Surface Water Resource Guide with more extensive information to assist public water systems in protecting their source waters is available at http://www.oregon.gov/deq/wq/programs/Pages/dwp.aspx. Please check it out and let us know if would like assistance with implementation.

For direct assistance and/or additional information regarding watershed protection, call Sheree Stewart at DEQ (503-229-5413). For more information on drinking water policies and procedures, call Casey Lyon at OHA (541-726-2587).

Sincerely,

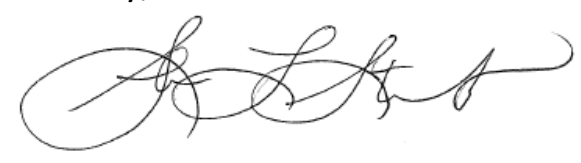

Sheree Stewart, Drinking Water Protection Coordinator Environmental Solutions Division

Cc: Casey Lyon, Technical Services Manager, Oregon Health Authority 


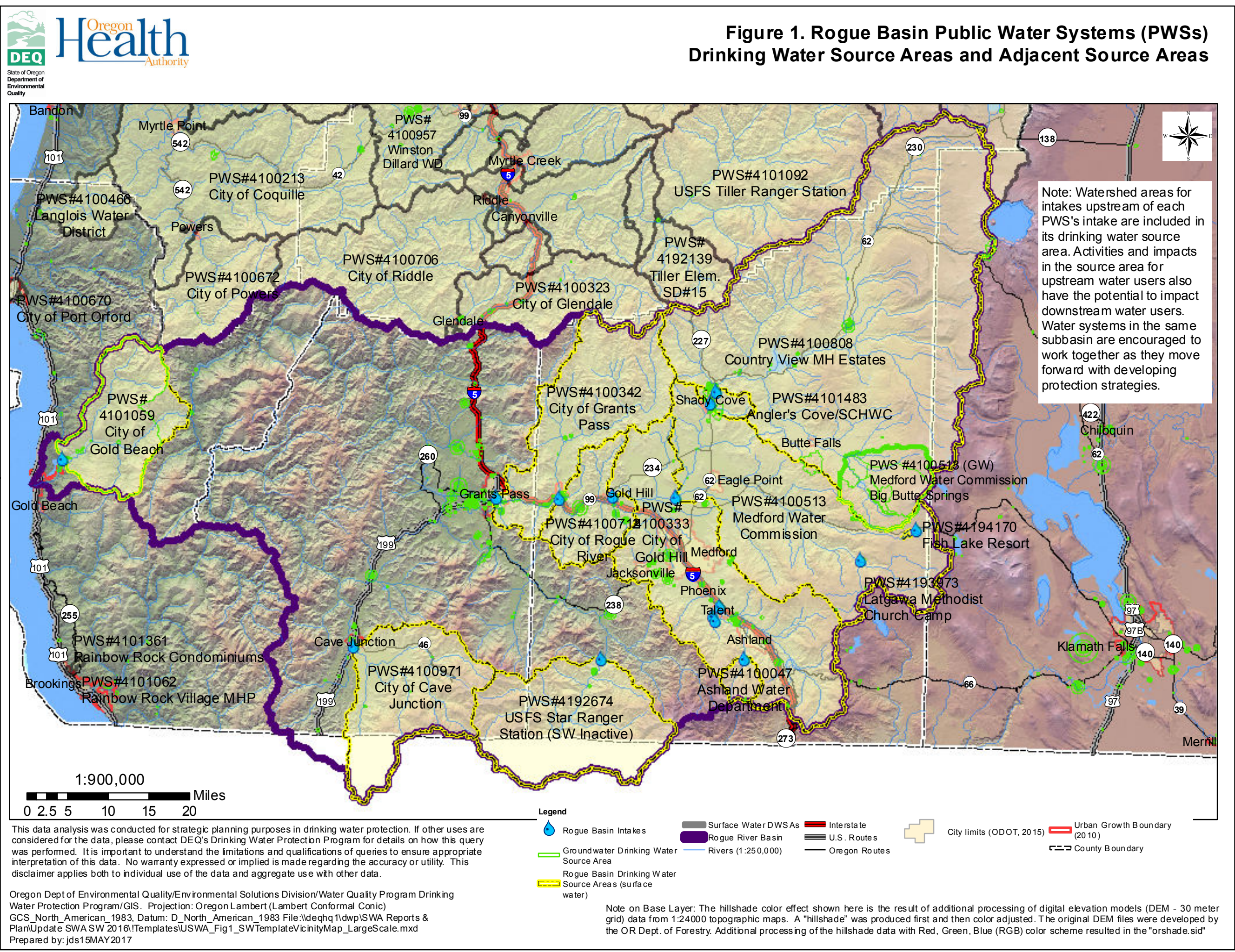


Figure 2a. City of Grants Pass (PWS 00342) Drinking Water Source Areas with Erosion Potential for Management Activities with Soil Surface Disturbance

(See Appendix 2 for Key to map details and metadata)

Legend

(.) City of Grants Pass Intake

- Surface Water Intake

$\checkmark$ City of Grants Pass: 8hr Time of Travel to intake (11.8mi Buffer)

- - 3 City of Grants Pass drinking water source area (including upstream areas)

Nearby drinking water source areas

Streams near soils with significant erosion potential. Erosion control measures

(BMPs) may be necessary for land management activites that disturb or leave bare soils in these areas.

Streams \& Lakes (NHD) with significant erosion potential from intensive $(>75 \%)$ soil surface disturbance (i.e.tilled or bare soils) (NRCS-RUSLE2/ODA-EVI; see Appendix 2 Note 4a)

Streams \& Lakes (NHD) with significant erosion potential from substantial (50-75\%) soil surface disturbance (NRCS off-road/off-trail ratings; see Appendix 2, Note 4b).

Streams (NHD) with significant erosion potential (slope $>30 \%$ using USFS SRI data, NRCS SSURGO data not avaliable; see Appendix 2 Note 4c).

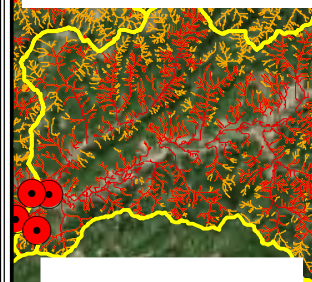

See Figure $2 b$.

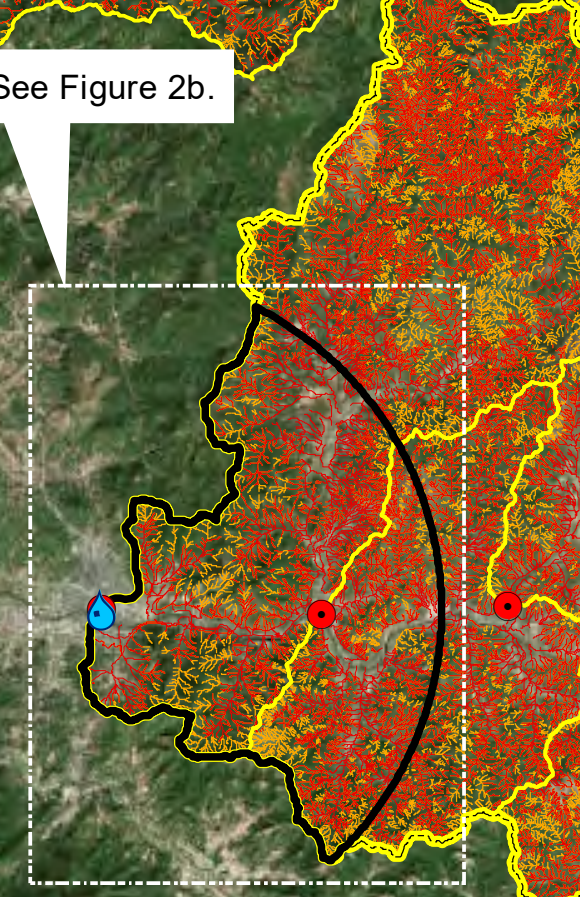

For this assessment, DEQ used three methods for evaluating soil erosion potential depending on the overall slope of the land surface, extent of soil disturbance and data availability. Streams and lakes/reservoirs that have moderate to very severe soil erosion hazard potential within 300 feet of surface water are mapped to provide an estimate of areas where land management activities may impact streams. Erosion control measures (BMPs) may be necessary in these areas. Maps and data of soil qualities without the 300 -foot stream buffer in local areas can be provided to public water systems and communities if additional detail or scale is needed for place based planning. See Appendix 2, Note 4 for additional information.

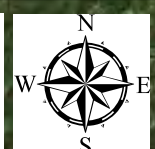


- City of Grants Pass Intake

- Surface Water Intake

$\boldsymbol{}$ City of Grants Pass: 8hr Time of Travel to intake (11.8mi Buffer)

$[-3$ City of Grants Pass drinking water source area (including upstream areas)

Nearby drinking water source areas

Streams near soils with significant erosion potential. Erosion control measures (BMPs) may be necessary for land management activites that disturb or leave bare soils in these areas.

Streams \& Lakes (NHD) with significant erosion potential from intensive $(>75 \%)$ soil surface disturbance (i.e.tilled or bare soils) (NRCS-RUSLE2/ODA-EVI; see Appendix 2 Note 4a).

Streams \& Lakes (NHD) with significant erosion potential from substantial (50$75 \%$ ) soil surface disturbance (NRCS offroad/off-trail ratings; see Appendix 2, Note $4 b)$.

Streams (NHD) with significant erosion potential (slope $>30 \%$ using USFS SRI data, NRCS SSURGO data not avaliable; see Appendix 2 Note 4c).

For this assessment, DEQ used three methods for evaluating soil erosion potential depending on the overall slope of the land surface, extent of soil disturbance and data availability. Streams and lakes/reservoirs that have moderate to very severe soil erosion hazard potential within 300 feet of surface water are mapped to provide an estimate of areas where land management activities may impact streams. Erosion control measures (BMPs) may be necessary in these areas. Maps and data of soil qualities without the $300-$ foot stream buffer in local areas can be provided to public water systems and communities if additional detail or scale is needed for place based planning. See Appendix 2, Note 4 for additional information.

The 8-hour time of travel area is provided as a planning tool for spills or releases at crossings or discharge points to the stream. Focus may need to extend further upstream for contaminants that are contributed to the stream over long time periods or recur frequently. See

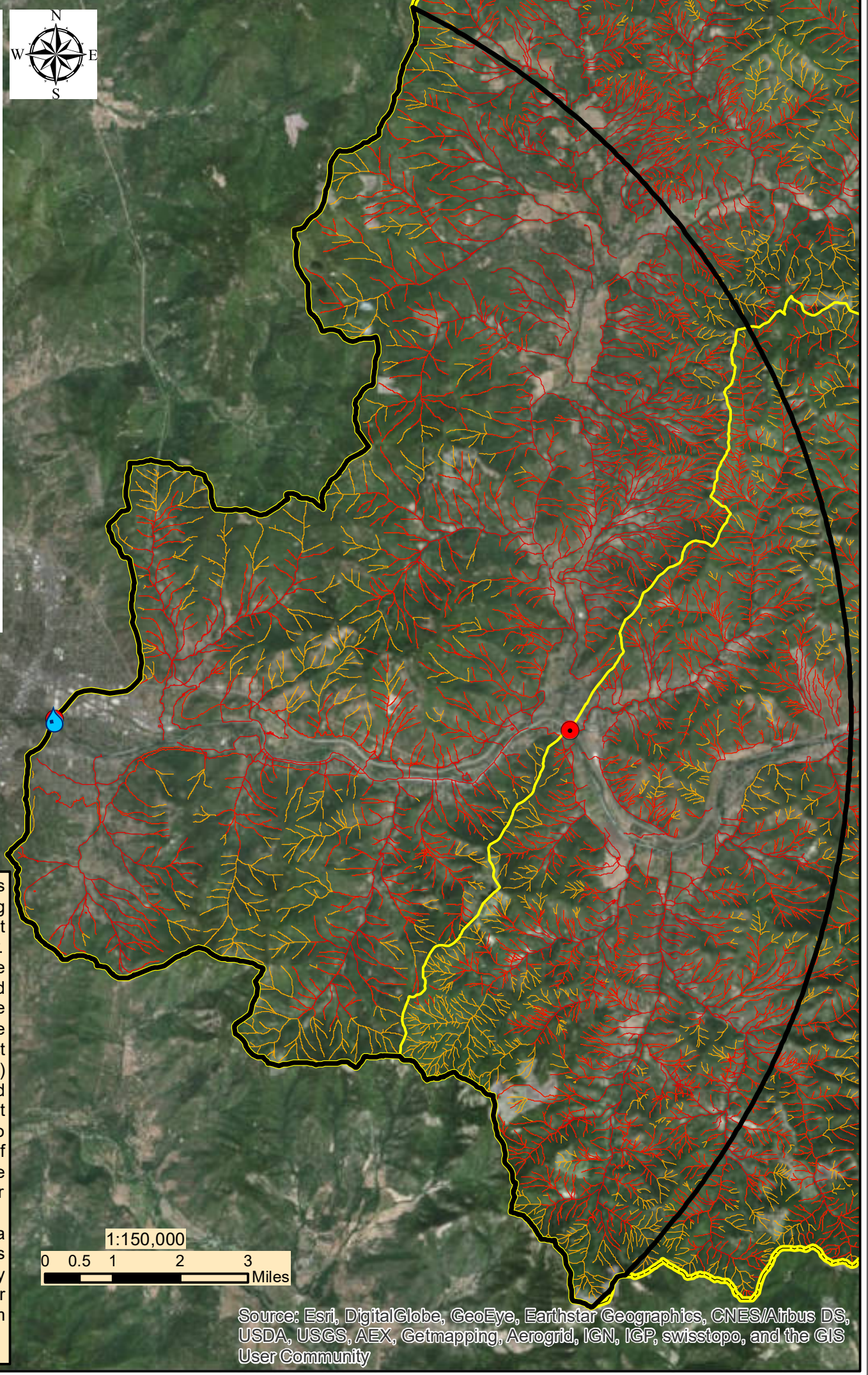


Figure 3a. City of Grants Pass (PWS \#00342) DEa

\section{Legend}

City of Grants Pass Intake

- Surface Water Intake

$\boldsymbol{C}$ City of Grants Pass: 8hr Time of City of Grants Pass drinking water 3 source area (including upstream areas)

Nearby drinking water source areas

$\sim$ Streams

Landslide Deposits (non-rock material, includes earth and debris slides, flows, slumps, falls and complex) (DOGAMI SLIDO3.2)

Scarp Flanks (DOGAMI SLIDO-3.2) Scarps (DOGAMI SLIDO-3.2)
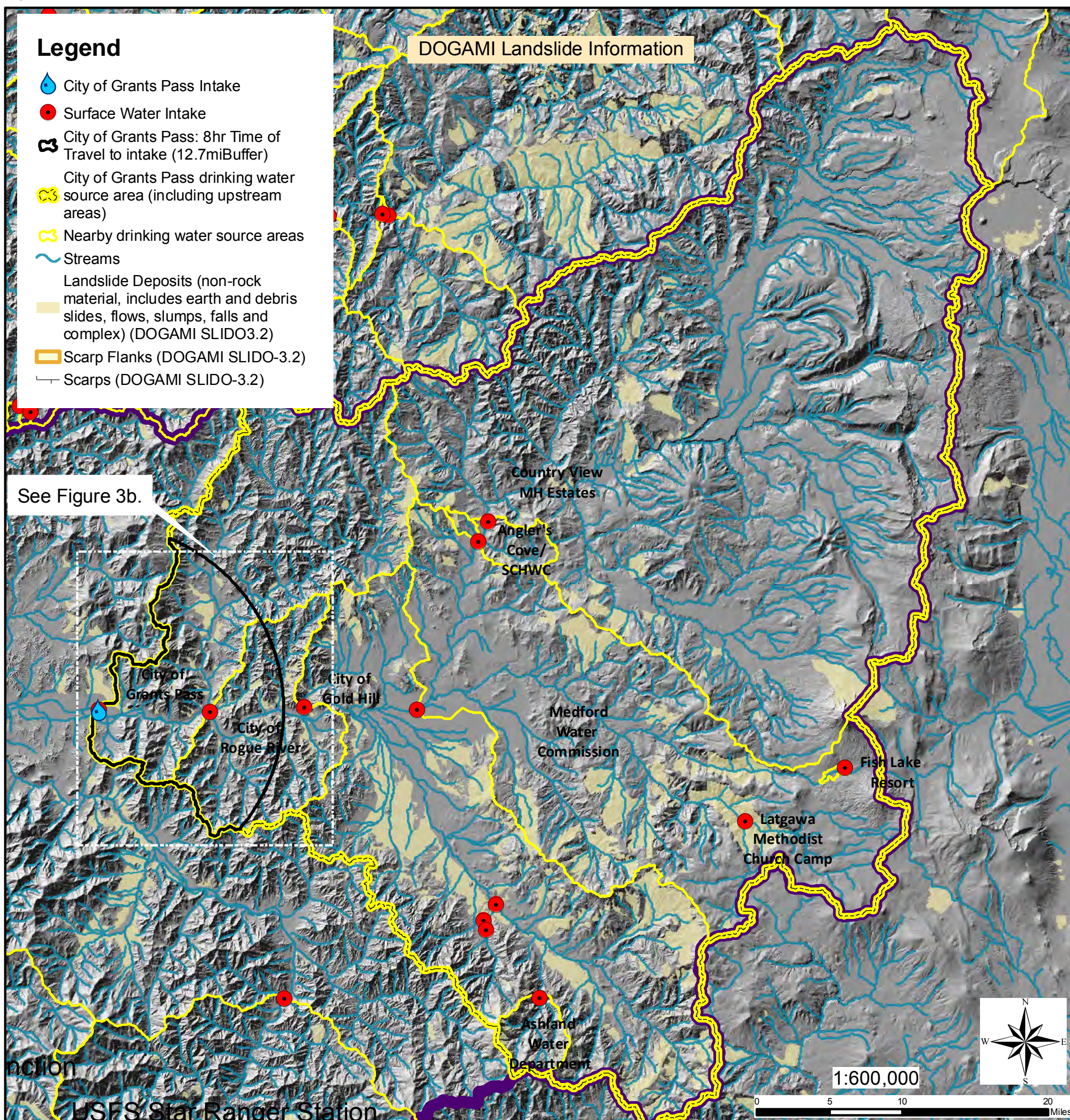

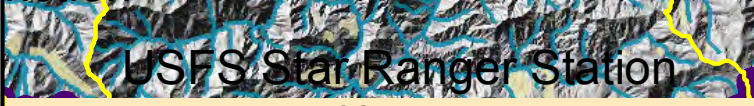

The data set is published by DOGAMI to improve the understanding of landslide hazards in Oregon and to provide a statewide base level of landslide data. This product is for
informational purposes and may not have been prepared for or be suitable for legal, engineering, or surveying purposes. Users of this information should review or consult the primary data and information sources to ascertain the usability of the information. This publication cannot substitute for site-specific investigations by qualified practitioners. Sitespecific data may give results that differ from the results shown in the publication. For more information see: http://www.oregongeology.org/sub/slido/ OR DEQ's Water Quality Program is currently working with DOGAMI to develop and provide a more detailed landslide potential analysis for public water systems. Contact Oregon DEQ's Environmental Solutions Division/Water Quality Program for further information on the analysis. If data is available for the specific area, DEQ will provide the more detailed landslide analysis to the PWS. 


\section{DED $H$ ealth}

Figure 3b. City of Grants Pass (PWS 00342)

8-hour Time-of-Travel Landslide Hazards Map (See Appendix 2 for Key to map details and metadata)

Legend

- City of Grants Pass Intake

- Surface Water Intake

$\boldsymbol{\Xi}$ City of Grants Pass: 8hr Time of

City of Grants Pass drinking

water source area (including

Nearby drinking water source

areas

Landslide Deposits (non-rock

material, includes earth and

debris slides, flows, slum
and complex) (DOGAMI

SLIDO3.2)

Sca

Scarps (DOGAMI SLIDO-3.2)
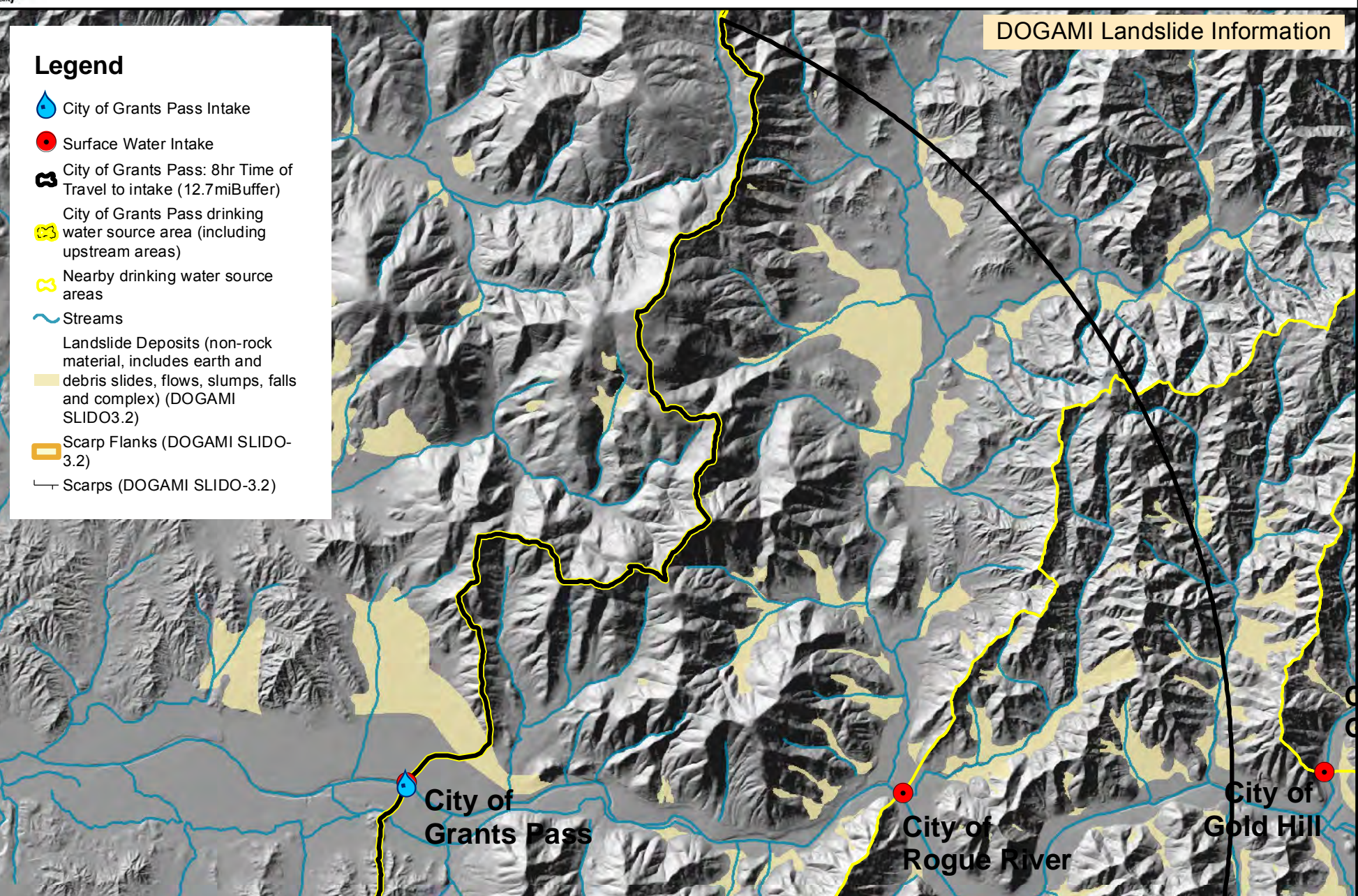

mis

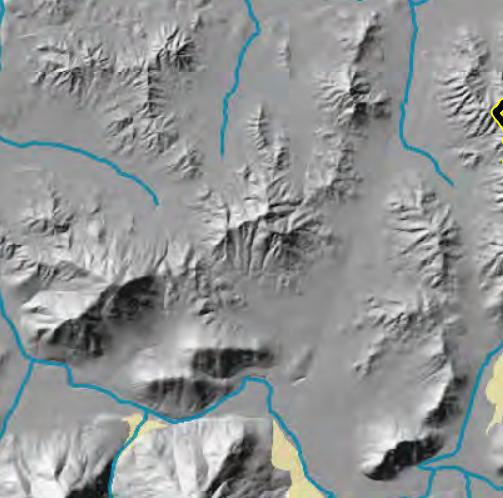

(2)
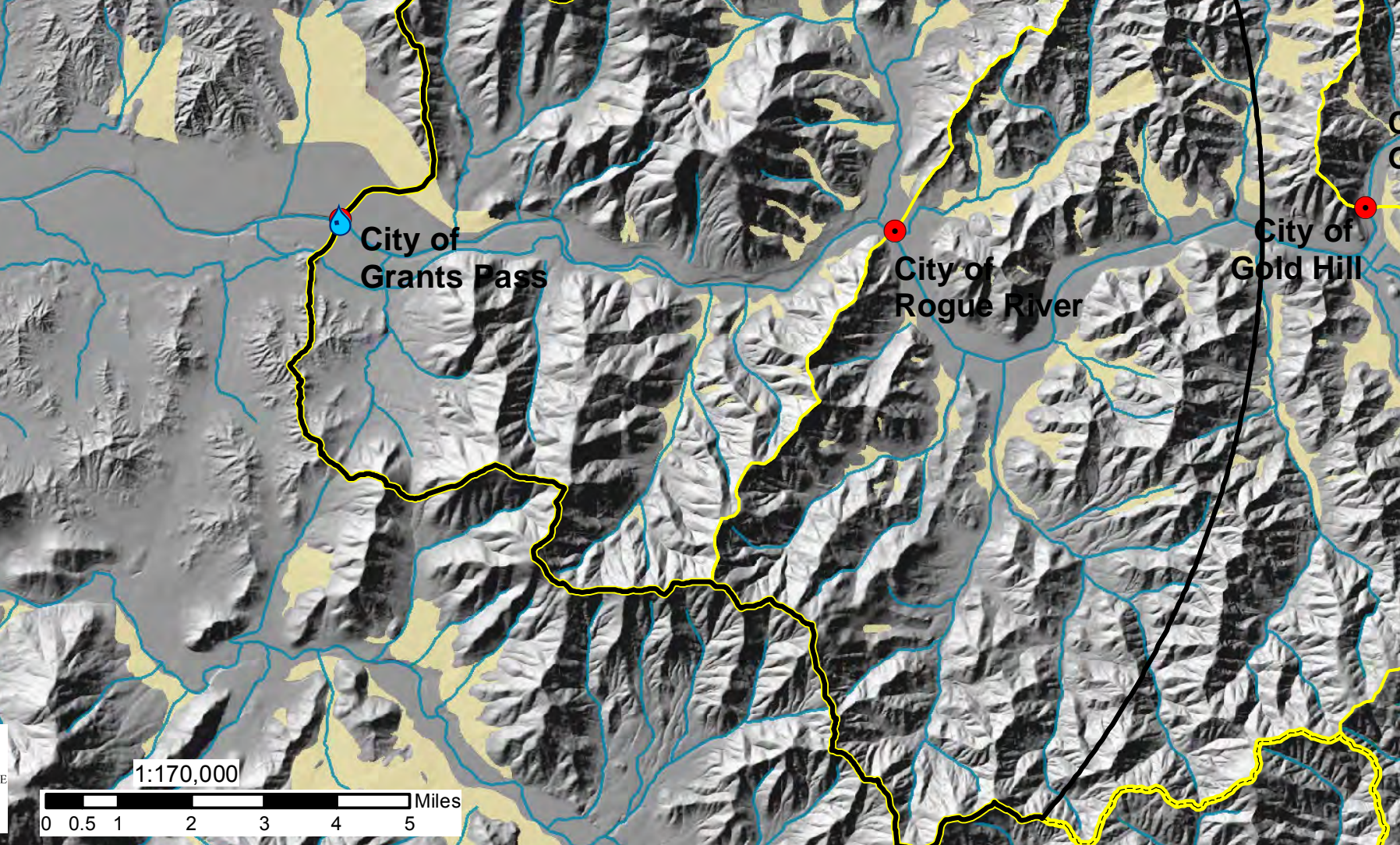

Gpta bil
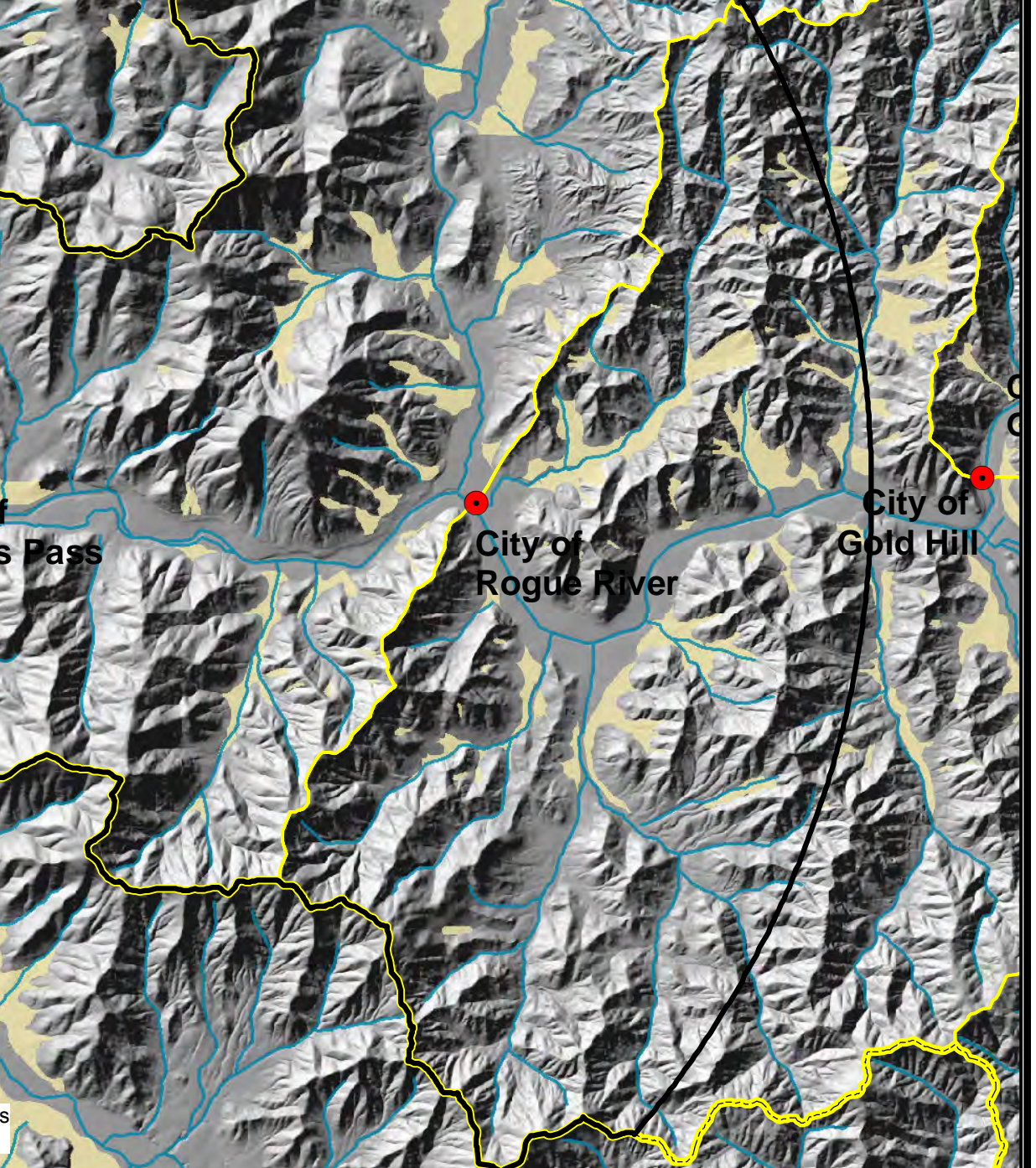

m.10

The data set is published by DOGAMI to improve the understanding of landslide hazards in Oregon and to provide a statewide base level of landslide data. This product is for informational purposes and may not have been prepared for or be suitable for legal, engineering, or surveying purposes. Users
this information should review or consult the primary data and information sources to ascertain the usability of the information. This publication cannot substitute for site-specific investigations by qualified practitioners. Site-specific data may give results that differ from the results shown in the publication. For more information see: http://www.oregongeology.org/sub/slido/ OR DEQ's Water Quality Program is currently working with DOGAMI to develop and provide a more detailed landslide potential analysis for public water systems. Contact Oregon DEQ's Environmental Solutions Division/Water Quality Program for further information on the analysis. If data is available for the specific area, DEQ will provide the more detailed landslide analysis to the PWS. The 8-hour time of travel area is provided as a planning tool for spills or releases at crossings or discharge points to the stream. Focus may need to 
Figure 4a. City of Grants Pass (PWS 00342) DEQ ] Calth

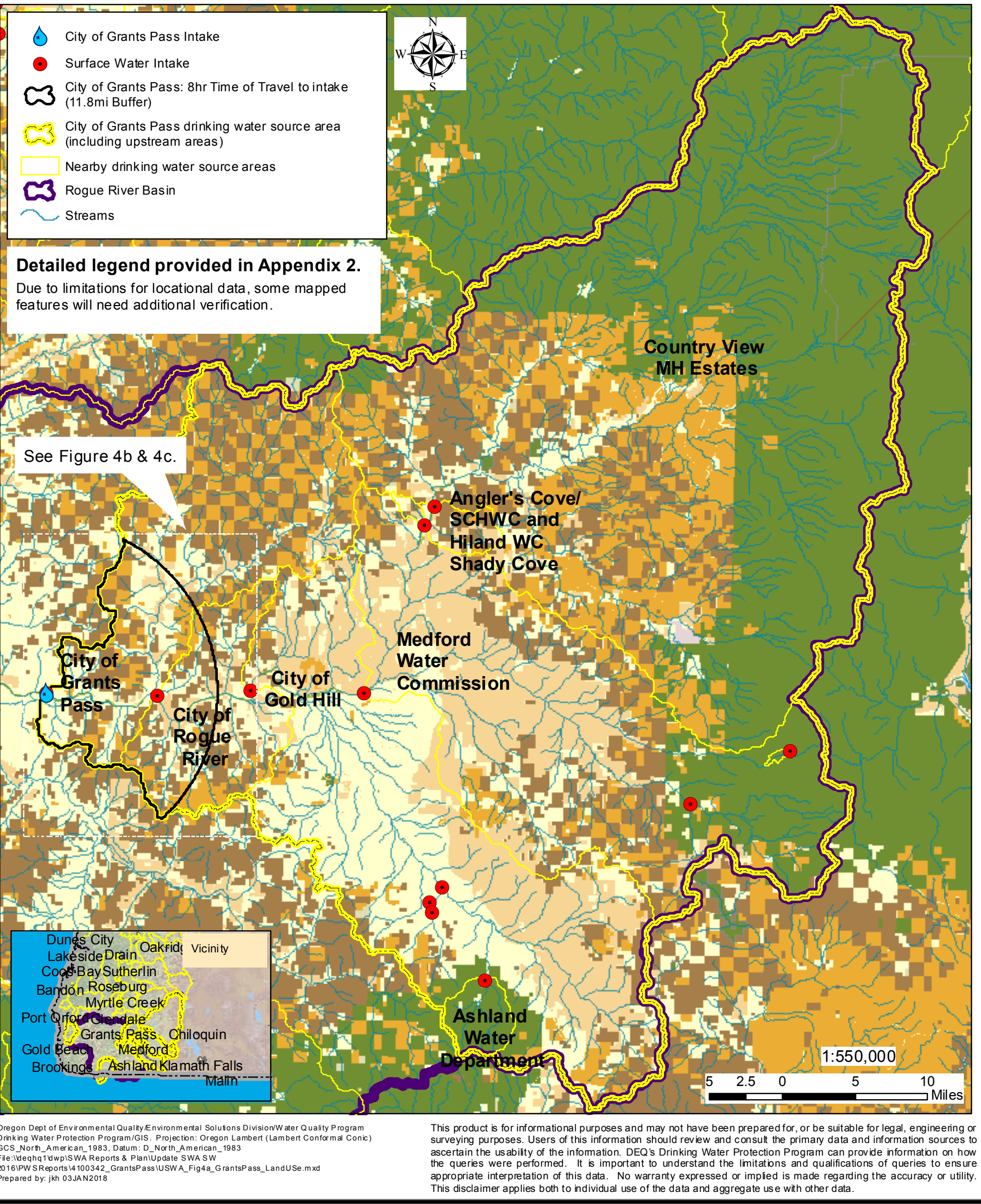


Figure 4b. City of Grants Pass (PWS 00342) DEo Health

8 Hour Time of Travel Potential Anthropogenic Sources and Land Ownership/Use (See Appendix 2 for Key to map details and metadata)

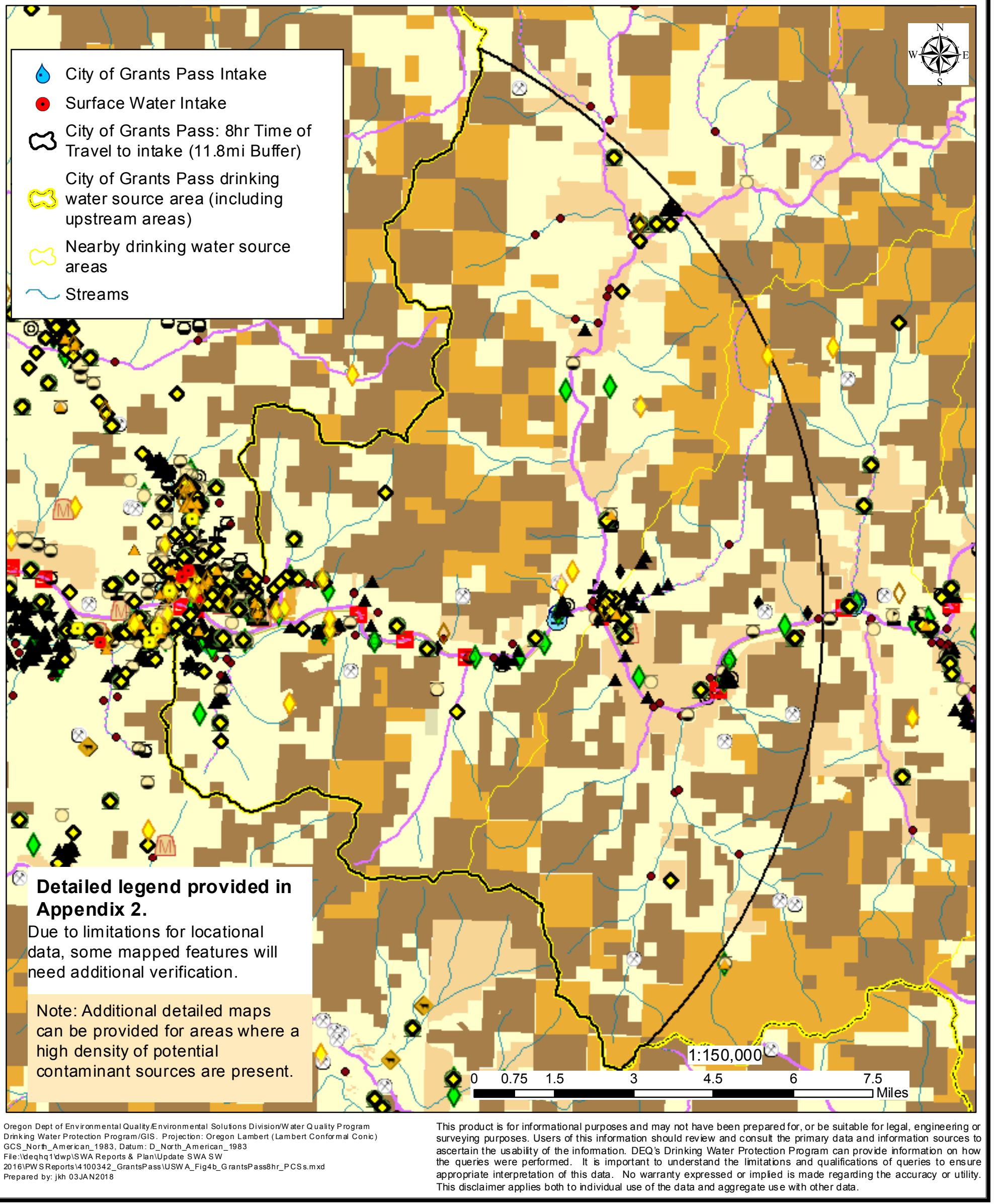




\section{Deo Health}

Figure 4c. City of Grants Pass (PWS 00342)

8 Hour Time of Travel -

Transportation Corridors and Land Ownership/Use

(See Appendix 2 for Key to map details and metadata)

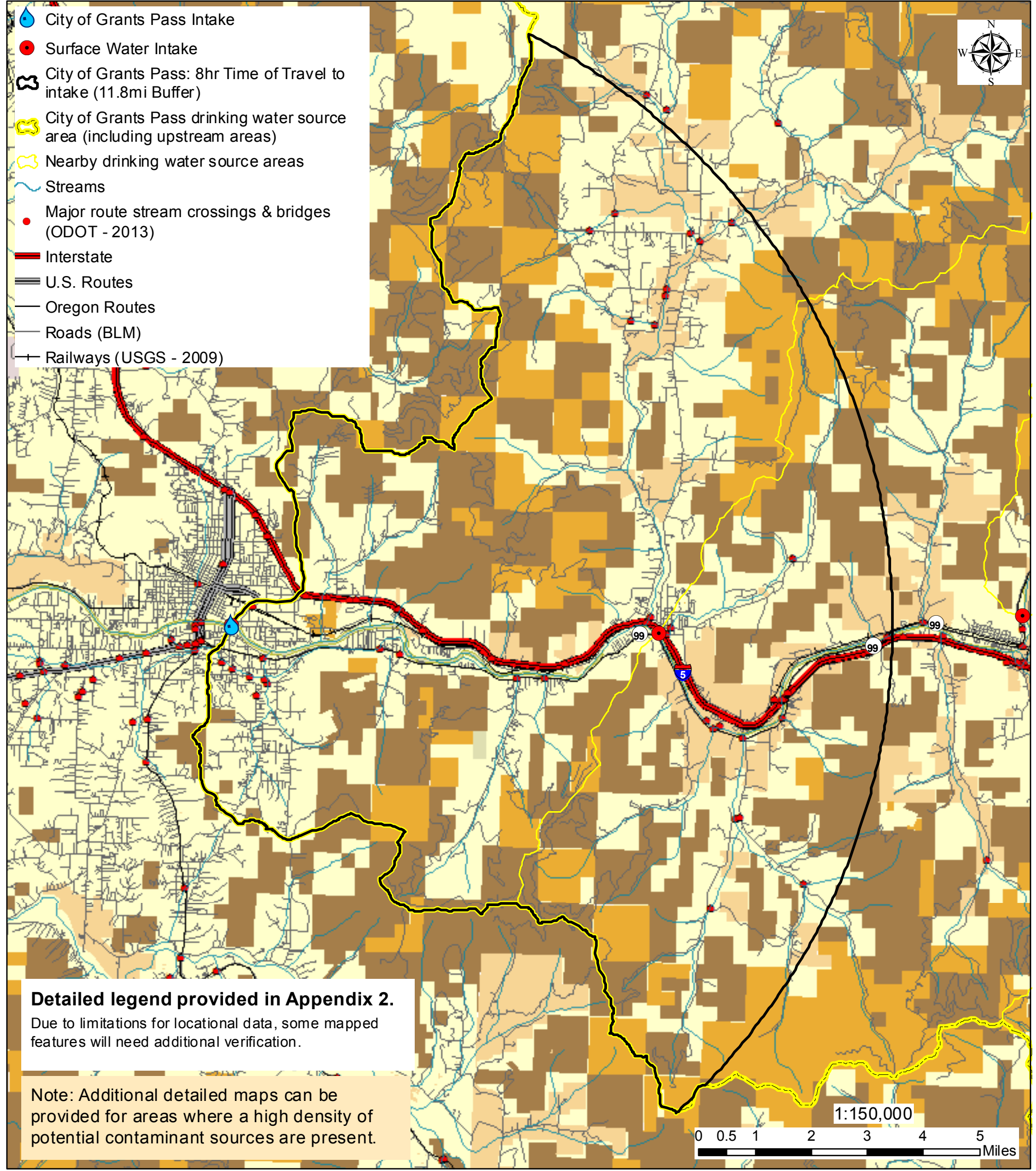




\section{Health}

Public Water System Name

PWS ID

State of Oregon Eepartment of

Ouality

County Served

Population (includes wholesale buyers) ${ }^{(2)}$

Number of Public Water Systems Served ${ }^{(2)}$
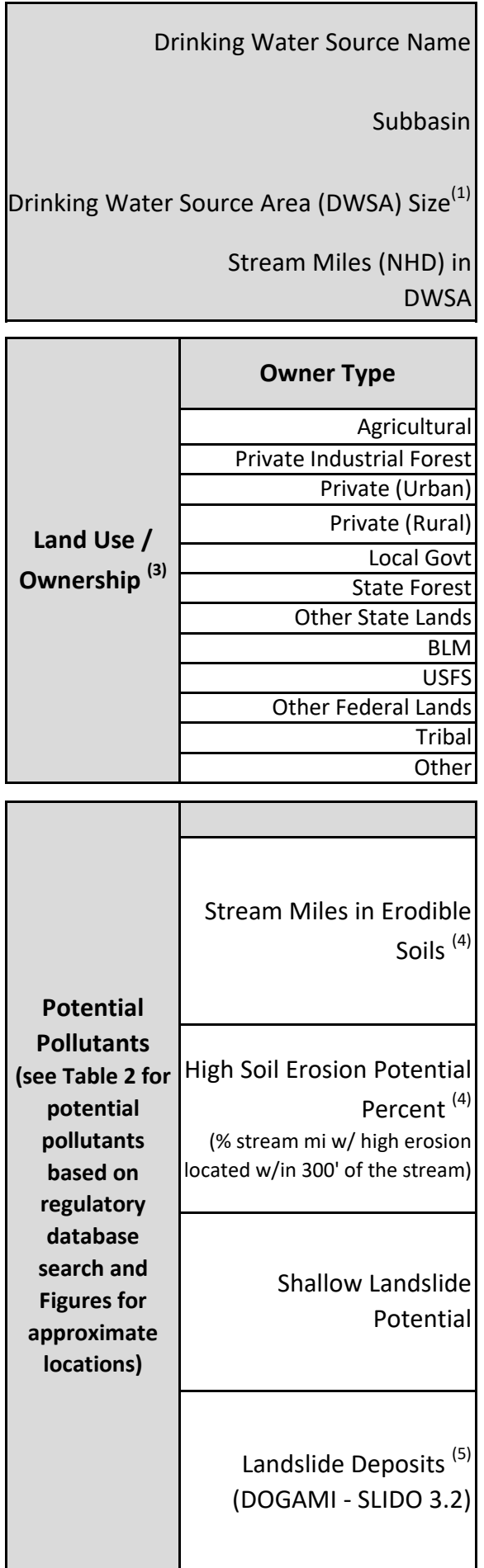

Table 1. Public Drinking Water System Land Use and Susceptibility Analysis Summary (See Appendix 2 for Key to Tables and Notes)

\section{City of Grants Pass}

00342

Josephine

37,088

1

\section{8-hr time-of-travel}

Rogue

13,134 miles

\begin{tabular}{|c|c|}
\hline $\begin{array}{c}\text { Area } \\
\text { (sq.mi.) }\end{array}$ & $\begin{array}{c}\% \text { of } \\
\text { DWSA }\end{array}$ \\
\hline 11.46 & $9 \%$ \\
\hline 21.69 & $16 \%$ \\
\hline 3.21 & $2 \%$ \\
\hline 51.79 & $39 \%$ \\
\hline & \\
\hline 0.12 & $<1 \%$ \\
\hline 0.73 & $<1 \%$ \\
\hline 45.39 & $34 \%$ \\
\hline & \\
\hline & \\
\hline & \\
\hline & \\
\hline
\end{tabular}

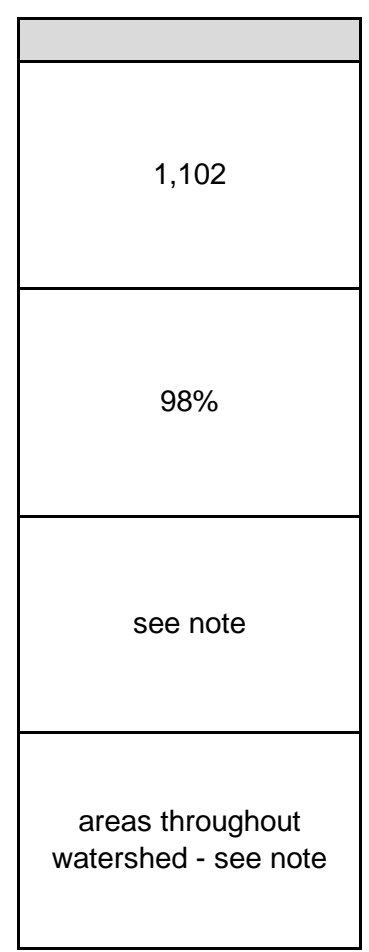

Rogue

\section{Full SW Source Area \\ (including upstream areas)}

$2456.80 \mathrm{sq} \mathrm{mi}$

The 8-hour time of travel area is provided as a planning tool for spills or releases at crossings or discharge points to the stream. Focus may need to extend further upstream for contaminants that are contributed to the stream over long time periods or recur frequently. See Note 1, Appendix 2.

\begin{tabular}{|c|c|}
\hline $\begin{array}{c}\text { Area } \\
\text { (sq.mi.) }\end{array}$ & \% of DWSA \\
\hline 343.47 & $14 \%$ \\
\hline 415.95 & $17 \%$ \\
\hline 47.56 & $2 \%$ \\
\hline 388.99 & $16 \%$ \\
\hline 2.00 & $<1 \%$ \\
\hline 1.69 & $<1 \%$ \\
\hline 1.48 & $<1 \%$ \\
\hline 435.16 & $18 \%$ \\
\hline 709.24 & $29 \%$ \\
\hline 111.25 & $5 \%$ \\
\hline & \\
\hline & \\
\hline
\end{tabular}

Notes

The data on land uses is only approximate due to limitations within the GIS layers. Public water systems and communities could use tax lot data available from the counties or other datasets to further refine the analysis if higher accuracy is needed.

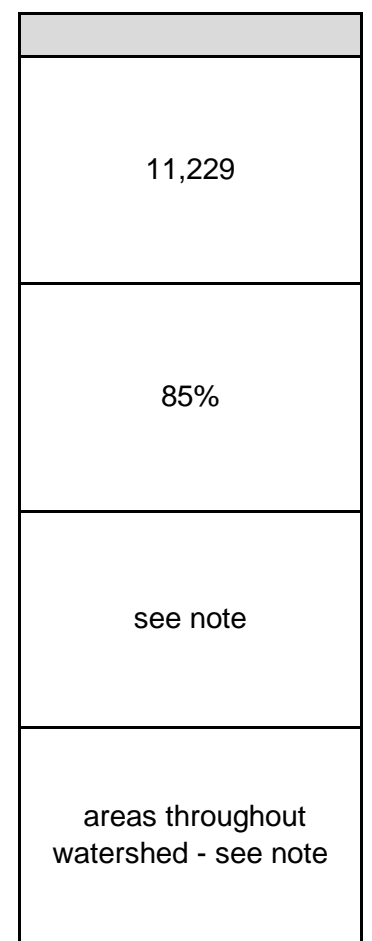

\section{Notes}

Erosion control measures ("best management practices") may be necessary for land management activities that disturb or leave bare soils in these areas. Maps and data of soil qualities without the 300 -foot stream buffer in local areas can be provided to public water systems and communities if additional detail or scale is needed for place-based planning. See Note 4 in Appendix 2.

More details on shallow landslide susceptibility may be available. Contact DEQ Drinking Water Protection for additional information.

Includes earth and debris slides, flows, slumps, falls and complex landslide types. Does not include rock material landslide deposits. 


\begin{tabular}{|c|c|c|}
\hline & Source Name & Rogue River (surface water) \\
\hline & Treatment Process & Rapid sand \\
\hline & $\underline{\text { Safe Drinking Water Information System Results }}{ }^{(6)}$ & $\frac{\text { MCL Violations and Significant Detections }}{\underline{(2005-2017)^{(6)}}}$ \\
\hline & $\begin{array}{r}\begin{array}{r}\text { Regulated volatile organic chemicals, synthetic organic } \\
\text { chemicals and inorganic compounds }\end{array} \\
\text { Disinfection byproducts } \\
\text { (Total Trihalomethanes (TTHM), } \\
\text { Haloacetic acids (five) (HAA5), bromate, and chlorite) } \\
\text { Bacteria (Ecoli and TCR=Total Coliform Rule) }\end{array}$ & 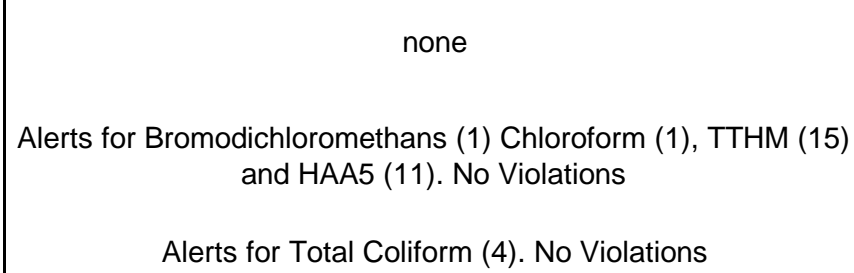 \\
\hline $\begin{array}{l}\text { Water Quality } \\
\text { Monitoring } \\
\text { Data and } \\
\text { Treatment } \\
\text { Method }\end{array}$ & $\begin{array}{r}\text { DEQ/OHA Source Water Monitoring } \\
\text { project test data }{ }^{(7)} \\
\text { ND = All parameters not detected } \\
\text { NA = source water not analyzed }\end{array}$ & $\begin{array}{l}\text { The following pesticides and typical wastewater constituents } \\
\text { were detected at low levels in drinking water source monitoring } \\
\text { in the Rogue River at the Grants Pass intake location: DEET, } \\
\text { Diuron, Sulfamethoxazole, Cholesterol, Coprostanol, beta- } \\
\text { Sitosterol, Stigmastanol, Bis(2-ethylhexyl)phthalate (2010). } \\
\text { These constituents were detected at low concentrations that are } \\
\text { well below EPA maximum contaminant levels (MCLs) for } \\
\text { drinking water or health based screening levels (where } \\
\text { established). The presence indicates a source and a pathway } \\
\text { and results can be used by public water systems to prioritize } \\
\text { protection activities. } \\
\text { Note that DEET, Atrazine, Diuron, Carbaryl, Fluometron, } \\
\text { Sulfamethoxazole, Butylbenzylphthalate, Carbamazepine, } \\
\text { Diphenhydramine, Cholesterol, Coprostanol, Bis(2- } \\
\text { ethylhexyl)phthalate and Aluminum (>SMCL) (2008) were also } \\
\text { detected at low levels in samples from the Rogue River at the } \\
\text { City of Gold Hill drinking water source intake (upstream of } \\
\text { Grants Pass). }\end{array}$ \\
\hline & $\begin{array}{l}\text { Additional raw water quality monitoring data for the drin } \\
\text { USGS, DEQ's LASAR database, individual water provi } \\
\text { watershed councils) or local volunteer monitoring. }\end{array}$ & $\begin{array}{l}\text { g water source may be available from other sources including } \\
\text { s, local partners (i.e. soil and water conservation districts or }\end{array}$ \\
\hline
\end{tabular}


Table 2: Summary of Potential Sources of Pollution identified in the 8-hour time-of-travel as identified in readily accessible state and federal databases and GIS layers Updated Source Water Assessment

see Appendix 2 for Key to Tables for Notes and Descriptions of Acronyms

See Appendix 5 for detailed list of potential sources

\section{PWS Name: City of Grants Pass PWS Number: 00342}

- This summary only includes potential sources of pollution within the estimated 8-hour time of travel area which is provided as a planning tool since eight hours should provide adequate response time to protect the integrity of the public water system intake after a spill or release at any crossing or discharge point to the stream. It is recommended the water system and community considers increased protection within this 8-hour travel time from the intake for spills and other acute risks. Focus may need to extend further upstream for contaminants that are contributed to the stream over long time periods or recur frequently.

- This information supplements the Original Source Water Assessment Inventory dated between 2000 and 2005 and should be used in conjunction with the original inventory to provide a more detailed analysis of potential sources of pollution.

- Note that due to state database limitations, some sites will require further research to verify presence and location.

\begin{tabular}{|c|c|c|c|}
\hline $\begin{array}{c}\text { Program and type of facility } \\
\text { - see Appendix } 2 \text { for Key to Acronyms }\end{array}$ & Count & $\begin{array}{c}\text { Program and type of facility } \\
\text { - see Appendix } 2 \text { for Key to Acronyms }\end{array}$ & Count \\
\hline CAFO & & SWMS & \\
\hline Large Concentrated - & 1 & 1109 Industrial - Wood - Terminated & 1 \\
\hline Small Confined - & 2 & 1185 Industrial - SW Treatment - Terminated & 1 \\
\hline State CAFO - Dairy & 1 & 1198 Industrial - Wood - Closure & 1 \\
\hline DOGAMI & & 1417 Waste Tire - Carrier - Active & 1 \\
\hline Closed - & 3 & UST & \\
\hline Permitted - rock & 1 & Sites with tanks with unknown status & 0 \\
\hline ECSI & & Sites with Decommissioned Tanks & 8 \\
\hline Inactive site with further action needed & 6 & Sites with Upgraded Tanks & 6 \\
\hline No further action required & 4 & WQ-SIS & \\
\hline Site active in Cleanup Program & 2 & GEN04 - INDUSTRIAL & 1 \\
\hline ECSI NFA & & GEN12C - STORMWATER & 3 \\
\hline No further action required & 34 & GEN12CA - STORMWATER & 1 \\
\hline HW & & GEN12Z - STORMWATER & 5 \\
\hline Conditionally Exempt Generator (CEG) & 7 & GEN15A - INDUSTRIAL & 1 \\
\hline LUST & & GEN17A - INDUSTRIAL & 1 \\
\hline CLEANUP_STARTED & 4 & GEN2401 - DOMESTIC & 1 \\
\hline REPORTED & & NPDES-DOM-Da - DOMESTIC & 1 \\
\hline LUST NFA & & WPCF-DOM-Da - DOMESTIC & 1 \\
\hline CLEANUP_COMPLETED & 19 & WPCFOS-Bii - DOMESTIC & 3 \\
\hline OSMB & & WPCFOS-Biii - DOMESTIC & 1 \\
\hline Boating access site & 4 & WPCFOS-BiiiRGF> - DOMESTIC & 4 \\
\hline Transportation & & WQ - WWTP & \\
\hline Stream crossing & 33 & DOMESTIC - NPDES-DOM-Da & 1 \\
\hline Highway & 2 & WQ - Outfalls & \\
\hline Railway & 2 & Outfalls & 1 \\
\hline Schools & 9 & WQ 303(d) list (number of categories and constituents) & \\
\hline SFM-HSIS-AST & & Birdseye Creek & 1 \\
\hline Aboveground storage tank(s) on site & 29 & Evans Creek & 26 \\
\hline UIC & & Fruitdale Creek & 3 \\
\hline 2-Industrial/Commercial (Minor-HW) / Abandoned & 1 & Jones Creek & 12 \\
\hline OnSite system / Info request & 1 & Rogue River & 13 \\
\hline OnSite system / Registered w/permit & 4 & Savage Creek & 1 \\
\hline OnSite system / Revoked & 1 & Ward Creek & 2 \\
\hline
\end{tabular}




\begin{tabular}{|c|c|c|c|}
\hline $\begin{array}{l}\text { Program and type of facility } \\
\text { - see Appendix } 2 \text { for Key to Acronyms }\end{array}$ & Count & $\begin{array}{c}\text { Program and type of facility } \\
\text { - see Appendix } 2 \text { for Key to Acronyms }\end{array}$ & Count \\
\hline \multicolumn{4}{|l|}{ SFM } \\
\hline All Other Automotive Repair \& Maint & 1 & Other Commercial \& Ind Machinery \& Eqp & 1 \\
\hline All Other Plastics Product Mfg & 1 & Other Gasoline Stations & 2 \\
\hline All Other Specialty Trade Contractors & 1 & Other General Gov Support & 7 \\
\hline Automotive Body, Paint, \& Interior Repair & 2 & Other Telecommunications & 1 \\
\hline Automotive Exhaust System Repair & 2 & Outdoor Power Eqp Stores & 1 \\
\hline Automotive Parts \& Accessories Stores & 1 & Paint \& Wallpaper Stores & 1 \\
\hline Automotive Transmission Repair & 1 & Petroleum \& Petroleum Products Whsle & 1 \\
\hline Bolt, Nut, Screw, Rivet, \& Washer Mfg & 1 & Pipeline Trans Of Natural Gas & 1 \\
\hline Ceramic Wall \& Floor Tile Mfg & 1 & Plastics Material \& Resin Mfg & 1 \\
\hline Const, Mining, \& Forestry Machinery \& Eqp & 1 & Plumbing \& Heating Eqp \& Supplies & 1 \\
\hline Cut Stone \& Stone Product Mfg & 1 & Ready-Mix Concrete Mfg & 1 \\
\hline Elementary \& Secondary Schools & 2 & Recreational Vehicle Dealers & 1 \\
\hline Exterminating \& Pest Control Svcs & 1 & Recyclable Material Whsle & 1 \\
\hline Fire Protection & 1 & Refrigerated Warehousing \& Storage & 1 \\
\hline Gasoline Stations With Convenience Store & 2 & School \& Employee Bus Trans & 1 \\
\hline General Automotive Repair & 6 & Site Preparation Contractors & 2 \\
\hline Hardware Stores & 1 & Softwood Veneer \& Plywood Mfg & 1 \\
\hline Hardwood Veneer \& Plywood Mfg & 1 & Supermarkets \& Other Grocery & 1 \\
\hline Highway, Street, \& Bridge Const & 1 & Timber Tract Operations & 1 \\
\hline Hydroelectric Power Generation & 1 & Wired Telecommunications Carriers & 1 \\
\hline Motor Vehicle Towing & 1 & Wood Kitchen Cabinet \& Countertop Mfg & 1 \\
\hline Ornamental \& Architectural MtI Work Mfg & 1 & Wood Window \& Door Mfg & 2 \\
\hline Other Bldg Material Dealers & 1 & & \\
\hline DWP-PCS & 451 & & \\
\hline $\begin{array}{l}\text { A04 Type; A - Crops - Irrigated (inc. orchards, } \\
\text { vineyards, nurseries, greenhouses) }\end{array}$ & 2 & C08 Type; P - Dry Cleaners & 4 \\
\hline $\begin{array}{l}\text { A05 Type; A - Crops - Nonirrigated (inc. Christmas } \\
\text { trees, grains, grass seed, pasture) }\end{array}$ & 2 & C09 Type; P - Electrical/Electronic Manufacturing & 9 \\
\hline $\begin{array}{l}\text { A07 Type; A - Grazing Animals (> } 5 \text { large animals or } \\
\text { equivalent/acre) }\end{array}$ & 2 & C10 Type; P - Fleet/Trucking/Bus Terminals & 3 \\
\hline $\begin{array}{l}\text { A11 Type; A - Managed Forest Land - Clearcut } \\
\text { Harvest }(<35 \text { yrs. })\end{array}$ & 1 & C12 Type; P - Furniture/Lumber/Parts Stores & 10 \\
\hline $\begin{array}{l}\text { A14 Type; P - Pesticide/Fertilizer/Petroleum Storage, } \\
\text { Handling, Mixing, \& Cleaning Areas - Exterminators }\end{array}$ & 1 & C14 Type; P - Junk/Scrap/Salvage Yards & 4 \\
\hline A52 Type; A - Irrigation Canal/Pond & 11 & C15 Type; P - Machine Shops & 2 \\
\hline C01 Type; P - Automobiles - Body Shops & 14 & C16 Type; P - Medical/Vet Offices & 16 \\
\hline C02 Type; P - Automobiles - Car Washes & 4 & C17 Type; P - Metal Plating/Finishing/Fabrication & 6 \\
\hline C03 Type; P - Automobiles - Gas Stations & 11 & C20 Type; Parking Lots/Malls (> 50 Spaces) & 9 \\
\hline C04 Type; P - Automobiles - Repair Shops & 60 & C21 Type; P - Photo Processing/Printing & 12 \\
\hline C05 Type; P - Boat Services/Repair/Refinishing & 2 & C22 Type; Plastics/Synthetics Producer & 2 \\
\hline C06 Type; P - Cement/Concrete Plants & 1 & C24 Type; P - RV/Mini Storage & 5 \\
\hline $\begin{array}{l}\text { C07 Type; A - Chemical/Petroleum } \\
\text { Processing/Storage }\end{array}$ & 1 & $\begin{array}{l}\text { C26 Type; P - Wood/Pulp/Paper Processing and } \\
\text { Mills }\end{array}$ & 4 \\
\hline
\end{tabular}




\begin{tabular}{|c|c|c|c|}
\hline $\begin{array}{c}\text { Program and type of facility } \\
\text { - see Appendix } 2 \text { for Key to Acronyms }\end{array}$ & Count & $\begin{array}{l}\text { Program and type of facility } \\
\text { - see Appendix } 2 \text { for Key to Acronyms }\end{array}$ & Count \\
\hline \multicolumn{4}{|l|}{ DWP-PCS (continued) } \\
\hline $\begin{array}{l}\text { C61 Type; P - Maintenance Shop/Equipment } \\
\text { Storage - Transportation Related - Highway Division } \\
\text { Grants Pass }\end{array}$ & 1 & M50 Type; A - Other - City of Rogue River & 1 \\
\hline C65 Type; Miscellaneous Manufacturing & 2 & M55 Type; P - Unknown Operation & 9 \\
\hline $\begin{array}{l}\text { M01 Type; P - Above Ground Storage Tanks - } \\
\text { Excluding Water and Residential ASTs }\end{array}$ & 6 & $\begin{array}{l}\text { M56 Type; A - Transportation Corridors - High Use } \\
\text { Roads/Streets }\end{array}$ & 2 \\
\hline M06 Type; P - Auto - Gas Stations - Historic & 9 & $\begin{array}{l}\text { M60 Type; A - Other Injection/Dry Wells, Sumps - } \\
\text { Class V UICs }\end{array}$ & 1 \\
\hline M07 Type; P - Historic Waste Dumps/Landfills & 1 & M61 Type; P - Wells - Abandoned & 4 \\
\hline $\begin{array}{l}\text { M09 Type; A - Homesteads - Rural - Septic Systems } \\
\text { (<1/acre) }\end{array}$ & 10 & $\begin{array}{l}\text { M62 Type; A - Lagoons/Liquid Wastes - } \\
\text { Municipal/Industrial Wastewater Treatment }\end{array}$ & 1 \\
\hline $\begin{array}{l}\text { M14 Type; A - River Recreation - Heavy Use (inc. } \\
\text { campgrounds) }\end{array}$ & 1 & R02 Type; A - Apartments and Condominiums & 2 \\
\hline M17 Type; A - Transmission Lines - Right-of-Ways & 2 & R03 Type; A - Campgrounds/RV Parks & 2 \\
\hline $\begin{array}{l}\text { M18 Type; A - Transportation - Freeways/State } \\
\text { Highways }\end{array}$ & 18 & R04 Type; P - Cemeteries - Pre-1945 & 5 \\
\hline M19 Type; A - Transportation - Railroads & 7 & R05 Type; P - Drinking Water Treatment Plants & 6 \\
\hline $\begin{array}{l}\text { M22 Type; Transportation - Stream Crossing - } \\
\text { Perennial }\end{array}$ & 5 & R06 Type; P - Fire Station & 5 \\
\hline M24 Type; A - UST - Decommissioned/Inactive & 3 & $\begin{array}{l}\text { R09 Type; A - Housing - High Density (> } 1 \\
\text { House/0.5 acres) }\end{array}$ & 31 \\
\hline M24 Type; P - UST - Decommissioned/Inactive & 17 & R11 Type; A - Lawn Care - Highly Maintained Areas & 2 \\
\hline $\begin{array}{l}\text { M26 Type; P - UST - Confirmed Leaking but listed as } \\
\text { NFA - DEQ LUST List }\end{array}$ & 14 & R13 Type; Parks & 5 \\
\hline M27 Type; A - UST - Upgraded/Registered - Active & 1 & R15 Type; P - Schools & 17 \\
\hline M27 Type; P - UST - Upgraded/Registered - Active & 14 & $\begin{array}{l}\text { R16 Type; A - Septic Systems - High Density ( > } 1 \\
\text { system/acre) }\end{array}$ & 6 \\
\hline M28 Type; P - UST - Status Unknown & 7 & $\begin{array}{l}\text { R17 Type; A - Sewer Lines - Close Proximity to } \\
\text { PWS }\end{array}$ & 15 \\
\hline M29 Type; P - Upstream Reservoirs/Dams & 1 & $\begin{array}{l}\text { R18 Type; P - Utility Stations/Powerplants - } \\
\text { Maintenance/Transformer Storage }\end{array}$ & 2 \\
\hline $\begin{array}{l}\text { M31 Type; Large Capacity Septic Systems (serves > } \\
20 \text { people) - Class V UICs }\end{array}$ & 8 & $\begin{array}{l}\text { R20 Type; P - Wastewater Treatment } \\
\text { Plants/Collection Stations }\end{array}$ & 1 \\
\hline M32 Type; Construction/Demolition Areas & 9 & & \\
\hline DWP - USWA (2016/17) Additional PCSs identified & 3 & & \\
\hline
\end{tabular}




\section{Developing Strategies For Drinking Water Protection}

Many ${ }^{1}$ public water systems in Oregon will receive an Updated Source Water Assessment (USWA) developed by the Oregon Department of Environmental Quality (DEQ) and the Oregon Health Authority (OHA) drinking water protection team by 2017. USWAs provide the water systems and communities more detailed information on the watershed or recharge area that supplies their well, spring or intake (the "drinking water source area”). Public water systems and local communities can use the information in the assessments to voluntarily develop and implement drinking water protection strategies.

Requirements for water quality monitoring of public water systems in Oregon provide some degree of assurance of safe drinking water; however, all systems are vulnerable to potential contamination. One of the best ways to ensure safe drinking water and minimize future treatment costs is to develop local strategies designed to protect against potential contamination. Not only will this add a margin of safety; it will also raise local community awareness of drinking water contamination risks and provide information about how communities and local landowners can help protect their drinking water sources.

\section{Using Place-Based Planning to Develop Protection Strategies}

The drinking water source area for most communities lies partially, if not entirely, outside of their jurisdiction and may include several different governing agencies as well as a diverse mix of landowners, businesses and residents. When developing protection

\footnotetext{
${ }^{1}$ All community water systems using surface water will receive a USWA. Because of the number of water systems using groundwater in Oregon, the Oregon Health Authority has prioritized completing assessments for new Community and Non-Transient Non-Community water systems and systems that have added a new water source since their original source water assessment was completed.
}

strategies, DEQ and OHA highly community involve potentially affected stakeholders early in the process to foster stakeholder awareness and trust in the resulting strategies.

Oregon adopted an "Integrated Water Resources Strategy (IWRS)” in 2012 that provides recommendations for how to do a place-based and integrated approach to water resources planning. This approach helps communities achieve the level of coordination and collaboration to

successfully address local water quality and water quantity challenges, such as developing and implementing strategies to protect their drinking water sources. The IWRS Place-Based Planning guidelines describe elements to consider for building a collaborative process, characterizing waterrelated issues, quantifying existing and future water needs, developing a suite of solutions, and adopting and implementing the plan. More information about the process can be found in this Water Resources Department document: http://www.oregon.gov/owrd/LAW/docs/IW RS/2015 February Draft Place_Based Gui delines.pdf

\section{Strategies to Achieve Risk Reduction}

The primary goal of the drinking water protection strategies should be to reduce or minimize the risks of pollution in the source water. It is highly improbable that one can eliminate risks in any area, but by applying one or more protection strategies, a community will be able to reduce the likelihood of pollutants affecting the water supply in the future. Potential strategies include both general management practices such as conservation or efficiency measures that will apply to the entire drinking water protection area and management practices that can be applied most appropriately by land-use category (commercial/industrial, agricultural/rural, forestry, recommend that the water system and

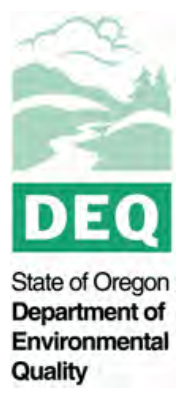

Water Quality Drinking Water Protection

700 NE Multnomah St. Suite 600

Portland, OR 97232

Phone: 503- 229-5413 800- 452-4011

Fax: $\quad$ 503- 229-6037

Contact: Sheree Stewart http://www.oregon.gov/DE $\mathrm{Q} /$ wq/programs/Pages/DW P.aspx

\section{Health}

Oregon Health Authority Drinking Water Program 444 "A" Street Springfield, OR 97477 Phone: 541- 726-2587 Fax: $\quad$ 541- 726-2596 Contact: Tom Pattee http://www.healthoregon.or g/dwp 
residential/municipal, and miscellaneous). The following list provides some of the most common management options as an example

to public water suppliers and communities:

\section{Example Strategies for Drinking Water Protection}

\section{Non-Regulatory Options}

Notify and Educate the Public: Contact property owners within the protection area so they are aware of the need for protection measures. Let them know this is voluntary. Focus educational efforts on basic information about the source water and the relationship between surface activities and the water quality; familiarity with the location of the protected area; basic information on sources of contamination; and effective strategies for safe management of all potential contaminants. Public education/notification can be accomplished using local news outlets, letters to residents, letters to land owners/operators, and bill stuffers/customer mailings. Information signs could be placed adjacent to roadways entering the protection area. Include on the sign the name of the water system/jurisdiction and a phone number where callers can obtain more information or report releases.

Use Technical Assistance Resources: Work with local or state providers of technical assistance (e.g., DEQ's regional offices, Soil and Water Conservation Districts, OSU Extension) to encourage the use of best management practices for pollution prevention and waste reduction. Apply for grants or funding to provide financial incentives such as pollution prevention tax credits, low-interest loans or direct subsidies/cost sharing. Provide recognition for environmental friendly businesses and operations (e.g., green awards, plaques/door signs).

Sponsor Hazardous Waste/Unused Chemical Collection: Establishing a permanent location or holding one-day events to collect hazardous wastes from community residents (including households and small businesses) is an effective way to reduce risks posed by storing hazardous wastes or other chemicals within the protection area. Hold an amnesty (free-disposal) event for unused business or agricultural chemicals stored in the protection area. Set up a local materials exchange program (or publicize existing programs).

Develop Spill Response Plans: Encourage and assist your local fire department and transportation department with spill response planning. Jurisdictions within protection areas could develop specific spill response procedures to allow quicker response and notifications should a hazardous material spill or release occur. These can be integrated into your county's Emergency Management Plan. Contact the Oregon Department of Transportation (ODOT) for state highways.

Acquire Land or Rights to Development: Community ownership of as much as possible of the critical land areas within the protection area and managed for water quality protection provides some of the best assurance of long-term protection of the public water supply. Protection could be provided by ownership accomplished through methods such as capital or bond fund programs, or through easements and deed restrictions. Private non-profit land conservation organizations or local land trusts in your area can assist you in acquiring land within your protection area by conveyance to a trust, seeking donations, or direct land purchases for conservation.

\section{Local Regulatory Options}

Existing Regulations and Permits: Take advantage of opportunities to provide public comment and input when existing regulatory programs are reviewing permits or programs which affect the siting, design, construction, operation or closure of facilities within your protection area. Ensure you are included on regulatory agency contact lists so that you receive announcements for public involvement opportunities. Consider participating in advisory group meetings for specific topics of interest. Ensure that the regulatory programs are aware of your protection area and request that compliance inspections or technical assistance is prioritized in critical areas.

Land Use Controls (Zoning/Health Ordinances): There are many different types of zoning tools. Your community can identify the protection area with an overlay map and enact specific requirements for land uses and development within these boundaries in order to protect public health. Ordinances applying to sites that pose a risk to water quality within the overlay area may include prohibition of various land uses (such as landfills or underground fuel storage tanks); subdivision controls (such as limiting density or requiring larger lot sizes); special permitting or siting requirements (i.e. placing limitations on the use of toxic and hazardous materials, pesticides, salts); and performance standards (i.e. requiring secondary containment for petroleum or chemical storage over a certain volume). 


\section{How do communities use the Updated Source Water Assessments?}

The Updated Source Water Assessment

(USWA) provides the information for

developing local protection strategies.

The USWAs include details

characterizing the source area and

potential source water risks. It also

provides key information that will allow

the community to focus limited resources on higher-risk areas within the watershed or recharge zones for wells. The USWA information should be supplemented with local knowledge of the water system and community. The water system and community can refine the delineation of sensitive areas and identification of potential contamination sources through further research, local input and coordination with state agencies.

The USWA source area characterization should be reviewed to clarify the presence, location, operational practices, and actual risks of the identified facilities and land-use activities. Additional potential contaminant sources or sensitive areas may also be added based on local knowledge or additional research. Potential sources with low or no risk (such as landowners who have already incorporated best management practices into their operations to protect your source of drinking water) can be screened out or selected for low priority outreach or technical assistance. Local and state resources can then be directed to the highest priority potential problems in the drinking water source area.

Another way to use the information in the USWA is in developing the water system's contingency plan. Contingency planning focuses on potential threats to the drinking water supply (such as mechanical problems, chemical detections in the source water, chemical spills in the source area, or natural disasters) and the development of procedures to be followed should these events occur. Guidance for preparing a contingency plan and examples are available from OHA. Many contingency plan elements may have already been completed by public water systems as part of their required Emergency

Response Plan. Additional elements can be added as drinking water source protection strategies are developed.

Public water systems may also find it necessary, as a result of either existing or projected increased demand, to explore the development of additional sources for drinking water. Drinking water source protection provides a mechanism that can be used to help select the best site and identify areas that should be protected now so they will provide quality drinking water in the future. Additionally, development of a new groundwater source in the vicinity of existing sources may modify the movement of groundwater in the subsurface, perhaps changing the shape and orientation of existing drinking water source areas. Evaluation of the significance of those changes should be addressed in the protection planning process to ensure that the management strategy in place will continue to protect the community's drinking water supply.

\section{Need assistance?}

Drinking water source protection is already at work in Oregon. A number of Oregon communities are currently developing and implementing strategies to protect their drinking water source areas. Successful drinking water protection plans developed in Oregon are available to communities as templates or examples. Staff members at $\mathrm{OHA}$ and DEQ are available to provide assistance, and extensive written materials are available to local community groups or consultants to assist in developing drinking water protection plans or strategies.

Detailed information about developing drinking water source protection strategies can be found on DEQ's Drinking Water Protection Program website. The website also includes Updated Source Water Assessment methods and results, sample Drinking Water Protection Plans, information for 
schools, and links to many other useful sites:http://www.oregon.gov/deq/wq/prog rams/Pages/DWP.aspx

The OHA - Drinking Water Program website includes system characteristics, monitoring data, contacts for all public water systems in Oregon, drinking water standards, fact sheets on contaminants, information on the Safe Drinking Water Revolving Loan Fund, Consumer Confidence Reports, and more: http://www.healthoregon.org/dwp

Water systems or community members interested in the potential of developing drinking water protection strategies should contact the respective DEQ and OHA coordinators. Those systems using surface water sources should initially contact Sheree Stewart, Drinking Water
Protection Program Coordinator, DEQ, Portland, (503) 229-5413. Groundwaterbased water systems should initially contact Tom Pattee, Groundwater Coordinator, OHA, Springfield, (541) 726-2587 x24. As the state moves further into the protection phase of the Oregon program, DEQ and OHA will be able to direct individual requests for assistance to specific staff trained and experienced in that area, both within the state agencies and in other partner organizations.

\section{Alternative formats}

Documents can be provided upon request in an alternate format for individuals with disabilities or in a language other than English for people with limited English skills. To request a document in another format or language, call DEQ in Portland at 503-229-5696, or toll-free in Oregon at 1-800-452-4011, ext. 5696; or email deqinfo@deq.state.or.us. 


\section{General Legend:}

(-) Public water system surface water

Public water system drinking water source

- Nearby public water system surface water

3 Nearby public surface water system drinking water source area

Stream (NHD)

Interstate

U.S. Routes

Oregon Routes

City limits (ODOT, 2013)

Urban Growth Boundary

County Boundary

\section{Erosion Potential:}

Streams near soils with significant erosion potential. Erosion control measures (BMPs) may be necessary for land management activites that disturb or leave bare soils in these areas.

Streams (NHD) with significant erosion potential from

$\sim$ intensive $(>75 \%)$ soil surface disturbance (i.e.tilled or bare soils) (NRCS-RUSLE2/ODA-EVI; see Note 4a).

Streams (NHD) with significant erosion potential from $\sim$ substantial $(50-75 \%)$ soil surface disturbance (NRCS offroad/off-trail ratings; see Note $4 \mathrm{~b}$ ).

Streams (NHD) with significant erosion potential (slope $>30 \%$ $\sim$ using USFS SRI data, NRCS SSURGO data not avaliable; see Note 4c).

\section{Landslide Information}

Landslide Deposits (non-rock material, includes earth and debris slides, flows, slumps, falls and complex) (DOGAMI SLIDO3.2)

Scarp Flanks (DOGAMI SLIDO-3.2)

Scarps (DOGAMI SLIDO-3.2)

\section{Land Ownership/Use:}

Private Urban Lands (within city limits)

Private Rural Lands (private non-industrial outside city limits)

Agriculture (Ag Zoning (BLM) and NASS 2013)

Private Industrial Forests (ODF data); Lands

Managed by Private Industry (BLM)

Local Government

State Dept. of Forestry

State - Other

Bureau of Land Management

U.S. Forest Service

Federal - Other

Bonneville Power Administration

Bureau of Indian Affairs

Undetermined

Water

\section{Potential Sources of Pollutants identified in} State and Federal Regulatory Databases:

- Confined Animal Feeding Operations (ODA as of 1/20/216)

- Dry Cleaner, Active (DEQ as of 2015)

- Dry Cleaner, Dry Store (DEQ as of 2015)

- Dry Cleaner, Closed (DEQ as of 2015)

- Dry Cleaner, Inactive (DEQ as of 2015)

- Dry Cleaner, Solvent Supplier (DEQ as of 2015)

Environmental cleanup site with known contamination (DEQ as of 01/2016)

Environmental cleanup site No Further Action required or otherwise lower risk (DEQ as of 01/2016)

Hazardous Material Large Quantity Generator (DEQ - HW as of 1/02/2016)

Hazardous Material Small Quantity or Conditionally Exempt - Generator (DEQ - HW as of 1/02/2016)

Hazardous Material Transport, Storage, and Disposal sites (DEQ

- HW as of 1/2016)

- Hazardous Substance Information System (OSFM as of 2009)

Hazardous Substance Information System - AST (OSFM as of 2009)

Leaking underground storage tank - Confirmed (DEQ as of

9/2012) (Locaton will likely need verification.)

Leaking underground storage tank with No Further Action

- required or otherwise lower risk (DEQ as of 9/2015) (Location will likely need verification.)

* Mining permits (DOGAMI as of 1/16/2014)

- Oil and Gas wells (permitted only) (DOGAMI as of 7/2016)

* Updated Source Water Assessment Potential Contaminant

Source - Area-wide source (DEQ as of 2017)

Updated Source Water Assessment Potential Contaminant

Source - Point source (DEQ as of 2017)

Original Source Water Assessment Potential Contaminant Source

- Area-wide source (DEQ as of 2005)

- Original Source Water Assessment Potential Contaminant Source

- Point source (DEQ as of 2005)

- School Locations OR (DHS as of 2015)

(11) Solid Waste sites (DEQ - SW as of $1 / 25 / 2016$ ) Underground Injection Control (DEQ as of 1/12/2016)

Underground Storage Tanks (DEQ as of 1/25/2016) (Location will likely need verificaton.)

Water Quality domestic wastewater treatment sites (DEQ - SIS as of $1 / 25 / 2016$ )

$\checkmark \quad$ Water Quality permits (DEQ - SIS as of $1 / 25 / 2016$ )

- Major route stream crossings and bridges (ODOT - 2013)

9 Water Quality effluent outfalls (DEQ -WQ as of 2009)

Water Quality Concern; lakes - Cat3 (DEQ - 2012)

- Major route stream crossings \& bridges (ODOT - 2013)

Water quality limited stream/lake, DEQ 303(d) list Cat 4A or 5, TMDL approved or needed (DEQ - 2012)

Water Quality Concern stream/lake, DEQ 303(d) Cat.3, Insufficient Data (DEQ - 2012) 


\section{Notes}

(1) DWSA - drinking water source area - delineated as the 5th-field watershed upstream of the intake. Oregon's surface water source areas are delineated intake to intake. For watersheds with more than one intake, the DWSA is the watershed segment from the PWSs intake to the next intake upstream. All protection areas upstream of a specific water system's intake are included in the drinking water source area for that water system and PWSs are encouraged to work with other water providers and other entities within the Subbasin as they evaluate potential sources and move forward with developing protection strategies.

Time of travel to the intake (8-hour TOT estimate): For surface water systems that encompass an area greater than 100 square miles DEQ has also estimated the area within an 8-hour time of travel from the intake. The 8-hour time of travel distance was estimated using Extended Unit Runoff Method (EROM) for the intake's stream segment in the National Hydrography Dataset (NHDPlus V2). Input data for EROM includes runoff, temperature, precipitation and the model is calibrated using stream gage flow records. For estimating the 8-hour time of travel distance, DEQ used the NHDPlus-V2 mean annual flow estimate (V0001E) attribute which provides a calculated stream velocity in the reach at mean flow. For purposes of this assessment, the 8 -hour time of travel is calculated only for the intake's reach segment and is shown as a radial ring originating at the intake which conservative estimate of the upstream 8-hr time of travel distance. The 8-hour time of travel area is provided as a planning tool for the public water system and community since eight hours should provide adequate response time to protect the integrity of the public water system intake after a spill or release at any crossing or discharge point to the stream. Although potential risks to the water supply can exist throughout the watershed, the area within an 8-hour time of travel from the intake is the area where contamination poses the greatest threat to the drinking water supply. It is recommended the water system and community considers increased protection within this 8-hour travel time from the intake for spills and other acute risks. Focus may need to extend further upstream for contaminants that are contributed to the stream over long time periods or recur frequently.

(2) There are independent public water systems that purchase water from the water systems listed and distribute it within their service areas. The total population served listed includes these "wholesale" customers and the total number of PWSs using the source water is also provided.

\section{(3) Land Ownership/Use}

The dataset is a combination of multiple datasets and was developed by DEQ in 02/2015 and updated 03/2017. The primary dataset is from Bureau of Land Management BLM (OWNERSHIP_POLY.shp dated 06/20/2013) obtained from BLM at: http://www.blm.gov/or/gis/data-details.php?id=425. Publication date: 20130718. The dataset has been modified by grouping land owner categories in order to simplify data display on the map and using geospatial techniques to add additional data to capture the following land uses:

- agricultural land using a combination of the National Agricultural Statistics Service (NASS) data from Natural Resource Conservation Service (2007 “cdl_awifs_r_or_2007.tif”) and agricultural land zoning from OR Dept. of Land Conservation and Development (note that public water systems may obtain more detailed information on potential crop types using the US Department of Agriculture National Agricultural Statistics Service "CropScape-cropland data layer." Available at https://nassgeodata.gmu.edu/CropScape/),

- private industrial forests using Oregon Dept. of Forestry's (ODF) Private_Industrial_2006_ORLambert.shp" last updated in 2013,

- local government land combined from BLM ownership, tax lot ownership information from local county tax lot data and "OR Map" on-line application: http://www.ormap.net/,

- private urban lands based on private lands located within 2016 city limits, and

- all other categories (BLM, USFS, State, etc) from BLM 06202013 data. Note that Private urban lands may include residential, municipal, commercial, and industrial land uses. Private non-urban lands typically include rural residential land but may also include commercial and industrial land uses.

Because of the nature of combining multiple datasets, minor discrepancies will be seen in some maps especially at larger scales. Public water systems and communities could use tax lot data available from the counties or other datasets to further refine the analysis if higher accuracy is needed. 


\section{(4) High Soil Erosion Potential}

For this updated source water assessment, DEQ used three different soil evaluation methods for estimating soil erosion potential depending on the overall slope of the land surface and data availability. These datasets are as follows:

(4a) For areas with $>\mathbf{7 5} \%$ soil disturbance (such as tilled or bare soils) AND lower slopes (generally $\leq \mathbf{3 0} \%$, i.e. valleys and agricultural lands): Potential erosion rates are predicted using USDA Natural Resource Conservation Service (NRCS) Revised Universal Soil Loss Equation -2 (RUSLE2), under conditions of exposed soil lacking both plant roots and conservation practices to reduce or control erosion. The Oregon Dept. of Agriculture's Erosion Vulnerability Index (EVI) utilizes the same approach and erosion rate classifications used are from ODA's EVI documentation. The NRCS-RUSLE2/ODA-EVI method utilizes the whole soil erodibility $\left(\mathrm{K}_{\mathrm{W}}\right)$, rainfall erosivity $(\mathrm{R})$, and length and gradient of slope (LS) factors from NRCS's RUSLE with the soil cover (C) and conservation practice $(\mathrm{P})$ factors set at a value of 1 . Setting soil cover and conservation practice factors to " 1 " illustrates a worst-case scenario where soil is uncovered and exposed directly to precipitation forces and where no conservation practices are in place. Therefore, this index reflects erosion risk from severe agricultural disturbance without mitigating measures in place. It does not evaluate delivery to surface waters. In the Updated Source Water Assessments, DEQ mapped locations where RUSLE2 values are $>5$ (indicating moderate to very high erosion vulnerability), slopes are low enough for intensive agriculture ( $\leq 30 \%)$, AND within 300 feet of surface water to estimate where delivery to water is possible. Maps and data of soil qualities without the 300-foot stream buffer in local areas can be provided to public water systems and communities if additional detail or scale is needed for place-based planning.

(4b) For management activities such as silviculture, grazing, mining, urban development, fire, firebreaks, etc. with $<\mathbf{7 5 \%}$ soil surface disturbance - the risk of soil loss after disturbance activities that expose the soil surface is based on the "off-road/off-trail erosion hazard rating" developed by the USDA NRCS as shown in the Web Soil Survey online viewer (https://websoilsurvey.sc.egov.usda.gov/App/WebSoilSurvey.aspx) . Surface erosion hazard potential for non-Forest Service lands with soil disturbance is determined by combining the effects of slope and the whole soil erodibility factor ("K $\mathrm{w}_{\mathrm{w}}$-factor") using 1:24,000 Soil Survey Geographic Database (SSURGO) data. The $\mathrm{K}_{\mathrm{w}}$-factor estimates the susceptibility of soil particles to detachment and movement by water including the effects of rainfall, surface runoff, and infiltration. Soils with erosion hazard ratings of Moderate, Severe, and Very Severe are more sensitive to extensive ground disturbance such as uncontrolled grazing, forestry, heavy equipment use, fire control, mining, and urban development. In the Updated Source Water Assessments, DEQ mapped locations where erosion hazard is moderate or higher AND that are within 300 feet of surface water to estimate where delivery to water is possible. Maps and data of soil qualities without the 300 -foot stream buffer in local areas can be provided to public water systems and communities if additional detail or scale is needed for place based planning.

According to NRCS, the ratings are:

Slight-Erosion is unlikely under ordinary climatic conditions.

Moderate-Some erosion is likely; control measures may be needed.

Severe-Erosion is very likely; control measures for vegetation re-establishment on bare areas and structural measures are advised.

Very Severe-Significant erosion is expected; loss of soil productivity and off-site damages are likely; control measures are costly and generally impractical.

Note that the off-road/off-trail erosion hazard rating assumes up to $75 \%$ of the soil surface is bare. Gully erosion, plowing or other disturbances that "disturb up to nearly 100 percent of the area and change the character of the soil", and Histosol soils containing primarily organic materials are not adequately characterized by this method and effects will be underestimated. Erosion hazard from roads and trails can also be accessed using the USDA NRCS Web Soil Survey online viewer (https://websoilsurvey.sc.egov.usda.gov/App/WebSoilSurvey.aspx). Areas with $>75 \%$ disturbance and lower slopes are addressed using the NRCS-RUSLE2/ODA-EVI method - see info in 4a). 
(4c) Where NRCS SSURGO data is not available (typically National Forest lands), Soil Resource Inventory (SRI) information from the US Forest Service was used to determine erosion potential. Erosion potential for soils represented in the SRI data is based on available representative data attributes such as sedimentation yield potential, sediment, or surface soil erosion potential. Specific information on the factors used for each National Forest to evaluate sensitivity is available from DEQ upon request. In the Updated Source Water Assessments, DEQ mapped locations where soils with erosion risk is within 300 feet of surface water to estimate where delivery to water is possible.

(5) Landslide Information

OR Department of Geology and Mineral Industries (DOGAMI) Statewide Landslide Information Database of Oregon Release 3.2 (SLIDO-3.2). Includes earth and debris slides, flows, slumps, falls and complex landslide types. Does not include rock material landslide deposits. The landslide data set is published to improve the understanding of landslide hazards in Oregon and to provide a statewide base level of landslide data. This product is for informational purposes and may not have been prepared for or be suitable for legal, engineering, or surveying purposes. Users of this information should review or consult the primary data and information sources to ascertain the usability of the information. This publication cannot substitute for site-specific investigations by qualified practitioners. Site-specific data may give results that differ from the results shown in the publication. For more information see: http://www.oregongeology.org/sub/slido/

OR DEQ's Water Quality Program is currently working with DOGAMI to develop and provide a more detailed landslide potential analysis for public water systems. Contact Oregon DEQ's Water Quality Program for further information on the analysis. If data is available for the specific area, DEQ will provide the more detailed landslide analysis to the public water system.

(6) Safe Drinking Water Information System (SDWIS) data is obtained from Oregon Health Authority's Data Online available at https://yourwater.oregon.gov/.

- $\quad$ "Significant detections" indicate water quality tests with analytical results greater than the detection limit (for volatile and synthetic organic compounds (VOCs and SOCs)) or one-half of the maximum allowable contaminant level (for inorganic compounds (IOCs), arsenic and nitrate). Significant detections are not water quality violations but may require follow-up actions by the OHA Drinking Water Program. Significant detections are available as "alerts" in OHAs Data Online.

- Maximum Contaminant Level (MCL) Violations indicate samples that exceed the MCL and may be based on an average of samples or violation of a treatment technique (i.e. lead and copper rule). Maximum Contaminant levels and action levels for chemicals are available OAR 333-061-0030. Does not include violations for late/non-reporting or treatment/distribution system deficiencies.

- A full list of tested and regulated volatile organic chemicals, synthetic organic chemicals and inorganic compounds and disinfection byproducts is provided in OAR 333-061-0030 and OAR 333-061-0036. Only regulated chemicals are reported in SDWIS. It is important to note that public water system compliance data is collected after drinking water treatment, typically at the entry point to the distribution system.

(7) DEQ/OHA source water monitoring project samples were collected between 2008 and 2012 and analyzed for several hundred compounds, including Oregon-specific herbicides, insecticides, pharmaceuticals, volatile organic compounds (including cleaners), fire retardants, polycyclic aromatic hydrocarbons (organic compounds produced as byproducts of fuel burning) and plasticizers. Only the contaminants that were detected are listed. The concentrations of compounds listed were detected at very low levels well below existing standards and guidelines and are well within acceptable limits. The primary objective of this ongoing monitoring program is to identify priorities for drinking water protection through water quality data. Water quality samples are taken from raw source waters, not treated drinking water. A comprehensive list of analytical methods, compounds, and detection limits is available in each Analytical Report (search DEQ database or by request) and information is summarized at http://www.oregon.gov/deq/wq/programs/Pages/DWPAssessments.aspx 


\section{Inventory of Potential Sources of Pollution (Table 2 and Figures)}

This information is intended to supplement the original Source Water Assessment completed for the water system between 2000 and 2005 by DEQ and Oregon Health Authority. This update should be used in conjunction with the original inventory. DEQ can provide more information on potential impact, risk and status as the public water system moves into developing protection strategies.

The inventory of potential sources of pollution is based on the readily-available state and federal regulatory databases listed below and general categories of land use/ownership. The primary intent is to identify and locate significant potential sources of contaminants of concern. Areas with agricultural, septic systems, or managed forests are generally not identified in the regulated databases but are presented in the figures as a factor of land ownership/use.

It is important to remember that the sites and areas identified are only potential sources of contamination to the drinking water. Water quality impacts are not likely to occur when contaminants are used and managed properly and land use activities occur in such a way as to minimize erosion and contaminant releases.

It is highly recommended that the community "enhance" or refine the delineation of the sensitive areas and the identification of the potential contamination sources through further research and local input. If there were no potential sources of contamination identified during the review of regulatory databases or community's enhanced inventory, the water system and community should consider the potential for future development to impact the source water.

\begin{tabular}{|l|l|}
\hline Table 2 Header & Description \\
\hline $\begin{array}{l}\text { Database Identifier } \\
\text { (DB_ID) }\end{array}$ & Database Type and site name for identified potential pollutant \\
\hline $\begin{array}{l}\text { Site Identifier (Site } \\
\text { ID) }\end{array}$ & $\begin{array}{l}\text { Program specific identifier. This is the number or name used to look the site up in the } \\
\text { programs regulatory database. }\end{array}$ \\
\hline Status & Select information on the site that helps to evaluate potential risk to water quality \\
\hline $\begin{array}{l}\text { Common Name, } \\
\text { Address, City }\end{array}$ & $\begin{array}{l}\text { Common Name, Address and City as listed in the regulatory database. Note that some sites } \\
\text { may have addresses associated with responsible party, not the physical location of the site. }\end{array}$ \\
\hline County & County site is located in \\
\hline $\begin{array}{l}\text { Retrieval Date } \\
\text { (Ret_Date) }\end{array}$ & Date the information was retrieved from the individual programs regulatory database \\
\hline Data Source & Source for geographic information system (GIS) data \\
\hline State and Federal Regulatory Database Information \\
\hline & $\begin{array}{l}\text { Oregon Department of Agriculture's Confined Animal Feeding Operation database of } \\
\text { livestock owners. Includes permitted, non-permitted, and applications. Status indicates } \\
\text { facility designation and animal type. Permits typically address conditions for animal waste } \\
\text { management. More information at } \\
\text { http://www.oregon.gov/ODA/programs/NaturalResources/Pages/CAFO.aspx }\end{array}$ \\
\hline CAFO & $\begin{array}{l}\text { Oregon Department of Geology and Mineral Industries list of mining sites. Status includes } \\
\text { permit status and primary material extracted. }\end{array}$ \\
\hline DoGAMI
\end{tabular}




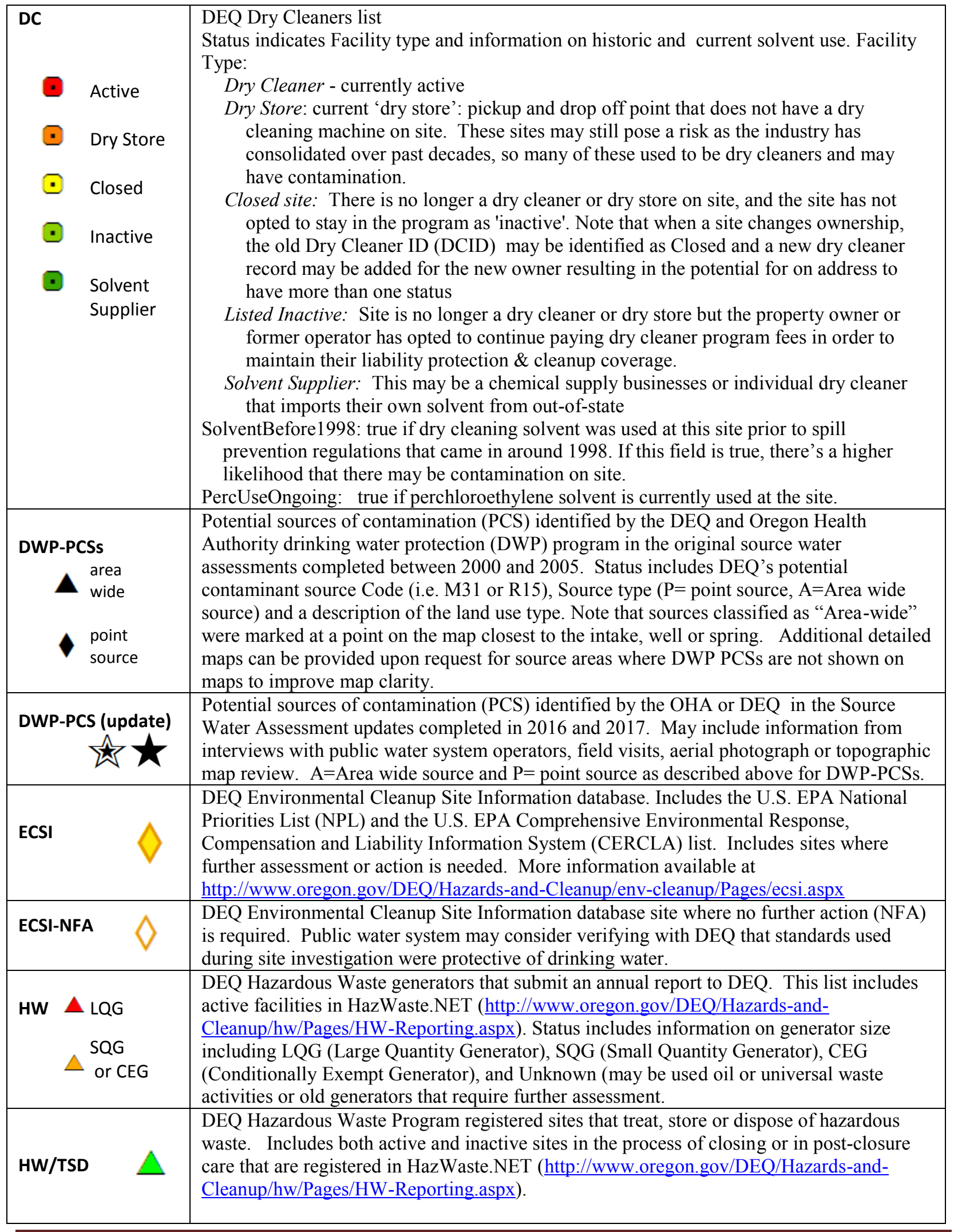




\begin{tabular}{|c|c|}
\hline LUST & $\begin{array}{l}\text { DEQ leaking underground storage tank (LUST) list - includes sites that have reported } \\
\text { releases from petroleum-containing underground storage tanks, including residential } \\
\text { heating oil tanks, regulated tanks at gas stations and other commercial facilities, and non- } \\
\text { regulated tanks. }\end{array}$ \\
\hline LUST-NFA & $\begin{array}{l}\text { DEQ leaking underground storage tank (LUST) list where no further action (NFA) is } \\
\text { required or cleanup is completed. PWS may consider verifying with DEQ that standards } \\
\text { used during site investigation were protective of drinking water. }\end{array}$ \\
\hline Oil \& Gas Wells & $\begin{array}{l}\text { Oil and Gas wells from OR Department of Geology and Mineral Industries. Only includes } \\
\text { wells with a status of "permitted". }\end{array}$ \\
\hline OSMB & Oregon State Marine Board's Boating Access Sites. \\
\hline School & $\begin{array}{l}\text { School as identified by Department of Human Services. Further evaluation may be needed } \\
\text { to identify if school has onsite/septic system, pesticide use, chemistry lab, vehicle } \\
\text { maintenance, or other potential contaminant sources. }\end{array}$ \\
\hline SFM-HSIS AST & $\begin{array}{l}\text { Aboveground storage tank(s) as identified in the State Fire Marshall Hazardous Material } \\
\text { Information System (HMIS) site list. Aboveground tanks storing gas products were not } \\
\text { included since gaseous compounds rarely pose a threat to surface water or groundwater. } \\
\text { Additional information on material stored and tank size is available upon request. }\end{array}$ \\
\hline SFM (HSIS) & $\begin{array}{l}\text { State Fire Marshall Hazardous Material Information System (HMIS) site list. Status } \\
\text { indicates number of different chemicals stored on site. A full list of chemicals with } \\
\text { information on storage type and a range of amounts is available on request. Information on } \\
\text { materials in a gas-form was not included in the chemical counts since gaseous compounds } \\
\text { rarely pose a threat to surface water or groundwater. }\end{array}$ \\
\hline $\begin{array}{l}\text { Stream } \\
\text { Crossing/Bridge }\end{array}$ & $\begin{array}{l}\text { Oregon Department of Transportation structure in the "Bridge" layer for interstates, } \\
\text { highways, or Oregon Routes. Does not include crossings over ODOT } 2012 \text { Roads layer. } \\
\text { Includes some culverts. Name indicates water body (or other structure) crossed and the } \\
\text { highway/route name. }\end{array}$ \\
\hline & $\begin{array}{l}\text { DEQ Active Solid Waste Disposal Permits list. Status includes permit type and activity } \\
\text { (active, terminated, closure, pending). Solid waste disposal site permits are issued for the } \\
\text { following facility types: landfill, solid waste treatment, transfer station/material recovery, } \\
\text { composting, incineration, conversion technology, and energy recovery. }\end{array}$ \\
\hline $\begin{array}{l}\text { UIC - } \\
\text { Stormwater }\end{array}$ & $\begin{array}{l}\text { DEQ Underground Injection Control (UIC) list of facilities with registered underground } \\
\text { injection control systems that manage Stormwater. Status includes type and number of UIC } \\
\text { wells registered. }\end{array}$ \\
\hline $\begin{array}{l}\text { UIC - Non- } \\
\text { Stormwater }\end{array}$ & $\begin{array}{l}\text { DEQ Underground Injection Control (UIC) facilities with registered underground injection } \\
\text { control systems that do not manage stormwater. Status includes type and number of UIC } \\
\text { wells registered. }\end{array}$ \\
\hline UST & $\begin{array}{l}\text { DEQ registered underground storage tank (UST) list with details on number of tanks } \\
\text { upgraded to current standards, decommissioned and with unknown status that require } \\
\text { further assessment. }\end{array}$ \\
\hline WQ SIS & $\begin{array}{l}\text { DEQ Site Information System (SIS) which includes Water Pollution Control Facility } \\
\text { (WPCF) permits where discharge to surface water is not allowed and National Pollutant } \\
\text { Discharge Elimination System (NPDES) permits for "point source" discharges into surface } \\
\text { water. Includes both individual permits (site specific) and general permits covering a } \\
\text { category of similar discharges. }\end{array}$ \\
\hline $\begin{array}{l}\text { WQ SIS- } \\
\text { WWTP }\end{array}$ & $\begin{array}{l}\text { Subset of water quality Site Information System (SIS) for domestic wastewater treatment } \\
\text { plants that discharge to surface water }\end{array}$ \\
\hline $\begin{array}{l}\text { WQ SIS } \\
\text { Outfalls }\end{array}$ & $\begin{array}{l}\text { Water quality effluent outfalls - location of permitted outfall to water body. May vary from } \\
\text { facility address or permitted activity location. }\end{array}$ \\
\hline
\end{tabular}




\begin{tabular}{|c|c|}
\hline $\begin{array}{l}\text { WQL Streams/ } \\
\text { Lakes } \\
\text { TMDL approved } \\
\text { or needed } \\
\text { Insuff- } \\
\text { icient data }\end{array}$ & $\begin{array}{l}\text { Streams and lakes identified by DEQ under Section 303(d) of the Clean Water Act as Water } \\
\text { Quality Limited and either having (Category 4A) or needing (Category 5) a Total } \\
\text { Maximum Daily Load pollutant load limit. Streams and lakes with insufficient data } \\
\text { (Category 3) to make a determination are also shown. Based on Oregon's } 2012 \text { Integrated } \\
\text { Report and 303(d) list. Contact DEQ basin coordinator for more information } \\
\text { (http://www.oregon.gov/deq/FilterDocs/basincoordinators.pdf) }\end{array}$ \\
\hline \multicolumn{2}{|c|}{ Transportation Sources } \\
\hline $\begin{array}{l}\text { Interstate/Highway } \\
\text { Interstate } \\
\text { U.S. Roads } \\
\text { Oregon Routes }\end{array}$ & $\begin{array}{l}\text { Oregon Department of Transportation interstate, highway, road or route identified in the } \\
\text { Integrated Transportation Information System database. }\end{array}$ \\
\hline Roads & $\begin{array}{l}\text { Oregon Department of Transportation } 2012 \text { Roads layer - note roads are usually mapped by } \\
\text { section so there will be many duplications of road names. }\end{array}$ \\
\hline Railways & Railways \\
\hline $\begin{array}{l}\text { Stream } \\
\text { Crossing/Bridge }\end{array}$ & $\begin{array}{l}\text { Oregon Department of Transportation structure in the "Bridge" layer for interstates, } \\
\text { highways, or Oregon Routes. Does not include crossings over ODOT } 2012 \text { Roads layer. } \\
\text { Includes some culverts. Name indicates water body (or other structure) crossed and the } \\
\text { highway/route name. }\end{array}$ \\
\hline
\end{tabular}




\section{Appendix \#3}

\section{Technical Information and Factsheets}

\section{DEQ for Water Quality}

State of Oregon

Department of

Environmental

Quality
PLEASE NOTE: The Internet URL Addresses listed in this document were included as a convenience for the users of this document. All URL Addresses were functional at the time this publication was last updated (January 2018). For active links, this list is located at http://www.oregon.gov/deq/wq/programs/Pages/DWPPubs.aspx see “A Summary of Technical Assistance Resources”

\begin{tabular}{|c|c|}
\hline \multicolumn{2}{|l|}{ General Water Quality Information } \\
\hline $\begin{array}{l}\text { Handbook for Developing Watershed Plans to } \\
\text { Restore and Protect Our Waters (EPA) }\end{array}$ & $\begin{array}{l}\text { https://www.epa.gov/polluted-runoff-nonpoint-source- } \\
\text { pollution/handbook-developing-watershed-plans-restore-and-protect }\end{array}$ \\
\hline $\begin{array}{l}\text { Water Quality Model Code and Guidebook } \\
\text { (DLCD) }\end{array}$ & http://www.oregon.gov/LCD/pages/waterqualitygb.aspx \\
\hline DEQ Toxics Reduction Strategy & $\begin{array}{l}\text { http://www.oregon.gov/deq/Hazards-and- } \\
\text { Cleanup/ToxicReduction/Pages/Reducing-Toxics.aspx }\end{array}$ \\
\hline $\begin{array}{l}\text { Oregon's Groundwater Protection Program - } \\
\text { who does what? (DEQ) }\end{array}$ & http://www.oregon.gov/DEQ/wq/programs/Pages/GWP-about.aspx \\
\hline $\begin{array}{l}\text { Groundwater Basics for Drinking Water } \\
\text { Protection (DEQ) }\end{array}$ & http://www.oregon.gov/deq/FilterDocs/dwpGwBasics.pdf \\
\hline $\begin{array}{l}\text { Protecting Oregon's Groundwater from } \\
\text { Contamination (OSU) }\end{array}$ & http://groundwater.orst.edu/groundwater/ \\
\hline Oregon Climate Change Research Institute & http://occri.net/ \\
\hline Climate Impacts in the Northwest (EPA) & $\begin{array}{l}\text { https://19january2017snapshot.epa.gov/climate-impacts/climate-impacts- } \\
\text { northwest_html }\end{array}$ \\
\hline $\begin{array}{l}\text { Climate science, data, tools, and information } \\
\text { (NOAA) }\end{array}$ & http://www.noaa.gov/climate.html \\
\hline $\begin{array}{l}\text { Harmful Algae Blooms (OHA) FAQs, } \\
\text { guidelines for lake managers and outreach } \\
\text { materials }\end{array}$ & $\begin{array}{l}\text { https://public.health.oregon.gov/HealthyEnvironments/Recreation/Harmfu } \\
\underline{\text { IAlgaeBlooms/Pages/index.aspx }}\end{array}$ \\
\hline $\begin{array}{l}\text { Harmful Algal Blooms (DEQ) - agency } \\
\text { strategy, actions to control/eliminate \& } \\
\text { prevention }\end{array}$ & http://www.oregon.gov/DEQ/wq/Pages/Harmful-Algal-Blooms.aspx \\
\hline \multicolumn{2}{|l|}{ Residential Areas, Parks and Golf Courses } \\
\hline $\begin{array}{l}\text { Domestic Well Safety Program (OHA) } \\
\text { Resources/ contacts for domestic/private wells }\end{array}$ & $\begin{array}{l}\text { http://public.health.oregon.gov/HealthyEnvironments/DrinkingWater/Sou } \\
\text { rceWater/DomesticWellSafety/Pages/index.aspx }\end{array}$ \\
\hline $\begin{array}{l}\text { Well Water Program (OSU)- tech. assistance } \\
\text { for domestic/private wells \& septic systems }\end{array}$ & http://wellwater.oregonstate.edu/ \\
\hline $\begin{array}{l}\text { Oregon's Domestic Well Testing Program for } \\
\text { Real Estate Transactions }\end{array}$ & $\begin{array}{l}\text { http://public.health.oregon.gov/HealthyEnvironments/DrinkingWater/Sou } \\
\text { rceWater/DomesticWellSafety/Pages/Testing-Regulations.aspx }\end{array}$ \\
\hline $\begin{array}{l}\text { Household Hazardous Waste Program website } \\
\text { (DEQ) }\end{array}$ & http://www.oregon.gov/DEQ/Hazards-and-Cleanup/hw/Pages/hhw.aspx \\
\hline $\begin{array}{l}\text { Household Hazardous Waste - locally- } \\
\text { sponsored and county collection programs }\end{array}$ & $\begin{array}{l}\text { http://www.oregon.gov/DEQ/Hazards-and-Cleanup/hw/Pages/HHW- } \\
\text { Events.aspx and } \\
\text { http://www.oregon.gov/DEQ/Hazards-and-Cleanup/hw/Pages/HHW-by- } \\
\text { County.aspx }\end{array}$ \\
\hline
\end{tabular}




\begin{tabular}{|c|c|}
\hline \multicolumn{2}{|c|}{ Residential Areas, Parks and Golf Courses (cont.) } \\
\hline $\begin{array}{l}\text { Household Pharmaceutical Waste Disposal } \\
\text { (OHA) }\end{array}$ & $\begin{array}{l}\text { https://public.health.oregon.gov/HealthyEnvironments/DrinkingWater/S } \\
\text { ourceWater/Pages/takeback.aspx }\end{array}$ \\
\hline Household Hazardous Wastes (EPA) & https://www.epa.gov/hw/household-hazardous-waste-hhw \\
\hline Recycle Used Motor Oil Resources (EPA) & https://www.epa.gov/recycle/managing-reusing-and-recycling-used-oil \\
\hline $\begin{array}{l}\text { Frequently Asked Questions About Heating Oil } \\
\text { Tanks (DEQ) }\end{array}$ & http://www.oregon.gov/DEQ/tanks/Pages/hot.aspx \\
\hline $\begin{array}{l}\text { Proper Care/Maintenance of Heating Oil and } \\
\text { Other Unregulated Tank Systems }\end{array}$ & http://www.oregon.gov/deq/FilterDocs/ProperCareMaintenance.pdf \\
\hline $\begin{array}{l}\text { Oregon resources for on-site septic systems } \\
\text { (DEQ) }\end{array}$ & http://www.oregon.gov/deq/Residential/Pages/Septic-Smart.aspx \\
\hline $\begin{array}{l}\text { Oregon’s Onsite Wastewater Management } \\
\text { Program (Septic Systems) (DEQ) }\end{array}$ & http://www.oregon.gov/DEQ/Residential/Pages/Onsite.aspx \\
\hline $\begin{array}{l}\text { Local Outreach Toolkit for Septic Systems } \\
\text { (EPA) }\end{array}$ & https://www.epa.gov/septic/septic-systems-outreach-toolkit \\
\hline A Homeowners Guide to Septic Systems (EPA) & http://www.nesc.wvu.edu/pdf/ww/septic/epa_septic_guide.pdf \\
\hline Septic Tank Maintenance (DEQ) & http://www.oregon.gov/deq/FilterDocs/septictankmaintFS.pdf \\
\hline Septic Systems OSU Extension website (OSU) & http://wellwater.oregonstate.edu/septic-systems-0 \\
\hline $\begin{array}{l}\text { Groundwater protection and your septic system } \\
\text { (National Small Flows Clearinghouse) }\end{array}$ & http://www.nesc.wvu.edu/pdf/ww/septic/septic_tank3.pdf \\
\hline Combating Illegal Dumping (DEQ) & $\begin{array}{l}\text { http://www.oregon.gov/DEQ/mm/Pages/Illegal-Dumping.aspx and } \\
\text { http://www.oregon.gov/DEQ/mm/Pages/lllegal-Dumping-Clean- } \\
\text { Up.aspx }\end{array}$ \\
\hline $\begin{array}{l}\text { Water Well Owner's Handbook \& other related } \\
\text { guidance documents (WRD) }\end{array}$ & http://www.oregon.gov/owrd/pages/pubs/index.aspx \\
\hline Oregon Water Resources Department & http://egov.oregon.gov/OWRD/ \\
\hline $\begin{array}{l}\text { Disposal of Chlorinated Water from Swimming } \\
\text { Pools and Hot Tubs (DEQ) }\end{array}$ & http://www.oregon.gov/deq/FilterDocs/bmpchlorwaterdisp.pdf \\
\hline $\begin{array}{l}\text { Source Water Protection Publications (EPA) for } \\
\text { managing various including: } \\
\text { Septic Systems } \\
\text { Turfgrass and Garden Fertilizer } \\
\text { Application } \\
\text { Small-Scale Application of Pesticides } \\
\text { Small Quantity Chemical Use } \\
\text { Pet and Wildlife Waste } \\
\text { Storm Water Runoff }\end{array}$ & $\underline{\text { http://www.oregon.gov/DEQ/wq/programs/Pages/DWP-Pubs.aspx }}$ \\
\hline Integrated Plant Protection Center (OSU) & http://ipmnet.org/ \\
\hline National Pesticide Information Center & http://npic.orst.edu/ \\
\hline $\begin{array}{l}\text { Integrated Pest Management and Pesticide } \\
\text { Safety for Schools (OSU) }\end{array}$ & http://www.ipmnet.org/Tim/PSEP_home.htm \\
\hline $\begin{array}{l}\text { Golf Course Integrated Pest Management (IPM) } \\
\text { tool and BMP Generator }\end{array}$ & http://www.greengolfusa.com/tiki-index.php \\
\hline $\begin{array}{l}\text { EcoBiz Certified Landscapers and Auto Repair } \\
\text { Shops }\end{array}$ & http://ecobiz.org/find-an-ecobiz/ \\
\hline
\end{tabular}




\begin{tabular}{|c|c|}
\hline \multicolumn{2}{|l|}{ Agriculture / Forestry Land Uses } \\
\hline $\begin{array}{l}\text { Tips for Small Acreages in Oregon (NRCS) - } \\
\text { Fact Sheets on wells, septic systems, animals, } \\
\text { crops, weeds, streamside erosion protection. } \\
\text { Includes specific factsheets for Eastern and } \\
\text { Western Oregon. }\end{array}$ & $\begin{array}{l}\text { https://www.nrcs.usda.gov/wps/portal/nrcs/detail/national/people/?cid=s } \\
\text { telprdb1167242 }\end{array}$ \\
\hline $\begin{array}{l}\text { Source Water Protection Pubs (EPA) for } \\
\text { managing various activities including: } \\
\text { Agricultural Fertilizer Application } \\
\text { Large-Scale and Small-Scale } \\
\text { Application of Pesticides } \\
\text { Livestock, Poultry and Horse Waste } \\
\text { Above Ground and Underground } \\
\text { Storage Tanks } \\
\text { Small Quantity Chemical Use } \\
\text { Turfgrass and Garden Fertilizer } \\
\text { Application }\end{array}$ & $\underline{\text { http://www.oregon.gov/DEQ/wq/programs/Pages/DWP-Pubs.aspx }}$ \\
\hline $\begin{array}{l}\text { Oregon Small Farms (OSU Extension) } \\
\text { Information on Crops, Grains, Livestock, } \\
\text { Pastures, and Soils (see tabs at top of page for } \\
\text { multiple resources) }\end{array}$ & http://smallfarms.oregonstate.edu/ \\
\hline $\begin{array}{l}\text { Oregon Pesticide Stewardship Partnerships and } \\
\text { Waste Pesticide Collection Events }\end{array}$ & $\begin{array}{l}\text { http://www.oregon.gov/oda/programs/pesticides/water/pages/pesticidest } \\
\text { ewardship.aspx }\end{array}$ \\
\hline Managing Waste Pesticide (DEQ) & $\begin{array}{l}\text { http://www.oregon.gov/DEQ/Hazards-and- } \\
\text { Cleanup/hw/Pages/Miscellaneous-Industries.aspx }\end{array}$ \\
\hline $\begin{array}{l}\text { Oregon Department of Agriculture (ODA) - } \\
\text { resources for reducing impacts }\end{array}$ & http://www.oregon.gov/oda/Pages/default.aspx \\
\hline $\begin{array}{l}\text { Soil and Water Conservation Districts (OACD) } \\
\text { - technical assistance for rural landowners, } \\
\text { family forests and growers }\end{array}$ & http://oacd.org/conservation-districts/directory \\
\hline $\begin{array}{l}\text { Natural Resources Conservation Service, } \\
\text { Oregon (NRCS) }\end{array}$ & http://www.or.nrcs.usda.gov/ \\
\hline NRCS Financial Assistance Programs & http://www.nrcs.usda.gov/wps/portal/nrcs/main/or/programs/financial/ \\
\hline $\begin{array}{l}\text { Oregon Department of Fish and Wildlife } \\
\text { Hatchery Information (ODFW) }\end{array}$ & http://www.dfw.state.or.us/fish/hatchery/ \\
\hline $\begin{array}{l}\text { Animal Care and Handling Facilities (from } \\
\text { California stormwater program) }\end{array}$ & $\begin{array}{l}\text { https://www.casqa.org/sites/default/files/BMPHandbooks/BMP_IndCo } \\
\text { mm_Appendix_D.pdf }\end{array}$ \\
\hline Managing Small-acreage Horse Farms (OSU) & https://catalog.extension.oregonstate.edu/ec1558/viewfile \\
\hline Irrigation well use and maintenance & $\begin{array}{l}\text { See resources for domestic wells under Information for Residential } \\
\text { Areas }\end{array}$ \\
\hline $\begin{array}{l}\text { Oregon State University Forestry \& Natural } \\
\text { Resources Extension Program }\end{array}$ & http://extensionweb.forestry.oregonstate.edu/ \\
\hline $\begin{array}{l}\text { Oregon Department of Forestry Stewardship } \\
\text { Foresters }\end{array}$ & http://www.oregon.gov/ODF/Working/Pages/FindAForester.aspx \\
\hline $\begin{array}{l}\text { Oregon Department of Forestry Grants and } \\
\text { Incentives }\end{array}$ & http://www.oregon.gov/ODF/AboutODF/Pages/GrantsIncentives.aspx \\
\hline $\begin{array}{l}\text { US Department of Agriculture Forest Incentive } \\
\text { Programs Available in Oregon }\end{array}$ & http://www.srs.fs.usda.gov/econ/data/forestincentives/or.htm \\
\hline
\end{tabular}




\begin{tabular}{|c|c|}
\hline \multicolumn{2}{|l|}{ Agriculture / Forestry Land Uses } \\
\hline $\begin{array}{l}\text { US Department of Agriculture Pacific Northwest } \\
\text { Research Station }\end{array}$ & http://www.fs.fed.us/pnw/ \\
\hline $\begin{array}{l}\text { US Forest Service State \& Private Forestry- } \\
\text { Cooperative Forestry, Forest Health Protection, } \\
\text { Sustainable Development \& Urban/ Community } \\
\text { Forestry }\end{array}$ & http://www.fs.fed.us/spf/ \\
\hline $\begin{array}{l}\text { Water quality impacts information from US } \\
\text { Forest Service - Part III: Chapter 10: Forest } \\
\text { Management; Chapter 13: Pesticides and Part } \\
\text { IV: Chapter 14-16 Animals }\end{array}$ & http://www.srs.fs.fed.us/pubs/gtr/gtr_srs039/ \\
\hline $\begin{array}{l}\text { National Management Measures to Control } \\
\text { Nonpoint Source Pollution from Forestry (EPA) }\end{array}$ & http://water.epa.gov/polwaste/nps/forestry/forestrymgmt_index.cfm \\
\hline $\begin{array}{l}\text { Managing Nonpoint Source Pollution from } \\
\text { Forestry (EPA) }\end{array}$ & $\begin{array}{l}\text { https://www.epa.gov/polluted-runoff-nonpoint-source- } \\
\text { pollution/forestry-additional-resources }\end{array}$ \\
\hline Oregon Forest Practices Act & https://www.oregon.gov/ODF/Working/Pages/FPA.aspx \\
\hline $\begin{array}{l}\text { Forest Practices Board Manual (Washington } \\
\text { Dept. of Natural Resources) }\end{array}$ & $\begin{array}{l}\text { http://www.dnr.wa.gov/about/boards-and-councils/forest-practices- } \\
\text { board/rules-and-guidelines/forest-practices-board-manual }\end{array}$ \\
\hline $\begin{array}{l}\text { Sustainable Forest Management } \\
\text { Programs/Certifications: }\end{array}$ & $\begin{array}{l}\text { https://www.oregon.gov/ODF/Documents/AboutODF/ForestCertificatio } \\
\text { nFactsheet.pdf }\end{array}$ \\
\hline American Tree Farm Systems (ATFS) & https://www.treefarmsystem.org/ \\
\hline Forest Stewardship Council (FSC) & https://us.fsc.org/en-us/certification \\
\hline Sustainable Forestry Initiative (SFI) & http://www.oregonsfi.org/ \\
\hline Dovetail Partners, Inc. & http://www.dovetailinc.org/ \\
\hline \multicolumn{2}{|l|}{ Commercial / Industrial / Municipal Land Uses } \\
\hline $\begin{array}{l}\text { Drinking Water Protection Strategies for } \\
\text { Commercial \& Industrial Land Uses (DEQ) }\end{array}$ & http://www.oregon.gov/deq/FilterDocs/DWPStrategiesComInd.pdf \\
\hline $\begin{array}{l}\text { Business and Industry tips for reducing water } \\
\text { quality impacts (DEQ) }\end{array}$ & http://www.oregon.gov/deq/FilterDocs/dwpbusindtips.pdf \\
\hline $\begin{array}{l}\text { Source Water Protection Publications (EPA) for } \\
\text { managing various including: } \\
\text { Above Ground and Underground } \\
\text { Storage Tanks } \\
\text { Aircraft and Airfield Deicing } \\
\text { Operations } \\
\text { Highway Deicing Operations } \\
\text { Vehicle Washing } \\
\text { Pet and Wildlife Waste } \\
\text { Small Quantity Chemical Use } \\
\text { Storm Water Runoff } \\
\end{array}$ & $\underline{\text { http://www.oregon.gov/DEQ/wq/programs/Pages/DWP-Pubs.aspx }}$ \\
\hline $\begin{array}{l}\text { Free Assistance from DEQ’s Toxics Use and } \\
\text { Waste Reduction Assistance }\end{array}$ & http://www.oregon.gov/deq/FilterDocs/TABrochure.pdf \\
\hline $\begin{array}{l}\text { Managing Used Computers and Other Electronic } \\
\text { Equipment (DEQ) }\end{array}$ & http://www.oregon.gov/DEQ/ecycles/Pages/default.aspx \\
\hline $\begin{array}{l}\text { Computer and Electronic Equipment Recyclers } \\
\text { (DEQ) }\end{array}$ & http://www.deq.state.or.us/ecsearch/Default.aspx \\
\hline $\begin{array}{l}\text { Underground Injection Control (UIC) Program } \\
\text { (DEQ) }\end{array}$ & http://www.oregon.gov/deq/wq/wqpermits/Pages/UIC.aspx \\
\hline
\end{tabular}




\begin{tabular}{|c|c|}
\hline \multicolumn{2}{|c|}{ Commercial / Industrial I Municipal Land Uses (cont.) } \\
\hline $\begin{array}{l}\text { Industrial Stormwater Best Management } \\
\text { Practices Manual (DEQ) }\end{array}$ & $\begin{array}{l}\text { http://www.oregon.gov/deq/FilterDocs/wq/wqpermit/docs/IndBMP02141 } \\
\text { 3.pdf }\end{array}$ \\
\hline $\begin{array}{l}\text { Illicit Discharge and Source Tracing Guidance } \\
\text { Manual (Washington Stormwater Center) }\end{array}$ & http://www.wastormwatercenter.org/illicit-connection-illicit-discharge \\
\hline $\begin{array}{l}\text { Construction Stormwater Best Management } \\
\text { Practices Manual (DEQ) }\end{array}$ & http://www.deq.state.or.us/wq/stormwater/docs/nwr/constrbmps.pdf \\
\hline $\begin{array}{l}\text { Low Impact Development O\&M guidance } \\
\text { (Washington Stormwater Center) }\end{array}$ & http://www.wastormwatercenter.org/lid-mando/ \\
\hline $\begin{array}{l}\text { Water quality impacts information from USFS } \\
\text { - Part V: Chapter 18-20 Mining and Oil/Gas }\end{array}$ & $\underline{\text { http://www.srs.fs.fed.us/pubs/gtr/gtr_srs039/ }}$ \\
\hline $\begin{array}{l}\text { Dam Safety Publications and Resources } \\
\text { FEMA website }\end{array}$ & https://www.fema.gov/dam-safety-publications-resources \\
\hline $\begin{array}{l}\text { Healthcare: Pollution Prevention \& Best } \\
\text { Management Practices (EPA) }\end{array}$ & http://www3.epa.gov/region9/waste/p2/hospart.html \\
\hline \multicolumn{2}{|l|}{ Boating / Marinas / Recreation Areas } \\
\hline Oregon Clean Boater Program (OSMB) & http://www.oregon.gov/OSMB/boater-info/Pages/Clean-Boater.aspx \\
\hline Clean Boater Guide (OSMB) & $\begin{array}{l}\text { http://www.oregon.gov/OSMB/boater- } \\
\text { info/Documents/2015_osmb_clean_boater_guide forweb.pdf }\end{array}$ \\
\hline $\begin{array}{l}\text { Best Management Practices for Oregon’s } \\
\text { Marinas (DEQ) }\end{array}$ & http://www.oregon.gov/deq/FilterDocs/marinas.pdf \\
\hline Clean Marina Program (OSMB) & http://www.oregon.gov/OSMB/boater-info/Pages/Clean-Marinas.aspx \\
\hline Clean Marina Guidebook (OSMB) & $\begin{array}{l}\text { http://www.oregon.gov/OSMB/forms- } \\
\text { library/Documents/Environmental/entire_clean_marina_guidebook.pdf }\end{array}$ \\
\hline $\begin{array}{l}\text { Marine Sewage and Wastewater Disposal } \\
\text { (DEQ) }\end{array}$ & http://www.oregon.gov/OSMB/Pages/Pumpout-and-Dump-Stations.aspx \\
\hline $\begin{array}{l}\text { Water quality impacts information from US } \\
\text { Forest Service - Part II: Chapters 7-8: } \\
\text { Recreation; Chapter 5: Dams and Chapter 9: } \\
\text { Roads }\end{array}$ & http://www.srs.fs.fed.us/pubs/gtr/gtr_srs039/ \\
\hline
\end{tabular}




\section{Appendix \#4}

\section{Funds and Resources for Drinking Water Source Protection}

This document provides brief descriptions and contact information for resources available to public water systems, including grants and loans to fund drinking water infrastructure and source protection projects. DEQ's list of "Technical Information and Factsheets for Water Quality Protection" provides other websites and resources available to public water systems and community members seeking to work on watershed protection.

Note: The Internet links listed in this document were included as a convenience for the users of this document. All URL Addresses were functional at the time this publication was last updated (January 2018).

\section{Oregon Health Authority (OHA)}

Drinking Water Services

Phone: 971-673-0405

Website: www.healthoregon.org/dwp

The Oregon Health Authority (OHA) is the primacy agency for the implementation of the federal Safe Drinking Water Act (SWDA) in Oregon. ORS 338.277 authorizes the OHA to administer the federal Safe Drinking Water Act in Oregon as the Primacy Agency in agreement with the federal government. ORS 448.131 further authorizes the adoption of standards necessary to protect public health through insuring safe drinking water within a water system. Standards in OAR 333-061 outlines requirements for systems to meet MCLs, submit to periodic inspections, and meet enforcement requirements as administered by OHA. As the primacy agency, OHA also approves drinking water treatment plans and sets construction standards, operator certification standards, and enforces rules to ensure safe drinking water. The OHA website has extensive information on drinking water treatment requirements: http://healthoregon.org/dwp

In order to assist systems in complying with standards, OHA also provides technical assistance and oversight of grants and loans from the Safe Drinking Water Act for public water system operation and improvements. For those Safe Drinking Water Act loans and grant funds, the Oregon Health Authority partners with Oregon Infrastructure Finance Authority to provide the financial services (see below).

\section{Business Oregon - Infrastructure Finance Authority (IFA)}

Infrastructure Finance Authority (IFA)

Municipal Infrastructure Funding

Phone: (503) 986-0123

Website: www.orinfrastructure.org

IFA is a division of Business Oregon that provides funding for municipally owned infrastructure projects. IFA manages federal infrastructure funds for agencies such as Oregon Health Authority and Housing and Urban Development. IFA is not a regulatory agency but collaborates and supports our state and federal partners with financing programs and technical assistance. 
The list of available funding programs for drinking water infrastructure and source protection is:

- $\quad$ Safe Drinking Water Revolving Loan Fund (SDWRLF)

- Drinking Water Source Protection Fund (DWSP)

- Water/Wastewater Financing Program (WWFP)

- Special Public Works Fund (SPWF)

- Community Development Block Grant Program (CDBG)

- $\quad$ Port Revolving Loan Fund (PRLF)

\section{Safe Drinking Water Revolving Loan Fund (SDWRLF)}

This loan program funds drinking water system improvements needed to maintain compliance with the Federal Safe Drinking Water Act. The Safe Drinking Water Fund is funded by annual grants from the U.S. Environmental Protection Agency (EPA) and matched with funds from the state Water/Wastewater Financing Program. The program is managed by the Oregon Health Authority (OHA), Drinking Water Services. The loans are managed by the Oregon Infrastructure Finance Authority (IFA).

The Safe Drinking Water Revolving Loan Fund (SDWRLF) is designed for water source, treatment, distribution, storage and related infrastructure projects. Funding is available for all sizes of water systems, although 15 percent of the funds are reserved for systems serving a population of fewer than 10,000. Eligible applicants can be owners of water systems that provide service to at least 25 year-round residents or systems that have 15 or more connections (or a nonprofit with 25 or more regular users). Owners can be a nonprofit, private party or municipality, but systems cannot be federally owned or operated.

To be eligible for funding, a project must solve an existing or potential health hazard or noncompliance issue under federal/state water quality standards. The following are the main types of eligible activities:

- Engineering, design, upgrade, construction or installation of system improvements and equipment for water intake, filtration, treatment, storage, transmission

- Acquisitions of property or easements

- Planning, surveys, legal/technical support and environmental review

- Investments to enhance the physical security of drinking water systems, as well as water sources

SDWRLF loan amount: The program provides up to \$6 million per project (more with additional approval) with the possibility of subsidized interest rate and principal forgiveness for a Disadvantaged Community. The standard loan term is 20 years or the useful life of project assets, whichever is less, and may be extended up to 30 years under SDWRLF for a Disadvantaged Community. Interest rates are 80 percent of state/local bond index rate.

To apply, the municipality should first submit a Letter of Interest to Oregon Health Authority where it will be rated and ranked. Call Oregon OHA Drinking Water Services at 971-673-0422 or go to the OHA website: www.healthoregon.org/srf

Projects placed on the Project Priority List will be invited to apply through IFA for funding. Contact your IFA Regional Coordinator for assistance and more information. Call IFA at 503-986-0123 or

http://www.orinfrastructure.org/

\section{Drinking Water Source Protection Fund (DWSP)}

From the Safe Drinking Water Act, loans and grants are also available for drinking water protection projects: low interest loans up to a maximum of $\$ 100,000$ per project, and grant funds up to $\$ 30,000$ per water system. Eligible systems include any public and privately-owned Community and Nonprofit Non-Community water systems with a completed Source Water Assessment are able to demonstrate a direct link between the proposed project and maintaining or improving drinking water quality. Eligible activities include those that lead to risk reduction within the delineated source water area or would contribute to a reduction in contaminant concentration within the drinking water source. Projects can take either a local or regional approach. Local projects are defined as activities that concentrate on a public water system's source area(s). Regional projects are defined as activities that involve multiple communities and/or water systems attempting to address a common source water issue or group of issues. 
The categories for eligible projects for DW Source Protection funding include the following:

Refined Delineation OHA and DEQ have completed delineations for most drinking water source areas (DWSA) for the community and non-community public water systems. DWSAs include aquifer recharge areas for groundwater sources and watershed areas for surface sources. DW Source Protection funding can be used to complete, update, or refine DWSA delineations using new or additional site-specific information as part of a more comprehensive protection strategy.

\section{Updated Assessment}

Inventory - Projects that improve upon existing potential contaminant source inventories available from the DEQ database, Geographic Information System, and Assessment Reports prepared by OHA/DEQ. A project could involve expanding or updating the inventory of land uses or existing and potential point and non-point contaminant sources.

Evaluation - Projects establishing a water quality monitoring project to evaluate existing and potential threats to water quality. This could include evaluating and prioritizing potential threats (or protection activities) based upon new or more detailed information.

\section{Source Protection Planning}

Projects designed to identify appropriate protection measures, including development of a comprehensive DW Source Protection plan, educational projects, projects to identify and ensure implementation of Best Management Practices (BMPs), development of local DW Source Protection ordinances, development of restoration or conservation plans for the source area for future easement or land acquisition.

\section{Implementation}

Funds can be used to implement many types of protection strategies in drinking water source areas. This can include implementation of any eligible activities that will reduce risks within the source water area or would contribute to a reduction of contaminant concentration within the drinking water source(s). Examples of the types of projects that can be funded include:

- Implementing drug-take-back projects in source areas

- Projects for reducing pesticide application rates and loadings in source area

- Implementing pesticide and household hazardous waste collection events

- Closure of high-risk abandoned or unused (private or irrigation) wells close to supply well

- Projects for reforestation or replanting in sensitive or riparian areas

- Installation of fencing to protect sensitive riparian source areas

- Installation of signs at boundaries of zones or protection areas

- Projects for assessing risks from onsite systems near supply wells, inspections, pump-outs, or decommissioning onsite systems.

- Secondary containment for high-risk ABOVE ground tanks

- Focused workshop events for household/business instruction for changing to alternative nonhazardous product usage ("green chemical" products)

- Seismic spill prevention or inspection project in proximate areas for high-risk sources

- Permanent abandonment (i.e. filling) of inadequately constructed private wells within the source area

- Installation of fencing around the immediate intake or well area to provide protection

- Structures to divert contaminated stormwater runoff affecting the source area

- Set up ecosystem services (or similar) project in watershed to fund preservation areas

- Implementation of pollution prevention or waste reduction projects

- Restoration and/or conservation projects within the drinking water source area

- Implementation of water reuse and other conservation measures related to source protection

- Implementation of best management practice projects

- Implementation of conservation easements to protect sensitive source areas

- Implementation of a drinking water source protection ordinance

- Establishing management plans for easements or lands purchased within source areas

- Development of educational flyers/brochures for purposes of public education 
- Purchase of lands within the drinking water source area (funded only via low interest loans)

Any Public and Privately-owned Community and Nonprofit Non-Community water systems with a completed Source Water Assessment are eligible for funds. A “community water system” is defined as a public water system that has 15 or more service connections used by year-round residents, or which regularly serves 25 or more year-round residents. This includes water systems that are owned privately, by non-profit or public entities such as a city, district, or port. A "nonprofit non-community water system" is a public water system that is not a community water system and that regularly serves at least 25 people (more than 6 months per year) and is legally recognized under Oregon law as a nonprofit entity.

For the source water protection funds, contact OHA regarding the letter of interest submittal schedule. Call Oregon OHA Drinking Water Services at 971-673-0422 or go to the OHA website: www.healthoregon.org/srf or contact IFA at 503-986-0123; www.orinfrastructure.org

\section{Water/Wastewater Funding Program (WWFP)}

This loan program funds the design and construction of public infrastructure needed to ensure compliance with the Safe Drinking Water Act or the Clean Water Act. The public entities that are eligible to apply for the program are cities, counties, county service districts, tribal councils, ports, and special districts as defined in ORS 198.010. Municipalities must either have a documented compliance issue or the potential of a compliance issue in the near future.

Allowable funded project activities may include:

- Construction costs, including Right of Way and Easements, for improvement or expansion of drinking water, wastewater or stormwater systems

- Design and construction engineering

- Planning/technical assistance for small communities

\section{WWFP Loans}

The maximum loan term is 25 years or the useful life of the infrastructure financed, whichever is less. The maximum loan amount is $\$ 10$ million per project (more with additional approval) through a combination of direct and/or bond funded loans. Loans are generally repaid with utility revenues or voter approved bond issues. A limited tax general obligation pledge also may be required. "Credit worthy" borrowers may be funded through the sale of state revenue bonds.

\section{WWFP Grants}

Grant awards up to $\$ 750,000$ may be awarded based on a financial review. An applicant is not eligible for grant funds if the applicant's annual median household income is equal or greater than 100 percent of the state average median household income for the same year.

\section{Funding for Technical Assistance}

The Infrastructure Finance Authority offers technical assistance financing for municipalities with populations of less than 15,000. The funds may be used to finance preliminary planning, engineering studies and economic investigations. Technical assistance projects must be in preparation for a construction project that is eligible and meets the established criteria.

Grants up to $\$ 20,000$ may be awarded per project.

Loans up to $\$ 60,000$ may be awarded per project.

To apply, call IFA at 503-986-0123, then contact your IFA Regional Coordinator for assistance and more information. http://www.orinfrastructure.org/

\section{Special Public Works Fund (SPWF)}

The Special Public Works Fund (SPWF) provides funds for publically owned facilities that support economic and community development in Oregon. Funds are available to public entities for planning, design, purchasing, improving and constructing publically owned facilities, replacing publically owned essential community facilities, emergency projects as a result of a disaster, and for planning. Public agencies that are eligible to apply for 
funding are cities, counties, county service districts (ORS 451), tribal councils, ports, districts as defined in ORS 198.010, and airport districts (ORS 838).

\section{SPWF Loans}

Loans for development (construction) projects range from less than $\$ 100,000$ to $\$ 10$ million (more with additional approval). The Infrastructure Finance Authority offers very attractive interest rates that reflect taxexempt market rates for highly qualified borrowers. Initial loan terms can be up to 25 years or the useful life of the project, whichever is less.

\section{SPWF Grants}

Grants are available for construction projects that create or retain traded-sector jobs. They are limited to $\$ 500,000$ or 85 percent of the project cost, whichever is less, and are based on up to $\$ 5,000$ per eligible job created or retained. Limited grants are available to plan industrial site development for publically owned sites and for feasibility studies.

To apply, call IFA at 503-986-0123, then contact your IFA Regional Coordinator for assistance and more information. http://www.orinfrastructure.org/

\section{Community Development Block Grant (CDBG)}

Grants and technical assistance are available to develop livable urban communities for persons of low and moderate incomes by expanding economic opportunities and providing housing and suitable living environments. Non-metropolitan cities and counties in rural Oregon can apply for and receive grants. [Oregon tribes, urban cities (Albany, Ashland, Bend, Corvallis, Eugene, Gresham, Hillsboro, Medford, Portland, Salem and Springfield) and counties (Clackamas, Multnomah, Washington) receive funds directly from HUD.] Funding amounts are based on the applicant's need, the availability of funds, and other restrictions defined in the program's guidelines. The maximum available grant for drinking water system projects is $\$ 3,000,000$.

All projects must meet one of three national objectives:

- The proposed activities must benefit low- and moderate-income individuals.

- The activities must aid in the prevention or elimination of slums or blight.

- There must be an urgent need that poses a serious and immediate threat to the health or welfare of the community.

To apply, call IFA at 503-986-0123, then contact your IFA Regional Coordinator for assistance and more information. http://www.orinfrastructure.org/

\section{Port Revolving Loan Fund (PRLF)}

The Port Revolving Loan Fund (PRLF) is a loan program to assist Oregon ports in the planning and construction of facilities and infrastructure. Ports must be incorporated under ORS Chapter 777 or 778 . The Fund may be used for port development projects (facilities or infrastructure) or to assist port-related private business development projects. The variety of eligible projects is very broad and may include water-oriented facilities, industrial parks, airports and commercial or industrial developments. Eligible project costs can include engineering, acquisition, improvement, rehabilitation, construction, operation, and maintenance or pre-project planning. Projects must be located within port district boundaries. The maximum loan amount is \$3 million at any one time. The loan term can be as long as 25 years or the useful life of the project, whichever is less. Interest rates are set by the IFA at market rates, but not less than Treasury Notes of a similar term minus one percent.

Note: Flexible manufacturing space projects will not accrue interest until the building is at least 25 percent occupied or until three years after the date of the loan contract, whichever is earlier.

To apply, call IFA at 503-986-0123, then contact your IFA Regional Coordinator for assistance and more information. http://www.orinfrastructure.org/ 


\section{Oregon Department of Environmental Quality (DEQ)}

\section{Clean Water State Revolving Fund (CWSRF)}

Clean Water State Revolving Fund

503-229-6412

Website: http://www.oregon.gov/DEQ/wq/cwsrf/Pages/default.aspx

Low-cost loans for planning, design, and construction projects to attain and maintain water quality standards, and necessary to protect beneficial uses such as fish habitat, drinking water sources, irrigation, and recreation. Eligible borrowers are public entities, such as cities and counties, Indian tribal governments, sanitary districts, soil and water conservation districts, irrigation districts, various special districts and some intergovernmental entities. CWSRF offers:

- Low-cost loans and bond purchases

- Lower than market interest rates

- Fixed interest rates

- Terms up to 30 years

- Up to $100 \%$ of eligible costs covered

- No match required

- Repayment begins after project is constructed

- No pre-payment penalty

- Additional financial incentives, including principle forgiveness

Applications are accepted year round with scheduled review and ranking in the first week of January, May and September. Contact the Oregon Department of Environmental Quality (DEQ); for a list of CWSRF project officers, go to http://www.oregon.gov/deq/wq/cwsrf/Pages/CWSRF-Contacts.aspx.

Financial incentives make CWSRF loans worth exploring. Principle forgiveness is available for communities meeting affordability criteria, or for meeting green project criteria. Implement a non-planning nonpoint source project and a traditional point source wastewater treatment project through the same application to reduce your interest rate on the combined two projects to as low as $1 \%$. This combined application is called a sponsorship option.

\section{CWSRF Pollution Reduction Funding}

The Clean Water State Revolving Fund loan program provides low-cost loans to public entities for the planning, design or construction of both point source and nonpoint source projects that prevent or mitigate water pollution. Wastewater facility improvements and stormwater management projects are funded with CWSRF.

CWSRF loans fund development of nonpoint source water quality improvement plans, such as an integrated water resources plan and a regional or municipality-wide stormwater management plan. Planning loans can also fund the establishment of watershed partnerships, local ordinances to implement a stormwater master/management plan, engineering and development standards for new and redevelopment, permanent riparian buffers, floodplains, wetlands and other natural features.

CWSRF offers a Local Community Loan, which allows the borrower to make loans to private entities like home owners and farmers. The Local Community Loans fund the repair and replacement of failing decentralized systems. This loan type can also fund nonpoint source agricultural best management practices such as building manure containment structures, manure digesters, and fences to protect riparian resources capture and convert methane, and purchase calibrated application equipment.

CWSRF loans fund a variety of nonpoint source watershed improvement implementation projects such as establishing or restoring permanent riparian buffers and floodplains, and daylighting streams from pipes. Loans can fund protecting and restoring streamside areas, wetlands and floodplains, and to acquire riparian land, wetlands, conservation easements, and land to protect drinking water sources.

More information on DEQ’s Clean Water State Revolving Fund program can be found here: http://www.oregon.gov/DEQ/wq/cwsrf/Pages/default.aspx. For specific information on the Sponsorship Option, Planning Loans, Nonpoint Source Loans, or Local Community Loans, see 
http://www.oregon.gov/DEQ/wq/cwsrf/Pages/CWSRF-Application.aspx. The application requirements for CWSRF loans may take some lead-time to develop and may require out-of-pocket expense to prepare.

Prospective CWSRF applicants should discuss any questions about the required content of these items with a regional DEQ CWSRF Project Officer at the earliest opportunity

(http://www.oregon.gov/DEQ/wq/cwsrf/Pages/CWSRF-Contacts.aspx).

\section{Supplemental Environmental Projects (SEPs)}

Supplemental Environmental Projects are administered by DEQ’s Office of Compliance and Enforcement. When DEQ assesses civil penalties for environmental law violations, violators can offset up to $80 \%$ of their monetary penalty by agreeing to pay for a Supplemental Environmental Project that improves Oregon's environment. SEPs can be for pollution prevention or reduction, public health protection, environmental restoration and protection as long as it is a project that the respondent is not already required to do by law or where the project would be financially self-serving for the respondent. The work can be completed by a third-party like a local government, watershed council, non-profit or private entity. Coastal PWSs can develop a "SEP Application" with general information that OCE can distribute to respondents. Community organizations with proposed projects are also free to contact respondents on their own initiative. The enforcement case does not necessarily have to be in the same area (watershed/county, etc.) as the environmental project or even address the same media (i.e. air/water/land). Interested parties can sign up for DEQ’s public notifications via email at http://www.oregon.gov/deq/Get-Involved/Pages/Public-Notices.aspx - when signing up, select types of information (select "enforcement actions") and which counties or subbasins are of interest.

\section{Nonpoint Source Implementation 319 Grants}

Nonpoint Source Grants support implementation and planning projects that address water quality problems in surface and groundwater resources resulting from nonpoint source pollution. Funds are appropriated by DEQ through the U.S. Environmental Protection Agency under Section 319 of the Clean Water Act and support a wide variety of management activities, including technical assistance, site assessment, public awareness and education, training, technology transfer, demonstration projects, and monitoring to assess the success of specific nonpoint source implementation projects. Eligible applicants include government agencies, tribal nations and nonprofit organizations. For more information including funding availability, eligible projects, and application requirements and timelines see http://www.oregon.gov/deq/wq/programs/Pages/Nonpoint-319-Grants.aspx

\section{Oregon Water Resources Department (WRD)}

Water Resources Development Program

725 Summer Street NE, Suite A

Salem, OR 97301

Phone: 503-986-0900

The Water Resources Department is the state agency charged with administration of the laws governing surface and ground water resources. The Department's core functions are to protect existing water rights, facilitate voluntary streamflow restoration, increase the understanding of the demands on the state's water resources, provide accurate and accessible water resource data, and facilitate water supply solutions. WRD is charged with carrying out the water management policies and rules set by the Water Resources Commission and with overseeing the enforcement of Oregon's water laws. By law, all surface and ground water in Oregon belongs to the public.

WRD's mission is to serve the public by practicing and promoting responsible water management through two key goals:

- to directly address Oregon's water supply needs, and

- to restore and protect streamflows and watersheds in order to ensure the long-term sustainability of Oregon's ecosystems, economy, and quality of life.

WRD developed Oregon's 2012 Integrated Water Resources Strategy to help individuals and communities address instream and out-of-stream needs now and into the future, including water quantity, water quality and 
ecosystem needs. More information can be found at:

https://www.oregon.gov/owrd/Pages/law/integrated_water_supply_strategy.aspx

There is funding available to support planning, feasibility studies, and implementation of water projects:

\section{Place-Based Integrated Water Resources Planning}

Place-based planning is a voluntary, locally initiated and led effort in which a balanced representation of water interests within a basin or watershed work in partnership with the state to: characterize current water resources and issues (water quantity, water quality, ecosystem health); understand current and future instream and out-of-stream water needs and demands; identify and prioritize strategic solutions to address water needs; and, develop a place-based integrated water resources plan that informs the statewide strategy.

Recent cycle of funding included \$750,000 in grants; requires $25 \%$ cost-share.

For more information, contact Harmony Burright at 503-986-0913.

\section{Feasibility Study Grants}

Once potential projects are identified, communities often find it difficult to secure funding to assess their viability. This program component addresses that need by providing grant funding to cover $50 \%$ of the cost of conducting feasibility studies for potential water conservation, storage and reuse projects. A feasibility study is an assessment of the practicality of a proposed project or plan and can be used to determine if and how a project should proceed to the implementation phase.

Recent cycle of funding included \$2.8 million in grants; 50\% cost share required.

For more information, contact Jon Unger at 503-986-0869.

\section{Water Project Grants \& Loans (formerly Water Supply Development Grants \& Loans)}

This account provides grants and loans to evaluate, plan and implement instream and out-of-stream water development projects that have economic, environmental and social/cultural benefits. Eligible projects include, but are not limited to projects that: increase water use efficiency; develop new or expanded storage; allocate federally stored water; promote water reuse or conservation; and protect or restore stream flows.

Recent cycle funding included \$14 million in grants or loans; 25\% cost share required; applications accepted year round.

For more information, contact Jon Unger at 503-986-0869.

More details and updates for these grants can be found at:

http://www.oregon.gov/OWRD/pages/Water_Resources_Development_Program.aspx

\section{Municipal Water Management and Conservation Planning}

Municipal water management and conservation planning provides a process through which cities and other municipal water suppliers estimate long-range water supply needs and identify alternatives, including water conservation programs, to meet those needs. The Department requires many municipal water suppliers to prepare plans as conditions of their water use permits or permit extensions.

\section{Water Rights}

Oregon's water laws are based on the principle of prior appropriation. This means the first person to obtain a water right on a stream is the last to be shut off in times of low streamflows. In water-short times, the water right holder with the oldest date of priority can demand the water specified in their water right regardless of the needs of junior users. The date of application for a permit to use water usually becomes the priority date of the right. Watermasters respond to complaints from water users and determine in times of water shortage, which generally occur every year, who has the right to use water. Each summer as streamflows drop, watermasters regulate junior users to provide water to the more senior users. On many streams throughout the state, by the end of summer, there is only enough water to supply users who established their rights in the late $1800 \mathrm{~s}$. All of the more recently established rights will have been regulated off by the watermaster. 
There are "watermaster" offices located around the state. The watermaster office is an excellent source of local information. Watermasters can research water rights for a particular stream reach and provide supporting maps (above). During critical flow periods, watermasters regulate water usage to enable senior water right holders to satisfy their water right. The watermaster may also provide information regarding instream leases, ground water rights, cancellations, transfers of water rights, streamflow data, and water right information in general (list as of 6/16).

\section{WRD Watermasters}

\section{District 1}

Nikki Hendricks

c/o Port of Tillamook Bay

4000 Blimp Blvd Ste 400

Tillamook, Oregon 97141

Ph: 503-815-1967

\section{District 2}

Michael Mattick

125 East 8th Avenue

Eugene, OR 97401-2926

Ph: 541-682-3620

\section{District 3}

Robert Wood

2705 E 2nd St

The Dalles, Oregon 97058

Ph: 541-506-2652

\section{District 4}

Eric Julsrud

201 S Humbolt, Suite 180

Grant County Courthouse

Canyon City, Oregon 97820

Ph: 541-575-0119

\section{District 5}

\section{Greg Silbernagel}

116 SE Dorion Ave

Pendleton, OR 97801

Ph: 541-278-5456

\section{District 6}

\section{Shad Hattan}

10507 N McAlister Rd \#6

La Grande, Oregon 97850

Ph: 541-963-1031

\section{District 7}

\section{David Bates}

401 NE First St., Suite 11

Enterprise, Oregon 97828

Ph: 541-426-4464

\section{District 8 \\ Rick Lusk \\ Baker County Courthouse \\ 1995 3rd Street, Suite 180 \\ Baker City, Oregon 97814 \\ Ph: 541-523-8224 ext 231}

District 9

Ron Jacobs

Malheur County Courthouse \#4

251 B St W

Vale, Oregon 97918

Ph: 541-473-5130

\section{District 10}

JR Johnson

Harney County Courthouse

450 N Buena Vista \#3

Burns, OR 97720

Ph: 541-573-2591

\section{District 11}

Jeremy Giffin

231 SW Scalehouse Loop,

Ste 103

Bend, Oregon 97702

Ph: 541-306-6885

\section{District 12 \\ Brian Mayer \\ 513 Center St \\ Lakeview, Oregon 97630 \\ Ph: 541-947-6038}

\section{District 13 \\ Travis Kelly \\ 10 S Oakdale, Rm 309A \\ Medford, Oregon 97501 \\ Ph: 541-774-6880 \\ District 14 \\ Kathy Smith \\ 700 NW Dimmick St. \\ Grants Pass, Oregon 97526 \\ Ph: 541-479-2401}

\section{District 15}

David Williams

Douglas County Courthouse, Room

306

Roseburg, Oregon 97470

Ph: 541-440-4255

\section{District 16}

Joel Plahn

725 Summer St NE, Ste A

Salem, Oregon 97301

Ph: 503-986-0889

\section{District 17}

Scott White

305 Main Street

Klamath Falls, Oregon 97601

Ph: 541-883-4182

\section{District 18}

Jake Constans

1400 SW Walnut St, Suite 240

Hillsboro, Oregon 97123

Ph: 503-846-7780

\section{District 19 \\ Greg Wacker \\ Physical Address: \\ $225 \mathrm{~N}$ Adams \\ Coquille, Oregon 97423 \\ Ph: 541-396-1905}

\section{District 20}

Amy Kim

10722 SE Highway 212

Clackamas, Oregon 97015

Ph: 503-722-1410

\section{District 21}

Ken Thiemann

221 S Oregon St.

P.O. Box 427

Condon, OR 97823

Ph: 541-384-4207 


\section{Oregon Department of Forestry (ODF)}

Salem Headquarters

2600 State Street

Salem, Oregon 97310

http://www.oregon.gov/ODF/Pages/index.aspx

The Oregon Department of Forestry manages and regulates activities on non-federal forestland in Oregon. There are three main divisions under ODF-- Fire Protection, Private Forests, and State Forests. The Private Forests Division administers the Forest Practices Act and various forestry incentive programs and employs the use of about 50 Stewardship Foresters who work closely with landowners and operators The State Forests Division is responsible for forest management to provide economic, environmental, and social benefits to Oregonians.

Financial incentive programs are aimed at encouraging and assisting landowners in managing their resources and meeting their objectives. Typical forestry projects can be aimed at protecting the landowner's resources/investment from fire or insect and disease infestation, to increasing its monetary and environmental value in the future.

Information about all ODF and federal forestry-related grants and incentive programs can be found at:

http://www.oregon.gov/ODF/AboutODF/Pages/GrantsIncentives.aspx

\section{Community Forest Program}

The Community Forest and Open Space Conservation Program is a federal financial assistance program with grants available to local governments, Indian tribes, and qualified nonprofit organizations to establish community forests and sustainably manage them for many public benefits, including recreation, income, wildlife habitat, stewardship demonstration sites, and environmental education.

\section{Conservation Stewardship Program}

To help landowners and operators maintain existing stewardship and adopt additional conservation on privatelyowned, non-industrial working forests and agricultural lands.

\section{Forest Legacy Program}

The Forest Legacy Program is a national program that addresses privately-owned forestlands that face threats of conversion to non-forest use by development pressures. The goal of the Forest Legacy Program is to promote stewardship and sustainable management of private forest lands by maintaining working forests that conserve important forest resource and conservation values. Forest Legacy provides funds for eligible private forestlands for the purchase of development rights through either conservation easement or fee-title acquisition into public ownership. All properties entered into Oregon's Forest Legacy Program - either through conservation easement, fee acquisition or donation - have their forest resources and conservation values protected and managed in accordance with a State Forester-approved Forest Stewardship Plan (see below).

\section{Forest Stewardship Program}

Oregon's Forest Management Planning System recognizes that forest management planning is a journey Pathways to Stewardship -- involving several distinct steps. A landowner's initial interest may be related to a specific project or action that is pressing on their property - such as reducing hazardous wildfire fuels or combating an invasive weed. Landowner assistance organizations and agencies usually first cross paths through outreach efforts defined around mutual interests or resource concerns. Landowners who are just beginning the management planning process begin a more formal journey by taking the Woodland Discovery step. Woodland Discovery consists of gathering basic property information and solidifying management goals. The remaining steps for completing your forest management plan include organizing the planning elements into specific management planning modules: soil and water, forest vegetation, fish and wildlife, access and protection, scenery and enjoyment and tax and business. Every step completed along the way results in the identification of specific actions that a landowner can take to improve conditions of the forestland or otherwise meet goals in owning forestland. Completion of a forest management plan opens up formal types of engagement such as forest certification and the enrollment of lands into specialized conservation programs that define a long-term commitment to sustainable forestry. 


\section{Healthy Forests Reserve Program (HFRP)}

The goal is to restore and enhance ecosystems and habitat for threatened and endangered species while promoting sustainable timber harvests on working forest lands.

\section{Department of Agriculture Natural Resources Program}

635 Capitol St. NE

Salem, OR 97301-2532

Phone: 503 986-4700

http://www.oregon.gov/ODA/programs/NaturalResources

The Oregon Department of Agriculture (ODA) is responsible for developing plans to prevent and control water pollution from agricultural activities and soil erosion on rural lands. ODA's Natural Resources Program aims to conserve, protect, and develop natural resources on public and private lands in order to ensure that agriculture will continue to be productive and economically viable in Oregon. Natural Resources Programs work to do the following:

- Address water quality and natural resource conservation on agricultural lands

- Protect Oregon's environment and public health by ensuring the proper and legal sale, use, and distribution of pesticide products

- Assist local soil and water conservation districts as they help landowners properly manage Oregon’s natural resources

More information on the Agricultural Plan Areas and Regulations can be found at: https://www.oregon.gov/ODA/programs/NaturalResources/AgWQ/Pages/AgWQPlans.aspx Information on local management plans and your area's ODA Water Quality Specialist can be found at: http://www.oregon.gov/ODA/programs/NaturalResources/AgWQ/Pages/AgWQPlans.aspx More information on the regulation and use of pesticides can be found at: http://www.oregon.gov/ODA/programs/Pesticides/Pages/default.aspx

\section{Department of Agriculture Pesticide Analytical and Response Center (PARC)} http://www.oregon.gov/ODA/programs/Pesticides/Pages/PARC.aspx

The Pesticide Analytical and Response Center (PARC) was created by executive order in 1978. The program was reauthorized under the Oregon Department of Agriculture (ODA) as ORS 634.550, in 1991.

PARC is mandated to perform the following activities with regard to pesticide-related incidents in Oregon that have suspected health or environmental effects: Collect incident information, mobilize expertise for investigations, identify trends and patterns of problems, make policy or other recommendations for action, report results of investigations, and prepare activity reports for each legislative session.

PARC does not have regulatory authority. Their primary function is to coordinate investigations to collect and analyze information about reported incidents. Investigation coordination includes collecting reports produced by member agencies and consultation as necessary with a toxicologist with Oregon State University. Member agencies conduct most of the investigations and take any necessary enforcement action(s). The eight member agencies include the following: Oregon Health Authority (OHA), Oregon Department of Fish and Wildlife ( ODF\&W), Oregon Department of Environmental Quality (DEQ), Oregon Department of Forestry (ODF), Oregon Occupational Safety and Health Administration (OR OSHA), Office of the State Fire Marshal (SFM), Oregon Poison Center (OPC), Oregon Department of Agriculture (ODA).

To report a pesticide incident that has impacted people, animals, or the environment, contact: Theodore Bunch Jr., PARC Coordination Team Leader at 503-986-6470 or toll-free at 844-688-7272 PARC@oda.state.or.us or Christina Higby, Citizen Advocate Liaison at 503-986-5105 chigby@oda.state.or.us 


\section{Department of Agriculture}

\section{Soil and Water Conservation Districts}

http://www.oregon.gov/ODA/SWCD/

SWCD Program and Water Quality Program Manager: John Byers, 503-986-4718

The Soil and Water Conservation District (SWCD) Program provides services to the 45 Soil and Water Conservation Districts throughout Oregon (list current as of 6/16). SWCDs are local government entities that have authorities to address soil, erosion, and water quality issues.

\section{Benton SWCD}

456 SW Monroe Ave., Suite 110

Corvallis, OR 97333

Phone: 541 753-7208

Website: www.bentonswcd.org

\section{Burnt River SWCD}

3990 Midway Drive

Baker City, OR 97814

Phone: 541 523-7121 Ext. 109

Email: whitney.collins@bakercountyswcds.com

\section{Clackamas SWCD}

221 Molalla Ave., Suite 102

Oregon City, OR 97045

Phone: 503 210-6000

Website: www.conservationdistrict.org

\section{Clatsop SWCD}

750 Commercial St., Room 207

Astoria, OR 97103

Phone: 503 325-4571

Website: www.clatsopswcd.org

\section{Columbia SWCD}

35285 Millard Road

St. Helens, OR 97051

Phone: 503 397-4555

Website: www.columbiaswcd.com

\section{Coos SWCD}

371 N Adams St.

Coquille, OR 97423

Phone: 541 396-6879

Website: www.coosswcd.org

\section{Crook County SWCD}

498 SE Lynn Blvd.

Prineville, OR 97754

Phone: 541 447-3548

\section{Curry County SWCD}

29692 Ellensburg Ave.

Gold Beach, OR 97444

Phone: 541 247-2755 Ext. 0\#

Website: www.currywatersheds.org

\section{Deschutes SWCD}

625 SE Salmon Ave., Suite 7

Redmond, OR 97756

Phone: 541 923-2204

Website: www.deschutesswcd.com

\section{Douglas SWCD}

2741 West Harvard Ave.

Roseburg, OR 97471

Phone: 541 957-5061

Website: www.douglasswcd.org

\section{Eagle Valley SWCD}

3990 Midway Drive

Baker City, OR 97814

Phone: 541 523-7121 Ext. 109

\section{East Multnomah SWCD}

5211 N Williams Ave.

Portland, OR 97217

Phone: 503 222-SOIL (7645)

Website: www.emswcd.org

\section{Fort Rock / Silver Lake SWCD}

17612 Highway 395

Lakeview, OR 97630

Phone: 541 947-5855

Email: LakeviewSWCD2@hotmail.com

\section{Gilliam County SWCD}

Dunn Office Building

333 S Main St.

Condon, OR 97823

Phone: 541 384-2672

Email: gilliamswcd@gmail.com

\section{Grant SWCD}

721 S Canyon Blvd.

John Day, OR 97845

Phone: 541 575-0135 Ext. 3

Email: jkehrberg@ortelco.net

\section{Harney SWCD}

530 Highway $20 \mathrm{~S}$

Hines, OR 97738

Phone: 541 573-5010

Email: marty.suter@or.nacdnet.net

\section{Hood River SWCD}

3007 Experiment Station Drive

Hood River, OR 97031

Phone: 541 386-4588 / 386-6719

Website: www.hoodriverswcd.org

\section{Illinois Valley SWCD}

Josephine Co. Office Building

102 S Redwood Highway

Cave Junction, OR 97523

Phone: 541 592-3731

Email: amy@ivstreamteam.org 
Jackson SWCD

89 Alder Street

Central Point, OR 97502

Phone: 541 664-1070

Website: www.jswcd.org

\section{Jefferson County SWCD}

625 SE Salmon Ave., Suite 6

Redmond, OR 97756

Phone: 541 923-4358 Ext. 101

Email: debbe.chadwick@oacd.org

\section{Josephine SWCD}

1440 Parkdale Drive

Grants Pass, OR 97527

Phone: 541 474-6840

Email: joswcd@outreachinternet.com

\section{Keating SWCD}

3990 Midway Drive

Baker City, OR 97814

Phone: 541 523-7121 Ext. 109

Email: whitney.collins@bakercountyswcds.com

\section{Klamath SWCD}

2316 S 6th St., Suite C

Klamath Falls, OR 97601

Phone: 541 883-6932 Ext. 101

Website: www.klamathswcd.org

\section{Lakeview SWCD}

17612 Highway 395

Lakeview, OR 97630

Phone: 541 947-5855

Email: lakeviewswcd2@hotmail.com

\section{Lincoln SWCD}

23 North Coast Highway

Newport, OR 97365

Phone: 541 265-2631

Website: www.lincolnswcd.org

\section{Linn SWCD}

33935 Highway 99E, Suite C

Tangent, OR 97389

Phone: 541 926-2483

Website: www.linnswcd.oacd.org

\section{Malheur County SWCD}

2925 SW Sixth Ave., Suite 2

Ontario, OR 97914

Phone: 541 889-2588 Ext. 101

Email: LRowe@malcoswcd.org

\section{Marion SWCD}

338 Hawthorne Ave. NE

Salem, OR 97301

Phone: 503 391-9927

Website: www.marionswcd.net

\section{Monument SWCD}

Columbia Power Building

311 Wilson St.

Monument, OR 97864

Phone: 541 934-2141

Website: www.monumentswcd.org

\section{Morrow SWCD}

430 Linden Way

Heppner, OR 97836

Phone: 541 676-5452

Email: swcdmanager@centurytel.net

\section{Polk SWCD}

580 Main St., Suite A

Dallas, OR 97338

Phone: 503 623-9680

Website: www.polkswcd.org

\section{Sherman County SWCD}

302 Scott St.

Moro, OR 97039

Phone: 541 565-3216 Ext. 3

Website: www.shermancountyswcd.com

\section{Siuslaw SWCD}

1775 Laurel Place, Suite 4

Florence, OR 97439

Phone: 541 997-1272

Website: www.siuswcd.com

\section{Tillamook SWCD}

4000 Blimp Blvd., Suite 200

Tillamook, OR 97141

Phone: 503 842-2240 Ext. 110

Website: tillamookcountyswcd.org/

\section{Tualatin SWCD}

1080 SW Baseline St., Suite B-2

Hillsboro, OR 97123

Phone: 503 648-3174 Ext. 4

Website: www.swcd.net

\section{Umatilla County SWCD}

1 SW Nye Ave., Suite 130

Pendleton, OR 97801

Phone: 541 278-8049

Website: www.umatillacountyswcd.com

\section{Umpqua SWCD}

1877 Winchester Ave.

Reedsport, OR 97467

Phone: 541 662-1341

Website: www.umpquasoilandwater.com

\section{Union SWCD}

10507 N McAlister Road, Room 7

La Grande, OR 97850

Phone: 541 963-1313

Website: unionswcd.org 
Upper Willamette SWCD

780 Bailey Hill Road, Suite 5

Eugene, OR 97402

Phone: 541 465-6443 Ext. 102

Website: www.uwswcd.org

Wallowa SWCD

401 NE 1st St., Suite E

Enterprise, OR 97828

Phone: 541 426-4521

Email: cynthia.a.warnock@gmail.com

Wasco County SWCD

2325 River Road, Suite 3

The Dalles, OR 97058

Phone: 541 296-6178 Ext. 3

Website: www.wascoswcd.org
West Multnomah SWCD

2701 NW Vaughn St., Suite 450

Portland, OR 97210

Phone: 503 238-4775

Website: www.wmswcd.org

Wheeler SWCD

40535 Highway 19

Fossil, OR 97830

Phone: 541 468-2990

Website: www.wheelerswcd.org

Yamhill SWCD

2200 SW Second St.

McMinnville, OR 97128

Phone: 503 472-6403

Fax: 503 472-6407

Website: www.yamhillswcd.org

\section{Oregon Watershed Enhancement Board (OWEB)}

775 Summer St. NE Suite 360

Salem, OR 97301

Phone: (503) 986-0178

Website: www.oregon.gov/OWEB

The Oregon Watershed Enhancement Board (OWEB) is a state agency that provides grants to help Oregonians take care of local streams, rivers, wetlands and natural areas. Community members and landowners use scientific criteria to decide jointly what needs to be done to conserve and improve rivers and natural habitat in the places where they live. OWEB grants are funded from the Oregon Lottery, federal dollars, and salmon license plate revenue. The agency is led by a 17 member citizen board drawn from the public at large, tribes, and federal and state natural resource agency boards and commissions. OWEB provides grants to projects that contribute to the Oregon Plan for Salmon and Watersheds and the Oregon Conservation Strategy by protecting, restoring and improving clean water and fish and wildlife habitat. See the OWEB website for more information on grants: http://www.oregon.gov/OWEB/GRANTS/pages/index.aspx.

\section{Oregon Sea Grant (OSG)}

Oregon State University

Corvallis, Oregon

Phone 541-737-2714

http://seagrant.oregonstate.edu/

Oregon Sea Grant serves Oregon coastal communities through integrated research, education and public engagement on ocean and coastal issues. Based at Oregon State University, OSG is part of the national network of NOAA Sea Grant College Programs, dedicated to promoting environmental stewardship, long-term economic development and responsible use of America's coastal, ocean and Great Lakes resources. OSG targets research on better defining the relationships between the many pressures that can degrade water quality: climate change, upland and coastal land use, fish and habitat restoration efforts, aquatic invasive species. OSG works with groups whose interests sometimes come in conflict - landowners, outdoor recreationists, farmers and woodland managers, local government, the general public - to seek solutions that will help sustain healthy watersheds and our precious water resources. OSG focuses on the question of resilience - the ability to plan, adapt and rebound in the face of change by supporting physical and social science research aimed at better understanding ocean and coastal processes and the socio-economic barriers to hazard and climate change preparation. Publications and resources available from OSG can be found here: http://seagrant.oregonstate.edu/sgpubs. 
OSG and OSU Extension produce textbooks and other publications on such topics as conservation-friendly gardening, sustainable living and low-impact development. OSG also partners with the Oregon State Marine Board to develop the Clean Vessel Act (CVA) Education Initiative. Funded by the Clean Vessel Act of 1992, the goal of the CVA Education Initiative is to improve boaters' awareness, accessibility and use of sewage pumpouts, dump stations, and floating toilets. Publications and resources available from OSG about watershed health can be found here: http://seagrant.oregonstate.edu/sgpubs by using "watersheds and wetlands" in the "Search by Subject" field.

Every two years, OSG awards approximately \$2 million in research grants addressing community preparedness for climate change, watershed health, other urgent or emerging regional needs with high relevance to coastal communities. For more information on grants, see: http://seagrant.oregonstate.edu/research

\section{Source Water Collaborative \\ led by U.S. Environmental Protection Agency}

Technical assistance and lists of resources and contacts are available from this national network that has worked to promote drinking water protection for several years. The Source Water Collaborative is a network of federal, state, and local organizations led by US EPA. Some of the key Source Water Collaborative members include the US EPA, US Department of Agriculture, AWWA, American Planning Association, ASDWA, ACWA, National Rural Water Association, Groundwater Protection Council, National Association of Counties, and The Trust for Public Land. Resources can be found here:

http://sourcewatercollaborative.org/

\section{U.S. Environmental Protection Agency Catalog of Federal Funding Sources for Watershed Protection}

This is an online, free searchable database of financial assistance sources (grants, loans, cost-sharing) available to fund a variety of watershed protection projects.

https://ofmpub.epa.gov/apex/watershedfunding/f?p=fedfund:1

\section{U.S. Environmental Protection Agency Environmental Finance Centers}

Free technical assistance is available through EPA's Environmental Finance Centers. There is currently no Environmental Finance Center for US EPA Region 10, but the resources are still available through the US EPA website. The program mission is to provide help to those facing the "how to pay" challenges of environmental protection. EFC is committed to helping the regulated community build and improve the technical, managerial, and financial capabilities needed to comply with federal and state environmental protection laws.

https://www.epa.gov/waterfinancecenter/efcn

\section{U.S. Environmental Protection Agency Community Action for a Renewed Environment (CARE) Grants}

Eligible Projects: Prevention of human exposure to harmful pollution; improve water quality. Form communitybased collaborative partnerships; identifying and developing an understanding of the many local sources of risk from toxic pollutants and environmental concerns; and setting priorities for the reduction of the identified risks and concerns of the community

Eligible Applicants: Local, public non-profit institution/organizations, federally-recognized Indian tribal government, Native American organizations, private non-profit institution/organization, quasi-public nonprofit institution/organization both interstate and intrastate, local government, colleges, and universities

Funding Available:\$75,000 to $\$ 100,000$ with an average project funding of about $\$ 90,000$

How To Apply: www.epa.gov/care 


\section{U.S. Bureau of Reclamation Cooperative Watershed Management Program}

Eligible Projects: Improve water quality; improve ecological resiliency of a river or stream; and to reduce conflicts over water at the watershed level by supporting the formation of watershed groups to develop local solutions to address water management issues

Eligible Applicants: States, Indian tribes, local and special districts (e.g., irrigation and water districts, county soil conservation districts, etc.), local governmental entities, interstate organizations, and non-profit organizations. To be eligible, applicants must also meet all of the following requirements: (1) Significantly affect or be affected by the quality or quantity of water in a watershed; (2) Be capable of promoting the sustainable use of water resources; (3) Be located in the western United States specifically: Arizona, California, Colorado, Idaho, Kansas, Montana, Nebraska, Nevada, New Mexico, North Dakota, Oklahoma, Oregon, South Dakota, Texas, Utah, Washington.

Funding Available: $\$ 22,000-\$ 100,000$ in the past

How To Apply: http://www.usbr.gov/WaterSMART/cwmp/index.html

\section{U.S. Department of Agriculture Farm Service Agency Conservation Programs}

http://www.fsa.usda.gov/programs-and-services/conservation-programs/index

USDA Farm Service Agency oversees a number of voluntary conservation-related programs. These programs work to address a large number of farming and ranching related conservation issues including:

- Drinking water protection

- Reducing soil erosion

- Wildlife habitat preservation

- Preservation and restoration of forests and wetlands

- Aiding farmers whose farms are damaged by natural disasters

\section{Source Water Protection Program (SWPP)}

The SWPP is designed to protect surface and ground water used as drinking water by rural residents. Through a partnership with the National Rural Water Association, local teams are formed to develop plans to reduce pollutant impacts in rural areas.

http://www.fsa.usda.gov/programs-and-services/conservation-programs/source-water-protection/index

\section{Conservation Reserve Program (CRP)}

The CRP pays a yearly rental payment in exchange for farmers removing environmentally sensitive land from agricultural production and planting species that will improve environmental quality. In exchange for a yearly rental payment, farmers enrolled in the program agree to remove environmentally sensitive land from agricultural production and plant species that will improve environmental health and quality. Contracts for land enrolled in CRP are 10-15 years in length. The long-term goal of the program is to re-establish valuable land cover to help improve water quality, prevent soil erosion, and reduce loss of wildlife habitat.

http://www.fsa.usda.gov/programs-and-services/conservation-programs/conservation-reserve-program/index

\section{Conservation Reserve Enhancement Program (CREP)}

The CREP, an offshoot of CRP, targets high-priority conservation issues identified by local, state, or tribal governments or non-governmental organizations. In exchange for removing environmentally sensitive land from production and introducing conservation practices, farmers, ranchers, and agricultural land owners are paid an annual rental rate. Participation is voluntary, and the contract period is typically 10-15 years, along with other federal and state incentives as applicable per each CREP agreement.

http://www.fsa.usda.gov/programs-and-services/conservation-programs/conservation-reserve-enhancement/index 


\section{Emergency Conservation Program (ECP)}

The ECP provides funding and technical assistance for farmers and ranchers to restore farmland damaged by natural disasters and for emergency water conservation measures in severe droughts. The ECP also provides funding and assistance to help ranchers and farmers install water conservation measures during severe drought.

http://www.fsa.usda.gov/programs-and-services/conservation-programs/emergency-conservation/index

\section{Emergency Forest Restoration Program (EFRP)}

The EFRP, which is very similar to the ECP, provides funding to restore privately owned forests damaged by natural disasters.

http://www.fsa.usda.gov/programs-and-services/disaster-assistance-program/emergency-forest-restoration/index

\section{Farmable Wetlands Program (FWP)}

The FWP is designed to restore wetlands and wetland buffer zones that are farmed. FWP gives farmers and ranchers annual rental payments in return for restoring wetlands and establishing plant cover.

http://www.fsa.usda.gov/programs-and-services/conservation-programs/farmable-wetlands/index

\section{U.S. Department of Agriculture Natural Resources Conservation Service}

NRCS provides farmers, ranchers and forest managers with free technical assistance, or advice, for their land. Common technical assistance includes: resource assessment, practice design and resource monitoring. The conservation planner will help you determine if financial assistance is right for you. Technical assistance is also available online through Conservation Client Gateway.

More information about NRCS can be found on their home page:

http://www.nrcs.usda.gov/wps/portal/nrcs/main/national/about/

\section{Environmental Quality Incentives Program (EQIP)}

Grants are available for best management practices and conservation on private, non-industrial forestland and agricultural lands. Financial assistance is available to help plan and implement conservation practices that address natural resource concerns and for opportunities to improve soil, water, plant, animal, air and related resources on agricultural land and non-industrial private forestland. In addition, EQIP can help producers meet Federal, State, Tribal and local environmental regulations.

http://www.nrcs.usda.gov/wps/portal/nrcs/main/national/programs/financial/eqip/

Eligible Applicants: Owners of land in agricultural or forest production or persons who are engaged in livestock, agricultural or forest production on eligible land and that have a natural resource concern on the land

Funding Available: Financial and technical assistance to agricultural and forestland producers through contracts up to 10 years. Not to exceed $\$ 300,000$ for all EQIP contracts entered into during any six-year period. If NRCS determines project has special environmental significance the payment limitation is a maximum of $\$ 450,000$.

\section{Conservation Stewardship Program (CSP)}

CSP helps agricultural producers maintain and improve their existing conservation systems and adopt additional conservation activities to address priority resources concerns. Through CSP, participants take additional steps to improve resource condition including soil quality, water quality, water quantity, air quality, and habitat quality, as well as energy. Participants earn CSP payments for conservation performance - the higher the performance, the higher the payment.

http://www.nrcs.usda.gov/wps/portal/nrcs/main/national/programs/financial/csp/ 


\section{Wetlands Reserve Easements (WRE)}

WRE provides habitat for fish and wildlife, including threatened and endangered species, improve water quality by filtering sediments and chemicals, reduce flooding, recharge groundwater, protect biological diversity and provide opportunities for educational, scientific and limited recreational activities.

NRCS also provides technical and financial assistance directly to private landowners and Indian tribes to restore, protect, and enhance wetlands through the purchase of a wetland reserve easement. http://www.nrcs.usda.gov/wps/portal/nrcs/detail/or/home/?cid=stelprdb1249312

\section{Agricultural Land Easements (ALE)}

ALE is designed to protect the long-term viability of the nation's food supply by preventing conversion of productive working lands to non-agricultural uses. Land protected by agricultural land easements provides additional public benefits, including environmental quality, historic preservation, wildlife habitat and protection of open space.

http://www.nrcs.usda.gov/wps/portal/nrcs/detail/or/home/?cid=stelprdb1249312

\section{Emergency Watershed Protection (EWP)}

The EWP program was set up by Congress to respond to emergencies created by natural disasters. The United States Department of Agriculture's Natural Resources Conservation Service is responsible for administering the program. EWP is designed to relieve imminent hazards to life and property caused by floods, fires, windstorms, and other natural occurrences. It is not necessary for a national emergency to be declared for an area to be eligible for assistance. Activities include providing financial and technical assistance to remove debris from streams, protect destabilized streambanks, establish cover on critically eroding lands, repairing conservation practices, and the purchase of flood plain easements. The purpose of EWP is to help groups of people with a common problem. EWP is generally not an individual assistance program. All projects undertaken must be sponsored by a political subdivision of the State, such as a city, county, general improvement district or conservation district, or by a tribal government.

http://www.nrcs.usda.gov/wps/portal/nrcs/main/or/programs/financial/ewp/

\section{Other NRCS Programs}

There are other NRCS programs that are specific to Oregon geographic areas---Wildfire Rehabilitation Initiative, Organic Initiative, drought funding, and restoration funding---see the Oregon NRCS link for more information on those: http://www.nrcs.usda.gov/wps/portal/nrcs/main/or/programs/financial/eqip/

Anyone applying for EQIP or any of the other NRCS grants for the first time should schedule a meeting with NRCS to discuss their options before moving forward.

\section{U.S. Department of Agriculture Rural Development Water and Waste Disposal Direct Loans and Grants}

Eligible Projects: Pre-construction and construction associated with building, repairing, or improving drinking water, solid waste facilities and wastewater facilities

\section{Eligible Applicants:}

- Cities or towns with fewer than 10,000 population

- Counties, special purpose districts, non-profit corporations or tribes unable to get funds from other sources at reasonable rates and terms

Funding Available: Loans (40-year term), grants in some cases, interest rates vary (currently 2.125 - 3.5\%)

How To Apply: Applications accepted year-round on a fund-available basis. http://www.rd.usda.gov/programsservices/water-waste-disposal-loan-grant-program 


\section{U.S. Department of Commerce \\ Community Development Block Grant Planning Program}

Region 10 HUD

Seattle Regional Office

Phone: (206) 220-5101

http://portal.hud.gov/hudportal/HUD?src=/states/washington/offices

http://portal.hud.gov/hudportal/HUD?src=/program_offices/comm_planning/communitydevelopment/programs

Eligible Projects: Comprehensive plans, Infrastructure plans, Feasibility studies, Community action plans, Lowincome housing assessments

Eligible Applicants: Projects must principally benefit low- to moderate-income people in non-entitlement cities and counties.

- Cities or towns with fewer than 50,000 people

- Counties with fewer than 200,000 people

Funding Available: Grants

- Up to $\$ 24,000$ for a single jurisdiction

- Up to $\$ 35,000$ for single jurisdiction projects that address urgent public health and safety needs

- Up to $\$ 40,000$ for multiple jurisdictions/joint application

How To Apply: http://portal.hud.gov/hudportal/HUD?src=/states/washington/offices

\section{Rural Community Assistance Corporation (RCAC)}

Environmental Programs

1020 S.W. Taylor Street Suite 450

Portland, OR 97205

\section{Local contacts:}

Chris Marko, Rural Development Specialist 503- 228-1780

RosAnna Noval, Rural Development Specialist 503-308-0207

Email: cmarko@rcac.org; rnoval@rcac.org

Website: www.rcac.org

At the national level, RCAC has a variety of loans for water and/or wastewater planning, environmental work, and other work to assist in developing an application for infrastructure improvements

Eligible Applicants: Non-profit organizations, public agencies, tribes, and low-income rural communities with a 50,000 population or less, or 10,000 or less if guaranteed by USDA Rural Development financing.

\section{Funding Available:}

- Maximum $\$ 50,000$ for feasibility loan

- Maximum \$350,000 for pre-development loan

- 1 year term

- $5.5 \%$ interest rate

How To Apply: Applications accepted anytime. www.rcac.org

National contact: Josh Griff, 720-951-2163, jgriff@rcac.org 


\section{Water Research Foundation - Source Water Protection Cost-Benefit Tool}

This is a free, online suite of tools designed to assist in evaluating the triple bottom-line costs and benefits of different source water protection options. Cost/benefit calculations help evaluate, prioritize, justify, and ultimately implement source water protection initiatives. http://www.swptool.org/index.cfm

\section{Healthy Watersheds Consortium}

The Healthy Watersheds Consortium Grant Program has just published a Request for Proposals (RFP) to support local projects that protect and sustain healthy watersheds (including drinking water sources). Through this program, EPA will provide approximately $\$ 3.75$ million over six years to the U.S. Endowment for Forestry and Communities for projects that develop and/or support state, interstate, and tribal healthy watersheds programs and enhance collaboration among the many groups who benefit from protecting healthy watersheds such as drinking water utilities, hunters and fisherman, foresters and farmers, and more. The Endowment is also matching a portion of EPA's financial commitment to the partnership and expects to leverage additional funding from other public and private sources.

The goal of the Healthy Watersheds Consortium Grant Program is to accelerate strategic protection of healthy, freshwater ecosystems and their watersheds. This goal will be achieved by: Funding key projects identified in existing watershed protection or conservation plans; Building the sustainable organizational infrastructure, social support, and long-term funding commitments necessary to implement large-scale protection of healthy watersheds; and supporting innovative or catalytic projects that may accelerate or broadly advance the field of practice for watershed protection efforts. For more information and to view and download the RFP and other helpful documents, visit the website: http://usendowment.org/partnerships/hwcgrantprogram.html

For questions, please contact Peter Stangel at peter@usendowment.org.

\section{Ecotrust}

http://www.ecotrust.org/

Ecotrust works to protect and restore watersheds and the economic and public health of the communities that depend upon them. Ecotrust develops and applies strategic approaches that improve habitat for native fish and wildlife, create local jobs and recreational opportunities, increase public awareness of the value of nature's services like water, and ensure a more reliable access to clean water for all members of the Oregon communities. Ecotrust provides Ecosystem Services, GIS Analysis, Mapping, Cartography, Data and Software Development, Economic Impact Assessment, etc.

\section{Ecotrust Forest Management}

\section{http://ecotrustforests.com}

Ecotrust Forest Management is a for-profit forestland investment management company that acquires and manages land on behalf of investors and forestland owners to enhance forest health and productivity, and to produce a diverse array of forest products and services including timber, biomass, carbon, and improved habitat and water quality. Where possible, our goal is to transition land to long-term, local, stewards of land like Tribes, Community Forests, Public or State Agencies etc. EFM is adept at using a wide array of financing sources- New Market Tax Credits, carbon credits, conservation easements, and restoration funding — to supplement private capital resources in the acquisition and management of forestland. Contact: info@ecotrustforests.com 


\title{
LAND TRUSTS
}

Resources to assist in locating a land trust can be found here:

http://findalandtrust.org/states/oregon41

\section{Coalition of Oregon Land Trusts}

The Coalition of Oregon Land Trusts (COLT) is a newly formed nonprofit representing and serving Oregon's land trusts. Its mission is to serve and strengthen the land trust community in Oregon. Oregon's land trust community is working at local, regional, and statewide scales with landowners, communities, public agencies and other partners to maintain the state's natural heritage and the economies it supports. COLT will accomplish its mission by strengthening public policies and programs that are supportive of land conservation, helping to build capacity within and across land trusts, and communicating to key audiences about the role of land trusts in conserving Oregon's natural heritage and healthy human communities that depend on it. There are currently 18 land trusts that are members of COLT.

Coalition of Oregon Land Trusts

322 NW 5th, Suite 312 Portland, OR 97209

Phone: 503-719-4732 http://oregonlandtrusts.org/

\section{Land Trust Alliance}

The Land Trust Alliance is a national conservation organization that works preserve land through conservation and easements, so land and natural resources get protected. The Alliance is based in Washington, D.C., and has several regional offices.

Northwest Conservation Manager

1353 Officers Row Vancouver, WA 98661

Phone: (971) 202-1483 http://www.landtrustalliance.org/

\section{Individual land trusts which may be of assistance include:}

\section{The Trust for Public Land}

\author{
http://www.tpl.org/services/conservation-transactions
}

\section{The Nature Conservancy}

http://www.nature.org/

\section{FOUNDATIONS}

\section{The Oregon Community Foundation I Community Grant Program}

Eligible Projects: Community Livability, Environment \& Citizen Engagement (10 to 20 percent of grants)

- Promote leadership development, volunteerism, immigrant integration, and civic participation

- Support stewardship and appreciation of Oregon's outdoor spaces and scenic beauty

- Address social, economic and environmental challenges or opportunities by bringing together disparate stakeholders

- Preserve places essential to communities' civic and historic identities

Eligible Applicants: nonprofits with tax-exempt status under Section 501(c)(3)

Funding Available: average grant is $\$ 20,000$

Contact: http://www.oregoncf.org/grants-scholarships/grants/community-grants 


\section{National Fish and Wildlife Foundation}

Eligible Projects: Environmental Solutions for Communities (1:1 match required)

- Supporting sustainable agricultural practices and private lands stewardship;

- Conserving critical land and water resources and improving local water quality;

- Restoring and managing natural habitat, species and ecosystems that are important to community livelihoods;

- Facilitating investments in green infrastructure, renewable energy and energy efficiency; and

- Encouraging broad-based citizen and targeted youth participation in project implementation.

Eligible Applicants: non-profit 501(c) organizations, state government agencies, local governments, municipal governments, Indian tribes, educational institutions

Funding Available: grants range from $\$ 25,000$ to $\$ 100,000$

Contact: 202-595-2434 - Community-Based Conservation

\section{Access Fund Foundation}

Eligible Projects: land acquisitions; considering the management and financial resources of land ownership, the Access Fund views land acquisitions as a tool of last resort and have adopted the following guidelines for land acquisition projects. If you are requesting funds for a land acquisitions please call the Access Fund before submitting your application.

- The area must be imminently threatened with permanent closure or sale to an outside party that may consider land development opportunities or other uses threatening its climbing and/or access resources.

- The area can be acquired for a reasonable price (reasonable price being one that falls within existing market values and is not in excess of appraised value), together with a reasonable budget (including secured funding) or secured exit-strategy for management by another land trust, local climbers organization or governmental agency.

- A fully executed purchase agreement stating how the project will be funded is required before Access Fund grant funds will be allocated to any acquisition.

- A high degree of matching funds is required. The Access Fund's role in land acquisitions is as an additional, not primary, funding resource.

- Applicants whose projects require continued payments and/or financing should submit a plan describing how these payments will be met in the future. These include, but are not limited to, property tax payments, loan payments, lease and mortgage payments. This payment plan will be taken into consideration during the grant review process.

Eligible Applicants: Local climbing groups, individuals or organizations (Note: tax exempt 501(c)(3) status is not a pre-requisite); governmental agencies that wish to sponsor or organize a local project; conservation organizations and land trusts.

Funding Available: $\$ 1,000$ to $\$ 4,000$. (The Access Fund considers requests for over $\$ 10,000$, but these projects should have national significance and utilize a high degree of matching funds.)

Contact: http://www.accessfund.org/

\section{The Collins Foundation}

Eligible Projects: land acquisitions; grants are for projects that directly benefit the residents of Oregon

Eligible Applicants: nonprofits with tax-exempt status under Section 501(c)(3) / agencies that have current registration with the offices of the Oregon State Attorney General and the Secretary of State

Funding Available: varies; grants may range from $\$ 3000$ to $\$ 150,000$

Contact: www.collinsfoundation.org 


\section{Giles W. and Elise G. Mead Foundation}

Eligible Projects: Preserving and improving the environment; primary emphasis forestry, fisheries and the sustainable use of natural resources in western North America

Eligible Applicants: nonprofits with tax-exempt status under Section 501(c)(3) in western North America

Funding Available: past grants ranged from $\$ 15,000$ to $\$ 100,000$

Contact: http://www.gileswmeadfoundation.org/

\section{Rose E. Tucker Charitable Trust}

Eligible Projects: giving limited to organizations and projects in Oregon, with emphasis on the metropolitan Portland area; land acquisition is eligible

Eligible Applicants: nonprofits with tax-exempt status under Section 501(c)(3)

Funding Available: past grants ranged from \$6,000 to \$150,000

How to Apply: apply anytime; board meets approximately every 2 months

Contact: Tuckertrust@stoel.com

\section{Doris Duke Charitable Foundation}

Eligible Projects: The foundation's grant-making is designed to provide frameworks and concrete examples of how practitioners can protect biodiversity in light of climate change through strategic land conservation. The program's adaptation efforts focus on three critical land conservation activities undertaken by non-profit organizations and government natural resource agencies:

- Habitat conservation planning (i.e., the identification of which sites should be conserved in their natural state to benefit wildlife);

- Permanent land protection (i.e., the acquisition of conservation easements or fee title to secure high priority sites); and C) Management of lands already in protected status. The goal for each of these activities is to encourage the conservation community to augment the dominant species-based approach to wildlife conservation with a focus on maintaining ecosystem functionality as climate change takes hold.

- The program has adopted three approaches to achieve its objectives: 1) Identifying resilient landscapes; 2) Protecting resilient landscapes; and 3) Managing conserved lands.

Eligible Applicants: nonprofits with tax-exempt status under Section 501(c)(3)

Funding Available: past grants ranged in the $\$ 100 \mathrm{~K}$

Contact: http://www.ddcf.org/what-we-fund/environment/

\section{Bonneville Environmental Foundation}

Eligible Projects: renewable power and acquire, maintain, preserve, restore, protect, and/or sustain fish and wildlife habitat within the Pacific Northwest.

Interest area: Watershed Restoration Program---supports restoration of damaged watershed ecosystems; supports communities trying to heal their local watersheds by supporting watershed restoration projects grounded in the best available watershed science

Eligible Applicants: nonprofit organizations

Funding Available: varies

Contact: www.b-e-f.org 


\section{The Bullitt Foundation}

\section{Program priorities:}

- Manage freshwater resources: control, use, distribution, conservation;

- Conserve and restore resilient watersheds, wetlands and estuaries;

- Maintain a working land base for sustainable agriculture and forestry;

- Enforce laws and policies intended to assure air and water quality;

- Create landowner incentives for maintaining and enhancing ecosystem services, including the development of market-based mechanisms.

Eligible Applicants: nonprofit organizations in Washington, Oregon, Idaho, western Montana, south-central Alaska, and British Columbia. Within that broad geographic range, work is targeted to specific sub-regions generally associated with major population centers.

Funding Available: varies---past grants ranged from $\$ 10,000$ to over $\$ 600,000$

Contact: http://www.bullitt.org/

\section{Weyerhaeuser Foundation}

Eligible Projects: forestry practices, manufacturing's effects on air, water and land; free trade, recycling, diversity, land conservation and environmental education; land acquisitions or conservation easement projects may fit with the Foundation's priorities and goals

Eligible Applicants: educational institutions, non-profit organizations, research institutions in Oregon and Washington

Funding Available: $\$ 1,000$ - $\$ 50,000$

Contact: http://www.wfamilyfoundation.org/

\section{Laird Norton Foundation}

Eligible Projects: projects contribute to a heightened awareness of the ecological, social and economic significance of water sources and watersheds. Preference will be given to projects which demonstrate innovative measures for protecting and restoring water resources and which involve local communities and/or regional institutions.

Eligible Applicants: nonprofit organizations working in Hood Canal (WA), Upper Deschutes (OR), and Rogue (OR) watersheds

Funding Available: varies; past grants ranged from $\$ 10 \mathrm{k}$ to $\$ 100 \mathrm{k}$

Contact: http://www.lairdnorton.org

\section{Alternative formats}

Documents can be provided upon request in an alternate format for individuals with disabilities or in a language other than English for people with limited English skills. To request a document in another format or language, call DEQ in Portland at 503-229-5696, or toll-free in Oregon at 1-800-452-4011, ext. 5696; or email deqinfo@deq.state.or.us. 


\section{Appendix 5 - Table 2 Detail, Inventory of Potential Sources of Pollution (8-Hour time-of-PWS Name: \\ Travel zone) as identified in readily accessible state and federal databases and GIS layers PWS Number:}

City of Grants Pass

Updated Source Water Assessment

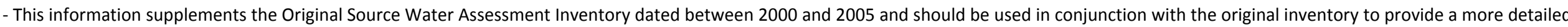
analysis of potential sources of pollution.

- Note that due to state database limitations, some sites will require further research to verify presence and location.

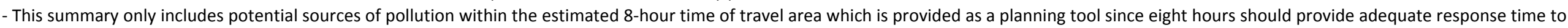

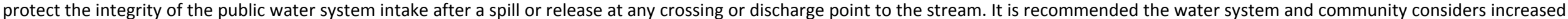

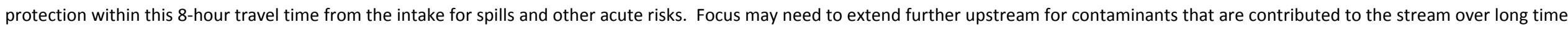
periods or recur frequently.

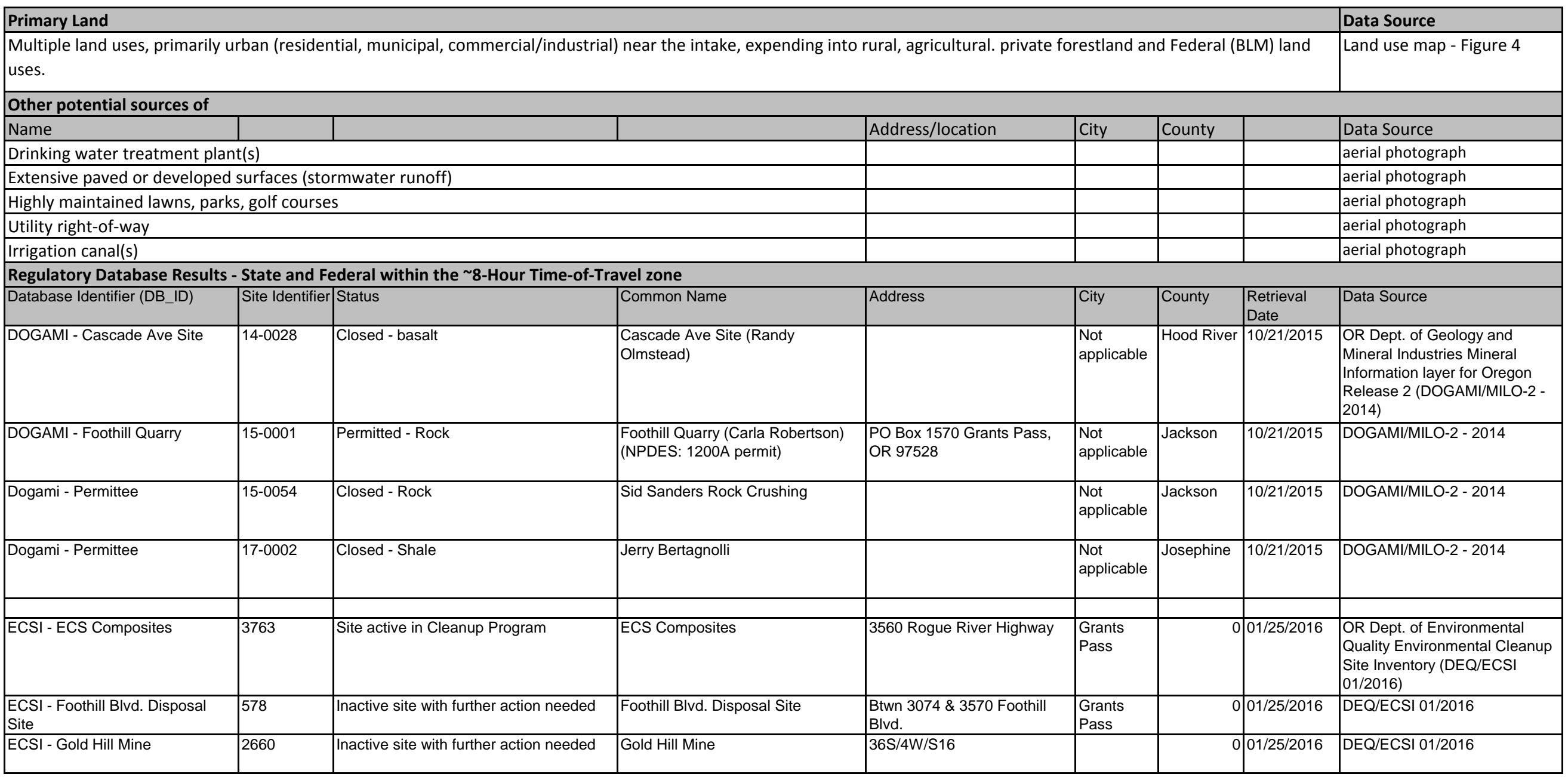




\begin{tabular}{|c|c|c|c|c|c|c|c|c|}
\hline Database Identifier (DB_ID) & Site Identifier & Status & Common Name & Address & City & County & \begin{tabular}{|l} 
Retrieval \\
Date
\end{tabular} & Data Source \\
\hline $\begin{array}{l}\text { ECSI - Hansen Site (Grants } \\
\text { Pass) }\end{array}$ & 5275 & Inactive site with further action needed & Hansen Site (Grants Pass) & Ament and N Sts. & \begin{tabular}{|l} 
Grants \\
Pass
\end{tabular} & 0 & $01 / 25 / 2016$ & DEQ/ECSI 01/2016 \\
\hline ECSI - Hidden Treasure Mine & 2661 & Inactive site with further action needed & Hidden Treasure Mine & $36 \mathrm{~S} / 4 \mathrm{~W} / \mathrm{S} 16$ & & 0 & $01 / 25 / 2016$ & DEQ/ECSI 01/2016 \\
\hline ECSI - Jewett Mine & 2662 & Inactive site with further action needed & Jewett Mine & $36 \mathrm{~S} / 5 \mathrm{~W} / \mathrm{S} 27$ & & 0 & $01 / 25 / 2016$ & DEQ/ECSI 01/2016 \\
\hline $\begin{array}{l}\text { ECSI - Laureldale Lane } \\
\text { Groundwater }\end{array}$ & 4520 & Site active in Cleanup Program & Laureldale Lane Groundwater & $\begin{array}{l}\text { 200-400 block Laureldale Ln. } \\
\text { \& 300-400 block Woodburn } \\
\text { Ln. }\end{array}$ & $\begin{array}{l}\text { Grants } \\
\text { Pass }\end{array}$ & 0 & $01 / 25 / 2016$ & DEQ/ECSI 01/2016 \\
\hline $\begin{array}{l}\text { ECSI - NFA - Grants Pass } \\
\text { Irrigation Dist. }\end{array}$ & 547 & No further action required & Grants Pass Irrigation Dist. & $36 \mathrm{~S} / 5 \mathrm{~W} / \mathrm{S} 23,24,14$ & $\begin{array}{l}\text { Grants } \\
\text { Pass }\end{array}$ & 0 & $01 / 25 / 2016$ & DEQ/ECSI 01/2016 \\
\hline $\begin{array}{l}\text { ECSI - NFA - Rogue River } \\
\text { Towing }\end{array}$ & 4202 & No further action required & Rogue River Towing & 107 Depot St. & \begin{tabular}{|l|} 
Rogue \\
River
\end{tabular} & 0 & $01 / 25 / 2016$ & DEQ/ECSI 01/2016 \\
\hline $\begin{array}{l}\text { ECSI - NFA - US Forest } \\
\text { Industries }\end{array}$ & 571 & No further action required & US Forest Industries & 1090 SE "M" St. & \begin{tabular}{|l|} 
Grants \\
Pass
\end{tabular} & 0 & $01 / 25 / 2016$ & DEQ/ECSI 01/2016 \\
\hline $\begin{array}{l}\text { ECSI - NFA - Warehouse } \\
\text { Rebuilders \& Manufacturing }\end{array}$ & 1516 & No further action required & $\begin{array}{l}\text { Warehouse Rebuilders \& } \\
\text { Manufacturing }\end{array}$ & 1961 SE "N" St. & \begin{tabular}{|l|} 
Grants \\
Pass
\end{tabular} & 0 & $01 / 25 / 2016$ & DEQ/ECSI 01/2016 \\
\hline \multirow[t]{2}{*}{ ECSI - Spalding \& Son Inc. } & 552 & Inactive site with further action needed & Spalding \& Son Inc. & 2345 SE "N" St. & $\begin{array}{l}\text { Grants } \\
\text { Pass }\end{array}$ & 0 & $01 / 25 / 2016$ & DEQ/ECSI 01/2016 \\
\hline & & & & & & 0 & & \\
\hline $\begin{array}{l}\text { Haz Waste Generator - Becklin } \\
\text { Holdings Inc. }\end{array}$ & $\begin{array}{l}\text { ORD059497 } \\
768\end{array}$ & Conditionally Exempt Generator (CEG) & Becklin Holdings Inc. & 3560 ROGUE RIVER HWY & \begin{tabular}{|l} 
GRANTS \\
PASS
\end{tabular} & Josephine & $01 / 29 / 2016$ & $\begin{array}{l}\text { OR Dept. of Environmental } \\
\text { Quality Hazardous Waste } \\
\text { Program database (DEQ/HW - } \\
01 / 2016 \text { ) }\end{array}$ \\
\hline \begin{tabular}{l|} 
Haz Waste Generator - First \\
Student, Inc. \#20204A
\end{tabular} & \begin{tabular}{|l|} 
ORD987199 \\
304
\end{tabular} & Conditionally Exempt Generator (CEG) & First Student, Inc. \#20204A & 1898 E EVANS CREEK RD & \begin{tabular}{|l} 
ROGUE \\
RIVER
\end{tabular} & Jackson & $01 / 29 / 2016$ & DEQ/HW - 01/2016 \\
\hline $\begin{array}{l}\text { Haz Waste Generator - Schnitzer } \\
\text { Steel Ind Inc For* }\end{array}$ & $\begin{array}{l}\text { ORQ000013 } \\
508\end{array}$ & Conditionally Exempt Generator (CEG) & $\begin{array}{l}\text { Schnitzer Steel Ind Inc Former } \\
\text { Sessler }\end{array}$ & 605 NE AGNES AVE & \begin{tabular}{|l|} 
GRANTS \\
PASS \\
\end{tabular} & Josephine & $01 / 29 / 2016$ & DEQ/HW - 01/2016 \\
\hline $\begin{array}{l}\text { Haz Waste Generator - Spalding } \\
\text { \& Son Inc }\end{array}$ & \begin{tabular}{|l|} 
ORD009411 \\
737
\end{tabular} & Conditionally Exempt Generator (CEG) & Spalding \& Son Inc & 2345 SE N ST & $\begin{array}{l}\text { GRANTS } \\
\text { PASS }\end{array}$ & Josephine & $01 / 29 / 2016$ & DEQ/HW - 01/2016 \\
\hline $\begin{array}{l}\text { Haz Waste Generator - Star } \\
\text { Collision Center }\end{array}$ & $\begin{array}{l}\text { ORD982659 } \\
575\end{array}$ & Conditionally Exempt Generator (CEG) & Star Collision Center & 210 GILMORE & \begin{tabular}{|l|} 
ROGUE \\
RIVER
\end{tabular} & Jackson & $01 / 29 / 2016$ & DEQ/HW - 01/2016 \\
\hline $\begin{array}{l}\text { Haz Waste Generator - } \\
\text { Swissmetric Inc }\end{array}$ & \begin{tabular}{|l|} 
ORD987198 \\
975
\end{tabular} & Conditionally Exempt Generator (CEG) & Swissmetric Inc & 1610 PARKDALE DR & $\begin{array}{l}\text { GRANTS } \\
\text { PASS }\end{array}$ & Josephine & $01 / 29 / 2016$ & DEQ/HW - 01/2016 \\
\hline $\begin{array}{l}\text { Haz Waste Generator - } \\
\text { Warehouse Rebuilding \& Mfg * }\end{array}$ & $\begin{array}{l}\text { ORD987202 } \\
447\end{array}$ & Conditionally Exempt Generator (CEG) & Warehouse Rebuilding \& Mfg Inc & 961 SE GLADIOLA DR & $\begin{array}{l}\text { GRANTS } \\
\text { PASS }\end{array}$ & Josephine & $01 / 29 / 2016$ & DEQ/HW - 01/2016 \\
\hline LUST - COLVIN OIL CO INC & $17-98-0086$ & CLEANUP_STARTED & COLVIN OIL CO INC & 2520 FOOTHILL BLVD & \begin{tabular}{|l} 
GRANTS \\
PASS
\end{tabular} & Josephine & $01 / 25 / 2016$ & $\begin{array}{l}\text { OR Dept. of Environmental } \\
\text { Quality Land Quality Leaking } \\
\text { Underground Storage Tank } \\
\text { database (DEQ/LUST - 2016) }\end{array}$ \\
\hline $\begin{array}{l}\text { LUST - POWELL DIST CO - } \\
\text { COLVIN/GRANTS PASS }\end{array}$ & $17-99-0050$ & CLEANUP_STARTED & \begin{tabular}{|l|} 
POWELL DIST CO - \\
COLVIN/GRANTS PASS
\end{tabular} & $\begin{array}{l}\text { 1919 ROGUE RIVER } \\
\text { HIGHWAY }\end{array}$ & \begin{tabular}{|l} 
GRANTS \\
PASS
\end{tabular} & Josephine & $01 / 25 / 2016$ & DEQ/LUST - 2016 \\
\hline LUST - SABO, BARBARA HOT & $34-99-1046$ & CLEANUP_STARTED & SABO, BARBARA HOT & 605 NE AGNESS AVE & \begin{tabular}{|l|} 
GRANTS \\
PASS
\end{tabular} & Josephine & $01 / 25 / 2016$ & DEQ/LUST - 2016 \\
\hline LUST - U-SAVE GAS & $17-98-0090$ & CLEANUP_STARTED & U-SAVE GAS & 935 ROGUE RIVER HWY & $\begin{array}{l}\text { GRANTS } \\
\text { PASS }\end{array}$ & Josephine & $01 / 25 / 2016$ & DEQ/LUST - 2016 \\
\hline & & & & & & & & DEQ/LUST - 2016 \\
\hline LUST NFA - ALTHEIDE, SYLVIA & $17-99-0056$ & CLEANUP_COMPLETED & ALTHEIDE, SYLVIA & 3660 ROGUE RIVER HWY & $\begin{array}{l}\text { GRANTS } \\
\text { PASS }\end{array}$ & Josephine & $01 / 25 / 2016$ & DEQ/LUST - 2016 \\
\hline $\begin{array}{l}\text { LUST NFA - CLOVER CREST } \\
\text { MARKET }\end{array}$ & $17-02-1517$ & CLEANUP_COMPLETED & CLOVER CREST MARKET & 2600 CLOVERLAWN DR & \begin{tabular}{|l} 
GRANTS \\
PASS
\end{tabular} & Josephine & $01 / 25 / 2016$ & DEQ/LUST - 2016 \\
\hline
\end{tabular}




\begin{tabular}{|c|c|c|c|c|c|c|c|c|}
\hline Database Identifier (DB_ID) & Site Identifier & Status & Common Name & Address & City & County & \begin{tabular}{|l|} 
Retrieval \\
Date
\end{tabular} & Data Source \\
\hline $\begin{array}{l}\text { LUST NFA - COLVIN OIL CO } \\
\text { INC }\end{array}$ & $17-99-0076$ & CLEANUP_COMPLETED & COLVIN OIL CO INC & 2520 FOOTHILL BLVD & \begin{tabular}{|l} 
GRANTS \\
PASS
\end{tabular} & Josephine & $01 / 25 / 2016$ & DEQ/LUST - 2016 \\
\hline $\begin{array}{l}\text { LUST NFA - COLVIN OIL CO., } \\
\text { ROGUE RIVER ARCO }\end{array}$ & $15-90-0110$ & CLEANUP_COMPLETED & $\begin{array}{l}\text { COLVIN OIL CO., ROGUE RIVER } \\
\text { ARCO }\end{array}$ & 95 PINE ST & $\begin{array}{l}\text { ROGUE } \\
\text { RIVER }\end{array}$ & Jackson & $01 / 25 / 2016$ & DEQ/LUST - 2016 \\
\hline $\begin{array}{l}\text { LUST NFA - FRUITDALE } \\
\text { SCHOOL HOT }\end{array}$ & $17-96-0064$ & CLEANUP_COMPLETED & FRUITDALE SCHOOL HOT & 1560 HAMILTON LN & $\begin{array}{l}\text { GRANTS } \\
\text { PASS } \\
\end{array}$ & Josephine & $01 / 25 / 2016$ & DEQ/LUST - 2016 \\
\hline $\begin{array}{l}\text { LUST NFA - GI JOES } \\
\text { PROPERTY }\end{array}$ & $17-94-0020$ & CLEANUP_COMPLETED & GI JOES PROPERTY & $\begin{array}{l}\text { SW CORNER OF } \\
\text { REDWOOD AVE \& AGNESS } \\
\text { AVE }\end{array}$ & $\begin{array}{l}\text { GRANTS } \\
\text { PASS }\end{array}$ & Josephine & $01 / 25 / 2016$ & DEQ/LUST - 2016 \\
\hline $\begin{array}{l}\text { LUST NFA - JOSEPHINE } \\
\text { COUNTY SHERIFF STORAGE } \\
\text { YARD }\end{array}$ & $17-03-2539$ & CLEANUP_COMPLETED & $\begin{array}{l}\text { JOSEPHINE COUNTY SHERIFF } \\
\text { STORAGE YARD }\end{array}$ & 1798 SE N ST & $\begin{array}{l}\text { GRANTS } \\
\text { PASS }\end{array}$ & Josephine & $01 / 25 / 2016$ & DEQ/LUST - 2016 \\
\hline $\begin{array}{l}\text { LUST NFA - LEW MERRILL } \\
\text { LUMBER CO. }\end{array}$ & $17-90-0022$ & CLEANUP_COMPLETED & LEW MERRILL LUMBER CO. & 1750 SE N ST & $\begin{array}{l}\text { GRANTS } \\
\text { PASS }\end{array}$ & Josephine & $01 / 25 / 2016$ & DEQ/LUST - 2016 \\
\hline $\begin{array}{l}\text { LUST NFA - MOORE, M HOT } \\
\text { (MOORE RESIDENCE) }\end{array}$ & $15-00-0025$ & CLEANUP_COMPLETED & $\begin{array}{l}\text { MOORE, M HOT (MOORE } \\
\text { RESIDENCE) }\end{array}$ & 6595 ROGUE RIVER HWY & $\begin{array}{l}\text { GRANTS } \\
\text { PASS }\end{array}$ & Jackson & $01 / 25 / 2016$ & DEQ/LUST - 2016 \\
\hline LUST NFA - NEGRO, GAYLE & $17-94-0037$ & CLEANUP_COMPLETED & NEGRO, GAYLE & 257 GORDON WAY & \begin{tabular}{|l|} 
GRANTS \\
PASS
\end{tabular} & Josephine & $01 / 25 / 2016$ & DEQ/LUST - 2016 \\
\hline $\begin{array}{l}\text { LUST NFA - OREGON STATE } \\
\text { HIGHWAY, 3-8 GRANTS PASS }\end{array}$ & 17-92-0092 & CLEANUP_COMPLETED & $\begin{array}{l}\text { OREGON STATE HIGHWAY, 3-8 } \\
\text { GRANTS PASS }\end{array}$ & 345 AGNESS AVE & $\begin{array}{l}\text { GRANTS } \\
\text { PASS }\end{array}$ & Josephine & $01 / 25 / 2016$ & DEQ/LUST - 2016 \\
\hline $\begin{array}{l}\text { LUST NFA - PETERSON, GARY } \\
\text { L. }\end{array}$ & $17-93-0037$ & CLEANUP_COMPLETED & PETERSON, GARY L. & 910 SE M ST & \begin{tabular}{|l} 
GRANTS \\
PASS
\end{tabular} & Josephine & $01 / 25 / 2016$ & DEQ/LUST - 2016 \\
\hline $\begin{array}{l}\text { LUST NFA - RIVERSIDE } \\
\text { SCHOOL HOT }\end{array}$ & $17-93-0074$ & CLEANUP_COMPLETED & RIVERSIDE SCHOOL HOT & 1200 SE HARVEY DR & \begin{tabular}{|l|} 
GRANTS \\
PASS
\end{tabular} & Josephine & $01 / 25 / 2016$ & DEQ/LUST - 2016 \\
\hline $\begin{array}{l}\text { LUST NFA - ROGUE RIVER } \\
\text { SCHOOL DISTRICT }\end{array}$ & $15-91-0121$ & CLEANUP_COMPLETED & $\begin{array}{l}\text { ROGUE RIVER SCHOOL } \\
\text { DISTRICT }\end{array}$ & 1898 E EVANS CREEK RD & $\begin{array}{l}\text { ROGUE } \\
\text { RIVER }\end{array}$ & Jackson & $01 / 25 / 2016$ & DEQ/LUST - 2016 \\
\hline $\begin{array}{l}\text { LUST NFA - ROGUE RIVER } \\
\text { TOW-LARRY'S TRIANGLE } \\
\text { SERVI }\end{array}$ & 15-93-0095 & CLEANUP_COMPLETED & $\begin{array}{l}\text { ROGUE RIVER TOW-LARRY'S } \\
\text { TRIANGLE SERVICE }\end{array}$ & MAIN \& DEPOT ST & $\begin{array}{l}\text { ROGUE } \\
\text { RIVER }\end{array}$ & Jackson & $01 / 25 / 2016$ & DEQ/LUST - 2016 \\
\hline $\begin{array}{l}\text { LUST NFA - ROGUE VALLEY } \\
\text { SASH \& DOOR }\end{array}$ & $17-93-0104$ & CLEANUP_COMPLETED & ROGUE VALLEY SASH \& DOOR & 123 NE BEACON DR & \begin{tabular}{|l|} 
GRANTS \\
PASS
\end{tabular} & Josephine & $01 / 25 / 2016$ & DEQ/LUST - 2016 \\
\hline LUST NFA - SMITH RESIDENCE & 15-94-0009 & CLEANUP_COMPLETED & SMITH RESIDENCE & 6916 W EVANS CREEK RD & \begin{tabular}{|l|} 
ROGUE \\
RIVER
\end{tabular} & Jackson & $01 / 25 / 2016$ & DEQ/LUST - 2016 \\
\hline $\begin{array}{l}\text { LUST NFA - SPALDING \& SON, } \\
\text { INC. }\end{array}$ & $17-90-0007$ & CLEANUP_COMPLETED & SPALDING \& SON, INC. & 2345 SE N ST & \begin{tabular}{|l} 
GRANTS \\
PASS
\end{tabular} & Josephine & $01 / 25 / 2016$ & DEQ/LUST - 2016 \\
\hline $\begin{array}{l}\text { LUST NFA - STONE FOREST } \\
\text { INDUSTRIES } \\
\end{array}$ & $17-93-0024$ & CLEANUP_COMPLETED & STONE FOREST INDUSTRIES & 851 SE MILBANK RD & \begin{tabular}{|l} 
GRANTS \\
PASS
\end{tabular} & Josephine & $01 / 25 / 2016$ & DEQ/LUST - 2016 \\
\hline OSMB - Chinook Park & $\begin{array}{l}\text { Chinook } \\
\text { Park }\end{array}$ & Boating access site & Chinook Park Boat Ramp & Chinook Park & Unknown & Josephine & $03 / 2016$ & $\begin{array}{l}\text { OR State Marine Board Boating } \\
\text { Access Sites Database (OSMB } \\
\text { 03/2016) }\end{array}$ \\
\hline OSMB - Coyote Evans Wayside & \begin{tabular}{|l|} 
Coyote \\
Evans \\
Wayside \\
\end{tabular} & Boating access site & $\begin{array}{l}\text { Coyote Evans Wayside City Boat } \\
\text { Ramp }\end{array}$ & Coyote Evans Wayside & Unknown & Jackson & $03 / 2016$ & OSMB 03/2016 \\
\hline OSMB - Pierce Riffle & Pierce Riffle & Boating access site & Pearce Riffle Park Boat Ramp & Pierce Riffle & Unknown & Josephine & $03 / 2016$ & OSMB 03/2016 \\
\hline $\begin{array}{l}\text { OSMB - Savage Rapids County } \\
\text { Park }\end{array}$ & \begin{tabular}{|l|} 
Savage \\
Rapids \\
County Park
\end{tabular} & Boating access site & Savage Rapids Boat Ramp & Savage Rapids County Park & Unknown & Douglas & $03 / 2016$ & OSMB 03/2016 \\
\hline & & & & & & & & \\
\hline
\end{tabular}




\begin{tabular}{|c|c|c|c|c|c|c|c|c|}
\hline Database Identifier (DB_ID) & Site Identifier & Status & Common Name & Address & City & County & \begin{tabular}{|l|} 
Retrieval \\
Date
\end{tabular} & Data Source \\
\hline $\begin{array}{l}\text { DHS/OHA - OFRA - Schools - } \\
\text { Fruitdale Elementary School }\end{array}$ & 445 & Unknown & Fruitdale Elementary School & 1560 Bill Baker Way & $\begin{array}{l}\text { Grants } \\
\text { Pass }\end{array}$ & Josephine & $06 / 02 / 2015$ & $\begin{array}{l}\text { OR Dept. of Human Services - } \\
\text { Office of Forecasting, } \\
\text { Research, and Analysis (via } \\
\text { OGDC (DHS/OHA - 2015) }\end{array}$ \\
\hline $\begin{array}{l}\text { DHS/OHA - OFRA - Schools - } \\
\text { Grants Pass High School }\end{array}$ & 442 & Unknown & Grants Pass High School & 1137 SE Gladiola Dr & $\begin{array}{l}\text { Grants } \\
\text { Pass }\end{array}$ & Josephine & $06 / 02 / 2015$ & DHS/OHA - 2015 \\
\hline $\begin{array}{l}\text { DHS/OHA - OFRA - Schools - } \\
\text { Newbridge High School }\end{array}$ & 2260 & Unknown & Newbridge High School & 2001 NE F St & \begin{tabular}{|l} 
Grants \\
Pass
\end{tabular} & Josephine & $06 / 02 / 2015$ & DHS/OHA - 2015 \\
\hline $\begin{array}{l}\text { DHS/OHA - OFRA - Schools - } \\
\text { Phagans' Grants Pass College of } \\
\text { Beauty }\end{array}$ & -1 & Unknown & $\begin{array}{l}\text { Phagans' Grants Pass College of } \\
\text { Beauty }\end{array}$ & 304 NE AGNESS & $\begin{array}{l}\text { GRANTS } \\
\text { PASS }\end{array}$ & Josephine & $06 / 02 / 2015$ & DHS/OHA - 2015 \\
\hline $\begin{array}{l}\text { DHS/OHA - OFRA - Schools - } \\
\text { Rivers Edge Academy Charter } \\
\text { School }\end{array}$ & 4856 & Unknown & $\begin{array}{l}\text { Rivers Edge Academy Charter } \\
\text { School }\end{array}$ & 270 W Evans Creek Rd & $\begin{array}{l}\text { Rogue } \\
\text { River }\end{array}$ & Jackson & $06 / 02 / 2015$ & DHS/OHA - 2015 \\
\hline $\begin{array}{l}\text { DHS/OHA - OFRA - Schools - } \\
\text { Riverside Elementary School }\end{array}$ & 440 & Unknown & Riverside Elementary School & 1200 SE Harvey Dr & $\begin{array}{l}\text { Grants } \\
\text { Pass }\end{array}$ & Josephine & $06 / 02 / 2015$ & $\mathrm{DHS} / \mathrm{OHA}-2015$ \\
\hline $\begin{array}{l}\text { DHS/OHA - OFRA - Schools - } \\
\text { Rogue River Elementary School }\end{array}$ & 399 & Unknown & Rogue River Elementary School & 300 Pine St & $\begin{array}{l}\text { Rogue } \\
\text { River }\end{array}$ & Jackson & $06 / 02 / 2015$ & DHS/OHA - 2015 \\
\hline $\begin{array}{l}\text { DHS/OHA - OFRA - Schools - } \\
\text { Rogue River Junior/Senior High }\end{array}$ & 401 & Unknown & Rogue River Junior/Senior High & 1898 E Evans Creek Rd & $\begin{array}{l}\text { Rogue } \\
\text { River }\end{array}$ & Jackson & $06 / 02 / 2015$ & DHS/OHA - 2015 \\
\hline $\begin{array}{l}\text { DHS/OHA - OFRA - Schools - } \\
\text { Rogue River SD } 35\end{array}$ & 2044 & Unknown & Rogue River SD 35 & 1898 E Evans Creek Rd & \begin{tabular}{|l|} 
Rogue \\
River \\
\end{tabular} & Jackson & $06 / 02 / 2015$ & DHS/OHA - 2015 \\
\hline $\begin{array}{l}\text { SFM - HSIS - ALPINE WEST } \\
\text { ENTERPRISES INC }\end{array}$ & 069658 & $\begin{array}{l}\text { CONST, MINING, \& FORESTRY } \\
\text { MACHINERY \& EQP with } 2 \text { different } \\
\text { chemicals reported on site (liquids and } \\
\text { solids only) }\end{array}$ & $\begin{array}{l}\text { ALPINE WEST ENTERPRISES } \\
\text { INC }\end{array}$ & 950 SAVAGE CREEK RD & $\begin{array}{l}\text { GRANTS } \\
\text { PASS }\end{array}$ & Josephine & $09 / 29 / 2008$ & $\begin{array}{l}\text { OR State Fire Marshall } \\
\text { Hazardous Substance } \\
\text { Information System database } \\
\text { (SFM/HSIS - 2009) }\end{array}$ \\
\hline $\begin{array}{l}\text { SFM - HSIS - BILL TERPENING } \\
\text { INC }\end{array}$ & 009950 & $\begin{array}{l}\text { OTHER GASOLINE STATIONS with } 3 \\
\text { different chemicals reported on site } \\
\text { (liquids and solids only) }\end{array}$ & BILL TERPENING INC & 505 ROGUE RIVER HWY & $\begin{array}{l}\text { GRANTS } \\
\text { PASS }\end{array}$ & Josephine & $09 / 29 / 2008$ & SFM/HSIS - 2009 \\
\hline SFM - HSIS - BRAKES PLUS & 083351 & $\begin{array}{l}\text { GENERAL AUTOMOTIVE REPAIR with } \\
1 \text { different chemicals reported on site } \\
\text { (liquids and solids only) }\end{array}$ & BRAKES PLUS & 1499 ROGUE RIVER HWY & $\begin{array}{l}\text { GRANTS } \\
\text { PASS }\end{array}$ & Josephine & $09 / 29 / 2008$ & SFM/HSIS - 2009 \\
\hline $\begin{array}{l}\text { SFM - HSIS - CABINET } \\
\text { CRAFTERS }\end{array}$ & 093327 & $\begin{array}{l}\text { WOOD KITCHEN CABINET \& } \\
\text { COUNTERTOP MFG with } 2 \text { different } \\
\text { chemicals reported on site (liquids and } \\
\text { solids only) }\end{array}$ & CABINET CRAFTERS & 1586 SE N ST & $\begin{array}{l}\text { GRANTS } \\
\text { PASS }\end{array}$ & Josephine & 09/29/2008 & SFM/HSIS - 2009 \\
\hline SFM - HSIS - CAVEMAN RV INC & 021796 & $\begin{array}{l}\text { RECREATIONAL VEHICLE DEALERS } \\
\text { with } 2 \text { different chemicals reported on } \\
\text { site (liquids and solids only) }\end{array}$ & CAVEMAN RV INC & 1190 ROGUE RIVER HWY & $\begin{array}{l}\text { GRANTS } \\
\text { PASS }\end{array}$ & Josephine & $09 / 29 / 2008$ & SFM/HSIS - 2009 \\
\hline $\begin{array}{l}\text { SFM - HSIS - COLVIN OIL CO } \\
\text { INC }\end{array}$ & 014478 & $\begin{array}{l}\text { PETROLEUM \& PETROLEUM } \\
\text { PRODUCTS WHSLE (EXC with } 3 \\
\text { different chemicals reported on site } \\
\text { (liquids and solids only) }\end{array}$ & COLVIN OIL CO INC & 2520 FOOTHILL BLVD & $\begin{array}{l}\text { GRANTS } \\
\text { PASS }\end{array}$ & Josephine & $09 / 29 / 2008$ & SFM/HSIS - 2009 \\
\hline $\begin{array}{l}\text { SFM - HSIS - COLVIN OIL CO } \\
\text { INC }\end{array}$ & 044949 & $\begin{array}{l}\text { GASOLINE STATIONS WITH } \\
\text { CONVENIENCE STORES with } 1 \\
\text { different chemicals reported on site } \\
\text { (liquids and solids only) }\end{array}$ & COLVIN OIL CO INC & 95 PINE ST & \begin{tabular}{|l} 
ROGUE \\
RIVER
\end{tabular} & Jackson & $09 / 29 / 2008$ & SFM/HSIS - 2009 \\
\hline
\end{tabular}




\begin{tabular}{|c|c|c|c|c|c|c|c|c|}
\hline Database Identifier (DB_ID) & Site Identifier & Status & Common Name & Address & City & County & \begin{tabular}{|l|} 
Retrieval \\
Date
\end{tabular} & Data Source \\
\hline $\begin{array}{l}\text { SFM - HSIS - E C S } \\
\text { COMPOSITES }\end{array}$ & 003080 & \begin{tabular}{|l|} 
ALL OTHER PLASTICS PRODUCT \\
MFG with 13 different chemicals reported \\
on site (liquids and solids only)
\end{tabular} & E C S COMPOSITES & 3560 ROGUE RIVER HWY & $\begin{array}{l}\text { GRANTS } \\
\text { PASS }\end{array}$ & Josephine & 09/29/2008 & SFM/HSIS - 2009 \\
\hline $\begin{array}{l}\text { SFM - HSIS - ECONOMY AUTO } \\
\text { SERVICE }\end{array}$ & 080625 & \begin{tabular}{|l|} 
GENERAL AUTOMOTIVE REPAIR with \\
2 different chemicals reported on site \\
(liquids and solids only)
\end{tabular} & ECONOMY AUTO SERVICE & 5482 N RIVER RD & $\begin{array}{l}\text { GOLD } \\
\text { HILL }\end{array}$ & Jackson & $09 / 29 / 2008$ & SFM/HSIS - 2009 \\
\hline $\begin{array}{l}\text { SFM - HSIS - ENCORE } \\
\text { CERAMICS INC }\end{array}$ & 086960 & $\begin{array}{l}\text { CERAMIC WALL \& FLOOR TILE MFG } \\
\text { with } 13 \text { different chemicals reported on } \\
\text { site (liquids and solids only) }\end{array}$ & ENCORE CERAMICS INC & 2245 NE SPALDING AVE & $\begin{array}{l}\text { GRANTS } \\
\text { PASS }\end{array}$ & Josephine & $09 / 29 / 2008$ & SFM/HSIS - 2009 \\
\hline $\begin{array}{l}\text { SFM - HSIS - FERGUSON } \\
\text { ENTERPRISES INC }\end{array}$ & 052651 & $\begin{array}{l}\text { PLUMBING \& HEATING EQP \& } \\
\text { SUPPLIES (HYDRONI with } 3 \text { different } \\
\text { chemicals reported on site (liquids and } \\
\text { solids only) }\end{array}$ & FERGUSON ENTERPRISES INC & 1470 SE M ST & \begin{tabular}{|l} 
GRANTS \\
PASS
\end{tabular} & Josephine & $09 / 29 / 2008$ & SFM/HSIS - 2009 \\
\hline $\begin{array}{l}\text { SFM - HSIS - FERGUSON } \\
\text { FABRICATION LLC }\end{array}$ & 070869 & $\begin{array}{l}\text { ORNAMENTAL \& ARCHITECTURAL } \\
\text { MTL WORK MFG with } 1 \text { different } \\
\text { chemicals reported on site (liquids and } \\
\text { solids only) }\end{array}$ & FERGUSON FABRICATION LLC & 959 SE GLADIOLA DR & $\begin{array}{l}\text { GRANTS } \\
\text { PASS }\end{array}$ & Josephine & $09 / 29 / 2008$ & SFM/HSIS - 2009 \\
\hline $\begin{array}{l}\text { SFM - HSIS - FINISH LINE } \\
\text { SERVICE CENTER }\end{array}$ & 104808 & $\begin{array}{l}\text { GENERAL AUTOMOTIVE REPAIR with } \\
3 \text { different chemicals reported on site } \\
\text { (liquids and solids only) }\end{array}$ & FINISH LINE SERVICE CENTER & 202 PARK ST & \begin{tabular}{|l} 
ROGUE \\
RIVER
\end{tabular} & Jackson & 09/29/2008 & SFM/HSIS - 2009 \\
\hline SFM - HSIS - FIRST STUDENT & 043766 & $\begin{array}{l}\text { SCHOOL \& EMPLOYEE BUS TRANS } \\
\text { with } 6 \text { different chemicals reported on } \\
\text { site (liquids and solids only) }\end{array}$ & FIRST STUDENT & 1898 E EVANS CREEK RD & \begin{tabular}{|l} 
ROGUE \\
RIVER
\end{tabular} & Jackson & $09 / 29 / 2008$ & SFM/HSIS - 2009 \\
\hline $\begin{array}{l}\text { SFM - HSIS - FRED MEYER } \\
\text { STORES INC }\end{array}$ & 018848 & $\begin{array}{l}\text { SUPERMARKETS \& OTHER } \\
\text { GROCERY (EXC CONVENIE with } 3 \\
\text { different chemicals reported on site } \\
\text { (liquids and solids only) } \\
\end{array}$ & FRED MEYER STORES INC & 1101 GRANTS PASS PKWY & $\begin{array}{l}\text { GRANTS } \\
\text { PASS }\end{array}$ & Josephine & $09 / 29 / 2008$ & SFM/HSIS - 2009 \\
\hline SFM - HSIS - G \& S EQUIP INC & 036540 & $\begin{array}{l}\text { OUTDOOR POWER EQP STORES with } \\
1 \text { different chemicals reported on site } \\
\text { (liquids and solids only) }\end{array}$ & G \& S EQUIP INC & 850 ROGUE RIVER HWY & $\begin{array}{l}\text { GRANTS } \\
\text { PASS }\end{array}$ & Josephine & $09 / 29 / 2008$ & SFM/HSIS - 2009 \\
\hline $\begin{array}{l}\text { SFM - HSIS - GARY PETERSON } \\
\text { EXCAVATING }\end{array}$ & 031607 & $\begin{array}{l}\text { READY-MIX CONCRETE MFG with } 2 \\
\text { different chemicals reported on site } \\
\text { (liquids and solids only) }\end{array}$ & GARY PETERSON EXCAVATING & 910 SE M ST & \begin{tabular}{|l} 
GRANTS \\
PASS
\end{tabular} & Josephine & $09 / 29 / 2008$ & SFM/HSIS - 2009 \\
\hline $\begin{array}{l}\text { SFM - HSIS - GRAN MAR } \\
\text { NATURAL STONE INC }\end{array}$ & 103553 & $\begin{array}{l}\text { OTHER BLDG MATERIAL DEALERS } \\
\text { with } 1 \text { different chemicals reported on } \\
\text { site (liquids and solids only) }\end{array}$ & $\begin{array}{l}\text { GRAN MAR NATURAL STONE } \\
\text { INC }\end{array}$ & 1470 SE M ST 2B & \begin{tabular}{|l} 
GRANTS \\
PASS
\end{tabular} & Josephine & $09 / 29 / 2008$ & SFM/HSIS - 2009 \\
\hline $\begin{array}{l}\text { SFM - HSIS - GRANTS PASS } \\
\text { CITY OF }\end{array}$ & 021089 & $\begin{array}{l}\text { OTHER GENERAL GOV SUPPORT with } \\
11 \text { different chemicals reported on site } \\
\text { (liquids and solids only) }\end{array}$ & GRANTS PASS CITY OF & 821 SE M ST & \begin{tabular}{|l|} 
GRANTS \\
PASS
\end{tabular} & Josephine & $09 / 29 / 2008$ & SFM/HSIS - 2009 \\
\hline $\begin{array}{l}\text { SFM - HSIS - GRANTS PASS } \\
\text { CITY OF }\end{array}$ & 110072 & $\begin{array}{l}\text { OTHER GENERAL GOV SUPPORT with } \\
1 \text { different chemicals reported on site } \\
\text { (liquids and solids only) }\end{array}$ & GRANTS PASS CITY OF & 2202 SE HAVILAND DR & $\begin{array}{l}\text { GRANTS } \\
\text { PASS }\end{array}$ & Josephine & $09 / 29 / 2008$ & SFM/HSIS - 2009 \\
\hline $\begin{array}{l}\text { SFM - HSIS - GRANTS PASS } \\
\text { EQUIPMENT RENTALS }\end{array}$ & 009021 & $\begin{array}{l}\text { OTHER COMMERCIAL \& IND } \\
\text { MACHINERY \& EQP REN with } 2 \\
\text { different chemicals reported on site } \\
\text { (liquids and solids only) }\end{array}$ & $\begin{array}{l}\text { GRANTS PASS EQUIPMENT } \\
\text { RENTALS }\end{array}$ & 851 SE MILLBANK RD & \begin{tabular}{|l|} 
GRANTS \\
PASS
\end{tabular} & Josephine & $09 / 29 / 2008$ & SFM/HSIS - 2009 \\
\hline $\begin{array}{l}\text { SFM - HSIS - GRANTS PASS } \\
\text { SCHOOL DISTRICT } 7\end{array}$ & 072527 & $\begin{array}{l}\text { ELEMENTARY \& SECONDARY } \\
\text { SCHOOLS with } 4 \text { different chemicals } \\
\text { reported on site (liquids and solids only) }\end{array}$ & $\begin{array}{l}\text { GRANTS PASS SCHOOL } \\
\text { DISTRICT } 7\end{array}$ & 1053 SE GLADIOLA ST & \begin{tabular}{|l|} 
GRANTS \\
PASS
\end{tabular} & Josephine & $09 / 29 / 2008$ & SFM/HSIS - 2009 \\
\hline
\end{tabular}




\begin{tabular}{|c|c|c|c|c|c|c|c|c|}
\hline Database Identifier (DB_ID) & Site Identifier & Status & Common Name & Address & City & County & \begin{tabular}{|l|} 
Retrieval \\
Date
\end{tabular} & Data Source \\
\hline $\begin{array}{l}\text { SFM - HSIS - HAMS BODY } \\
\text { SHOP INC }\end{array}$ & 011600 & $\begin{array}{l}\text { AUTOMOTIVE BODY, PAINT, \& } \\
\text { INTERIOR REPAIR with } 1 \text { different } \\
\text { chemicals reported on site (liquids and } \\
\text { solids only) }\end{array}$ & HAMS BODY SHOP INC & 2560 FOOTHILL BLVD & \begin{tabular}{|l|} 
GRANTS \\
PASS
\end{tabular} & Josephine & $09 / 29 / 2008$ & SFM/HSIS - 2009 \\
\hline SFM - HSIS - HAYS OIL CO & 067018 & $\begin{array}{l}\text { OTHER GASOLINE STATIONS with } 2 \\
\text { different chemicals reported on site } \\
\text { (liquids and solids only) }\end{array}$ & HAYS OIL CO & $\begin{array}{l}3033 \text { FOOTHILL BLVD } \\
\text { BLDG D }\end{array}$ & \begin{tabular}{|l} 
GRANTS \\
PASS
\end{tabular} & Josephine & $09 / 29 / 2008$ & SFM/HSIS - 2009 \\
\hline $\begin{array}{l}\text { SFM - HSIS - INDIAN CREEK } \\
\text { AUTO }\end{array}$ & 010824 & $\begin{array}{l}\text { MOTOR VEHICLE TOWING with } 1 \\
\text { different chemicals reported on site } \\
\text { (liquids and solids only) }\end{array}$ & INDIAN CREEK AUTO & 1098 SE M ST & \begin{tabular}{|l} 
GRANTS \\
PASS
\end{tabular} & Josephine & $09 / 29 / 2008$ & SFM/HSIS - 2009 \\
\hline $\begin{array}{l}\text { SFM - HSIS - JERRYS CLUTCH } \\
\text { \& TRANS INC }\end{array}$ & 011524 & $\begin{array}{l}\text { AUTOMOTIVE TRANSMISSION } \\
\text { REPAIR with } 4 \text { different chemicals } \\
\text { reported on site (liquids and solids only) }\end{array}$ & JERRYS CLUTCH \& TRANS INC & 209 MACNEW LN & \begin{tabular}{|l} 
GRANTS \\
PASS
\end{tabular} & Josephine & $09 / 29 / 2008$ & SFM/HSIS - 2009 \\
\hline $\begin{array}{l}\text { SFM - HSIS - JOSEPHINE } \\
\text { COUNTY }\end{array}$ & 023946 & $\begin{array}{l}\text { OTHER GENERAL GOV SUPPORT with } \\
2 \text { different chemicals reported on site } \\
\text { (liquids and solids only) }\end{array}$ & JOSEPHINE COUNTY & 3700 PEARCE PARK RD & \begin{tabular}{|l} 
GRANTS \\
PASS
\end{tabular} & Josephine & 09/29/2008 & SFM/HSIS - 2009 \\
\hline $\begin{array}{l}\text { SFM - HSIS - KINGS } \\
\text { PROTECTIVE COATINGS }\end{array}$ & 092840 & \begin{tabular}{|l|} 
ALL OTHER AUTOMOTIVE REPAIR \& \\
MAINT with 2 different chemicals \\
reported on site (liquids and solids only)
\end{tabular} & KINGS PROTECTIVE COATINGS & 869 ROGUE RIVER HWY & \begin{tabular}{|l|} 
GRANTS \\
PASS
\end{tabular} & Josephine & 09/29/2008 & SFM/HSIS - 2009 \\
\hline $\begin{array}{l}\text { SFM - HSIS - MACES } \\
\text { EXCAVATION \& CONST }\end{array}$ & 027111 & \begin{tabular}{|l|} 
SITE PREPARATION CONTRACTORS \\
with 2 different chemicals reported on \\
site (liquids and solids only)
\end{tabular} & MACES EXCAVATION \& CONST & 597 AURORA AVE & \begin{tabular}{|l|} 
GRANTS \\
PASS
\end{tabular} & Josephine & 09/29/2008 & SFM/HSIS - 2009 \\
\hline $\begin{array}{l}\text { SFM - HSIS - MAIN BUILDING } \\
\text { SUPPLY INC }\end{array}$ & 015358 & $\begin{array}{l}\text { HARDWARE STORES with } 1 \text { different } \\
\text { chemicals reported on site (liquids and } \\
\text { solids only) }\end{array}$ & MAIN BUILDING SUPPLY INC & 100 BROADWAY & \begin{tabular}{|l} 
ROGUE \\
RIVER
\end{tabular} & Jackson & $09 / 29 / 2008$ & SFM/HSIS - 2009 \\
\hline $\begin{array}{l}\text { SFM - HSIS - MOCKS BODY } \\
\text { SHOP }\end{array}$ & 091231 & $\begin{array}{l}\text { GENERAL AUTOMOTIVE REPAIR with } \\
3 \text { different chemicals reported on site } \\
\text { (liquids and solids only) }\end{array}$ & MOCKS BODY SHOP & 2185 SPALDING AVE & \begin{tabular}{|l|} 
GRANTS \\
PASS
\end{tabular} & Josephine & $09 / 29 / 2008$ & SFM/HSIS - 2009 \\
\hline SFM - HSIS - MUFFLER KING & 039175 & $\begin{array}{l}\text { AUTOMOTIVE EXHAUST SYSTEM } \\
\text { REPAIR with } 2 \text { different chemicals } \\
\text { reported on site (liquids and solids only) }\end{array}$ & MUFFLER KING & 1492 ROGUE RIVER HWY & \begin{tabular}{|l} 
GRANTS \\
PASS
\end{tabular} & Josephine & $09 / 29 / 2008$ & SFM/HSIS - 2009 \\
\hline $\begin{array}{l}\text { SFM - HSIS - NAPA AUTO } \\
\text { PARTS }\end{array}$ & 068573 & $\begin{array}{l}\text { AUTOMOTIVE PARTS \& } \\
\text { ACCESSORIES STORES with } 3 \\
\text { different chemicals reported on site } \\
\text { (liquids and solids only) }\end{array}$ & NAPA AUTO PARTS & 200 FIRST ST & \begin{tabular}{|l|} 
ROGUE \\
RIVER
\end{tabular} & Jackson & $09 / 29 / 2008$ & SFM/HSIS - 2009 \\
\hline $\begin{array}{l}\text { SFM - HSIS - NORTHWEST } \\
\text { PIPELINE GP }\end{array}$ & 023491 & $\begin{array}{l}\text { PIPELINE TRANS OF NATURAL GAS } \\
\text { with } 1 \text { different chemicals reported on } \\
\text { site (liquids and solids only) }\end{array}$ & NORTHWEST PIPELINE GP & 737 W JONES CREEK RD & \begin{tabular}{|l|} 
GRANTS \\
PASS
\end{tabular} & Josephine & $09 / 29 / 2008$ & SFM/HSIS - 2009 \\
\hline SFM - HSIS - ODOT & 006044 & $\begin{array}{l}\text { OTHER GENERAL GOV SUPPORT with } \\
8 \text { different chemicals reported on site } \\
\text { (liquids and solids only) }\end{array}$ & ODOT & 345 AGNESS AVE & \begin{tabular}{|l} 
GRANTS \\
PASS
\end{tabular} & Josephine & $09 / 29 / 2008$ & SFM/HSIS - 2009 \\
\hline $\begin{array}{l}\text { SFM - HSIS - OREGON SWISS } \\
\text { PRECISION INC }\end{array}$ & 056542 & $\begin{array}{l}\text { BOLT, NUT, SCREW, RIVET, \& } \\
\text { WASHER MFG with } 1 \text { different } \\
\text { chemicals reported on site (liquids and } \\
\text { solids only) }\end{array}$ & OREGON SWISS PRECISION INC & 2143 NE SPALDING AVE & \begin{tabular}{|l|} 
GRANTS \\
PASS
\end{tabular} & Josephine & $09 / 29 / 2008$ & SFM/HSIS - 2009 \\
\hline SFM - HSIS - PACIFICORP & 110990 & \begin{tabular}{|l|} 
HYDROELECTRIC POWER \\
GENERATION with 1 different chemicals \\
reported on site (liquids and solids only)
\end{tabular} & PACIFICORP & E JONES CREEK RD & \begin{tabular}{|l} 
GRANTS \\
PASS
\end{tabular} & Josephine & $09 / 29 / 2008$ & SFM/HSIS - 2009 \\
\hline
\end{tabular}




\begin{tabular}{|c|c|c|c|c|c|c|c|c|}
\hline Database Identifier (DB_ID) & Site Identifier & Status & Common Name & Address & City & County & \begin{tabular}{|l|} 
Retrieval \\
Date
\end{tabular} & Data Source \\
\hline $\begin{array}{l}\text { SFM - HSIS - PANEL } \\
\text { PRODUCTS LLC }\end{array}$ & 017396 & $\begin{array}{l}\text { SOFTWOOD VENEER \& PLYWOOD } \\
\text { MFG with } 22 \text { different chemicals reported } \\
\text { on site (liquids and solids only) }\end{array}$ & PANEL PRODUCTS LLC & 5205 N RIVER RD & $\begin{array}{l}\text { GOLD } \\
\text { HILL }\end{array}$ & Jackson & 09/29/2008 & SFM/HSIS - 2009 \\
\hline $\begin{array}{l}\text { SFM - HSIS - QWEST } \\
\text { CORPORATION }\end{array}$ & 020962 & $\begin{array}{l}\text { OTHER TELECOMMUNICATIONS with } \\
2 \text { different chemicals reported on site } \\
\text { (liquids and solids only) }\end{array}$ & QWEST CORPORATION & 310 BROADWAY ST & \begin{tabular}{|l} 
ROGUE \\
RIVER
\end{tabular} & Jackson & $09 / 29 / 2008$ & SFM/HSIS - 2009 \\
\hline SFM - HSIS - ROBCO INC & 012007 & $\begin{array}{l}\text { HIGHWAY, STREET, \& BRIDGE } \\
\text { CONST with } 3 \text { different chemicals } \\
\text { reported on site (liquids and solids only) }\end{array}$ & ROBCO INC & 1935 ROGUE RIVER HWY & $\begin{array}{l}\text { GRANTS } \\
\text { PASS }\end{array}$ & Josephine & $09 / 29 / 2008$ & SFM/HSIS - 2009 \\
\hline SFM - HSIS - ROGER HANSEN & 009180 & $\begin{array}{l}\text { GENERAL AUTOMOTIVE REPAIR with } \\
4 \text { different chemicals reported on site } \\
\text { (liquids and solids only) }\end{array}$ & ROGER HANSEN & 1560 SE M ST & \begin{tabular}{|l} 
GRANTS \\
PASS
\end{tabular} & Josephine & $09 / 29 / 2008$ & SFM/HSIS - 2009 \\
\hline $\begin{array}{l}\text { SFM - HSIS - ROGUE RIVER } \\
\text { CITY OF }\end{array}$ & 016600 & $\begin{array}{l}\text { OTHER GENERAL GOV SUPPORT with } \\
5 \text { different chemicals reported on site } \\
\text { (liquids and solids only) }\end{array}$ & ROGUE RIVER CITY OF & 417 SECOND ST & \begin{tabular}{|l|} 
ROGUE \\
RIVER
\end{tabular} & Jackson & 09/29/2008 & SFM/HSIS - 2009 \\
\hline $\begin{array}{l}\text { SFM - HSIS - ROGUE RIVER } \\
\text { CITY OF }\end{array}$ & 023266 & $\begin{array}{l}\text { OTHER GENERAL GOV SUPPORT with } \\
2 \text { different chemicals reported on site } \\
\text { (liquids and solids only) }\end{array}$ & ROGUE RIVER CITY OF & 5680 FOOTHILL BLVD & \begin{tabular}{|l|} 
GRANTS \\
PASS
\end{tabular} & Jackson & 09/29/2008 & SFM/HSIS - 2009 \\
\hline $\begin{array}{l}\text { SFM - HSIS - ROGUE RIVER } \\
\text { CITY OF }\end{array}$ & 043940 & $\begin{array}{l}\text { OTHER GENERAL GOV SUPPORT with } \\
2 \text { different chemicals reported on site } \\
\text { (liquids and solids only) }\end{array}$ & ROGUE RIVER CITY OF & 333 CLASSICK DR & \begin{tabular}{|l} 
ROGUE \\
RIVER
\end{tabular} & Jackson & 09/29/2008 & SFM/HSIS - 2009 \\
\hline $\begin{array}{l}\text { SFM - HSIS - ROGUE RIVER } \\
\text { RURAL FIRE DIST }\end{array}$ & 069637 & $\begin{array}{l}\text { FIRE PROTECTION with } 3 \text { different } \\
\text { chemicals reported on site (liquids and } \\
\text { solids only) }\end{array}$ & ROGUE RIVER RURAL FIRE DIST & 5474 N RIVER RD & $\begin{array}{l}\text { GOLD } \\
\text { HILL }\end{array}$ & Jackson & $09 / 29 / 2008$ & SFM/HSIS - 2009 \\
\hline $\begin{array}{l}\text { SFM - HSIS - ROGUE RIVER } \\
\text { SCHOOL DISTRICT } 35\end{array}$ & 042776 & $\begin{array}{l}\text { ELEMENTARY \& SECONDARY } \\
\text { SCHOOLS with } 1 \text { different chemicals } \\
\text { reported on site (liquids and solids only) }\end{array}$ & $\begin{array}{l}\text { ROGUE RIVER SCHOOL } \\
\text { DISTRICT } 35\end{array}$ & 1898 E EVANS CREEK RD & \begin{tabular}{|l} 
ROGUE \\
RIVER
\end{tabular} & Jackson & $09 / 29 / 2008$ & SFM/HSIS - 2009 \\
\hline $\begin{array}{l}\text { SFM - HSIS - ROGUE VALLEY } \\
\text { MARBLE INC }\end{array}$ & 069644 & $\begin{array}{l}\text { CUT STONE \& STONE PRODUCT MFG } \\
\text { with } 6 \text { different chemicals reported on } \\
\text { site (liquids and solids only) }\end{array}$ & ROGUE VALLEY MARBLE INC & 2375 FOOTHILL BLVD & \begin{tabular}{|l} 
GRANTS \\
PASS
\end{tabular} & Josephine & $09 / 29 / 2008$ & SFM/HSIS - 2009 \\
\hline $\begin{array}{l}\text { SFM - HSIS - ROGUE VALLEY } \\
\text { SASH \& DOOR }\end{array}$ & 005620 & $\begin{array}{l}\text { WOOD WINDOW \& DOOR MFG with } 7 \\
\text { different chemicals reported on site } \\
\text { (liquids and solids only) }\end{array}$ & ROGUE VALLEY SASH \& DOOR & 123 NE BEACON DR & \begin{tabular}{|l} 
GRANTS \\
PASS
\end{tabular} & Josephine & $09 / 29 / 2008$ & SFM/HSIS - 2009 \\
\hline $\begin{array}{l}\text { SFM - HSIS - ROGUE VALLEY } \\
\text { SASH \& DOOR }\end{array}$ & 090924 & $\begin{array}{l}\text { WOOD WINDOW \& DOOR MFG with } 7 \\
\text { different chemicals reported on site } \\
\text { (liquids and solids only) }\end{array}$ & ROGUE VALLEY SASH \& DOOR & 124 NE BEACON DR & \begin{tabular}{|l} 
GRANTS \\
PASS
\end{tabular} & Josephine & $09 / 29 / 2008$ & SFM/HSIS - 2009 \\
\hline $\begin{array}{l}\text { SFM - HSIS - SCHNITZER } \\
\text { STEEL }\end{array}$ & 015527 & $\begin{array}{l}\text { RECYCLABLE MATERIAL WHSLE with } \\
2 \text { different chemicals reported on site } \\
\text { (liquids and solids only) }\end{array}$ & SCHNITZER STEEL & 605 NE AGNESS AVE & $\begin{array}{l}\text { GRANTS } \\
\text { PASS }\end{array}$ & Josephine & $09 / 29 / 2008$ & SFM/HSIS - 2009 \\
\hline $\begin{array}{l}\text { SFM - HSIS - SHERWIN- } \\
\text { WILLIAMS CO }\end{array}$ & 108146 & $\begin{array}{l}\text { PAINT \& WALLPAPER STORES with } 3 \\
\text { different chemicals reported on site } \\
\text { (liquids and solids only) }\end{array}$ & SHERWIN-WILLIAMS CO & 1072 ROGUE RIVER HWY & \begin{tabular}{|l} 
GRANTS \\
PASS
\end{tabular} & Josephine & $09 / 29 / 2008$ & SFM/HSIS - 2009 \\
\hline SFM - HSIS - SK8 LEVOR INC & 088067 & $\begin{array}{l}\text { PLASTICS MATERIAL \& RESIN MFG } \\
\text { with } 8 \text { different chemicals reported on } \\
\text { site (liquids and solids only) }\end{array}$ & SK8 LEVOR INC & 1470 SE M ST STE 2G & \begin{tabular}{|l} 
GRANTS \\
PASS
\end{tabular} & Josephine & $09 / 29 / 2008$ & SFM/HSIS - 2009 \\
\hline $\begin{array}{l}\text { SFM - HSIS - SLAYDEN } \\
\text { CONSTRUCTION GROUP INC }\end{array}$ & 107441 & $\begin{array}{l}\text { SITE PREPARATION CONTRACTORS } \\
\text { with } 1 \text { different chemicals reported on } \\
\text { site (liquids and solids only) }\end{array}$ & $\begin{array}{l}\text { SLAYDEN CONSTRUCTION } \\
\text { GROUP INC }\end{array}$ & 5645 ROGUE RIVER HWY & \begin{tabular}{|l} 
GRANTS \\
PASS
\end{tabular} & Josephine & $09 / 29 / 2008$ & SFM/HSIS - 2009 \\
\hline
\end{tabular}




\begin{tabular}{|c|c|c|c|c|c|c|c|c|}
\hline Database Identifier (DB_ID) & Site Identifier & Status & Common Name & Address & City & County & \begin{tabular}{|l} 
Retrieval \\
Date
\end{tabular} & Data Source \\
\hline $\begin{array}{l}\text { SFM - HSIS - SPALDING \& SON } \\
\text { INC }\end{array}$ & 006656 & $\begin{array}{l}\text { TIMBER TRACT OPERATIONS with } 2 \\
\text { different chemicals reported on site } \\
\text { (liquids and solids only) }\end{array}$ & SPALDING \& SON INC & 2345 NE N ST & $\begin{array}{l}\text { GRANTS } \\
\text { PASS }\end{array}$ & Josephine & $09 / 29 / 2008$ & SFM/HSIS - 2009 \\
\hline $\begin{array}{l}\text { SFM - HSIS - STAR COLLISION } \\
\text { CENTERS }\end{array}$ & 009934 & $\begin{array}{l}\text { AUTOMOTIVE BODY, PAINT, \& } \\
\text { INTERIOR REPAIR with } 2 \text { different } \\
\text { chemicals reported on site (liquids and } \\
\text { solids only) }\end{array}$ & STAR COLLISION CENTERS & 210 GILMORE & \begin{tabular}{|l} 
ROGUE \\
RIVER
\end{tabular} & Jackson & $09 / 29 / 2008$ & SFM/HSIS - 2009 \\
\hline $\begin{array}{l}\text { SFM - HSIS - STEVES } \\
\text { MUFFLER SHOP }\end{array}$ & 039171 & $\begin{array}{l}\text { AUTOMOTIVE EXHAUST SYSTEM } \\
\text { REPAIR with } 1 \text { different chemicals } \\
\text { reported on site (liquids and solids only) }\end{array}$ & STEVES MUFFLER SHOP & 860 ROGUE RIVER HWY & $\begin{array}{l}\text { GRANTS } \\
\text { PASS }\end{array}$ & Josephine & $09 / 29 / 2008$ & SFM/HSIS - 2009 \\
\hline $\begin{array}{l}\text { SFM - HSIS - SWANSON } \\
\text { GROUP AVIATION LLC }\end{array}$ & 091466 & $\begin{array}{l}\text { GENERAL AUTOMOTIVE REPAIR with } \\
3 \text { different chemicals reported on site } \\
\text { (liquids and solids only) }\end{array}$ & $\begin{array}{l}\text { SWANSON GROUP AVIATION } \\
\text { LLC }\end{array}$ & 2790 FOOTHILL BLVD & \begin{tabular}{|l} 
GRANTS \\
PASS
\end{tabular} & Josephine & $09 / 29 / 2008$ & SFM/HSIS - 2009 \\
\hline $\begin{array}{l}\text { SFM - HSIS - THREE RIVERS } \\
\text { PAVING COMPANY INC }\end{array}$ & 099150 & $\begin{array}{l}\text { ALL OTHER SPECIALTY TRADE } \\
\text { CONTRACTORS with } 1 \text { different } \\
\text { chemicals reported on site (liquids and } \\
\text { solids only) }\end{array}$ & $\begin{array}{l}\text { THREE RIVERS PAVING } \\
\text { COMPANY INC }\end{array}$ & 1935 ROGUE RIVER HWY & $\begin{array}{l}\text { GRANTS } \\
\text { PASS }\end{array}$ & Josephine & 09/29/2008 & SFM/HSIS - 2009 \\
\hline $\begin{array}{l}\text { SFM - HSIS - TIMBER } \\
\text { PRODUCTS COMPANY }\end{array}$ & 095404 & \begin{tabular}{|l|} 
HARDWOOD VENEER \& PLYWOOD \\
MFG with 33 different chemicals reported \\
on site (liquids and solids only)
\end{tabular} & TIMBER PRODUCTS COMPANY & 1090 SE M ST & $\begin{array}{l}\text { GRANTS } \\
\text { PASS }\end{array}$ & Josephine & $09 / 29 / 2008$ & SFM/HSIS - 2009 \\
\hline $\begin{array}{l}\text { SFM - HSIS - UMPQUA DAIRY } \\
\text { PRODUCTS CO }\end{array}$ & 060875 & \begin{tabular}{|l|} 
REFRIGERATED WAREHOUSING \& \\
STORAGE with 2 different chemicals \\
reported on site (liquids and solids only)
\end{tabular} & UMPQUA DAIRY PRODUCTS CO & 1686 SE N ST & \begin{tabular}{|l} 
GRANTS \\
PASS
\end{tabular} & Josephine & $09 / 29 / 2008$ & SFM/HSIS - 2009 \\
\hline $\begin{array}{l}\text { SFM - HSIS - U-SAVE GAS \& } \\
\text { TACKLE }\end{array}$ & 105267 & $\begin{array}{l}\text { GASOLINE STATIONS WITH } \\
\text { CONVENIENCE STORES with } 4 \\
\text { different chemicals reported on site } \\
\text { (liquids and solids only) } \\
\end{array}$ & U-SAVE GAS \& TACKLE & 935 ROGUE RIVER HWY & \begin{tabular}{|l} 
GRANTS \\
PASS
\end{tabular} & Josephine & $09 / 29 / 2008$ & SFM/HSIS - 2009 \\
\hline $\begin{array}{l}\text { SFM - HSIS - VALLEY } \\
\text { INSPECTIONS AND PESTS }\end{array}$ & 098351 & \begin{tabular}{l|} 
EXTERMINATING \& PEST CONTROL \\
SVCS with 2 different chemicals reported \\
on site (liquids and solids only)
\end{tabular} & $\begin{array}{l}\text { VALLEY INSPECTIONS AND } \\
\text { PESTS }\end{array}$ & 1360 SUMMIT LP & \begin{tabular}{|l} 
GRANTS \\
PASS
\end{tabular} & Josephine & $09 / 29 / 2008$ & SFM/HSIS - 2009 \\
\hline $\begin{array}{l}\text { SFM - HSIS - VERIZON } \\
\text { BUSINESS }\end{array}$ & 078265 & $\begin{array}{l}\text { WIRED TELECOMMUNICATIONS } \\
\text { CARRIERS with } 2 \text { different chemicals } \\
\text { reported on site (liquids and solids only) }\end{array}$ & VERIZON BUSINESS & 4070 B FOOTHILLS BLVD & $\begin{array}{l}\text { GRANTS } \\
\text { PASS }\end{array}$ & Josephine & $09 / 29 / 2008$ & SFM/HSIS - 2009 \\
\hline SFM-HSIS AST - BRAKES PLUS & 083351 & Aboveground storage tank(s) on site & BRAKES PLUS & 1499 ROGUE RIVER HWY & \begin{tabular}{|l} 
GRANTS \\
PASS
\end{tabular} & Josephine & $09 / 29 / 2008$ & $\begin{array}{l}\text { OR State Fire Marshall } \\
\text { Hazardous Substance } \\
\text { Information System database } \\
\text { (SFM/HSIS - 2009) }\end{array}$ \\
\hline $\begin{array}{l}\text { SFM-HSIS AST - FERGUSON } \\
\text { FABRICATION LLC }\end{array}$ & 070869 & Aboveground storage tank(s) on site & FERGUSON FABRICATION LLC & 959 SE GLADIOLA DR & $\begin{array}{l}\text { GRANTS } \\
\text { PASS }\end{array}$ & Josephine & $09 / 29 / 2008$ & SFM/HSIS - 2009 \\
\hline $\begin{array}{l}\text { SFM-HSIS AST - FINISH LINE } \\
\text { SERVICE CENTER }\end{array}$ & 104808 & Aboveground storage tank(s) on site & FINISH LINE SERVICE CENTER & 202 PARK ST & \begin{tabular}{|l} 
ROGUE \\
RIVER \\
\end{tabular} & Jackson & $09 / 29 / 2008$ & SFM/HSIS - 2009 \\
\hline $\begin{array}{l}\text { SFM-HSIS AST - FIRST } \\
\text { STUDENT }\end{array}$ & 043766 & Aboveground storage tank(s) on site & FIRST STUDENT & 1898 E EVANS CREEK RD & \begin{tabular}{|l} 
ROGUE \\
RIVER
\end{tabular} & Jackson & $09 / 29 / 2008$ & SFM/HSIS - 2009 \\
\hline $\begin{array}{l}\text { SFM-HSIS AST - GARY } \\
\text { PETERSON EXCAVATING }\end{array}$ & 031607 & Aboveground storage tank(s) on site & GARY PETERSON EXCAVATING & 910 SE M ST & $\begin{array}{l}\text { GRANTS } \\
\text { PASS }\end{array}$ & Josephine & $09 / 29 / 2008$ & SFM/HSIS - 2009 \\
\hline $\begin{array}{l}\text { SFM-HSIS AST - GRANTS } \\
\text { PASS EQUIPMENT RENTALS }\end{array}$ & 009021 & Aboveground storage tank(s) on site & $\begin{array}{l}\text { GRANTS PASS EQUIPMENT } \\
\text { RENTALS }\end{array}$ & 851 SE MILLBANK RD & \begin{tabular}{|l} 
GRANTS \\
PASS
\end{tabular} & Josephine & $09 / 29 / 2008$ & SFM/HSIS - 2009 \\
\hline
\end{tabular}




\begin{tabular}{|c|c|c|c|c|c|c|c|c|}
\hline Database Identifier (DB_ID) & Site Identifier & Status & Common Name & Address & City & County & \begin{tabular}{|l} 
Retrieval \\
Date
\end{tabular} & Data Source \\
\hline $\begin{array}{l}\text { SFM-HSIS AST - GRANTS } \\
\text { PASS SCHOOL DISTRICT } 7\end{array}$ & 072527 & Aboveground storage tank(s) on site & $\begin{array}{l}\text { GRANTS PASS SCHOOL } \\
\text { DISTRICT } 7\end{array}$ & 1053 SE GLADIOLA ST & \begin{tabular}{|l} 
GRANTS \\
PASS
\end{tabular} & Josephine & 09/29/2008 & SFM/HSIS - 2009 \\
\hline SFM-HSIS AST - HAYS OIL CO & 067018 & Aboveground storage tank(s) on site & HAYS OIL CO & $\begin{array}{l}3033 \text { FOOTHILL BLVD } \\
\text { BLDG D }\end{array}$ & \begin{tabular}{|l|} 
GRANTS \\
PASS
\end{tabular} & Josephine & $09 / 29 / 2008$ & SFM/HSIS - 2009 \\
\hline $\begin{array}{l}\text { SFM-HSIS AST - JERRYS } \\
\text { CLUTCH \& TRANS INC }\end{array}$ & 011524 & Aboveground storage tank(s) on site & JERRYS CLUTCH \& TRANS INC & 209 MACNEW LN & \begin{tabular}{|l} 
GRANTS \\
PASS
\end{tabular} & Josephine & $09 / 29 / 2008$ & SFM/HSIS - 2009 \\
\hline $\begin{array}{l}\text { SFM-HSIS AST - JOSEPHINE } \\
\text { COUNTY }\end{array}$ & 023946 & Aboveground storage tank(s) on site & JOSEPHINE COUNTY & 3700 PEARCE PARK RD & $\begin{array}{l}\text { GRANTS } \\
\text { PASS }\end{array}$ & Josephine & $09 / 29 / 2008$ & SFM/HSIS - 2009 \\
\hline $\begin{array}{l}\text { SFM-HSIS AST - MAIN } \\
\text { BUILDING SUPPLY INC }\end{array}$ & 015358 & Aboveground storage tank(s) on site & MAIN BUILDING SUPPLY INC & 100 BROADWAY & \begin{tabular}{|l|} 
ROGUE \\
RIVER
\end{tabular} & Jackson & $09 / 29 / 2008$ & SFM/HSIS - 2009 \\
\hline $\begin{array}{l}\text { SFM-HSIS AST - MUFFLER } \\
\text { KING }\end{array}$ & 039175 & Aboveground storage tank(s) on site & MUFFLER KING & 1492 ROGUE RIVER HWY & \begin{tabular}{|l|} 
GRANTS \\
PASS
\end{tabular} & Josephine & $09 / 29 / 2008$ & SFM/HSIS - 2009 \\
\hline $\begin{array}{l}\text { SFM-HSIS AST - NORTHWEST } \\
\text { PIPELINE GP }\end{array}$ & 023491 & Aboveground storage tank(s) on site & NORTHWEST PIPELINE GP & 737 W JONES CREEK RD & \begin{tabular}{|l|} 
GRANTS \\
PASS \\
\end{tabular} & Josephine & 09/29/2008 & SFM/HSIS - 2009 \\
\hline $\begin{array}{l}\text { SFM-HSIS AST - PANEL } \\
\text { PRODUCTS LLC }\end{array}$ & 017396 & Aboveground storage tank(s) on site & PANEL PRODUCTS LLC & 5205 N RIVER RD & $\begin{array}{l}\text { GOLD } \\
\text { HILL } \\
\end{array}$ & Jackson & $09 / 29 / 2008$ & SFM/HSIS - 2009 \\
\hline $\begin{array}{l}\text { SFM-HSIS AST - QWEST } \\
\text { CORPORATION }\end{array}$ & 020962 & Aboveground storage tank(s) on site & QWEST CORPORATION & 310 BROADWAY ST & \begin{tabular}{|l} 
ROGUE \\
RIVER
\end{tabular} & Jackson & $09 / 29 / 2008$ & SFM/HSIS - 2009 \\
\hline SFM-HSIS AST - ROBCO INC & 012007 & Aboveground storage tank(s) on site & ROBCO INC & 1935 ROGUE RIVER HWY & \begin{tabular}{|l} 
GRANTS \\
PASS
\end{tabular} & Josephine & $09 / 29 / 2008$ & SFM/HSIS - 2009 \\
\hline \begin{tabular}{l|} 
SFM-HSIS AST - ROGER \\
HANSEN
\end{tabular} & 009180 & Aboveground storage tank(s) on site & ROGER HANSEN & 1560 SE M ST & $\begin{array}{l}\text { GRANTS } \\
\text { PASS } \\
\end{array}$ & Josephine & $09 / 29 / 2008$ & SFM/HSIS - 2009 \\
\hline $\begin{array}{l}\text { SFM-HSIS AST - ROGUE RIVER } \\
\text { CITY OF }\end{array}$ & 023266 & Aboveground storage tank(s) on site & ROGUE RIVER CITY OF & 5680 FOOTHILL BLVD & $\begin{array}{l}\text { GRANTS } \\
\text { PASS }\end{array}$ & Jackson & $09 / 29 / 2008$ & SFM/HSIS - 2009 \\
\hline $\begin{array}{l}\text { SFM-HSIS AST - ROGUE RIVER } \\
\text { RURAL FIRE DIST }\end{array}$ & 069637 & Aboveground storage tank(s) on site & ROGUE RIVER RURAL FIRE DIST & 5474 N RIVER RD & \begin{tabular}{|l|} 
GOLD \\
HILL
\end{tabular} & Jackson & $09 / 29 / 2008$ & SFM/HSIS - 2009 \\
\hline $\begin{array}{l}\text { SFM-HSIS AST - ROGUE RIVER } \\
\text { SCHOOL DISTRICT } 35\end{array}$ & 042776 & Aboveground storage tank(s) on site & $\begin{array}{l}\text { ROGUE RIVER SCHOOL } \\
\text { DISTRICT } 35\end{array}$ & 1898 E EVANS CREEK RD & \begin{tabular}{|l} 
ROGUE \\
RIVER
\end{tabular} & Jackson & 09/29/2008 & SFM/HSIS - 2009 \\
\hline $\begin{array}{l}\text { SFM-HSIS AST - ROGUE } \\
\text { VALLEY SASH \& DOOR }\end{array}$ & 090924 & Aboveground storage tank(s) on site & ROGUE VALLEY SASH \& DOOR & 124 NE BEACON DR & $\begin{array}{l}\text { GRANTS } \\
\text { PASS }\end{array}$ & Josephine & $09 / 29 / 2008$ & SFM/HSIS - 2009 \\
\hline $\begin{array}{l}\text { SFM-HSIS AST - SCHNITZER } \\
\text { STEEL }\end{array}$ & 015527 & Aboveground storage tank(s) on site & SCHNITZER STEEL & 605 NE AGNESS AVE & $\begin{array}{l}\text { GRANTS } \\
\text { PASS } \\
\end{array}$ & Josephine & $09 / 29 / 2008$ & SFM/HSIS - 2009 \\
\hline $\begin{array}{l}\text { SFM-HSIS AST - SLAYDEN } \\
\text { CONSTRUCTION GROUP INC }\end{array}$ & 107441 & Aboveground storage tank(s) on site & $\begin{array}{l}\text { SLAYDEN CONSTRUCTION } \\
\text { GROUP INC }\end{array}$ & 5645 ROGUE RIVER HWY & \begin{tabular}{|l} 
GRANTS \\
PASS
\end{tabular} & Josephine & $09 / 29 / 2008$ & SFM/HSIS - 2009 \\
\hline $\begin{array}{l}\text { SFM-HSIS AST - SPALDING \& } \\
\text { SON INC }\end{array}$ & 006656 & Aboveground storage tank(s) on site & SPALDING \& SON INC & 2345 NE N ST & \begin{tabular}{|l} 
GRANTS \\
PASS
\end{tabular} & Josephine & $09 / 29 / 2008$ & SFM/HSIS - 2009 \\
\hline $\begin{array}{l}\text { SFM-HSIS AST - SWANSON } \\
\text { GROUP AVIATION LLC }\end{array}$ & 091466 & Aboveground storage tank(s) on site & $\begin{array}{l}\text { SWANSON GROUP AVIATION } \\
\text { LLC }\end{array}$ & 2790 FOOTHILL BLVD & \begin{tabular}{|l|} 
GRANTS \\
PASS
\end{tabular} & Josephine & 09/29/2008 & SFM/HSIS - 2009 \\
\hline $\begin{array}{l}\text { SFM-HSIS AST - TIMBER } \\
\text { PRODUCTS COMPANY }\end{array}$ & 095404 & Aboveground storage tank(s) on site & TIMBER PRODUCTS COMPANY & 1090 SE M ST & $\begin{array}{l}\text { GRANTS } \\
\text { PASS } \\
\end{array}$ & Josephine & 09/29/2008 & SFM/HSIS - 2009 \\
\hline $\begin{array}{l}\text { SFM-HSIS AST - UMPQUA } \\
\text { DAIRY PRODUCTS CO }\end{array}$ & 060875 & Aboveground storage tank(s) on site & UMPQUA DAIRY PRODUCTS CO & 1686 SE N ST & \begin{tabular}{|l} 
GRANTS \\
PASS
\end{tabular} & Josephine & $09 / 29 / 2008$ & SFM/HSIS - 2009 \\
\hline $\begin{array}{l}\text { SFM-HSIS AST - U-SAVE GAS \& } \\
\text { TACKLE }\end{array}$ & 105267 & Aboveground storage tank(s) on site & U-SAVE GAS \& TACKLE & 935 ROGUE RIVER HWY & $\begin{array}{l}\text { GRANTS } \\
\text { PASS }\end{array}$ & Josephine & $09 / 29 / 2008$ & SFM/HSIS - 2009 \\
\hline \begin{tabular}{l|} 
SFM-HSIS AST - VERIZON \\
BUSINESS
\end{tabular} & 078265 & Aboveground storage tank(s) on site & VERIZON BUSINESS & 4070 B FOOTHILLS BLVD & $\begin{array}{l}\text { GRANTS } \\
\text { PASS } \\
\end{array}$ & Josephine & $09 / 29 / 2008$ & SFM/HSIS - 2009 \\
\hline $\begin{array}{l}\text { SWMS - Clearwater Co-Op } \\
\text { TERMINATED }\end{array}$ & 109568 & $\begin{array}{l}1185 \text { Industrial - SW Treatment - } \\
\text { Terminated }\end{array}$ & Clearwater Co-Op TERMINATED & 1130 GLADIOLA DR & $\begin{array}{l}\text { GRANTS } \\
\text { PASS }\end{array}$ & Josephine & $01 / 25 / 2016$ & $\begin{array}{l}\text { OR Dept. of Environmental } \\
\text { Quality Solid Waste } \\
\text { Management System } \\
\text { (DEQ/SWMS - 2016) }\end{array}$ \\
\hline SWMS - Roger Hansen & 112143 & 1417 Waste Tire - Carrier - Active & Roger Hansen & 1560 SE M ST & $\begin{array}{l}\text { GRANTS } \\
\text { PASS }\end{array}$ & Josephine & $01 / 25 / 2016$ & DEQ/SWMS - 2016 \\
\hline
\end{tabular}




\begin{tabular}{|c|c|c|c|c|c|c|c|c|}
\hline Database Identifier (DB_ID) & Site Identifier $\leq$ & Status & Common Name & Address & City & County & \begin{tabular}{|l} 
Retrieval \\
Date
\end{tabular} & Data Source \\
\hline $\begin{array}{l}\text { SWMS - Rogue River Plywood } \\
\text { Division }\end{array}$ & 104315 & 1109 Industrial - Wood - Terminated & Rogue River Plywood Division & 5205 N ROGUE RIVER RD & \begin{tabular}{|l} 
ROGUE \\
RIVER
\end{tabular} & Jackson & $01 / 25 / 2016$ & DEQ/SWMS - 2016 \\
\hline $\begin{array}{l}\text { SWMS - Rogue River Plywood } \\
\text { Division }\end{array}$ & 104315 & 1198 Industrial - Wood - Closure & Rogue River Plywood Division & 5205 N ROGUE RIVER RD & \begin{tabular}{|l|} 
ROGUE \\
RIVER
\end{tabular} & Jackson & $01 / 25 / 2016$ & DEQ/SWMS - 2016 \\
\hline UIC - ECS Composites & 10243 & OnSite system / Registered w/permit & ECS Composites & 3560 Rogue River Highway & $\begin{array}{l}\text { Grants } \\
\text { Pass }\end{array}$ & Josephine & $01 / 12 / 2016$ & $\begin{array}{l}\text { OR Dept. of Environmental } \\
\text { Quality Underground Injection } \\
\text { Control database (DEQ/UIC - } \\
\text { 2016) }\end{array}$ \\
\hline UIC - Frontier Mobile Home Park & 10413 & OnSite system / Registered w/permit & Frontier Mobile Home Park & 7001 Rogue River Highway & $\begin{array}{l}\text { Rogue } \\
\text { River }\end{array}$ & Jackson & $01 / 12 / 2016$ & DEQ/UIC - 201 \\
\hline UIC - Pine Villa MHP & 12717 & OnSite system / Revoked & Pine Villa MHP & $\begin{array}{l}3381 \text { Rogue River Highway } \\
\# 28\end{array}$ & \begin{tabular}{|l|} 
Grants \\
Pass
\end{tabular} & Josephine & $01 / 12 / 2016$ & DEQ/UIC - 201 \\
\hline UIC - R River Place & 11541 & OnSite system / Registered w/permit & R River Place & 4550 Rogue River HWY & \begin{tabular}{|l|} 
Grants \\
Pass
\end{tabular} & Josephine & $01 / 12 / 2016$ & DEQ/UIC - 201 \\
\hline UIC - Rogue River School District & 11186 & OnSite system / Info request & Rogue River School District & 1898 E Evans Creek Rd & $\begin{array}{l}\text { Rogue } \\
\text { River }\end{array}$ & Jackson & $01 / 12 / 2016$ & DEQ/UIC - 201 \\
\hline \begin{tabular}{l|} 
UIC - Rogue River Valley Mobile \\
Estates
\end{tabular} & 10293 & OnSite system / Registered w/permit & Rogue River Valley Mobile Estates & 5648 Foothill Blvd. & $\begin{array}{l}\text { Grants } \\
\text { Pass }\end{array}$ & Jackson & $01 / 12 / 2016$ & DEQ/UIC - 201 \\
\hline $\begin{array}{l}\text { UIC - US Forest Industries (Stone } \\
\text { Forest Industries) }\end{array}$ & 10236 & $\begin{array}{l}\text { 2-Industrial/Commercial (Minor-HW) / } \\
\text { Abandoned }\end{array}$ & $\begin{array}{l}\text { US Forest Industries (Stone Forest } \\
\text { Industries) }\end{array}$ & 1090 SE "M" Street & $\begin{array}{l}\text { Grants } \\
\text { Pass }\end{array}$ & Josephine & $01 / 12 / 2016$ & DEQ/UIC - 201 \\
\hline UST - ALTHEIDE, SYLVIA & 9390 & $\begin{array}{l}0 \text { Upgraded, } 2 \text { Decommissioned, } 0 \\
\text { Unknown }\end{array}$ & ALTHEIDE, SYLVIA & 3660 ROGUE RIVER HWY & $\begin{array}{l}\text { GRANTS } \\
\text { PASS }\end{array}$ & Josephine & $01 / 25 / 2016$ & $\begin{array}{l}\text { OR Dept. of Environmental } \\
\text { Quality Underground Storage } \\
\text { Tank Program (DEQ/UST - } \\
\text { 2016) }\end{array}$ \\
\hline $\begin{array}{l}\text { UST - CLOVER CREST } \\
\text { MARKET }\end{array}$ & 2292 & $\begin{array}{l}0 \text { Upgraded, } 4 \text { Decommissioned, } 0 \\
\text { Unknown }\end{array}$ & CLOVER CREST MARKET & 2600 CLOVERLAWN DR & $\begin{array}{l}\text { GRANTS } \\
\text { PASS }\end{array}$ & Josephine & $01 / 25 / 2016$ & DEQ/UST - 2016 \\
\hline $\begin{array}{l}\text { UST - COLVIN OIL CO DBA } \\
\text { ROGUE RIVER CHEVRON }\end{array}$ & 6633 & $\begin{array}{l}3 \text { Upgraded, } 4 \text { Decommissioned, } 0 \\
\text { Unknown }\end{array}$ & $\begin{array}{l}\text { COLVIN OIL CO DBA ROGUE } \\
\text { RIVER CHEVRON }\end{array}$ & 95 PINE ST & \begin{tabular}{|l|} 
ROGUE \\
RIVER
\end{tabular} & Jackson & $01 / 25 / 2016$ & DEQ/UST - 2016 \\
\hline UST - COLVIN OIL CO INC & 6745 & $\begin{array}{l}4 \text { Upgraded, } 1 \text { Decommissioned, } 0 \\
\text { Unknown }\end{array}$ & COLVIN OIL CO INC & 2520 FOOTHILL BLVD & \begin{tabular}{|l|} 
GRANTS \\
PASS
\end{tabular} & Josephine & $01 / 25 / 2016$ & DEQ/UST - 2016 \\
\hline $\begin{array}{l}\text { UST - CONCORDE } \\
\text { INVESTMENTS }\end{array}$ & 5547 & $\begin{array}{l}0 \text { Upgraded, } 1 \text { Decommissioned, } 0 \\
\text { Unknown }\end{array}$ & CONCORDE INVESTMENTS & 1686 SE 'N' & \begin{tabular}{|l|} 
GRANTS \\
PASS
\end{tabular} & Josephine & $01 / 25 / 2016$ & DEQ/UST - 2016 \\
\hline $\begin{array}{l}\text { UST - ENVIRONMENTAL } \\
\text { CONTAINER SYSTEMS }\end{array}$ & 3153 & $\begin{array}{l}0 \text { Upgraded, } 3 \text { Decommissioned, } 0 \\
\text { Unknown }\end{array}$ & $\begin{array}{l}\text { ENVIRONMENTAL CONTAINER } \\
\text { SYSTEMS }\end{array}$ & 3560 ROGUE RIVER HWY & \begin{tabular}{|l|} 
GRANTS \\
PASS \\
\end{tabular} & Josephine & $01 / 25 / 2016$ & DEQ/UST - 2016 \\
\hline UST - FOURPLY TRUCK SHOP & 6727 & $\begin{array}{l}0 \text { Upgraded, } 2 \text { Decommissioned, } 0 \\
\text { Unknown }\end{array}$ & FOURPLY TRUCK SHOP & 124 NE BEACON DR & \begin{tabular}{|l} 
GRANTS \\
PASS
\end{tabular} & Josephine & $01 / 25 / 2016$ & DEQ/UST - 2016 \\
\hline $\begin{array}{l}\text { UST - FRED MEYER - GRANTS } \\
\text { PASS SHOPPING CENTER }\end{array}$ & 8883 & $\begin{array}{l}4 \text { Upgraded, } 0 \text { Decommissioned, } 0 \\
\text { Unknown }\end{array}$ & $\begin{array}{l}\text { FRED MEYER - GRANTS PASS } \\
\text { SHOPPING CENTER }\end{array}$ & 1101 NE REDWOOD HWY & \begin{tabular}{|l} 
GRANTS \\
PASS
\end{tabular} & Josephine & $01 / 25 / 2016$ & DEQ/UST - 2016 \\
\hline UST - GI JOES PROPERTY & 11356 & $\begin{array}{l}0 \text { Upgraded, } 1 \text { Decommissioned, } 0 \\
\text { Unknown }\end{array}$ & GI JOES PROPERTY & $\begin{array}{l}\text { SW CORNER OF } \\
\text { REDWOOD AVE \& AGNESS } \\
\text { AVE }\end{array}$ & $\begin{array}{l}\text { GRANTS } \\
\text { PASS }\end{array}$ & Josephine & $01 / 25 / 2016$ & DEQ/UST - 2016 \\
\hline UST - GRANTS PASS ARCO & 11300 & $\begin{array}{l}4 \text { Upgraded, } 0 \text { Decommissioned, } 0 \\
\text { Unknown }\end{array}$ & GRANTS PASS ARCO & 242 NE TERRY LN & $\begin{array}{l}\text { GRANTS } \\
\text { PASS }\end{array}$ & Josephine & $01 / 25 / 2016$ & DEQ/UST - 2016 \\
\hline $\begin{array}{l}\text { UST - GRANTS PASS } \\
\text { MOULDING INC }\end{array}$ & 4220 & $\begin{array}{l}0 \text { Upgraded, } 3 \text { Decommissioned, } 0 \\
\text { Unknown }\end{array}$ & GRANTS PASS MOULDING INC & 123 NE BEACON DR & $\begin{array}{l}\text { GRANTS } \\
\text { PASS }\end{array}$ & Josephine & $01 / 25 / 2016$ & DEQ/UST - 2016 \\
\hline \begin{tabular}{l|} 
UST - GRANTS PASS SCHOOL \\
DISTRICT TRANSPORTATI
\end{tabular} & 12093 & $\begin{array}{l}1 \text { Upgraded, } 0 \text { Decommissioned, } 0 \\
\text { Unknown }\end{array}$ & $\begin{array}{l}\text { GRANTS PASS SCHOOL } \\
\text { DISTRICT TRANSPORTATI }\end{array}$ & 1053 SE GLADIOLA DR & \begin{tabular}{|l} 
GRANTS \\
PASS
\end{tabular} & Josephine & $01 / 25 / 2016$ & DEQ/UST - 2016 \\
\hline $\begin{array}{l}\text { UST - JOSEPHINE COUNTY } \\
\text { SHERIFF STORAGE YARD }\end{array}$ & 12184 & $\begin{array}{l}0 \text { Upgraded, } 1 \text { Decommissioned, } 0 \\
\text { Unknown }\end{array}$ & $\begin{array}{l}\text { JOSEPHINE COUNTY SHERIFF } \\
\text { STORAGE YARD }\end{array}$ & 1798 SE N ST & $\begin{array}{l}\text { GRANTS } \\
\text { PASS }\end{array}$ & Josephine & $01 / 25 / 2016$ & DEQ/UST - 2016 \\
\hline
\end{tabular}




\begin{tabular}{|c|c|c|c|c|c|c|c|c|}
\hline Database Identifier (DB_ID) & Site Identifier & Status & Common Name & Address & City & County & \begin{tabular}{|l} 
Retrieval \\
Date
\end{tabular} & Data Source \\
\hline $\begin{array}{l}\text { UST - LARRY'S TRIANGLE } \\
\text { SERVICE }\end{array}$ & 3736 & $\begin{array}{l}\text { 0 Upgraded, } 3 \text { Decommissioned, } 0 \\
\text { Unknown }\end{array}$ & LARRY'S TRIANGLE SERVICE & MAIN \& DEPOT ST & $\begin{array}{l}\text { ROGUE } \\
\text { RIVER }\end{array}$ & Jackson & $01 / 25 / 2016$ & DEQ/UST - 2016 \\
\hline $\begin{array}{l}\text { UST - LEW MERRILL LUMBER } \\
\text { SALES INC }\end{array}$ & 3797 & $\begin{array}{l}0 \text { Upgraded, } 1 \text { Decommissioned, } 0 \\
\text { Unknown }\end{array}$ & $\begin{array}{l}\text { LEW MERRILL LUMBER SALES } \\
\text { INC }\end{array}$ & 1750 SE N ST & \begin{tabular}{|l|} 
GRANTS \\
PASS
\end{tabular} & Josephine & $01 / 25 / 2016$ & DEQ/UST - 2016 \\
\hline $\begin{array}{l}\text { UST - LIL'PANTRY MARKET \& } \\
\text { DELI-GRANTS PASS }\end{array}$ & 12364 & $\begin{array}{l}3 \text { Upgraded, } 0 \text { Decommissioned, } 0 \\
\text { Unknown }\end{array}$ & $\begin{array}{l}\text { LIL'PANTRY MARKET \& DELI- } \\
\text { GRANTS PASS }\end{array}$ & 979 ROGUE RIVER HWY & \begin{tabular}{|l|} 
GRANTS \\
PASS
\end{tabular} & Josephine & $01 / 25 / 2016$ & DEQ/UST - 2016 \\
\hline $\begin{array}{l}\text { UST - MAIN BUILDING SUPPLY } \\
\text { INC }\end{array}$ & 4537 & $\begin{array}{l}0 \text { Upgraded, } 1 \text { Decommissioned, } 0 \\
\text { Unknown }\end{array}$ & MAIN BUILDING SUPPLY INC & 100 BROADWAY & \begin{tabular}{|l|} 
ROGUE \\
RIVER
\end{tabular} & Jackson & $01 / 25 / 2016$ & DEQ/UST - 2016 \\
\hline \begin{tabular}{|l|} 
UST - NEGRO, GAYLE \\
\end{tabular} & 11358 & $\begin{array}{l}0 \text { Upgraded, } 1 \text { Decommissioned, } 0 \\
\text { Unknown }\end{array}$ & NEGRO, GAYLE & 257 GORDON WAY & \begin{tabular}{|l|} 
GRANTS \\
PASS
\end{tabular} & Josephine & $01 / 25 / 2016$ & DEQ/UST - 2016 \\
\hline $\begin{array}{l}\text { UST - OR STATE HWY 3-8 - } \\
\text { GRANTS PASS }\end{array}$ & 1112 & $\begin{array}{l}0 \text { Upgraded, } 5 \text { Decommissioned, } 0 \\
\text { Unknown }\end{array}$ & $\begin{array}{l}\text { OR STATE HWY 3-8 - GRANTS } \\
\text { PASS }\end{array}$ & 345 AGNESS AVE & \begin{tabular}{|l|} 
GRANTS \\
PASS
\end{tabular} & Josephine & $01 / 25 / 2016$ & DEQ/UST - 2016 \\
\hline UST - PETERSON, GARY L & 2602 & $\begin{array}{l}0 \text { Upgraded, } 2 \text { Decommissioned, } 0 \\
\text { Unknown }\end{array}$ & PETERSON, GARY L & 910 SE M ST & \begin{tabular}{|l|} 
GRANTS \\
PASS \\
\end{tabular} & Josephine & $01 / 25 / 2016$ & DEQ/UST - 2016 \\
\hline UST - PORTOLA MARKET & 7455 & $\begin{array}{l}0 \text { Upgraded, } 1 \text { Decommissioned, } 0 \\
\text { Unknown }\end{array}$ & PORTOLA MARKET & 1215 SE ROUGE DR & $\begin{array}{l}\text { GRANTS } \\
\text { PASS }\end{array}$ & Josephine & $01 / 25 / 2016$ & DEQ/UST - 2016 \\
\hline $\begin{array}{l}\text { UST - POWELL DIST CO - } \\
\text { COLVIN/GRANTS PASS }\end{array}$ & 6037 & $\begin{array}{l}0 \text { Upgraded, } 4 \text { Decommissioned, } 0 \\
\text { Unknown }\end{array}$ & \begin{tabular}{|l|} 
POWELL DIST CO - \\
COLVIN/GRANTS PASS
\end{tabular} & 1741 ROGUE RIVER HWY & \begin{tabular}{|l|} 
GRANTS \\
PASS
\end{tabular} & Josephine & $01 / 25 / 2016$ & DEQ/UST - 2016 \\
\hline UST - RADIO DESIGN GROUP & 12404 & $\begin{array}{l}0 \text { Upgraded, } 1 \text { Decommissioned, } 0 \\
\text { Unknown }\end{array}$ & RADIO DESIGN GROUP & $\begin{array}{l}\text { 8925 ROGUE RIVER } \\
\text { HIGHWAY }\end{array}$ & \begin{tabular}{|l} 
ROGUE \\
RIVER
\end{tabular} & Jackson & $01 / 25 / 2016$ & DEQ/UST - 2016 \\
\hline UST - ROGUE RIVER 76 & 4759 & $\begin{array}{l}4 \text { Upgraded, } 5 \text { Decommissioned, } 0 \\
\text { Unknown }\end{array}$ & ROGUE RIVER 76 & 125 DEPOT ST & \begin{tabular}{|l|} 
ROGUE \\
RIVER
\end{tabular} & Jackson & $01 / 25 / 2016$ & DEQ/UST - 2016 \\
\hline UST - ROGUE RIVER DIVISION & 8968 & $\begin{array}{l}0 \text { Upgraded, } 1 \text { Decommissioned, } 0 \\
\text { Unknown }\end{array}$ & ROGUE RIVER DIVISION & 5205 N RIVER RD & \begin{tabular}{|l} 
ROGUE \\
RIVER
\end{tabular} & Jackson & $01 / 25 / 2016$ & DEQ/UST - 2016 \\
\hline $\begin{array}{l}\text { UST - ROGUE RIVER EXXON } \\
\# 9316\end{array}$ & 2433 & $\begin{array}{l}0 \text { Upgraded, } 4 \text { Decommissioned, } 0 \\
\text { Unknown }\end{array}$ & ROGUE RIVER EXXON \#9316 & 1 PINE ST & \begin{tabular}{|l} 
ROGUE \\
RIVER
\end{tabular} & Jackson & $01 / 25 / 2016$ & DEQ/UST - 2016 \\
\hline $\begin{array}{l}\text { UST - ROGUE RIVER SCHOOL } \\
\text { DISTRICT }\end{array}$ & 9462 & $\begin{array}{l}0 \text { Upgraded, } 2 \text { Decommissioned, } 0 \\
\text { Unknown }\end{array}$ & $\begin{array}{l}\text { ROGUE RIVER SCHOOL } \\
\text { DISTRICT }\end{array}$ & 1898 E EVANS CREEK RD & \begin{tabular}{|l|} 
ROGUE \\
RIVER
\end{tabular} & Jackson & $01 / 25 / 2016$ & DEQ/UST - 2016 \\
\hline UST - SMITH, D C & 11346 & $\begin{array}{l}0 \text { Upgraded, } 1 \text { Decommissioned, } 0 \\
\text { Unknown }\end{array}$ & SMITH, D C & 6916 W EVANS CREEK RD & \begin{tabular}{|l} 
ROGUE \\
RIVER
\end{tabular} & Jackson & $01 / 25 / 2016$ & DEQ/UST - 2016 \\
\hline UST - SPALDING \& SON INC & 5759 & $\begin{array}{l}0 \text { Upgraded, } 2 \text { Decommissioned, } 0 \\
\text { Unknown }\end{array}$ & SPALDING \& SON INC & 2345 SE N ST & $\begin{array}{l}\text { GRANTS } \\
\text { PASS }\end{array}$ & Josephine & $01 / 25 / 2016$ & DEQ/UST - 2016 \\
\hline $\begin{array}{l}\text { UST - STONE FOREST } \\
\text { INDUSTRIES }\end{array}$ & 6411 & $\begin{array}{l}0 \text { Upgraded, } 1 \text { Decommissioned, } 0 \\
\text { Unknown }\end{array}$ & STONE FOREST INDUSTRIES & 1090 SE M ST & $\begin{array}{l}\text { GRANTS } \\
\text { PASS }\end{array}$ & Josephine & $01 / 25 / 2016$ & DEQ/UST - 2016 \\
\hline $\begin{array}{l}\text { UST - STONE FOREST } \\
\text { INDUSTRIES }\end{array}$ & 11201 & $\begin{array}{l}0 \text { Upgraded, } 4 \text { Decommissioned, } 0 \\
\text { Unknown }\end{array}$ & STONE FOREST INDUSTRIES & 851 SE MILBANK RD & \begin{tabular}{|l|} 
GRANTS \\
PASS
\end{tabular} & Josephine & $01 / 25 / 2016$ & DEQ/UST - 2016 \\
\hline UST - TAYLOR, DORAN & 11079 & $\begin{array}{l}0 \text { Upgraded, } 4 \text { Decommissioned, } 0 \\
\text { Unknown }\end{array}$ & TAYLOR, DORAN & 2820 FOOTHILL BLVD & \begin{tabular}{|l|} 
GRANTS \\
PASS
\end{tabular} & Josephine & $01 / 25 / 2016$ & DEQ/UST - 2016 \\
\hline $\begin{array}{l}\text { UST - U-SAVE GAS \& TACKLE } \\
\text { LLC }\end{array}$ & 7651 & $\begin{array}{l}3 \text { Upgraded, } 0 \text { Decommissioned, } 0 \\
\text { Unknown }\end{array}$ & U-SAVE GAS \& TACKLE LLC & 935 ROGUE RIVER HWY & \begin{tabular}{|l|l} 
GRANTS \\
PASS
\end{tabular} & Josephine & $01 / 25 / 2016$ & DEQ/UST - 2016 \\
\hline $\begin{array}{l}\text { UST - USWEST - WOLF CREEK } \\
\text { - } 010169\end{array}$ & 5981 & $\begin{array}{l}0 \text { Upgraded, } 1 \text { Decommissioned, } 0 \\
\text { Unknown }\end{array}$ & $\begin{array}{l}\text { USWEST - WOLF CREEK - } \\
010169\end{array}$ & $\begin{array}{l}\text { NW 1/4 SW 1/4 S26 T33S } \\
\text { R6W }\end{array}$ & MEDFORD & Josephine & $01 / 25 / 2016$ & DEQ/UST - 2016 \\
\hline UST - VALLEY OF THE ROGUE & 797 & $\begin{array}{l}0 \text { Upgraded, } 3 \text { Decommissioned, } 0 \\
\text { Unknown }\end{array}$ & VALLEY OF THE ROGUE & 3792 N RIVER RD & $\begin{array}{l}\text { GOLD } \\
\text { HILL }\end{array}$ & Jackson & $01 / 25 / 2016$ & DEQ/UST - 2016 \\
\hline $\begin{array}{l}\text { WQ - SIS outfalls - ROGUE } \\
\text { RIVER STP }\end{array}$ & 76030 & See WQ permit file & ROGUE RIVER STP & Not applicable & \begin{tabular}{|l} 
ROGUE \\
RIVER
\end{tabular} & Jackson & 2009 & $\begin{array}{l}\text { OR Dept. of Environmental } \\
\text { Quality Water Quality SIS } \\
\text { Outfall location data (DEQ/WQ } \\
\text { SIS - 2009) }\end{array}$ \\
\hline WQ - WWTP & 76030 & DOMESTIC - NPDES-DOM-Da & ROGUE RIVER STP & FOOTHILL BOULEVARD & $\begin{array}{l}\text { ROGUE } \\
\text { RIVER }\end{array}$ & Jackson & $01 / 25 / 2016$ & DEDQ/WQ WWTP - 2016 \\
\hline WQ SIS - COUNTRY ESTATES & 112611 & WPCFOS-BiiiRGF> - DOMESTIC & COUNTRY ESTATES & 4697 E EVANS CREEK RD & $\begin{array}{l}\text { ROGUE } \\
\text { RIVER }\end{array}$ & Jackson & $01 / 25 / 2016$ & DEDQ/WQ WWTP - 2016 \\
\hline WQ SIS - ESC CASE & 119529 & GEN12Z - STORMWATER & ESC CASE & $\begin{array}{l}\text { 3560 ROGUE RIVER } \\
\text { HIGHWAY }\end{array}$ & \begin{tabular}{|l} 
GRANTS \\
PASS
\end{tabular} & Josephine & $01 / 25 / 2016$ & DEDQ/WQ WWTP - 2016 \\
\hline
\end{tabular}




\begin{tabular}{|c|c|c|c|c|c|c|c|c|}
\hline Database Identifier (DB_ID) & Site Identifier $\leq$ & Status & Common Name & Address & City & County & \begin{tabular}{|l} 
Retrieval \\
Date
\end{tabular} & Data Source \\
\hline WQ SIS - FIREBALL GAS & 110721 & GEN15A - INDUSTRIAL & FIREBALL GAS & $\begin{array}{l}\text { 1919 ROGUE RIVER } \\
\text { HIGHWAY }\end{array}$ & $\begin{array}{l}\text { GRANTS } \\
\text { PASS }\end{array}$ & Josephine & $01 / 25 / 2016$ & DEDQ/WQ WWTP - 2016 \\
\hline $\begin{array}{l}\text { WQ SIS - FRONTIER MOBILE } \\
\text { ESTATES }\end{array}$ & 106858 & WPCFOS-BiiiRGF> - DOMESTIC & FRONTIER MOBILE ESTATES & $\begin{array}{l}\text { 7001 ROGUE RIVER } \\
\text { HIGHWAY }\end{array}$ & \begin{tabular}{|l|} 
ROGUE \\
RIVER \\
\end{tabular} & Jackson & $01 / 25 / 2016$ & DEDQ/WQ WWTP - 2016 \\
\hline $\begin{array}{l}\text { WQ SIS - GRANTS PASS CITY } \\
\text { PROJECTS }\end{array}$ & 113651 & GEN12CA - STORMWATER & GRANTS PASS CITY PROJECTS & $\begin{array}{l}\text { PROJECTS WITHIN } \\
\text { GRANTS PASS UGB }\end{array}$ & \begin{tabular}{|l|} 
GRANTS \\
PASS \\
\end{tabular} & Josephine & $01 / 25 / 2016$ & DEDQ/WQ WWTP - 2016 \\
\hline $\begin{array}{l}\text { WQ SIS - HAVE A NICE DAY } \\
\text { CAMPGROUND }\end{array}$ & 111539 & WPCFOS-BiiiRGF> - DOMESTIC & $\begin{array}{l}\text { HAVE A NICE DAY } \\
\text { CAMPGROUND }\end{array}$ & 7275 ROGUE RIVER HWY. & $\begin{array}{l}\text { GRANTS } \\
\text { PASS }\end{array}$ & Jackson & $01 / 25 / 2016$ & DEDQ/WQ WWTP - 2016 \\
\hline WQ SIS - HELGA M BUSH & 122655 & GEN2401 - DOMESTIC & HELGA M BUSH & 4733 W EVANS CRK RD & \begin{tabular}{|l|} 
ROGUE \\
RIVER \\
\end{tabular} & Jackson & 01/25/2016 & DEDQ/WQ WWTP - 2016 \\
\hline $\begin{array}{l}\text { WQ SIS - HUMMINGBIRD } \\
\text { MOBILE HOME PARK }\end{array}$ & 111768 & WPCFOS-Bii - DOMESTIC & $\begin{array}{l}\text { HUMMINGBIRD MOBILE HOME } \\
\text { PARK }\end{array}$ & 8330 ROGUE RIVER HWY. & \begin{tabular}{|l|} 
GRANTS \\
PASS \\
\end{tabular} & Jackson & $01 / 25 / 2016$ & DEDQ/WQ WWTP - 2016 \\
\hline \begin{tabular}{l|} 
WQ SIS - MEADOW WOOD \\
SUBDIVISION
\end{tabular} & 111273 & GEN12C - STORMWATER & MEADOW WOOD SUBDIVISION & 2221 HAVILAND DR & \begin{tabular}{|l|} 
GRANTS \\
PASS \\
\end{tabular} & Josephine & $01 / 25 / 2016$ & DEDQ/WQ WWTP - 2016 \\
\hline $\begin{array}{l}\text { WQ SIS - MOON MOUNTAIN RV } \\
\text { RESORT }\end{array}$ & 109162 & WPCFOS-BiiiRGF> - DOMESTIC & MOON MOUNTAIN RV RESORT & 3298 PEARCE PARK RD. & $\begin{array}{l}\text { GRANTS } \\
\text { PASS }\end{array}$ & Josephine & $01 / 25 / 2016$ & DEDQ/WQ WWTP - 2016 \\
\hline \begin{tabular}{l|l} 
WQ SIS - MURPHY ROGUE \\
RIVER PLYWOOD DIVISION
\end{tabular} & 55170 & GEN04 - INDUSTRIAL & $\begin{array}{l}\text { MURPHY ROGUE RIVER } \\
\text { PLYWOOD DIVISION }\end{array}$ & 5205 N ROGUE RIVER RD & \begin{tabular}{|l} 
ROGUE \\
RIVER
\end{tabular} & Jackson & $01 / 25 / 2016$ & DEDQ/WQ WWTP - 2016 \\
\hline $\begin{array}{l}\text { WQ SIS - MURPHY ROGUE } \\
\text { RIVER PLYWOOD DIVISION }\end{array}$ & 55170 & GEN12Z - STORMWATER & $\begin{array}{l}\text { MURPHY ROGUE RIVER } \\
\text { PLYWOOD DIVISION } \\
\end{array}$ & 5205 N ROGUE RIVER RD & \begin{tabular}{|l|} 
ROGUE \\
RIVER
\end{tabular} & Jackson & $01 / 25 / 2016$ & DEDQ/WQ WWTP - 2016 \\
\hline $\begin{array}{l}\text { WQ SIS - OPRD - VALLEY OF } \\
\text { THE ROGUE STATE PARK }\end{array}$ & 64770 & WPCF-DOM-Da - DOMESTIC & $\begin{array}{l}\text { OPRD - VALLEY OF THE ROGUE } \\
\text { STATE PARK }\end{array}$ & 2 MILES SOUTH OF CITY & $\begin{array}{l}\text { ROGUE } \\
\text { RIVER } \\
\end{array}$ & Jackson & $01 / 25 / 2016$ & DEDQ/WQ WWTP - 2016 \\
\hline $\begin{array}{l}\text { WQ SIS - PARTIN FOREST } \\
\text { PRODUCTS, LLC }\end{array}$ & 121507 & GEN12Z - STORMWATER & $\begin{array}{l}\text { PARTIN FOREST PRODUCTS, } \\
\text { LLC }\end{array}$ & 2263 NE SPALDING AVE & $\begin{array}{l}\text { GRANTS } \\
\text { PASS }\end{array}$ & Josephine & $01 / 25 / 2016$ & DEDQ/WQ WWTP - 2016 \\
\hline $\begin{array}{l}\text { WQ SIS - PINE VILLA MOBILE } \\
\text { ESTATES }\end{array}$ & 111111 & WPCFOS-Biii - DOMESTIC & PINE VILLA MOBILE ESTATES & 3381 ROGUE RIVER HWY. & \begin{tabular}{|l|} 
GRANTS \\
PASS
\end{tabular} & Josephine & $01 / 25 / 2016$ & DEDQ/WQ WWTP - 2016 \\
\hline WQ SIS - R. RIVER PLACE & 108977 & WPCFOS-Bii - DOMESTIC & R. RIVER PLACE & $\begin{array}{l}\text { 4550 ROGUE RIVER } \\
\text { HIGHWAY }\end{array}$ & $\begin{array}{l}\text { GRANTS } \\
\text { PASS } \\
\end{array}$ & Josephine & $01 / 25 / 2016$ & DEDQ/WQ WWTP - 2016 \\
\hline $\begin{array}{l}\text { WQ SIS - RANDOLPH ESTATES } \\
\text { SUBDIVISION }\end{array}$ & 116842 & GEN12C - STORMWATER & $\begin{array}{l}\text { RANDOLPH ESTATES } \\
\text { SUBDIVISION }\end{array}$ & WALKER RD/ HESSER ST & $\begin{array}{l}\text { GRANTS } \\
\text { PASS } \\
\end{array}$ & Josephine & $01 / 25 / 2016$ & DEDQ/WQ WWTP - 2016 \\
\hline WQ SIS - ROGUE RIVER STP & 76030 & NPDES-DOM-Da - DOMESTIC & ROGUE RIVER STP & FOOTHILL BOULEVARD & \begin{tabular}{|l} 
ROGUE \\
RIVER
\end{tabular} & Jackson & $01 / 25 / 2016$ & DEDQ/WQ WWTP - 2016 \\
\hline $\begin{array}{l}\text { WQ SIS - ROGUE RIVER } \\
\text { VALLEY MOBILE HOME } \\
\text { ESTATES }\end{array}$ & 106857 & WPCFOS-Bii - DOMESTIC & $\begin{array}{l}\text { ROGUE RIVER VALLEY MOBILE } \\
\text { HOME ESTATES }\end{array}$ & $\begin{array}{l}5648 \text { FOOTHILL } \\
\text { BOULEVARD }\end{array}$ & \begin{tabular}{|l} 
ROGUE \\
RIVER
\end{tabular} & Jackson & $01 / 25 / 2016$ & DEDQ/WQ WWTP - 2016 \\
\hline $\begin{array}{l}\text { WQ SIS - ROGUE VALLEY } \\
\text { DOOR }\end{array}$ & 109145 & GEN12Z - STORMWATER & ROGUE VALLEY DOOR & 123 NE BEACON DR & $\begin{array}{l}\text { GRANTS } \\
\text { PASS } \\
\end{array}$ & Josephine & $01 / 25 / 2016$ & DEDQ/WQ WWTP - 2016 \\
\hline $\begin{array}{l}\text { WQ SIS - SEQUOIA VILLAGE } \\
\text { P.U.D. }\end{array}$ & 115304 & GEN12C - STORMWATER & SEQUOIA VILLAGE P.U.D. & SE N ST./AGNESS AVE. & $\begin{array}{l}\text { GRANTS } \\
\text { PASS }\end{array}$ & Josephine & $01 / 25 / 2016$ & DEDQ/WQ WWTP - 2016 \\
\hline $\begin{array}{l}\text { WQ SIS - TP GRANTS PASS, } \\
\text { LLC }\end{array}$ & 107300 & GEN12Z - STORMWATER & TP GRANTS PASS, LLC & 1090 SE M ST & $\begin{array}{l}\text { GRANTS } \\
\text { PASS }\end{array}$ & Josephine & $01 / 25 / 2016$ & DEDQ/WQ WWTP - 2016 \\
\hline $\begin{array}{l}\text { WQ SIS - UNITED PARCEL } \\
\text { SERVICE - GRANTS PASS } \\
\end{array}$ & 108132 & GEN17A - INDUSTRIAL & $\begin{array}{l}\text { UNITED PARCEL SERVICE - } \\
\text { GRANTS PASS } \\
\end{array}$ & $\begin{array}{l}2552 \text { FOOTHILL } \\
\text { BOULEVARD }\end{array}$ & \begin{tabular}{|l|} 
GRANTS \\
PASS \\
\end{tabular} & Josephine & $01 / 25 / 2016$ & DEDQ/WQ WWTP - 2016 \\
\hline $\begin{array}{l}\text { DHS/OHA - OFRA - Schools - } \\
\text { Fruitdale Elementary School }\end{array}$ & 445 & Unknown & Fruitdale Elementary School & 1560 Bill Baker Way & $\begin{array}{l}\text { Grants } \\
\text { Pass }\end{array}$ & Josephine & $06 / 02 / 2015$ & $\begin{array}{l}\text { OR Dept. of Human Services - } \\
\text { Office of Forecasting, } \\
\text { Research, and Analysis (via } \\
\text { OGDC (DHS/OHA - 2015) }\end{array}$ \\
\hline $\begin{array}{l}\text { DHS/OHA - OFRA - Schools - } \\
\text { Grants Pass High School }\end{array}$ & 442 & Unknown & Grants Pass High School & 1137 SE Gladiola Dr & $\begin{array}{l}\text { Grants } \\
\text { Pass }\end{array}$ & Josephine & $06 / 02 / 2015$ & $\mathrm{DHS} / \mathrm{OHA}-2015$ \\
\hline $\begin{array}{l}\text { DHS/OHA - OFRA - Schools - } \\
\text { Newbridge High School }\end{array}$ & 2260 & Unknown & Newbridge High School & 2001 NE F St & $\begin{array}{l}\text { Grants } \\
\text { Pass }\end{array}$ & Josephine & $06 / 02 / 2015$ & DHS/OHA - 2015 \\
\hline
\end{tabular}




\begin{tabular}{|c|c|c|c|c|c|c|c|c|}
\hline Database Identifier (DB_ID) & Site Identifier & Status & Common Name & Address & City & County & \begin{tabular}{|l|} 
Retrieval \\
Date
\end{tabular} & Data Source \\
\hline $\begin{array}{l}\text { DHS/OHA - OFRA - Schools - } \\
\text { Phagans' Grants Pass College of } \\
\text { Beauty }\end{array}$ & -1 & Unknown & $\begin{array}{l}\text { Phagans' Grants Pass College of } \\
\text { Beauty }\end{array}$ & 304 NE AGNESS & \begin{tabular}{|l|} 
GRANTS \\
PASS
\end{tabular} & Josephine & $06 / 02 / 2015$ & DHS/OHA - 2015 \\
\hline $\begin{array}{l}\text { DHS/OHA - OFRA - Schools - } \\
\text { Rivers Edge Academy Charter } \\
\text { School }\end{array}$ & 4856 & Unknown & $\begin{array}{l}\text { Rivers Edge Academy Charter } \\
\text { School }\end{array}$ & 270 W Evans Creek Rd & $\begin{array}{l}\text { Rogue } \\
\text { River }\end{array}$ & Jackson & $06 / 02 / 2015$ & DHS/OHA - 2015 \\
\hline $\begin{array}{l}\text { DHS/OHA - OFRA - Schools - } \\
\text { Riverside Elementary School }\end{array}$ & 440 & Unknown & Riverside Elementary School & 1200 SE Harvey Dr & \begin{tabular}{|l|} 
Grants \\
Pass
\end{tabular} & Josephine & $06 / 02 / 2015$ & DHS/OHA - 2015 \\
\hline $\begin{array}{l}\text { DHS/OHA - OFRA - Schools - } \\
\text { Rogue River Elementary School }\end{array}$ & 399 & Unknown & Rogue River Elementary School & 300 Pine St & $\begin{array}{l}\text { Rogue } \\
\text { River }\end{array}$ & Jackson & $06 / 02 / 2015$ & DHS/OHA - 2015 \\
\hline $\begin{array}{l}\text { DHS/OHA - OFRA - Schools - } \\
\text { Rogue River Junior/Senior High }\end{array}$ & 401 & Unknown & Rogue River Junior/Senior High & 1898 E Evans Creek Rd & \begin{tabular}{|l} 
Rogue \\
River
\end{tabular} & Jackson & $06 / 02 / 2015$ & DHS/OHA - 2015 \\
\hline $\begin{array}{l}\text { DHS/OHA - OFRA - Schools - } \\
\text { Rogue River SD } 35\end{array}$ & 2044 & Unknown & Rogue River SD 35 & 1898 E Evans Creek Rd & \begin{tabular}{|l|} 
Rogue \\
River
\end{tabular} & Jackson & $06 / 02 / 2015$ & DHS/OHA - 2015 \\
\hline $\begin{array}{l}\text { Water Quality Limited streams, } \\
\text { Cat4A \& Cat5, DEQ-2012 - } \\
\text { Birdseye Creek }\end{array}$ & $\begin{array}{l}1231506424 \\
080\end{array}$ & $\begin{array}{l}\text { Cat 4A: Water quality limited, TMDL } \\
\text { approved - Temperature }\end{array}$ & Birdseye Creek & Not applicable & $\begin{array}{l}\text { Not } \\
\text { applicable }\end{array}$ & $\begin{array}{l}\text { Not } \\
\text { applicable }\end{array}$ & $10 / 31 / 2014$ & $\begin{array}{l}\text { OR Dept. of Environmental } \\
\text { Quality Water Quality } \\
\text { Assessment } 2012 \text { - (DEQ/WQ } \\
\text { 10/31/2014) }\end{array}$ \\
\hline $\begin{array}{l}\text { Water Quality Limited streams, } \\
\text { Cat3 - Evans Creek }\end{array}$ & $\begin{array}{l}1231755424 \\
330\end{array}$ & $\begin{array}{l}\text { Cat 5: Water quality limited, 303(d) list, } \\
\text { TMDL needed - Biological CriteriaCat. } \\
\text { 4A: Water quality limited, TMDL } \\
\text { approved - Fecal Coliform. Cat 3B: } \\
\text { Insufficient data, potential concern - } \\
\text { Temperature. Cat 3: Insufficient data - } \\
\text { Alkalinity, Ammonia, Antimony,Arsenic, } \\
\text { Barium, Cadmium, Chromium, Copper, } \\
\text { Dissolved Oxygen, E.Coli, Flow } \\
\text { Modification, Habitat modification, Iron, } \\
\text { Lead, Manganese, Nickel, Phosphate } \\
\text { Phosphorus, Sedimentation, Selenium, } \\
\text { Silver, Thallium, Zinc }\end{array}$ & Evans Creek & Not applicable & \begin{tabular}{|l|} 
Not \\
applicable
\end{tabular} & $\begin{array}{l}\text { Not } \\
\text { applicable }\end{array}$ & $10 / 31 / 2014$ & DEQ/WQ - 10/31/2014 \\
\hline $\begin{array}{l}\text { Water Quality Limited streams, } \\
\text { Cat3 - Fruitdale Creek }\end{array}$ & $\begin{array}{l}1233054424 \\
240\end{array}$ & $\begin{array}{l}\text { Cat 3: Insufficient data - Flow } \\
\text { Modification, Sedimentation, } \\
\text { Temperature }\end{array}$ & Fruitdale Creek & Not applicable & $\begin{array}{l}\text { Not } \\
\text { applicable }\end{array}$ & $\begin{array}{l}\text { Not } \\
\text { applicable }\end{array}$ & $10 / 31 / 2014$ & DEQ/WQ - 10/31/2014 \\
\hline $\begin{array}{l}\text { Water Quality Limited streams, } \\
\text { Cat3 - Jones Creek }\end{array}$ & \begin{tabular}{|l|}
1232878424 \\
260
\end{tabular} & $\begin{array}{l}\text { Cat 5: Water quality limited, 303(d) list, } \\
\text { TMDL needed - Dissolved Oxygen. Cat } \\
\text { 4A: Water quality limited, TMDL } \\
\text { approved - E. Coli. Cat 3: Insufficient } \\
\text { data - Alkalinity, Ammonia, Chlorophyll a, } \\
\text { Dissolved Oxygen, E.Coli, Flow } \\
\text { Modificaiton, pH, Phosphate } \\
\text { Phosphorus, Sedimentation }\end{array}$ & Jones Creek & Not applicable & $\begin{array}{l}\text { Not } \\
\text { applicable }\end{array}$ & $\begin{array}{l}\text { Not } \\
\text { applicable }\end{array}$ & $10 / 31 / 2014$ & DEQ/WQ - 10/31/2014 \\
\hline
\end{tabular}




\begin{tabular}{|c|c|c|c|c|c|c|c|c|}
\hline Database Identifier (DB_ID) & Site Identifier & Status & Common Name & Address & City & County & \begin{tabular}{|l|} 
Retrieval \\
Date
\end{tabular} & Data Source \\
\hline $\begin{array}{l}\text { Water Quality Limited streams, } \\
\text { Cat3 - Rogue River }\end{array}$ & $\begin{array}{l}1244292424 \\
210\end{array}$ & $\begin{array}{l}\text { Cat 5: Water quality limited, 303(d) list, } \\
\text { TMDL needed - Dissolved Oxygen, } \\
\text { Mercury. Cat 4A: Water quality limited, } \\
\text { TMDL approved - Fecal Coliform, } \\
\text { Temperature. Cat 3B: Insufficient data, } \\
\text { potential concern - Phosphate } \\
\text { Phosphorus. Cat 3: Insufficient data - } \\
\text { Aquatic Weeds Or Algae, Chlorophylla, } \\
\text { E.Coli, Flow Modification, Nutrients, } \\
\text { Sedimentation }\end{array}$ & Rogue River & Not applicable & $\begin{array}{l}\text { Not } \\
\text { applicable }\end{array}$ & $\begin{array}{l}\text { Not } \\
\text { applicable }\end{array}$ & $10 / 31 / 2014$ & DEQ/WQ - 10/31/2014 \\
\hline $\begin{array}{l}\text { Water Quality Limited streams, } \\
\text { Cat4A \& Cat5, DEQ-2012 - } \\
\text { Savage Creek }\end{array}$ & $\begin{array}{l}1232199424 \\
200\end{array}$ & $\begin{array}{l}\text { Cat 4A: Water quality limited, TMDL } \\
\text { approved - Temperature }\end{array}$ & Savage Creek & Not applicable & $\begin{array}{l}\text { Not } \\
\text { applicable }\end{array}$ & $\begin{array}{l}\text { Not } \\
\text { applicable }\end{array}$ & $10 / 31 / 2014$ & DEQ/WQ - 10/31/2014 \\
\hline $\begin{array}{l}\text { Water Quality Limited streams, } \\
\text { Cat3 - Ward Creek }\end{array}$ & $\begin{array}{l}1231708424 \\
310\end{array}$ & $\begin{array}{l}\text { Cat 3: Insufficient data - Flow } \\
\text { Modification, Temperature }\end{array}$ & Ward Creek & Not applicable & \begin{tabular}{|l|} 
Not \\
applicable
\end{tabular} & $\begin{array}{l}\text { Not } \\
\text { applicable }\end{array}$ & $10 / 31 / 2014$ & DEQ/WQ - 10/31/2014 \\
\hline Cities & \begin{tabular}{|l|} 
City of \\
Grants Pass
\end{tabular} & 30550 & City of Grants Pass & & & & & \\
\hline Cities & \begin{tabular}{|l|} 
City of \\
Rogue River
\end{tabular} & 63450 & City of Rogue River & & & & & \\
\hline Highways & \begin{tabular}{|l|} 
Highways - I- \\
5
\end{tabular} & 001 & Highway/Interstate & PACIFIC & $\begin{array}{l}\text { Not } \\
\text { applicable }\end{array}$ & $\begin{array}{l}\text { Not } \\
\text { applicable }\end{array}$ & $\begin{array}{l}\text { Not } \\
\text { Applicable }\end{array}$ & 2008 \\
\hline Highways & $\begin{array}{l}\text { Highways - } \\
\text { OR-99 }\end{array}$ & 060 & Highway/Interstate & ROGUE RIVER & $\begin{array}{l}\text { Not } \\
\text { applicable }\end{array}$ & $\begin{array}{l}\text { Not } \\
\text { applicable }\end{array}$ & $\begin{array}{l}\text { Not } \\
\text { Applicable }\end{array}$ & 2008 \\
\hline Railway & \begin{tabular}{|l|} 
Railway - \\
Cental \\
Oregon \& \\
Pacific \\
Railroad, \\
Inc.
\end{tabular} & 2525 & Railway & $\begin{array}{l}\text { Cental Oregon \& Pacific } \\
\text { Railroad, Inc. }\end{array}$ & Unknown & $\begin{array}{l}\text { Not } \\
\text { applicable }\end{array}$ & Josephine & $03 / 14 / 2016$ \\
\hline Railway & \begin{tabular}{|l|} 
Railway - \\
Cental \\
Oregon \& \\
Pacific \\
Railroad, \\
Inc.
\end{tabular} & 2526 & Railway & $\begin{array}{l}\text { Cental Oregon \& Pacific } \\
\text { Railroad, Inc. }\end{array}$ & Unknown & $\begin{array}{l}\text { Not } \\
\text { applicable }\end{array}$ & Jackson & $03 / 14 / 2016$ \\
\hline Bridge - Birdseye Creek, Hwy 60 & $00412 \mathrm{~A}$ & $\begin{array}{l}\text { Highway, major road, bridge, or stream } \\
\text { crossing }\end{array}$ & Birdseye Creek, Hwy 60 & Not Applicable & $\begin{array}{l}\text { UNKNOW } \\
\text { N }\end{array}$ & Jackson & 2013 & $\begin{array}{l}\text { Oregon Dept. of Transportation, } \\
\text { Technical Services Branch, } \\
\text { Bridges Section (ODOT - 2013) }\end{array}$ \\
\hline $\begin{array}{l}\text { Bridge - Equipment Pass, Hwy } 1 \\
\text { at MP } 50.80\end{array}$ & 08673 & $\begin{array}{l}\text { Highway, major road, bridge, or stream } \\
\text { crossing }\end{array}$ & $\begin{array}{l}\text { Equipment Pass, Hwy } 1 \text { at MP } \\
50.80\end{array}$ & Not Applicable & $\begin{array}{l}\text { UNKNOW } \\
\mathrm{N}\end{array}$ & \begin{tabular}{|l|} 
Jackson \\
\end{tabular} & 2013 & ODOT - 2013 \\
\hline $\begin{array}{l}\text { Bridge - Equipment Pass, Hwy } 1 \\
\text { at MP } 52.12\end{array}$ & 08672 & $\begin{array}{l}\text { Highway, major road, bridge, or stream } \\
\text { crossing }\end{array}$ & $\begin{array}{l}\text { Equipment Pass, Hwy } 1 \text { at MP } \\
52.12\end{array}$ & Not Applicable & \begin{tabular}{|l|} 
UNKNOW \\
$N$
\end{tabular} & Jackson & 2013 & ODOT - 2013 \\
\hline $\begin{array}{l}\text { Bridge - Equipment Pass, Hwy } 1 \\
\text { at MP } 53.51\end{array}$ & 08336 & $\begin{array}{l}\text { Highway, major road, bridge, or stream } \\
\text { crossing }\end{array}$ & $\begin{array}{l}\text { Equipment Pass, Hwy } 1 \text { at MP } \\
53.51\end{array}$ & Not Applicable & $\begin{array}{l}\text { UNKNOW } \\
\mathrm{N}\end{array}$ & Josephine & 2013 & ODOT - 2013 \\
\hline Bridge - Evans Creek, Hwy 1 & 08376 & $\begin{array}{l}\text { Highway, major road, bridge, or stream } \\
\text { crossing }\end{array}$ & Evans Creek, Hwy 1 & Not Applicable & \begin{tabular}{|l|} 
ROGUE \\
RIVER
\end{tabular} & Jackson & 2013 & ODOT - 2013 \\
\hline
\end{tabular}




\begin{tabular}{|c|c|c|c|c|c|c|c|c|}
\hline Database Identifier (DB_ID) & Site Identifier & Status & Common Name & Address & City & County & \begin{tabular}{|l} 
Retrieval \\
Date
\end{tabular} & Data Source \\
\hline Bridge - Evans Creek, W Main St & 20054 & $\begin{array}{l}\text { Highway, major road, bridge, or stream } \\
\text { crossing }\end{array}$ & Evans Creek, W Main St & Not Applicable & \begin{tabular}{|l|} 
ROGUE \\
RIVER
\end{tabular} & Jackson & 2013 & ODOT - 2013 \\
\hline $\begin{array}{l}\text { Bridge - Fruitdale Creek, Hamiltin } \\
\text { Ln }\end{array}$ & 322006 & $\begin{array}{l}\text { Highway, major road, bridge, or stream } \\
\text { crossing }\end{array}$ & Fruitdale Creek, Hamiltin Ln & Not Applicable & $\begin{array}{l}\text { UNKNOW } \\
\mathrm{N}\end{array}$ & Josephine & 2013 & ODOT - 2013 \\
\hline Bridge - Fruitdale Creek, Hwy 60 & 03799 & $\begin{array}{l}\text { Highway, major road, bridge, or stream } \\
\text { crossing }\end{array}$ & Fruitdale Creek, Hwy 60 & Not Applicable & $\begin{array}{l}\text { UNKNOW } \\
\mathrm{N}\end{array}$ & Josephine & 2013 & ODOT - 2013 \\
\hline Bridge - Green Creek, Hwy 60 & 01606 & $\begin{array}{l}\text { Highway, major road, bridge, or stream } \\
\text { crossing }\end{array}$ & Green Creek, Hwy 60 & Not Applicable & $\begin{array}{l}\text { UNKNOW } \\
\mathrm{N}\end{array}$ & Josephine & 2013 & ODOT - 2013 \\
\hline $\begin{array}{l}\text { Bridge - Hwy } 1 \text { NB over Foothill } \\
\text { Blvd }\end{array}$ & $08335 \mathrm{~N}$ & $\begin{array}{l}\text { Highway, major road, bridge, or stream } \\
\text { crossing }\end{array}$ & Hwy 1 NB over Foothill Blvd & Not Applicable & $\begin{array}{l}\text { UNKNOW } \\
\mathrm{N}\end{array}$ & Josephine & 2013 & ODOT - 2013 \\
\hline Bridge - Hwy 1 over Depot St & 08377 & $\begin{array}{l}\text { Highway, major road, bridge, or stream } \\
\text { crossing }\end{array}$ & Hwy 1 over Depot St & Not Applicable & \begin{tabular}{|l|} 
ROGUE \\
RIVER
\end{tabular} & Jackson & 2013 & ODOT - 2013 \\
\hline Bridge - Hwy 1 over Foothill Blvd & 08333 & $\begin{array}{l}\text { Highway, major road, bridge, or stream } \\
\text { crossing }\end{array}$ & Hwy 1 over Foothill Blvd & Not Applicable & $\begin{array}{l}\text { UNKNOW } \\
\text { N }\end{array}$ & Josephine & 2013 & ODOT - 2013 \\
\hline Bridge - Hwy 1 over Hwy 25 Spur & 20551 & $\begin{array}{l}\text { Highway, major road, bridge, or stream } \\
\text { crossing }\end{array}$ & Hwy 1 over Hwy 25 Spur & Not Applicable & $\begin{array}{l}\text { UNKNOW } \\
\mathrm{N}\end{array}$ & Josephine & 2013 & ODOT - 2013 \\
\hline $\begin{array}{l}\text { Bridge - Hwy } 1 \text { SB over Foothill } \\
\text { Blvd }\end{array}$ & $08335 S$ & $\begin{array}{l}\text { Highway, major road, bridge, or stream } \\
\text { crossing }\end{array}$ & Hwy 1 SB over Foothill Blvd & Not Applicable & $\begin{array}{l}\text { UNKNOW } \\
\mathrm{N}\end{array}$ & Josephine & 2013 & ODOT - 2013 \\
\hline Bridge - Hwy 25 Spur over CORP & 16845 & $\begin{array}{l}\text { Highway, major road, bridge, or stream } \\
\text { crossing }\end{array}$ & Hwy 25 Spur over CORP & Not Applicable & $\begin{array}{l}\text { GRANTS } \\
\text { PASS }\end{array}$ & Josephine & 2013 & ODOT - 2013 \\
\hline $\begin{array}{l}\text { Bridge - Irrigation Canal, } \\
\text { Cloverlawn Dr }\end{array}$ & 320005 & $\begin{array}{l}\text { Highway, major road, bridge, or stream } \\
\text { crossing }\end{array}$ & Irrigation Canal, Cloverlawn Dr & Not Applicable & \begin{tabular}{|l|} 
UNKNOW \\
N
\end{tabular} & Josephine & 2013 & ODOT - 2013 \\
\hline $\begin{array}{l}\text { Bridge - Irrigation Canal, Drury } \\
\text { Lane }\end{array}$ & 312005 & $\begin{array}{l}\text { Highway, major road, bridge, or stream } \\
\text { crossing }\end{array}$ & Irrigation Canal, Drury Lane & Not Applicable & $\begin{array}{l}\text { UNKNOW } \\
\text { N }\end{array}$ & Josephine & 2013 & ODOT - 2013 \\
\hline $\begin{array}{l}\text { Bridge - Irrigation Canal, Gaffney } \\
\text { Way }\end{array}$ & 313005 & $\begin{array}{l}\text { Highway, major road, bridge, or stream } \\
\text { crossing }\end{array}$ & Irrigation Canal, Gaffney Way & Not Applicable & $\begin{array}{l}\text { GRANTS } \\
\text { PASS }\end{array}$ & Josephine & 2013 & ODOT - 2013 \\
\hline \begin{tabular}{|l|l} 
Bridge - Irrigation Canal, \\
Hamilton Ln
\end{tabular} & 322015 & $\begin{array}{l}\text { Highway, major road, bridge, or stream } \\
\text { crossing }\end{array}$ & Irrigation Canal, Hamilton Ln & Not Applicable & \begin{tabular}{|l|} 
UNKNOW \\
N
\end{tabular} & Josephine & 2013 & ODOT - 2013 \\
\hline $\begin{array}{l}\text { Bridge - Jones Creek, Foothill } \\
\text { Blvd }\end{array}$ & 20723 & $\begin{array}{l}\text { Highway, major road, bridge, or stream } \\
\text { crossing }\end{array}$ & Jones Creek, Foothill Blvd & Not Applicable & $\begin{array}{l}\text { UNKNOW } \\
\mathrm{N}\end{array}$ & Josephine & 2013 & ODOT - 2013 \\
\hline Bridge - Jones Creek, Hwy 1 & 08334 & $\begin{array}{l}\text { Highway, major road, bridge, or stream } \\
\text { crossing }\end{array}$ & Jones Creek, Hwy 1 & Not Applicable & $\begin{array}{l}\text { UNKNOW } \\
\mathrm{N}\end{array}$ & Josephine & 2013 & ODOT - 2013 \\
\hline $\begin{array}{l}\text { Bridge - Main Canal, Cloverlawn } \\
\text { Dr }\end{array}$ & 320010 & $\begin{array}{l}\text { Highway, major road, bridge, or stream } \\
\text { crossing }\end{array}$ & Main Canal, Cloverlawn Dr & Not Applicable & $\begin{array}{l}\text { UNKNOW } \\
\mathrm{N}\end{array}$ & Josephine & 2013 & ODOT - 2013 \\
\hline Bridge - Main Low Canal, Hwy 60 & 01605 & $\begin{array}{l}\text { Highway, major road, bridge, or stream } \\
\text { crossing }\end{array}$ & Main Low Canal, Hwy 60 & Not Applicable & $\begin{array}{l}\text { UNKNOW } \\
\mathrm{N}\end{array}$ & Josephine & 2013 & ODOT - 2013 \\
\hline $\begin{array}{l}\text { Bridge - Owl Creek, Hwy } 60 \text { (Little } \\
\text { Savage Creek) }\end{array}$ & 00071 & $\begin{array}{l}\text { Highway, major road, bridge, or stream } \\
\text { crossing }\end{array}$ & $\begin{array}{l}\text { Owl Creek, Hwy } 60 \text { (Little Savage } \\
\text { Creek) }\end{array}$ & Not Applicable & \begin{tabular}{|l} 
UNKNOW \\
N
\end{tabular} & Jackson & 2013 & ODOT - 2013 \\
\hline \begin{tabular}{|l|} 
Bridge - Rogue River Greenway \\
Trail Bridge A
\end{tabular} & 21658 & $\begin{array}{l}\text { Highway, major road, bridge, or stream } \\
\text { crossing }\end{array}$ & $\begin{array}{l}\text { Rogue River Greenway Trail Bridge } \\
\text { A }\end{array}$ & Not Applicable & $\begin{array}{l}\text { UNKNOW } \\
\mathrm{N}\end{array}$ & Jackson & 2013 & ODOT - 2013 \\
\hline $\begin{array}{l}\text { Bridge - Rogue River Greenway } \\
\text { Trail Bridge B }\end{array}$ & 21659 & $\begin{array}{l}\text { Highway, major road, bridge, or stream } \\
\text { crossing }\end{array}$ & $\begin{array}{l}\text { Rogue River Greenway Trail Bridge } \\
\text { B }\end{array}$ & Not Applicable & $\begin{array}{l}\text { UNKNOW } \\
\text { N }\end{array}$ & Jackson & 2013 & ODOT - 2013 \\
\hline Bridge - Rogue River, Depot St & 19273 & $\begin{array}{l}\text { Highway, major road, bridge, or stream } \\
\text { crossing }\end{array}$ & Rogue River, Depot St & Not Applicable & $\begin{array}{l}\text { ROGUE } \\
\text { RIVER }\end{array}$ & Jackson & 2013 & ODOT - 2013 \\
\hline $\begin{array}{l}\text { Bridge - Rogue River, Hwy } 25 \\
\text { Spur }\end{array}$ & 16844 & $\begin{array}{l}\text { Highway, major road, bridge, or stream } \\
\text { crossing }\end{array}$ & Rogue River, Hwy 25 Spur & Not Applicable & $\begin{array}{l}\text { GRANTS } \\
\text { PASS } \\
\end{array}$ & Josephine & 2013 & ODOT - 2013 \\
\hline Bridge - Savage Creek, Hwy 60 & $00072 A$ & $\begin{array}{l}\text { Highway, major road, bridge, or stream } \\
\text { crossing }\end{array}$ & Savage Creek, Hwy 60 & Not Applicable & $\begin{array}{l}\text { UNKNOW } \\
\mathrm{N}\end{array}$ & Jackson & 2013 & ODOT - 2013 \\
\hline Bridge - Tokay Canal, Hwy 1 & $0 \mathrm{P} 412$ & $\begin{array}{l}\text { Highway, major road, bridge, or stream } \\
\text { crossing }\end{array}$ & Tokay Canal, Hwy 1 & Not Applicable & $\begin{array}{l}\text { UNKNOW } \\
\mathrm{N}\end{array}$ & Josephine & 2013 & ODOT - 2013 \\
\hline Bridge - Ward Creek, Classic Dr & 19196 & $\begin{array}{l}\text { Highway, major road, bridge, or stream } \\
\text { crossing }\end{array}$ & Ward Creek, Classic Dr & Not Applicable & $\begin{array}{l}\text { ROGUE } \\
\text { RIVER }\end{array}$ & Jackson & 2013 & ODOT - 2013 \\
\hline Bridge - Ward Creek, Hwy 1 & 08378 & $\begin{array}{l}\text { Highway, major road, bridge, or stream } \\
\text { crossing }\end{array}$ & Ward Creek, Hwy 1 & Not Applicable & \begin{tabular}{|l} 
ROGUE \\
RIVER
\end{tabular} & Jackson & 2013 & ODOT - 2013 \\
\hline
\end{tabular}




\begin{tabular}{|c|c|c|c|c|c|c|c|c|}
\hline Database Identifier (DB_ID) & Site Identifier & Status & Common Name & Address & City & County & \begin{tabular}{|l|} 
Retrieval \\
Date
\end{tabular} & Data Source \\
\hline Bridge - Wards Creek, Main St & 07694 & $\begin{array}{l}\text { Highway, major road, bridge, or stream } \\
\text { crossing }\end{array}$ & Wards Creek, Main St & Not Applicable & \begin{tabular}{|l} 
ROGUE \\
RIVER
\end{tabular} & Jackson & 2013 & ODOT - 2013 \\
\hline 2016_USWA_PCSs & 43229 & & $\begin{array}{l}\text { Campgrounds/RV Park Septic - } \\
\text { Leaks or spills of automotive fluids } \\
\text { or improperly managed septic } \\
\text { systems and wastewater disposal } \\
\text { may impact drinking water supply. }\end{array}$ & 7275 Rogue River Highway & $\begin{array}{l}\text { Grants } \\
\text { Pass }\end{array}$ & Jackson & 42906 & $\begin{array}{l}\text { OHA/DEQ Updated Source } \\
\text { Water Assessment (DEQ/OHA } \\
\text { USWA 2016/2017) }\end{array}$ \\
\hline 2017_USWA_PCSs & 26827 & & $\begin{array}{l}\text { Grants Pass Irrigation Canal - } \\
\text { Seasonal - the irrigation district } \\
\text { pumps water into the canal } 3 \\
\text { months of the year }\end{array}$ & 250 Sky Crest Drive & $\begin{array}{l}\text { Grants } \\
\text { Pass }\end{array}$ & Josephine & 42821 & $\begin{array}{l}\text { OHA/DEQ Updated Source } \\
\text { Water Assessment (DEQ/OHA } \\
\text { USWA 2016/2017) }\end{array}$ \\
\hline 2017_USWA_PCSs & 26828 & & $\begin{array}{l}\text { Grants Pass Irrigation Canal - } \\
\text { Irrigation district pumps water into } \\
\text { this canal } 3 \text { months of the year }\end{array}$ & 250 Sky Crest Drive & $\begin{array}{l}\text { Grants } \\
\text { Pass }\end{array}$ & Josephine & 42821 & $\begin{array}{l}\text { OHA/DEQ Updated Source } \\
\text { Water Assessment (DEQ/OHA } \\
\text { USWA 2016/2017) }\end{array}$ \\
\hline $\begin{array}{l}\text { DWP - PCS - "M" Street } \\
\text { Industrial Park }\end{array}$ & 10416 & $\begin{array}{l}\text { C65 Type; P - Miscellaneous } \\
\text { Manufacturing - Industrial Complex }\end{array}$ & "M" Street Industrial Park & Off "M" Street & $\begin{array}{l}\text { Grants } \\
\text { Pass }\end{array}$ & Josephine & 2005 & $\begin{array}{l}\text { OR Dept. of Environmental } \\
\text { Quality and OR Health Authority } \\
\text { Source Water Assessment } \\
\text { database (DEQ/OHA SWA } \\
2000 \text { - 2005) }\end{array}$ \\
\hline $\begin{array}{l}\text { DWP - PCS - A-1 } \\
\text { EXTERMINATORS }\end{array}$ & 773 & $\begin{array}{l}\text { A14 Type; P - } \\
\text { Pesticide/Fertilizer/Petroleum Storage, } \\
\text { Handling, Mixing, \& Cleaning Areas - } \\
\text { Exterminators } \\
\end{array}$ & A-1 EXTERMINATORS & $\begin{array}{l}\text { NORTHWEST CORNER OF } \\
\text { ROGUE RIVER HWY AND } \\
\text { HAMILTON L }\end{array}$ & $\begin{array}{l}\text { Grants } \\
\text { Pass }\end{array}$ & Josephine & 2005 & DEQ/OHA SWA 2000 - 2005 \\
\hline $\begin{array}{l}\text { DWP - PCS - Aboveground } \\
\text { Storage Tanks }\end{array}$ & 14889 & $\begin{array}{l}\text { M32 Type; P - Construction/Demolition } \\
\text { Areas }\end{array}$ & Aboveground Storage Tanks & $\begin{array}{l}\text { Corner of Leyen Drive and } \mathrm{N} . \\
\text { River Road }\end{array}$ & \begin{tabular}{|l} 
Rogue \\
River
\end{tabular} & Jackson & 2005 & DEQ/OHA SWA 2000 - 2005 \\
\hline $\begin{array}{l}\text { DWP - PCS - Aboveground } \\
\text { Storage Tanks }\end{array}$ & 14889 & $\begin{array}{l}\text { M32 Type; P - Construction/Demolition } \\
\text { Areas }\end{array}$ & Aboveground Storage Tanks & $\begin{array}{l}\text { Corner of Leyen Drive and N. } \\
\text { River Road }\end{array}$ & \begin{tabular}{|l} 
Rogue \\
River
\end{tabular} & Jackson & 2005 & DEQ/OHA SWA 2000 - 2005 \\
\hline $\begin{array}{l}\text { DWP - PCS - Aboveground } \\
\text { Storage Tanks }\end{array}$ & 14889 & $\begin{array}{l}\text { M32 Type; P - Construction/Demolition } \\
\text { Areas }\end{array}$ & Aboveground Storage Tanks & $\begin{array}{l}\text { Corner of Leyen Drive and N. } \\
\text { River Road }\end{array}$ & \begin{tabular}{|l} 
Rogue \\
River
\end{tabular} & Jackson & 2005 & DEQ/OHA SWA 2000 - 2005 \\
\hline $\begin{array}{l}\text { DWP - PCS - Aboveground } \\
\text { Storage Tanks }\end{array}$ & 14889 & $\begin{array}{l}\text { M32 Type; P - Construction/Demolition } \\
\text { Areas }\end{array}$ & Aboveground Storage Tanks & $\begin{array}{l}\text { Corner of Leyen Drive and } \mathrm{N} \text {. } \\
\text { River Road }\end{array}$ & $\begin{array}{l}\text { Rogue } \\
\text { River }\end{array}$ & Jackson & 2005 & DEQ/OHA SWA 2000 - 2005 \\
\hline DWP - PCS - Ace Hardware & 10006 & $\begin{array}{l}\text { C12 Type; P - Furniture/Lumber/Parts } \\
\text { Stores }\end{array}$ & Ace Hardware & Off Broadway Street & $\begin{array}{l}\text { Rogue } \\
\text { River }\end{array}$ & Jackson & 2005 & DEQ/OHA SWA 2000 - 2005 \\
\hline DWP - PCS - Ace Hardware & 10006 & $\begin{array}{l}\text { M24 Type; P - UST - } \\
\text { Decommissioned/Inactive }\end{array}$ & Ace Hardware & Off Broadway Street & $\begin{array}{l}\text { Rogue } \\
\text { River }\end{array}$ & Jackson & 2005 & DEQ/OHA SWA 2000 - 2005 \\
\hline DWP - PCS - Ace Hardware & 10006 & $\begin{array}{l}\text { C12 Type; P - Furniture/Lumber/Parts } \\
\text { Stores }\end{array}$ & Ace Hardware & Off Broadway Street & $\begin{array}{l}\text { Rogue } \\
\text { River }\end{array}$ & Jackson & 2005 & DEQ/OHA SWA 2000 - 2005 \\
\hline DWP - PCS - Ace Hardware & 10006 & $\begin{array}{l}\text { M24 Type; P - UST - } \\
\text { Decommissioned/Inactive }\end{array}$ & Ace Hardware & Off Broadway Street & $\begin{array}{l}\text { Rogue } \\
\text { River }\end{array}$ & Jackson & 2005 & DEQ/OHA SWA 2000 - 2005 \\
\hline DWP - PCS - Ace Hardware & 10006 & $\begin{array}{l}\text { C12 Type; P - Furniture/Lumber/Parts } \\
\text { Stores }\end{array}$ & Ace Hardware & Off Broadway Street & $\begin{array}{l}\text { Rogue } \\
\text { River }\end{array}$ & Jackson & 2005 & DEQ/OHA SWA 2000 - 2005 \\
\hline DWP - PCS - Ace Hardware & 10006 & $\begin{array}{l}\text { M24 Type; P - UST - } \\
\text { Decommissioned/Inactive }\end{array}$ & Ace Hardware & Off Broadway Street & $\begin{array}{l}\text { Rogue } \\
\text { River }\end{array}$ & Jackson & 2005 & DEQ/OHA SWA 2000 - 2005 \\
\hline DWP - PCS - Ace Hardware & 10006 & $\begin{array}{l}\text { C12 Type; P - Furniture/Lumber/Parts } \\
\text { Stores }\end{array}$ & Ace Hardware & Off Broadway Street & \begin{tabular}{|l|} 
Rogue \\
River
\end{tabular} & Jackson & 2005 & DEQ/OHA SWA 2000 - 2005 \\
\hline DWP - PCS - Ace Hardware & 10006 & $\begin{array}{l}\text { M24 Type; P - UST - } \\
\text { Decommissioned/Inactive }\end{array}$ & Ace Hardware & Off Broadway Street & $\begin{array}{l}\text { Rogue } \\
\text { River }\end{array}$ & Jackson & 2005 & DEQ/OHA SWA 2000 - 2005 \\
\hline DWP - PCS - Ace Hardware & 10006 & $\begin{array}{l}\text { C12 Type; P - Furniture/Lumber/Parts } \\
\text { Stores }\end{array}$ & Ace Hardware & Off Broadway Street & $\begin{array}{l}\text { Rogue } \\
\text { River }\end{array}$ & Jackson & 2005 & DEQ/OHA SWA 2000 - 2005 \\
\hline
\end{tabular}




\begin{tabular}{|c|c|c|c|c|c|c|c|c|}
\hline Database Identifier (DB_ID) & Site Identifier & Status & Common Name & Address & City & County & \begin{tabular}{|l} 
Retrieval \\
Date
\end{tabular} & Data Source \\
\hline DWP - PCS - Ace Hardware & 10006 & $\begin{array}{l}\text { M24 Type; P - UST - } \\
\text { Decommissioned/Inactive }\end{array}$ & Ace Hardware & Off Broadway Street & \begin{tabular}{|l} 
Rogue \\
River
\end{tabular} & Jackson & 2005 & DEQ/OHA SWA 2000 - 2005 \\
\hline $\begin{array}{l}\text { DWP - PCS - ALIGNMENT AND } \\
\text { BRAKE SERVICE }\end{array}$ & 1385 & $\begin{array}{l}\text { C04 Type; P - Automobiles - Repair } \\
\text { Shops }\end{array}$ & $\begin{array}{l}\text { ALIGNMENT AND BRAKE } \\
\text { SERVICE }\end{array}$ & $\begin{array}{l}\text { SECOND BUILDING EAST } \\
\text { FROM JUNCTION OF } \\
\text { PARKDALE AND ROGUE } \\
\text { RIVER HWY/NORTH SIDE }\end{array}$ & $\begin{array}{l}\text { Grants } \\
\text { Pass }\end{array}$ & Josephine & 2005 & DEQ/OHA SWA $2000-2005$ \\
\hline $\begin{array}{l}\text { DWP - PCS - ALIGNMENT AND } \\
\text { BRAKE SERVICE }\end{array}$ & 1385 & $\begin{array}{l}\text { C04 Type; P - Automobiles - Repair } \\
\text { Shops }\end{array}$ & $\begin{array}{l}\text { ALIGNMENT AND BRAKE } \\
\text { SERVICE }\end{array}$ & $\begin{array}{l}\text { SECOND BUILDING EAST } \\
\text { FROM JUNCTION OF } \\
\text { PARKDALE AND ROGUE } \\
\text { RIVER HWY/NORTH SIDE }\end{array}$ & $\begin{array}{l}\text { Grants } \\
\text { Pass }\end{array}$ & Josephine & 2005 & DEQ/OHA SWA 2000 - 2005 \\
\hline DWP - PCS - Allied Auto & 14884 & $\begin{array}{l}\text { C04 Type; P - Automobiles - Repair } \\
\text { Shops }\end{array}$ & Allied Auto & Pine Street & \begin{tabular}{|l} 
Rogue \\
River
\end{tabular} & Jackson & 2005 & DEQ/OHA SWA $2000-2005$ \\
\hline DWP - PCS - Allied Auto & 14884 & $\begin{array}{l}\text { C04 Type; P - Automobiles - Repair } \\
\text { Shops }\end{array}$ & Allied Auto & Pine Street & \begin{tabular}{|l|} 
Rogue \\
River
\end{tabular} & Jackson & 2005 & DEQ/OHA SWA $2000-2005$ \\
\hline DWP - PCS - Allied Auto & 14884 & $\begin{array}{l}\text { C04 Type; P - Automobiles - Repair } \\
\text { Shops }\end{array}$ & Allied Auto & Pine Street & \begin{tabular}{|l|} 
Rogue \\
River
\end{tabular} & Jackson & 2005 & DEQ/OHA SWA $2000-2005$ \\
\hline DWP - PCS - Allied Auto & 14884 & $\begin{array}{l}\text { C04 Type; P - Automobiles - Repair } \\
\text { Shops }\end{array}$ & Allied Auto & Pine Street & \begin{tabular}{|l|} 
Rogue \\
River
\end{tabular} & Jackson & 2005 & DEQ/OHA SWA $2000-2005$ \\
\hline $\begin{array}{l}\text { DWP - PCS - AMERICAN } \\
\text { BUILDING SUPPLY STORE }\end{array}$ & 1087 & $\begin{array}{l}\text { C12 Type; P - Furniture/Lumber/Parts } \\
\text { Stores }\end{array}$ & $\begin{array}{l}\text { AMERICAN BUILDING SUPPLY } \\
\text { STORE }\end{array}$ & $\begin{array}{l}\text { SOUTH SIDE OF HIGHWAY } \\
\text { AT EXTREME WESTERN } \\
\text { EDGE OF 15-YEAR TOT }\end{array}$ & $\begin{array}{l}\text { Grants } \\
\text { Pass }\end{array}$ & Josephine & 2005 & DEQ/OHA SWA $2000-2005$ \\
\hline $\begin{array}{l}\text { DWP - PCS - AMERICAN } \\
\text { BUILDING SUPPLY STORE }\end{array}$ & 1087 & $\begin{array}{l}\text { C12 Type; P - Furniture/Lumber/Parts } \\
\text { Stores }\end{array}$ & $\begin{array}{l}\text { AMERICAN BUILDING SUPPLY } \\
\text { STORE }\end{array}$ & $\begin{array}{l}\text { SOUTH SIDE OF HIGHWAY } \\
\text { AT EXTREME WESTERN } \\
\text { EDGE OF 15-YEAR TOT }\end{array}$ & $\begin{array}{l}\text { Grants } \\
\text { Pass }\end{array}$ & Josephine & 2005 & DEQ/OHA SWA $2000-2005$ \\
\hline DWP - PCS - Animal Clinic & 14873 & C16 Type; P - Medical/Vet Offices & Animal Clinic & Main Street & $\begin{array}{l}\text { Rogue } \\
\text { River }\end{array}$ & Jackson & 2005 & DEQ/OHA SWA $2000-2005$ \\
\hline DWP - PCS - Animal Clinic & 14873 & C16 Type; P - Medical/Vet Offices & Animal Clinic & Main Street & \begin{tabular}{|l|} 
Rogue \\
River
\end{tabular} & Jackson & 2005 & DEQ/OHA SWA $2000-2005$ \\
\hline DWP - PCS - Animal Clinic & 14873 & C16 Type; P - Medical/Vet Offices & Animal Clinic & Main Street & $\begin{array}{l}\text { Rogue } \\
\text { River }\end{array}$ & Jackson & 2005 & DEQ/OHA SWA $2000-2005$ \\
\hline DWP - PCS - Animal Clinic & 14873 & C16 Type; P - Medical/Vet Offices & Animal Clinic & Main Street & \begin{tabular}{|l|} 
Rogue \\
River
\end{tabular} & Jackson & 2005 & DEQ/OHA SWA $2000-2005$ \\
\hline \begin{tabular}{|l|} 
DWP - PCS - APARTMENTS \\
JUST EAST OF TRAILER PARK
\end{tabular} & 1092 & $\begin{array}{l}\text { R02 Type; A - Apartments and } \\
\text { Condominiums }\end{array}$ & $\begin{array}{l}\text { APARTMENTS JUST EAST OF } \\
\text { TRAILER PARK }\end{array}$ & $\begin{array}{l}\text { JUST EAST OF TRAILER } \\
\text { PARK ON SOUTH SIDE OF } \\
\text { HWY }\end{array}$ & $\begin{array}{l}\text { Grants } \\
\text { Pass }\end{array}$ & Josephine & 2005 & DEQ/OHA SWA $2000-2005$ \\
\hline \begin{tabular}{|l|} 
DWP - PCS - APARTMENTS \\
JUST EAST OF TRAILER PARK
\end{tabular} & 1092 & $\begin{array}{l}\text { R02 Type; A - Apartments and } \\
\text { Condominiums }\end{array}$ & $\begin{array}{l}\text { APARTMENTS JUST EAST OF } \\
\text { TRAILER PARK }\end{array}$ & $\begin{array}{l}\text { JUST EAST OF TRAILER } \\
\text { PARK ON SOUTH SIDE OF } \\
\text { HWY }\end{array}$ & $\begin{array}{l}\text { Grants } \\
\text { Pass }\end{array}$ & Josephine & 2005 & DEQ/OHA SWA 2000 - 2005 \\
\hline DWP - PCS - ARCO & 10421 & $\begin{array}{l}\text { C03 Type; P - Automobiles - Gas } \\
\text { Stations }\end{array}$ & ARCO & NE Terry Ln & $\begin{array}{l}\text { Grants } \\
\text { Pass }\end{array}$ & Josephine & 2005 & DEQ/OHA SWA $2000-2005$ \\
\hline DWP - PCS - ARCO & 10421 & $\begin{array}{l}\text { M27 Type; P - UST - } \\
\text { Upgraded/Registered - Active }\end{array}$ & ARCO & NE Terry Ln & $\begin{array}{l}\text { Grants } \\
\text { Pass }\end{array}$ & Josephine & 2005 & DEQ/OHA SWA $2000-2005$ \\
\hline $\begin{array}{l}\text { DWP - PCS - AUTO BODY AND } \\
\text { PAINT/REPAIR/GENERAL } \\
\text { AUTO REPAIR }\end{array}$ & 1376 & C01 Type; P - Automobiles - Body Shops & $\begin{array}{l}\text { AUTO BODY AND } \\
\text { PAINT/REPAIR/GENERAL AUTO } \\
\text { REPAIR }\end{array}$ & \begin{tabular}{|l|} 
849 ROGUE RIVER \\
HWY/SOUTH SIDE OF \\
BLOCK MIDWAY BETWEEN \\
PLAYFORD AND \\
FLORENCE LAN
\end{tabular} & $\begin{array}{l}\text { Grants } \\
\text { Pass }\end{array}$ & Josephine & 2005 & DEQ/OHA SWA $2000-2005$ \\
\hline $\begin{array}{l}\text { DWP - PCS - AUTO BODY AND } \\
\text { PAINT/REPAIR/GENERAL } \\
\text { AUTO REPAIR }\end{array}$ & 1376 & $\begin{array}{l}\text { C04 Type; P - Automobiles - Repair } \\
\text { Shops }\end{array}$ & $\begin{array}{l}\text { AUTO BODY AND } \\
\text { PAINT/REPAIR/GENERAL AUTO } \\
\text { REPAIR }\end{array}$ & \begin{tabular}{|l|} 
849 ROGUE RIVER \\
HWY/SOUTH SIDE OF \\
BLOCK MIDWAY BETWEEN \\
PLAYFORD AND \\
FLORENCE LAN
\end{tabular} & $\begin{array}{l}\text { Grants } \\
\text { Pass }\end{array}$ & Josephine & 2005 & DEQ/OHA SWA $2000-2005$ \\
\hline
\end{tabular}




\begin{tabular}{|c|c|c|c|c|c|c|c|c|}
\hline Database Identifier (DB_ID) & Site Identifier & Status & Common Name & Address & City & County & \begin{tabular}{|l|} 
Retrieval \\
Date
\end{tabular} & Data Source \\
\hline $\begin{array}{l}\text { DWP - PCS - AUTO BODY AND } \\
\text { PAINT/REPAIR/GENERAL } \\
\text { AUTO REPAIR }\end{array}$ & 1376 & C01 Type; P - Automobiles - Body Shops & $\begin{array}{l}\text { AUTO BODY AND } \\
\text { PAINT/REPAIR/GENERAL AUTO } \\
\text { REPAIR }\end{array}$ & \begin{tabular}{|l|} 
849 ROGUE RIVER \\
HWY/SOUTH SIDE OF \\
BLOCK MIDWAY BETWEEN \\
PLAYFORD AND \\
FLORENCE LAN
\end{tabular} & $\begin{array}{l}\text { Grants } \\
\text { Pass }\end{array}$ & Josephine & 2005 & DEQ/OHA SWA 2000 - 2005 \\
\hline $\begin{array}{l}\text { DWP - PCS - AUTO BODY AND } \\
\text { PAINT/REPAIR/GENERAL } \\
\text { AUTO REPAIR }\end{array}$ & 1376 & $\begin{array}{l}\text { C04 Type; P - Automobiles - Repair } \\
\text { Shops }\end{array}$ & $\begin{array}{l}\text { AUTO BODY AND } \\
\text { PAINT/REPAIR/GENERAL AUTO } \\
\text { REPAIR }\end{array}$ & \begin{tabular}{|l|}
849 ROGUE RIVER \\
HWY/SOUTH SIDE OF \\
BLOCK MIDWAY BETWEEN \\
PLAYFORD AND \\
FLORENCE LAN
\end{tabular} & $\begin{array}{l}\text { Grants } \\
\text { Pass }\end{array}$ & Josephine & 2005 & DEQ/OHA SWA $2000-2005$ \\
\hline DWP - PCS - Auto Detail & 14245 & C01 Type; P - Automobiles - Body Shops & Auto Detail & Cloverlawn Drive & $\begin{array}{l}\text { Grants } \\
\text { Pass }\end{array}$ & Josephine & 2005 & DEQ/OHA SWA $2000-2005$ \\
\hline DWP - PCS - Auto Works & 14834 & $\begin{array}{l}\text { C04 Type; P - Automobiles - Repair } \\
\text { Shops }\end{array}$ & Auto Works & Depot Street & \begin{tabular}{|l|} 
Rogue \\
River
\end{tabular} & Jackson & 2005 & DEQ/OHA SWA $2000-2005$ \\
\hline DWP - PCS - Auto Works & 14834 & $\begin{array}{l}\text { C04 Type; P - Automobiles - Repair } \\
\text { Shops }\end{array}$ & Auto Works & Depot Street & $\begin{array}{l}\text { Rogue } \\
\text { River }\end{array}$ & Jackson & 2005 & DEQ/OHA SWA $2000-2005$ \\
\hline DWP - PCS - Auto Works & 14834 & $\begin{array}{l}\text { C04 Type; P - Automobiles - Repair } \\
\text { Shops }\end{array}$ & Auto Works & Depot Street & $\begin{array}{l}\text { Rogue } \\
\text { River }\end{array}$ & Jackson & 2005 & DEQ/OHA SWA $2000-2005$ \\
\hline DWP - PCS - Auto Works & 14834 & $\begin{array}{l}\text { C04 Type; P - Automobiles - Repair } \\
\text { Shops }\end{array}$ & Auto Works & Depot Street & $\begin{array}{l}\text { Rogue } \\
\text { River }\end{array}$ & Jackson & 2005 & DEQ/OHA SWA $2000-2005$ \\
\hline DWP - PCS - Auto Wrecker & 10413 & C14 Type; P - Junk/Scrap/Salvage Yards & Auto Wrecker & Off "M" Street & \begin{tabular}{|l|} 
Grants \\
Pass
\end{tabular} & Josephine & 2005 & DEQ/OHA SWA $2000-2005$ \\
\hline $\begin{array}{l}\text { DWP - PCS - AUTOMANIA CAR } \\
\text { SALES }\end{array}$ & 774 & $\begin{array}{l}\text { C04 Type; P - Automobiles - Repair } \\
\text { Shops }\end{array}$ & AUTOMANIA CAR SALES & \begin{tabular}{|l|}
1455 ROGUE RIVER HWY/2 \\
BUSINESSES EAST OF \\
REVOLVO
\end{tabular} & $\begin{array}{l}\text { Grants } \\
\text { Pass }\end{array}$ & Josephine & 2005 & DEQ/OHA SWA $2000-2005$ \\
\hline $\begin{array}{l}\text { DWP - PCS - Big Sam's Auto } \\
\text { Repair }\end{array}$ & 14246 & $\begin{array}{l}\text { C04 Type; P - Automobiles - Repair } \\
\text { Shops }\end{array}$ & Big Sam's Auto Repair & \begin{tabular}{|l|l} 
Highway 99 \\
\end{tabular} & $\begin{array}{l}\text { Grants } \\
\text { Pass }\end{array}$ & Josephine & 2005 & DEQ/OHA SWA $2000-2005$ \\
\hline $\begin{array}{l}\text { DWP - PCS - Bills Auto \& } \\
\text { Exhaust }\end{array}$ & 10007 & $\begin{array}{l}\text { C04 Type; P - Automobiles - Repair } \\
\text { Shops }\end{array}$ & Bills Auto \& Exhaust & Off Park Street & $\begin{array}{l}\text { Rogue } \\
\text { River }\end{array}$ & Jackson & 2005 & DEQ/OHA SWA $2000-2005$ \\
\hline $\begin{array}{l}\text { DWP - PCS - Bills Auto \& } \\
\text { Exhaust }\end{array}$ & 10007 & $\begin{array}{l}\text { C04 Type; P - Automobiles - Repair } \\
\text { Shops }\end{array}$ & Bills Auto \& Exhaust & Off Park Street & \begin{tabular}{|l|} 
Rogue \\
River
\end{tabular} & Jackson & 2005 & DEQ/OHA SWA $2000-2005$ \\
\hline $\begin{array}{l}\text { DWP - PCS - Bills Auto \& } \\
\text { Exhaust }\end{array}$ & 10007 & $\begin{array}{l}\text { C04 Type; P - Automobiles - Repair } \\
\text { Shops }\end{array}$ & Bills Auto \& Exhaust & Off Park Street & $\begin{array}{l}\text { Rogue } \\
\text { River }\end{array}$ & Jackson & 2005 & DEQ/OHA SWA $2000-2005$ \\
\hline $\begin{array}{l}\text { DWP - PCS - Bills Auto \& } \\
\text { Exhaust }\end{array}$ & 10007 & $\begin{array}{l}\text { C04 Type; P - Automobiles - Repair } \\
\text { Shops }\end{array}$ & Bills Auto \& Exhaust & Off Park Street & $\begin{array}{l}\text { Rogue } \\
\text { River }\end{array}$ & Jackson & 2005 & DEQ/OHA SWA $2000-2005$ \\
\hline \begin{tabular}{l|l} 
DWP - PCS - Bills Auto \& \\
Exhaust
\end{tabular} & 10007 & $\begin{array}{l}\text { C04 Type; P - Automobiles - Repair } \\
\text { Shops }\end{array}$ & Bills Auto \& Exhaust & Off Park Street & $\begin{array}{l}\text { Rogue } \\
\text { River }\end{array}$ & Jackson & 2005 & DEQ/OHA SWA $2000-2005$ \\
\hline $\begin{array}{l}\text { DWP - PCS - BP STATION (AKA } \\
\text { BILL TERPENING) }\end{array}$ & 1387 & $\begin{array}{l}\text { C03 Type; P - Automobiles - Gas } \\
\text { Stations }\end{array}$ & $\begin{array}{l}\text { BP STATION (AKA BILL } \\
\text { TERPENING) }\end{array}$ & $\begin{array}{l}\text { 515 ROGUE RIVER HWY/ } \\
\text { HALF BLOCK WEST OF } \\
\text { PARKDALE }\end{array}$ & \begin{tabular}{|l|} 
Grants \\
Pass
\end{tabular} & Josephine & 2005 & DEQ/OHA SWA $2000-2005$ \\
\hline $\begin{array}{l}\text { DWP - PCS - BP STATION (AKA } \\
\text { BILL TERPENING) }\end{array}$ & 1387 & \begin{tabular}{|l|} 
M27 Type; P - UST - \\
Upgraded/Registered - Active
\end{tabular} & $\begin{array}{l}\text { BP STATION (AKA BILL } \\
\text { TERPENING) }\end{array}$ & $\begin{array}{l}\text { 515 ROGUE RIVER HWY/ } \\
\text { HALF BLOCK WEST OF } \\
\text { PARKDALE }\end{array}$ & $\begin{array}{l}\text { Grants } \\
\text { Pass }\end{array}$ & Josephine & 2005 & DEQ/OHA SWA $2000-2005$ \\
\hline $\begin{array}{l}\text { DWP - PCS - BP STATION (AKA } \\
\text { BILL TERPENING) }\end{array}$ & 1387 & $\begin{array}{l}\text { C03 Type; P - Automobiles - Gas } \\
\text { Stations }\end{array}$ & $\begin{array}{l}\text { BP STATION (AKA BILL } \\
\text { TERPENING) }\end{array}$ & $\begin{array}{l}\text { 515 ROGUE RIVER HWY/ } \\
\text { HALF BLOCK WEST OF } \\
\text { PARKDALE }\end{array}$ & $\begin{array}{l}\text { Grants } \\
\text { Pass }\end{array}$ & Josephine & 2005 & DEQ/OHA SWA $2000-2005$ \\
\hline $\begin{array}{l}\text { DWP - PCS - BP STATION (AKA } \\
\text { BILL TERPENING) }\end{array}$ & 1387 & $\begin{array}{l}\text { M27 Type; P - UST - } \\
\text { Upgraded/Registered - Active }\end{array}$ & $\begin{array}{l}\text { BP STATION (AKA BILL } \\
\text { TERPENING) }\end{array}$ & $\begin{array}{l}515 \text { ROGUE RIVER HWY/ } \\
\text { HALF BLOCK WEST OF } \\
\text { PARKDALE }\end{array}$ & $\begin{array}{l}\text { Grants } \\
\text { Pass }\end{array}$ & Josephine & 2005 & DEQ/OHA SWA $2000-2005$ \\
\hline $\begin{array}{l}\text { DWP - PCS - BURGAN } \\
\text { AUTOMOTIVE } \\
\text { BRAKES/ALIGNMENT }\end{array}$ & 1399 & $\begin{array}{l}\text { C04 Type; P - Automobiles - Repair } \\
\text { Shops }\end{array}$ & \begin{tabular}{|l} 
BURGAN AUTOMOTIVE \\
BRAKES/ALIGNMENT
\end{tabular} & \begin{tabular}{|l|}
838 FRUITDALE DR/SOUTH \\
OF JOSE'S AUTOBODY
\end{tabular} & $\begin{array}{l}\text { Grants } \\
\text { Pass }\end{array}$ & Josephine & 2005 & DEQ/OHA SWA $2000-2005$ \\
\hline
\end{tabular}




\begin{tabular}{|c|c|c|c|c|c|c|c|c|}
\hline Database Identifier (DB_ID) & Site Identifier & Status & Common Name & Address & City & County & \begin{tabular}{|l} 
Retrieval \\
Date
\end{tabular} & Data Source \\
\hline $\begin{array}{l}\text { DWP - PCS - BURGAN } \\
\text { AUTOMOTIVE } \\
\text { BRAKES/ALIGNMENT }\end{array}$ & 1399 & $\begin{array}{l}\text { C04 Type; P - Automobiles - Repair } \\
\text { Shops }\end{array}$ & $\begin{array}{l}\text { BURGAN AUTOMOTIVE } \\
\text { BRAKES/ALIGNMENT }\end{array}$ & $\begin{array}{l}838 \text { FRUITDALE DR/SOUTH } \\
\text { OF JOSE'S AUTOBODY }\end{array}$ & $\begin{array}{l}\text { Grants } \\
\text { Pass }\end{array}$ & Josephine & 2005 & DEQ/OHA SWA 2000 - 2005 \\
\hline DWP - PCS - Car Wash & 14890 & $\begin{array}{l}\text { C02 Type; P - Automobiles - Car } \\
\text { Washes }\end{array}$ & Car Wash & N. River Road & \begin{tabular}{|l|} 
Rogue \\
River
\end{tabular} & Jackson & 2005 & DEQ/OHA SWA $2000-2005$ \\
\hline DWP - PCS - Car Wash & 14890 & $\begin{array}{l}\text { C02 Type; P - Automobiles - Car } \\
\text { Washes }\end{array}$ & Car Wash & N. River Road & \begin{tabular}{|l|} 
Rogue \\
River
\end{tabular} & Jackson & 2005 & DEQ/OHA SWA $2000-2005$ \\
\hline DWP - PCS - Car Wash & 14890 & $\begin{array}{l}\text { C02 Type; P - Automobiles - Car } \\
\text { Washes }\end{array}$ & Car Wash & N. River Road & $\begin{array}{l}\text { Rogue } \\
\text { River }\end{array}$ & Jackson & 2005 & DEQ/OHA SWA 2000 - 2005 \\
\hline DWP - PCS - Car Wash & 14890 & $\begin{array}{l}\text { C02 Type; P - Automobiles - Car } \\
\text { Washes }\end{array}$ & Car Wash & N. River Road & $\begin{array}{l}\text { Rogue } \\
\text { River }\end{array}$ & Jackson & 2005 & DEQ/OHA SWA $2000-2005$ \\
\hline $\begin{array}{l}\text { DWP - PCS - Caveman Mobile } \\
\text { Home Park }\end{array}$ & 9365 & $\begin{array}{l}\text { M31 Type; A - Large Capacity Septic } \\
\text { Systems (serves }>20 \text { people) - Class V } \\
\text { UICs }\end{array}$ & Caveman Mobile Home Park & Throughout DWPA & $\begin{array}{l}\text { Grants } \\
\text { Pass }\end{array}$ & Josephine & 2005 & DEQ/OHA SWA $2000-2005$ \\
\hline $\begin{array}{l}\text { DWP - PCS - Caveman Mobile } \\
\text { Home Park }\end{array}$ & 9365 & $\begin{array}{l}\text { R09 Type; A - Housing - High Density (> } \\
1 \text { House/0.5 acres) }\end{array}$ & Caveman Mobile Home Park & Throughout DWPA & $\begin{array}{l}\text { Grants } \\
\text { Pass }\end{array}$ & Josephine & 2005 & DEQ/OHA SWA $2000-2005$ \\
\hline $\begin{array}{l}\text { DWP - PCS - CAVEMAN RV } \\
\text { REPAIR, PARTS \& SERVICE }\end{array}$ & 1083 & $\begin{array}{l}\text { C04 Type; P - Automobiles - Repair } \\
\text { Shops }\end{array}$ & $\begin{array}{l}\text { CAVEMAN RV REPAIR, PARTS \& } \\
\text { SERVICE }\end{array}$ & $\begin{array}{l}\text { NORTHWEST OF TRAILER } \\
\text { PARK/1190 ROGUE RIVER } \\
\text { HWY }\end{array}$ & $\begin{array}{l}\text { Grants } \\
\text { Pass }\end{array}$ & Josephine & 2005 & DEQ/OHA SWA $2000-2005$ \\
\hline $\begin{array}{l}\text { DWP - PCS - CAVEMAN RV } \\
\text { REPAIR, PARTS \& SERVICE }\end{array}$ & 1083 & $\begin{array}{l}\text { C12 Type; P - Furniture/Lumber/Parts } \\
\text { Stores }\end{array}$ & $\begin{array}{l}\text { CAVEMAN RV REPAIR, PARTS \& } \\
\text { SERVICE }\end{array}$ & $\begin{array}{l}\text { NORTHWEST OF TRAILER } \\
\text { PARK/1190 ROGUE RIVER } \\
\text { HWY }\end{array}$ & $\begin{array}{l}\text { Grants } \\
\text { Pass }\end{array}$ & Josephine & 2005 & DEQ/OHA SWA $2000-2005$ \\
\hline $\begin{array}{l}\text { DWP - PCS - CAVEMAN RV } \\
\text { REPAIR, PARTS \& SERVICE }\end{array}$ & 1083 & $\begin{array}{l}\text { C04 Type; P - Automobiles - Repair } \\
\text { Shops }\end{array}$ & $\begin{array}{l}\text { CAVEMAN RV REPAIR, PARTS \& } \\
\text { SERVICE }\end{array}$ & $\begin{array}{l}\text { NORTHWEST OF TRAILER } \\
\text { PARK/1190 ROGUE RIVER } \\
\text { HWY }\end{array}$ & $\begin{array}{l}\text { Grants } \\
\text { Pass }\end{array}$ & Josephine & 2005 & DEQ/OHA SWA $2000-2005$ \\
\hline $\begin{array}{l}\text { DWP - PCS - CAVEMAN RV } \\
\text { REPAIR, PARTS \& SERVICE }\end{array}$ & 1083 & $\begin{array}{l}\text { C12 Type; P - Furniture/Lumber/Parts } \\
\text { Stores }\end{array}$ & $\begin{array}{l}\text { CAVEMAN RV REPAIR, PARTS \& } \\
\text { SERVICE }\end{array}$ & $\begin{array}{l}\text { NORTHWEST OF TRAILER } \\
\text { PARK/1190 ROGUE RIVER } \\
\text { HWY }\end{array}$ & $\begin{array}{l}\text { Grants } \\
\text { Pass }\end{array}$ & Josephine & 2005 & DEQ/OHA SWA $2000-2005$ \\
\hline $\begin{array}{l}\text { DWP - PCS - Caveman Salvage } \\
\text { Yard }\end{array}$ & 10423 & C14 Type; P - Junk/Scrap/Salvage Yards & Caveman Salvage Yard & Off Agness Ave & $\begin{array}{l}\text { Grants } \\
\text { Pass }\end{array}$ & Josephine & 2005 & DEQ/OHA SWA $2000-2005$ \\
\hline $\begin{array}{l}\text { DWP - PCS - Central Oregon } \\
\text { Pacific Railroad }\end{array}$ & 10003 & M19 Type; A - Transportation - Railroads & Central Oregon Pacific Railroad & $\begin{array}{l}\text { Runs along I-5 and Rogue } \\
\text { River }\end{array}$ & \begin{tabular}{|l|} 
Rogue \\
River
\end{tabular} & Jackson & 2005 & DEQ/OHA SWA $2000-2005$ \\
\hline $\begin{array}{l}\text { DWP - PCS - Central Oregon } \\
\text { Pacific Railroad }\end{array}$ & 10003 & M19 Type; A - Transportation - Railroads & Central Oregon Pacific Railroad & $\begin{array}{l}\text { Runs along I-5 and Rogue } \\
\text { River }\end{array}$ & \begin{tabular}{|l|} 
Rogue \\
River
\end{tabular} & Jackson & 2005 & DEQ/OHA SWA $2000-2005$ \\
\hline $\begin{array}{l}\text { DWP - PCS - Central Oregon } \\
\text { Pacific Railroad }\end{array}$ & 10003 & M19 Type; A - Transportation - Railroads & Central Oregon Pacific Railroad & $\begin{array}{l}\text { Runs along I-5 and Rogue } \\
\text { River }\end{array}$ & \begin{tabular}{|l|} 
Rogue \\
River
\end{tabular} & Jackson & 2005 & DEQ/OHA SWA $2000-2005$ \\
\hline $\begin{array}{l}\text { DWP - PCS - Central Oregon } \\
\text { Pacific Railroad }\end{array}$ & 10003 & M19 Type; A - Transportation - Railroads & Central Oregon Pacific Railroad & $\begin{array}{l}\text { Runs along } 1-5 \text { and Rogue } \\
\text { River }\end{array}$ & $\begin{array}{l}\text { Rogue } \\
\text { River }\end{array}$ & Jackson & 2005 & DEQ/OHA SWA 2000 - 2005 \\
\hline $\begin{array}{l}\text { DWP - PCS - Central Oregon } \\
\text { Pacific Railroad }\end{array}$ & 10003 & M19 Type; A - Transportation - Railroads & Central Oregon Pacific Railroad & $\begin{array}{l}\text { Runs along I-5 and Rogue } \\
\text { River }\end{array}$ & $\begin{array}{l}\text { Rogue } \\
\text { River }\end{array}$ & Jackson & 2005 & DEQ/OHA SWA $2000-2005$ \\
\hline DWP - PCS - Central Printing & 14882 & C21 Type; P - Photo Processing/Printing & Central Printing & Pine Street & \begin{tabular}{|l|} 
Rogue \\
River
\end{tabular} & Jackson & 2005 & DEQ/OHA SWA $2000-2005$ \\
\hline DWP - PCS - Central Printing & 14882 & C21 Type; P - Photo Processing/Printing & Central Printing & Pine Street & $\begin{array}{l}\text { Rogue } \\
\text { River }\end{array}$ & Jackson & 2005 & DEQ/OHA SWA $2000-2005$ \\
\hline DWP - PCS - Central Printing & 14882 & C21 Type; P - Photo Processing/Printing & Central Printing & Pine Street & \begin{tabular}{|l|} 
Rogue \\
River
\end{tabular} & Jackson & 2005 & DEQ/OHA SWA $2000-2005$ \\
\hline DWP - PCS - Central Printing & 14882 & C21 Type; P - Photo Processing/Printing & Central Printing & Pine Street & $\begin{array}{l}\text { Rogue } \\
\text { River }\end{array}$ & Jackson & 2005 & DEQ/OHA SWA $2000-2005$ \\
\hline DWP - PCS - City of Rogue River & 10443 & $\begin{array}{l}\text { M50 Type; A - Other - City of Rogue } \\
\text { River }\end{array}$ & City of Rogue River & East of intake & \begin{tabular}{|l|} 
Rogue \\
River
\end{tabular} & Josephine & 2005 & DEQ/OHA SWA $2000-2005$ \\
\hline DWP - PCS - City of Rogue River & 10443 & $\begin{array}{l}\text { R09 Type; A - Housing - High Density (> } \\
1 \text { House/0.5 acres) }\end{array}$ & City of Rogue River & East of intake & $\begin{array}{l}\text { Rogue } \\
\text { River }\end{array}$ & Josephine & 2005 & DEQ/OHA SWA 2000 - 2005 \\
\hline DWP - PCS - City of Rogue River & 14874 & $\begin{array}{l}\text { C09 Type; P - Electrical/Electronic } \\
\text { Manufacturing }\end{array}$ & City of Rogue River & 417 2nd Street & $\begin{array}{l}\text { Rogue } \\
\text { River }\end{array}$ & Jackson & 2005 & DEQ/OHA SWA $2000-2005$ \\
\hline
\end{tabular}




\begin{tabular}{|c|c|c|c|c|c|c|c|c|}
\hline Database Identifier (DB_ID) & Site Identifier & Status & Common Name & Address & City & County & \begin{tabular}{|l} 
Retrieval \\
Date
\end{tabular} & Data Source \\
\hline DWP - PCS - City of Rogue River & 14874 & M61 Type; P - Wells - Abandoned & City of Rogue River & 417 2nd Street & \begin{tabular}{|l|} 
Rogue \\
River
\end{tabular} & Jackson & 2005 & DEQ/OHA SWA 2000 - 2005 \\
\hline DWP - PCS - City of Rogue River & 14874 & $\begin{array}{l}\text { C09 Type; } \mathrm{P} \text { - Electrical/Electronic } \\
\text { Manufacturing }\end{array}$ & City of Rogue River & 417 2nd Street & $\begin{array}{l}\text { Rogue } \\
\text { River }\end{array}$ & Jackson & 2005 & DEQ/OHA SWA $2000-2005$ \\
\hline DWP - PCS - City of Rogue River & 14874 & M61 Type; P - Wells - Abandoned & City of Rogue River & 417 2nd Street & $\begin{array}{l}\text { Rogue } \\
\text { River }\end{array}$ & Jackson & 2005 & DEQ/OHA SWA $2000-2005$ \\
\hline DWP - PCS - City of Rogue River & 14874 & $\begin{array}{l}\text { C09 Type; } \mathrm{P} \text { - Electrical/Electronic } \\
\text { Manufacturing }\end{array}$ & City of Rogue River & 417 2nd Street & $\begin{array}{l}\text { Rogue } \\
\text { River }\end{array}$ & Jackson & 2005 & DEQ/OHA SWA 2000 - 2005 \\
\hline DWP - PCS - City of Rogue River & 14874 & M61 Type; P - Wells - Abandoned & City of Rogue River & 417 2nd Street & $\begin{array}{l}\text { Rogue } \\
\text { River }\end{array}$ & Jackson & 2005 & DEQ/OHA SWA $2000-2005$ \\
\hline DWP - PCS - City of Rogue River & 14874 & $\begin{array}{l}\text { C09 Type; } \mathrm{P} \text { - Electrical/Electronic } \\
\text { Manufacturing }\end{array}$ & City of Rogue River & 417 2nd Street & $\begin{array}{l}\text { Rogue } \\
\text { River }\end{array}$ & Jackson & 2005 & DEQ/OHA SWA $2000-2005$ \\
\hline DWP - PCS - City of Rogue River & 14874 & M61 Type; P - Wells - Abandoned & City of Rogue River & 417 2nd Street & $\begin{array}{l}\text { Rogue } \\
\text { River }\end{array}$ & Jackson & 2005 & DEQ/OHA SWA $2000-2005$ \\
\hline $\begin{array}{l}\text { DWP - PCS - City of Rogue River } \\
\text { Wastewater Plant }\end{array}$ & 10442 & $\begin{array}{l}\text { R20 Type; P - Wastewater Treatment } \\
\text { Plants/Collection Stations }\end{array}$ & $\begin{array}{l}\text { City of Rogue River Wastewater } \\
\text { Plant }\end{array}$ & East of intake & $\begin{array}{l}\text { Rogue } \\
\text { River }\end{array}$ & Josephine & 2005 & DEQ/OHA SWA 2000 - 2005 \\
\hline DWP - PCS - Clearwater Co-op & 10430 & C14 Type; P - Junk/Scrap/Salvage Yards & Clearwater Co-op & 1130 Gladiola Dr & $\begin{array}{l}\text { Grants } \\
\text { Pass }\end{array}$ & Josephine & 2005 & DEQ/OHA SWA $2000-2005$ \\
\hline $\begin{array}{l}\text { DWP - PCS - CLOSED } \\
\text { IRRIGATION DITCH }\end{array}$ & 787 & A52 Type; A - Irrigation Canal/Pond & CLOSED IRRIGATION DITCH & \begin{tabular}{|l|} 
EAST OF SCHOOL/RUNS \\
NORTH/SOUTH AND \\
PERPENDICULAR \\
\end{tabular} & \begin{tabular}{|l|} 
Grants \\
Pass
\end{tabular} & Josephine & 2005 & DEQ/OHA SWA $2000-2005$ \\
\hline DWP - PCS - Clovercrest Market & 12333 & $\begin{array}{l}\text { M26 Type; P - UST - Confirmed Leaking } \\
\text { but listed as NFA - DEQ LUST List }\end{array}$ & Clovercrest Market & $\begin{array}{l}\text { Cloverlawn Dr. and Crestview } \\
\text { Loop }\end{array}$ & $\begin{array}{l}\text { Grants } \\
\text { Pass }\end{array}$ & Josephine & 2005 & DEQ/OHA SWA 2000 - 2005 \\
\hline DWP - PCS - Clovercrest Market & 12333 & \begin{tabular}{|l|} 
M26 Type; P - UST - Confirmed Leaking \\
but listed as NFA - DEQ LUST List
\end{tabular} & Clovercrest Market & $\begin{array}{l}\text { Cloverlawn Dr. and Crestview } \\
\text { Loop }\end{array}$ & $\begin{array}{l}\text { Grants } \\
\text { Pass }\end{array}$ & Josephine & 2005 & DEQ/OHA SWA $2000-2005$ \\
\hline $\begin{array}{l}\text { DWP - PCS - Clovercrest Service } \\
\text { Center }\end{array}$ & 12334 & $\begin{array}{l}\text { C04 Type; P - Automobiles - Repair } \\
\text { Shops }\end{array}$ & Clovercrest Service Center & $\begin{array}{l}\text { Cloverlawn Dr. and Crestview } \\
\text { Loop }\end{array}$ & $\begin{array}{l}\text { Grants } \\
\text { Pass }\end{array}$ & Josephine & 2005 & DEQ/OHA SWA $2000-2005$ \\
\hline $\begin{array}{l}\text { DWP - PCS - Clovercrest Service } \\
\text { Center }\end{array}$ & 12334 & $\begin{array}{l}\text { C04 Type; P - Automobiles - Repair } \\
\text { Shops }\end{array}$ & Clovercrest Service Center & $\begin{array}{l}\text { Cloverlawn Dr. and Crestview } \\
\text { Loop }\end{array}$ & $\begin{array}{l}\text { Grants } \\
\text { Pass }\end{array}$ & Josephine & 2005 & DEQ/OHA SWA $2000-2005$ \\
\hline \begin{tabular}{|l|} 
DWP - PCS - Colvin Oil \\
Company/Rogue River Arco
\end{tabular} & 14883 & $\begin{array}{l}\text { C03 Type; P - Automobiles - Gas } \\
\text { Stations }\end{array}$ & $\begin{array}{l}\text { Colvin Oil Company/Rogue River } \\
\text { Arco }\end{array}$ & \begin{tabular}{|l|} 
Pine Street \\
\end{tabular} & $\begin{array}{l}\text { Rogue } \\
\text { River }\end{array}$ & Jackson & 2005 & DEQ/OHA SWA $2000-2005$ \\
\hline $\begin{array}{l}\text { DWP - PCS - Colvin Oil } \\
\text { Company/Rogue River Arco }\end{array}$ & 14883 & $\begin{array}{l}\text { C04 Type; P - Automobiles - Repair } \\
\text { Shops }\end{array}$ & $\begin{array}{l}\text { Colvin Oil Company/Rogue River } \\
\text { Arco }\end{array}$ & Pine Street & $\begin{array}{l}\text { Rogue } \\
\text { River }\end{array}$ & Jackson & 2005 & DEQ/OHA SWA $2000-2005$ \\
\hline $\begin{array}{l}\text { DWP - PCS - Colvin Oil } \\
\text { Company/Rogue River Arco }\end{array}$ & 14883 & \begin{tabular}{|l|} 
M26 Type; P - UST - Confirmed Leaking \\
but listed as NFA - DEQ LUST List
\end{tabular} & $\begin{array}{l}\text { Colvin Oil Company/Rogue River } \\
\text { Arco }\end{array}$ & Pine Street & \begin{tabular}{|l|} 
Rogue \\
River
\end{tabular} & Jackson & 2005 & DEQ/OHA SWA 2000 - 2005 \\
\hline $\begin{array}{l}\text { DWP - PCS - Colvin Oil } \\
\text { Company/Rogue River Arco }\end{array}$ & 14883 & $\begin{array}{l}\text { M27 Type; P - UST - } \\
\text { Upgraded/Registered - Active }\end{array}$ & $\begin{array}{l}\text { Colvin Oil Company/Rogue River } \\
\text { Arco }\end{array}$ & Pine Street & \begin{tabular}{|l|} 
Rogue \\
River
\end{tabular} & Jackson & 2005 & DEQ/OHA SWA $2000-2005$ \\
\hline $\begin{array}{l}\text { DWP - PCS - Colvin Oil } \\
\text { Company/Rogue River Arco }\end{array}$ & 14883 & $\begin{array}{l}\text { C03 Type; P - Automobiles - Gas } \\
\text { Stations }\end{array}$ & $\begin{array}{l}\text { Colvin Oil Company/Rogue River } \\
\text { Arco }\end{array}$ & Pine Street & $\begin{array}{l}\text { Rogue } \\
\text { River }\end{array}$ & Jackson & 2005 & DEQ/OHA SWA 2000 - 2005 \\
\hline $\begin{array}{l}\text { DWP - PCS - Colvin Oil } \\
\text { Company/Rogue River Arco }\end{array}$ & 14883 & $\begin{array}{l}\text { C04 Type; P - Automobiles - Repair } \\
\text { Shops }\end{array}$ & $\begin{array}{l}\text { Colvin Oil Company/Rogue River } \\
\text { Arco }\end{array}$ & Pine Street & $\begin{array}{l}\text { Rogue } \\
\text { River }\end{array}$ & Jackson & 2005 & DEQ/OHA SWA $2000-2005$ \\
\hline $\begin{array}{l}\text { DWP - PCS - Colvin Oil } \\
\text { Company/Rogue River Arco }\end{array}$ & 14883 & $\begin{array}{l}\text { M26 Type; P - UST - Confirmed Leaking } \\
\text { but listed as NFA - DEQ LUST List }\end{array}$ & $\begin{array}{l}\text { Colvin Oil Company/Rogue River } \\
\text { Arco }\end{array}$ & Pine Street & $\begin{array}{l}\text { Rogue } \\
\text { River }\end{array}$ & Jackson & 2005 & DEQ/OHA SWA 2000 - 2005 \\
\hline $\begin{array}{l}\text { DWP - PCS - Colvin Oil } \\
\text { Company/Rogue River Arco }\end{array}$ & 14883 & $\begin{array}{l}\text { M27 Type; P - UST - } \\
\text { Upgraded/Registered - Active }\end{array}$ & $\begin{array}{l}\text { Colvin Oil Company/Rogue River } \\
\text { Arco }\end{array}$ & Pine Street & $\begin{array}{l}\text { Rogue } \\
\text { River }\end{array}$ & Jackson & 2005 & DEQ/OHA SWA 2000 - 2005 \\
\hline $\begin{array}{l}\text { DWP - PCS - Colvin Oil } \\
\text { Company/Rogue River Arco }\end{array}$ & 14883 & $\begin{array}{l}\text { C03 Type; P - Automobiles - Gas } \\
\text { Stations }\end{array}$ & $\begin{array}{l}\text { Colvin Oil Company/Rogue River } \\
\text { Arco }\end{array}$ & Pine Street & $\begin{array}{l}\text { Rogue } \\
\text { River }\end{array}$ & Jackson & 2005 & DEQ/OHA SWA 2000 - 2005 \\
\hline $\begin{array}{l}\text { DWP - PCS - Colvin Oil } \\
\text { Company/Rogue River Arco }\end{array}$ & 14883 & $\begin{array}{l}\text { C04 Type; P - Automobiles - Repair } \\
\text { Shops }\end{array}$ & $\begin{array}{l}\text { Colvin Oil Company/Rogue River } \\
\text { Arco }\end{array}$ & Pine Street & \begin{tabular}{|l|} 
Rogue \\
River
\end{tabular} & Jackson & 2005 & DEQ/OHA SWA $2000-2005$ \\
\hline
\end{tabular}




\begin{tabular}{|c|c|c|c|c|c|c|c|c|}
\hline Database Identifier (DB_ID) & Site Identifier & Status & Common Name & Address & City & County & \begin{tabular}{|l|} 
Retrieval \\
Date
\end{tabular} & Data Source \\
\hline $\begin{array}{l}\text { DWP - PCS - Colvin Oil } \\
\text { Company/Rogue River Arco }\end{array}$ & 14883 & $\begin{array}{l}\text { M26 Type; P - UST - Confirmed Leaking } \\
\text { but listed as NFA - DEQ LUST List }\end{array}$ & $\begin{array}{l}\text { Colvin Oil Company/Rogue River } \\
\text { Arco }\end{array}$ & Pine Street & \begin{tabular}{|l} 
Rogue \\
River
\end{tabular} & Jackson & 2005 & DEQ/OHA SWA 2000 - 2005 \\
\hline $\begin{array}{l}\text { DWP - PCS - Colvin Oil } \\
\text { Company/Rogue River Arco }\end{array}$ & 14883 & $\begin{array}{l}\text { M27 Type; P - UST - } \\
\text { Upgraded/Registered - Active }\end{array}$ & $\begin{array}{l}\text { Colvin Oil Company/Rogue River } \\
\text { Arco }\end{array}$ & Pine Street & $\begin{array}{l}\text { Rogue } \\
\text { River }\end{array}$ & Jackson & 2005 & DEQ/OHA SWA 2000 - 2005 \\
\hline \begin{tabular}{|l} 
DWP - PCS - Colvin Oil \\
Company/Rogue River Arco
\end{tabular} & 14883 & $\begin{array}{l}\text { C03 Type; P - Automobiles - Gas } \\
\text { Stations }\end{array}$ & $\begin{array}{l}\text { Colvin Oil Company/Rogue River } \\
\text { Arco }\end{array}$ & Pine Street & \begin{tabular}{|l|} 
Rogue \\
River
\end{tabular} & Jackson & 2005 & DEQ/OHA SWA 2000 - 2005 \\
\hline $\begin{array}{l}\text { DWP - PCS - Colvin Oil } \\
\text { Company/Rogue River Arco }\end{array}$ & 14883 & $\begin{array}{l}\text { C04 Type; P - Automobiles - Repair } \\
\text { Shops }\end{array}$ & $\begin{array}{l}\text { Colvin Oil Company/Rogue River } \\
\text { Arco }\end{array}$ & Pine Street & \begin{tabular}{|l|} 
Rogue \\
River
\end{tabular} & Jackson & 2005 & DEQ/OHA SWA 2000 - 2005 \\
\hline $\begin{array}{l}\text { DWP - PCS - Colvin Oil } \\
\text { Company/Rogue River Arco }\end{array}$ & 14883 & $\begin{array}{l}\text { M26 Type; P - UST - Confirmed Leaking } \\
\text { but listed as NFA - DEQ LUST List }\end{array}$ & $\begin{array}{l}\text { Colvin Oil Company/Rogue River } \\
\text { Arco }\end{array}$ & Pine Street & $\begin{array}{l}\text { Rogue } \\
\text { River }\end{array}$ & Jackson & 2005 & DEQ/OHA SWA 2000 - 2005 \\
\hline $\begin{array}{l}\text { DWP - PCS - Colvin Oil } \\
\text { Company/Rogue River Arco }\end{array}$ & 14883 & \begin{tabular}{|l|} 
M27 Type; P - UST - \\
Upgraded/Registered - Active
\end{tabular} & $\begin{array}{l}\text { Colvin Oil Company/Rogue River } \\
\text { Arco }\end{array}$ & Pine Street & $\begin{array}{l}\text { Rogue } \\
\text { River }\end{array}$ & Jackson & 2005 & DEQ/OHA SWA 2000 - 2005 \\
\hline \begin{tabular}{|l|} 
DWP - PCS - Construction - \\
Panoramic View Estates
\end{tabular} & 12337 & $\begin{array}{l}\text { M32 Type; A - Construction/Demolition } \\
\text { Areas }\end{array}$ & $\begin{array}{l}\text { Construction - Panoramic View } \\
\text { Estates }\end{array}$ & Panoramic Ln. & $\begin{array}{l}\text { Grants } \\
\text { Pass }\end{array}$ & Josephine & 2005 & DEQ/OHA SWA 2000 - 2005 \\
\hline $\begin{array}{l}\text { DWP - PCS - DRURY LANE } \\
\text { CROSSING MAIN CANAL }\end{array}$ & 1394 & $\begin{array}{l}\text { M22 Type; P - Transportation - Stream } \\
\text { Crossing - Perennial }\end{array}$ & $\begin{array}{l}\text { DRURY LANE CROSSING MAIN } \\
\text { CANAL }\end{array}$ & $\begin{array}{l}\text { SOUTH OF FRUITDALE } \\
\text { DR/NORTH OF BAILEY DR }\end{array}$ & $\begin{array}{l}\text { Grants } \\
\text { Pass }\end{array}$ & Josephine & 2005 & DEQ/OHA SWA 2000 - 2005 \\
\hline $\begin{array}{l}\text { DWP - PCS - DRURY LANE } \\
\text { CROSSING MAIN CANAL }\end{array}$ & 1394 & $\begin{array}{l}\text { M22 Type; P - Transportation - Stream } \\
\text { Crossing - Perennial }\end{array}$ & $\begin{array}{l}\text { DRURY LANE CROSSING MAIN } \\
\text { CANAL }\end{array}$ & $\begin{array}{l}\text { SOUTH OF FRUITDALE } \\
\text { DR/NORTH OF BAILEY DR }\end{array}$ & $\begin{array}{l}\text { Grants } \\
\text { Pass }\end{array}$ & Josephine & 2005 & DEQ/OHA SWA 2000 - 2005 \\
\hline DWP - PCS - Economy Auto & 14891 & $\begin{array}{l}\text { C04 Type; P - Automobiles - Repair } \\
\text { Shops }\end{array}$ & Economy Auto & N. River Road & $\begin{array}{l}\text { Rogue } \\
\text { River }\end{array}$ & Jackson & 2005 & DEQ/OHA SWA 2000 - 2005 \\
\hline DWP - PCS - Economy Auto & 14891 & $\begin{array}{l}\text { C04 Type; P - Automobiles - Repair } \\
\text { Shops }\end{array}$ & Economy Auto & N. River Road & $\begin{array}{l}\text { Rogue } \\
\text { River }\end{array}$ & Jackson & 2005 & DEQ/OHA SWA 2000 - 2005 \\
\hline DWP - PCS - Economy Auto & 14891 & $\begin{array}{l}\text { C04 Type; P - Automobiles - Repair } \\
\text { Shops }\end{array}$ & Economy Auto & N. River Road & $\begin{array}{l}\text { Rogue } \\
\text { River }\end{array}$ & Jackson & 2005 & DEQ/OHA SWA 2000 - 2005 \\
\hline DWP - PCS - Economy Auto & 14891 & $\begin{array}{l}\text { C04 Type; P - Automobiles - Repair } \\
\text { Shops }\end{array}$ & Economy Auto & N. River Road & \begin{tabular}{|l|} 
Rogue \\
River
\end{tabular} & Jackson & 2005 & DEQ/OHA SWA 2000 - 2005 \\
\hline DWP - PCS - ECS Composites & 10437 & $\begin{array}{l}\text { C22 Type; P - Plastics/Synthetics } \\
\text { Producer }\end{array}$ & ECS Composites & 3560 Rogue River Highway & $\begin{array}{l}\text { Grants } \\
\text { Pass }\end{array}$ & Josephine & 2005 & DEQ/OHA SWA 2000 - 2005 \\
\hline DWP - PCS - ECS Composites & 10437 & $\begin{array}{l}\text { M24 Type; P - UST - } \\
\text { Decommissioned/Inactive }\end{array}$ & ECS Composites & 3560 Rogue River Highway & $\begin{array}{l}\text { Grants } \\
\text { Pass } \\
\end{array}$ & Josephine & 2005 & DEQ/OHA SWA 2000 - 2005 \\
\hline DWP - PCS - ECS Composites & 10437 & $\begin{array}{l}\text { M31 Type; P - Large Capacity Septic } \\
\text { Systems (serves }>20 \text { people) - Class V } \\
\text { UICs }\end{array}$ & ECS Composites & 3560 Rogue River Highway & $\begin{array}{l}\text { Grants } \\
\text { Pass }\end{array}$ & Josephine & 2005 & DEQ/OHA SWA 2000 - 2005 \\
\hline DWP - PCS - EMC Headware & 14870 & M55 Type; P - Unknown Operation & EMC Headware & Park Street & \begin{tabular}{|l|} 
Rogue \\
River
\end{tabular} & Jackson & 2005 & DEQ/OHA SWA 2000 - 2005 \\
\hline DWP - PCS - EMC Headware & 14870 & M55 Type; P - Unknown Operation & EMC Headware & Park Street & \begin{tabular}{|l|} 
Rogue \\
River
\end{tabular} & Jackson & 2005 & DEQ/OHA SWA 2000 - 2005 \\
\hline DWP - PCS - EMC Headware & 14870 & M55 Type; P - Unknown Operation & EMC Headware & Park Street & \begin{tabular}{|l|} 
Rogue \\
River
\end{tabular} & Jackson & 2005 & DEQ/OHA SWA 2000 - 2005 \\
\hline DWP - PCS - EMC Headware & 14870 & M55 Type; P - Unknown Operation & EMC Headware & Park Street & \begin{tabular}{|l|} 
Rogue \\
River
\end{tabular} & Jackson & 2005 & DEQ/OHA SWA 2000 - 2005 \\
\hline $\begin{array}{l}\text { DWP - PCS - Environmental } \\
\text { Container Systems }\end{array}$ & 9368 & $\begin{array}{l}\text { C07 Type; A - Chemical/Petroleum } \\
\text { Processing/Storage }\end{array}$ & Environmental Container Systems & West of well & $\begin{array}{l}\text { Grants } \\
\text { Pass }\end{array}$ & Josephine & 2005 & DEQ/OHA SWA 2000 - 2005 \\
\hline $\begin{array}{l}\text { DWP - PCS - Environmental } \\
\text { Container Systems }\end{array}$ & 9368 & $\begin{array}{l}\text { C22 Type; A - Plastics/Synthetics } \\
\text { Producer }\end{array}$ & Environmental Container Systems & West of well & $\begin{array}{l}\text { Grants } \\
\text { Pass }\end{array}$ & Josephine & 2005 & DEQ/OHA SWA 2000 - 2005 \\
\hline $\begin{array}{l}\text { DWP - PCS - Environmental } \\
\text { Container Systems }\end{array}$ & 9368 & $\begin{array}{l}\text { M24 Type; A - UST - } \\
\text { Decommissioned/Inactive }\end{array}$ & Environmental Container Systems & West of well & $\begin{array}{l}\text { Grants } \\
\text { Pass }\end{array}$ & Josephine & 2005 & DEQ/OHA SWA 2000 - 2005 \\
\hline $\begin{array}{l}\text { DWP - PCS - Environmental } \\
\text { Container Systems }\end{array}$ & 9368 & $\begin{array}{l}\text { M31 Type; A - Large Capacity Septic } \\
\text { Systems (serves > } 20 \text { people) - Class V } \\
\text { UICs }\end{array}$ & Environmental Container Systems & West of well & $\begin{array}{l}\text { Grants } \\
\text { Pass }\end{array}$ & Josephine & 2005 & DEQ/OHA SWA 2000 - 2005 \\
\hline
\end{tabular}




\begin{tabular}{|c|c|c|c|c|c|c|c|c|}
\hline Database Identifier (DB_ID) & Site Identifier & Status & Common Name & Address & City & County & \begin{tabular}{|l} 
Retrieval \\
Date
\end{tabular} & Data Source \\
\hline DWP - PCS - ESAM & 10427 & $\begin{array}{l}\text { C09 Type; P - Electrical/Electronic } \\
\text { Manufacturing }\end{array}$ & ESAM & 2100 NE Spaulding Ave & $\begin{array}{l}\text { Grants } \\
\text { Pass }\end{array}$ & Josephine & 2005 & DEQ/OHA SWA 2000 - 2005 \\
\hline DWP - PCS - Foothill Dump & 10429 & $\begin{array}{l}\text { M07 Type; P - Historic Waste } \\
\text { Dumps/Landfills }\end{array}$ & Foothill Dump & Along Foothill Blvd & $\begin{array}{l}\text { Grants } \\
\text { Pass }\end{array}$ & Josephine & 2005 & DEQ/OHA SWA 2000 - 2005 \\
\hline DWP - PCS - Foothill Dump & 10429 & $\begin{array}{l}\text { M24 Type; P - UST - } \\
\text { Decommissioned/Inactive }\end{array}$ & Foothill Dump & Along Foothill Blvd & $\begin{array}{l}\text { Grants } \\
\text { Pass }\end{array}$ & Josephine & 2005 & DEQ/OHA SWA 2000 - 2005 \\
\hline DWP - PCS - Foothill Dump & 10429 & $\begin{array}{l}\text { M27 Type; P - UST - } \\
\text { Upgraded/Registered - Active }\end{array}$ & Foothill Dump & Along Foothill Blvd & $\begin{array}{l}\text { Grants } \\
\text { Pass }\end{array}$ & Josephine & 2005 & DEQ/OHA SWA 2000 - 2005 \\
\hline DWP - PCS - Four Ply Inc. & 10418 & $\begin{array}{l}\text { C04 Type; P - Automobiles - Repair } \\
\text { Shops }\end{array}$ & Four Ply Inc. & 124 NE Beacon & $\begin{array}{l}\text { Grants } \\
\text { Pass }\end{array}$ & Josephine & 2005 & DEQ/OHA SWA 2000 - 2005 \\
\hline DWP - PCS - Four Ply Inc. & 10418 & $\begin{array}{l}\text { M01 Type; P - Above Ground Storage } \\
\text { Tanks - Excluding Water and Residential } \\
\text { ASTs }\end{array}$ & Four Ply Inc. & 124 NE Beacon & $\begin{array}{l}\text { Grants } \\
\text { Pass }\end{array}$ & Josephine & 2005 & DEQ/OHA SWA 2000 - 2005 \\
\hline DWP - PCS - Four Ply Inc. & 10418 & $\begin{array}{l}\text { M24 Type; P - UST - } \\
\text { Decommissioned/Inactive }\end{array}$ & Four Ply Inc. & 124 NE Beacon & $\begin{array}{l}\text { Grants } \\
\text { Pass }\end{array}$ & Josephine & 2005 & DEQ/OHA SWA 2000 - 2005 \\
\hline DWP - PCS - Fred Meyers & 10419 & $\begin{array}{l}\text { C20 Type; P - Parking Lots/Malls (> } 50 \\
\text { Spaces) }\end{array}$ & Fred Meyers & Beacon and NE "F" Street & $\begin{array}{l}\text { Grants } \\
\text { Pass }\end{array}$ & Josephine & 2005 & DEQ/OHA SWA 2000 - 2005 \\
\hline DWP - PCS - Fred Meyers & 10419 & $\begin{array}{l}\text { M27 Type; P - UST - } \\
\text { Upgraded/Registered - Active }\end{array}$ & Fred Meyers & Beacon and NE "F" Street & $\begin{array}{l}\text { Grants } \\
\text { Pass }\end{array}$ & Josephine & 2005 & DEQ/OHA SWA 2000 - 2005 \\
\hline $\begin{array}{l}\text { DWP - PCS - FRIENDLY } \\
\text { MOTORS }\end{array}$ & 1102 & $\begin{array}{l}\text { C04 Type; P - Automobiles - Repair } \\
\text { Shops }\end{array}$ & FRIENDLY MOTORS & $\begin{array}{l}\text { JUST WEST OF } \\
\text { CLOVERLAWN }\end{array}$ & $\begin{array}{l}\text { Grants } \\
\text { Pass }\end{array}$ & Josephine & 2005 & DEQ/OHA SWA $2000-2005$ \\
\hline $\begin{array}{l}\text { DWP - PCS - FRIENDLY } \\
\text { MOTORS }\end{array}$ & 1102 & $\begin{array}{l}\text { C04 Type; P - Automobiles - Repair } \\
\text { Shops }\end{array}$ & FRIENDLY MOTORS & $\begin{array}{l}\text { JUST WEST OF } \\
\text { CLOVERLAWN }\end{array}$ & $\begin{array}{l}\text { Grants } \\
\text { Pass }\end{array}$ & Josephine & 2005 & DEQ/OHA SWA 2000 - 2005 \\
\hline $\begin{array}{l}\text { DWP - PCS - Frontier Mobile } \\
\text { Estates }\end{array}$ & 12312 & $\begin{array}{l}\text { M27 Type; A - UST - } \\
\text { Upgraded/Registered - Active }\end{array}$ & Frontier Mobile Estates & Rogue River Hwy & $\begin{array}{l}\text { Rogue } \\
\text { River }\end{array}$ & Jackson & 2005 & DEQ/OHA SWA 2000 - 2005 \\
\hline $\begin{array}{l}\text { DWP - PCS - Frontier Mobile } \\
\text { Estates }\end{array}$ & 12312 & $\begin{array}{l}\text { M31 Type; A - Large Capacity Septic } \\
\text { Systems (serves }>20 \text { people) - Class V } \\
\text { UICs }\end{array}$ & Frontier Mobile Estates & Rogue River Hwy & $\begin{array}{l}\text { Rogue } \\
\text { River }\end{array}$ & Jackson & 2005 & DEQ/OHA SWA 2000 - 2005 \\
\hline $\begin{array}{l}\text { DWP - PCS - Frontier Mobile } \\
\text { Estates }\end{array}$ & 12312 & $\begin{array}{l}\text { R09 Type; A - Housing - High Density (> } \\
1 \text { House/0.5 acres) }\end{array}$ & Frontier Mobile Estates & Rogue River Hwy & $\begin{array}{l}\text { Rogue } \\
\text { River }\end{array}$ & Jackson & 2005 & DEQ/OHA SWA 2000 - 2005 \\
\hline $\begin{array}{l}\text { DWP - PCS - FRUITDALE } \\
\text { CREEK }\end{array}$ & 789 & $\begin{array}{l}\text { M22 Type; A - Transportation - Stream } \\
\text { Crossing - Perennial }\end{array}$ & FRUITDALE CREEK & $\begin{array}{l}\text { EAST OF SCHOOL/RUNS } \\
\text { NORTH - SOUTH EAST OF } \\
\text { HAMILTON }\end{array}$ & $\begin{array}{l}\text { Grants } \\
\text { Pass }\end{array}$ & Josephine & 2005 & DEQ/OHA SWA 2000 - 2005 \\
\hline $\begin{array}{l}\text { DWP - PCS - FRUITDALE } \\
\text { ELEMENTARY SCHOOL }\end{array}$ & 783 & $\begin{array}{l}\text { C20 Type; P - Parking Lots/Malls (> } 50 \\
\text { Spaces) }\end{array}$ & $\begin{array}{l}\text { FRUITDALE ELEMENTARY } \\
\text { SCHOOL }\end{array}$ & 1560 HAMILTON LANE & $\begin{array}{l}\text { Grants } \\
\text { Pass }\end{array}$ & Josephine & 2005 & DEQ/OHA SWA 2000 - 2005 \\
\hline $\begin{array}{l}\text { DWP - PCS - FRUITDALE } \\
\text { ELEMENTARY SCHOOL }\end{array}$ & 783 & M28 Type; P - UST - Status Unknown & $\begin{array}{l}\text { FRUITDALE ELEMENTARY } \\
\text { SCHOOL }\end{array}$ & 1560 HAMILTON LANE & $\begin{array}{l}\text { Grants } \\
\text { Pass }\end{array}$ & Josephine & 2005 & DEQ/OHA SWA 2000 - 2005 \\
\hline $\begin{array}{l}\text { DWP - PCS - FRUITDALE } \\
\text { ELEMENTARY SCHOOL }\end{array}$ & 783 & R15 Type; P - Schools & $\begin{array}{l}\text { FRUITDALE ELEMENTARY } \\
\text { SCHOOL }\end{array}$ & 1560 HAMILTON LANE & $\begin{array}{l}\text { Grants } \\
\text { Pass }\end{array}$ & Josephine & 2005 & DEQ/OHA SWA 2000 - 2005 \\
\hline DWP - PCS - Fruitdale School & 14249 & M28 Type; P - UST - Status Unknown & Fruitdale School & Hamilton Lane & $\begin{array}{l}\text { Grants } \\
\text { Pass }\end{array}$ & Josephine & 2005 & DEQ/OHA SWA 2000 - 2005 \\
\hline DWP - PCS - Fruitdale School & 14249 & R15 Type; P - Schools & Fruitdale School & Hamilton Lane & $\begin{array}{l}\text { Grants } \\
\text { Pass }\end{array}$ & Josephine & 2005 & DEQ/OHA SWA 2000 - 2005 \\
\hline $\begin{array}{l}\text { DWP - PCS - FRUITDALE } \\
\text { TRAILER PARK }\end{array}$ & 1090 & $\begin{array}{l}\text { R09 Type; A - Housing - High Density (> } \\
1 \text { House/0.5 acres) }\end{array}$ & FRUITDALE TRAILER PARK & 1275 ROGUE RIVER HWY & $\begin{array}{l}\text { Grants } \\
\text { Pass }\end{array}$ & Josephine & 2005 & DEQ/OHA SWA 2000 - 2005 \\
\hline $\begin{array}{l}\text { DWP - PCS - FRUITDALE } \\
\text { TRAILER PARK }\end{array}$ & 1090 & $\begin{array}{l}\text { R09 Type; A - Housing - High Density (> } \\
1 \text { House/0.5 acres) }\end{array}$ & FRUITDALE TRAILER PARK & 1275 ROGUE RIVER HWY & $\begin{array}{l}\text { Grants } \\
\text { Pass }\end{array}$ & Josephine & 2005 & DEQ/OHA SWA 2000 - 2005 \\
\hline $\begin{array}{l}\text { DWP - PCS - GAFFNEY WAY } \\
\text { CROSSING MAIN CANAL } \\
\end{array}$ & 1378 & $\begin{array}{l}\text { M22 Type; P - Transportation - Stream } \\
\text { Crossing - Perennial }\end{array}$ & $\begin{array}{l}\text { GAFFNEY WAY CROSSING MAIN } \\
\text { CANAL }\end{array}$ & $\begin{array}{l}\text { AT SOUTHEAST EDGE OF } \\
15 \text { YR TOT }\end{array}$ & $\begin{array}{l}\text { Grants } \\
\text { Pass }\end{array}$ & Josephine & 2005 & DEQ/OHA SWA 2000 - 2005 \\
\hline $\begin{array}{l}\text { DWP - PCS - GAFFNEY WAY } \\
\text { CROSSING MAIN CANAL }\end{array}$ & 1378 & $\begin{array}{l}\text { M22 Type; P - Transportation - Stream } \\
\text { Crossing - Perennial }\end{array}$ & $\begin{array}{l}\text { GAFFNEY WAY CROSSING MAIN } \\
\text { CANAL }\end{array}$ & $\begin{array}{l}\text { AT SOUTHEAST EDGE OF } \\
15 \text { YR TOT }\end{array}$ & $\begin{array}{l}\text { Grants } \\
\text { Pass }\end{array}$ & Josephine & 2005 & DEQ/OHA SWA 2000 - 2005 \\
\hline $\begin{array}{l}\text { DWP - PCS - Gary Peterson } \\
\text { Excavating }\end{array}$ & 10411 & C06 Type; P - Cement/Concrete Plants & Gary Peterson Excavating & 910 SE M Street & $\begin{array}{l}\text { Grants } \\
\text { Pass }\end{array}$ & Josephine & 2005 & DEQ/OHA SWA 2000 - 2005 \\
\hline
\end{tabular}




\begin{tabular}{|c|c|c|c|c|c|c|c|c|}
\hline Database Identifier (DB_ID) & Site Identifier & Status & Common Name & Address & City & County & \begin{tabular}{|l} 
Retrieval \\
Date
\end{tabular} & Data Source \\
\hline $\begin{array}{l}\text { DWP - PCS - Grants Pass } \\
\text { Irrigation District }\end{array}$ & 10436 & \begin{tabular}{|l|} 
M26 Type; P - UST - Confirmed Leaking \\
but listed as NFA - DEQ LUST List
\end{tabular} & Grants Pass Irrigation District & 200 Fruitdale Drive & \begin{tabular}{|l} 
Grants \\
Pass
\end{tabular} & Josephine & 2005 & DEQ/OHA SWA 2000 - 2005 \\
\hline $\begin{array}{l}\text { DWP - PCS - Grants Pass } \\
\text { Irrigation District }\end{array}$ & 10436 & \begin{tabular}{|l|} 
M29 Type; P - Upstream \\
Reservoirs/Dams
\end{tabular} & Grants Pass Irrigation District & 200 Fruitdale Drive & \begin{tabular}{|l|} 
Grants \\
Pass
\end{tabular} & Josephine & 2005 & DEQ/OHA SWA $2000-2005$ \\
\hline $\begin{array}{l}\text { DWP - PCS - Grants Pass } \\
\text { Parkway }\end{array}$ & 10404 & $\begin{array}{l}\text { M56 Type; A - Transportation Corridors - } \\
\text { High Use Roads/Streets }\end{array}$ & Grants Pass Parkway & Runs east of intake & \begin{tabular}{|l|} 
Grants \\
Pass
\end{tabular} & Josephine & 2005 & DEQ/OHA SWA $2000-2005$ \\
\hline $\begin{array}{l}\text { DWP - PCS - Grants Pass Water } \\
\text { Treatment Plant }\end{array}$ & 10402 & $\begin{array}{l}\text { R05 Type; P - Drinking Water Treatment } \\
\text { Plants }\end{array}$ & Grants Pass Water Treatment Plant & Next to intake & \begin{tabular}{|l|} 
Grants \\
Pass
\end{tabular} & Josephine & 2005 & DEQ/OHA SWA $2000-2005$ \\
\hline DWP - PCS - Grazing Animals & 10021 & $\begin{array}{l}\text { A07 Type; A - Grazing Animals (> } 5 \text { large } \\
\text { animals or equivalent/acre) }\end{array}$ & Grazing Animals & Throughout DWPA & $\begin{array}{l}\text { Rogue } \\
\text { River }\end{array}$ & Jackson & 2005 & DEQ/OHA SWA $2000-2005$ \\
\hline DWP - PCS - Grazing Animals & 10440 & $\begin{array}{l}\text { A05 Type; A - Crops - Nonirrigated (inc. } \\
\text { Christmas trees, grains, grass seed, } \\
\text { pasture) }\end{array}$ & Grazing Animals & Upper River Road & $\begin{array}{l}\text { Grants } \\
\text { Pass }\end{array}$ & Josephine & 2005 & DEQ/OHA SWA $2000-2005$ \\
\hline DWP - PCS - Grazing Animals & 10440 & $\begin{array}{l}\text { A07 Type; A - Grazing Animals (> } 5 \text { large } \\
\text { animals or equivalent/acre) }\end{array}$ & Grazing Animals & Upper River Road & $\begin{array}{l}\text { Grants } \\
\text { Pass }\end{array}$ & Josephine & 2005 & DEQ/OHA SWA $2000-2005$ \\
\hline DWP - PCS - HAMILTON LANE & 790 & $\begin{array}{l}\text { M56 Type; A - Transportation Corridors - } \\
\text { High Use Roads/Streets }\end{array}$ & HAMILTON LANE & $\begin{array}{l}\text { IMMEDIATELY EAST OF } \\
\text { SCHOOL RUNNING } \\
\text { NORTH/SOUTH }\end{array}$ & $\begin{array}{l}\text { Grants } \\
\text { Pass }\end{array}$ & Josephine & 2005 & DEQ/OHA SWA $2000-2005$ \\
\hline $\begin{array}{l}\text { DWP - PCS - Have a Nice Day } \\
\text { Campground }\end{array}$ & 12317 & R03 Type; A - Campgrounds/RV Parks & Have a Nice Day Campground & East of Well on Hwy 99 & $\begin{array}{l}\text { Rogue } \\
\text { River }\end{array}$ & Jackson & 2005 & DEQ/OHA SWA $2000-2005$ \\
\hline $\begin{array}{l}\text { DWP - PCS - HIGH DENSITY } \\
\text { HOUSING }\end{array}$ & 1088 & $\begin{array}{l}\text { R09 Type; } A \text { - Housing - High Density (> } \\
1 \text { House/0.5 acres) }\end{array}$ & HIGH DENSITY HOUSING & $\begin{array}{l}\text { NORTHWEST OF HIGHWAY } \\
\text { AND CLARA }\end{array}$ & $\begin{array}{l}\text { Grants } \\
\text { Pass }\end{array}$ & Josephine & 2005 & DEQ/OHA SWA $2000-2005$ \\
\hline $\begin{array}{l}\text { DWP - PCS - HIGH DENSITY } \\
\text { HOUSING }\end{array}$ & 1088 & $\begin{array}{l}\text { R09 Type; A - Housing - High Density (> } \\
1 \text { House/0.5 acres) }\end{array}$ & HIGH DENSITY HOUSING & $\begin{array}{l}\text { NORTHWEST OF HIGHWAY } \\
\text { AND CLARA }\end{array}$ & $\begin{array}{l}\text { Grants } \\
\text { Pass }\end{array}$ & Josephine & 2005 & DEQ/OHA SWA $2000-2005$ \\
\hline $\begin{array}{l}\text { DWP - PCS - High Density } \\
\text { Housing }\end{array}$ & 10005 & $\begin{array}{l}\text { R09 Type; A - Housing - High Density (> } \\
1 \text { House/0.5 acres) }\end{array}$ & High Density Housing & Within Rogue River/Gold Hill & $\begin{array}{l}\text { Rogue } \\
\text { River }\end{array}$ & Jackson & 2005 & DEQ/OHA SWA $2000-2005$ \\
\hline $\begin{array}{l}\text { DWP - PCS - High Density } \\
\text { Housing }\end{array}$ & 10005 & $\begin{array}{l}\text { R09 Type; A - Housing - High Density (> } \\
1 \text { House/0.5 acres) }\end{array}$ & High Density Housing & Within Rogue River/Gold Hill & $\begin{array}{l}\text { Rogue } \\
\text { River }\end{array}$ & Jackson & 2005 & DEQ/OHA SWA 2000 - 2005 \\
\hline $\begin{array}{l}\text { DWP - PCS - High Density } \\
\text { Housing }\end{array}$ & 10005 & $\begin{array}{l}\text { R09 Type; A - Housing - High Density (> } \\
1 \text { House/0.5 acres) }\end{array}$ & High Density Housing & Within Rogue River/Gold Hill & $\begin{array}{l}\text { Rogue } \\
\text { River }\end{array}$ & Jackson & 2005 & DEQ/OHA SWA $2000-2005$ \\
\hline $\begin{array}{l}\text { DWP - PCS - High Density } \\
\text { Housing }\end{array}$ & 10005 & $\begin{array}{l}\text { R09 Type; A - Housing - High Density (> } \\
1 \text { House/0.5 acres) }\end{array}$ & High Density Housing & Within Rogue River/Gold Hill & $\begin{array}{l}\text { Rogue } \\
\text { River }\end{array}$ & Jackson & 2005 & DEQ/OHA SWA $2000-2005$ \\
\hline $\begin{array}{l}\text { DWP - PCS - High Density } \\
\text { Housing }\end{array}$ & 10005 & $\begin{array}{l}\text { R09 Type; A - Housing - High Density (> } \\
1 \text { House/0.5 acres) }\end{array}$ & High Density Housing & Within Rogue River/Gold Hill & $\begin{array}{l}\text { Rogue } \\
\text { River }\end{array}$ & Jackson & 2005 & DEQ/OHA SWA 2000 - 2005 \\
\hline $\begin{array}{l}\text { DWP - PCS - High Density } \\
\text { Housing }\end{array}$ & 10406 & $\begin{array}{l}\text { R09 Type; A - Housing - High Density (> } \\
1 \text { House/0.5 acres) }\end{array}$ & High Density Housing & Throughout DWPA & \begin{tabular}{|l|} 
Grants \\
Pass
\end{tabular} & Josephine & 2005 & DEQ/OHA SWA $2000-2005$ \\
\hline $\begin{array}{l}\text { DWP - PCS - High Density } \\
\text { Housing }\end{array}$ & 12316 & $\begin{array}{l}\text { R09 Type; A - Housing - High Density (> } \\
1 \text { House/0.5 acres) }\end{array}$ & High Density Housing & $\begin{array}{l}\text { Along Rogue River and east } \\
\text { of well }\end{array}$ & \begin{tabular}{|l|} 
Rogue \\
River
\end{tabular} & Jackson & 2005 & DEQ/OHA SWA 2000 - 2005 \\
\hline $\begin{array}{l}\text { DWP - PCS - High Density } \\
\text { Housing }\end{array}$ & 12318 & $\begin{array}{l}\text { R09 Type; A - Housing - High Density (> } \\
1 \text { House/0.5 acres) }\end{array}$ & High Density Housing & Throughout DWPA & Medford & Josephine & 2005 & DEQ/OHA SWA $2000-2005$ \\
\hline $\begin{array}{l}\text { DWP - PCS - High Density } \\
\text { Housing }\end{array}$ & 14239 & $\begin{array}{l}\text { R09 Type; A - Housing - High Density (> } \\
1 \text { House/0.5 acres) }\end{array}$ & High Density Housing & Throughout DWPA & $\begin{array}{l}\text { Grants } \\
\text { Pass }\end{array}$ & Josephine & 2005 & DEQ/OHA SWA $2000-2005$ \\
\hline $\begin{array}{l}\text { DWP - PCS - High Density } \\
\text { Housing }\end{array}$ & 14774 & $\begin{array}{l}\text { R09 Type; A - Housing - High Density (> } \\
1 \text { House/0.5 acres) }\end{array}$ & High Density Housing & Throughout DWPA & $\begin{array}{l}\text { Grants } \\
\text { Pass }\end{array}$ & Josephine & 2005 & DEQ/OHA SWA $2000-2005$ \\
\hline $\begin{array}{l}\text { DWP - PCS - High Density } \\
\text { Housing }\end{array}$ & 14774 & $\begin{array}{l}\text { R09 Type; A - Housing - High Density (> } \\
1 \text { House/0.5 acres) }\end{array}$ & High Density Housing & Throughout DWPA & \begin{tabular}{|l} 
Grants \\
Pass
\end{tabular} & Josephine & 2005 & DEQ/OHA SWA $2000-2005$ \\
\hline $\begin{array}{l}\text { DWP - PCS - High Density } \\
\text { Housing }\end{array}$ & 14774 & $\begin{array}{l}\text { R09 Type; A - Housing - High Density (> } \\
1 \text { House/0.5 acres) }\end{array}$ & High Density Housing & Throughout DWPA & \begin{tabular}{|l|} 
Grants \\
Pass
\end{tabular} & Josephine & 2005 & DEQ/OHA SWA $2000-2005$ \\
\hline $\begin{array}{l}\text { DWP - PCS - High Density } \\
\text { Housing }\end{array}$ & 14774 & $\begin{array}{l}\text { R09 Type; A - Housing - High Density (> } \\
1 \text { House/0.5 acres) }\end{array}$ & High Density Housing & Throughout DWPA & $\begin{array}{l}\text { Grants } \\
\text { Pass }\end{array}$ & Josephine & 2005 & DEQ/OHA SWA $2000-2005$ \\
\hline $\begin{array}{l}\text { DWP - PCS - High Density } \\
\text { Housing Areas }\end{array}$ & 14896 & $\begin{array}{l}\text { R09 Type; } A \text { - Housing - High Density (> } \\
1 \text { House/0.5 acres) }\end{array}$ & High Density Housing Areas & Within City of Rogue River & $\begin{array}{l}\text { Rogue } \\
\text { River }\end{array}$ & Jackson & 2005 & DEQ/OHA SWA $2000-2005$ \\
\hline $\begin{array}{l}\text { DWP - PCS - HIGH DENSITY } \\
\text { HOUSING EAST OF SCHOOL }\end{array}$ & 775 & $\begin{array}{l}\text { R09 Type; A - Housing - High Density (> } \\
1 \text { House/0.5 acres) }\end{array}$ & $\begin{array}{l}\text { HIGH DENSITY HOUSING EAST } \\
\text { OF SCHOOL }\end{array}$ & $\begin{array}{l}\text { EAST OF SCHOOL/EAST } \\
\text { SIDE OF HAMILTON LANE }\end{array}$ & $\begin{array}{l}\text { Grants } \\
\text { Pass }\end{array}$ & Josephine & 2005 & DEQ/OHA SWA $2000-2005$ \\
\hline
\end{tabular}




\begin{tabular}{|c|c|c|c|c|c|c|c|c|}
\hline Database Identifier (DB_ID) & Site Identifier & Status & Common Name & Address & City & County & \begin{tabular}{|l} 
Retrieval \\
Date
\end{tabular} & Data Source \\
\hline \begin{tabular}{l|} 
DWP - PCS - HIGH DENSITY \\
HOUSING NORTH OF SCHOOL
\end{tabular} & 776 & $\begin{array}{l}\text { R09 Type; A - Housing - High Density (> } \\
1 \text { House/0.5 acres) }\end{array}$ & $\begin{array}{l}\text { HIGH DENSITY HOUSING NORTH } \\
\text { OF SCHOOL }\end{array}$ & $\begin{array}{l}\text { NORTH OF } \\
\text { SCHOOL/NORTH OF } \\
\text { EASEMENT }\end{array}$ & $\begin{array}{l}\text { Grants } \\
\text { Pass }\end{array}$ & Josephine & 2005 & DEQ/OHA SWA 2000 - 2005 \\
\hline $\begin{array}{l}\text { DWP - PCS - HIGH DENSITY } \\
\text { HOUSING SOUTH OF SCHOOL }\end{array}$ & 784 & $\begin{array}{l}\text { R09 Type; A - Housing - High Density (> } \\
1 \text { House/0.5 acres) }\end{array}$ & $\begin{array}{l}\text { HIGH DENSITY HOUSING SOUTH } \\
\text { OF SCHOOL }\end{array}$ & $\begin{array}{l}\text { SOUTH OF SCHOOL ON } \\
\text { WEST SIDE OF HAMILTON } \\
\text { LANE }\end{array}$ & $\begin{array}{l}\text { Grants } \\
\text { Pass }\end{array}$ & Josephine & 2005 & DEQ/OHA SWA $2000-2005$ \\
\hline $\begin{array}{l}\text { DWP - PCS - HIGH DENSITY } \\
\text { HOUSING SOUTH OF TRAILER } \\
\text { PARK }\end{array}$ & 1084 & $\begin{array}{l}\text { R09 Type; A - Housing - High Density (> } \\
1 \text { House/0.5 acres) }\end{array}$ & $\begin{array}{l}\text { HIGH DENSITY HOUSING SOUTH } \\
\text { OF TRAILER PARK }\end{array}$ & $\begin{array}{l}\text { WEST OF CLOVERLAWN } \\
\text { AND SOUTH OF TRAILER } \\
\text { PARK }\end{array}$ & $\begin{array}{l}\text { Grants } \\
\text { Pass }\end{array}$ & Josephine & 2005 & DEQ/OHA SWA $2000-2005$ \\
\hline $\begin{array}{l}\text { DWP - PCS - HIGH DENSITY } \\
\text { HOUSING SOUTH OF TRAILER } \\
\text { PARK }\end{array}$ & 1084 & $\begin{array}{l}\text { R09 Type; A - Housing - High Density (> } \\
1 \text { House/0.5 acres) }\end{array}$ & $\begin{array}{l}\text { HIGH DENSITY HOUSING SOUTH } \\
\text { OF TRAILER PARK }\end{array}$ & $\begin{array}{l}\text { WEST OF CLOVERLAWN } \\
\text { AND SOUTH OF TRAILER } \\
\text { PARK }\end{array}$ & \begin{tabular}{|l} 
Grants \\
Pass
\end{tabular} & Josephine & 2005 & DEQ/OHA SWA $2000-2005$ \\
\hline \begin{tabular}{|l|} 
DWP - PCS - High Density Septic \\
Systems
\end{tabular} & 9366 & $\begin{array}{l}\text { R16 Type; A - Septic Systems - High } \\
\text { Density ( > } 1 \text { system/acre) }\end{array}$ & High Density Septic Systems & Throughout DWPA & $\begin{array}{l}\text { Grants } \\
\text { Pass }\end{array}$ & Josephine & 2005 & DEQ/OHA SWA $2000-2005$ \\
\hline DWP - PCS - HIGHWAY 99 & 1381 & $\begin{array}{l}\text { M18 Type; A - Transportation - } \\
\text { Freeways/State Highways }\end{array}$ & HIGHWAY 99 & $\begin{array}{l}\text { RUNS FROM EAST TO } \\
\text { WEST THROUGH } 15 \text { YR } \\
\text { TOT }\end{array}$ & $\begin{array}{l}\text { Grants } \\
\text { Pass }\end{array}$ & Josephine & 2005 & DEQ/OHA SWA 2000 - 2005 \\
\hline DWP - PCS - HIGHWAY 99 & 1381 & $\begin{array}{l}\text { M18 Type; A - Transportation - } \\
\text { Freeways/State Highways }\end{array}$ & HIGHWAY 99 & $\begin{array}{l}\text { RUNS FROM EAST TO } \\
\text { WEST THROUGH } 15 \text { YR } \\
\text { TOT }\end{array}$ & $\begin{array}{l}\text { Grants } \\
\text { Pass }\end{array}$ & Josephine & 2005 & DEQ/OHA SWA $2000-2005$ \\
\hline DWP - PCS - Highway 99 & 9372 & $\begin{array}{l}\text { M18 Type; A - Transportation - } \\
\text { Freeways/State Highways }\end{array}$ & Highway 99 & North of well & $\begin{array}{l}\text { Grants } \\
\text { Pass }\end{array}$ & Josephine & 2005 & DEQ/OHA SWA 2000 - 2005 \\
\hline DWP - PCS - Highway 99 & 9374 & $\begin{array}{l}\text { M18 Type; A - Transportation - } \\
\text { Freeways/State Highways }\end{array}$ & Highway 99 & North of well & $\begin{array}{l}\text { Grants } \\
\text { Pass }\end{array}$ & Josephine & 2005 & DEQ/OHA SWA $2000-2005$ \\
\hline DWP - PCS - Highway 99 & 10433 & $\begin{array}{l}\text { M18 Type; A - Transportation - } \\
\text { Freeways/State Highways }\end{array}$ & Highway 99 & Runs east/west along Rogue & $\begin{array}{l}\text { Grants } \\
\text { Pass }\end{array}$ & Josephine & 2005 & DEQ/OHA SWA $2000-2005$ \\
\hline DWP - PCS - Highway 99 & 14240 & $\begin{array}{l}\text { M18 Type; A - Transportation - } \\
\text { Freeways/State Highways }\end{array}$ & Highway 99 & Runs Through DWPA & $\begin{array}{l}\text { Grants } \\
\text { Pass }\end{array}$ & Josephine & 2005 & DEQ/OHA SWA $2000-2005$ \\
\hline $\begin{array}{l}\text { DWP - PCS - Highway } 99 \text { (Rogue } \\
\text { River Hwy) }\end{array}$ & 10002 & $\begin{array}{l}\text { M18 Type; A - Transportation - } \\
\text { Freeways/State Highways }\end{array}$ & Highway 99 (Rogue River Hwy) & \begin{tabular}{|l} 
Runs along I-5 and Rogue \\
River
\end{tabular} & $\begin{array}{l}\text { Rogue } \\
\text { River }\end{array}$ & Jackson & 2005 & DEQ/OHA SWA 2000 - 2005 \\
\hline \begin{tabular}{l|l} 
DWP - PCS - Historic Gas \\
Station
\end{tabular} & 14837 & $\begin{array}{l}\text { M06 Type; P - Auto - Gas Stations - } \\
\text { Historic }\end{array}$ & Historic Gas Station & W. Main Street & $\begin{array}{l}\text { Rogue } \\
\text { River }\end{array}$ & Jackson & 2005 & DEQ/OHA SWA $2000-2005$ \\
\hline $\begin{array}{l}\text { DWP - PCS - Historic Gas } \\
\text { Station }\end{array}$ & 14837 & $\begin{array}{l}\text { M06 Type; P - Auto - Gas Stations - } \\
\text { Historic }\end{array}$ & Historic Gas Station & W. Main Street & $\begin{array}{l}\text { Rogue } \\
\text { River }\end{array}$ & Jackson & 2005 & DEQ/OHA SWA $2000-2005$ \\
\hline $\begin{array}{l}\text { DWP - PCS - Historic Gas } \\
\text { Station }\end{array}$ & 14837 & $\begin{array}{l}\text { M06 Type; P - Auto - Gas Stations - } \\
\text { Historic }\end{array}$ & Historic Gas Station & W. Main Street & $\begin{array}{l}\text { Rogue } \\
\text { River }\end{array}$ & Jackson & 2005 & DEQ/OHA SWA $2000-2005$ \\
\hline $\begin{array}{l}\text { DWP - PCS - Historic Gas } \\
\text { Station }\end{array}$ & 14837 & $\begin{array}{l}\text { M06 Type; P - Auto - Gas Stations - } \\
\text { Historic }\end{array}$ & Historic Gas Station & W. Main Street & $\begin{array}{l}\text { Rogue } \\
\text { River }\end{array}$ & Jackson & 2005 & DEQ/OHA SWA 2000 - 2005 \\
\hline $\begin{array}{l}\text { DWP - PCS - Holiday Mobile } \\
\text { Manor }\end{array}$ & 9373 & $\begin{array}{l}\text { M09 Type; A - Homesteads - Rural - } \\
\text { Septic Systems (< 1/acre) }\end{array}$ & Holiday Mobile Manor & Surrounding Well & $\begin{array}{l}\text { Grants } \\
\text { Pass }\end{array}$ & Josephine & 2005 & DEQ/OHA SWA $2000-2005$ \\
\hline $\begin{array}{l}\text { DWP - PCS - Holiday Mobile } \\
\text { Manor }\end{array}$ & 9373 & $\begin{array}{l}\text { M31 Type; A - Large Capacity Septic } \\
\text { Systems (serves }>20 \text { people) - Class V } \\
\text { UICs }\end{array}$ & Holiday Mobile Manor & Surrounding Well & $\begin{array}{l}\text { Grants } \\
\text { Pass }\end{array}$ & Josephine & 2005 & DEQ/OHA SWA $2000-2005$ \\
\hline DWP - PCS - Housing & 12336 & $\begin{array}{l}\text { R09 Type; A - Housing - High Density (> } \\
1 \text { House/0.5 acres) }\end{array}$ & Housing & $\begin{array}{l}\text { Roslington Lane and } \\
\text { throughout DWPA }\end{array}$ & $\begin{array}{l}\text { Grants } \\
\text { Pass }\end{array}$ & Josephine & 2005 & DEQ/OHA SWA 2000 - 2005 \\
\hline DWP - PCS - Housing & 12336 & $\begin{array}{l}\text { R09 Type; A - Housing - High Density (> } \\
1 \text { House/0.5 acres) }\end{array}$ & Housing & $\begin{array}{l}\text { Roslington Lane and } \\
\text { throughout DWPA }\end{array}$ & $\begin{array}{l}\text { Grants } \\
\text { Pass }\end{array}$ & Josephine & 2005 & DEQ/OHA SWA $2000-2005$ \\
\hline DWP - PCS - Hwy 99 & 12313 & $\begin{array}{l}\text { M18 Type; A - Transportation - } \\
\text { Freeways/State Highways }\end{array}$ & Hwy 99 & Runs Through DWPA & $\begin{array}{l}\text { Rogue } \\
\text { River }\end{array}$ & Jackson & 2005 & DEQ/OHA SWA 2000 - 2005 \\
\hline $\begin{array}{l}\text { DWP - PCS - Hwy } 99 \text { Industrial } \\
\text { Companies }\end{array}$ & 10434 & $\begin{array}{l}\text { C65 Type; A - Miscellaneous } \\
\text { Manufacturing - PCS along Hwy } 99 \text { East }\end{array}$ & Hwy 99 Industrial Companies & Along Highway 99 & $\begin{array}{l}\text { Grants } \\
\text { Pass }\end{array}$ & Josephine & 2005 & DEQ/OHA SWA $2000-2005$ \\
\hline DWP - PCS - I-5 & 12309 & $\begin{array}{l}\text { M18 Type; A - Transportation - } \\
\text { Freeways/State Highways }\end{array}$ & $1-5$ & South of Well & $\begin{array}{l}\text { Rogue } \\
\text { River }\end{array}$ & Jackson & 2005 & DEQ/OHA SWA $2000-2005$ \\
\hline
\end{tabular}




\begin{tabular}{|c|c|c|c|c|c|c|c|c|}
\hline Database Identifier (DB_ID) & Site Identifier & Status & Common Name & Address & City & County & \begin{tabular}{|l|} 
Retrieval \\
Date
\end{tabular} & Data Source \\
\hline DWP - PCS - Interstate 5 & 10001 & $\begin{array}{l}\text { M18 Type; A - Transportation - } \\
\text { Freeways/State Highways }\end{array}$ & Interstate 5 & Runs along the Rogue River & \begin{tabular}{|l} 
Rogue \\
River
\end{tabular} & Jackson & 2005 & DEQ/OHA SWA 2000 - 2005 \\
\hline DWP - PCS - Interstate 5 & 10001 & $\begin{array}{l}\text { M18 Type; A - Transportation - } \\
\text { Freeways/State Highways }\end{array}$ & Interstate 5 & Runs along the Rogue River & $\begin{array}{l}\text { Rogue } \\
\text { River }\end{array}$ & Jackson & 2005 & DEQ/OHA SWA 2000 - 2005 \\
\hline DWP - PCS - Interstate 5 & 10001 & $\begin{array}{l}\text { M18 Type; A - Transportation - } \\
\text { Freeways/State Highways }\end{array}$ & Interstate 5 & Runs along the Rogue River & \begin{tabular}{|l|} 
Rogue \\
River
\end{tabular} & Jackson & 2005 & DEQ/OHA SWA 2000 - 2005 \\
\hline DWP - PCS - Interstate 5 & 10001 & $\begin{array}{l}\text { M18 Type; A - Transportation - } \\
\text { Freeways/State Highways }\end{array}$ & Interstate 5 & Runs along the Rogue River & \begin{tabular}{|l|} 
Rogue \\
River
\end{tabular} & Jackson & 2005 & DEQ/OHA SWA 2000 - 2005 \\
\hline DWP - PCS - Interstate 5 & 10001 & $\begin{array}{l}\text { M18 Type; A - Transportation - } \\
\text { Freeways/State Highways }\end{array}$ & Interstate 5 & Runs along the Rogue River & \begin{tabular}{|l|} 
Rogue \\
River
\end{tabular} & Jackson & 2005 & DEQ/OHA SWA 2000 - 2005 \\
\hline DWP - PCS - Interstate 5 & 10438 & \begin{tabular}{|l|} 
M18 Type; A - Transportation - \\
Freeways/State Highways
\end{tabular} & Interstate 5 & East of intake & $\begin{array}{l}\text { Grants } \\
\text { Pass }\end{array}$ & Josephine & 2005 & DEQ/OHA SWA 2000 - 2005 \\
\hline DWP - PCS - Irrigated Crops & 10018 & $\begin{array}{l}\text { A04 Type; A - Crops - Irrigated (inc. } \\
\text { orchards, vineyards, nurseries, } \\
\text { greenhouses) }\end{array}$ & Irrigated Crops & Throughout DWPA & $\begin{array}{l}\text { Rogue } \\
\text { River }\end{array}$ & Jackson & 2005 & DEQ/OHA SWA 2000 - 2005 \\
\hline DWP - PCS - Irrigated Crops & 10441 & $\begin{array}{l}\text { A04 Type; A - Crops - Irrigated (inc. } \\
\text { orchards, vineyards, nurseries, } \\
\text { greenhouses) }\end{array}$ & Irrigated Crops & Upper River Road & $\begin{array}{l}\text { Grants } \\
\text { Pass }\end{array}$ & Josephine & 2005 & DEQ/OHA SWA 2000 - 2005 \\
\hline DWP - PCS - Irrigation Canal & 9367 & A52 Type; A - Irrigation Canal/Pond & Irrigation Canal & Runs N-S through DWPA & \begin{tabular}{|l} 
Grants \\
Pass
\end{tabular} & Josephine & 2005 & DEQ/OHA SWA 2000 - 2005 \\
\hline DWP - PCS - Irrigation Canal & 9371 & A52 Type; A - Irrigation Canal/Pond & Irrigation Canal & North of Well & $\begin{array}{l}\text { Grants } \\
\text { Pass }\end{array}$ & Josephine & 2005 & DEQ/OHA SWA 2000 - 2005 \\
\hline DWP - PCS - Irrigation Canal & 9375 & A52 Type; A - Irrigation Canal/Pond & Irrigation Canal & North of well & $\begin{array}{l}\text { Grants } \\
\text { Pass }\end{array}$ & Josephine & 2005 & DEQ/OHA SWA 2000 - 2005 \\
\hline DWP - PCS - Irrigation Canal & 10022 & A52 Type; A - Irrigation Canal/Pond & Irrigation Canal & Northeast/Southeast of intake & $\begin{array}{l}\text { Rogue } \\
\text { River }\end{array}$ & Jackson & 2005 & DEQ/OHA SWA 2000 - 2005 \\
\hline DWP - PCS - Irrigation Canal & 12308 & A52 Type; A - Irrigation Canal/Pond & Irrigation Canal & North of Well & \begin{tabular}{|l|} 
Rogue \\
River
\end{tabular} & Jackson & 2005 & DEQ/OHA SWA 2000 - 2005 \\
\hline DWP - PCS - Irrigation Ditch & 14773 & A52 Type; A - Irrigation Canal/Pond & Irrigation Ditch & Runs Through DWPA & $\begin{array}{l}\text { Grants } \\
\text { Pass }\end{array}$ & Josephine & 2005 & DEQ/OHA SWA 2000 - 2005 \\
\hline DWP - PCS - Irrigation Ditch & 14773 & A52 Type; A - Irrigation Canal/Pond & Irrigation Ditch & Runs Through DWPA & $\begin{array}{l}\text { Grants } \\
\text { Pass }\end{array}$ & Josephine & 2005 & DEQ/OHA SWA 2000 - 2005 \\
\hline DWP - PCS - Irrigation Ditch & 14773 & A52 Type; A - Irrigation Canal/Pond & Irrigation Ditch & Runs Through DWPA & $\begin{array}{l}\text { Grants } \\
\text { Pass }\end{array}$ & Josephine & 2005 & DEQ/OHA SWA 2000 - 2005 \\
\hline DWP - PCS - Irrigation Ditch & 14773 & A52 Type; A - Irrigation Canal/Pond & Irrigation Ditch & Runs Through DWPA & $\begin{array}{l}\text { Grants } \\
\text { Pass }\end{array}$ & Josephine & 2005 & DEQ/OHA SWA 2000 - 2005 \\
\hline $\begin{array}{l}\text { DWP - PCS - JERRY'S } \\
\text { APPLIANCE REPAIR }\end{array}$ & 1391 & C15 Type; P - Machine Shops & JERRY'S APPLIANCE REPAIR & 1077 ROGUE RIVER HWY & $\begin{array}{l}\text { Grants } \\
\text { Pass }\end{array}$ & Josephine & 2005 & DEQ/OHA SWA 2000 - 2005 \\
\hline $\begin{array}{l}\text { DWP - PCS - JERRY'S } \\
\text { APPLIANCE REPAIR } \\
\end{array}$ & 1391 & C15 Type; P - Machine Shops & JERRY'S APPLIANCE REPAIR & 1077 ROGUE RIVER HWY & $\begin{array}{l}\text { Grants } \\
\text { Pass }\end{array}$ & Josephine & 2005 & DEQ/OHA SWA 2000 - 2005 \\
\hline $\begin{array}{l}\text { DWP - PCS - JOSEPHINE } \\
\text { COUNTY SCHOOL }\end{array}$ & 1384 & R15 Type; P - Schools & JOSEPHINE COUNTY SCHOOL & $\begin{array}{l}\text { NORTHEAST CORNER OF } \\
\text { FRUITDALE AND } \\
\text { PARKDALE }\end{array}$ & $\begin{array}{l}\text { Grants } \\
\text { Pass }\end{array}$ & Josephine & 2005 & DEQ/OHA SWA 2000 - 2005 \\
\hline $\begin{array}{l}\text { DWP - PCS - JOSEPHINE } \\
\text { COUNTY SCHOOL }\end{array}$ & 1384 & R15 Type; P - Schools & JOSEPHINE COUNTY SCHOOL & $\begin{array}{l}\text { NORTHEAST CORNER OF } \\
\text { FRUITDALE AND } \\
\text { PARKDALE } \\
\end{array}$ & $\begin{array}{l}\text { Grants } \\
\text { Pass }\end{array}$ & Josephine & 2005 & DEQ/OHA SWA $2000-2005$ \\
\hline $\begin{array}{l}\text { DWP - PCS - JOSE'S } \\
\text { AUTOBODY }\end{array}$ & 1390 & C01 Type; P - Automobiles - Body Shops & JOSE'S AUTOBODY & $\begin{array}{l}\text { SOUTH SIDE OF ROGUE } \\
\text { RIVER HWY/JUST EAST OF } \\
\text { JUNCTION WITH OAKHILL } \\
\text { LANE }\end{array}$ & $\begin{array}{l}\text { Grants } \\
\text { Pass }\end{array}$ & Josephine & 2005 & DEQ/OHA SWA 2000 - 2005 \\
\hline $\begin{array}{l}\text { DWP - PCS - JOSE'S } \\
\text { AUTOBODY }\end{array}$ & 1390 & C01 Type; P - Automobiles - Body Shops & JOSE'S AUTOBODY & $\begin{array}{l}\text { SOUTH SIDE OF ROGUE } \\
\text { RIVER HWY/JUST EAST OF } \\
\text { JUNCTION WITH OAKHILL } \\
\text { LANE }\end{array}$ & $\begin{array}{l}\text { Grants } \\
\text { Pass }\end{array}$ & Josephine & 2005 & DEQ/OHA SWA $2000-2005$ \\
\hline
\end{tabular}




\begin{tabular}{|c|c|c|c|c|c|c|c|c|}
\hline Database Identifier (DB_ID) & Site Identifier & Status & Common Name & Address & City & County & \begin{tabular}{|l|} 
Retrieval \\
Date
\end{tabular} & Data Source \\
\hline $\begin{array}{l}\text { DWP - PCS - Kens Auto Body \& } \\
\text { Paint }\end{array}$ & 10008 & C01 Type; P - Automobiles - Body Shops & Kens Auto Body \& Paint & Off Gilmore Street & $\begin{array}{l}\text { Rogue } \\
\text { River }\end{array}$ & Jackson & 2005 & DEQ/OHA SWA 2000 - 2005 \\
\hline $\begin{array}{l}\text { DWP - PCS - Kens Auto Body \& } \\
\text { Paint }\end{array}$ & 10008 & C01 Type; P - Automobiles - Body Shops & Kens Auto Body \& Paint & Off Gilmore Street & $\begin{array}{l}\text { Rogue } \\
\text { River }\end{array}$ & Jackson & 2005 & DEQ/OHA SWA 2000 - 2005 \\
\hline $\begin{array}{l}\text { DWP - PCS - Kens Auto Body \& } \\
\text { Paint }\end{array}$ & 10008 & C01 Type; P - Automobiles - Body Shops & Kens Auto Body \& Paint & Off Gilmore Street & $\begin{array}{l}\text { Rogue } \\
\text { River }\end{array}$ & Jackson & 2005 & DEQ/OHA SWA 2000 - 2005 \\
\hline $\begin{array}{l}\text { DWP - PCS - Kens Auto Body \& } \\
\text { Paint }\end{array}$ & 10008 & C01 Type; P - Automobiles - Body Shops & Kens Auto Body \& Paint & Off Gilmore Street & $\begin{array}{l}\text { Rogue } \\
\text { River }\end{array}$ & Jackson & 2005 & DEQ/OHA SWA 2000 - 2005 \\
\hline $\begin{array}{l}\text { DWP - PCS - Kens Auto Body \& } \\
\text { Paint }\end{array}$ & 10008 & C01 Type; P - Automobiles - Body Shops & Kens Auto Body \& Paint & Off Gilmore Street & \begin{tabular}{|l|} 
Rogue \\
River
\end{tabular} & Jackson & 2005 & DEQ/OHA SWA 2000 - 2005 \\
\hline DWP - PCS - Laidlaw Bus Barn & 10431 & \begin{tabular}{|l|} 
C10 Type; $\mathrm{P}$ - Fleet/Trucking/Bus \\
Terminals
\end{tabular} & Laidlaw Bus Barn & Gladiola Ave & $\begin{array}{l}\text { Grants } \\
\text { Pass }\end{array}$ & Josephine & 2005 & DEQ/OHA SWA 2000 - 2005 \\
\hline DWP - PCS - Laidlaw Bus Barn & 10431 & $\begin{array}{l}\text { M01 Type; P - Above Ground Storage } \\
\text { Tanks - Excluding Water and Residential } \\
\text { ASTs }\end{array}$ & Laidlaw Bus Barn & Gladiola Ave & $\begin{array}{l}\text { Grants } \\
\text { Pass }\end{array}$ & Josephine & 2005 & DEQ/OHA SWA 2000 - 2005 \\
\hline DWP - PCS - Laidlaw Bus Barn & 10431 & \begin{tabular}{|l|} 
M27 Type; P - UST - \\
Upgraded/Registered - Active
\end{tabular} & Laidlaw Bus Barn & Gladiola Ave & $\begin{array}{l}\text { Grants } \\
\text { Pass }\end{array}$ & Josephine & 2005 & DEQ/OHA SWA 2000 - 2005 \\
\hline DWP - PCS - Laidlaw Transit Inc. & 10444 & $\begin{array}{l}\text { C10 Type; P - Fleet/Trucking/Bus } \\
\text { Terminals }\end{array}$ & Laidlaw Transit Inc. & Evans Creek & $\begin{array}{l}\text { Rogue } \\
\text { River }\end{array}$ & Josephine & 2005 & DEQ/OHA SWA 2000 - 2005 \\
\hline $\begin{array}{l}\text { DWP - PCS - Lindsay Auto } \\
\text { Repair }\end{array}$ & 14243 & \begin{tabular}{|l|} 
C04 Type; P - Automobiles - Repair \\
Shops
\end{tabular} & Lindsay Auto Repair & Highway 99 & $\begin{array}{l}\text { Grants } \\
\text { Pass }\end{array}$ & Josephine & 2005 & DEQ/OHA SWA 2000 - 2005 \\
\hline $\begin{array}{l}\text { DWP - PCS - LINDSAY } \\
\text { AUTOBODY }\end{array}$ & 1082 & C01 Type; P - Automobiles - Body Shops & LINDSAY AUTOBODY & $\begin{array}{l}\text { 1349 ROGUE RIVER } \\
\text { HWY/EAST OF TRAILER } \\
\text { PARK }\end{array}$ & $\begin{array}{l}\text { Grants } \\
\text { Pass }\end{array}$ & Josephine & 2005 & DEQ/OHA SWA 2000 - 2005 \\
\hline $\begin{array}{l}\text { DWP - PCS - LINDSAY } \\
\text { AUTOBODY }\end{array}$ & 1082 & C01 Type; P - Automobiles - Body Shops & LINDSAY AUTOBODY & $\begin{array}{l}\text { 1349 ROGUE RIVER } \\
\text { HWY/EAST OF TRAILER } \\
\text { PARK }\end{array}$ & $\begin{array}{l}\text { Grants } \\
\text { Pass }\end{array}$ & Josephine & 2005 & DEQ/OHA SWA 2000 - 2005 \\
\hline DWP - PCS - Louisiana Pacific & 10445 & $\begin{array}{l}\text { C26 Type; P - Wood/Pulp/Paper } \\
\text { Processing and Mills }\end{array}$ & Louisiana Pacific & Just outside DWPA & $\begin{array}{l}\text { Rogue } \\
\text { River }\end{array}$ & Josephine & 2005 & DEQ/OHA SWA 2000 - 2005 \\
\hline DWP - PCS - Louisiana Pacific & 10445 & \begin{tabular}{|l|} 
M01 Type; P - Above Ground Storage \\
Tanks - Excluding Water and Residential \\
ASTs
\end{tabular} & Louisiana Pacific & Just outside DWPA & \begin{tabular}{|l} 
Rogue \\
River
\end{tabular} & Josephine & 2005 & DEQ/OHA SWA 2000 - 2005 \\
\hline $\begin{array}{l}\text { DWP - PCS - Louisiana Pacific } \\
\text { Mill }\end{array}$ & 10011 & \begin{tabular}{|l|} 
C26 Type; P - Wood/Pulp/Paper \\
Processing and Mills
\end{tabular} & Louisiana Pacific Mill & East of intake & $\begin{array}{l}\text { Rogue } \\
\text { River }\end{array}$ & Jackson & 2005 & DEQ/OHA SWA 2000 - 2005 \\
\hline $\begin{array}{l}\text { DWP - PCS - Louisiana Pacific } \\
\text { Mill }\end{array}$ & 10011 & \begin{tabular}{|l|} 
M01 Type; P - Above Ground Storage \\
Tanks - Excluding Water and Residential \\
ASTs
\end{tabular} & Louisiana Pacific Mill & East of intake & $\begin{array}{l}\text { Rogue } \\
\text { River }\end{array}$ & Jackson & 2005 & DEQ/OHA SWA 2000 - 2005 \\
\hline \begin{tabular}{|l|} 
DWP - PCS - Managed \\
Forestlands-Harvest Units
\end{tabular} & 10016 & \begin{tabular}{|l|} 
A11 Type; A - Managed Forest Land - \\
Clearcut Harvest $(<35$ yrs.)
\end{tabular} & $\begin{array}{l}\text { Managed Forestlands-Harvest } \\
\text { Units }\end{array}$ & Wards Creek Drainage & $\begin{array}{l}\text { Rogue } \\
\text { River }\end{array}$ & Jackson & 2005 & DEQ/OHA SWA 2000 - 2005 \\
\hline $\begin{array}{l}\text { DWP - PCS - MIKE'S RADIATOR } \\
\text { REPAIR AND SERVICE }\end{array}$ & 1393 & $\begin{array}{l}\text { C04 Type; P - Automobiles - Repair } \\
\text { Shops }\end{array}$ & $\begin{array}{l}\text { MIKE'S RADIATOR REPAIR AND } \\
\text { SERVICE }\end{array}$ & 1077 ROGUE RIVER HWY & $\begin{array}{l}\text { Grants } \\
\text { Pass }\end{array}$ & Josephine & 2005 & DEQ/OHA SWA 2000 - 2005 \\
\hline $\begin{array}{l}\text { DWP - PCS - MIKE'S RADIATOR } \\
\text { REPAIR AND SERVICE }\end{array}$ & 1393 & $\begin{array}{l}\text { C04 Type; P - Automobiles - Repair } \\
\text { Shops }\end{array}$ & $\begin{array}{l}\text { MIKE'S RADIATOR REPAIR AND } \\
\text { SERVICE }\end{array}$ & 1077 ROGUE RIVER HWY & $\begin{array}{l}\text { Grants } \\
\text { Pass }\end{array}$ & Josephine & 2005 & DEQ/OHA SWA 2000 - 2005 \\
\hline $\begin{array}{l}\text { DWP - PCS - MILD TO WILD } \\
\text { SUZUKI SHOP }\end{array}$ & 1086 & $\begin{array}{l}\text { C04 Type; P - Automobiles - Repair } \\
\text { Shops }\end{array}$ & MILD TO WILD SUZUKI SHOP & $\begin{array}{l}\text { WEST OF TRAILER PARK } \\
\text { AND SOUTH OF HIGHWAY }\end{array}$ & $\begin{array}{l}\text { Grants } \\
\text { Pass }\end{array}$ & Josephine & 2005 & DEQ/OHA SWA 2000 - 2005 \\
\hline $\begin{array}{l}\text { DWP - PCS - MILD TO WILD } \\
\text { SUZUKI SHOP }\end{array}$ & 1086 & $\begin{array}{l}\text { C04 Type; P - Automobiles - Repair } \\
\text { Shops }\end{array}$ & MILD TO WILD SUZUKI SHOP & $\begin{array}{l}\text { WEST OF TRAILER PARK } \\
\text { AND SOUTH OF HIGHWAY }\end{array}$ & $\begin{array}{l}\text { Grants } \\
\text { Pass }\end{array}$ & Josephine & 2005 & DEQ/OHA SWA 2000 - 2005 \\
\hline DWP - PCS - Milo's Auto & 14835 & $\begin{array}{l}\text { C04 Type; P - Automobiles - Repair } \\
\text { Shops }\end{array}$ & Milo's Auto & Depot Street & $\begin{array}{l}\text { Rogue } \\
\text { River }\end{array}$ & Jackson & 2005 & DEQ/OHA SWA 2000 - 2005 \\
\hline
\end{tabular}




\begin{tabular}{|c|c|c|c|c|c|c|c|c|}
\hline Database Identifier (DB_ID) & Site Identifier & Status & Common Name & Address & City & County & $\begin{array}{l}\text { Retrieval } \\
\text { Date }\end{array}$ & Data Source \\
\hline DWP - PCS - Milo's Auto & 14835 & $\begin{array}{l}\text { C04 Type; P - Automobiles - Repair } \\
\text { Shops }\end{array}$ & Milo's Auto & Depot Street & \begin{tabular}{|l} 
Rogue \\
River
\end{tabular} & Jackson & 2005 & DEQ/OHA SWA 2000 - 2005 \\
\hline DWP - PCS - Milo's Auto & 14835 & $\begin{array}{l}\text { C04 Type; P - Automobiles - Repair } \\
\text { Shops }\end{array}$ & Milo's Auto & Depot Street & \begin{tabular}{|l|} 
Rogue \\
River
\end{tabular} & Jackson & 2005 & DEQ/OHA SWA $2000-2005$ \\
\hline DWP - PCS - Milo's Auto & 14835 & $\begin{array}{l}\text { C04 Type; P - Automobiles - Repair } \\
\text { Shops }\end{array}$ & Milo's Auto & Depot Street & \begin{tabular}{|l|} 
Rogue \\
River
\end{tabular} & Jackson & 2005 & DEQ/OHA SWA $2000-2005$ \\
\hline DWP - PCS - Morningstar Press & 14829 & C21 Type; P - Photo Processing/Printing & Morningstar Press & West Main Street & \begin{tabular}{|l|} 
Rogue \\
River
\end{tabular} & Jackson & 2005 & DEQ/OHA SWA 2000 - 2005 \\
\hline DWP - PCS - Morningstar Press & 14829 & C21 Type; P - Photo Processing/Printing & Morningstar Press & West Main Street & $\begin{array}{l}\text { Rogue } \\
\text { River }\end{array}$ & Jackson & 2005 & DEQ/OHA SWA $2000-2005$ \\
\hline DWP - PCS - Morningstar Press & 14829 & C21 Type; P - Photo Processing/Printing & Morningstar Press & West Main Street & \begin{tabular}{|l|} 
Rogue \\
River
\end{tabular} & Jackson & 2005 & DEQ/OHA SWA $2000-2005$ \\
\hline DWP - PCS - Morningstar Press & 14829 & C21 Type; P - Photo Processing/Printing & Morningstar Press & West Main Street & \begin{tabular}{|l|} 
Rogue \\
River
\end{tabular} & Jackson & 2005 & DEQ/OHA SWA $2000-2005$ \\
\hline DWP - PCS - Muffler King & 14247 & $\begin{array}{l}\text { C04 Type; P - Automobiles - Repair } \\
\text { Shops }\end{array}$ & Muffler King & Highway 99 & $\begin{array}{l}\text { Grants } \\
\text { Pass }\end{array}$ & Josephine & 2005 & DEQ/OHA SWA $2000-2005$ \\
\hline $\begin{array}{l}\text { DWP - PCS - MUFFLER KING } \\
\text { MUFFLER/BRAKE SHOP }\end{array}$ & 786 & $\begin{array}{l}\text { C04 Type; P - Automobiles - Repair } \\
\text { Shops }\end{array}$ & $\begin{array}{l}\text { MUFFLER KING } \\
\text { MUFFLER/BRAKE SHOP }\end{array}$ & $\begin{array}{l}1492 \text { ROGUE RIVER } \\
\text { HWY/JUST WEST OF } \\
\text { EXTERMINATOR }\end{array}$ & $\begin{array}{l}\text { Grants } \\
\text { Pass }\end{array}$ & Josephine & 2005 & DEQ/OHA SWA $2000-2005$ \\
\hline DWP - PCS - Non-Irrigated Crops & 10019 & $\begin{array}{l}\text { A05 Type; A - Crops - Nonirrigated (inc. } \\
\text { Christmas trees, grains, grass seed, } \\
\text { pasture) }\end{array}$ & Non-Irrigated Crops & Throughout DWPA & $\begin{array}{l}\text { Rogue } \\
\text { River }\end{array}$ & Jackson & 2005 & DEQ/OHA SWA $2000-2005$ \\
\hline $\begin{array}{l}\text { DWP - PCS - ODOT Highway } \\
\text { Division }\end{array}$ & 10424 & $\begin{array}{l}\text { C61 Type; P - Maintenance } \\
\text { Shop/Equipment Storage - } \\
\text { Transportation Related - Highway } \\
\text { Division Grants Pass }\end{array}$ & ODOT Highway Division & Off Agness Ave & $\begin{array}{l}\text { Grants } \\
\text { Pass }\end{array}$ & Josephine & 2005 & DEQ/OHA SWA 2000 - 2005 \\
\hline $\begin{array}{l}\text { DWP - PCS - ODOT Highway } \\
\text { Division }\end{array}$ & 10424 & $\begin{array}{l}\text { M24 Type; P - UST - } \\
\text { Decommissioned/Inactive }\end{array}$ & ODOT Highway Division & Off Agness Ave & $\begin{array}{l}\text { Grants } \\
\text { Pass }\end{array}$ & Josephine & 2005 & DEQ/OHA SWA $2000-2005$ \\
\hline $\begin{array}{l}\text { DWP - PCS - OPEN } \\
\text { IRRIGATION CHANNEL }\end{array}$ & 788 & A52 Type; A - Irrigation Canal/Pond & OPEN IRRIGATION CHANNEL & $\begin{array}{l}\text { NORTH OF SCHOOL/RUNS } \\
\text { EAST WEST PARALLEL TO } \\
\text { HWY ON }\end{array}$ & $\begin{array}{l}\text { Grants } \\
\text { Pass }\end{array}$ & Josephine & 2005 & DEQ/OHA SWA $2000-2005$ \\
\hline $\begin{array}{l}\text { DWP - PCS - Oregon Swiss } \\
\text { Precision }\end{array}$ & 10412 & $\begin{array}{l}\text { C17 Type; P - Metal } \\
\text { Plating/Finishing/Fabrication }\end{array}$ & Oregon Swiss Precision & 1080 SE M Street & \begin{tabular}{|l} 
Grants \\
Pass
\end{tabular} & Josephine & 2005 & DEQ/OHA SWA $2000-2005$ \\
\hline DWP - PCS - Pacific Marine Inc. & 10414 & $\begin{array}{l}\text { C05 Type; P - Boat } \\
\text { Services/Repair/Refinishing }\end{array}$ & Pacific Marine Inc. & Off "M" Street & $\begin{array}{l}\text { Grants } \\
\text { Pass }\end{array}$ & Josephine & 2005 & DEQ/OHA SWA $2000-2005$ \\
\hline DWP - PCS - Park & 14877 & R13 Type; P - Parks & Park & Pine Street & $\begin{array}{l}\text { Rogue } \\
\text { River }\end{array}$ & Jackson & 2005 & DEQ/OHA SWA 2000 - 2005 \\
\hline DWP - PCS - Park & 14877 & R13 Type; P - Parks & Park & Pine Street & \begin{tabular}{|l|} 
Rogue \\
River
\end{tabular} & Jackson & 2005 & DEQ/OHA SWA $2000-2005$ \\
\hline DWP - PCS - Park & 14877 & R13 Type; P - Parks & Park & Pine Street & \begin{tabular}{|l|} 
Rogue \\
River
\end{tabular} & Jackson & 2005 & DEQ/OHA SWA $2000-2005$ \\
\hline DWP - PCS - Park & 14877 & R13 Type; P - Parks & Park & Pine Street & \begin{tabular}{|l|} 
Rogue \\
River
\end{tabular} & Jackson & 2005 & DEQ/OHA SWA $2000-2005$ \\
\hline $\begin{array}{l}\text { DWP - PCS - PARKDALE } \\
\text { SUBDIVISION }\end{array}$ & 1382 & $\begin{array}{l}\text { R09 Type; A - Housing - High Density (> } \\
1 \text { House/0.5 acres) }\end{array}$ & PARKDALE SUBDIVISION & \begin{tabular}{|l|} 
AT SOUTH END OF \\
PARKDALE/WELL ON WEST \\
SIDE OF ROAD
\end{tabular} & $\begin{array}{l}\text { Grants } \\
\text { Pass }\end{array}$ & Josephine & 2005 & DEQ/OHA SWA 2000 - 2005 \\
\hline $\begin{array}{l}\text { DWP - PCS - PARKDALE } \\
\text { SUBDIVISION }\end{array}$ & 1382 & $\begin{array}{l}\text { R11 Type; A - Lawn Care - Highly } \\
\text { Maintained Areas }\end{array}$ & PARKDALE SUBDIVISION & \begin{tabular}{|l|} 
AT SOUTH END OF \\
PARKDALE/WELL ON WEST \\
SIDE OF ROAD
\end{tabular} & $\begin{array}{l}\text { Grants } \\
\text { Pass }\end{array}$ & Josephine & 2005 & DEQ/OHA SWA $2000-2005$ \\
\hline $\begin{array}{l}\text { DWP - PCS - PARKDALE } \\
\text { SUBDIVISION }\end{array}$ & 1382 & $\begin{array}{l}\text { R09 Type; A - Housing - High Density (> } \\
1 \text { House/0.5 acres) }\end{array}$ & PARKDALE SUBDIVISION & \begin{tabular}{|l|} 
AT SOUTH END OF \\
PARKDALE/WELL ON WEST \\
SIDE OF ROAD
\end{tabular} & $\begin{array}{l}\text { Grants } \\
\text { Pass }\end{array}$ & Josephine & 2005 & DEQ/OHA SWA 2000 - 2005 \\
\hline
\end{tabular}




\begin{tabular}{|c|c|c|c|c|c|c|c|c|}
\hline Database Identifier (DB_ID) & Site Identifier & Status & Common Name & Address & City & County & \begin{tabular}{|l|} 
Retrieval \\
Date
\end{tabular} & Data Source \\
\hline $\begin{array}{l}\text { DWP - PCS - PARKDALE } \\
\text { SUBDIVISION }\end{array}$ & 1382 & $\begin{array}{l}\text { R11 Type; A - Lawn Care - Highly } \\
\text { Maintained Areas }\end{array}$ & PARKDALE SUBDIVISION & $\begin{array}{l}\text { AT SOUTH END OF } \\
\text { PARKDALE/WELL ON WEST } \\
\text { SIDE OF ROAD }\end{array}$ & $\begin{array}{l}\text { Grants } \\
\text { Pass }\end{array}$ & Josephine & 2005 & DEQ/OHA SWA $2000-2005$ \\
\hline DWP - PCS - Parking Lot & 9369 & $\begin{array}{l}\text { C20 Type; A - Parking Lots/Malls (> } 50 \\
\text { Spaces) }\end{array}$ & Parking Lot & North of well & $\begin{array}{l}\text { Grants } \\
\text { Pass }\end{array}$ & Josephine & 2005 & DEQ/OHA SWA 2000 - 2005 \\
\hline $\begin{array}{l}\text { DWP - PCS - Pine Villa Mobile } \\
\text { Park }\end{array}$ & 9370 & $\begin{array}{l}\text { M09 Type; A - Homesteads - Rural - } \\
\text { Septic Systems (<1/acre) }\end{array}$ & Pine Villa Mobile Park & Surrounding Well & \begin{tabular}{|l|} 
Grants \\
Pass
\end{tabular} & Josephine & 2005 & DEQ/OHA SWA 2000 - 2005 \\
\hline $\begin{array}{l}\text { DWP - PCS - Pine Villa Mobile } \\
\text { Park }\end{array}$ & 9370 & $\begin{array}{l}\text { M31 Type; A - Large Capacity Septic } \\
\text { Systems (serves > } 20 \text { people) - Class V } \\
\text { UICs }\end{array}$ & Pine Villa Mobile Park & Surrounding Well & $\begin{array}{l}\text { Grants } \\
\text { Pass }\end{array}$ & Josephine & 2005 & DEQ/OHA SWA 2000 - 2005 \\
\hline $\begin{array}{l}\text { DWP - PCS - Potential } \\
\text { Development }\end{array}$ & 14897 & $\begin{array}{l}\text { R16 Type; A - Septic Systems - High } \\
\text { Density ( > 1 system/acre) }\end{array}$ & Potential Development & $\begin{array}{l}\text { North of Wards Creek Road - } \\
\text { East of City Limits }\end{array}$ & \begin{tabular}{|l|} 
Rogue \\
River
\end{tabular} & Jackson & 2005 & DEQ/OHA SWA 2000 - 2005 \\
\hline $\begin{array}{l}\text { DWP - PCS - Potential New } \\
\text { Residential Development }\end{array}$ & 14895 & $\begin{array}{l}\text { R16 Type; A - Septic Systems - High } \\
\text { Density ( > } 1 \text { system/acre) }\end{array}$ & $\begin{array}{l}\text { Potential New Residential } \\
\text { Development }\end{array}$ & $\begin{array}{l}\text { North of Wards Creek Road - } \\
\text { East of City Limits }\end{array}$ & $\begin{array}{l}\text { Rogue } \\
\text { River }\end{array}$ & Jackson & 2005 & DEQ/OHA SWA 2000 - 2005 \\
\hline $\begin{array}{l}\text { DWP - PCS - Potential New } \\
\text { Residential Development }\end{array}$ & 14895 & $\begin{array}{l}\text { R16 Type; A - Septic Systems - High } \\
\text { Density ( > 1 system/acre) }\end{array}$ & \begin{tabular}{|l} 
Potential New Residential \\
Development
\end{tabular} & $\begin{array}{l}\text { North of Wards Creek Road - } \\
\text { East of City Limits }\end{array}$ & \begin{tabular}{|l|} 
Rogue \\
River
\end{tabular} & Jackson & 2005 & DEQ/OHA SWA 2000 - 2005 \\
\hline $\begin{array}{l}\text { DWP - PCS - Potential New } \\
\text { Residential Development }\end{array}$ & 14895 & $\begin{array}{l}\text { R16 Type; A - Septic Systems - High } \\
\text { Density ( > } 1 \text { system/acre) }\end{array}$ & $\begin{array}{l}\text { Potential New Residential } \\
\text { Development }\end{array}$ & $\begin{array}{l}\text { North of Wards Creek Road - } \\
\text { East of City Limits }\end{array}$ & \begin{tabular}{|l|} 
Rogue \\
River
\end{tabular} & Jackson & 2005 & DEQ/OHA SWA 2000 - 2005 \\
\hline \begin{tabular}{|l|} 
DWP - PCS - Potential New \\
Residential Development
\end{tabular} & 14895 & $\begin{array}{l}\text { R16 Type; A - Septic Systems - High } \\
\text { Density ( >1 system/acre) }\end{array}$ & $\begin{array}{l}\text { Potential New Residential } \\
\text { Development }\end{array}$ & $\begin{array}{l}\text { North of Wards Creek Road - } \\
\text { East of City Limits }\end{array}$ & \begin{tabular}{|l|} 
Rogue \\
River
\end{tabular} & Jackson & 2005 & DEQ/OHA SWA 2000 - 2005 \\
\hline $\begin{array}{l}\text { DWP - PCS - POWDER WORKS } \\
\text { CAR PAINTING }\end{array}$ & 1403 & C01 Type; P - Automobiles - Body Shops & $\begin{array}{l}\text { POWDER WORKS CAR } \\
\text { PAINTING }\end{array}$ & \begin{tabular}{|l} 
JUST EAST OF BURGAN \\
AUTOMOTIVE ON \\
FRUITDALE \\
\end{tabular} & $\begin{array}{l}\text { Grants } \\
\text { Pass }\end{array}$ & Josephine & 2005 & DEQ/OHA SWA 2000 - 2005 \\
\hline $\begin{array}{l}\text { DWP - PCS - POWDER WORKS } \\
\text { CAR PAINTING }\end{array}$ & 1403 & C01 Type; P - Automobiles - Body Shops & $\begin{array}{l}\text { POWDER WORKS CAR } \\
\text { PAINTING }\end{array}$ & $\begin{array}{l}\text { JUST EAST OF BURGAN } \\
\text { AUTOMOTIVE ON } \\
\text { FRUITDALE } \\
\end{array}$ & $\begin{array}{l}\text { Grants } \\
\text { Pass }\end{array}$ & Josephine & 2005 & DEQ/OHA SWA 2000 - 2005 \\
\hline DWP - PCS - Qwest Corporation & 14875 & $\begin{array}{l}\text { C09 Type; P - Electrical/Electronic } \\
\text { Manufacturing }\end{array}$ & Qwest Corporation & 301 Broadway & \begin{tabular}{|l|} 
Rogue \\
River
\end{tabular} & Jackson & 2005 & DEQ/OHA SWA 2000 - 2005 \\
\hline DWP - PCS - Qwest Corporation & 14875 & $\begin{array}{l}\text { C09 Type; P - Electrical/Electronic } \\
\text { Manufacturing }\end{array}$ & Qwest Corporation & 301 Broadway & \begin{tabular}{|l|} 
Rogue \\
River
\end{tabular} & Jackson & 2005 & DEQ/OHA SWA 2000 - 2005 \\
\hline DWP - PCS - Qwest Corporation & 14875 & $\begin{array}{l}\text { C09 Type; P - Electrical/Electronic } \\
\text { Manufacturing }\end{array}$ & Qwest Corporation & 301 Broadway & \begin{tabular}{|l|} 
Rogue \\
River
\end{tabular} & Jackson & 2005 & DEQ/OHA SWA 2000 - 2005 \\
\hline DWP - PCS - Qwest Corporation & 14875 & $\begin{array}{l}\text { C09 Type; P - Electrical/Electronic } \\
\text { Manufacturing }\end{array}$ & Qwest Corporation & 301 Broadway & \begin{tabular}{|l|} 
Rogue \\
River
\end{tabular} & Jackson & 2005 & DEQ/OHA SWA 2000 - 2005 \\
\hline DWP - PCS - Railroad & 10408 & M19 Type; A - Transportation - Railroads & Railroad & East of intake & $\begin{array}{l}\text { Grants } \\
\text { Pass }\end{array}$ & Josephine & 2005 & DEQ/OHA SWA 2000 - 2005 \\
\hline DWP - PCS - Railroad & 12311 & M19 Type; A - Transportation - Railroads & Railroad & South of I-5 & \begin{tabular}{|l|} 
Rogue \\
River
\end{tabular} & Jackson & 2005 & DEQ/OHA SWA 2000 - 2005 \\
\hline $\begin{array}{l}\text { DWP - PCS - Randy' Shamblin } \\
\text { Truck and RV }\end{array}$ & 14887 & $\begin{array}{l}\text { C04 Type; P - Automobiles - Repair } \\
\text { Shops }\end{array}$ & Randy' Shamblin Truck and RV & Pine Street & \begin{tabular}{|l|} 
Rogue \\
River
\end{tabular} & Jackson & 2005 & DEQ/OHA SWA 2000 - 2005 \\
\hline $\begin{array}{l}\text { DWP - PCS - Randy' Shamblin } \\
\text { Truck and RV }\end{array}$ & 14887 & $\begin{array}{l}\text { C04 Type; P - Automobiles - Repair } \\
\text { Shops }\end{array}$ & Randy' Shamblin Truck and RV & Pine Street & \begin{tabular}{|l|} 
Rogue \\
River
\end{tabular} & Jackson & 2005 & DEQ/OHA SWA 2000 - 2005 \\
\hline $\begin{array}{l}\text { DWP - PCS - Randy' Shamblin } \\
\text { Truck and RV }\end{array}$ & 14887 & $\begin{array}{l}\text { C04 Type; P - Automobiles - Repair } \\
\text { Shops }\end{array}$ & Randy' Shamblin Truck and RV & Pine Street & $\begin{array}{l}\text { Rogue } \\
\text { River }\end{array}$ & Jackson & 2005 & DEQ/OHA SWA 2000 - 2005 \\
\hline $\begin{array}{l}\text { DWP - PCS - Randy' Shamblin } \\
\text { Truck and RV }\end{array}$ & 14887 & $\begin{array}{l}\text { C04 Type; P - Automobiles - Repair } \\
\text { Shops }\end{array}$ & Randy' Shamblin Truck and RV & Pine Street & \begin{tabular}{|l|} 
Rogue \\
River
\end{tabular} & Jackson & 2005 & DEQ/OHA SWA 2000 - 2005 \\
\hline $\begin{array}{l}\text { DWP - PCS - Redding Re-Load } \\
\text { and Dispatch }\end{array}$ & 14244 & $\begin{array}{l}\text { C10 Type; P - Fleet/Trucking/Bus } \\
\text { Terminals }\end{array}$ & Redding Re-Load and Dispatch & Highway 99 & $\begin{array}{l}\text { Grants } \\
\text { Pass }\end{array}$ & Josephine & 2005 & DEQ/OHA SWA 2000 - 2005 \\
\hline DWP - PCS - REVOLVO WEST & 772 & $\begin{array}{l}\text { C04 Type; P - Automobiles - Repair } \\
\text { Shops }\end{array}$ & REVOLVO WEST & $\begin{array}{l}\text { SOUTHWEST CORNER OF } \\
\text { ROGUE RIVER HWY AND } \\
\text { HAMILTON L }\end{array}$ & \begin{tabular}{|l} 
Grants \\
Pass
\end{tabular} & Josephine & 2005 & DEQ/OHA SWA 2000 - 2005 \\
\hline DWP - PCS - REVOLVO WEST & 772 & $\begin{array}{l}\text { M06 Type; P - Auto - Gas Stations - } \\
\text { Historic }\end{array}$ & REVOLVO WEST & $\begin{array}{l}\text { SOUTHWEST CORNER OF } \\
\text { ROGUE RIVER HWY AND } \\
\text { HAMILTON L }\end{array}$ & $\begin{array}{l}\text { Grants } \\
\text { Pass }\end{array}$ & Josephine & 2005 & DEQ/OHA SWA 2000 - 2005 \\
\hline
\end{tabular}




\begin{tabular}{|c|c|c|c|c|c|c|c|c|}
\hline Database Identifier (DB_ID) & Site Identifier & Status & Common Name & Address & City & County & \begin{tabular}{|l} 
Retrieval \\
Date
\end{tabular} & Data Source \\
\hline $\begin{array}{l}\text { DWP - PCS - River Wild } \\
\text { Aluminum Boats }\end{array}$ & 10415 & $\begin{array}{l}\text { C05 Type; P - Boat } \\
\text { Services/Repair/Refinishing }\end{array}$ & River Wild Aluminum Boats & Off "M" Street & \begin{tabular}{|l} 
Grants \\
Pass
\end{tabular} & Josephine & 2005 & DEQ/OHA SWA $2000-2005$ \\
\hline DWP - PCS - Riverfront RV Park & 12315 & R03 Type; A - Campgrounds/RV Parks & Riverfront RV Park & Hwy 99 & $\begin{array}{l}\text { Rogue } \\
\text { River }\end{array}$ & Jackson & 2005 & DEQ/OHA SWA $2000-2005$ \\
\hline DWP - PCS - Riverside School & 10432 & $\begin{array}{l}\text { M26 Type; P - UST - Confirmed Leaking } \\
\text { but listed as NFA - DEQ LUST List }\end{array}$ & Riverside School & Harvey Drive & $\begin{array}{l}\text { Grants } \\
\text { Pass }\end{array}$ & Josephine & 2005 & DEQ/OHA SWA $2000-2005$ \\
\hline DWP - PCS - Riverside School & 10432 & R15 Type; P - Schools & Riverside School & Harvey Drive & $\begin{array}{l}\text { Grants } \\
\text { Pass }\end{array}$ & Josephine & 2005 & DEQ/OHA SWA $2000-2005$ \\
\hline $\begin{array}{l}\text { DWP - PCS - Rogue River } \\
\text { Center }\end{array}$ & 10010 & $\begin{array}{l}\text { C20 Type; P - Parking Lots/Malls (> } 50 \\
\text { Spaces) }\end{array}$ & Rogue River Center & Northeast of intake & $\begin{array}{l}\text { Rogue } \\
\text { River }\end{array}$ & Jackson & 2005 & DEQ/OHA SWA $2000-2005$ \\
\hline $\begin{array}{l}\text { DWP - PCS - Rogue River } \\
\text { Center }\end{array}$ & 10010 & $\begin{array}{l}\text { C20 Type; P - Parking Lots/Malls (> } 50 \\
\text { Spaces) }\end{array}$ & Rogue River Center & Northeast of intake & $\begin{array}{l}\text { Rogue } \\
\text { River }\end{array}$ & Jackson & 2005 & DEQ/OHA SWA $2000-2005$ \\
\hline $\begin{array}{l}\text { DWP - PCS - Rogue River } \\
\text { Center }\end{array}$ & 10010 & $\begin{array}{l}\text { C20 Type; P - Parking Lots/Malls (> } 50 \\
\text { Spaces) }\end{array}$ & Rogue River Center & Northeast of intake & $\begin{array}{l}\text { Rogue } \\
\text { River }\end{array}$ & Jackson & 2005 & DEQ/OHA SWA $2000-2005$ \\
\hline $\begin{array}{l}\text { DWP - PCS - Rogue River } \\
\text { Center }\end{array}$ & 10010 & $\begin{array}{l}\text { C20 Type; P - Parking Lots/Malls (> } 50 \\
\text { Spaces) }\end{array}$ & Rogue River Center & Northeast of intake & $\begin{array}{l}\text { Rogue } \\
\text { River }\end{array}$ & Jackson & 2005 & DEQ/OHA SWA $2000-2005$ \\
\hline $\begin{array}{l}\text { DWP - PCS - Rogue River } \\
\text { Center }\end{array}$ & 10010 & $\begin{array}{l}\text { C20 Type; P - Parking Lots/Malls (> } 50 \\
\text { Spaces) }\end{array}$ & Rogue River Center & Northeast of intake & $\begin{array}{l}\text { Rogue } \\
\text { River }\end{array}$ & Jackson & 2005 & DEQ/OHA SWA $2000-2005$ \\
\hline DWP - PCS - Rogue River CO & 14880 & $\begin{array}{l}\text { M24 Type; P - UST - } \\
\text { Decommissioned/Inactive }\end{array}$ & Rogue River CO & Broadway & $\begin{array}{l}\text { Rogue } \\
\text { River }\end{array}$ & Jackson & 2005 & DEQ/OHA SWA $2000-2005$ \\
\hline DWP - PCS - Rogue River CO & 14880 & M55 Type; P - Unknown Operation & Rogue River CO & Broadway & \begin{tabular}{|l|} 
Rogue \\
River
\end{tabular} & Jackson & 2005 & DEQ/OHA SWA $2000-2005$ \\
\hline DWP - PCS - Rogue River CO & 14880 & $\begin{array}{l}\text { M24 Type; P - UST - } \\
\text { Decommissioned/Inactive }\end{array}$ & Rogue River CO & Broadway & \begin{tabular}{|l|} 
Rogue \\
River
\end{tabular} & Jackson & 2005 & DEQ/OHA SWA $2000-2005$ \\
\hline DWP - PCS - Rogue River CO & 14880 & M55 Type; P - Unknown Operation & Rogue River CO & Broadway & $\begin{array}{l}\text { Rogue } \\
\text { River }\end{array}$ & Jackson & 2005 & DEQ/OHA SWA $2000-2005$ \\
\hline DWP - PCS - Rogue River CO & 14880 & $\begin{array}{l}\text { M24 Type; P - UST - } \\
\text { Decommissioned/Inactive }\end{array}$ & Rogue River CO & Broadway & $\begin{array}{l}\text { Rogue } \\
\text { River }\end{array}$ & Jackson & 2005 & DEQ/OHA SWA $2000-2005$ \\
\hline DWP - PCS - Rogue River CO & 14880 & M55 Type; P - Unknown Operation & Rogue River CO & Broadway & $\begin{array}{l}\text { Rogue } \\
\text { River }\end{array}$ & Jackson & 2005 & DEQ/OHA SWA $2000-2005$ \\
\hline DWP - PCS - Rogue River CO & 14880 & $\begin{array}{l}\text { M24 Type; P - UST - } \\
\text { Decommissioned/Inactive }\end{array}$ & Rogue River CO & Broadway & \begin{tabular}{|l|} 
Rogue \\
River
\end{tabular} & Jackson & 2005 & DEQ/OHA SWA $2000-2005$ \\
\hline DWP - PCS - Rogue River CO & 14880 & M55 Type; P - Unknown Operation & Rogue River CO & Broadway & $\begin{array}{l}\text { Rogue } \\
\text { River }\end{array}$ & Jackson & 2005 & DEQ/OHA SWA $2000-2005$ \\
\hline $\begin{array}{l}\text { DWP - PCS - Rogue River Fire } \\
\text { Station }\end{array}$ & 10012 & R06 Type; P - Fire Station & Rogue River Fire Station & East of intake & $\begin{array}{l}\text { Rogue } \\
\text { River }\end{array}$ & Jackson & 2005 & DEQ/OHA SWA $2000-2005$ \\
\hline $\begin{array}{l}\text { DWP - PCS - Rogue River Fire } \\
\text { Station }\end{array}$ & 10012 & R06 Type; P - Fire Station & Rogue River Fire Station & East of intake & \begin{tabular}{|l|} 
Rogue \\
River
\end{tabular} & Jackson & 2005 & DEQ/OHA SWA $2000-2005$ \\
\hline $\begin{array}{l}\text { DWP - PCS - Rogue River Fire } \\
\text { Station }\end{array}$ & 10012 & R06 Type; P - Fire Station & Rogue River Fire Station & East of intake & \begin{tabular}{|l|} 
Rogue \\
River
\end{tabular} & Jackson & 2005 & DEQ/OHA SWA $2000-2005$ \\
\hline $\begin{array}{l}\text { DWP - PCS - Rogue River Fire } \\
\text { Station }\end{array}$ & 10012 & R06 Type; P - Fire Station & Rogue River Fire Station & East of intake & \begin{tabular}{|l|} 
Rogue \\
River
\end{tabular} & Jackson & 2005 & DEQ/OHA SWA 2000 - 2005 \\
\hline $\begin{array}{l}\text { DWP - PCS - Rogue River Fire } \\
\text { Station }\end{array}$ & 10012 & R06 Type; P - Fire Station & Rogue River Fire Station & East of intake & $\begin{array}{l}\text { Rogue } \\
\text { River }\end{array}$ & Jackson & 2005 & DEQ/OHA SWA $2000-2005$ \\
\hline $\begin{array}{l}\text { DWP - PCS - Rogue River Health } \\
\text { Clinic }\end{array}$ & 14838 & C16 Type; P - Medical/Vet Offices & Rogue River Health Clinic & W. Main Street & \begin{tabular}{|l|} 
Rogue \\
River
\end{tabular} & Jackson & 2005 & DEQ/OHA SWA $2000-2005$ \\
\hline $\begin{array}{l}\text { DWP - PCS - Rogue River Health } \\
\text { Clinic }\end{array}$ & 14838 & C16 Type; P - Medical/Vet Offices & Rogue River Health Clinic & W. Main Street & \begin{tabular}{|l|} 
Rogue \\
River
\end{tabular} & Jackson & 2005 & DEQ/OHA SWA $2000-2005$ \\
\hline $\begin{array}{l}\text { DWP - PCS - Rogue River Health } \\
\text { Clinic }\end{array}$ & 14838 & C16 Type; P - Medical/Vet Offices & Rogue River Health Clinic & W. Main Street & \begin{tabular}{|l|} 
Rogue \\
River \\
\end{tabular} & Jackson & 2005 & DEQ/OHA SWA 2000 - 2005 \\
\hline $\begin{array}{l}\text { DWP - PCS - Rogue River Health } \\
\text { Clinic }\end{array}$ & 14838 & C16 Type; P - Medical/Vet Offices & Rogue River Health Clinic & W. Main Street & $\begin{array}{l}\text { Rogue } \\
\text { River }\end{array}$ & Jackson & 2005 & DEQ/OHA SWA $2000-2005$ \\
\hline $\begin{array}{l}\text { DWP - PCS - Rogue River Health } \\
\text { Clinic }\end{array}$ & 14869 & C16 Type; P - Medical/Vet Offices & Rogue River Health Clinic & West Main Street & $\begin{array}{l}\text { Rogue } \\
\text { River }\end{array}$ & Jackson & 2005 & DEQ/OHA SWA $2000-2005$ \\
\hline
\end{tabular}




\begin{tabular}{|c|c|c|c|c|c|c|c|c|}
\hline Database Identifier (DB_ID) & Site Identifier & Status & Common Name & Address & City & County & \begin{tabular}{|l} 
Retrieval \\
Date
\end{tabular} & Data Source \\
\hline $\begin{array}{l}\text { DWP - PCS - Rogue River Health } \\
\text { Clinic }\end{array}$ & 14869 & C16 Type; P - Medical/Vet Offices & Rogue River Health Clinic & West Main Street & \begin{tabular}{|l} 
Rogue \\
River
\end{tabular} & Jackson & 2005 & DEQ/OHA SWA 2000 - 2005 \\
\hline $\begin{array}{l}\text { DWP - PCS - Rogue River Health } \\
\text { Clinic }\end{array}$ & 14869 & C16 Type; P - Medical/Vet Offices & Rogue River Health Clinic & West Main Street & \begin{tabular}{|l|} 
Rogue \\
River
\end{tabular} & Jackson & 2005 & DEQ/OHA SWA $2000-2005$ \\
\hline $\begin{array}{l}\text { DWP - PCS - Rogue River Health } \\
\text { Clinic }\end{array}$ & 14869 & C16 Type; P - Medical/Vet Offices & Rogue River Health Clinic & West Main Street & \begin{tabular}{|l|} 
Rogue \\
River
\end{tabular} & Jackson & 2005 & DEQ/OHA SWA $2000-2005$ \\
\hline $\begin{array}{l}\text { DWP - PCS - Rogue River High } \\
\text { School }\end{array}$ & 14894 & R15 Type; P - Schools & Rogue River High School & Valley View Drive & \begin{tabular}{|l|} 
Rogue \\
River
\end{tabular} & Jackson & 2005 & DEQ/OHA SWA $2000-2005$ \\
\hline $\begin{array}{l}\text { DWP - PCS - Rogue River High } \\
\text { School }\end{array}$ & 14894 & R15 Type; P - Schools & Rogue River High School & Valley View Drive & \begin{tabular}{|l|} 
Rogue \\
River
\end{tabular} & Jackson & 2005 & DEQ/OHA SWA $2000-2005$ \\
\hline $\begin{array}{l}\text { DWP - PCS - Rogue River High } \\
\text { School }\end{array}$ & 14894 & R15 Type; P - Schools & Rogue River High School & Valley View Drive & \begin{tabular}{|l|} 
Rogue \\
River
\end{tabular} & Jackson & 2005 & DEQ/OHA SWA $2000-2005$ \\
\hline $\begin{array}{l}\text { DWP - PCS - Rogue River High } \\
\text { School }\end{array}$ & 14894 & R15 Type; P - Schools & Rogue River High School & Valley View Drive & \begin{tabular}{|l|} 
Rogue \\
River
\end{tabular} & Jackson & 2005 & DEQ/OHA SWA $2000-2005$ \\
\hline $\begin{array}{l}\text { DWP - PCS - ROGUE RIVER } \\
\text { HIGHWAY }\end{array}$ & 785 & $\begin{array}{l}\text { M18 Type; A - Transportation - } \\
\text { Freeways/State Highways }\end{array}$ & ROGUE RIVER HIGHWAY & $\begin{array}{l}\text { RUNS EAST/WEST NORTH } \\
\text { OF SCHOOL }\end{array}$ & $\begin{array}{l}\text { Grants } \\
\text { Pass }\end{array}$ & Josephine & 2005 & DEQ/OHA SWA $2000-2005$ \\
\hline $\begin{array}{l}\text { DWP - PCS - ROGUE RIVER } \\
\text { HIGHWAY }\end{array}$ & 1091 & $\begin{array}{l}\text { M18 Type; A - Transportation - } \\
\text { Freeways/State Highways }\end{array}$ & ROGUE RIVER HIGHWAY & $\begin{array}{l}\text { RUNS EAST TO WEST } \\
\text { THROUGH } 2 \text { YR TOT }\end{array}$ & $\begin{array}{l}\text { Grants } \\
\text { Pass }\end{array}$ & Josephine & 2005 & DEQ/OHA SWA 2000 - 2005 \\
\hline $\begin{array}{l}\text { DWP - PCS - ROGUE RIVER } \\
\text { HIGHWAY }\end{array}$ & 1091 & $\begin{array}{l}\text { M18 Type; A - Transportation - } \\
\text { Freeways/State Highways }\end{array}$ & ROGUE RIVER HIGHWAY & $\begin{array}{l}\text { RUNS EAST TO WEST } \\
\text { THROUGH } 2 \text { YR TOT }\end{array}$ & $\begin{array}{l}\text { Grants } \\
\text { Pass }\end{array}$ & Josephine & 2005 & DEQ/OHA SWA $2000-2005$ \\
\hline $\begin{array}{l}\text { DWP - PCS - Rogue River Middle } \\
\text { School }\end{array}$ & 14886 & R15 Type; P - Schools & Rogue River Middle School & Pine Street & $\begin{array}{l}\text { Rogue } \\
\text { River }\end{array}$ & Jackson & 2005 & DEQ/OHA SWA $2000-2005$ \\
\hline $\begin{array}{l}\text { DWP - PCS - Rogue River Middle } \\
\text { School }\end{array}$ & 14886 & R15 Type; P - Schools & Rogue River Middle School & Pine Street & \begin{tabular}{|l|} 
Rogue \\
River
\end{tabular} & Jackson & 2005 & DEQ/OHA SWA $2000-2005$ \\
\hline $\begin{array}{l}\text { DWP - PCS - Rogue River Middle } \\
\text { School }\end{array}$ & 14886 & R15 Type; P - Schools & Rogue River Middle School & Pine Street & \begin{tabular}{|l|} 
Rogue \\
River
\end{tabular} & Jackson & 2005 & DEQ/OHA SWA $2000-2005$ \\
\hline $\begin{array}{l}\text { DWP - PCS - Rogue River Middle } \\
\text { School }\end{array}$ & 14886 & R15 Type; P - Schools & Rogue River Middle School & Pine Street & $\begin{array}{l}\text { Rogue } \\
\text { River }\end{array}$ & Jackson & 2005 & DEQ/OHA SWA $2000-2005$ \\
\hline $\begin{array}{l}\text { DWP - PCS - Rogue River Mobile } \\
\text { Estates }\end{array}$ & 12307 & $\begin{array}{l}\text { M24 Type; A - UST - } \\
\text { Decommissioned/Inactive }\end{array}$ & Rogue River Mobile Estates & Foothill Blvd. & $\begin{array}{l}\text { Rogue } \\
\text { River }\end{array}$ & Jackson & 2005 & DEQ/OHA SWA $2000-2005$ \\
\hline $\begin{array}{l}\text { DWP - PCS - Rogue River Mobile } \\
\text { Estates }\end{array}$ & 12307 & $\begin{array}{l}\text { M31 Type; A - Large Capacity Septic } \\
\text { Systems (serves }>20 \text { people) - Class V } \\
\text { UICs }\end{array}$ & Rogue River Mobile Estates & Foothill Blvd. & $\begin{array}{l}\text { Rogue } \\
\text { River }\end{array}$ & Jackson & 2005 & DEQ/OHA SWA $2000-2005$ \\
\hline $\begin{array}{l}\text { DWP - PCS - Rogue River Mobile } \\
\text { Estates }\end{array}$ & 12307 & $\begin{array}{l}\text { R09 Type; A - Housing - High Density (> } \\
1 \text { House/0.5 acres) }\end{array}$ & Rogue River Mobile Estates & Foothill Blvd. & \begin{tabular}{|l} 
Rogue \\
River
\end{tabular} & Jackson & 2005 & DEQ/OHA SWA 2000 - 2005 \\
\hline DWP - PCS - Rogue River Press & 14885 & C21 Type; P - Photo Processing/Printing & Rogue River Press & Pine Street & $\begin{array}{l}\text { Rogue } \\
\text { River }\end{array}$ & Jackson & 2005 & DEQ/OHA SWA $2000-2005$ \\
\hline DWP - PCS - Rogue River Press & 14885 & C21 Type; P - Photo Processing/Printing & Rogue River Press & Pine Street & \begin{tabular}{|l|} 
Rogue \\
River
\end{tabular} & Jackson & 2005 & DEQ/OHA SWA $2000-2005$ \\
\hline DWP - PCS - Rogue River Press & 14885 & C21 Type; P - Photo Processing/Printing & Rogue River Press & Pine Street & \begin{tabular}{|l|} 
Rogue \\
River
\end{tabular} & Jackson & 2005 & DEQ/OHA SWA $2000-2005$ \\
\hline DWP - PCS - Rogue River Press & 14885 & C21 Type; P - Photo Processing/Printing & Rogue River Press & Pine Street & $\begin{array}{l}\text { Rogue } \\
\text { River }\end{array}$ & Jackson & 2005 & DEQ/OHA SWA $2000-2005$ \\
\hline $\begin{array}{l}\text { DWP - PCS - Rogue River } \\
\text { Storage }\end{array}$ & 10014 & C24 Type; P - RV/Mini Storage & Rogue River Storage & East of intake & $\begin{array}{l}\text { Rogue } \\
\text { River }\end{array}$ & Jackson & 2005 & DEQ/OHA SWA $2000-2005$ \\
\hline $\begin{array}{l}\text { DWP - PCS - Rogue River } \\
\text { Storage }\end{array}$ & 10014 & C24 Type; P - RV/Mini Storage & Rogue River Storage & East of intake & \begin{tabular}{|l|} 
Rogue \\
River
\end{tabular} & Jackson & 2005 & DEQ/OHA SWA $2000-2005$ \\
\hline $\begin{array}{l}\text { DWP - PCS - Rogue River } \\
\text { Storage }\end{array}$ & 10014 & C24 Type; P - RV/Mini Storage & Rogue River Storage & East of intake & \begin{tabular}{|l|} 
Rogue \\
River
\end{tabular} & Jackson & 2005 & DEQ/OHA SWA $2000-2005$ \\
\hline $\begin{array}{l}\text { DWP - PCS - Rogue River } \\
\text { Storage }\end{array}$ & 10014 & C24 Type; P - RV/Mini Storage & Rogue River Storage & East of intake & $\begin{array}{l}\text { Rogue } \\
\text { River }\end{array}$ & Jackson & 2005 & DEQ/OHA SWA 2000 - 2005 \\
\hline $\begin{array}{l}\text { DWP - PCS - Rogue River } \\
\text { Storage }\end{array}$ & 10014 & C24 Type; P - RV/Mini Storage & Rogue River Storage & East of intake & $\begin{array}{l}\text { Rogue } \\
\text { River }\end{array}$ & Jackson & 2005 & DEQ/OHA SWA $2000-2005$ \\
\hline $\begin{array}{l}\text { DWP - PCS - Rogue River } \\
\text { Texaco }\end{array}$ & 14878 & $\begin{array}{l}\text { M06 Type; P - Auto - Gas Stations - } \\
\text { Historic }\end{array}$ & Rogue River Texaco & Depot Street & \begin{tabular}{|l|} 
Rogue \\
River
\end{tabular} & Jackson & 2005 & DEQ/OHA SWA $2000-2005$ \\
\hline
\end{tabular}




\begin{tabular}{|c|c|c|c|c|c|c|c|c|}
\hline Database Identifier (DB_ID) & Site Identifier & Status & Common Name & Address & City & County & \begin{tabular}{|l} 
Retrieval \\
Date
\end{tabular} & Data Source \\
\hline $\begin{array}{l}\text { DWP - PCS - Rogue River } \\
\text { Texaco }\end{array}$ & 14878 & $\begin{array}{l}\text { M24 Type; P - UST - } \\
\text { Decommissioned/Inactive }\end{array}$ & Rogue River Texaco & Depot Street & $\begin{array}{l}\text { Rogue } \\
\text { River }\end{array}$ & Jackson & 2005 & DEQ/OHA SWA 2000 - 2005 \\
\hline $\begin{array}{l}\text { DWP - PCS - Rogue River } \\
\text { Texaco }\end{array}$ & 14878 & $\begin{array}{l}\text { M27 Type; P - UST - } \\
\text { Upgraded/Registered - Active }\end{array}$ & Rogue River Texaco & Depot Street & $\begin{array}{l}\text { Rogue } \\
\text { River }\end{array}$ & Jackson & 2005 & DEQ/OHA SWA $2000-2005$ \\
\hline $\begin{array}{l}\text { DWP - PCS - Rogue River } \\
\text { Texaco }\end{array}$ & 14878 & M28 Type; P - UST - Status Unknown & Rogue River Texaco & Depot Street & \begin{tabular}{|l|} 
Rogue \\
River
\end{tabular} & Jackson & 2005 & DEQ/OHA SWA $2000-2005$ \\
\hline $\begin{array}{l}\text { DWP - PCS - Rogue River } \\
\text { Texaco }\end{array}$ & 14878 & $\begin{array}{l}\text { M06 Type; P - Auto - Gas Stations - } \\
\text { Historic }\end{array}$ & Rogue River Texaco & Depot Street & \begin{tabular}{|l|} 
Rogue \\
River
\end{tabular} & Jackson & 2005 & DEQ/OHA SWA $2000-2005$ \\
\hline $\begin{array}{l}\text { DWP - PCS - Rogue River } \\
\text { Texaco }\end{array}$ & 14878 & \begin{tabular}{|l|} 
M24 Type; P - UST - \\
Decommissioned/Inactive
\end{tabular} & Rogue River Texaco & Depot Street & $\begin{array}{l}\text { Rogue } \\
\text { River }\end{array}$ & Jackson & 2005 & DEQ/OHA SWA 2000 - 2005 \\
\hline $\begin{array}{l}\text { DWP - PCS - Rogue River } \\
\text { Texaco }\end{array}$ & 14878 & $\begin{array}{l}\text { M27 Type; P - UST - } \\
\text { Upgraded/Registered - Active }\end{array}$ & Rogue River Texaco & Depot Street & $\begin{array}{l}\text { Rogue } \\
\text { River }\end{array}$ & Jackson & 2005 & DEQ/OHA SWA $2000-2005$ \\
\hline $\begin{array}{l}\text { DWP - PCS - Rogue River } \\
\text { Texaco }\end{array}$ & 14878 & M28 Type; P - UST - Status Unknown & Rogue River Texaco & Depot Street & $\begin{array}{l}\text { Rogue } \\
\text { River }\end{array}$ & Jackson & 2005 & DEQ/OHA SWA $2000-2005$ \\
\hline $\begin{array}{l}\text { DWP - PCS - Rogue River } \\
\text { Texaco }\end{array}$ & 14878 & $\begin{array}{l}\text { M06 Type; P - Auto - Gas Stations - } \\
\text { Historic }\end{array}$ & Rogue River Texaco & Depot Street & $\begin{array}{l}\text { Rogue } \\
\text { River }\end{array}$ & Jackson & 2005 & DEQ/OHA SWA $2000-2005$ \\
\hline $\begin{array}{l}\text { DWP - PCS - Rogue River } \\
\text { Texaco }\end{array}$ & 14878 & \begin{tabular}{|l|} 
M24 Type; P - UST - \\
Decommissioned/Inactive
\end{tabular} & Rogue River Texaco & Depot Street & $\begin{array}{l}\text { Rogue } \\
\text { River }\end{array}$ & Jackson & 2005 & DEQ/OHA SWA $2000-2005$ \\
\hline $\begin{array}{l}\text { DWP - PCS - Rogue River } \\
\text { Texaco }\end{array}$ & 14878 & $\begin{array}{l}\text { M27 Type; P - UST - } \\
\text { Upgraded/Registered - Active }\end{array}$ & Rogue River Texaco & Depot Street & $\begin{array}{l}\text { Rogue } \\
\text { River }\end{array}$ & Jackson & 2005 & DEQ/OHA SWA $2000-2005$ \\
\hline $\begin{array}{l}\text { DWP - PCS - Rogue River } \\
\text { Texaco }\end{array}$ & 14878 & M28 Type; P - UST - Status Unknown & Rogue River Texaco & Depot Street & $\begin{array}{l}\text { Rogue } \\
\text { River }\end{array}$ & Jackson & 2005 & DEQ/OHA SWA $2000-2005$ \\
\hline $\begin{array}{l}\text { DWP - PCS - Rogue River } \\
\text { Texaco }\end{array}$ & 14878 & $\begin{array}{l}\text { M06 Type; P - Auto - Gas Stations - } \\
\text { Historic }\end{array}$ & Rogue River Texaco & Depot Street & \begin{tabular}{|l|} 
Rogue \\
River
\end{tabular} & Jackson & 2005 & DEQ/OHA SWA $2000-2005$ \\
\hline $\begin{array}{l}\text { DWP - PCS - Rogue River } \\
\text { Texaco }\end{array}$ & 14878 & $\begin{array}{l}\text { M24 Type; P - UST - } \\
\text { Decommissioned/Inactive }\end{array}$ & Rogue River Texaco & Depot Street & $\begin{array}{l}\text { Rogue } \\
\text { River }\end{array}$ & Jackson & 2005 & DEQ/OHA SWA $2000-2005$ \\
\hline $\begin{array}{l}\text { DWP - PCS - Rogue River } \\
\text { Texaco }\end{array}$ & 14878 & $\begin{array}{l}\text { M27 Type; P - UST - } \\
\text { Upgraded/Registered - Active } \\
\end{array}$ & Rogue River Texaco & Depot Street & \begin{tabular}{|l|} 
Rogue \\
River
\end{tabular} & Jackson & 2005 & DEQ/OHA SWA 2000 - 2005 \\
\hline $\begin{array}{l}\text { DWP - PCS - Rogue River } \\
\text { Texaco }\end{array}$ & 14878 & M28 Type; P - UST - Status Unknown & Rogue River Texaco & Depot Street & $\begin{array}{l}\text { Rogue } \\
\text { River }\end{array}$ & Jackson & 2005 & DEQ/OHA SWA $2000-2005$ \\
\hline $\begin{array}{l}\text { DWP - PCS - Rogue River Tow - } \\
\text { Larry's Triangle Service }\end{array}$ & 14879 & $\begin{array}{l}\text { C04 Type; P - Automobiles - Repair } \\
\text { Shops }\end{array}$ & $\begin{array}{l}\text { Rogue River Tow - Larry's Triangle } \\
\text { Service }\end{array}$ & Main and Depot Streets & \begin{tabular}{|l|} 
Rogue \\
River
\end{tabular} & Jackson & 2005 & DEQ/OHA SWA $2000-2005$ \\
\hline $\begin{array}{l}\text { DWP - PCS - Rogue River Tow - } \\
\text { Larry's Triangle Service }\end{array}$ & 14879 & \begin{tabular}{|l|} 
M26 Type; P - UST - Confirmed Leaking \\
but listed as NFA - DEQ LUST List
\end{tabular} & \begin{tabular}{|l|} 
Rogue River Tow - Larry's Triangle \\
Service
\end{tabular} & Main and Depot Streets & $\begin{array}{l}\text { Rogue } \\
\text { River }\end{array}$ & Jackson & 2005 & DEQ/OHA SWA $2000-2005$ \\
\hline $\begin{array}{l}\text { DWP - PCS - Rogue River Tow - } \\
\text { Larry's Triangle Service }\end{array}$ & 14879 & $\begin{array}{l}\text { C04 Type; P - Automobiles - Repair } \\
\text { Shops }\end{array}$ & $\begin{array}{l}\text { Rogue River Tow - Larry's Triangle } \\
\text { Service }\end{array}$ & Main and Depot Streets & $\begin{array}{l}\text { Rogue } \\
\text { River }\end{array}$ & Jackson & 2005 & DEQ/OHA SWA $2000-2005$ \\
\hline $\begin{array}{l}\text { DWP - PCS - Rogue River Tow - } \\
\text { Larry's Triangle Service }\end{array}$ & 14879 & $\begin{array}{l}\text { M26 Type; P - UST - Confirmed Leaking } \\
\text { but listed as NFA - DEQ LUST List }\end{array}$ & $\begin{array}{l}\text { Rogue River Tow - Larry's Triangle } \\
\text { Service }\end{array}$ & Main and Depot Streets & $\begin{array}{l}\text { Rogue } \\
\text { River }\end{array}$ & Jackson & 2005 & DEQ/OHA SWA $2000-2005$ \\
\hline $\begin{array}{l}\text { DWP - PCS - Rogue River Tow - } \\
\text { Larry's Triangle Service }\end{array}$ & 14879 & $\begin{array}{l}\text { C04 Type; P - Automobiles - Repair } \\
\text { Shops }\end{array}$ & $\begin{array}{l}\text { Rogue River Tow - Larry's Triangle } \\
\text { Service }\end{array}$ & Main and Depot Streets & \begin{tabular}{|l} 
Rogue \\
River
\end{tabular} & Jackson & 2005 & DEQ/OHA SWA $2000-2005$ \\
\hline $\begin{array}{l}\text { DWP - PCS - Rogue River Tow - } \\
\text { Larry's Triangle Service }\end{array}$ & 14879 & \begin{tabular}{|l|} 
M26 Type; P - UST - Confirmed Leaking \\
but listed as NFA - DEQ LUST List
\end{tabular} & $\begin{array}{l}\text { Rogue River Tow - Larry's Triangle } \\
\text { Service }\end{array}$ & Main and Depot Streets & $\begin{array}{l}\text { Rogue } \\
\text { River }\end{array}$ & Jackson & 2005 & DEQ/OHA SWA $2000-2005$ \\
\hline $\begin{array}{l}\text { DWP - PCS - Rogue River Tow - } \\
\text { Larry's Triangle Service }\end{array}$ & 14879 & $\begin{array}{l}\text { C04 Type; P - Automobiles - Repair } \\
\text { Shops }\end{array}$ & \begin{tabular}{|l|} 
Rogue River Tow - Larry's Triangle \\
Service
\end{tabular} & Main and Depot Streets & $\begin{array}{l}\text { Rogue } \\
\text { River }\end{array}$ & Jackson & 2005 & DEQ/OHA SWA $2000-2005$ \\
\hline $\begin{array}{l}\text { DWP - PCS - Rogue River Tow - } \\
\text { Larry's Triangle Service }\end{array}$ & 14879 & \begin{tabular}{|l|} 
M26 Type; P - UST - Confirmed Leaking \\
but listed as NFA - DEQ LUST List
\end{tabular} & $\begin{array}{l}\text { Rogue River Tow - Larry's Triangle } \\
\text { Service }\end{array}$ & Main and Depot Streets & $\begin{array}{l}\text { Rogue } \\
\text { River }\end{array}$ & Jackson & 2005 & DEQ/OHA SWA $2000-2005$ \\
\hline $\begin{array}{l}\text { DWP - PCS - Rogue River Water } \\
\text { Treatment Plant }\end{array}$ & 10009 & $\begin{array}{l}\text { R05 Type; P - Drinking Water Treatment } \\
\text { Plants }\end{array}$ & Rogue River Water Treatment Plant & Off Classick Drive & \begin{tabular}{|l} 
Rogue \\
River
\end{tabular} & Jackson & 2005 & DEQ/OHA SWA $2000-2005$ \\
\hline $\begin{array}{l}\text { DWP - PCS - Rogue River Water } \\
\text { Treatment Plant }\end{array}$ & 10009 & $\begin{array}{l}\text { R05 Type; P - Drinking Water Treatment } \\
\text { Plants }\end{array}$ & Rogue River Water Treatment Plant & Off Classick Drive & $\begin{array}{l}\text { Rogue } \\
\text { River }\end{array}$ & Jackson & 2005 & DEQ/OHA SWA $2000-2005$ \\
\hline
\end{tabular}




\begin{tabular}{|c|c|c|c|c|c|c|c|c|}
\hline Database Identifier (DB_ID) & Site Identifier & Status & Common Name & Address & City & County & \begin{tabular}{|l} 
Retrieval \\
Date
\end{tabular} & Data Source \\
\hline $\begin{array}{l}\text { DWP - PCS - Rogue River Water } \\
\text { Treatment Plant }\end{array}$ & 10009 & $\begin{array}{l}\text { R05 Type; P - Drinking Water Treatment } \\
\text { Plants }\end{array}$ & Rogue River Water Treatment Plant & Off Classick Drive & \begin{tabular}{|l} 
Rogue \\
River
\end{tabular} & Jackson & 2005 & DEQ/OHA SWA 2000 - 2005 \\
\hline $\begin{array}{l}\text { DWP - PCS - Rogue River Water } \\
\text { Treatment Plant }\end{array}$ & 10009 & $\begin{array}{l}\text { R05 Type; P - Drinking Water Treatment } \\
\text { Plants }\end{array}$ & Rogue River Water Treatment Plant & Off Classick Drive & $\begin{array}{l}\text { Rogue } \\
\text { River }\end{array}$ & Jackson & 2005 & DEQ/OHA SWA 2000 - 2005 \\
\hline $\begin{array}{l}\text { DWP - PCS - Rogue River Water } \\
\text { Treatment Plant }\end{array}$ & 10009 & $\begin{array}{l}\text { R05 Type; P - Drinking Water Treatment } \\
\text { Plants }\end{array}$ & Rogue River Water Treatment Plant & Off Classick Drive & \begin{tabular}{|l|} 
Rogue \\
River
\end{tabular} & Jackson & 2005 & DEQ/OHA SWA 2000 - 2005 \\
\hline $\begin{array}{l}\text { DWP - PCS - Rogue Valley Sash } \\
\text { and Door }\end{array}$ & 10422 & $\begin{array}{l}\text { C12 Type; P - Furniture/Lumber/Parts } \\
\text { Stores }\end{array}$ & Rogue Valley Sash and Door & $123 \mathrm{NE}$ Beacon & $\begin{array}{l}\text { Grants } \\
\text { Pass }\end{array}$ & Josephine & 2005 & DEQ/OHA SWA 2000 - 2005 \\
\hline $\begin{array}{l}\text { DWP - PCS - Rogue Valley Sash } \\
\text { and Door }\end{array}$ & 10422 & $\begin{array}{l}\text { M01 Type; P - Above Ground Storage } \\
\text { Tanks - Excluding Water and Residential } \\
\text { ASTs }\end{array}$ & Rogue Valley Sash and Door & 123 NE Beacon & $\begin{array}{l}\text { Grants } \\
\text { Pass }\end{array}$ & Josephine & 2005 & DEQ/OHA SWA 2000 - 2005 \\
\hline $\begin{array}{l}\text { DWP - PCS - Rogue Valley Sash } \\
\text { and Door }\end{array}$ & 10422 & $\begin{array}{l}\text { M26 Type; P - UST - Confirmed Leaking } \\
\text { but listed as NFA - DEQ LUST List }\end{array}$ & Rogue Valley Sash and Door & $123 \mathrm{NE}$ Beacon & $\begin{array}{l}\text { Grants } \\
\text { Pass }\end{array}$ & Josephine & 2005 & DEQ/OHA SWA 2000 - 2005 \\
\hline \begin{tabular}{|l|} 
DWP - PCS - Rolling Art \\
Restorations
\end{tabular} & 14242 & $\begin{array}{l}\text { C04 Type; P - Automobiles - Repair } \\
\text { Shops }\end{array}$ & Rolling Art Restorations & Highway 99 & $\begin{array}{l}\text { Grants } \\
\text { Pass }\end{array}$ & Josephine & 2005 & DEQ/OHA SWA 2000 - 2005 \\
\hline DWP - PCS - Rural Homes & 12314 & $\begin{array}{l}\text { M09 Type; A - Homesteads - Rural - } \\
\text { Septic Systems (<1/acre) }\end{array}$ & Rural Homes & Throughout DWPA & $\begin{array}{l}\text { Rogue } \\
\text { River }\end{array}$ & Jackson & 2005 & DEQ/OHA SWA 2000 - 2005 \\
\hline DWP - PCS - Rural Homes & 14771 & $\begin{array}{l}\text { M09 Type; A - Homesteads - Rural - } \\
\text { Septic Systems (<1/acre) }\end{array}$ & Rural Homes & Throughout DWPA & $\begin{array}{l}\text { Grants } \\
\text { Pass }\end{array}$ & Josephine & 2005 & DEQ/OHA SWA 2000 - 2005 \\
\hline DWP - PCS - Rural Homes & 14771 & $\begin{array}{l}\text { M09 Type; A - Homesteads - Rural - } \\
\text { Septic Systems (<1/acre) }\end{array}$ & Rural Homes & Throughout DWPA & $\begin{array}{l}\text { Grants } \\
\text { Pass }\end{array}$ & Josephine & 2005 & DEQ/OHA SWA 2000 - 2005 \\
\hline DWP - PCS - Rural Homes & 14771 & $\begin{array}{l}\text { M09 Type; A - Homesteads - Rural - } \\
\text { Septic Systems (<1/acre) }\end{array}$ & Rural Homes & Throughout DWPA & $\begin{array}{l}\text { Grants } \\
\text { Pass }\end{array}$ & Josephine & 2005 & DEQ/OHA SWA 2000 - 2005 \\
\hline DWP - PCS - Rural Homes & 14771 & $\begin{array}{l}\text { M09 Type; } A \text { - Homesteads - Rural - } \\
\text { Septic Systems (<1/acre) }\end{array}$ & Rural Homes & Throughout DWPA & $\begin{array}{l}\text { Grants } \\
\text { Pass }\end{array}$ & Josephine & 2005 & DEQ/OHA SWA 2000 - 2005 \\
\hline DWP - PCS - Rural Homesteads & 10015 & $\begin{array}{l}\text { M09 Type; } A \text { - Homesteads - Rural - } \\
\text { Septic Systems (<1/acre) }\end{array}$ & Rural Homesteads & Throughout DWPA & \begin{tabular}{|l|} 
Rogue \\
River
\end{tabular} & Jackson & 2005 & DEQ/OHA SWA 2000 - 2005 \\
\hline DWP - PCS - Rural Homesteads & 10015 & $\begin{array}{l}\text { M09 Type; } A \text { - Homesteads - Rural - } \\
\text { Septic Systems (<1/acre) }\end{array}$ & Rural Homesteads & Throughout DWPA & $\begin{array}{l}\text { Rogue } \\
\text { River }\end{array}$ & Jackson & 2005 & DEQ/OHA SWA 2000 - 2005 \\
\hline DWP - PCS - Rural Homesteads & 10435 & $\begin{array}{l}\text { M09 Type; A - Homesteads - Rural - } \\
\text { Septic Systems (<1/acre) }\end{array}$ & Rural Homesteads & Throughout DWPA & $\begin{array}{l}\text { Grants } \\
\text { Pass }\end{array}$ & Josephine & 2005 & DEQ/OHA SWA 2000 - 2005 \\
\hline $\begin{array}{l}\text { DWP - PCS - Sampson's Rogue } \\
\text { River Automotive }\end{array}$ & 12310 & $\begin{array}{l}\text { C04 Type; P - Automobiles - Repair } \\
\text { Shops }\end{array}$ & $\begin{array}{l}\text { Sampson's Rogue River } \\
\text { Automotive }\end{array}$ & Foothill Blvd & \begin{tabular}{|l|} 
Rogue \\
River
\end{tabular} & Jackson & 2005 & DEQ/OHA SWA 2000 - 2005 \\
\hline DWP - PCS - Schnitzer Steel & 10425 & C14 Type; P - Junk/Scrap/Salvage Yards & Schnitzer Steel & Off Agness Ave & $\begin{array}{l}\text { Grants } \\
\text { Pass }\end{array}$ & Josephine & 2005 & DEQ/OHA SWA 2000 - 2005 \\
\hline DWP - PCS - School & 14876 & R15 Type; P - Schools & School & Pine Street & $\begin{array}{l}\text { Rogue } \\
\text { River }\end{array}$ & Jackson & 2005 & DEQ/OHA SWA 2000 - 2005 \\
\hline DWP - PCS - School & 14876 & R15 Type; P - Schools & School & Pine Street & \begin{tabular}{|l|} 
Rogue \\
River
\end{tabular} & Jackson & 2005 & DEQ/OHA SWA 2000 - 2005 \\
\hline DWP - PCS - School & 14876 & R15 Type; P - Schools & School & Pine Street & $\begin{array}{l}\text { Rogue } \\
\text { River }\end{array}$ & Jackson & 2005 & DEQ/OHA SWA 2000 - 2005 \\
\hline DWP - PCS - School & 14876 & R15 Type; P - Schools & School & Pine Street & $\begin{array}{l}\text { Rogue } \\
\text { River }\end{array}$ & Jackson & 2005 & DEQ/OHA SWA $2000-2005$ \\
\hline DWP - PCS - Sewer Lines & 10004 & $\begin{array}{l}\text { R17 Type; A - Sewer Lines - Close } \\
\text { Proximity to PWS }\end{array}$ & Sewer Lines & Within Rogue River/Gold Hill & \begin{tabular}{|l|} 
Rogue \\
River
\end{tabular} & Jackson & 2005 & DEQ/OHA SWA 2000 - 2005 \\
\hline DWP - PCS - Sewer Lines & 10004 & $\begin{array}{l}\text { R17 Type; A - Sewer Lines - Close } \\
\text { Proximity to PWS }\end{array}$ & Sewer Lines & Within Rogue River/Gold Hill & \begin{tabular}{|l|} 
Rogue \\
River
\end{tabular} & Jackson & 2005 & DEQ/OHA SWA 2000 - 2005 \\
\hline DWP - PCS - Sewer Lines & 10004 & $\begin{array}{l}\text { R17 Type; A - Sewer Lines - Close } \\
\text { Proximity to PWS }\end{array}$ & Sewer Lines & Within Rogue River/Gold Hill & $\begin{array}{l}\text { Rogue } \\
\text { River }\end{array}$ & Jackson & 2005 & DEQ/OHA SWA $2000-2005$ \\
\hline DWP - PCS - Sewer Lines & 10004 & $\begin{array}{l}\text { R17 Type; A - Sewer Lines - Close } \\
\text { Proximity to PWS }\end{array}$ & Sewer Lines & Within Rogue River/Gold Hill & $\begin{array}{l}\text { Rogue } \\
\text { River }\end{array}$ & Jackson & 2005 & DEQ/OHA SWA 2000 - 2005 \\
\hline DWP - PCS - Sewer Lines & 10004 & $\begin{array}{l}\text { R17 Type; A - Sewer Lines - Close } \\
\text { Proximity to PWS }\end{array}$ & Sewer Lines & Within Rogue River/Gold Hill & \begin{tabular}{|l|} 
Rogue \\
River
\end{tabular} & Jackson & 2005 & DEQ/OHA SWA 2000 - 2005 \\
\hline
\end{tabular}




\begin{tabular}{|c|c|c|c|c|c|c|c|c|}
\hline Database Identifier (DB_ID) & Site Identifier & Status & Common Name & Address & City & County & \begin{tabular}{|l}
$\begin{array}{l}\text { Retrieval } \\
\text { Date }\end{array}$ \\
\end{tabular} & Data Source \\
\hline DWP - PCS - Sewer Lines & 12319 & $\begin{array}{l}\text { R17 Type; A - Sewer Lines - Close } \\
\text { Proximity to PWS }\end{array}$ & Sewer Lines & Throughout DWPA & Medford & Josephine & 2005 & DEQ/OHA SWA 2000 - 2005 \\
\hline DWP - PCS - Sewer Lines & 12335 & $\begin{array}{l}\text { R17 Type; A - Sewer Lines - Close } \\
\text { Proximity to PWS }\end{array}$ & Sewer Lines & Throughout DWPA & $\begin{array}{l}\text { Grants } \\
\text { Pass }\end{array}$ & Josephine & 2005 & DEQ/OHA SWA 2000 - 2005 \\
\hline DWP - PCS - Sewer Lines & 12335 & $\begin{array}{l}\text { R17 Type; A - Sewer Lines - Close } \\
\text { Proximity to PWS }\end{array}$ & Sewer Lines & Throughout DWPA & $\begin{array}{l}\text { Grants } \\
\text { Pass }\end{array}$ & Josephine & 2005 & DEQ/OHA SWA 2000 - 2005 \\
\hline DWP - PCS - Sewer Lines & 14241 & $\begin{array}{l}\text { R17 Type; A - Sewer Lines - Close } \\
\text { Proximity to PWS }\end{array}$ & Sewer Lines & Throughout DWPA & $\begin{array}{l}\text { Grants } \\
\text { Pass }\end{array}$ & Josephine & 2005 & DEQ/OHA SWA 2000 - 2005 \\
\hline DWP - PCS - Sewer Lines & 14772 & $\begin{array}{l}\text { R17 Type; A - Sewer Lines - Close } \\
\text { Proximity to PWS }\end{array}$ & Sewer Lines & Sky Crest Drive & $\begin{array}{l}\text { Grants } \\
\text { Pass }\end{array}$ & Josephine & 2005 & DEQ/OHA SWA 2000 - 2005 \\
\hline DWP - PCS - Sewer Lines & 14772 & $\begin{array}{l}\text { R17 Type; A - Sewer Lines - Close } \\
\text { Proximity to PWS }\end{array}$ & Sewer Lines & Sky Crest Drive & $\begin{array}{l}\text { Grants } \\
\text { Pass }\end{array}$ & Josephine & 2005 & DEQ/OHA SWA 2000 - 2005 \\
\hline DWP - PCS - Sewer Lines & 14772 & $\begin{array}{l}\text { R17 Type; A - Sewer Lines - Close } \\
\text { Proximity to PWS }\end{array}$ & Sewer Lines & Sky Crest Drive & $\begin{array}{l}\text { Grants } \\
\text { Pass }\end{array}$ & Josephine & 2005 & DEQ/OHA SWA 2000 - 2005 \\
\hline DWP - PCS - Sewer Lines & 14772 & $\begin{array}{l}\text { R17 Type; A - Sewer Lines - Close } \\
\text { Proximity to PWS }\end{array}$ & Sewer Lines & Sky Crest Drive & $\begin{array}{l}\text { Grants } \\
\text { Pass }\end{array}$ & Josephine & 2005 & DEQ/OHA SWA 2000 - 2005 \\
\hline DWP - PCS - Sewer Lines & 14898 & $\begin{array}{l}\text { R17 Type; A - Sewer Lines - Close } \\
\text { Proximity to PWS }\end{array}$ & Sewer Lines & \begin{tabular}{|l|} 
Throughout the City of Rogue \\
River
\end{tabular} & \begin{tabular}{|l|} 
Rogue \\
River
\end{tabular} & Jackson & 2005 & DEQ/OHA SWA 2000 - 2005 \\
\hline DWP - PCS - Sewerlines & 10407 & $\begin{array}{l}\text { R17 Type; A - Sewer Lines - Close } \\
\text { Proximity to PWS }\end{array}$ & Sewerlines & East half of DWPA & $\begin{array}{l}\text { Grants } \\
\text { Pass }\end{array}$ & Josephine & 2005 & DEQ/OHA SWA 2000 - 2005 \\
\hline DWP - PCS - Shell Gasoline & 14836 & $\begin{array}{l}\text { C03 Type; P - Automobiles - Gas } \\
\text { Stations }\end{array}$ & Shell Gasoline & Depot Street & $\begin{array}{l}\text { Rogue } \\
\text { River }\end{array}$ & Jackson & 2005 & DEQ/OHA SWA 2000 - 2005 \\
\hline DWP - PCS - Shell Gasoline & 14836 & $\begin{array}{l}\text { C03 Type; P - Automobiles - Gas } \\
\text { Stations }\end{array}$ & Shell Gasoline & Depot Street & \begin{tabular}{|l|} 
Rogue \\
River
\end{tabular} & Jackson & 2005 & DEQ/OHA SWA 2000 - 2005 \\
\hline DWP - PCS - Shell Gasoline & 14836 & $\begin{array}{l}\text { C03 Type; P - Automobiles - Gas } \\
\text { Stations }\end{array}$ & Shell Gasoline & Depot Street & \begin{tabular}{|l|} 
Rogue \\
River
\end{tabular} & Jackson & 2005 & DEQ/OHA SWA 2000 - 2005 \\
\hline DWP - PCS - Shell Gasoline & 14836 & $\begin{array}{l}\text { C03 Type; P - Automobiles - Gas } \\
\text { Stations }\end{array}$ & Shell Gasoline & Depot Street & $\begin{array}{l}\text { Rogue } \\
\text { River }\end{array}$ & Jackson & 2005 & DEQ/OHA SWA 2000 - 2005 \\
\hline $\begin{array}{l}\text { DWP - PCS - Southern Oregon } \\
\text { Dental Health and Hygiene }\end{array}$ & 14871 & C16 Type; P - Medical/Vet Offices & $\begin{array}{l}\text { Southern Oregon Dental Health and } \\
\text { Hygiene }\end{array}$ & Park Street & $\begin{array}{l}\text { Rogue } \\
\text { River }\end{array}$ & Jackson & 2005 & DEQ/OHA SWA 2000 - 2005 \\
\hline $\begin{array}{l}\text { DWP - PCS - Southern Oregon } \\
\text { Dental Health and Hygiene }\end{array}$ & 14871 & C16 Type; P - Medical/Vet Offices & $\begin{array}{l}\text { Southern Oregon Dental Health and } \\
\text { Hygiene }\end{array}$ & Park Street & $\begin{array}{l}\text { Rogue } \\
\text { River }\end{array}$ & Jackson & 2005 & DEQ/OHA SWA 2000 - 2005 \\
\hline $\begin{array}{l}\text { DWP - PCS - Southern Oregon } \\
\text { Dental Health and Hygiene }\end{array}$ & 14871 & C16 Type; P - Medical/Vet Offices & $\begin{array}{l}\text { Southern Oregon Dental Health and } \\
\text { Hygiene }\end{array}$ & Park Street & $\begin{array}{l}\text { Rogue } \\
\text { River }\end{array}$ & Jackson & 2005 & DEQ/OHA SWA 2000 - 2005 \\
\hline $\begin{array}{l}\text { DWP - PCS - Southern Oregon } \\
\text { Dental Health and Hygiene }\end{array}$ & 14871 & C16 Type; P - Medical/Vet Offices & $\begin{array}{l}\text { Southern Oregon Dental Health and } \\
\text { Hygiene }\end{array}$ & Park Street & $\begin{array}{l}\text { Rogue } \\
\text { River }\end{array}$ & Jackson & 2005 & DEQ/OHA SWA 2000 - 2005 \\
\hline DWP - PCS - Spaulding \& Son & 10428 & $\begin{array}{l}\text { C26 Type; P - Wood/Pulp/Paper } \\
\text { Processing and Mills }\end{array}$ & Spaulding \& Son & Spaulding Ave/NE "N" Street & $\begin{array}{l}\text { Grants } \\
\text { Pass }\end{array}$ & Josephine & 2005 & DEQ/OHA SWA 2000 - 2005 \\
\hline DWP - PCS - Spaulding \& Son & 10428 & $\begin{array}{l}\text { M26 Type; P - UST - Confirmed Leaking } \\
\text { but listed as NFA - DEQ LUST List }\end{array}$ & Spaulding \& Son & Spaulding Ave/NE "N" Street & $\begin{array}{l}\text { Grants } \\
\text { Pass }\end{array}$ & Josephine & 2005 & DEQ/OHA SWA 2000 - 2005 \\
\hline DWP - PCS - Spaulding \& Son & 10428 & M28 Type; P - UST - Status Unknown & Spaulding \& Son & Spaulding Ave/NE "N" Street & $\begin{array}{l}\text { Grants } \\
\text { Pass }\end{array}$ & Josephine & 2005 & DEQ/OHA SWA 2000 - 2005 \\
\hline $\begin{array}{l}\text { DWP - PCS - STEVE'S } \\
\text { MUFFLER AUTOMOTIVE }\end{array}$ & 1402 & $\begin{array}{l}\text { C04 Type; P - Automobiles - Repair } \\
\text { Shops }\end{array}$ & $\begin{array}{l}\text { STEVE'S MUFFLER } \\
\text { AUTOMOTIVE }\end{array}$ & \begin{tabular}{|l|} 
NORTH SIDE OF ROGUE \\
RIVER HWY/ACROSS FROM \\
JOSE'S AUTOBODY
\end{tabular} & $\begin{array}{l}\text { Grants } \\
\text { Pass }\end{array}$ & Josephine & 2005 & DEQ/OHA SWA 2000 - 2005 \\
\hline
\end{tabular}




\begin{tabular}{|c|c|c|c|c|c|c|c|c|}
\hline Database Identifier (DB_ID) & Site Identifier & Status & Common Name & Address & City & County & \begin{tabular}{|l} 
Retrieval \\
Date
\end{tabular} & Data Source \\
\hline $\begin{array}{l}\text { DWP - PCS - STEVE'S } \\
\text { MUFFLER AUTOMOTIVE }\end{array}$ & 1402 & $\begin{array}{l}\text { C04 Type; P - Automobiles - Repair } \\
\text { Shops }\end{array}$ & $\begin{array}{l}\text { STEVE'S MUFFLER } \\
\text { AUTOMOTIVE }\end{array}$ & \begin{tabular}{|l|} 
NORTH SIDE OF ROGUE \\
RIVER HWY/ACROSS FROM \\
JOSE'S AUTOBODY
\end{tabular} & $\begin{array}{l}\text { Grants } \\
\text { Pass }\end{array}$ & Josephine & 2005 & DEQ/OHA SWA 2000 - 2005 \\
\hline DWP - PCS - Substation & 10023 & \begin{tabular}{|l|} 
R18 Type; P - Utility \\
Stations/Powerplants - \\
Maintenance/Transformer Storage \\
\end{tabular} & Substation & Directly across from intake & $\begin{array}{l}\text { Rogue } \\
\text { River }\end{array}$ & Jackson & 2005 & DEQ/OHA SWA 2000 - 2005 \\
\hline DWP - PCS - Substation & 10405 & \begin{tabular}{|l|} 
R18 Type; $\mathrm{P}$ - Utility \\
Stations/Powerplants - \\
Maintenance/Transformer Storage \\
\end{tabular} & Substation & Opposite side of intake & $\begin{array}{l}\text { Grants } \\
\text { Pass }\end{array}$ & Josephine & 2005 & DEQ/OHA SWA 2000 - 2005 \\
\hline DWP - PCS - Suds N Duds & 14872 & C08 Type; P - Dry Cleaners & Suds N Duds & W. Main Street & \begin{tabular}{|l} 
Rogue \\
River
\end{tabular} & Jackson & 2005 & DEQ/OHA SWA 2000 - 2005 \\
\hline DWP - PCS - Suds N Duds & 14872 & C08 Type; P - Dry Cleaners & Suds N Duds & W. Main Street & $\begin{array}{l}\text { Rogue } \\
\text { River }\end{array}$ & Jackson & 2005 & DEQ/OHA SWA $2000-2005$ \\
\hline DWP - PCS - Suds N Duds & 14872 & C08 Type; P - Dry Cleaners & Suds N Duds & W. Main Street & $\begin{array}{l}\text { Rogue } \\
\text { River }\end{array}$ & Jackson & 2005 & DEQ/OHA SWA 2000 - 2005 \\
\hline DWP - PCS - Suds N Duds & 14872 & C08 Type; P - Dry Cleaners & Suds N Duds & W. Main Street & $\begin{array}{l}\text { Rogue } \\
\text { River }\end{array}$ & Jackson & 2005 & DEQ/OHA SWA 2000 - 2005 \\
\hline DWP - PCS - Transmission Lines & 10017 & $\begin{array}{l}\text { M17 Type; A - Transmission Lines - } \\
\text { Right-of-Ways }\end{array}$ & Transmission Lines & Throughout DWPA & \begin{tabular}{|l|} 
Rogue \\
River
\end{tabular} & Jackson & 2005 & DEQ/OHA SWA 2000 - 2005 \\
\hline DWP - PCS - Transmission Lines & 10439 & $\begin{array}{l}\text { M17 Type; A - Transmission Lines - } \\
\text { Right-of-Ways }\end{array}$ & Transmission Lines & East of intake & $\begin{array}{l}\text { Grants } \\
\text { Pass }\end{array}$ & Josephine & 2005 & DEQ/OHA SWA 2000 - 2005 \\
\hline DWP - PCS - Trask Industries & 10013 & $\begin{array}{l}\text { C17 Type; P - Metal } \\
\text { Plating/Finishing/Fabrication }\end{array}$ & Trask Industries & East of intake & $\begin{array}{l}\text { Rogue } \\
\text { River }\end{array}$ & Jackson & 2005 & DEQ/OHA SWA 2000 - 2005 \\
\hline DWP - PCS - Trask Industries & 10013 & $\begin{array}{l}\text { C17 Type; P - Metal } \\
\text { Plating/Finishing/Fabrication }\end{array}$ & Trask Industries & East of intake & $\begin{array}{l}\text { Rogue } \\
\text { River }\end{array}$ & Jackson & 2005 & DEQ/OHA SWA 2000 - 2005 \\
\hline DWP - PCS - Trask Industries & 10013 & $\begin{array}{l}\text { C17 Type; } P \text { - Metal } \\
\text { Plating/Finishing/Fabrication }\end{array}$ & Trask Industries & East of intake & $\begin{array}{l}\text { Rogue } \\
\text { River }\end{array}$ & Jackson & 2005 & DEQ/OHA SWA 2000 - 2005 \\
\hline DWP - PCS - Trask Industries & 10013 & $\begin{array}{l}\text { C17 Type; } P \text { - Metal } \\
\text { Plating/Finishing/Fabrication }\end{array}$ & Trask Industries & East of intake & $\begin{array}{l}\text { Rogue } \\
\text { River }\end{array}$ & Jackson & 2005 & DEQ/OHA SWA $2000-2005$ \\
\hline DWP - PCS - Trask Industries & 10013 & $\begin{array}{l}\text { C17 Type; P - Metal } \\
\text { Plating/Finishing/Fabrication }\end{array}$ & Trask Industries & East of intake & $\begin{array}{l}\text { Rogue } \\
\text { River }\end{array}$ & Jackson & 2005 & DEQ/OHA SWA 2000 - 2005 \\
\hline $\begin{array}{l}\text { DWP - PCS - Unknown } \\
\text { Operation }\end{array}$ & 14248 & M55 Type; P - Unknown Operation & Unknown Operation & Hamilton Lane & $\begin{array}{l}\text { Grants } \\
\text { Pass }\end{array}$ & Josephine & 2005 & DEQ/OHA SWA $2000-2005$ \\
\hline $\begin{array}{l}\text { DWP - PCS - US Forest } \\
\text { Industries }\end{array}$ & 10417 & $\begin{array}{l}\text { C26 Type; P - Wood/Pulp/Paper } \\
\text { Processing and Mills }\end{array}$ & US Forest Industries & 1090 SE "M" Street & $\begin{array}{l}\text { Grants } \\
\text { Pass }\end{array}$ & Josephine & 2005 & DEQ/OHA SWA 2000 - 2005 \\
\hline $\begin{array}{l}\text { DWP - PCS - US Forest } \\
\text { Industries }\end{array}$ & 10417 & $\begin{array}{l}\text { M01 Type; P - Above Ground Storage } \\
\text { Tanks - Excluding Water and Residential } \\
\text { ASTs }\end{array}$ & US Forest Industries & 1090 SE "M" Street & $\begin{array}{l}\text { Grants } \\
\text { Pass }\end{array}$ & Josephine & 2005 & DEQ/OHA SWA 2000 - 2005 \\
\hline $\begin{array}{l}\text { DWP - PCS - Valley of the Rogue } \\
\text { State Park }\end{array}$ & 10020 & $\begin{array}{l}\text { M14 Type; A - River Recreation - Heavy } \\
\text { Use (inc. campgrounds) }\end{array}$ & Valley of the Rogue State Park & Southeast of intake. & \begin{tabular}{|l|} 
Rogue \\
River
\end{tabular} & Jackson & 2005 & DEQ/OHA SWA $2000-2005$ \\
\hline $\begin{array}{l}\text { DWP - PCS - Valley of the Rogue } \\
\text { State Park }\end{array}$ & 10020 & $\begin{array}{l}\text { M24 Type; A - UST - } \\
\text { Decommissioned/Inactive }\end{array}$ & Valley of the Rogue State Park & Southeast of intake. & $\begin{array}{l}\text { Rogue } \\
\text { River }\end{array}$ & Jackson & 2005 & DEQ/OHA SWA $2000-2005$ \\
\hline $\begin{array}{l}\text { DWP - PCS - Valley of the Rogue } \\
\text { State Park }\end{array}$ & 10020 & $\begin{array}{l}\text { M31 Type; A - Large Capacity Septic } \\
\text { Systems (serves }>20 \text { people) - Class V } \\
\text { UICs }\end{array}$ & Valley of the Rogue State Park & Southeast of intake. & $\begin{array}{l}\text { Rogue } \\
\text { River }\end{array}$ & Jackson & 2005 & DEQ/OHA SWA $2000-2005$ \\
\hline $\begin{array}{l}\text { DWP - PCS - Valley of the Rogue } \\
\text { State Park }\end{array}$ & 10020 & $\begin{array}{l}\text { M60 Type; A - Other Injection/Dry Wells, } \\
\text { Sumps - Class V UICs }\end{array}$ & Valley of the Rogue State Park & Southeast of intake. & \begin{tabular}{|l|} 
Rogue \\
River \\
\end{tabular} & Jackson & 2005 & DEQ/OHA SWA $2000-2005$ \\
\hline $\begin{array}{l}\text { DWP - PCS - Valley of the Rogue } \\
\text { State Park }\end{array}$ & 10020 & $\begin{array}{l}\text { M62 Type; A - Lagoons/Liquid Wastes - } \\
\text { Municipal/Industrial Wastewater } \\
\text { Treatment } \\
\end{array}$ & Valley of the Rogue State Park & Southeast of intake. & $\begin{array}{l}\text { Rogue } \\
\text { River }\end{array}$ & Jackson & 2005 & DEQ/OHA SWA 2000 - 2005 \\
\hline $\begin{array}{l}\text { DWP - PCS - Valley of the Rogue } \\
\text { State Park }\end{array}$ & 10020 & R13 Type; A - Parks & Valley of the Rogue State Park & Southeast of intake. & $\begin{array}{l}\text { Rogue } \\
\text { River }\end{array}$ & Jackson & 2005 & DEQ/OHA SWA $2000-2005$ \\
\hline
\end{tabular}




\begin{tabular}{|c|c|c|c|c|c|c|c|c|}
\hline Database Identifier (DB_ID) & Site Identifier & Status & Common Name & Address & City & County & \begin{tabular}{|l} 
Retrieval \\
Date
\end{tabular} & Data Source \\
\hline DWP - PCS - WalMart & 10420 & $\begin{array}{l}\text { C20 Type; P - Parking Lots/Malls (> } 50 \\
\text { Spaces) }\end{array}$ & WalMart & Off Beacon and Terry Street & $\begin{array}{l}\text { Grants } \\
\text { Pass }\end{array}$ & Josephine & 2005 & DEQ/OHA SWA 2000 - 2005 \\
\hline DWP - PCS - Whitestone Estates & 14888 & $\begin{array}{l}\text { M32 Type; A - Construction/Demolition } \\
\text { Areas }\end{array}$ & Whitestone Estates & 719 3rd Street & $\begin{array}{l}\text { Rogue } \\
\text { River }\end{array}$ & Jackson & 2005 & DEQ/OHA SWA $2000-2005$ \\
\hline DWP - PCS - Whitestone Estates & 14888 & $\begin{array}{l}\text { M32 Type; A - Construction/Demolition } \\
\text { Areas }\end{array}$ & Whitestone Estates & 719 3rd Street & \begin{tabular}{|l|} 
Rogue \\
River
\end{tabular} & Jackson & 2005 & DEQ/OHA SWA $2000-2005$ \\
\hline DWP - PCS - Whitestone Estates & 14888 & $\begin{array}{l}\text { M32 Type; A - Construction/Demolition } \\
\text { Areas }\end{array}$ & Whitestone Estates & 719 3rd Street & \begin{tabular}{|l|} 
Rogue \\
River
\end{tabular} & Jackson & 2005 & DEQ/OHA SWA $2000-2005$ \\
\hline DWP - PCS - Whitestone Estates & 14888 & $\begin{array}{l}\text { M32 Type; A - Construction/Demolition } \\
\text { Areas }\end{array}$ & Whitestone Estates & 719 3rd Street & $\begin{array}{l}\text { Rogue } \\
\text { River }\end{array}$ & Jackson & 2005 & DEQ/OHA SWA 2000 - 2005 \\
\hline $\begin{array}{l}\text { DWP - PCS - Woodville } \\
\text { Cemetery }\end{array}$ & 14893 & R04 Type; P - Cemeteries - Pre-1945 & Woodville Cemetery & Wards Creek Drive & $\begin{array}{l}\text { Rogue } \\
\text { River }\end{array}$ & Jackson & 2005 & DEQ/OHA SWA $2000-2005$ \\
\hline $\begin{array}{l}\text { DWP - PCS - Woodville } \\
\text { Cemetery }\end{array}$ & 14893 & R04 Type; P - Cemeteries - Pre-1945 & Woodville Cemetery & Wards Creek Drive & $\begin{array}{l}\text { Rogue } \\
\text { River }\end{array}$ & Jackson & 2005 & DEQ/OHA SWA $2000-2005$ \\
\hline $\begin{array}{l}\text { DWP - PCS - Woodville } \\
\text { Cemetery }\end{array}$ & 14893 & R04 Type; P - Cemeteries - Pre-1945 & Woodville Cemetery & Wards Creek Drive & $\begin{array}{l}\text { Rogue } \\
\text { River }\end{array}$ & Jackson & 2005 & DEQ/OHA SWA $2000-2005$ \\
\hline $\begin{array}{l}\text { DWP - PCS - Woodville } \\
\text { Cemetery }\end{array}$ & 14893 & R04 Type; P - Cemeteries - Pre-1945 & Woodville Cemetery & Wards Creek Drive & $\begin{array}{l}\text { Rogue } \\
\text { River }\end{array}$ & Jackson & 2005 & DEQ/OHA SWA $2000-2005$ \\
\hline $\begin{array}{l}\text { DWP - PCS - Woodville } \\
\text { Cemetery }\end{array}$ & 14893 & R04 Type; P - Cemeteries - Pre-1945 & Woodville Cemetery & Wards Creek Drive & $\begin{array}{l}\text { Rogue } \\
\text { River }\end{array}$ & Jackson & 2005 & DEQ/OHA SWA $2000-2005$ \\
\hline
\end{tabular}


APPENDIX B. Memorandum of Agreement - Rogue Drinking Water Partnership 


\title{
Memorandum of Agreement - Rogue Drinking Water Partnership
}

\author{
February 3, 2020
}

\section{Background}

The overall goal of the Rogue Drinking Water Partnership (RDWP) is to protect sources of drinking water and improve drinking water quality in the Rogue River Basin by sustaining a collaborative partnership of drinking water providers and other organizations concerned with clean drinking water. Protecting the quality and quantity of our drinking water resources not only serves public health, but also benefits nature. It is the underlying intent of the RDWP to develop and support strategies designed to protect the lakes, streams, rivers and aquifers used for drinking water and the land which protects and recharges these sources of water. To ensure that the RDWP is useful to our members and other users, we have developed a Memorandum of Agreement (MOA), the Rogue Drinking Water Partnership Agreement, between participating drinking water providers and other interested organizations committing to engagement and cooperation in drinking water source protection. This agreement was created through an open and collaborative process in RDWP meetings and discussions between members. This MOA asks for a commitment to engage and cooperate. A major benefit of the collaboration will be a common understanding of the greatest threats to drinking water sources and agreement on the highest priority actions to protect source water. This MOA describes the purpose, vision, structure, principles, operations and outputs of the RDWP.

\section{Purpose and Vision of RDWP}

The RDWP meets quarterly to share information, determine ways to effectively collaborate on mutually beneficial projects, assess RDWP progress on projects, and act in an advisory capacity on a wide range of decisions and products, such as current and projected water quality conditions; monitoring; data analysis; water quality treatment; agency initiatives and requirements; geographic areas of interest in the Rogue source watersheds; sociological and political areas of concern for drinking water; communications and public outreach plans, messages and means; technical assistance; training opportunities; grant funding; and identification of high priority collaborative projects, e.g., emergency response and contingency plans and programs.

RDWP Vision

$>$ To foster closer relationships with each other.

$>$ To collectively coordinate and obtain funding for source water protection efforts, public outreach and education.

$>$ To realize economies of scale and save money by sharing in costs.

$>$ To speak in one voice regarding drinking water resource issues.

$>$ To protect the Rogue River Basin's drinking water resources to provide safe, sustainable drinking water for the benefit of all citizens. 
The Rogue Drinking Water Partnership Agreement is:

- A living document to be maintained and modified by the RDWP.

- A procedural guide, describing the operation of the RDWP's collaborative work and how RDWP members commit to interact constructively in good faith.

- An informal, non-binding agreement among RDWP members.

- A public document, available for anyone to read and review. Comments about this document should be addressed to the RDWP Coordinator.

\section{RDWP Structure, Composition}

The RDWP is comprised of drinking water providers and other key interest groups that represent a wide set of sectors and perspectives. Organizations can become members of the RDWP by adopting this MOA and regularly attending RDWP meetings and activities. The following list includes organizations that have attended RDWP meetings and expressed interest in membership, but it is not an exhaustive list. Other organizations may become members of the RDWP by adopting the MOA and actively participating in RDWP meetings and activities.

RDWP organizations and team members:

Drinking Water Providers -

City of Ashland

City of Central Point

City of Eagle Point

City of Gold Hill

City of Grants Pass

City of Jacksonville

City of Phoenix

City of Rogue River

City of Talent

Medford Water Commission

Anglers Cove/Shady Cove Hiland Water Co.

Country View Mobile Home Estates

Other Organizations -

City of Cave Junction

City of Shady Cove

Jackson Soil and Water Conservation District

Oregon Department of Environmental Quality

Rogue River Watershed Council

Rogue Valley Council of Governments

Rogue Valley Sewer Services

\section{Roles and Responsibilities}

The RDWP will be coordinated by an organization or individual acceptable to all members of the RDWP. The Coordinator will be responsible for convening and facilitating meetings;

summarizing meetings; soliciting, researching, and summarizing end user needs and feedback; 
developing and disseminating RDWP-related materials; and other coordinating tasks as necessary.

RDWP organizations will appoint a member who will serve as the main point of contact to their respective organizations, gathering needs and suggestions from their organizations, keeping their organizations informed of relevant RDWP activities; and bringing information and perspectives from their organizations to inform RDWP business. Another person from the member organization will be able to fill in for the primary member if he or she is unable to attend a meeting or participate in an activity.

RDWP members are expected to read all meeting preparation materials before the meetings take place and to attend all meetings, if possible. Additional roles and tasks for individual RDWP members may be identified and agreed upon by the RDWP.

\section{Meetings and Communications}

Meetings will be held as frequently as the RDWP decides is useful to advancing the goals of the RDWP and ensuring a productive collaborative process. As of January 2020, the anticipated schedule is quarterly meetings of approximately 2 hours in length. All meetings will be held in locations convenient for the majority of the RDWP, and off-site participation will be made available upon request when possible.

Meeting notes will be drafted by the Coordinator and reviewed at the following meeting by the RDWP before being considered a part of the RDWP record or being distributed.

Each meeting will end with a brief and informal dialogue to ensure that progress is being made and that RDWP members feel that their time is used wisely.

In addition to regularly scheduled meetings, RDWP members may be asked from time to time to review documents, offer opinions, or consult individually or in groups with the Coordinator and other RDWP members.

\section{Operating Protocols}

\section{General Principles of Collaboration}

Using the following general principles of collaboration, RDWP members agree to:

- Commit to expending the time, energy, and organizational resources necessary to meet objectives mutually agreed upon by the RDWP.

- Recognize the validity of differing points of view.

- Be prepared to listen intently to understand others' views.

- Regard disagreements as problems to be solved, not battles to be won.

\section{Decision Making}

RDWP members will strive for consensus when making decisions on substantive issues and recommendations. If consensus cannot be reached, decisions will be made on a super-majority vote (at least $2 / 3$ of members present). 


\section{Conflict Resolution}

Should disagreement arise among RDWP members (e.g., over data sources or interpretation, RDWP outputs, etc.), the group will strive to first resolve the disagreement internally with the assistance of the RDWP Coordinator. Should conflict remain, other appropriate conflict resolution methods will be explored, e.g., professional facilitation or mediation.

\section{Outputs}

\section{Meeting Records}

Meeting agendas and meeting notes with action items, decisions, and recommendations will be distributed to RDWP members and kept by the Coordinator. These notes and other documents produced by the RDWP will serve as the sources of documentation for the collaborative process. RDWP members must review and refine these notes and documents before they are made public.

\section{Needs Assessment}

RDWP members and Coordinator will gather input from RDWP members and draft a concise needs assessment describing the RDWP niche in drinking water source protection, including primary measurable objectives, existing organizations and programs, barriers, target audiences, etc. The role of the RDWP is to provide feedback and input on drafts, to agree that the documents accurately reflect pressing needs, and to recommend findings from this needs assessment to be incorporated into the next stages of RDWP development.

\section{RDWP Action Plan}

An Action Plan will be developed by the RDWP identifying short- and long-term high priority measures for collaborative source water protection to be implemented by the RDWP and its members. The Action Plan will identify measures that:

$>$ Prevent, minimize and mitigate activities that have known or potentially harmful impacts on drinking water quality.

$>$ Promote public awareness and stewardship of drinking water sources and healthy watersheds in collaboration with other stakeholders.

$>$ Develop resources and financial support for RDWP Action Plan implementation.

The Action Plan will be reviewed and revised as needed on a five-year basis. 
APPENDIX C. Conceptual Model - Risk Matrix 


\section{Conceptual Model - Risk Matrix}

Using interactive maps* from the City of Grants Pass (City) and the source water assessments (SWA) for Grants Pass from the Oregon Department of Environmental Quality (ODEQ), the following components and scoring were created to identify high priority potential contaminant sources (PCS). As a result of using this risk matrix, site-specific best management practices (BMP) recommendations will be determined for each high priority PCS within Grants Pass, which the City may offer to businesses in order to protect drinking water quality.

*The interactive maps used included the Public Works - Water, Sewer and Stormwater Systems and Hazards Fire, Flood, Earthquake, \& Steep Slope, which can be found here:

https://grantspassoregon.maps.arcgis.com/home/index.html.

\section{Scoring Ranges:}

- 1-4: $\quad$ Low (L) risk; green circle on map*

- 5-8: $\quad$ Moderate (M) risk; yellow circle on map*

- 9-13: High $(\mathrm{H})$ risk; red circle on map*

*Colored circles will also be labeled with either an "A," for area of interest, or "P," for point of interest on the associated map.*

\section{Stormwater Basin:}

\begin{tabular}{|l|l|}
\hline \multicolumn{1}{|c|}{ Score: } & \multicolumn{1}{c|}{ General Description: } \\
\hline 0 & $\begin{array}{l}\text { Gilbert/Allen/Sand creeks are downstream of the } \\
\text { drinking water intake }\end{array}$ \\
\hline 1 & $\begin{array}{l}\text { "Needs Basin ID" is upstream of the drinking water } \\
\text { intake, but does not cover any urban or industrial } \\
\text { areas }\end{array}$ \\
\hline 2 & $\begin{array}{l}\text { Jones Creek is farther upstream from the city center, } \\
\text { but does cover industrial areas }\end{array}$ \\
\hline 3 & $\begin{array}{l}\text { Skunk/Fruitdale creeks cover the city center, } \\
\text { industrial areas, and are both mostly upstream of the } \\
\text { drinking water intake }\end{array}$ \\
\hline
\end{tabular}

Stormwater Basin Data from the Interactive Maps, City of Grants Pass. 
2. Proximity to Waterway (river, tributary, irrigation canal):

\begin{tabular}{|l|l|}
\hline \multicolumn{1}{|c|}{ Score: } & \multicolumn{1}{|c|}{ General Description: } \\
\hline 0 & $1,500^{\prime}$ and beyond \\
\hline 1 & $1,001^{\prime}-1,499^{\prime}$ \\
\hline 2 & $501^{\prime}-1,000^{\prime}$ \\
\hline 3 & $0-500^{\prime}$ \\
\hline
\end{tabular}

\section{Surface Water (SW) Risk:}

\begin{tabular}{|l|l|}
\hline \multicolumn{1}{|c|}{ Score: } & \multicolumn{1}{|c|}{ General Description: } \\
\hline 0 & Low $(\mathrm{L})$ risk \\
\hline 1 & Medium $(\mathrm{M})$ risk \\
\hline 2 & High $(\mathrm{H})$ risk \\
\hline
\end{tabular}

Surface Water (SW) Risk Data from the SWAs, ODEQ.

\section{Flood Hazard:}

\begin{tabular}{|l|l|}
\hline \multicolumn{1}{|c|}{ Score: } & \multicolumn{1}{c|}{ General Description: } \\
\hline 0 & Beyond the flood hazard area \\
\hline 1 & 500 -year flood \\
\hline 2 & 100 -year flood \\
\hline 3 & Floodway \\
\hline
\end{tabular}

Flood Hazard Data from the Interactive Maps, City of Grants Pass.

\section{Jurisdiction:}

\begin{tabular}{|l|l|}
\hline \multicolumn{1}{|c|}{ Score: } & \multicolumn{1}{c|}{ General Description: } \\
\hline 1 & Outside the urban growth boundary (UGB) \\
\hline 2 & Inside the UGB \\
\hline
\end{tabular}

Jurisdiction Data from the Interactive Maps, City of Grants Pass. 
APPENDIX D. Directions - Applying the Risk Matrix to GIS 
Rogue Drinking Water Partnership

Directions - Applying the Risk Matrix to GIS

Amie Siedlecki

$7 / 30 / 19$

\section{$\underline{\text { Area of Interest: }}$}

1. Start the "Editor" tool,

2. Create a CityName_AOI polygon layer by clicking on four points on the map (creates a square or rectangular area) that captures both the City Limits and/or Urban Growth Boundary (UGB) layers,

3. Stop editing, and

4. Clip all layers to be completely within the CityName_AOI.

\section{PCS (Surface Water Risk):}

1. Select by location,

2. Use RDWP_PCS layer,

3. Select "completely within" the AOI layer, and

4. Create a new layer: RDWP_PCS_selection (rename if needed).

\section{Proximity to Waterway:}

1. Turn on both the Ditches and Waterlines layers,

2. Create one layer that combines the two layers,

3. Select by location,

4. Use the combined Ditches and Waterlines layer,

5. Select "completely within" the AOI layer,

6. Use the "Buffer" tool to create $500 \mathrm{ft} ., 1,000 \mathrm{ft}$., and 1,500 ft. buffers around the CityName_AOI_Water layer. (500 ft., FULL, FLAT; 1,000 ft., FULL, FLAT; 1,500ft., FULL, FLAT, etc.),

7. Save each layer as CityName_500ft_Buffer, CityName_1000ft_Buffer, CityName_1500ft_Buffer, etc.

8. Use the "Overlay Analysis - Overlay Erase" tool to erase the 1,000 ft. buffer from the $1,500 \mathrm{ft}$. buffer, and the $500 \mathrm{ft}$. buffer from the 1,000 ft. buffer, which separates each buffer out from the others. (Example: 1,500 ft. is the input, 1,000 ft. is the layer to be erased; larger number erases the smaller number).

\section{Floodplain (Flood Hazard):}

1. Turn on the Floodplain layer,

2. Use the "Clip" tool to show only the Floodplain area that lies within the AOI layer, and

3. Create a new layer: CityName_Floodplain.

4. Select by attributes to visually show the "floodway," "100-year flood zone," "500-year flood zone," and beyond: "Flood" = "Floodway," etc., and

5. Create a selection layer for each of the flood zones. 


\section{Attribute Table Preparation:}

1. Using the "Editor" tool, add risk component column headers into the original RDWP_PCS layer attribute table: SWScore, Jurisd., Proximity, and Floodzone,

2. Select by attributes,

3. Use RDWP_PCS layer,

4. Select "H" risk "within" the CityName_500ft_Buffer,

5. Enter the scores associated with where the PCS fall on the map,

6. Repeat for CityName_1000ft_Buffer and CityName_1500ft_Buffer, as well as "M" risk,

7. Use the "Editor" tool to add scores into the attribute tables, save, and "Stop Editing,"

8. Merge the "H" and "M" PCS into one shapefile,

9. Export data to an Excel spreadsheet, and

10. Create a "Total Score" column in Excel and add up the scores to determine overall risk and where windshield surveys will be conducted. 
APPENDIX E. Windshield Survey Sites 


\section{Grants Pass Stormwater Project Windshield Survey Sites}

Amie Siedlecki

$12 / 30 / 18$

\section{Visited in the Following Order:}

1. Swiss Metric - 1610 Parkdale.

2. Parkdale Subdivision - Established at time of survey; no perceived surface water (SW) risk.

3. Jerry's Appliance and Repair - 1077 Rogue River Highway; closed.

4. Automania Car Sales - 1455 Rogue River Highway; moved to Gladiola.

5. Brakes Plus - 1499 Rogue River Highway.

6. High density housing - 1560 Hamilton Lane - Established at time of survey; no perceived SW risk.

7. Heating Oil Tank - 1560 Hamilton Lane; could not find.

8. Unknown Operation - Hamilton Lane; could not find.

9. Clovercrest Service Center - 2600 Cloverlawn Lane; now NAPA Autocare Center.

10. Panoramic Loop Construction - Established at time of survey; no perceived SW risk.

11. Willow Development - 2221 Haviland - Established at time of survey; no perceived SW risk.

12. Grants Pass Irrigation District (GPID) Easement - 2202 Haviland.

13. Powell Distributing - 1919 Rogue River Highway; now Timber Mountain Construction, Inc.

14. Robco/Three Rivers Paving - 1935 Rogue River Highway; now Timber Mountain Construction, Inc.

15. Big Sam's Auto Repair - Highway 99; could not find.

16. Heating Oil Tank - 1980 Rogue River Highway; could not find.

17. Muffler King/A-1 Exterminators - 1492 Rogue River Highway; closed.

18. High density housing - SE Mystic Drive - Established at time of survey; no perceived SW risk.

19. Caveman RV - 1182/1190 Rogue River Highway.

20. Gary Peterson - 910 SE M Street.

21. Auto Wrecker - M Street; could not determine by listing.

22. Warehouse Rebuilding - 961 SE N Street; moved to Gladiola.

23. Grants Pass Equipment Rentals - 851 SE Milbank; now Southern Oregon Tire.

24. Oregon Swiss Precision - 1080 SE M Street; moved. Now Caveman Fence.

25. Timber Products Company - 1090 SE M Street.

26. Indian Creek Auto Dismantlers - 1098 SE M Street.

27. Cabinet Crafters - 1586 SE N Street; could not find.

28. Umpqua Dairy Products Company - 1686 SE N Street.

29. Ferguson Fabrication - 959 Gladiola; moved. Now Warehouse Rebuilders.

30. Grants Pass School District 7 - 1053 Gladiola.

31. Clearwater Co-op - 1130 Gladiola; appeared closed.

32. Gladiola Campus - 1137 Gladiola.

33. Riverside Elementary School - 1200 Harvey.

34. Spalding and Son - 2345 SE N Street; also on Spalding Ave.

35. Roger Hansen - 450 Ament; could not find. Illegal dump site on the other side of the road.

36. RV Marble/Castle Marble and Granite LLC - 2375 Foothill Boulevard.

37. Oregon Department of Transportation - 345 Agness.

38. Edgewater Christian Office - 101 Assembly Circle - Established at time of survey; no perceived SW risk.

39. Edmond Corporation/Schnitzer Steel - 605 Agness; moved. 
APPENDIX F. Source Water Protection Inspection Worksheet 
Grants Pass Stormwater Project:

Source Water Protection Inspection Worksheet

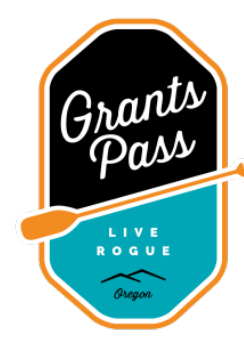

\begin{tabular}{|l|c|}
\hline \multicolumn{2}{|c|}{ Facility Information } \\
\hline Facility Name & \\
\hline Facility Address/Phone Number & \\
\hline Receiving Waterbody & \\
\hline
\end{tabular}

\begin{tabular}{|l|l|l|}
\hline \multicolumn{3}{|c|}{ General Information } \\
\hline Date & Name(s) & \\
\hline Weather Conditions & Time & \\
\end{tabular}

\begin{tabular}{|l|l|}
\hline \multicolumn{2}{|c|}{ Site Information } \\
\hline Facility Description & \\
\hline Activity at Site & \\
\hline
\end{tabular}


Grants Pass Stormwater Project:

Source Water Protection Inspection Worksheet

\section{Observations:}

\begin{tabular}{|c|c|c|c|c|}
\hline Item & & & & Notes/Describe Observation \\
\hline $\begin{array}{l}\text { Evidence of visual sheen } \\
\text { (oil/grease) on ground or } \\
\text { other surfaces? }\end{array}$ & & $\begin{array}{l}\text { No } \\
\square\end{array}$ & $\begin{array}{l}\text { N/A } \\
\square\end{array}$ & \\
\hline $\begin{array}{l}\text { Evidence of spilled } \\
\text { materials? Note location, } \\
\text { substance, and container. }\end{array}$ & & $\begin{array}{l}\text { No } \\
\square\end{array}$ & $\begin{array}{l}\text { N/A } \\
\square\end{array}$ & \\
\hline $\begin{array}{l}\text { Evidence of erosion, } \\
\text { water movement, pooling } \\
\text { water? }\end{array}$ & $\begin{array}{l}\text { Ye } \\
\square\end{array}$ & $\begin{array}{l}\text { No } \\
\square\end{array}$ & $\begin{array}{l}\text { N/A } \\
\square\end{array}$ & \\
\hline $\begin{array}{l}\text { Bulk storage areas } \\
\text { present (tanks, drums, } \\
\text { pallets, other } \\
\text { materials/supplies, etc.)? }\end{array}$ & $\begin{array}{l}\text { Ye } \\
\square\end{array}$ & $\begin{array}{l}\text { No } \\
\square\end{array}$ & $\begin{array}{l}\text { N/A } \\
\square\end{array}$ & \\
\hline $\begin{array}{l}\text { Loading/unloading or } \\
\text { shipping/receiving areas } \\
\text { present? }\end{array}$ & $\begin{array}{l}\text { Ye } \\
\square \\
\text { Co } \\
\text { Ye } \\
\square\end{array}$ & $\begin{array}{c}\text { No } \\
\square \\
\text { ed? } \\
\text { No } \\
\square\end{array}$ & $\begin{array}{l}\mathrm{N} / \mathrm{A} \\
\square\end{array}$ & \\
\hline $\begin{array}{l}\text { Are raw materials, } \\
\text { intermediate product, by- } \\
\text { product, and/or final } \\
\text { product storage on site? }\end{array}$ & $\begin{array}{l}\text { Ye } \\
\square\end{array}$ & $\begin{array}{l}\text { No } \\
\square\end{array}$ & $\begin{array}{l}\text { N/A } \\
\square\end{array}$ & \\
\hline
\end{tabular}




\begin{tabular}{|c|c|c|}
\hline Item & & Notes/Describe Observation \\
\hline $\begin{array}{l}\text { Evidence of excavation or } \\
\text { ground disturbance? }\end{array}$ & 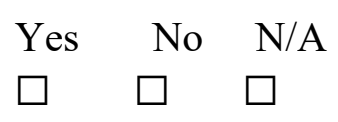 & \\
\hline $\begin{array}{l}\text { Vehicle maintenance } \\
\text { areas present? }\end{array}$ & 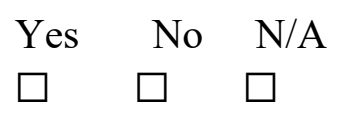 & \\
\hline $\begin{array}{l}\text { Gas, oil, and grease } \\
\text { storage and management } \\
\text { areas present? }\end{array}$ & $\begin{array}{ll}\text { Yes } & \text { No } \quad \text { N/A } \\
\square & \square \quad \square\end{array}$ & \\
\hline $\begin{array}{l}\text { Chemical storage and } \\
\text { management areas } \\
\text { present? Describe. }\end{array}$ & $\begin{array}{ll}\text { Yes } & \text { No } \quad \text { N/A } \\
\square & \square \quad \square\end{array}$ & \\
\hline $\begin{array}{l}\text { Any erosion and } \\
\text { sediment control } \\
\text { features? Are they } \\
\text { functioning? }\end{array}$ & $\begin{array}{l}\text { Yes No N/A } \\
\square \quad \square \quad \square \\
\text { Functioning? } \\
\text { Yes No } \\
\square \quad \square\end{array}$ & \\
\hline $\begin{array}{l}\text { Storm drains or } \\
\text { catchment basins } \\
\text { present? }\end{array}$ & $\begin{array}{l}\text { Yes No N/A } \\
\square \quad \square \quad \square \\
\text { Functioning? } \\
\text { Yes No } \\
\square \quad \square\end{array}$ & \\
\hline $\begin{array}{l}\text { Wash and drainage } \\
\text { areas present? Describe } \\
\text { plumbing of wash area: } \\
\text { Sanitary? Storm? } \\
\text { Unknown? }\end{array}$ & 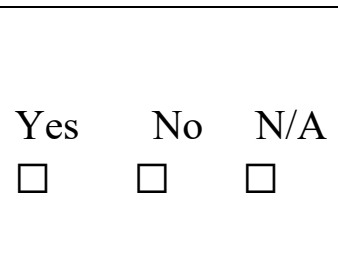 & \\
\hline $\begin{array}{l}\text { How was plumbing } \\
\text { verified? Verbal? Dye } \\
\text { test? As-builts? }\end{array}$ & $\begin{array}{ll}\text { Yes } & \text { No } \quad \text { N/A } \\
\square & \square \quad \square\end{array}$ & \\
\hline
\end{tabular}




\begin{tabular}{|l|l|l|}
\hline \multicolumn{3}{|c|}{ Stormwater Runoff } \\
\hline $\begin{array}{l}\text { Was stormwater } \\
\text { discharging from the } \\
\text { site at time of } \\
\text { inspection? }\end{array}$ & $\begin{array}{l}\text { Yes No } \\
\text { N/A } \square \\
\square\end{array}$ & If yes, describe volume (estimated CFS), color, and odor: \\
\hline $\begin{array}{l}\text { Does there appear to be } \\
\text { any stormwater BMPs } \\
\text { in place? }\end{array}$ & $\begin{array}{l}\text { Yes No } \\
\text { N/A } \\
\square\end{array}$ & \\
\hline $\begin{array}{l}\text { If there are BMPs, are } \\
\text { they functioning as } \\
\text { intended? }\end{array}$ & $\begin{array}{l}\text { Yes No } \\
\text { N/A } \square\end{array}$ & \\
\hline & \\
\hline
\end{tabular}

\begin{tabular}{|l|l|}
\hline \multicolumn{1}{|c|}{ Stormwater Controls Observed } \\
\hline & $\square$ Catch basin or other inlet treatment/filter system \\
& $\square$ Oil/water separator \\
& $\square$ On-site bio-swale \\
& $\square$ Constructed wetlands \\
& $\square$ Roof downspout (gutter) treatment/filter system \\
Control Measures & $\square$ Dumpster and equipment cover \\
Observed (BMPs) & $\square$ Drum and container containment \\
& $\square$ Sweeping (manual or mechanical) \\
& $\square$ Stormwater diversion \\
& $\square$ Vegetated buffer zone \\
& $\square$ Storm treatment system (stormwater is sent to the log pond) \\
& $\square$ Multi-chambered treatment train \\
\hline
\end{tabular}

\section{Stormwater Pollution Control Plan (SWPCP) or Spill Plan}

\begin{tabular}{|c|c|}
\hline Is there a SWPCP? & $\begin{array}{lll}\text { Yes } & \text { No } & \text { N/A } \\
\square & \square & \square\end{array}$ \\
\hline $\begin{array}{l}\text { Is there a spill } \\
\text { prevention and } \\
\text { response plan? }\end{array}$ & $\begin{array}{lll}\text { Yes } & \text { No } & \text { N/A } \\
\square & \square & \square\end{array}$ \\
\hline $\begin{array}{l}\text { Are employees aware } \\
\text { of and understand } \\
\text { spill plan? }\end{array}$ & $\begin{array}{lll}\text { Yes } & \text { No } & \text { N/A } \\
\square & \square & \square\end{array}$ \\
\hline
\end{tabular}


Photo Log: Photo naming convention: Date, Facility Name, and photo \#.

Photo \# Describe general location Describe what you are trying to show in photo

\begin{tabular}{|l|l|l|}
\hline & & \\
\hline & & \\
\hline & & \\
\hline & & \\
\hline & & \\
\hline & & \\
\hline
\end{tabular}


APPENDIX G. BMP Database 


\begin{tabular}{|c|c|c|c|c|}
\hline PCS Code & Activity at Site & SW Risk & BMP Code & BMP Name \\
\hline \begin{tabular}{l|l}
$\mathrm{A} 03$ \\
\end{tabular} & Confined Animal Feeding Operations (CAFOs) & \begin{tabular}{|l|l}
$\mathrm{H}$ & $\mathrm{r}$ \\
\end{tabular} & \begin{tabular}{|l|l} 
BMP17 \\
\end{tabular} & Stormwater Wetland \\
\hline A03 & Confined Animal Feeding Operations (CAFOs) & $\mathrm{H}$ & BMP20 & Bio-Retention Practices \\
\hline A04 & Crops - Irrigated - Berries, Hops, Mint, Orchards, Vineyards/Nurseries, Green Houses, Vegetables, etc. & $\mathrm{H}$ & BMP3 & Preservation of Existing Vegetation \\
\hline A04 & Crops - Irrigated - Berries, Hops, Mint, Orchards, Vineyards/Nurseries, Green Houses, Vegetables, etc. & $\mathrm{H}$ & BMP9 & Inlet Protection \\
\hline $\mathrm{A} 04$ & Crops - Irrigated - Berries, Hops, Mint, Orchards, Vineyards/Nurseries, Green Houses, Vegetables, etc. & $\mathrm{H}$ & BMP10 & Fiber Rolls or Wattles \\
\hline A04 & Crops - Irrigated - Berries, Hops, Mint, Orchards, Vineyards/Nurseries, Green Houses, Vegetables, etc. & $\mathrm{H}$ & BMP12 & Stockpile and Materials Management \\
\hline A04 & Crops - Irrigated - Berries, Hops, Mint, Orchards, Vineyards/Nurseries, Green Houses, Vegetables, etc. & $\mathrm{H}$ & BMP14 & Wet Pond \\
\hline A04 & Crops - Irrigated - Berries, Hops, Mint, Orchards, Vineyards/Nurseries, Green Houses, Vegetables, etc. & $\mathrm{H}$ & BMP15 & Wet Extended Detention Pond \\
\hline A04 & Crops - Irrigated - Berries, Hops, Mint, Orchards, Vineyards/Nurseries, Green Houses, Vegetables, etc. & $\mathrm{H}$ & BMP16 & Dry Pond \\
\hline A04 & Crops - Irrigated - Berries, Hops, Mint, Orchards, Vineyards/Nurseries, Green Houses, Vegetables, etc. & $\mathrm{H}$ & BMP17 & Stormwater Wetland \\
\hline A04 & Crops - Irrigated - Berries, Hops, Mint, Orchards, Vineyards/Nurseries, Green Houses, Vegetables, etc. & $\mathrm{H}$ & BMP18 & Infiltration Trench \\
\hline A04 & Crops - Irrigated - Berries, Hops, Mint, Orchards, Vineyards/Nurseries, Green Houses, Vegetables, etc. & $\mathrm{H}$ & BMP19 & Infiltration Basin \\
\hline $\mathrm{A} 04$ & Crops - Irrigated - Berries, Hops, Mint, Orchards, Vineyards/Nurseries, Green Houses, Vegetables, etc. & $\mathrm{H}$ & BMP47 & Proper Garden and Lawn Maintenance \\
\hline A04 & Crops - Irrigated - Berries, Hops, Mint, Orchards, Vineyards/Nurseries, Green Houses, Vegetables, etc. & $\mathrm{H}$ & BMP48 & Use Pesticide Alternatives \\
\hline A04 & Crops - Irrigated - Berries, Hops, Mint, Orchards, Vineyards/Nurseries, Green Houses, Vegetables, etc. & $\mathrm{H}$ & BMP51 & Materials Safety Data Sheets (MSDS) On-Hand \\
\hline A07 & Grazing Animals - > 5 Large Animals or Equivalent/Acre & $\mathrm{H}$ & BMP17 & Stormwater Wetland \\
\hline A07 & Grazing Animals - > 5 Large Animals or Equivalent/Acre & $\mathrm{H}$ & BMP20 & Bio-Retention Practices \\
\hline A11 & Managed Forest Lands - Clearcut Harvested $-<35$ yrs & $\mathrm{H}$ & BMP3 & Preservation of Existing Vegetation \\
\hline A11 & Managed Forest Lands - Clearcut Harvested $-<35$ yrs & $\mathrm{H}$ & BMP4 & Mulches \\
\hline A11 & Managed Forest Lands - Clearcut Harvested $-<35$ yrs & $\mathrm{H}$ & BMP5 & Seeding and Planting \\
\hline A11 & Managed Forest Lands - Clearcut Harvested $-<35$ yrs & $\mathrm{H}$ & BMP52 & Site Stabilization \\
\hline A14 & Pesticide/Fertilizer/Petroleum Storage, Handling, Mixing, \& Cleaning Areas & $\mathrm{H}$ & BMP12 & Stockpile and Materials Management \\
\hline A14 & Pesticide/Fertilizer/Petroleum Storage, Handling, Mixing, \& Cleaning Areas & $\mathrm{H}$ & BMP30 & Spill and Leak Prevention \\
\hline A14 & Pesticide/Fertilizer/Petroleum Storage, Handling, Mixing, \& Cleaning Areas & $\mathrm{H}$ & BMP31 & Spill Clean-Up \\
\hline A14 & Pesticide/Fertilizer/Petroleum Storage, Handling, Mixing, \& Cleaning Areas & $\mathrm{H}$ & BMP32 & Proper Fluids Handling \\
\hline A14 & Pesticide/Fertilizer/Petroleum Storage, Handling, Mixing, \& Cleaning Areas & $\mathrm{H}$ & BMP36 & Housekeeping \\
\hline A14 & Pesticide/Fertilizer/Petroleum Storage, Handling, Mixing, \& Cleaning Areas & $\mathrm{H}$ & BMP37 & Proper Wash Water Disposal \\
\hline A14 & Pesticide/Fertilizer/Petroleum Storage, Handling, Mixing, \& Cleaning Areas & $\mathrm{H}$ & BMP41 & Minimize Chlorinated Cleaners and Solvents \\
\hline A14 & Pesticide/Fertilizer/Petroleum Storage, Handling, Mixing, \& Cleaning Areas & $\mathrm{H}$ & BMP49 & Secondary Containment \\
\hline A14 & Pesticide/Fertilizer/Petroleum Storage, Handling, Mixing, \& Cleaning Areas & $\mathrm{H}$ & BMP51 & Materials Safety Data Sheets (MSDS) On-Hand \\
\hline A14 & Pesticide/Fertilizer/Petroleum Storage, Handling, Mixing, \& Cleaning Areas & $\mathrm{H}$ & BMP13 & Emergency Spillway \\
\hline A14 & Pesticide/Fertilizer/Petroleum Storage, Handling, Mixing, \& Cleaning Areas & $\mathrm{H}$ & BMP22 & Hydrodynamic Separators \\
\hline A14 & Pesticide/Fertilizer/Petroleum Storage, Handling, Mixing, \& Cleaning Areas & $\mathrm{H}$ & BMP23 & Catch Basin Media Inserts \\
\hline A14 & Pesticide/Fertilizer/Petroleum Storage, Handling, Mixing, \& Cleaning Areas & $\mathrm{H}$ & BMP24 & Filtration Systems \\
\hline A14 & Pesticide/Fertilizer/Petroleum Storage, Handling, Mixing, \& Cleaning Areas & $\mathrm{H}$ & BMP9 & Inlet Protection \\
\hline A14 & Pesticide/Fertilizer/Petroleum Storage, Handling, Mixing, \& Cleaning Areas & $\mathrm{H}$ & BMP10 & Fiber Rolls or Wattles \\
\hline A14 & Pesticide/Fertilizer/Petroleum Storage, Handling, Mixing, \& Cleaning Areas & $\mathrm{H}$ & BMP27 & Clean and Maintain Storm Drainage System \\
\hline A52 & Irrigation Canal/Pond & $\mathrm{H}$ & BMP3 & Preservation of Existing Vegetation \\
\hline A52 & Irrigation Canal/Pond & $\mathrm{H}$ & BMP13 & Emergency Spillway \\
\hline A52 & Irrigation Canal/Pond & $\mathrm{H}$ & BMP52 & Site Stabilization \\
\hline A52 & Irrigation Canal/Pond & $\mathrm{H}$ & BMP53 & Protecting Slopes \\
\hline $\mathrm{CO4}$ & Auto - Repair Shops & $M$ & BMP30 & Spill and Leak Prevention \\
\hline $\mathrm{CO4}$ & Auto - Repair Shops & $M$ & BMP31 & Spill Clean-Up \\
\hline $\mathrm{CO4}$ & Auto - Repair Shops & $M$ & BMP32 & Proper Fluids Handling \\
\hline $\mathrm{CO4}$ & Auto - Repair Shops & M & BMP33 & Drain, Cover, and Contain Oily Parts Stored Outside \\
\hline $\mathrm{CO4}$ & Auto - Repair Shops & $\mathrm{M}$ & BMP34 & Equipment Maintenance \\
\hline $\mathrm{CO} 4$ & Auto - Repair Shops & $M$ & BMP36 & Housekeeping \\
\hline $\mathrm{CO} 4$ & Auto - Repair Shops & M & BMP38 & Vehicle Owner Education \\
\hline $\mathrm{CO4}$ & Auto - Repair Shops & M & BMP39 & Proper Grease and Oil Disposal \\
\hline $\mathrm{CO4}$ & Auto - Repair Shops & $M$ & BMP40 & Dumpster Maintenance \\
\hline $\mathrm{CO4}$ & Auto - Repair Shops & $M$ & BMP49 & Secondary Containment \\
\hline $\mathrm{CO4}$ & Auto - Repair Shops & M & BMP51 & Materials Safety Data Sheets (MSDS) On-Hand \\
\hline $\mathrm{CO4}$ & Auto - Repair Shops & M & BMP13 & Emergency Spillway \\
\hline $\mathrm{CO} 4$ & Auto - Repair Shops & $M$ & BMP20 & Bio-Retention Practices \\
\hline $\mathrm{CO} 4$ & Auto - Repair Shops & $M$ & BMP22 & Hydrodynamic Separators \\
\hline $\mathrm{CO4}$ & Auto - Repair Shops & $M$ & BMP23 & Catch Basin Media Inserts \\
\hline $\mathrm{CO5}$ & Boat Services/Repair/Refinishing & $\mathrm{H}$ & BMP12 & Stockpile and Materials Management \\
\hline $\mathrm{CO5}$ & Boat Services/Repair/Refinishing & $\mathrm{H}$ & BMP13 & Emergency Spillway \\
\hline $\mathrm{CO5}$ & Boat Services/Repair/Refinishing & $\mathrm{H}$ & BMP22 & Hydrodynamic Separators \\
\hline $\mathrm{CO5}$ & Boat Services/Repair/Refinishing & $\mathrm{H}$ & BMP23 & Catch Basin Media Inserts \\
\hline $\mathrm{CO5}$ & Boat Services/Repair/Refinishing & $\mathrm{H}$ & BMP30 & Spill and Leak Prevention \\
\hline $\mathrm{CO5}$ & Boat Services/Repair/Refinishing & $\mathrm{H}$ & BMP31 & Spill Clean-Up \\
\hline $\mathrm{CO5}$ & Boat Services/Repair/Refinishing & $\mathrm{H}$ & BMP32 & Proper Fluids Handling \\
\hline $\mathrm{CO5}$ & Boat Services/Repair/Refinishing & $\mathrm{H}$ & BMP33 & Drain, Cover, and Contain Oily Parts Stored Outside \\
\hline $\mathrm{CO5}$ & Boat Services/Repair/Refinishing & $\mathrm{H}$ & BMP34 & Equipment Maintenance \\
\hline $\mathrm{CO5}$ & Boat Services/Repair/Refinishing & $\mathrm{H}$ & BMP36 & Housekeeping \\
\hline $\mathrm{CO5}$ & Boat Services/Repair/Refinishing & $\mathrm{H}$ & BMP37 & Proper Wash Water Disposal \\
\hline $\mathrm{CO5}$ & Boat Services/Repair/Refinishing & $\mathrm{H}$ & BMP39 & Proper Grease and Oil Disposal \\
\hline $\mathrm{CO5}$ & Boat Services/Repair/Refinishing & $\mathrm{H}$ & BMP40 & Dumpster Maintenance \\
\hline $\mathrm{CO5}$ & Boat Services/Repair/Refinishing & $\mathrm{H}$ & BMP41 & Minimize Chlorinated Cleaners and Solvents \\
\hline $\mathrm{CO5}$ & Boat Services/Repair/Refinishing & $\mathrm{H}$ & BMP49 & Secondary Containment \\
\hline $\mathrm{CO5}$ & Boat Services/Repair/Refinishing & $\mathrm{H}$ & BMP50 & Proper Hazardous Waste Disposal \\
\hline $\mathrm{CO5}$ & Boat Services/Repair/Refinishing & $\mathrm{H}$ & BMP51 & Materials Safety Data Sheets (MSDS) On-Hand \\
\hline $\mathrm{CO6}$ & Cement/Concrete Plants & $M$ & BMP11 & Concrete Management \\
\hline
\end{tabular}




\begin{tabular}{|c|c|c|}
\hline $\mathrm{CO6}$ & Cement/Concrete Plants & M \\
\hline $\mathrm{CO6}$ & Cement/Concrete Plants & M \\
\hline $\mathrm{CO6}$ & Cement/Concrete Plants & M \\
\hline $\mathrm{CO6}$ & Cement/Concrete Plants & M \\
\hline $\mathrm{CO6}$ & Cement/Concrete Plants & M \\
\hline $\mathrm{CO6}$ & Cement/Concrete Plants & M \\
\hline $\mathrm{CO6}$ & Cement/Concrete Plants & M \\
\hline $\mathrm{CO6}$ & Cement/Concrete Plants & M \\
\hline $\mathrm{CO6}$ & Cement/Concrete Plants & M \\
\hline C06 & Cement/Concrete Plants & M \\
\hline $\mathrm{CO6}$ & Cement/Concrete Plants & M \\
\hline $\mathrm{CO}$ & Chemical/Petroleum Processing/Storage & $\mathrm{H}$ \\
\hline $\mathrm{CO}$ & Chemical/Petroleum Processing/Storage & $\mathrm{H}$ \\
\hline $\mathrm{CO}$ & Chemical/Petroleum Processing/Storage & $\mathrm{H}$ \\
\hline $\mathrm{CO7}$ & Chemical/Petroleum Processing/Storage & $\mathrm{H}$ \\
\hline $\mathrm{CO}$ & Chemical/Petroleum Processing/Storage & $\mathrm{H}$ \\
\hline $\mathrm{CO}$ & Chemical/Petroleum Processing/Storage & $\mathrm{H}$ \\
\hline $\mathrm{CO} 7$ & Chemical/Petroleum Processing/Storage & $\mathrm{H}$ \\
\hline $\mathrm{CO7}$ & Chemical/Petroleum Processing/Storage & H \\
\hline $\mathrm{CO}$ & Chemical/Petroleum Processing/Storage & $\mathrm{H}$ \\
\hline $\mathrm{CO7}$ & Chemical/Petroleum Processing/Storage & $\mathrm{H}$ \\
\hline $\mathrm{CO} 7$ & Chemical/Petroleum Processing/Storage & $\mathrm{H}$ \\
\hline $\mathrm{CO7}$ & Chemical/Petroleum Processing/Storage & $\mathrm{H}$ \\
\hline $\mathrm{CO}$ & Chemical/Petroleum Processing/Storage & $\mathrm{H}$ \\
\hline $\mathrm{CO}$ & Chemical/Petroleum Processing/Storage & $\mathrm{H}$ \\
\hline $\mathrm{CO}$ & Chemical/Petroleum Processing/Storage & $\mathrm{H}$ \\
\hline $\mathrm{C} 10$ & Fleet/Trucking/Bus Terminals & M \\
\hline $\mathrm{C} 10$ & Fleet/Trucking/Bus Terminals & M \\
\hline $\mathrm{C} 10$ & Fleet/Trucking/Bus Terminals & M \\
\hline $\mathrm{C} 10$ & Fleet/Trucking/Bus Terminals & M \\
\hline $\mathrm{C} 10$ & Fleet/Trucking/Bus Terminals & M \\
\hline $\mathrm{C} 10$ & Fleet/Trucking/Bus Terminals & M \\
\hline $\mathrm{C} 10$ & Fleet/Trucking/Bus Terminals & M \\
\hline $\mathrm{C} 10$ & Fleet/Trucking/Bus Terminals & M \\
\hline $\mathrm{C} 10$ & Fleet/Trucking/Bus Terminals & M \\
\hline $\mathrm{C} 10$ & Fleet/Trucking/Bus Terminals & M \\
\hline $\mathrm{C} 10$ & Fleet/Trucking/Bus Terminals & M \\
\hline $\mathrm{C} 10$ & Fleet/Trucking/Bus Terminals & M \\
\hline $\mathrm{C} 12$ & Furniture/Lumber/Parts Stores & M \\
\hline $\mathrm{C} 12$ & Furniture/Lumber/Parts Stores & M \\
\hline $\mathrm{C} 12$ & Furniture/Lumber/Parts Stores & M \\
\hline $\mathrm{C} 13$ & Home Manufacturing & $\mathrm{H}$ \\
\hline $\mathrm{C} 13$ & Home Manufacturing & $\mathrm{H}$ \\
\hline $\mathrm{C} 13$ & Home Manufacturing & $\mathrm{H}$ \\
\hline $\mathrm{C} 13$ & Home Manufacturing & $\mathrm{H}$ \\
\hline $\mathrm{C} 13$ & Home Manufacturing & $\mathrm{H}$ \\
\hline $\mathrm{C} 13$ & Home Manufacturing & $\mathrm{H}$ \\
\hline $\mathrm{C} 13$ & Home Manufacturing & $\mathrm{H}$ \\
\hline $\mathrm{C} 13$ & Home Manufacturing & $\mathrm{H}$ \\
\hline $\mathrm{C} 13$ & Home Manufacturing & $\mathrm{H}$ \\
\hline $\mathrm{C} 13$ & Home Manufacturing & $\mathrm{H}$ \\
\hline $\mathrm{C} 13$ & Home Manufacturing & $\mathrm{H}$ \\
\hline C13 & Home Manufacturing & $\mathrm{H}$ \\
\hline $\mathrm{C} 13$ & Home Manufacturing & $\mathrm{H}$ \\
\hline $\mathrm{C} 13$ & Home Manufacturing & $\mathrm{H}$ \\
\hline $\mathrm{C} 13$ & Home Manufacturing & $\mathrm{H}$ \\
\hline $\mathrm{C} 13$ & Home Manufacturing & $\mathrm{H}$ \\
\hline C13 & Home Manufacturing & $\mathrm{H}$ \\
\hline $\mathrm{C} 13$ & Home Manufacturing & H \\
\hline $\mathrm{C} 13$ & Home Manufacturing & $\mathrm{H}$ \\
\hline $\mathrm{C} 13$ & Home Manufacturing & $\mathrm{H}$ \\
\hline $\mathrm{C} 13$ & Home Manufacturing & $\mathrm{H}$ \\
\hline $\mathrm{C} 13$ & Home Manufacturing & $\mathrm{H}$ \\
\hline $\mathrm{C} 13$ & Home Manufacturing & $\mathrm{H}$ \\
\hline $\mathrm{C} 13$ & Home Manufacturing & $\mathrm{H}$ \\
\hline $\mathrm{C} 13$ & Home Manufacturing & $\mathrm{H}$ \\
\hline $\mathrm{C} 13$ & Home Manufacturing & $\mathrm{H}$ \\
\hline $\mathrm{C} 13$ & Home Manufacturing & $\mathrm{H}$ \\
\hline $\mathrm{C} 13$ & Home Manufacturing & $\mathrm{H}$ \\
\hline $\mathrm{C} 13$ & Home Manufacturing & $\mathrm{H}$ \\
\hline $\mathrm{C} 13$ & Home Manufacturing & $\mathrm{H}$ \\
\hline $\mathrm{C} 13$ & Home Manufacturing & $\mathrm{H}$ \\
\hline $\mathrm{C} 13$ & Home Manufacturing & $\mathrm{H}$ \\
\hline C14 & Junk/Scrap/Salvage Yar & \\
\hline
\end{tabular}

\begin{tabular}{|c|c|}
\hline BMP12 & Stockpile and Materials Management \\
\hline BMP31 & Spill Clean-Up \\
\hline BMP36 & Housekeeping \\
\hline BMP37 & Proper Wash Water Disposal \\
\hline BMP40 & Dumpster Maintenance \\
\hline BMP41 & Minimize Chlorinated Cleaners and Solvents \\
\hline BMP51 & Materials Safety Data Sheets (MSDS) On-Hand \\
\hline BMP13 & Emergency Spillway \\
\hline BMP20 & Bio-Retention Practices \\
\hline BMP22 & Hydrodynamic Separators \\
\hline BMP23 & Catch Basin Media Inserts \\
\hline BMP12 & Stockpile and Materials Management \\
\hline BMP13 & Emergency Spillway \\
\hline BMP22 & Hydrodynamic Separators \\
\hline BMP23 & Catch Basin Media Inserts \\
\hline BMP30 & Spill and Leak Prevention \\
\hline BMP31 & Spill Clean-Up \\
\hline BMP32 & Proper Fluids Handling \\
\hline BMP34 & Equipment Maintenance \\
\hline BMP36 & Housekeeping \\
\hline BMP37 & Proper Wash Water Disposal \\
\hline BMP40 & Dumpster Maintenance \\
\hline BMP41 & Minimize Chlorinated Cleaners and Solvents \\
\hline BMP49 & Secondary Containment \\
\hline BMP50 & Proper Hazardous Waste Disposal \\
\hline BMP51 & Materials Safety Data Sheets (MSDS) On-Hand \\
\hline BMP30 & Spill and Leak Prevention \\
\hline BMP31 & Spill Clean-Up \\
\hline BMP32 & Proper Fluids Handling \\
\hline BMP35 & Pave Refueling Areas \\
\hline BMP36 & Housekeeping \\
\hline BMP37 & Proper Wash Water Disposal \\
\hline BMP39 & Proper Grease and Oil Disposal \\
\hline BMP40 & Dumpster Maintenance \\
\hline BMP41 & Minimize Chlorinated Cleaners and Solvents \\
\hline BMP20 & Bio-Retention Practices \\
\hline BMP22 & Hydrodynamic Separators \\
\hline BMP23 & Catch Basin Media Inserts \\
\hline BMP12 & Stockpile and Materials Management \\
\hline BMP36 & Housekeeping \\
\hline BMP51 & Materials Safety Data Sheets (MSDS) On-Hand \\
\hline BMP3 & Preservation of Existing Vegetation \\
\hline BMP4 & Mulches \\
\hline BMP5 & Seeding and Planting \\
\hline BMP6 & Erosion Blankets and Mats \\
\hline BMP7 & Entrance/Exit Tracking \\
\hline BMP8 & Sediment/Silt Fence \\
\hline BMP9 & Inlet Protection \\
\hline BMP10 & Fiber Rolls or Wattles \\
\hline BMP11 & Concrete Management \\
\hline BMP12 & Stockpile and Materials Management \\
\hline BMP18 & Infiltration Trench \\
\hline BMP19 & Infiltration Basin \\
\hline BMP20 & Bio-Retention Practices \\
\hline BMP21 & Underground Infiltration Devices \\
\hline BMP22 & Hydrodynamic Separators \\
\hline BMP23 & Catch Basin Media Inserts \\
\hline BMP24 & Filtration Systems \\
\hline BMP25 & Infiltration Systems \\
\hline BMP26 & Porous Pavement \\
\hline BMP27 & Clean and Maintain Storm Drainage System \\
\hline BMP28 & Stormdrain Inlet Labels \\
\hline BMP29 & Illicit Connection Identification and Elimination \\
\hline BMP31 & Spill Clean-Up \\
\hline BMP37 & Proper Wash Water Disposal \\
\hline BMP40 & Dumpster Maintenance \\
\hline BMP42 & Properly Working Portable Toilets \\
\hline BMP44 & Proper Timing for Construction \\
\hline BMP45 & Reduce Soil Compaction \\
\hline BMP46 & Downspout Extenders \\
\hline BMP51 & Materials Safety Data Sheets (MSDS) On-Hand \\
\hline BMP52 & Site Stabilization \\
\hline BMP53 & Protecting Slopes \\
\hline BMP12 & Stockpile and Materials Management \\
\hline
\end{tabular}




\begin{tabular}{|c|c|c|c|c|}
\hline $\mathrm{C} 14$ & Junk/Scrap/Salvage Yards & $\mathrm{H}$ & BMP13 & Emergency Spillway \\
\hline $\mathrm{C} 14$ & Junk/Scrap/Salvage Yards & $\mathrm{H}$ & BMP22 & Hydrodynamic Separators \\
\hline $\mathrm{C} 14$ & Junk/Scrap/Salvage Yards & $\mathrm{H}$ & BMP23 & Catch Basin Media Inserts \\
\hline $\mathrm{C} 14$ & Junk/Scrap/Salvage Yards & $\mathrm{H}$ & BMP24 & Filtration Systems \\
\hline $\mathrm{C} 14$ & Junk/Scrap/Salvage Yards & $\mathrm{H}$ & BMP28 & Stormdrain Inlet Labels \\
\hline $\mathrm{C} 14$ & Junk/Scrap/Salvage Yards & $\mathrm{H}$ & BMP30 & Spill and Leak Prevention \\
\hline $\mathrm{C} 14$ & Junk/Scrap/Salvage Yards & $\mathrm{H}$ & BMP31 & Spill Clean-Up \\
\hline $\mathrm{C} 14$ & Junk/Scrap/Salvage Yards & $\mathrm{H}$ & BMP32 & Proper Fluids Handling \\
\hline $\mathrm{C} 14$ & Junk/Scrap/Salvage Yards & $\mathrm{H}$ & BMP33 & Drain, Cover, and Contain Oily Parts Stored Outside \\
\hline $\mathrm{C} 14$ & Junk/Scrap/Salvage Yards & $\mathrm{H}$ & BMP34 & Equipment Maintenance \\
\hline $\mathrm{C} 14$ & Junk/Scrap/Salvage Yards & $\mathrm{H}$ & BMP37 & Proper Wash Water Disposal \\
\hline $\mathrm{C} 14$ & Junk/Scrap/Salvage Yards & $\mathrm{H}$ & BMP39 & Proper Grease and Oil Disposal \\
\hline $\mathrm{C} 14$ & Junk/Scrap/Salvage Yards & $\mathrm{H}$ & BMP40 & Dumpster Maintenance \\
\hline C14 & Junk/Scrap/Salvage Yards & $\mathrm{H}$ & BMP41 & Minimize Chlorinated Cleaners and Solvents \\
\hline $\mathrm{C} 14$ & Junk/Scrap/Salvage Yards & $\mathrm{H}$ & BMP49 & Secondary Containment \\
\hline $\mathrm{C} 14$ & Junk/Scrap/Salvage Yards & $\mathrm{H}$ & BMP50 & Proper Hazardous Waste Disposal \\
\hline $\mathrm{C} 14$ & Junk/Scrap/Salvage Yards & $\mathrm{H}$ & BMP51 & Materials Safety Data Sheets (MSDS) On-Hand \\
\hline $\mathrm{C} 15$ & Machine Shops & $\mathrm{H}$ & BMP12 & Stockpile and Materials Management \\
\hline $\mathrm{C} 15$ & Machine Shops & $\mathrm{H}$ & BMP22 & Hydrodynamic Separators \\
\hline $\mathrm{C} 15$ & Machine Shops & $\mathrm{H}$ & BMP23 & Catch Basin Media Inserts \\
\hline $\mathrm{C} 15$ & Machine Shops & $\mathrm{H}$ & BMP31 & Spill Clean-Up \\
\hline $\mathrm{C} 15$ & Machine Shops & $\mathrm{H}$ & BMP33 & Drain, Cover, and Contain Oily Parts Stored Outside \\
\hline $\mathrm{C} 15$ & Machine Shops & $\mathrm{H}$ & BMP34 & Equipment Maintenance \\
\hline $\mathrm{C} 15$ & Machine Shops & $\mathrm{H}$ & BMP36 & Housekeeping \\
\hline $\mathrm{C} 15$ & Machine Shops & $\mathrm{H}$ & BMP37 & Proper Wash Water Disposal \\
\hline $\mathrm{C} 15$ & Machine Shops & $\mathrm{H}$ & BMP40 & Dumpster Maintenance \\
\hline $\mathrm{C} 15$ & Machine Shops & $\mathrm{H}$ & BMP41 & Minimize Chlorinated Cleaners and Solvents \\
\hline $\mathrm{C} 15$ & Machine Shops & $\mathrm{H}$ & BMP49 & Secondary Containment \\
\hline $\mathrm{C} 15$ & Machine Shops & $\mathrm{H}$ & BMP50 & Proper Hazardous Waste Disposal \\
\hline $\mathrm{C} 15$ & Machine Shops & $\mathrm{H}$ & BMP51 & Materials Safety Data Sheets (MSDS) On-Hand \\
\hline $\mathrm{C} 17$ & Metal Plating/Finishing/Fabrication & $\mathrm{H}$ & BMP12 & Stockpile and Materials Management \\
\hline $\mathrm{C} 17$ & Metal Plating/Finishing/Fabrication & $\mathrm{H}$ & BMP22 & Hydrodynamic Separators \\
\hline $\mathrm{C} 17$ & Metal Plating/Finishing/Fabrication & $\mathrm{H}$ & BMP23 & Catch Basin Media Inserts \\
\hline $\mathrm{C} 17$ & Metal Plating/Finishing/Fabrication & $\mathrm{H}$ & BMP31 & Spill Clean-Up \\
\hline $\mathrm{C} 17$ & Metal Plating/Finishing/Fabrication & $\mathrm{H}$ & BMP33 & Drain, Cover, and Contain Oily Parts Stored Outside \\
\hline $\mathrm{C} 17$ & Metal Plating/Finishing/Fabrication & $\mathrm{H}$ & BMP34 & Equipment Maintenance \\
\hline $\mathrm{C} 17$ & Metal Plating/Finishing/Fabrication & $\mathrm{H}$ & BMP36 & Housekeeping \\
\hline $\mathrm{C} 17$ & Metal Plating/Finishing/Fabrication & $\mathrm{H}$ & BMP37 & Proper Wash Water Disposal \\
\hline $\mathrm{C} 17$ & Metal Plating/Finishing/Fabrication & $\mathrm{H}$ & BMP40 & Dumpster Maintenance \\
\hline $\mathrm{C} 17$ & Metal Plating/Finishing/Fabrication & $\mathrm{H}$ & BMP41 & Minimize Chlorinated Cleaners and Solvents \\
\hline $\mathrm{C} 17$ & Metal Plating/Finishing/Fabrication & $\mathrm{H}$ & BMP49 & Secondary Containment \\
\hline $\mathrm{C} 17$ & Metal Plating/Finishing/Fabrication & $\mathrm{H}$ & BMP50 & Proper Hazardous Waste Disposal \\
\hline $\mathrm{C} 17$ & Metal Plating/Finishing/Fabrication & $\mathrm{H}$ & BMP51 & Materials Safety Data Sheets (MSDS) On-Hand \\
\hline $\mathrm{C} 18$ & Mining Activities - Gravel Mines/Gravel Pits & $\mathrm{H}$ & BMP12 & Stockpile and Materials Management \\
\hline $\mathrm{C} 18$ & Mining Activities - Gravel Mines/Gravel Pits & $\mathrm{H}$ & BMP7 & Entrance/Exit Tracking \\
\hline $\mathrm{C} 18$ & Mining Activities - Gravel Mines/Gravel Pits & $\mathrm{H}$ & BMP8 & Sediment/Silt Fence \\
\hline $\mathrm{C} 18$ & Mining Activities - Gravel Mines/Gravel Pits & $\mathrm{H}$ & BMP9 & Inlet Protection \\
\hline $\mathrm{C} 18$ & Mining Activities - Gravel Mines/Gravel Pits & $\mathrm{H}$ & BMP10 & Fiber Rolls or Wattles \\
\hline $\mathrm{C} 18$ & Mining Activities - Gravel Mines/Gravel Pits & $\mathrm{H}$ & BMP27 & Clean and Maintain Storm Drainage System \\
\hline $\mathrm{C} 18$ & Mining Activities - Gravel Mines/Gravel Pits & $\mathrm{H}$ & BMP45 & Reduce Soil Compaction \\
\hline $\mathrm{C} 18$ & Mining Activities - Gravel Mines/Gravel Pits & $\mathrm{H}$ & BMP52 & Site Stabilization \\
\hline $\mathrm{C} 18$ & Mining Activities - Gravel Mines/Gravel Pits & $\mathrm{H}$ & BMP53 & Protecting Slopes \\
\hline $\mathrm{C} 20$ & Parking Lots/Malls - > 50 Spaces & $\mathrm{H}$ & BMP3 & Preservation of Existing Vegetation \\
\hline $\mathrm{C} 20$ & Parking Lots/Malls - > 50 Spaces & $\mathrm{H}$ & BMP28 & Stormdrain Inlet Labels \\
\hline $\mathrm{C} 20$ & Parking Lots/Malls - > 50 Spaces & $\mathrm{H}$ & BMP31 & Spill Clean-Up \\
\hline $\mathrm{C} 20$ & Parking Lots/Malls - > 50 Spaces & $\mathrm{H}$ & BMP36 & Housekeeping \\
\hline $\mathrm{C} 20$ & Parking Lots/Malls - > 50 Spaces & $\mathrm{H}$ & BMP20 & Bio-Retention Practices \\
\hline $\mathrm{C} 20$ & Parking Lots/Malls - > 50 Spaces & $\mathrm{H}$ & BMP22 & Hydrodynamic Separators \\
\hline $\mathrm{C} 20$ & Parking Lots/Malls - > 50 Spaces & $\mathrm{H}$ & BMP23 & Catch Basin Media Inserts \\
\hline $\mathrm{C} 20$ & Parking Lots/Malls - > 50 Spaces & $\mathrm{H}$ & BMP26 & Porous Pavement \\
\hline $\mathrm{C} 20$ & Parking Lots/Malls - > 50 Spaces & $\mathrm{H}$ & BMP9 & Inlet Protection \\
\hline $\mathrm{C} 20$ & Parking Lots/Malls - > 50 Spaces & $\mathrm{H}$ & BMP27 & Clean and Maintain Storm Drainage System \\
\hline $\mathrm{C} 21$ & Photo Processing/Printing & $\mathrm{H}$ & BMP12 & Stockpile and Materials Management \\
\hline $\mathrm{C} 21$ & Photo Processing/Printing & $\mathrm{H}$ & BMP30 & Spill and Leak Prevention \\
\hline $\mathrm{C} 21$ & Photo Processing/Printing & $\mathrm{H}$ & BMP31 & Spill Clean-Up \\
\hline $\mathrm{C} 21$ & Photo Processing/Printing & $\mathrm{H}$ & BMP32 & Proper Fluids Handling \\
\hline $\mathrm{C} 21$ & Photo Processing/Printing & $\mathrm{H}$ & BMP34 & Equipment Maintenance \\
\hline $\mathrm{C} 21$ & Photo Processing/Printing & $\mathrm{H}$ & BMP36 & Housekeeping \\
\hline $\mathrm{C} 21$ & Photo Processing/Printing & $\mathrm{H}$ & BMP50 & Proper Hazardous Waste Disposal \\
\hline $\mathrm{C} 21$ & Photo Processing/Printing & $\mathrm{H}$ & BMP51 & Materials Safety Data Sheets (MSDS) On-Hand \\
\hline $\mathrm{C} 21$ & Photo Processing/Printing & $\mathrm{H}$ & BMP13 & Emergency Spillway \\
\hline $\mathrm{C} 21$ & Photo Processing/Printing & $\mathrm{H}$ & BMP22 & Hydrodynamic Separators \\
\hline $\mathrm{C} 21$ & Photo Processing/Printing & $\mathrm{H}$ & BMP23 & Catch Basin Media Inserts \\
\hline $\mathrm{C} 21$ & Photo Processing/Printing & $\mathrm{H}$ & BMP24 & Filtration Systems \\
\hline
\end{tabular}




\begin{tabular}{|c|c|c|c|c|}
\hline $\mathrm{C} 21$ & Photo Processing/Printing & H & BMP9 & Inlet Protection \\
\hline $\mathrm{C} 21$ & Photo Processing/Printing & $\mathrm{H}$ & BMP10 & Fiber Rolls or Wattles \\
\hline $\mathrm{C} 21$ & Photo Processing/Printing & $\mathrm{H}$ & BMP27 & Clean and Maintain Storm Drainage System \\
\hline $\mathrm{C} 22$ & Plastic/Synthetics Producer & $\mathrm{H}$ & BMP12 & Stockpile and Materials Management \\
\hline $\mathrm{C} 22$ & Plastic/Synthetics Producer & $\mathrm{H}$ & BMP34 & Equipment Maintenance \\
\hline $\mathrm{C} 22$ & Plastic/Synthetics Producer & $\mathrm{H}$ & BMP36 & Housekeeping \\
\hline $\mathrm{C} 22$ & Plastic/Synthetics Producer & $\mathrm{H}$ & BMP37 & Proper Wash Water Disposal \\
\hline $\mathrm{C} 22$ & Plastic/Synthetics Producer & $\mathrm{H}$ & BMP41 & Minimize Chlorinated Cleaners and Solvents \\
\hline $\mathrm{C} 22$ & Plastic/Synthetics Producer & H & BMP49 & Secondary Containment \\
\hline $\mathrm{C} 22$ & Plastic/Synthetics Producer & H & BMP50 & Proper Hazardous Waste Disposal \\
\hline $\mathrm{C} 22$ & Plastic/Synthetics Producer & $\mathrm{H}$ & BMP51 & Materials Safety Data Sheets (MSDS) On-Hand \\
\hline $\mathrm{C} 22$ & Plastic/Synthetics Producer & $\mathrm{H}$ & BMP13 & Emergency Spillway \\
\hline $\mathrm{C} 22$ & Plastic/Synthetics Producer & H & BMP22 & Hydrodynamic Separators \\
\hline $\mathrm{C} 22$ & Plastic/Synthetics Producer & $\mathrm{H}$ & BMP23 & Catch Basin Media Inserts \\
\hline $\mathrm{C} 22$ & Plastic/Synthetics Producer & $\mathrm{H}$ & BMP24 & Filtration Systems \\
\hline $\mathrm{C} 22$ & Plastic/Synthetics Producer & $\mathrm{H}$ & BMP9 & Inlet Protection \\
\hline $\mathrm{C} 26$ & Wood/Pulp/Paper Processing and Mills & $\mathrm{H}$ & BMP12 & Stockpile and Materials Management \\
\hline $\mathrm{C} 26$ & Wood/Pulp/Paper Processing and Mills & $\mathrm{H}$ & BMP31 & Spill Clean-Up \\
\hline $\mathrm{C} 26$ & Wood/Pulp/Paper Processing and Mills & $\mathrm{H}$ & BMP32 & Proper Fluids Handling \\
\hline $\mathrm{C} 26$ & Wood/Pulp/Paper Processing and Mills & $\mathrm{H}$ & BMP34 & Equipment Maintenance \\
\hline $\mathrm{C} 26$ & Wood/Pulp/Paper Processing and Mills & $\mathrm{H}$ & BMP36 & Housekeeping \\
\hline $\mathrm{C} 26$ & Wood/Pulp/Paper Processing and Mills & H & BMP37 & Proper Wash Water Disposal \\
\hline $\mathrm{C} 26$ & Wood/Pulp/Paper Processing and Mills & $\mathrm{H}$ & BMP41 & Minimize Chlorinated Cleaners and Solvents \\
\hline $\mathrm{C} 26$ & Wood/Pulp/Paper Processing and Mills & $\mathrm{H}$ & BMP49 & Secondary Containment \\
\hline $\mathrm{C} 26$ & Wood/Pulp/Paper Processing and Mills & $\mathrm{H}$ & BMP50 & Proper Hazardous Waste Disposal \\
\hline $\mathrm{C} 26$ & Wood/Pulp/Paper Processing and Mills & $\mathrm{H}$ & BMP51 & Materials Safety Data Sheets (MSDS) On-Hand \\
\hline $\mathrm{C} 26$ & Wood/Pulp/Paper Processing and Mills & $\mathrm{H}$ & BMP13 & Emergency Spillway \\
\hline $\mathrm{C} 26$ & Wood/Pulp/Paper Processing and Mills & $\mathrm{H}$ & BMP22 & Hydrodynamic Separators \\
\hline $\mathrm{C} 26$ & Wood/Pulp/Paper Processing and Mills & H & BMP23 & Catch Basin Media Inserts \\
\hline $\mathrm{C} 26$ & Wood/Pulp/Paper Processing and Mills & $\mathrm{H}$ & BMP24 & Filtration Systems \\
\hline $\mathrm{C} 26$ & Wood/Pulp/Paper Processing and Mills & $\mathrm{H}$ & BMP9 & Inlet Protection \\
\hline $\mathrm{C} 26$ & Wood/Pulp/Paper Processing and Mills & H & BMP10 & Fiber Rolls or Wattles \\
\hline $\mathrm{C} 26$ & Wood/Pulp/Paper Processing and Mills & $\mathrm{H}$ & BMP27 & Clean and Maintain Storm Drainage System \\
\hline $\mathrm{C} 51$ & Pipelines - Chemical and Petroleum & $\mathrm{H}$ & BMP3 & Preservation of Existing Vegetation \\
\hline $\mathrm{C51}$ & Pipelines - Chemical and Petroleum & H & BMP30 & Spill and Leak Prevention \\
\hline C51 & Pipelines - Chemical and Petroleum & $\mathrm{H}$ & BMP31 & Spill Clean-Up \\
\hline $\mathrm{C51}$ & Pipelines - Chemical and Petroleum & $\mathrm{H}$ & BMP34 & Equipment Maintenance \\
\hline C51 & Pipelines - Chemical and Petroleum & $\mathrm{H}$ & BMP49 & Secondary Containment \\
\hline $\mathrm{C51}$ & Pipelines - Chemical and Petroleum & $\mathrm{H}$ & BMP13 & Emergency Spillway \\
\hline $\mathrm{C51}$ & Pipelines - Chemical and Petroleum & $\mathrm{H}$ & BMP18 & Infiltration Trench \\
\hline C51 & Pipelines - Chemical and Petroleum & H & BMP52 & Site Stabilization \\
\hline C51 & Pipelines - Chemical and Petroleum & $\mathrm{H}$ & BMP53 & Protecting Slopes \\
\hline $\mathrm{C} 58$ & Known Contamination Sites/Plumes/Spills (ECSI) & $\mathrm{H}$ & BMP2 & Diversion of Run-On \\
\hline $\mathrm{C} 58$ & Known Contamination Sites/Plumes/Spills (ECSI) & H & BMP13 & Emergency Spillway \\
\hline $\mathrm{C} 58$ & Known Contamination Sites/Plumes/Spills (ECSI) & $\mathrm{H}$ & BMP24 & Filtration Systems \\
\hline $\mathrm{C} 58$ & Known Contamination Sites/Plumes/Spills (ECSI) & $\mathrm{H}$ & BMP31 & Spill Clean-Up \\
\hline $\mathrm{C} 60$ & Maintenance Shop/Equipment Storage - Not Transportation Related & M & BMP12 & Stockpile and Materials Management \\
\hline $\mathrm{C} 60$ & Maintenance Shop/Equipment Storage - Not Transportation Related & M & BMP31 & Spill Clean-Up \\
\hline $\mathrm{C60}$ & Maintenance Shop/Equipment Storage - Not Transportation Related & M & BMP34 & Equipment Maintenance \\
\hline $\mathrm{C} 60$ & Maintenance Shop/Equipment Storage - Not Transportation Related & M & BMP36 & Housekeeping \\
\hline $\mathrm{C} 60$ & Maintenance Shop/Equipment Storage - Not Transportation Related & M & BMP37 & Proper Wash Water Disposal \\
\hline $\mathrm{C} 60$ & Maintenance Shop/Equipment Storage - Not Transportation Related & M & BMP39 & Proper Grease and Oil Disposal \\
\hline $\mathrm{C} 60$ & Maintenance Shop/Equipment Storage - Not Transportation Related & M & BMP40 & Dumpster Maintenance \\
\hline $\mathrm{C} 60$ & Maintenance Shop/Equipment Storage - Not Transportation Related & M & BMP41 & Minimize Chlorinated Cleaners and Solvents \\
\hline $\mathrm{C} 60$ & Maintenance Shop/Equipment Storage - Not Transportation Related & M & BMP49 & Secondary Containment \\
\hline $\mathrm{C} 60$ & Maintenance Shop/Equipment Storage - Not Transportation Related & M & BMP50 & Proper Hazardous Waste Disposal \\
\hline $\mathrm{C} 60$ & Maintenance Shop/Equipment Storage - Not Transportation Related & M & BMP51 & Materials Safety Data Sheets (MSDS) On-Hand \\
\hline $\mathrm{C} 60$ & Maintenance Shop/Equipment Storage - Not Transportation Related & M & BMP13 & Emergency Spillway \\
\hline $\mathrm{C} 60$ & Maintenance Shop/Equipment Storage - Not Transportation Related & M & BMP20 & Bio-Retention Practices \\
\hline $\mathrm{C} 60$ & Maintenance Shop/Equipment Storage - Not Transportation Related & M & BMP22 & Hydrodynamic Separators \\
\hline $\mathrm{C} 60$ & Maintenance Shop/Equipment Storage - Not Transportation Related & M & BMP23 & Catch Basin Media Inserts \\
\hline $\mathrm{C} 61$ & Maintenance Shop/Equipment Storage - Transportation Related & M & BMP12 & Stockpile and Materials Management \\
\hline $\mathrm{C} 61$ & Maintenance Shop/Equipment Storage - Transportation Related & M & BMP30 & Spill and Leak Prevention \\
\hline $\mathrm{C61}$ & Maintenance Shop/Equipment Storage - Transportation Related & M & BMP31 & Spill Clean-Up \\
\hline $\mathrm{C} 61$ & Maintenance Shop/Equipment Storage - Transportation Related & M & BMP32 & Proper Fluids Handling \\
\hline $\mathrm{C} 61$ & Maintenance Shop/Equipment Storage - Transportation Related & M & BMP33 & Drain, Cover, and Contain Oily Parts Stored Outside \\
\hline $\mathrm{C61}$ & Maintenance Shop/Equipment Storage - Transportation Related & M & BMP34 & Equipment Maintenance \\
\hline $\mathrm{C61}$ & Maintenance Shop/Equipment Storage - Transportation Related & M & BMP36 & Housekeeping \\
\hline $\mathrm{C} 61$ & Maintenance Shop/Equipment Storage - Transportation Related & M & BMP37 & Proper Wash Water Disposal \\
\hline $\mathrm{C61}$ & Maintenance Shop/Equipment Storage - Transportation Related & M & BMP38 & Vehicle Owner Education \\
\hline $\mathrm{C61}$ & Maintenance Shop/Equipment Storage - Transportation Related & M & BMP39 & Proper Grease and Oil Disposal \\
\hline $\mathrm{C} 61$ & Maintenance Shop/Equipment Storage - Transportation Related & M & BMP40 & Dumpster Maintenance \\
\hline $\mathrm{C61}$ & Maintenance Shop/Equipment Storage - Transportation Related & M & BMP41 & Minimize Chlorinated Cleaners and Solvents \\
\hline $\mathrm{C61}$ & Maintenance Shop/Equipment Storage - Transportation Related & M & BMP49 & Secondary Containment \\
\hline
\end{tabular}




\begin{tabular}{|c|c|c|}
\hline C61 & Maintenance Shop/Equipment Storage - Transportation Related & M \\
\hline $\mathrm{C} 61$ & Maintenance Shop/Equipment Storage - Transportation Related & M \\
\hline $\mathrm{C} 61$ & Maintenance Shop/Equipment Storage - Transportation Related & M \\
\hline C61 & Maintenance Shop/Equipment Storage - Transportation Related & M \\
\hline $\mathrm{C} 61$ & Maintenance Shop/Equipment Storage - Transportation Related & M \\
\hline $\mathrm{C} 63$ & Mining Activities - Active - other than gravel & $\mathrm{H}$ \\
\hline $\mathrm{C} 63$ & Mining Activities - Active - other than gravel & $\mathrm{H}$ \\
\hline $\mathrm{C} 63$ & Mining Activities - Active - other than gravel & $\mathrm{H}$ \\
\hline $\mathrm{C} 63$ & Mining Activities - Active - other than gravel & $\mathrm{H}$ \\
\hline $\mathrm{C} 63$ & Mining Activities - Active - other than gravel & $\mathrm{H}$ \\
\hline $\mathrm{C} 63$ & Mining Activities - Active - other than gravel & $\mathrm{H}$ \\
\hline $\mathrm{C} 63$ & Mining Activities - Active - other than gravel & $\mathrm{H}$ \\
\hline $\mathrm{C} 63$ & Mining Activities - Active - other than gravel & $\mathrm{H}$ \\
\hline $\mathrm{C} 63$ & Mining Activities - Active - other than gravel & $\mathrm{H}$ \\
\hline $\mathrm{C} 65$ & Miscellaneous Manufacturing & $\mathrm{H}$ \\
\hline $\mathrm{C} 65$ & Miscellaneous Manufacturing & $\mathrm{H}$ \\
\hline $\mathrm{C} 65$ & Miscellaneous Manufacturing & $\mathrm{H}$ \\
\hline C65 & Miscellaneous Manufacturing & $\mathrm{H}$ \\
\hline $\mathrm{C} 65$ & Miscellaneous Manufacturing & $\mathrm{H}$ \\
\hline $\mathrm{C} 65$ & Miscellaneous Manufacturing & $\mathrm{H}$ \\
\hline $\mathrm{C} 65$ & Miscellaneous Manufacturing & $\mathrm{H}$ \\
\hline $\mathrm{C} 65$ & Miscellaneous Manufacturing & $\mathrm{H}$ \\
\hline $\mathrm{C} 65$ & Miscellaneous Manufacturing & $\mathrm{H}$ \\
\hline $\mathrm{C} 65$ & Miscellaneous Manufacturing & $\mathrm{H}$ \\
\hline $\mathrm{C} 65$ & Miscellaneous Manufacturing & $\mathrm{H}$ \\
\hline $\mathrm{C} 65$ & Miscellaneous Manufacturing & $\mathrm{H}$ \\
\hline $\mathrm{C} 65$ & Miscellaneous Manufacturing & $\mathrm{H}$ \\
\hline $\mathrm{C} 65$ & Miscellaneous Manufacturing & $\mathrm{H}$ \\
\hline $\mathrm{C} 65$ & Miscellaneous Manufacturing & $\mathrm{H}$ \\
\hline $\mathrm{C} 65$ & Miscellaneous Manufacturing & $\mathrm{H}$ \\
\hline $\mathrm{C} 65$ & Miscellaneous Manufacturing & $\mathrm{H}$ \\
\hline $\mathrm{C} 65$ & Miscellaneous Manufacturing & $\mathrm{H}$ \\
\hline $\mathrm{C} 65$ & Miscellaneous Manufacturing & $\mathrm{H}$ \\
\hline $\mathrm{C} 65$ & Miscellaneous Manufacturing & $\mathrm{H}$ \\
\hline $\mathrm{C} 65$ & Miscellaneous Manufacturing & $\mathrm{H}$ \\
\hline $\mathrm{C} 65$ & Miscellaneous Manufacturing & $\mathrm{H}$ \\
\hline C67 & Warehouses & $\mathrm{L}$ \\
\hline $\mathrm{C} 67$ & Warehouses & $\mathrm{L}$ \\
\hline $\mathrm{C} 67$ & Warehouses & $\mathrm{L}$ \\
\hline C67 & Warehouses & $\mathrm{L}$ \\
\hline C67 & Warehouses & $\mathrm{L}$ \\
\hline C67 & Warehouses & $\mathrm{L}$ \\
\hline $\mathrm{C} 67$ & Warehouses & $\mathrm{L}$ \\
\hline $\mathrm{C} 67$ & Warehouses & $\mathrm{L}$ \\
\hline C68 & Miscellaneous Equipment Maintenance & $\mathrm{H}$ \\
\hline C68 & Miscellaneous Equipment Maintenance & $\mathrm{H}$ \\
\hline $\mathrm{C} 68$ & Miscellaneous Equipment Maintenance & $\mathrm{H}$ \\
\hline C68 & Miscellaneous Equipment Maintenance & $\mathrm{H}$ \\
\hline C68 & Miscellaneous Equipment Maintenance & $\mathrm{H}$ \\
\hline C68 & Miscellaneous Equipment Maintenance & $\mathrm{H}$ \\
\hline $\mathrm{C} 68$ & Miscellaneous Equipment Maintenance & $\mathrm{H}$ \\
\hline $\mathrm{C} 68$ & Miscellaneous Equipment Maintenance & $\mathrm{H}$ \\
\hline C68 & Miscellaneous Equipment Maintenance & $\mathrm{H}$ \\
\hline C68 & Miscellaneous Equipment Maintenance & $\mathrm{H}$ \\
\hline $\mathrm{C} 68$ & Miscellaneous Equipment Maintenance & $\mathrm{H}$ \\
\hline $\mathrm{C} 68$ & Miscellaneous Equipment Maintenance & $\mathrm{H}$ \\
\hline $\mathrm{C} 68$ & Miscellaneous Equipment Maintenance & $\mathrm{H}$ \\
\hline C68 & Miscellaneous Equipment Maintenance & $\mathrm{H}$ \\
\hline C68 & Miscellaneous Equipment Maintenance & $\mathrm{H}$ \\
\hline $\mathrm{C} 68$ & Miscellaneous Equipment Maintenance & $\mathrm{H}$ \\
\hline $\mathrm{C} 68$ & Miscellaneous Equipment Maintenance & $\mathrm{H}$ \\
\hline $\mathrm{C} 68$ & Miscellaneous Equipment Maintenance & $\mathrm{H}$ \\
\hline C68 & Miscellaneous Equipment Maintenance & $\mathrm{H}$ \\
\hline C68 & Miscellaneous Equipment Maintenance & $\mathrm{H}$ \\
\hline M06 & Auto - Gas Stations - Historic & $\mathrm{H}$ \\
\hline M06 & Auto - Gas Stations - Historic & $\mathrm{H}$ \\
\hline M06 & Auto - Gas Stations - Historic & $\mathrm{H}$ \\
\hline M06 & Auto - Gas Stations - Historic & $\mathrm{H}$ \\
\hline M06 & Auto - Gas Stations - Historic & $\mathrm{H}$ \\
\hline M06 & Auto - Gas Stations - Historic & $\mathrm{H}$ \\
\hline M06 & Auto - Gas Stations - Historic & $\mathrm{H}$ \\
\hline M06 & Auto - Gas Stations - Historic & $\mathrm{H}$ \\
\hline M06 & Auto - Gas Stations - Historic & H \\
\hline M07 & Historic Waste Dumps/Landfil & \\
\hline
\end{tabular}

\begin{tabular}{|c|c|}
\hline BMP51 & Materials Safety Data Sheets (MSDS) On-Hand \\
\hline BMP13 & Emergency Spillway \\
\hline BMP20 & Bio-Retention Practices \\
\hline BMP22 & Hydrodynamic Separators \\
\hline BMP23 & Catch Basin Media Inserts \\
\hline BMP12 & Stockpile and Materials Management \\
\hline BMP7 & \begin{tabular}{|l|} 
Entrance/Exit Tracking \\
\end{tabular} \\
\hline BMP8 & Sediment/Silt Fence \\
\hline BMP9 & Inlet Protection \\
\hline BMP10 & Fiber Rolls or Wattles \\
\hline BMP27 & Clean and Maintain Storm Drainage System \\
\hline BMP45 & Reduce Soil Compaction \\
\hline BMP52 & Site Stabilization \\
\hline BMP53 & Protecting Slopes \\
\hline BMP12 & Stockpile and Materials Management \\
\hline BMP28 & Stormdrain Inlet Labels \\
\hline BMP30 & Spill and Leak Prevention \\
\hline BMP31 & Spill Clean-Up \\
\hline BMP32 & Proper Fluids Handling \\
\hline BMP33 & Drain, Cover, and Contain Oily Parts Stored Outside \\
\hline BMP34 & Equipment Maintenance \\
\hline BMP35 & Pave Refueling Areas \\
\hline BMP36 & Housekeeping \\
\hline BMP37 & Proper Wash Water Disposal \\
\hline BMP39 & Proper Grease and Oil Disposal \\
\hline BMP41 & Minimize Chlorinated Cleaners and Solvents \\
\hline BMP49 & Secondary Containment \\
\hline BMP50 & Proper Hazardous Waste Disposal \\
\hline BMP51 & Materials Safety Data Sheets (MSDS) On-Hand \\
\hline BMP13 & Emergency Spillway \\
\hline BMP22 & Hydrodynamic Separators \\
\hline BMP23 & Catch Basin Media Inserts \\
\hline BMP24 & Filtration Systems \\
\hline BMP9 & Inlet Protection \\
\hline BMP10 & Fiber Rolls or Wattles \\
\hline BMP27 & Clean and Maintain Storm Drainage System \\
\hline BMP12 & Stockpile and Materials Management \\
\hline BMP36 & Housekeeping \\
\hline BMP40 & Dumpster Maintenance \\
\hline BMP49 & Secondary Containment \\
\hline BMP51 & Materials Safety Data Sheets (MSDS) On-Hand \\
\hline BMP13 & Emergency Spillway \\
\hline BMP22 & Hydrodynamic Separators \\
\hline BMP23 & Catch Basin Media Inserts \\
\hline BMP28 & Stormdrain Inlet Labels \\
\hline BMP30 & Spill and Leak Prevention \\
\hline BMP31 & Spill Clean-Up \\
\hline BMP32 & Proper Fluids Handling \\
\hline BMP33 & Drain, Cover, and Contain Oily Parts Stored Outside \\
\hline BMP34 & Equipment Maintenance \\
\hline BMP35 & Pave Refueling Areas \\
\hline BMP36 & Housekeeping \\
\hline BMP37 & Proper Wash Water Disposal \\
\hline BMP39 & Proper Grease and Oil Disposal \\
\hline BMP41 & Minimize Chlorinated Cleaners and Solvents \\
\hline BMP49 & Secondary Containment \\
\hline BMP50 & Proper Hazardous Waste Disposal \\
\hline BMP51 & Materials Safety Data Sheets (MSDS) On-Hand \\
\hline BMP22 & Hydrodynamic Separators \\
\hline BMP23 & Catch Basin Media Inserts \\
\hline BMP24 & Filtration Systems \\
\hline BMP9 & Inlet Protection \\
\hline BMP10 & Fiber Rolls or Wattles \\
\hline BMP27 & Clean and Maintain Storm Drainage System \\
\hline BMP12 & Stockpile and Materials Management \\
\hline BMP13 & Emergency Spillway \\
\hline BMP22 & Hydrodynamic Separators \\
\hline BMP23 & Catch Basin Media Inserts \\
\hline BMP31 & Spill Clean-Up \\
\hline BMP32 & Proper Fluids Handling \\
\hline BMP34 & Equipment Maintenance \\
\hline BMP35 & Pave Refueling Areas \\
\hline BMP49 & Secondary Containment \\
\hline BMP9 & Stockpile and Materials Management \\
\hline
\end{tabular}




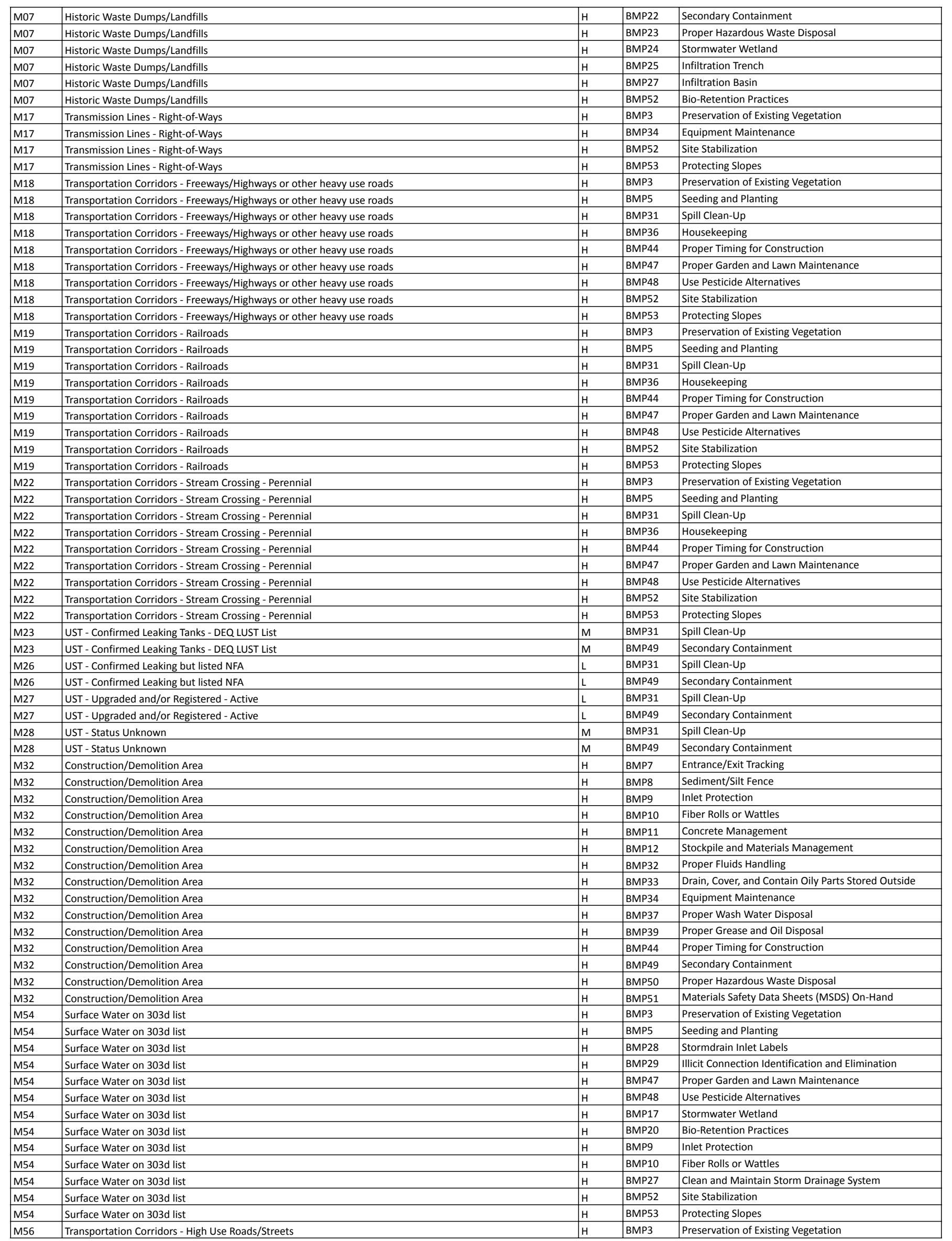




\begin{tabular}{|c|c|c|c|c|}
\hline M56 & Transportation Corridors - High Use Roads/Streets & $\mathrm{H}$ & BMP5 & Seeding and Planting \\
\hline M56 & Transportation Corridors - High Use Roads/Streets & $\mathrm{H}$ & BMP31 & Spill Clean-Up \\
\hline M56 & Transportation Corridors - High Use Roads/Streets & $\mathrm{H}$ & BMP36 & Housekeeping \\
\hline M56 & Transportation Corridors - High Use Roads/Streets & $\mathrm{H}$ & BMP44 & Proper Timing for Construction \\
\hline M56 & Transportation Corridors - High Use Roads/Streets & $\mathrm{H}$ & BMP47 & Proper Garden and Lawn Maintenance \\
\hline M56 & Transportation Corridors - High Use Roads/Streets & $\mathrm{H}$ & BMP48 & Use Pesticide Alternatives \\
\hline M56 & Transportation Corridors - High Use Roads/Streets & $\mathrm{H}$ & BMP52 & Site Stabilization \\
\hline M56 & Transportation Corridors - High Use Roads/Streets & $\mathrm{H}$ & BMP53 & Protecting Slopes \\
\hline M61 & Wells - Abandoned & $\mathrm{H}$ & BMP34 & Equipment Maintenance \\
\hline M61 & Wells - Abandoned & $\mathrm{H}$ & BMP49 & Secondary Containment \\
\hline M61 & Wells - Abandoned & $\mathrm{H}$ & BMP52 & Site Stabilization \\
\hline M62 & Lagoons/Liquid Wastes - Municipal/Industrial Wastewater Treatment & $\mathrm{H}$ & BMP2 & Diversion of Run-On \\
\hline M62 & Lagoons/Liquid Wastes - Municipal/Industrial Wastewater Treatment & $\mathrm{H}$ & BMP13 & Emergency Spillway \\
\hline M62 & Lagoons/Liquid Wastes - Municipal/Industrial Wastewater Treatment & $\mathrm{H}$ & BMP24 & Filtration Systems \\
\hline M62 & Lagoons/Liquid Wastes - Municipal/Industrial Wastewater Treatment & $\mathrm{H}$ & BMP31 & Spill Clean-Up \\
\hline R10 & Municipal/Industrial Landfill & $\mathrm{H}$ & BMP12 & Stockpile and Materials Management \\
\hline R10 & Municipal/Industrial Landfill & $\mathrm{H}$ & BMP49 & Secondary Containment \\
\hline R10 & Municipal/Industrial Landfill & $\mathrm{H}$ & BMP50 & Proper Hazardous Waste Disposal \\
\hline R10 & Municipal/Industrial Landfill & H & BMP17 & Stormwater Wetland \\
\hline R10 & Municipal/Industrial Landfill & $\mathrm{H}$ & BMP18 & Infiltration Trench \\
\hline R10 & Municipal/Industrial Landfill & $\mathrm{H}$ & BMP19 & Infiltration Basin \\
\hline R10 & Municipal/Industrial Landfill & H & BMP20 & Bio-Retention Practices \\
\hline R15 & Schools & M & BMP3 & Preservation of Existing Vegetation \\
\hline R15 & Schools & M & BMP46 & Downspout Extenders \\
\hline R15 & Schools & M & BMP12 & Stockpile and Materials Management \\
\hline R15 & Schools & M & BMP28 & Stormdrain Inlet Labels \\
\hline R15 & Schools & M & BMP29 & Illicit Connection Identification and Elimination \\
\hline R15 & Schools & M & BMP36 & Housekeeping \\
\hline R15 & Schools & M & BMP37 & Proper Wash Water Disposal \\
\hline R15 & Schools & M & BMP40 & Dumpster Maintenance \\
\hline R15 & Schools & M & BMP41 & Minimize Chlorinated Cleaners and Solvents \\
\hline R15 & Schools & M & BMP47 & Proper Garden and Lawn Maintenance \\
\hline R15 & Schools & M & BMP48 & Use Pesticide Alternatives \\
\hline R15 & Schools & M & BMP51 & Materials Safety Data Sheets (MSDS) On-Hand \\
\hline R15 & Schools & M & BMP13 & Emergency Spillway \\
\hline R15 & Schools & M & BMP17 & Stormwater Wetland \\
\hline R15 & Schools & M & BMP20 & Bio-Retention Practices \\
\hline R15 & Schools & M & BMP22 & Hydrodynamic Separators \\
\hline R15 & Schools & M & BMP23 & Catch Basin Media Inserts \\
\hline R15 & Schools & M & BMP26 & Porous Pavement \\
\hline R15 & Schools & M & BMP27 & Clean and Maintain Storm Drainage System \\
\hline R15 & Schools & M & BMP52 & Site Stabilization \\
\hline R15 & Schools & M & BMP53 & Protecting Slopes \\
\hline R18 & Utility Stations - Maintenance/Transformer Storage (inc. Hydroelectric Power Equip.) & H & BMP3 & Preservation of Existing Vegetation \\
\hline R18 & Utility Stations - Maintenance/Transformer Storage (inc. Hydroelectric Power Equip.) & $\mathrm{H}$ & BMP12 & Stockpile and Materials Management \\
\hline R18 & Utility Stations - Maintenance/Transformer Storage (inc. Hydroelectric Power Equip.) & H & BMP31 & Spill Clean-Up \\
\hline R18 & Utility Stations - Maintenance/Transformer Storage (inc. Hydroelectric Power Equip.) & $\mathrm{H}$ & BMP32 & Proper Fluids Handling \\
\hline R18 & Utility Stations - Maintenance/Transformer Storage (inc. Hydroelectric Power Equip.) & $\mathrm{H}$ & BMP33 & Drain, Cover, and Contain Oily Parts Stored Outside \\
\hline R18 & Utility Stations - Maintenance/Transformer Storage (inc. Hydroelectric Power Equip.) & H & BMP34 & Equipment Maintenance \\
\hline R18 & Utility Stations - Maintenance/Transformer Storage (inc. Hydroelectric Power Equip.) & $\mathrm{H}$ & BMP36 & Housekeeping \\
\hline R18 & Utility Stations - Maintenance/Transformer Storage (inc. Hydroelectric Power Equip.) & $\mathrm{H}$ & BMP49 & Secondary Containment \\
\hline R18 & Utility Stations - Maintenance/Transformer Storage (inc. Hydroelectric Power Equip.) & $\mathrm{H}$ & BMP50 & Proper Hazardous Waste Disposal \\
\hline R18 & Utility Stations - Maintenance/Transformer Storage (inc. Hydroelectric Power Equip.) & $\mathrm{H}$ & BMP51 & Materials Safety Data Sheets (MSDS) On-Hand \\
\hline R18 & Utility Stations - Maintenance/Transformer Storage (inc. Hydroelectric Power Equip.) & $\mathrm{H}$ & BMP13 & Emergency Spillway \\
\hline R18 & Utility Stations - Maintenance/Transformer Storage (inc. Hydroelectric Power Equip.) & $\mathrm{H}$ & BMP22 & Hydrodynamic Separators \\
\hline R18 & Utility Stations - Maintenance/Transformer Storage (inc. Hydroelectric Power Equip.) & $\mathrm{H}$ & BMP23 & Catch Basin Media Inserts \\
\hline R18 & Utility Stations - Maintenance/Transformer Storage (inc. Hydroelectric Power Equip.) & $\mathrm{H}$ & BMP24 & Filtration Systems \\
\hline R18 & Utility Stations - Maintenance/Transformer Storage (inc. Hydroelectric Power Equip.) & H & BMP9 & Inlet Protection \\
\hline R18 & Utility Stations - Maintenance/Transformer Storage (inc. Hydroelectric Power Equip.) & $\mathrm{H}$ & BMP27 & Clean and Maintain Storm Drainage System \\
\hline R20 & Wastewater Treatment Plants/Collection Stations & $\mathrm{H}$ & BMP12 & Stockpile and Materials Management \\
\hline R20 & Wastewater Treatment Plants/Collection Stations & $\mathrm{H}$ & BMP31 & Spill Clean-Up \\
\hline R20 & Wastewater Treatment Plants/Collection Stations & $\mathrm{H}$ & BMP32 & Proper Fluids Handling \\
\hline R20 & Wastewater Treatment Plants/Collection Stations & $\mathrm{H}$ & BMP34 & Equipment Maintenance \\
\hline R20 & Wastewater Treatment Plants/Collection Stations & $\mathrm{H}$ & BMP36 & Housekeeping \\
\hline R20 & Wastewater Treatment Plants/Collection Stations & H & BMP49 & Secondary Containment \\
\hline R20 & Wastewater Treatment Plants/Collection Stations & $\mathrm{H}$ & BMP50 & Proper Hazardous Waste Disposal \\
\hline R20 & Wastewater Treatment Plants/Collection Stations & $\mathrm{H}$ & BMP51 & Materials Safety Data Sheets (MSDS) On-Hand \\
\hline R20 & Wastewater Treatment Plants/Collection Stations & $\mathrm{H}$ & BMP13 & Emergency Spillway \\
\hline R20 & Wastewater Treatment Plants/Collection Stations & $\mathrm{H}$ & BMP17 & Stormwater Wetland \\
\hline R20 & Wastewater Treatment Plants/Collection Stations & $\mathrm{H}$ & BMP22 & Hydrodynamic Separators \\
\hline R20 & Wastewater Treatment Plants/Collection Stations & $\mathrm{H}$ & BMP23 & Catch Basin Media Inserts \\
\hline R20 & Wastewater Treatment Plants/Collection Stations & $\mathrm{H}$ & BMP24 & Filtration Systems \\
\hline R20 & Wastewater Treatment Plants/Collection Stations & H & BMP9 & Inlet Protection \\
\hline R20 & Wastewater Treatment Plants/Collection Stations & $\mathrm{H}$ & BMP10 & Fiber Rolls or Wattles \\
\hline
\end{tabular}




\begin{tabular}{|c|c|c|c|c|c|c|}
\hline 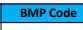 & BMP Name & BMP Description & & & & \\
\hline вмР1 & check Dam & 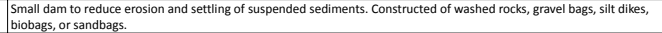 & Runoff Controls & ACWA & & \\
\hline BMP2 & Diversion of Run-0n & 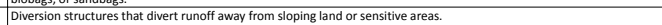 & Runoff Controls & ACWA & & \\
\hline вмРз & \begin{tabular}{|l|l} 
Preservation of Existing Vegetation \\
\end{tabular} & 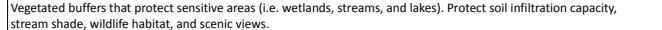 & Erosion Prevention & ACWA & & \\
\hline $\begin{array}{l}\text { BMPP4 } \\
\text { BMP5 }\end{array}$ & $\begin{array}{l}\text { Mulches } \\
\text { Seeding and Planting } \\
\end{array}$ & 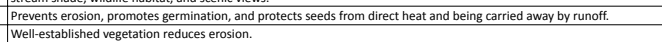 & 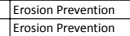 & 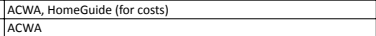 & $\$ 15-565$ per vard & \\
\hline BMPG & Erosion Blankets and Mats & Covers the soil to prevent tersioion and assist in the establishment to vegetation. & Erosion Prevention & ACWA, Minnestota Stormwater Manual ffor costs) & $\$ 1.02$-\$2.90 per square yard & 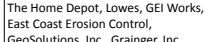 \\
\hline BMP7 & Entrance//Exit Tracking & 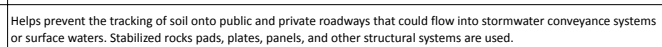 & Sediment Controls & ACWA, Minnessta stormwater Manual ffor costs) & |55,763.08 & 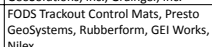 \\
\hline вмРв & Sediment/sit Fence & 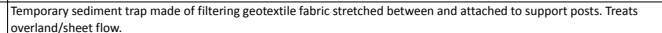 & Sediment Controls & ACWA, EPA & \$1.1.14-\$4.88 per linear foot & \\
\hline BMP9 & Inlmet rotetction & Devices that fititer course sediments from runoff, preventing them from entering storm draingage systems. & |Sediment controls & ACWA & & \\
\hline Bmp10 & Fiber Rolls or Wattles & 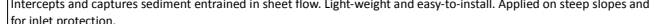 & Sediment Controls & ACWA, After Wildfrie - A Guide for New Mexicic Communtities & $\$ 1,100-54,000$ per acce & \\
\hline BMP11 & Concrete Management & Prevents or reductuces discharge of pollutants to stormwater from concretete waste. & Pollution Prevention & ACWA & & \\
\hline BMP12 & Stockpile and Materials Management & 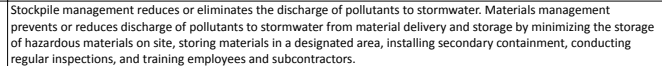 & Pollution Prevention & ACWA & . & - \\
\hline вмр13 & Emergency Spillway & 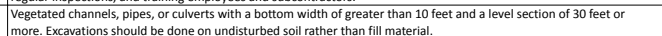 & Runoff Controls & Minnesota Pollution Control Agency & & \\
\hline BMp14 & Wet Pond & 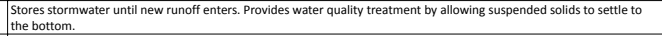 & Runoff Controls & Minnesota Pollution Control Agency & & \\
\hline BMP15 & Wet Extended Detention Pond & 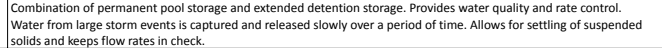 & Runoff Controls & Minnesota Pollution Control Agency & & \\
\hline вмр16 & ory pond & 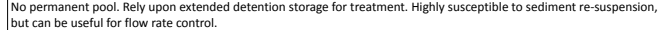 & Runoff Controls & Minnesota Pollution Control Agency & & \\
\hline BMP17 & Stormwater Wetland & 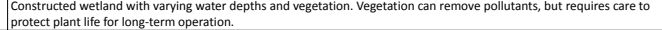 & Runoff Controls & Minnesta Pollution Control Agency & & \\
\hline BмР18 & Infittration Trench & 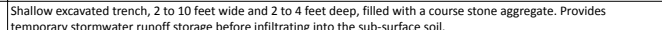 & Runoffif controls & Minnesta Pollution Control Agency & & \\
\hline 8мР19 & Infiltration Basin & 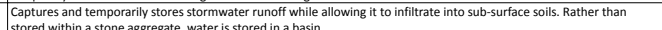 & Runoff controls & Minnesota Polutuion Control Agency & & \\
\hline BMP20 & Bio-Retention Practices & 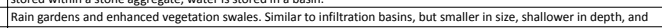 & Runoff Controls & Minnesota Pollution Control A Agency, Reduce Runoff, Interstate & 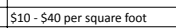 & \\
\hline BMP21 & 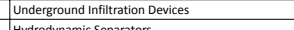 & 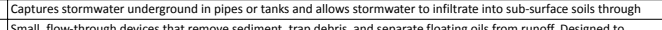 & 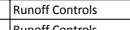 & Minnesstat Pollution Control Agency & & \\
\hline BMP23 & Catch Basin Medial Inserts & 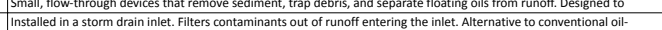 & $\begin{array}{l}\text { Runoff controls } \\
\text { Runofft }\end{array}$ & 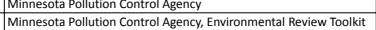 & $\$ 100-\$ 1,500$ per unit & \\
\hline BMP24 & Filtration Systems & 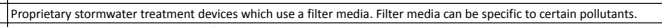 & Runoff Controls & Minnesotat Pollution Control ABgency & & Hydro International, contech \\
\hline 皿PN25 & 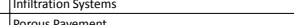 & 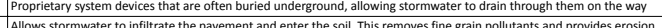 & Runoff Controls & Mininestat Polutution control A A Bency & & \\
\hline BMP27 & Clean and Maintain Storm Drainage System & Clean and maintain catch basins anvually, or more frequently at sites with activities generating a lot of sediment and & Sedimentent Controls & King county storom & & \\
\hline BMP28 & Stormdrain Inlet tabels & 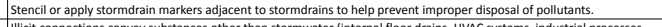 & Pollution Prevention & King County Stormwater Services & & \\
\hline 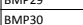 & 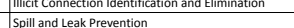 & 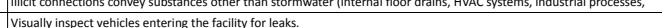 & $\begin{array}{l}\text { Poolution Prevention } \\
\text { Pollution Prevention } \\
\end{array}$ & $\begin{array}{l}\text { King gonnty Stormwater rervices } \\
\text { Sustainable Conservation }\end{array}$ & & \\
\hline & & 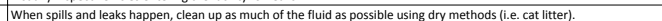 & & istainable Conservation & & \\
\hline BMP32 & Proper fluids thandling & 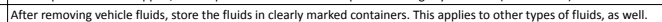 & Pollution Prevention & Sustainable Conservation & & \\
\hline BMP33 & Orain, Cover, and Contain Oily Patts Stored Outside & 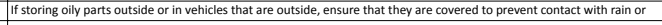 & Pollution Prevention & Uustainable Conservation & & \\
\hline 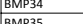 & 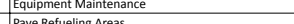 & & Pollution Prevention & Sustainable Consenation & & \\
\hline 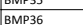 & $\begin{array}{l}\text { Pave Refueling areas } \\
\text { Housekeeping }\end{array}$ & 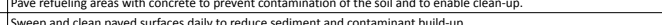 & 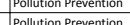 & $\begin{array}{l}\text { Sustatabalele Conservation } \\
\text { Sustanalie Conseration }\end{array}$ & & \\
\hline & Proper Wash Water Disososal & 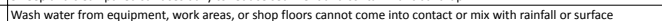 & & Sustainable Conservation & & \\
\hline BMP38 & Venicle Owner Education & Inform customers who remove parts to do so properly and to a pproporiately dissosose of fluids. & Polution Prevention & Sustainable consereation & & \\
\hline BMP39 & Proper Grease and Oil Disposal & Recrcle grease and oli instead of pouring down sinks, floor drains, and/or into parking lots and streets. & Pollution Prevention & Environmental Services, City of Portland & & \\
\hline 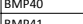 & Dumpster Maintenance & Keep pumpster areas clean and the lidis closed. Don' fill with hiquid waste or hose them out. & |polulutio Prevention & Envirommental Services, city of Portlan & & \\
\hline BMP42 & 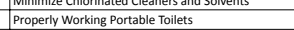 & Make sure portable toilets are in good working order and not leaking. & $\begin{array}{l}\text { Pollution Priovention Prevention } \\
\end{array}$ & 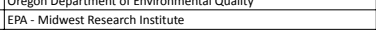 & & \\
\hline & Water Conservation & Use as intitl water as possible for dust control. & & EPA-Midwest Research histitute & & \\
\hline BMP445 & $\begin{array}{l}\text { Proper Timinin for Construction } \\
\text { Reduce Soil Compaction }\end{array}$ & 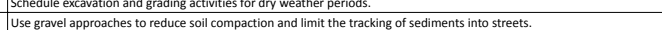 & $\begin{array}{l}\text { Poplution Preventiton } \\
\text { Sediment Controls } \\
\end{array}$ & $\begin{array}{l}\text { EAP-Midevest Research institute } \\
\text { EPA-Midwest Research Institute }\end{array}$ & & \\
\hline BMP46 & Downspout Extenders & Prevents erossion from roof funoff and allows for groundwater recharge. & Erosion Prevention & University of Wisconsin - Extension & $\sim \$ 5-\sim 50$ per unit & \begin{tabular}{|l|l|l} 
The Home Depot, Lowes, Menards, \\
\end{tabular} \\
\hline 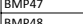 & $\begin{array}{l}\text { Proper Garden and Lawn Maintenance } \\
\end{array}$ & 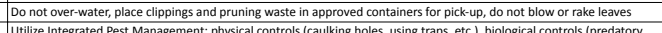 & Pollution Prevention & EPA-Midwest Research Institute & & \\
\hline BMP49 & Secondary Contaimment & 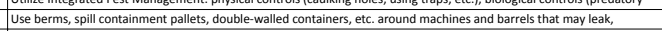 & $\begin{array}{l}\text { Pollutution Prevention } \\
\text { Prention } \\
\end{array}$ & Santa Clara Valleyey Urban Rununoff Pollution Pre & 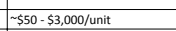 & Instate Products, ULINE, New Pig, \\
\hline & Proper Hazardous Waste isposal & Metalworking fluids that can't be recycded, cleaning solutions, and absorbent materials used to clean & Poolution Prevention & Santa Clar valley urban Runoff Pollution Prevention Program & & \\
\hline BMP51 & Materials Safety Data Sheets (MSDS) On-Hand & obtain MSDS for al chemicals and metals stored in the facility and keep them accessible atall times. & Pollution Prevention & Santa Clara valley Urban Runoff Pollution Prevention Program & & \\
\hline $\begin{array}{l}\text { BMP52 } \\
\text { BMP53 }\end{array}$ & $\begin{array}{l}\text { Site Stabilization } \\
\text { Protecting liopes }\end{array}$ & 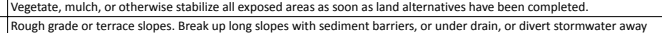 & $\begin{array}{l}\text { Sediment Controls } \\
\text { Sediment Contros }\end{array}$ & 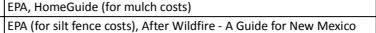 & 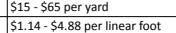 & $\begin{array}{l}\text { The Home Depot, Ground } \\
\text { The Home Depot Gringe }\end{array}$ \\
\hline
\end{tabular}

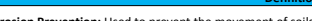

Pollution Prevention: Used to prevent pollution from reaching the storm drain system or waterbodies. 
Activity at Site

Above Ground Storage Tanks-Excluding Water Tanks and Residential ASTs

Aboveground Storage Tanks - Residential

Apartments and Condominium

Auto - Body Shops

Auto - Car Washes

Auto - Gas Stations

Auto - Gas Stations - Historic

Auto - Repair Shops

Automobiles - Body Shops

Automobiles - Gas Stations

Boat Services/Repair/Refinishing

Campgrounds/RV Parks

Cement/Concrete Plants

Cemeteries - Pre-1945

Chemical/Petroleum Processing/Storage

Communications Office

Confined Animal Feeding Operations (CAFOs)

Construction Company

Construction/Demolition Area

Crops - Irrigated - Berries, Hops, Mint, Orchards, Vineyards/Nurseries, Green Houses, Vegetables, etc.

Crops - Nonirrigated - Christmas Trees, Grains, Grass Seeds, Hay, Pasture

Drinking Water Treatment Plants

Dry Cleaners

Electrical/Electronic Manufacturing

Fire Station

Fleet/Trucking/Bus Terminals

Furniture/Lumber/Parts Stores

Graywater reuse/disposal or Industrial reuse

Grazing Animals - > 5 Large Animals or Equivalent/Acre

Historic Waste Dumps/Landfills

Home Manufacturing

Homesteads - Rural - Septic Systems $<1 /$ Acre

Housing - High Density - $>1$ House/0.5 Acres

Industrial/Commercial Injection Wells/Drywells/Sumps - Class V UICS

Irrigation Canal/Pond

Junk/Scrap/Salvage Yards

Known Contamination listed as NFA (Sites/Plumes/Spills from ECSI)

Known Contamination Sites/Plumes/Spills (ECSI)

Lagoons/Liquid Wastes - Municipal/Industrial Wastewater Treatmen

Large Capacity Septic Systems -Class V UIC (serves >20)

Lawn Care - Highly Maintained Areas

Machine Shops

Maintenance Shop/Equipment Storage - Not Transportation Related

Maintenance Shop/Equipment Storage - Transportation Related

Managed Forest Lands - Clearcut Harvested $-<35$ yrs

Medical/Vet Offices

Metal Plating/Finishing Fabrication

Mining Activities - Active - sand/gravel/rock/soil

Mining Activities - Gravel Mines/Gravel Pits

Mining Activities - Inactive - other than sand/gravel/rock/sol

Mining Activities - Inactive - sand/gravel/rock/soil

Miscellaneous Equipment Maintenance

Miscellaneous Manufacturing

Municipal/Industrial Landfill

Parking Lots/Malls $->50$ Spaces

Parks

Pesticide/Fertilizer/Petroleum Storage, Handling, Mixing, \& Cleaning Areas

Photo Processing/Printing

Pipelines - Chemical and Petroleum

Plastic/Synthetics Producer

Ponds/Streams/Wetlands

River Recreation - Heavy Use (inc Campgrounds)

RV/Mini Storage

Schools

Septic Systems - High Density - > 1/Acre

Sewer Lines - Close Proximity to PWS

Stormwater Wastewater Injection/Dry Wells, Sumps - Class V UIC

Surface Water on 303d list

Transmission Lines - Right-of-Ways

Transportation - Railroads

Transportation Corridors - Freeways/Highways or other heavy use roads

Transportation Corridors - High Use Roads/Streets

Transportation Corridors - Railroads

Transportation Corridors - Stream Crossing - Perennial

Unknown Operation

Upstream Reservoirs/Dams

UST - Confirmed Leaking but listed as NFA - DEQ LUST Lis

UST - Confirmed Leaking Tanks - DEQ LUST List

UST - Decommissioned - Inactive

UST - Status Unknown

UST - Upgraded and/or Registered - Active

Utility Stations - Maintenance/Transformer Storage (inc. Hydroelectric Power Equip.)

Warehouses

Wastewater Treatment Plants/Collection Stations

Wells - Abandoned

Wells - Production

Wood/Pulp/Paper Processing and Mills
Runoff Controls

Erosion Prevention Practices

Sediment Controls

Pollution Prevention Practices 
APPENDIX H. USWA Excel File with PCS Information 


\begin{tabular}{|c|c|c|c|}
\hline & & & \\
\hline & $\begin{array}{l}\text { City } \\
\text { uknown }\end{array}$ & $\begin{array}{l}\text { County } \\
\text { loseppine }\end{array}$ & $\begin{array}{l}\text { RET_DATE } \\
20213\end{array}$ \\
\hline Not Applicable & $\begin{array}{l}\text { UnkNOWN } \\
\text { unkNOWN }\end{array}$ & $\begin{array}{l}\text { Jossphne } \\
\text { Josphine }\end{array}$ & 2013 \\
\hline Not Applicable & unkNOWN & Josephine & 2013 \\
\hline & & & $\begin{array}{l}2013 \\
2013\end{array}$ \\
\hline Not Applicable & $\begin{array}{l}\text { UNRNOOWN } \\
\text { unkNown }\end{array}$ & $\begin{array}{l}\text { Jackson } \\
\text { Jackson }\end{array}$ & 2013 \\
\hline Not Applicable & $\begin{array}{l}\text { unkNOOWN } \\
\text { unNNOWN }\end{array}$ & $\begin{array}{l}\text { Jackson } \\
\text { Jackson }\end{array}$ & 2013 \\
\hline $\begin{array}{l}\text { Not AtAppicicable } \\
\text { Not Aplicable }\end{array}$ & & $\begin{array}{l}\text { Jackson } \\
\text { Jackson }\end{array}$ & \\
\hline Not Applicable & ASHLAND & Jackson & 2013 \\
\hline $\begin{array}{l}\text { Not Applicable } \\
\text { Not Applicable }\end{array}$ & $\begin{array}{l}\text { UnkNNown } \\
\text { unkNOWN }\end{array}$ & $\begin{array}{l}\text { Jackson } \\
\text { Jackson }\end{array}$ & $\begin{array}{l}2013 \\
2013\end{array}$ \\
\hline Not Applicable & UnkNown & Jackson & 2013 \\
\hline $\begin{array}{l}\text { Not Applicable } \\
\text { Not Appicicble }\end{array}$ & $\begin{array}{l}\text { UNKNOOWN } \\
\text { unkNOWNA }\end{array}$ & Jackson & $\begin{array}{l}2013 \\
2013 \\
2013\end{array}$ \\
\hline $\begin{array}{l}\text { Not AtApplicable } \\
\text { Not Applicable }\end{array}$ & SHADY COVE & $\begin{array}{l}\text { Jackson } \\
\text { Jackson }\end{array}$ & ${ }_{2013}^{2013}$ \\
\hline & & Jackson & \\
\hline Not Applicable & UNKNOWN & Jackson & 2013 \\
\hline $\begin{array}{l}\text { Not A Applicable } \\
1 \text { CLARK ST }\end{array}$ & 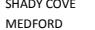 & $\begin{array}{l}\text { Jackson } \\
\text { JaCKSON }\end{array}$ & $\begin{array}{l}2013 \\
09 / 2 / 2 / 2008\end{array}$ \\
\hline Not Applicable & unknown & Jackson & 2013 \\
\hline $\begin{array}{l}\text { Not Applicable } \\
\text { Not Applicable }\end{array}$ & $\begin{array}{l}\text { unkNown } \\
\text { unkNOWNA }\end{array}$ & $\begin{array}{l}\text { Jackson } \\
\text { Josephine }\end{array}$ & 2013 \\
\hline $\begin{array}{l}\text { Not Applicable } \\
\text { Not Aplicable }\end{array}$ & $\begin{array}{l}\text { UNNNEONN } \\
\text { UNKNNOWNA }\end{array}$ & $\begin{array}{l}\text { Jossephine } \\
\text { losenhine }\end{array}$ & $\begin{array}{l}2013 \\
2013\end{array}$ \\
\hline Not Applicable & $\begin{array}{l}\text { UnNRNOWN } \\
\text { unkNown }\end{array}$ & 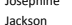 & $\begin{array}{l}2013 \\
2013\end{array}$ \\
\hline Not Applicable & unkNown & Jackson & 2013 \\
\hline Not Applicable & unknown & Jackson & 2013 \\
\hline Not Applicable & UnkNown & Jackson & 2013 \\
\hline $\begin{array}{l}\text { Not Applicable } \\
\text { Not Appichale }\end{array}$ & $\begin{array}{l}\text { UNKNOWN } \\
\text { CENTALPOONT }\end{array}$ & Jackson & $\begin{array}{l}2013 \\
2013 \\
2013\end{array}$ \\
\hline Not Appicicale & $\begin{array}{l}\text { CENTRAL POINT } \\
\text { uNKNOWN }\end{array}$ & $\begin{array}{l}\text { Jackson } \\
\text { Jackson }\end{array}$ & 2013 \\
\hline $\begin{array}{l}\text { Not Appolicolie } \\
\text { Not Appicable }\end{array}$ & 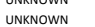 & 势 & $\begin{array}{l}2013 \\
2013 \\
2013\end{array}$ \\
\hline Not Applicable & unkNown & Jackson & 2013 \\
\hline Not Applicable & unknown & Jackson & 2013 \\
\hline Not Applicable & UnkNown & Douglas & 2013 \\
\hline $\begin{array}{l}\text { Not Applicable } \\
\text { Not Applicable }\end{array}$ & & Jackson & 2013 \\
\hline $\begin{array}{l}\text { Not Applicable } \\
\text { Not Applicable }\end{array}$ & $\begin{array}{l}\text { UNNNEONN } \\
\text { UNKNNOWN }\end{array}$ & $\begin{array}{l}\text { Jackson } \\
\text { Jackson }\end{array}$ & $\begin{array}{l}2013 \\
2013 \\
2013\end{array}$ \\
\hline $\begin{array}{l}\text { Not Appplicane } \\
\text { Not Aplicable }\end{array}$ & $\begin{array}{l}\text { uns } \\
\text { unkNown }\end{array}$ & 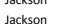 & $\begin{array}{l}2013 \\
2013\end{array}$ \\
\hline 1 E CLARK ST & MEEFORD & $\begin{array}{l}\text { Jackson } \\
\text { Jackon }\end{array}$ & o9/29/2008 \\
\hline 10 S OAKDALE AVE & Medford & JackSON & 11/1/2018 \\
\hline Not Applicable & UnkNown & Jackson & 2013 \\
\hline 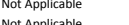 & ASHLAND & Jackson & 2013 \\
\hline - & $\begin{array}{l}\text { UNNNOONN } \\
\text { UNKNOWN }\end{array}$ & $\begin{array}{l}\text { Jackson } \\
\text { ackson }\end{array}$ & 2013 \\
\hline Not Applicable & $\begin{array}{l}\text { unkNown } \\
\text { uns }\end{array}$ & $\begin{array}{l}\text { Sackon } \\
\text { Jactson }\end{array}$ & $\begin{array}{l}2013 \\
2013\end{array}$ \\
\hline Not Applicable & unkNown & Jackson & 2013 \\
\hline Not Applicable & unknown & Jackson & 2013 \\
\hline Not Applicable & UnkNOWN & Jackson & 2013 \\
\hline - & 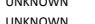 & Jackson & 2013 \\
\hline Not Appocicabe & 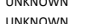 & $\begin{array}{l}\text { Jackson } \\
\text { accsen }\end{array}$ & 2013 \\
\hline Not Applicable & unkNown & Jackson & 2013 \\
\hline Not Applicable & UnkNown & Jackson & 2013 \\
\hline Not Applicable & UNKNOWN & Jackson & 2013 \\
\hline Not Applicable & JAACKSOVVIULE & Jackson & 2013 \\
\hline Not Appocicabe & 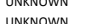 & $\begin{array}{l}\text { Jackson } \\
\text { accsen }\end{array}$ & 2013 \\
\hline Not Applicable & - & 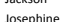 & $\begin{array}{l}2013 \\
2013\end{array}$ \\
\hline Not Applicable & unkNown & Josechine & 2013 \\
\hline Not Applicable & unkNown & Josephine & 2013 \\
\hline Not Applicable & TALENT & Jackson & 2013 \\
\hline $\begin{array}{l}\text { Not Applicicalle } \\
\text { Not Alichle }\end{array}$ & UnWNNOWN & Jackson & 2013 \\
\hline 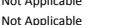 & 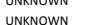 & $\begin{array}{l}\text { Sacsenon } \\
\text { lackson }\end{array}$ & $\begin{array}{l}2013 \\
2013\end{array}$ \\
\hline Not popiciable & EAGLE POINT & 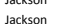 & $\begin{array}{l}2013 \\
2013\end{array}$ \\
\hline 1005 OAKDALE ST & Medford & Jackson & 10/31/2018 \\
\hline 100 WIILG WAY & Medfordd & JaCKSON & $10 / 31 / 2018$ \\
\hline Not Applicable & UnKNoWNN & & \\
\hline Not Applicable & 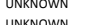 & Jactson & 2013 \\
\hline Not Applicibere & 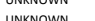 & 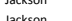 & 2013 \\
\hline Not Appliciabe & unkNown & 势 & $\begin{array}{l}2013 \\
2013\end{array}$ \\
\hline Not Applicable & unkNown & Jackson & 2013 \\
\hline Not Applicable & UnkNown & Jackson & 2013 \\
\hline 1000 S CENTRAL AVE & $\begin{array}{l}\text { Medford } \\
\text { Metford }\end{array}$ & $\begin{array}{l}\text { Jackson } \\
\text { Jacsoon }\end{array}$ & $\begin{array}{l}10 / / 31 / 2018 \\
101 / 212018\end{array}$ \\
\hline $\begin{array}{l}1000 \text { S SENTTAL LVE } \\
1000 \text { S CENTRA AVEF }\end{array}$ & $\begin{array}{l}\text { Metotorl } \\
\text { Metford }\end{array}$ & 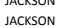 & $\begin{array}{l}101 / 3 / 21 / 2018 \\
10 / 131 / 2018\end{array}$ \\
\hline 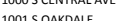 & 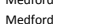 & 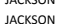 & $\begin{array}{l}101 / 1 / 2018 \\
101312018\end{array}$ \\
\hline 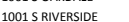 & MEFFRO & $\begin{array}{l}\text { IICCSSON } \\
\text { I. }\end{array}$ & $\begin{array}{l}10 / 131 / 2018 \\
09 / 29 / 2008\end{array}$ \\
\hline 10015 RIVERSID AVE & Mefford & & 10/31/2018 \\
\hline Not Applicable & UnkNown & Jackson & 2013 \\
\hline Not Applicable & UNRNoWn & & 2013 \\
\hline $\begin{array}{l}\text { A Applicable } \\
\text { Applicable }\end{array}$ & $\begin{array}{l}\text { UNKNNOWN } \\
\text { UNKNOWN }\end{array}$ & & $\begin{array}{l}2013 \\
2013\end{array}$ \\
\hline - & - & 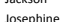 & 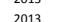 \\
\hline $\begin{array}{l}\text { Not Applicable } \\
\text { Pate }\end{array}$ & unkNown & Jackstun & \\
\hline
\end{tabular}

Data_sourc

regenn Dept. of Transportation, Technicial Servicices raranch, Brideses section (1000O)

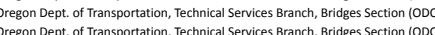
Deson Dept. of Transportation, Technical Services Branch, Bridges Section (ODOOO) (a) tion, Technical Services Branch, Bridges Section (ODOOO) Oregon Dept. of Transportation, Technical Services Branch, Bridges Section (ODOO

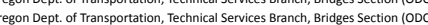

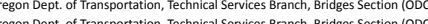
(a) (a)

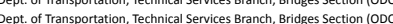
regon Dept. of Transportation, Technical Services Branch, Brideses Section (1000) OR State Fire Marshall Hazardous Substance Information System databasese (SFM/HSIS- 2009

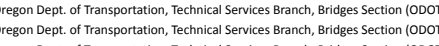
Oregon Dept. of Transportation, Technical Sevvices Branch, Brideses Section (ODDO) oregon Dept. of Transportation, Technical Services Branch, Brideses Section looof regeon Dept. of Transportation, Technical Services Branch, Bridees Section $1000 \mathrm{O})$

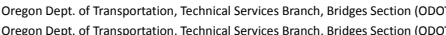

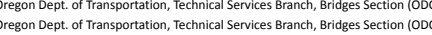

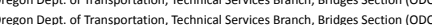
Oregon Dept. of Transsortation, Technical Sevices rranch, Bridges Section $1000 \mathrm{O})$

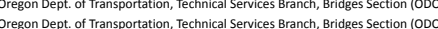
oregon Dept. of Transportation, Technical Services Branch, Brideses Section $(000)$

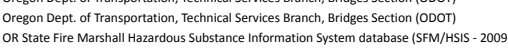

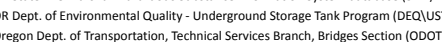

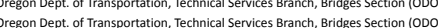

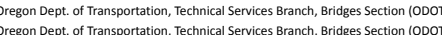

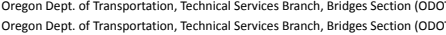

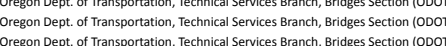

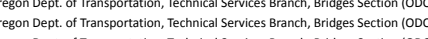

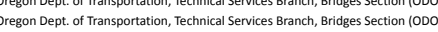

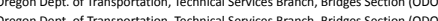

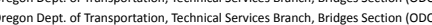
Oregon Dept. of Transsortation, Technical Services rranch, Bridges Section $1000 \mathrm{O})$

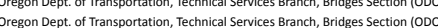
Oregon Dept. of Transportation, Technical Sevices rranch, Bridges Section (ODOON) oregon Dept. of Transsortation, Technical Services Branch, Bridges Section food Oregon Deent of Transsortation. Techicial Sevices Branch, Brideses Section (1000)

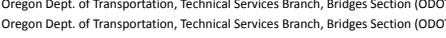

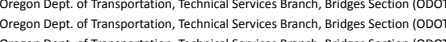

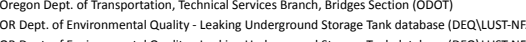

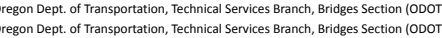

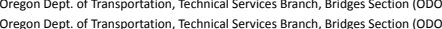
Oregon Dept. of Transsortation, Technical Services Branch, Bridges Section $1000 \mathrm{O}$

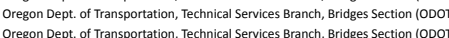

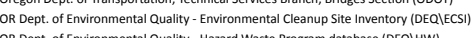
OR Dept. of Environmental Q Quality - Hazard Waste Program database (DEQQ|HW)

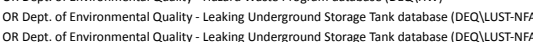

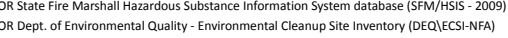
, Technical Services rranch, Bridges Section (1000T) Oregon Dept. of Transportation, Technical Services rranch, Bridides Section (lood

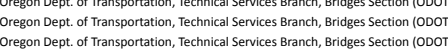

\begin{tabular}{|c|c|c|}
\hline \multicolumn{3}{|r|}{ k Common_nm } \\
\hline M22 & $\begin{array}{l}\mathrm{L} \\
\mathrm{L}\end{array} \mathrm{H}_{\mathrm{H}}^{\mathrm{H}}$ & $\begin{array}{l}\text { Chapmana Creek, Hwy } 38 \\
\text { culvert Hyw } 38\end{array}$ \\
\hline 2022 & $\begin{array}{lll}L & H \\
L & H\end{array}$ & Tycer reek, Awy 38 \\
\hline & & $\begin{array}{l}\text { Culvert, Hwy } 38 \text { a t MP } 3.47 \\
\text { Rogue River Hhy } 72 \text { (Dodge) }\end{array}$ \\
\hline & & 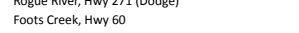 \\
\hline & ${ }_{H}^{H}$ & 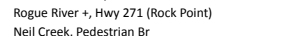 \\
\hline & H & $\begin{array}{l}\text { Neilicreke, Pedestrtran Br } \\
\text { Jackson creek, Hwy } 63\end{array}$ \\
\hline & & Hwy 21 over CORP \\
\hline & ${ }_{H}^{H}$ & 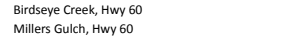 \\
\hline M222 & ${ }_{4}^{H}$ & 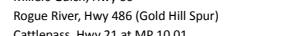 \\
\hline & H & 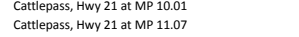 \\
\hline M2: & ${ }_{H}^{\mathrm{H}}$ & 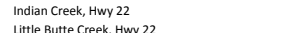 \\
\hline & H & 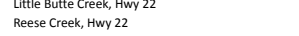 \\
\hline & H & 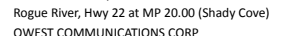 \\
\hline & H & $\begin{array}{l}\text { aWEST COMMUNNCATIONS CORP } \\
\text { Elk Creek, HWy } 62\end{array}$ \\
\hline & & $\begin{array}{l}\text { Lewis Creek, Hwy } 62 \\
\text { Kelly Creak Hwy } 38\end{array}$ \\
\hline & ᄂ & $\begin{array}{l}\text { Kelly r ceek, hHy } 38 \\
\text { Bear reek, Hwy } 38\end{array}$ \\
\hline 1222 & H & CORP over Hwy 1 (Wall Creek) \\
\hline & & $\begin{array}{l}\text { Neil rreek, Hww } 1 \\
\text { Hamition Creek, Hwy } 63\end{array}$ \\
\hline & L & 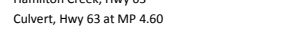 \\
\hline & & 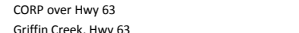 \\
\hline & ᄂ & 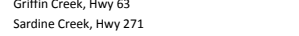 \\
\hline & H & Caste Creek, Hwy 233 \\
\hline & & $\begin{array}{l}\text { Bybee ereee, } \text {, why } 2333 \\
\text { Rogue River Hwy } 233\end{array}$ \\
\hline & i & Muir Creek, Hwy 233 \\
\hline & & 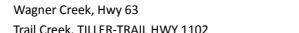 \\
\hline & t & 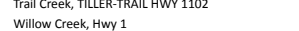 \\
\hline & ᄂ & Hog Creek, Agate Rd \\
\hline & H & QWEST COMMUNICATIONS CORP \\
\hline M22 & L & 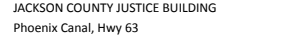 \\
\hline M22 & ᄂ & $\begin{array}{l}\text { Hamiton Creek, Huy } 21 \\
\text { Cation }\end{array}$ \\
\hline 122 & & 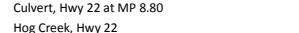 \\
\hline & ᄂ & 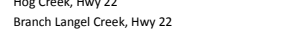 \\
\hline & H & Cricket creek, Huy 22 \\
\hline & & $\begin{array}{l}\text { Flat Creek, Hwy } 22 \\
\text { Union reeak Hiy } 22\end{array}$ \\
\hline & L & 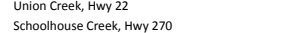 \\
\hline & & 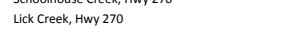 \\
\hline & L & Salt creek, Hwy 270 \\
\hline & ᄂ & 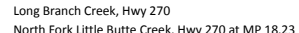 \\
\hline & & 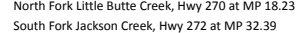 \\
\hline M2: & $\mathrm{L}$ & 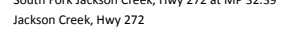 \\
\hline & $\begin{array}{l}\mathrm{L} \\
\mathrm{L}\end{array}$ & 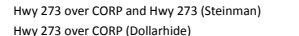 \\
\hline & i & 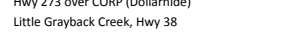 \\
\hline & & Little Lake Creek, HWy 38 \\
\hline & & Grayback creek, Hwy 38 \\
\hline & & 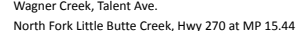 \\
\hline & & $\begin{array}{l}\text { Dead horse creek, } \text {, wy } 230 \\
\text { Do }\end{array}$ \\
\hline & & Buck Rock Creek, Hwy 230 \\
\hline & L & Little Butte Creek, Main St (Eagle Point) \\
\hline & & 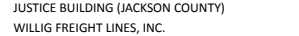 \\
\hline & H & Whetstone Creek, kirkland Rd \\
\hline & & $\begin{array}{l}\text { Daisy Creek, Hwy } 272 \text { at MP 35.68 } \\
\text { Sindercreak Hww 271 }\end{array}$ \\
\hline & ᄂ & West Fork Snider Creek, Hwy 271 \\
\hline & & East Fork Snider Creek, Hwy 271 \\
\hline & t & 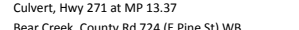 \\
\hline & н & 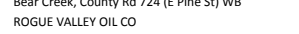 \\
\hline & & SHELL OLL PRODUCTS US \\
\hline & & ROGUE VALLEY OIL CO \\
\hline & & $\begin{array}{l}\text { Heatitg gi IT Tank } \\
\text { REGENCY CAR WASH }\end{array}$ \\
\hline & & REGENCY CARWASH \\
\hline & & Bear Creek, HWy 1 NB at MP 23.07 \\
\hline & & Bear Creek, Hwy 1 SB at MP 23.07 \\
\hline & L & 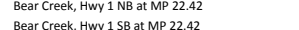 \\
\hline & H & Briggs rreek, Holland Loop Rd \\
\hline & & Table Rock creek, HWy 277 at MP .86 \\
\hline
\end{tabular}

pstype

Tansportation Corridors - Stream Crossing - Perenn Transportatation Corrididors - Stream Crorsising - Perennial transportation Corridors- Stream Crossing- Perennial
transportation Corridors - Stream Crossing - Perennial

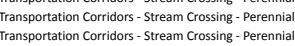
rensportation Corridors - Stream Crossing - Peerennie Transportation Corridors- Stream Crossing- Perennial
Transportation Corridors - Stream Crossing - Perennial ransportation Corridors - Stream Crossing - Perennia

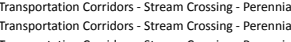
Transportation Corridiors- Stream Crossing- Perennial
Transportation Corridors - Stream Crossing- - Perennial

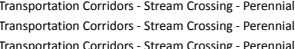

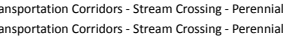

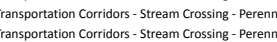
Iransportation Corridors- - Stream Crossing- Perennial
ranspsportation Corridors - Stream Crossing - Perennia Transportation Corridors- Strteam Crossing- Perennial

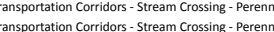

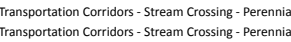
Iransportation Corridors- Stream Crossing - Perenni-
Transportation Corridors - Stream Crossing - PerenniUST - Upgraded and/or Registered-Active Transportation Corridors -stream Crossing - Perennial rransportation Corridors- Strteam Crossing- Perenntian rransportation Corridors-Stream Crossing - Perenn Inssortation Corridors - stream Crossing - Perenne Transoortation Corridors-Stream Crossing - Perenn

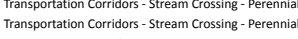
rransportation Corridors - Stream Crossing- Perennial Transportation Corddors - Stream crossing - Perennial
Transportation Corridors - Stream Crossing - Perennial Iransportation Corridors- Stream Crossing- - Perennial

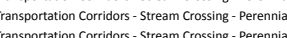
Transportation Corridors- Stream Crossing - Perennial
Transportation Corridors - Stream Crossing - Perennia Transortation Corridors- Stream Crossing - Perent

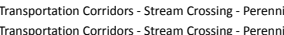

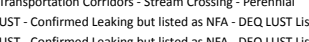

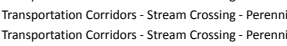

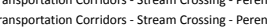
Transportation Corridors- Stream Crossing - Perennial
Transportation Corridors - Stream C rossing - Perennit Chemical/Petroloum Processing/Storage
UST- Confirmed Leaking but tisted as NAA - DEQ LUST List Automobiles - Gas Stations
Known Contamination lised as NFA (Stites/Plumes/spills from ECSI transportation Corridors - Stream crossing - Perenn Transsortation Corridors- Stream Crossing- Perennia Transportation Corridors- Stream Crossing- Perennial
ransportation Corridors - Stream Crossing - Perennial 


\begin{tabular}{|c|c|c|c|}
\hline Address & City & County & RET_DATE \\
\hline $\begin{array}{l}\text { Not Applicable } \\
\text { Not Applicable }\end{array}$ & $\begin{array}{l}\text { UNkNOOWN } \\
\text { unkNOWON }\end{array}$ & Jackson & $\begin{array}{l}2013 \\
2013\end{array}$ \\
\hline $\begin{array}{l}\text { Not At Applicable } \\
\text { Not Appicable }\end{array}$ & $\begin{array}{l}\text { UNNNNOWN } \\
\text { UNKNOWN }\end{array}$ & $\begin{array}{l}\text { Jackson } \\
\text { Jackson }\end{array}$ & $\begin{array}{l}2013 \\
2013\end{array}$ \\
\hline Not Applicable & $\begin{array}{l}\text { UnkNOWN } \\
\text { unNow }\end{array}$ & & 2013 \\
\hline Not Applicable & unknown & Jackson & 2013 \\
\hline Not Applicable & unknown & Jackson & 2013 \\
\hline Not Applicable & unkNown & Jackson & 2013 \\
\hline $\begin{array}{l}\text { Not Applicable } \\
\text { Not Applicable }\end{array}$ & $\begin{array}{l}\text { UNKNNOONN } \\
\text { ukNNOWN }\end{array}$ & $\begin{array}{l}\text { Jackson } \\
\text { Jackson }\end{array}$ & 2013 \\
\hline Not Applicable & $\begin{array}{l}\text { UNNNOONN } \\
\text { UNKNOWN }\end{array}$ & $\begin{array}{l}\text { Jackson } \\
\text { Jackson }\end{array}$ & 2013 \\
\hline Not Applicable & unkNown & $\begin{array}{l}\text { Jackson } \\
\text { Jackon }\end{array}$ & \\
\hline Not Applicable & unkNown & & 2013 \\
\hline Not Applicable & unknown & Jackson & 2013 \\
\hline Not Applicable & UNKNOWN & Jackson & 2013 \\
\hline $\begin{array}{l}\text { Not Applicable } \\
\text { Not Aplicable }\end{array}$ & ROGUE RIVER & Jackson & 2013 \\
\hline Not Applicable & 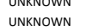 & $\begin{array}{l}\text { Jackson } \\
\text { Jackson }\end{array}$ & $\begin{array}{l}2013 \\
2013\end{array}$ \\
\hline Not Applicable & unkNown & $\begin{array}{l}\text { Jackson } \\
\text { Jackon }\end{array}$ & $\begin{array}{l}2013 \\
2013\end{array}$ \\
\hline Not Applicable & UnkNown & Jackson & 2013 \\
\hline Not Applicable & unknown & Jackson & 2013 \\
\hline Not Applicable & UnNNOWOWN & Jackson & 2013 \\
\hline Not Appocicabe & UNKNOOWN & Jackson & 2013 \\
\hline $\begin{array}{l}\text { Not Appicicable } \\
\text { Pate }\end{array}$ & $\begin{array}{l}\text { unkNown } \\
\text { uns }\end{array}$ & $\begin{array}{l}\text { sacsson } \\
\text { lackson }\end{array}$ & $\begin{array}{l}2013 \\
2013\end{array}$ \\
\hline Not Applicable & unkNown & Jackson & 2013 \\
\hline Not Applicable & unknown & Jackson & 2013 \\
\hline Not Applicable & unkNown & Jackson & 2013 \\
\hline Not Applicable & unkNOWN & Jackson & 2013 \\
\hline $\begin{array}{l}\text { Not AtApplicable } \\
\text { Not Aplicable }\end{array}$ & unkNown & $\begin{array}{l}\text { Jackson } \\
\text { Jackson }\end{array}$ & 2013 \\
\hline $\begin{array}{l}\text { Not Applicable } \\
\text { Pate }\end{array}$ & ASHLAND & $\begin{array}{l}\text { Jackson } \\
\text { Jackson }\end{array}$ & 2013 \\
\hline Not Applicable & unkNown & Jackson & 2013 \\
\hline Not Applicable & unkNown & Jackson & 2013 \\
\hline Not Applicable & UnkNown & Jackson & 2013 \\
\hline $\begin{array}{l}\text { Not Applicicale } \\
\text { Notacolichle }\end{array}$ & $\begin{array}{l}\text { UNNNEONN } \\
\text { UNKNOWWN }\end{array}$ & & 2013 \\
\hline $\begin{array}{l}\text { Not Appildabe } \\
1001 \text { R RIVERSIDE AV }\end{array}$ & $\begin{array}{l}\text { UNkNNown } \\
\text { Medford }\end{array}$ & $\begin{array}{l}\text { Jackson } \\
\text { N/A }\end{array}$ & $\begin{array}{l}2013 \\
10 / 31 / 2018\end{array}$ \\
\hline Not Applicable & UNKNOWN & Jackson & 2013 \\
\hline Not Applicable & $\begin{array}{l}\text { JAAKSONVILLE } \\
\text { unKNOWN }\end{array}$ & $\begin{array}{l}\text { Jackson } \\
\text { Jackson }\end{array}$ & 2013 \\
\hline $\begin{array}{l}\text { Not Apppicicable } \\
\text { Not Aplicicble }\end{array}$ & $\begin{array}{l}\text { UNRNOWN } \\
\text { ROGUE RIVER }\end{array}$ & $\begin{array}{l}\text { Jacksson } \\
\text { Jackson }\end{array}$ & $\begin{array}{l}2013 \\
2013\end{array}$ \\
\hline Not Applicable & $\begin{array}{l}\text { UNKNOWN } \\
\text { UNAOER }\end{array}$ & $\begin{array}{l}\text { Jackson } \\
\text { Jack }\end{array}$ & 2013 \\
\hline Not Applicable & UNKNoWN & Jackson & 2013 \\
\hline Not Applicable & UnkNOWN & Jackson & 2013 \\
\hline $\begin{array}{l}\text { Not Applicable } \\
\text { Not Applicable }\end{array}$ & $\begin{array}{l}\text { UNNNNOWN } \\
\text { UNKNOWNN }\end{array}$ & $\begin{array}{l}\text { Jackson } \\
\text { Jackson }\end{array}$ & $\begin{array}{l}2013 \\
2013\end{array}$ \\
\hline $\begin{array}{l}\text { Not AtAppicicale } \\
\text { Not Aplicable }\end{array}$ & unkNown & Jackson & $\begin{array}{l}2013 \\
2013\end{array}$ \\
\hline Not Applicable & unkNown & Jackson & $\begin{array}{l}2013 \\
2013\end{array}$ \\
\hline Not Applicable & unknown & Jackson & 2013 \\
\hline Not Applicable & UnkNown & Jackson & 2013 \\
\hline $\begin{array}{l}\text { Not Applicable } \\
\text {. }\end{array}$ & UnkNown & Jackson & 2013 \\
\hline 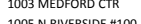 & & & $10 / 31 / 2018$ \\
\hline 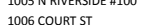 & 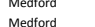 & 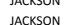 & $\begin{array}{l}10 / 3 / 1 / 2018 \\
1013 / 2018\end{array}$ \\
\hline Not Applicable & unkNown & Jackson & $\begin{array}{l}101 / 1 / 2018 \\
2013\end{array}$ \\
\hline Not Applicable & unknown & Jackson & 2013 \\
\hline Not Applicable & unknown & Jackson & 2013 \\
\hline Not Applicable & UnkNown & Jackson & 2013 \\
\hline Not Applicable & & & 2013 \\
\hline Not Applicibate & 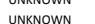 & 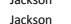 & $\begin{array}{l}2013 \\
2013\end{array}$ \\
\hline Not Applicable & unkNown & Jackson & $\begin{array}{l}2013 \\
2013\end{array}$ \\
\hline Not Applicable & unknown & Jackson & 2013 \\
\hline Not Applicable & unknown & Jackson & 2013 \\
\hline Not Applicable & UnNNOWONN & Jackson & 2013 \\
\hline 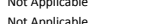 & (1) & Jackson & 2013 \\
\hline & 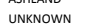 & 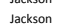 & $\begin{array}{l}2013 \\
2013\end{array}$ \\
\hline Not Applicable & $\begin{array}{l}\text { ASHLAND } \\
\text { ASt }\end{array}$ & Jackson & 2013 \\
\hline Not Applicable & unkNown & Jackson & 2013 \\
\hline Not Applicable & UnkNown & Jackson & 2013 \\
\hline Not Applicable & UnkNOWOWN & Jackson & 2013 \\
\hline $\begin{array}{l}\text { Not AtApplicable } \\
\text { Not Aplicable }\end{array}$ & $\begin{array}{l}\text { UNRNowN } \\
\text { unkNOWN }\end{array}$ & $\begin{array}{l}\text { Jackson } \\
\text { Jackson }\end{array}$ & $\begin{array}{l}2013 \\
2013\end{array}$ \\
\hline Not Applicable & unknown & Jackson & 2013 \\
\hline Not Applicable & unkNown & & 2013 \\
\hline Not Applicable & UnkNown & Jackson & 2013 \\
\hline Not Applicable & UnkNoWNN & Jackson & 2013 \\
\hline Not Applicable & UNKNNOWN & Jackson & 2013 \\
\hline 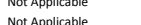 & 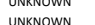 & $\begin{array}{l}\text { sackson } \\
\text { backson }\end{array}$ & 2013 \\
\hline Not Applicable & UNkNown & 作 & 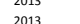 \\
\hline 1006 Court St & Medford & Jackson & $8 / 27 / 2018$ \\
\hline pplicable & UnkNown & Jackson & 2013 \\
\hline $\begin{array}{l}\text { Not Applicable } \\
\text { Notapelicable }\end{array}$ & $\begin{array}{l}\text { UNkNOOWN } \\
\text { unkNOWWN }\end{array}$ & $\begin{array}{l}\text { Jackson } \\
\text { Jackson }\end{array}$ & \\
\hline
\end{tabular}

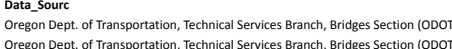

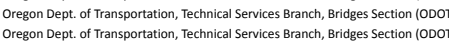

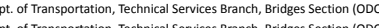

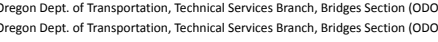
Egon Dept. of Transportation, Technical serices

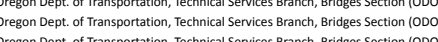

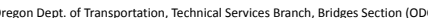

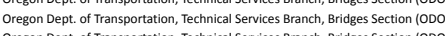
Oregon Dept. of Transportation, Technical Sevicices Branch, Bridges Section (OODO)

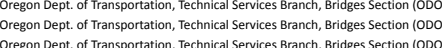

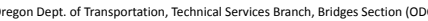
Oregon Dept. of Transportation, Technical Services r ranch, Bridides Section (ODOO Dept. of Transportation, Technical Services Branch, , Bridges Section (000)

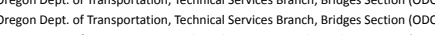

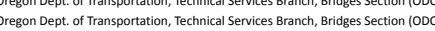
Oregon Dept. of Transportation, Technical Services Branch, Bridges Section (1000

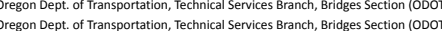
oregon Dept. of Transportation, Technica Services Branch, Bridges Section (ODOO Oregon Dept. of Transportation, Technical Sevicices Branch, Bridges Section (1000)

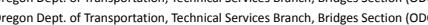

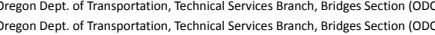

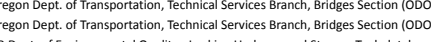

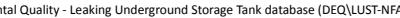

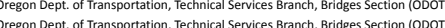
Dept. of Transportation, Technical Services Branch, Bridges Section (ODOCO

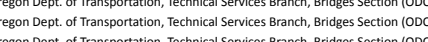
Oregon Dept. of Transportation, Technical Services Branch, Bridges Section (ODOO Oregon Dept. of Transportation, Technical Services Branch, Bridides Section (ODOO Oregon Dept. of Transportation, Technical Services Branch, Brideses Section (1000)

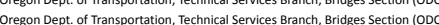

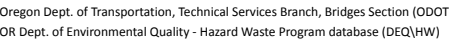

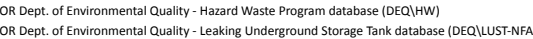

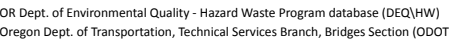

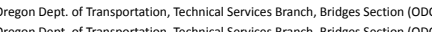
Oregon Dept. of Transsortation, Technical Services Branch, Bridges Section $(1000)$

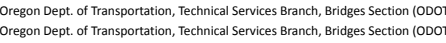

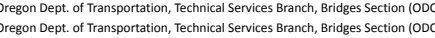

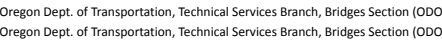

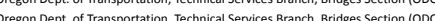
Oregon Dept. of Transportation, Technical Sevices rranch, Bridges Section (1000) Oregon Dept. of Transportation, Technical Services Branch, Bridges Section 1000 O Oregon Dept. of Transsortation, Technical Services Branch, Brideses Section looo oregon Dept. of Transsortation, Technical Services Branch, Bridges Section $1000 \mathrm{C})$

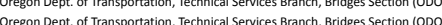
Oregon Dept. of Transportation, Technical Sevices rranch, Bridges Section $(000)$ Oregon Dept. of Transportation, Technical Serviceses reanch, Brrdges Section $(000)$ Oregon Dept. of Transportation, Technical sevices rranch, ridideses section looo

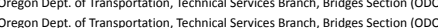

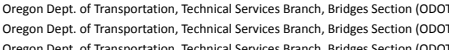

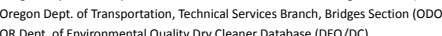

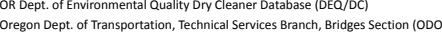

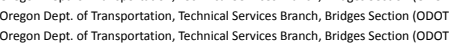

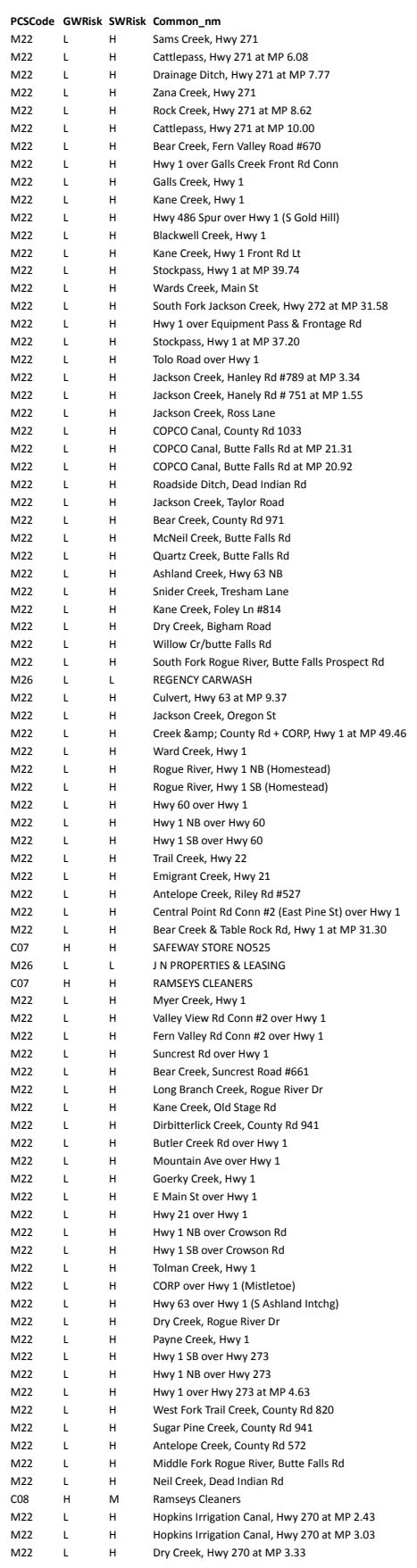

PCSType Transportation Corridors - Stream Crossing - Perennial Transportation Corridors-s Stream crossing- Perennt

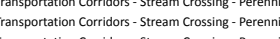
transportation Corrididrs - Stream Crosing - Perennie Tansportation Corridors-stream Crossing- Perent Transportation Corridors- Strteam Crossing- - Perennial
Transportation Corridors -stream Crossing- - Perennial

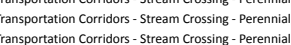

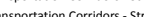

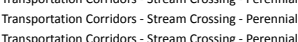

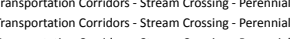
ransportation Coridors - stream Crossing - Perenn Transsortation Corridors- Stream C Crossing - Perennial
Transportation Corridors - Strteam Crossing - Perernial Transportation Corridors - Stream Crossing- Perernnia Transportation Corridors - Stream Crossing - Perennial
Transportation Coridors -stream Crossing - Perennial Transportation Corridors- Stream Crossing- Perennial Iransportation Corridors- Stream Crossing - Perennial
UST- Confirmed Leaking but listed as NFA- DEa UST Lis Transportation Corridors-Stream Crossing - Perennial
Transportation Corridors - Stream Crossing - Perennial Transportation Corridors - Stream Crossing - Perennial

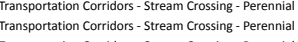
ransportation Corididor- Strteam Crossing - Perenni

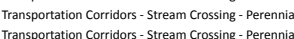

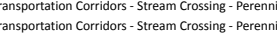
Transporation Corridors - Stream crossing- Perent Transportation Corridors- Stream Crossing- Perennia Chemical/Pertroleum Processing//5orage
UST - Confirmed Leaking but listed a s NFA - DEQ L UST List Chemical/Petroleum Processing//torage Transportation Corridors- Stream Crossing - Perennial
Transportation Corridors - Stream Crosing- Perennie

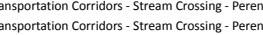

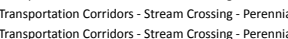
Transportation Corridors- Stream C Crossing - Perenniai
Transportation Corridors - Stream Crossing - Perennia Transportation Corridors-S Stream Crossing - Perennt
Transportation Corridors - Stream Crossing- Perennial

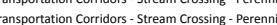

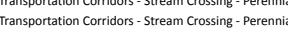
transportation Corridors - Stream Crossing - Perenn

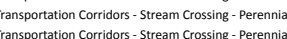

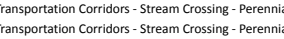
Transportation Corridors - Stream Crossing - Perenni

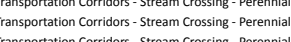
ransportation Corridors-Stream Crossing- Pererenter Transportation Corridors- Stream Crossing - Perenn Dry Cleaners

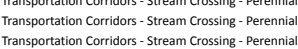




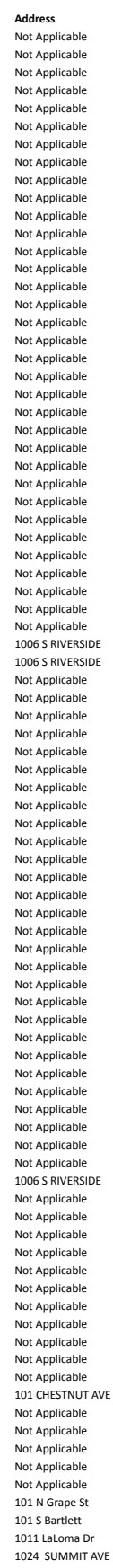

Oata_ Sourc
Oregon Dept. of Transportation, Technical Sevices Branch, Bridges Section (000T)

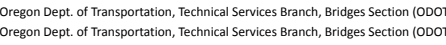
of Transportation, Technical Services Branch, Bridges Section (ODOO) Jregon Dept. of Transportation, Technical Services rranch, Bridges Section (ODo)

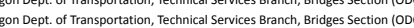

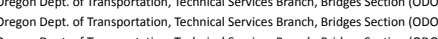

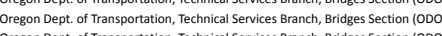

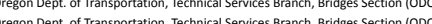
Oregon Dept. of Transsortation, Technical Sevicese rranch, Bridges Section (100

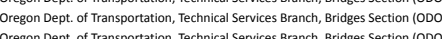

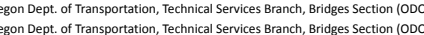
(a) Oregon Dept. of Transsortation, Technical Services rranch, Bridges Section (1000 oregon Dept. of Transsortation, Technical Services Branch, Bridges Section $1000 \mathrm{Cr})$

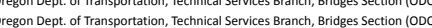
regenn Dept. of Transportation, Technical Services Branch, Bridges Section (ODDO)

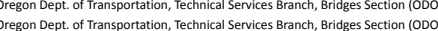
oregon Dept. of Transportation, Technical Services rranch, Brideses Section (ODDOO) Oregon Dept. of Transportation, Technical Services Branch, Bridges Section lood

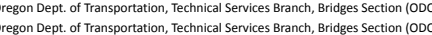

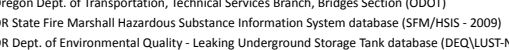
R Dept. of Environmental Quality - Leaking Underground Storage Tank database (DEQ) ULST-NES)

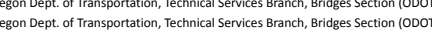

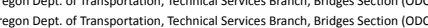
regenn Dept. of fransportation, Technical Senvices rranch, Bridges Section (ODoO)

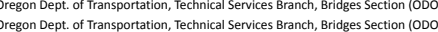
Gegon Dept. of Transportation, Technical Services Branch, Bridges Section (ODOO) Oregon Dept. of Transportation, Technical Services Branch, Bridides Section (ODOO Oregon Dept. of Transportation, Technical Seviceses rranch, Bridges Section (ODOOOOF)

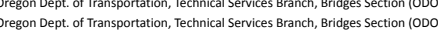
regenn Dept. of Transportation, Technical Services Branch, Brideses Section $1000 \mathrm{O})$

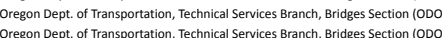
Gregon Dept. of Transportation, Technica Services Branch, Bridges Section (000)

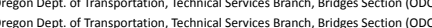
Oregon Dept. of Transportation, Technical Services Branch, Brideses Section (ODOO)

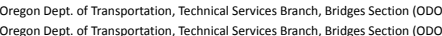

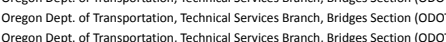

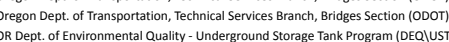

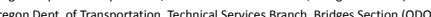
Oregon Dept. of Transportation, Technical Services Branch, Brideses Section (000) Oregon Dept. of Transportation, Technical Services Branch, Bridges Section 1000 O Oregon Dept. of transportation, Technical Services rranch, Brideses Section looo regenn Dept. of Transportation, Technical Services Branch, Bridees Section $(000)$

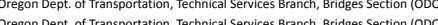

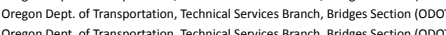
Oregon Dept. of Transportation, Technical Services Branch, Bridges Section (ODOOT)
OR Dept. of Environmental Quality - Leaking Underground Storage Tank database (DEQ) LUST-NFA)

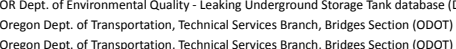

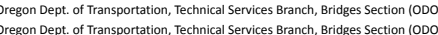

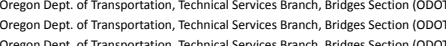

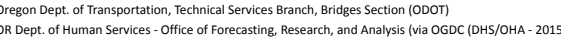

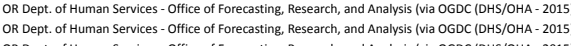

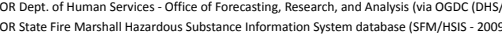

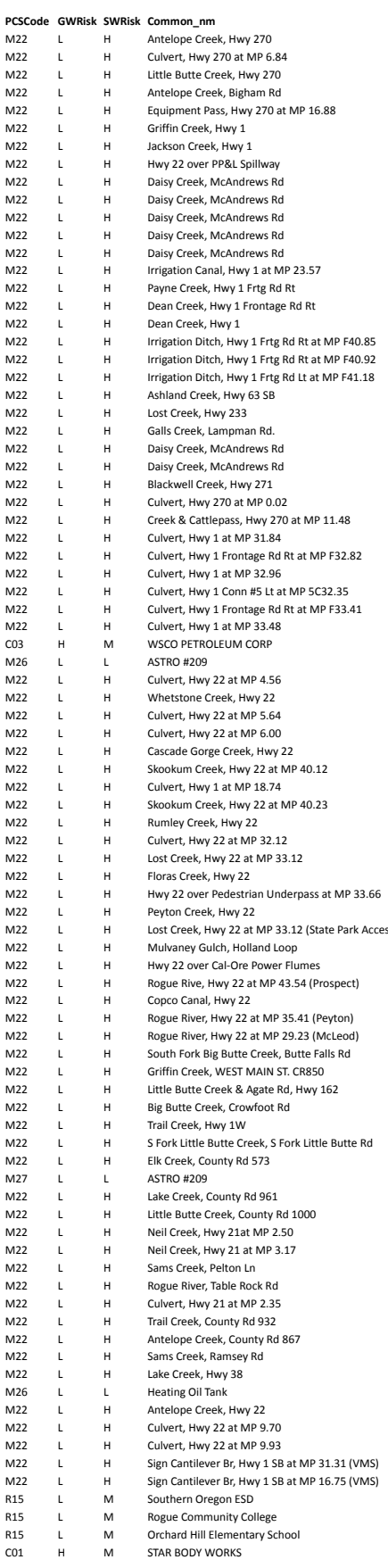

PCSType Transportation Corridors - Stream Crossing - Perennial ransportation Corridors- Stream crossing - Perenent Transportation Corridors-s Stream crossing- Perennt Transportation Corridors - Stream Crossing - Perennie

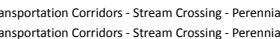
Transolration Corididor-s Stream crossing - Perenn Iransportation Corridors- Stream Crossing- Perennial
Transportation Corridors - Stream Crossing- Perennni

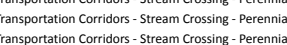

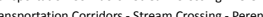

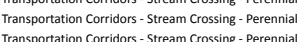

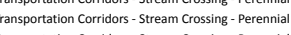
ransportation Corridors- Stream Crossing - Perennt Iransportation Corridors- Stream Crossing - Perennila
Transportation Corridors - Stream Crossing- Perennial ransporatation Corididars- Strteam Crossing - Perennt Transportation Corridors- S Stream Crossing - Perennit
Transportation Corridors - Stream Crossing- Perennial Lutomobilies-Gas station

Confirmed Leaking but listed as NFA- DEQ Q UST List

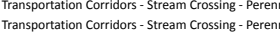
Transportation Corrididrs - Stream Crossing - Perennial Transportatiton Corridors- Stream Crossing- Perennial
Transportation Corridors S-Stream Crossing - Peerennial Fransportation Corridors - Stream Crossing- Perennia

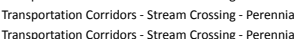

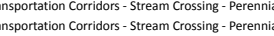

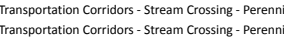
Iransportation Corridors- - Stream Crossing- Perennial
ranspsportation Corridors - Stream Crossing - Perennia Transsortation Corridors - Stream Crossing - Perennia
tansportation Corridors - stream Crossing - Perenna

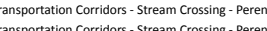
Transportation Corridors - Stream Crossing- - Perennial
rransportation Corridors - Stream Crossing - Perennit

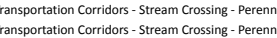
UST - Upgraded and/or Registered- Active (may also have decommissioned tanks on S

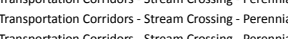
ransportation Corridors - Stream Crossing - Perennter

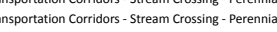

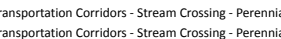

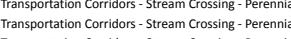
Trnsportatioion Corridors- Stream Crossing - Perennial

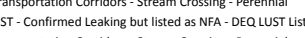
Tansportation Corridors- Stream Crossing - Perennie

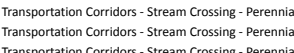
Schools
Schools Schools
Automobiles - Body Shoos 


\begin{tabular}{|c|c|c|c|}
\hline $\begin{array}{l}\text { Address } \\
1024 \text { SUMMit AVE }\end{array}$ & $\begin{array}{l}\text { City } \\
\text { MEOFORD }\end{array}$ & $\begin{array}{l}\text { County } \\
\text { JACKSON }\end{array}$ & $\begin{array}{l}\text { RET_DATE } \\
09 / 29 / 2008\end{array}$ \\
\hline 1024 S RIVERSIDE & MEFFORD & JACKSON & $\begin{array}{l}0.992 / 2008 \\
09 / 29 / 2008\end{array}$ \\
\hline 1024 S RIVERSIDE AVE & $\begin{array}{l}\text { Mefford } \\
\text { Mefford }\end{array}$ & Jackson & $\begin{array}{l}10 / 31 / 2018 \\
10 / 2028\end{array}$ \\
\hline $\begin{array}{l}12044 \text { UUMMIT TVE } \\
1025 \text { N CENTRAL BLG B }\end{array}$ & $\begin{array}{l}\text { Mefiford } \\
\text { MEFFRD }\end{array}$ & $\begin{array}{l}\text { JaCSSON } \\
\text { JACSSON }\end{array}$ & $\begin{array}{l}101 / 1 / 2018 \\
09 / 29 / 2008\end{array}$ \\
\hline $\begin{array}{l}\text { Not Applicable } \\
\end{array}$ & UNkNOWN & Jackson & \\
\hline $\begin{array}{l}\text { Not Applicable } \\
\text { Not Appicable }\end{array}$ & ASHLAND & & 2013 \\
\hline $\begin{array}{l}\text { Not Apppicicable } \\
\text { Not Aplicable }\end{array}$ & $\begin{array}{l}\text { UNNNEONN } \\
\text { UNKNOWWN }\end{array}$ & $\begin{array}{l}\text { Jackson } \\
\text { Jackson }\end{array}$ & $\begin{array}{l}2013 \\
2013\end{array}$ \\
\hline & $\begin{array}{l}\text { UNNNNOWN } \\
\text { unkNOWN }\end{array}$ & $\begin{array}{l}\text { Jacksson } \\
\text { Jackson }\end{array}$ & \\
\hline 103 W M CANDREWS RD & Medford & $\begin{array}{l}\text { Jactson } \\
\text { Jacson }\end{array}$ & 10/31/2018 \\
\hline 103 W MCANDREWS RD & Medford & & $10 / 31 / 2018$ \\
\hline 1031 NARREGAN ST & MEDFORD & JaCKSON & 09/29/2008 \\
\hline 104 FLORENCE ST & $\begin{array}{l}\text { Mefford } \\
\text { MEFFogD }\end{array}$ & $\begin{array}{l}\text { JACKSON } \\
\text { IACCSOON }\end{array}$ & $\begin{array}{l}10 / 131 / 2018 \\
0992 / 2008\end{array}$ \\
\hline $\begin{array}{l}1050 \text { B BAATYYST } \\
1050 \text { RIVERSIDE AVE }\end{array}$ & $\begin{array}{l}\text { MEEFOROD } \\
\text { Mefford }\end{array}$ & $\begin{array}{l}\text { JaCSSON } \\
\text { ICCCSON }\end{array}$ & $\begin{array}{l}09 / 2 / 2 / 2008 \\
1 / 131 / 2018 \\
\end{array}$ \\
\hline 1068 S RIVERSIDE & $\begin{array}{l}\text { Meforord } \\
\text { MEOFORD }\end{array}$ & $\begin{array}{l}\text { JaCSSON } \\
\text { IACCSON }\end{array}$ & $\begin{array}{l}101 / 3 / 21 / 818 \\
09 / 29 / 2008\end{array}$ \\
\hline 1068 S RVERSDID & & $\begin{array}{l}\text { S } \\
\text { IACCSSON }\end{array}$ & $10 / 31 / 2018$ \\
\hline Not Applicable & & Jackson & \\
\hline Not Applicable & UnkNown & Jackson & 2013 \\
\hline $\begin{array}{l}\text { Not At Appicicable } \\
\text { Nate }\end{array}$ & $\begin{array}{l}\text { UNKNNOWN } \\
\text { ukNNown }\end{array}$ & $\begin{array}{l}\text { Jackson } \\
\text { Jackson }\end{array}$ & 2013 \\
\hline Not Appicicable & $\begin{array}{l}\text { UNNNOONN } \\
\text { UNKNOWN }\end{array}$ & $\begin{array}{l}\text { Jackson } \\
\text { Jackson }\end{array}$ & $\begin{array}{l}2013 \\
2013\end{array}$ \\
\hline Not Applicable & 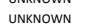 & & $\begin{array}{l}2013 \\
2013\end{array}$ \\
\hline Not Applicable & unkNown & Jackson & 2013 \\
\hline Not Applicable & unkNown & Jackson & 2013 \\
\hline Not Applicable & UNKNOWN & Jackson & 2013 \\
\hline 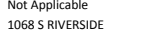 & 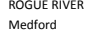 & $\begin{array}{l}\text { Jackson } \\
\text { JaCKSON }\end{array}$ & $\begin{array}{l}2013 \\
11 / 1 / 2018\end{array}$ \\
\hline 107 Newtown St. & Medford & Jackson & $1 / 11 / 2018$ \\
\hline 1078 CourT ST & Medford & JACKSONN & 10/31/2018 \\
\hline 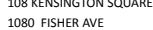 & $\begin{array}{l}\text { Mefford } \\
\text { MEOFORD }\end{array}$ & $\begin{array}{l}\text { JaCSKON } \\
\text { JaCKSON }\end{array}$ & 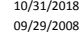 \\
\hline Not Applicable & $\begin{array}{l}\text { MEFFRODO } \\
\text { UNKNOWN }\end{array}$ & & $\begin{array}{l}09912 / 2008 \\
2013\end{array}$ \\
\hline Not Applicable & unkNown & Jackson & ${ }_{2013}$ \\
\hline Not Applicable & UNkNoWn & Jackson & 2013 \\
\hline Not Applicable & JACKSONVILLE & Jackson & 2013 \\
\hline $\begin{array}{l}\text { Not Applicicable } \\
\text { Not Appicichle }\end{array}$ & JACKSONVILLE & Jackson & 2013 \\
\hline Not Applicable & $\begin{array}{l}\text { UNKNNDWN } \\
\text { EAGIE POINT }\end{array}$ & & $\begin{array}{l}2013 \\
2013\end{array}$ \\
\hline Not Applicable & 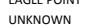 & $\begin{array}{l}\text { Jacsson } \\
\text { Jackson }\end{array}$ & $\begin{array}{l}2013 \\
2013\end{array}$ \\
\hline Not Appicicable & unkNown & Jackson & 2013 \\
\hline Not Applicable & CENTRAL POINT & Jackson & 2013 \\
\hline Not Applicable & ASHLAND & Jackson & 2013 \\
\hline & & & 2013 \\
\hline $\begin{array}{l}\text { Not Applicable } \\
\text { The }\end{array}$ & $\begin{array}{l}\text { JACSKONVIILE } \\
\text { ASHLLND }\end{array}$ & $\begin{array}{l}\text { Jackson } \\
\text { Jackson }\end{array}$ & $\begin{array}{l}2013 \\
2013\end{array}$ \\
\hline $\begin{array}{l}\text { Not Applicable } \\
\text { Pate }\end{array}$ & $\begin{array}{l}\text { UnkNown } \\
\text { Unt }\end{array}$ & $\begin{array}{l}\text { Jackson } \\
\text { Jack }\end{array}$ & 2013 \\
\hline Not Applicable & UnkNOWN & Jackson & 2013 \\
\hline Not Applicable & unkNown & Jackson & \\
\hline $\begin{array}{l}1083 \text { A K KUSTSON } \\
\text { Not Applicable }\end{array}$ & $\begin{array}{l}\text { MEEFORD } \\
\text { UNKNNWWN }\end{array}$ & $\begin{array}{l}\text { Jackson } \\
\text { Jackson }\end{array}$ & $\begin{array}{l}0901 / 2 / 2008 \\
2013\end{array}$ \\
\hline $\begin{array}{l}\text { Not AtAppicicable } \\
\text { Not Aplicable }\end{array}$ & $\begin{array}{l}\text { UNRNOONN } \\
\text { UnkNOWN }\end{array}$ & $\begin{array}{l}\text { Jackson } \\
\text { Jackson }\end{array}$ & 2013 \\
\hline Not Applicable & UnkNown & Jackson & 2013 \\
\hline Not Applicable & UNkNOWN & Jackson & 2013 \\
\hline $\begin{array}{l}\text { Not Appolicable } \\
\text { Not Anplicable }\end{array}$ & $\begin{array}{l}\text { TALENT } \\
\text { UKNNOWN }\end{array}$ & $\begin{array}{l}\text { Jackson } \\
\text { Jackson }\end{array}$ & $\begin{array}{l}2013 \\
2013\end{array}$ \\
\hline $\begin{array}{l}\text { Not Appplicalie } \\
\text { Not Aplicable }\end{array}$ & 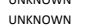 & 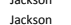 & $\begin{array}{l}2013 \\
2013\end{array}$ \\
\hline Not Applicable & unkNown & Jackson & 2013 \\
\hline Not Applicable & UnkNown & Jackson & \\
\hline Not Applicable & UnkNown & Jackson & \\
\hline $\begin{array}{l}1085 \text { STEWART AVE } \\
1055 \text { STFWAT AVE }\end{array}$ & $\begin{array}{l}\text { Mefford } \\
\text { Mefford }\end{array}$ & & $10 / 31 / 2018$ \\
\hline 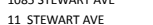 & $\begin{array}{l}\text { Meforord } \\
\text { MEOFORD }\end{array}$ & 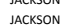 & 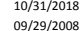 \\
\hline 11 STEWART AVE & MEFFORD & $\begin{array}{l}\text { SACKSON } \\
\text { ICCSON }\end{array}$ & $\begin{array}{l}0.9929 / 2008 \\
09 / 29 / 2008\end{array}$ \\
\hline 1100 BARNETT & Medford & JaCKSON & $10 / 31 / 2018$ \\
\hline 1101 COURT ST & MEOFORD & JACKSON & $09 / 29 / 2008$ \\
\hline 1101 COURT ST & MEOFORD & JACKSON & 09/29/2008 \\
\hline 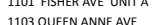 & $\begin{array}{l}\text { MEFOFODD } \\
\text { Mefford }\end{array}$ & JACSSON & $\begin{array}{l}09 / 29 / 2008 \\
103 / 2 / 2018\end{array}$ \\
\hline $\begin{array}{l}105 \text { COURT ST } \\
110 \text { COA }\end{array}$ & Menford & $\begin{array}{l}\text { IACKSON } \\
\text { IA }\end{array}$ & $\begin{array}{l}101 / 3 / 21 / 18 \\
10131 / 2018\end{array}$ \\
\hline Not Applicable & unkNown & Jackson & ${ }_{2013}^{1 / 3 / 2018}$ \\
\hline Not Applicable & unkNown & Josephine & 2013 \\
\hline Not Applicable & unkNown & Jackson & 2013 \\
\hline Not Applicable & CENTRAL POINT & Jackson & \\
\hline 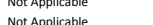 & 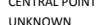 & 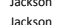 & ${ }_{2013}^{2013}$ \\
\hline 10109 COUTS ST & $\begin{array}{l}\text { MEFFORD } \\
\text { MEF }\end{array}$ & $\begin{array}{l}\text { IACKSON } \\
\text { IASON }\end{array}$ & $\begin{array}{l}20013 \\
09 / 29 / 2008\end{array}$ \\
\hline Not Applicable & UnkNOWN & Jackson & 2013 \\
\hline Not Applicable & unkNown & Jackson & \\
\hline Not Applicable & UNKNoWN & Jackson & 2013 \\
\hline Appilcable & UnkNOWNN & Jackson & 2013 \\
\hline $\begin{array}{l}\text { AApplicable } \\
\text { Applicble }\end{array}$ & $\begin{array}{l}\text { UNNGWND } \\
\text { ASHLAND }\end{array}$ & $\begin{array}{l}\text { Jacsson } \\
\text { Jackson }\end{array}$ & $\begin{array}{l}2013 \\
2013\end{array}$ \\
\hline Apolicable & UNOWN & & \\
\hline Not Applicable & UnkNown & Jackson & \\
\hline
\end{tabular}

(a)

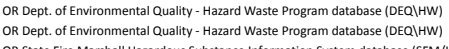

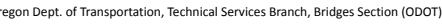

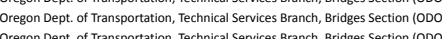

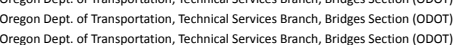

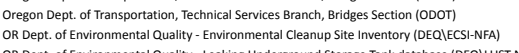

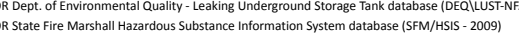

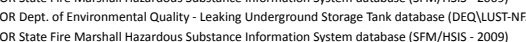

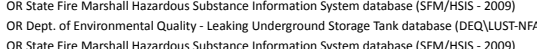

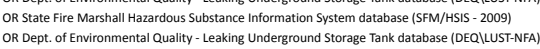

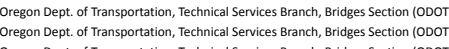

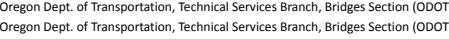

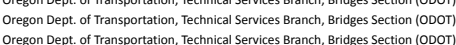

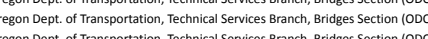

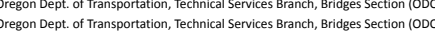

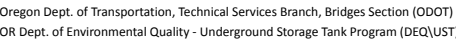

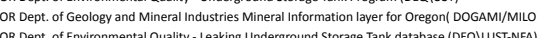

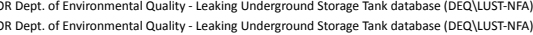

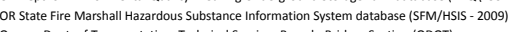

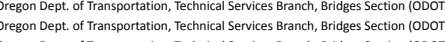

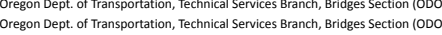

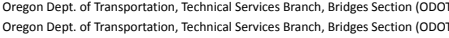

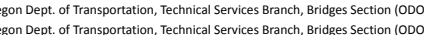

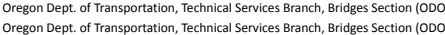

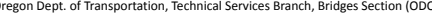

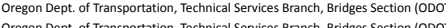

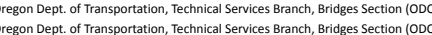

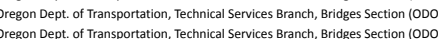

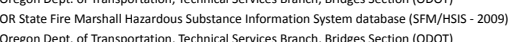

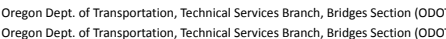

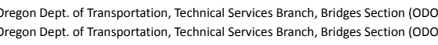

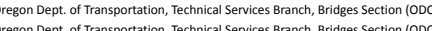

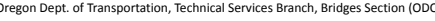

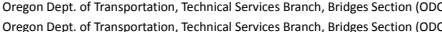

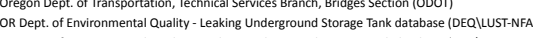

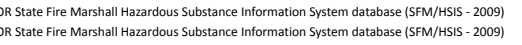

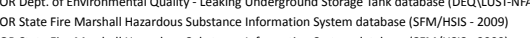

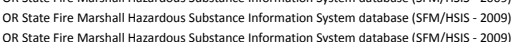

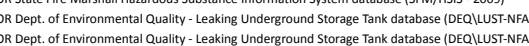

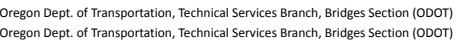

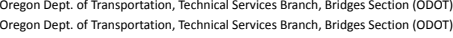

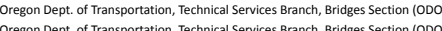

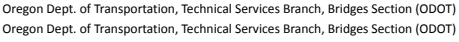

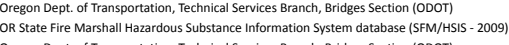

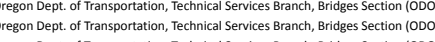

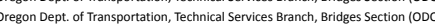

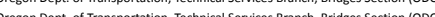

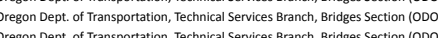

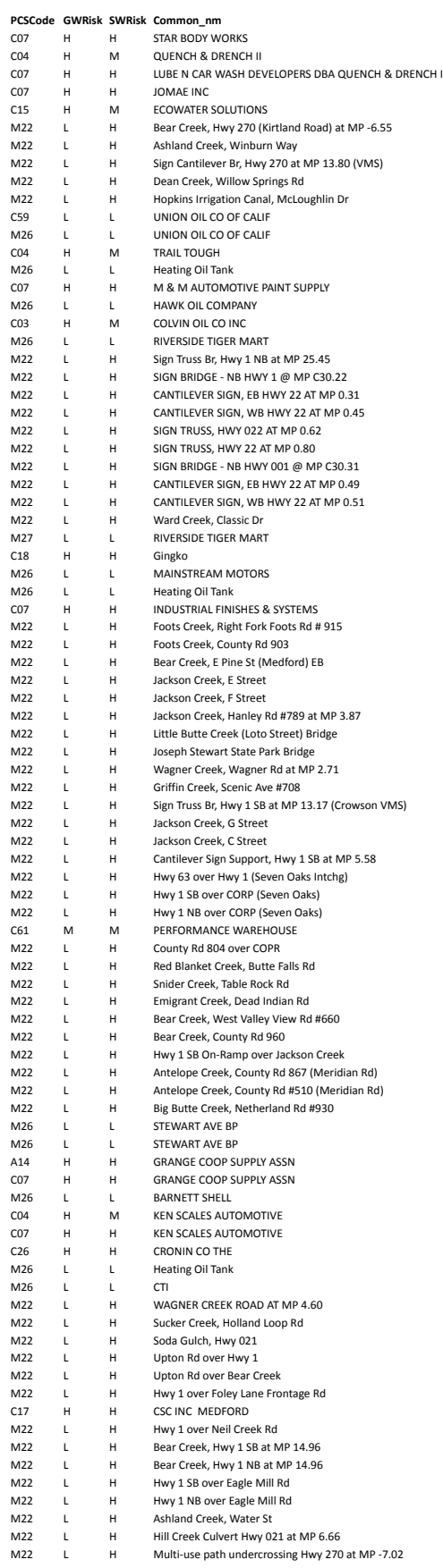

PCSType
Chemical

al/Petroleum Processing/Storage

o- Repair Sh

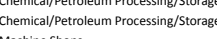

Transportation Corridors Stream Crossing - Perennial
Transportation Corridors - Stream Crossing - Perennial

Transportation Corridors- Stream Crossing- Perennia
Transportation Corridors - Strteam Crossing - Perennia

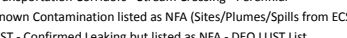

Auto- Repari Shoos

UST - Confirmed Leaking but listed as NFA- DEQ L LST Lis Automobiles- Gas stations

IS NFA- DEQ LUST List

Transportation Corridors - Stream Crossing- Perennial
Transportation Corridors - Stream Crossing - Perennial

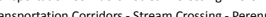

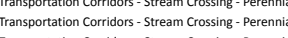

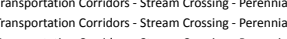

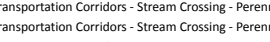

usT - Upgraded and/or Registered- - Active (may also have decommissioned tanks on site)

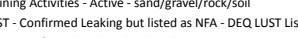

IS NAA- DEQ LUST L

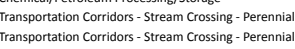

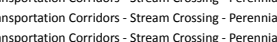

Transportation Corridors-Stream Crossing - Perennial
Transportation Corridors - Stream Crossing - Perennial

Transoortation Corridors- Strtram Crossing - Perennial

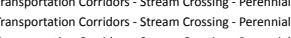

Transportation Corridors- Strteam Crossing - Perennial

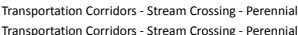

Transportation Corridors - Stream Crossing - Perennia

Transportation Corridors - Stream Crossing- Perernnial
Transportation Corridors - Stream Crossing - Perennial

Maintenance Shop/Equipment Storage - Transsortation
rensportation Corridors - Stream Crosing - Perennial

Transportation Corridors - Stream Crossing - Perennial

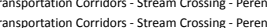

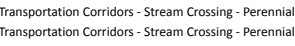

ransportation Corridors-SStream Crossing- Perennial
UST - Confirmed Leaking but isted as NFA - DEQ UST Lis

Pesticide//Frtilizer/Petroleuum Sterage, Handling, Mixisin, \& Cleaning Areas

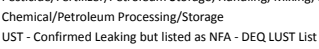

SST- Confirmed Leal
Auto- Repair Shops

Chemical/Petroleum Processing/storage

UST - Confirmed Leaking but listed as NFA - DEQ LUST ULS

UST - Confirmed Leaking but listed as NFA - DEQ LUST List

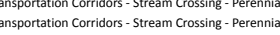

Transportation Corridors- Strteam Crossing- -Perenta

Transportation Corridors - Stream Crossing - Perennial
Transporataion Corridors - Stream Crossing - Perennial

Metal Plating//Finishing Fabrication

Transportatation Corrididors - Strteam Crossing - Perennnial

Transportation Corridors- Stream Crossing - Perennia

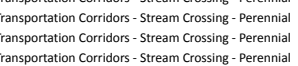




\begin{tabular}{|c|c|c|}
\hline \multirow{2}{*}{\multicolumn{3}{|c|}{$\begin{array}{l}\text { Address } \\
\text { Not Asplicable } \\
\text { Not Applicable }\end{array}$}} \\
\hline & & \\
\hline & $\begin{array}{l}\text { UNRNNOWN } \\
\text { unkNOWN }\end{array}$ & $\begin{array}{l}\text { Jackson } \\
\text { Jackson }\end{array}$ \\
\hline $\begin{array}{l}\text { Not Applicable } \\
\text { Nat Appliable }\end{array}$ & UnkNOWN & Josephine \\
\hline $\begin{array}{l}\text { NotA Applifable } \\
\text { Not Applicable }\end{array}$ & $\begin{array}{l}\text { UNRNAONN } \\
\text { unkNOWN }\end{array}$ & $\begin{array}{l}\text { Jackson } \\
\text { Jactson }\end{array}$ \\
\hline $\begin{array}{l}\text { Not Applicable } \\
\text { e }\end{array}$ & UnkNOWN & Jackson \\
\hline $\begin{array}{l}\text { NotApplicable } \\
\text { Not Applicable }\end{array}$ & $\begin{array}{l}\text { UNNNNOWN } \\
\text { UNKNOWWN }\end{array}$ & $\begin{array}{l}\text { Jackson } \\
\text { Jackson }\end{array}$ \\
\hline Not Applicable & UnkNOWN & Jackson \\
\hline $\begin{array}{l}\text { Not Applicable } \\
\text { Net Appliable }\end{array}$ & UNKNOWN & $\begin{array}{l}\text { Jackson } \\
\text { Jackon }\end{array}$ \\
\hline $\begin{array}{l}\text { NotA Applicable } \\
\text { Not Applicable }\end{array}$ & $\begin{array}{l}\text { UNRNNOWN } \\
\text { unkNOWN }\end{array}$ & $\begin{array}{l}\text { Jackson } \\
\text { Jackson }\end{array}$ \\
\hline Not Applicable & & Jackson \\
\hline $\begin{array}{l}\text { Not Applicable } \\
\text { Not Applicale }\end{array}$ & $\begin{array}{l}\text { UNNNOONN } \\
\text { UKNNOWWN }\end{array}$ & $\begin{array}{l}\text { Jackson } \\
\text { Jackson }\end{array}$ \\
\hline $\begin{array}{l}\text { NotAppindabe } \\
\text { Not Applicable }\end{array}$ & $\begin{array}{l}\text { unkNown } \\
\text { uns }\end{array}$ & $\begin{array}{l}\text { Jacsonon } \\
\text { Jackson }\end{array}$ \\
\hline Not Applicable & UnNNOWN & Jackson \\
\hline Not Applicable & UnkNown & Jackson \\
\hline Not Applicable & UnkNOWN & Jackson \\
\hline $\begin{array}{l}\text { Not Applifable } \\
\text { Not Applicable }\end{array}$ & $\begin{array}{l}\text { UNNNNONN } \\
\text { UNKNOWWN }\end{array}$ & $\begin{array}{l}\text { Jackson } \\
\text { Jackson }\end{array}$ \\
\hline Not Applicable & UnkNown & Jackson \\
\hline Not Applicable & UNKNOWN & Jackson \\
\hline $\begin{array}{l}\text { Not Applicable } \\
\text { Not Applicale }\end{array}$ & $\begin{array}{l}\text { UNNENOWN } \\
\text { UNKNOWWN }\end{array}$ & $\begin{array}{l}\text { Jackson } \\
\text { Jackson }\end{array}$ \\
\hline Not Applicable & CENTRAL POINT & $\begin{array}{l}\text { Jackcon } \\
\text { Jackson }\end{array}$ \\
\hline Not Applicable & $\begin{array}{l}\text { UNKNOWN } \\
\text { und }\end{array}$ & Jackson \\
\hline Not Applicable & unkNown & Jackson \\
\hline Not Applicable & UnkNown & Jackson \\
\hline Not Applicable & UNENOWN & \\
\hline $\begin{array}{l}\text { Not Applicable } \\
\text { Not Applicable }\end{array}$ & $\begin{array}{l}\text { UNNKNOWN } \\
\text { unNNOWWN }\end{array}$ & $\begin{array}{l}\text { Jackson } \\
\text { Jackson }\end{array}$ \\
\hline Not Applicable & $\begin{array}{l}\text { UNNKNOWW } \\
\text { UnNOWN }\end{array}$ & $\begin{array}{l}\text { Jackson } \\
\text { Jackson }\end{array}$ \\
\hline Not Applicable & unkNown & $\begin{array}{l}\text { Jackson } \\
\text { Jat }\end{array}$ \\
\hline Not Applicable & UnkNOWN & Jackson \\
\hline Not Applicable & unkNown & Jackson \\
\hline 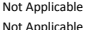 & UnkNown & \\
\hline $\begin{array}{l}\text { Not Applicable } \\
\text { Not Applice }\end{array}$ & $\begin{array}{l}\text { UNNKNOWW } \\
\text { UnNOWN }\end{array}$ & $\begin{array}{l}\text { Jackson } \\
\text { Jackson }\end{array}$ \\
\hline Not Applicable & unkNown & $\begin{array}{l}\text { Jackson } \\
\text { Jat }\end{array}$ \\
\hline Not Applicable & unkNown & $\begin{array}{l}\text { Jackson } \\
\text { D. }\end{array}$ \\
\hline Not Applicable & UnkNown & Jackson \\
\hline Not Applicable & UNKNOOWN & Jackson \\
\hline & & \\
\hline $\begin{array}{l}\text { Not Applifable } \\
\text { Not Applice }\end{array}$ & 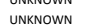 & 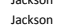 \\
\hline Not Applicable & & $\begin{array}{l}\text { Jackson } \\
\text { Jackon }\end{array}$ \\
\hline Not Applicable & UnkNown & Jackson \\
\hline Not Applicable & UnkNown & Jackson \\
\hline Not Applicable & UNENNOWN & Jackson \\
\hline Not Applicable & UNWNOWNW & \\
\hline $\begin{array}{l}\text { Not Applifable } \\
\text { Not Applicale }\end{array}$ & $\begin{array}{l}\text { UNNNOOWN } \\
\text { Une }\end{array}$ & 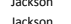 \\
\hline Not Applicable & 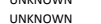 & $\begin{array}{l}\text { sackon } \\
\text { Jackon. }\end{array}$ \\
\hline Not Applicable & UnkNown & Jackson \\
\hline Not Applicable & UnkNown & Jackson \\
\hline Not Applicable & UNKNOWN & Jackson \\
\hline $\begin{array}{l}\text { Not Applicable } \\
\text { Not Applicable }\end{array}$ & 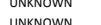 & Jackson \\
\hline $\begin{array}{l}\text { Not Applifable } \\
\text { Not Applicale }\end{array}$ & $\begin{array}{l}\text { UNKNNOWN } \\
\text { Une }\end{array}$ & Jacsson \\
\hline Not Applicable & UnkNown & $\begin{array}{l}\text { Sackson } \\
\text { Jackon }\end{array}$ \\
\hline Not Applicable & UnkNOWN & Jackson \\
\hline Not Applicable & UNKNOWNN & Jackson \\
\hline $\begin{array}{l}\text { Not Appliachle } \\
\text { Not Applicable }\end{array}$ & $\begin{array}{l}\text { UNNNEONN } \\
\text { UNKNOWWN }\end{array}$ & $\begin{array}{l}\text { Jackson } \\
\text { Jackon }\end{array}$ \\
\hline $\begin{array}{l}\text { Not Appinablobe } \\
\text { Not Applicale }\end{array}$ & UnNNOWN & $\begin{array}{l}\text { Jackson } \\
\text { Jackson }\end{array}$ \\
\hline & & \\
\hline Not Applicable & UnkNown & Jackson \\
\hline Not Applicable & UnkNOWN & Jackson \\
\hline Not Applicable & UNRNOWN & Jackson \\
\hline Not Applicable & TALENT & Jacksonn \\
\hline $\begin{array}{l}\text { Not Applicabbe } \\
\text { Not Applicas }\end{array}$ & T. & 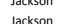 \\
\hline 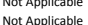 & $\begin{array}{l}\text { AateNA } \\
\text { ASHAND }\end{array}$ & Jatson \\
\hline Not Applicable & ASHLAND & Jackson \\
\hline Not Applicable & unkNown & Josephine \\
\hline Not Applicable & UNKNOWN & Josephine \\
\hline $\begin{array}{l}\text { Not Applicabbe } \\
\text { Not Applicas }\end{array}$ & $\begin{array}{l}\text { UNNENOWN } \\
\text { UNKNOWWN }\end{array}$ & $\begin{array}{l}\text { Joseppine } \\
\text { Jossphine }\end{array}$ \\
\hline $\begin{array}{l}\text { Not Appinablobe } \\
\text { Not Applicale }\end{array}$ & $\begin{array}{l}\text { UNRNOOWN } \\
\text { unN }\end{array}$ & $\begin{array}{l}\text { Jospentine } \\
\text { Josephine }\end{array}$ \\
\hline Not Applicable & UnkNOWN & \\
\hline Not Applicable & UNKNOWN & $\begin{array}{l}\text { Josepphine } \\
\text { ons }\end{array}$ \\
\hline Applicable & UnkNown & \\
\hline Not Applicable & & \\
\hline
\end{tabular}

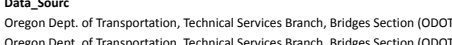

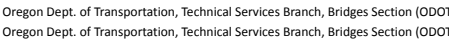
ef Transportation, Technical Services rranch, Bridges Section (ODOCOn) Oregon Dept. of Transportation, Technical Services rranch, Bridges Section (ODOO) Oregon Dept. of Transportation, Technical Services r ranch, ,ridides Section (ODOO

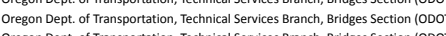
Oregon Dept. of Transportation, Technical Sevicices Branch, Bridges Section (OODO)

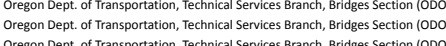
Oregon Dept of

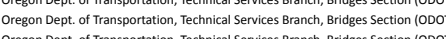

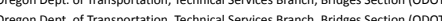

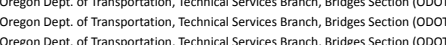

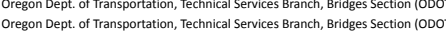

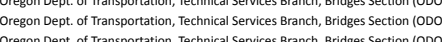
Oregon Dept. of Transportation, Technical Services branch, Brideges Section (ODOO

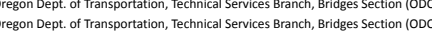
Oregon Dept. of ranssortation, Technical S Services Branch, Bridges Section $(000)$

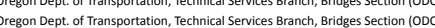
Oregon Dept. of Transportation, Technical Services Branch, Brideses Section (ODoO

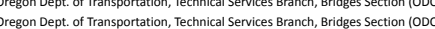
Oregon Dept. of Transportation, Technical Services Branch, Bridiges Section (ODOCON Oregon Dept. of ranssortation, Technical S Services Branch, Bridges Section $(000)$

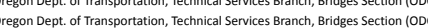
Oregon Dept. of Transportation, Technical Senvices rranch, Brideges Section $(000)$

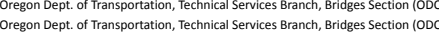
Oregon Dept. of Transportation, Technical Services Branch, Brideses Section (ODOOO Oregon Dept. of Transportation, Technical Services Branch, Bridges Section $1000 \mathrm{O})$ Oregon Dept. of Transportation, Technical Sevices branch, Bridges Section (ODOCON

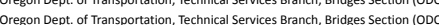
Oregon Dept. of Transportation, Technical Services rranch, Bridges Section (OoO

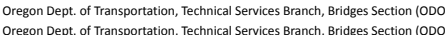

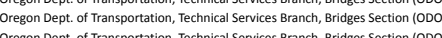

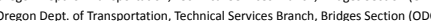
Oregon Dept. of Transsortation, Technical Services rranch, Bridges Section $1000 \mathrm{O}$

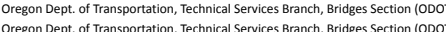

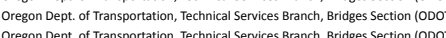

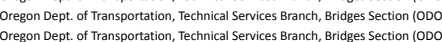

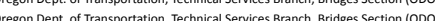
oregon Dept. of Transportation, Technical Services Branch, Brideses Section (1000 Oregon Dept. of Transportation, Technical Services Branch, Bridges Section $(000)$

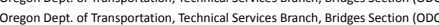
Oregon Dept. of Transportation, Technical Senvices rranch, Bridiges Section $1000 \mathrm{O}$

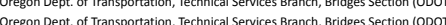
Oregon Dept. of Transportation, Technical Sevices rranch, Bridges Section (O00O) Oregon Dept. of Transportation, Technical Serviceses reanch, Brrdges Section $(000)$ Oregon Dept. of Transsortation, Technical S Services Branch, Brideses Section loDof Oregon Dept. of Transsortation, Technical services rranch, Bridges Section $(000)$

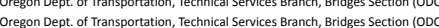
Oregon Dept. of Transportation, Technical Sevices Branch, Bridges Section (ODOO

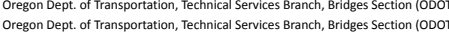

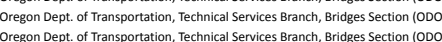

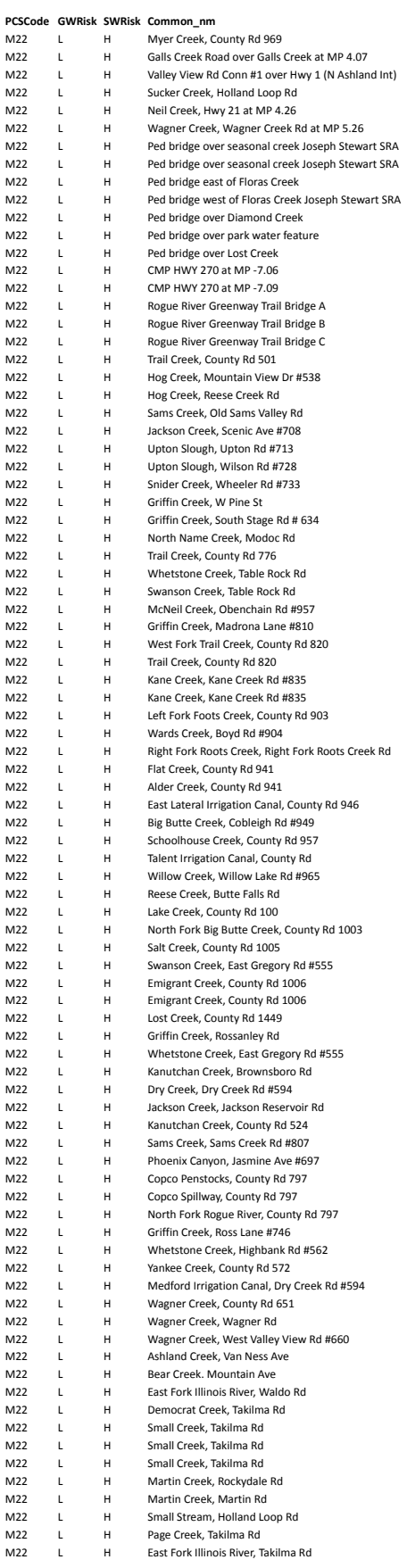

PCSType Transportation Corrididers - Stream Crossing - Perennia Transportation Corididor-stream Crossing - Perenni

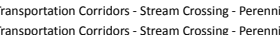
Iransportation Corridors- Stream Crossing - Perennnia

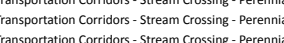
fansportation Corridors- Strteam Crossing - Perenn Transportation Corridors- Stream Crossing - Perennia
Transportation Corridors - Stream Crosing - Perennial Transportation Corridors - Stream Crossing- Perennial
transportation Corridors - Stream Crossing- - Perenn

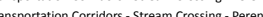

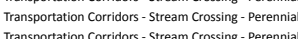

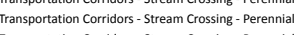

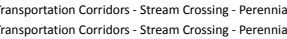
Transportatition Corrididors - Strteam Crom Crossing - Pererenn Iransoortation Corridors-S Stream Crossing - Perennial
Transportation Corridors - Stream Crossing - Perennis ransportation Corridors- Stream Crossing - Perennial Transportation Corrididors - Stream Crossing - Perennil
Transportation Corridors - Stream Crosing rransoortation Corridors - Stream crossing - Perenent Transportation Corridors-S Stream Crossing- Perennial
Transportation Corridors - Stream Crossing- - Perennial

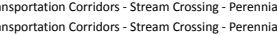
Transportation Corridors- Stream Crossing - Perennila
Transportation Corridors - Stream Crossing - Peren

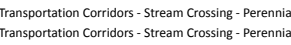
Transportatainion Corridors - Stream Crossing - Perennia Transportation Corridors- Stream Crossing - Perennial
Transportation Corridors - Stream Crossing - Perennial nsportation Corridors - Stream Crossing - Perenn Transportation Corridors- - Stream Crossing- Perennial
ransportation Corridors - Stream Crossing - Perennni

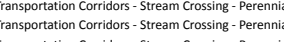

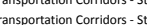
Transportation Corridors - Stream Crossing - Perennia
ranssportation Corridors - Stream Crossing - Perenn

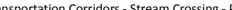

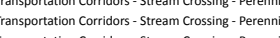
Transportation Corridors - Stream Crossing - Perennt
Transportation Corridors - Stream Crossing- Perennial Transportation Corridors-s - Stream Crossing - Perennia ransportation Corridorss - Stream Crossing - Perenn Iransportation Corridors- Stream Crossing- Pererniti Tansportation Corridors- Stream Crosing - Perenn Transportation Corridors - Stream Crossing - Perenni ransportation Corridors - Strteam Crosing - Perennit

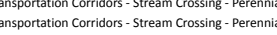
Transportatation Corrididors - Stream Crossing - Perennia Iransportation Corridors- Stream Crossing - Perenn
Iranssortation Coridors - Stream Crossing - Perennt reansportation Corridors - Stream crossing - Perennia
ransportation Corridors - Stream Crossing - Perenni 


\begin{tabular}{|c|c|c|c|}
\hline $\begin{array}{l}\text { Address } \\
\text { NotApplicale }\end{array}$ & City & County & RET_DATE \\
\hline $\begin{array}{l}\text { Not Appolicable } \\
\text { Not Applicable }\end{array}$ & $\begin{array}{l}\text { UnNNNOWN } \\
\text { UNRNOWN }\end{array}$ & & $\begin{array}{l}2013 \\
2013\end{array}$ \\
\hline Not Applicable & 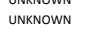 & $\begin{array}{l}\text { Jospepnine } \\
\text { Josphine }\end{array}$ & 2013 \\
\hline $\begin{array}{l}\text { Agate Lake } \\
\text { Casey state Park }\end{array}$ & Unknown & Jackson & 03/2016 \\
\hline 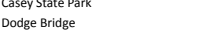 & $\begin{array}{l}\text { Unnkown } \\
\text { Unknown }\end{array}$ & $\begin{array}{l}\text { Jacksson } \\
\text { Jackson }\end{array}$ & $\begin{array}{l}033 / 21016 \\
03 / 2016\end{array}$ \\
\hline Dowden Falls & & & \\
\hline Emigrant Lake North & Unknown & Jackson & . \\
\hline Emigrant Lake West & Unknown & Jackson & 03/2016 \\
\hline Fish Lake Recreation Area & Unknown & Jackson & 03/2016 \\
\hline $\begin{array}{l}\text { Fishers ferry } \\
\text { Gold Hill }\end{array}$ & $\begin{array}{l}\text { Unknown } \\
\text { Unknown }\end{array}$ & $\begin{array}{l}\text { Jackson } \\
\text { Jackson }\end{array}$ & $\begin{array}{l}03 / 2016 \\
0.320216\end{array}$ \\
\hline $\begin{array}{l}\text { Gold Hill } \\
\text { oseph Stewart State Park }\end{array}$ & $\begin{array}{l}\text { Unknown } \\
\text { Unknown }\end{array}$ & $\begin{array}{l}\text { Jackson } \\
\text { Jackson }\end{array}$ & $\begin{array}{l}03 / 2016 \\
00 / 2016\end{array}$ \\
\hline $\begin{array}{l}\text { oloseph Shewart trate Park } \\
\text { ost reek Marina }\end{array}$ & $\begin{array}{l}\text { Unknown } \\
\text { Unknown }\end{array}$ & $\begin{array}{l}\text { Jackson } \\
\text { Jackson }\end{array}$ & $\begin{array}{l}0.03721016 \\
03 / 2016\end{array}$ \\
\hline & Unknown & $\begin{array}{l}\text { Jackson } \\
\text { Jassons }\end{array}$ & 然/2016 \\
\hline Patrick Dunn & Unknown & Jackson & 03/2016 \\
\hline Rogue Elk & Unknown & Jackson & 03/2016 \\
\hline $\begin{array}{l}\text { Sardiden Creek } \\
\text { Shany Cove }\end{array}$ & $\begin{array}{l}\text { Unknown } \\
\text { Unknown }\end{array}$ & Jackson & o3/2016 \\
\hline $\begin{array}{l}\text { Shady Cover } \\
\text { Songer Wayside }\end{array}$ & $\begin{array}{l}\text { Unknown } \\
\text { Unknown }\end{array}$ & $\begin{array}{l}\text { Jackson } \\
\text { Jackson }\end{array}$ & $\begin{array}{l}03 / 2016 \\
00 / 2016\end{array}$ \\
\hline $\begin{array}{l}\text { Takelma } \\
\text { Takelmats }\end{array}$ & $\begin{array}{l}\text { ONknown } \\
\text { Unknown }\end{array}$ & $\begin{array}{l}\text { Jackson } \\
\text { Jackson }\end{array}$ & $\begin{array}{l}0.03721016 \\
03 / 2016\end{array}$ \\
\hline Takelma Park & Unknown & Jackson & . \\
\hline Tou Velle State Park & Unknown & Jackson & $03 / 2016$ \\
\hline Valley of the Rogue State Par & Unknown & $\begin{array}{l}\text { Jackson } \\
\text { Jackson }\end{array}$ & $\begin{array}{l}03 / 2016 \\
032021616\end{array}$ \\
\hline 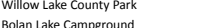 & $\begin{array}{l}\text { Unknown } \\
\text { Unnknown }\end{array}$ & $\begin{array}{l}\text { Jackson } \\
\text { Jesephine }\end{array}$ & $\begin{array}{l}03 / 2016 \\
0.320216\end{array}$ \\
\hline $\begin{array}{l}\text { Bilan Lake Campgrround } \\
\text { Fish Lake Resort }\end{array}$ & $\begin{array}{l}\text { Unknown } \\
\text { Unknown }\end{array}$ & $\begin{array}{l}\text { Josesphine } \\
\text { Klamath }\end{array}$ & $\begin{array}{l}03 / 216 \\
03 / 2016\end{array}$ \\
\hline $\begin{array}{l}\text { Fiss Lake Resort } \\
1017 \text { WHEELER RD }\end{array}$ & $\begin{array}{l}\text { Unknown } \\
\text { CENTRAL POINT }\end{array}$ & $\begin{array}{l}\text { Klamatn } \\
\text { Jackson }\end{array}$ & $\begin{array}{l}83 / 14 / 2018 \\
8018\end{array}$ \\
\hline 11161 TABLE ROCK RD & CENTRAL POINT & Jackson & $8 / 14 / 2018$ \\
\hline 11161 TABLE ROCK RD & CENTRAL POINT & JACKSON & $8 / 14 / 2018$ \\
\hline 615 Butte Falls Highway & $\begin{array}{l}\text { Eagle Point } \\
\text { CEvTrup }\end{array}$ & Jackson & $\begin{array}{l}8 / 14 / 2018 \\
8 / 142018\end{array}$ \\
\hline 5325 TOLO RD & $\begin{array}{l}\text { CENTTA POONT } \\
\text { CNETRA POONT }\end{array}$ & $\begin{array}{l}\text { JACKSON } \\
\text { JACKSON }\end{array}$ & $\begin{array}{l}8 / 14 / 2 / 218 \\
88 / 1 / 2018\end{array}$ \\
\hline $\begin{array}{l}70000 \text { HIG B HANSS RD } \\
13794 \text { PERRY RD }\end{array}$ & $\begin{array}{l}\text { CENTRAA POINT } \\
\text { CENTRA POINT }\end{array}$ & $\begin{array}{l}\text { JAACSSON } \\
\text { JACKSON }\end{array}$ & $\begin{array}{l}8 / 1442018 \\
8 / 1 / 2018\end{array}$ \\
\hline 11161 TABLE ROCK RD & CENTRAL POINT & JACKSON & $\begin{array}{l}8 / 1 / 42018 \\
8 / 14 / 2018\end{array}$ \\
\hline 1701 CHAPEL DRIVE & Cartion & Benton & 10/131/2018 \\
\hline 1109 COURT ST & Medtord & JaCKSON & $10 / 31 / 2018$ \\
\hline 3100 WILOW SPRINGS RD & N/A & JACKSON & $\begin{array}{l}10 / 31 / 2018 \\
1013 / 2018\end{array}$ \\
\hline $\begin{array}{l}2000 \text { ROGUUE RVVER DR } \\
306 \text { ST }\end{array}$ & $\begin{array}{l}\text { N/A } \\
\text { phoenix }\end{array}$ & $\begin{array}{l}\text { JaCSSON } \\
\text { IACCSON }\end{array}$ & $\begin{array}{l}101 / 3 / 21 / 2018 \\
10131 / 2018\end{array}$ \\
\hline 146 MISTLETOE RD & $\begin{array}{l}\text { Phoenix } \\
\text { Ashland }\end{array}$ & $\begin{array}{l}\text { SACSSON } \\
\text { IACSSON }\end{array}$ & $\begin{array}{l}10131 / 2018 \\
10 / 11 / 2018\end{array}$ \\
\hline $\begin{array}{l}1110 \text { E VILAS RD } \\
\text { lat }\end{array}$ & MEDFORD & Jackson & $\begin{array}{l}1 \\
09 / 29 / 20008\end{array}$ \\
\hline 7050 бтн ST & $\mathrm{N} / \mathrm{A}$ & JaCksoN & $10 / 31 / 2018$ \\
\hline 2655 S PACIFIC HWV & N/A & JACKSON & 10/31/2018 \\
\hline $\begin{array}{l}11111 \text { CARTER LAKK AVE } \\
3959 \text { HAMRICK RD }\end{array}$ & $\begin{array}{l}\text { MEFOORD } \\
\text { N/A }\end{array}$ & $\begin{array}{l}\text { JaCSSON } \\
\text { JaCSSON }\end{array}$ & $\begin{array}{l}099 / 2 / 2008 \\
10 / 31 / 2018 \\
\end{array}$ \\
\hline 1790 AVE G & N/A & JACKSON & $10 / 31 / 2018$ \\
\hline 25 E. McAndrews & N/A & JACKSON & $10 / 31 / 2018$ \\
\hline 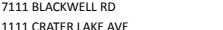 & $\begin{array}{l}\text { N/A } \\
\text { Metford }\end{array}$ & $\begin{array}{l}\text { JaCKSON } \\
\text { IACCSON }\end{array}$ & $\begin{array}{l}10 / 31 / 2018 \\
10 / 132018\end{array}$ \\
\hline 1400 ANTELOPE RD & $\begin{array}{l}\text { Weatora } \\
\text { N/A }\end{array}$ & $\begin{array}{l}\text { JaCSSON } \\
\text { IACSSON }\end{array}$ & $\begin{array}{l}10 / 13 / 212018 \\
10 / 11 / 2018\end{array}$ \\
\hline 1111 AVE C & $N / A$ & JACKSON & $\begin{array}{l}101 / 17 / 218 \\
10 / 21 / 218\end{array}$ \\
\hline 4386 AVENUE A & N/A & JaCkSON & $10 / 31 / 2018$ \\
\hline 2291 AVE G & N/A & JACKSON & 10/31/2018 \\
\hline 11111 CRATER LAKE AVE & Mentored & JACKSON & 10/31/2018 \\
\hline $\begin{array}{l}111111 \text { CRATER LAAKE AVE } \\
1-5 \text { SUEPST } 36\end{array}$ & $\begin{array}{l}\text { Netorord } \\
\text { N/A }\end{array}$ & $\begin{array}{l}\text { JaCKSON } \\
\text { IACCSON }\end{array}$ & $\begin{array}{l}10 / 13 / 21 / 218 \\
10 / 11 / 2018\end{array}$ \\
\hline 441 West Antelope Rd. & $\begin{array}{l}N / A \\
N / A\end{array}$ & $\begin{array}{l}\text { IACCSON } \\
\text { Ion }\end{array}$ & $\begin{array}{l}1 / 131 / 2018 \\
10 / 31 / 2018\end{array}$ \\
\hline 42991 14TH ST & N/A & JaCkSON & $\begin{array}{l}101 / 17 / 218 \\
10 / 1218\end{array}$ \\
\hline 45865 PACIFIC HWY & Phoenix & JACKSON & 10/31/2018 \\
\hline 930 ANTELLOPE RD & N/A & JACKSON & 10/31/2018 \\
\hline & N/A & IACCSONO & 10/31/2018 \\
\hline 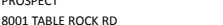 & 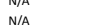 & 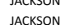 & $\begin{array}{l}10 / 31 / 2018 \\
10 / 31212018\end{array}$ \\
\hline 8001 TABLE ROCK RD & N/A & IACKSON & $\begin{array}{l}10 / 131 / 2018 \\
10 / 11 / 2018\end{array}$ \\
\hline 1385 ANTELOPE RD & N/A & JaCkSON & $10 / 11 / 2018$ \\
\hline 1111 CRATTR LAKE AVE & Medford & JACKSON & 10/31/2018 \\
\hline 1111 CRATER LAKE AVE & Medford & JACKSON & 11/1/2018 \\
\hline 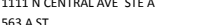 & 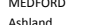 & 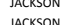 & 09/29/2008 \\
\hline 11111 N CENTRAL AVE STEF & MEFRO & 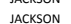 & $\begin{array}{l}1 / 131 / 2018 \\
09 / 29 / 2008\end{array}$ \\
\hline 1111 N CENTRA AVE UNTT & Mefford & JACKSON & $\begin{array}{l}0.9 / 299 / 2008 \\
10 / 31 / 2018\end{array}$ \\
\hline 9464 BLACKWEL RD & N/A & JaCKSON & $10 / 31 / 2018$ \\
\hline 1111 N CENTRALLAVE UNITI & Mefford & JaCKSON & 10/31/2018 \\
\hline 1116 N RVVESSID AVE & MEDFORD & JACKSON & 09/29/2008 \\
\hline 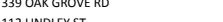 & Nat & 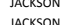 & 10/31/2018 \\
\hline 17 & & 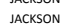 & $\begin{array}{l}10 / 7131 / 218 \\
10 / 31 / 2018\end{array}$ \\
\hline 105 WATER ST & Asthand & JacksoN & $\begin{array}{l}10 / 311 / 218 \\
10 / 31 / 2018\end{array}$ \\
\hline 2310 vo & & JACKSON & $10 / 31 / 2018$ \\
\hline 1125 KNUTSON AVE & MEFFORD & JACKSON & 09/29/2008 \\
\hline 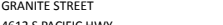 & N/A & JACCSONO & 10/31/2018 \\
\hline 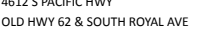 & 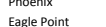 & 年 & $\begin{array}{l}101 / 3 / 2018 \\
01 / 31 / 2018\end{array}$ \\
\hline & & JACKSON & 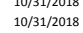 \\
\hline & & JACKSON & \\
\hline
\end{tabular}

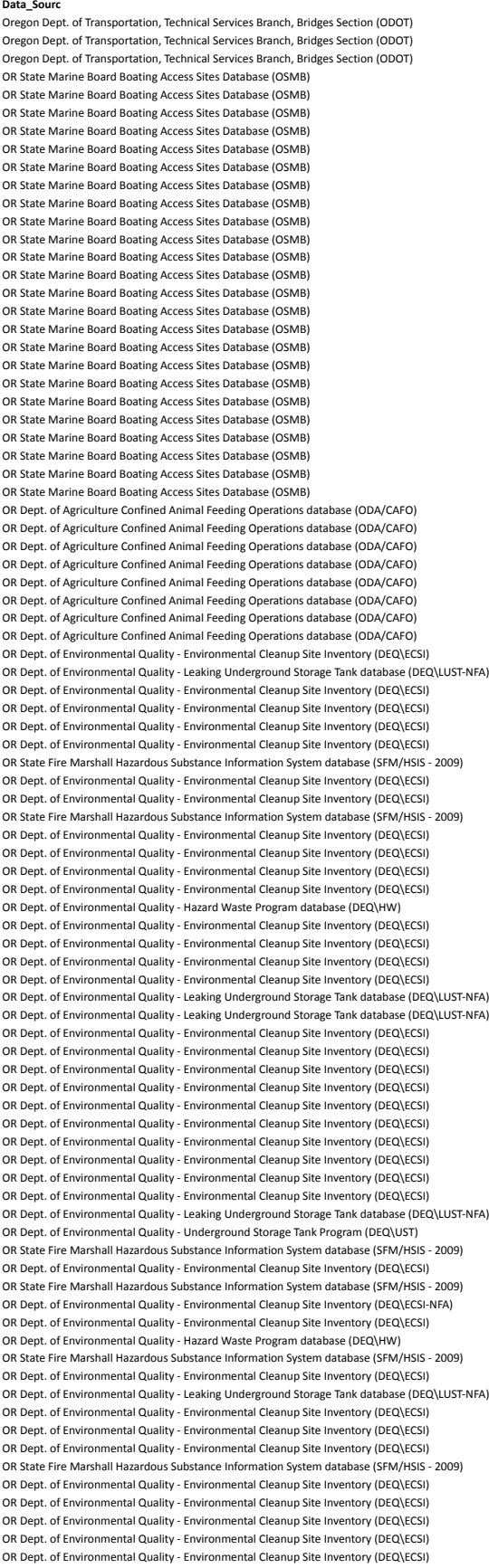

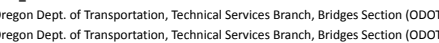

titions database (ODA/CAF

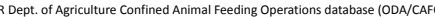
OR Dept. of Africuture Confined Animal Feeding Operations database (ODA/CAAE

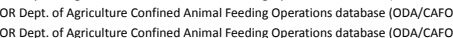

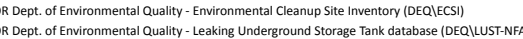

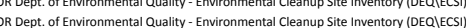

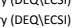

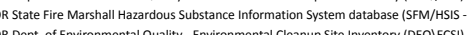

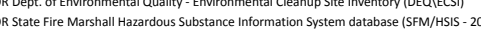
ality- Hazard Waste Program database (DEQOHW)

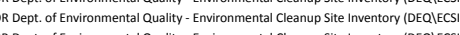
OR Dept. of Environmental Quality - Leaking Underground Storgege Tank database (DEQQI LUST-N QR Dept. of Environme R Dept. of Environmental Quality - Environmental Cleanup Site Inventory (DEQQlECSS) OR Dept. of Environmental Q Quality - Environmental Cleanup Site Inventory (DEQQ|KCSSI)

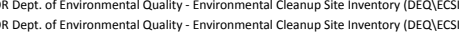
OR Dept. of Environmental Quality - Leaking Underground Storage Tank database (DEQ) IUST-NEA) (a)

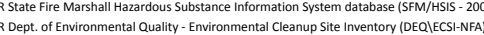

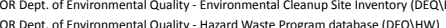

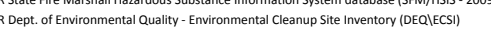

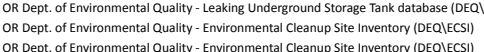
OR Dept. of Enviro

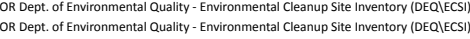

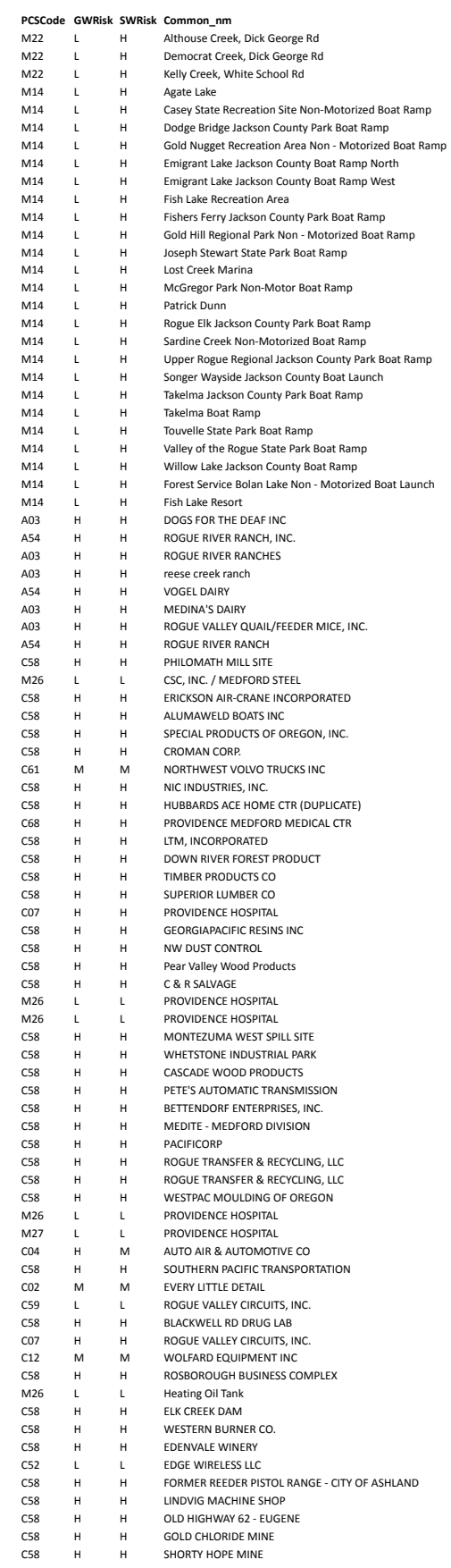

PCSType

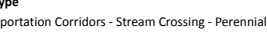

Iransportation Corridors - Stream Crossing - Perennial
transportation Corridors - Stream Crossing - Perenn

er Recreation - Heary Use (inc Campgrounds

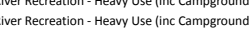

River Recreation- Heary Use inc Campgrounds
River Recceration - Heavy Use (inc Campgrounds

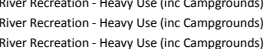

River Recreation - Heary Use (inc Campgrounds
River Recreation - Heavy Use linc Camprounds

River Recreation - Heavy Use (inc Campgrounds)
River Recreation- Heavy Use (inc Campgrounds

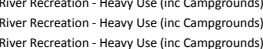

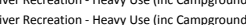

River Recreation- Heary Use (inc Camprogunds
River Recreation - Heary Use (inc Campground

Niver Recreation- Hear Use (inc Campgrounn
River Recreation - Heary Use (inc Campground

River Recreation- Heary Use (inc Camprogrounds)

Concentrated Animal Feeding Operations (CAFOS) - Fed Regulated Confined Animal Feeding operations (CAFs)

Concentrated Animal Feeding Operations (CAFS)- - Fed Regulated
Confines A (a) Concentrated Animal Feeding Operations (CAFS). Fed Regulated
Known Contamination Sites Pllumes/Spills $(E S S)$ )

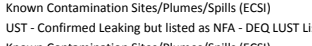

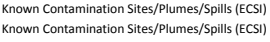

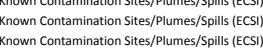
Maintenance Shop/Equipment Storage- Transportat Known Contamination Sitess'Plumes/spipils EESS Miscellaneous Equipment Maintenance

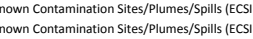

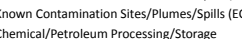
nown Contamination Sittes/Plumes/spills (ECS)

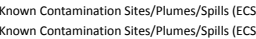
UST - Confirmed Leaking but listed as NFA- DEQ LUST L hown Contamination Sites Pllumes/Spills (ECSI) Known Contamination Sites/Plumes/Spills (ECSS)

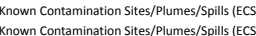

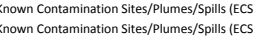
Known Contamination Sites/Plumeses/Spillis \& ECSS)

UST - Confirmed Leaking but listed as NFA. DEQ LUST Lis

UST - Upgraded and/or Registered-Active (may also have decommissioned tanks on Site)

known Contanination Sites/Plumes/Spills (ECS)

Enown Contamination listed as NFA SSites/Plumes/Spills from ECSII

\&nown Contamination Sitess Plumes/Spills $(E C C)$

Furniture/Lumber//Parts Stores

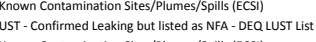

Known Contamination Stess Plumes/Spills EEST)
Communications office

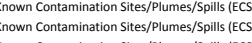




\begin{tabular}{|c|c|c|}
\hline datress & city & County \\
\hline $355 / 3 \mathrm{~W} / / 229$ & N/A & $\begin{array}{l}\text { JACKSON } \\
\text { JACKSON }\end{array}$ \\
\hline 37 S CHOOLHOUSE LANE & Shady Cove & JACKSON \\
\hline 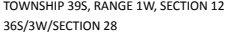 & N/A & $\begin{array}{l}\text { JAAKKON } \\
\text { JACKSON }\end{array}$ \\
\hline $37 / / 3 w / 36$ & N/A & JACKSON \\
\hline 251 W VALLEY VIEW RD & Talent & JACKSON \\
\hline $\begin{array}{l}27220 \text { HIGH ARNAS RDD. } \\
\text { KESHAW RD. }\end{array}$ & N/A & $\begin{array}{l}\text { A ACCCSSON } \\
\text { AACKON }\end{array}$ \\
\hline 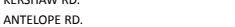 & N/A & 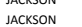 \\
\hline 1125 KNUTSON AVE & MEDFORD & JACKSON \\
\hline 850 S FRONT ST & Talent & Jackson \\
\hline 1127 COURT ST & Metford & Jackson \\
\hline $\begin{array}{l}14475 \text { HIGHWWAV } 234 \\
113 \text { Ross IN }\end{array}$ & $\begin{array}{l}\text { Gold dill } \\
\text { MEFFORO }\end{array}$ & IACCSSON \\
\hline $\begin{array}{l}11313 \text { RoSS LN } \\
1130 \text { N RIVRSIDE AVE }\end{array}$ & $\begin{array}{l}\text { MEFEOODD } \\
\text { MEFFRD }\end{array}$ & $\begin{array}{l}\text { A ACCSSON } \\
\text { AACKSON }\end{array}$ \\
\hline 8425 AGATE RD & N/A & JACKSON \\
\hline 395/2E//23 & $\begin{array}{l}\text { N/A } \\
N / A\end{array}$ & $\begin{array}{l}\text { IACCSSON } \\
\text { AACKSON }\end{array}$ \\
\hline 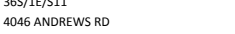 & N/A & AACKSON \\
\hline 1130 NIANTIC ST & Medtord & Jackson \\
\hline 718 B BEEBE ROAD & N/A & \\
\hline 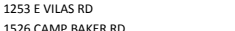 & N/A & IACCSSON \\
\hline $\begin{array}{l}11256 \text { CAMP PARKR RD } \\
1134 \text { COURT ST }\end{array}$ & $\begin{array}{l}\text { N/A } \\
\text { MEDFORD }\end{array}$ & $\begin{array}{l}\text { A ACCCSON } \\
\text { AACSON }\end{array}$ \\
\hline 1200 AVENUE G & N/A & JACKSON \\
\hline $\begin{array}{ll}7675 \text { AGATE RD. } \\
\text { VOCTTGSEST }\end{array}$ & N/A & IACCSON \\
\hline 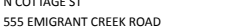 & N/A & $\begin{array}{l}\text { IACCSSON } \\
\text { AACKSON }\end{array}$ \\
\hline 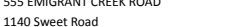 & Metford & $\begin{array}{l}\text { Jaccsson } \\
\text { Jackson }\end{array}$ \\
\hline 1775 suncrest food & N/A & JACKSON \\
\hline 3210 CHEITENHAM WAY & N/A & JACKSON \\
\hline 1111 MILL CREEK DR. & N/A & JACKSON \\
\hline & N/A & $\begin{array}{l}\text { J JSSPEPINE } \\
\text { JOSPHNE }\end{array}$ \\
\hline $405 / 8 \mathrm{~W} / 36$ & $\mathrm{~N} / \mathrm{A}$ & JOSEPHINE \\
\hline 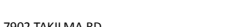 & N/A & JOSEPHINE \\
\hline 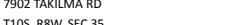 & N/A & $\begin{array}{l}\text { J JOSEPHNN } \\
\text { OSSPPINE }\end{array}$ \\
\hline $\begin{array}{l}1736 \text { NE } 45 \text { TH AVE } \\
1\end{array}$ & $\begin{array}{l}\text { Nortland } \\
\text { Portand }\end{array}$ & $\begin{array}{l}\text { J JSSPFINE } \\
\text { Mulnomah }\end{array}$ \\
\hline 117 s. Central Ave & Metford & JaCkson \\
\hline 1170 BIDOLE RD & Medford & JACKSON \\
\hline 7905 AGATE RD & N/A & IACCSON \\
\hline 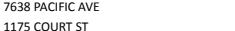 & $\begin{array}{l}\text { N/A } \\
\text { Medford }\end{array}$ & $\begin{array}{l}\text { JAAKSON } \\
\text { JACKSON }\end{array}$ \\
\hline 1175 East Main Sutite 1-C & Medford & Jackson \\
\hline 1187 W MCANDREWS RD & MEFFRD & JACKSON \\
\hline 1187 W MCANDREWS RD & $\begin{array}{l}\text { MEEFOOD } \\
\text { MEFPOODD }\end{array}$ & JACKSON \\
\hline 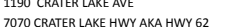 & $\begin{array}{l}\text { MEFEORO } \\
\text { NA }\end{array}$ & 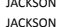 \\
\hline 10175 AGATE RD FWV 10. & $\begin{array}{l}N / A \\
N / A\end{array}$ & 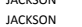 \\
\hline 1401 SISKIYYOU BLVD & Ashland & JAckson \\
\hline 2809 JACKSONVILLE HWY & N/A & IACKSON \\
\hline 11900 CRATER LARE AVE & Medford & \\
\hline D. & N/A & IACCSON \\
\hline 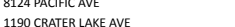 & Metord & JACKSON \\
\hline 1190 C CRATER L LAK AVE & Medtord & \\
\hline 12 GLEN OAK COURT & Medtord & KSON \\
\hline 7475 HIGH BANK RD & N/A & JACKSON \\
\hline 1200 MIRA MAR AVE & МEFFORD & AACKSON \\
\hline 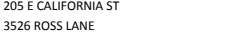 & $\begin{array}{l}\text { Jacksonvile } \\
\text { N/S }\end{array}$ & $\begin{array}{l}\text { A ACCCSON } \\
\text { AACKSON }\end{array}$ \\
\hline 8705 CRATER LAKE HWY & N/A & JACKSON \\
\hline 1200 MIRA MAR AVE & Medford & JACKSON \\
\hline 90 N MOUNTAIN AVE & $\begin{array}{l}\mathrm{N} / \mathrm{A} \\
\text { Medford }\end{array}$ & IACCSSON \\
\hline $\begin{array}{l}1200 \text { MIRA A ARR AVE } \\
1200 \text { MRAA AR AEE }\end{array}$ & $\begin{array}{l}\text { Medeford } \\
\text { Medford }\end{array}$ & $\begin{array}{l}\text { APCCSSON } \\
\text { ACKSON }\end{array}$ \\
\hline $\begin{array}{l}12000 \text { MIRA MARR AV AVE } \\
120 \text { AVE }\end{array}$ & Medford & Jackson \\
\hline 1200 MIRA MAR AVE & Medtord & Jackson \\
\hline 7890 AGATE RD & N/A & JACKSON \\
\hline 1200 MIRA MAR AVE & Meefford & IACCSON \\
\hline 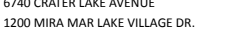 & $\begin{array}{l}\mathrm{N} / \mathrm{A} \\
\text { Metford }\end{array}$ & $\begin{array}{l}\text { AACCSSON } \\
\text { AACKON }\end{array}$ \\
\hline $1-5$ MILEPOST 14.5 & $\mathrm{~N} / \mathrm{A}$ & \\
\hline ST \& FREEMAN RD & Central Point & \\
\hline 1201 ARMOOPY OR & Medford & JACKSON \\
\hline R LN \& SHAFER LN & ssonville & JACKSON \\
\hline 1208 S SF & & \\
\hline & $\begin{array}{l}\text { MEOFORD } \\
\text { MEOT }\end{array}$ & \\
\hline WAL & Ashland & IACKSON \\
\hline PIEFIN CREEKK, & N/A & \\
\hline 13003 HWY 6 2 AKA C CRATER LAKE HWY & $\mathrm{N} / \mathrm{A}$ & JACKSON \\
\hline $\begin{array}{l}\text { EEEEER R \& HAMRRCK RD } \\
11 \text { FIST ST }\end{array}$ & $\begin{array}{l}\text { N/A } \\
\text { N/A }\end{array}$ & $\begin{array}{l}\text { JaACSONON } \\
\text { JACKSOON }\end{array}$ \\
\hline
\end{tabular}

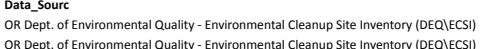

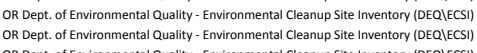

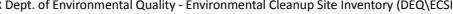

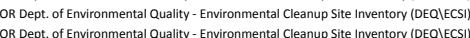
OR Dept. of Environmental Quality - Environmental Cleanup Site Inventory (DEQQECESS)

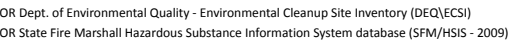

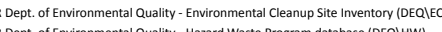

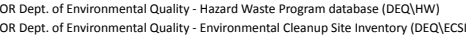

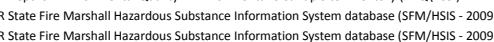

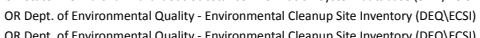

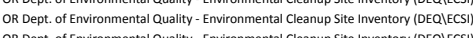

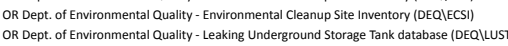

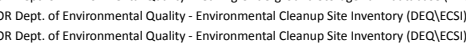

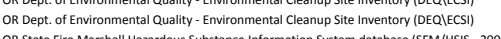

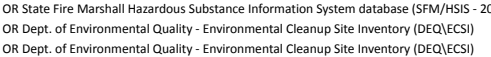

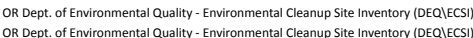

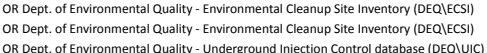

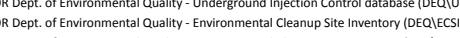

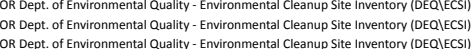

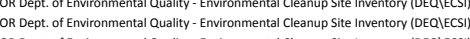

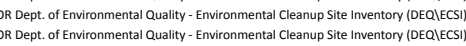

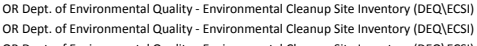

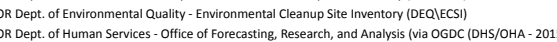

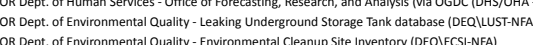

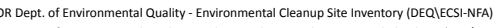

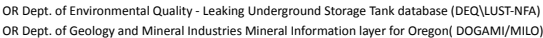

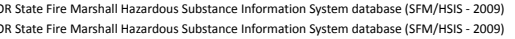

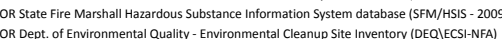

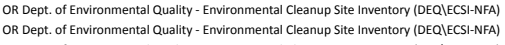

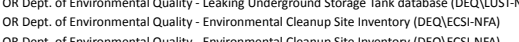

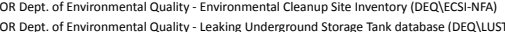

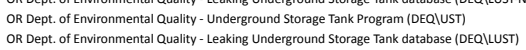

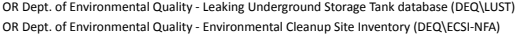

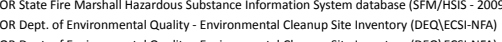

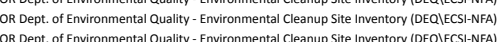

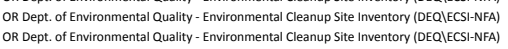

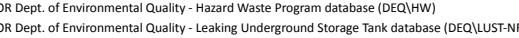
OR Dept. of Environmental Quality - Leaking Underground Storage Tank database (DEQ) LUST-NF

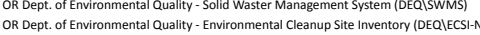

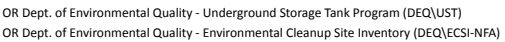
OR Dept. of Environmentata Quality - Water Quality SIS datababse
OR

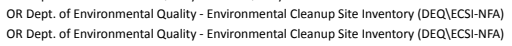

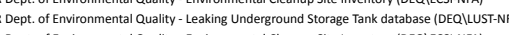

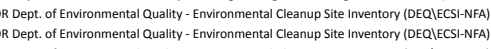

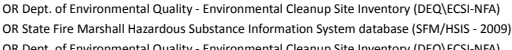

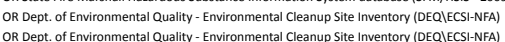

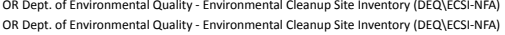

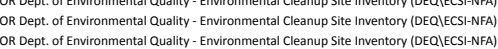

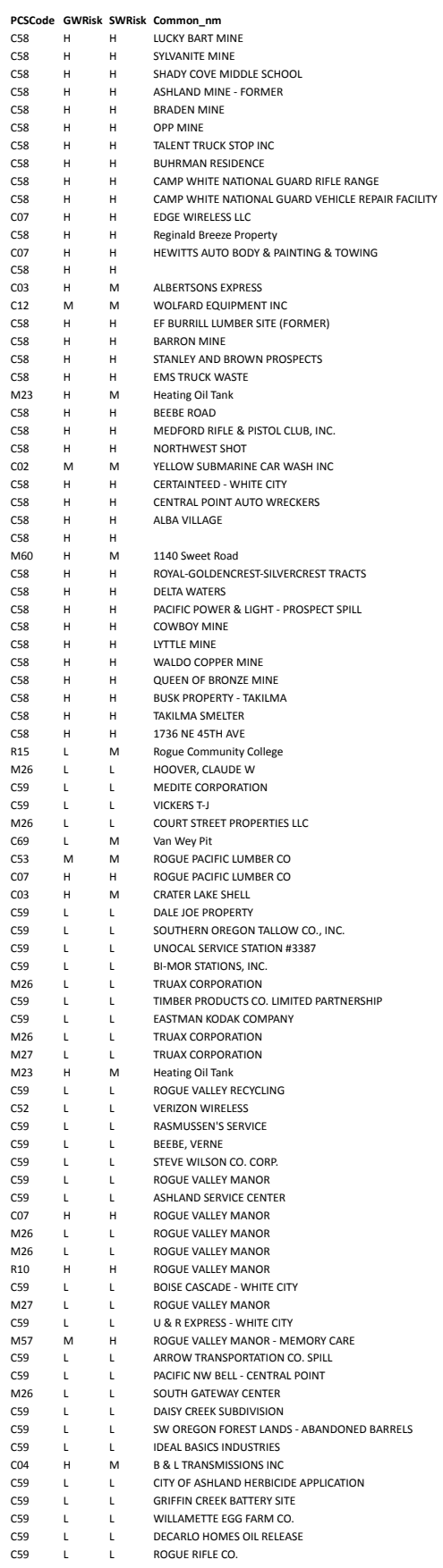

PCSType

Swn Contamination Stes/Plumes/spills $(E C S)$

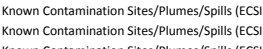

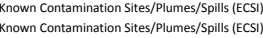

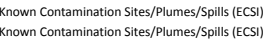
/Storage

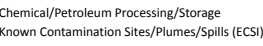

Known Contamination Stites/Plumes/spills (ESS) Rown Contamination Sites/Plumes/spills ECCS)

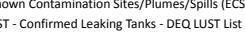

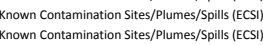
Automobiles-Car Washes

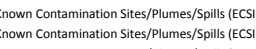

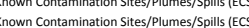
Other Injection/Dry Wells, Sumps- class v Vilcs

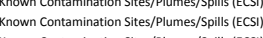

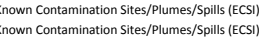

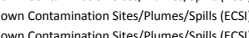

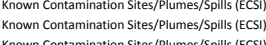

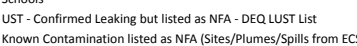

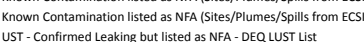

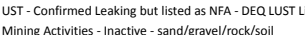

Cremical/Petroleum Processing/5torage

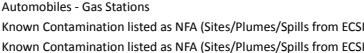

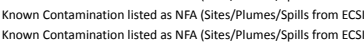
UST - Confirmed Leaking but listed a as NA - DEQ LUST List known Contaminatition listed as NFA (Sites/Plumes/SPillis from ECSI)

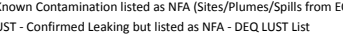
UST - Upgraded and/or Registered-Active (may also h UST - confirmed Leaking Tanks - DEQ Lust List Known Contamination listed as NFA (Sites/Plumes/Spills from ECSS

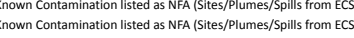
Chemical/Petroleum Processing/Storage

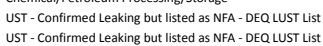
Municipal/Industrial Landifil

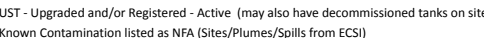

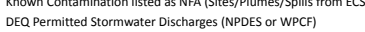

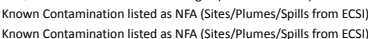

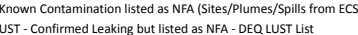
Known Contamination Iisted as NFA (Sites/Plumes/Spills from ESG Known Contamination isted as N NAA SSites/Plumes/Spills from ECSS Auto- Repair Shops

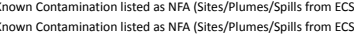

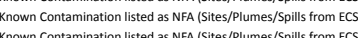




\begin{tabular}{|c|c|c|c|}
\hline $\begin{array}{l}\text { Adrdess } \\
1204 \text { SToWE AVE } 110 \\
1212 \text { Queen Anne Ave }\end{array}$ & $\begin{array}{l}\text { City } \\
\text { MEFFoRD } \\
\text { Medford }\end{array}$ & $\begin{array}{l}\text { County } \\
\text { Jackson } \\
\text { JaCSSON }\end{array}$ & $\begin{array}{l}\text { RET_DATE } \\
\text { o9/2/2/2008 } \\
0 . / 2 / 2015\end{array}$ \\
\hline & & & \\
\hline 2415 GLORY C RD & N/A & JaCKSON & $\begin{array}{l}101 / 1 / 71818 \\
10 / 31 / 2018\end{array}$ \\
\hline GRANITE STREET & N/A & JACKSON & $10 / 31 / 2018$ \\
\hline 8385 Division Rd. & N/A & JaCKSON & $10 / 31 / 2018$ \\
\hline $\begin{array}{l}1215 \text { South Columbus } \\
5550 \text { Hwy }\end{array}$ & Mefford & $\begin{array}{l}\text { Josepphine } \\
\text { Aacrsor }\end{array}$ & $\begin{array}{l}1 / 11 / 2018 \\
{ }_{10 / 3 / 2018}\end{array}$ \\
\hline $\begin{array}{l}51500 \text { HWV } 140 \\
1225 \text { SENTPAL }\end{array}$ & $\begin{array}{l}\text { N/A } \\
\text { Medford }\end{array}$ & $\begin{array}{l}\text { J JACSSON } \\
\text { JACKSON }\end{array}$ & $\begin{array}{l}10 / 3 / 1 / 2018 \\
10131 / 2018\end{array}$ \\
\hline 3213 HAMRICK RD. & N/A & 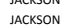 & $\begin{array}{l}101 / 3 / 21 / 2018 \\
10 / 131 / 2018\end{array}$ \\
\hline 1225 COURT ST & Medford & 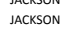 & $\begin{array}{l}10 / 131 / 2018 \\
1018\end{array}$ \\
\hline E PINE ST \& 4TH ST & Central Point & Jackson & $10 / 31 / 2018$ \\
\hline 1007 S PACIFIC HWY & N/A & JACKSON & $10 / 31 / 2018$ \\
\hline 1-5 MP 6 & N/A & $\begin{array}{l}\text { JaCkson } \\
\text { Iacsson }\end{array}$ & $\begin{array}{l}10 / 13 / 2018 \\
1013 / 2018\end{array}$ \\
\hline $\begin{array}{l}1717 \text { W. GLENWOOOD RD. } \\
1231 \\
1 \text { IISK DR }\end{array}$ & $\begin{array}{l}\text { N/A } \\
\text { MEFFRD }\end{array}$ & $\begin{array}{l}\text { JaCKSON } \\
\text { JACKSON }\end{array}$ & 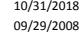 \\
\hline 3285 N PACIFIC HWY & & $\begin{array}{l}\text { JaCSSON } \\
\text { IACCSON }\end{array}$ & $\begin{array}{l}0992 / 2 / 2008 \\
10 / 1312018\end{array}$ \\
\hline 3285 N PACIFIC HWY & & 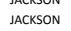 & $\begin{array}{l}10 / 31 / 2018 \\
1018\end{array}$ \\
\hline 1234 CORONA AVE & MEFFRD & JACKSON & 09/29/2008 \\
\hline AVENUE G & N/A & JACKSON & $10 / 31 / 2018$ \\
\hline $\begin{array}{l}165 \text { WATER ST. } \\
\text { 217 N RSSS ANE }\end{array}$ & Ashland & $\begin{array}{l}\text { JACKSON } \\
\text { IACSSON }\end{array}$ & $\begin{array}{l}10 / 13 / 2018 \\
1013 / 2018\end{array}$ \\
\hline $\begin{array}{l}2177 \text { ROSS LAAE } \\
1234 \text { CORONA AVE }\end{array}$ & $\begin{array}{l}\text { N/A } \\
\text { MEFORD }\end{array}$ & $\begin{array}{l}\text { JaCKSON } \\
\text { IACCSON }\end{array}$ & 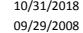 \\
\hline $\begin{array}{l}12344 \text { CoROOANA AVE } \\
1234 \text { Corona Avenue }\end{array}$ & $\begin{array}{l}\text { MEEFRORO } \\
\text { Medford }\end{array}$ & $\begin{array}{l}\text { IJacsison } \\
\text { Jackson }\end{array}$ & $\begin{array}{l}09929 / 2008 \\
1 / 11 / 2018\end{array}$ \\
\hline 124 GREENWAY CRCLE & Mefford & 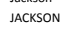 & $\begin{array}{l}10 / 11 / 2018 \\
1 / 21\end{array}$ \\
\hline 22015 PACFIC HWY. & N/A & JACKSON & 10/31/2018 \\
\hline ROSSANLEY DRIVE & N/A & Jackson & $\begin{array}{l}10 / 31 / 2018 \\
103 / 212018\end{array}$ \\
\hline $\begin{array}{l}72800 \text { AACFFCCAAE } \\
1244 \text { S Riverside Ave }\end{array}$ & N/A & JACKSON & $\begin{array}{l}10 / 31 / 2018 \\
8 / 27 / 2018\end{array}$ \\
\hline $\begin{array}{l}12444 \text { S Riversidid Ave } \\
125 \text { W FOURTH ST }\end{array}$ & $\begin{array}{l}\text { Mefford } \\
\text { MEOFORD }\end{array}$ & $\begin{array}{l}\text { Jackson } \\
\text { IacrsoN }\end{array}$ & $\begin{array}{l}8 / 27 / 21218 \\
0 / 292008\end{array}$ \\
\hline 1250 Biddle Rd Ste G & Medford & $\begin{array}{l}\text { Sacsoun } \\
\text { Jackson }\end{array}$ & $\begin{array}{l}8 / 27 / 2018 \\
8 / 2018\end{array}$ \\
\hline 1250 CRATER LAKE AVE & Mefford & 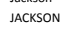 & 10/11/2018 \\
\hline 1251 S RIVERSIDE AVE & Medtord & JACKSON & 10/31/2018 \\
\hline 1253 E VILAS RD & $\begin{array}{l}\text { Mefford } \\
\text { Seropon }\end{array}$ & Jackson & 10/31/2018 \\
\hline 1271 STOWE AVE & $\begin{array}{l}\text { МEFFRO } \\
\text { MEFOODD }\end{array}$ & JaCKSON & $\begin{array}{l}09 / 29 / 2008 \\
09929 / 2008\end{array}$ \\
\hline $\begin{array}{l}12829 \text { SENS LN LNE } \\
1289\end{array}$ & $\begin{array}{l}\text { MEFFRO } \\
\text { MEFFRD }\end{array}$ & $\begin{array}{l}\text { JaCSSON } \\
\text { JaCSSON }\end{array}$ & $\begin{array}{l}09929 / 2008 \\
09 / 29 / 2008\end{array}$ \\
\hline 129 N Oakdale Ave & Medford & Jackson & $\begin{array}{l}06 / 292 / 2015 \\
0015\end{array}$ \\
\hline 1299 STOWE AVE & MEFFRD & Jackson & 09/29/2008 \\
\hline 4594 S PACIFIC HWY & Phoenix & JaCKSON & 10/31/2018 \\
\hline 801 AVENUE C & N/A & JaCKSON & 10/31/2018 \\
\hline $\begin{array}{l}1299 \text { STTOWE AVE } \\
1493 \text { KINGSEF DR }\end{array}$ & $\begin{array}{l}\text { MEFFORD } \\
\text { N/A }\end{array}$ & $\begin{array}{l}\text { J JCCSSON } \\
\text { JACKSON }\end{array}$ & $\begin{array}{l}09 / 29 / 2008 \\
101312018\end{array}$ \\
\hline $\begin{array}{l}12 \mathrm{~A} \text { W JACKSON } \\
1 \text {. }\end{array}$ & $\begin{array}{l}\text { NARA } \\
\text { MEFFRD }\end{array}$ & 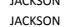 & $\begin{array}{l}1 / 131 / 2018 \\
09 / 29 / 2008\end{array}$ \\
\hline 250 LOZER LN & $\mathrm{N} / \mathrm{A}$ & JACKSON & $\begin{array}{l}0.979 / 2008 \\
10 / 11 / 218\end{array}$ \\
\hline 8399 14TH ST & N/A & JACKSON & 10/31/2018 \\
\hline 3100 WILOW SPRINGS RD & N/A & JACKSON & 10/31/2018 \\
\hline $\begin{array}{l}1977 \text { HWW } 99 \mathrm{~N} \\
197\end{array}$ & N/A & JACKSON & 10/31/2018 \\
\hline (2000 RGGUE RUER DR & $\begin{array}{l}\text { Metord } \\
N \text { A }\end{array}$ & $\begin{array}{l}\text { JaCCSON } \\
\text { ACCSSON }\end{array}$ & $\begin{array}{l}10 / 13 / 21 / 218 \\
1 / 131 / 2018\end{array}$ \\
\hline 1250 SISkYYOU BVIVD & $\begin{array}{l}\text { Ashiand } \\
\text { Ashland }\end{array}$ & 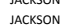 & $\begin{array}{l}10 / 131 / 2018 \\
10 / 11 / 2018\end{array}$ \\
\hline 11111 EVILAS RD & N/A & JACCSON & $101 / 11 / 2018$ \\
\hline 1300 CENTRAL & Medford & JACKSON & 10/31/2018 \\
\hline 4511 2NDSST & Central Point & JACKSON & 10/31/2018 \\
\hline 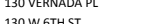 & 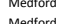 & 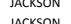 & 10/11/2018 \\
\hline D. & N/A & 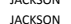 & $\begin{array}{l}10 / 13 / 21 / 218 \\
1 / 1312018\end{array}$ \\
\hline 7111 ВАСККWЕL ВО & N/A & JACCSON & $\begin{array}{l}10 / 131 / 2018 \\
10 / 11 / 2018\end{array}$ \\
\hline 130 W CLARK & MEOFORD & JaCKSON & 09/29/2008 \\
\hline 130 W CLARK & MEFFORD & JACKSON & 09/29/2008 \\
\hline 2045 HWr $99 \mathrm{~N}$ & N/A & JACKSSON & 10/31/2018 \\
\hline 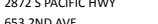 & N/A & 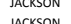 & 10/31/2018 \\
\hline (7) & N/A & $\begin{array}{l}\text { S } \\
\text { ACCSSON }\end{array}$ & $\begin{array}{l}10 / 13 / 21218 \\
10 / 11 / 2018\end{array}$ \\
\hline 130 W CLARK ST & Medford & JaCKSON & $\begin{array}{l}10 / 131 / 2018 \\
10 / 11 / 2018\end{array}$ \\
\hline 100 COLE M RIVERS DR & N/A & JaCKSON & $10 / 31 / 2018$ \\
\hline 8250 AGATE RD & N/A & JACCSON & 10/31/2018 \\
\hline 1990 E MANST ST & Ashland & JACCSONO & 10/31/2018 \\
\hline 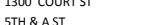 & $\begin{array}{l}\text { MEEFHono } \\
\text { Asthan }\end{array}$ & 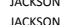 & $\begin{array}{l}09 / 29 / 2008 \\
101312 / 2018\end{array}$ \\
\hline $\begin{array}{l}1300 \text { COURT ST } \\
13\end{array}$ & $\begin{array}{l}\text { MEFFRO } \\
\text { MEFO }\end{array}$ & $\begin{array}{l}\text { JaCKSON } \\
\text { Jack }\end{array}$ & $\begin{array}{l}1 / 131 / 2018 \\
09 / 29 / 2008\end{array}$ \\
\hline 1300 COURT ST & Medford & JaCKSON & $\begin{array}{l}0.979 / 2008 \\
10 / 11 / 2018\end{array}$ \\
\hline 640 TOLMAN CREEK RD & Ashland & JACKSON & 10/31/2018 \\
\hline 2095 СоREY RD & $N / A$ & JaCKSON & 10/31/2018 \\
\hline 1 S PACFFC HWV & N/A & JACCSONO & 10/31/2018 \\
\hline 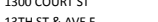 & Nevorord & 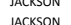 & $\begin{array}{l}10 / 13 / 21 / 2018 \\
1 / 1312018\end{array}$ \\
\hline 1301 CEETER D & (N) & $\begin{array}{l}\text { JaCkSON } \\
\text { Jack }\end{array}$ & $\begin{array}{l}1 / 131 / 2018 \\
09 / 29 / 2008\end{array}$ \\
\hline 1301 CENTER DR & MEFORD & Jackson & $\begin{array}{l}09792 / 2008 \\
09 / 29 / 208\end{array}$ \\
\hline SFRONT ST & Central Point & JACKSON & 10/11/2018 \\
\hline 1301 CENTER DR & Medford & JACKSON & 10/31/2018 \\
\hline 1301 CENTER DR & Mefford & IACCSON & 11/1/2018 \\
\hline & - & 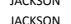 & $\begin{array}{l}09 / 29 / 2008 \\
0.929 / 2008\end{array}$ \\
\hline $\begin{array}{l}1301 \text { S HOLY } \\
1311 \text { N RIVERS }\end{array}$ & MEFFOO & $\begin{array}{l}\text { JACKSON } \\
\text { Jas }\end{array}$ & 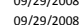 \\
\hline $\begin{array}{l}1109 \text { COURT ST } \\
1 \text {. AVt }\end{array}$ & & JaCKSON & 10/11/2018 \\
\hline
\end{tabular}

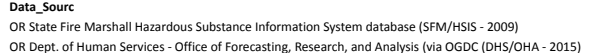

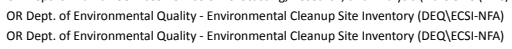

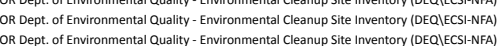

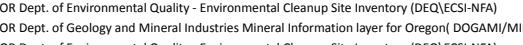

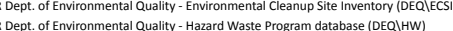

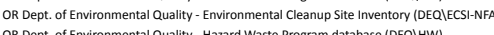

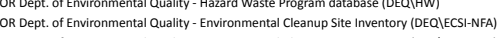

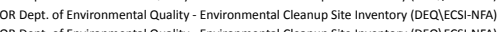

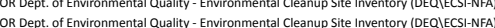
OR State fire Marshall Hazardous Substance Intormation System database (SFM/HSIS- 2009

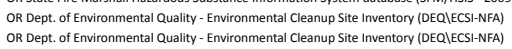

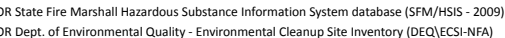
OR Dept. of Environmental Luality - Environmental Cleanup Site Inventory (DEQQE|ECS-NFA)

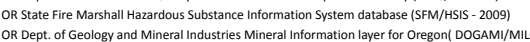

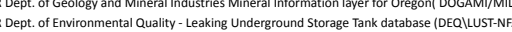
OR Dept. of Environmental Q Quality - Environmental Cleanup Site Inventor (DEQ)ECES-NAFA)

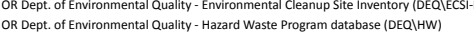
OR State fire Marshall Hazard dous Substance information System database (SFM/MSIS- 2009

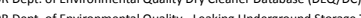

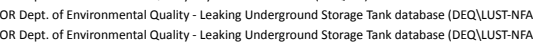
OR Dept. of Environmental auality - Hazard Waste Program database (DEQQ1HW)

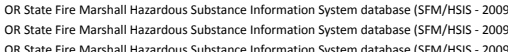

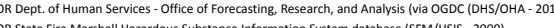
OR Dept. of Environmental Q Quality - Hazard Waste Program datatabsese (DEEO)

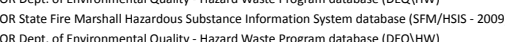

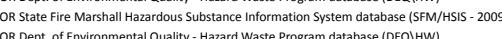

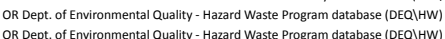
OR Dept. of Environmental Q Quality - Hazard Waste Program dis bras

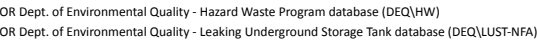

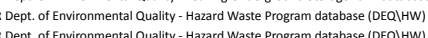

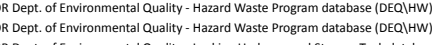

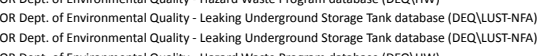
OR State Fire Marshall Hazardous Substance Information System database (SFFM/HIS - 2009

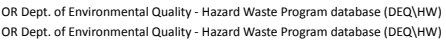

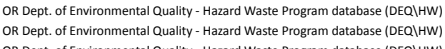

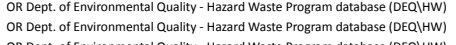
OR Dept. of Environmental Quality - Hazard Waste Program database (DEQ1HW)
OR State fire Marshall Hazardous Substance Information System database (SFM/HIIS- 2009 S

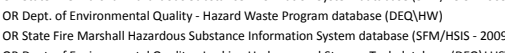

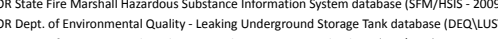

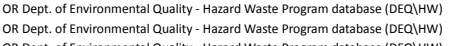

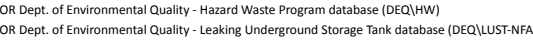

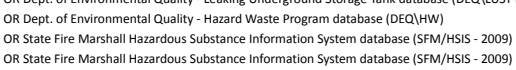

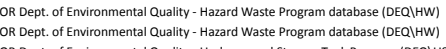
OR Dept. of Environmental Quality - Underground Storage Tank F rogram (OEEQUUST) OR State Fire Marshall Hazard dus Substance Infformation System database (SFM/HIIS-2009)

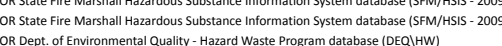

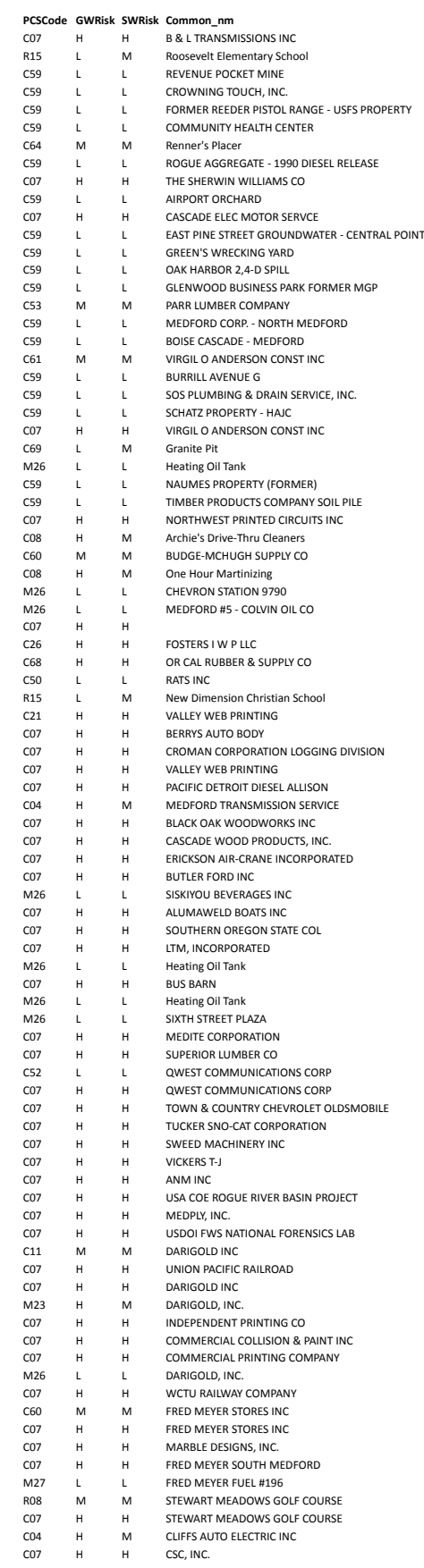

PcSTye
chemical/Petroleum Processine/storage

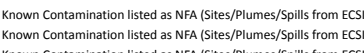

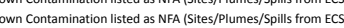

Mining Activites - Inactive - other than sand/frave/rock/soil

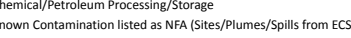

known Contamination listed as NFA (Sites/P/lumes/Spills from ECSI)

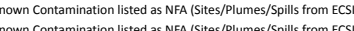

Construction Company
Known Contamination listed as NFA (Stites/Plumes/SPills from ECSS)

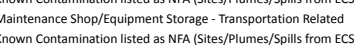
Kown Contamination listed as NEA (Sites/Pllumes/Spills from ECSPS

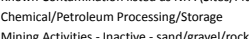

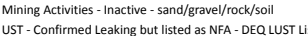

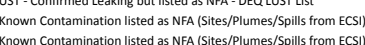
Mnown Contamination listed as NFA A Sites

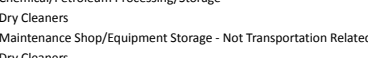
UST - Confirmed Leaking but listed as NFA - DEQ L UST Lis Chemical/Petroleum Processing//storage
Wood/Pulp/Paper Processing and Mills Wood/Pulp/Paper Processing and Mills
Miscellaneous Equipment Maintenance others-6ov't/Business/NoO Services Schools
Photo Processing//Printing
.

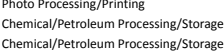
Chemical/Petroleum Processing/storage
Chemical/Petroleum Processing//storage Auto- Repari Shops
Chemical/Petroleum Processing//storage

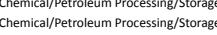

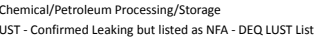
Chemical/Petroleaum Processing//storage
Chemical/Petroleum Processing/Storage

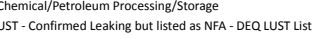

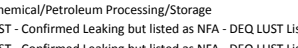
Chemical/Petroleum Processing/storage
chemical/Petroleum Processing/Storage Communications office
chemical Petroleum Procss

Chemical/Petroleum Processsing//tororage
Chemical/Petroleum Processing//torage

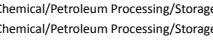
Chemica//Petroleum Processing//torage
Chemica//etroleum Processin/storage
Chemical/Petroleum Processing/Storage Food rocessing

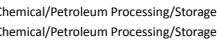
UST - Confirmed Leaking Tanks - DEQ LUST Lis Chemical/Petroleum Processing//storage
Chemical/Petroleum Processing//storage

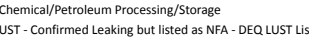

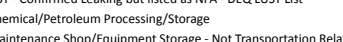
Chemical/Petroleum Processing//Storage

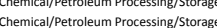
UT - Upgraded and/or Registered - Active (may also have decommissioned tanks on site) Goll Courses
chemicalperoleum Processing/storarae Auto- Repair hops
Chemical Petrolum Processing/s/storage 


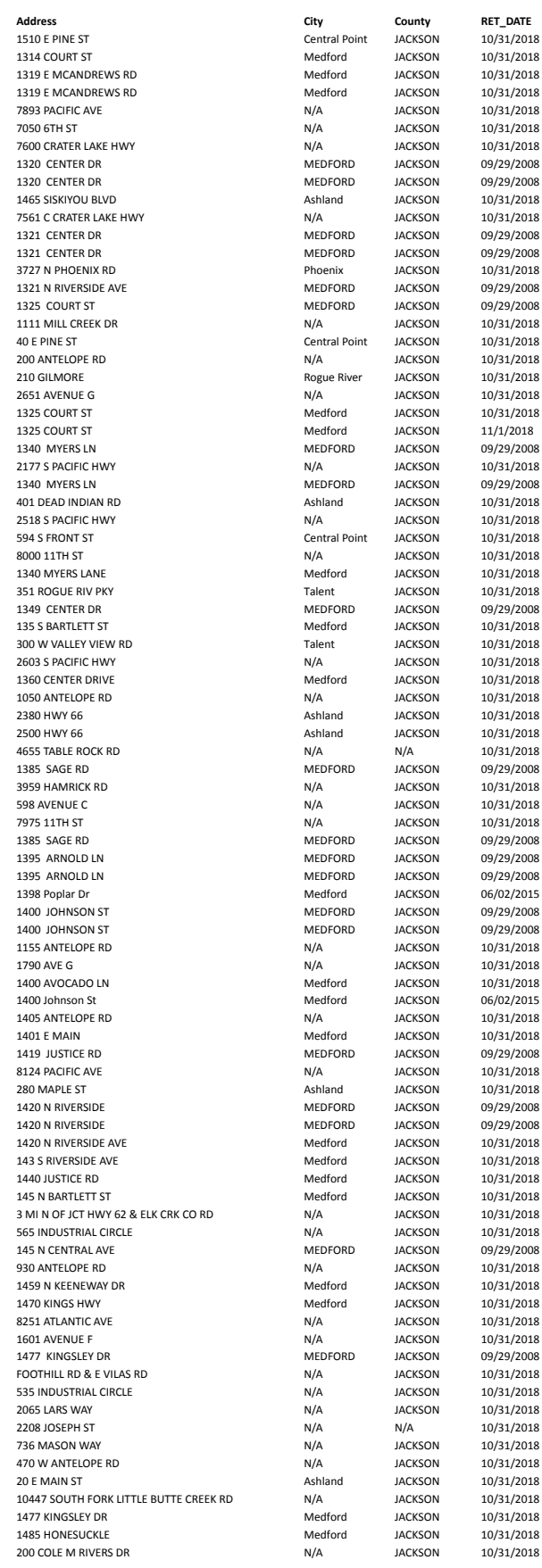

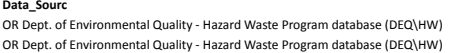

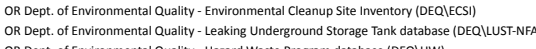

OR Dept. of Environmental Q Quality - Hazard Waste Program databasese (DEQQ HWW

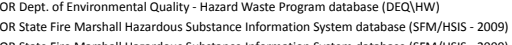

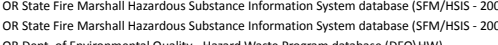

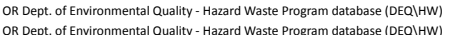

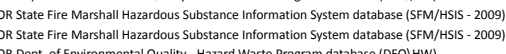

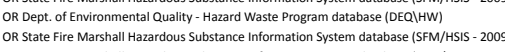
OR S State fire Marshall Hazarddous Substance Information System databases SSFM/HISIS- 2009

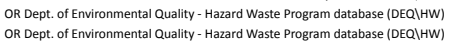
OR Dept. of Environmental luality - Hazard Waste Program databasse (DEOCHWW'

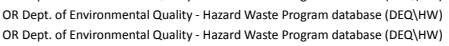

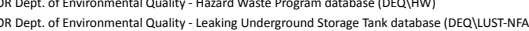

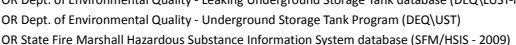

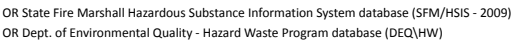

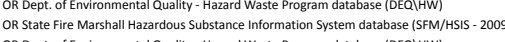

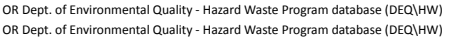

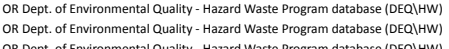

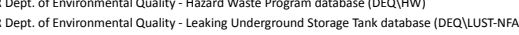
OR D State fire Marshall Hazardous Substance Infformation System database (SFM/HSIS - 2009)

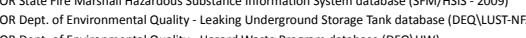
OR Dept. of Environmentat Quality- Hazard Waste Program database (DEQR|HW) OR Dept. of Environmental Quality - Hazard Waste Program database (DEQQHWW) OR Dept. of Environmentata Quality - Hazard Waste Program database (DEQQIHW)

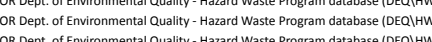
OR State fire Marshall Hazardous Substance Inffrermation System database OR Dept. of Environmental Quality - Hazard Waste Program database (DEQ1HW) OR Dept. of Environmentat Q uality- Hazard Waste Program database (DEQQ1HW)

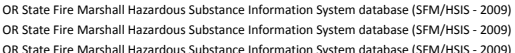

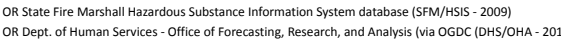

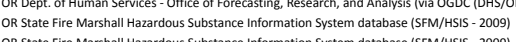

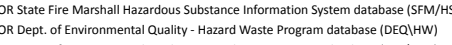

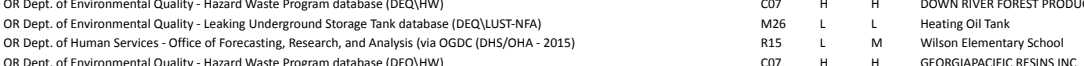
OR Dept. of Environmental Quality L Leaking Underground Storage Tank databasese (DEQQ| LSST-NES OR State fire Marshall Hazardous Substance information System database SSFM/HIS - 200 S

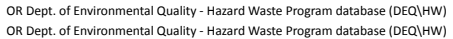

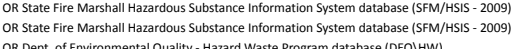
OR Dept. of Env

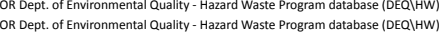
a OR Dept. of Environmental auality - Hazard Waste Progeram datababse (DEQRHW) OR State fire Marshall Hazardous Substance information System databases (SFM/HSIS - 2009)

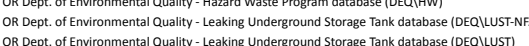

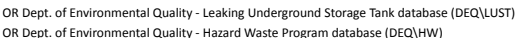

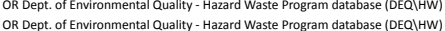
OR S State fire Marshall Hazardous Substance Information System database (SFM/HISIS - 2009) OR Dept. of Environmental luality - Hazard Waste Program database (DEQQ HWW)

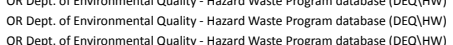

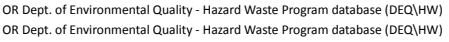

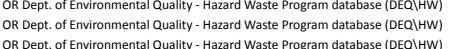

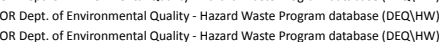

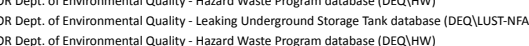

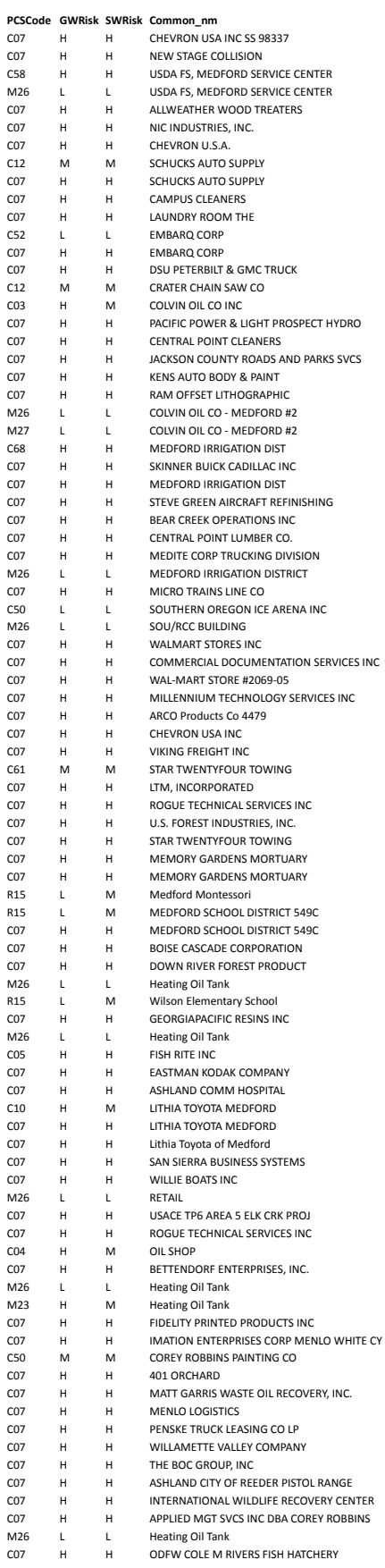

PCSType

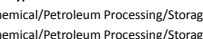

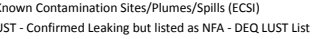
Cremical/Petroleum Processing/stsorage

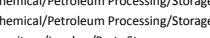
Chemical/Petroleum Processing/tororage
Chemical/Petroleum Processing/storage emical/Petroleum Processing/Storage Chemical/Petroleum Processing//torage
Chemical/Petroleum Processing/storage furntiture/Lumber/Parts Storees
Automobiles - Cas stations

Chemicallertroleum Processing/5torage

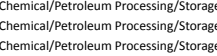

UST-Confirmed Leaking but Iisted as NFA- DEQ L UST Lis UST - Upgraded and/or Registered -Active Miscellaneous Equipment Maintenance
chemical/Petroleum Processing/storage Chemical/Petroleum Processing/storage
Chemical/Petroleum Processing/storage

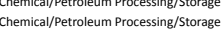

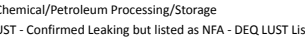
UST - Confirmed Leaking but listed as NFA-
Chemical/petroleum Procoessing /Storage UST - Confirmed Leaking but listed as NFA - DEQ L UST Lis

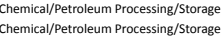
chenical/Petroleum Processing/storoge Chemical/Petroleum Processing//torage

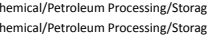
Chemical/Petroleum Processing/Storage
Maintenance Shop/Equipment- Transportation Rels Chemical/pertoleum Processing $/$ /storage chenicalpetroleum Processing/s/torage Chemical/Pertoleum Processing/Storage
Chemical/Pertolum Processing / Storage Schools
schools

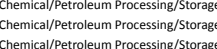

UST - Confifmed Leaking but listed as NFA- DEQ LUST Lis Schools
Chemical

Chemical/petroleum Processing//5torage
UST - Confirmed Leaking but listed as NFA- DEQ LUST L Boat Services/Repair/Refinissing
Chemical/Petroleum Processing/storage Fleet//Tucketin//um Trerminals
chemical/Petroloum Processing/Storage

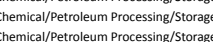

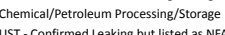

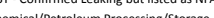
chemical/Petroleum Processing / stororage Auto- Repair Shops

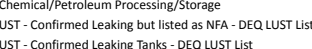

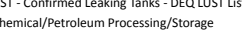
Chemical/Petrolum Processing//torage

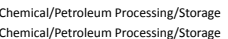
Chemical/Petroleum Processing/5torage chemical/Petroleum Processing/storage Chemical/Petroleum Processing//storage
Cheminal/Petroleum Processing/storage
chemical/Petroleum Processing/storage chemical/Petrolum Processing/storare UST - Confirmed Leaking but listed as NAA - DEQ LUST Lis 


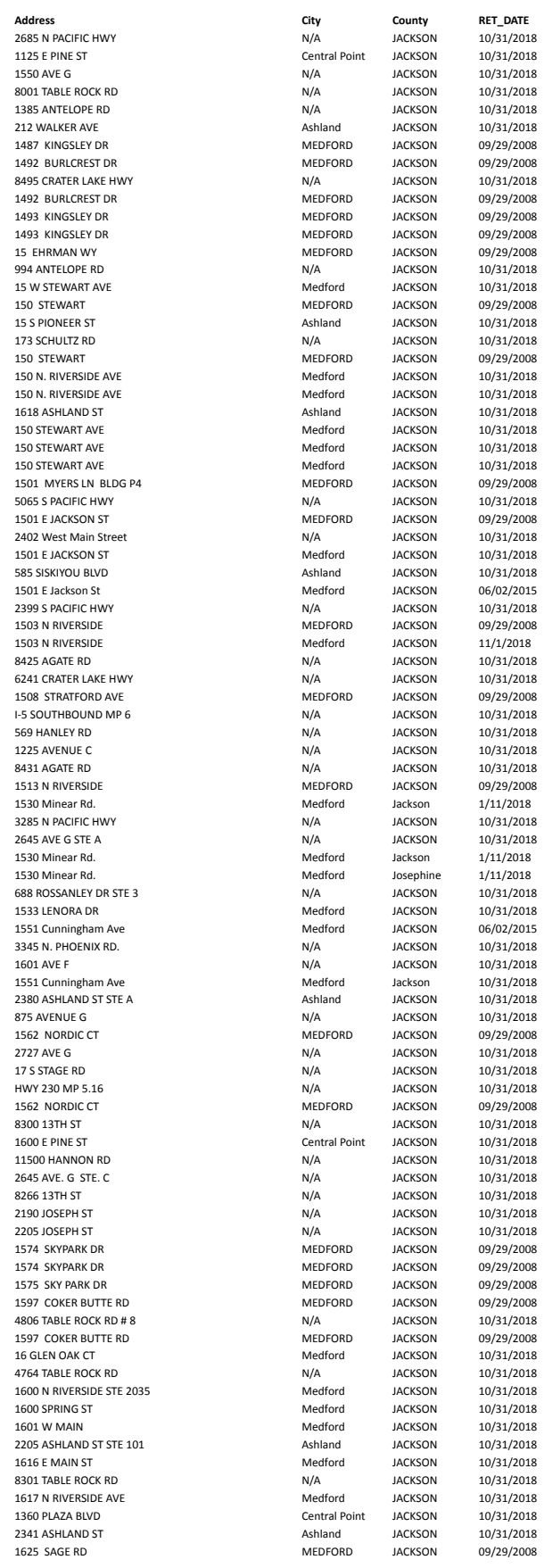

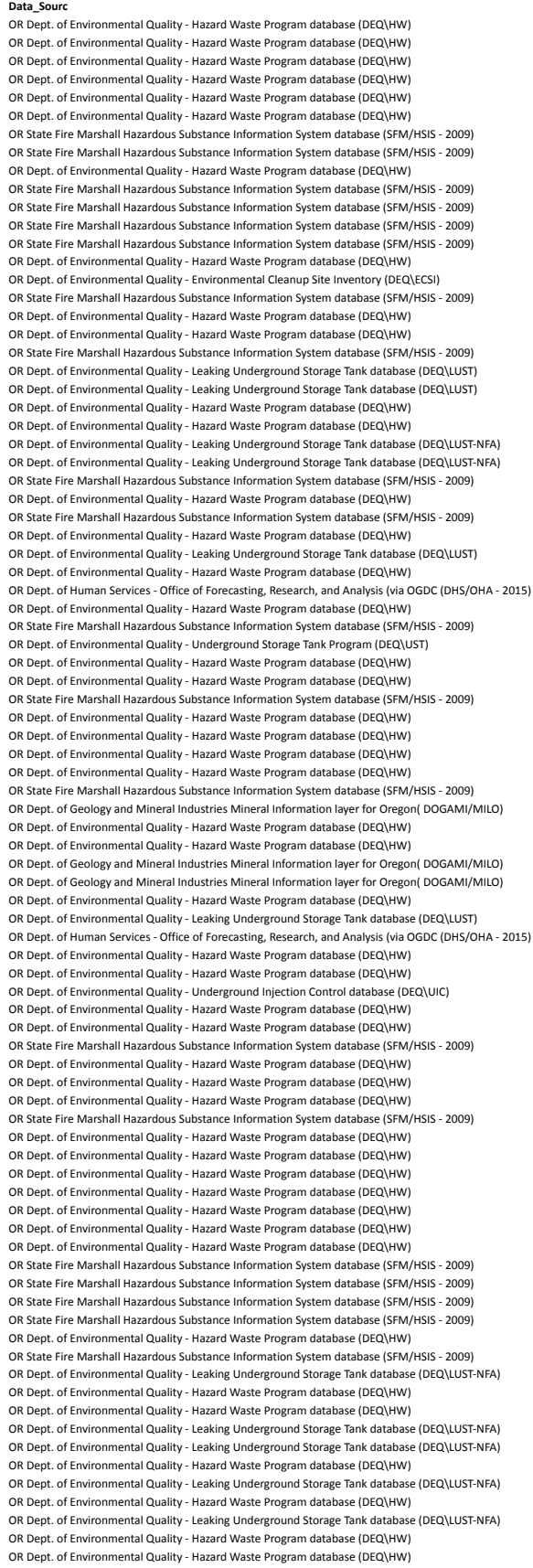

Pcstrye Chemical/Petroleum Processingi//totorage
chemical/Petroleum Processing//torage chemical/Petroluum Processing/storage Miscellaneous Manufacturing
Fleet/Tructin/

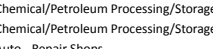
to- Repair Shops

Auto- Repair Shops
Chemical/Petroleum Processing/storage

Communications officice
chemical/Petroleum Processing/storage

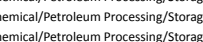

UST - Confirmed Leaking Tanks- DEQ LUST Lis
UST - Confirmed Leaking Tanks - DEQ ULST Lis

Chemical/Petrolum Processing/storogere

UST - Confirmed Leaking but listed as NAA - DEQ Q UST Lis

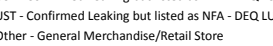

Schools

Chemical/Petrolum Processing//Storage
UST - Confirmed Leaking Tanks - DEQ UST Lis

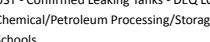

Chemicalpertroleum Processing//Storage

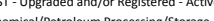

Chemical/Petroleum Processsing/Stororage

Others - Miscelaneous bulding H Home Contracting

Chemical/Petroleum Processing//5torage

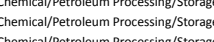

Mining Activities - Active - other than sand/gravel/rock/soil

Chemical/Petroleum Processing/storage
Chemical/Pentroleum Processin/storese

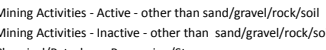

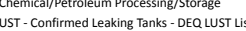

Chemical/Petroleum Processing/storage

Other hijection/ory Wells, Sumps-Class V Vics

chemical/Petroleum Processing//torage

Chemica//Petroleum Processing
Others - Miscerage

Chemical/Petroleum Processing/storage
Chemical/Petroleum Processing/storage

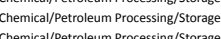

Chemiaca///Petroleum Processsing//torarge
chemical/Petroleum Processing/Storage

chemical/Petroleum Processing/5torage

Chemical/Pertroleum Processing/storage

Construction Company
Chemical/Petroleum Processing/Storage

Farm Machinery Repar

Chemical/Petroleum Processing/storate

UST - Confirmed Leaking but listed as NFA- DEQ LUST Lis

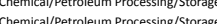

UST - Confirmed Leaking but listed a s NFA- DEQ Q UST List
UST - Confirmed Leaking but listed as NFA - DEQ LUST List

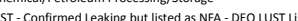

chemical/Petroleum Processing//torage UST - Confirmed Leaking but listed as NFA- DEQ LUST Lis

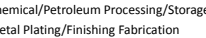




\begin{tabular}{|c|c|c|c|c|}
\hline $\begin{array}{l}\text { Address } \\
1625 \text { EMAIN ST }\end{array}$ & 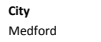 & $\begin{array}{l}\text { County } \\
\text { Jackson }\end{array}$ & $\begin{array}{l}\text { RTEDDARE } \\
\text { 10/31/2018 }\end{array}$ & 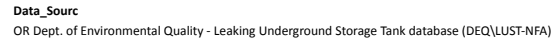 \\
\hline 164 GRENWWAY CIR & Medford & JACKSON & 10/31/2018 & 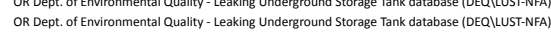 \\
\hline 1656 SPRING ST & $\begin{array}{l}\text { Medford } \\
\text { MEDSORP }\end{array}$ & Jackson & 10/31/2018 & OR Dept. of Environmental Q Quality - Leaking Underground Storage Tank database (DERQIUST) \\
\hline $\begin{array}{l}18699 \text { SAGG RD C } \\
17 \text { STAAE RD }\end{array}$ & $\begin{array}{l}\text { MEFORD } \\
\text { MEOFORD }\end{array}$ & $\begin{array}{l}\text { JaCCSON } \\
\text { JACKSON }\end{array}$ & $\begin{array}{l}0.0 / 99 / 2008 \\
09 / 29 / 2008\end{array}$ & 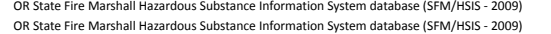 \\
\hline 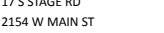 & $\begin{array}{l}\text { MEEPORD } \\
\text { NAS }\end{array}$ & $\begin{array}{l}\text { JaCGSON } \\
\text { IACSSON }\end{array}$ & 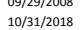 & 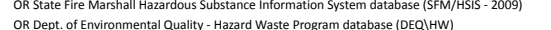 \\
\hline 17 SSTAGE RD & MEDFC & $\begin{array}{l}\text { JaCGSONO } \\
\text { JACKSON }\end{array}$ & $\begin{array}{l}11 / 7172 / 218 \\
09 / 29 / 2008\end{array}$ & 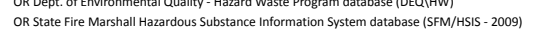 \\
\hline 2406 ANTELOPE RD & & JACKSON & 10/31/2018 & 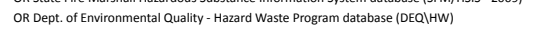 \\
\hline 404 N MAIN ST & Phoenix & JACKSON & 10/31/2018 & OR Dept. of Environmental Quality - Hazard Waste Program database (DEQQ1HW) \\
\hline 2000 EAGLE MILL RD & N/A & JaCKSON & 10/31/2018 & OR Dept. of Environmental Q uality - Hazard Waste Program databasese (OEQQ HWW \\
\hline $\begin{array}{l}\text { 1701 PANOAAMA DR } \\
\text { 640A MASON WAY }\end{array}$ & $\begin{array}{l}\text { Metford } \\
N / A\end{array}$ & JACKSON & 10/31/2018 1018 & 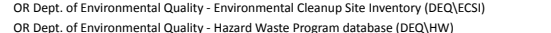 \\
\hline $\begin{array}{l}\text { C20A MAAONANAY } 15 \\
201 \text { BAEMAN DR No } 15\end{array}$ & N/A & $\begin{array}{l}\text { JaCCSON } \\
\text { JACKSON }\end{array}$ & 10/31/2018 11018 & 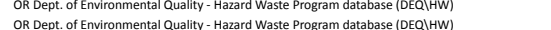 \\
\hline $226 \mathrm{NROSSLN}$ & N/A & JACKSON & 10/31/2018 & 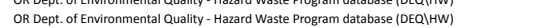 \\
\hline 1-5 SB MLLEPOST 8 & N/A & JACKSON & 10/31/2018 & OR Dept. of Environmental Luality - Hazard Waste Program database (DEQQHWW) \\
\hline 1701 PANORAMA DR & Medtord & JaCKSON & 10/31/2018 & 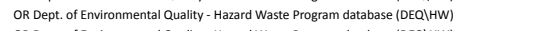 \\
\hline 3075 HAMRICK RD & Central Point & JaCKSON & 10/31/2018 & 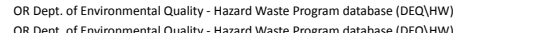 \\
\hline $\begin{array}{l}15 \text { P PCAFIC CWV } \\
\text { KELER RD }\end{array}$ & 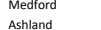 & JACSSON & 1/1/31/2018 & 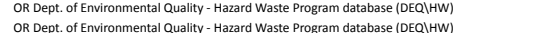 \\
\hline 66 COBURG & $\begin{array}{l}\text { Assland } \\
\text { Portland }\end{array}$ & Lane & 10/31/21/2018 & 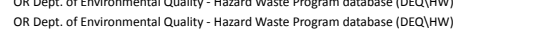 \\
\hline SPAC & MEOFORD & JACKSON & o9/29/2008 & OR State Fire Marshall Hazardous Substance Information System database (SFM/HSIS- 2009) \\
\hline 2000 ROGUE RIVER D & EAGLE POINT & JaCkson & 09/29/2008 & OR State Fire Marshall Hazardous Substance Information System database (SFM/HIS - 2009) \\
\hline 6301 CRATER LAKE HWY & CENTRAL POINT & JACKSON & 09/29/2008 & OR State Fire Marshall Hazardous Substance Information System database (SFM/HIS- 2009) \\
\hline & $\begin{array}{l}\text { MEDOFR } \\
\text { WHITE CITY }\end{array}$ & JACKSON & 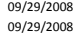 & 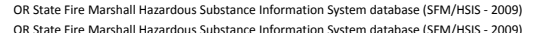 \\
\hline $\begin{array}{l}\text { 8250 AGATRD RD RD } \\
\text { 8087 BAACKWEL RD }\end{array}$ & $\begin{array}{l}\text { WHITE EIVIN } \\
\text { CENTRAL POINT }\end{array}$ & $\begin{array}{l}\text { JACCSON } \\
\text { JACKSON }\end{array}$ & $\begin{array}{l}0.9 / 99 / 2 / 208 \\
09 / 29 / 2008\end{array}$ & 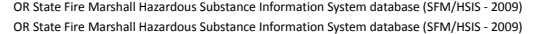 \\
\hline & ASHLAND & 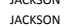 & 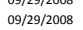 & 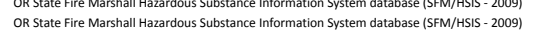 \\
\hline 1701 S PACIFIC HWY & Medtord & JACKSON & 10/31/2018 & 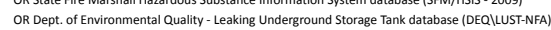 \\
\hline 640 TOMAN CREE & ASHLAND & JacksoN & 09/29/2008 & OR State Fire Marshall Hazarddous Substance Information System database (SFM/HSIS- 2009) \\
\hline 1705 PANORAMA DR & MEOFORD & JACKSON & 09/29/2008 & OR State fire Marshall Hazardous Substance Information System database (SFM/HSIS - 2009) \\
\hline 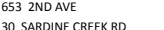 & GOLD HLL & JaCKSON & 09/29/2008 & 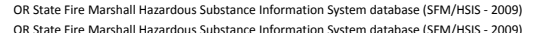 \\
\hline $\begin{array}{l}30 \text { SARINEE CREEK RD } \\
1712 \text { W Main St }\end{array}$ & Medford & $\begin{array}{l}\text { Jacksoon } \\
\text { Jackson }\end{array}$ & 8/27/2012008 & 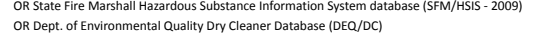 \\
\hline 1729 N RVERSIOE & $\begin{array}{l}\text { MEOFORD } \\
\text { MEOTO }\end{array}$ & $\begin{array}{l}\text { Jackson } \\
\text { JacsoN }\end{array}$ & 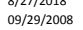 & 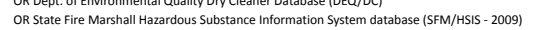 \\
\hline бтн ST & WHITE CITY & JACKSON & $09 / 29 / 2008$ & 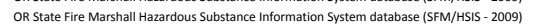 \\
\hline ROGUE RII & TALENT & JACKSON & 09/29/2008 & 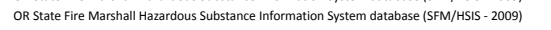 \\
\hline N RIVERSIDE & MEDFORD & JACKSON & 09/29/2008 & OR State fire Marshall Hazardous Substance Information SSystem database (SFM/HSIS- 2009) \\
\hline $\begin{array}{l}2 \text { CRATER LARE AVE } \\
\text { TOLAN CREEK RD }\end{array}$ & ENTRALPOINT & & 09/29/2008 & 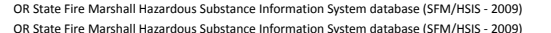 \\
\hline $\begin{array}{l}\text { 435 TOLMANACREERD } \\
2350 \text { AVENUE F }\end{array}$ & $\begin{array}{l}\text { AHALANO } \\
\text { WHITE CITY }\end{array}$ & $\begin{array}{l}\text { JaCCSONO } \\
\text { ACCSSON }\end{array}$ & $\begin{array}{l}0.9 / 992 / 2008 \\
09 / 29 / 2008\end{array}$ & 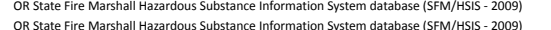 \\
\hline C CRATRR LAKE & WHITE CITY & Jackson & 09/29/2008 & 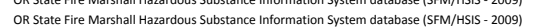 \\
\hline AVERUEC & WHITE CITY & Jackson & $09 / 29 / 2008$ & 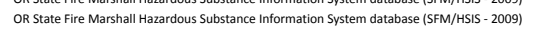 \\
\hline 5300 CRATER L. & CENTRAL POINT & JACKSON & 09/29/2008 & 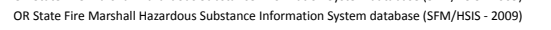 \\
\hline $\begin{array}{l}\text { 55610 TABEL ROCKK RD } \\
\text { 8425 AGAT RD }\end{array}$ & $\begin{array}{l}\text { CENTRA LOINT } \\
\text { WHTICETY }\end{array}$ & $\begin{array}{l}\text { JACCSSON } \\
\text { JACKON }\end{array}$ & 09/29/2008 0008 & 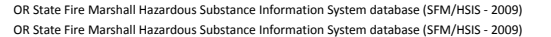 \\
\hline $\begin{array}{l}85258 \text { AABLE ROCK RD } \\
5580\end{array}$ & CENTRAL POINT & JACKSON & 09/29/2008 & 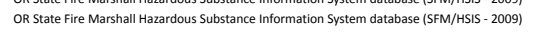 \\
\hline 3072 OLD MLLTARY RD & CENTRAL POINT & JACKSON & 09/29/2008 & OR State fire Marshall Hazardous Substance Information System database (SFM/HSIS- 2009) \\
\hline 789 EIEFFERSON AVE & $\begin{array}{l}\text { ASHLAND } \\
\text { WHITE CITY }\end{array}$ & JACKSON & 09/29/2008 0 & 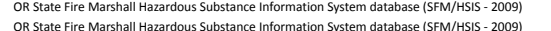 \\
\hline 7260 BLACKWELL RD & CENTRAL POINT & $\begin{array}{l}\text { JACCSSON } \\
\text { JACKSON }\end{array}$ & 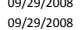 & 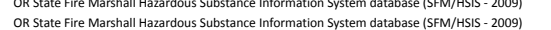 \\
\hline S R RUERSIID AVE & Medford & 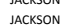 & 10/31/2018 & 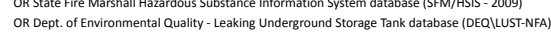 \\
\hline p siskry & SHLAND & JACKSON & 09/29/2008 & 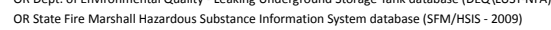 \\
\hline b Justice & CENTRAL POINT & JACKSON & 09/29/2008 & OR Satat fire Marshall Hazardous Substance Information System databasese (SFM/HSIS- 20099 \\
\hline 1759 ALLAN & $\begin{array}{l}\text { Medford } \\
\text { centent }\end{array}$ & JACKSON & 10/31/2018 & 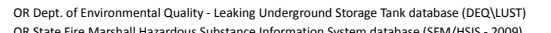 \\
\hline 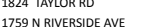 & $\begin{array}{l}\text { EENRAL POINT } \\
\text { Medford }\end{array}$ & $\begin{array}{l}\text { ATCCSSON } \\
\text { ACKSON }\end{array}$ & 09/29/2/2008 & 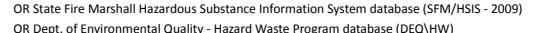 \\
\hline 5731 CRATER LAKE & $\begin{array}{l}\text { NeErora } \\
\text { CENTRAL POINT }\end{array}$ & IACKSON & 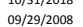 & 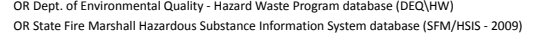 \\
\hline 51 BARTOI & GLEPINT & JACKSON & 09/29/2008 & \\
\hline 1787 SPRING & edford & JACKSON & 10/31/2018 & OR Dept. of Envirionmental Quality - Leaking Underground Storage Tank database (DEQQIUSTT-NFA) \\
\hline 1022 NEWLAND & CENTRAL POINT & JACKSON & 09/29/2008 & OR State Fire Marshall Hazardous Substance Information System database (SFM/HSIS- 2009) \\
\hline 1022 NEWLAND RD & CENTRAL POINT & JAACKON & 09/29/2008 & 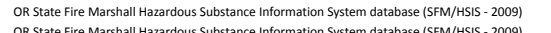 \\
\hline $\begin{array}{l}\text { SAGG RD } \\
\text { MISTLEOE RD }\end{array}$ & $\begin{array}{l}\text { MEEFORD } \\
\text { ASHLAND }\end{array}$ & $\begin{array}{l}\text { A ACCSSON } \\
\text { ACKSON }\end{array}$ & 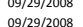 & 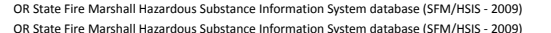 \\
\hline $3100 \mathrm{~V}$ & NTFAL POINT & 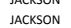 & 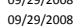 & 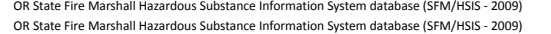 \\
\hline & entom. & JACKSON & 0 & 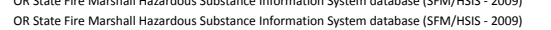 \\
\hline 17171 N Rо & CAVE JUNCTION & JOSEPHINE & 09//29/2008 & 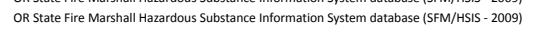 \\
\hline & edford & JACKSON & 10/31/2018 & OR Dept. of Environmental Quality - Environmental Cleanup Site Inventory (DEQQECSSI) \\
\hline & carir & JACKSON & 10/1/12018 & 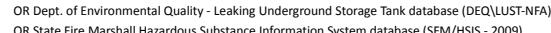 \\
\hline & NTRALPOIN & $\begin{array}{l}\text { AACCSSON } \\
\text { IACKSON }\end{array}$ & $\begin{array}{l}00 / 7292 / 2008 \\
09 / 2 / 2008\end{array}$ & 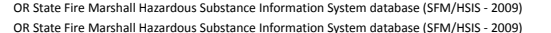 \\
\hline & & $\begin{array}{l}\text { AICCKSON } \\
\end{array}$ & $\begin{array}{l}0 \\
00 / 2 / 29 / 2008\end{array}$ & 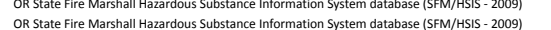 \\
\hline 1495 E GREGC & ENTRAL POINT & JACKSON & 09/29/2008 & 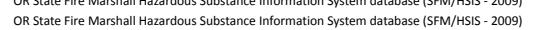 \\
\hline 379 & Вьн нाL & Jackson & 59/29/20008 & 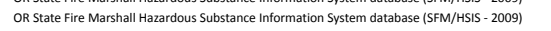 \\
\hline & HLAND & JACKSON & 09/29/2008 & OR State fire Marshall Hazard dous Substance Information System database (SFM/HISS - 2009) \\
\hline & & & 09/29/2008 & ce Information System databasese (SFM/HISIS-2009) \\
\hline & 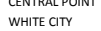 & $\begin{array}{l}\text { ATCCSSON } \\
\text { JACKSON }\end{array}$ & $099 / 29 / 20008$ & 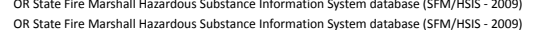 \\
\hline & & & $\begin{array}{l}0 \\
00 / 2 / 29 / 2008\end{array}$ & 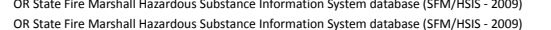 \\
\hline CAVE & & & $09 / 29 / 2008$ & Information System database (SFM/HISIS- 2009) \\
\hline & AND & & /29/2008 & entromation System databasase (SFM/HISIS-2009) \\
\hline RR & & JACKSON & 10/31/2018 & tal Quality - Hazardd Waste Program database (DEQQIHW) \\
\hline 8380 AGATE RD & $\begin{array}{l}\text { CENTRALPOINT } \\
\text { WHITE CITY }\end{array}$ & & 09/29/20008 2008 & 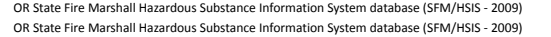 \\
\hline 1801 SAGE RD & MEFFORD & JaCKSON & 09/29/2008 & 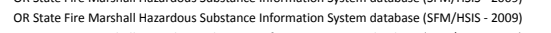 \\
\hline $\begin{array}{l}7638 \text { PAAFIC AVE } \\
1801 \text { SAGE RD }\end{array}$ & $\begin{array}{l}\text { WHITIC CITY } \\
\text { MEEFORD }\end{array}$ & $\begin{array}{l}\text { JACCSSON } \\
\text { JACKON }\end{array}$ & $\begin{array}{l}09 / 29 / 2008 \\
00 / 29 / 2008\end{array}$ & 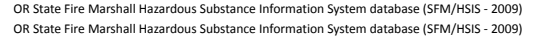 \\
\hline
\end{tabular}

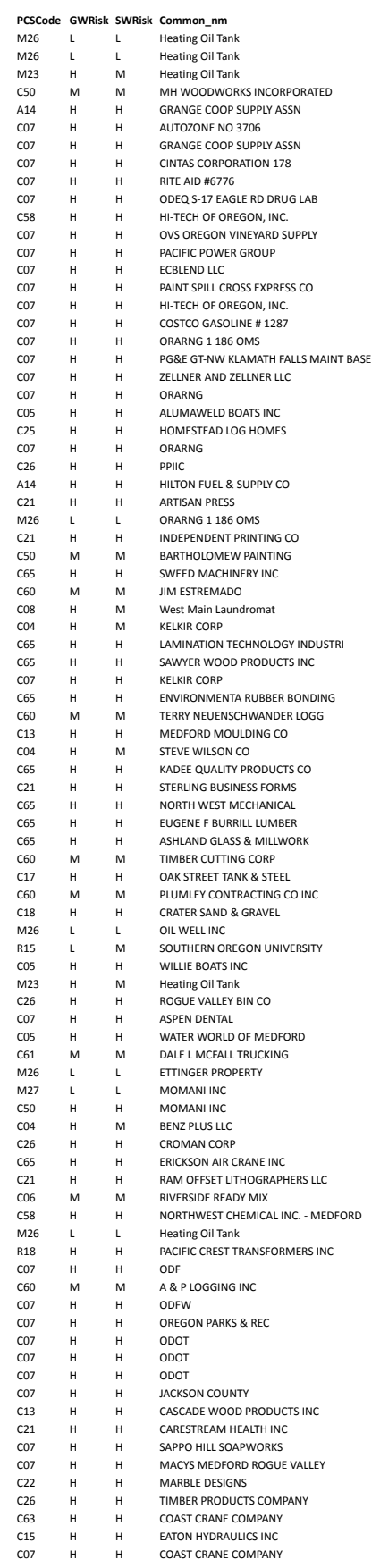

PCSType
UST-Confirm

UST - Confirmed Leaking but listed as NFA - DEQ LUST List

UST- Confirmed Leaking Tanks - DEQ Q USS L List

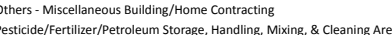
Chemical/Petrolum Processin//5trage
Chemical/Petroleum Processing/storage

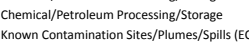

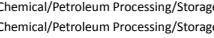

chemical/Petroleum Processing//storage

Chemical/Petroleum Processing/storage
Chemical/Petroleum Processing/storage

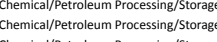

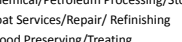

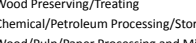

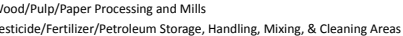

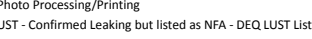

Photo Processing/P Printing

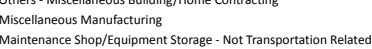

Dry Cleaners
Auto Reaair Shoops

Miscellaneous Manufatcturing
Miscellaneous Manufacturing

Chemical/Petroleum Processing/storage

Miscellaneous Manufacturing
Maintenance Shop/Equipment Storage - Not Transportation Related

to Manufacturing

Miscellaneous Manufacturing
Photo Processing (Printing

Miscellaneous Manufacturing

Miscelaneous Manulacturng
Miscellaneous Manufacturing
Miscellaneous Manufacturing

Maintenance Shop/Equipment Stontal

Metal Plating/Frinis

Mining Ativities- Gravel Mines/Grovel Pits

Soat Services/Repair/ Refininhing

Wood/Pulp/Paper Processing and Mills

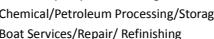

Maintenance Shop/Equipment Storoge- -Transportation Relate

UST - Confirmed Leaking but listed a s NFA - DEQ L UST List
UST - Uppraded andor Regitsered - Active Imay als have

Others- - nnknown
Auto Repair Shops

Auto- Repair Shops
Nood/Pulp/Paper rocessing and Mill
Wiscellaneous Manutacturing

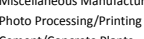

Known Contamination Stes/P/lumes/spillil (ECSS)
UST - Confirmed Leaking but Iisted as NFA - DEQ LUS List

Utility Stationss Powerphans -Mantenance/Transformer

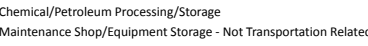

Chemical/Petroleum Processing/stsorage

Chemical/Petrolum Processing/stororage

chemical/Petroluum Processing/storage

chemical/Petroleum Processing/storage
chemical

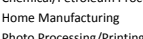

Choto Processing//rinting
Chemica//Petroleum Proessing//torage
Chemical/Petroleum Processing/Storage

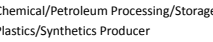

Wood/Pup/paperer Processing and Mills
Mining Activities - Active - other than grave

Macchine Shops 


\begin{tabular}{|c|c|c|c|c|}
\hline \multicolumn{5}{|r|}{ 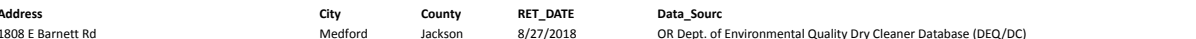 } \\
\hline \multicolumn{5}{|r|}{ 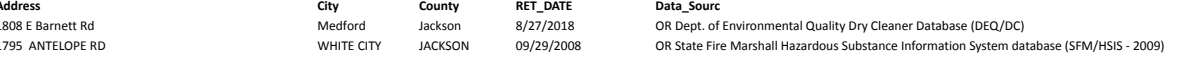 } \\
\hline 612 N MAIN & PHOENIX & JACKSON & 09/29/2/2008 & OR State fire Marshall Hazarddous Substance Information System database (SFM/HIS - 2009) \\
\hline $\begin{array}{l}1802 \text { ANETLOPP RD } \\
1711 \text { ROCKYDALE RD }\end{array}$ & $\begin{array}{l}\text { WHITE EIVY } \\
\text { CAVE UUCTION }\end{array}$ & $\begin{array}{l}\text { JACSSON } \\
\text { JOSEPHINE }\end{array}$ & $\begin{array}{l}09 / 2 / 2 / 208 \\
00 / 2 / 2008\end{array}$ & 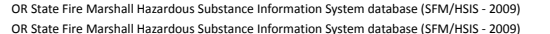 \\
\hline $\begin{array}{l}1762 \text { DELTA WATERES RD } \\
1860 .\end{array}$ & & & 10/31/2018 & 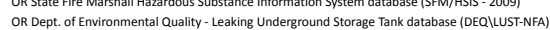 \\
\hline 025 GTH ST & 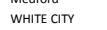 & JACKSON & 09/29/2008 & 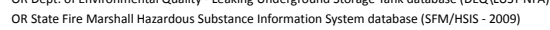 \\
\hline 278 HELMAN & ASHLAND & JACKSON & 09/29/2008 & OR State Fire Marshall Hazardous Substance Information System database (SFM/HISS - 2009) \\
\hline 1865 SIIY CIRCLE & Medford & JaCKSON & 10/31/2018 & OR Dept. of Environmental Quality - Leaking Underground Storage Tank databasese (DEQQIUST) \\
\hline 1890 S PACIFIC HWY & $\begin{array}{l}\text { Medford } \\
\text { MEFPORD }\end{array}$ & JaCKSON & 10/31/2018 & 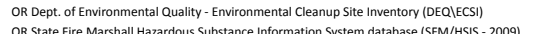 \\
\hline 98 AVENUEC C & $\begin{array}{l}\text { MEFROR } \\
\text { WHITECITY }\end{array}$ & JACKSON & $\begin{array}{l}09929 / 27208 \\
00 / 292008\end{array}$ & 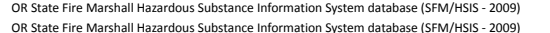 \\
\hline 890 S PACFICL & MEFFRD & JACKSON & 09/29/2008 & 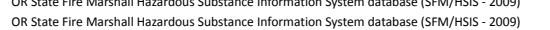 \\
\hline 20 CRATER LAK & CENTRALPOIN & JACKSON & 09/29/2008 & 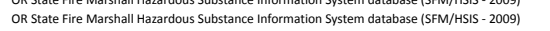 \\
\hline 390 S Pacifictiwy s & Mefford & Jackson & 8/27/2018 & 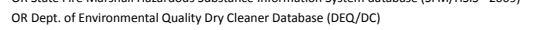 \\
\hline $\begin{array}{lll}565 \text { INDUSTRRAL CIR } \\
\text { 100 N RSSS SN }\end{array}$ & $\begin{array}{l}\text { WHITECTY } \\
\text { MEFFOD }\end{array}$ & JaCKSON & 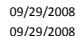 & 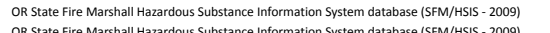 \\
\hline 3959 HAMRICK RD & CENTRAL POINT & JACKSON & . & 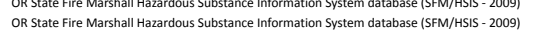 \\
\hline 419 NRONT ST & CENTRAL POINT & JACKSON & $09 / 29 / 2008$ & OR State Fire Marstall Hazardous Substance Information System database (SFM/HISIS - 2009) \\
\hline 3727 N PHOENIX RD & PHOENIX & JACKSON & 09/29/2008 & OR State Fire Marshall Hazardous Substance Information System database (SFM/HSIS- 2009) \\
\hline 61212N MAAN & $\begin{array}{l}\text { PHONNX } \\
\text { MEDORD }\end{array}$ & JACCSSON & 罂9/29/2/2008 & 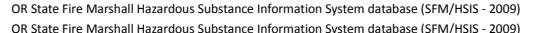 \\
\hline 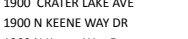 & $\begin{array}{l}\text { MEBORO } \\
\text { MEDFORD }\end{array}$ & $\begin{array}{l}\text { SACSSON } \\
\text { JACKSON }\end{array}$ & $\begin{array}{l}099292 / 2008 \\
09 / 29 / 2008\end{array}$ & 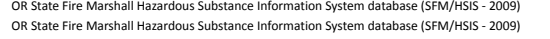 \\
\hline $\begin{array}{l}1900 \mathrm{~N} \mathrm{Keene} \mathrm{Way} \mathrm{Dr} \\
1900 \mathrm{~N} \text { PHONNIX RD }\end{array}$ & $\begin{array}{l}\text { Mefiord } \\
\text { MEFFRO }\end{array}$ & JaCKSON & $\begin{array}{l}06 / 102 / 212015 \\
099202008\end{array}$ & 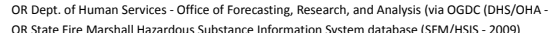 \\
\hline $\begin{array}{l}1900 \text { N PHOENIIX RD } \\
210 \text { GLMORE }\end{array}$ & $\begin{array}{l}\text { MMDOFRD } \\
\text { ROGUE RIVR }\end{array}$ & $\begin{array}{l}\text { JaCGSON } \\
\text { JACSSON }\end{array}$ & 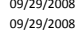 & 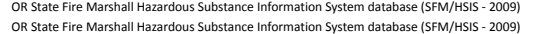 \\
\hline 1900 N PHOENI & MEDFORD & JACKSON & 09/29/2008 & 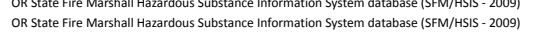 \\
\hline 900 TABLE ROCK RD & Medford & Jackson & 10/31/2018 & 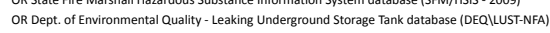 \\
\hline 1901 UNITED WY & MEOFORD & JACKSON & 09/29/2008 & OR State Fire Marshall Hazarddous Substance Information System database (SFM/HSIS - 2009) \\
\hline 1356 JUSTICE R R & CENTAA POINT & JACKSON & $09 / 29 / 2008$ & OR State Fire Marshall Hazardous Substance Information System database (SFM/HSIS- 2009) \\
\hline 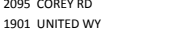 & & JACKSON & 09/29/2008 008 - & 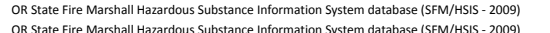 \\
\hline 1901 CRATER LAKE HWY & Medford & JACKSON & $10 / 31 / 2018$ & 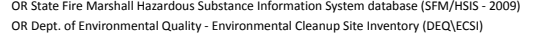 \\
\hline 1901 CRATER LAKE HWY & Medford & JACKSON & $10 / 11 / 2018$ & 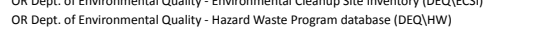 \\
\hline 100 BEALL LN & CENTRAL POINT & JACKSON & 09/29/2008 & 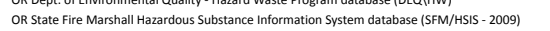 \\
\hline 1901 CRATER LAKE HWY & Medford & JACKSON & 10/31/2018 & OR Dept. of Environmental Quality - Leaking Underground Storage Tank databasese (DEQQUULST-NFA) \\
\hline $\begin{array}{l}19035 \text { SKYYARR DR } 105 \\
215 \text { WAER ST }\end{array}$ & $\begin{array}{l}\text { MEDOFRD } \\
\text { ASHLAND }\end{array}$ & JACKSSN & 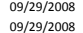 & 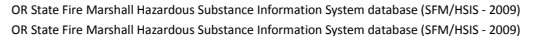 \\
\hline 217 BATEMAN DR & CENTRAL POINT & JACKSON & 09/29/2008 & 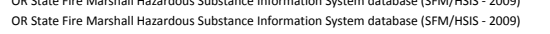 \\
\hline 1903 SKYPARK DR 105 & MEDFORD & Jackson & $09 / 29 / 2008$ & OR State Fire Marshall Hazardous S ubstance Information System database (SFM/HSIS- 2009) \\
\hline 1904 HAZZEL & $\begin{array}{l}\text { MEFOOOD } \\
\text { MEFPORD }\end{array}$ & JACKSON & 09/29/2008 & OR State Fire Marshall Hazardous Substance Information System database (SFM/HSIS- 2009) \\
\hline 1100 KRTTLAND RD & $\begin{array}{l}\text { MEFBOBD } \\
\text { CENTRALOINT }\end{array}$ & JACKSON & 09/29/2/2008 008 & 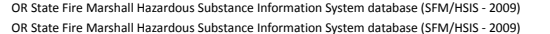 \\
\hline 1908 WESTERLUNS & Mefford & JACKSON & 10/31/2018 & 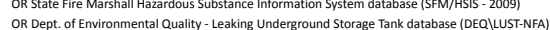 \\
\hline 1 BATEMAN DR & Metorard & JACCSON & 11/1/2018 & 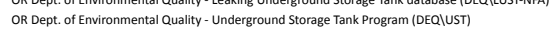 \\
\hline 1910 TABLE ROCKR R & MEDFORD & JaCKSON & 09/29/2008 & OR State fire Marshall Hazarddous Substance Information System database (SFM/HSIS- 2009) \\
\hline 1910 TABLE ROCK RC & MEOFORD & JaCKSON & $09 / 29 / 2008$ & OR State Fire Marshall Hazardous Substance Information System database (SFM/HSIS-2009) \\
\hline 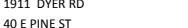 & $\begin{array}{l}\text { MEFOOD } \\
\text { CENTRAL POINT }\end{array}$ & JACKSON & 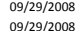 & OR State fire Marshall Hazardous Substance Information System database (SFM/HIIS- 2009) \\
\hline 1922 UNITEO WY & MEOFORD & JaCKSON & 09/29/2008 & 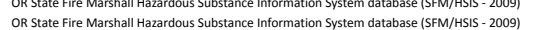 \\
\hline 923 SAGE RD & MEOFORD & JACKSON & 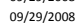 & 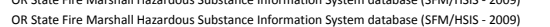 \\
\hline 923 SAGE RD & MEDFORD & Jackson & 09/29/2008 & 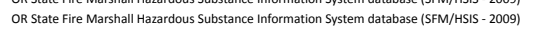 \\
\hline 1923 DELTA WAT & Metford & JACCSON & 10/31/2018 & OR Dept. of Environmental Quality - Leaking Underground Storage Tank databasese (DEQQUULST-NFA) \\
\hline 1923 SAGE RD & 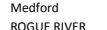 & JACKSSON & 10/31/2018 & OR Dept. of Environmental Quality - Hazard Waste Program database (DEQOHW) \\
\hline $\begin{array}{l}1000 \text { BROAAWWAY } \\
1923 \text { SAGE RD }\end{array}$ & $\begin{array}{l}\text { RoOUU RVVR } \\
\text { Medford }\end{array}$ & JaCSSON & 09/29/2008 & OR State Fire Marshall Hazarddous Substance Information System database (SFM/HSIS-2009) \\
\hline 625 AVENUEG G & $\begin{array}{l}\text { Wellor } \\
\text { WHITY IT }\end{array}$ & JACKSON & 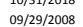 & 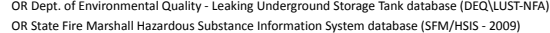 \\
\hline 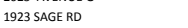 & Medford & JACCSSON & 11/1/2018 & 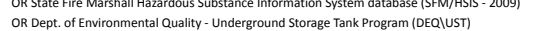 \\
\hline 191 BATEMAN & CENTRAL POI & JACKSON & 09/29/2008 & OR S State fire Marshall Hazardous Substance Information System database 1 SFM/HSIS -2 \\
\hline 1923 SAGE RD & Medford & JACKSON & 10/31/2018 & OR Dept. of Environmental Q Quality - Water Quality SIS database \\
\hline 1465 SISKKYOu ST & ASHLAND & JACKSSON & 09/29/2008 & OR State Fire Marshall Hazardous Substance Information System database (SFM/HSIS- 2009) \\
\hline $\begin{array}{l}25 \\
255 \text { EMMENUE } \\
\end{array}$ & $\begin{array}{l}\text { MEEFRO } \\
\text { WHIT CITY }\end{array}$ & 年CCSSONO & 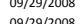 & OR State fire Marshall Hazardous Substance Information System database (SFM/HIIS-2009) \\
\hline 227 ELM AVE & MEEFORD & JACKSON & 09/29/2008 & 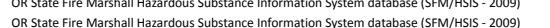 \\
\hline 27 EMAVE & Medford & JACKSON & 10/31/2018 & 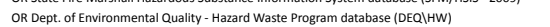 \\
\hline 1928 TABLE & MEOFORD & JaCKSON & 09/29/2008 & OR State Fire Marshall Hazardous Substance Information System database (SFM/HSIS -20 \\
\hline CKRD & MEOFFRO & JACCSON & 09/29/2008 & OR State Fire Marshall Hazardous S ubstance Information System database (SFM/HSIS - 2009) \\
\hline 1945 w main & $\begin{array}{l}\text { Metford } \\
\text { Menogera }\end{array}$ & Jackson & 8/27/2018 & OR Dept. of Environmental Quality Ory Cleaner Databases (DEQ/DC) \\
\hline 1945 WWAIN & $\begin{array}{l}\text { MEEFRO } \\
\text { BUTTE FALS }\end{array}$ & $\begin{array}{l}\text { JaCSSON } \\
\text { IACKSON }\end{array}$ & 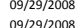 & 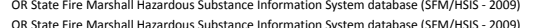 \\
\hline & 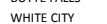 & 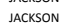 & 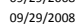 & 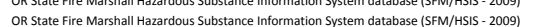 \\
\hline & MEFFORD & JACKSON & 09/29/2008 & 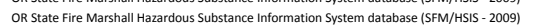 \\
\hline $417 \mathrm{~s}$ & ROGUE RUER & JACKSON & 09/29/2008 & OR State Fire Marshall Hazardous Substance Information System database (SFM/HSIS- 2009) \\
\hline & EAGLE POINT & JACKSON & 09/29/2008 & OR State fire Marshall Hazardous Substance Information System database (SFM/HSIS- 2009) \\
\hline & ASHALND & IACCSON & 09/29/2008 & OR State Fire Marshall Hazardous Substance Information System database (SFM/HSIS- 2009) \\
\hline LEVRD & $\begin{array}{l}\text { GOLD HILL } \\
\text { CENTRAL POIN }\end{array}$ & 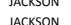 & 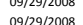 & 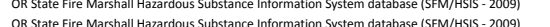 \\
\hline & $\begin{array}{l}\text { CENARL PIIN } \\
\text { WHITE CIY }\end{array}$ & $\begin{array}{l}\text { Jacksoo } \\
\text { Jack }\end{array}$ & 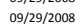 & 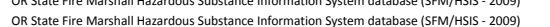 \\
\hline & WHITE CITY & JACCSON & 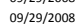 & 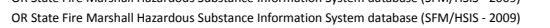 \\
\hline :RD & CENTRAL POINT & JaCKSON & 09/29/2008 & OR State fire Marshall Harardous Substance Intormation System databasese (SFM/HISS - 2009) \\
\hline & WHITE CITY & JACKSON & 09/29/2008 & OR State Fire Marstsall Hazardous Substance Information System database (SFM/HSIS - 2009) \\
\hline TEWARt AVE & HITE CITY & JACKSON & 09/29/2008 & 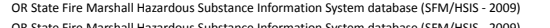 \\
\hline 1948 N PACFICIC HWY & Mefford & 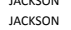 & 10/31/2018 & 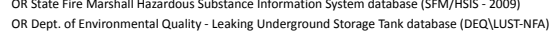 \\
\hline 1111 MILL CREEK RD & PROSPECT & 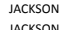 & $09 / 29 / 2008$ & OR State Fire Marshall Hazardous Substance Information System database (SFM/HSIS- 2009) \\
\hline & & & 10/31/2018 & 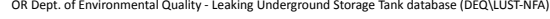 \\
\hline
\end{tabular}

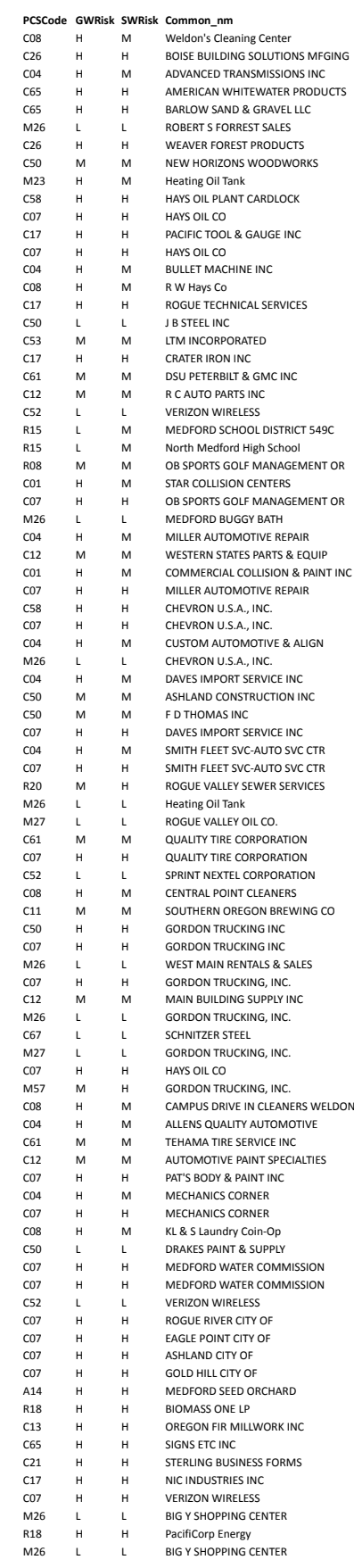

PCSType

Wood/Pulp/Paper P $P$
Auto - Repair Shops
Miscellaneous Man

Auto- Repair Shops
Miscellaneous Manufacturing
Micselnanesum

UST - Confirmed Leaking but isted a S NFA- DEQ U UST List

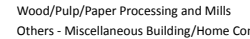

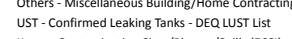

Known Contamination Sites/Plumes/Spills (ESSI)

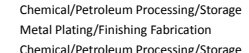

Chemical/Petroleum
Auto - Repair Shops
Dry Cleaners

Metal Plating//Finsting F Frbication
Other - General Merchandise/Retail store

Construction Company
Metal Plating//Finishing Fabrication
Mation

Metal Plating/ finshing fabrication
Maintenance Shop/Equipment Storage - Transportation Related

Furiture/lumber/fParts Stores

Communicic
Schools
Schools

Golf Courses
Automobiles - Body Shops
Chemical/Petroleum Proses

Chemical/Petroleum Processing/Storage
UST - Confirmed Leaking but listed as NFA - DEQ LUST List

UST - Confirmed Leaking but
Auto- Repari Shops
Furniture/rente

Auto-Repair Shops
furutiture/umberparts Stores
Automobiles - Body Shops

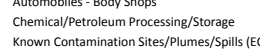

Rnown Contamination Sites/Plumes/spilils
Chemical/Pertroleum Processing/Storage

Chemical/Petroleum
Auto Repari Shops
UST- Confirmed Lea

Whe but listed as NFA- DEQ Q UST Lis

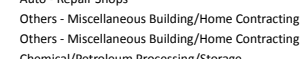

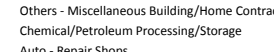

Auto- Repair hoos
Chemical/Pertoleum Processing/5tororge

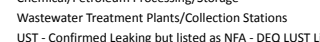

UST - Upgraded and/or Registered-Active (may also have decomm

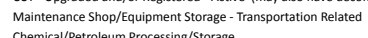

\section{Chemicalpetroleum Prices
Communictions Offic
Dry cleaners}

Communications
Ory cleaners
food rocessing

Others- - Trassporation Support Activties
chenical/Petroleum Processing/Storage

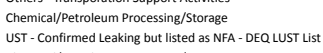
Chemical/Petroleum Processing/5tororge

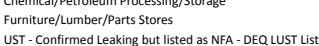

Warehours
UST - Upraded and/or Registered - Active (may also ha

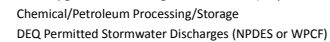

Dery Cleaners
Auto-Repair Shops
Wut

Maintenance Shop/Equipment Storage - Transsortation Relatat

Furniture//umber//Parts Stores
Chemical/Petroleum Processing/storage

Chemicalpetroleum
Auto - Repari Shops
Chemical Petroleum

Dry Cleaners

Ory Cleaners
Other - General Merchandise/Retail store
Chemical/Petroleum Processing/storage
Chemical/Petroleum Processing/storage

Communications office

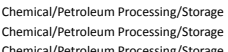

Chemical/Petroleum Procosesing//Storage

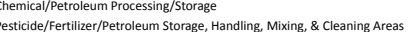

Utility Stations/Powerplants - Maintenance//ransformer Storage

Miscellaneous Manufacturing
Photo Processing $/$ Printing

Metal Platingef fFinshining Fabrication

Chemical/Petroleum Processing/Stororge
UST- Confirmed Leaking but listed as NFA - DEQ UST List

Utility Stations/Powerplants - Maintenance/TrTansformer Storage
UST - Confirmed Leaking but listed as NAA - DEQ LUST List 


\begin{tabular}{|c|c|c|c|c|}
\hline $\begin{array}{l}\text { Address } \\
\text { 1948 N PACFIC HWY }\end{array}$ & 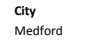 & $\begin{array}{l}\text { County } \\
\text { JAckson }\end{array}$ & $\begin{array}{l}\text { RET-DARE } \\
\text { 10/31/2018 }\end{array}$ & 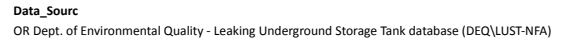 \\
\hline 340 S PIONEER ST & ASHLAND & JACKSON & 09/29/2008 & 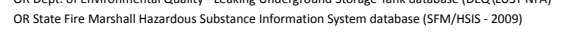 \\
\hline 14745 HWY 234 & GOLO HILL & JACKSON & 09/29/2008 & OR State fire Marshall Hazardous Substance Information System database (SFM/HIIS - 2009) \\
\hline 1968 CRAAER LAAEE HWY & $\begin{array}{l}\text { MEFEFOD } \\
\text { Mefford }\end{array}$ & $\begin{array}{l}\text { IACCSON } \\
\text { ACCKSON }\end{array}$ & $\begin{array}{l}090 / 299 / 2008 \\
10 / 31 / 2018\end{array}$ & 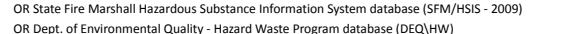 \\
\hline 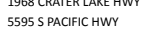 & $\begin{array}{l}\text { Metord } \\
\text { PHOENIX }\end{array}$ & $\begin{array}{l}\text { TaCCSON } \\
\text { IACKSON }\end{array}$ & $\begin{array}{l}109 / 3 / 2018 \\
09 / 29 / 2008\end{array}$ & 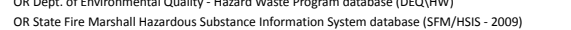 \\
\hline 7890 AGATE & WHITEE CITY & JACKSON & $09 / 29 / 2008$ & 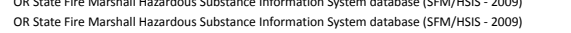 \\
\hline 4000 HAMRICK RD & ETRAL POINT & JACKSON & 09/29/2008 & 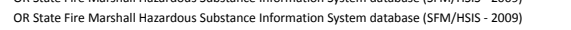 \\
\hline 1968 CRATER LAKE HWY & edford & JACKSON & 10/31/2018 & OR Dept. of Environmental Quality - Leaking Underground Storage Tank database (DEQQLUST-NFA) \\
\hline $\begin{array}{l}1981 \text { 1RAGINAAT } \\
\text { 5630 TABLE ROCK RD }\end{array}$ & ENFFRD & $\begin{array}{l}\text { JACCSON } \\
\text { JACKOSON }\end{array}$ & $090 / 29 / 2008$ & OR State Fire Marshall Hazardous Substance Information System databasese (SFM/HISIS-2009) \\
\hline $\begin{array}{l}\text { I961 VRRG RONA ST } \\
1980\end{array}$ & $\begin{array}{l}\text { CENTARPOONT } \\
\text { MEDFORD }\end{array}$ & $\begin{array}{l}\text { JAAKSON } \\
\text { JACKSON }\end{array}$ & $\begin{array}{l}09 / 29 / 2008 \\
09 / 29 / 2008\end{array}$ & \\
\hline 1155 ANTELOPE RD & $\begin{array}{l}\text { MEEFRD } \\
\text { WHITE CITY }\end{array}$ & $\begin{array}{l}\text { A ACCSSON } \\
\text { IACKSON }\end{array}$ & $\begin{array}{l}09 / 29922008 \\
09 / 29 / 2008\end{array}$ & 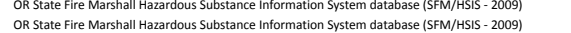 \\
\hline 1981 CAMELLA AVE & Metford & JACKSON & 10/31/2018 & 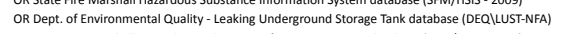 \\
\hline 7600 CRATER LAKE HWY & & & 09/29/2008 & \\
\hline 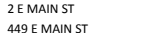 & $\begin{array}{l}\text { Medford } \\
\text { AstlaND }\end{array}$ & Jackson & 10/31/2018 & OR Dept. of Environmental Quality - Leaking Underground Storage Tank database (DEQQ|lusT-NFA) \\
\hline 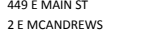 & $\begin{array}{l}\text { HEALND } \\
\text { NEFORD }\end{array}$ & $\begin{array}{l}\text { IACCSSON } \\
\text { ACCSON }\end{array}$ & og//29/2008 & \\
\hline $\begin{array}{l}\text { 2EMACNOREWS } \\
2 \text { EMAANREWS }\end{array}$ & $\begin{array}{l}\text { MEFORD } \\
\text { MEDFRD }\end{array}$ & $\begin{array}{l}\text { AaCCSSON } \\
\text { IACCSON }\end{array}$ & $\begin{array}{llll} & \end{array}$ & 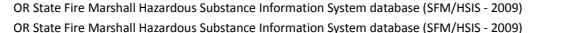 \\
\hline 1ST\&CST & $\begin{array}{l}\text { MEEFRO } \\
\text { PHOENIX }\end{array}$ & Jackson & 09/29/2008 & 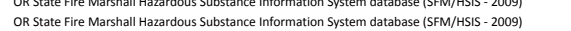 \\
\hline 20 S STAGE & MEFFRD & Jackson & 09/29/2008 & 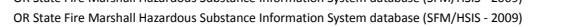 \\
\hline 930 ANTELC & WHITE CITY & JaCKSON & /29/2008 & 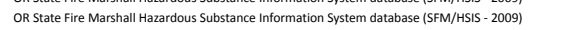 \\
\hline 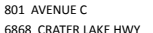 & $\begin{array}{l}\text { WHITECTIY } \\
\text { WHTIT GITV }\end{array}$ & Jackson & 09/29/2008 & OR State fire Marshall Hazard dous Substance Information System database (SFM/HISIS - 2009) \\
\hline 8868 CRATE & 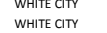 & JACCSSON & 年/29/2008 & 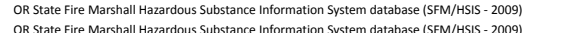 \\
\hline $\begin{array}{l}1820 \text { WATNELC } \\
2050 \text { ANTELOP }\end{array}$ & 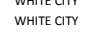 & 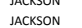 & 9/29/29008 & 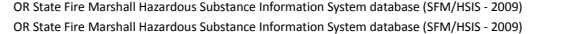 \\
\hline 5200 CRATER LAKE & CENTRALPOI & Jackson & 09/29/2008 & 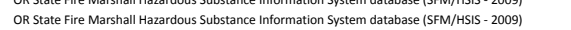 \\
\hline 2325 MERRY LN & wHITE CITY & Jackson & $09 / 29 / 2008$ & 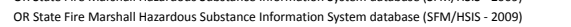 \\
\hline 7640 CRATER LAKE & WHITE CITY & JACKSON & 09/2/290008 & 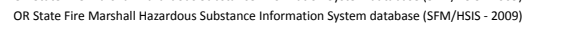 \\
\hline 8425 AGATE RD & 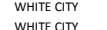 & JACKSON & 09/29/2008 & 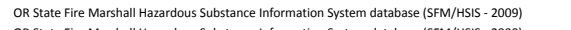 \\
\hline 1550 AVENUEG G & HHEAND & JACCSSON & /21/202008 & 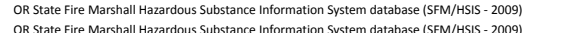 \\
\hline 6260 DRY CREEKRD & EALLE POINT & JACKSON & 9/29/2008 & \\
\hline 20 S STAGE RD & EDFORD & SACCSSON & $\begin{array}{l}9 / 2 / 2 / 2008 \\
\end{array}$ & 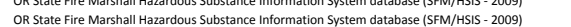 \\
\hline 2308 ASHLAND ST & ASHLAND & Jackson & 年/29/2008 & 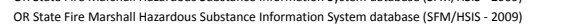 \\
\hline 2510 G AVE & WHITE CITY & Jackson & 09/29/2008 & OR State Fire Marstall Hazardous Substance Information System databasese (SFM/HSIS- 2009 ) \\
\hline 20 W JACKSON & Medford & JACKSON & 10/31/2018 & OR Dept. of Environmental Quality - Leaking Underground Storgag Tank database (DEQQ LuST-NFA) \\
\hline 120 LOWE RD & $\begin{array}{l}\text { ASHLAND } \\
\text { WHITECTIV }\end{array}$ & JACKSON & 09/29/2008 090 & 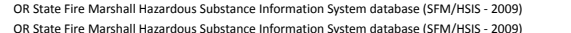 \\
\hline $\begin{array}{l}\text { 2094 ANTELLPR RD } \\
\text { NIST AVE \& TH ST }\end{array}$ & $\begin{array}{l}\text { WHITE CIITY } \\
\text { GoOD HIIL }\end{array}$ & JACKSON & o9/29/2/2008 0 & 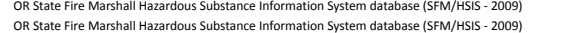 \\
\hline $\begin{array}{l}\text { 50 GTH ST } \\
\text { STITST }\end{array}$ & ASHLAND & $\begin{array}{l}\text { JACSKON } \\
\text { JACKSON }\end{array}$ & $\begin{array}{l}09 / 2992008 \\
09 / 29 / 2008\end{array}$ & 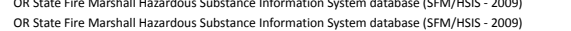 \\
\hline 336 MANZANITA ST & CENTRAL POINT & JACKSON & 09/29/2008 & 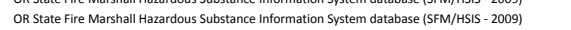 \\
\hline 200 CANON STREET & Medford & JACKSON & 10/31/2018 & 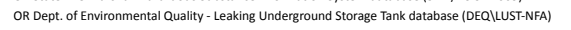 \\
\hline 200 HILHOUSE AVE & $\begin{array}{l}\text { Medford } \\
\text { CETTBP }\end{array}$ & $\begin{array}{l}\text { JaCKSON } \\
\text { AACSSON }\end{array}$ & $10 / 31 / 2018$ & 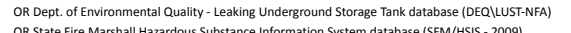 \\
\hline $\begin{array}{l}1150 \text { EINE ST } \\
\text { 200 R RIVERSIDE AVE }\end{array}$ & $\begin{array}{l}\text { CENTRALLOINT } \\
\text { Mefford }\end{array}$ & & $\begin{array}{l}090 / 29 / 2008 \\
10 / 31 / 2018\end{array}$ & \\
\hline 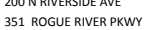 & $\begin{array}{l}\text { Mentord } \\
\text { TALNT }\end{array}$ & $\begin{array}{l}\text { JACKSON } \\
\text { JACSON }\end{array}$ & $\begin{array}{l}1093 / 172018 \\
09 / 29 / 2008\end{array}$ & 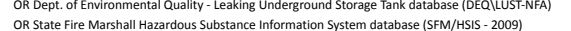 \\
\hline ALAVE & edford & JACKSON & 10/31/2018 & \\
\hline 200 FRONT ST & Medford & JACKSON & 10/31/2018 & 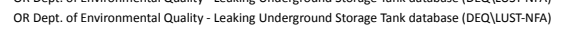 \\
\hline 200 WEST BA: & MEDFORD & JaCKSON & 06/02/2015 & OR Dept. of Human Services - Office of foreceasting, Research, and Analysis viva OGDC (DHS/OHA - 20 \\
\hline 135 MSSTLET & ASHLAND & JaCKSON & 09/29/2008 & OR State fire Marshall Hazardous Substance Information System database (SFM/HISIS - 2009) \\
\hline 2575 PACAFEC & $\begin{array}{l}\text { MEEFRO } \\
\text { WHITECIT }\end{array}$ & JACKSSON & $\begin{array}{l}099 / 2992008 \\
09 / 29 / 2008\end{array}$ & 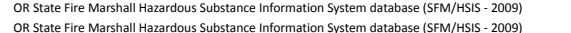 \\
\hline 2030 ANTELO & 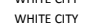 & JACKSON & 09/29/2008 & 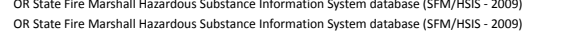 \\
\hline IEW RD & ASHLAND & JACKSON & 9/29/2008 & 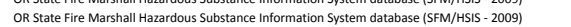 \\
\hline 2000 CRAT & edford & JACKSON & 1/1/2018 & 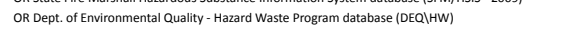 \\
\hline 2000 W STE & EEFOORD & JaCKSON & 09/29/2008 & OR Satet fire Marshall Hazard dous Substance Information System database (SFM/HISS - 2009) \\
\hline $2000 \mathrm{WST}$ & MEEFORD & JACKSON & 09/2/29008 & 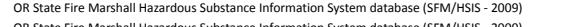 \\
\hline 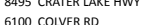 & $\begin{array}{l}\text { WHIIICETY } \\
\text { TALNT }\end{array}$ & 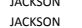 & 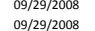 & 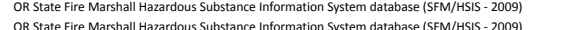 \\
\hline 2001 BIDDLE RD & 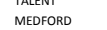 & 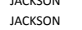 & 9/29/2/2008 & 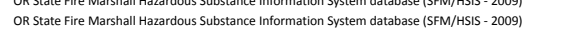 \\
\hline 2001 BIDOLE RD & MEFFORD & JACKSON & 9/29/2008 & 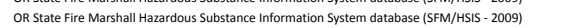 \\
\hline 2045 N HWr 99 & ILAND & JACKSON & 09/29/2008 & 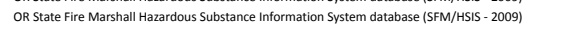 \\
\hline 2001 BIDD & 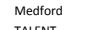 & JACKSON & 10/31/2018 & OR Dept. of Environmental Quality - Leaking Underground Storgag Tank database (DEQQ|lusT-NFA) \\
\hline & IITET CITY & IACCSSON & 09/29/2008 & 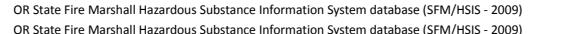 \\
\hline & edford & JACKSON & $10 / 31 / 2018$ & 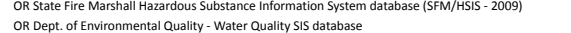 \\
\hline & ledford & JACKSON & $10 / 31 / 2018$ & 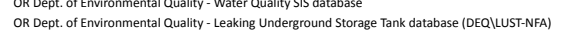 \\
\hline 2015 con & IEFFORD & IACKSON & 09/29/2008 0 & 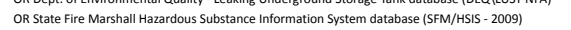 \\
\hline 202 CANON STREET & deford & IACKSON & 0/31/2018 & OR Dept. of Environmental Quality - Leaking Underground Storage Tank database (DEQQ|LST-NFA) \\
\hline 2026 LARS Wr & EOFORD & JACKSON & 09/29/2008 & OR State Fire Marshall Hazarddous Substance Information System database (SFM/HISS- 2009) \\
\hline & TRAD PO & & 的/2/2/2008 & 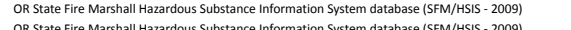 \\
\hline & TRALPOI & JACKSON & 99/2/2/2008 & 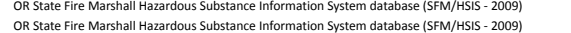 \\
\hline & & JACKSON & 99/2/2/2008 & 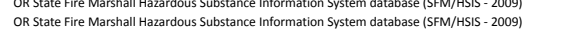 \\
\hline & ORD & HUTES & 09/2/29008 & or State fire Marsh \\
\hline 2059 IAAS WT & ORD & JACKSON & 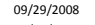 & OR State Fire Marshall Hazardous Substance Information System da \\
\hline 2061 LAASS WV & MEEFORD & JACKSON & 9/29/2008 & (SFM/HIS - 2009) \\
\hline 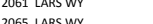 & & & 0008 & (SFM/HIST- 2009) \\
\hline & RALP PS & IACKSON & 9/29/29008 & 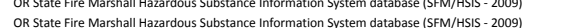 \\
\hline & RAL POINT & IACKSON & 09/2//2008 & e (SFM/HSIS- 2009) \\
\hline 431 & UTRAL POINT & & 9/29/2008 & (SFM/HISIS- 2009) \\
\hline 2067 COMMERCE DR & $\begin{array}{l}\text { Mefford } \\
\text { CETTBP }\end{array}$ & JACKSON & 10/31/2018 & Q Quality - Hazard Waste Program database (D) \\
\hline $\begin{array}{l}44455 \text { SALBR ROCKC RD } \\
5179 \text { CRATER LAE HWY }\end{array}$ & $\begin{array}{l}\text { CENTRALPONT } \\
\text { CENTRAL POINT }\end{array}$ & $\begin{array}{l}\text { JAAKKON } \\
\text { JACKSON }\end{array}$ & $\begin{array}{l}09 / 299 / 2008 \\
099 / 29 / 2008\end{array}$ & 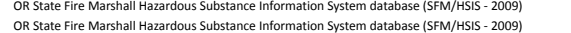 \\
\hline
\end{tabular}

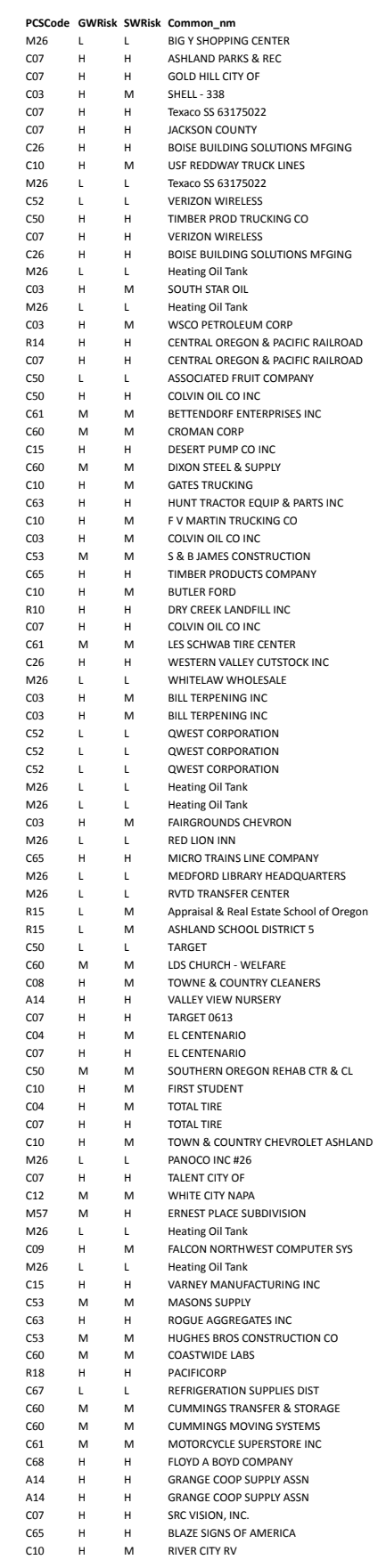

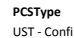

UST - Confirmed Leaking but listed as NFA - DR U

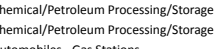
Chemical/Petroleum Processing//storage

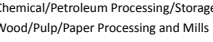
but listed as NFA - DEQ LUST List Communications office

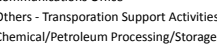
Wood/Pulp/Paper Processing and Milis UST - Confirmed Leaking but listed as NFA- DEQ LUST L UST - Confirmed Leaking but Iisted as NFA- DEQ Q LST List Automobiles - Gas Stations
Railrod Vards/Maintenance//Fueling Areas

Onther- Groceries/Marcests
Others - Unknown Commercial Others -Unknown Commercial
Maintenance Shop/EGuipment Storage - Transportation Related

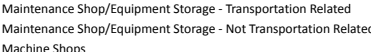
Maintenance Shop/Equipment Storage - Not Transsortation Related

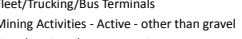
Fleet/Trucking/Bus Terminals
Automobiles - Gas Stations Construction Company Miscellaneous Manufacturing
Fleet//rucking/Bus Terminals

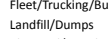

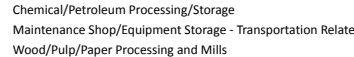
Wood/Pul//Paper Processing and Mills
UST - Confirmed Leaking but listed as NFA - DEQ L UST Lis

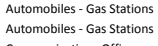
Automobiles- Gas statios
Communications ofice
Communications fofice Communications office

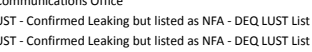

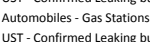
Dingut lised as N NFA - DEQ Lust Lis

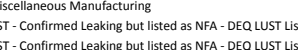
Schools
Other- General Merchandise/Retail Store
Maintenance Shop/Fquipment Storase -

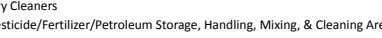
roleum Processing/storage

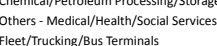
Auto- Repair Shops

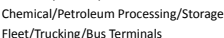

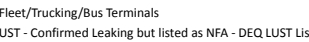

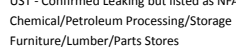
Furniture/Lumber//Part Stores
DEQ Perninted Stormwater Discharges (NPDES or WPCF)

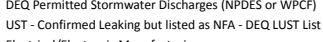

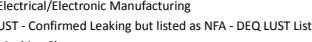

Mininuz
Metivitities - Active - other than gravel
Construction Comanyy Maintenance Shop/Equipment Storage - Not Transportation Related Utility Stations/Powerplants - Maintenance/Transformer Storagee Maintenance Shop/Equipment Storage - Not Transportation Related
Maititenance Shop/Equipment Storage - Not Transportation Related Maintenance Shop/Equipment Storage - Trass ration Related Pesticide/Fertilizer/Petroleum Storage, Handling, Mixing, \& Cleaning Areas
Pesticide//ertilizer/Petrolum Stsorge, Handling, Mixing, \& cleaning Areas Miscelalaneous Manufoccustring
Fleet/Trucking/Bus Terminals 


\begin{tabular}{|c|c|c|c|}
\hline $\begin{array}{l}\text { Address } \\
2078 \text { LARS Wr }\end{array}$ & $\begin{array}{l}\text { City } \\
\text { MEOFORD }\end{array}$ & $\begin{array}{l}\text { County } \\
\text { JACKSON }\end{array}$ & $\begin{array}{l}\text { RET_DATE } \\
\text { OEg/2/2/2008 }\end{array}$ \\
\hline 3000 VALLEY VIEW RD & ASHLAND & JACKSON & $\begin{array}{l}09912 / 2008 \\
09 / 29 / 2008\end{array}$ \\
\hline 2079 W MAIN ST & $\begin{array}{l}\text { MEDOROD } \\
\text { CETTPB PONT }\end{array}$ & JACKSON & 09/29/2008 \\
\hline $\begin{array}{l}3479 \text { ODD MLLITAYY RD } \\
2079 \text { W MAII ST }\end{array}$ & $\begin{array}{l}\text { CEETRAPLOINT } \\
\text { MEDFORD }\end{array}$ & $\begin{array}{l}\text { JAACCSON } \\
\text { JACKSON }\end{array}$ & $\begin{array}{l}099 / 2 / 20088 \\
09 / 2 / 2008\end{array}$ \\
\hline 208 BRADFORD WAY & Medford & JACKSON & 10/31/2018 \\
\hline 2080 COMMERCE DR & MEDFORD & JACKSON & 09/2/2/2008 \\
\hline $\begin{array}{l}20200 \text { S Sacafific CWWy. } \\
5740 \text { CRATER LAKE AVE }\end{array}$ & 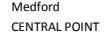 & $\begin{array}{l}\text { JACCSSON } \\
\text { JACKSON }\end{array}$ & $\begin{array}{l}100 / 3 / 1 / 2018 \\
0992 / 2008\end{array}$ \\
\hline 6779 CRATER LAKE HWY & $\begin{array}{l}\text { CERTRALLOLINT } \\
\text { WHITE CITY }\end{array}$ & & $\begin{array}{l}099 / 2 / 2008 \\
09 / 29 / 2008\end{array}$ \\
\hline 2086 LARS Wr & MEDFORD & $\begin{array}{l}\text { SaCKSON } \\
\text { Jas }\end{array}$ & og/29/2008 \\
\hline 2087 LARS Wr & MEDFORD & & \\
\hline 32 SRRONT ST & CENTRAL POINT & JACKSON & 09/29/2008 \\
\hline & $\begin{array}{l}\text { ASHLAND } \\
\text { MEDEOODO }\end{array}$ & JACKSON & $\begin{array}{l}09 / 29 / 2008 \\
0992 / 2008\end{array}$ \\
\hline $\begin{array}{l}2087 \text { 2095 COME RD } \\
209 \text { COMERE DR }\end{array}$ & $\begin{array}{l}\text { MEDEORD } \\
\text { MERFORD }\end{array}$ & $\begin{array}{l}\text { JaCKSON } \\
\text { JACKSON }\end{array}$ & $\begin{array}{l}\text { o9 } 09 / 2 / 2 / 2008 \\
09 / 2 / 2008\end{array}$ \\
\hline $\begin{array}{l}2110 \text { WHITTLE } \\
2 \text { OK }\end{array}$ & & $\begin{array}{l}\text { JaCSSON } \\
\text { IACCSON }\end{array}$ & 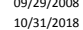 \\
\hline 2111 ROBERTS RD & $\begin{array}{l}\text { METrora } \\
\text { MEOFORD }\end{array}$ & $\begin{array}{l}\text { S } \\
\text { IACCSSON }\end{array}$ & $\begin{array}{l}1 \\
09 / 29 / 20008\end{array}$ \\
\hline 2111 ROBERTS RD & Medford & JACKSON & $10 / 31 / 2018$ \\
\hline 2111 Roberts Rd & Medford & Jackson & $8 / 27 / 2018$ \\
\hline $\begin{array}{l}21115 \text { OAKWWOOD ST } \\
21220 \text { OAKOOD }\end{array}$ & $\begin{array}{l}\text { Metford } \\
\text { Mefford }\end{array}$ & JACKSON & $10 / 31 / 2018$ \\
\hline 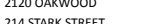 & $\begin{array}{l}\text { Metford } \\
\text { Mether }\end{array}$ & JACKSON & $100 / 31 / 2018$ \\
\hline 217 BATEMAN DR & $\begin{array}{l}\text { Metiored } \\
\text { Metford }\end{array}$ & $\begin{array}{l}\text { JaCSSON } \\
\text { IACCSON }\end{array}$ & $\begin{array}{l}10 / 3 / 1 / 2018 \\
10 / 13 / 2018\end{array}$ \\
\hline 2176 CRESTBROOK RD & & JACKSON & $10 / 11 / 2018$ \\
\hline 2177 S PACIFIC HWY & MEDFORD & JACKSON & 09/29/2008 \\
\hline 218 W GTH ST & Medford & JaCKSON & 10/31/2018 \\
\hline 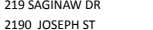 & $\begin{array}{l}\text { Mederord } \\
\text { MEFORD }\end{array}$ & Jackson & $\begin{array}{l}10 / 31 / 2018 \\
0\end{array}$ \\
\hline 20294 SAGER RD & $\begin{array}{l}\text { MMEDFROD } \\
\text { MEDFORD }\end{array}$ & $\begin{array}{l}\text { JACCSSON } \\
\text { JACKSON }\end{array}$ & $\begin{array}{l}099 / 2 / 2008 \\
09 / 29 / 2008\end{array}$ \\
\hline 250 N FRON ST & CENTRAL POINT & JACKSON & $\begin{array}{l}0 \\
09 / 29 / 20008 \\
0\end{array}$ \\
\hline 145 N MAIN ST & ASHLAND & JACKSON & $\begin{array}{l}09 / 29 / 2008 \\
0\end{array}$ \\
\hline 2445 SISKIYOU BIVD & ASHLAND & JACKSON & 09/29/2008 \\
\hline 6615 ROCKYDALE RD & CAVE JUNCTION & JOSEPHINE & 09/2/2/2008 \\
\hline $\begin{array}{l}6371 \text { 1 CRATER LAAE HWY } \\
\text { 201 N PHOENIX R D }\end{array}$ & CENTRAL POINT & $\begin{array}{l}\text { JACKSON } \\
\text { JACSSON }\end{array}$ & o9/2/2/2008 \\
\hline 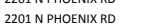 & $\begin{array}{l}\text { MEERORD } \\
\text { MEEFORD }\end{array}$ & $\begin{array}{l}\text { JACCSSON } \\
\text { JACKSON }\end{array}$ & $\begin{array}{l}\text { o9 } 09 / 2 / 2 / 2008 \\
09 / 2 / 2008\end{array}$ \\
\hline 2206 ARCTIC CIRCLE & Medford & JACKSON & 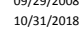 \\
\hline 2938 HANMEr Ro & CENTRAL POINT & JACKSON & 09/29/2008 \\
\hline 22214 TOWER EAST & MEDFORD & JACKSON & $06 / 102 / 2015$ \\
\hline 2216 W MAIIST & MEDFORD & Jackson & 09/2/2/2008 \\
\hline $\begin{array}{l}22121 \text { W MAANT } \\
2218 \text { CAPPAL LVE }\end{array}$ & $\begin{array}{l}\text { MEBOFoRD } \\
\text { Meftard }\end{array}$ & Jackson & $\begin{array}{l}099 / 29 / 2008 \\
103 / 212018\end{array}$ \\
\hline 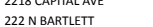 & $\begin{array}{l}\text { Mefoford } \\
\text { Mefford }\end{array}$ & $\begin{array}{l}\text { JaCSSOON } \\
\text { IACSSON }\end{array}$ & $\begin{array}{l}10 / 3 / 1 / 2018 \\
10 / 31 / 2018\end{array}$ \\
\hline 2221 JOSEPH ST & $\begin{array}{l}\text { Meatord } \\
\text { MEOFORD }\end{array}$ & JACKSON & $\begin{array}{l}1 \\
09 / 29 / 2008 \\
0\end{array}$ \\
\hline 2222 TABlE ROCKK RD & MEDFORD & JaCKSON & 09/29/2008 \\
\hline 22311 BDODLE RD & MEOFORD & JACKSON & 09/29/2008 \\
\hline 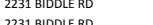 & Medford & JACKSON & $10 / 31 / 2018$ \\
\hline 作 & $\begin{array}{l}\text { Mefoford } \\
\text { MEFFORD }\end{array}$ & $\begin{array}{l}\text { JaCSSON } \\
\text { ICCCSON }\end{array}$ & 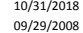 \\
\hline 7837 HALUE WY & 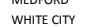 & 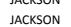 & $\begin{array}{l}099 / 2 / 2008 \\
09 / 29 / 2008\end{array}$ \\
\hline 705 HEMANST & ASHLAND & JACKSON & $\begin{array}{l}09 / 29 / 2008 \\
0\end{array}$ \\
\hline 2015 MOUNTAIIN AVE & ASHLAND & JaCKSON & 09/29/2008 \\
\hline 100 WAKKRR AVE & ASHLAND & JACKSON & 09/29/2008 \\
\hline 3299 MANA ST & BUTTE FALLS & JACKSON & 09/29/20008 \\
\hline The & $\begin{array}{l}\text { ASHAAN } \\
\text { PHOENIX }\end{array}$ & $\begin{array}{l}\text { JaCSSON } \\
\text { ICCCSON }\end{array}$ & $\begin{array}{l}09 / 29 / 2008 \\
09929 / 2008\end{array}$ \\
\hline 591 ЕІК СREEK RD & TrAll & 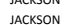 & $\begin{array}{l}0 \\
09 / 29 / 20008 \\
0\end{array}$ \\
\hline 37 SCHOOL HOUSE LN & SHADY COVE & JaCKSON & $\begin{array}{l}0 \\
09 / 29 / 20008 \\
0\end{array}$ \\
\hline 2232 BIDDLE RD & Medford & JaCKSON & $10 / 31 / 2018$ \\
\hline 1201 AVENUEC C & WHITE CITY & JACKSON & 09/29/2008 \\
\hline & $\begin{array}{l}\text { Mefford } \\
\text { Mereseng }\end{array}$ & JACKSON & 11/1/2018 \\
\hline 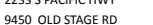 & $\begin{array}{l}\text { MEEGOFB } \\
\text { CENTRAL POINT }\end{array}$ & $\begin{array}{l}\text { JAACSSON } \\
\text { JACKSON }\end{array}$ & $\begin{array}{l}09 / 2 / 2 / 2008 \\
0 / 292 / 2008\end{array}$ \\
\hline 6061 CRATER LARE HWY & CENTRAL POINT & $\begin{array}{l}\text { Jackson } \\
\text { JACKSON }\end{array}$ & $\begin{array}{l}0.09 / 2 / 27008 \\
09 / 29 / 2008\end{array}$ \\
\hline 1004 GIBBON RD & CENTRAL POINT & JACKSON & $\begin{array}{l}09 / 29 / 2008 \\
0\end{array}$ \\
\hline 22335 PACAFIC HWV & MEOFORD & JACKSON & 09/29/2008 \\
\hline 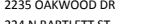 & Medford & JaCKSON & $10 / 31 / 2018$ \\
\hline 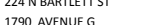 & 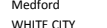 & IACCSSON & $\begin{array}{l}10131 / 212018 \\
10208\end{array}$ \\
\hline 2248 DELWOOOD AVE & Metford & 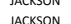 & 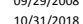 \\
\hline 225 E GTH \& BARTLETT & Mesford & $\begin{array}{l}\text { IACKSON } \\
\text { Ians }\end{array}$ & $\begin{array}{l}10131 / 21 / 2018 \\
10131 / 2018\end{array}$ \\
\hline 225 S RUVERSIDE & MEEFORD & Jackson & $\begin{array}{l}09 / 29 / 2008 \\
0\end{array}$ \\
\hline 333 CLASSICK DR & ROGUE RVER & JACKSON & 09/29/2008 \\
\hline 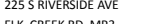 & Medford & JACKSON & 10/31/2018 \\
\hline 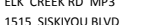 & ASAL & 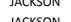 & $\begin{array}{l}09929 / 2 / 2008 \\
00298\end{array}$ \\
\hline 2295 ASHANDST & $\begin{array}{l}\text { ASHLAND } \\
\text { ASH }\end{array}$ & Jacsoos & $\begin{array}{l}0 \\
099 / 29 / 2 / 2008 \\
0.008\end{array}$ \\
\hline 345 LTHIA WY & ASHLAND & JACKSON & 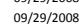 \\
\hline 460 S VALLEY VIEW RD & ASHLAND & JACKSON & 09/29/2008 \\
\hline ITHA Wr & ASHLLAND & JACKSON & 09/29/2008 \\
\hline 326 BROAD ST & BUTTE FALIS & JACKSON & 09/29/20008 \\
\hline 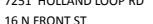 & CETPA PONT & 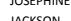 & $\begin{array}{l}09 / 29 / 2008 \\
0.929 / 2008\end{array}$ \\
\hline 5600 BUTTE FAL & EAGLE POINT & $\begin{array}{l}\text { IACKSON } \\
\text { IACSO }\end{array}$ & $\begin{array}{l}0.9792 / 2008 \\
09 / 29 / 2008\end{array}$ \\
\hline 945 N STH ST & JACKSONYILE & JACKSON & 09/29/2008 \\
\hline
\end{tabular}

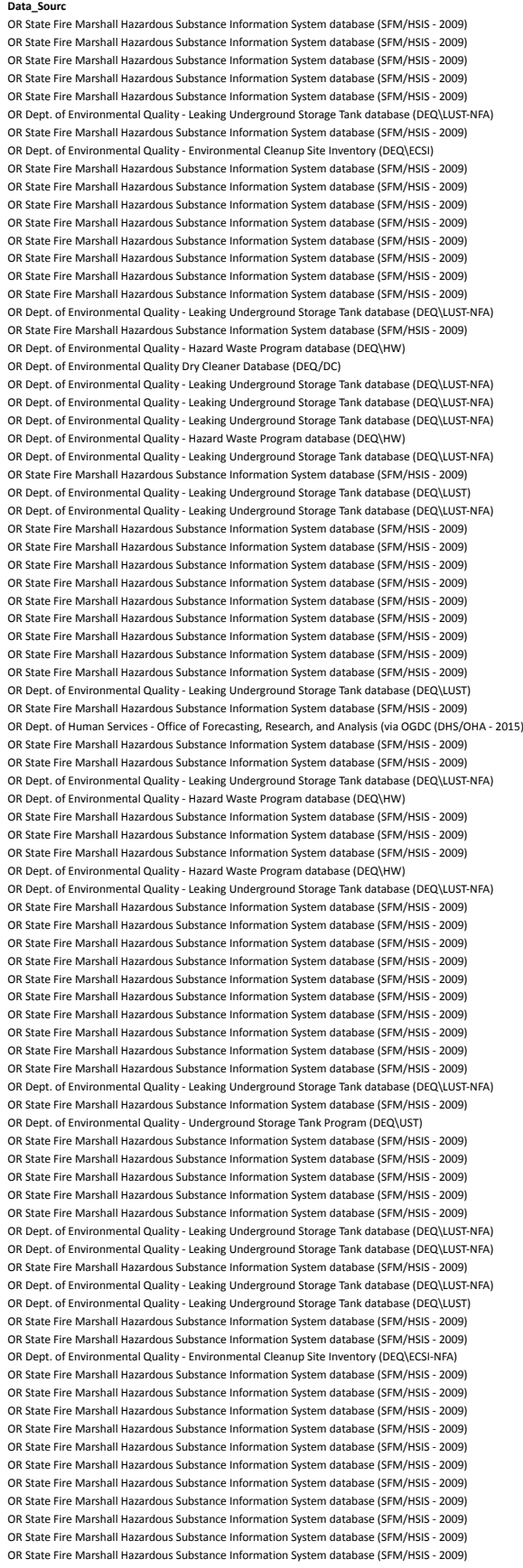

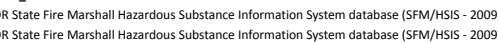

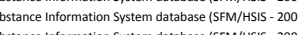

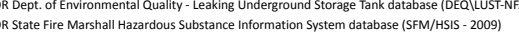

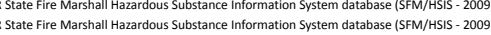

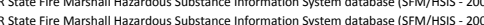
OR S State fire Marshall Hazardous Substance Information System database (SFM/HSIS - 2009)

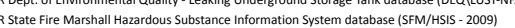
R Dept. of Environmental Quality Dry Cleaner Database (DEQ/DC

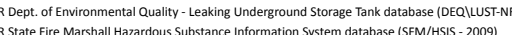
(a)

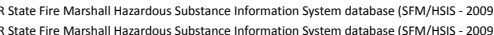

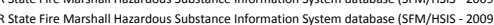

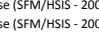
政 Leaking Underground Storage Tank database (DEQQ) LUS

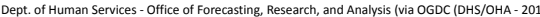
RR State fire Marshall Hazard ous Substance Information S System database (SFM/HSIS - 2009)

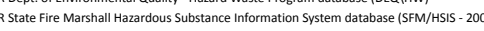
Information System database (SFM/HSIS- 2000

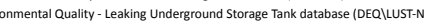
(1) OR State fire Marshall Hazardous Substance Intormation System database (SFM/HSIS - 2009 OR State fire Marshall Hazardous Substance Information System database (SFM/HSIS - 2009)

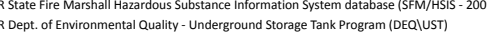
(SFMM/HIS - 2009)

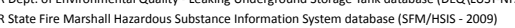

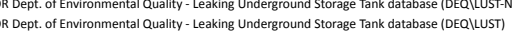
作

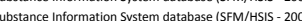

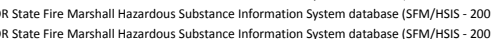

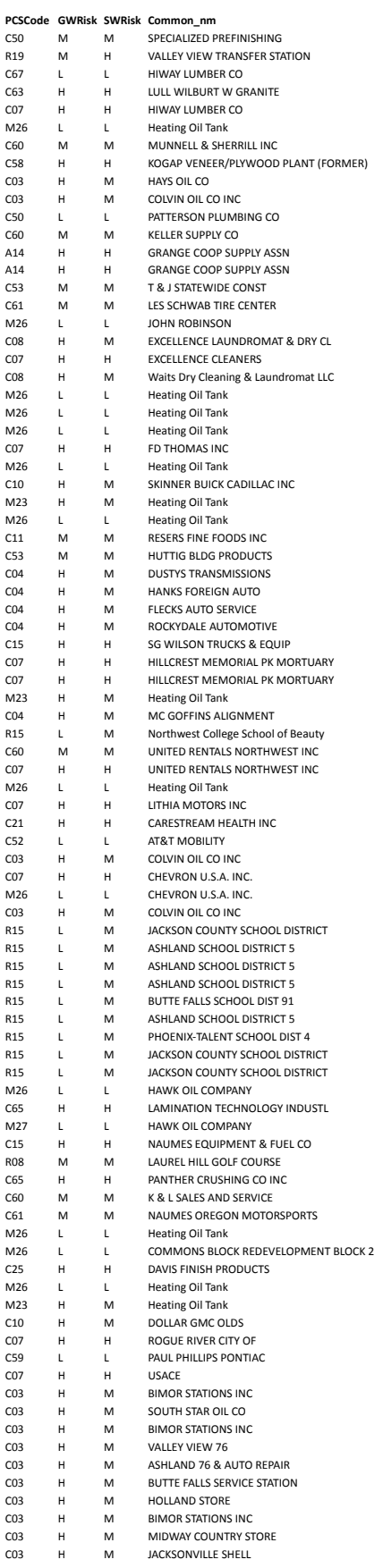

Pcstype

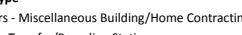

Warehouses
Mining Activities - Active - other than grave

UST - Confirmed Leaking but listed as NFA - DEQ LUST List

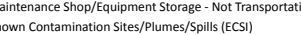
Automobiles- - Gas Stations
Automobiles- Gas stations

Maintenance Shop/Equipment Storage - Not Transportation Related Pesticide/fertilizer/Petroleum Storage, Handling, Mixing, \& Cleaning Areas
Pesticide/fFrtilizer/Petroloum Storage, Handling, Mixing, \& cleaning Areas construction compentis

Maintenance Shop/Equipment Storage - Transportation Rela
UST - Confirmed Leaking but Iisted as NFA - DEQ USST List

Dry Cleaners
chemicalpe

UST - Confirimed Leaking but isted as NFA- DEQ LUST Lis UST - Confirmed Leaking but listed as NFA- DEQ LUST Lis

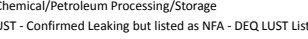
Fleet/Trucking//Uus Terminals

UST - Confirmed Leaking Tanks - DEQ UNT List
UST - Confirmed Leaking but listed as NAA - DEQ LUST Lit Food Processing
constructin Compent

Construction compary
Auto- Repair Shops

Auto- Repair shops
Auto - Repair shops
Auto- Repair Shops

Machine Shops
chemical/Petroleum Processing/storage UST - Confirmed Leaking Tanks - DEQ LuST Lis

Maintenance Shop/Equipment Storage -
Chemical/Petroleum Processing/Storage

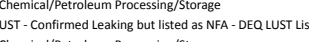
Chemical/Petroleum Processing/s/torage
photo Processing $/$ Printins Photo Processing/Prining
Communications office Chemical/Petroleum Processing/Storoge
UST- Confirmed Leaking uut isted as SNA - DEQ L LST List

Automol
Schools
Schools
Schools

Schools
Schools
Schools

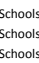

sctholl
ust-C.

UST - Uppradeded and/or Regisitered -Active (may also have decommissioned tanks on stite)

Golf Courses
Miscellaneous Manufacturing

UST - Confirmed Leaking but listed as NFA- DEQ Q UST L List UST - Confirmed Leang G SU ISted as NAA - DEQ LUST L

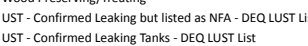
UST - Confirmed Leaking Tanks - DEQ LUST Lis
Fleet/Trucking//Uus Terminals

Fleet/Trucking//us Terminals
Chemical/Petroleum Processing/storage Known Contanination listed as NFA SSteses/Plumes/Spills from ECSI) Automobilies - Gas Sortcessing / Storage Automobiles - Gas Stations
Automobiles - cas tsations

Automobiles - Gas Station
Automobies - Gas stations

Automobiles - Gas stations

Automobiles - Gas Station
Automobiles - Gas stations 


\begin{tabular}{|c|c|c|c|c|}
\hline $\begin{array}{l}\text { Address } \\
225 \text { S SNERSID AVE }\end{array}$ & 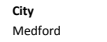 & $\begin{array}{l}\text { County } \\
\text { JACKSON }\end{array}$ & $\begin{array}{l}\text { RET-DARE } \\
\text { 10/31/2018 }\end{array}$ & 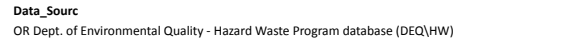 \\
\hline 225 SRIVERSIDE AVE & Mettord & IACKSON & 10/31/2018 & OR Dept. of Envirommental Q Quality - Leaking Underground Storage Tank database (DEQQ|LUST-NFA) \\
\hline 2250 CRATER LAKE AVE & Medford & Jackson & $100 / 31 / 2018$ & OR Dept. of Environmental Quality - Environmental Cleanup Site Inventory (OEQQIECSI) \\
\hline $\begin{array}{l}22206 \text { SAGER D } \\
2260 \text { SAGE RD }\end{array}$ & $\begin{array}{l}\text { MEDEFRD } \\
\text { MEDFORD }\end{array}$ & $\begin{array}{l}\text { IACKSON } \\
\text { ICCCSON }\end{array}$ & 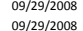 & 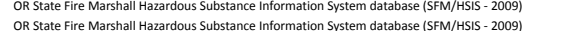 \\
\hline 2274 S STAGE RD & MEDFORD & $\begin{array}{l}\text { IACSSON } \\
\text { IACKSON }\end{array}$ & $\begin{array}{l}0.9929 / 2008 \\
09 / 29 / 2008\end{array}$ & 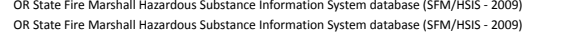 \\
\hline 2274 S STAC & MEDFORD & 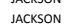 & 09/29/2008 & 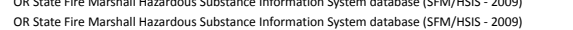 \\
\hline 800 N MAIN ST & PHOENIX & JACKSON & 09/29/2008 & 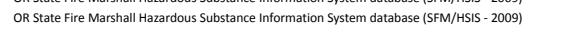 \\
\hline 540 MILL CREEK RD & PROSPECT & JACKSON & 09/29/2008 & OR State fire Marshall Hazarddous Substance Information System database (SFM/HSIS- 2009) \\
\hline $\begin{array}{l}21 \text { TALENT AVE } \\
990 \\
\text { INDSTSALC CIR }\end{array}$ & TALENT & & 09/29/2008 & OR State fire Marshall Hazard dous Substance Information System database (SFM/HSIS- 2009) \\
\hline $\begin{array}{l}9000 \text { INDSUSRTALL IR } \\
229 \text { NARTLET }\end{array}$ & 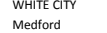 & $\begin{array}{l}\text { IACKSON } \\
\text { ICCCSON }\end{array}$ & $\begin{array}{l}09 / 29 / 2008 \\
101312018\end{array}$ & \\
\hline $\begin{array}{l}229 \text { NARRL TOE } \\
7020 \text { TOLO RD }\end{array}$ & $\begin{array}{l}\text { Medord } \\
\text { CENTRAL POINT }\end{array}$ & $\begin{array}{l}\text { JACCSSON } \\
\text { JACKSON }\end{array}$ & $\begin{array}{ll}103 / 1 / 2018 \\
09 / 29 / 2008\end{array}$ & 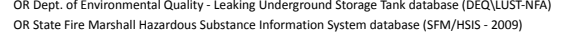 \\
\hline 440 NORMAL ST & ASHLAND & JACKSON & 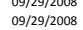 & 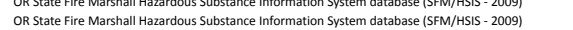 \\
\hline 1195 OAK ST & ASHLAND & JACKSON & 09/29/2008 & OR State Fire Marshall Hazard ous Substance Information System database (SFM/HSIS- 2009) \\
\hline 2294 SAGE RD & MEDFORD & IACKSON & 09/29/2008 & OR State fire Marshall Hazarddous Substance Information System database (SFM/HSIS - 2009) \\
\hline 2294 SAGE RD & $\begin{array}{l}\text { MEDFORD } \\
\text { Mefford }\end{array}$ & IACKSON & $09 / 29 / 2008$ & \\
\hline $\begin{array}{l}23 \text { WLILAMTTE } \\
2300 \text { CAATR LAKE AVE }\end{array}$ & $\begin{array}{l}\text { Mefford } \\
\text { Metford }\end{array}$ & $\begin{array}{l}\text { IACKSON } \\
\text { ICCCSON }\end{array}$ & $\begin{array}{lll}10 / 31 / 2018 \\
101312018\end{array}$ & 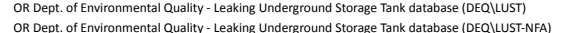 \\
\hline & $\begin{array}{l}\text { Mefford } \\
\text { Medford }\end{array}$ & $\begin{array}{l}\text { JaCKSON } \\
\text { ICCKSON }\end{array}$ & $\begin{array}{lll}100 / 3 / 2018 \\
10 / 13 / 2018\end{array}$ & \\
\hline $\begin{array}{l}23009 \text { SAGE RD LAEE AVE } \\
2305\end{array}$ & $\begin{array}{l}\text { Metarora } \\
\text { MEFFRD }\end{array}$ & $\begin{array}{l}\text { IACCSON } \\
\text { ICSON }\end{array}$ & 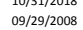 & 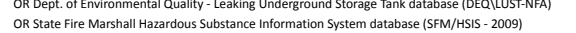 \\
\hline 2309 SAGE RD & Medford & JACKSON & 10/31/2018 & 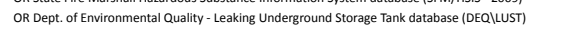 \\
\hline 2490 ASHLAND ST & ASHLAND & JACKSON & 09/29/2008 & OR State Fire Marshall Hazard dus S Substance Information System database (SFM/HSIS - 2009) \\
\hline 2309 SAGE RD & $\begin{array}{l}\text { Medford } \\
\text { Mefford }\end{array}$ & $\begin{array}{l}\text { IACKSON } \\
\text { ACCSSON }\end{array}$ & 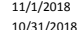 & OR Dept. of Environmental Quality - Underground Storage Tank Program (DEQQUST) \\
\hline $\begin{array}{l}232 \text { BRAAFRODD WAY } \\
1952 \text { ASHLAND ST }\end{array}$ & $\begin{array}{l}\text { Medford } \\
\text { ASHLAND }\end{array}$ & $\begin{array}{l}\text { JACKSON } \\
\text { JACKSON }\end{array}$ & $\begin{array}{l}109 / 1 / 212018 \\
0 / 292 / 2008\end{array}$ & 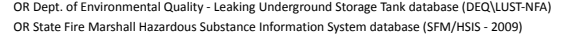 \\
\hline 280 MAPLE ST & ASHLAND & 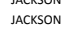 & 09/29/2008 & 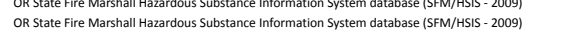 \\
\hline 1618 ASHLAND ST & ASHLAND & JACKSON & 09/29/2008 & 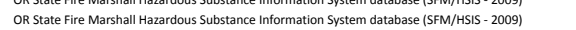 \\
\hline 270 WILSON RD & CENTRAL POINT & JACKSON & 09/29/2008 & 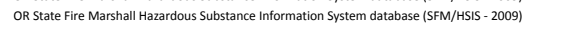 \\
\hline 5758 CRATER LAKE & CENTRAL POINT & JACKSON & 09/29/2008 & 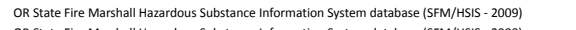 \\
\hline 4600 SARDINE CREEK RD & 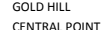 & JACKSON & 09/29/2008 0 & 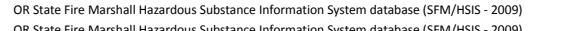 \\
\hline 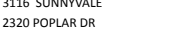 & $\begin{array}{l}\text { CEETRA LOINT } \\
\text { MEPFORD }\end{array}$ & $\begin{array}{l}\text { JACCSSON } \\
\text { JACKSON }\end{array}$ & $\begin{array}{llll}0 \\
09 / 29 / 2 / 2008 \\
0 . / 2015\end{array}$ & 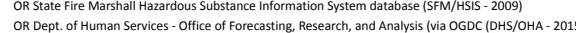 \\
\hline 2323 SISKIYYOU BLVD & MEFFRD & JACKSON & 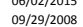 & 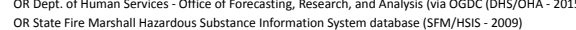 \\
\hline 2323 SISKIYYOU BLVD & MEDFORD & Jackson & 09/29/2008 & 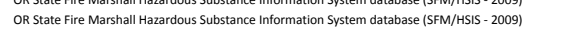 \\
\hline 2323 Siskiyou Blvd & Medford & JACKSON & 06/102/2015 & OR Dept. of Human Services - Office of Forecasting, Research, and Analysis (via OGOC (DHS/OHA \\
\hline 2429 BROPHY RD & EAGLE POINT & JACKSON & 09/29/2008 & OR State Fire Marshall Hazarddous Substance Information System database (SFM/HISS - 2009) \\
\hline 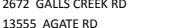 & $\begin{array}{l}\text { GODD HIL } \\
\text { EAGE PONT }\end{array}$ & JACSSON & & 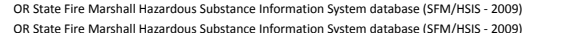 \\
\hline $\begin{array}{l}13555 \text { AGGARED } \\
2325 \text { STEWART AVE }\end{array}$ & $\begin{array}{l}\text { EAGE POINT } \\
\text { Medford }\end{array}$ & $\begin{array}{l}\text { JaCCSONO } \\
\text { ACCSSON }\end{array}$ & $\begin{array}{lll}090 / 2 / 2 / 208 \\
101312018\end{array}$ & 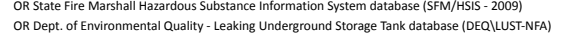 \\
\hline 301 W VALLEYV & $\begin{array}{l}\text { Teatero } \\
\text { TALNT }\end{array}$ & 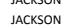 & $\begin{array}{lll}10131 / 2018 \\
09 / 29 / 2008\end{array}$ & 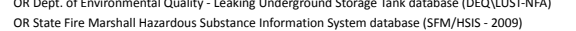 \\
\hline 654 KENDAL & CAVE JUNCTION & JOSEPHINE & $09 / 29 / 2008$ & 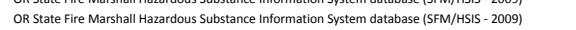 \\
\hline 2341 BIDOLE & Medford & JACKSON & 10/31/2018 & 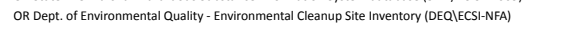 \\
\hline $7701111 \mathrm{H}$ & 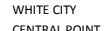 & JACKSON & 09/29/2008 & 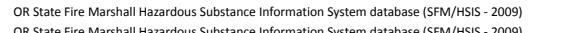 \\
\hline $\begin{array}{l}52355 \text { ARINBOW DR } \\
1502 \text { ANTELOPE }\end{array}$ & $\begin{array}{l}\text { CEETRAP LOINT } \\
\text { WHITE CIIY }\end{array}$ & & 09/29/2/2008 0 & 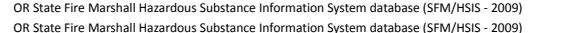 \\
\hline 1502 ANTELOPE & WHITEC CITY & IACKSON & 09/29/2008 & \\
\hline 2341 BIDOLE & Medford & JACKSON & 11/1/2018 & OR Dept. of Envirom nental Q Quality - Underground Storage Tank Program (DERQUST) \\
\hline 515 BROAD ST & BUTTE FALS & JACKSON & 09/29/2008 & 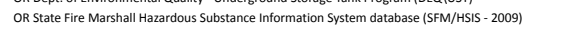 \\
\hline 2343 BIDLLE RD & MEFFORD & JACKSON & 09/29/2008 & 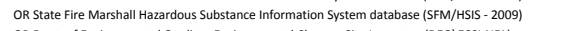 \\
\hline 2335 EARNETT RD & $\begin{array}{l}\text { Medford } \\
\text { Meffordi }\end{array}$ & IACKSON & 10/11/2018 & 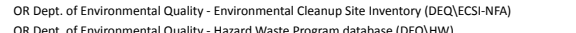 \\
\hline 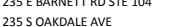 & $\begin{array}{l}\text { Metarord } \\
\text { MEOFORD }\end{array}$ & $\begin{array}{l}\text { JaCSSON } \\
\text { IACSSON }\end{array}$ & $\begin{array}{l}100131 / 2018 \\
09 / 292008\end{array}$ & 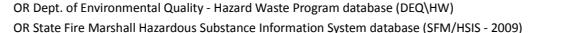 \\
\hline 1396 ANTELOPE RD & WHITECIYY & JACKSON & 09/29/2008 & 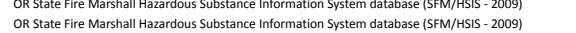 \\
\hline 2355 S PACIFIC HWY & MEFORD & JACKSON & 09/29/2008 & 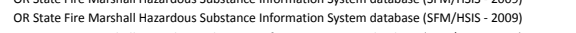 \\
\hline 5742 CAATER LAK & CENTRAL PO & JACKSON & 09/29/2008 & OR State fire Marstall Hazarddous Substance Information System database (SFM/HSIS- 2009) \\
\hline 1213 AVENUEC C & WHITE CITY & JACCSON & 09/29/2008 & OR State Fire Marshall Hazardous Substance Information System database (SFM/HISIS -2009) \\
\hline 9201 OLD ST & CENTRAL POINT & JACKSON & 09/29/2008 & OR State fire Marshall Hazardous Substance Information System database (SFM/HISIS-2009) \\
\hline 2366 Happy 20. & $\begin{array}{l}\text { MEFFoRD } \\
\text { Mefford }\end{array}$ & IACKSON & 109/12//2008 & 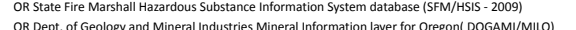 \\
\hline 2370 POPLAR DR & $\begin{array}{l}\text { MEDORORD } \\
\text { MEOPO }\end{array}$ & $\begin{array}{l}\text { Jackson } \\
\text { JACKSON }\end{array}$ & 09/29/2008 & 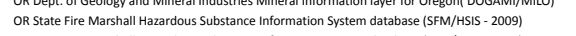 \\
\hline 100 HOUSTON RD & PHOENIX & JaCKSON & & 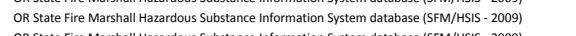 \\
\hline $1860 \mathrm{NHWY} 99$ & ASHLAND & JACKSON & 09/29/2008 & OR State Fire Marshall Hazardous Substance Information System database (SFM/HISIS-2009) \\
\hline 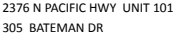 & $\begin{array}{l}\text { MEFOORD } \\
\text { CENTRALP }\end{array}$ & JaCKSON & 09/29/2008 & OR State Fire Marstall Hazardous Substance Information System database (SFM/HISIS-2009) \\
\hline & & JACSSON & 909/2/2/20008 1008 & 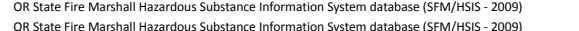 \\
\hline 885 Inous & 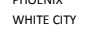 & JACKSON & 09/29/2008 & 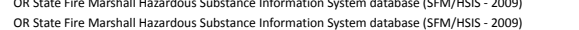 \\
\hline & CENTRAL POINT & JACKSON & $09 / 29 / 2008$ & 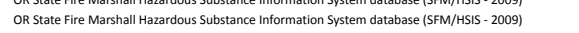 \\
\hline 3295 HANLEY RD & CENTRAL POINT & JACKSON & 09/29/2008 & 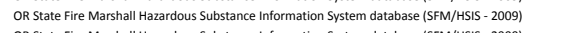 \\
\hline 51265 PACFIC HWY & $\begin{array}{l}\text { PHOENIX } \\
\text { WHTTEGT }\end{array}$ & JaCKSON & 09/29/2008 & 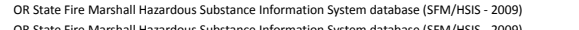 \\
\hline & $\begin{array}{l}\text { WHITE CIIY } \\
\text { EGGE PONT }\end{array}$ & JACKSON & 09/29/2008 & OR State fire Marshall Hazarddous Substance Information System database (SFM/HISIS - 2009) \\
\hline & IEPONT & & & 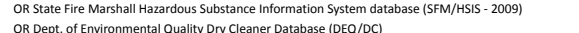 \\
\hline 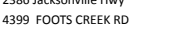 & 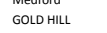 & $\begin{array}{l}\text { Jackson } \\
\text { Jackson }\end{array}$ & 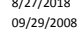 & ation S(DQQDStem database (SFM/HSII - 2009) \\
\hline & CENTRAL POINT & JACKSON & 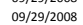 & ation System database (SFM/HIS - 2009) \\
\hline & CENTRA POINT & Jackson & 9/2/2/2008 & e (SFM/HSIS - 2009) \\
\hline 2389 TERRIL & MEFFRD & JACKSON & 09/29/2008 & ormation System database (SFM/HSIS- 2009) \\
\hline twe & MEOPORD & JACKSON & 09/29/2008 & Idatabase (SFM/HSIS - 2009) \\
\hline & & & & $\begin{array}{l}\text { abase (DEQQ LUST-NFA) } \\
\text { ans }\end{array}$ \\
\hline NSINGTON Sa & forod & JACKSON & 10/31/2018 & 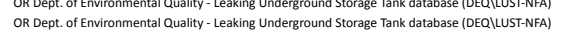 \\
\hline MAlinst & FORD & JACKSON & & W/HSIS - 2009) \\
\hline 234 & CENTRAL POINT & JACKSON & 99/2/2/2008 & (HISIS-2009) \\
\hline $\begin{array}{l}20399 \text { SAAWER RD } \\
2 \text { 2008 W Main St }\end{array}$ & $\begin{array}{l}\text { SHADY COVE } \\
\text { Medford }\end{array}$ & Jackson & 09/29/2008 8018 & $\begin{array}{l}\text { OR State fire Marshall } \\
\text { OR Reta of Envirome }\end{array}$ \\
\hline $\begin{array}{l}2408 \text { W Manist St } \\
447 \text { WILIAMSON Wr }\end{array}$ & $\begin{array}{l}\text { Medford } \\
\text { ASHLAND }\end{array}$ & $\begin{array}{l}\text { Jackson } \\
\text { JACKSON }\end{array}$ & 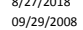 & 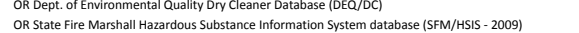 \\
\hline
\end{tabular}

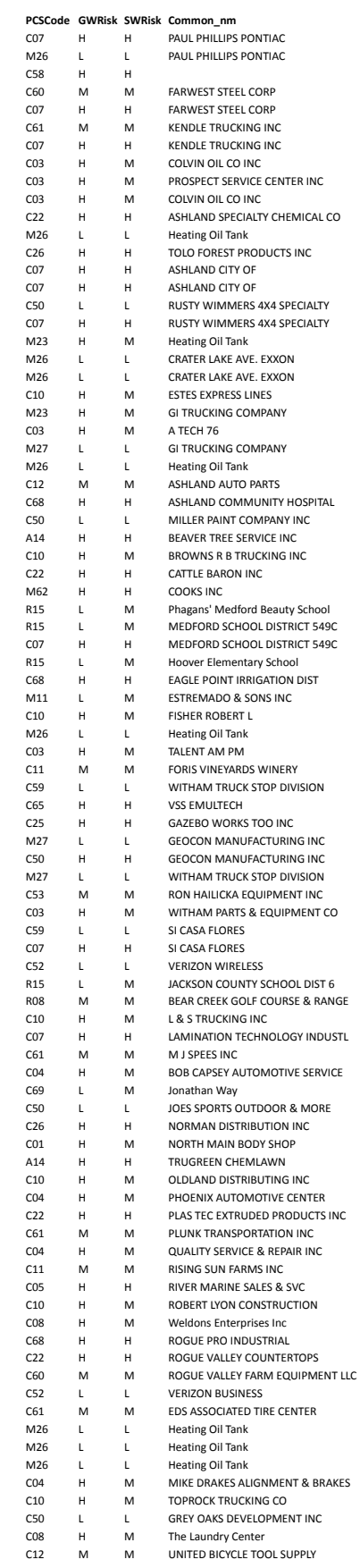

PcSType
Chemical/Pe

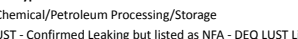

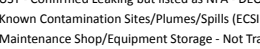

Maintenance Shop/Equipment Storage- Not Transportation Related
Chemical/Pertoleum Processing/Storage Maintenance Shop/Equipment Storage -
Chemical Pertolum processine/storage chemical/Petroleum Processing/Storage

Automobiles - Gas Stations
Automobiles- Gas stations

UST- Confirmed Leaking but listed as NFA - DEQ LUST Lis Wood/Pulu/Paper Processing and Mills
Cheminacl/Petroleum Processing//storage
Chemical/Petroleum Processing/Storage

Other- General Merchandise/Retail Store
Chemical Petroleum Processing

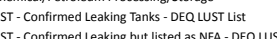
UST - Confirmed Leaking but listed as NFA- DEQ W LST Lis

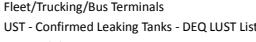

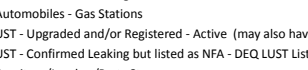
furniture/lumber/Parts Stores

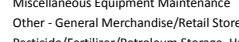

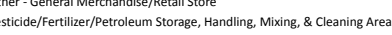
jeet/Trucking//Bus Terminals

政

Lasoons/lid
School
Schools

Chemical/Petrolum Processing//Storage
Schools

Miscellaneous Equipm
Kennels $(>20$ Pens)
Fens

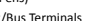

UST - Confirmed Leaking but listed as NFA - DEQ LUST Lis
Antomobiles - Gas Stations

Food rocessing

NFA (Sites/Plumes/Spills from ECSI)

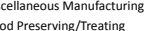

Wood Preserning /reating
UST - Upgraded and/or Registered- Active (may also have decommissioned tanks on site)

athers - Unknown Commercial - Active (may also have decoommissioned tanks on

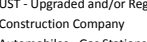

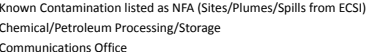

Schools
Golf Courses

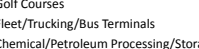

Maintenance Shop//Equipment Stororge- - Transportation Related

Auto- Repair Shops

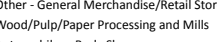

un storge, Handling Miving \& cleaning Artess

Pesticide/fertilizer/Petroleum
Fleet/Trucking//us Terminals
Auto- Repair shops

Auto- Repair Shops
Plastics/snnthetics rodoucer

Maintenance Shoo//Euipment Storgae - Transportation Related

Auto- Repair shops
food rocesing

Food Processing
Boat Serices Repair/ Refinishing
Fleet/Trucking//Bus Terminals

Dry Cleaners
Miscellaneous Equipment Maintenare

Miscellaneous Equipment Main
Plastics/syntheteics Producer

Maintenance Shop/Equipment Storage - No

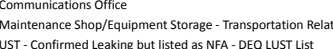

UST - Confirmed Leaking but ilsted as SNA- - EEQ LUST L

Fleet/Trucking//us Terminals
Others - Govit/Uusiness/Noo Services

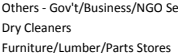




\begin{tabular}{|c|c|c|c|}
\hline \multicolumn{4}{|c|}{$\begin{array}{l}\text { Cetiy } \\
\text { EAGL POINT }\end{array}$} \\
\hline 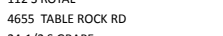 & $\begin{array}{l}\text { CENTRAL POINT } \\
\text { Cont }\end{array}$ & $\begin{array}{l}\text { S JACKSON } \\
\text { JACSON }\end{array}$ & $\begin{array}{ll}09 / 29 / 2008 \\
0.008\end{array}$ \\
\hline $\begin{array}{l}\text { 24-1/2 } 2 \text { SRAPE } \\
7730 \text { CRATE LAKE HWY }\end{array}$ & 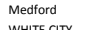 & $\begin{array}{l}\text { JACKSON } \\
\text { ACKSON }\end{array}$ & $\begin{array}{l}10 / 31 / 2018 \\
0992 / 2008\end{array}$ \\
\hline $\begin{array}{l}7130 \text { C C CARERE LAAE HWY } \\
2105 \text { CORY RD }\end{array}$ & 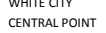 & $\begin{array}{l}\text { JAACCSON } \\
\text { JACKSON }\end{array}$ & $\begin{array}{l}099 / 2 / 2008 \\
09 / 29 / 2008\end{array}$ \\
\hline 2410 N PACFFIC HWY & MEFFORD & JACKSON & $09 / 29 / 2008$ \\
\hline $\begin{array}{l}2410 \text { NA PACIFI HWY } \\
2220 \text { W MAAN ST }\end{array}$ & $\begin{array}{l}\text { Mefford } \\
\text { MEDFORD }\end{array}$ & $\begin{array}{l}\text { JACKSON } \\
\text { JACSSON }\end{array}$ & $\begin{array}{l}10 / 31 / 2018 \\
092 / 2 / 2008\end{array}$ \\
\hline $\begin{array}{l}20220 \text { W MAANST } \\
4865 \text { HWY } 234\end{array}$ & $\begin{array}{l}\text { MEDEORD } \\
\text { WHITE CITY }\end{array}$ & $\begin{array}{l}\text { JaCGSON } \\
\text { JACSSON }\end{array}$ & $\begin{array}{l}099 / 2 / 2008 \\
09 / 29 / 2008\end{array}$ \\
\hline 100 EAGLE POINT DR & EAGLE POINT & $\begin{array}{l}\text { JaCGSON } \\
\text { JACCSON }\end{array}$ & $\begin{array}{l}09929 / 2008 \\
09 / 29 / 2008\end{array}$ \\
\hline 12451 MODOC RD & Whilre cIrT & Jackson & $\begin{array}{ll}09 / 29 / 2008 \\
0\end{array}$ \\
\hline 2120 AVENUE H & WHITE CITY & JaCKSON & 09/29/2008 \\
\hline $\begin{array}{l}24244 \text { CRATER LAKE HWY } \\
114199 \text { USTCC RD }\end{array}$ & $\begin{array}{l}\text { MEFFRD } \\
\text { CETTA PONT }\end{array}$ & JACKSON & $09 / 29 / 2008$ \\
\hline 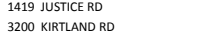 & $\begin{array}{l}\text { CENTTAA POONT } \\
\text { CENTRA POINT }\end{array}$ & $\begin{array}{l}\text { JACKSON } \\
\text { JACKSON }\end{array}$ & 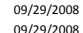 \\
\hline 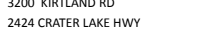 & $\begin{array}{l}\text { CENRFA PolNI } \\
\text { Medford }\end{array}$ & $\begin{array}{l}\text { JAACSSON } \\
\text { JACKSON }\end{array}$ & $\begin{array}{l}09929 / 2008 \\
10 / 31 / 2018\end{array}$ \\
\hline 2424 CRATER L LAKE HWY & $\begin{array}{l}\text { Meterof } \\
\text { Metford }\end{array}$ & $\begin{array}{l}\text { JaCKSON } \\
\text { Jan }\end{array}$ & $\begin{array}{l}10 / 131 / 2018 \\
10 / 31 / 2018\end{array}$ \\
\hline 2424 CRATER L LAKE HWY & Medford & Jackson & $\begin{array}{l}11 / 1 / 2018 \\
11818\end{array}$ \\
\hline 2430 Griffin Creek Rd & Medford & JACKSON & 06/202/2015 \\
\hline 635 AVENUE C & $\begin{array}{l}\text { WHITE CITY } \\
\text { CETTPOPTI }\end{array}$ & JACKSON & $09 / 29 / 2008$ \\
\hline $\begin{array}{l}3750 \text { KIRTLAND RD } \\
2433 \text { LNDOO GIR }\end{array}$ & CENTRAL POINT & $\begin{array}{l}\text { JACKSON } \\
\text { A CKSSON }\end{array}$ & $\begin{array}{l}09 / 29 / 2008 \\
103 / 2 / 2018\end{array}$ \\
\hline $\begin{array}{l}2433 \text { L LNDONO CRT } \\
420 \text { FRONT ST }\end{array}$ & $\begin{array}{l}\text { Mefordd } \\
\text { CENTRAL POINT }\end{array}$ & $\begin{array}{l}\text { JACCSSON } \\
\text { JACKSON }\end{array}$ & 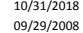 \\
\hline $\begin{array}{l}422 \text { N FRRNT TS } \\
4400 \text { LIVINSTON RD }\end{array}$ & $\begin{array}{l}\text { CENRRA POLONT } \\
\text { CENTRAL POINT }\end{array}$ & $\begin{array}{l}\text { JAACSSON } \\
\text { JACKSON }\end{array}$ & $\begin{array}{l}0.0929 / 2008 \\
09 / 29 / 2008\end{array}$ \\
\hline 11 S FRONTAGE RD & GOLD HILL & JACKSON & $\begin{array}{ll}09 / 29 / 2008 \\
0\end{array}$ \\
\hline 2441 N KEENEWAY DR & Medford & JACKSON & 10/31/2018 \\
\hline 1993 KIRTLAND RD & CENTRAL POINT & JACKSON & $09 / 29 / 2008$ \\
\hline $\begin{array}{l}2455 \text { S GRAAE ST } \\
\text { 633 BARTON RD }\end{array}$ & $\begin{array}{l}\text { MEEFRO } \\
\text { EAGE POINT }\end{array}$ & JACKSON & $\begin{array}{l}09 / 29 / 2008 \\
0992 / 2 / 208\end{array}$ \\
\hline $\begin{array}{l}\text { 6533 BARRON RD } \\
12294 \text { AGATE RD }\end{array}$ & $\begin{array}{l}\text { EAGL POONT } \\
\text { EAGL POINT }\end{array}$ & $\begin{array}{l}\text { JaCSSON } \\
\text { IACSSON }\end{array}$ & $\begin{array}{l}099 / 2 / 2008 \\
09 / 29 / 2008\end{array}$ \\
\hline 245 VALLEY VIEW DR & Medford & Jackson & $\begin{array}{l}0.971 / 2008 \\
10 / 21 / 218\end{array}$ \\
\hline 212 E MAIN ST & ASHLAND & JACKSON & 09/29/2008 \\
\hline 8333 AGATE RD & WHITE CITY & JACKSON & 09/29/2008 \\
\hline 245 YALL DR & $\begin{array}{l}\text { Medford } \\
\text { CETrespont }\end{array}$ & $\begin{array}{l}\text { JACKSON } \\
\text { JACSSON }\end{array}$ & $10 / 31 / 2018$ \\
\hline 52626 CRATER L LAKE AVE & CENTRAL POINT & JACKSON & $09 / 29 / 2008$ \\
\hline $\begin{array}{l}2455 \text { Savivi Lane } \\
1500 \text { ANTELOPE RD }\end{array}$ & $\begin{array}{l}\text { Mefoford } \\
\text { WHitr CITY }\end{array}$ & $\begin{array}{l}\text { Jackson } \\
\text { jackson }\end{array}$ & $\begin{array}{l}1 / 11 / 2018 \\
0 / 292 / 2008\end{array}$ \\
\hline 2490 FOOTHILL RD & Medford & 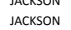 & $\begin{array}{l}\text { 10/31/2018 } \\
109128\end{array}$ \\
\hline 2498 COKER BUTTE RD & MEEFORD & JACKSON & $\begin{array}{ll}09 / 29 / 2008 \\
0\end{array}$ \\
\hline 1450 ANTELOPE RD & WHITE CITY & JaCKSON & 09/29/2008 \\
\hline 5704 ADAMS ST & TALENT & JaCKSON & 09/29/2008 \\
\hline 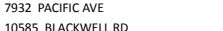 & $\begin{array}{l}\text { WHITECIIY } \\
\text { CNETRAP PONT }\end{array}$ & JACKSON & 09/29/2008 $1-20$ \\
\hline 2498 COKER BUTTE RD & $\begin{array}{l}\text { CENTFA POINT } \\
\text { MEFFORD }\end{array}$ & $\begin{array}{l}\text { JAACSSON } \\
\text { JACKSON }\end{array}$ & $\begin{array}{l}09929 / 2008 \\
09 / 29 / 2008\end{array}$ \\
\hline 1605 BROWNSBORO-MER IDIA N R & EAGLE POINT & $\begin{array}{l}\text { JaCKSON } \\
\text { JASON }\end{array}$ & $\begin{array}{ll}0 & 09 / 29 / 2008 \\
09 / 2908 & \end{array}$ \\
\hline 25 E MCANDREWS RD & Medford & JACKSON & $10 / 31 / 2018$ \\
\hline 25 E MCANDREWS RD & MEDFORD & JACKSON & $09 / 29 / 2008$ \\
\hline 25 E MCANDREWS RD & MEDFORD & JaCKSON & $09 / 29 / 2008$ \\
\hline $\begin{array}{l}25 \text { Easas McAnAdrews Rd } \\
25 \text { East McAndrews Rd }\end{array}$ & $\begin{array}{l}\text { Medoford } \\
\text { Metford }\end{array}$ & $\begin{array}{l}\text { Jackson } \\
\text { Jackson }\end{array}$ & $\begin{array}{l}2005 \\
2005\end{array}$ \\
\hline $\begin{array}{l}2054 \text { ANTELOPE RD } \\
200 \text { TIS }\end{array}$ & $\begin{array}{l}\text { Wetror } \\
\text { WHITE CITY }\end{array}$ & $\begin{array}{l}\text { Sactson } \\
\text { JACKSON }\end{array}$ & $\begin{array}{l}2005 \\
09 / 29 / 2008\end{array}$ \\
\hline 25 East McAndrews Rd & Medford & Jackson & 2005 \\
\hline 626 HARTLEY RD & PHOENIX & JACKSON & 09/29/2008 \\
\hline 25 East McAndrews Rd & 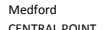 & Jackson & 2005 \\
\hline 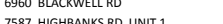 & $\begin{array}{l}\text { CENTTAA POONT } \\
\text { CNNTRA POINT }\end{array}$ & JACKSON & $\begin{array}{l}09 / 29 / 2008 \\
0.9929 / 2008\end{array}$ \\
\hline 6241 CRATER LAKE HWY & $\begin{array}{l}\text { CENRTAA POONI } \\
\text { CENTRAL POINT }\end{array}$ & 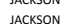 & $\begin{array}{l}09929 / 2008 \\
00 / 29 / 2008\end{array}$ \\
\hline 5800 P PACFIC HWY & PHOENI & JACKSON & $\begin{array}{l}099929 / 2008 \\
09 / 29 / 2008\end{array}$ \\
\hline 250 LOZZRR LN & MEEFORD & JACKSON & $\begin{array}{l}09 / 29 / 2008 \\
0\end{array}$ \\
\hline 1024-B NEWLLND RD & CENTRAL POINT & JACKSON & 09/29/2008 \\
\hline 7905 AGATE RD & WHITE CITY & JAAKSON & 09/29/2008 \\
\hline 6061 CRATER LAKE HWY & CENTRAL POINT & JACKSON & 09/29/2008 \\
\hline 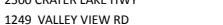 & $\begin{array}{l}\text { Wearora } \\
\text { ASHLAND }\end{array}$ & $\begin{array}{l}\text { S } \\
\text { ACCSSON }\end{array}$ & $\begin{array}{l}10 / 131 / 2018 \\
09 / 29 / 2008\end{array}$ \\
\hline 2500 S PACFFC HWY & MEFFORD & JACKSON & $\begin{array}{l}09992 / 2008 \\
09 / 29 / 2008\end{array}$ \\
\hline 2500 S PACFIC HWY & MEFFRD & JaCKSON & $\begin{array}{l}09 / 29 / 2008 \\
0\end{array}$ \\
\hline 386 BATEMAN DR & CENTRAL POINT & JACKSON & 09/29/2008 \\
\hline 25017 CRATER LLKE HWY & $\begin{array}{l}\text { Medford } \\
\text { Mats }\end{array}$ & JACCSONO & $\begin{array}{l}10 / 31 / 2018 \\
\end{array}$ \\
\hline $\begin{array}{l}2371 \text { ASALAAD ST } \\
251 \text { EARNETT RD }\end{array}$ & $\begin{array}{l}\text { A AHFALNO } \\
\text { Metford }\end{array}$ & 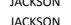 & $\begin{array}{l}\text { 09/292/2008 } \\
1 / 31 / 2018\end{array}$ \\
\hline $\begin{array}{l}\text { 1065 PPNNEST } \\
1005\end{array}$ & $\begin{array}{l}\text { CENTRA PONTT } \\
\text { The }\end{array}$ & JACSSON & $\begin{array}{l}1 / 131 / 2018 \\
09 / 29 / 2008\end{array}$ \\
\hline 6034 CRATER LAKE AVE & CENTRAL POINT & JACKSON & $\begin{array}{l}0.979 / 2008 \\
09 / 29 / 2008\end{array}$ \\
\hline 8422 HIGH BANSS RD & CENTRAL POINT & JACKSON & 09/29/2008 \\
\hline 2517 BIDDDE RD & MEFFORD & JACKSON & 09/29/2008 \\
\hline STS & HUAND & & 09/29/2008 \\
\hline 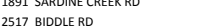 & $\begin{array}{l}\text { MEDFORD } \\
\text { MEOLA }\end{array}$ & 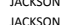 & $\begin{array}{l}09929 / 2008 \\
00 / 29 / 2008\end{array}$ \\
\hline $\begin{array}{l}2517 \text { BIDOLE RO RD } \\
250\end{array}$ & Medford & Accsoov & $\begin{array}{l}0.9 / 3 / 2008 \\
10 / 121818\end{array}$ \\
\hline 1200 AVENUE G & & JaCKSON & 09/29/2008 \\
\hline 2288 OLD STAGE RD & ITRAL POINT & JACKSON & $09 / 29 / 2008$ \\
\hline SONVILE! & EFFRD & JACKSON & 09/29/2008 \\
\hline 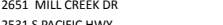 & & & 09/2/29/2008 \\
\hline (1) & 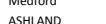 & 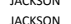 & $\begin{array}{l}10 / 11 / 2018 \\
00 / 292008\end{array}$ \\
\hline c cHWr & fForo & SAcrson & $\begin{array}{l}0.979 / 2008 \\
09 / 29 / 2008\end{array}$ \\
\hline 500 E ANTELOPE RD & EAGLE POINT & JaCKSON & 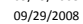 \\
\hline
\end{tabular}

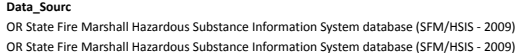
OR Dept. of Environmental Quality- Leaking Underground Storage Tank database (DEQ) LUST)
OR State fire Marshall Hazardous Substance Information System database (SFM/HIS - 2009) OR State fire Marshall Hazardous Substance Ifitrmation System database (SFM/HISS- - 2009)

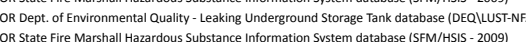

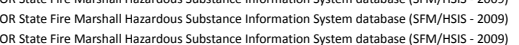

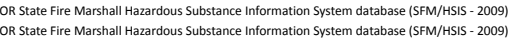

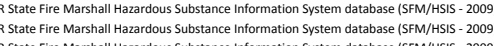

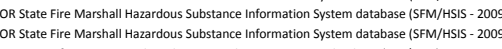

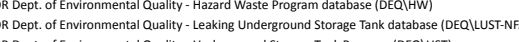

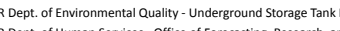

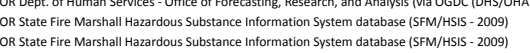

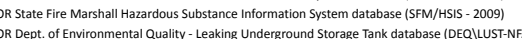

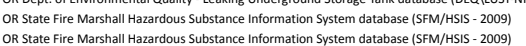
OR State fire Marshall Hazardous Substance Information System database (SFM/SISIS - 2009)

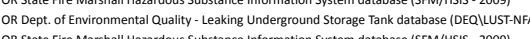

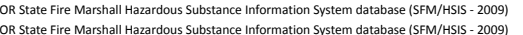

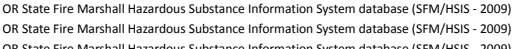

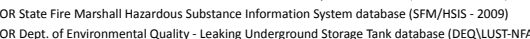

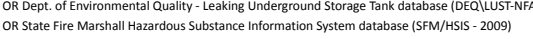

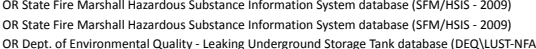

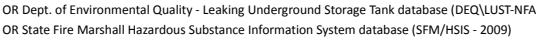

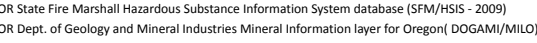
OR State fire Marshall Hazard ous Substance Information System database (SFMM/HIS - 2009)

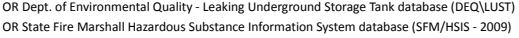

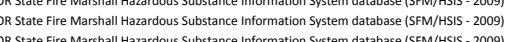

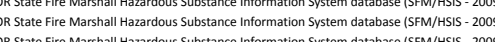

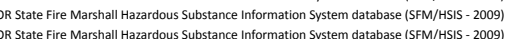

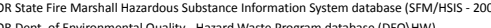

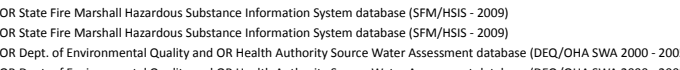

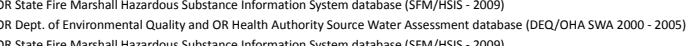

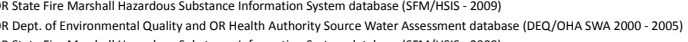

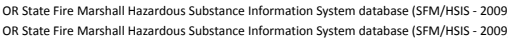

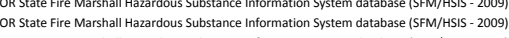

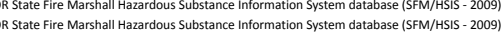

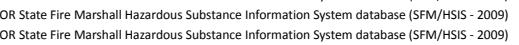

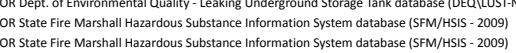
intormation System database (SFM/HSIS - 2009)

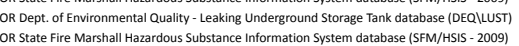

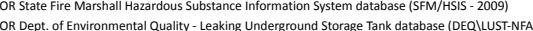

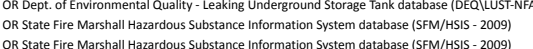

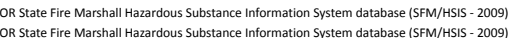

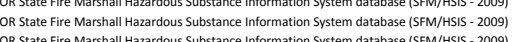

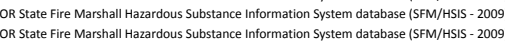

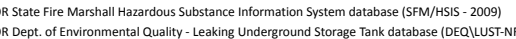
OR S State fire Marshall Hazardous Substance Information System database (SFM/HIS - 2000 -

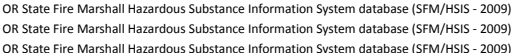
OR State fire Marshall Hazarddous Substance Information System database (SFM/HISIS-2000

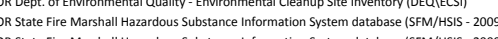

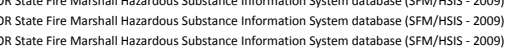

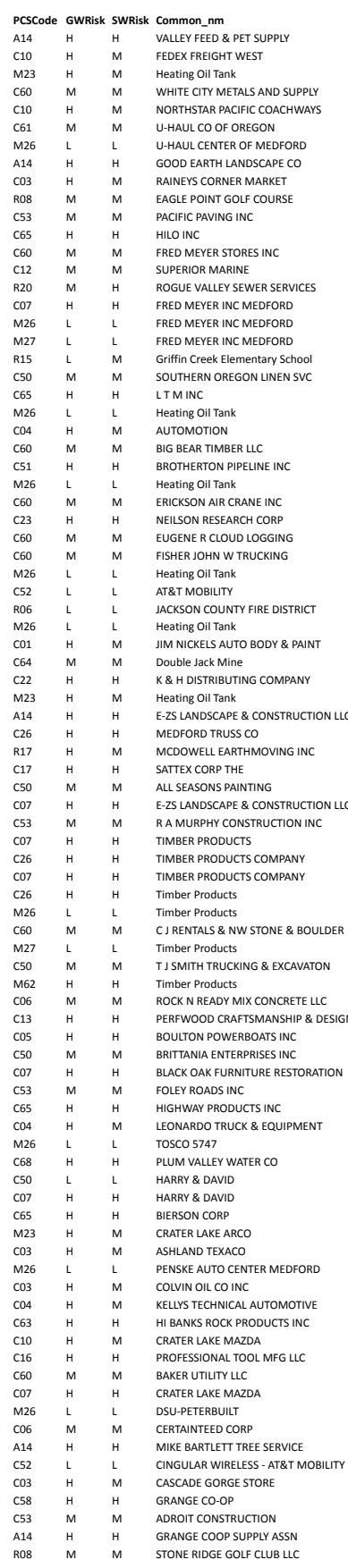

Pestype

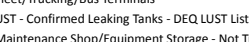

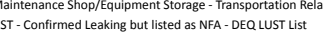

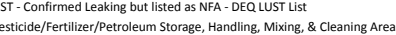
Automobiles - Gas Station
Golf Cuurses

Construction company
Miscellaneous Manufacturing Maintenance Shop/Lquipment Storage - Not Transportation Related Furniture/Lumber/Parts Stores
Wastewater r reatment Plants/col UST - Confirmed Leaking but listed as N NAA - DEQ WUST Lis Other - Cleaning and Supply Services

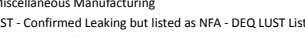
Auto- Repair Shops helines chemical and Petroloum UST-Confirmed Leaking but lised as N NA - DEQ Q WST Lis wissearch Maintenance Shoo/Equipment Storage - Not Transsortation Relatated UST- Confirmed Leaking but listed as NFA- DEQ LUST Lis

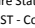

Automobiles - Body Shops

sand/grave//rock/soil

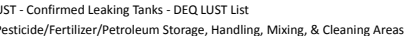

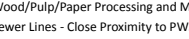

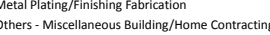
Chemical/Petroleum Processing/ $/$ tororge
Coge

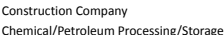
Wood/PPlu//Paper Procoessing and Mills
Chemical/Petroleum Processing/Storage

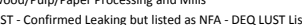
Maintenance Shop/Gquipment Storage - Not Transportation Related
UST - Upgraded and/or Registered - Active aggons/Liquid Wastes - Municicial/ndustrial Wastewater r reatment cement/Concetete Plants
Home Manufaturine

others-Miscelaneous suidining /Home Contracting Cemical/ertoloum Processing/storoage

Miscelaneous Manufacturing

Auto- Repair Shops
UST-Confirmed Lea

Iisted a s NFA - DEQ LuST List Miscellaneous Equipment Maintenance
other - General Merchandise/Retail Store

Miscellaneous Manufacturing
UST - Confirmed Leaking Tanks - DEQ L UST Lis

Automobilies- Gas Stations
UST - Confirmed Leaking but listed as NFA- DEQ LUST Lis

Auto - Repair Shops

Mining Activities- -Active- other than gravel
Fleet/rucking/us Terminals

Fleet frucking $/$ Uus Term
Machine Shops Waintenance shop/fquipment storage - Not Transportation Related

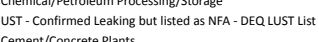
Cement/Concrete Plants

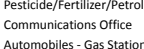
known Contamination Sites/PPlumes/spills EECS)

Construction Company
Pesticid/Fertilizer/Petroleum Storage, Handling, Mixing, \& Cleaning Areas
Colf Courses 


\begin{tabular}{|c|c|c|c|}
\hline \multirow{2}{*}{\multicolumn{4}{|c|}{ RET_DATE }} \\
\hline \multicolumn{3}{|l|}{ 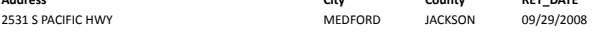 } & \\
\hline \multicolumn{4}{|c|}{$\begin{array}{ll}\text { MEDDORD } & \text { JACKSON } \\
\text { ASHLAND } & \text { JACKSON }\end{array}$} \\
\hline \multirow{2}{*}{\multicolumn{4}{|c|}{ 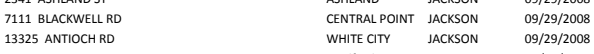 }} \\
\hline & $\begin{array}{l}\text { WHTTE CITY } \\
\text { Mefford }\end{array}$ & JACKSON & 09/2/2/2008 \\
\hline $\begin{array}{l}25511 \text { PAAFFCICHV } \\
1085 \text { BENSON WY STE }\end{array}$ & $\begin{array}{l}\text { Mefford } \\
\text { AsHLAND }\end{array}$ & JACKSON & $\begin{array}{l}10 / 31 / 2018 \\
0.0120208\end{array}$ \\
\hline 19000 CAVES HWY & $\begin{array}{l}\text { ASALAND } \\
\text { CAVE UUNCTION }\end{array}$ & & $\begin{array}{l}0.0929 / 2008 \\
09 / 29 / 2008\end{array}$ \\
\hline 4210 HOLLAND LOOP RD & $\begin{array}{l}\text { CAVE UUNCTION } \\
\text { S }\end{array}$ & JOSEPHINE & o9/20/2008 \\
\hline 4865 HWY 234 & WHITE CITY & JACKSON & 09/29/2008 \\
\hline 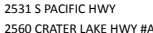 & Medford & JACKSON & $\begin{array}{l}11 / 1 / 2018 \\
10 / 312028\end{array}$ \\
\hline 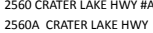 & $\begin{array}{l}\text { Medford } \\
\text { MEDFFRD }\end{array}$ & JACKSON & $\begin{array}{l}100 / 3 / 1 / 2018 \\
092 / 2 / 2008\end{array}$ \\
\hline 2577 W MAIN ST & $\begin{array}{l}\text { MEEFRO } \\
\text { MEFFORD }\end{array}$ & $\begin{array}{l}\text { JAAKSON } \\
\text { JACKSON }\end{array}$ & $\begin{array}{l}09 / 2 / 2 / 2008 \\
0 / 929 / 2008\end{array}$ \\
\hline 461 V VALEYY VIEW RD & & & \\
\hline 2600 BIDDLE RD & MEFFORD & Jackson & $\begin{array}{l}09 / 29 / 2008 \\
0\end{array}$ \\
\hline 2600 BIDOLE RD & MEDFORD & JaCKSON & 09/29/2008 \\
\hline 2600 BIDDL RD & $\begin{array}{l}\text { Medford } \\
\text { Mefford }\end{array}$ & JACKSON & $\begin{array}{l}10 / 31 / 2018 \\
103 / 212018\end{array}$ \\
\hline 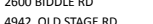 & $\begin{array}{l}\text { Medford } \\
\text { CESTTPRPPINT }\end{array}$ & JACKSON & 10/13/2018 \\
\hline $\begin{array}{l}2600 \text { OID SIAE RD RD } \\
2600\end{array}$ & $\begin{array}{l}\text { CENIRALOINT } \\
\text { Mefford }\end{array}$ & $\begin{array}{l}\text { JaCSSON } \\
\text { IACSSON }\end{array}$ & $\begin{array}{l}09 / 2 / 2 / 2008 \\
10 / 1 / 2018 \\
\end{array}$ \\
\hline 2603 S PACIFIC HWY & MEOFORO & JACKSON & $\begin{array}{l}1 \\
09 / 29 / 20008 \\
0\end{array}$ \\
\hline 2611 BIDDLE RD & MEDFORD & JACKSON & $\begin{array}{l}09 / 29 / 2008 \\
0\end{array}$ \\
\hline 2611 BIDDLE RD & 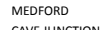 & JACKSON & 09/29/2008 \\
\hline 41919DICK GEORGE RD & $\begin{array}{l}\text { CAVE UNNCTION } \\
\text { PROSEET }\end{array}$ & JOSEPHINE & $\begin{array}{l}09 / 29 / 2008 \\
099292008\end{array}$ \\
\hline $\begin{array}{l}1980 \text { MILL CREEK DR } \\
7675 \text { 4TH ST }\end{array}$ & $\begin{array}{l}\text { PROOPPET } \\
\text { WHITE CITY }\end{array}$ & $\begin{array}{l}\text { JAAKSON } \\
\text { JACKSON }\end{array}$ & $\begin{array}{l}09 / 29 / 2008 \\
09929 / 2008\end{array}$ \\
\hline 2611 BIDDE RD & & 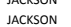 & $\begin{array}{l}0992 / 2 / 2008 \\
10 / 1 / 2018\end{array}$ \\
\hline 528 W LINN RD & EAGLE POINT & 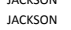 & $\begin{array}{l}109 / 1 / 212008 \\
09 / 2008\end{array}$ \\
\hline 2611 BIDOLE RD & Medford & JaCksoN & $10 / 31 / 2018$ \\
\hline 2611 BIDDLE RD & Medford & JaCKSON & 10/31/2018 \\
\hline 2620-K E Barnett Rd & $\begin{array}{l}\text { Mefford } \\
\text { PRPSOSPTT }\end{array}$ & $\begin{array}{l}\text { Jackson } \\
\text { Nacsson }\end{array}$ & 8/27/2018 \\
\hline 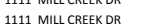 & $\begin{array}{l}\text { PRRSOEECT } \\
\text { PROSPECT }\end{array}$ & $\begin{array}{l}\text { JaCCSON } \\
\text { ACCSSON }\end{array}$ & $\begin{array}{l}09 / 29 / 2008 \\
09929 / 2008\end{array}$ \\
\hline 203 N PLATT AVE & EAGLE POINT & 作 & $\begin{array}{l}099 / 2 / 2 / 2008 \\
09292 / 2008\end{array}$ \\
\hline 140 E MAIN ST & EAGLE POINT & Jackson & o9/29/2008 \\
\hline 1570 AVENUE F & WHITE CITY & JACKSON & 09/29/2008 \\
\hline 1011 SUNNY ST & & & 09/29/2008 \\
\hline 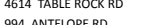 & 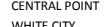 & JACKSON & 09/29/2008 \\
\hline 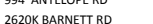 & 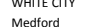 & $\begin{array}{l}\text { JaCSSON } \\
\text { ICCCSON }\end{array}$ & $09 / 29 / 2008$ \\
\hline $264 \mathrm{~s} \mathrm{MODOC}$ & & 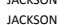 & $\begin{array}{l}101 / 3 / 21 / 2018 \\
10131 / 2018\end{array}$ \\
\hline 6740 CRATER L LakE AVE A & CENTRAL POINT & JACKSON & $\begin{array}{l}09 / 29 / 2008 \\
0\end{array}$ \\
\hline 2655 PACFIC HWY & MEDFRD & JaCKSON & $09 / 29 / 2008$ \\
\hline 1065 BENSON WAY STE 9 & ASHLAND & JACKSON & 09/29/2008 \\
\hline 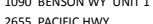 & $\begin{array}{l}\text { ASHLAND } \\
\text { MEFEOPO }\end{array}$ & IACCSON & 09/29/2008 \\
\hline $\begin{array}{l}2565 \text { P PCCAFCC HWV } \\
599 \text { EVILS RD STE A }\end{array}$ & $\begin{array}{l}\text { MEEFRORD } \\
\text { CENTRALOINT Pat }\end{array}$ & 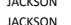 & $\begin{array}{l}09 / 29 / 2008 \\
099292008\end{array}$ \\
\hline 2660 HILLCREST RD & $\begin{array}{l}\text { MEFFORD } \\
\text { MED }\end{array}$ & $\begin{array}{l}\text { IACKSON } \\
\text { Iats }\end{array}$ & $\begin{array}{l}0 \\
09 / 29 / 2 / 2008 \\
0908\end{array}$ \\
\hline 1070 TOLMAN CREEK RD & ASHLAND & JACKSON & $\begin{array}{l}09 / 29 / 2008 \\
0\end{array}$ \\
\hline 311 N FRONT ST & CENTRAL POINT & & 09/29/2008 \\
\hline 1313 BoOCKOALE RD & CAVE UUNCTION & JOSEPHINE & 09/29/2008 \\
\hline 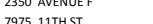 & 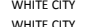 & JAACKONON & $\begin{array}{l}099 / 2 / 2 / 2008 \\
00208\end{array}$ \\
\hline 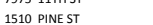 & CENTRAL POINT & 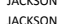 & $\begin{array}{l}09 / 29 / 2008 \\
0.929 / 2008\end{array}$ \\
\hline 175 HANLEY RD & $\begin{array}{l}\text { CENTRA POINT } \\
\text { Clat }\end{array}$ & $\begin{array}{l}\text { IACKSON } \\
\text { IACS }\end{array}$ & $\begin{array}{l}0.9919 / 2008 \\
09 / 29 / 2008\end{array}$ \\
\hline 1401 BEENTWOOD RO & EAGLE POINT & JACKSON & 09/29/2008 \\
\hline 2660 HILCREST RD & MEFFORD & JACKSON & 09/29/2008 \\
\hline $\begin{array}{l}2660 \\
260 \text { HULCRESTS RO }\end{array}$ & MEDEORD & JACCSON & 09/29/2008 \\
\hline 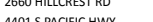 & 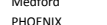 & A & 10/31/2018 \\
\hline 275 CTIADE RD & TRAMAn & 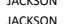 & 等 \\
\hline 7905 AGATE RD & WHITE CITY & JACKSON & $\begin{array}{l}0 \\
09 / 29 / 20008 \\
0972 / 20\end{array}$ \\
\hline 1899 HWY 99 N & ASHLLND & JaCKSON & 09/29/2008 \\
\hline 26615 PACIFIC HWY & MEOFORD & & 09/29/2008 \\
\hline 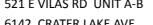 & 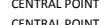 & ACCSSON & o9/29/20008 \\
\hline THST & ASt ASHD & 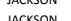 & 09/29/20008 \\
\hline 1890 HWr Y $99 \mathrm{~N}$ & S & 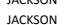 & $\begin{array}{l}099292 / 2008 \\
09 / 29 / 2008\end{array}$ \\
\hline 69096 6T ST & WHITE CITY & JaCkSON & $\begin{array}{l}0 \\
09 / 29 / 20008 \\
0\end{array}$ \\
\hline 8001 TABLE ROCK RD & WHITE CITY & JACKSON & 09/29/2008 \\
\hline 2665 вULLOCK RD & MEFFORD & JACKSON & 09/29/2008 \\
\hline 2665 BUULOCK RD & $\begin{array}{l}\text { Mefford } \\
\text { wytroc city }\end{array}$ & JACKSON & 10/31/2018 \\
\hline (4) & $\begin{array}{l}\text { WHITECTIY } \\
\text { MEFFORD }\end{array}$ & 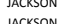 & $\begin{array}{l}099 / 29 / 2008 \\
0 . / 292 / 2008\end{array}$ \\
\hline 5 5.42A CRATER LAKE AVE & CENTRA POINT & IACKSON & 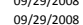 \\
\hline 2685 N PACFFC HWY & MEFFORD & JaCkSON & $\begin{array}{l}09 / 29 / 2008 \\
098\end{array}$ \\
\hline 2685 N PACFFC HWY & Mefford & & $10 / 31 / 2018$ \\
\hline 2690 C CRATER L LAKE HWY & MEFERO & & 09/29/2008 \\
\hline 2690 CRAATER LAKE HWY & 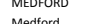 & JACCSONO & 09/29/2/2008 \\
\hline (2) & Netrorar & 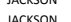 & $\begin{array}{l}10131 / 2018 \\
1013218\end{array}$ \\
\hline 26922 PACIFC HWY & Medford & JACKSON & $\begin{array}{l}1 / 131 / 2018 \\
1018\end{array}$ \\
\hline 2518 SARDINE CREEK RD & GOLD HILL & JaCKSON & 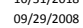 \\
\hline 2692 N Pacific Hwy & Medford & Jackson & 1/1/1/2018 \\
\hline $2692 \mathrm{~N}$ P Pactific Hur & Mefford & Jackson & 1/1/1/2018 \\
\hline
\end{tabular}

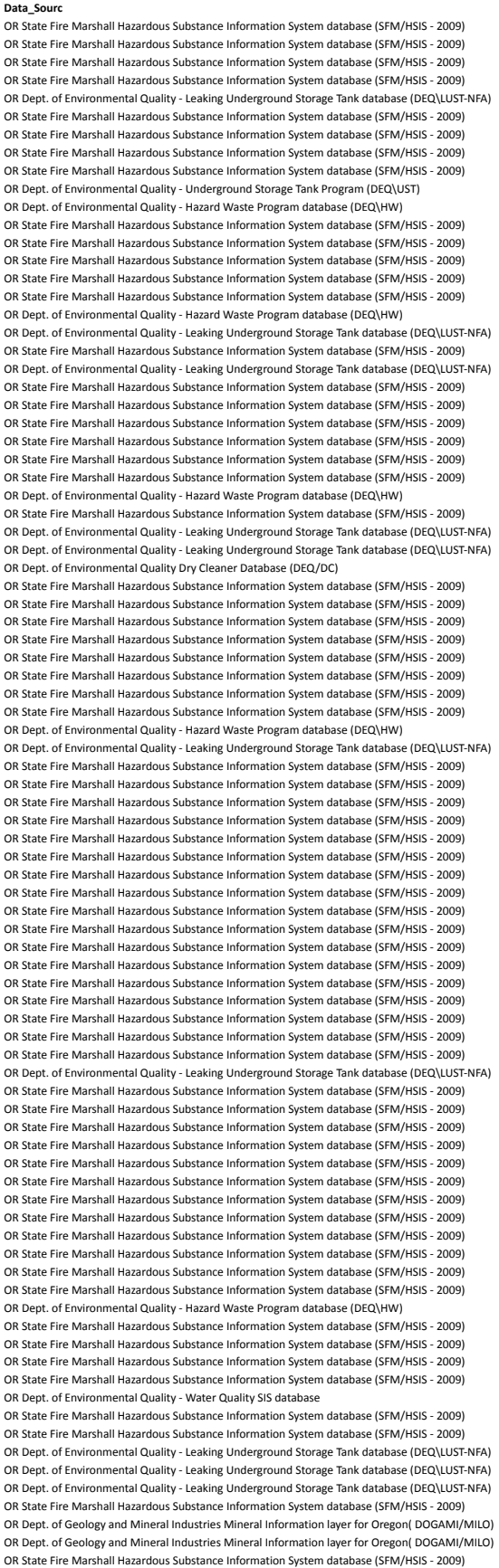

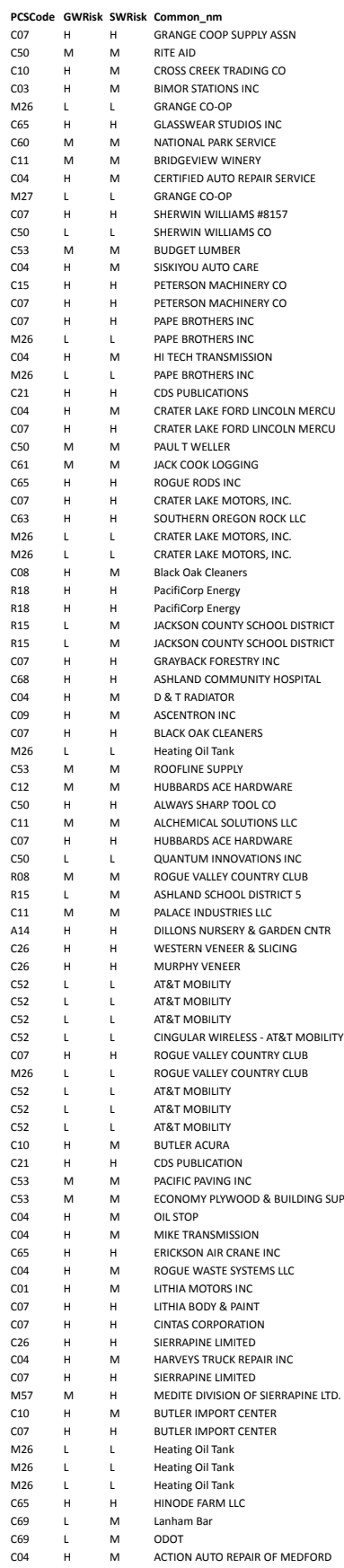

PCSType

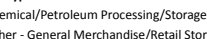

Freet/Trucking//Uus Terminal
Automobiliss - Gas Stations

UST - Confirmed Leaking but listed as NFA- DEQ LUST List

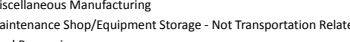

UST - Upgraded and/or Registered-Active (may also have decommissioned tanks on site)

Other- General Merchandise/Retail Store

Construction Compani
Auto- $\operatorname{Repari}$ Shops

Chemical/pertolueum Processing/storage

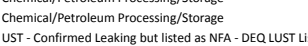

Auto- Repair Shops

UST - Confirmed Leaking but

Photo Processing//Printing
Auto- Repair Shoos

Chemical/Petroleum Processing//5torage

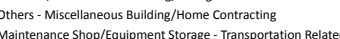

Maintenance Shop/Guipment Storase
Miscellaneous Manuracturing

Mininicalpetrotolum Privecessing/s/torage

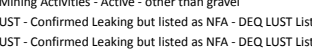

Dry Cleaners
Utility Stations/Powerplants - Maintenance/Transformer Storage
Utility Stations/Powerplants - Maintenance//Transformer storage

Utility St
Schools
Schools

Schools
Cheminal//Petroleum Processing//storage
Miscellaneous Equipment Maintenance

uto- Repair Shops

ic Manufacturing

chemica/Petroleum Processing/Storage
UST- Confirmed Leaking but Isted as NFA - DEQ Q UST List

Construction Company
Furniture/Lumber/Parts Stores

Others - Unknown Commercial
Fod Processing

Food Processing
Chemical/Petrolum Processing//5orage

others - Gov't

Golf Courses
sheols

schools

Pesticide/Fertilizer/Petroleum Storage, Handling, Mixing, \& Cleaning Areas

Wood/Pulp/Paper Processing and Mills
Wood/Pulp/paper Processing and Mills

Communications Office
Communications office
Communications office

communications office

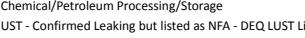

communications office

Communications Office

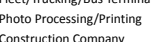

Construction compen

Auto-Repair Shops
Auto- Repair Shops

Miscellaneous Manutaturturn
Auto - Repair Shops

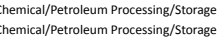

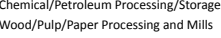

Auto - Repair Shops Proessing and Mills

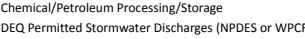

DEQ Permitited Stormuatet Discha
Fleet/rrucking/tus Terninals

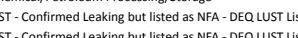
UST - Confirmed Leaking but listed as NFA- DEQ LUST Lis
UST - Confirmed Leaking but isted as NFA- DEQ UST Lis

Miscelanenous Manutucturing
Mining Activitites - Inactive- sand/ravel/rock/soil

Mining Activities - Inactive - sand/fravel//rock/f/soil 


\begin{tabular}{|c|c|c|c|c|}
\hline $\begin{array}{l}\text { Address } \\
2708 \text { N PACIFIC HWY }\end{array}$ & 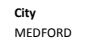 & $\begin{array}{l}\text { County } \\
\text { JaCKSON }\end{array}$ & $\begin{array}{l}\text { RET_DATE } \\
09 / 29 / 2008\end{array}$ & $\begin{array}{l}\text { Data_Sourc } \\
\text { OR State fire Marshall Hazardous Substance Information System database (SFM/HSIS - 2009) }\end{array}$ \\
\hline 2720 CRATER LAKE HWY & MEFFRD & JACKSON & 09/29/2008 & 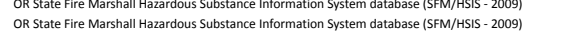 \\
\hline 3762 AVENUEH & WHITE CITY & JACKSON & 09/29/2008 & 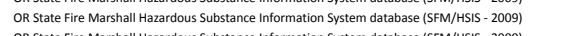 \\
\hline $\begin{array}{llll}1423 \\
1423 \text { BENTTOOOA RD }\end{array}$ & $\begin{array}{l}\text { EAGLE PNINT } \\
\text { ASHLAD }\end{array}$ & $\begin{array}{l}\text { JACKSON } \\
\text { JACKSON }\end{array}$ & $\begin{array}{l}09 / 29 / 2008 \\
00 / 2920008\end{array}$ & \\
\hline 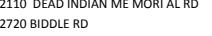 & $\begin{array}{l}\text { AAHHAND } \\
\text { Medford }\end{array}$ & $\begin{array}{l}\text { JAACKON } \\
\text { JACKSON }\end{array}$ & 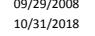 & 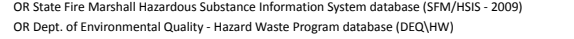 \\
\hline 2720 BIDDLE RD & 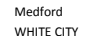 & JACKSON & 10/31/2018 & OR Dept. of Environmental Quality - Leaking Underground Storage Tank datababse (DEQQIUST-NFA) \\
\hline $\begin{array}{l}877 \text { AVENAUE G } \\
\text { 27220 CRATRR LAKE HWY }\end{array}$ & $\begin{array}{l}\text { WhHTrt Ciry } \\
\text { Medford }\end{array}$ & JACKSON & $\begin{array}{l}09 / 29 / 2008 \\
10 / 1 / 2018\end{array}$ & 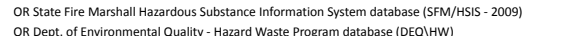 \\
\hline 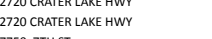 & $\begin{array}{l}\text { Medoror } \\
\text { Medford }\end{array}$ & $\begin{array}{l}\text { JACKSON } \\
\text { JACSON }\end{array}$ & $\begin{array}{l}10 / 3172018 \\
10 / 31 / 2018\end{array}$ & 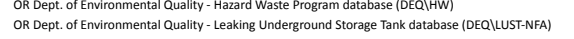 \\
\hline $\begin{array}{l}7750 \text { TTH ST } \\
2730 \mathrm{NPCFCHWY}\end{array}$ & $\begin{array}{l}\text { WHITE CITY } \\
\text { MEEFRD }\end{array}$ & JACKSON & $\begin{array}{l}09 / 29 / 2008 \\
009 / 292008\end{array}$ & 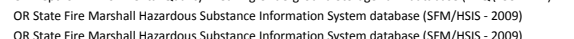 \\
\hline 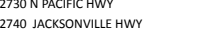 & $\begin{array}{l}\text { OFORB } \\
\text { OFORD }\end{array}$ & $\begin{array}{l}\text { JAAKSON } \\
\text { JACKSON }\end{array}$ & 29/20008 & 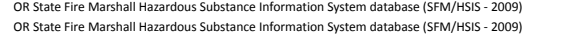 \\
\hline 8251 194H ST & ITE CII & $\begin{array}{l}\text { Jackson } \\
\text { IACKSON }\end{array}$ & $29 / 2008$ & 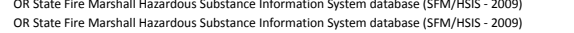 \\
\hline 6473 CRATER LAK & IRALPOIN & JACKSON & 29/2008 & 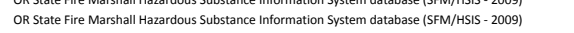 \\
\hline 2740 JACKSONVIL & PFORD & JACKSON & 09/29/2008 & 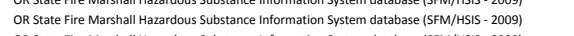 \\
\hline 1385 JUSTCEC RD & 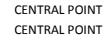 & JAckson & $\begin{array}{l}09 / 29 / 2008 \\
00 / 202008\end{array}$ & 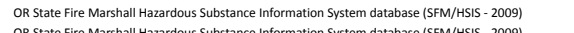 \\
\hline 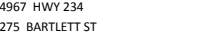 & 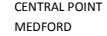 & JACCKSON & 220/2/2008 & \\
\hline 2456 AST RTLT 20. & $\begin{array}{l}\text { DOFRO } \\
\text { HAND }\end{array}$ & $\begin{array}{l}\text { JAACKSON } \\
\text { JACKSON }\end{array}$ & 292/2008 & 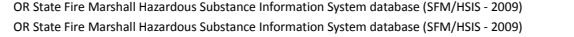 \\
\hline & EJUNC & JOSEPHINE & 219/2008 & 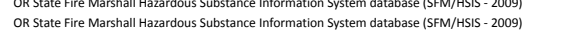 \\
\hline & 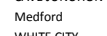 & JackSON & 31/2018 & 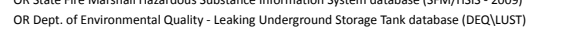 \\
\hline 27004 4TH: & Tie Ch & Jackson & /29/2008 & State Fire Marshall Hazardous Substance Information System databasese (SFM/HSIS- 2009) \\
\hline $\begin{array}{l}2755 \text { SSH ST } \\
\text { 5598 TABLE ROCK RD STE } 102\end{array}$ & dford & JACKSON & 13/1/2018 & \\
\hline $\begin{array}{l}55998 \text { 8ABLER ROCK RD STE } 102 \\
1099 \text { B ST }\end{array}$ & 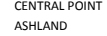 & $\begin{array}{l}\text { SACCSSON } \\
\text { JACKSON }\end{array}$ & 212/2008 & 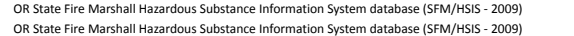 \\
\hline 30 KEE LN & SHADY COVE & JACKSON & 09/29/2008 & 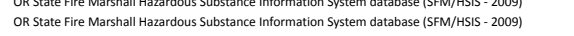 \\
\hline $\begin{array}{l}1508 \text { W WATTLOPE RD } \\
\text { 2764 W MAIN ST }\end{array}$ & 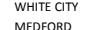 & JACKSON & 09/29/2008 & 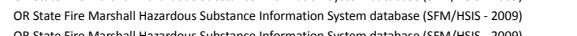 \\
\hline 2764 W MAIN ST & MEDFORD & JACKSON & 20/2008 & 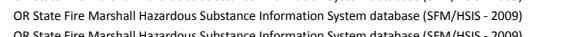 \\
\hline 2266 W MAANS & $\begin{array}{l}\text { MEEFORD } \\
\text { TALENT }\end{array}$ & 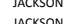 & $29 / 2008$ & 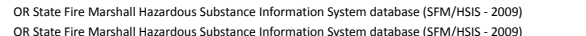 \\
\hline 3590 N RIVER RD & (GOD HIL & $\begin{array}{l}\text { JAAKSON } \\
\text { JACKSON }\end{array}$ & $\begin{array}{l}090 / 2992008 \\
09 / 29 / 2008\end{array}$ & 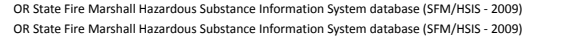 \\
\hline 2770 CRATER LAKE HWY & MEDFORD & JACKSON & 09/29/2008 & 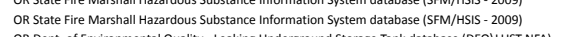 \\
\hline 2790 CRATERR LAKE HWY & $\begin{array}{l}\text { Mefford } \\
\text { Mefford }\end{array}$ & JACKSON & $\begin{array}{l}10 / 31 / 2018 \\
10 / 312018\end{array}$ & \\
\hline $\begin{array}{l}2797 \text { MRERRAMN RD } \\
285 \text { GROVELAND }\end{array}$ & deford & JACKSON & $\begin{array}{l}10 / / 11 / 218 \\
10 / 31 / 2018\end{array}$ & 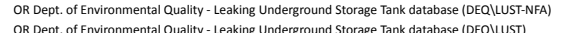 \\
\hline $\begin{array}{l}288 \text { GROVVELAND } \\
2800 \text { CRAER LAKE HWY }\end{array}$ & $\begin{array}{l}\text { Metord } \\
\text { MEEFORD }\end{array}$ & $\begin{array}{l}\text { JAAKSON } \\
\text { JACKSON }\end{array}$ & 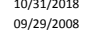 & 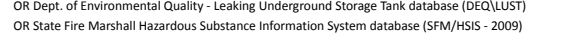 \\
\hline SOLE RO & $\begin{array}{l}\text { Mefford } \\
\text { cefrop }\end{array}$ & JACKSON & 年 & 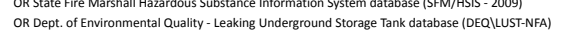 \\
\hline 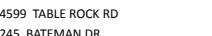 & NTRALPC & JACKSON & 9/2008 & 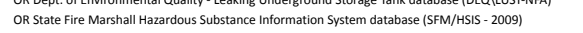 \\
\hline & NTRAL POINT & JACKSON & 9/2008 & R State Fire Marshall Hazardous Substance Infformation System databasese (SFM/HISIS - 2009) \\
\hline $\begin{array}{l}1970 \text { CANES HWV } \\
990 \\
9 \text { UPERR RVER RD }\end{array}$ & $\begin{array}{l}\text { CAVEUNCTION } \\
\text { GOLD HIL }\end{array}$ & IOSEPHINE & o9/29/2/2008 0 & 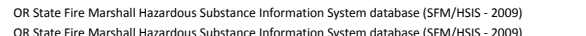 \\
\hline 2825 E BARNETT RD & Medford & JACKSON & 10/31/2018 & 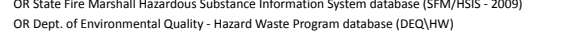 \\
\hline $\begin{array}{l}2825 \text { E BARNETT RD } \\
\text { 2825 E BRBNTT RD }\end{array}$ & $\begin{array}{l}\text { MEFFORD } \\
\text { MEFORD }\end{array}$ & JACKSON & $\begin{array}{l}09 / 29 / 2008 \\
002 / 20008\end{array}$ & OR State Fire Marshall Hazardous Substance Information System database (SFM/HISIS-2009) \\
\hline $\begin{array}{l}2825 \text { BAANEETI } \\
1050 \text { BENSON }\end{array}$ & $\begin{array}{l}\text { MEFFORD } \\
\text { ASHLAND }\end{array}$ & JACKSON & $\begin{array}{l}0.9 / 29 / 2 / 2008 \\
00 / 2 / 2008\end{array}$ & 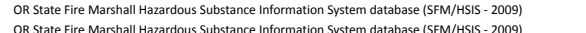 \\
\hline 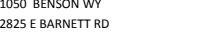 & ChaAOord & $\begin{array}{l}\text { JaCCSSON } \\
\text { IACCSON }\end{array}$ & 13/2008 & 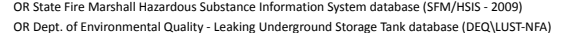 \\
\hline ЕTRD & OFFR & Jackson & 102/2015 & 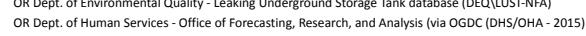 \\
\hline ITIN & 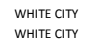 & JacksoN & 9/2008 & 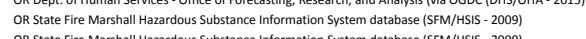 \\
\hline & HITE CITY & JaCKSON & 9/2008 & 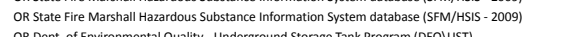 \\
\hline 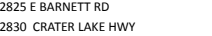 & $\begin{array}{l}\text { Mefford } \\
\text { MEOFORD }\end{array}$ & JACKSON & 1/2018 & 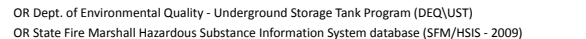 \\
\hline 2830 CRATER LAKE HWY & MEFFORD & JACKSON & 09/29/2008 & 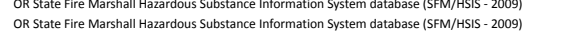 \\
\hline 2833 N PACFIC HWY & MEDFORD & JACKSON & 09/29/2008 & OR Sate fire Marshall Hazardous Substance Information System database (SFM/HISIS-2009) \\
\hline 4764 TABLE ROCK RD & $\begin{array}{l}\text { CENTARAL POINT } \\
\text { CENTRA PONT }\end{array}$ & AACKSON & & 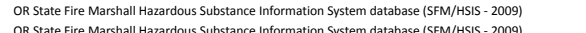 \\
\hline RD & 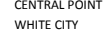 & JACCKSON & $\begin{array}{l}0.9 / 29 / 2 / 2008 \\
00 / 2 / 2008\end{array}$ & 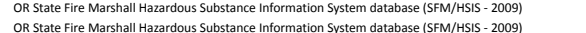 \\
\hline & NTRALPOINT & $\begin{array}{l}\text { JACCSON } \\
\text { JaCKON }\end{array}$ & 292/2008 & 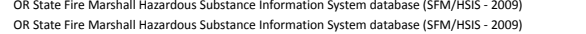 \\
\hline & dford & JacksoN & 102/2015 & 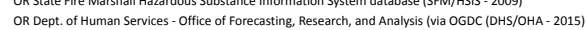 \\
\hline & OFORD & JACKSON & 9/29/2008 & 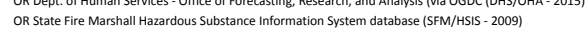 \\
\hline 2840 & EFFRO & JACKSON & 09/29/2008 & 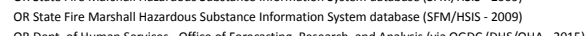 \\
\hline & diford & & & R Dept. of Human Services - Office of Forecasting, Research, and Analysis (via OGDC COHS/OHA- 201 \\
\hline & TrRAL & $\begin{array}{l}\text { PACCSSON } \\
\text { JACKSON }\end{array}$ & 2/201015 & 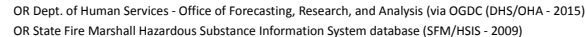 \\
\hline & & JACKSON & 1/2018 & 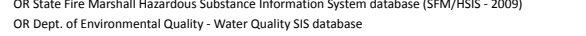 \\
\hline & & & & 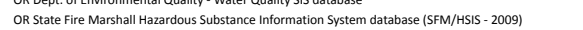 \\
\hline & & JACKSON & 9/2008 & SState Fire Marshall Hazardous Substance Information System database (SFM/HSIS - 2009) \\
\hline & $\begin{array}{l}\text { ASHLAND } \\
\text { MEOFORD }\end{array}$ & JACKSON & & 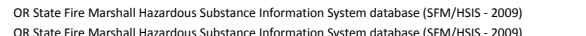 \\
\hline & $\begin{array}{l}\text { MEFORD } \\
\text { ROGUERI }\end{array}$ & AACCSSON & & \\
\hline & ASHLAND & JACKSON & 09/29/2008 & \\
\hline OR & MEDFORD & JACKSON & 129/2008 & 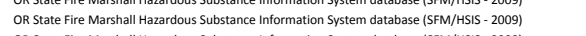 \\
\hline LOOP RD & NEE JUNCTION & JOSEPHINE & 0008 & 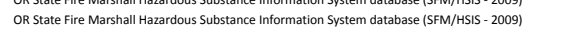 \\
\hline & & JACKS. & t/2008 & tance Information System database (SFM/HSIS - 2009) \\
\hline & & JaCKSON & & 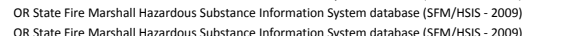 \\
\hline $\begin{array}{l}\text { NSEE DR } \\
\text { BER ROCK RD }\end{array}$ & $\begin{array}{l}\text { MEDOOD } \\
\text { CENTRALOINT }\end{array}$ & $\begin{array}{l}\text { JAACSSON } \\
\text { JACKSON }\end{array}$ & $\begin{array}{l}09 / 29 / 2008 \\
09 / 29 / 2008\end{array}$ & 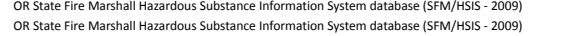 \\
\hline ANSENST & Medford & JACKSON & 10/31/2018 & 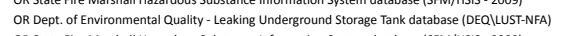 \\
\hline 212 EAST MAIN ST & ASHLAND & JaCKSON & 09/29/2008 & OR State Fire Marshall Hazardous Substance Information S System database (SFM/HISIS-2009) \\
\hline $\begin{array}{l}29 \text { MYRTLEST } \\
652 \text { S VALEY VIEW R }\end{array}$ & $\begin{array}{l}\text { MEEFFRD } \\
\text { ASHAND }\end{array}$ & JACKSON & $\begin{array}{l}0.9 / 29 / 2 / 2008 \\
00 / 2 / 2008\end{array}$ & 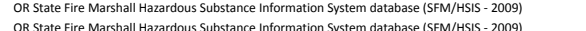 \\
\hline $\begin{array}{l}3464 \text { WILOOW SPRIN } \\
345\end{array}$ & $\begin{array}{l}\text { ASHALNO } \\
\text { CENTRAL POINT }\end{array}$ & JACKSON & 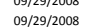 & 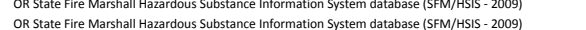 \\
\hline 150 NICK YOUNG RD & EAGLE POINT & JACKSON & $\begin{array}{l}0.01 / 29 / 20008 \\
09 / 2008\end{array}$ & 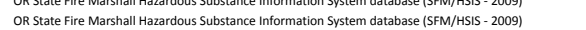 \\
\hline 29 W MAIN ST & Medford & Jackson & 10/31/2018 & OR Dept. of Environmental Quallity - Leaking Underground Storage Tank database (DEQ| UUST-NFA) \\
\hline 794 OD STAGE RD & $\begin{array}{l}\text { CENTRAL POINT } \\
\text { ASLLAD }\end{array}$ & JACKSSON & $\begin{array}{l}09 / 29 / 2008 \\
090 / 2920008\end{array}$ & 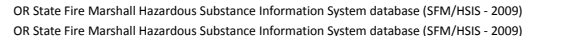 \\
\hline
\end{tabular}

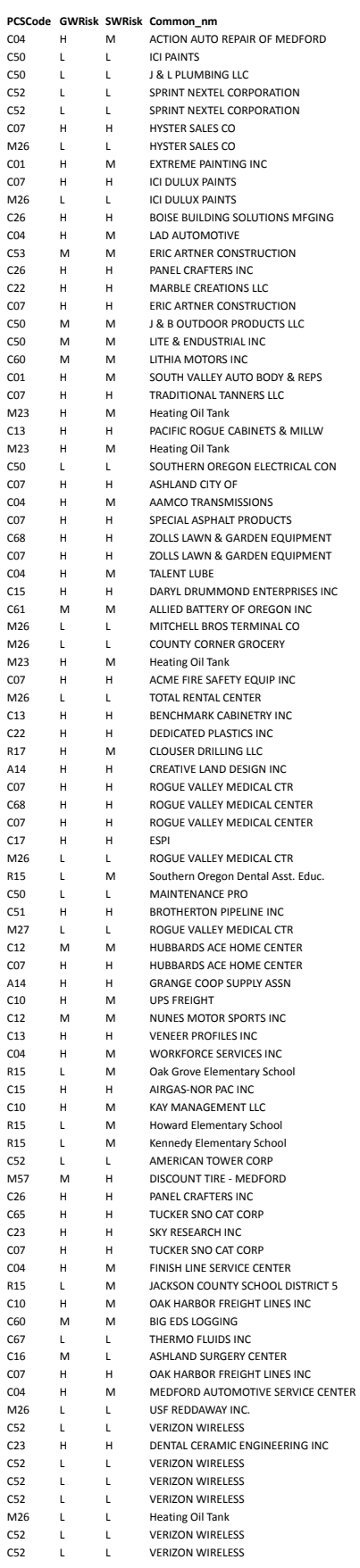

PCSType
Auto- Repair Shops

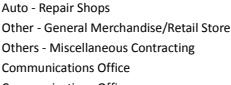
Chemical/Petroleum Processing/Strorage
UST - confirmed Leaking but isted as NAA - DEQ LUST List

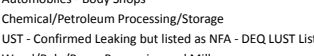

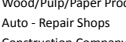

construction Compary Wood/Pull/Paper Processing and Mills
Plastics/Synthetics Proeducer

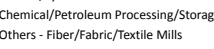
gi/Home contracting

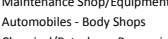

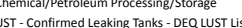
Home Manufacturing
UST - Confirmed Leaking Tanks - DEQ LusT Lis Others- - Miscellaneous Contracting
chemical Petrolum Processing StStrage uuto- Repair Shops Chemical/Petroleuu Processing//torage
Miscellaneus Equipment taintenance
Chemical Petroleum Processing/Storage Auto- Repair Shops
Machine Shops

Maintenance Shop//Fuipment- Transportation Related
UST - Confirmed Leaking but listed a s NFA - DEQ WuST L
$L$

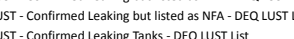
Chemical/Petroleum Processing UST - Confirmed Leaking but listed as N NFA- DEQ Q WST Lis Home Manutacturing
Plastics/lynnthetics
roducuer

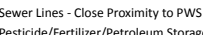
Pesticided frettizen, Miscellaneous Equipment Maintenance Chemiea/petroleum Processing/storarge UST- Confirmed Leaking but listed a N NAA - DEQ LUST Lit Schools
Others- Miscellaneous Contracting
Pipelines - Chemical and Petroleum UST - Upgraded andor Registered -Active (may also have decommissioned tanks on site)

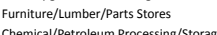
Hrage, Handling, Mixing, \& Cleaning Areas Fleet//Tucking//us Terminals
Furniture/Lumber/Parts Stores

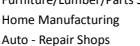
Auto-Repair Shoors
Schools Schools
Machine Sho Machine Shops
Fleet/Trucking/Bus Terminats
Schools Schools
Schools

Schools
Communications office
DEQ Permitted Storm (NDES or WPC)

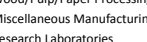
enical/Petroleum Processing/Stororae Auto- Repair Shops
schools schools
Fleet/Trucking/Bus Terminals Warehouses
Medical/vet Offices

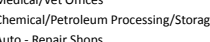
Auto- Repair Shops
UST- Confirmed Leaking but listed a s NFA- DEQ Q UST List Communications Office
Research haboratories Communications Officice
Communications office
Communications offerite UST- Confirmed Leaking but Isted as N NFA- DEQQ LUST Lis communications office
communications office 


\begin{tabular}{|c|c|c|c|c|}
\hline $\begin{array}{l}\text { Address } \\
29 \text { W MAIN ST }\end{array}$ & $\begin{array}{l}\text { City } \\
\text { Mediord } \\
\text { tats }\end{array}$ & $\begin{array}{l}\text { County } \\
\text { JACKSON }\end{array}$ & $\begin{array}{l}\text { RET-DARE } \\
\text { 10/31/2018 }\end{array}$ & 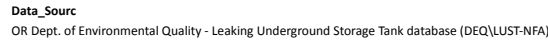 \\
\hline 2901 E BARNETT RD & MEDFORD & JACKSON & . & 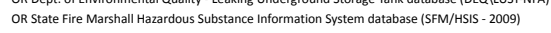 \\
\hline 2901 E BARNETT RD & MEDFORD & JaAKSON & 09/29/2008 & OR State fire Marshall Hazard dous Substance Information System database (SFM/HSIS - 2009) \\
\hline $\begin{array}{l}2917 \text { COMCCD DR } \\
940 \text { AYRES RD }\end{array}$ & $\begin{array}{l}\text { Medford } \\
\text { EAGLE POINT }\end{array}$ & $\begin{array}{l}\text { JAAKSON } \\
\text { JACKSON }\end{array}$ & $\begin{array}{ll}103 / 1 / 2018 \\
0 / 29 / 2008\end{array}$ & 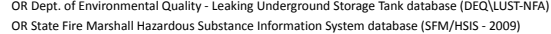 \\
\hline 327 BROAD ST & 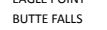 & JACKSON & 09/29/2008 & 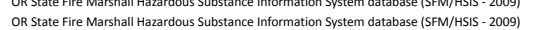 \\
\hline 324 MILL CREEK RD & PROSPECT & 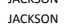 & 09/29/2008 & 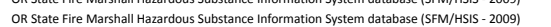 \\
\hline 2650 AVENUE G & WHITE CITY & JaCKSON & 09/29/2008 & 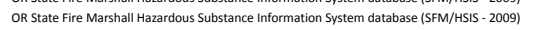 \\
\hline 441 W ANTELOPE RD & WHITE CITY & JACKSON & 09/29/2008 & OR State fire Marshall Hazarddous Substance Information System database (SFM/HSIS - 2009) \\
\hline $\begin{array}{l}2917 \text { N PACFIC HWY } \\
2919 \text { N PCAFC HWY }\end{array}$ & $\begin{array}{l}\text { Mefford } \\
\text { Mefrogi }\end{array}$ & IACKSON & 10/11/2018 & OR Dept. of Environmental Quality - Leaking Underground Storage Tank database (DEQQIUSST-N \\
\hline & $\begin{array}{l}\text { MEDEFRD } \\
\text { MEDFORD }\end{array}$ & $\begin{array}{l}\text { JACKSON } \\
\text { ICCKSON }\end{array}$ & 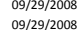 & 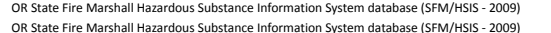 \\
\hline 15 S PIONERR ST & $\begin{array}{l}\text { MEDEFRO } \\
\text { ASHLAND }\end{array}$ & $\begin{array}{l}\text { JACSKON } \\
\text { IACKSON }\end{array}$ & $\begin{array}{l}0.9929 / 2008 \\
09 / 29 / 2008\end{array}$ & 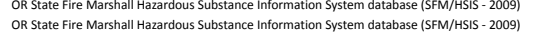 \\
\hline 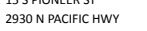 & MEDFORD & IACKSON & 09/29/2008 & 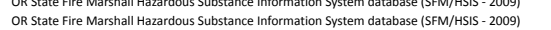 \\
\hline 4495 TABLE ROCK RD A & CENTRAL POINT & JACKSON & 09/29/2008 & \\
\hline S49 EVILAS RD & CENTRAL POINT & JACKSON & 09/29/2008 & OR State fire Marshall Hazarddous Substance Information System database (SFM/HIS - 2009) \\
\hline 2930 N PACFIC HWY & $\begin{array}{l}\text { MEOEORD } \\
\text { MEPOOPRO }\end{array}$ & IACKSON & 09/29/2008 & \\
\hline $\begin{array}{l}2961 \text { N A ACIFI HWY } \\
2978 \text { C RATER LAKE HWY }\end{array}$ & MEOFORD & IACKSON & 09/29/2008 & 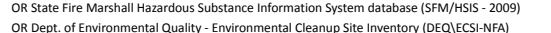 \\
\hline 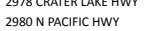 & $\begin{array}{l}\text { MNedord } \\
\text { MEOFORD }\end{array}$ & $\begin{array}{l}\text { JACKSON } \\
\text { ICCKSON }\end{array}$ & $\begin{array}{ll}109 / 1 / 21 / 2018 \\
0 / 292 / 2008\end{array}$ & 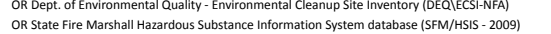 \\
\hline 2980 N PACFFC HWY & $\begin{array}{l}\text { MEUERK } \\
\text { MEOFORD }\end{array}$ & $\begin{array}{l}\text { IACCSON } \\
\text { ICSON }\end{array}$ & 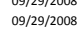 & 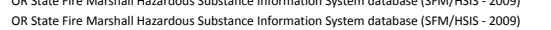 \\
\hline 2985 CRATER LAKE HWY & Medford & JACKSON & 10/11/2018 & 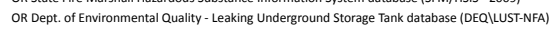 \\
\hline 875 AVE G STE B & WHITE CITY & JACKSON & $09 / 29 / 2008$ & OR State Fire Marshall Hazardous Substance Information System database (SFM/HSIS - 2009) \\
\hline 2989 SECKEL ST & Medford & JACKSON & $10 / 13 / 21 / 218$ & \\
\hline $\begin{array}{l}2900 \text { N PAAFICICHWY } \\
608 \text { N MAI ST }\end{array}$ & $\begin{array}{l}\text { Medford } \\
\text { PHOENIX }\end{array}$ & JACKSON & $\begin{array}{l}109 / 1 / 212018 \\
0 / 292 / 2008\end{array}$ & 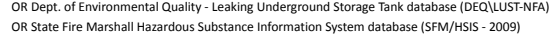 \\
\hline 540 YMCAWY & ASHLAND & JACKSON & 09/29/2008 & 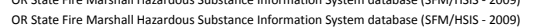 \\
\hline 300 S OAKDALE & Medford & JACKSON & 10/13/2018 & 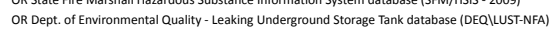 \\
\hline 6422 BLACKWELL RD & CENTRAL POINT & Jackson & $09 / 29 / 2008$ & OR State fire Marshall Hazarddous Substance information System database (SFM/HSIS- 2009) \\
\hline $4410 \mathrm{~N} \mathrm{PACFFC} \mathrm{HWY}$ & CENTRAL POINT & JACKSON & $09 / 29 / 2008$ & OR State fire Marshall Hazarddous Substance Information System database (SFM/HSIS- 2009) \\
\hline 3000 CRATER LAKE & MEDFRO & IACKSON & $09 / 29 / 2008$ & oR State fire Marshall Hazard dous Substance Information System database (SFM/HISIS- 2009) \\
\hline $\begin{array}{l}3000 \text { CRAARE LAKE HWY } \\
3000 \text { CRATR LAKE HWY }\end{array}$ & $\begin{array}{l}\text { Medoford } \\
\text { Mefford }\end{array}$ & $\begin{array}{l}\text { JaCKSON } \\
\text { JACKSON }\end{array}$ & 101/1/2018 $11 / 2018$ & 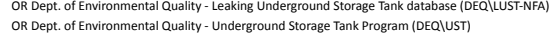 \\
\hline 11500 HANNON RD & EAGLE POINT & 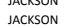 & $\begin{array}{l}111 / 12018 \\
09 / 29 / 2008\end{array}$ & 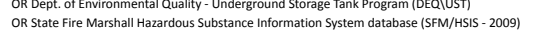 \\
\hline 1111 MILL CREEK DR & PROSPECT & Jackson & 09/29/2008 & 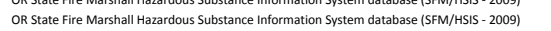 \\
\hline 3001 BIDDLE RD & MEDFORD & JACKSON & 09/29/2008 & OR State fire Marshall Hazarddous Substance Information System database (SFM/HSIS - 2009) \\
\hline 3001 BIDOLE RD & Medford & JACKSON & 10/31/2018 & OR Dept. of Environmental Quality - Hazard Waste Program database (DEQ|HW) \\
\hline 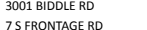 & $\begin{array}{l}\text { Medford } \\
\text { GoD HIL }\end{array}$ & JAAKSON & $\begin{array}{l}10 / 13 / 21 / 2018 \\
0992 / 2008\end{array}$ & OR Dept. of Environmental Quality - Leaking Underground Storage Tank database (DEQQ|lust-NFA) \\
\hline $\begin{array}{l}7351 \text { OANGASER RD } \\
431\end{array}$ & 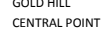 & $\begin{array}{l}\text { JaCSSON } \\
\text { IACKSON }\end{array}$ & 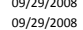 & 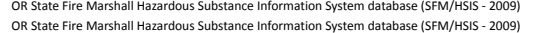 \\
\hline 7893 PACFIC AVE & WHITE CITY & 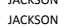 & 09/29/2008 & 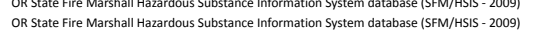 \\
\hline 5186 CRATE LAKE HWY & CENTRAL POINT & JACKSON & $09 / 29 / 2008$ & 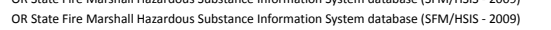 \\
\hline 470 W ANTELOPE RD & WHITE CITY & JACKSON & 09/29/2008 & OR State fire Marshall Hazarddous Substance Information System database (SFM/HSIS - 2009) \\
\hline 8205 CRATER LAKE HWY & $\begin{array}{l}\text { WHITE CIVY } \\
\text { MESEFogD }\end{array}$ & JACKSON & 09/29/2008 & OR State fire Marshall Hazard dous Substance Information System database (SFM/HSIS - 2009) \\
\hline 3504 NORTH RIVER RD & $\begin{array}{l}\text { MEFORD } \\
\text { GOODHIL }\end{array}$ & JACKSON & 09/29/2/2008 0 & 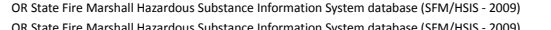 \\
\hline 5465 HOLAND LP & 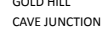 & JOSEPHINE & 09/29/2008 & $\begin{array}{l}\text { of } \\
\text { of }\end{array}$ \\
\hline 25320 REDWOOD HWY & CAVE UUNCTION & JOSEPHINE & 09/29/2008 & 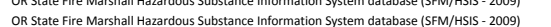 \\
\hline 112 REDWOOD HWY & CAVE JUNCTION & JOSEPHINE & $09 / 29 / 2008$ & 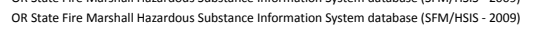 \\
\hline 232 NREDWOOD HWY & CAVE JUNCTION & JOSEPHINE & 09/29/2008 & OR State fire Marshall Hazarddous Substance Information System database (SFM/HIS - 2009) \\
\hline 4095 REDWODOH HWY & CAVE JUNCTION & JOSEPHINE & 09/29/2008 & OR State fire Marshall Hazardous Substance Information System database (SFM/HSIS- 2009) \\
\hline $\begin{array}{l}11001 \text { C CVESHWY } \\
1713 \text { SISKYYOU BLVD }\end{array}$ & 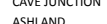 & 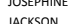 & 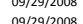 & 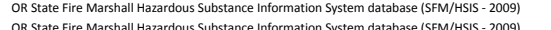 \\
\hline 300 DEAD INDIAN RD & ASHANO & 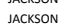 & 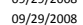 & 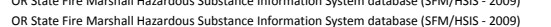 \\
\hline 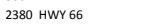 & ASHLAND & JACKSON & 09/29/2008 & 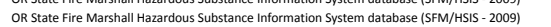 \\
\hline $2500 \mathrm{HWr} 66$ & ASHLAND & JACKSON & 09/29/2008 & OR State Fire Marshall Hazardous Substance Information System database (SFM/HSIS- 2009) \\
\hline 60 SHAMROCK LN & ASHLAND & JACKSON & 09/29/2008 & OR State fire Marshall Hazarddous Substance Information System database (SFM/HSIS - 2009) \\
\hline 403 DEAD INDIAN RD & ASHANDO & JACCSSON & 09/292/2008 & OR State Fire Marshall Hazard dous Substance Information System database (SFM/HSIS- 2009) \\
\hline 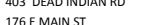 & 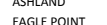 & 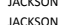 & 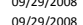 & 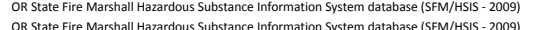 \\
\hline 500 MOUNTAIN AVE & AStlaNo" & $\begin{array}{l}\text { IACKSON } \\
\text { ICSO }\end{array}$ & 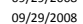 & 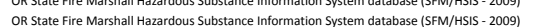 \\
\hline 2110 DEAD INDIAN RD & ASHLAND & Jackson & 09/29/2008 & 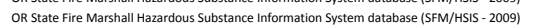 \\
\hline 2275 DEAD INDIAN RD & ASHLAND & JACKSON & 09/29/2008 & OR State fire Marshall Hazardous Substance Information System database (sFM/HIS- 2009) \\
\hline 2275 DEAD INDIAN RD & ASHLAND & JACKSON & 09/29/2008 & OR State fire Marshall Hazarddous Substance Information System database (SFM/HSIS- 2009) \\
\hline 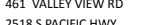 & ASHANO & A IACSSONO & 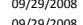 & OR State fire Marshall Hazarddous Substance Information System database (SFM/HISIS- 2009) \\
\hline 1309 S PACIFIC HWY & 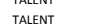 & 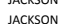 & 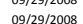 & 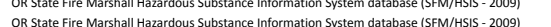 \\
\hline & TALENT & JACKSON & 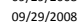 & 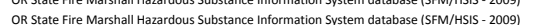 \\
\hline 11199 PACIFC HWY & TALENT & JACKSON & $09 / 29 / 2008$ & OR State Fire Marshall Hazardous Substance Information System database (SFM/HISS- 2009) \\
\hline 1119 S PACIFIC HWr & TALENT & JACKSON & 09/29/2008 & OR State fire Marshall Hazardous Substance Information System database (SFM/HIS- 2009) \\
\hline 11200 PACIFIC HWV & TALENT & JACKSSON & 09/29/2008 & OR State Fire Marshall Hazard dus Substance Information System database (SFM/HIS - 2009) \\
\hline 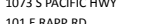 & TA & 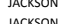 & 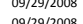 & OR State fire Marshall Hazardous Substance Information System database (SFM/HSIS - 2009) \\
\hline 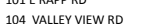 & TALENT & 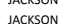 & 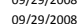 & 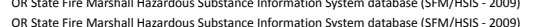 \\
\hline & TALENT & JACKSON & 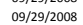 & 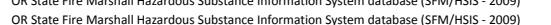 \\
\hline NEW RD & TALENT & JACKSON & 09/29/2008 & tion System datababese (SFM/HASIS- 2009) \\
\hline 251 VALEYY VIEW RD & TALlent & JACKSON & 09/29/2008 & OR State Fire Marshall Hazardous Substance Information System database (SFM/HSIS- 2009) \\
\hline 251 WVaLU & TALENT & JACKSON & 09/29/2008 & 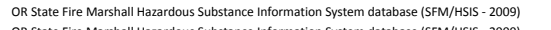 \\
\hline & & & & ISe (SFM/HSIS - 2009) \\
\hline & 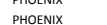 & 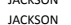 & 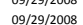 & 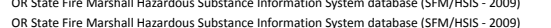 \\
\hline & & JACCSON & & $\begin{array}{lll} & \end{array}$ \\
\hline Fic HWr & & JACKSON & & tabase (SFNM/HSIS- 20099) \\
\hline & OFFRO & JACKSON & 29/2008 & tem database (SFM/HSIS - 2009) \\
\hline & $\begin{array}{l}\text { Medford } \\
\text { MEDFop }\end{array}$ & JaCKSON & 10/11/2018 & (2) \\
\hline 301 N CENTRAL AVE & MEOPORD & JACKSON & 09/29/2008 & OR State Fire Marshall Hazarddous Substance Information System databasese (SFM/HSIS - 20099) \\
\hline
\end{tabular}

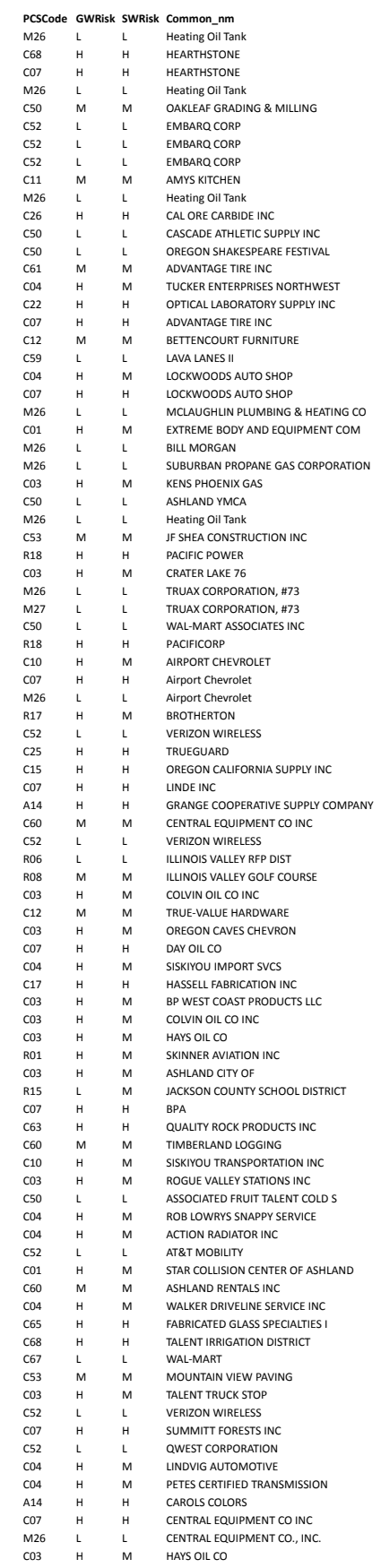

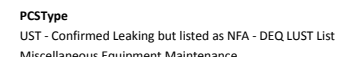

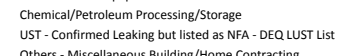
communications office
communications office

Communications
Food Processing

Food Processing
UST - Confirmed Leaking but listed as NFA- DEQ LUST List

Wood/Pulp/Paper Processing and Mills
Other- General Merchandise/Retail Store
others - Sports//Entertainment Activities

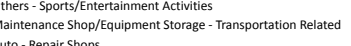

Auto- Repair Shops
Plastics/snnthetics roducer

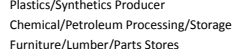

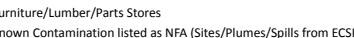

Auto- Repair Shops

Chemical/Pertoleum Processing/Stororge
UST - Confirmed Leaking but listed as NFA - DEQ LusT Liss

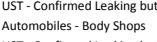

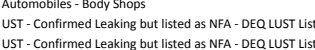

Automobilies- Gas trations

Others - Sports/ ntertatinment

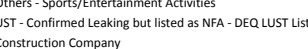

Construction Company
Utility Stations/Powerplants - Maintenance/Transformer Storage

Automobilies - Gas Stations
UST - Confirmed Leaking but listed as NFA - DEQ W UST Lis

UST- Confirmed Leaking but listed as NFA- DEQ Lust List
UST - Upgraded and/or Registered - Active (may also have decommissioned tanks on site)

Other- General Merchandise/Retail Store
Utility Stations/Powerplants - Maintenance/Tran sormer S Storage

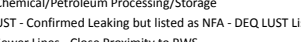

Sewer Lines - Close Proximity to PUS
Communications office

Wood Presenving/rireating

Machine Shops
Chemical/Petroleum Processing/Storage

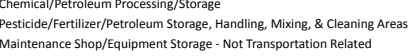

Eire Station

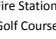

Automobilies - Gas Stations
Eurniture/Lumber//Parts Stores

Autritureabiles - Gers statitions
Chemical/Petroleum Processing/storase

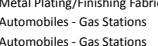

Automobilies - Gas Stations
Automobilies - Gas stations

Airport-Maintenance/fueling Are
Automobiles - Gas tsations

Chemical/Petrolum Processing/storage
Mining Activities - Active - other than grave

the

Fleet/Trucking//Bus Terminals
Automobiles - Gas stations

Other- Groceries/Marlest
Auto - Repari Shops
Wuto- Repair Shooss

Communications office

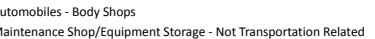

Auto Repair shops

Miscellaneous Manufacturing
Miscellaneous Gquipment Maintenance

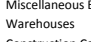

Construction company
Automobiles - Gas Stations
Communictions office

Chemical/Petrotolum
Chrice

Communications office
Auto- Repair Shos
Auto - Repair Shops

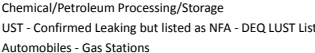




\begin{tabular}{|c|c|c|c|c|}
\hline $\begin{array}{ll}\text { Address } \\
204 \\
\text { MAN ST }\end{array}$ & $\begin{array}{l}\text { City } \\
\text { PAULINA }\end{array}$ & $\begin{array}{l}\text { County } \\
\text { CROoK }\end{array}$ & $\begin{array}{l}\text { RE_DATE } \\
09 / 29 / 2008\end{array}$ & $\begin{array}{l}\text { Data_sourc } \\
\text { OR State fire Marshall Hazarddous Substance Information System database (SFM/HISI- 2009) }\end{array}$ \\
\hline 301 N CENTRAL AVE & Metford & JACKSON & 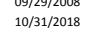 & 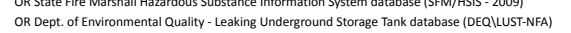 \\
\hline 301 N CENTRAL AVE & Medford & JACKSON & $111 / 1 / 2018$ & OR Dept. of Envirommental Q quality - Underground Storage Tank Program (DEQQUSST) \\
\hline 3011 Crater Lake Hwy & $\begin{array}{l}\text { Medford } \\
\text { Mestort }\end{array}$ & & $1 / 1 / 1 / 2018$ & OR Dept. of Geology and Mineral Industries Mineral Information layer for Oregon( DOGAMI/MLO) \\
\hline & $\begin{array}{l}\text { Medford } \\
\text { MEOFORD }\end{array}$ & $\begin{array}{l}\text { Jackson } \\
\text { Accsson }\end{array}$ & $\begin{array}{l}1 / 1 / 1 / 2018 \\
09 / 29 / 2008\end{array}$ & 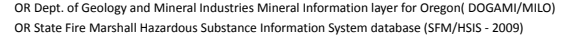 \\
\hline $\begin{array}{l}3022 \text { N FRONT ST } \\
\text { 302 HWY }\end{array}$ & $\begin{array}{l}\text { MEOOORO } \\
\text { MEOFORD }\end{array}$ & $\begin{array}{l}\text { IACSSON } \\
\text { IACSSON }\end{array}$ & $\begin{array}{l}0 \text { 90/299/2008 } \\
09 / 29 / 2008\end{array}$ & 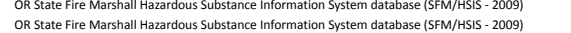 \\
\hline 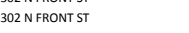 & doford & JACKSON & 年 & \\
\hline O BULLOCK RD & DFORD & JACKSON & 09/29/2008 & OR State Fire Marshall Hazardous Substance Information System database (SFMM/HIS- 2009) \\
\hline 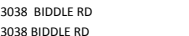 & MEDFORD & JACKSON & 09/29/2008 & 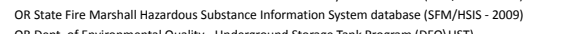 \\
\hline 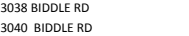 & $\begin{array}{l}\text { efford } \\
\text { EFFoRD }\end{array}$ & IACKSON & $\begin{array}{l}11 / 1 / 2018 \\
00 / 292 / 2008\end{array}$ & 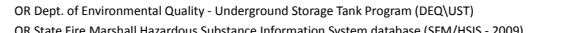 \\
\hline $\begin{array}{l}3040 \text { BBDDER RD } \\
3040 \text { BIIDOE RD }\end{array}$ & efford & $\begin{array}{l}\text { JaCKSON } \\
\text { ICCKSON }\end{array}$ & 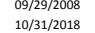 & 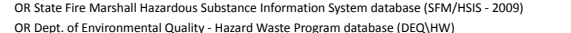 \\
\hline 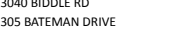 & edford & $\begin{array}{l}\text { IACSSON } \\
\text { IACSSON }\end{array}$ & $\begin{array}{l}10 / 1 / 12018 \\
10 / 11 / 2018\end{array}$ & 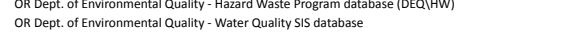 \\
\hline 305 SFIR ST & EDFRD & IACCSON & $09 / 29 / 2008$ & 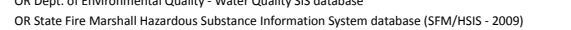 \\
\hline 3050 S PACIFIC HWY & doford & JACKSON & 10/31/2018 & 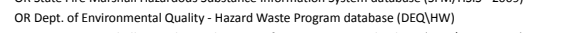 \\
\hline $\begin{array}{l}3050 \text { S PACFIC HWY } \\
3070 \text { ROSS }\end{array}$ & $\begin{array}{l}\text { MEDFORD } \\
\text { Mefford }\end{array}$ & JACKSON & 09/29/2008 & OR State Fire Marshall Hazardous Substance Information System database (SFM/HSIS - 2009) \\
\hline $\begin{array}{l}3070 \text { OOSS LN } \\
3070 \text { Ross Ln }\end{array}$ & edford & JACKSON & $\begin{array}{l}10 / 31 / 2018 \\
060 / 022015\end{array}$ & OR Dept. of Environmental Quality - Leaking Underground Storage Tank database (IEQQLUST-NFA) \\
\hline $\begin{array}{l}3070 \text { Ross In LARE AVE } \\
3074 \text { CRATE LAK AVE }\end{array}$ & $\begin{array}{l}\text { Nefford } \\
\text { UEFORD }\end{array}$ & JaCSKON & $\begin{array}{l}66 / 2 / 2015 \\
9 / 2 / 2008\end{array}$ & 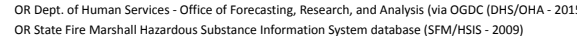 \\
\hline 3074 CRATER & Nefford & 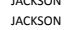 & 0/31/2018 & 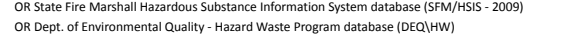 \\
\hline 3074 CRATE & edford & IACCSON & 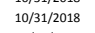 & \\
\hline 3088 EARNE & $\begin{array}{l}\text { deford } \\
\text { DFopg }\end{array}$ & & //11/2018 & 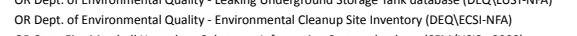 \\
\hline & Oforo & Jackson & 00/29/2008 & 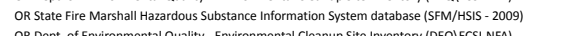 \\
\hline $\begin{array}{l}3089 \text { C CAREER LAKE HWY } \\
30 \text { BAANT RD }\end{array}$ & & $\begin{array}{l}\text { JaCKSON } \\
\text { ICCSSON }\end{array}$ & $\begin{array}{l}10 / 31 / 2018 \\
10 / 1 / 2018\end{array}$ & 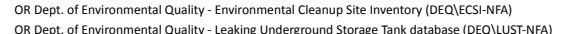 \\
\hline $\begin{array}{l}309 \text { BRANTIRD } \\
315 \text { GRAPE }\end{array}$ & elford & $\begin{array}{l}\text { JaCSSON } \\
\text { ICCKSON }\end{array}$ & $\begin{array}{l}10 / 31 / 2018 \\
10 / 31 / 2018\end{array}$ & 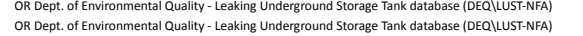 \\
\hline 310 W GTH ST & edforard & 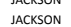 & $10 / 31 / 2018$ & 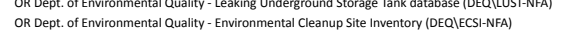 \\
\hline 399 S STH ST CITY SHOPS & CENTRAL POINT & JACKSON & 09/29/2008 & 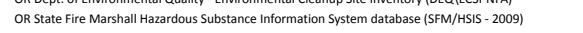 \\
\hline 310 W 6 GH ST & Medford & JACKSON & 10/31/2018 & OR Dept. of Environmental Quality - Hazard Waste Program databasese (DEQQHWW) \\
\hline 310 W GTH ST & edford & IACKSON & $10 / 31 / 2018$ & OR Dept. of Environmental Quality- Leaking Underground Storage Tank database (DEQQ LUST-NFA) \\
\hline 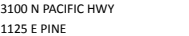 & ENTRA POANT & $\begin{array}{l}\text { JACKSON } \\
\text { JAKSSON }\end{array}$ & $\begin{array}{l}10 / 311 / 218 \\
00 / 29 / 2008\end{array}$ & 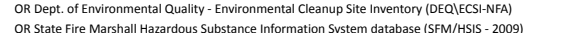 \\
\hline $\begin{array}{l}1125 \text { E PNE } \\
1600 \text { P PIN ST }\end{array}$ & $\begin{array}{l}\text { NTRAQDONT } \\
\text { NTAL PONT }\end{array}$ & $\begin{array}{l}\text { JACCSSON } \\
\text { JACKSON }\end{array}$ & 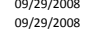 & 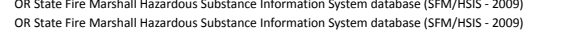 \\
\hline 4500 ROGUE VALLEY & NTFAL POINT & JACKSON & //29/2008 & 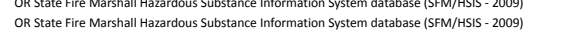 \\
\hline 4500 ROGUEVA & CENTRAL POINT & JACKSON & 9/29/2008 & 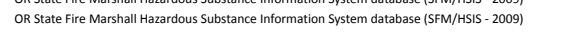 \\
\hline 155 SCHULZ RD & CENTRAL POINT & JACKSON & 9/29/2008 & OR State fire Marshall Hazarddous Substance Information System database (SFM/HSIS- 2009) \\
\hline 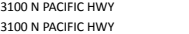 & edford & JACKSON & 年 & 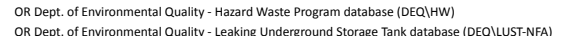 \\
\hline $\begin{array}{l}3 \text { 3010N P PACFIFC CWY } \\
\text { 1A PENN }\end{array}$ & ENTRAL POINT & $\begin{array}{l}\text { JACKSON } \\
\text { JAKSON }\end{array}$ & 年/2/2018 & 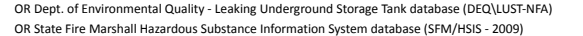 \\
\hline 3101 Mcloughlin Dr & $\begin{array}{l}\text { CNETRAL LOINT } \\
\text { Medford }\end{array}$ & $\begin{array}{l}\text { JACCSSON } \\
\text { JACKSON }\end{array}$ & 1/202/2015 & 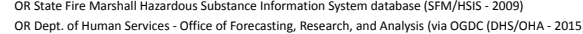 \\
\hline 5020 TABLC & CENTRAL POINT & JACKSON & /1/29/2008 & 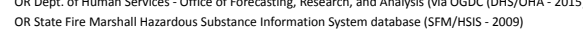 \\
\hline 3103 8100 & EFFRD & JACKSON & 09/29/2008 & 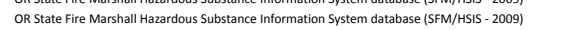 \\
\hline 4849 ARWAY DR STE 10 & ENTRAA POINT & JACKSON & 09/29/2008 & 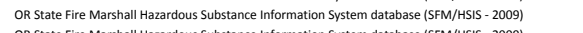 \\
\hline $\begin{array}{l}47311 \text { N N OUSSRR DR } \\
4822 \text { INDUSTR DR }\end{array}$ & $\begin{array}{l}\text { CENTTALPONT } \\
\text { CENTRAL POINT }\end{array}$ & JACKSON & 99/2/29/2008 & 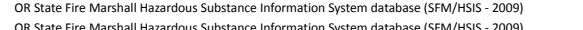 \\
\hline 3533 N MEEFFORO IN & 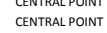 & $\begin{array}{l}\text { JACCSSON } \\
\text { JAKSON }\end{array}$ & $\begin{array}{l}90 / 29 / 2008 \\
092 / 2008\end{array}$ & 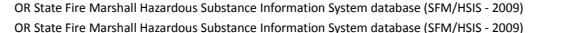 \\
\hline & NNTRAL POINT & JACKSSON & 年/29/2008 & 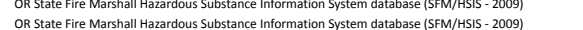 \\
\hline 7420 KESHAW RD & HITE CITY & JACKSON & //29/2008 & 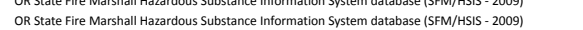 \\
\hline 555 Mosqui & ENTRAL POINT & JACKSON & 09/29/2008 & 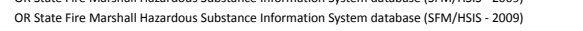 \\
\hline & OLD HILL & JACKSON & 9/29/2008 & 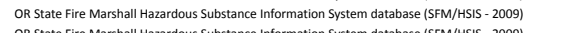 \\
\hline S474 N RIVER RD & $\begin{array}{l}\text { GoOD HLL } \\
\text { GOLD HILL }\end{array}$ & IACCSSON & /29/2008 & 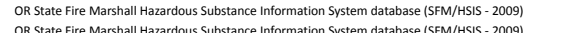 \\
\hline $\begin{array}{l}2882 \text { N RAVRER RO COURT DR } \\
283 \text { M }\end{array}$ & $\begin{array}{l}\text { Gold HIL } \\
\text { WHITE CITY }\end{array}$ & $\begin{array}{l}\text { IACCSSON } \\
\text { IACSSON }\end{array}$ & $\begin{array}{l}99 / 2 / 2008 \\
9 / 29 / 2008\end{array}$ & 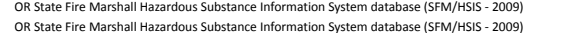 \\
\hline & 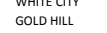 & 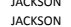 & $0909 / 29 / 2008$ & 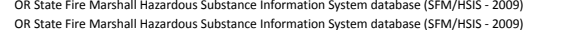 \\
\hline $3103 \mathrm{BIOD}$ & EDFORD & JACKSON & 09/29/2008 & \\
\hline & HITE CITY & JACKSON & 09/29/2008 & 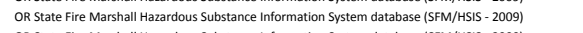 \\
\hline 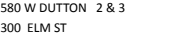 & $\begin{array}{l}\text { EAGLE POINT } \\
\text { CHLOQUNN }\end{array}$ & JACKSON & $\begin{array}{l}09 / 29 / 2008 \\
00 / 292008\end{array}$ & 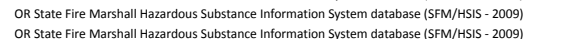 \\
\hline 40 W ROLING HILLS & EAGLE POINT & JACKSON & $09 / 29 / 2008$ & 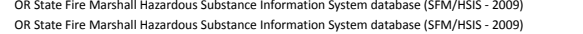 \\
\hline 100 FISH LAKE RD & BUTTE FALLS & JACKSON & 09/29/2008 & OR State Fire Marshall Hazardous Substance Information System database (SFM/HISIS-2009) \\
\hline $\begin{array}{l}580 \text { FISH LAKE RD } \\
101 \text { ODDHWY } 2\end{array}$ & BUTTE FALL & JaCKSON & 09/29/2008 & OR State Fire Marshall Hazarddus Substance Information System database (SFM/HISIS-2009) \\
\hline $\begin{array}{l}101 \text { OLD HWY } 62 \\
200 \text { COLE M RIVES DR }\end{array}$ & $\begin{array}{l}\text { EAGAE POINT } \\
\text { TRAL }\end{array}$ & JACKSON & $\begin{array}{l}09 / 29 / 2 / 2008 \\
00 / 292008\end{array}$ & 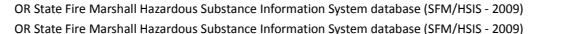 \\
\hline 100 COLE M RIVERS DR & $\begin{array}{l}\text { TARAL } \\
\text { TRAL }\end{array}$ & $\begin{array}{l}\text { IACSSON } \\
\text { IACCSON }\end{array}$ & 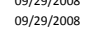 & \\
\hline $17525 \mathrm{EL}$ & & JACKSON & //29/2008 & 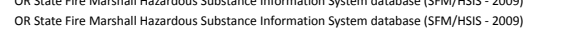 \\
\hline & TALENT & JACKSON & 09/29/2008 & 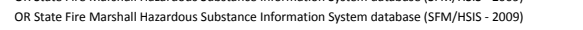 \\
\hline 599 & & JACKSON & 09/29/2008 & OR State Fire Marstall Hazardous Substance Ifrormation System database (SFM/HISIS-2009) \\
\hline LAT: & PROSPECT & IACKSON & 09/29/2008 & 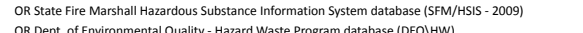 \\
\hline & $\begin{array}{l}\text { Medford } \\
\text { MEOFFRD }\end{array}$ & $\begin{array}{l}\text { JACKSON } \\
\text { ICCKSON }\end{array}$ & $\begin{array}{l}1 / 131 / 218 \\
09 / 2 / 2008\end{array}$ & \\
\hline & ROSPECT & IACKSON & 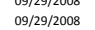 & 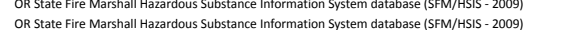 \\
\hline & & & 09/29/2008 & 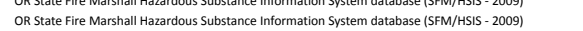 \\
\hline & dfford & JACKSON & 10/31/2018 & OR Dept. of Environmental Q Quallity - Hazard Waste Program database (DER|HW) \\
\hline & & Jackson & 10/31/2018 & 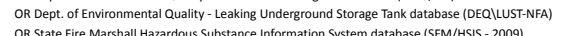 \\
\hline 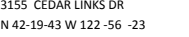 & $\begin{array}{l}\text { MEPEORD } \\
\text { JACKSONVILE }\end{array}$ & 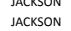 & $\begin{array}{l}09 / 2992008 \\
099 / 29 / 2008\end{array}$ & 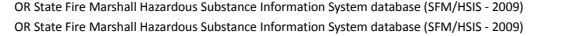 \\
\hline 6301 CRATER LAKE HWY & CENTRAL POINT & JACKSON & 09/29/2008 & 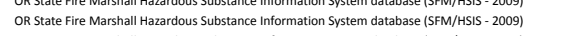 \\
\hline 8087 BLACKWELLR R & CENTRAL POINT & JACKSON & 09/29/2008 & 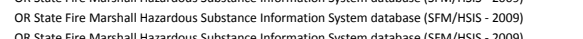 \\
\hline & & & 年9292008 & 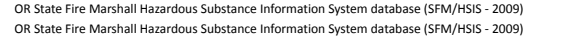 \\
\hline 235 & & & 127/20008 & 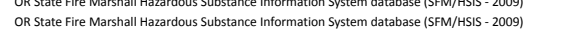 \\
\hline & & & & \\
\hline 8425 AGATE RD & HITE CITY & JACKSON & t/29/2008 & istate fire Marshall Hazarddous Substance Information System daz \\
\hline $\begin{array}{l}7289 \text { AGACE D } \\
726 \text { BACKWEL RD }\end{array}$ & $\begin{array}{l}\text { WEITEE CIY } \\
\text { CETTALPOINT }\end{array}$ & $\begin{array}{l}\text { JACCSSON } \\
\text { JAKSON }\end{array}$ & 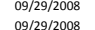 & 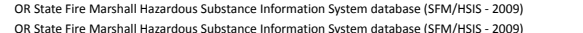 \\
\hline
\end{tabular}

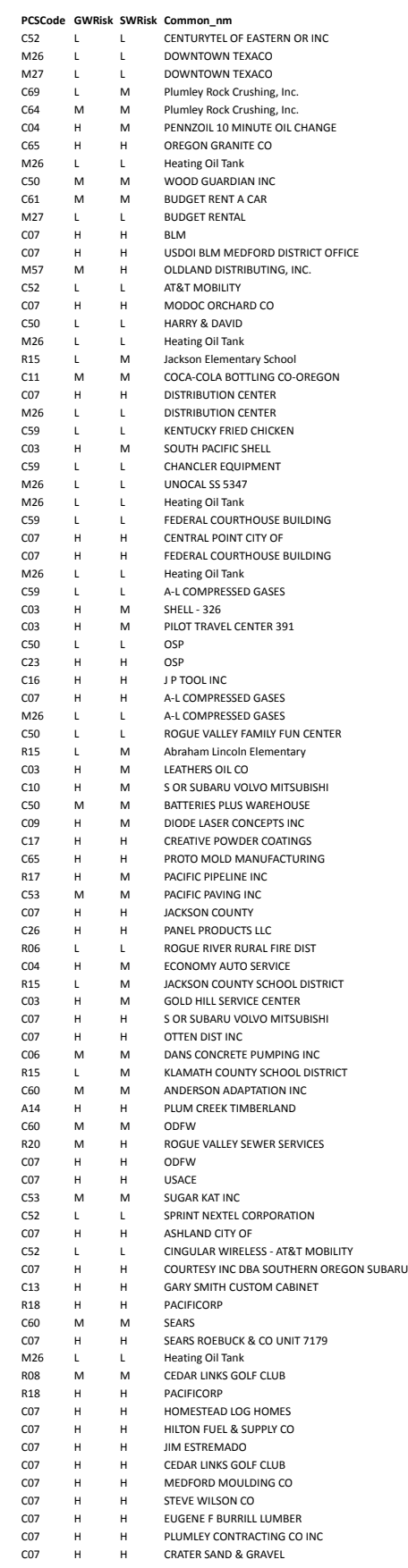

PCSType

UST- Confirmed Leaking but listed as NFA - DEQ L LST List

Ming Activities - Inactive- sand/gravel/rock/soil

Miscelaneous Manutacturng

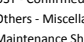

Maintenance Shop/FGuipment Storage- - Transsportation Related

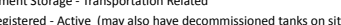
Chemical/Petroleum Processing//Ptorage DEQ Permitted Stormwater Discharges (NPDES or WPCF) Chemical/Petroleum Processing//storage

Other- General Merchandise/Retail StSore
UST - Confirmed Leaking but listed as NFA- DEQ LuST List

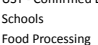

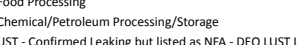
Krown Contanination listed as NFA S Stites Pllumes/SPills from ECSI) Automobiles - Gas Stations
Known Contamination listed as NFA (Steses/Plumes/Spills from ECSII

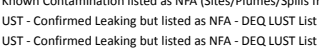
Nown Contamination listed as NFA (Siteses Plumeses/Spills from ECSI Chemical/Petroleum Processing/storage UST - Confirmed Leaking but listed as NFA - DEQ LUST List

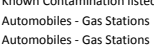

Automobilies-Gas Stations
Others - Govit/Business/NGo Services Research laboratores
Machine Shops

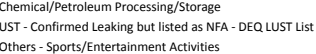

Schools
Automobiles - Gas Stations
Hleet/Truckin/Bus Terminals

Other- General Merchandise
Electrica//Eectronic Manufactur

Electrical/Eectronic Manufacturing
Metal Plating /Finshing Fabrication

Miscellaneus Manufacturing
Sewer Lines - Close roximity to Pws

Construction Company
Chemical/Petroleum Processing//storage

Wood/Pulf/Paper Processing and $N$

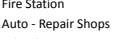

Schools
Automobiles - Gas Stations

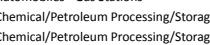

Cement/concrete Plants
Schools

Maintenance Shop/Equipment Storozge- Not Transsortation Related

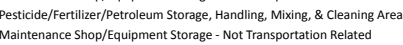
Maintenance Shop/Equipment Storgege - Not Tran
Wastewater Treatment Plants/Collection Stations

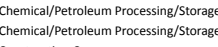
Construction Company
Communications office

chemical/Petroluum Processing/storage Communications office
Chemical/Petroleum Processing/storage Home Manufacturing Utility Stations/Powerplants - Maintenance//ransformer Storage

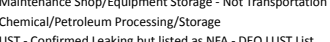
UST - Confirmed Leaking but listed as NFA- DEQ Q LST L List Golf Courses
Utility Station Utility Stations/Powerplants - Maintenan
Chemica///etroleum Processing/torage
Chemical/Petroleum Processing/Storage

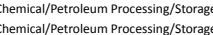
Chemical/Petroleum Processing/Storage

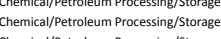

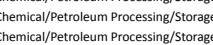




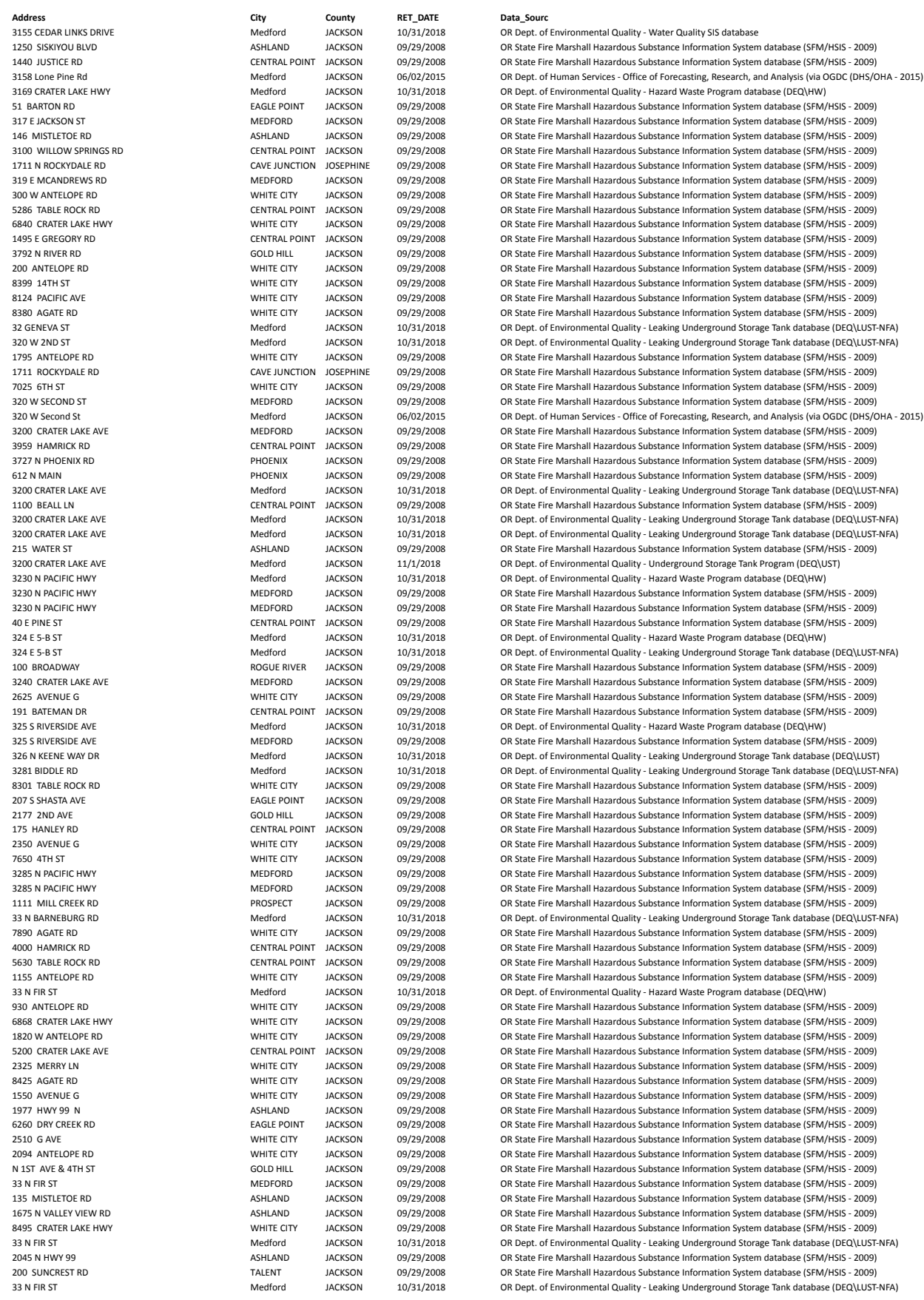

\begin{tabular}{|c|c|c|c|}
\hline \multicolumn{4}{|c|}{$\operatorname{cscos}^{2}$} \\
\hline & M & & CEDAR LANDING PUD \\
\hline & ${ }_{H}^{H}$ & ${ }_{H}^{H}$ & $\begin{array}{l}\text { SOUTHERN ORRGNO UNIVESSTI } \\
\text { WILIE BOATS INC }\end{array}$ \\
\hline & L & M & \\
\hline & H & H & SAEEWAY STORE NO 1643 \\
\hline & & & $\begin{array}{l}\text { DALE L MCFALL TRUCKING } \\
\end{array}$ \\
\hline & & & $\begin{array}{l}\text { MODERN PLUMBING SERVICE } 1 \\
\text { CROANAN CORP }\end{array}$ \\
\hline & $\mathrm{H}$ & H & ERICSON ARR CRANE INC \\
\hline & & & 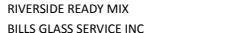 \\
\hline & & & $\begin{array}{l}\text { BLILS GASSSERVRCE ENC } \\
\text { PACFIC CREST TRANSFRMERS INC }\end{array}$ \\
\hline & & r & ODF \\
\hline & & & \\
\hline & H & H & OREGON PARKS \& REC \\
\hline & & & $\begin{array}{l}\text { JACKSON COUNTY } \\
\text { CASCDE WOOD PRODUCTS INC }\end{array}$ \\
\hline & н & + & CARESTREAM HEALTH INC \\
\hline & & & $\begin{array}{l}\text { TIMBER PRODUCTS COMPANY } \\
\text { Heatiog OOTITKK }\end{array}$ \\
\hline & & & $\begin{array}{l}\text { Heatitgg il Tink } \\
\text { Heating Oil Tank }\end{array}$ \\
\hline & 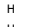 & H & BOISE BULLIIING SOUUTIONS MFGING \\
\hline & & & 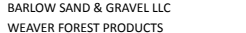 \\
\hline & t & M & MEOFRO SCHOOL DISTRICT $549 \mathrm{C}$ \\
\hline & M & & 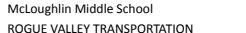 \\
\hline & H & н & $\begin{array}{l}\text { LTM INCORPORATED } \\
\text { LeVATIN }\end{array}$ \\
\hline & & & 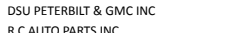 \\
\hline & & & 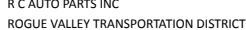 \\
\hline & н & H & 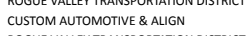 \\
\hline & & & 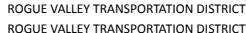 \\
\hline & H & н & 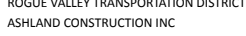 \\
\hline & $H$ & & ROGUE VALLEY TRANSPORTATION DISTRIC \\
\hline & & & $\begin{array}{l}\text { ROEBRTS MOOOR R NN OF OOEEGON } \\
\text { ROEERTS MOTOR INC-ORE }\end{array}$ \\
\hline & H & H & $\begin{array}{l}\text { R ROERTS MOTOR INC-ORE } \\
\text { ROEE }\end{array}$ \\
\hline & $\mathrm{H}$ & & 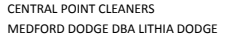 \\
\hline & L & L & 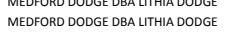 \\
\hline & H & & 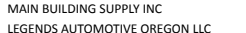 \\
\hline & & & $\begin{array}{l}\text { LGGENDA AUTOOOTTVE OREGON LLC } \\
\text { SCHNITZR STEEL }\end{array}$ \\
\hline & н & H & HaYs OLl CO \\
\hline & & & $\begin{array}{l}\text { Dollar GMC Oldsmobile } \\
\text { DolAR GMC }\end{array}$ \\
\hline & H & M & Heating oil Tank \\
\hline & L & & $\begin{array}{l}\text { HAMLIN MOTOR CO } \\
\text { MEDFORD WATER COMMISSION }\end{array}$ \\
\hline & H & H & EAGLE POINT CITY OF FISTSION \\
\hline & & & $\begin{array}{l}\text { GOLD HLL CITY OF } \\
\text { MEDFORD EED ORCAAD }\end{array}$ \\
\hline & & & $\begin{array}{l}\text { MEEFROD DEED ORCAARD } \\
\text { BOOMASS ONE LP }\end{array}$ \\
\hline & H & H & OREGON FIR MLLWORK INC \\
\hline & & & 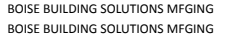 \\
\hline & н & H & $\begin{array}{l}\text { Pacificorp Energy } \\
\text { Parl }\end{array}$ \\
\hline & $H_{H}$ & & 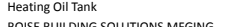 \\
\hline & & & 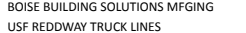 \\
\hline & H & H & TIMEER PROD TRUCKING CO \\
\hline & H & & 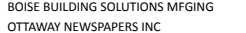 \\
\hline & н & H & BETTENDORE ENTERPRISES INC \\
\hline & ${ }_{H}^{H}$ & & $\begin{array}{l}\text { DEESRT PUMP PO IIC } \\
\text { DIXON STEEL \& SUPPIY }\end{array}$ \\
\hline & H & H & $\begin{array}{l}\text { HUNT TRACTOR EQUIP \& PARTS INC } \\
\text { Hers }\end{array}$ \\
\hline & $H_{H}$ & & $\begin{array}{l}\text { FV MARTIN TRUCKING CO } \\
\text { s\& B B ALES COSSTRUCTION }\end{array}$ \\
\hline & & & $\begin{array}{l}\text { S\& \& BAAESS CONSFUUCCION } \\
\text { TIMBER PRODUCTS COMPANY }\end{array}$ \\
\hline & ${ }^{H}$ & H & BUTLER FORD \\
\hline & H & 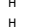 & $\begin{array}{l}\text { DRY CREER LANOFLI IIC } \\
\text { WESTRE VALLY CUSTOC }\end{array}$ \\
\hline & H & 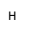 & $\begin{array}{l}\text { WESERNVAUE CUIST } \\
\text { BILT TERENING INC }\end{array}$ \\
\hline & & & $\begin{array}{l}\text { OWEST CORPORATION } \\
\text { MEEFRD MAATRBBUNE }\end{array}$ \\
\hline & $\mathrm{H}$ & H & 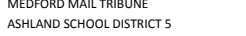 \\
\hline & 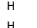 & & $\begin{array}{l}\text { VALLEY VEW NURSERY } \\
\text { SOLTHEPRO }\end{array}$ \\
\hline & n & L & $\begin{array}{l}\text { SOUTHERN OREGGNR RHABA CTR \&CL } \\
\text { OTTAWAY NEWSPAPES INC }\end{array}$ \\
\hline & H & & TOWN \& COUNTRY CHEVROLET ASHLLND \\
\hline & & & $\begin{array}{l}\text { TALENT IIV OF } \\
\text { OTTANAY NEWSAPERS INC }\end{array}$ \\
\hline
\end{tabular}

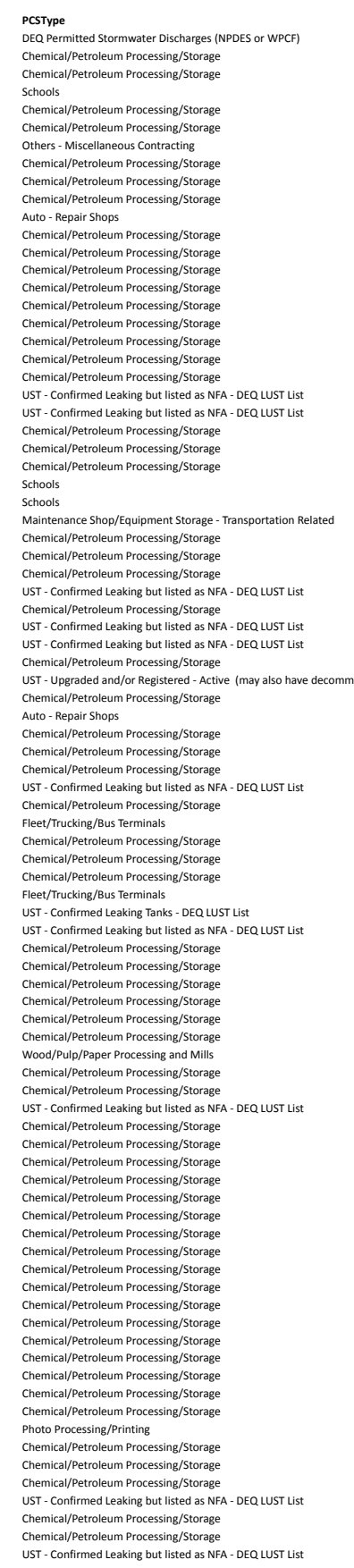


Address
33 NIR ST
33 W. 8 TH ST.

3300 CRATRR LAKE AVE
5550 TABLE ROCK RD

5550 TABLE ROCK RD
3300 CRATER LAKE AVE

5759 CRATRER LAKE HW

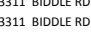

332 W GTH ST
333 HOLMES AVE

333 Holmes
333 W 8 RTS
33 W

335 E MCANDREWS
145 N MAIN ST

145 N MAIN ST
6615 R RCCYALE RD
3366 CRATER LAKE AV

2938 HANLEY RD
3384 CRATER LAKE AVE

ATER LAKE AVE

34 SFIR ST

3400 Crater Lake Avenue
9450 OD STAGE RD
6051 CRATEB AAKE HWV

3425 LNE PNE RO
460 SVALEV VEW

75 LTTHA WV
7251 HOLLAND LOOP RD

5600 BUTTE FALL HW

3450 CRATER L LAK AVV
3450 O GRATR L LKE AVE

$3460 \mathrm{~N} \mathrm{PACFFCHW}$

$3460 \mathrm{~N} \mathrm{PACFICC} \mathrm{HWY}$
280 MAPLE ST

277 WILSON RD
5758 CRATER L LAEE AVE
3116 SUNWYYAEE

3116 SUNNVALE
348 N RVERSDIE

348 NRIEESIDE AVE
2672 GALIS CREEK RO

$770111 T H$
515 BROAD ST
348 WHTE

348 WHTTE OAK CIR
1396 ANTELOPE RD
9201 OLDSTAGE RD

9201 OLD STAGE RD
35 W BTH ST

35 W 8 8THST
305 BATEMAN DR

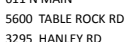

3295 HANLEY RD
4777 BUTTE FALLS HW

3531 AVION
3550 BIDOLE

4444 HWY 234
20399 SAWVER RE

20399 SAWVER RD
3551 AVOON OR

7130 C CRATER LAKE HWV
2105 S SOEV PD

2205 COREY RD
3555 CRATRR LAKE HWV
${ }_{100}$ ESAGE PONT

100 EAGLE POINT D
12451 MODOC RD

3200 KRTLAND

3200 KRTTLAND RD
3555 CRAER LAKE HWN
635 AVENUE C

635 AVENUEC C
3750 KRTLAND RD
3555 CRATER LAEE HWY

3555 CRATER L LAK HWU
420 N FRONT ST

4400
11 SFRONGTAGE RD RD

3560 S PACFCC HW

12294 AGATE RD
3590 FRE STATION SPUR STE 102
3333 AGATE RD

\begin{tabular}{|c|c|c|c|}
\hline $\begin{array}{l}\text { Citiv } \\
\text { Medford }\end{array}$ & $\begin{array}{l}\text { County } \\
\text { JaCKSON }\end{array}$ & $\begin{array}{l}\text { RET_DATE } \\
10 / 31 / 2018\end{array}$ & $\begin{array}{l}\text { Data_Sourc } \\
\text { OR Dept. of Environmental Q Quality - Leaking Underground Storage Tank databasese (DEQQ|lusT-NAA) }\end{array}$ \\
\hline & JACKSON & 10/131/2018 & OR Dept. of Environmental Quality - Environmental Cleanup Site Inventor (DEQ|ECESINFA) \\
\hline MEEFORD & JACKSON & 09/29/2008 & OR State fire Marshal Hazardous Substance Information System datababe (SFM/HISIS- 2009) \\
\hline & & & 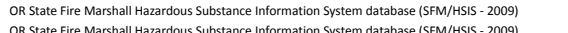 \\
\hline & 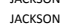 & 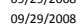 & 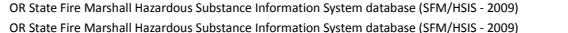 \\
\hline & JACKSON & 09/29/2008 & 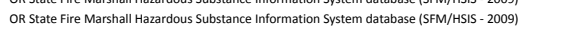 \\
\hline & Jackso & 09/29/2008 & OR State Fire Marssall Hazarddous Substance Information System database (SFM/HSIS- 2009) \\
\hline & & & formation System database (SFM/HSIS - 2009) \\
\hline 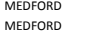 & JaCGSON & 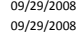 & 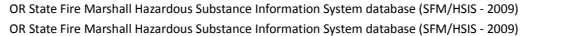 \\
\hline & & 09/29/2008 & 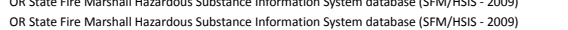 \\
\hline & JACKSON & 10/31/2018 & 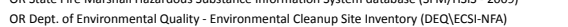 \\
\hline DFORD & son & 09/29/2008 & OR State fire Marshall Harardous Substance Information System databasese (SFM/HISIS - 2009) \\
\hline dford & Jackson & 1/1/1/2018 & OR Dept. of Geelogy and Mineral Industries Mineral Information laye for Oregon( DOGAMI/MLO) \\
\hline & & 06/02/2015 & OR Dept. of Human Services-Office of forecestring, Research, and Analysis (via OGDC (OHS/OHA- 20 \\
\hline fiford & $\begin{array}{l}\text { JaCGSON } \\
\text { IACKSON }\end{array}$ & 10/311/2018 & 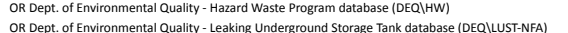 \\
\hline OFORD & 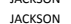 & $\begin{array}{l}1.1713 / 21818 \\
09 / 29 / 2008\end{array}$ & 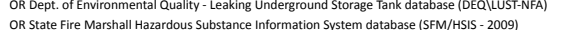 \\
\hline laAND & JACKSON & 09/29/2008 008 & 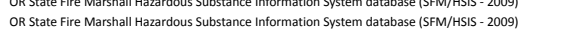 \\
\hline CAI & JOSEP & 09/29/2008 & 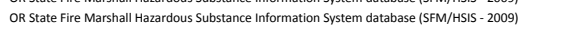 \\
\hline PFORD & JACKSON & 09/29/2008 & OR State Fire Marssall Hazarddous Substance Information System database (SFM/HSIS- 2009) \\
\hline & JACKS & 09/29/2008 0 & 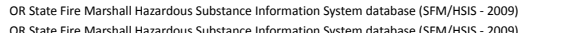 \\
\hline Medford & JACKSON & $\begin{array}{lll}09929 / 2008 \\
10 / 11 / 2018\end{array}$ & 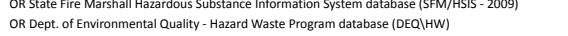 \\
\hline TRAll & JACKSON & $09 / 29 / 2008$ & OR State Fire Marshall Hazardous Substance Information System database (SFM/HIS - 2009) \\
\hline 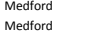 & Jackson & 10/11/2018 $11 / 12018$ & 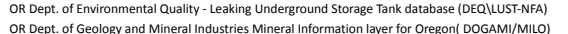 \\
\hline & $\begin{array}{l}\text { Jackson } \\
\text { Jackson }\end{array}$ & $\begin{array}{l}1 / 1 / 1 / 2018 \\
09 / 29 / 2008\end{array}$ & 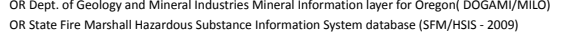 \\
\hline & JACKSON & 09/29/2008 008 & 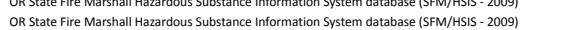 \\
\hline Aford & JACKSON & 10/31/2018 & 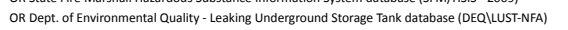 \\
\hline Medford & JACKSON & 10/31/2018 & OR Dept. of Environmental Quality - Leaking Underground Storage Tank database (DEQQ LUST-NFA) \\
\hline LAND & JaCKSON & 09/29/2008 & OR State fire Marshal Hazard dous Substance Information System database (SFM/HIS - 2009) \\
\hline $\begin{array}{l}\text { AHSALND } \\
\text { CAVE UUCTION }\end{array}$ & $\begin{array}{l}\text { JACSSON } \\
\text { JOSEPINE }\end{array}$ & $\begin{array}{l}09929 / 2008 \\
09 / 292 / 2008\end{array}$ & 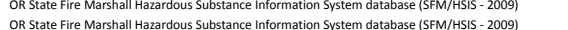 \\
\hline LE PONT & 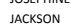 & 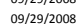 & 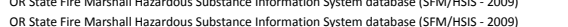 \\
\hline SPECT & JaCKSON & 09/29/2008 008 & 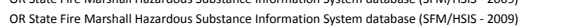 \\
\hline CENTRAL PO & JACKSON & 09/29/2008 & OR State fire Marstall Hazardous Substance Information System database (SFM/HSIS- 2009) \\
\hline MEDFORD & JaCKSON & 09/29/2008 & OR State fire Marssall Hazarddous Substance Information System database (SFM/HSIS- 2009 ) \\
\hline & IACCSON & 09/29/2008 & OR State Fire Marstall Hazardous Substance Information Sistem database (SFM/HISIS-2009) \\
\hline $\begin{array}{l}\text { MEFOOOD } \\
\text { MEDFORD }\end{array}$ & $\begin{array}{l}\text { JaCCSONO } \\
\text { IACKSON }\end{array}$ & $\begin{array}{l}09929 / 2008 \\
09 / 292 / 2008\end{array}$ & \\
\hline LaNo & 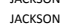 & 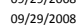 & 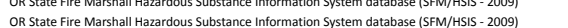 \\
\hline CENTRALP & JACKSON & $09 / 29 / 2008$ & 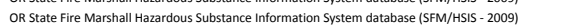 \\
\hline ITRAL POINT & JACKSON & 09/29/2008 & 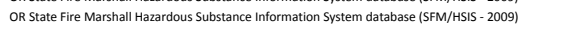 \\
\hline CENTRAL POINT & JACKSON & 09/29/2008 & OR State fire Marshall Hazarddous Substance Information System database (SFM/HSIS- 2009) \\
\hline $\begin{array}{l}\text { Medford } \\
\text { Metford } \\
\text {. }\end{array}$ & JaCSSON & 10/13/2018 & 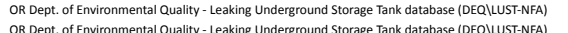 \\
\hline $\begin{array}{l}\text { Medford } \\
\text { GOODHIL }\end{array}$ & 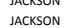 & $\begin{array}{l}10 / 7121 / 218 \\
09 / 29008\end{array}$ & 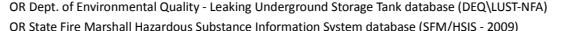 \\
\hline & JACKSON & 09/29/2008 & 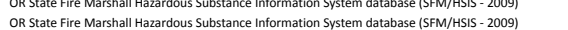 \\
\hline WHITE CITY & JaCKSON & $09 / 29 / 2008$ & 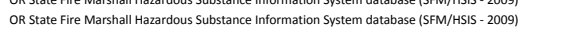 \\
\hline TEF Fall & JACKSON & 09/29/2008 & 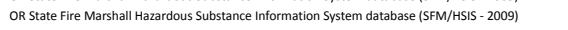 \\
\hline & JACKSON & 10/31/2018 & OR Dept. of Environmental Quality- Leaking Underground Storage Tank database (DEQQ LUST-NFA) \\
\hline 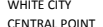 & JACCSSON & 09/29/2008 & 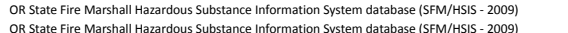 \\
\hline Medford & 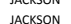 & 10/31/2018 & 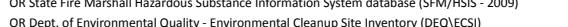 \\
\hline 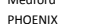 & 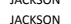 & 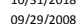 & 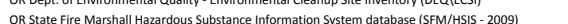 \\
\hline Medford & JaCKSON & 10/31/2018 & 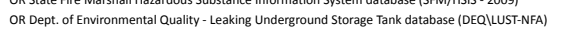 \\
\hline & JACKSON & 09/29/2008 & 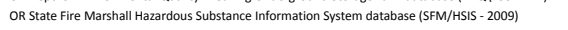 \\
\hline & & & OR State fire Marstall Hazardous Substance Information System databasese (SFM/HISIs- 2009) \\
\hline & $\begin{array}{l}\text { JACCSSON } \\
\text { JACKSON }\end{array}$ & $\begin{array}{l}09 / 29 / 2008 \\
00 / 292008\end{array}$ & 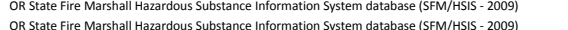 \\
\hline & & 09/29/2008 & 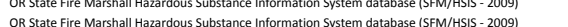 \\
\hline MEFFORD & JACCSON & 09/29/2008 & 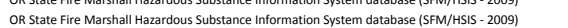 \\
\hline Me & JACKSON & 10/31/2018 & 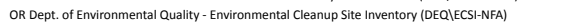 \\
\hline & JACKSON & 09/29/2008 & OR State fire Marshall Hazardous Substance Information System database (SFM/HISI - 2009) \\
\hline OY COVE & JACKSON & 09/29/2008 & OR State Fite Marshall Hazardous Substance Information S Sistem database (SFM/HISIS - 2009) \\
\hline & $\begin{array}{l}\text { JaCSSON } \\
\text { IACSSON }\end{array}$ & $\begin{array}{l}09929 / 2008 \\
09 / 292 / 2008\end{array}$ & 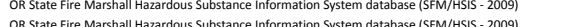 \\
\hline & 列 & 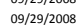 & 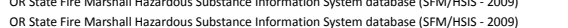 \\
\hline ME & & & 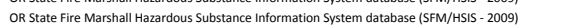 \\
\hline & JACKSON & 09/29/2008 & OR State fire Marstall Hazardous Substance Information System database (SFM/HSIS- 2009) \\
\hline & JACKSON & 09/29/2008 & OR State fire Marshall Hazardous Substance Information System database (SFM/HISI - 2009) \\
\hline & JAACKSON & & Fire Marshall Hazardous Substance Information System database!! \\
\hline & 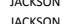 & 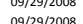 & 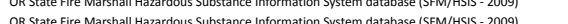 \\
\hline & 列 & 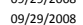 & 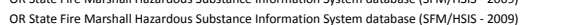 \\
\hline & & & (t) \\
\hline & JACKSON & 10/31/2018 & OR Dep \\
\hline & & & 009) \\
\hline 刑 & 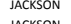 & & 009) \\
\hline & & & 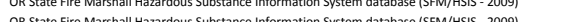 \\
\hline & & & \\
\hline ford & & & \\
\hline & & & rshall Hazardious Substance Information System database (SFM/HSIS - 2009) \\
\hline
\end{tabular}

\begin{tabular}{|c|c|c|c|}
\hline PCsco & GWRis & sWRisk cor & \\
\hline $\begin{array}{l}\text { M26 } \\
\text { c99 }\end{array}$ & & & OTTAWAY NEWSPAPERS INC \\
\hline C26 & H & 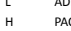 & $\begin{array}{l}\text { ADDINGTON SUED CARS } \\
\text { PACIIIC SUPPIY }\end{array}$ \\
\hline c07 & H & H & $\begin{array}{l}\text { HUGHES BROS CONSTRUCTION CO } \\
\text { Hes }\end{array}$ \\
\hline c07 & $\mathrm{H}$ & PAC & PACIFIC SUPPLY \\
\hline 007 & H & FLC & FLOYD A BOYD COMPANY \\
\hline col & H & VAI & VALLEY VIEW TRANSFER STATION \\
\hline c04 & H & Jor & 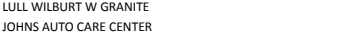 \\
\hline c07 & & & \\
\hline c50 & M & DR & 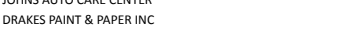 \\
\hline 007 & H & GR. & GRANGE COOP SUPPYY ASSN \\
\hline c59 & ᄂ & & LORETO DEVELOPMENT PROPERTY \\
\hline R15 & L & ME & MEEFORD SCHOOL DISTRRCT $549 \mathrm{C}$ \\
\hline $\begin{array}{l}6199 \\
\text { R15 }\end{array}$ & t & Jeff $14 \mathrm{C}$ & $\begin{array}{l}1904 \text { Rocco Quarry } \\
\text { Jefferson Elementary School }\end{array}$ \\
\hline COT & H & Ro & 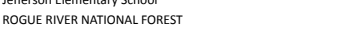 \\
\hline M26 & $\mathrm{L}$ & & 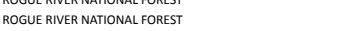 \\
\hline c12 & M & m & CARQUEST \\
\hline co7 & H & H & 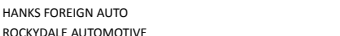 \\
\hline $\begin{array}{c}007 \\
004\end{array}$ & 音 & & 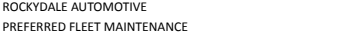 \\
\hline c07 & H & H & MC GOFFINS ALIGNMENT \\
\hline c53 & M & & 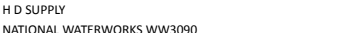 \\
\hline $\begin{array}{c}c 07 \\
007\end{array}$ & ${ }_{H}^{H}$ & ${ }_{H}^{H}$ & 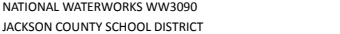 \\
\hline M26 & & & 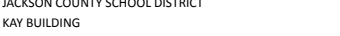 \\
\hline $\mathrm{c64}$ & m & $o_{w}$ & Owen Bar \\
\hline c07 & H & & LAUREL HILL GOLF COURSE \\
\hline$c_{026}$ & H & & $\begin{array}{l}\text { PANTHER RRUSHING CO INC } \\
\text { Heating O. OIJKK }\end{array}$ \\
\hline${ }_{\text {M26 }}^{\text {Me }}$ & t & L & $\begin{array}{l}\text { Heatinin Oili Inak } \\
\text { Heating oil Tank }\end{array}$ \\
\hline 007 & H & H & $\begin{array}{l}\text { VALES VIEW } 76 \\
\text { VALE }\end{array}$ \\
\hline co7 & H & & ASHLAND 76 \& AUTO REPAIR \\
\hline $\begin{array}{c}007 \\
007\end{array}$ & & & $\begin{array}{l}\text { HOLLAAD STORE } \\
\text { MIDWAY COUNTYY STORE }\end{array}$ \\
\hline cor & H & H & $\begin{array}{l}\text { PROSPECT SERVICE CENTER INC } \\
\text { PROSE }\end{array}$ \\
\hline c07 & H & & $\begin{array}{l}\text { TOLO FOREST PRODUCTS INC } \\
\text { WEFEOT TSC }\end{array}$ \\
\hline $\begin{array}{l}c 10 \\
007\end{array}$ & ${ }_{H}^{H}$ & & 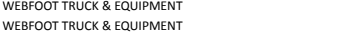 \\
\hline co4 & H & M & $\begin{array}{l}\text { WEFOOT RUCKC E EUUPMENT } \\
\text { valEY AUTO REPAIR }\end{array}$ \\
\hline co7 & H & H & VALLEY AUTO REPAIR \\
\hline c07 & н & Ast & ASHLANO COMMUNITY HOSPTTAL \\
\hline cor & H & BE, & BEAVER TREE SERVICE INC \\
\hline c07 & ${ }_{H}^{H}$ & $\begin{array}{l}\text { BR } \\
\text { Co }\end{array}$ & $\begin{array}{l}\text { BROWWN R B TRUCKING INC } \\
\text { CoOKK INC }\end{array}$ \\
\hline M26 & L & PA! & 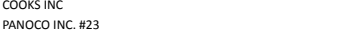 \\
\hline M26 & i & PA & $\begin{array}{l}\text { PandOCOINC. \#23 } \\
\text { PANOCO }\end{array}$ \\
\hline c07 & н & EST & ESTREMADO \& SONS INC \\
\hline cor & H & FIS & FISHER ROBERT L \\
\hline cor & H & vis & VSS EMULTECH \\
\hline M26 & & $\begin{array}{l}\text { Ro } \\
\text { He. }\end{array}$ & $\begin{array}{l}\text { RON AAILCICA EQUIPMENT INC } \\
\text { Heating Oil }\end{array}$ \\
\hline c07 & H & 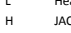 & 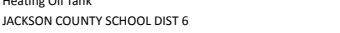 \\
\hline c07 & н & m. & 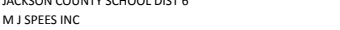 \\
\hline c58 & H & $\mathrm{AD}$ & ADDINGTON'S USED CARS \\
\hline cor & H & No & NORMAN DISTRRBUTION INC \\
\hline 留26 & L & AD & ADDINGTON'S USED CARS \\
\hline c07 & ${ }_{H}^{H}$ & PH & $\begin{array}{l}\text { OLDLAND DISTRRBUTTVG INC } \\
\text { POHENA AUTTMOTTE CENTER }\end{array}$ \\
\hline$c 07$ & $\mathrm{H}$ & PLL & 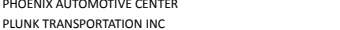 \\
\hline C07 & H & au & $\begin{array}{l}\text { QUA LIT SERVICE \& REPAIR INC } \\
\text { Q }\end{array}$ \\
\hline c07 & H & Ro & ROBERT LYON CONSTRUCTON \\
\hline c53 & $\mathrm{m}$ & OR & OREGON TLE \& MARBLE \\
\hline (c99 & 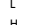 & ME & MEDFRO-AACKON COUNTT INTT'L AIRPORT (APRON PRTT) \\
\hline cor & $\mathrm{H}$ & TR & $\begin{array}{l}\text { TOPROCK TRUCKING CO } \\
\text { GERY OKKS DEVELOPMENT INC }\end{array}$ \\
\hline C65 & H & sis & $\begin{array}{l}\text { SISKKYOU BUCKLE CO INC } \\
\text { Sul IN }\end{array}$ \\
\hline c07 & н & wh & $\begin{array}{l}\text { WHIE CITY METALS AND SUPPLY } \\
\text { WhI }\end{array}$ \\
\hline C07 & H & No & NORTHSTAR PACIFIC COACHWAYS \\
\hline $\mathrm{C}_{12}$ & m & sch & SCHUCKS AUTO SUPPLY \\
\hline 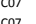 & H & EAC & EAGLE POING GOLF COURSE \\
\hline 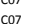 & H & PAt & 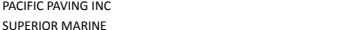 \\
\hline c07 & H & Ro & $\begin{array}{l}\text { ROGUE VALLYY SEWER SERVICES } \\
\text { ROELE }\end{array}$ \\
\hline C07 & H & sct & $\begin{array}{l}\text { SCHUCKS AUTO SUPPLY } \\
\text { STUCS }\end{array}$ \\
\hline c07 & H & so & SOUTHERN OREGON LINEN SVC \\
\hline Co7 & H & LT & LTMINC \\
\hline 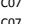 & H & Au & AUTOZONE NO 6268 \\
\hline 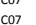 & & & $\begin{array}{l}\text { AUUOMOITON } \\
\text { BIG BEAR TIMBER LLC }\end{array}$ \\
\hline c07 & $\mathrm{H}$ & BR & B. \\
\hline A14 & & ALL & ALL NATURA PEST ELMINATION \\
\hline C07 & H & Fis & FISHER J OHN W TRUCKING \\
\hline 管7 & H & t & 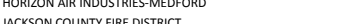 \\
\hline
\end{tabular}

PCSType
ust-cont

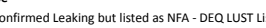

Wood/Pulp/Paper Processing and Mills
Chemical/Petrolum Processing/storage

enica/Petroleum Processing/Storage

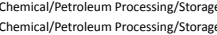

Auto- Repair Shops

chemicalp Petroleum Processinge/storage

nown Contamination listed as NFA S Sites PlPumes/Spills from ECS

Schools
Mining Attivities - Inactive - sand//gravel/rock/soil

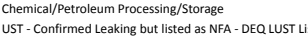

UST-Confirmed Leaking but
Furniture/Lumber/Parts Stores

Chemical/Petroleum Processing/storage

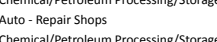

Construction Company
chemical Pertoleum Processing / storage

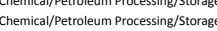

UST- Confirmed Leaking but isted as NFA- DEQ LUST List

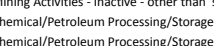

UST - Confirmed Leaking but listed a N NFA- DEQ Q UST L List
UST - Confirmed Leakin but isted as NFA - DEQ LUST Lis

chemical/Petroleum Processing \& S/torage

Chemical/Petroleum Processing//storage
Chemical/Petroleum Processing/storage

chemical/Petroleum Processing/Stororage

Chemical/Petroleum Processing//torage
Fleet/Trucking/Bus Terminals

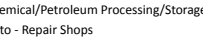

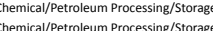

Chemical/Petroleum Processing/Ststrage

chenical/Petroleum Procossing//Storage

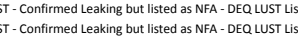

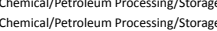

chemical/Petrolum Processing/s:orarage

Unemical/Petrolueum Processing/Storage

Chemical/Petroleum Processing/storage

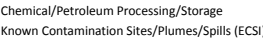

Chemica/Petroleum Processing//torage
UST - Confirmed Leaking but listed as NFA - DEQ LUST Lis

chemical/Petroleum Processing / /

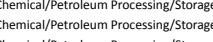

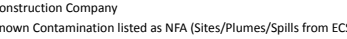

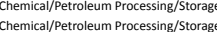

Miscellaneous Manufacturing

Chemica//Petroleuum Processing//torage
Chemical//Petroleum Processing//torage

Chemical/Petroleum Processing / Sto

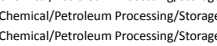

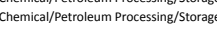

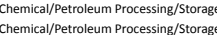

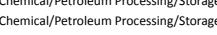

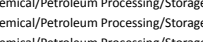

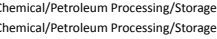

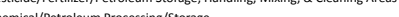

Chemical/Petroleum Processing/Storage
chemical/Petroleum Processing//torage
chemical/Petroleum Processing/storage 


\begin{tabular}{|c|c|c|c|c|}
\hline $\begin{array}{l}\text { Address } \\
3598 \text { S PACFIC HWY }\end{array}$ & 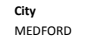 & $\begin{array}{l}\text { Connty } \\
\text { JACKSON }\end{array}$ & $\begin{array}{l}\text { RET_DARE } \\
\text { O0/29/2008 }\end{array}$ & 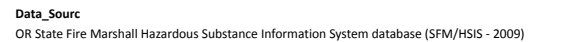 \\
\hline 1450 ANTELOPE RD & WHITECITY & JACKSON & 09/29/2008 & OR State Fire Marshall Hazardous Substance Information System datababse (SFM/HSIS- 2009) \\
\hline 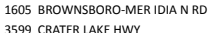 & $\begin{array}{l}\text { EAGLE POINT } \\
\text { MEFFRD }\end{array}$ & Jackson & 09/29/2008 & OR State Fire Marshall Hazard dous Substance Information System database (SFM/HISIS - 2009) \\
\hline 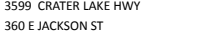 & $\begin{array}{l}\text { MEEFFoRD } \\
\text { Medford }\end{array}$ & JACKSON & & 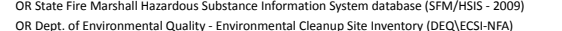 \\
\hline $\begin{array}{l}360 \text { EEACACSONS ST } \\
\text { 360 ST }\end{array}$ & edford & $\begin{array}{l}\text { JACSSON } \\
\text { ACCKSON }\end{array}$ & $\begin{array}{ll}10 / 31 / 2018 \\
10 / 31 / 2018\end{array}$ & 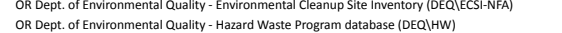 \\
\hline 4 ANTEL & IITE CITY & JACKSON & $09 / 29 / 2008$ & 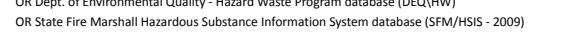 \\
\hline EAACKON ST & OFORD & JACKSON & 09/29/2008 & OR State Fire Marshall Hazardous Substance Information System database (SFM/HIS - 2009) \\
\hline O BLACKWEL RD & NTRAL POINT & JACKSON & 09/29/2008 & \\
\hline $\begin{array}{l}5800 \text { S PACIFC HWY } \\
360 \text { FACKSONS }\end{array}$ & $\begin{array}{l}\text { PHOENIX } \\
\text { MEFORD }\end{array}$ & $\begin{array}{l}\text { JACKSON } \\
\text { ACCSOOH }\end{array}$ & & \\
\hline $\begin{array}{l}\text { 306 EAACKSSNNST } \\
360 \text { EAAKSON ST }\end{array}$ & $\begin{array}{l}\text { MEDEFRD } \\
\text { Medford }\end{array}$ & JACKSON & $\begin{array}{l}09 / 29 / 2008 \\
10 / 312018\end{array}$ & 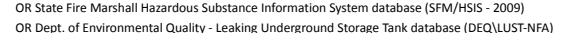 \\
\hline $\begin{array}{l}3600 \text { ICACSSONST } \\
360 \text { EACKSON ST }\end{array}$ & edford & $\begin{array}{l}\text { JAAKSON } \\
\text { JaCSSON }\end{array}$ & $\begin{array}{l}10 / 311 / 2018 \\
10 / 31 / 2018\end{array}$ & 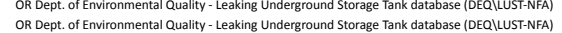 \\
\hline 3600 CRATER LLAEE & deford & $\begin{array}{l}\text { JACSOON } \\
\text { IACSSON }\end{array}$ & $10 / 31 / 2018$ & 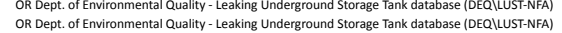 \\
\hline 6034 CRATER LAKE AV & NTRAL POINT & JACKSON & 09/29/2008 & 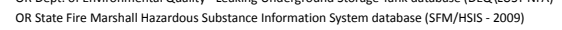 \\
\hline 3600 S PACIFC WY STE & MEDORD & JACKSON & 09/29/2008 & OR State fire Marshall Hazardous Substance Infrormation System databasese (SFM/HISIS- 2009 ) \\
\hline 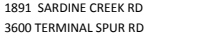 & GOLD HILL & 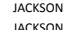 & $09 / 29 / 2008$ & 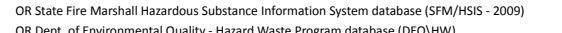 \\
\hline $\begin{array}{l}3600 \text { TRRMNNALSPUURD } \\
360 \text { TERMNALL SPUR RD }\end{array}$ & $\begin{array}{l}\text { Metford } \\
\text { Mefford }\end{array}$ & $\begin{array}{l}\text { JACKSON } \\
\text { ICASOON }\end{array}$ & $\begin{array}{l}10 / 31 / 21 / 218 \\
10 / 31 / 218\end{array}$ & 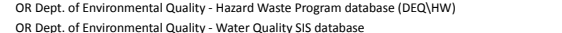 \\
\hline 1200 AVENUE G & $\begin{array}{l}\text { Metord } \\
\text { WhIT CITY }\end{array}$ & $\begin{array}{l}\text { JAASSON } \\
\text { JACKSON }\end{array}$ & $\begin{array}{l}10 / 31 / 2018 \\
09 / 29 / 2008\end{array}$ & 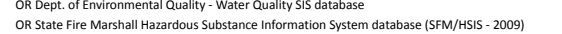 \\
\hline 3601 CRATER LAKE & MEOFORD & JACKSON & $09 / 29 / 2008$ & 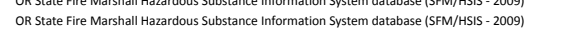 \\
\hline 500 EANTE & GLEOINT & JACKSON & 09/29/2008 & 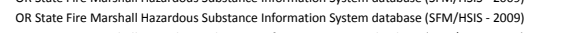 \\
\hline 7111 BLAC & CENTRAL POINT & JACKSON & 09/29/2008 & 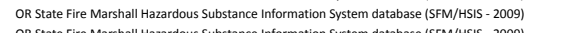 \\
\hline 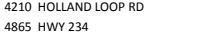 & $\begin{array}{l}\text { CAVE JUNCTION } \\
\text { WHTIE STV }\end{array}$ & JOSEPHINE & $\begin{array}{l}09 / 29 / 2008 \\
00 / 292008\end{array}$ & 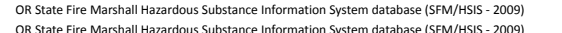 \\
\hline $\begin{array}{l}4865 \text { HWW } 234 \text { CRTER LAKE HWY } \\
3602\end{array}$ & efford & $\begin{array}{l}\text { AACCSSON } \\
\text { JACCSON }\end{array}$ & $\begin{array}{l}0 / 0 / 29 / 2008 \\
10 / 31 / 2018\end{array}$ & 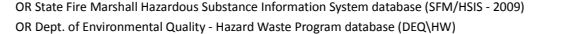 \\
\hline $3602 \mathrm{NPACF}$ & Mefford & IACKSON & $10 / 31 / 2018$ & 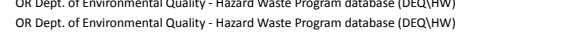 \\
\hline $3602 \mathrm{NPAC}$ & MEDFORD & JACKSON & 09/29/2008 & 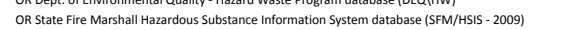 \\
\hline $3602 \mathrm{NPA}$ & Medford & Jackson & 10/31/2018 & OR Dept. of Environmental Quality - Leaking Underground Storage Tank database (DEQ|luss-NFA) \\
\hline 4942 OLD & CENTRAL POINT & JACKSON & 09/29/2008 & OR State Fire Marshall Hazardous Substance Information System database (SFM/HISIS-2009) \\
\hline $\begin{array}{l}\text { GOERGE RD } \\
\text { LREEK DR }\end{array}$ & 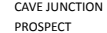 & JOSEPHINE & $\begin{array}{l}09 / 29 / 2008 \\
00 / 292008\end{array}$ & 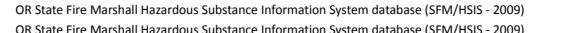 \\
\hline $\begin{array}{l}1488 \text { MILCREE D DR } \\
528 \text { W LNN RD }\end{array}$ & $\begin{array}{l}\text { OOPBET } \\
\text { GLE POINT }\end{array}$ & $\begin{array}{l}\text { A ACCCSON } \\
\text { JACKSON }\end{array}$ & $\begin{array}{l}0 / 999 / 2 / 2008 \\
09 / 29 / 2008\end{array}$ & 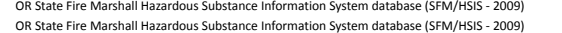 \\
\hline 3602 N PACIFC HWY & ledford & & $11 / 1 / 2018$ & 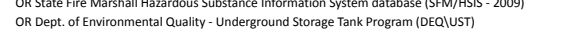 \\
\hline 1570 AVE & HITE CITY & Jackson & 09/29/2008 & 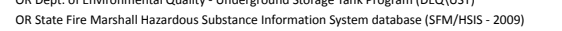 \\
\hline 3607 C RAA & edford & JACKSON & 11/1/2018 & 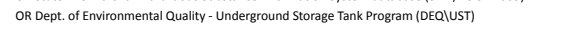 \\
\hline 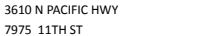 & ledford & JaCKSON & 10/31/2018 & OR Dept. of Environmental Quality - Leaking Underground Storage Tank database (DEQQ) UST-NFA) \\
\hline 175 HAN 111 & $\begin{array}{l}\text { Hite CIT } \\
\text { NTRALP }\end{array}$ & JACKSON & 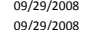 & 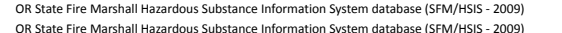 \\
\hline $1899 \mathrm{HW}$ & HLAADO & $\begin{array}{l}\text { JaCGSON } \\
\text { JACKSON }\end{array}$ & //29/29008 & 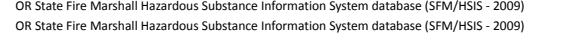 \\
\hline $3615 \mathrm{CP}$ & EDFORD & Jackson & $09 / 29 / 2008$ & 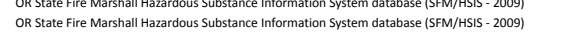 \\
\hline $6142 \mathrm{CR}$ & NTRALPC & JACKSON & $09 / 29 / 2008$ & 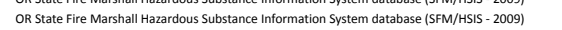 \\
\hline 3615 CRATER LAKE HWY & 1edford & JACKSON & 10/31/2018 & 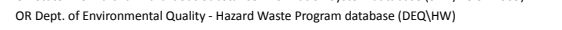 \\
\hline $\begin{array}{l}3630 \text { CRAARE LAK AVE } \\
3630 \text { CRATER LAKE AVE }\end{array}$ & $\begin{array}{l}\text { MEOBORD } \\
\text { MERPORD }\end{array}$ & JACKSON & $\begin{array}{l}09 / 299 / 2008 \\
090 / 29 / 2008\end{array}$ & 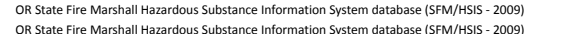 \\
\hline 2518 SAROINE CREEK RD & GOLOHILL & Jackson & $09 / 29 / 2008$ & 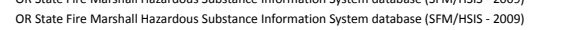 \\
\hline 3639 CRATER LAKE HWY & MEEFORD & IACKSON & 09/29/2008 & OR State fire Marshall Hazardous Substance Information System database (SFM/HISS - 2009) \\
\hline 3639 CRATER LAK & 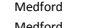 & JACKSON & $\begin{array}{l}10 / 31 / 2018 \\
10 / 31 / 2018\end{array}$ & 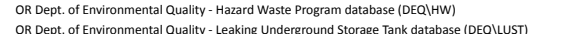 \\
\hline $\begin{array}{l}\text { RaAE HWY } \\
\text { ERD }\end{array}$ & $\begin{array}{l}\text { Mefford } \\
\text { MEOFORD }\end{array}$ & $\begin{array}{l}\text { JaCKSON } \\
\text { JACKSON }\end{array}$ & $\begin{array}{l}10 / 311 / 2018 \\
09 / 29 / 2008\end{array}$ & $\begin{array}{l}\text { of } \\
\text { of }\end{array}$ \\
\hline 369981000 & EEFORD & JACKSON & 09/29/2008 & 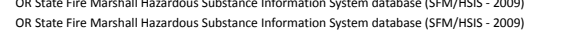 \\
\hline 365 EHRMAN WY & EDFORD & JACKSON & $09 / 29 / 2008$ & 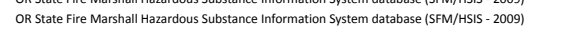 \\
\hline $3650 \mathrm{BDOD}$ & MEFFORD & JACKSON & 09/29/2008 & 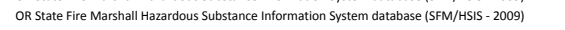 \\
\hline $1508 \mathrm{WAN}$ & WHITE CITY & JACKSON & 09/29/2008 & OR State Fire Marshall Hazardous Substance Information S Sistem database (SFM/HISIS-2009) \\
\hline & UeNT & JACKSON & 09/29/2008 & 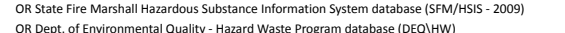 \\
\hline 36508081002 & $\begin{array}{l}\text { edford } \\
\text { edford }\end{array}$ & $\begin{array}{l}\text { JaCSSON } \\
\text { JACKSON }\end{array}$ & $\begin{array}{ll}10 / 31 / 2018 \\
10 / 31 / 2018\end{array}$ & \\
\hline 245 BATEN & NTRALP & SACCSSON & 09/29/2008 & 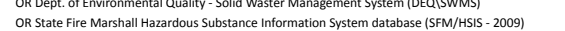 \\
\hline 1470 CA & UE Junctrion & JOSEPHINE & $09 / 29 / 2008$ & 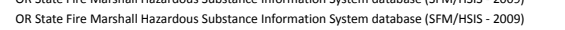 \\
\hline & ОІО нІІL & JACKSON & $09 / 29 / 2008$ & OR State fire Marshall Hazardous Substance Information System database (SFM/HISIS-2009) \\
\hline & HITE CITY & JACKSON & 09/29/2008 & OR State Fire Marshal Hazardous Substance Information System databasese (SFM/HISIS-2009) \\
\hline & & IACCSONO & & 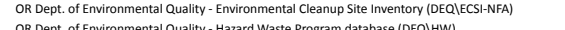 \\
\hline 3650 fire sta & edford & IACSTSON & $\begin{array}{l}10 / 31 / 2018 \\
10 / 31 / 2018\end{array}$ & 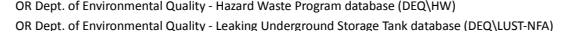 \\
\hline 445 DEA & 促 & JACKSON & $09 / 29 / 2008$ & 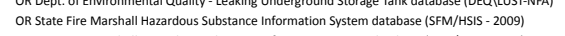 \\
\hline & & & 9/29/2008 & OR State fire Marshall Hazardous Substance Information System databasese (SFM/HISS- 2009) \\
\hline 212 WALL & Asth & JACKSON & 09//29/2008 & OR Sate fire Marshall Hazardous Substance Information System database (SFM/HISIS- 2009) \\
\hline 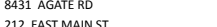 & WHTE CITY & JACKSON & 9/29/2008 & OR State Fire Marshall Hazarddus Substance Information S System database (SFM/HISIS - 2009) \\
\hline & edford & & 09/129/2008 & 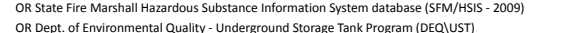 \\
\hline & & JACKSON & 09/29/2008 & 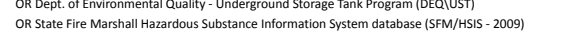 \\
\hline & & JaCKSON & $09 / 29 / 2008$ & 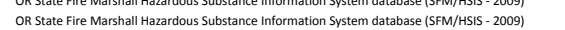 \\
\hline & $\mathrm{PO}$ & & 9/29/2008 & or State fire Marshall Hazardous Substance Information System databasese (SFM/HISIS - 2009) \\
\hline & ALP & & 09/29/2008 & OR State fire Marshall Hazardous Substance Information System database (SFM/HISIS- 2009) \\
\hline & & & 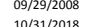 & R State fire Marshall Hazardous Substance Information System database (SFM/HISI - 2009) \\
\hline 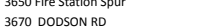 & & & & 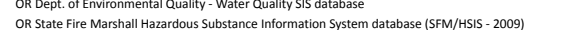 \\
\hline 940 AYRES RD & 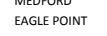 & JACKSON & 09/29/2008 & 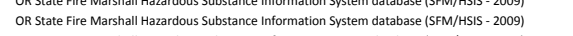 \\
\hline 20550 AVENUEG & $\begin{array}{l}\text { WHIT CITY } \\
\text { WHIF CITY }\end{array}$ & JACKSON & $09 / 29 / 2008$ & 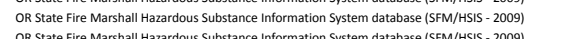 \\
\hline $\begin{array}{l}\text { 441 WNANELLPP RD } \\
3675 \text { Stage Rd }\end{array}$ & $\begin{array}{l}\text { WhHII Cird } \\
\text { Medford }\end{array}$ & $\begin{array}{l}\text { J ACCSSON } \\
\text { JACSSON }\end{array}$ & $\begin{array}{l}09 / 2992 / 208 \\
06 / 02 / 2015\end{array}$ & 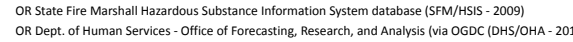 \\
\hline 3675 South Stage & ford & Jackson & $1 / 11 / 2018$ & \\
\hline 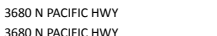 & $\begin{array}{l}\text { MEEFforo } \\
\text { Metrod }\end{array}$ & JACKSON & 09/29/2008 & \\
\hline $\begin{array}{l}36800 \text { NACFICL HWY } \\
3860 \text { P PACFCCWY }\end{array}$ & $\begin{array}{l}\text { Medoford } \\
\text { Mefford }\end{array}$ & JACKSON & 10/31/2018 & $\begin{array}{l}O R \text { Dept. of En } \\
\text { OR Dept of } E n\end{array}$ \\
\hline $\begin{array}{l}3680 \text { NAPCFFCCWY } \\
875 \text { AVE G STE B }\end{array}$ & $\begin{array}{l}\text { Wearora } \\
\text { WHITE CITY }\end{array}$ & $\begin{array}{l}\text { SACSOUN } \\
\text { JACSSON }\end{array}$ & 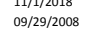 & 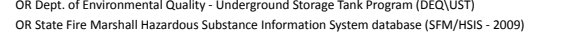 \\
\hline
\end{tabular}

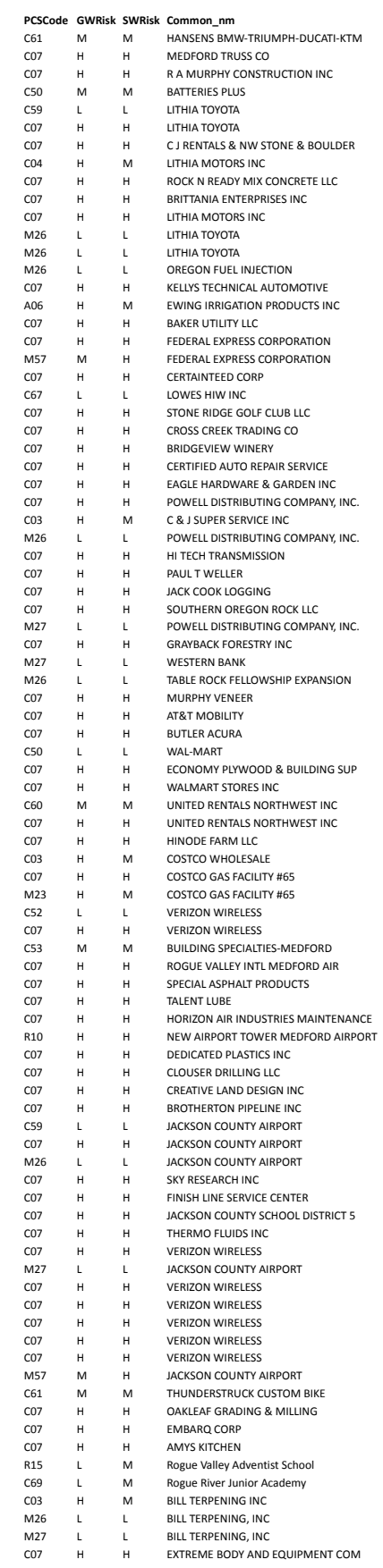

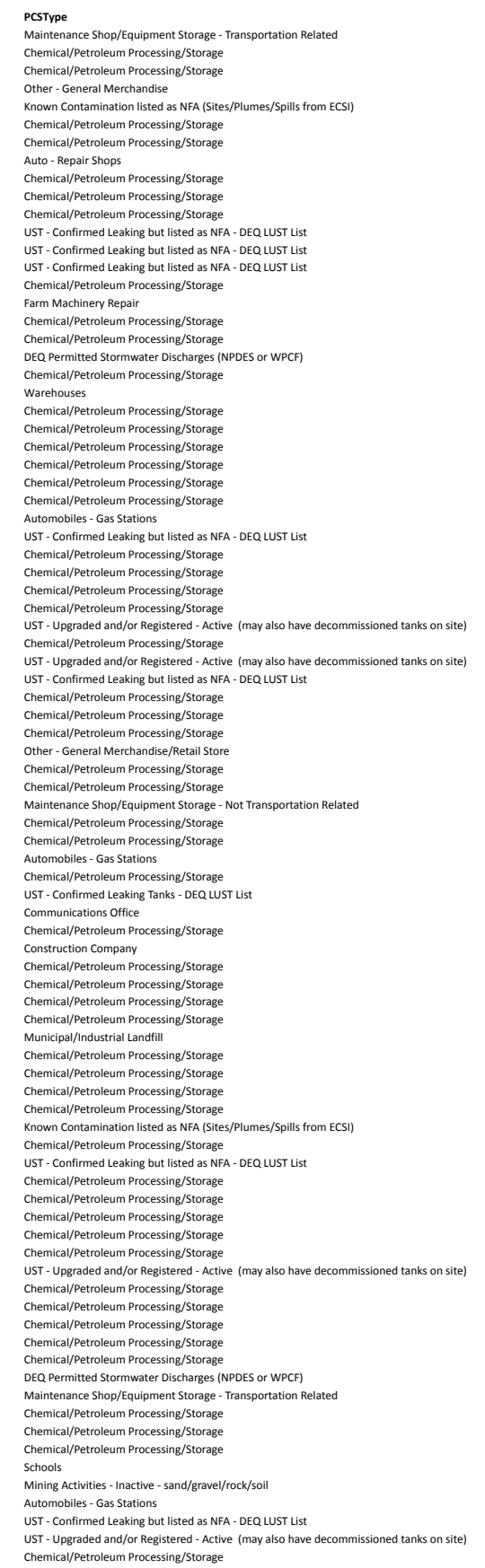




\begin{tabular}{|c|c|c|c|}
\hline & & & \\
\hline $\begin{array}{l}\text { Address } \\
3777 \text { STAGE RD }\end{array}$ & 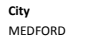 & $\begin{array}{l}\text { County } \\
\text { IACSSON }\end{array}$ & 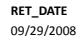 \\
\hline 6422 BLACKWELL RD & $\begin{array}{l}\text { MEEFROBD } \\
\text { CENTRALOINT PT }\end{array}$ & $\begin{array}{l}\text { JACSSON } \\
\text { IACSSON }\end{array}$ & $\begin{array}{ll}0.99192 / 2008 \\
09 / 29 / 2008\end{array}$ \\
\hline 11500 HANNON RD & EAGL POINT & Jackson & 09/29/2008 \\
\hline $\begin{array}{l}7 \text { S FFoNTAGE RD } \\
7893 \text { PACICIC AVE }\end{array}$ & $\begin{array}{l}\text { Gold HLL } \\
\text { WHITECITY }\end{array}$ & JaCKSON & $\begin{array}{l}09 / 29 / 2008 \\
0.992 / 2 / 208\end{array}$ \\
\hline $\begin{array}{l}7893 \text { PACCCCACAVE HWE } \\
5186 \text { CRATR LAKE HWY }\end{array}$ & 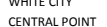 & $\begin{array}{l}\text { JaCSSON } \\
\text { IACSSON }\end{array}$ & $\begin{array}{l}09 / 29 / 2008 \\
0.9292 / 2008\end{array}$ \\
\hline 470 W ANTELOPE RD & $\begin{array}{l}\text { WHITE CITY } \\
\text { WH }\end{array}$ & JACKSON & \\
\hline 3504 NORTH RIVER RD & GOLD HIL & $\begin{array}{l}\text { JACKSON } \\
\text { Jack }\end{array}$ & 09/29/2008 \\
\hline 5465 HOLAND LP & CAVE Junction & JOSEPHINE & 09/29/2008 \\
\hline $\begin{array}{l}25320 \text { REDWOOD HWY } \\
1001 \text { CCEVS WWY }\end{array}$ & $\begin{array}{l}\text { CAVE JUNCTION } \\
\text { CAEE UNTTOO }\end{array}$ & JOSEPHINE & $09 / 29 / 2008$ \\
\hline $\begin{array}{l}17011 \text { 1 A AVES HWY } \\
1713 \text { B SISKIYOU BIVD }\end{array}$ & $\begin{array}{l}\text { CASE UNCTION } \\
\text { ASHLND }\end{array}$ & JOSEPHINE & $\begin{array}{l}09 / 29 / 2008 \\
0.992 / 2008\end{array}$ \\
\hline $\begin{array}{l}1173 \text { DEAD INDOANAN RD } \\
300\end{array}$ & $\begin{array}{l}\text { ASHLLND } \\
\text { ASHLAND }\end{array}$ & $\begin{array}{l}\text { JAAKSON } \\
\text { JACKSON }\end{array}$ & $\begin{array}{l}09 / 29 / 2008 \\
0.9292 / 2008\end{array}$ \\
\hline 403 DEAD INDIAN RD & ASHLAND & JACKSON & 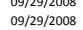 \\
\hline 403 DEAD INDIAN RD & ASHLAND & JACKSON & $\begin{array}{l}09 / 29 / 2008 \\
0\end{array}$ \\
\hline 176 E MAIN ST & EAGLE POINT & JACKSON & 09/29/2008 \\
\hline 2110 DEAD INDIAN RD & ASHLAND & JACKSON & $09 / 29 / 2008$ \\
\hline $\begin{array}{l}2275 \text { DEAD DNDINAN RD } \\
2518 \text { PACFIC HWY }\end{array}$ & ASHLAND & JACKSON & $\begin{array}{l}09 / 29 / 2008 \\
0.90202008\end{array}$ \\
\hline $\begin{array}{l}2185 \text { SAACFIC HWY } \\
1309 \text { SACIFIC HWY }\end{array}$ & $\begin{array}{l}\text { TAAENT } \\
\text { TALNAT }\end{array}$ & JAAKSON & $\begin{array}{l}099 / 2 / 2 / 2008 \\
00 / 29 / 2008\end{array}$ \\
\hline 1119 S PACIFIC HWY & $\begin{array}{l}\text { TALEN } \\
\text { TALNT }\end{array}$ & $\begin{array}{l}\text { JaCSSON } \\
\text { IACSSON }\end{array}$ & $\begin{array}{l}0 \\
09 / 29 / 2008 \\
0\end{array}$ \\
\hline 1120 S PACIFIC HWY & TAlent & JACKSON & 09/29/2008 \\
\hline 1073 S PACIFIC HWY & TALENT & JACKSON & $09 / 29 / 2008$ \\
\hline 101 E RAPP RD & $\begin{array}{l}\text { TALENT } \\
\text { TALENT }\end{array}$ & JACKSON & $09 / 29 / 2008$ \\
\hline $\begin{array}{l}104 \text { VALLFY VEWW RD } \\
300 \text { W VALEY VIEW }\end{array}$ & $\begin{array}{l}\text { TAAENT } \\
\text { TALENT }\end{array}$ & $\begin{array}{l}\text { JAAKSON } \\
\text { JACKSON }\end{array}$ & $\begin{array}{l}09 / 29 / 2008 \\
009292008\end{array}$ \\
\hline 530 VALLEY VIEW RD & $\begin{array}{l}\text { SALENT } \\
\text { TALENT }\end{array}$ & $\begin{array}{l}\text { JAAKSON } \\
\text { JACKSON }\end{array}$ & $\begin{array}{l}09929 / 2008 \\
09 / 29 / 2008\end{array}$ \\
\hline 251 VALLYY VIEW RD & $\begin{array}{l}\text { TALENT } \\
\text { TALENT }\end{array}$ & 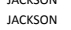 & $\begin{array}{lll}0 & 09 / 29 / 2008 \\
0\end{array}$ \\
\hline 5065 S PACFFC HWY & PHOENIX & JaCksoN & 09/29/2008 \\
\hline 3719 INTERNATIONAL WY & MEEFORD & JaCKSON & 09/29/2008 \\
\hline 3724 CRATER LAKE AVE & $\begin{array}{l}\text { MEEFORD } \\
\text { MEFERO }\end{array}$ & JACKSON & 09/29/2008 \\
\hline $\begin{array}{l}3724 \text { CRARER LAAEE AVE } \\
3730 \text { FERN VALLY RD }\end{array}$ & $\begin{array}{l}\text { MEOBORD } \\
\text { MEFEORD }\end{array}$ & JAAKSON & $\begin{array}{l}09 / 29 / 2008 \\
0.9292 / 2008\end{array}$ \\
\hline 3735 CRATER LAKE HWY & $\begin{array}{l}\text { MEEFOODO } \\
\text { MEOFORD }\end{array}$ & $\begin{array}{l}\text { JaCSSON } \\
\text { IACCSON }\end{array}$ & $\begin{array}{l}09929 / 2008 \\
09 / 29 / 2008\end{array}$ \\
\hline 3736 EARNETT RD & MEDFORD & 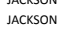 & $\begin{array}{lll}0 & 09 / 29 / 2008 \\
0\end{array}$ \\
\hline 3736 E BARNETT RD & MEDFORO & JACKSON & 09/29/2008 \\
\hline $37 / 2 \mathrm{~W} / 524$ & $\begin{array}{l}\text { Medford } \\
\text { Menopon }\end{array}$ & JACKSON & 10/13/2018 \\
\hline 3811 CRATER LAKE HWY STE C & MEDFORD & Jackson & $09 / 29 / 2008$ \\
\hline $\begin{array}{l}3811 \text { CARÉER L LAKE RAOD } \\
3825 \text { CRATER AKE HWY }\end{array}$ & $\begin{array}{l}\text { Mefford } \\
\text { MEFFoRD }\end{array}$ & JAAKSON & $\begin{array}{l}10 / 13 / 2018 \\
019292008\end{array}$ \\
\hline 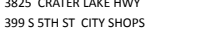 & $\begin{array}{l}\text { MEEFOOD } \\
\text { CENTRAL POINT }\end{array}$ & $\begin{array}{l}\text { JAAKSON } \\
\text { JACKSON }\end{array}$ & $\begin{array}{l}09929 / 2008 \\
09 / 29 / 2008\end{array}$ \\
\hline 4500 ROGUE VALLEY HWY & CENTRAL POINT & JACKSON & $\begin{array}{ll}09 / 29 / 2008 \\
0\end{array}$ \\
\hline 3841 CRATER LAKE HWY & Medford & JACKSON & $10 / 31 / 2018$ \\
\hline 555 MosQuito LN & CENTRAL POINT & JACKSON & $09 / 29 / 2008$ \\
\hline 5205 N RIVER RD & GOLD HILL & JACKSON & 09/29/2008 \\
\hline $\begin{array}{l}\text { 53474 R RVVRR RD } \\
\text { 3843 CRATER LAKE HWY }\end{array}$ & Metford & $\begin{array}{l}\text { JAAKSON } \\
\text { JACKSON }\end{array}$ & $\begin{array}{l}09 / 2 / 2 / 2008 \\
10 / 13 / 2018\end{array}$ \\
\hline 100 FISH LAKE RD & BUTTE FALLS & JACKSON & $\begin{array}{l}1 \\
09 / 29 / 2008\end{array}$ \\
\hline 101 OLD HWY 62 & EAGLE POINT & Jackson & $\begin{array}{l}09 / 29 / 2008 \\
0\end{array}$ \\
\hline 200 COLE M RIVERS DR & TRAIL & JaCKSON & 09/29/2008 \\
\hline $\begin{array}{ll}100 \\
100\end{array}$ & TRAAL & JACKSON & 09/29/2008 \\
\hline LAT T2 4813 LINGG 1223255 & PROSPECT & JACKSON & 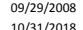 \\
\hline 3846 S PACIFIC HWY & $\begin{array}{l}\text { Morree } \\
\text { MEDFORD }\end{array}$ & $\begin{array}{l}\text { Benton } \\
\text { AaCKSON }\end{array}$ & $\begin{array}{l}\begin{array}{l}101 / 1 / 2018 \\
09 / 29 / 2008\end{array} \\
0\end{array}$ \\
\hline 200 STAGE RD & N/A & JACKSON & $\begin{array}{l}\text { 10/11/2018 } \\
1097 / 208\end{array}$ \\
\hline 205 STAGE RD & N/A & JACKSON & 10/31/2018 \\
\hline 2015 MOUNTAIN AVE & Astland & JACKSON & 10/31/2018 \\
\hline 3846 SAACFCC HWV & MEFFRO & JACKSON & 09/29/2008 \\
\hline 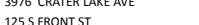 & $\begin{array}{l}\text { MEEFrok } \\
\text { Centroint }\end{array}$ & JACCSON & $\begin{array}{l}09 / 29 / 2008 \\
10 / 1312018\end{array}$ \\
\hline $\begin{array}{l}3976 \text { CRATER LAKE AVE } \\
3.9\end{array}$ & MEFFRD & $\begin{array}{l}\text { JACKSONON } \\
\text { JACKSON }\end{array}$ & $\begin{array}{l}1 / 131 / 2018 \\
09 / 29 / 2008\end{array}$ \\
\hline 205 ECALIFORNA ST & Jacksonvile & JACKSON & $\begin{array}{l}0.0797 / 2008 \\
10 / 31 / 2018\end{array}$ \\
\hline 2008 JoSEPH ST & $\mathrm{N} / \mathrm{A}$ & N/A & $10 / 31 / 2018$ \\
\hline 62700 CRATER LAKE HWY 62 & $\begin{array}{l}\text { N/A } \\
\text { Thent }\end{array}$ & $\begin{array}{l}\text { JACKSON } \\
\text { IACCSON }\end{array}$ & $\begin{array}{l}10 / 13 / 21 / 2018 \\
101312018\end{array}$ \\
\hline $\begin{array}{l}2000 \text { TALLNA TVE } \\
740 \text { MIL CREEK DR }\end{array}$ & $\begin{array}{l}\text { Talent } \\
\text { N/A }\end{array}$ & $\begin{array}{l}\text { JaCSSON } \\
\text { JaCKSON }\end{array}$ & $\begin{array}{l}101 / 13 / 218018 \\
10 / 11 / 2018\end{array}$ \\
\hline 3989 CRATER LAKE HWY & MEDFORD & 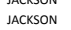 & 09/29/2008 \\
\hline 3995 S PACAFIC HWY & MEDFORD & JACKSON & 09/29/2008 \\
\hline 5800 PACAFIC HWY & N/A & JACKSON & 10/31/2018 \\
\hline $\begin{array}{l}2366 \text { HWY } 66 \\
236 \text { HWY } 66\end{array}$ & $\begin{array}{l}\text { Assland } \\
\text { Assland }\end{array}$ & $\begin{array}{l}\text { JACKSON } \\
\text { JACKON }\end{array}$ & $\begin{array}{l}10 / 13 / 21 / 2018 \\
101312018\end{array}$ \\
\hline $\begin{array}{l}3266 \text { HWV } 66 \\
3995 \text { S PACIFIC HWY }\end{array}$ & $\begin{array}{l}\text { ASSlan } \\
\text { MEDFORO }\end{array}$ & $\begin{array}{l}\text { JAAKSON } \\
\text { JACKON }\end{array}$ & $\begin{array}{l}1 \\
09 / 29 / 29008 \\
0\end{array}$ \\
\hline 4 W $3 R D$ ST & Medford & JACKSON & 10/31/2018 \\
\hline 40 S BARTLETT ST & Mefford & JACKSON & 10/31/2018 \\
\hline 15 WVILASRD & N/A & JaCKSON & 10/31/2018 \\
\hline 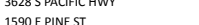 & 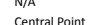 & IACCSONO & $\begin{array}{l}10 / 31 / 2018 \\
10 / 312018\end{array}$ \\
\hline 00 & $\begin{array}{ll}1 \\
\text { OFFRR }\end{array}$ & 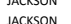 & $\begin{array}{l}10 / 11 / 2018 \\
0.1292008\end{array}$ \\
\hline T TSS, R BE, SE, 33, TAX LOT 7000 & & 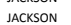 & $\begin{array}{l}0.01991 / 2008 \\
10 / 312018\end{array}$ \\
\hline 400 E MCANDREWS RD & & JaCkSON & 10/31/2018 \\
\hline 1300 WIISON R & & JACKSON & 10/31/2018 \\
\hline & $\begin{array}{l}\text { Mecoford } \\
\text { Ashland }\end{array}$ & $\begin{array}{l}\text { J IACCSON } \\
\text { JACKSON }\end{array}$ & $\begin{array}{l}06 / 02 / 21 / 2015 \\
10 / 1312018\end{array}$ \\
\hline ILEY VIEW RD & & $\begin{array}{l}\text { JackSON } \\
\text { JACSON }\end{array}$ & $101 / 1 / 2018$ \\
\hline 400 N RIVERSIDE & EEFFRD & $\begin{array}{l}\text { JACKSON } \\
\text { A }\end{array}$ & 09/29/2008 \\
\hline & & & \\
\hline
\end{tabular}

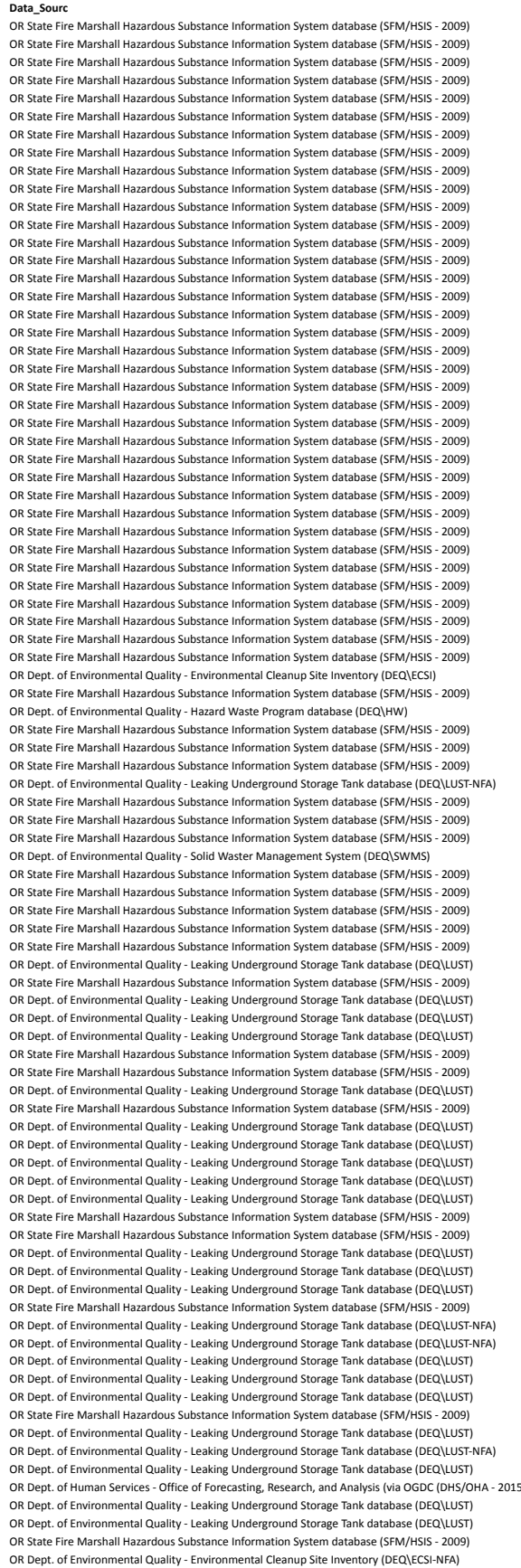
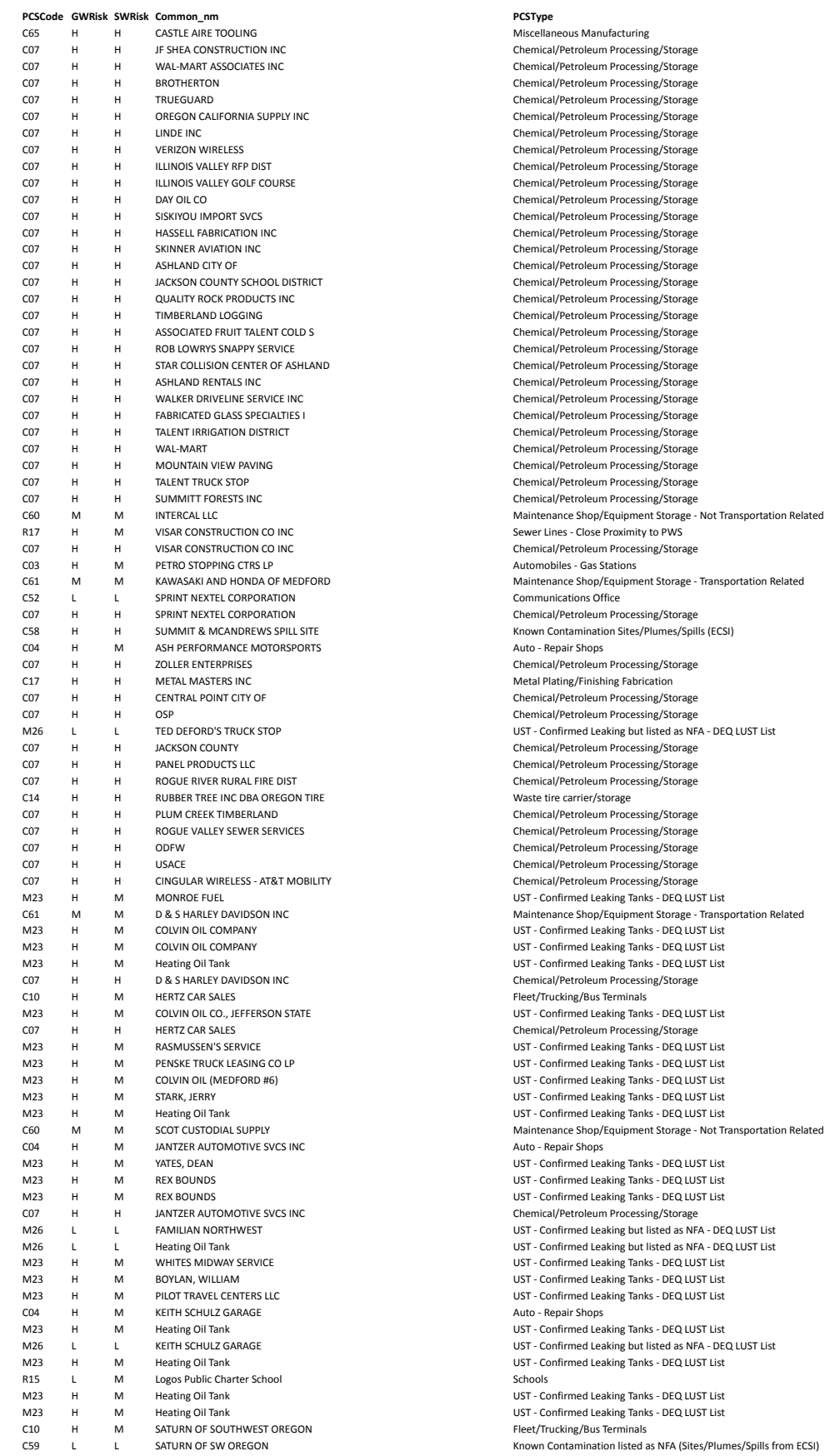


\begin{tabular}{|c|c|c|c|}
\hline $\begin{array}{l}\text { Address } \\
\text { 355 SCENIC }\end{array}$ & $\begin{array}{l}\text { city } \\
\text { Asshand }\end{array}$ & $\begin{array}{l}\text { County } \\
\text { JACKSON }\end{array}$ & $\begin{array}{l}\text { RET_DATE } \\
10 / 31 / 2018\end{array}$ \\
\hline $215 \mathrm{~N} \mathrm{ROSE}$ & $\begin{array}{l}\text { Phoenix } \\
\text { Phom }\end{array}$ & 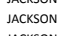 & $10 / 31 / 2018$ \\
\hline $\begin{array}{l}1116 \text { IOWA STREET } \\
400 \text { NIIESSDIF AVE }\end{array}$ & $\begin{array}{l}\text { Ashland } \\
\text { netsers }\end{array}$ & Jackson & $10 / 31 / 2018$ \\
\hline $\begin{array}{l}400 \mathrm{NRRVESSIDA} \mathrm{AVE} \\
4002 \text { CIRRUS DR }\end{array}$ & 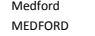 & $\begin{array}{l}\text { JaCSSON } \\
\text { JACSSON }\end{array}$ & $\begin{array}{l}101 / 1 / 2018 \\
09 / 29 / 2008\end{array}$ \\
\hline 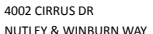 & & Jackson & $10 / 31 / 2018$ \\
\hline $\begin{array}{l}\text { NUTLYY \& WNINURN WAY W } \\
10663 \text { MEADOWS RD }\end{array}$ & Asthand & $\begin{array}{l}\text { JACKSON } \\
\text { JAAKSON }\end{array}$ & $\begin{array}{l}10 / 31 / 2018 \\
1013 / 2018\end{array}$ \\
\hline $\begin{array}{l}106633 \text { MEADDOWS RD } \\
2911 \text { EMAN }\end{array}$ & $\begin{array}{l}\mathrm{N} / \mathrm{A} \\
\text { Asthand }\end{array}$ & $\begin{array}{l}\text { JAAKSON } \\
\text { JACKSON }\end{array}$ & $\begin{array}{l}101 / 3 / 21 / 2018 \\
10131 / 2018\end{array}$ \\
\hline 4002 CIRRUS DR & Medford & & $\begin{array}{l}10 / 131 / 2018 \\
10138\end{array}$ \\
\hline 201 RACHEL DR & Central Point & Jackson & $\begin{array}{l}10 / 131 / 2018 \\
1018\end{array}$ \\
\hline 4002 CIRRUS DR & Medford & Jackson & $11 / 1 / 2018$ \\
\hline 121 REAGER ST & N/A & JaCKSON & $10 / 31 / 2018$ \\
\hline 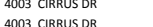 & $\begin{array}{l}\text { MEDEORD } \\
\text { MEEDORD }\end{array}$ & $\begin{array}{l}\text { JACKSON } \\
\text { IACCSOON }\end{array}$ & $\begin{array}{l}09 / 29 / 2008 \\
0992 / 2008\end{array}$ \\
\hline 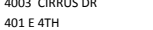 & $\begin{array}{l}\text { MEFrofi } \\
\text { Mefford }\end{array}$ & $\begin{array}{l}\text { IJCCSSON } \\
\text { JACKSON }\end{array}$ & $\begin{array}{l}09929 / 2008 \\
10 / 1 / 2018 \\
\end{array}$ \\
\hline 509 N LAUREL ST & $\begin{array}{l}\text { Asshland } \\
\text { Astat }\end{array}$ & 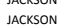 & $\begin{array}{l}101 / 3 / 21 / 2018 \\
10 / 131 / 2018\end{array}$ \\
\hline 4013 CRATER LAKE HWY & MEDFORD & Jackson & $\begin{array}{l}1 \\
09 / 29 / 20008\end{array}$ \\
\hline 3626 Ross LN & $\mathrm{N} / \mathrm{A}$ & JACKSON & $10 / 31 / 2018$ \\
\hline 194 TIMBERRLAKE DR & $\mathrm{N} / \mathrm{A}$ & JACKSON & $10 / 31 / 2018$ \\
\hline 148 E VALLEY VIEW RD & N/A & JaCKSON & $\begin{array}{l}10 / 13 / 2018 \\
1013 / 2018\end{array}$ \\
\hline 4013 CRATER LK HWY & $\begin{array}{l}\text { Jacksonolile } \\
\text { Medford }\end{array}$ & $\begin{array}{l}\text { JaCSSON } \\
\text { IACCSON }\end{array}$ & $\begin{array}{l}101 / 3 / 21 / 2018 \\
10 / 13 / 2018\end{array}$ \\
\hline & Medford & $\begin{array}{l}\text { Sactson } \\
\text { Jackson }\end{array}$ & $\begin{array}{l}10131 / 2018 \\
1 / 11 / 2018\end{array}$ \\
\hline 9 HILLCREST ST & Ashland & 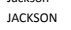 & $\begin{array}{l}10 / 11 / 2018 \\
1 / 2170\end{array}$ \\
\hline 3444 HOLLYWOOD AVE & N/A & JaCkSON & 10/31/2018 \\
\hline 4017 Jacksonville Hwy & $\begin{array}{l}\text { Medford } \\
\text { Meffores }\end{array}$ & $\begin{array}{l}\text { Jackson } \\
\text { Jucssoos }\end{array}$ & $\begin{array}{l}1 / 11 / 2018 \\
103 / 218\end{array}$ \\
\hline $\begin{array}{l}4025 \text { CENTRAL AVE } \\
302 \text { 2ND ST }\end{array}$ & $\begin{array}{l}\text { Mefford } \\
\text { Ashland }\end{array}$ & $\begin{array}{l}\text { JaCSSON } \\
\text { IACKSON }\end{array}$ & $\begin{array}{l}10 / 31 / 2018 \\
10131 / 2018\end{array}$ \\
\hline 4045 CRATER LAKE HWY & $\begin{array}{l}\text { Asshand } \\
\text { MEDFORD }\end{array}$ & $\begin{array}{l}\text { JaCSSONO } \\
\text { IACSSON }\end{array}$ & 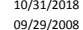 \\
\hline 800 MalN ST & Phoenix & JACKSON & 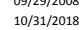 \\
\hline 414 E PINE ST & Central point & JACKSON & $10 / 11 / 2018$ \\
\hline 4045 CRATER LAKE HWY & Medford & JACKSON & 10/31/2018 \\
\hline 407 BOARDMAN ST & Medford & JaCKSON & $10 / 31 / 2018$ \\
\hline $\begin{array}{l}8400 \text { AGAEE ROAD } \\
41 \text { STEWART AVE }\end{array}$ & N/A & JACKSON & $\begin{array}{l}10 / 11 / 2018 \\
\end{array}$ \\
\hline $\begin{array}{l}41 \text { ESE EWATT AVE } \\
41 \text { ETEWART AVE }\end{array}$ & $\begin{array}{l}\text { MEEFORD } \\
\text { MEOFORD }\end{array}$ & $\begin{array}{l}\text { JaCSSON } \\
\text { JaCSSON }\end{array}$ & $\begin{array}{l}09929 / 2008 \\
09 / 29 / 2008\end{array}$ \\
\hline 945 HILUVEW DR & Ashland & Jackson & $\begin{array}{l}0.979 / 2008 \\
10 / 11 / 2018\end{array}$ \\
\hline 412 AST & Ashland & JaCKSON & $10 / 31 / 2018$ \\
\hline 1401 CENTER DR & N/A & JACKSON & 10/31/2018 \\
\hline 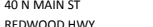 & Assland & JACKSON & 10/31/2018 \\
\hline $\begin{array}{l}\text { REDWOOOH HWY } \\
19000 \text { CAVES HIGHWAY }\end{array}$ & N/A & 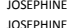 & $\begin{array}{l}10 / 31 / 2018 \\
101312018 \\
\end{array}$ \\
\hline 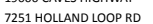 & N/A & $\begin{array}{l}\text { JOSEFPHINE } \\
\text { JOSEHNE }\end{array}$ & $\begin{array}{l}10 / 13 / 21 / 2018 \\
101312018\end{array}$ \\
\hline 1025 REDWOOD HWY & N/A & JOSEPHINE & $\begin{array}{l}10 / 31 / 2018 \\
1018\end{array}$ \\
\hline CRATER LAKE & N/A & КLAMATH & $10 / 31 / 2018$ \\
\hline HOUSE 19 & N/A & КІАМАTH & 10/31/2018 \\
\hline $\begin{array}{l}6055 \text { SW CANYNON LN } \\
41 \text { HAWTHORNE ST }\end{array}$ & $\begin{array}{l}\text { Portland } \\
\text { Menfford }\end{array}$ & 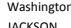 & $10 / 31 / 2018$ \\
\hline 41 ROSE AVE & 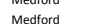 & $\begin{array}{l}\text { A ACCSSON } \\
\text { Accson }\end{array}$ & $\begin{array}{l}101 / 1 / 2018 \\
101312018\end{array}$ \\
\hline 4 15 SARNABURG RD & Medford & 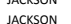 & $\begin{array}{l}1 / 1 / 13 / 212018 \\
1 / 31 / 2018\end{array}$ \\
\hline 4100 CRATER Lake AVE & MEFORD & Jackson & $\begin{array}{l}09 / 29 / 2008 \\
0\end{array}$ \\
\hline 4100 CRATER LAKE AVE & MEDFORD & Jackson & 09/29/2008 \\
\hline 411 W 8 8TH ST & Medtord & JACKSON & 10/31/2018 \\
\hline 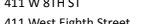 & $\begin{array}{l}\text { Metiord } \\
\text { Metrid }\end{array}$ & JACKSON & 10/31/2018 \\
\hline 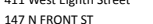 & 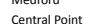 & $\begin{array}{l}\text { Jackson } \\
\text { accsoN }\end{array}$ & $\begin{array}{l}1 / 11 / 2018 \\
1 / 131 / 2018\end{array}$ \\
\hline 414 EADS ST & МЕFORO & 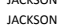 & $\begin{array}{l}10 / 131 / 2018 \\
09 / 29 / 2008\end{array}$ \\
\hline 3100 WILOW SPRINGS RD & $\mathrm{N} / \mathrm{A}$ & JaCKSON & 10/11/2018 \\
\hline 1977 HWY $99 \mathrm{~N}$ & $\mathrm{~N} / \mathrm{A}$ & JACKSON & 10/31/2018 \\
\hline 11111EVVILASRD & N/A & JACKSON & 10/31/2018 \\
\hline 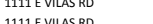 & N/A & JACKSON & 10/31/2018 \\
\hline 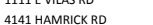 & Metford & 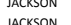 & $\begin{array}{l}1 / 1 / 31 / 2018 \\
10 / 31 / 2018\end{array}$ \\
\hline 7235 KELER RD & Ashland & 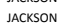 & $\begin{array}{l}10 / 131 / 21 / 818 \\
10 / 31 / 2018\end{array}$ \\
\hline 451 2 2NOST & Central Point & JaCKSON & $101 / 1 / 2018$ \\
\hline 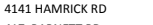 & Metford & JACKSON & 10/31/2018 \\
\hline 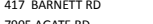 & MEFFRO & JACKSON & 09/29/2008 \\
\hline $\begin{array}{l}7 \\
7\end{array}$ & N/A & JACSSON & $\begin{array}{l}10 / 13 / 21 / 218 \\
1 / 1312018\end{array}$ \\
\hline 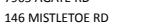 & Asthand & 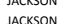 & $\begin{array}{l}10 / 131 / 218 \\
10 / 31 / 2018\end{array}$ \\
\hline 4177 BLACK OAK DR & Medford & $\begin{array}{l}\text { IACKSON } \\
\text { Ians }\end{array}$ & $101 / 1 / 2018$ \\
\hline 100 COLE M RVVES DR & N/A & Jackson & 10/31/2018 \\
\hline 8250 AGATE RD & N/A & JACKSON & 10/31/2018 \\
\hline 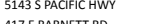 & N/A & JACCSONO & 10/31/2018 \\
\hline 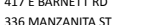 & $\begin{array}{l}\text { Meetrord } \\
\text { Centroint }\end{array}$ & 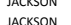 & $\begin{array}{l}10 / 13 / 21 / 2018 \\
1 / 1312018\end{array}$ \\
\hline 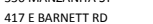 & Medford & Jacsoos & $\begin{array}{l}10 / 131 / 218 \\
10 / 31 / 2018\end{array}$ \\
\hline 13TH ST \& AVE F & & JaCKSON & $\begin{array}{l}101 / 1 / 2018 \\
1018\end{array}$ \\
\hline A17 E BARNETT RD & Medford & JACKSON & $11 / 1 / 2018$ \\
\hline 425 GROVEEAND ST & Medford & JACKSON & 10/31/2018 \\
\hline & Medford & JACKSON & 10/31/2018 \\
\hline & 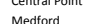 & 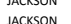 & $\begin{array}{l}10 / 13 / 21 / 218 \\
1 / 1312018\end{array}$ \\
\hline ARNETTRD & 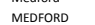 & SACKSoN & $\begin{array}{l}101711 / 2018 \\
09 / 29 / 2008\end{array}$ \\
\hline 7600 CRATER LAKE HWY & & JACKSON & 10/13/2018 \\
\hline
\end{tabular}

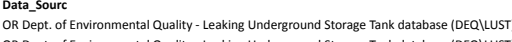

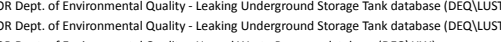
Be stare

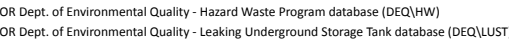

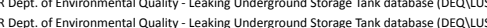

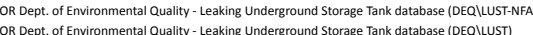

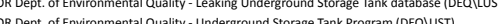
OR Dept. of Environmental Quality - Leakinin Underground Storage Tank database (IEQQ1 USS

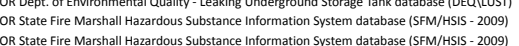

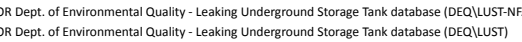
Se (SFM/HIS- 2000

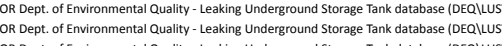

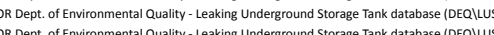
OR Dept. of Environmental Q uality - Hazard Waste Program database (DEQQHW) OR Dept. of Geology and Mineral Industries Mineral Information layer for Oregon( DOGAMI/MLIL

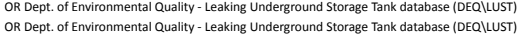

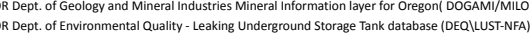

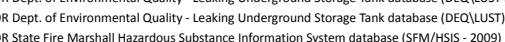

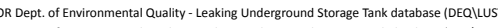

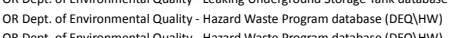

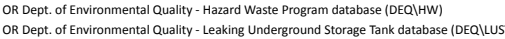

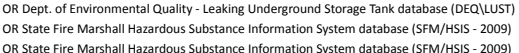
OR State Fire Marshall Hazard ous Substance Information System database (SFM/HSIS- 2000

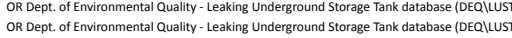

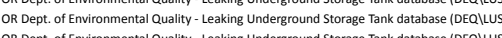

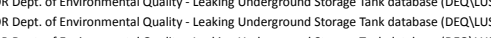

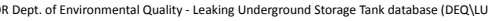

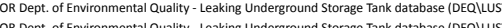

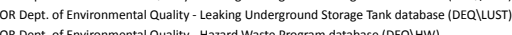

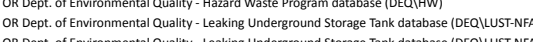

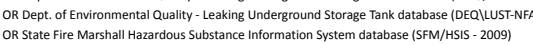

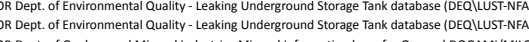

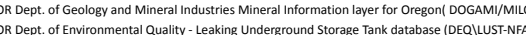
OR State fire Marshall Hazardous Substance Information System database (SFM/HSIS - 2009)

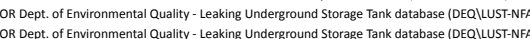

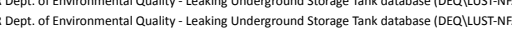
(o Depent. of OR Dept. of Environmental a uality - Leakin B Underground Storage Tank database (DEQQ IUST-NFF)

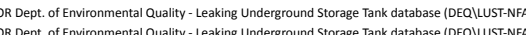
OR State fire Marshall Hazardous Substance Information System database (SFM/HSIS- 2009) OR Dept. of Environmental Quality - Leaking Underground Storage Tank database IDEQQIUST-NF

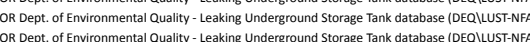
OR Dept. of Environmental Q Qualty - Leaking Underground Storage Tank database (DEQQ LIUST-N

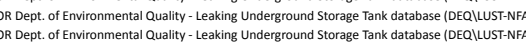
OR Dept. of Environmental Q Quality - Hazard Waste Program databases (DEQQ1HW)

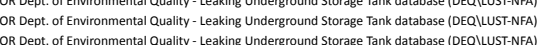

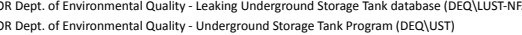

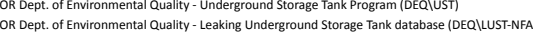
OR Dept. of Environmental Quality - Hazard Waste Program database (DEQOHW)

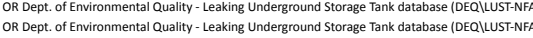
OR State fire Marshall Hazardous Substance Information System database (SFM)/FIS - 2009)
OR Dept. of Environmental Quality L Leaking Underground Storage Tank database (DEQ IUST-NFA)

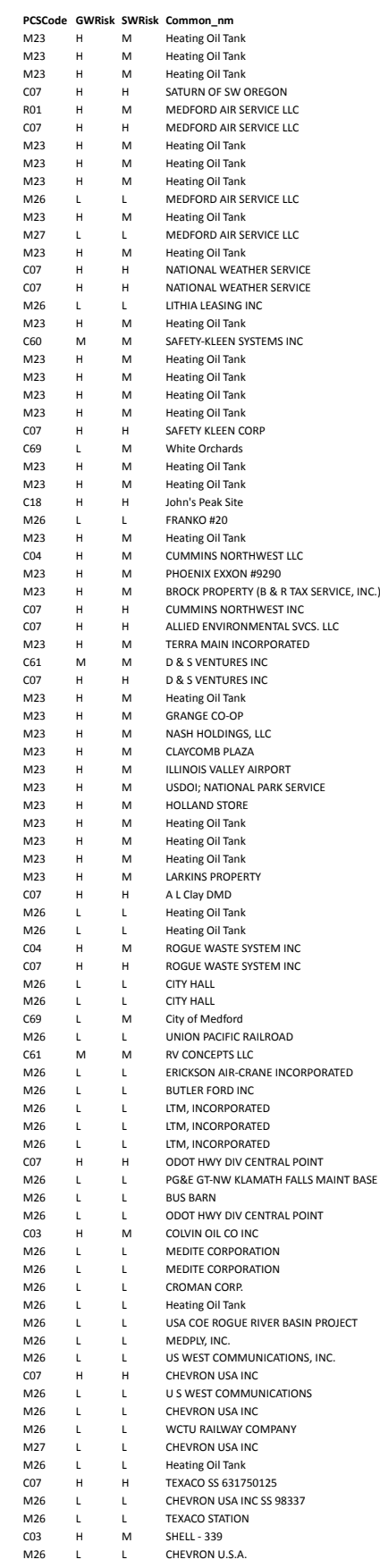

USTT-Confirmed Leaking Tanks - DEQ L LST Lis

UST - Confirmed Leaking Tanks - DEQ LUST Lis UST - Confirmed leakinin Tanks - DEQ LUST
Chemical/Petroleum Processing/storage

Chemical/Petroleum Processing//storage
UST - Confirmed Leaking Tanks - DEQ LUST

UST - Confirmed Leaking Tanks - DEQ USST List

UST- Confirmed Leaking but listed as NFA- DEQ LUST Lis

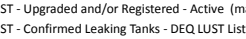
Chenical/Petroleum Processing//Storage

UST- Confirmed Leaking but listed as NFA- DEQ LuST Lis
UST - Confirmed Leaking Tanks DEQ LUST List

UST- Confirmed Leaking Tanks - DEQ LUST List

UST - Confirmed Leaking Tanks - DEQ LUST Lis

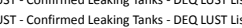

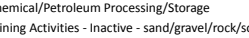

UST- Confirmed Leaking Tanks - DEQ LUST Lis
UST - Confirmed Leaking Tanks - DEQ UST LiS

Mining Activities - Active - sand/fravel/rock/soil
UST- Confirmed Leaking but listed as NFA- DEQ LST

UST - Confirmed Leaking Tanks - DEQ LUST Lit

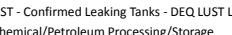

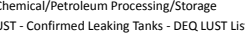

Maintenance Shop/Equipment Storage. - Trass

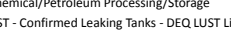

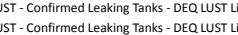

UST C Confirmed Leaking Tanks - DEQ Q UST Lis
UST - Confirmed Leaking Tanks - DEQ LUST L L

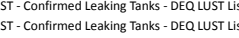

UST- Confirmed Leaking Tanks - DEQ LUST Lis

Chemicalpertoleum Processing/ StStrage
UST - Confirmed Leaking but listed as NFA- DEQ L LST Lis

UST - Confirmed Leak
Auto - Repair Shops

UST - Confirmed Leaking but listed as NFA- DEQ LUST Lis

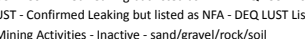
UST - Confirmed Leaking but listed as NFA- DEQ LUST Lis

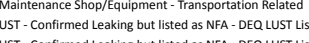

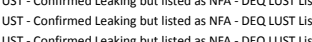

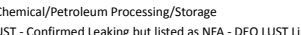
WST-COT UST- Confrimene Leaknin bu
Automobilies - Gas stations

UST- Confirmed Leaking but isted as NFA - DEQ LUST LIS UST - Confirmed Leaking but listed as NFA - DEQ LUST T UST - Confirmed Leaking but listed as NFA - DEQ LUST Li

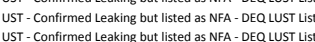
UST - confirmed Leaking but ist isted as N NAA - DEQ LUST Lis UST- Confirimed Led Leaking but tisteded as NFA- DEQQ USST Lis

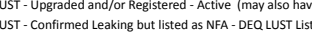

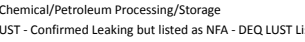
UST - Confirmed Leaking but listed a S NFA- DEQ QUST List
UST - Confirmed Leaking but isted as NFA - DEQ Q LSS L List Automobiles - Gas Stations
UST - Confirmed Leaking b 


\begin{tabular}{|c|c|c|c|}
\hline $\begin{array}{l}\text { Address } \\
7600 \text { CRATER LAKE HWY }\end{array}$ & $\begin{array}{l}\text { City } \\
N / A\end{array}$ & $\begin{array}{l}\text { County } \\
\text { JACKSON }\end{array}$ & $\begin{array}{l}\text { RET_DATE } \\
10 / 11 / 2018\end{array}$ \\
\hline 5100 CRATER L LKE AVE & & JACKSON & \\
\hline $\begin{array}{l}4300 \text { HADLEY DR } \\
200 \text { ATTEOOPF RD }\end{array}$ & $\begin{array}{l}\text { MEFOORD } \\
\text { NA }\end{array}$ & $\begin{array}{l}\text { JaCKSON } \\
\text { IACCSSON }\end{array}$ & $\begin{array}{l}09 / 29 / 2008 \\
101 / 12012\end{array}$ \\
\hline $\begin{array}{l}200 \text { ANTELOOER RD } \\
4300 \text { HADLEY DR }\end{array}$ & $\begin{array}{l}\text { N/A } \\
\text { MEFFRD }\end{array}$ & $\begin{array}{l}\text { JACSKON } \\
\text { JACKSON }\end{array}$ & $\begin{array}{l}1 / 131 / 212018 \\
09 / 29 / 2008\end{array}$ \\
\hline $\begin{array}{l}431 \text { s livst } \\
\text { sofy }\end{array}$ & Medford & JACKSON & 06/102/2015 \\
\hline $\begin{array}{l}50 \text { 6TH ST } \\
435 \text { N BAPTLETT }\end{array}$ & $\begin{array}{l}\text { Assland } \\
\text { MEDFORD }\end{array}$ & $\begin{array}{l}\text { IJCCSON } \\
\text { ACCKSON }\end{array}$ & $\begin{array}{l}10 / 31 / 2018 \\
0.9292 / 2008\end{array}$ \\
\hline $\begin{array}{l}\text { 435 B BARRLTTT } \\
\text { 435 N BARTLETT ST }\end{array}$ & $\begin{array}{l}\text { MEForok } \\
\text { Mefford }\end{array}$ & & $\begin{array}{l}09 / 29 / 2 / 2008 \\
10 / 312018\end{array}$ \\
\hline 2518 PACFIC CWUY & N/A & & $\begin{array}{l}10 / 131 / 218 \\
10 / 31 / 2018\end{array}$ \\
\hline 2518 PACIFIC HWY & $\mathrm{N} / \mathrm{A}$ & Jackson & $\begin{array}{l}10 / 31 / 21 / 2018 \\
10018\end{array}$ \\
\hline 435 N BARTLETT ST & Medford & & 11/1/2018 \\
\hline 4399 CENTRAL & Medford & JaCKSON & 10/31/2018 \\
\hline $\begin{array}{l}238000 \text { HWY } 66 \\
2506\end{array}$ & $\begin{array}{l}\text { Asshland } \\
\text { Asthand }\end{array}$ & JACSSON & $\begin{array}{l}10 / 31 / 2018 \\
10 / 212018\end{array}$ \\
\hline 2500 HWY 66 & 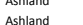 & 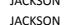 & $\begin{array}{l}1 / 131 / 2018 \\
10 / 312018\end{array}$ \\
\hline 4655 TABLE ROCK RD & N/A & & $\begin{array}{l}10 / 1131 / 218 \\
10 / 31 / 2018\end{array}$ \\
\hline 3959 HAMRICK RD & $\mathrm{N} / \mathrm{A}$ & & $\begin{array}{l}10 / 31 / 2018 \\
101818\end{array}$ \\
\hline 7975 11TH ST & & JACKSON & 10/31/2018 \\
\hline 439 N CENTRAL AVE & MEDFORD & Jackson & 09/29/2008 \\
\hline 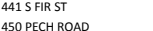 & $\begin{array}{l}\text { MEFFORD } \\
\text { Mefford }\end{array}$ & $\begin{array}{l}\text { JACKSON } \\
\text { JACSSON }\end{array}$ & $\begin{array}{l}09 / 29 / 2008 \\
11 / 12018\end{array}$ \\
\hline $\begin{array}{l}450 \text { PECH ROAD } \\
454 \text { S WEXEORD CIR }\end{array}$ & $\begin{array}{l}\text { MEETCOA } \\
\text { MEDFRO }\end{array}$ & $\begin{array}{l}\text { JaCGSON } \\
\text { JACCSON }\end{array}$ & $\begin{array}{l}1 / 1 / 1 / 2018 \\
09 / 29 / 2008\end{array}$ \\
\hline 10175 AGATE RD & W/A & 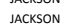 & \\
\hline 4540 GRUMMAN DR & MEDFORD & JaCKSON & 09/29/2008 \\
\hline 4540 GRUMMAN DR & MEFFRD & JACKSON & 09/29/2008 \\
\hline 4621 GRUMMAN DR & $\begin{array}{l}\text { Medtord } \\
\text { Metroper }\end{array}$ & JACKSON & $\begin{array}{l}10 / 31 / 2018 \\
0.9202008\end{array}$ \\
\hline 4643 GRUMMAN DR & & $\begin{array}{l}\text { IACCSSON } \\
\text { ACCSON }\end{array}$ & $\begin{array}{l}09 / 29 / 2 / 2008 \\
10 / 312018\end{array}$ \\
\hline 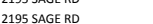 & N/A & 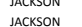 & $\begin{array}{l}1 / 131 / 218 \\
10 / 31 / 2018\end{array}$ \\
\hline 1001 ISSkrou Blvo & Ashland & Jackson & $\begin{array}{l}10 / 31 / 21 / 2018 \\
1018\end{array}$ \\
\hline 2809 J ACKSONVILE HWY & $\mathrm{N} / \mathrm{A}$ & JACKSON & 10/31/2018 \\
\hline 2606 OLD STAGE RD & N/A & JACKSON & 10/31/2018 \\
\hline 4643 GRUMMAN DRIVE & $\begin{array}{l}\text { Medtord } \\
\text { Centrant pons }\end{array}$ & JaCKSON & $\begin{array}{l}10 / 31 / 2018 \\
101 / 212018\end{array}$ \\
\hline 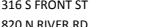 & & JACKSON & $\begin{array}{l}10 / 31 / 2018 \\
101312018\end{array}$ \\
\hline 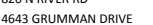 & Metord & 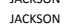 & $\begin{array}{l}1 / 131 / 218 \\
10 / 31 / 2018 \\
\end{array}$ \\
\hline $25 \mathrm{E}$. Mcandrews & N/A & JACKSON & $\begin{array}{l}1.0711 / 21 / 218 \\
10 / 31 / 2018\end{array}$ \\
\hline 25 E. McAndrews & $N / A$ & JACKSON & 10/31/2018 \\
\hline 4819 HELO DR & Medford & JACKSON & 10/31/2018 \\
\hline 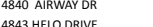 & MEFFORD & IACCSON & 09/29/2008 \\
\hline 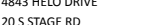 & Nevorord & 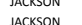 & $\begin{array}{l}10 / 31 / 2018 \\
10 / 11212018\end{array}$ \\
\hline$\triangle 485$ & MEDОов & 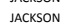 & $\begin{array}{l}1 / 131 / 218 \\
09 / 2 / 2008\end{array}$ \\
\hline 2350 AVE G & N/A & JACKSON & 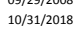 \\
\hline 2355 AVE G & N/A & JacksoN & 10/31/2018 \\
\hline 4845 COLLMAN CREEK R R & MEFFORD & JACKSON & 09/29/2008 \\
\hline $\begin{array}{l}\text { 8380 GAAAED } \\
\text { 830 }\end{array}$ & N/A & 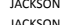 & $\begin{array}{l}10 / 31 / 2018 \\
11 / 212018\end{array}$ \\
\hline A990 CCARE LKK AVE & МЕFОRD & 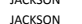 & $\begin{array}{l}1 / 131 / 21218 \\
0.129202008\end{array}$ \\
\hline 4950 CRATR LAKE AVE & MEFFRD & 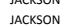 & 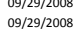 \\
\hline 4950 CRATER LAKE AVE & MEEFORD & Jackson & 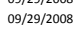 \\
\hline 3750 KRTLLAND ROAD & N/A & JACKSON & 10/31/2018 \\
\hline 49999 CRATER LLKE HWY & MEFFORD & JACKSON & 09/29/2008 \\
\hline 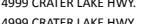 & $\begin{array}{l}\text { Medtord } \\
\text { Metrat }\end{array}$ & 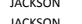 & $\begin{array}{l}10 / 31 / 2018 \\
10121019\end{array}$ \\
\hline 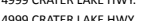 & 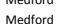 & 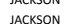 & $\begin{array}{l}10 / 31 / 2018 \\
11 / 1212018\end{array}$ \\
\hline 500 PECH RD & МЁFоㅇ & 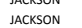 & $\begin{array}{l}111 / 1 / 2018 \\
09 / 2 / 2008\end{array}$ \\
\hline 500 PECH RD & MEOFORD & JACKSON & 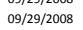 \\
\hline FOOTHHL RD \& VVILAS RD & N/A & JACKSON & 10/31/2018 \\
\hline 500 PEECH RD & Meefford & JACKSSON & 10/31/2018 \\
\hline 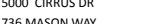 & MEFORO & $\begin{array}{l}\text { PaCCSSON } \\
\text { ACCSON }\end{array}$ & 09/29/2008 \\
\hline T) & 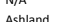 & $\begin{array}{l}\text { S } \\
\text { IICCSSON }\end{array}$ & $\begin{array}{l}1 / 131 / 21218 \\
10 / 312018\end{array}$ \\
\hline 200 COLE M RIVERS DR & N/A & JaCCSSON & $\begin{array}{l}10 / 317 / 218 \\
10 / 3 / 2018\end{array}$ \\
\hline 19000 CAVES HIGHWAY & N/A & JOSEPHINE & 10/31/2018 \\
\hline 706 TOLIMAN CREER ROAD & N/A & JACKSON & 10/31/2018 \\
\hline 706 TOLOMAN CREER ROAD & N/A & JACCSONO & 10/31/2018 \\
\hline 2490 ASHANAN BIVO & & 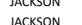 & 10/31/2018 \\
\hline 2730 OREN VULFY ROAD & Phoenix & actson & $\begin{array}{l}1 / 131 / 218 \\
10 / 312018\end{array}$ \\
\hline $\begin{array}{l}3730 \text { EREN VALLF R RAD } \\
\end{array}$ & Phoenix & 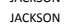 & $\begin{array}{l}1.1711 / 218 \\
10 / 31 / 2018\end{array}$ \\
\hline 3730 FERN VALLEY ROAD & Phoenix & JaCKSON & 10/31/2018 \\
\hline 3730 FERN VALLEY ROAD & Phoenix & JACKSON & 10/31/2018 \\
\hline 18 STEWART AVE. & N/A & JACCSONO & 10/31/2018 \\
\hline 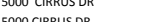 & MEEFERO & 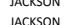 & 09/29/2008 \\
\hline 5 & Medford & Jackson & $\begin{array}{l}10 / 31 / 2018 \\
101 / 2112018\end{array}$ \\
\hline 5000 CRATER LAKE HWY & Medford & Jackson & $\begin{array}{l}10 / 3172018 \\
10 / 21 / 2018\end{array}$ \\
\hline 5009 GRIFFIN CREEK RD & MEFFORD & JaCKSON & 09/29/2008 \\
\hline 501 AlRPORT RD & & JACKSON & 09/29/2008 \\
\hline 3813 CRATER LAKE HWY & manpons & JACCSONO & 10/31/2018 \\
\hline & (1) & 年 & 10/3112012018 \\
\hline 501 MEDFORD CTR & diford & Jackson & $\begin{array}{l}10 / 111 / 218 \\
10 / 31 / 2018\end{array}$ \\
\hline 501 MEDFORD CTR & Medford & & 101/11/2018 \\
\hline
\end{tabular}

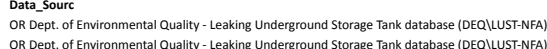
OR State fire Marshall Hazarddous Substance Informatiotio System database (SFM/HSIS - 2009)

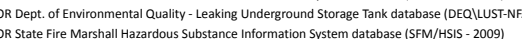

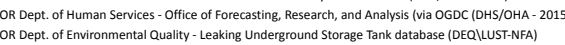

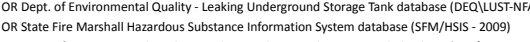

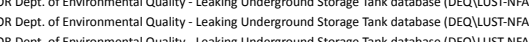

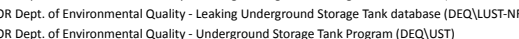

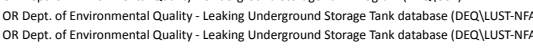

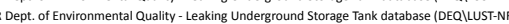

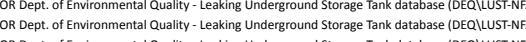
OR Dept. of Environmental Qually- Leakin

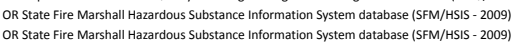

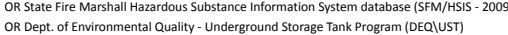

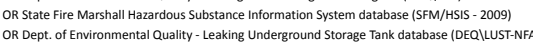

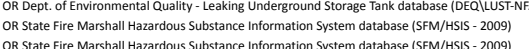

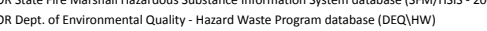

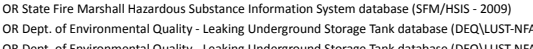
OR Dept. of Environmental Q uality - Leaking Underground Storage Tank database (DEQ) LUST-NFA

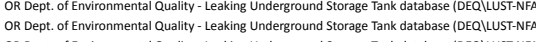

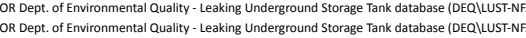

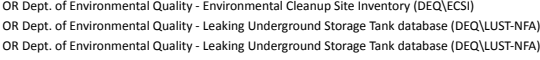
OR Dept. of Environmental Quality- Leaking Underground Storage Tank database (DEQQILUST-NFFA

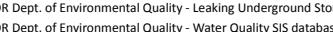
OR State fire Marshall Hazardous Substance Information Sys D. Dept. of Environmental Q Quality - Water Ruality SIS database

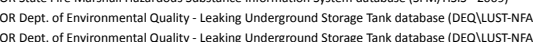

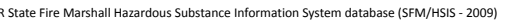

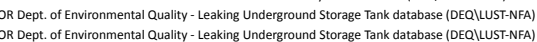

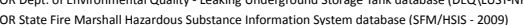

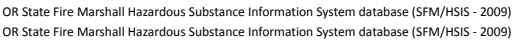

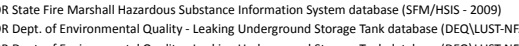

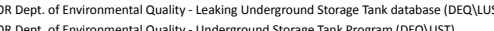

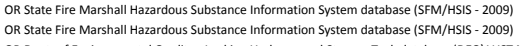

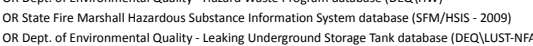

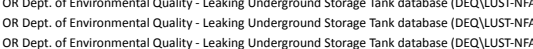

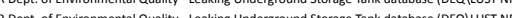
OR Dept. of Environmental Quality - Leaking Underground Storage Tank database (DEQQLLST-NFA

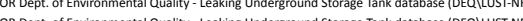

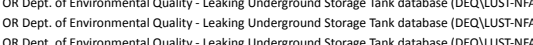

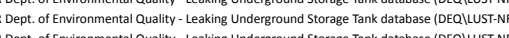

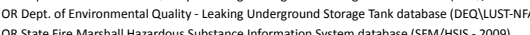

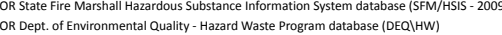

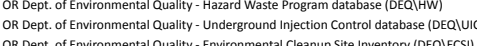

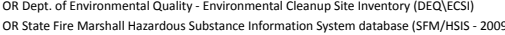

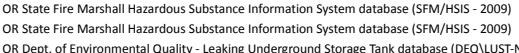

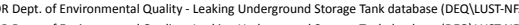

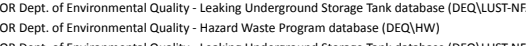

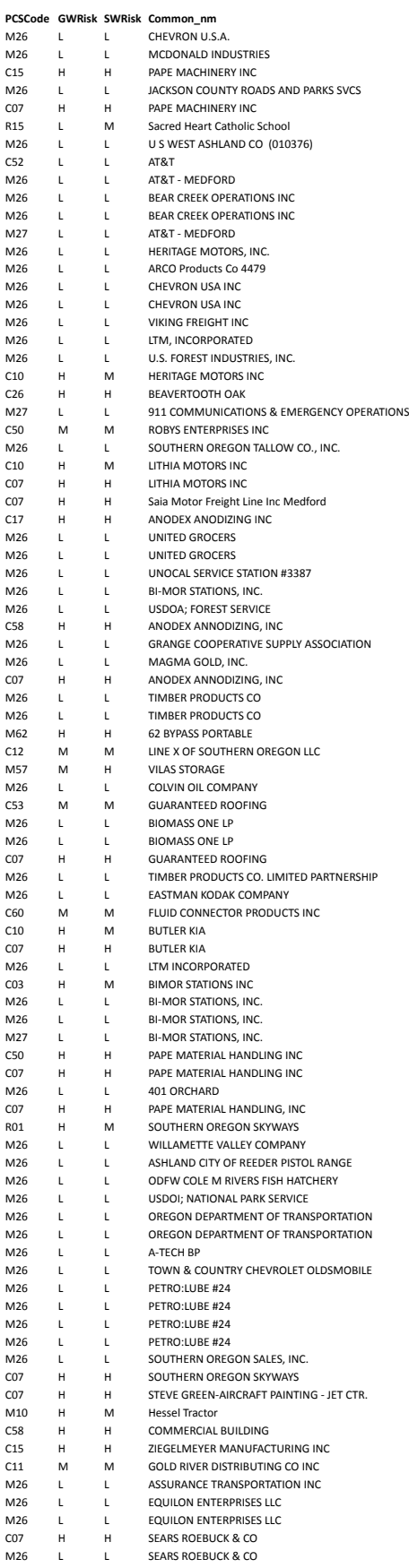

USTYPe
UST Confirmed Leaking but listed as NFA- DEQ LUST List UST- Confirmed Leakin Machine Shops
uST - Confirmed Leaking but listed as N NFA- DEQ LuST List Schools
UST- Confirmed Leaking but listed as NFA- DEQ LUST List
Communication S Office ST - Confirmed Les

UST - Confirmed Leaking but listed a a N NAA- DEQ UST Lis

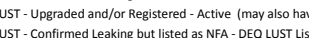
UST - Confirmed Leaking but listed a S NFA - DEQ LUST Lis UST - Confirmed Leaking but listed as NFA - DEQ LUST L UST - Confirmed Leaking but listed a S NFA- DEQ LUST Lis UST - Confirmed Leaking but listed a NFA - DEQ WST T Fleet/Trucking/us Terminals

Wood/Pulp/Paper Processing and Mills
UST - Uperaded and/or Registered - Active

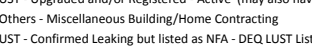
chemical/petroleum Processing / Stororage

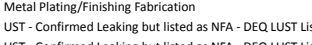
UST - Confirmed Leaking but listed a N NFA - DEQ LUST Lis

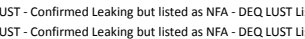

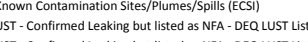
chemicalpetroloum Processing $/$ tororge UST - Confirmed Leaking but listed as NFA- DEQ LUST Lis
UST- Confirmed Leaking but isted as NAF- DEQ OUST ndustrial or Process W

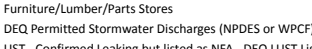

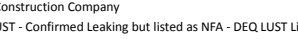

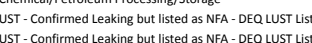
Maintenance Shop/Equipments storge- Not Tansportation Hleet/Trucking/Bus Terminals
Chemical/Petroleum Processing/st UST- confirmed Leaking but tisted as N NAA - DEQ L LST Lis UST - Confirmed Leaking but listed as NAA - DEQ LUST List
UST - Confirmed leaking but Iised as NAA - DEQ WUST List UST - Upgraded and/or Registered-Active (may also have daco Others - Unknown Commercial

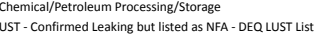

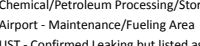

UST - Confirmed Leaking but Isted as NFA- DEQ LUST Lis UST - Confirmed Leaking but listed as NFA - DEQ L UST List

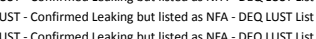
WST-Confirmed Leaking but IIsted a s NAA- DEQ LSSTL UST - Confirmed Leaking but listed as NAA-DF W WST UST - Confirmed Leaking but listed a N NFA - DEQQ LUST Lis UST- Confirmed Leaking but isted as a SFA- ERQ ULST Lis Chemical/Petrolum Processing/Storage

Industrial/Commercial Injection Wells/Sorvwells/Sumps - Class V VICS Mnown Contamm
Machie Shops
Food rocessing

UST- Confirmed Leaking but Iisted as NFA- DEQ Q UST List UST- Confirmed Leaking but isted as NFA- DEQ UUST Lis

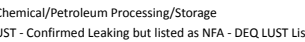




\begin{tabular}{|c|c|c|c|}
\hline $\begin{array}{l}\text { Address } \\
7260 \text { BLACKWELL RD. }\end{array}$ & City & $\begin{array}{l}\text { County } \\
\text { JACKSON }\end{array}$ & $\begin{array}{l}\text { RET_DATE } \\
\text { 10131/2018 }\end{array}$ \\
\hline 21 BALL RD RO & N/A & N/A & $10 / 31 / 2018$ \\
\hline $\begin{array}{l}5015 \text { M MEDFRD CENTER } \\
\text { OAK\& \&'A'ST }\end{array}$ & $\begin{array}{l}\text { MEDFORD } \\
\text { Asshand }\end{array}$ & $\begin{array}{l}\text { JaCKSON } \\
\text { JACKSON }\end{array}$ & $\begin{array}{l}09 / 29 / 2008 \\
101 / 2 / 2018\end{array}$ \\
\hline $\begin{array}{l}\text { OAA\& A A ST } \\
\text { OAK\& A'ST }\end{array}$ & $\begin{array}{l}\text { Assinna } \\
\text { Ashland }\end{array}$ & & $\begin{array}{l}10 / 131 / 2018 \\
1018\end{array}$ \\
\hline 6740 CRATER L LAKE AVE & & JackSON & $10 / 31 / 2018$ \\
\hline $\begin{array}{l}431 \text { S FRONT ST } \\
413 \text { SRONT ST }\end{array}$ & $\begin{array}{l}\text { Central Point } \\
\text { Centra Poits }\end{array}$ & 作 & $\begin{array}{l}10 / 31 / 2018 \\
1013 / 2018\end{array}$ \\
\hline $\begin{array}{l}\text { 431 S R ROONT ST } \\
5746 \text { CRATER LAKE AVE }\end{array}$ & $\begin{array}{l}\text { Central Point } \\
\text { N/A }\end{array}$ & N/A & $\begin{array}{l}101 / 3 / 21 / 2018 \\
10131 / 2018\end{array}$ \\
\hline 2350 AVENUE F FVE & $\begin{array}{l}N / A \\
N / A\end{array}$ & $\begin{array}{l}\text { NACKSON } \\
\text { JAC }\end{array}$ & $\begin{array}{l}101 / 3 / 21 / 2018 \\
10 / 131 / 2018\end{array}$ \\
\hline 7640 CRATER LAKE HWY & N/A & 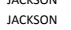 & $\begin{array}{l}10 / 131 / 2018 \\
1018\end{array}$ \\
\hline 7640 CRATER LAKE HWY & $\mathrm{N} / \mathrm{A}$ & Jackson & $10 / 31 / 2018$ \\
\hline 7640 CRATER L LAKE HWY & N/A & Jackson & $10 / 31 / 2018$ \\
\hline 5015 SIIVERSIDE AVE & $\begin{array}{l}\text { Medford } \\
\text { MEDFORD }\end{array}$ & $\begin{array}{l}\text { JACKSON } \\
\text { IACCSOON }\end{array}$ & $\begin{array}{l}10 / 131 / 2018 \\
0992 / 2008\end{array}$ \\
\hline $\begin{array}{l}5015 \text { S RRVILRSIDE A AE } \\
70 \text { MILC CREE DR }\end{array}$ & $\begin{array}{l}\text { MEFFORD } \\
\text { N/A }\end{array}$ & $\begin{array}{l}\text { JaCCSSON } \\
\text { JACKSON }\end{array}$ & $\begin{array}{l}09 / 2 / 2 / 2008 \\
1 / 131 / 2018 \\
\end{array}$ \\
\hline 28 S FRONT ST & & $\begin{array}{l}\text { JACKSON } \\
\text { Jask }\end{array}$ & $\begin{array}{l}101 / 3 / 21 / 2018 \\
10 / 131 / 2018\end{array}$ \\
\hline 5015 SRIVERSIDE AVE & MEDFORD & Jackson & $\begin{array}{l}1 \\
09 / 29 / 20008\end{array}$ \\
\hline 3629 S PACIFIC HWY & & JACKSON & $10 / 31 / 2018$ \\
\hline 501 W MCANDREWS S & MEDFORD & JACKSON & $09 / 29 / 2008$ \\
\hline & $\begin{array}{l}\text { Ashland } \\
\text { Shayd cove }\end{array}$ & $\begin{array}{l}\text { JACKSON } \\
\text { IACSSON }\end{array}$ & $\begin{array}{l}10 / 13 / 2018 \\
1013 / 2018\end{array}$ \\
\hline 501 W N MCANR LANDREWS S & $\begin{array}{l}\text { Shadd Cove } \\
\text { MEYFoRD }\end{array}$ & $\begin{array}{l}\text { JAAKSON } \\
\text { JACKSON }\end{array}$ & 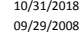 \\
\hline 5019 TABLE ROCK RD & $\begin{array}{l}\text { MEEFForo } \\
\text { Mefiord }\end{array}$ & $\begin{array}{l}\text { JAAKSON } \\
\text { JACKSON }\end{array}$ & $\begin{array}{l}09929 / 2008 \\
10 / 31 / 2018\end{array}$ \\
\hline 2864 TAYLOR RD & & JACKSON & $10 / 11 / 2018$ \\
\hline CORNER OF GARFELLD AND & Ashland & JACKSON & 10/31/2018 \\
\hline 75010 OL HWW 99 S S & N N/A & JACKSON & $100 / 31 / 2018$ \\
\hline $\begin{array}{l}5022 \text { N CENTRAL } \\
502 \text { CENTRAL AVE }\end{array}$ & $\begin{array}{l}\text { MEDEFORD } \\
\text { Mefford }\end{array}$ & $\begin{array}{l}\text { JACKSON } \\
\text { IACCSON }\end{array}$ & $\begin{array}{l}09 / 29 / 2008 \\
10131 / 2018 \\
\end{array}$ \\
\hline $\begin{array}{l}502 \text { N CENNRALAVE } \\
502 \text { N CENTRAL AVE }\end{array}$ & $\begin{array}{l}\text { Metatord } \\
\text { Mefford }\end{array}$ & $\begin{array}{l}\text { PaCSSON } \\
\text { IACCSON }\end{array}$ & $\begin{array}{l}101 / 3 / 2 / 2018 \\
11 / 1 / 2018\end{array}$ \\
\hline 502 S RIVERSIDE & $\begin{array}{l}\text { Metarld } \\
\text { MEOFORD }\end{array}$ & 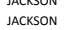 & $\begin{array}{l}1 \\
09 / 29 / 2008 \\
0\end{array}$ \\
\hline 502 S RIVERSIDE & Mefford & JACKSON & $10 / 11 / 2018$ \\
\hline 181 APPLEGATE RD & Jacksonvile & JACKSON & 10/31/2018 \\
\hline 181 APPLEGATE RD & Jacksonville & JaCKSON & $10 / 31 / 2018$ \\
\hline 1385 ANTELOPE RD & N/A & $\begin{array}{l}\text { JACKSON } \\
\text { IACCSON }\end{array}$ & $\begin{array}{l}10 / 13 / 21 / 2018 \\
10131 / 2018\end{array}$ \\
\hline 5020 TABLE ROCK RD & $\begin{array}{l}\text { Assland } \\
\text { Mefford } \\
\text { Mets }\end{array}$ & $\begin{array}{l}\text { JaCSSON } \\
\text { IACSSON }\end{array}$ & $\begin{array}{l}101 / 3 / 21 / 2018 \\
10 / 131 / 2018\end{array}$ \\
\hline 2651 MILL CREEK DR & N/A & Jackson & $\begin{array}{l}10 / 31 / 2018 \\
10 / 218\end{array}$ \\
\hline 5020 TABLE ROCK RD & Medford & JACKSON & $11 / 1 / 2018$ \\
\hline 5050 TABLE ROCK RD & MEDFORD & JaCKSON & $09 / 29 / 2008$ \\
\hline 509 WEST 11TH & $\begin{array}{l}\text { MEDFORO } \\
\text { MEROEPB }\end{array}$ & JaCKSON & 06/102/2015 \\
\hline 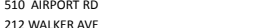 & $\begin{array}{l}\text { MEDEFRD } \\
\text { Ashland }\end{array}$ & 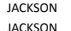 & $\begin{array}{l}09 / 29 / 2008 \\
10131 / 2018 \\
\end{array}$ \\
\hline $\begin{array}{l}\text { L2W WA ARKRER AV } \\
510 \text { ARPORT RD }\end{array}$ & $\begin{array}{l}\text { ASSland } \\
\text { MEDFORD }\end{array}$ & $\begin{array}{l}\text { JaCSSOON } \\
\text { IACSSON }\end{array}$ & 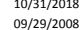 \\
\hline 160 LOWE RD & $\begin{array}{l}\text { MEFOOKD } \\
\text { N/A }\end{array}$ & $\begin{array}{l}\text { SACSOCON } \\
\text { IACSSON }\end{array}$ & $\begin{array}{l}\text { 10/31/2018 } \\
1091 / 208\end{array}$ \\
\hline 5100 Crater Lake Avenue & Medford & Jackson & $10 / 31 / 2018$ \\
\hline 21824 HWY 62 & Shady cove & JACKSON & $10 / 31 / 2018$ \\
\hline $\begin{array}{l}500 \text { W W BUTLER LANE } \\
135 \text { MISTELTE RD }\end{array}$ & N N/A & JACKSON & $\begin{array}{l}10 / 31 / 2018 \\
1013 / 2018\end{array}$ \\
\hline $\begin{array}{l}133 \text { MISTLLTOE RD } \\
135 \text { MISTLETOE RD }\end{array}$ & $\begin{array}{l}N^{N / A} \\
N / A\end{array}$ & $\begin{array}{l}\text { JaCSSON } \\
\text { ICCCSON }\end{array}$ & $\begin{array}{l}10 / 31 / 2018 \\
1013 / 2018\end{array}$ \\
\hline 34 MIL CREER OR & $\begin{array}{l}\text { N/A } \\
N / A\end{array}$ & 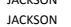 & $\begin{array}{l}10 / 13 / 21 / 2018 \\
10 / 18\end{array}$ \\
\hline S11 SFFR & Medford & Jackson & $10 / 11 / 2018$ \\
\hline $716 \mathrm{~S}$ PACIFIC HWY & $\mathrm{N} / \mathrm{A}$ & JaCKSON & 10/31/2018 \\
\hline S1115FRST & MEDFORD & JACKSON & 09/29/2008 \\
\hline 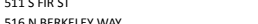 & MEDFORD & JACKSON & 09/29/2008 \\
\hline 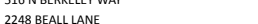 & $\begin{array}{l}\text { Medford } \\
\text { Central Point }\end{array}$ & $\begin{array}{l}\text { JaCCSON } \\
\text { ACCSSON }\end{array}$ & $\begin{array}{l}10 / 13 / 21 / 218 \\
1 / 1312018\end{array}$ \\
\hline 15625 F FRK LITTLE BUTTE CR RD & $\mathrm{N} / \mathrm{A}$ & 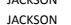 & $\begin{array}{l}10 / 131 / 2018 \\
10 / 11 / 2018\end{array}$ \\
\hline 8705 CRATE LAKE HWY & $\mathrm{N} / \mathrm{A}$ & JaCKSON & $10 / 11 / 2018$ \\
\hline 9625 OLD STAGE RD & N/A & JaCKSON & 10/31/2018 \\
\hline 2015 HANEKY RD & N/A & JACKSON & 10/31/2018 \\
\hline 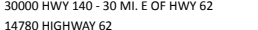 & N/A & JACKSON & 10/31/2018 \\
\hline 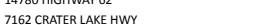 & 酸 & $\begin{array}{l}\text { JaCCSON } \\
\text { IACCSON }\end{array}$ & $\begin{array}{l}10 / 13 / 21 / 218 \\
10 / 1312018\end{array}$ \\
\hline 2185 GRIFIFIN CREEK RD & N/A & 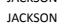 & $\begin{array}{l}10 / 131 / 21 / 8 \\
10 / 31 / 2018\end{array}$ \\
\hline 2185 GRIFFIN CREEK RD & $\mathrm{N} / \mathrm{A}$ & JaCKSON & $10 / 31 / 2018$ \\
\hline 10668 HWV 62 & Eagle Point & JACKSON & 10/31/2018 \\
\hline 3175 BOGUE RVER HWVY & N/A & JACKSON & 10/31/2018 \\
\hline 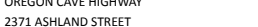 & N/A & JOSEPHINE & $\begin{array}{l}10 / 31 / 2018 \\
1 / 1312018\end{array}$ \\
\hline $\begin{array}{l}120 \text { MIL CREEK OR } \\
120\end{array}$ & N/A & $\begin{array}{l}\text { JAKSON } \\
\text { N/A }\end{array}$ & $\begin{array}{l}10 / 131 / 2018 \\
10 / 31 / 2018\end{array}$ \\
\hline 5186 CRATER LAKE AVE & Medford & JACKSON & $\begin{array}{l}10 / 131 / 2018 \\
10 / 31 / 2018\end{array}$ \\
\hline 5199 TABLE ROCK RD & Medford & JaCKSON & 10/31/2018 \\
\hline 326 BROAD ST & Butte Falls & JACKSON & 10/31/2018 \\
\hline 326 BROAOST ST & Butte Fals & JaCKSON & 10/31/2018 \\
\hline 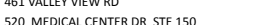 & N/A & JACSSON & $\begin{array}{l}10 / 31 / 2018 \\
0.9292008\end{array}$ \\
\hline & MEFFRO & 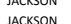 & $\begin{array}{l}0 \\
09 / 29 / 20008 \\
0\end{array}$ \\
\hline & MEEFORD & $\begin{array}{l}\text { JaCKSON } \\
\text { Jas }\end{array}$ & $\begin{array}{l}09792 / 2008 \\
09 / 2908\end{array}$ \\
\hline 523 ROSSANEEF OR & MEDFORD & JaCKSON & 09/29/2008 \\
\hline & Medfior & JACKSON & 10/31/2018 \\
\hline CORN & N & JACKSSON & 10/31/2018 \\
\hline 460 VALLEYVEW & & 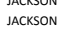 & $\begin{array}{l}10 / 3171 / 2018 \\
101312818\end{array}$ \\
\hline HWr 62 & & JACKSON & 10/31/2018 \\
\hline 525 N CENTRAL & MEDFORO & JACKSON & $09 / 29 / 2008$ \\
\hline
\end{tabular}

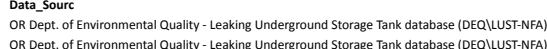

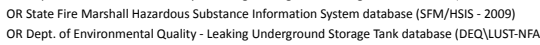
OR Dept. of Environmental Quality - Leaking Underg round Storage Tank database (DEQQILUST-NFF OR Dept. of Environmental Q Quality - Leaking Underground Storage Tank database (DEQQIULST-NFF

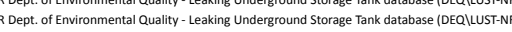

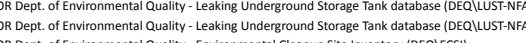

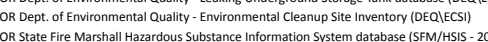

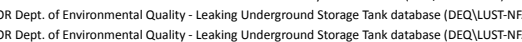

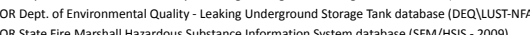

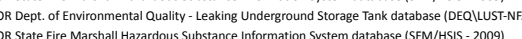

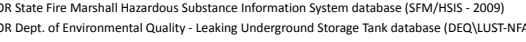
OR State fire Marshall Hazardous Substance Information System database (SFM/HSIS - 2009) OR Dept. of Environmental Quality - Leaking Underground Storage Tank database (DEQQLILST-NF

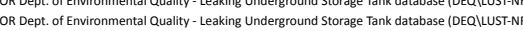
OR Dept. of Environmental Q Quality - Leaking Underground Storage Tank database (DEQQ LUST-N

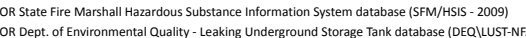

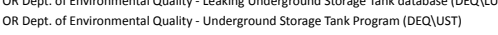

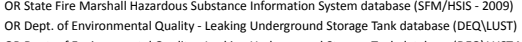
Se Tank database (DEQQ LUST-NFA

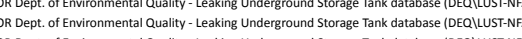
OR Dept. of Environmental Quality - Leaking Underground Storgage Tank database (DEQQ LUST) OR Dept. of Environmental Q Quality - Leaking Underground Storage Tank databases (DEQQ LIUST-N.

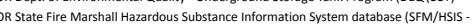

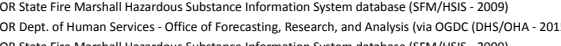
OR S State Fire Marshall Hazardous Substance Information System database (SFM/HSIS - 2009)
OR Dept of Environmental

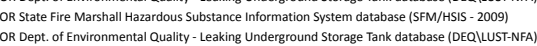

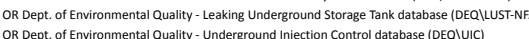

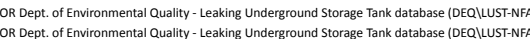

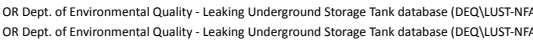
QR Dept. of Environmental Quality - Leaking Underground Storage Tank database (DEQQ) UUST-NFA

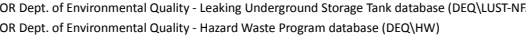

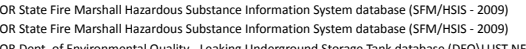

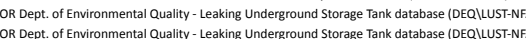
OR Dept. of Environmental Q Quality - Leaking Underground Storage Tank database (DEQQIUST-NFA

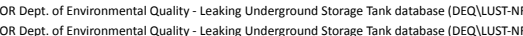

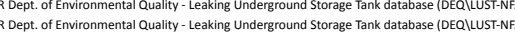

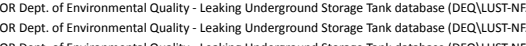
(0) OR Dept. of Environmental Q Quality - Leaking Underground Storage Tank database (DEQQLUST-NFA

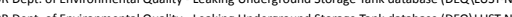

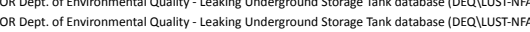

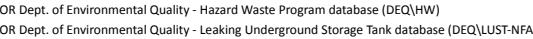

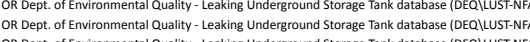
OR Dept. of Environmental Q Quality - Leaking Underground Storage Tank database (DEQQ LUST-NFA)

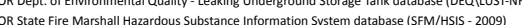
OR State fire Marshall Hazardous Substance informmation System datababase (SFM/MISIS - 2009) OR State fire Marshall Hazardous Substance Information System database (SFM/HISS -2009)

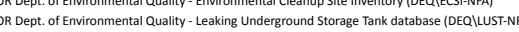

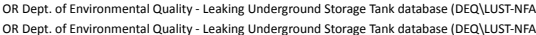

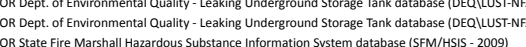

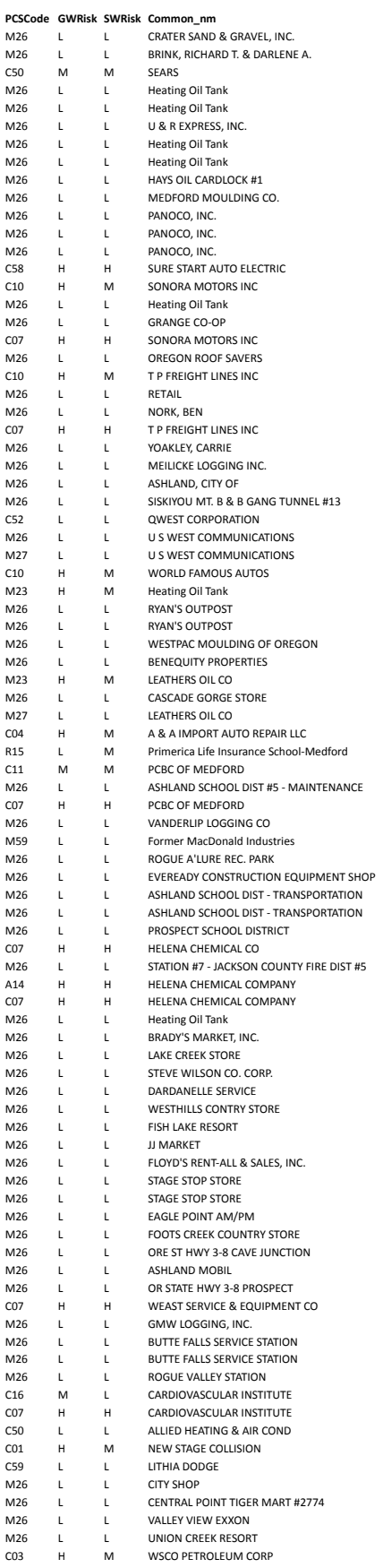

UST- Confirmed Leaking but listed as N NAA- DEQ UST Lis UT- Confirmed Leaking but listed as NFA - DEQ LUST L

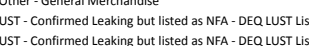
UST - Confirmed Leaking but listed as NFA- DEQ LUST Lis WT - Confirmed Leaking but listed as NFA- DEQ LSSTH

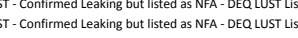

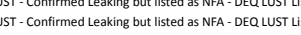
Known Contamination Stestiplumes/spills ERST)

UST- Confirmed Leaking but listed a s NFA- DEQ Q UST Lis
UST - Confirmed Leaking but isted as NFA - DEQ LUS Lis Fleet/Trucking//us Terminals

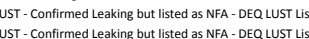
hemical/Petroleum Processing/storage UST - Conffirmed Leaking but isted as NFA- DEQ LUST LS

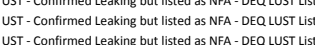
UST- Confirmed leaking
communications

WT - Confirimed Leaking but isted as NFA - DEQ LUST List Hiet/Trucking/(Bus Terminals UST - Confirmed Leaking but listed as NFA. DEQ LUST LIT UST - Confirmed Leaking but listed as NFA- DEQ LUST Lis

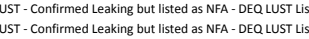
UST - Confirmed Leaking Tanks - DEQ LUST List

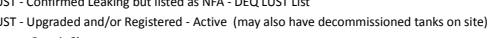

Food Processing
UST - confirmed Leaking but listed as NFA - DEQ L LST List

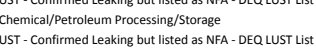

UST - Confirmed Leaking but listed as NFA - DEQ L LSTS Lis

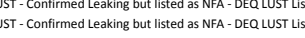

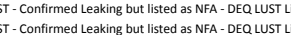
chemical/Petroleum Processinin/storage \& Cleaning Areas Chemical/Petroleum Processing/Storage
uST - Confirmed Leaking but isted as N NAA - DEQ Q UST List ST - Confirmed Leaking but listed a N NA - DEQ LUST Lis UST - Confirmed Leaking but listed as NFA- DEQ LUST LS

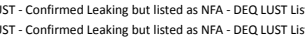

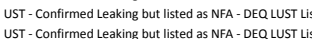
UST - Confirmed Leaking but listed as NFA - DEQ LUST Lis

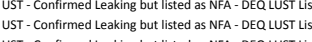

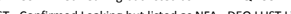

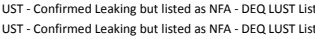

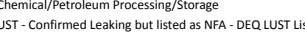
UST - Confirmed Leaking but listed as NFA - DEQ LUST Lis WST - Confirmed Leaking but listed as NFA - DEQ LUST L chemical/Petroleum Processing/Storage Others - Miscellaneous Contracting
Automobiles - Body Shops Automobilies - Body Shops
Known Contamination listed as NFA A Sites/P/Plumes/Spills from ECSI UST - Confirmed Leaking but listed a S NFA - DEQ LSST Lis
UST - Confirmed Leaking but listed as NFA- DEQ LUST Lis UST - Confirmed Leaking but listed a S NAA- DEQ Q UST LS UST- Confirmed Leaking but listed as NFA - DEQ USST List 


\begin{tabular}{|c|c|c|}
\hline Address & city & County \\
\hline $\begin{array}{l}555 \text { N NEENARAL } \\
75 \text { LTHA WAY }\end{array}$ & $\begin{array}{l}\text { Medford } \\
\text { Ashland }\end{array}$ & $\begin{array}{l}\text { JAAKSON } \\
\text { JACKSON }\end{array}$ \\
\hline 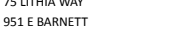 & & JAcKSON \\
\hline 525 N CENTRAL & Medford & JACKSON \\
\hline 1765 SIIKKYYOU BIVD & $\begin{array}{l}\text { Ashland } \\
\text { Metfors }\end{array}$ & IACKSON \\
\hline $\begin{array}{l}\text { S25 N NENTFAL } \\
3380 \text { HWY } 22\end{array}$ & $\begin{array}{l}\text { Netotord } \\
N / A\end{array}$ & $\begin{array}{l}\text { JACKSON } \\
\text { JACKSON }\end{array}$ \\
\hline 525 N CENTRAL & Medford & JACKSON \\
\hline 529 E MAIN ST & Medford & JACKSON \\
\hline 529 E MAIN ST & $\begin{array}{l}\text { Medford } \\
\text { Metfors }\end{array}$ & JACKSON \\
\hline $\begin{array}{l}2599 \text { MAANS } \\
240 \text { COVER LANE }\end{array}$ & $\begin{array}{l}\text { Metford } \\
\text { Ashland } \\
\text { M }\end{array}$ & $\begin{array}{l}\text { IACCSSON } \\
\text { AACKSON }\end{array}$ \\
\hline 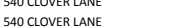 & $\begin{array}{l}\text { Asshand } \\
\text { Ashland }\end{array}$ & $\begin{array}{l}\text { JAAKSON } \\
\text { JACKSON }\end{array}$ \\
\hline 530 CRATER LAKE AVE & Metford & $\begin{array}{l}\text { JACKSON } \\
\text { JaCSON }\end{array}$ \\
\hline 180 ANTELOPE RD & & Jackson \\
\hline 530 CRATER LAKE AVE & Medtord & JACKSON \\
\hline 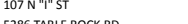 & N/A & IACCSSON \\
\hline $\begin{array}{l}26869 \text { ABSEL ROCK RD } \\
1649 \text { SHAND AVE }\end{array}$ & $\begin{array}{l}\text { N/A } \\
\text { Ashland }\end{array}$ & $\begin{array}{l}\text { IJCCSSON } \\
\text { ACKSON }\end{array}$ \\
\hline 608 N MAN ST & $\begin{array}{l}\text { Asshalad } \\
\text { Phoenix }\end{array}$ & $\begin{array}{l}\text { JACCSSON } \\
\text { JACKSON }\end{array}$ \\
\hline 5300 C RATTE L LAK AVE & & JACKSON \\
\hline 335 ROSSANLEY & MEDFORD & JACKSON \\
\hline 535 ROSSAMLEY & MEOFFRO & JACKSON \\
\hline $\begin{array}{l}1515 \text { SISKKYYOU UBVD } \\
1515 \text { SISKYYO BiVD }\end{array}$ & $\begin{array}{l}\text { Asshland } \\
\text { Ashrand }\end{array}$ & $\begin{array}{l}\text { JACCSON } \\
\text { IACCSON }\end{array}$ \\
\hline 16 N FRONT ST & $\begin{array}{l}\text { Assland } \\
\text { Central Point }\end{array}$ & JACCSON \\
\hline 2727 AVENUE G & & 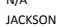 \\
\hline 2216 W MAIN ST & $\mathrm{N} / \mathrm{A}$ & Jackson \\
\hline S41 BUSINESS PARK DR STE $G$ & MEFFRD & Jackson \\
\hline S4600 SW SOUTH ROAD & Medford & Washington \\
\hline 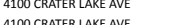 & $N / A$ & JaCKSON \\
\hline 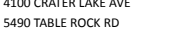 & $\begin{array}{l}\text { NA } \\
\text { Medford } \\
\text { S }\end{array}$ & $\begin{array}{l}\text { A ACCCSON } \\
\text { JACKSON }\end{array}$ \\
\hline 60 SHAMROCK LANE & $\mathrm{N} / \mathrm{A}$ & Jackson \\
\hline $\begin{array}{l}5 \text { SSTAGE RD } \\
\text { 555 }\end{array}$ & N/A & JACKSON \\
\hline $\begin{array}{l}550 \text { ARPPRT RD } \\
55 \text { ARPORT RD }\end{array}$ & $\begin{array}{l}\text { MEFEORD } \\
\text { MEFFORD }\end{array}$ & $\begin{array}{l}\text { JACKSON } \\
\text { JACKSON }\end{array}$ \\
\hline 550 BUSINESS PARK DR & $\begin{array}{l}\text { MEFOCOR } \\
\text { MEOFO }\end{array}$ & $\begin{array}{l}\text { JACKSON } \\
\text { Jas }\end{array}$ \\
\hline 24231 HWr 62 & & \\
\hline 551 BUSINESS PARK DR & MEDFORD & JACKSON \\
\hline 551 BUSINESS PARK DR & MEFFORD & JACKSON \\
\hline $\begin{array}{l}21 \text { T2ALNT AAE } \\
21 \text { TALENT AVE }\end{array}$ & 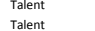 & $\begin{array}{l}\text { IACCSSON } \\
\text { AACKSON }\end{array}$ \\
\hline 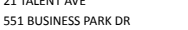 & $\begin{array}{l}\text { Talant } \\
\text { Mefford }\end{array}$ & 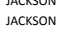 \\
\hline 555 AIRPORT RD & MEFFRD & JACKSON \\
\hline 136 MAIN ST W & Eagle Point & JACKSON \\
\hline $\begin{array}{l}136 \text { MAII ST W } \\
136 \text { MANSTW }\end{array}$ & $\begin{array}{l}\text { Eagle Point } \\
\text { Eagle point }\end{array}$ & $\begin{array}{l}\text { IACCSON } \\
\text { IACCSON }\end{array}$ \\
\hline $\begin{array}{l}136 \text { MANNS W W } \\
5550 \text { TABLE ROCK RD }\end{array}$ & $\begin{array}{l}\text { Eagle point } \\
\text { Medford }\end{array}$ & $\begin{array}{l}\text { JACKSON } \\
\text { JACKSON }\end{array}$ \\
\hline 562 PARSONS DR & MEFFRO & JACKSON \\
\hline 569 HANLEY RD & MEFFRD & JACKSON \\
\hline 569 HANLEY RD & $\begin{array}{l}\text { MEFFORD } \\
\text { Gold dill }\end{array}$ & $\begin{array}{l}\text { JAAKSON } \\
\text { JACKON }\end{array}$ \\
\hline 652 2ND AVE & $\begin{array}{l}\text { Gold dill } \\
\text { lackonvile }\end{array}$ & JACKSON \\
\hline $\begin{array}{l}\text { 945 N S SH ST ST } \\
3875 \text { FENN VALEY RD }\end{array}$ & $\begin{array}{l}\text { Phonix } \\
\text { Phonte }\end{array}$ & JACKSON \\
\hline 5742 CRATER LAKE AVE & Medford & JACKSON \\
\hline AGATE ROAD \& AVE A & N/A & IACKSON \\
\hline $\begin{array}{l}5600 \text { BUTTE F FWLL HWY } \\
578 \text { MASON WY }\end{array}$ & $\begin{array}{l}\text { N/A } \\
\text { MEFFORD }\end{array}$ & $\begin{array}{l}\text { N/A } \\
\text { JACKSON }\end{array}$ \\
\hline $\begin{array}{l}6079 \text { TABLL ROCKK RD } \\
6\end{array}$ & N/A & JACKSON \\
\hline 90 N MOUNTAIN AVE & $\mathrm{N} / \mathrm{A}$ & JACKSON \\
\hline 90 N MOUNTAIN AVE & N/A & \\
\hline 403 DEAD INDIAN MEMORIAL RD & $\begin{array}{l}\text { N/A } \\
\text { Mefford }\end{array}$ & IACCSSON \\
\hline $\begin{array}{l}6 \text { N MODDC AVE } \\
600 \text { BIDDLE RD }\end{array}$ & $\begin{array}{l}\text { Metotord } \\
\text { Metford }\end{array}$ & $\begin{array}{l}\text { AACCSSON } \\
\text { AACSON }\end{array}$ \\
\hline 600 BIDOLE RD & Medford & JACKSON \\
\hline 21825 HWY 62 & Shady cove & Jackson \\
\hline $\begin{array}{l}\text { 550 MOLL REEKK } \\
2650 \text { AVENUE G }\end{array}$ & N/A & A ACCSSON \\
\hline $\begin{array}{l}2755 \text { AVENGUE G } \\
1776 \text { ROGUE RIVER HWY }\end{array}$ & N/A & $\begin{array}{l}\text { JACSSON } \\
\text { JACKSON }\end{array}$ \\
\hline 600 E MAIN & MEOFORD & JACKSON \\
\hline 2073 N PACIFIC HWY & & JACKSON \\
\hline 600 E MAIN ST & Meefford & IACCSON \\
\hline 600 EAIN ST & $\begin{array}{l}\text { Mefford } \\
\text { Mefford }\end{array}$ & $\begin{array}{l}\text { IACCSSON } \\
\text { AACKSON }\end{array}$ \\
\hline $\begin{array}{l}6000 \text { MANAN ST } \\
600 \text { N CENTRAL }\end{array}$ & $\begin{array}{l}\text { Metarord } \\
\text { MEOFORD }\end{array}$ & JAcrson \\
\hline 2260 W MAIN & & Jackson \\
\hline 2260 W MAIN & N/A & JackSON \\
\hline 499 E MAIN ST & Ashland & JACKSON \\
\hline 6100 COVIVR RD & N/A & IACCSSON \\
\hline 600 NCENTRAL & $\begin{array}{l}\text { MEFPORD } \\
\text { NA }\end{array}$ & $\begin{array}{l}\text { AaCCSSON } \\
\text { AACKSON }\end{array}$ \\
\hline $\begin{array}{l}\text { EMIGRANT LAKE } \\
\text { GOON NENTRAL AVE }\end{array}$ & Mefford & JAcKSON \\
\hline 600 CENTRAL AVE & Medford & Jackson \\
\hline 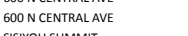 & Medford & JACKSON \\
\hline ISIIYOU SUMMIT & & \\
\hline
\end{tabular}

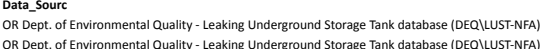

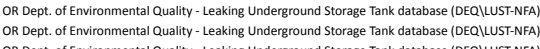

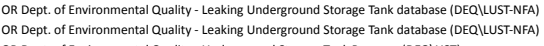

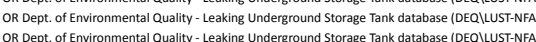
R Dept. of Environmental Q Quallity - Leaking Underground Storgage Tank database (DEQ) UUST-NFA)

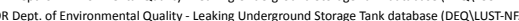

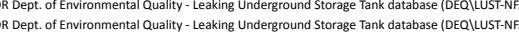

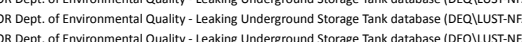
Dept. of Environmental Luality - Haz

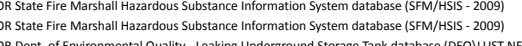

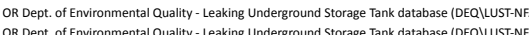

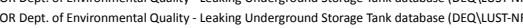
OR Dept. of Environmental Quality - Leaking Underground Storage Tank database (DEQQ LUST-N

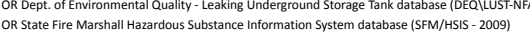
OR Dept. of Environmental Q Quality - Water Q Quality SIS database

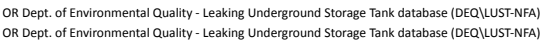

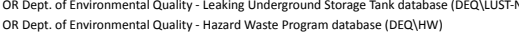

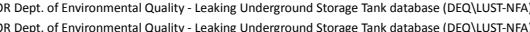

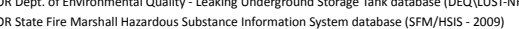

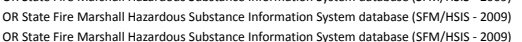

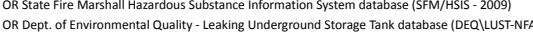

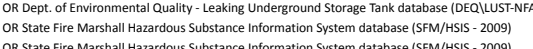

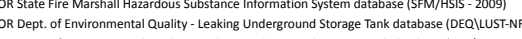
OR Dept. of Environmental Q Quality - Leaking Underground Storage Tank database (DEQQ L LST-NFFin

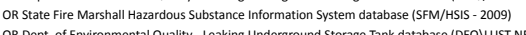

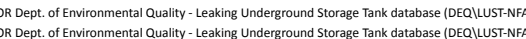

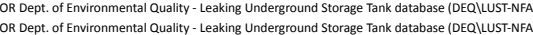
OR State fire Marshall Hazarddous Substance Information S System datatbase (SFM/HISIs - 2009) OR State Fire Marshall Hazard dus Substance Information System database (SFM/HSIS - 2009)
OR State fire Marshall Hazardous Substance Information System database (SFM/HSIS - 2009)

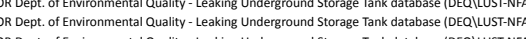
OR Dept. of Environmental Q Quality - Leaking Underground Storage Tank database (DEQQ L LSTS-N OR Dept. of Environmental Q Quallity - Leaking Underground Storage Tank database (DEQQ L LST-N

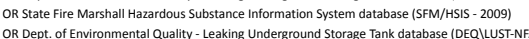

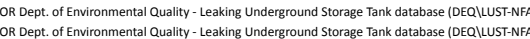

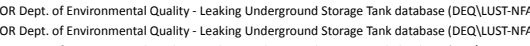

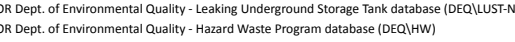

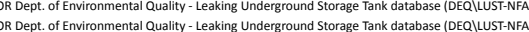

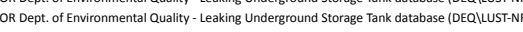

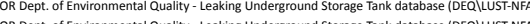
OR State fire Marshall Hazardous Substance Information System database (SFM/HISS - 2009)

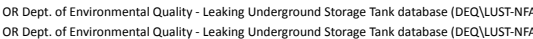

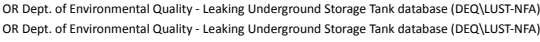

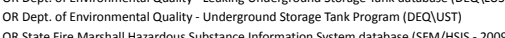

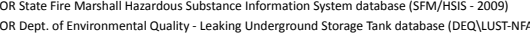

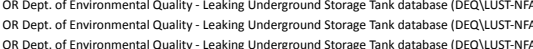

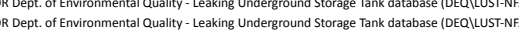

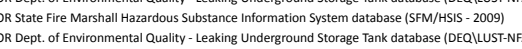

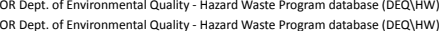

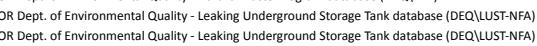

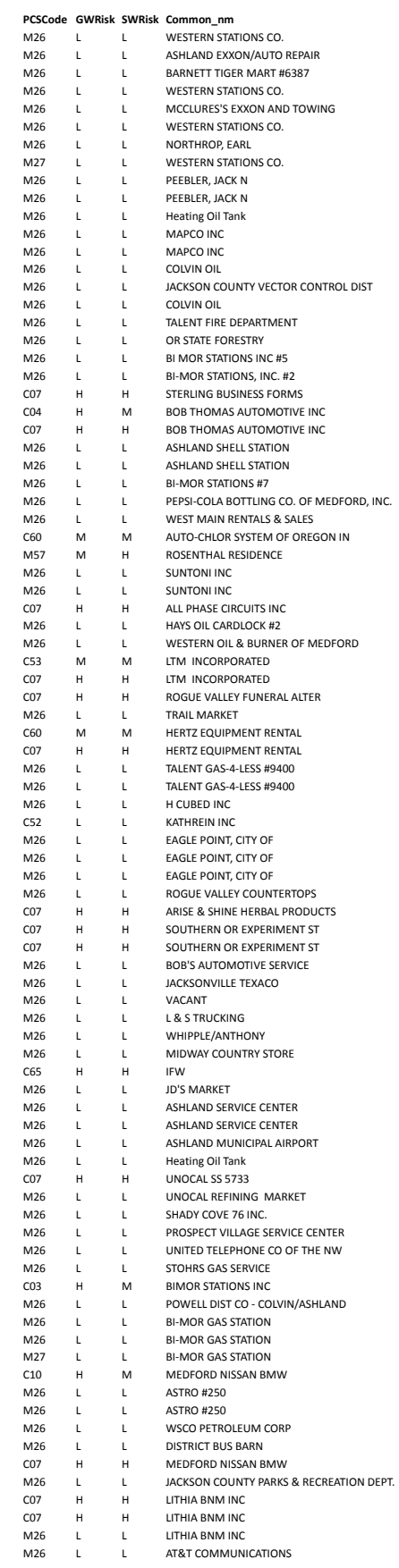

PCSTYpe
UST - Confirmed Leaking but listed as N NA- - DEQ LUST List WST-Confirmed Leaking but listed a a NAA - DEE Q LUST L L UST - Confirmed Leaking but listed as NEA- DEQ WUST TS UST- Confirmed Leaking but listed as NAF- DEQ LUST List
UST - Confirmed Leaking but Iisted as NFA- DEQ UST Lis UST - Confirmed Leaking but listed as NFA- DEQ LUST Lis UST - Confirmed Leaking but listed a S NFA - DEQ LUST LiS UST - Confirmed Leaking but listed as NFA- DEQ LUST Lis UST - Confirmed Leaking but listed as NFA - DEQ LUST L

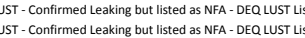
UST- Confirmed Leaking but listed as NFA- DEQ LUST List

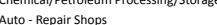

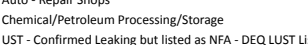
UST-Confirmed Leaking but lived as SNA - DEQ UST UST - Confirmed Leaking but listed as NFA - DEQ LUST Lis

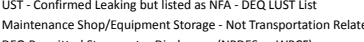
DEQ Permitted Stormwater Discharges (NNDES or WPCF)
UST- Confirmed Leaking but isted as NFA- DEQ UST L UST - Confirmed Leaking but listed a s NFA- DEQ LUST List
UST - Confirmed Leaking but listed a s NFA - DEQ UST List Chemical/Petroleum Processing/storage UST - Confirmed Leaking but listed a N NFA- DEQ Q UST List
UST - Confirmed Leaking but isted as NFA - DEQ LUST List UST - Confirmed Leak
Construction Compary

Chemical/Petroleum Processing//torage

Chemical/Petroleum Processing/Storage
uST- confirmed Leaking but isted as NFA- DEQ Q LuST List Chemical/Petroleum Processing /Storage

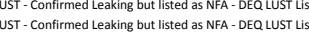
UST - Confirmed Leaking but listed as NFA- DEQ Q WST Lis Communications office
UST-Confirmed Leaking

Itted a s NFA- DEQ Q Wust list

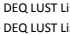
chemical/Petroleum Processing/storage
chemicalpertoleum Processinglstorage

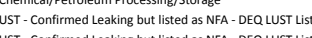
UST - Confirmed Leaking but listed a S NFA- DEQ L LST Lis

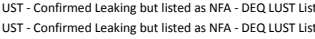

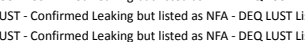

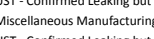

Jut lised as NFA- DEQ LUST Lis UST - Confirmed Leaking but Iisted as NFA- DEQ Q UST L L

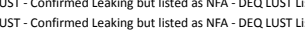

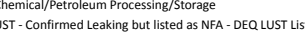

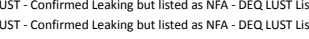
UTT - Confirmed Leaking but listed as NAA- DEQ L UST LIT Automobilies- - Gas stations

STT - Confirmed Leaking but listed as NFA - DEQ LUST Lis

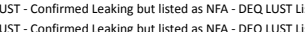
UST - Upgraded and/or Registered - Active (may also have decommissioned tanks on site) UST- Confirmed Leaking but listed as NFA - DEQ LUST Lis UST - Confirmed Leaking but listed as NFA- DEQ WST

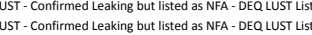

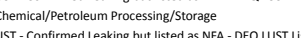
chemical/Petroleum Processing//storage

UST - Confirmed Leaking but listed a N NFA- DEQ Q UST List
UST - Confirmed Leaking but Isted as NFA - DEQ LUST List 


\begin{tabular}{|c|c|c|c|}
\hline $\begin{array}{l}\text { Address } \\
\text { Aaro ENA }\end{array}$ & 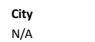 & $\begin{array}{l}\text { County } \\
\text { JACKSON }\end{array}$ & $\begin{array}{l}\text { RET_DATE } \\
\text { 10131/2018 }\end{array}$ \\
\hline 601 E MAN ST & Medford & 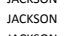 & $10 / 31 / 2018$ \\
\hline 601 E MAIN ST & MEDFORD & Jackson & $09 / 29 / 2008$ \\
\hline $\begin{array}{l}601 \text { EMAIN ST } \\
1065 \text { E PINE ST }\end{array}$ & $\begin{array}{l}\text { Medford } \\
\text { Central Point. }\end{array}$ & $\begin{array}{l}\text { JACCSON } \\
\text { ACCKSON }\end{array}$ & $\begin{array}{l}101 / 3 / 21 / 2018 \\
10131 / 2018\end{array}$ \\
\hline 60598 CRAATES LAKE HIGHWAY & $\begin{array}{l}\text { Central point } \\
\text { N/A }\end{array}$ & $\begin{array}{l}\text { JaCCSONO } \\
\text { IACKSON }\end{array}$ & $\begin{array}{l}101 / 3 / 21 / 2018 \\
10 / 131 / 2018\end{array}$ \\
\hline 6840 C RATER LAKE HWY & N/A & $\begin{array}{l}\text { PaCSSON } \\
\text { IACCSON }\end{array}$ & $\begin{array}{l}10131 / 218018 \\
10 / 31 / 2018\end{array}$ \\
\hline $601 \mathrm{E}$ Main St & Medford & & 8 \\
\hline 399 N STH ST & Central Point & JACKSON & $10 / 31 / 2018$ \\
\hline $\begin{array}{l}601 \text { NEBUUA WAY } \\
602 \text { SBIVESSDF AVE }\end{array}$ & Medford & JaCKSON & $\begin{array}{l}10 / 31 / 2018 \\
0\end{array}$ \\
\hline $\begin{array}{l}6022 \text { SIVERSSDD AVE } \\
602 \text { SIVERSID AVE AVE }\end{array}$ & $\begin{array}{l}\text { MEFFFo } \\
\text { Mefford }\end{array}$ & $\begin{array}{l}\text { JACKSON } \\
\text { IACCSON }\end{array}$ & $\begin{array}{l}09 / 29 / 2008 \\
10131 / 2018\end{array}$ \\
\hline 345 LTHIA WAY AVE & $\begin{array}{l}\text { Metatord } \\
\text { Ashland }\end{array}$ & $\begin{array}{l}\text { PaCSSON } \\
\text { IACCSON }\end{array}$ & $\begin{array}{l}101 / 3 / 21 / 2018 \\
10 / 131 / 2018\end{array}$ \\
\hline 345 LTHIA WAY & & JACKSON & $\begin{array}{l}10 / 1 / 21 / 2018 \\
1013\end{array}$ \\
\hline 2194 SAGE RD & $\mathrm{N} / \mathrm{A}$ & JACKSON & $10 / 11 / 2018$ \\
\hline 7625 CRATER LAKE HWY & $\mathrm{N} / \mathrm{A}$ & JACKSON & 10/31/2018 \\
\hline 1355 SISKKYY BIVD & $\begin{array}{l}\text { Astland } \\
\text { Asthand }\end{array}$ & JACKSON & $\begin{array}{l}10 / 31 / 2018 \\
103 / 212018\end{array}$ \\
\hline $\begin{array}{l}1350 \text { SIIIFKYYOU B BVD } \\
604 \text { Crater Lake Ave }\end{array}$ & $\begin{array}{l}\text { Assland } \\
\text { MEDFORD }\end{array}$ & $\begin{array}{l}\text { JACKSON } \\
\text { IACCSON }\end{array}$ & $\begin{array}{l}10 / 31 / 2018 \\
06 / 201 / 2015\end{array}$ \\
\hline $\begin{array}{l}604 \text { Catare Lake Ave } \\
608 \text { MEDFRD CENTER }\end{array}$ & $\begin{array}{l}\text { MEEFOFB } \\
\text { Mefford }\end{array}$ & $\begin{array}{l}\text { JaCSSON } \\
\text { JaCSSON }\end{array}$ & $\begin{array}{l}06 / 20 / 21 / 2015 \\
10 / 1312018\end{array}$ \\
\hline 47201 Crater Lake Hwy & & 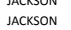 & $\begin{array}{l}10 / 31 / 2018 \\
10138\end{array}$ \\
\hline 608 MEDFORD CENTER & Medford & JACKSON & $10 / 11 / 2018$ \\
\hline 2723 RLLEY RD & N/A & JACKSON & $10 / 31 / 2018$ \\
\hline 608 Medford Center & $\begin{array}{l}\text { Medford } \\
\text { MEDFORD }\end{array}$ & $\begin{array}{l}\text { Jackson } \\
\text { Jacksoy }\end{array}$ & $\begin{array}{l}8 / 27 / 2018 \\
099292008\end{array}$ \\
\hline $\begin{array}{l}6088 \text { 8 RIVERSSDE EVE } \\
\text { 4762 TABLEROCK RD }\end{array}$ & $\begin{array}{l}\text { MEDFFRD } \\
\text { N/A }\end{array}$ & $\begin{array}{l}\text { JaCGSON } \\
\text { JaCKSON }\end{array}$ & $\begin{array}{l}09 / 2 / 2 / 2008 \\
10 / 11 / 2018 \\
\end{array}$ \\
\hline 610 KING ST & & $\begin{array}{l}\text { JaCSSON } \\
\text { IACCSON }\end{array}$ & $\begin{array}{l}101 / 3 / 21 / 2018 \\
10 / 131 / 2018\end{array}$ \\
\hline 610 OAKDALE & Medford & 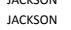 & 10/13/2018 \\
\hline РО вох 100 & & JaCksoN & $10 / 31 / 2018$ \\
\hline 43 N FRONT & Central Point & JaCKSON & $10 / 31 / 2018$ \\
\hline $\begin{array}{l}610 \text { S PEACH ST } \\
107 \text { MANW }\end{array}$ & Medford & $\begin{array}{l}\text { JaCKSON } \\
\text { Jacsson }\end{array}$ & $\begin{array}{l}10 / 13 / 2018 \\
1013 / 2018\end{array}$ \\
\hline $\begin{array}{l}\text { 1070 MAIA W } \\
640 \text { MASN WAY }\end{array}$ & Eagle Point & $\begin{array}{l}\text { JaCCSON } \\
N / \text { A }\end{array}$ & $\begin{array}{l}10 / 31 / 2018 \\
1013 / 2018\end{array}$ \\
\hline 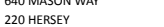 & $\begin{array}{l}\text { N/A } \\
\text { Ashland }\end{array}$ & $\begin{array}{l}\text { NACKSON } \\
\text { JAA }\end{array}$ & $\begin{array}{l}101 / 3 / 21 / 2018 \\
10 / 131 / 2018\end{array}$ \\
\hline 595 N MAIN & Ashland & Jackson & $\begin{array}{l}10 / 31 / 2018 \\
1018\end{array}$ \\
\hline 610 S Peach St & Medford & JaCksoN & $06 / 02 / 2015$ \\
\hline 351 MILL CREEK DR & N/A & JaCKSON & $10 / 31 / 2018$ \\
\hline $\begin{array}{l}2499 \text { Assland Street } \\
102 \text { TALENT AVE }\end{array}$ & $\begin{array}{l}\text { Ashland } \\
\text { Thalent } \\
\text { S }\end{array}$ & $\begin{array}{l}\text { JaCKSON } \\
\text { IACCSON }\end{array}$ & $\begin{array}{l}10 / 31 / 2018 \\
1013 / 2018\end{array}$ \\
\hline MT ASHLAND ACCESS RD & & 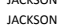 & $\begin{array}{l}10 / 131 / 2018 \\
10 / 31 / 2018\end{array}$ \\
\hline 1291 OAKST & Ashland & 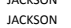 & $\begin{array}{l}10 / 131 / 2018 \\
10 / 11 / 2018\end{array}$ \\
\hline 5322 BROWNSBORO HWY & $\mathrm{N} / \mathrm{A}$ & JACKSON & $101 / 11 / 2018$ \\
\hline 613 EACKSON & MEDFORD & JaCKSON & 09/29/2008 \\
\hline 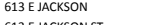 & MEDFORD & JACKSON & 09/29/2008 \\
\hline $\begin{array}{l}613 \text { EACRSON S ST } \\
1590 \text { P ST }\end{array}$ & $\begin{array}{l}\text { Medford } \\
\text { entran Pant }\end{array}$ & IACCSON & $10 / 13 / 2018$ \\
\hline $\begin{array}{l}19500 \text { P PNINEST } \\
1590 \text { P PIN ST }\end{array}$ & $\begin{array}{l}\text { Centrapoint } \\
\text { Central Point }\end{array}$ & $\begin{array}{l}\text { JaCCSONO } \\
\text { JACKSON }\end{array}$ & $\begin{array}{l}1 / 131 / 2018 \\
1 / 1 / 1 / 2018 \\
\end{array}$ \\
\hline 21222 W MAIN & N/A & $\begin{array}{l}\text { JaCKSON } \\
\text { Jactor }\end{array}$ & $\begin{array}{l}10 / 131 / 218 \\
10 / 31 / 2018\end{array}$ \\
\hline 8495 CRATER L LAEE HWY & $\mathrm{N} / \mathrm{A}$ & JaCKSON & $10 / 11 / 2018$ \\
\hline 8495 CRATER LAKE HWY & N/A & JACKSON & 10/31/2018 \\
\hline 5100 CRATER LAKE AVENUE & N/A & JACKSON & 10/31/2018 \\
\hline $\begin{array}{l}115 \text { JHARSMAN } \\
115 \text { SHAN }\end{array}$ & $\begin{array}{l}\text { Metorod } \\
\text { Methat }\end{array}$ & JACKSON & $\begin{array}{l}10 / 31 / 2018 \\
101312018\end{array}$ \\
\hline 617 SRIVERSIDE & 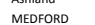 & $\begin{array}{l}\text { JACKSONON } \\
\text { JACKSON }\end{array}$ & $\begin{array}{l}1 \\
1 / 1131 / 218 \\
09 / 29 / 2008\end{array}$ \\
\hline HIGH BANKS ROAD & N/A & $\begin{array}{l}\text { JaCKSON } \\
\text { Jack }\end{array}$ & $\begin{array}{l}0.9 / 29 / 2008 \\
10 / 11 / 2018\end{array}$ \\
\hline HWr. 140 & $\mathrm{~N} / \mathrm{A}$ & JaCKSON & 10/31/2018 \\
\hline HWY.99 \& VALLEY VEWW & N/A & JACKSON & 10/31/2018 \\
\hline 6195 SRAPEST & Meaford & JACKSON & 10/31/2018 \\
\hline $\begin{array}{l}622 \text { Crater Lake Ave } \\
1 \text { N FOURTH }\end{array}$ & $\begin{array}{l}\text { Meetord } \\
\text { Centapiont }\end{array}$ & $\begin{array}{l}\text { Jackson } \\
\text { Jacrsoon }\end{array}$ & $\begin{array}{l}8 / 27 / 2018 \\
1 / 1312018\end{array}$ \\
\hline 13080 THREE OAKS DRVEE & N/A & $\begin{array}{l}\text { JACKSONON } \\
\text { JACKSON }\end{array}$ & $\begin{array}{l}1 / 131 / 2018 \\
10 / 11 / 2018 \\
\end{array}$ \\
\hline 563AST. & Ashland & JACKSON & $\begin{array}{l}101013 / 21818 \\
10 / 11 / 2018\end{array}$ \\
\hline 625 MAREE ST & Medford & JaCKSON & 10/31/2018 \\
\hline 64 LOZIER LN & MEOFORD & JACKSON & 09/29/2008 \\
\hline $\begin{array}{l}640 \text { MASON WY } \\
1800 \text { ROSSANIY DRIIE }\end{array}$ & & $\begin{array}{l}\text { JACKSON } \\
\text { IACSSON }\end{array}$ & 09/29/2008 \\
\hline 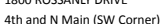 & $\begin{array}{l}\text { N/A } \\
\text { Phoenix }\end{array}$ & 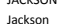 & $\begin{array}{l}10 / 131 / 2018 \\
1 / 31 / 2018\end{array}$ \\
\hline 6040 MASON Wr & MEEFORD & JACKSON & $\begin{array}{l}10 / 131 / 2018 \\
09 / 29 / 2008\end{array}$ \\
\hline 6415 SGAAE ST & MEEFORD & JaCKSON & $09 / 29 / 2008$ \\
\hline 2883 Ross LN & $\mathrm{N} / \mathrm{A}$ & JaCksoN & $10 / 31 / 2018$ \\
\hline 644 Crater Lake Ave & Meefford & Jackson & 8/27/2018 \\
\hline $\begin{array}{l}646 \text { GRAPE ST S } \\
493 \text { N MAIN ST }\end{array}$ & Metiorod & JACKSON & 10/31/2018 \\
\hline 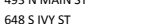 & 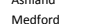 & 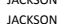 & $\begin{array}{l}10 / 131 / 2018 \\
1 / 131 / 2018\end{array}$ \\
\hline 649 Crater Lake Ave & Mefford & IACKSON & 作 \\
\hline 660 S GRAPE ST & Medfford & JACKSON & $10 / 11 / 2018$ \\
\hline 132 N MAIN & Ashland & JACKSON & 10/31/2018 \\
\hline 661 АाRPORT RD & Medford & JACKSON & 10/31/2018 \\
\hline 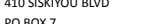 & Asshand & 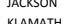 & 10/31/2018 \\
\hline 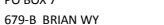 & $\begin{array}{l}\text { N/A } \\
\text { MEDFORD }\end{array}$ & 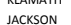 & $\begin{array}{l}1 / 1 / 31 / 2018 \\
09 / 29 / 2008\end{array}$ \\
\hline 78 FOURTH STREET & Asthand & JACKSON & $\begin{array}{l}099929 / 2008 \\
10 / 11 / 2018\end{array}$ \\
\hline PACIFIC HWY & Central Point & JaCKSON & $10 / 11 / 2018$ \\
\hline 680 BIDDLE RD & Metford & JACKSON & 10/31/2018 \\
\hline $\begin{array}{l}1968 \text { MEEED ST } \\
682 \text { BRAN WY }\end{array}$ & $\begin{array}{l}\text { Assland } \\
\text { MEEFORD }\end{array}$ & $\begin{array}{l}\text { JaCKSON } \\
\text { ICCKSON }\end{array}$ & $\begin{array}{l}10 / 1 / 2 / 2018 \\
00 / 29 / 2008\end{array}$ \\
\hline
\end{tabular}

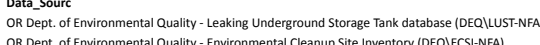

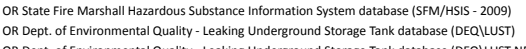
OR Dept. of Environmental Q Quality - Leaking Underground Storage Tank databasese (DEQQ IUST-NFA

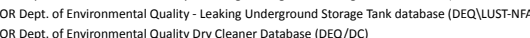
be Tank database (DEQQLUST-NFA)
base (DEQQHW)

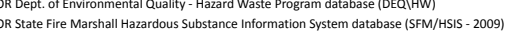

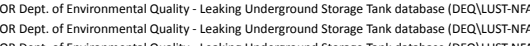

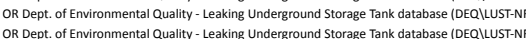

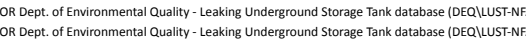
R Dept. of Environmental Quality - Leaking Wisteng

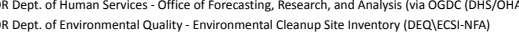

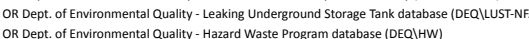

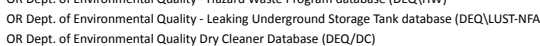

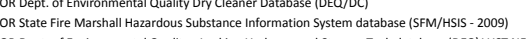

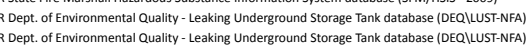

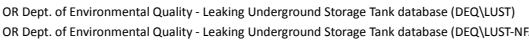
OR Dept. of Environmental Quality - Leaking Underground Storage Tank database (DEQQ LUST.NFA

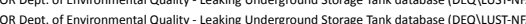

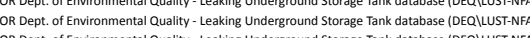

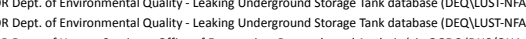

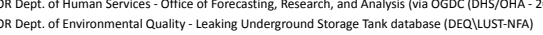

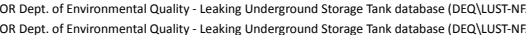
OR Dept. of Environmental Quallty - Leaking Underground Storage Tank databasese (DEQQ L LUST-NNA

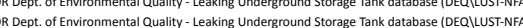

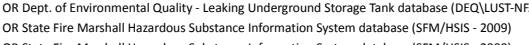

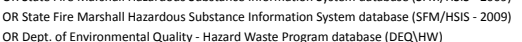

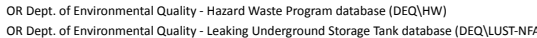
OR Dept. of Environmental Quality - Leaking Underground Storage Tank database (DEQQ) UUST-NFA)

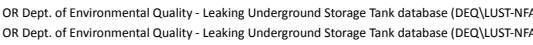

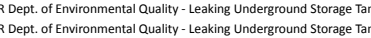

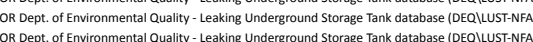

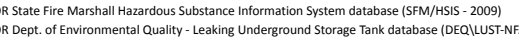

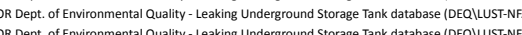
OR Dept. of Environmental Quality - Hazard Waste Program database (DEQQ1HWI OR Dept. of Environmental Quality Ory Cleaner Database (DEC/DCC) OR Dept. of Envirionmentatal Q Quality

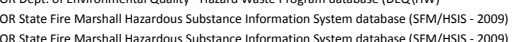
OR State fire Marshall Hazardous Substance Ifformation System database (SFMM/HIS - 2009)

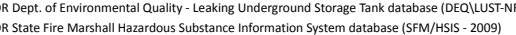
OR State Fire Marshall Hazardous Substance InG

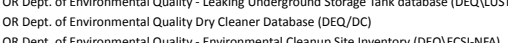
OR Dept. of Environmental Quality- Environmental Cleanup Site Inventory (DEQ) ECCSIN-NAA)

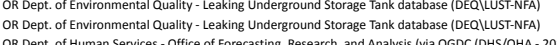

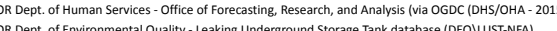

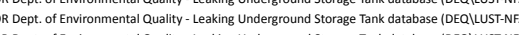

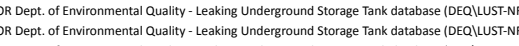

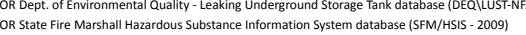
OR Dept. of Environmental Quality - Leaking Underground Storage Tank database (DEQQLIUST-NFA

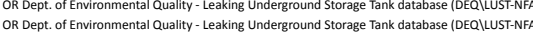

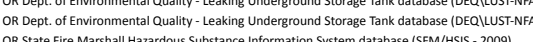

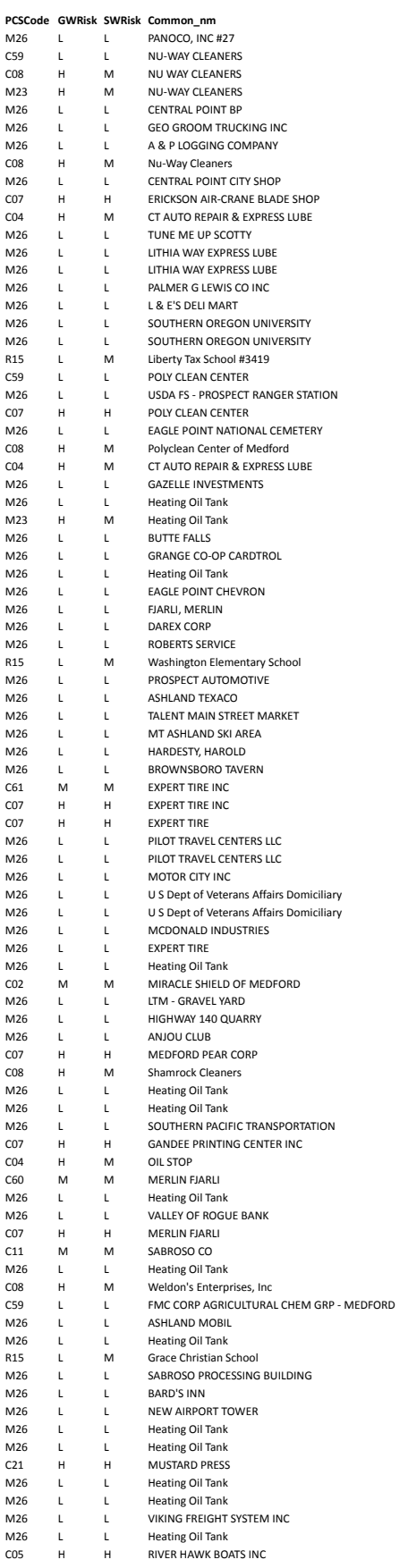

PCSTYpe
UST-Confirmed Leaking but listed as N NAA- DEQ Q UST Lis

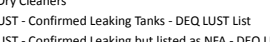
UST - Confirmed Leaking but listed a N NFA - DEQ LUST L UST - Confirmed Leaking but listed as NFA - DEQ LUST L UST - Confirmed Leaking but listed as NFA- DEQ LUST Lis Chemical/Petroleum Processing//5torage
Auto- Repair shops

(lis

UST - Confirmed Leaking but Isteded as NAA - DEQ LELUST LST UST - Confirmed Leaking but Iisted as NFA- DEQ LUST L

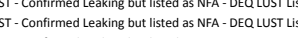
Known Contamination listed as NFA (Stites/Plumes/Spills from ECS
UST - Confirmed Leaking but listed as NFA - DEQ UST List W mical/Petroleum Processing//Storage Dry Cleaners

Auto - Repair Shops
UST - Confirmed Leaking but listed as NFA - DEQ LUST UST - Confirmed Leaking but listed a S NFA - DEQ LUST Lis UST- Confirmed Leaking Tanks - DEQ LUST List
UST - Confirmed Leaking but listed as NFA - DEQ WST UST-Confirmed Leaking but isted a s N NA - DEQ Q UST L Lis WST - Confirmed Leaking but Iisted a SN NAA - DEQ LUST LIS UST - Confirmed Leaking but listed as N NEA - DEQ WWST L UST - Confirmed Leaking but listed as NFA - DEQ LUST Lis schools UST - Confirmed Leaking but listed as NAA- DEQ LUST Lis UST - Confirmed Leaking but listed as NAA- DEQ LUST LIS UST - Confirmed Leaking but listed a SNEA - DEQ WUST UST - Confirmed Leaking but Iisted as NFA- DEQ USTT Lis

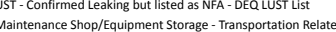
Chemical/Petroleum Processing/ststorge

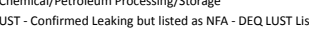
UST - Confirmed Leaking but listed as NAA-DEQ WST H UST - Confirmed Leaking but listed a S NFA - DEQ LUST Lis

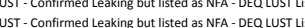
UST - Confirmed Leaking but listed a N NFA - DEQ LUST L

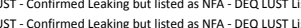

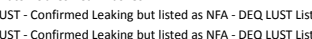
UST - Confirmed Leaking but listed as NNA - DEO UWS chemical/Petroleum Processing/Storage Dry Cleaners
ust- - onfirmete

Ped a NFA- DEQ Lust Lis UST - Confirmed Leaking but Isted a a N NFA- DEQ Q WUST Lis

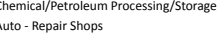
Maintenance Shoo/Equipment Storage - Not Transportation Related WST - Confirmed Leaking but listed as NFA- DEQ LUST LIS
UST - Confirmed Leaking but listed a s NFA- DEQ UST Lis Food Processing
UST - Confirmed Leaking but listed as NFA - DEQ LUST Lis

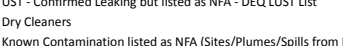

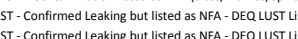
UST- Confirmed Leaking but listed as NFA- DEQ LUST Lis

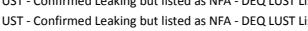
UST - Confirmed Leaking but listed as NFA - DEQ LUST LIS Shato processine/Printing

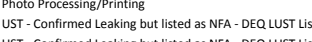
UST - Confifrmed Leaking but isted a s NAA- DEQ LUST Lis

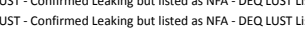




\begin{tabular}{|c|c|c|c|}
\hline $\begin{array}{l}\text { Address } \\
810 \text { cRown }\end{array}$ & $\begin{array}{l}\text { City } \\
\text { Central point }\end{array}$ & $\begin{array}{l}\text { County } \\
\text { JACKSON }\end{array}$ & $\begin{array}{l}\text { RET_DATE } \\
\text { 10131/2018 }\end{array}$ \\
\hline 810 CROWN & 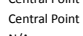 & 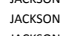 & $10 / 31 / 2018$ \\
\hline $\begin{array}{l}7352 \text { ADAMS D D } \\
688 \text { A ARREE ST }\end{array}$ & $\begin{array}{l}\text { N/A } \\
\text { Medford }\end{array}$ & $\begin{array}{l}\text { JACKSON } \\
\text { IACCSON }\end{array}$ & $\begin{array}{l}10 / 31 / 2018 \\
10131 / 2018\end{array}$ \\
\hline 690 BIDOLL RD RO & $\begin{array}{l}\text { MEDFORD } \\
\text { MEFT }\end{array}$ & 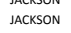 & $\begin{array}{l}09 / 29 / 2008 \\
098\end{array}$ \\
\hline 3784 COLEMAN CREEK ROAD & N/A & Jackson & $10 / 31 / 2018$ \\
\hline 690 BIIDLE RD & $\begin{array}{l}\text { MEFFORD } \\
\text { ASthand }\end{array}$ & $\begin{array}{l}\text { JaCKSON } \\
\text { IacsSoO }\end{array}$ & $\begin{array}{l}09 / 29 / 2008 \\
1013 / 2018\end{array}$ \\
\hline & $\begin{array}{l}\text { Assland } \\
N / A\end{array}$ & $\begin{array}{l}\text { JaCKSON } \\
\text { IACCSON }\end{array}$ & $\begin{array}{l}10 / 3 / 1 / 2018 \\
10 / 131 / 2018\end{array}$ \\
\hline $\begin{array}{l}17277 \text { CARERENTER HILL ROAD } \\
690 \text { S GAPE ST }\end{array}$ & $\begin{array}{l}\text { NAE MEFORD } \\
\text { MES }\end{array}$ & $\begin{array}{l}\text { JaCSSON } \\
\text { IACCSON }\end{array}$ & $\begin{array}{l}10131 / 2018 \\
09 / 29 / 2008\end{array}$ \\
\hline G90 SGAAPE ST & Medford & 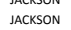 & $10 / 31 / 2018$ \\
\hline 690 S GRAPE ST & Medford & Jackson & $10 / 31 / 2018$ \\
\hline 1188 N VALLEY VIEW & N/A & JaCKSON & 10/31/2018 \\
\hline 101 OAK STREET & $\begin{array}{l}\text { Ashland } \\
\text { MEFERO }\end{array}$ & JaCKSON & $\begin{array}{l}10 / 13 / 2018 \\
092 / 2 / 2008\end{array}$ \\
\hline $\begin{array}{l}6 \text { 6RATRR LAN WY } \\
\text { cRAM DRIVE }\end{array}$ & $\begin{array}{l}\text { MEFFORD } \\
\text { N/A }\end{array}$ & JACKSON & \\
\hline 2717 ORINOELL DRVE & & 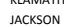 & $\begin{array}{l}10131 / 2018 \\
10 / 1312018\end{array}$ \\
\hline 691 S FRR ST & MËFORD & Jackson & $\begin{array}{ll}0 & 09 / 29 / 2008 \\
0\end{array}$ \\
\hline 627 OAK ST & Ashland & JACKSON & $10 / 31 / 2018$ \\
\hline 199 TEARWOOD & $\begin{array}{l}\text { Eagle Point } \\
\text { Nefopon }\end{array}$ & JACKSON & $10 / 31 / 2018$ \\
\hline $\begin{array}{l}691 \text { SEIR ST } \\
\text { 7. LEN OAKCT }\end{array}$ & $\begin{array}{l}\text { MEFFORD } \\
\text { Mefford }\end{array}$ & JACKSON & $\begin{array}{l}09 / 29 / 2008 \\
103 / 2 / 2018\end{array}$ \\
\hline $\begin{array}{l}796 \text { GEN MAKCF } \\
293 \text { EAIN }\end{array}$ & $\begin{array}{l}\text { Meditord } \\
\text { Ashland }\end{array}$ & $\begin{array}{l}\text { JaCGSON } \\
\text { JACCSON }\end{array}$ & $\begin{array}{l}1 / 131 / 2018 \\
1 / 31 / 2018\end{array}$ \\
\hline 6588 SISKIYYOU BIVD & $\begin{array}{l}\text { Asshina } \\
\text { Ashland }\end{array}$ & 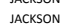 & $\begin{array}{l}10 / 131 / 2018 \\
10 / 11 / 2018\end{array}$ \\
\hline 1605-7 SISKIYYO BLVD & Ashland & JaCKSON & $10 / 31 / 2018$ \\
\hline 700 N CENTRAL & Mefford & JACKSON & 10/31/2018 \\
\hline 700 N CENTRAL AVE & МЕFFRD & JaCKSON & 09/29/2008 \\
\hline 700 N CENTRAL AVE & МЁFОRD & JACKSON & $\begin{array}{l}09 / 29 / 2008 \\
10131 / 2018\end{array}$ \\
\hline $\begin{array}{l}1410 \text { 1 DAK G GOVVE RD } \\
1555 \text { S PEACH ST }\end{array}$ & N/A & $\begin{array}{l}\text { JaCSSON } \\
\text { ICCKSON }\end{array}$ & $\begin{array}{l}101 / 3 / 21 / 2018 \\
10131 / 2018\end{array}$ \\
\hline 701 N CENTRAL & MEOFORD & $\begin{array}{l}\text { JaCKSON } \\
\text { JACSON }\end{array}$ & $\begin{array}{l}1 \\
09 / 29 / 20008\end{array}$ \\
\hline & Ashland & JACKSON & 10/31/2018 \\
\hline 1716 PARKER ST & Ashland & JACKSON & 10/31/2018 \\
\hline 701 N COLUMBUS AVE & Medtord & JaCKSON & $10 / 31 / 2018$ \\
\hline 12008 BLCCC OF FISKKYOU AT INDIANA & $\begin{array}{l}\text { N/A } \\
\text { Medford }\end{array}$ & JACKSON & $\begin{array}{l}10 / 13 / 21 / 2018 \\
10131 / 2018\end{array}$ \\
\hline $\begin{array}{l}734 \text { NoWOUMABS SUS AVE } \\
345 \text { IOWT }\end{array}$ & 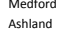 & $\begin{array}{l}\text { JaCSSON } \\
\text { IACCSON }\end{array}$ & $\begin{array}{l}101 / 3 / 2018 \\
10 / 131 / 2018\end{array}$ \\
\hline 7017 Dark Hollow Road & $\begin{array}{l}\text { Assinan } \\
\text { Medford }\end{array}$ & $\begin{array}{l}\text { Sacsson } \\
\text { Jackson }\end{array}$ & $\begin{array}{l}1 / 11 / 2 / 2018 \\
1 / 1 / 20\end{array}$ \\
\hline 7017 Dark Hollow Road & Medford & Jackson & 1/1/12018 \\
\hline 2182 TAYLOR RD & N/A & JaCKSON & 10/31/2018 \\
\hline 154 FOOTHILL RD & N/A & JaCKSON & 10/13/2018 \\
\hline $\begin{array}{l}4530 \text { OLD STAGE RD } \\
7025 \text { GRAPE ST }\end{array}$ & $\begin{array}{l}\text { N/A } \\
\text { Medford }\end{array}$ & JACKSON & $\begin{array}{l}10 / 31 / 2018 \\
1 / 131212018\end{array}$ \\
\hline 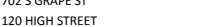 & $\begin{array}{l}\text { Medford } \\
\text { Ashland } \\
\text { S }\end{array}$ & $\begin{array}{l}\text { JaCCSON } \\
\text { JACKSON }\end{array}$ & $\begin{array}{l}1 / 131 / 2018 \\
10 / 31 / 2018\end{array}$ \\
\hline 520 BLACKSTONE ALLEY & Jacksonville & JACKSON & $\begin{array}{l}100131 / 2018 \\
10 / 1 / 2018\end{array}$ \\
\hline 119 ORCHARD LANE & N/A & JACKSON & 10/31/2018 \\
\hline 4607 DARK HOLLOW RD & N/A & JACKSON & $10 / 31 / 2018$ \\
\hline $\begin{array}{l}\text { 467 BEACA ST } \\
\text { 7025 SGRAPE ST }\end{array}$ & $\begin{array}{l}\text { Ashland } \\
\text { Mentford }\end{array}$ & JACKSON & $\begin{array}{l}10 / 31 / 2018 \\
1 / 1312018\end{array}$ \\
\hline $\begin{array}{l}7292 \text { S SRAAEST } \\
1295 \text { OAK ST }\end{array}$ & N/A & $\begin{array}{l}\text { JaCCSONO } \\
\text { ACCSSON }\end{array}$ & $\begin{array}{l}10 / 13 / 21 / 218 \\
1 / 131 / 2018\end{array}$ \\
\hline 7025 GRAPE ST & $\begin{array}{l}\text { MEDFORD } \\
\text { MEA }\end{array}$ & 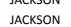 & $\begin{array}{l}10 / 131 / 2018 \\
09 / 29 / 2008\end{array}$ \\
\hline 702 S GRAPE ST & MEFFORD & Jackson & $\begin{array}{l}09 / 29 / 2008 \\
0\end{array}$ \\
\hline 2984 N PHOENII RD & N/A & JACKSON & 10/31/2018 \\
\hline INDOANA \& SISKKYYU BLVDS & Ashland & JaCKSON & 10/31/2018 \\
\hline $\begin{array}{l}7 \\
7022 \text { W ANANST }\end{array}$ & 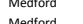 & JACKSON & 10/11/2018 \\
\hline T) & $\begin{array}{l}\text { Waterora } \\
\text { Ashland }\end{array}$ & $\begin{array}{l}\text { Jactson } \\
\text { accsson }\end{array}$ & $\begin{array}{l}8 / 27 / 201818 \\
1 / 31 / 2018\end{array}$ \\
\hline 14675 HIGGWAY 234 & Gold till & 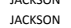 & $\begin{array}{l}10 / 131 / 2018 \\
10 / 11 / 2018\end{array}$ \\
\hline 703 LAWNSSDAE RD & MEFFRD & JACKSON & 09/29/2008 \\
\hline 703 N CENTRAL AVE & Medford & JACKSON & 10/31/2018 \\
\hline 2055 HHRMAN STRETT & Ashland & JACKSSON & 10/31/2018 \\
\hline 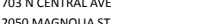 & Mentord & A ACCSTON & 10/31/2018 \\
\hline (2370 MORADA AN & Asthand & $\begin{array}{l}\text { S } \\
\text { ACCSSON }\end{array}$ & $\begin{array}{l}1 / 1 / 31 / 2018 \\
10 / 31 / 2018\end{array}$ \\
\hline 199 RAPP RD & N/A & JACKSON & $\begin{array}{l}10 / 131 / 21 / 818 \\
10 / 31 / 2018\end{array}$ \\
\hline 3817 GRANT RD & N/A & JaCKSON & $101 / 1 / 2018$ \\
\hline BULLDING 2222 & N/A & KLAMATH & 10/31/2018 \\
\hline 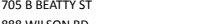 & Metford & JACCSONO & 10/31/2018 \\
\hline 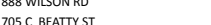 & S & $\begin{array}{l}\text { SaCGSON } \\
\text { JaCSSON }\end{array}$ & $\begin{array}{l}10 / 11 / 2018 \\
0.929 / 2008\end{array}$ \\
\hline 705 WTSEWART AVE & $\begin{array}{l}\text { MEFFRD } \\
\text { MEFo }\end{array}$ & $\begin{array}{l}\text { JaCKSON } \\
\text { Jack }\end{array}$ & $\begin{array}{l}0.9919 / 2008 \\
09 / 29 / 2008\end{array}$ \\
\hline 705 W STEWART AVE & Mefford & JaCKSON & $\begin{array}{l}0.979 / 2008 \\
10 / 11 / 2018\end{array}$ \\
\hline 157 N MAIN ST & Ashland & JaCKSON & $10 / 31 / 2018$ \\
\hline 1757 JASMINE & ssonville & JaCKSON & 10/31/2018 \\
\hline SMODOC RD & N & IACKSON & 10/31/2018 \\
\hline & tand & 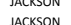 & $\begin{array}{l}10 / 31 / 2018 \\
1 / 1312018\end{array}$ \\
\hline STEWART AYE & Medford & $\begin{array}{l}\text { JaCkSON } \\
\text { Jack }\end{array}$ & $\begin{array}{l}11 / 11 / 21 / 218 \\
11 / 1 / 2018\end{array}$ \\
\hline Central AVE & MEFFORD & Jackson & $\begin{array}{l}11 / 1 / 2018 \\
09 / 29 / 2008\end{array}$ \\
\hline IskYou Bivo & land & Jackson & $10 / 31 / 2018$ \\
\hline 457 IISKYOU BIVO & Ashland & JACKSON & 10/31/2018 \\
\hline NOEPENDENCE SCF & & Son & 10/31/2018 \\
\hline & Medford & 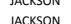 & $\begin{array}{l}10 / 11 / 218 \\
10 / 312018\end{array}$ \\
\hline & & $\begin{array}{l}\text { JACKSON } \\
\text { Jas }\end{array}$ & \\
\hline 145 CENTRAL & Ashland & JaCKSON & $\begin{array}{l}10 / 11 / 2018 \\
1 / 20\end{array}$ \\
\hline
\end{tabular}

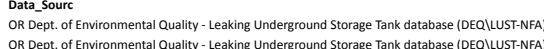

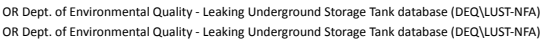
OR State Fire Marshall Hazardous Substance Information System database (SFM/HSIS- 2009)

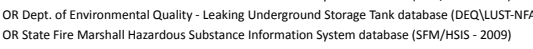
OR State fire Marshall Hazardouu Substance Information System database (SFM/HSIS - 2009)

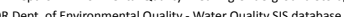
OR Dept. of Environmental Quality - Leaking Underground Storage Tank databasese (DEQQLLST-NFA

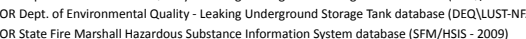
OR Dept. of Environmental Q Quality - Leaking Underground StStrage Tank databases (DEEQ) LUST-NFFA

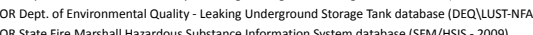

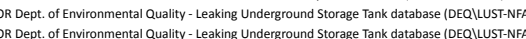

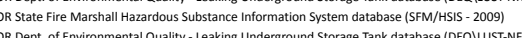

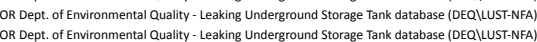

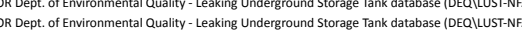

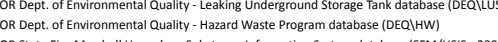

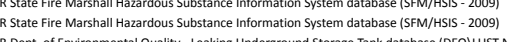

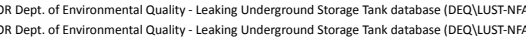
OR State fire Marshall Hazarddus Substance Infformation System database (SFM/HSIS - 2009)

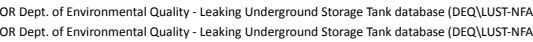

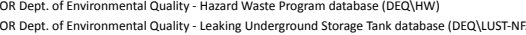

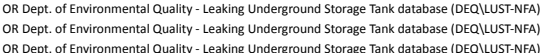

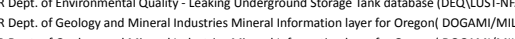

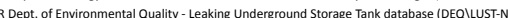

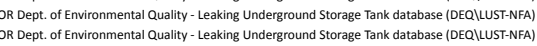

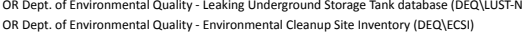

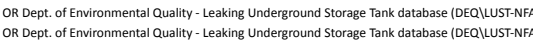

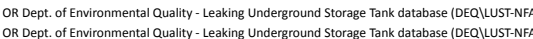

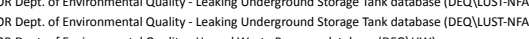
OR Dept. of Environmental Quality- Hazard Waste Program databases (DEQQ HWW)

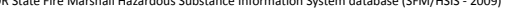

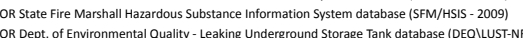

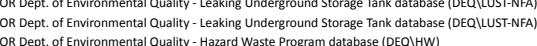
OR Dept. of Environmental Quality - Hazard Waste Program database (DEQQ1HWW

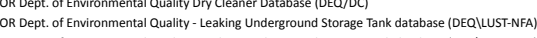
OR Dept. of Environmental Quality - Leaking Underground Storage Tank database (DEQQ) LUST-N OR State fire Marshal Hazardous Substance Information System database (SFM/HISIS-2009)

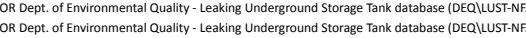

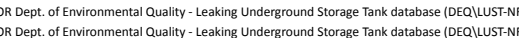

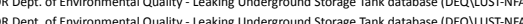
OR Dept. of Environmental Quality - Hazard Waste Program database (DEQQHWW)

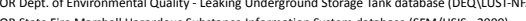

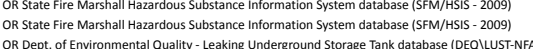

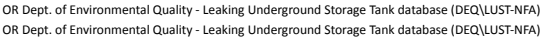

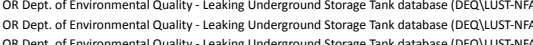

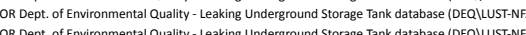

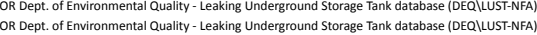

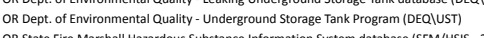

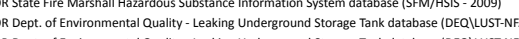
OR Dept. of Environmental Quality - Leaking Underground Storage Tank database (DEQQ L UST-NFA

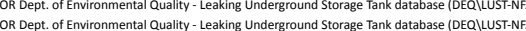

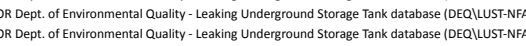

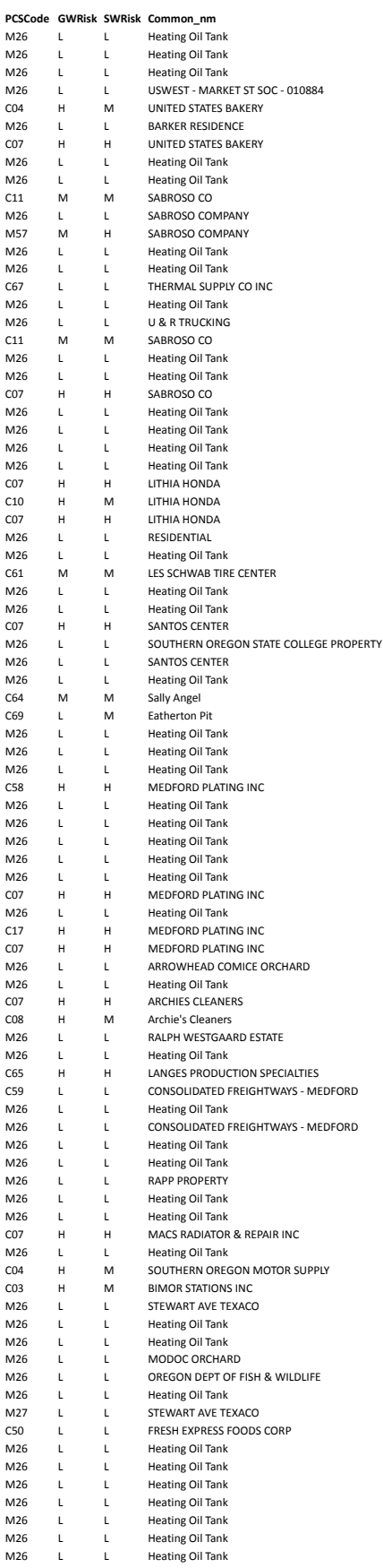

CSTYye
UST - Confirmed Leaking but lised as NFA- DEQ LUST Lis

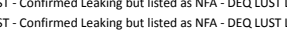
UST - Confirmed Leaking but listed as NFA- DEQ L UST Lis

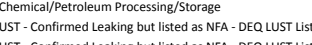
UST - Confirmed Leaking but listed as NFA - DEQ LUST Lis Food Processing
uST- Confirmed Leaking but listed as N NFA- -DEQ L LST Lis UST - Confirmed Leaking but listed a SNAA- DEQ Q UST List
UST - Confirmed Leaking but Isted as NFA - DEQ LUST List UST - Confirmed Leaking but listed a a NFA- DEQ LUST Lis
UST- Confirmed Leaking but isted as NFA - DEQ L UST Lis UST - Confimed Leaking but listed as NFA- DEQ L UST List
UST - Confirmed Leaking but listed as NFA- DEQ UST List Chemical/etroleum Processing/storage UST - Confirmed Leaking but listed as NFA - DEQ LUST L UST - Confirmed Leaking but listed as NFA - DEQ LUST Lis

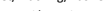

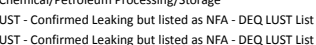
UST - Confirmed Leaking but listed a s NFA- DEQ UST Lis
UST - Confirmed Leaking but isted as NFA - DEQ UST Lis

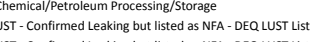
UST - Confirmed Leaking but listed as NFA- DEQ LUST List Mining Activites - Inactive - other han sand/fravel/rock/son UST - Confirmed Leaking but listed as N NFA - DEQ WUST UST - Confirmed Leaking but listed as NFA - DEQ LUST Lis WST - Confirmed Leaking but isted as NFA - DEQ LUST L UST - Confirmed Leaking but listed as NFA- DEQ LUST $L$ UST - Confirmed Leaking but isted a s NFA- DEQ LUST Lis

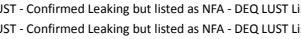
chemica/Petroleum Processing//Storage

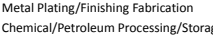

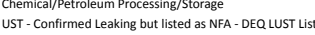

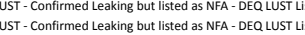
Chemical/Petroleum Processing/Storage

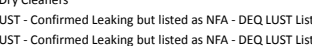
Miscelaneous Manufacturing

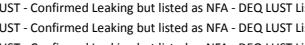
UST - Confirmed Leaking but listed as NFA - DEQ LUST UST - Confirmed Leaking but listed as NNA- DEQ USST Lis

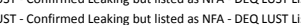

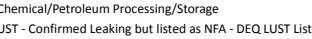
UST- Confirmed Leaking bu
Auto- Repair Shops

UST - Confirmed Leaking but listed as NFA - DEQ LUST List

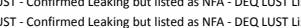
UST - Confirmed Leaking but listed a s NFA - DEQ LUST Lis

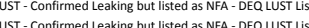
(a) UST- Confirmed Leaking but listed as NFA - DEQ LUST List

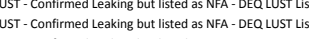
UST - Confirmed Leaking but listed as NFA- DEQ LUST Lis
UST - Confirmed leaking but isted as SNA - DEO WuST List UST- Confirmed Leaking but isted as NAA- DEQ LST Lis
UST - Confirmed Leakin but isted as NFA- DEQ LUS Lis 


\begin{tabular}{|c|c|c|c|}
\hline $\begin{array}{l}\text { Address } \\
710 \text { SGAPE ST }\end{array}$ & 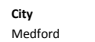 & $\begin{array}{l}\text { County } \\
\text { JackSON }\end{array}$ & $\begin{array}{l}\text { RET_DATE } \\
10 / 31 / 2018\end{array}$ \\
\hline 711 STEWART AVE & MEFPORD & JACKSON & . $09 / 29 / 2008$ \\
\hline $\begin{array}{l}7890 \text { A AATET RD } \\
7890 \text { GAATE RD }\end{array}$ & N/A & $\begin{array}{l}\text { JACKSON } \\
\text { JACKON }\end{array}$ & $\begin{array}{l}10 / 31 / 2018 \\
10131 / 2018\end{array}$ \\
\hline 7890 AGATE RD RD & N/A & JACKSON & $10 / 31 / 2018$ \\
\hline 7890 AGATE RD & N/A & JaCkSON & $10 / 31 / 2018$ \\
\hline $\begin{array}{l}711 \text { tewart Ave } \\
711 \text { w WTEWART AVE }\end{array}$ & $\begin{array}{l}\text { Metford } \\
\text { Metford }\end{array}$ & $\begin{array}{l}\text { Jackson } \\
\text { Jacksoy }\end{array}$ & $\begin{array}{l}8 / 27 / 2018 \\
101 / 212018\end{array}$ \\
\hline $\begin{array}{l}711 \text { w STSWWARR AVE } \\
711 \text { W STEWART AVE }\end{array}$ & $\begin{array}{l}\text { Mefford } \\
\text { Metford }\end{array}$ & $\begin{array}{l}\text { JaCKSON } \\
\text { JACKSON }\end{array}$ & $\begin{array}{l}101 / 3 / 21 / 2018 \\
10131 / 2018\end{array}$ \\
\hline 200 MOWETZA & $\begin{array}{l}\text { Wertor } \\
\text { NA }\end{array}$ & 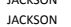 & $\begin{array}{l}101 / 3 / 21 / 2018 \\
10 / 131 / 2018\end{array}$ \\
\hline 780 RETTEN DR & & JACKSON & $\begin{array}{l}10 / 131 / 2018 \\
1018\end{array}$ \\
\hline 711A STEWART AVE & Medford & Jackson & $10 / 31 / 2018$ \\
\hline 234 NFRONT ST & Central Point & & $10 / 31 / 2018$ \\
\hline $\begin{array}{l}715 \text { W M MiN ST } \\
71599 \text { Pineridge Drive }\end{array}$ & $\begin{array}{l}\text { Medford } \\
\text { Medford }\end{array}$ & JACKSON & $\begin{array}{l}10 / 13 / 2018 \\
1013 / 2018\end{array}$ \\
\hline 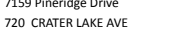 & $\begin{array}{l}\text { Mefoford } \\
\text { MEFFoRD }\end{array}$ & $\begin{array}{l}\text { Jacason } \\
\text { IACKSON }\end{array}$ & 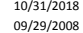 \\
\hline 722 ROSSANLEY DR & $\begin{array}{l}\text { MEFUCOO } \\
\text { MEDFOD }\end{array}$ & $\begin{array}{l}\text { JaCSSON } \\
\text { IACCSON }\end{array}$ & $\begin{array}{l}099 / 2 / 2008 \\
09 / 29 / 2008\end{array}$ \\
\hline 722 WELCH ST & MEFFORD & Jackson & $\begin{array}{l}09 / 29 / 2008 \\
0\end{array}$ \\
\hline 1816 OLD STAGE ROAD & & JACKSON & $10 / 31 / 2018$ \\
\hline 726 Royal No. 31 & Medford & Jackson & $1 / 11 / 2018$ \\
\hline $\begin{array}{l}1400 \text { MAIN ST } \\
\text { 331 FRONSTT }\end{array}$ & $\begin{array}{l}\text { Eagle Point } \\
\text { Centra Point }\end{array}$ & $\begin{array}{l}\text { JACKSON } \\
\text { IACSSON }\end{array}$ & $\begin{array}{l}10 / 13 / 2018 \\
1013 / 2018\end{array}$ \\
\hline $\begin{array}{l}313 \text { FRRNTS ST } \\
727 \text { CARDLY AVE }\end{array}$ & $\begin{array}{l}\text { Centra Point } \\
\text { MEDFORD }\end{array}$ & $\begin{array}{l}\text { JaCKSON } \\
\text { JACKSON }\end{array}$ & 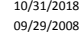 \\
\hline 727 N. CENTRAL AVE. & & $\begin{array}{l}\text { JaCSSON } \\
\text { IACCSON }\end{array}$ & $\begin{array}{l}09929 / 2008 \\
10 / 31 / 2018\end{array}$ \\
\hline 727 W MCANDREWS RD & MEFFORD & $\begin{array}{l}\text { Jackson } \\
\text { Jas }\end{array}$ & $\begin{array}{l}09 / 29 / 2008 \\
0\end{array}$ \\
\hline 13673 HIGHWAY 234 & N/A & JACKSON & 10/31/2018 \\
\hline 1291 N VALLY VIEW RD & N/A & Jackson & 10/131/2018 \\
\hline 737 W WCANDDDERWS RD & $\begin{array}{l}\text { MEDEORD } \\
\text { MEFOORD }\end{array}$ & $\begin{array}{l}\text { JACKSON } \\
\text { IACCSON }\end{array}$ & $\begin{array}{l}09 / 29 / 2008 \\
09929 / 2008\end{array}$ \\
\hline $\begin{array}{l}1100 \text { KIRTANLAND DR } \\
110 \text { RT }\end{array}$ & & $\begin{array}{l}\text { JaCSSON } \\
\text { IACSSON }\end{array}$ & $\begin{array}{l}09 / 29 / 2008 \\
10131 / 2018 \\
\end{array}$ \\
\hline 730 BIDOLE RD & Medford & Jackson & $\begin{array}{l}10 / 31 / 218018 \\
10 / 2128\end{array}$ \\
\hline 3290 ACCSONVVILE HWY & $\mathrm{N} / \mathrm{A}$ & JaCKSON & $10 / 11 / 2018$ \\
\hline 835 TIMBERRLAKE DR & $\mathrm{N} / \mathrm{A}$ & JACKSON & 10/31/2018 \\
\hline 736 MASON WY & MEOFORD & JaCKSON & $09 / 29 / 2008$ \\
\hline 203 NPLAT & $\begin{array}{l}\text { Eage pont } \\
\text { Eage Pojt }\end{array}$ & JACKSON & $\begin{array}{l}10 / 13 / 2018 \\
101312018\end{array}$ \\
\hline 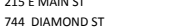 & M MEFORD & $\begin{array}{l}\text { JaCSSON } \\
\text { IACSSON }\end{array}$ & $\begin{array}{l}1 / 131 / 2018 \\
09 / 29 / 2008\end{array}$ \\
\hline 744 DIAMONO ST & MEOFORO & Jackson & $\begin{array}{l}0 \\
09 / 29 / 29 / 2008 \\
0\end{array}$ \\
\hline 744 Cardley Ave. Ste. 100 & Medford & Jackson & $1 / 11 / 2018$ \\
\hline 744 Cardley Ave. Ste. 100 & Medford & Jackson & 1/11/2018 \\
\hline 745 W STEWART AVE & $\begin{array}{l}\text { Metford } \\
N \text { e }\end{array}$ & & 10/31/2018 \\
\hline 24 EHRMAN WAY & N/A & Jackson & $\begin{array}{l}10 / 13 / 21 / 218 \\
1 / 1312018\end{array}$ \\
\hline $\begin{array}{l}750 \text { N COLUMBUS AVE } \\
750\end{array}$ & Mefford & $\begin{array}{l}\text { JaCSSOON } \\
\text { IACSSON }\end{array}$ & $\begin{array}{l}10 / 13 / 21 / 218 \\
1 / 1312018\end{array}$ \\
\hline 750 N COLUMBUS AVE & Mefford & JACKSON & $\begin{array}{l}101 / 11 / 218 \\
10 / 11 / 2018\end{array}$ \\
\hline 944 E DUTTON RD & $N / A$ & JaCKSON & 10/31/2018 \\
\hline 4031 PIONERR RD & N/A & JACKSON & 10/31/2018 \\
\hline 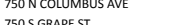 & 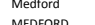 & JACKSON & 10/31/2018 \\
\hline 750 W STEWART AVE & $\begin{array}{l}\text { MEEROEO } \\
\text { MEDFORO }\end{array}$ & $\begin{array}{l}\text { JaCSSON } \\
\text { ICCCSON }\end{array}$ & $\begin{array}{l}099292 / 2008 \\
0 . / 292 / 2008\end{array}$ \\
\hline 135 CARPENTER HIL R RAD & N/A & 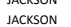 & $\begin{array}{l}09929 / 2008 \\
10 / 31 / 2018\end{array}$ \\
\hline 765 N PHOENIX RD & Medford & Jackson & $101 / 11 / 2018$ \\
\hline 775 MEOFORD CENTER & MEOFORD & JACKSON & 09/29/2008 \\
\hline 785 STEWART AVE & MEFPORD & JACKSON & 09/29/2008 \\
\hline $\begin{array}{l}7855 \text { SENWWARA AVE } \\
785 \text { SEFWAT AVE }\end{array}$ & $\begin{array}{l}\text { Medeford } \\
\text { Mefford }\end{array}$ & 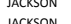 & 10/31/2018 \\
\hline 1251 OLDARIGHWEAY $99 \mathrm{~S}$ & N/A & 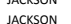 & $\begin{array}{l}111 / 12018 \\
1 / 1 / 21 / 2018\end{array}$ \\
\hline 800 MAlN ST & $\begin{array}{l}\text { Phoenix } \\
\text { Pho }\end{array}$ & IACSSON & 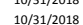 \\
\hline 795 S RIVERSID AVE & Medford & JACKSON & $10 / 11 / 2018$ \\
\hline 795 S RIVERSID AVE & Medfordd & JACKSON & 10/31/2018 \\
\hline "B'sT \& \& MUNTAAN AVE & Asthland & JACKSON & 10/31/2018 \\
\hline 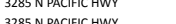 & N/A & 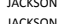 & 10/31/2018 \\
\hline 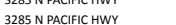 & 酸 & 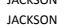 & $\begin{array}{l}1 / 1 / 31 / 2018 \\
10 / 31 / 2018\end{array}$ \\
\hline $3285 \mathrm{~N}$ PACIFIC HWY & $\mathrm{N} / \mathrm{A}$ & $\begin{array}{l}\text { SACKSON } \\
\text { ICCSON }\end{array}$ & 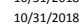 \\
\hline 32255 N PACFFC HWY & N/A & JaCKSON & $101 / 1 / 2018$ \\
\hline 1150 C CREST DR & Central Point & JaCKSON & 10/31/2018 \\
\hline 1135 LOZIER LN & N/A & IACCSSON & 10/31/2018 \\
\hline (7) & $\begin{array}{l}\text { Eaget ront } \\
\text { vela }\end{array}$ & 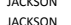 & $\begin{array}{l}10 / 13 / 21 / 218 \\
1 / 1312018\end{array}$ \\
\hline 580 OAK KNOL DR & Astland & $\begin{array}{l}\text { IACKSON } \\
\text { IA }\end{array}$ & $\begin{array}{l}10 / 131 / 218 \\
10 / 31 / 2018\end{array}$ \\
\hline 1975 CAMP BAKER RD & $\mathrm{N} / \mathrm{A}$ & JACKSON & 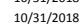 \\
\hline GRANT RD & N/A & JaCKSON & 10/31/2018 \\
\hline 52 GAANITE ST & Ashland & JaCKSON & 10/31/2018 \\
\hline TEWART AVE AT & Meefford & IACCSSON & 10/31/2018 \\
\hline 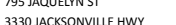 & Astand & 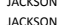 & $\begin{array}{l}10 / 13 / 21 / 2018 \\
1 / 1312018\end{array}$ \\
\hline 3921 DARK ННООW RD & N/A & Incsoon & $\begin{array}{l}10 / 131 / 218 \\
10 / 31 / 2018\end{array}$ \\
\hline ANNEY LN & N/A & JACKSON & $\begin{array}{l}101 / 1 / 2018 \\
1018\end{array}$ \\
\hline 8001 TABLE RoCK RD & MEDFORD & JaCkSON & 09/29/2008 \\
\hline 4642 RISING GLEN DR & N/A & JACKSON & 10/31/2018 \\
\hline MEBREARE R R & N/A & JACCSONO & 10/31/2018 \\
\hline N & MEOFORO & 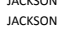 & $\begin{array}{l}0 \\
09 / 29 / 20008 \\
0\end{array}$ \\
\hline $\begin{array}{l}1958 \text { OLD MLLTARY RD } \\
800 \text { poss IN }\end{array}$ & $N / A$ & JACKSON & $\begin{array}{l}10 / 31 / 2018 \\
\end{array}$ \\
\hline 801 POSSE LN & & & 09/29/2008 \\
\hline
\end{tabular}

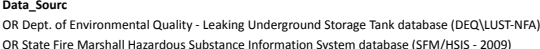
OR Dept. of Environmental Quality - Leakinin Underground Storage Tank databasese (DEQRIUUS-NFFA OR Dept. of Environmental Q Quality - Leaking Underground Storage Tank database (DEQQIUST-NFA) OR Dept. of Environmental Quality Dry Cleaner Database (DEQ/LC C

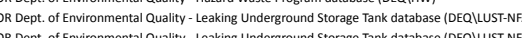

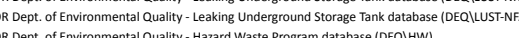
OR Dept. of Environmental Quality L Leaking Underground Storage Tank database (DEQQIUST-NFA OR Dept. of Environmental Quality - Hazard Waste Program database (DEQOAWW)

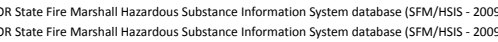

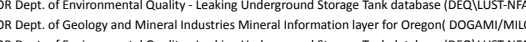

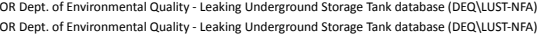

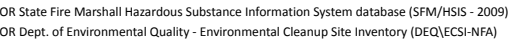

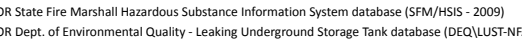

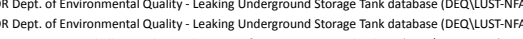

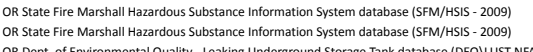
OR Dept. of Environmental Q Quality - Leakinin Underground Storage Tank database (DEQQ ULST-NFA)

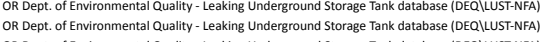

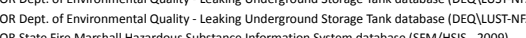

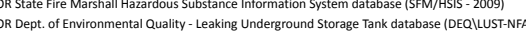
OR State Fire Marshall Hazardous Substance Information System database (SFM/HIS - 2009) OR State fire Marshall Hazardous Substance Ifformation System database (SFM/HISIS-2009)

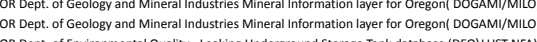

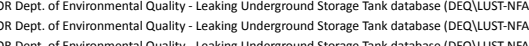
R Dept. of Environmental Q Quality - Hazard Waste Program database ( $D E Q \mathrm{O} H \mathrm{HW}$ -

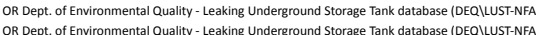

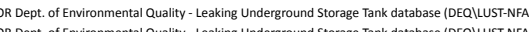
OR Dept. of Environmental Q Quality - Leaking Underground Storage Tank database (DEQRI) UST-N OR Dept. of Environmental Quality L Leaking Underground Storage Tank database (DEQ) IUST-NFA) OR Dept. of Environmental Quality - Water Quality IIS database

database (SFMM/HIS. -2009)

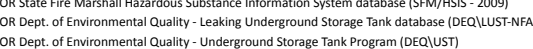

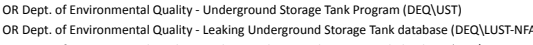

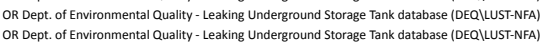

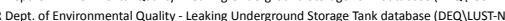

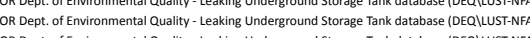

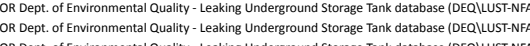

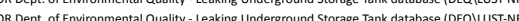
OR Dept. of Environmental Quality - Leaking Underground Storage Tank database (DEQQLLUST-NFA

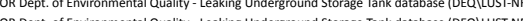
OR Dept. of Environmental Q Quality - Leaking Underground Storage Tank databasese (DEQQI LUST-NFA

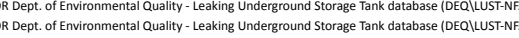

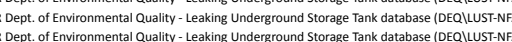
OR Dept. of Environmental Quality - Leaking Underground Storage Tank database (DEQ) ULST-N

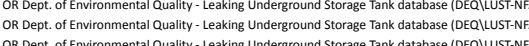

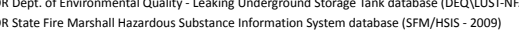

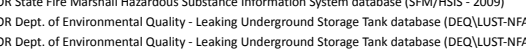

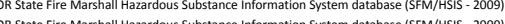

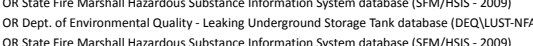

\begin{tabular}{|c|c|c|c|}
\hline \multicolumn{4}{|c|}{ GWRisk SWR } \\
\hline & L & & QUALTYY PRODUCTS \\
\hline & H & & WELDONS CLEANING CENTER \\
\hline & L & & $\begin{array}{l}\text { BOOSEC CASCADE-WHITE CIT } \\
\text { BOISE CASCADE-WHITE CITY }\end{array}$ \\
\hline & ¿ & & BOISE CASCADE - WHITE CITY \\
\hline & L & L & BOISE CASCADE - WHITE CITY \\
\hline & & & $\begin{array}{l}\text { WELDONS CLEANING CENTER } \\
\text { WeES }\end{array}$ \\
\hline & H & H & WELDONS CLEANING CENTER \\
\hline & & & Heating Oil Tank \\
\hline & $\begin{array}{l}\mathrm{L} \\
\mathrm{H}\end{array}$ & 4 & $\begin{array}{l}\text { Heating Oil Tank } \\
\text { Pang }\end{array}$ \\
\hline & ז & & 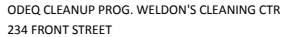 \\
\hline & & H & 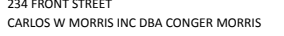 \\
\hline & H & & Robino Well 2 (ASR) \\
\hline & н & & 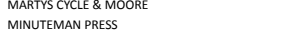 \\
\hline & н & M & GALE HASZ AUTOMOTIVE \\
\hline & t & 列 & Heating Oil Tank \\
\hline & & & \\
\hline & & & $\begin{array}{l}\text { Heating Oi Tink } \\
\text { RoGUE CREAMERY }\end{array}$ \\
\hline & L & & 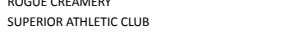 \\
\hline & & & AYALA PROPERRTY \\
\hline & t' & ${ }_{L}^{M}$ & $\begin{array}{l}\text { CAACADE BOLCC MEDFORD } \\
\text { Heating Oil Tank }\end{array}$ \\
\hline & L & & Heating Oil Tank \\
\hline & H & 正 & CASCADE BLOCK MEDFORD \\
\hline & M & ${ }_{1}^{M}$ & TEA SUPPY COMPANY INC \\
\hline & t & & JAY ALEN CO-GLEAVES VW \\
\hline & & & $\begin{array}{ll}\text { Heating oil Tank } \\
\text { Hetritio }\end{array}$ \\
\hline & H & & $\begin{array}{l}\text { Heeating oil Tank } \\
\text { WLLAMETEV VALEY COMPANY }\end{array}$ \\
\hline & ᄂ & L & SCHOOL OPTIONS ALERNATTVE SCH \\
\hline & L & & Heating Oil Tank \\
\hline & & & B \& M WHEEL ALIGNMENT \& AUTOMOTIVE \\
\hline & 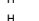 & & B \& M WHEEL ALGGNMENT \& AUTOMOTIVE \\
\hline & L & & $\begin{array}{l}\text { Galpinitighbanks } \\
\text { Buurill }\end{array}$ \\
\hline & ᄂ & & $\begin{array}{l}\text { Burfun Oit Tank } \\
\text { Heating }\end{array}$ \\
\hline & L & & \\
\hline & & & Heating Oil Tank \\
\hline & L & & MEDFORD SCHOOL DISTRRCT 549-C \\
\hline & L & & MEFFORD SCHOOL DISTRICT 599-C \\
\hline & L & L & 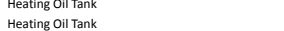 \\
\hline & L & L & 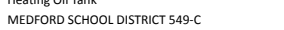 \\
\hline & H & M & \\
\hline & & & VIKING Pools \\
\hline & 2 & 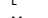 & Heating Oil Tank \\
\hline & M & & LEVITT, MARCUSC C \\
\hline & ${ }_{H}^{M}$ & $\begin{array}{c}M \\
M\end{array}$ & 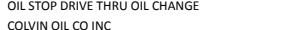 \\
\hline & L & & 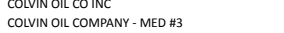 \\
\hline & ᄂ & L & COLVIN OIL COMPANY - MED H3 \\
\hline & 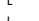 & & Heating Oil Tank \\
\hline & & & PHOENIX EXXON \#9290 \\
\hline & 1 & 2 & $\begin{array}{l}\text { Heating Oil Tank } \\
\text { Heating Oil Inak }\end{array}$ \\
\hline & L & ¿ & $\begin{array}{l}\text { Heatitig Oi Tank } \\
\text { CITr OA ASLLAND, STREET DIVISION }\end{array}$ \\
\hline & L & & 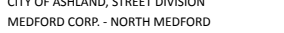 \\
\hline & L & & MEDFORD CORP. NORTH MEDFORD \\
\hline & L & & BOISE CASCADE-MEDFORD \\
\hline & L & & BOISE CASCADE-MEDFORD \\
\hline & 2 & & 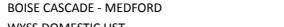 \\
\hline & t & & $\begin{array}{l}\text { WYSSD DOMESTTC UST } \\
\text { STEWART MARKET }\end{array}$ \\
\hline & ᄂ & L & Heating Oil Tank \\
\hline & 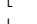 & & Heating Oil Tank \\
\hline & & & Heating Oil Tank \\
\hline & $\mathrm{L}$ & & HUNT PROPERTY \\
\hline & (2) & & TWIN CREEKS DEVELOPMENT \\
\hline & ᄂ & & $\begin{array}{l}\text { Heating Oil Tank } \\
\text { MoBIL OLL \#10-610 }\end{array}$ \\
\hline & L & L & Heating Oil Tank \\
\hline & 1 & & 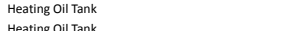 \\
\hline & & & $\begin{array}{l}\text { Heazitigg in lank } \\
\text { Heating Oil Tank }\end{array}$ \\
\hline & m $>2>>$ & & ROGUE MATERIALS \& RECOVERY \\
\hline & L & & Heating Oil Tank \\
\hline & L & & Heating Oil Tank \\
\hline & H & 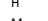 & ROGUE MAERRIALLS \& RECOVERY \\
\hline & & Wh & 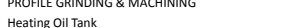 \\
\hline & H & $\mathrm{H}$ & $\begin{array}{l}\text { PROFLE GRINING \& MACHNING } \\
\text { PROLE }\end{array}$ \\
\hline
\end{tabular}

PCSTYpe
UST- Confirmed Leaking but listed as NFA - DEQ LUST Lis

UST - Confirmed Leaking but listed as NFA- DEQ LUST Lis UST-Confirmed Leaking but listed as as NA- DEQ DEQ USTS LS UST- Confirmed Leaking but listed as NFA- DEQ LUST Lis Dry Cleaners

UST- Confirmed Leaking but listed as NFA- DEQ UST L is

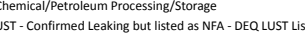
Oher lijiection/ /ry Wells, Sumps - class V Vics Other- Generar Merchand
Photo Processing/Printing
Auto

UST - Confirmed Leaking but listed as NFA- DEQ LUST Mining Activities - Inactive - sand//rrave//rock/soil WTT - Confirmed Leaking but listed a N NAA - DEQ LUST Lis Others - Sports//Fntertainment Activities
Known Contamination listed as NFA Astes/Plumes/Spills from

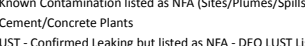

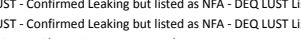
Construction Company

UST - Confirmed Leaking but listed as NFA- DEQ UST Lis

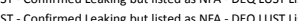

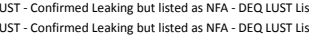
Thoto Processing/Printing
UST - Confirmed Leaking but listed as NFA- DEQ LUST Lis Auto - Repair Shops Mininical/Petroleum Processing//Storage UT- Confirmed Leaking but listed as NFA- DEQ LUST Lis

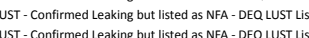

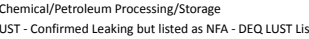
UST - Confirmed Leaking but listed as NFA- DEQ LUST Lis UST - Confirmed Leaking but I Iseded as NFA - DEQ WUST Lis Fleet/rucking

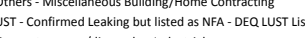
Gaywater revese/disposal or or industrial rester

UST- Confirmed Leaking but listed as NFA - DEQ LUST Lis UST - Confirmed Leaking but Iisted a S NFA - DEQ LUST L UST - Confirmed Leaking but Iisted a S NFA - DEQ LUST LI

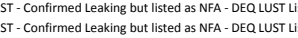

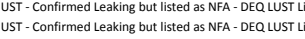
UST - Confirmed Leaking but listed as NFA - DEQ LUST L UST - Confirmed Leaking but isted a S N NAA - EEQ LUST Lis

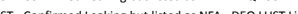
UST - Confirmed Leaking but listed as N NAA - DEQ USST UST - Confirmed Leaking but listed as NFA- DEQ LUST Lis

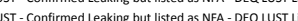
UST - Confirmed Leaking but listed a s NFA - DEQ LUST Lis STT- Confirmed Leaking but listed as NAA - DEO LST Lis

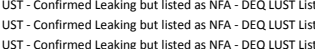
Transer/Recyching stations UST - Confirmed Leaking but listed as NFA- DEQ LUST Lis
UST - Confirmed Leaking but isted as NFA- DEQ UST Lis Chemical/Petroleum Processing//5torage
Machine Shoos

UST - Confirmed Leaking but listed as NFA- DEQ LUST List 


\begin{tabular}{|c|c|c|c|}
\hline $\begin{array}{l}\text { Address } \\
\text { Bo1 N RIVERSIDE }\end{array}$ & $\begin{array}{l}\text { City } \\
\text { MEDFORD }\end{array}$ & $\begin{array}{l}\text { County } \\
\text { JACKSON }\end{array}$ & $\begin{array}{l}\text { RET_DATE } \\
099 / 29 / 200\end{array}$ \\
\hline 801 NRIVERSIDE & MEFFORD & JACKSON & $\begin{array}{l}099 / 2 / 200808 \\
09 / 20 / 2008\end{array}$ \\
\hline 625 Houlys & Ashland & Jackson & $10 / 31 / 2018$ \\
\hline $\begin{array}{l}68840 \text { HIGHWWA } 66 \\
3930 \text { COAL MINE RD }\end{array}$ & N/A & $\begin{array}{l}\text { JaCKSON } \\
\text { IACCSON }\end{array}$ & $\begin{array}{l}10 / 31 / 10218 \\
10 / 31 / 2018\end{array}$ \\
\hline 114 RACHEL DR & $\begin{array}{l}\text { Central point } \\
\text { Cht }\end{array}$ & $\begin{array}{l}\text { JaCSSON } \\
\text { JaCKSON }\end{array}$ & $\begin{array}{l}10 / 3 / 1 / 2018 \\
10 / 31 / 2018\end{array}$ \\
\hline 204 W. MAIN STREET & Eagle Point & JACKSON & $\begin{array}{l}10 / 31 / 10218 \\
10 / 31 / 2018\end{array}$ \\
\hline 1996 GRIFFFI CREEK RD & & JACKSON & $10 / 31 / 2018$ \\
\hline 8495 CRATER LAKE HWY & N/A & JACKSON & $10 / 31 / 2018$ \\
\hline 3015 MARIGOLD LN & N/A & JaCKSON & $\begin{array}{l}10 / 31 / 2018 \\
10 / 312018\end{array}$ \\
\hline 110 E MAIN ST & Talent & $\begin{array}{l}\text { JaCSKON } \\
\text { IACKSON }\end{array}$ & $\begin{array}{l}10 / 31 / 12018 \\
1013 / 12018\end{array}$ \\
\hline $552 \mathrm{BACH} S \mathrm{TST}$ & $\begin{array}{l}\text { Metorod } \\
\text { Ashland }\end{array}$ & $\begin{array}{l}\text { JaCSSON } \\
\text { IACKSON }\end{array}$ & $\begin{array}{l}10 / 3 / 1 / 2018 \\
10 / 31 / 2018\end{array}$ \\
\hline 870 S FRONT ST & N/A & 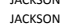 & $\begin{array}{l}10 / 3 / 1 / 2018 \\
10 / 28\end{array}$ \\
\hline 870 S FRONT ST & N/A & JACKSON & $10 / 31 / 2018$ \\
\hline NCENTRAL AVE, 5 MI SO & N/A & JACKSON & $10 / 31 / 2018$ \\
\hline 1996 GRANTFE ST & Ashland & JaCKSON & $10 / 31 / 2018$ \\
\hline $\begin{array}{l}20070 \text { P POLEERER RD } \\
1055 \text { CRES RD }\end{array}$ & N/A & $\begin{array}{l}\text { JACKSON } \\
\text { IACCSON }\end{array}$ & $\begin{array}{l}10 / 3 / 1 / 2018 \\
1013 / 12018\end{array}$ \\
\hline $\begin{array}{l}1056 \text { C CERSW RO } \\
320 \text { CATALA OR }\end{array}$ & $\begin{array}{l}\text { NA/ } \\
\text { Ashland }\end{array}$ & $\begin{array}{l}\text { PaCSSON } \\
\text { IACCSON }\end{array}$ & $\begin{array}{l}10 / 31 / 10218 \\
10 / 31 / 2018\end{array}$ \\
\hline 8022 Griffin Creek & Metford & Jackson & $\begin{array}{l}101 / 1 / 2018 \\
1 / 1 / 2018\end{array}$ \\
\hline 803 S CENTRAL & MEDFORD & JACKSON & $\begin{array}{l}09 / 29 / 2008 \\
0\end{array}$ \\
\hline $803 \mathrm{~S}$ CENTRAL AVE & Medford & Jackson & 10/31/2018 \\
\hline 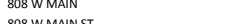 & $\begin{array}{l}\text { Mefford } \\
\text { MEDORD }\end{array}$ & $\begin{array}{l}\text { JaCKSON } \\
\text { IACCSOON }\end{array}$ & $\begin{array}{l}11 / 1 / 2018 \\
092 / 2 / 2008\end{array}$ \\
\hline $\begin{array}{l}808 \text { W WAANST } \\
808 \text { W MAIN ST }\end{array}$ & $\begin{array}{l}\text { MEFOORD } \\
\text { MEDFORD }\end{array}$ & $\begin{array}{l}\text { JaCCSON } \\
\text { JACKSON }\end{array}$ & $\begin{array}{l}09 / 2 / 2 / 2008 \\
00 / 292008\end{array}$ \\
\hline 2447 RosS LN & N/A & 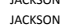 & $\begin{array}{l}09929 / 2008 \\
10 / 31 / 2018 \\
\end{array}$ \\
\hline 812 BROOKOALE & Medford & Jackson & $\begin{array}{l}10 / 31 / 2018 \\
1018\end{array}$ \\
\hline 2444 Ross LN & N/A & JaCkSON & $10 / 31 / 2018$ \\
\hline 1070 TOLMAN CREEK RD & Asshland & JaCKSON & $10 / 31 / 2018$ \\
\hline 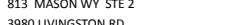 & MEDFORD & JaCKSON & $09 / / 29 / 2008$ \\
\hline 3475 PAYNE RD & N/A & $\begin{array}{l}\text { JaCKSON } \\
\text { ACCKSON }\end{array}$ & $\begin{array}{l}10 / 3 / 1 / 2018 \\
10 / 31 / 2018\end{array}$ \\
\hline 813 MASON WY STE 2 & MEDFORD & $\begin{array}{l}\text { JaCKSON } \\
\text { Jats }\end{array}$ & $\begin{array}{l}10101312121 \\
09 / 29 / 200\end{array}$ \\
\hline 813 MASON WAY UNIT 2 & Medford & Jackson & $\begin{array}{l}10 / 31 / 201 \\
1019\end{array}$ \\
\hline 813 MASON WAY UNIT 2 & Medtord & JACKSON & $10 / 31 / 20$ \\
\hline 815 S OAKDALE AVE & MEDFORD & Jackson & 09/2/29/200 \\
\hline 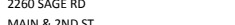 & & $\begin{array}{l}\text { JaCKSON } \\
\text { IACCSON }\end{array}$ & $\begin{array}{l}10 / 31 / 20 \\
103 / 20\end{array}$ \\
\hline 475 ENEVADA ST & $\begin{array}{l}\text { Asstanca } \\
\text { Ashland }\end{array}$ & 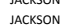 & $\begin{array}{l}101 / 3 / 20 \\
10 / 31 / 20\end{array}$ \\
\hline 785 BEACH ST & Assland & JACKSON & $\begin{array}{l}10131 / 20 \\
10 / 31 / 20\end{array}$ \\
\hline 8155 Oakdale Ave & Medford & JACKSON & 06/102/20. \\
\hline 580 FISH LAKE RD & N/A & JACKSON & $10 / 31 / 20$ \\
\hline 99 UNION ST & $\begin{array}{l}\text { Ashland } \\
\text { Mefford }\end{array}$ & $\begin{array}{l}\text { Jackson } \\
\text { Jacsoon }\end{array}$ & $\begin{array}{l}10 / 31 / 20 \\
0602720\end{array}$ \\
\hline $\begin{array}{l}8155 \text { S Oakadale Ave } \\
8 \text { BLACK OAK DR }\end{array}$ & $\begin{array}{l}\text { Metaford } \\
\text { MEDFORD }\end{array}$ & $\begin{array}{l}\text { JaCSKON } \\
\text { IACKSON }\end{array}$ & $\begin{array}{l}06 / 102 / 20 \\
09292 / 20\end{array}$ \\
\hline 816 Black Oak Dr & Menterord & IACKSON & $\begin{array}{l}0.9929 / 200 \\
06 / 02 / 20\end{array}$ \\
\hline 750 LOZZER LN & N/A & JaCKSON & $\begin{array}{l}0.102 / 20 \\
101 / 20\end{array}$ \\
\hline 816 BLACK OAK DRIVE & Medford & JACKSON & $10 / 31 / 20$ \\
\hline 816 W 10THST & Medtord & JACKSON & $10 / 31 / 20$ \\
\hline $\begin{array}{l}819 \text { BENNET TVVE } \\
\text { 89 BNE }\end{array}$ & MEFPORD & IACCSSON & $09 / 29 / 20 \mathrm{C}$ \\
\hline 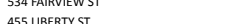 & $\begin{array}{l}\text { Assand } \\
\text { Aschad }\end{array}$ & JACKSON & $\begin{array}{l}10 / 31 / 20 \\
103\end{array}$ \\
\hline 1800 ROSSANLEY OR & N/A & 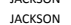 & $\begin{array}{l}10 / 11 / 20 \\
10 / 21 / 20\end{array}$ \\
\hline 819 BENNET AVE & MEDFORD & JACKSON & $\begin{array}{l}109 / 3 / 1 / 20 \\
09 / 20\end{array}$ \\
\hline 10170 TABLE ROCK RD & & JACKSON & $10 / 31 / 20$ \\
\hline 2038 BOESA AVE & Central Point & JACKSON & $10 / 31 / 20$ \\
\hline 2000 SERA ST & Ashland & IACCSSON & $10 / 31 / 20$ \\
\hline 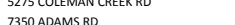 & N/A & $\begin{array}{l}\text { JaCKSON } \\
\text { JACKSON }\end{array}$ & $\begin{array}{l}10 / 13 / 20 \\
1013120\end{array}$ \\
\hline 90 MEADEST & & $\begin{array}{l}\text { IACKSON } \\
\text { IACS }\end{array}$ & $\begin{array}{l}10 / 13 / 20 \\
1 / 13120\end{array}$ \\
\hline 821 N COLUMBUS AVE & Medford & N/A & $\begin{array}{l}10 / 11 / 20 \\
10 / 121\end{array}$ \\
\hline 821 COOUMBUU AVE & Medford & & $10 / 31 / 20$ \\
\hline 75 DONNA WAY & Central Point & JACKSON & $10 / 31 / 20$ \\
\hline 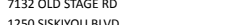 & & & $10 / 31 / 20$ \\
\hline $\begin{array}{l}{ }_{1840} \text { CRESTVIFEF DR } \\
\end{array}$ & Aschland & 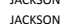 & $\begin{array}{l}10131 / 20 \\
1 / 131 / 20\end{array}$ \\
\hline 821 N COLUMBUS AVE & Menfford & N/A & $\begin{array}{l}10131 / 201 \\
1 / 1 / 2018\end{array}$ \\
\hline 3355 MADRONA LN & N/A & JACKSON & $\begin{array}{l}171 / 1201 \\
10 / 31 / 20\end{array}$ \\
\hline 2978 N FOOTHILL RD & N/A & JaCkSON & $10 / 31 / 20$ \\
\hline 821 NORTH RIVERSIDE & Mefford & Jackson & 10/31/20 \\
\hline 823 W JACKSON & MEEFORD & JACKSON & $09 / 29 / 200$ \\
\hline 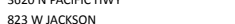 & DEF & 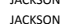 & $\begin{array}{l}10 / 131 / 20 \\
0.929200\end{array}$ \\
\hline & & JACKSON & $\begin{array}{l}0.9929 / 200 \\
10 / 31 / 20\end{array}$ \\
\hline 40 ATTIX CIRCLE & Central Point & JACKSON & $10 / 31 / 20$ \\
\hline${ }_{4840 \text { HIGHWAV } 66}$ & N/A & JACKSON & $10 / 31 / 20$ \\
\hline ORTONST & land & JACKSON & 10/131/20: \\
\hline 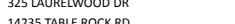 & & 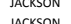 & 10/31/201 \\
\hline & Central Point & 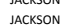 & $\begin{array}{l}10 / 31 / 201 \\
10 / 131201\end{array}$ \\
\hline $\begin{array}{l}1 \\
\text { Po Box } 389\end{array}$ & Berokins & 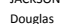 & $\begin{array}{l}100131 / 20 \\
1 / 11 / 2018\end{array}$ \\
\hline $\begin{array}{l}\text { OB Box } 389 \\
3500 \mathrm{NW} \text { Stewart Parl }\end{array}$ & Roseburg & & $\begin{array}{l}11 / 2018 \\
11720\end{array}$ \\
\hline 3497 Military Road & ntral Point & sson & 111/2018 \\
\hline $\begin{array}{l}2632 \text { HWw } 2588 \\
208\end{array}$ & aacksonville & Jackson & 1/1/1/2018 \\
\hline & Medford & & \\
\hline
\end{tabular}

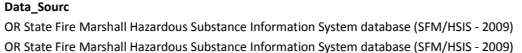

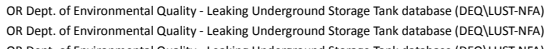
R Dept. of Environmental Quality - Leaking Underground Storage Tank database (DEQQLUST-NFFA

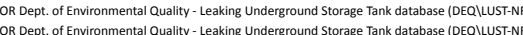
OR Dept. of Environmental Quality - Leaking Underground Storage Tank databases (DEQQ| LUST-N N

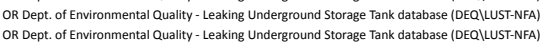

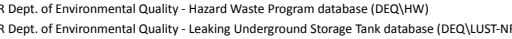

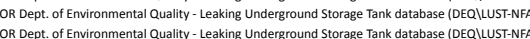
OR Dept. of Environmental Q Quality - Leaking Underground Storage Tank database (DEO) WUS-NA

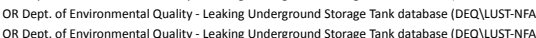

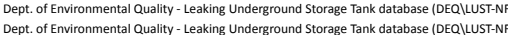

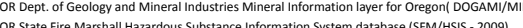

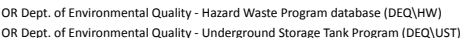

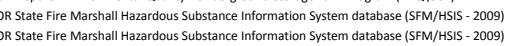

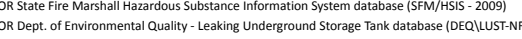

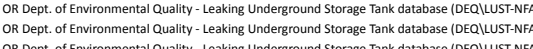

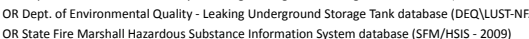

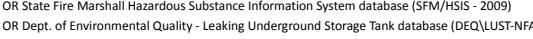

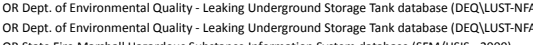

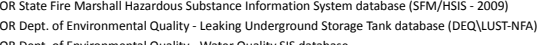

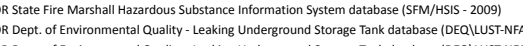

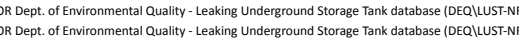

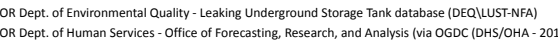

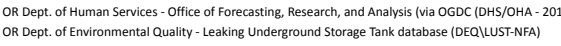

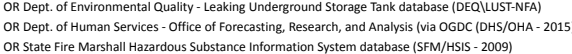

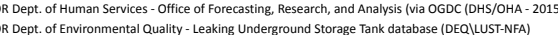

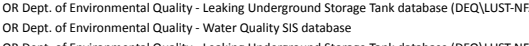
OR State Fire Marshall Hazardous S ubstance Information System database (SFM/HISS- 2009)

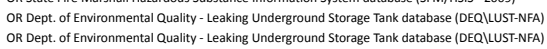

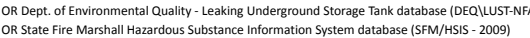
OR State fire Marshall Hazardous Substance Information System database (SFM) IFIS - 2009) OR Dept. of Environmental Quality - Leaking Underground Storage Tank database (DEQQLUST-NFA

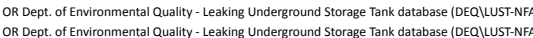

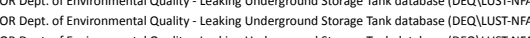

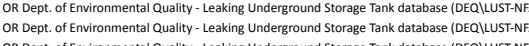

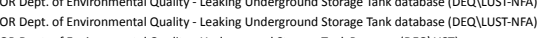

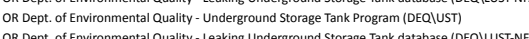

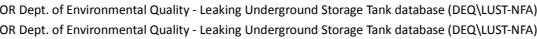
OR Dept. of Environmental Quality - Water Quality SIS datatbase

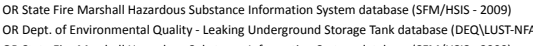

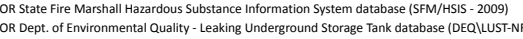

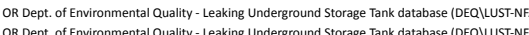

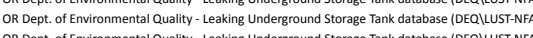

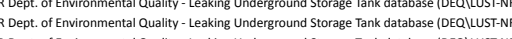

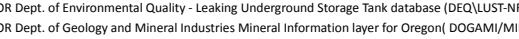

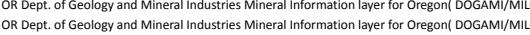

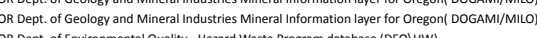

\begin{tabular}{|c|c|c|c|}
\hline & \\
\hline \multicolumn{4}{|r|}{ LTHIA VOLKSWAGEN MEDFORD } \\
\hline$c^{c 07}$ & & & LTHHA VOLKSWAGEN MEDFORD \\
\hline & L & & $\begin{array}{l}\text { Heating Oil Tank } \\
\text { Heating OII Inkk }\end{array}$ \\
\hline M26 & L & & $\begin{array}{l}\text { Heazing Bif ankn } \\
\text { Heating Oil Tank }\end{array}$ \\
\hline & t & & 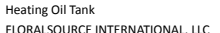 \\
\hline M26 & t & & $\begin{array}{l}\text { FoLAALSOCOREE ITTENATIONAL, LLC } \\
\text { Heating Oil Tank }\end{array}$ \\
\hline M26 & i & & $\begin{array}{l}\text { Heding } \\
\text { Heating Oil Tank Tank }\end{array}$ \\
\hline $\begin{array}{l}\text { M26 } \\
\text { M26 }\end{array}$ & L & & 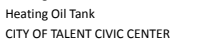 \\
\hline co7 & & & \\
\hline & L & 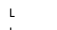 & Heating Oil Tank \\
\hline & & L & Heating oil Tank \\
\hline & & & 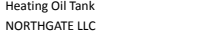 \\
\hline & $\mathrm{L}$ & 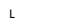 & Heating Oil Tank \\
\hline & & & Heating Oil Tank \\
\hline & L & & Heating oil Tank \\
\hline C69 & t & 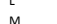 & $\begin{array}{l}\text { Heating il Tank } \\
\text { Ganatite Pit }\end{array}$ \\
\hline c50 & i & L & MLLER PANT COMPANY INC \\
\hline 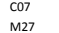 & ${ }^{H}$ & & $\begin{array}{l}\text { Milier Paint Companyy Inc } \\
\text { Jackson counry }\end{array}$ \\
\hline c07 & $\mathrm{H}$ & H & JACKSON COUNTY PUBUIC WORKKS \\
\hline & H & & JACKSON COUNTY PUBULC WORKS \\
\hline M26 & t & & $\begin{array}{l}\text { HeEFFRENAN, ROBERTT } \\
\text { Heating Oil Tank }\end{array}$ \\
\hline M26 & L & L & Heating oil Tank \\
\hline & L & & 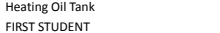 \\
\hline M26 & $\mathrm{L}$ & th & Heating Oil Tank \\
\hline & L & L & $\begin{array}{l}\text { Heating oil Tank } \\
\text { FisfSTSPONT }\end{array}$ \\
\hline & t & & $\begin{array}{l}\text { FRS STSUDNT } \\
\text { MAVFLWER CONTRACT SERVICE INC }\end{array}$ \\
\hline M57 & м & & $\begin{array}{l}\text { MAPFLOWR CONTRACT SERVICES INC } \\
\text { M }\end{array}$ \\
\hline & L & M & MEEFORD SCHOOL DISTRICT $549 \mathrm{C}$ \\
\hline & L & & $\begin{array}{l}\text { FARWEST TAELL CORP ORATION } \\
\text { OD SIGNAA GAS SATIN }\end{array}$ \\
\hline & t & & $\begin{array}{l}\text { olo SIGGAA LAAS STATION } \\
\text { Heating Oill Tank }\end{array}$ \\
\hline & t & & 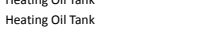 \\
\hline & L & m $>>>$ & Central Mediford high School \\
\hline M26 & L & & Heating Oil Tank \\
\hline $\begin{array}{l}\text { M26 } \\
\text { R15 }\end{array}$ & $\begin{array}{l}\mathrm{L} \\
\mathrm{L}\end{array}$ & M & 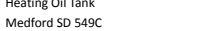 \\
\hline & $\mathrm{t}$ & m & ST MARY'S OF MEDFORD, INC. \\
\hline & L & M & St. Mary's school \\
\hline M57 & M & L & 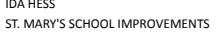 \\
\hline & L & L & $\begin{array}{l}\text { Heating oil Tank } \\
\text { Vegrzonwigs }\end{array}$ \\
\hline M26 & t & L & $\begin{array}{l}\text { VERRZZN WIIEELESS } \\
\text { Heating Oil Tank }\end{array}$ \\
\hline & L & & $\begin{array}{l}\text { Heatumg Oil tank } \\
\text { Heank }\end{array}$ \\
\hline & ᄂ & ᄂ & Heating Oil Tank \\
\hline & $H$ & H & VERIZON WIRELESS \\
\hline $\begin{array}{l}\text { M26 } \\
\text { M26 }\end{array}$ & L & ᄂ & Heating oil Tank \\
\hline M26 & t & 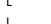 & $\begin{array}{ll}\text { Heating Oil Tank } \\
\text { Heation }\end{array}$ \\
\hline M26 & t & & $\begin{array}{l}\text { Heating in lank } \\
\text { Heating Oil Tank }\end{array}$ \\
\hline & i & & 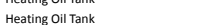 \\
\hline M26 & i & ᄂ & Heating Oil Tank \\
\hline M26 & ᄂ & L & CITY OF MEDFORO/PUBULC WORKS \\
\hline M26 & & & CITY OF MEDFORD/PUBULC WORKS \\
\hline${ }^{\mathrm{M} 26}$ & t & 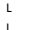 & $\begin{array}{l}\text { Heating Oil Tank } \\
\text { Heatitio Oil Tank }\end{array}$ \\
\hline & $i$ & & $\begin{array}{l}\text { Heating gil Trank } \\
\text { Heating Oil Tank }\end{array}$ \\
\hline & L & & 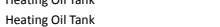 \\
\hline & 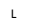 & & 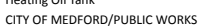 \\
\hline M26 & t & & Heating Oil Tank \\
\hline M26 & $x-2>0$ & 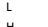 & Heating Oil Tank \\
\hline $\begin{array}{l}\text { M57 } \\
\text { Co4 }\end{array}$ & $\begin{array}{c}M \\
H \\
H\end{array}$ & H & KIDS UNLIMTED OF OREGON \\
\hline & & & $\begin{array}{l}\text { ROTH PROPERTY } \\
\text { ROST }\end{array}$ \\
\hline & H & H & $\begin{array}{l}\text { JRS WRENCH WORKS } \\
\text { JRE TR T }\end{array}$ \\
\hline M26 & 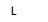 & L & Heating Oil Tank \\
\hline M26 & t & 1 & Heating Oil Tank \\
\hline 策26 & 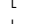 & 2 & Heating Oil Tank \\
\hline M26 & 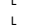 & 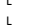 & $\begin{array}{l}\text { Heating oi fi Tank } \\
\text { Heating Oil Tank }\end{array}$ \\
\hline & & & 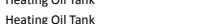 \\
\hline M26 & & 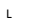 & $\begin{array}{l}\text { Heacumg } \\
\text { Heating Oil Tank }\end{array}$ \\
\hline & & $M$ & Russell I. Ralls \\
\hline & & M & Cascade Creek Quart \\
\hline & H & & Lull Grante \\
\hline & H & H & \\
\hline
\end{tabular}
WTT - Confifrmed Leaking but listed a S NFA - DEQ L LST Lis ing but listed as NFA- DEQ LUST Lis WT- Confirmed Leaking but listed as NFA- DEQ LUST L WST - Confirmed Leaking but listed as NFA- DEQ L LST L L UST - Confirmed Leaking but listed as NFA - DEQ LUST LI UST - Confirmed Leaking but listed a s N FA- DEQ Q UST List
UST - Confirmed Leaking but listed as NFA - DEQ UST List chemica/petroleum Processing/storage
UST - confirmed Leaking but isted as NFA - DEQ LuST Lis UST - Confirmed Leaking but listed as N NA - DER Q UST Lis STT - Confirmed Leaking but listed as NFA - DEQ LUST Lis UST - Confirmed Leaking but IItsed as NNA A DEQ L LST Lis

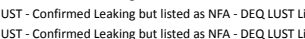
Mining Activities - Inactive- sand/fravel/rock/

Chemical/Petroleum Processing/Storage

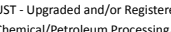
chemicalpertroleum Processing/5tsorage

UST - Confirmed Leaking but listed a N NA- DEQ LST Lis

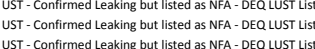
UST- Confirmed Leaking but listed as NFA - DEQQ LuST Lis UST - Confirmed Leaking but listed as NAA- DEQ UST Lis
DEQ Perritted Stormwater Dischares (NPDES or WPCCF Schools

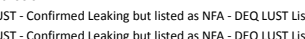
UST- Confirmed Leaking but listed as as NAA - DEQ OQ WST L L L UST - Confirmed Leaking but listed as NFA- DEQ LUST Lis

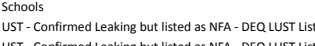
UST- Con
Schools
Schools
Schools

UST- Confirmed Leaking but listed as NFA- DEQ LUST List

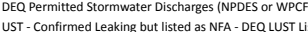
Communications office
UST - Confirmed Leaking but listed as NFA - DEQ L UST List UST - Confirmed Leaking but listed a SNEA- DEQ WST Lit

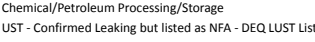
UST - Confirmed Leaking but Isted as N NAA- DEQ Q UST Lis

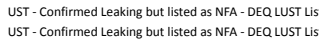
UST - Confirmed Leaking but Iisted a S NFA - DEQ L UST L UST- Confirmed Leaking but listed a S NFA- DEQ UST Lis
UST - Confirmed Leaking but isted as NFA - DEQ UST LiS

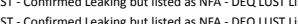
UST - Upgraded and/or Registered-Active (may also have decommisisioned tanks on sitte)

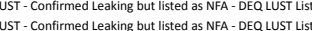
DEQ Perritited Storrmwater Discharges (NPDES Or WPCCF)

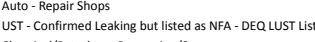
UST - Confirmed Leaking but listed as NFA - DEQ LUST Lis

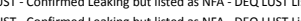

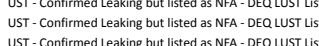

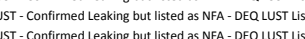
Mining Activities - Inactive - other than sand/gravel/rock/sol

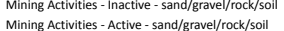
Mining Activities - Inactive - sand//Fravel/rocks/soil
Chemical/Pertolum Processing/Storage 


\begin{tabular}{|c|c|c|c|}
\hline Address & city & $\begin{array}{l}\text { County } \\
\text { Jackson } \\
\text { Jacson }\end{array}$ & $\begin{array}{l}\text { RET_DATE } \\
1 / 111 / 2018\end{array}$ \\
\hline РО Вох 27 & Spookane & $\begin{array}{l}\text { Jackson } \\
\text { Jackson }\end{array}$ & $\begin{array}{l}1 / 1 / 12018 \\
1 / 1 / 2018\end{array}$ \\
\hline 827 C ALDER CREEK DR & MEDFORD & JACKSON & $06 / 102 / 2015$ \\
\hline 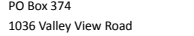 & $\begin{array}{l}\text { Wadena } \\
\text { Talent }\end{array}$ & $\begin{array}{l}\text { Jacksson } \\
\text { Jackson }\end{array}$ & $\begin{array}{l}1 / 1 / 12018 \\
1 / 1 / 2018\end{array}$ \\
\hline $\begin{array}{l}360 \text { Wildish Lane } \\
3{ }_{34} \text { lammman Read }\end{array}$ & $\begin{array}{l}\text { Eugene } \\
\text { Gold dill }\end{array}$ & $\begin{array}{l}\text { Jackson } \\
\text { Jackson }\end{array}$ & $\begin{array}{l}1 / 11 / 2018 \\
1 / 1 / 2018\end{array}$ \\
\hline & & & \\
\hline 200 Antelope Road & White city & Jackson & $1 / 11 / 2018$ \\
\hline 2000 Antelope Raad & White city & Jackson & 1/11/2018 \\
\hline 835 Alder Creek Drive & $\begin{array}{l}\begin{array}{l}\text { Medford } \\
\text { White City }\end{array} \\
\end{array}$ & Jackson & $\begin{array}{l}1 / 11 / 2018 \\
1 / 1112018\end{array}$ \\
\hline $\begin{array}{l}200 \text { Antelope Road } \\
200 \text { Antelope Road }\end{array}$ & $\begin{array}{l}\text { White City } \\
\text { White city }\end{array}$ & $\begin{array}{l}\text { Jackson } \\
\text { Jackson }\end{array}$ & $\begin{array}{l}1 / 11 / 2018 \\
1 / 1 / 2018\end{array}$ \\
\hline 200 Antelope Road & White city & $\begin{array}{l}\text { Jacsoun } \\
\text { Jackson }\end{array}$ & $\begin{array}{l}1 / 1 / 12018 \\
1 / 1 / 2018\end{array}$ \\
\hline 200 Antelope Road & White city & Jackson & $\begin{array}{l}1 / 11 / 2018 \\
1 / 18\end{array}$ \\
\hline 839 E MAIN ST STE B & MEDFORD & JACKSON & $09 / 29 / 2008$ \\
\hline $\begin{array}{l}\text { 843E E.MAANST } \\
\text { Po Box89 }\end{array}$ & 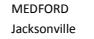 & $\begin{array}{l}\text { Iackson } \\
\text { Jackson }\end{array}$ & $\begin{array}{l}06 / 12 / 2015 \\
1 / 11 / 2018\end{array}$ \\
\hline & & $\begin{array}{l}\text { Jackson } \\
\text { Achros }\end{array}$ & $\begin{array}{l}1 / 1 / 1 / 2018 \\
1 / 1 / 2018\end{array}$ \\
\hline $\begin{array}{l}\text { 844 W RIVEESTDE AVE } \\
\text { PO BOx } 2444\end{array}$ & white city & $\begin{array}{l}\text { Jacsson } \\
\text { Jackson }\end{array}$ & $\begin{array}{l}10 / 31 / 2018 \\
1 / 1 / 2018\end{array}$ \\
\hline PO Box 3085 & Central Point & Jackson & $\begin{array}{l}1 / 11 / 2018 \\
1 / 218\end{array}$ \\
\hline РО B०० 2515 & White City & Jackson & 1/1/1/2018 \\
\hline 8535 SRIERSIDE & Medtord & Jackson & $10 / 31 / 2018$ \\
\hline $\begin{array}{l}885 \text { C Chery Wy } \\
867 \text { BEATTS ST }\end{array}$ & $\begin{array}{l}\text { Meforord } \\
\text { MEFFRD }\end{array}$ & $\begin{array}{l}\text { JacksoN } \\
\text { JACKSON }\end{array}$ & $\begin{array}{l}06 / 2 / 2 / 2015 \\
0 / 292 / 2008\end{array}$ \\
\hline 874 BEATTY ST & Medford & JACKSON & 10/31/2018 \\
\hline 877 BEATTY ST & Medford & $\begin{array}{l}\text { Jackson } \\
\text { Jackson }\end{array}$ & $\begin{array}{l}101 / 3 / 212018 \\
1 / 1 / 2018\end{array}$ \\
\hline PO Box 1088 & Eagle Point & Jackson & 1/11/2018 \\
\hline $\begin{array}{l}\text { PO Bох } 2298 \\
883 \text { MEDFR SНОРG }\end{array}$ & $\begin{array}{l}\text { White City } \\
\text { MEFPODD }\end{array}$ & $\begin{array}{l}\text { Jackson } \\
\text { Iackson }\end{array}$ & $1 / 11 / 2018$ \\
\hline 883 MEDEOORD SHOPG CENTER & $\begin{array}{l}\text { MEFEORD } \\
\text { MEFEOPD }\end{array}$ & $\begin{array}{l}\text { JaCKSON } \\
\text { ICGSON }\end{array}$ & $\begin{array}{l}09 / 29 / 2008 \\
099292 / 208\end{array}$ \\
\hline 887 GLLMAN RD & $\begin{array}{l}\text { MEFEORO } \\
\text { MEDFRD }\end{array}$ & $\begin{array}{l}\text { JAASKON } \\
\text { JACSON }\end{array}$ & $\begin{array}{l}0992 / 2 / 2008 \\
09 / 292 / 2008\end{array}$ \\
\hline 2311 Old Military Road & Central Point & Jackson & $\begin{array}{l}1 \\
1 / 11 / 2018\end{array}$ \\
\hline 313 Rene Drive & $\begin{array}{l}\text { Shaty cove } \\
\text { Shat }\end{array}$ & Jackson & $1 / 11 / 2018$ \\
\hline 893 BEATTY ST & MEEFORD & JACKSON & 09/29/2008 \\
\hline 901 MASON WY & MEFFORD & JACKSON & $09 / 29 / 2008$ \\
\hline 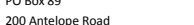 & $\begin{array}{l}\text { Jacksonnlile } \\
\text { White city }\end{array}$ & $\begin{array}{l}\text { Jackson } \\
\text { Jackson }\end{array}$ & $\begin{array}{l}1 / 11 / 2018 \\
1 / 1 / 2018\end{array}$ \\
\hline g01 MASON WAY & $\begin{array}{l}\text { Mntiectiry } \\
\text { Mefford }\end{array}$ & $\begin{array}{l}\text { Jackson } \\
\text { Jackson }\end{array}$ & $\begin{array}{l}1 / 1112018 \\
10 / 31 / 2018\end{array}$ \\
\hline 901 Mason WAY & Medford & JACKSON & $\begin{array}{l}10 / 31 / 2018 \\
128\end{array}$ \\
\hline 901 MASON WAY & Medford & JACKSON & $11 / 1 / 2018$ \\
\hline 907 N CENTRAL AVE & MEDFORD & JACKSON & $09 / 29 / 2008$ \\
\hline $\begin{array}{l}11526 \text { Camp Paker Road } \\
907 \text { SOAKDAE AVE }\end{array}$ & $\begin{array}{l}\text { Phoneni } \\
\text { Mentrord }\end{array}$ & $\begin{array}{l}\text { Jackson } \\
\text { Iacsson }\end{array}$ & 1/11/2018 \\
\hline $\begin{array}{l}9075 \text { OARDAALE AVE } \\
8087 \text { Blackwell Road }\end{array}$ & $\begin{array}{l}\text { Meadtord } \\
\text { Central Point }\end{array}$ & $\begin{array}{l}\text { JaAskson } \\
\text { Jackson }\end{array}$ & $\begin{array}{l}10 / 31 / 2018 \\
1 / 1 / 2018\end{array}$ \\
\hline P० B०х 319 & $\begin{array}{l}\text { Talent } \\
\text { Tomint }\end{array}$ & Jackson & $\begin{array}{l}1 / 1 / 12018 \\
1 / 1 / 2018\end{array}$ \\
\hline $910 \mathrm{~N} \mathrm{PHOENIX} \mathrm{RD}$ & MEOFFRD & JACKSON & $\begin{array}{l}09 / 29 / 2008 \\
0\end{array}$ \\
\hline РО Вох 3790 & Central Point & Jackson & $1 / 11 / 2018$ \\
\hline 910 N. PHOENIX ROAD & Medtord & JaCKSON & 11/1/2018 \\
\hline $\begin{array}{l}914 \text { QUUEN ANNE AVE } \\
\text { PO }\end{array}$ & $\begin{array}{l}\text { Mefford } \\
\text { Meresfield }\end{array}$ & JACKSON & $10 / 13 / 2018$ \\
\hline 2916 Orchard Heights NW & $\begin{array}{l}\text { Bakerstield } \\
\text { Salem }\end{array}$ & $\begin{array}{l}\text { Jacsson } \\
\text { Jackson }\end{array}$ & $\begin{array}{l}1 / 1112018 \\
1 / 1 / 2018\end{array}$ \\
\hline & & $\begin{array}{l}\text { Jackson } \\
\text { Jackon }\end{array}$ & $\begin{array}{l}1 / 11 / 2018 \\
1 / 112018\end{array}$ \\
\hline Po Box 3085 & Central Point & $\begin{array}{l}\text { Jackson } \\
\text { Jackson }\end{array}$ & $\begin{array}{l}1 / 1 / 12018 \\
1 / 1 / 2018\end{array}$ \\
\hline PO Box 3009 & $\begin{array}{l}\text { Assland } \\
\text { Ashand }\end{array}$ & Jackson & $\begin{array}{l}1 / 1 / 2018 \\
1 / 172018\end{array}$ \\
\hline 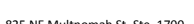 & & Jackson & 1/11/2018 \\
\hline 825 NE Multromah St. Ste. & Portland & Jackson & 1/11/2018 \\
\hline 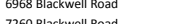 & Central Point & Jackson & 1/1/1/2018 \\
\hline $\begin{array}{l}7260 \text { Blackwwell Road } \\
313 \text { Rene Drive }\end{array}$ & $\begin{array}{l}\text { Centar Point } \\
\text { Sente }\end{array}$ & Jackson & 1/11/2018 \\
\hline 313 Rene Orive & Shady cove & $\begin{array}{l}\text { Jacsono } \\
\text { Jackson }\end{array}$ & $\begin{array}{l}1 / 1 / 12018 \\
1 / 1 / 2018\end{array}$ \\
\hline 2270 Rogue River Drive & Eagle Point & & 1/11/2018 \\
\hline 313 Rene Drive & & Jackson & 1/11/2018 \\
\hline 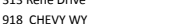 & MEFORD & $\begin{array}{l}\text { Jackson } \\
\text { JacksoN }\end{array}$ & 1/11/2018 \\
\hline wave & Central Point & $\begin{array}{l}\text { Jackson } \\
\text { Jackson }\end{array}$ & $\begin{array}{l}09 / 2 / 2 / 2018 \\
1 / 1 / 2018\end{array}$ \\
\hline 920 N PHOENIX RD & Medtord & JACKSON & $\begin{array}{l}11 / 1 / 201 / 2018 \\
10 / 218\end{array}$ \\
\hline 920 S GRAPE ST & MEOFORD & JaCKSON & 09/29/2008 \\
\hline 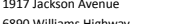 & La Grande & Jackson & $1 / 11 / 2018$ \\
\hline 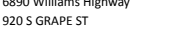 & $\begin{array}{l}\text { Grantin pass } \\
\text { Meeford }\end{array}$ & $\begin{array}{l}\text { Jackson } \\
\text { Jackson }\end{array}$ & $\begin{array}{l}1 / 1 / 12018 \\
10 / 31 / 2018\end{array}$ \\
\hline & Shady Cove & Jackson & \\
\hline 925 S GRAPE ST & Metford & JACKSON & $\begin{array}{l}10 / 11 / 2018 \\
1018\end{array}$ \\
\hline & & Jackson & 1/11/2018 \\
\hline & $\begin{array}{l}\text { Shaycover } \\
\text { Mefford }\end{array}$ & $\begin{array}{l}\text { Jackson } \\
\text { Jacsson }\end{array}$ & 1/11/2018 \\
\hline & & & 10/31/2018 \\
\hline 925 S GRAPE ST & MEFFORD & $\begin{array}{l}\text { JackSSON } \\
\text { JACSON }\end{array}$ & $\begin{array}{l}1 / 1 / 12018 \\
09 / 29 / 2008\end{array}$ \\
\hline ne Drive & Shady cove & & $1 / 1 / 1 / 2018$ \\
\hline GRAPE ST & MEFORD & JacksoN & \\
\hline 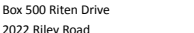 & $\begin{array}{l}\text { Assland } \\
\text { Eale point }\end{array}$ & $\begin{array}{l}\text { Jackson } \\
\text { lackson }\end{array}$ & $\begin{array}{l}1 / 11 / 2018 \\
1 / 11120218\end{array}$ \\
\hline
\end{tabular}

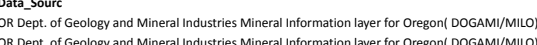

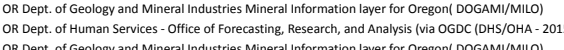

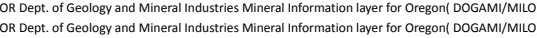
of Geology and Mineral Industries Mineral

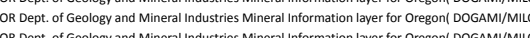

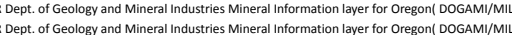

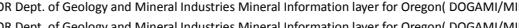

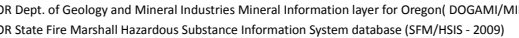

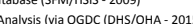
OR Dept. of Geelogy and Mineral Industries Mineral Information laver for oregonn (DOGAMI/MLOO OR Dept. of Geology and Mineral Industries Mineral I hformation layer for Oregono( DOGAMIMIMLO

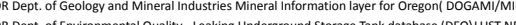

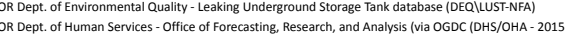

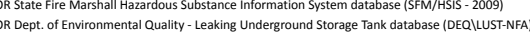

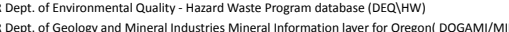
OR Dept. of Geelogy and Mineral Industries Mineral Information layer for Oregon(DOGAMM/MLO OR S State fire Marshall Hazardous Substance Information System database (SFMM/HSIS-2009]

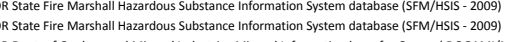

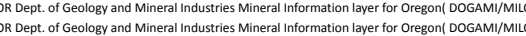

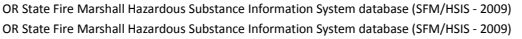
OR Dept. of Geology and Mineral Industries Mineral linformation layer for Oregon( DOGAMI/MILO

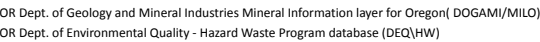

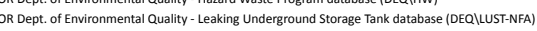

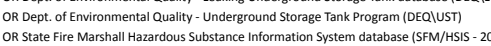

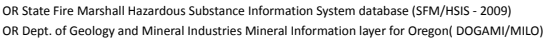

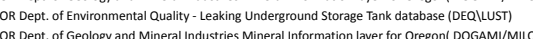

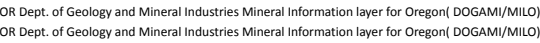

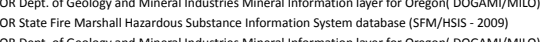

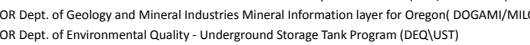

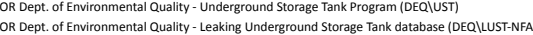

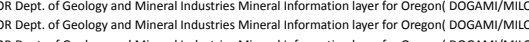
(1)

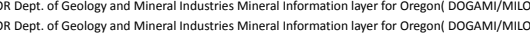

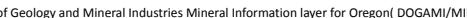

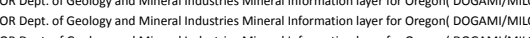

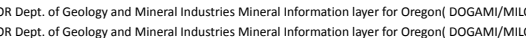
(1) OR Dept. of Geology and Mineral Industries Mineral Information layer for Oregenon DOGAMI/MLILO

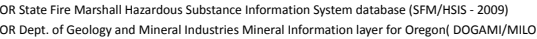
OR Dept. of Environmental Quality - Hazard Waste Program database (DEO) HW)

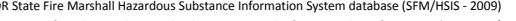

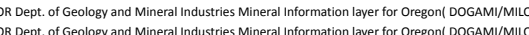
OR Dept. of Environmental Quality - Leaking Underground Storage Tank databse (IEQ) LIUST) OR Dept. of Geology and Mineral industries Mineral Information layer for Oregon( DOGAM/ML

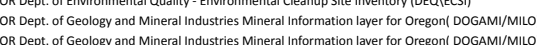
OR Dept. of Gelology and Mineral Industries Mineral Information lave for Oregon DOGAMIMIMLO

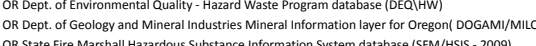

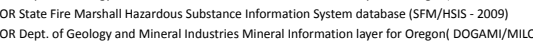
OR Dept. of Geology and Mineral Industries Mineral hnformation layer for Oregon (DOGAM/MiL OR Dept. of Geology and Mineral Industries Mineral Information layer for Oregon( DOGAMM/MILO

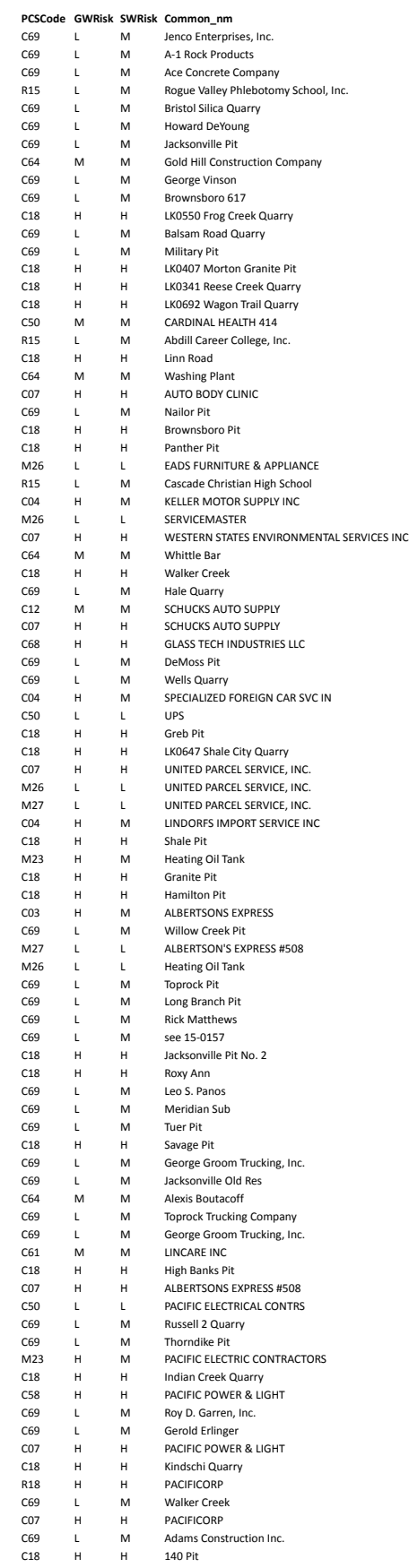

PCSType

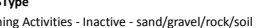

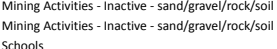

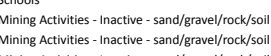
Mining Activities - Inactive - Sand/Fravel/rock/soil

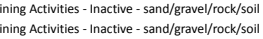
Mining Attivities- - Inactive - sand/gravel/rock/soil Ming Activities - Active - sand/gravel/rock/soil

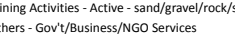

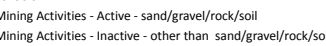
Minining Activitites - Inactive- -sand/gravel/rock/soil

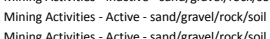

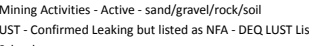

Luto- Repair Shops
SST - Confirmed Leaking but listed as NFA- DEQ L UST Lis

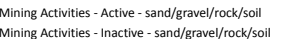
Mining Activities - Inactive- Sand/fravel/roch/
Eurniture/ Lumber/Parts stores

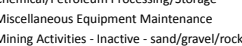

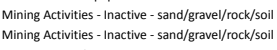
Auto- Repair Shops
Others- Govt//Usiness/NGo Services

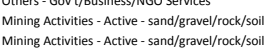

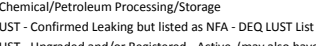
UST- Upgraded andor Registered - Active (may als
Auto UST - Confirmed Leaking Tanks - DEQ LUST List Mining Atctivities - Active- sand/gravel/rock/sol Mining Activities - Inactive - sand/fravel/rock/soil
UST - Upgraded and/or Registered - Active (may als Mining Activities- Inactive- sand//ravel/rock/soil

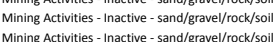

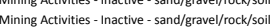
Mining Activities-Active- Sand/grave//rock/soil Mininin Ativities - Ative- - sand/fravel/rock/soil

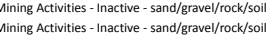

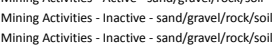
Mining Activities - Inactive - sand/Fravel/rockssoil Mining Activities - Inactive - sand/gravel/rock/soil

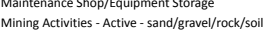

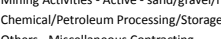

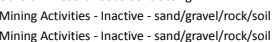
UST - Confirmed Leaking Tanks - DEQ WUST List

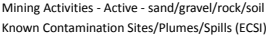

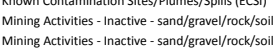

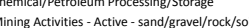
Utility Stations/Powerplants - Maintenance//ransformer storase

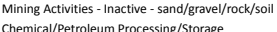

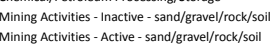




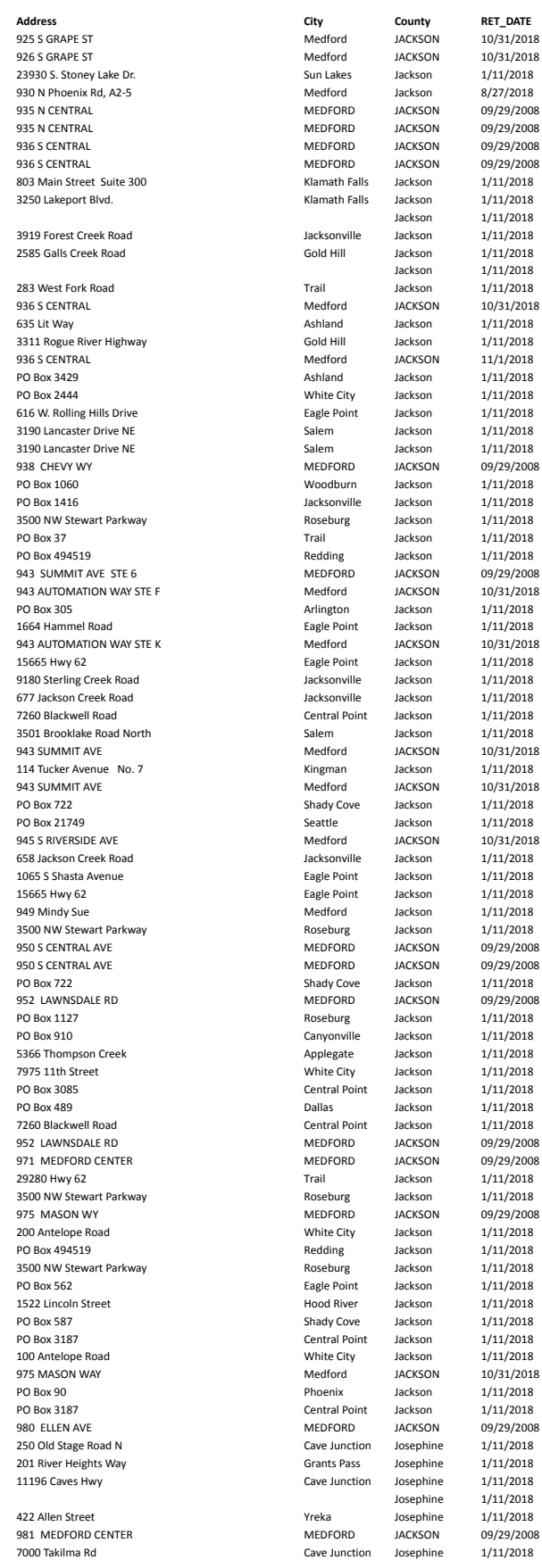

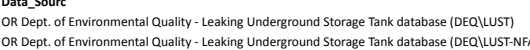

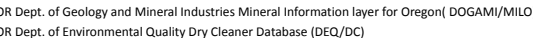

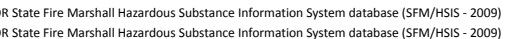

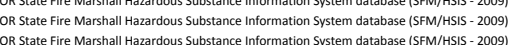
OR Dept. of Geology and Mineral Industries Mineral Information layer for Oregonn( DOGAM/MMLO RR Dept. of Geology and Mir OR Dept. of Geology and Mineral Industries Mineral Information layer for Oregon( DDGAMI/MILO

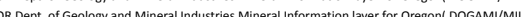

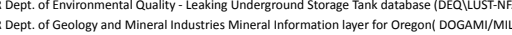
for regenon DOGAMM/MLIL

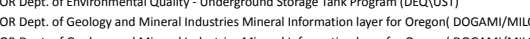

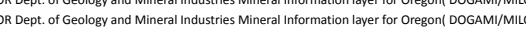

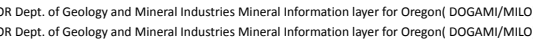

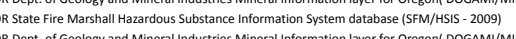
OR Dept. of Geology and Mineral Industries Mineral Information laye f for regeonn( DDGAMI/MILO

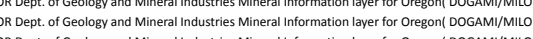
OR Dept. of Geelogy and Mineral ndustries Mineral Information layer for Oregon( DOGAM/MIL

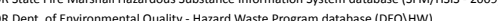

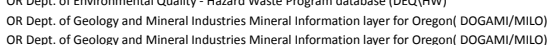
OR Dept. of Geology and Mineral Industries Mineral Information layer for Oregon(DOGAMIMILO

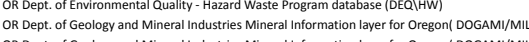
OR Dept. of Geology and Mineral Industries Mineral Information layer for Oregonn DOGAM/MLILO

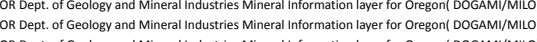

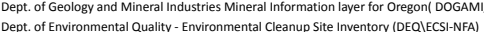
OR Dept. of Geology and Mineral Industries Mineral Information laver for Oregon( DOGAMIMIMLOF) OR Dept. of Geology and Mineral Industries Mineral Information layer for Oregon( DOGAM/MILO

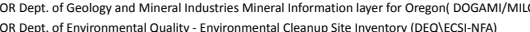

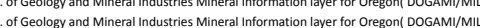
R Rept. of Geology and Mineral Industries Mineral Information layer for Oregonn (DOGAM//MLO

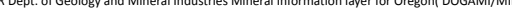

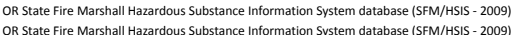
OR Dept. of Geology and Mineral Industries Mineral Intrormation laver for oregonon(DOGAMM/MILO

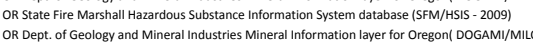

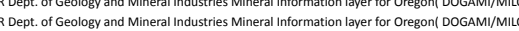
OR Dept. of Geology and Mineral Industries Mineral Information layer for Oregonn(DOGAMI/MLL

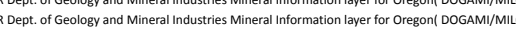

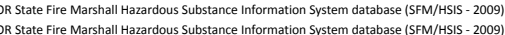

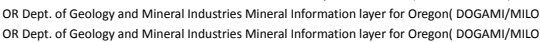
OR State fire Marshall Hazardous Substance Information System databasese (SFM/HISI- 2009)

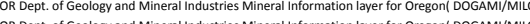

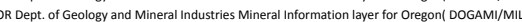

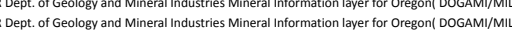
OR Dept. of Geology and Mineral Industries Mineral Information laver for Oregonn DOGAMM/MLIO

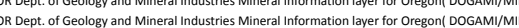
OR State Fire Marshall Hazarddous Substance Information System database (SFM/HSIS - 2009)

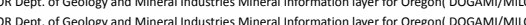
OR Dept. of Geelogy and Mineral Industries Mineral Information layer for Oregon( DDGAMI/MILO

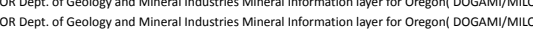

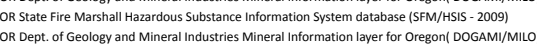

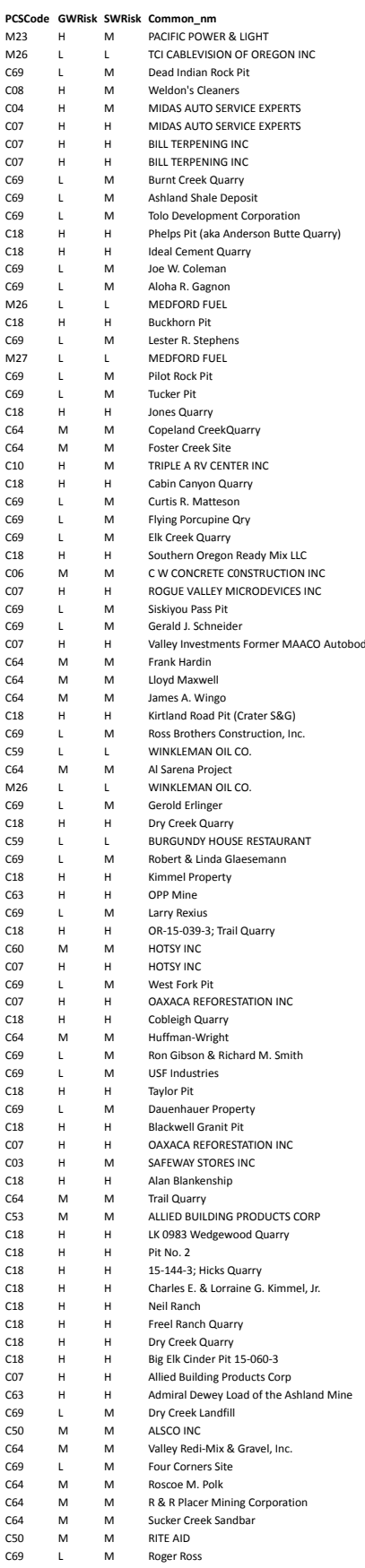

PCSType
UST-Cont

Cofirmed Leaking Tanks - DEQ LusT List 政 Dry Cleaners
Auto - Repair Shoops

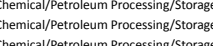

Mining Activities - Inactive - sand//ravel/rock/sol
Mining Activities - Inactive - sand/gravel/rock/sol Mining Activities - Active - sand/gravel/rock/sol

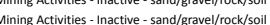

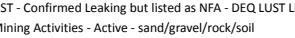

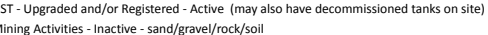
Mning Activities - Inactive - sand/gravel/rock/)

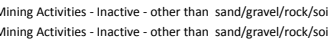

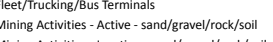

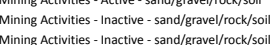

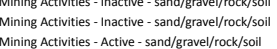

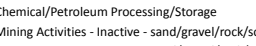
Mining Activities - Inactive- sand/gravel/rockl/sol Mining A Ativitites - Inactive - other thanan sand/fravel/rock/sol

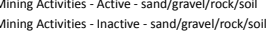

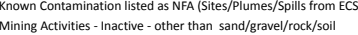

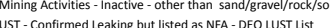
Mining Activities - Inactive- sand//ravel/rock/soil
Mining Activities - Active - sand/gravel/rock/soill

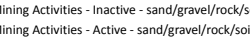
Ming Activites - Active - other than sand/frave/rock/soil Mining Atcivities - Active- sand//ravel/rock/soil Maintenance Shop/Equipment Storage - Not Transportation Related

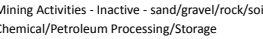
Chemical/Petroleum Processing/Storage Minin A Ativities- - Inactive- other than sand/fravel/rock/sol

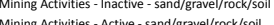

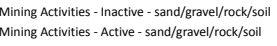
Minino Activities - Activive -sand/fravel/rock/soil

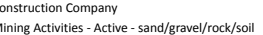
Mining Activities - Active- sand/gravel/rock/s/so

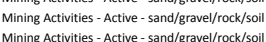

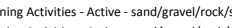
Mining Activities - Active- sand/Fravel/rock/soil

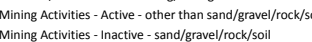
other- Clearing and Supply Services

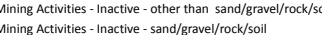
Mining Atcivities - Inactive - other than sand/fravel/rocklsoil

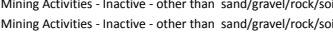
Other- General Merchandise/Retail Store
Mining Activities -Inactive - sand/grave//rock/sos 


\begin{tabular}{|c|c|c|}
\hline Address & city & county \\
\hline $\begin{array}{l}\text { Po Box } 147 \\
\text { Po Box } 1170\end{array}$ & Paisley & Josephine \\
\hline 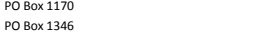 & $\begin{array}{l}\text { Cresenent tity } \\
\text { Grants Pass }\end{array}$ & $\begin{array}{l}\text { Josspopine } \\
\text { Josehine }\end{array}$ \\
\hline 1730 NE Burris & Corvallis & Josephine \\
\hline $\begin{array}{l}\text { Po Po } 20275 \\
\text { Po Box } 488\end{array}$ & $\begin{array}{l}\text { Mound } \\
\text { Coeur d'Alene }\end{array}$ & $\begin{array}{l}\text { Jossphine } \\
\text { osesehine }\end{array}$ \\
\hline 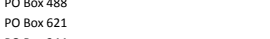 & $\begin{array}{l}\text { Coeur daAnene } \\
\text { Cave Junction }\end{array}$ & $\begin{array}{l}\text { Josseppine } \\
\text { Josphine }\end{array}$ \\
\hline PO Box 344 & Cave Junction & Josephine \\
\hline $\begin{array}{l}5938 \text { B HOlaland Loop Road } \\
981 \text { MEDFOR CTR }\end{array}$ & 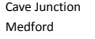 & $\begin{array}{l}\text { Josephine } \\
\text { Jacrson }\end{array}$ \\
\hline PO Box 488 & Coeur d'Alene & Josephine \\
\hline $\begin{array}{l}\text { 681-1/2 Caves Highway } \\
\text { po oox } 1514\end{array}$ & $\begin{array}{l}\text { Cave Junction } \\
\text { Cave unction }\end{array}$ & $\begin{array}{l}\text { Jossphine } \\
\text { ossenhine }\end{array}$ \\
\hline 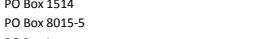 & $\begin{array}{l}\text { Cave Junction } \\
\text { Blaine }\end{array}$ & $\begin{array}{l}\text { Josspopine } \\
\text { Josephine }\end{array}$ \\
\hline PO Box 1 & Scappoose & $\begin{array}{l}\text { Jossphine } \\
\text { Jossehine }\end{array}$ \\
\hline 695 SE J St. & Grants Pass & Josephine \\
\hline 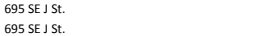 & $\begin{array}{l}\text { Grants Pass } \\
\text { Gants a pass }\end{array}$ & $\begin{array}{l}\text { Jossephine } \\
\text { Josephine }\end{array}$ \\
\hline 695 SE S St. & 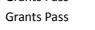 & Josephine \\
\hline PO BO BOX 273 & Cave Junction & Josepphine \\
\hline 63055 N. Highway 97, Bldg M & & Klamath \\
\hline $\begin{array}{l}\text { Off forck Dell Ravd } \\
\text { of Focky Dell Road }\end{array}$ & $\begin{array}{l}\text { Cave uuction } \\
\text { Cave Junction }\end{array}$ & $\begin{array}{l}\text { Jossephine } \\
\text { osesphine }\end{array}$ \\
\hline $\begin{array}{l}\text { Tft Rocky Dell Rada } \\
\text { Throughout OWPA }\end{array}$ & $\begin{array}{l}\text { Cave Junction } \\
\text { Cave Junction }\end{array}$ & $\begin{array}{l}\text { Joseppine } \\
\text { Josphine }\end{array}$ \\
\hline Throughout DWPA & Cave Junction & \\
\hline Off Caves Hwy & Cave Junction & Josephine \\
\hline Off Rocky Dell Road & Cave Junction & Josephine \\
\hline $\begin{array}{l}\text { Throughout OWPA } \\
\text { Next tointake bridepe }\end{array}$ & $\begin{array}{l}\text { Cave Junction } \\
\text { Cave unction }\end{array}$ & $\begin{array}{l}\text { Joseppine } \\
\text { Josenpine }\end{array}$ \\
\hline $\begin{array}{l}\text { Next to intake biridge) } \\
101 \text { Caves Highway }\end{array}$ & $\begin{array}{l}\text { Cave enction } \\
\text { Cave Junction }\end{array}$ & $\begin{array}{l}\text { Joseppine } \\
\text { Josphine }\end{array}$ \\
\hline $\begin{array}{l}101 \text { Coves Higinay } \\
101 \text { Caves Highway }\end{array}$ & $\begin{array}{l}\text { Cave Junction } \\
\text { Cave Junction }\end{array}$ & $\begin{array}{l}\text { Joseppone } \\
\text { Josphine }\end{array}$ \\
\hline Throughout DWPA & Cave Junction & $\begin{array}{l}\text { Josephine } \\
\text { Josphe }\end{array}$ \\
\hline South of intake & Cave Junction & Josephine \\
\hline South of intake & Cave Junction & Josepphine \\
\hline $\begin{array}{l}\text { Runs Through DWPA } \\
\text { Throughout DWPA }\end{array}$ & $\begin{array}{l}\text { Cave Junction } \\
\text { Cave enction }\end{array}$ & $\begin{array}{l}\text { Jossephine } \\
\text { Josenhine }\end{array}$ \\
\hline $\begin{array}{l}\text { Throughout DWPA } \\
\text { Redwood Highway }\end{array}$ & $\begin{array}{l}\text { Cave Junction } \\
\text { Cave Junction }\end{array}$ & $\begin{array}{l}\text { Jussephine } \\
\text { olosenhine }\end{array}$ \\
\hline Off Martin Road & $\begin{array}{l}\text { Cave Junction } \\
\text { Cave Junction }\end{array}$ & $\begin{array}{l}\text { Josephine } \\
\text { Josephe }\end{array}$ \\
\hline Throughout DWPA & Cave Junction & $\begin{array}{l}\text { Josephine } \\
\text { Josphes }\end{array}$ \\
\hline Off Bridgeview Road & Cave Junction & Josephine \\
\hline Directly across from intake & Rogue River & Jackson \\
\hline $\begin{array}{l}\text { Runs along } 1 \text {-5 and Rogegu River } \\
\text { Runs alang the Rogue River }\end{array}$ & Rogue River & Jackson \\
\hline $\begin{array}{l}\text { Runs alang the Rogue River } \\
\text { Runs along 1-5 and Rogue River }\end{array}$ & $\begin{array}{l}\text { Rogove iver } \\
\text { Rouve River }\end{array}$ & $\begin{array}{l}\text { Jaccsson } \\
\text { Jackson }\end{array}$ \\
\hline Within Rogue River/Gold Hill & 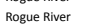 & Jackson \\
\hline Main and Deport Streets & 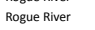 & Jackson \\
\hline Main and Depot Streets & Rogue River & Jackson \\
\hline $\begin{array}{l}\text { W. Main Street } \\
\text { West tain street }\end{array}$ & & Jackson \\
\hline $\begin{array}{l}\text { West Main Street } \\
\text { Broadway }\end{array}$ & $\begin{array}{l}\text { Rogge viver } \\
\text { Rogue River }\end{array}$ & $\begin{array}{l}\text { Jackson } \\
\text { Jackson }\end{array}$ \\
\hline Broadway & Rogue River & Jackson \\
\hline $\begin{array}{l}\text { off froadway Street } \\
\text { off Broadway Street }\end{array}$ & $\begin{array}{l}\text { Rogue River } \\
\text { Rover }\end{array}$ & Jackson \\
\hline $\begin{array}{l}\text { Off BBoadway Street } \\
\text { Off Classick Drive }\end{array}$ & $\begin{array}{l}\text { Rogove iver } \\
\text { Rouve River }\end{array}$ & $\begin{array}{l}\text { Jaccsson } \\
\text { Jackson }\end{array}$ \\
\hline Off Park Street & 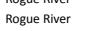 & $\begin{array}{l}\text { Jackson } \\
\text { Jackon }\end{array}$ \\
\hline Park Street & Rogue River & \\
\hline Park street & Rogue River & Jackson \\
\hline $\begin{array}{l}\text { Within Rogute River//Gold till } \\
\text { W. Min street }\end{array}$ & $\begin{array}{l}\text { Rogue River } \\
\text { Rogue }\end{array}$ & $\begin{array}{l}\text { Jackson } \\
\text { Jackson }\end{array}$ \\
\hline $\begin{array}{l}\text { W. Main istreet } \\
\text { Main Street }\end{array}$ & $\begin{array}{l}\text { Rogue iver } \\
\text { Rogue River }\end{array}$ & $\begin{array}{l}\text { Jackson } \\
\text { Jackson }\end{array}$ \\
\hline Off Gilmore Street & Rogue River & Jackson \\
\hline $\begin{array}{l}447 \text { 2nd dtreet } \\
447 \text { d nd Street }\end{array}$ & Rogue River & Jackson \\
\hline $\begin{array}{l}\text { 417 2nd Strteet } \\
\text { Northeast//outheast of intake }\end{array}$ & $\begin{array}{l}\text { Rogute viver } \\
\text { Rogue River }\end{array}$ & $\begin{array}{l}\text { Jackson } \\
\text { Jackson }\end{array}$ \\
\hline Just outside DWPA & 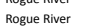 & Josephine \\
\hline Just outside DWPA & $\begin{array}{l}\text { Rogue River } \\
\text { Rover }\end{array}$ & Josephine \\
\hline Northeast of intake & Rogue River & Jackson \\
\hline East of intate & Rogue River & Jackson \\
\hline $\begin{array}{l}\text { tast of intake } \\
\text { 719 3rd Street }\end{array}$ & $\begin{array}{l}\text { Rogue River } \\
\text { Rogue River }\end{array}$ & Jackson \\
\hline Comero of teven Drive & $\begin{array}{l}\text { Rogute iner } \\
\text { Roune River }\end{array}$ & $\begin{array}{l}\text { Jackson } \\
\text { Jackson }\end{array}$ \\
\hline $\begin{array}{l}\text { N. River Road } \\
\text { lof }\end{array}$ & 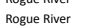 & 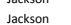 \\
\hline vghout OWPA & jue River & \\
\hline Eass of intake & ue River & Jackson \\
\hline 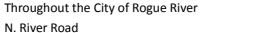 & $\begin{array}{l}\text { Rogue River } \\
\text { Rogiver }\end{array}$ & $\begin{array}{l}\text { Jackson } \\
\text { Jackson }\end{array}$ \\
\hline $\begin{array}{l}\text { Ne. River Road } \\
\text { East of intake }\end{array}$ & $\begin{array}{l}\text { gue River } \\
\text { uve River }\end{array}$ & $\begin{array}{l}\text { Jackson } \\
\text { Jackson }\end{array}$ \\
\hline East of intake & ue River & \\
\hline ds Creek Drive & ue River & Jackson \\
\hline Wit & ue River & Jackson \\
\hline \& creek Road- East of City Limits & ue River & Jackson \\
\hline Wards sreek Ro & ue River & Jackson \\
\hline 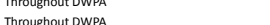 & & $\begin{array}{l}\text { Sactson } \\
\text { Jackson }\end{array}$ \\
\hline 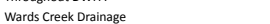 & Rogue River & Jackson \\
\hline
\end{tabular}

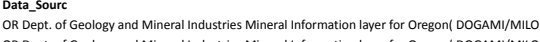

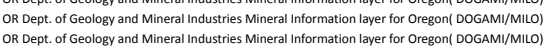
OR Dept. of Geology and Mineral Industries Mineral Information layer for Oregonn( DOGAM/MIMLO

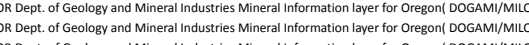

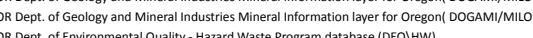

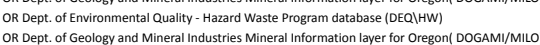

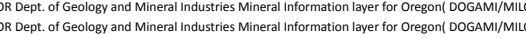
OR Dept. of Geology and Mineral Industries Mineral Information layer for Oregonn DOGAM/MLIL

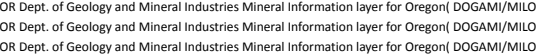

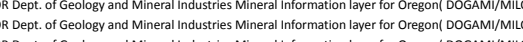

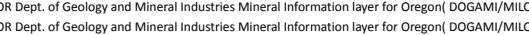

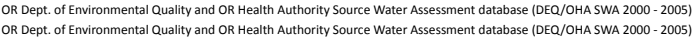

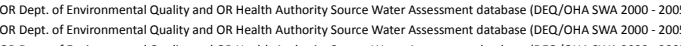

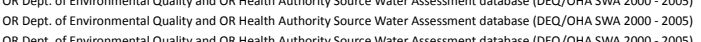

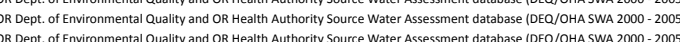

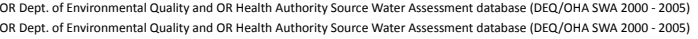

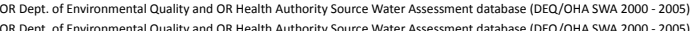

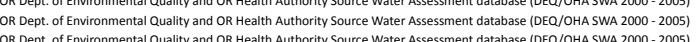

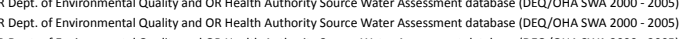

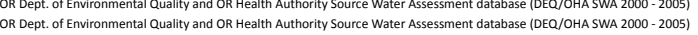

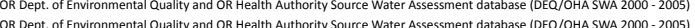

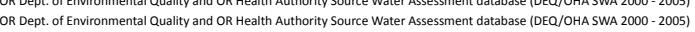
OR Dept. of Environmental Q vallity and OR Heath Authority Source Water Assessment database (DEE//OHA SWA 2000- 2005)

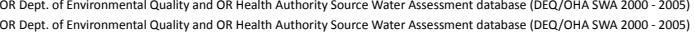
OR Dept. of Environmental Q Quality and OR Heath Authority Source Water ASSessment database (DEC/OHA SWA 2000- 2005)

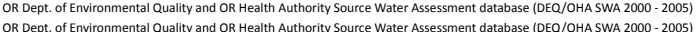

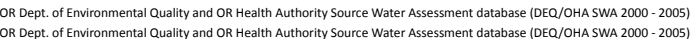

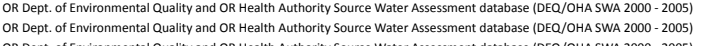

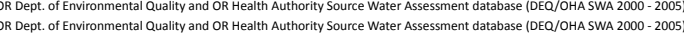

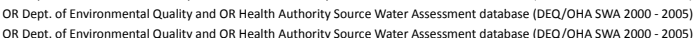

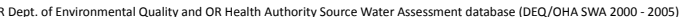

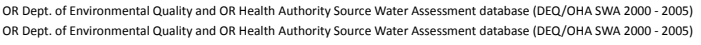

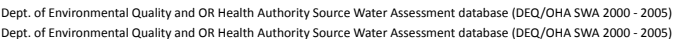

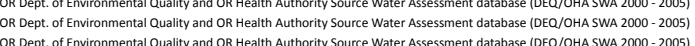

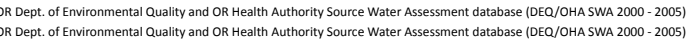
(1)

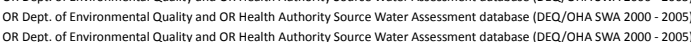

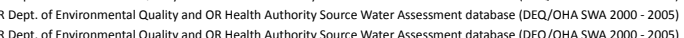

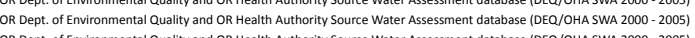
OR Dept. of Environmental Q Quality and OR Heath Authority Source Water A Assessment databasese (DEQ/OHA SWA 2000 - 2005) OR Dept. of Environmental Q Quality and OR Heath Authority Source Water Assessment database (DEQ/OHA SWA 2000- 2005)

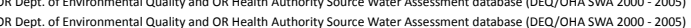
OR Dept. of Environmental Q Quality and OR Heath Authority Source Water Assessment database (DEQ/OHA SWA 2000- 2005) OR Dept. of Environmental Q Quality and OR Heath Authorty Source Water A Asesesment database (DEE/OHA SWA 2000 - 2005)

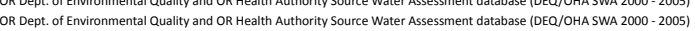
OR Dept. of Environmental Q Quality and OR Health Authority Source Water Assessment database (DEC/OHA SWA 2000- 2005)

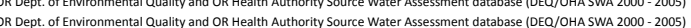
OR Dept. of Evironmental Quality and OR Health Authority Source Water Assessment database (DEQ//OHA SWA 2000- 2005)

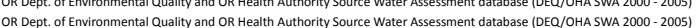

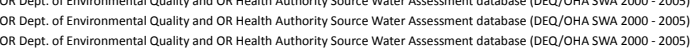

\begin{tabular}{|c|c|c|c|}
\hline PCSCOO & GWRi: & & \\
\hline c63 & $\stackrel{H}{M}$ & $\stackrel{H}{M}$ & $\begin{array}{l}\text { Cartor's Gravel Pit } \\
\text { Inter-american Nickel Inc. }\end{array}$ \\
\hline $\begin{array}{c}664 \\
664\end{array}$ & M & M & $\begin{array}{l}\text { Inter-manercanan ickel, nc. } \\
\text { Rich Gallagher }\end{array}$ \\
\hline & $\mathrm{M}$ & $\mathrm{M}$ & 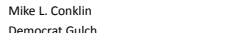 \\
\hline 664 & $M_{M}^{M}$ & $\begin{array}{l}M \\
M\end{array}$ & $\begin{array}{l}\text { Democrat Gulch } \\
\text { Bromutonn Site }\end{array}$ \\
\hline & & M & $\begin{array}{l}\text { Brombtown Site } \\
\text { Tracy Claims }\end{array}$ \\
\hline 664 & M & M & Esterly Placers Mining Company \\
\hline & M & M & A.E. Windsor \\
\hline co7 & ${ }_{n}^{H}$ & ${ }^{H}$ & PAVY LSS 1410 \\
\hline & m & m & $\begin{array}{l}\text { Slug Bar } \\
\text { Gary. Grifing }\end{array}$ \\
\hline C64 & M & M & $\begin{array}{l}\text { Gary E. G Gififin } \\
\text { Mark IIII }\end{array}$ \\
\hline & & M & National Pro Gold, Inc. \\
\hline 644 & M & M & Robert Webster \\
\hline 664 & m & M & Part of Tracy Claims \\
\hline 699 & L & $\mathrm{M}$ & I.V. Ranch Bar \\
\hline & & M & $\begin{array}{l}\text { LLttle Elm Ranch } \\
\text { Little Em Ranch Corp. }\end{array}$ \\
\hline C69 & & M & $\begin{array}{l}\text { Luttele Rim Ranch Corp. } \\
\text { Little Elm Ranch Pond \#2 }\end{array}$ \\
\hline & H & H & Avatar Mining \\
\hline & L & M & \\
\hline M61 & н & H & Abandoned Wells \\
\hline co1 & H & $\mathrm{M}$ & Lamb-Baaa-Dy Shop \\
\hline A11 & M & H & $\begin{array}{l}\text { Rural Homesteads } \\
\text { Clear Cuts }\end{array}$ \\
\hline & & $\mathrm{H}$ & Future Development Site \\
\hline c18 & H & $\mathrm{H}$ & Barlow Rock \\
\hline A05 & 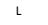 & t & Non-irrigated crops \\
\hline M18 & M & ${ }^{H}$ & Highway 199 \\
\hline col & H & M & Day oi Company \\
\hline AO7 & M & H & 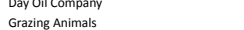 \\
\hline & & L & $\begin{array}{l}\text { Gazning Animails } \\
\text { Takima }\end{array}$ \\
\hline R16 & н & m $>2>>$ & Takilma \\
\hline M18 & m & н & Redwood Highway \\
\hline Mo9 & L & L & Rural Homes \\
\hline & L & ${ }_{\mathrm{H}}^{\mathrm{M}}$ & $\begin{array}{l}\text { Dome school } \\
\text { Jukn/Scrap Yard }\end{array}$ \\
\hline $\begin{array}{ccc}c 14 \\
c 63\end{array}$ & H & $\mathrm{H}$ & $\begin{array}{l}\text { Junk//Fcrap Vard } \\
\text { Mining Claims }\end{array}$ \\
\hline A04 & M & H & Bridgeview Winery \\
\hline R18 & H & ${ }_{H}^{H}$ & Substation \\
\hline M18 & m & ${ }_{H}^{H}$ & $\begin{array}{l}\text { Highway } 99 \text { (Rogue River Hwy) } \\
\text { lnterstate }\end{array}$ \\
\hline M19 & M & $\mathrm{H}$ & $\begin{array}{l}\text { Interestate } 5 \\
\text { Central Oregon Pacific Railiroad }\end{array}$ \\
\hline R17 & H & м & Sewer lines \\
\hline c04 & $\mathrm{H}$ & M & Rogue River Tow - Larry's Triangle Service \\
\hline & L & L & Rogue River Tow - Larry's Triangle Service \\
\hline 16 & $m$ & t & $\begin{array}{l}\text { Rogue River teath hilinic } \\
\text { Rogue River Heath hinic }\end{array}$ \\
\hline M24 & t & L & $\begin{array}{l}\text { Kogutu Rvertheath Linic } \\
\text { Rogue River co }\end{array}$ \\
\hline M55 & M & M & Rogue River co \\
\hline${ }_{12}$ & M $>2>>$ & M & Ace Hardware \\
\hline 孟24 & L & L & Ace Hardware \\
\hline 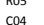 & $\begin{array}{c}M \\
H\end{array}$ & M & 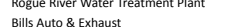 \\
\hline M55 & M & $\mathrm{M}$ & EMC Headware \\
\hline & M & L & Southern Oregon Dental Health and Hygien \\
\hline R09 & M & m & High Density Housing \\
\hline & H & M & Suds N Duds \\
\hline $\begin{array}{l}\text { c16 } \\
\text { c01 }\end{array}$ & ${ }_{M}^{M}$ & 要 & Animal Clinic \\
\hline cog & H & 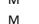 & $\begin{array}{l}\text { Kens Auto Body \& \&aint } \\
\text { City of Rogue River. }\end{array}$ \\
\hline M61 & H & H & City of Rogue River \\
\hline & M & H & Irrigation Canal \\
\hline & & & Louisiana Paccific \\
\hline & m & wh & Louisiana Pacticic \\
\hline & H & H & Rogue River Center \\
\hline mo1 & M & M & $\begin{array}{l}\text { Lousisana Pacticic Mili } \\
\text { Lousiana Pacific }\end{array}$ \\
\hline M32 & M & н & Whitestone Estates \\
\hline M32 & M $>2>>$ & H & Abovergound Storage Tanks \\
\hline C02 & m $>2>>$ & $m$ & Carwash \\
\hline A07 & $\mathrm{M}$ & $H$ & Grazing Animals \\
\hline Ro6 & H & , $>0$ & $\begin{array}{l}\text { Rogue River Fire Station } \\
\text { Sever }\end{array}$ \\
\hline c04 & H & m & $\begin{array}{l}\text { Sewer Lnines } \\
\text { Economy Auto }\end{array}$ \\
\hline & & & Rogue River Storage \\
\hline$c_{17}$ & н & H & $\begin{array}{l}\text { Trask Industries } \\
\text { S }\end{array}$ \\
\hline & $\mathrm{m}$ & $\mathrm{t}$ & Woodville Cemetery \\
\hline Ro9 & $\mathrm{m}$ & $m$ & High Density Housing Areas \\
\hline & & & $\begin{array}{l}\text { Potential Develolomentent } \\
\text { Pote }\end{array}$ \\
\hline M17 & L & H & $\begin{array}{l}\text { Transmisision Lines } \\
\text { Thes }\end{array}$ \\
\hline & & & Rural Homesteads \\
\hline A11 & M & $\mathrm{H}$ & Managed forestlat \\
\hline
\end{tabular}

Pcstype

Wing Actwites - hactive other han sud

hactive - other than sand//grave//rock/sol

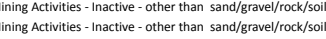

Ming Activities - Inactive - other than sand//Frave//rock/sol

Chemical/Petroleum Processing/s Storogege

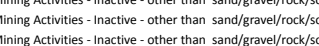

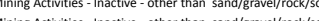

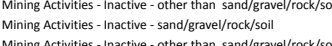

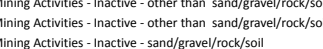

Wells - Abandoned
Auto - Body Shops

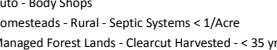

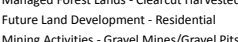

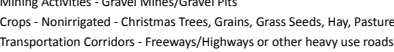

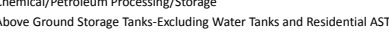
Gazing Animals $>5$ Large Animals or Equivalent/Acre

Homesteads- - Rural - Septic Systems $<1 /$ Acre
Septic systems - High Density $\rightarrow 1 /$ Acre

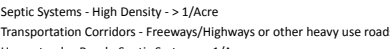

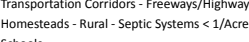

Junk/scrap/Salvage vards

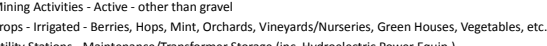

Utility Stations- Maintenance/Transformer Storgege (inc. Hydroelectric P Power LG

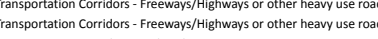

Transportation Corridors - Railroads

Leaking but listed as NFA- DEQ Wust Lis

Medicall tet offices
Medich

UST Decommissioned - Inactive
Unknown Operation

furniture/Lumber/Parts Stores
ust - Decommissioned - Inactive

Drinking Water Treatmen
Auto Repair Shops

Unknown Operation
Medicalvet offices

Housing-High Density $\rightarrow 1$ House/0.5 Acres

Medical/vet Offices

Muto- Body Shops
Electic/lectionic

Wectrica/l/lectronic
Werigation Canoned
Irigation Cana/Pond

hoveve Ground Storagere Tanks -Excluding W Water Tanks and Residential ASS

Construction/Demolition Area
Construction/Demolition Area

Swer Lines - Close Proximity to PWs

Auto- Repair shops
RV/Mini storage

Metal Pating/Finishing/Fabrication
Cemeterese- Pre-1945

Housing - High Density $>1$ House/.5.5 Acres
Septic systems - High Density $\rightarrow 1 /$ Ace

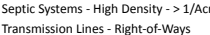

Transmisision Lines - Right-of-Ways
Homesteads - Rural
Managed 


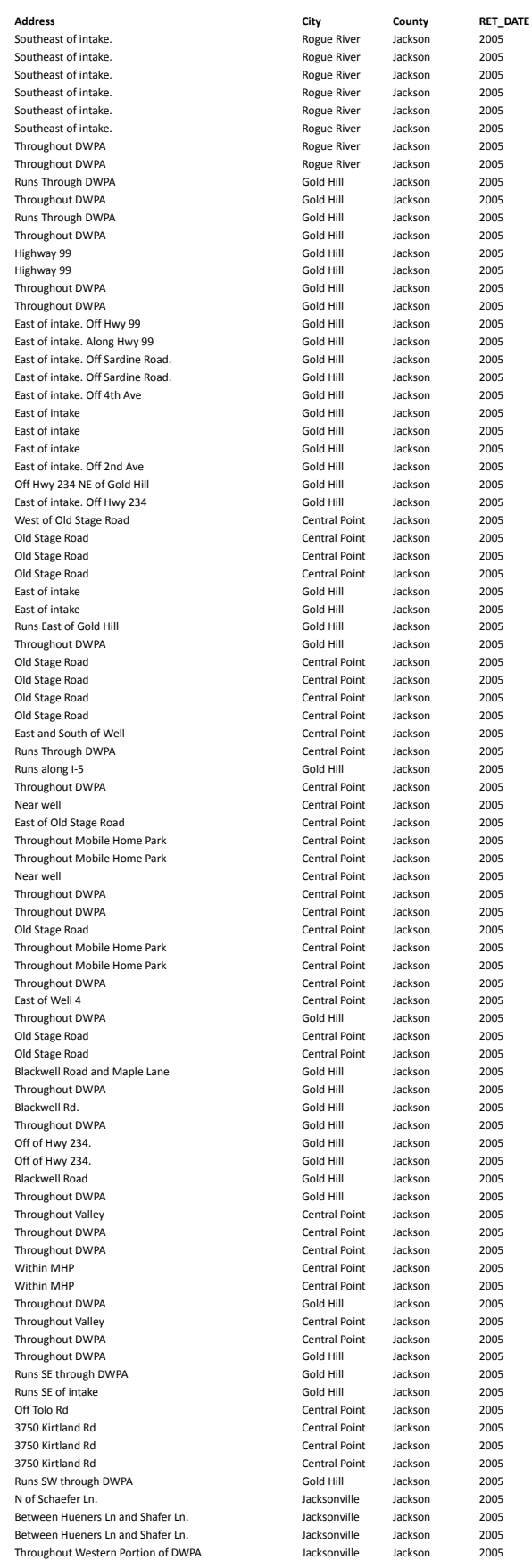

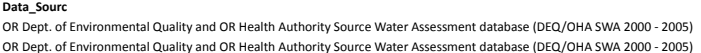

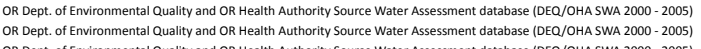

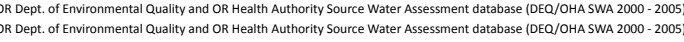

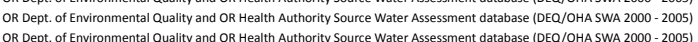

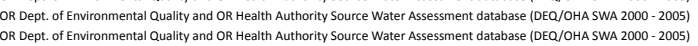

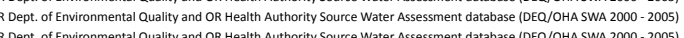

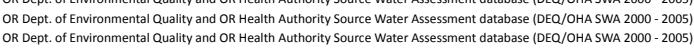

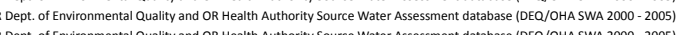

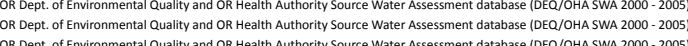

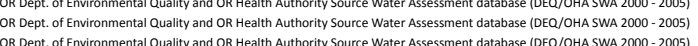

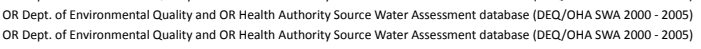

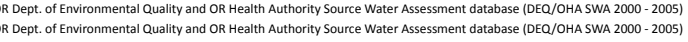
OR Dept. of Environmental Q ualitity and OR Heath Authority Source Water Assessment database (DEQ/OHA SWA 2000-2005)

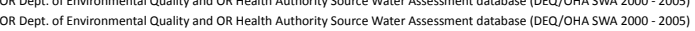
OR Dept. of Environmental Q Quality and OR Heath Authority Source Water AsSessment database (DEQ/OHA SWA 2000- 2005)

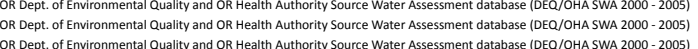
OR Dept. of Environmental Q Quality and OR Health Authority Source Water Assessment database (DEE/OHA SWA 2000- 2005)

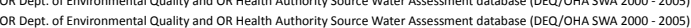
OR Dept. of Environmental Q Quality and OR Health Authority Source Water Assessment database (DEC/OHA SWA 2000- 2005)

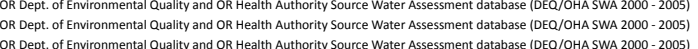
OR Dept. of Environmental Q Quality and OR Heath Authority Source Water A Asessment database (DEG/OHA SWA 2000- 2005)

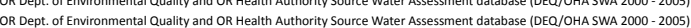
OR Dept. of Environmental Quality and OR Health Authority Source Water Assessment database (DEE/OHA SWA 2000- 2005)

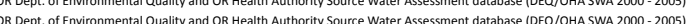
OR Dept. of Environmental Quality and OR Heath Authority Source Water Assessment database (DE//OHA SWA 2000 - 2005 OR Dept. of Environmental Q Quality and OR Heath Authority Source Water Assessment database (DEE//OHA SWA 2000 - 2005)

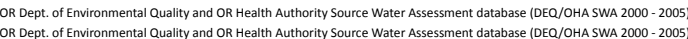

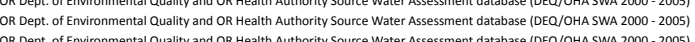
OR Dept. of Environmental Q Quality and OR Health Authority Source Water Assessment database (DEQ/OHA SWA 2000- 2005)

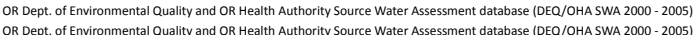
OR Dept. of Environmental Quality and OR Health Authority Source Water Assessment database (DEQ//OHA SWA 2000- 2005)

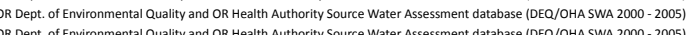

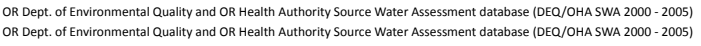

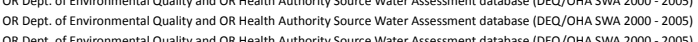

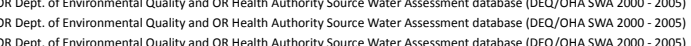

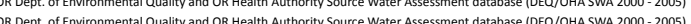
OR Dept. of Environmental Q aulity and OR Health Authority S Surce Water Assessment database (DEQ/OHA SWA 2000- 2005)

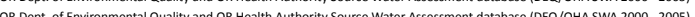
OR Dept. of Environmental Q Quality and OR Heath Authority Source Water Assessment database (DEC/OHA SWA 2000 - 2005) OR Dept. of Environmental Q Quality and OR Heath Authority Source Water A Assessment database (DEQ/OHA SWA 2000- 2005)

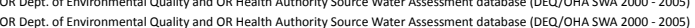
OR Dept. of Environmental Q Quality and OR Heath Authority Source Water Assessment database (DEQ/OHA SWA 2000- 2005) OR Dept. of Environmental Q uality ynd OR Heath Authority Source Water Assessment database (DEQ/JOHA SWA 2000- 2005)

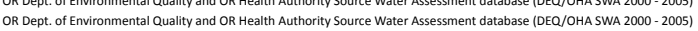

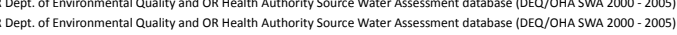

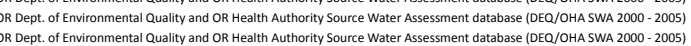
OR Dept. of Environmental Qualtry ynd OR Heath Authority Source Water Assessment database (DEQ/JHA SWA 2000- 2005)

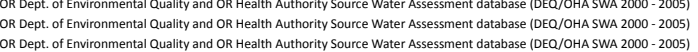

\begin{tabular}{|c|c|c|c|}
\hline Pcscode & & & $\begin{array}{l}\text { Common_nm } \\
\text { Valley of the Rogue State Park }\end{array}$ \\
\hline M24 & L & & $\begin{array}{l}\text { Valaye of the Roguve State } \\
\text { Valley of the Rogue State }\end{array}$ \\
\hline M31 & H & m & Valley of the Rogue State Park \\
\hline 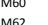 & ${ }_{H}^{H}$ & & $\begin{array}{l}\text { Valley of the Rogue State Park } \\
V\end{array}$ \\
\hline & M & 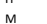 & $\begin{array}{l}\text { Valle of the Rogue e State Park Park } \\
\text { Valley of the Rogue State Park }\end{array}$ \\
\hline 1004 & M & H & Irrigated Crops \\
\hline A05 & L & L & $\begin{array}{l}\text { Non-Irigated Crops } \\
\text { Hiphwa } 99\end{array}$ \\
\hline Mo9 & L & L & $\begin{array}{l}\text { Highway } 99 \\
\text { Rural Homes }\end{array}$ \\
\hline M18 & м & H & Interstate 5 \\
\hline م31 & ${ }_{H}^{M}$ & $M_{M}^{M}$ & 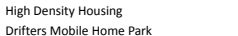 \\
\hline Rо9 & м & M & $\begin{array}{l}\text { Drifters Mobile Home Park } \\
\text { Denter }\end{array}$ \\
\hline A04 & M & H & Irrigated crops \\
\hline A04 & 等 & H & Irrigated Crops \\
\hline 004 & M & H & $\begin{array}{l}\text { Avdanced A Agrgegate Inc. } \\
\text { Del Rio Vineyard }\end{array}$ \\
\hline M24 & L & & 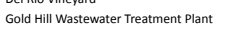 \\
\hline & M & & Gold Hill Wastewater r Treatment Plant \\
\hline & H & H & Ideal Basic Industries \\
\hline cos & H & & Gold Hill Texaco \\
\hline M24 & L & thes & $\begin{array}{ll}\text { Gold dill Texaco } \\
\text { Gold Hill Texaco }\end{array}$ \\
\hline $\mathrm{c} 17$ & H & H & Sweed Machinery \\
\hline & m & $\mathrm{M}$ & 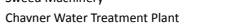 \\
\hline & м & $m$ & Gold Hill Water Treatment Plant \\
\hline A04 & m & H & Irrigated Crops \\
\hline$c_{03}^{03}$ & H & m & Dardanelle Service Station \\
\hline & L & & $\begin{array}{l}\text { Dardanelle Service Station } \\
\text { Dardanale erevice catrition }\end{array}$ \\
\hline 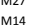 & t & 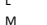 & $\begin{array}{l}\text { Dardanelle Service Station } \\
\text { Ropoue }\end{array}$ \\
\hline M54 & t & th & $\begin{array}{l}\text { Kogute hiver } \\
\text { Rogue River }\end{array}$ \\
\hline M56 & m & H & $\begin{array}{l}\text { Kogue River } \\
\text { Highway 234/Upper River Road }\end{array}$ \\
\hline mo9 & $\mathrm{t}$ & $\mathrm{t}$ & Rural Homestead \\
\hline M31 & $H$ & m & High Density Housing \\
\hline R99 & M & m & $\begin{array}{l}\text { High Density Housing } \\
\text { Conveyerd }\end{array}$ \\
\hline 124 & L & M & 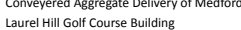 \\
\hline & m & m & $\begin{array}{l}\text { Laure Hill Golf Course Building } \\
\text { Golf Course }\end{array}$ \\
\hline M18 & m & H & $\begin{array}{l}\text { Sinterstate } 5 \\
\text { Inte }\end{array}$ \\
\hline M19 & m & H & Railroad \\
\hline no9 & L & L & Rural Homes \\
\hline $\begin{array}{l}\text { Ma61 } \\
0.4\end{array}$ & $\stackrel{H}{M}$ & H & $\begin{array}{l}\text { Abandoned Well } \\
\text { Arrigated d rops }\end{array}$ \\
\hline 管1 & H & $\mathrm{M}$ & $\begin{array}{l}\text { Irrigated Crops } \\
\text { High Density Housing }\end{array}$ \\
\hline Rоя & m & $\mathrm{m}$ & $\begin{array}{l}\text { High Density Housing } \\
\text { Hons }\end{array}$ \\
\hline & H & H & Abandoned Well \\
\hline мо9 & L & L & Rural Homes \\
\hline & L & L & \\
\hline M31 & M & M & $\begin{array}{l}\text { Grazing Animals } \\
\text { High Density Housing }\end{array}$ \\
\hline Rо9 & m & M & $\begin{array}{l}\text { High bensity yousing } \\
\text { High Density Housing }\end{array}$ \\
\hline м09 & 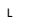 & L & Rural Homes \\
\hline A04 & M & H & $\begin{array}{l}\text { Irrigated Crops } \\
\text { Ires }\end{array}$ \\
\hline A05 & L & t & Non-Irrigated Crops \\
\hline M08 & H & H & $\begin{array}{l}\text { Home Machine Shop } \\
\text { Heripe }\end{array}$ \\
\hline A02 & $\mathrm{m}$ & $\mathrm{H}$ & $\begin{array}{l}\text { Fire Departrment } \\
\text { Gold cost Arena }\end{array}$ \\
\hline & & $\mathrm{i}$ & $\begin{array}{l}\text { Gold Coaast Arena } \\
\text { Non-Irigated Crops }\end{array}$ \\
\hline & $M$ & $\mathrm{M}$ & Leisure Pines Mobile Ranch \\
\hline mo9 & & L & Rural Homes \\
\hline 663 & H & H & Sylvanite Mine \\
\hline M024 & is $>0$ & $\mathrm{H}_{\mathrm{H}}$ & $\begin{array}{l}\text { Sylvantite Mine } \\
\text { Grazin Animals }\end{array}$ \\
\hline & & $\mathrm{H}$ & \\
\hline 104 & $\mathrm{~m}$ & H & 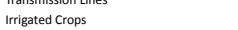 \\
\hline mo9 & t & L & Rural Homes \\
\hline Mog & t & L & Rural Homes \\
\hline & H & m & Nestled in Hills \\
\hline م09 & $\begin{array}{l}m \\
m\end{array}$ & M & $\begin{array}{l}\text { Nestled in } \text { Nills } \\
\text { Granina Animals }\end{array}$ \\
\hline A04 & m & $\mathrm{H}$ & 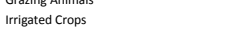 \\
\hline мо9 & & & Rural Homes \\
\hline A04 & $\begin{array}{l}m \\
M\end{array}$ & H & Irrigated Crops \\
\hline $\begin{array}{lll}\text { Mi8 } & \end{array}$ & & t & $\begin{array}{l}\text { Interstate } 5 \\
\text { Beacr creek }\end{array}$ \\
\hline $\mathrm{C}_{24} 4$ & H & H & $\begin{array}{l}\text { Bear Creek } \\
\text { Tolo Forest Products }\end{array}$ \\
\hline c18 & H & H & Rogue Aggregates \\
\hline M26 & & L & Roguve Aggregates \\
\hline & M & 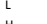 & Rogue Agreregates \\
\hline 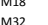 & n & 型 & $\begin{array}{l}\text { Highway 99/Blackwell Rd } \\
\text { Construction }\end{array}$ \\
\hline & $\mathrm{t}$ & t & Daisy Creek Subdivisision \\
\hline M24 & L & L & $\begin{array}{l}\text { Daisy Creek Subdivision } \\
\text { Hirith }\end{array}$ \\
\hline
\end{tabular}

Tistrpe

Large Capacity Septic Systems - Class V UIC (serves >

crops-Irigited- Berries, Hops, Mint, Orchards, Vineyards,

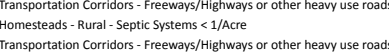
Transportation Corridors - Freeways/Highways
Housing - High Density $\rightarrow 1$ House/.5. Acres

Large Capacity Septic Systems - Class V UIC (serves 20)

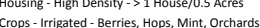
Vineyards/Nurseries, Green Houses, Vegetables, etc 政 Wastewater Treatment Plants/Collection Stations Metal Plating/tinshing//rabication UST - Decocominissioned - Inactive
UST - Upgraded and/or Registered - Active

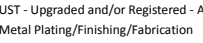
Metal Plating/finisthing//Fabricas
Drinking Water Treatment Plants

cops- Irrigated Berries, Hops, Mint orchers

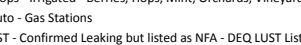
UST- Upgraded and/or Registered- - Active
River Recreation - Heary Use (inc. Camprogrounds River Recreation - Heavy Use (inc. Campgrounds)
Surface Water on $303 \mathrm{~d}$ list Iransportation Corridors - High Use Roads/Streets
Homesteads - Rural - Septic Systems $<1 /$ Acre

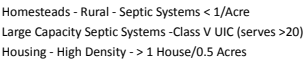

Fleet Trucking//us Terminals
UST- Decommisisioned-Inactive

Solf Courses
Transportation Corridors - Freeways/His Iransportation Corridors- Railroads
Homesteads - Rural - Septic Systems $<1 /$ Acre

Wells - Abandoned

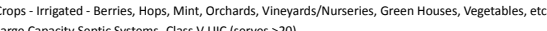

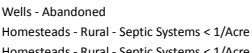
Grazing Animals s s L Large Animals or Equivalent/Acre
Large capacity Septic Systems - Class V ViC (serves 220$)$ fousing - High Density $\rightarrow 1$ House/ 0.5 Acres

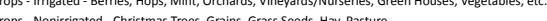
(a)

Fire Station

Soarding stables

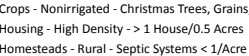

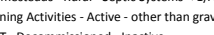

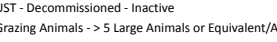

crops- -Irigiated- - Berries, Hops, Mint, Orchards, Vineyards/Nurseries, Green Houses, vegetables, etc

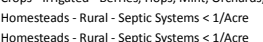

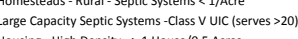

Cops- Irrigated - Berries, Hops, Mint, orchards, Vineyards/Wurseries Green Houses, Vegetables, etc

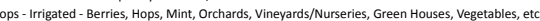
Tansportation Corridors - Freewaysitigh ways or other heavy use roadc

Mining Activities - Gravel Mines/Gravel Pits

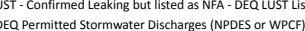

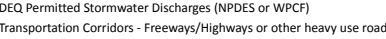

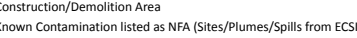

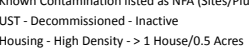




\begin{tabular}{|c|c|c|c|}
\hline $\begin{array}{l}\text { Address } \\
\text { Throuhhout DWPA }\end{array}$ & City & County & RET_DATE \\
\hline $\begin{array}{l}\text { Trrounhhuot DWPA } \\
\text { Runs through DWPA }\end{array}$ & $\begin{array}{l}\text { Jacksonvile } \\
\text { Jacksonille }\end{array}$ & $\begin{array}{l}\text { Jackson } \\
\text { Jackson }\end{array}$ & $\begin{array}{l}2005 \\
2005\end{array}$ \\
\hline $\begin{array}{l}\text { Carriage Ln } \\
\text { L }\end{array}$ & $\begin{array}{l}\text { Tacksonvilie } \\
\text { Jacksonvile }\end{array}$ & $\begin{array}{l}\text { Jacsson } \\
\text { Jackson }\end{array}$ & 2005 \\
\hline $\begin{array}{l}\text { Carriage Ln } \\
\text { Off Blackwell Road }\end{array}$ & & $\begin{array}{l}\text { Jackson } \\
\text { Jackson }\end{array}$ & $\begin{array}{l}2005 \\
2005\end{array}$ \\
\hline $\begin{array}{l}\text { Of Blackwell Raad } \\
\text { Off Blackwell Road }\end{array}$ & $\begin{array}{l}\text { Centrat Point } \\
\text { Central Point }\end{array}$ & $\begin{array}{l}\text { Jackson } \\
\text { Jackson }\end{array}$ & $\begin{array}{l}2005 \\
2005\end{array}$ \\
\hline Eastern Portion of DWPA & $\begin{array}{l}\text { Centrat Pont } \\
\text { Jacksonville }\end{array}$ & $\begin{array}{l}\text { Jackson } \\
\text { Jackson }\end{array}$ & ${ }_{2005}^{2005}$ \\
\hline $\begin{array}{l}\text { Sw of Well } \\
\text { Sor }\end{array}$ & Jacksonville & Jackson & 2005 \\
\hline 997 Medford Center & Medford & Jackson & 8/27/2018 \\
\hline 999 CREWS RD & MEFFRD & JaCKSON & $09 / 29 / 2008$ \\
\hline 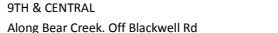 & $\begin{array}{l}\text { Mefford } \\
\text { Mefford }\end{array}$ & $\begin{array}{l}\text { Jackson } \\
\text { Jackson }\end{array}$ & $\begin{array}{l}10 / 31 / 2018 \\
2005\end{array}$ \\
\hline $\begin{array}{l}\text { Along Bear Creek. of Blacklwel Id } \\
\text { Runs along Hanley Rd. }\end{array}$ & $\begin{array}{l}\text { Medotord } \\
\text { Jacksonille }\end{array}$ & $\begin{array}{l}\text { Jacasson } \\
\text { Jackson }\end{array}$ & $\begin{array}{l}2005 \\
2005\end{array}$ \\
\hline $\begin{array}{l}\text { Kinns along Haniey ka. } \\
\text { Huy } 238\end{array}$ & $\begin{array}{l}\text { Sacksonnie } \\
\text { Jacksonvile }\end{array}$ & $\begin{array}{l}\text { Jackson } \\
\text { Jackson }\end{array}$ & 2005 \\
\hline Hwy 238 & & & 2005 \\
\hline South and East of wells & Jacksonville & Jackson & 2005 \\
\hline $\begin{array}{ll}H w y \\
H\end{array}$ & Jacksonville & Jackson & 2005 \\
\hline $\begin{array}{l}\text { Hww } 238 \\
\text { Hanley Rd. }\end{array}$ & $\begin{array}{l}\text { Jacksonnilile } \\
\text { alcksonvile }\end{array}$ & $\begin{array}{l}\text { Jackson } \\
\text { Jackson }\end{array}$ & $\begin{array}{l}2005 \\
2005\end{array}$ \\
\hline Throughout DWPA & $\begin{array}{l}\text { Jacksonnilue } \\
\text { Jacksonvile }\end{array}$ & $\begin{array}{l}\text { Jackson } \\
\text { Jackson }\end{array}$ & $\begin{array}{l}2005 \\
2005\end{array}$ \\
\hline Knowles Rd. & $\begin{array}{l}\text { Tacksonniue } \\
\text { Jacksonvile }\end{array}$ & $\begin{array}{l}\text { Jackson } \\
\text { Jackson }\end{array}$ & 2005 \\
\hline Hanley Rd. & Jacksonville & Jackson & 2005 \\
\hline Hwy 238 & Jacksonvile & Jackson & 2005 \\
\hline $\begin{array}{l}\text { Throughout OWWAPA } \\
\text { The }\end{array}$ & $\begin{array}{l}\text { Jacksonville } \\
\text { acchowele }\end{array}$ & Jackson & 2005 \\
\hline $\begin{array}{l}\text { Throuuhhout DWPA } \\
\text { East of Well }\end{array}$ & $\begin{array}{l}\text { Jacksonsilie } \\
\text { Jacksonvile }\end{array}$ & $\begin{array}{l}\text { Jackson } \\
\text { Jackson }\end{array}$ & $\begin{array}{l}2005 \\
2005\end{array}$ \\
\hline & 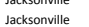 & $\begin{array}{l}\text { Jackson } \\
\text { Jackson }\end{array}$ & $\begin{array}{l}2005 \\
2005\end{array}$ \\
\hline North of Well & $\begin{array}{l}\text { Jacksonville } \\
\text { Jastes }\end{array}$ & $\begin{array}{l}\text { Jackson } \\
\text { Jackson }\end{array}$ & 2005 \\
\hline North of well & Sams valley & Jackson & 2005 \\
\hline North of well & Sams valley & Jackson & 2005 \\
\hline South of well outside the DWPA & Sams valley & Jackson & 2005 \\
\hline $\begin{array}{l}\text { North o well } \\
\text { South of well }\end{array}$ & $\begin{array}{l}\text { Sams saley } \\
\text { Sams valley }\end{array}$ & $\begin{array}{l}\text { Jackson } \\
\text { Jackson }\end{array}$ & $\begin{array}{l}2005 \\
2005\end{array}$ \\
\hline South of well outside the DWPA & $\begin{array}{l}\text { Sams valey } \\
\text { Sams valey }\end{array}$ & $\begin{array}{l}\text { Jacksson } \\
\text { Jackson }\end{array}$ & $\begin{array}{l}2005 \\
2005\end{array}$ \\
\hline Throughout the DWPA & sams valley & $\begin{array}{l}\text { Jackson } \\
\text { Jackson }\end{array}$ & 2005 \\
\hline Southeast of well & Sams valley & Jackson & 2005 \\
\hline West of Well & Jacksonvile & Jackson & 2005 \\
\hline 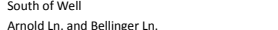 & Jacksonville & Jackson & 2005 \\
\hline 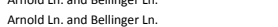 & $\begin{array}{l}\text { Jacksonswlile } \\
\text { Jacksonvile }\end{array}$ & $\begin{array}{l}\text { Jacksson } \\
\text { Jackson }\end{array}$ & $\begin{array}{l}2005 \\
2005\end{array}$ \\
\hline Arrold Ln & $\begin{array}{l}\text { sactsonite } \\
\text { Jacksonvile }\end{array}$ & $\begin{array}{l}\text { Jackson } \\
\text { Jackson }\end{array}$ & $\begin{array}{l}2005 \\
2005\end{array}$ \\
\hline Arnold tn. and Bellinger Ln. & $\begin{array}{l}\text { Jacksonville } \\
\text { J }\end{array}$ & Jackson & 2005 \\
\hline Along Bear Creek. off Blackwell Rd & Medford & Jackson & \\
\hline 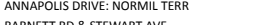 & Medtord & & 10/31/2018 \\
\hline $\begin{array}{l}\text { BAANEET R R \& STTWART AVE } \\
\text { BEAR C REEK PARK }\end{array}$ & $\begin{array}{l}\text { Medford } \\
\text { Medford }\end{array}$ & JACSSON & $\begin{array}{l}10 / 31 / 2018 \\
101312018\end{array}$ \\
\hline 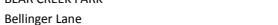 & $\begin{array}{l}\text { Mectorer } \\
\text { Mettord }\end{array}$ & $\begin{array}{l}\text { Jackson } \\
\text { Jackson }\end{array}$ & $\begin{array}{l}10005 \\
2001 / 2018\end{array}$ \\
\hline Bellinger Lane & Medtord & Jackson & 2005 \\
\hline Bellinger Lane & Medtord & Jackson & 2005 \\
\hline BensLn & Medford & Jackson & 2005 \\
\hline North of well, between rood a & $\begin{array}{l}\text { Jacksonville } \\
\text { Jacksewile. }\end{array}$ & Jackson & 2005 \\
\hline $\begin{array}{l}\text { Suthth Stage Rd. } \\
\text { North of S outh Stage Rd. }\end{array}$ & & Jackson & $\begin{array}{l}2005 \\
2005\end{array}$ \\
\hline $\begin{array}{l}\text { North of Jouth htage Ka. } \\
\text { South Stage Rd. }\end{array}$ & $\begin{array}{l}\text { Jacksonnue } \\
\text { Jacksonvile }\end{array}$ & $\begin{array}{l}\text { Jacksson } \\
\text { Jackson }\end{array}$ & $\begin{array}{l}2005 \\
2005\end{array}$ \\
\hline $\begin{array}{l}\text { BIDDE RD } \\
\text { Bon }\end{array}$ & Medford & JACCSON & 10/31/2018 \\
\hline BIDDLE RD \&STE VENS RD & MEDFORD & JACKSON & $009 / 29 / 2008$ \\
\hline BIDDLE RD \&STE VENS RD & MEFFORD & JACKSON & 09/29/2008 \\
\hline 1100 kirtland Road & Central Point & Jackson & 2005 \\
\hline 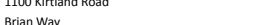 & $\begin{array}{l}\text { centara pont } \\
\text { Melford }\end{array}$ & Jackson & $\begin{array}{l}2005 \\
2005\end{array}$ \\
\hline Brian Way & Medtord & $\begin{array}{l}\text { Jackson } \\
\text { Jackson }\end{array}$ & 2005 \\
\hline 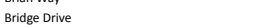 & Medford & Jackson & 10/31/2018 \\
\hline CADEt DRUE & Medford & JaCKSON & 10/31/2018 \\
\hline CORNER OF MAIN \& CENTRAL & Medford & JACKSON & 10/31/2018 \\
\hline CCAAER LAKE AVEFROBERTS ROAD & 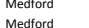 & Jackson & 201051/2018 \\
\hline & $\begin{array}{l}\text { Weterorla } \\
\text { Metford }\end{array}$ & 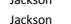 & 2005 \\
\hline $\begin{array}{l}\text { D. } \\
\text { DOWNTOWN MEDFORD } \\
\text { DOSU. }\end{array}$ & Medtord & JaCKSON & 1005/1/2018 \\
\hline EAST MAIN AND TRIPP STREET & Medtord & JACKSON & $\begin{array}{l}10 / 31 / 2018 \\
1018\end{array}$ \\
\hline EAST MAIN AND TRIPP STREET & Metford & JaCKSON & 10/31/2018 \\
\hline END OF STONEGATE DRVE & Medoford & JACCSON & $10 / 31 / 2018$ \\
\hline 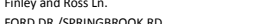 & 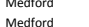 & $\begin{array}{l}\text { Jackson } \\
\text { accsson }\end{array}$ & 2005 \\
\hline . & $\begin{array}{l}\text { Weterora } \\
\text { Metford }\end{array}$ & 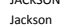 & $\begin{array}{l}1 / 131 / 2018 \\
2005\end{array}$ \\
\hline o Sewage Treatment Plant & Central Point & Jackson & 2005 \\
\hline to Sewage Treatment Plant & Central Point & Jackson & 2005 \\
\hline Next to Sewage Treatment Plant & Central Point & Jackson & 2005 \\
\hline in Creek Roadd & Medford & Jackson & 2005 \\
\hline nctrex foad & $\begin{array}{l}\text { Medtord } \\
\text { Mentrat }\end{array}$ & 官on & 2005 \\
\hline & $\begin{array}{l}\text { Mentorad } \\
\text { Melford }\end{array}$ & $\begin{array}{l}\text { Sacson } \\
\text { Jactson }\end{array}$ & $\begin{array}{l}2005 \\
2005\end{array}$ \\
\hline fin Creek Road & Medford & Jackson & ${ }_{2005}^{2005}$ \\
\hline ncreeek Road & & & \\
\hline |LANE & ledford & JACKSON & 10/31/2018 \\
\hline HONOELEAU IN. \& SPR & Meafford & & 10/31/2018 \\
\hline 1100 Kirtland Road & White city & & \\
\hline
\end{tabular}

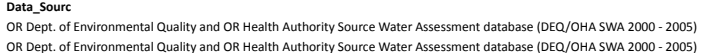

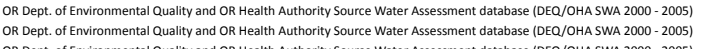

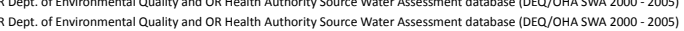

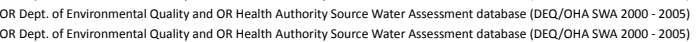
OR Dept. of Environmental Quality Dry Cleaner Database (DEQ/DC

OR State fire Marshall Hazardous Substance Information System database (SFM/HISIS 2009)

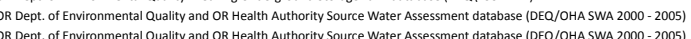

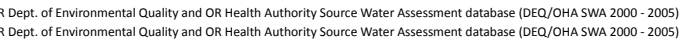

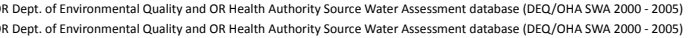

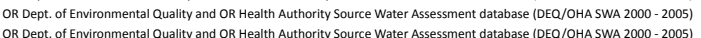

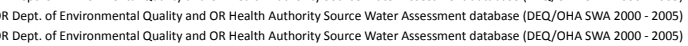

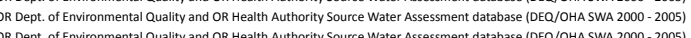

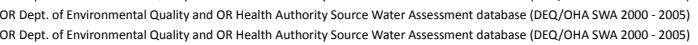

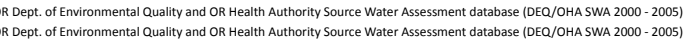
OR Dept. of Environmental Q Quality and OR Heath Authority Source Water A Asesssment database (DE//OHA SWA 2000-2005)

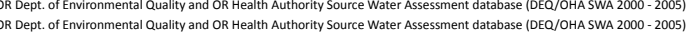
OR Dept. of Environmental Q Quality and OR Health Authority S Surce Water Assessment database (DER/OHA SWA 20000- 2005) OR Dept. of Environmental Q Quality and OR Heath Authority Source Water ASSessment database (DEE//OHA SWA $2000-2005)$

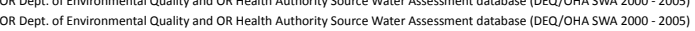
(N)

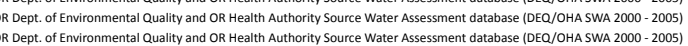

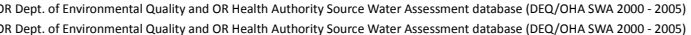
OR Dept. of Environmentatal auality and OR H Heath Authority SOurce Water Assessment database (DEC/OOHA SWA 2000- 2005)

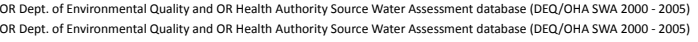
OR Dept. of Environmental Quality ynd OR Heelth Authority SOurce Water Assessment database (DEQ/OHA AWA 2000 - 2005) OR Dept. of Environmental Quality - Water Quality IIS database

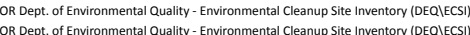

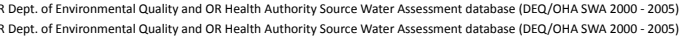

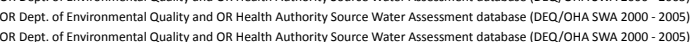

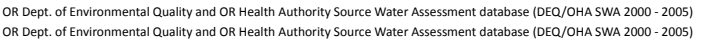

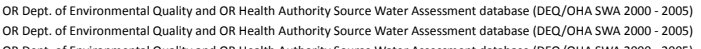

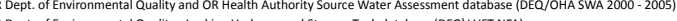

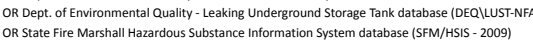

SSe (DEQ/OHA SWA 2000- 2005)

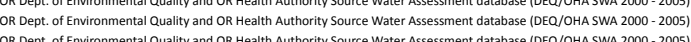

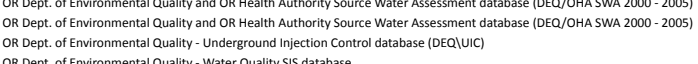

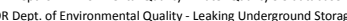

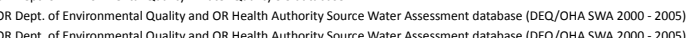

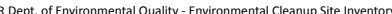

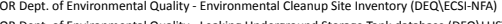

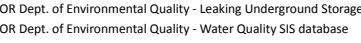

OR Dept. of Environmental Quality and OR Health Authority Source Water Assessment database (DEQ/OHA SWA 2000- 2005) OR Dept. of Environmental Q Quality - Water Quality SIS database

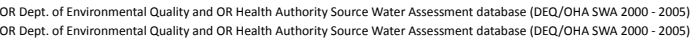
OR Dept. of Environmentat Q Quality and OR Heath Authority Source Water A ASsessment database (DER/OHA SWA 2000 - 2005)

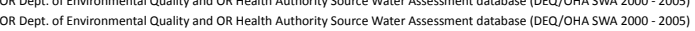

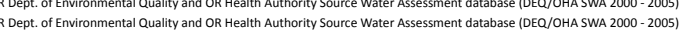

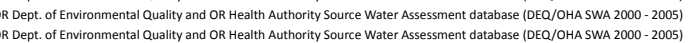

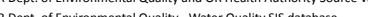

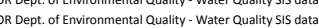

OR Dept. of Environmental Quality and OR Health Authority Source Water Assessment database (DEQ/OHA SWA 2000-2005)

\begin{tabular}{|c|c|c|}
\hline Pcscode & GWRisk SWRis & $\begin{array}{l}\text { Sk Common_nm } \\
\text { Sewertiles }\end{array}$ \\
\hline & $\begin{array}{ll}H & M \\
M & H\end{array}$ & $\begin{array}{l}\text { Sewer tines } \\
\text { Irrigation Canal }\end{array}$ \\
\hline Ro3 & L M & covered Wagon RV Park \\
\hline & $\begin{array}{ll}M & M \\
H & H\end{array}$ & $\begin{array}{l}\text { Covered Wagon nV Park } \\
\text { Rock \& Ready Mix }\end{array}$ \\
\hline & м & Rock \& Ready Mix \\
\hline & เ & Non-Irrigated Crops \\
\hline & m & Jacksonville Sewage Disposal Ponds \\
\hline $\begin{array}{c}c 08 \\
c 04\end{array}$ & $M_{M}^{M}$ & 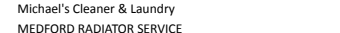 \\
\hline & L & CENTRAL AVENUE PARKING LOT B \\
\hline A03 & $\mathrm{H}$ & County Park/Exposition \\
\hline & M & $\begin{array}{l}\text { Irrigation Ditch } \\
\text { Irrigated reps }\end{array}$ \\
\hline & H & $\begin{array}{l}\text { IIrigageted Crops } \\
\text { Swerer Lines }\end{array}$ \\
\hline & M & 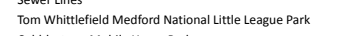 \\
\hline M01 & M & $\begin{array}{l}\text { Cobblestone Mobile Home Park } \\
\text { Aboveround storaeg Tans }\end{array}$ \\
\hline & m & 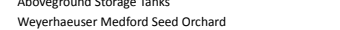 \\
\hline & L & Non-rrigigated Crops \\
\hline & m & Grazing Animals \\
\hline & H & Pacific Power J Jacksonville Substation \\
\hline & L & $\begin{array}{l}\text { Abovegrorund Storage Tanks } \\
\text { Non-Irigated Crops }\end{array}$ \\
\hline M95 & L & Rural Homes \\
\hline $\begin{array}{l}242 \\
\text { R17 }\end{array}$ & ᄂ & $\begin{array}{l}\text { RV Storage } \\
\text { Sewer Lines }\end{array}$ \\
\hline A52 & м & Talent Irrigation Ditch \\
\hline & L & $\begin{array}{l}\text { Pasture } \\
\text { Pasture }\end{array}$ \\
\hline M53 & M & $\begin{array}{l}\text { Pasture } \\
\text { Sams zalley Elementary School - Water Well }\end{array}$ \\
\hline M53 & m & $\begin{array}{l}\text { Sams valey ylementary School- Water Well } \\
\text { Valley Vista WID }\end{array}$ \\
\hline & L & Sams Valley Elementary school \\
\hline & H & Sewage Lagoon \\
\hline & t & $\begin{array}{l}\text { Valey Vista WID } \\
\text { clata wlo }\end{array}$ \\
\hline & M & 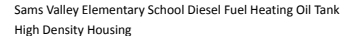 \\
\hline & $\mathrm{H}$ & $\begin{array}{l}\text { High Denstityousing } \\
\text { Phoenix Irigation Ditch }\end{array}$ \\
\hline 24 & ᄂ & Memory Gardens Memorial Park \\
\hline & M & Memory Gardens Memorial Park \\
\hline & M & $\begin{array}{l}\text { Nursery } \\
\text { Irrigated crops }\end{array}$ \\
\hline & $m$ & $\begin{array}{l}\text { Irigtated crops } \\
\text { County Park/xpostition }\end{array}$ \\
\hline & m & 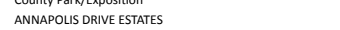 \\
\hline & н & SOUTH GATEWAY CENTER \\
\hline & H & BEAR CREEK HISTORIC LANDFILL \\
\hline Ro9 & M & $\begin{array}{l}\text { Blue Spruce Estates } \\
\text { Bulu Spruce statase }\end{array}$ \\
\hline & M & $\begin{array}{l}\text { Bulu Spruce states } \\
\text { Blue Spruce Estates }\end{array}$ \\
\hline & H & 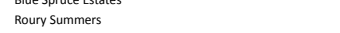 \\
\hline & M & Orchard \\
\hline & H & Sewer Lines \\
\hline & m & Phoenix xrrigation Ditch \\
\hline & L & $\begin{array}{l}\text { Rogue Valley Adventist School } \\
\text { JI \& PRROPERTES }\end{array}$ \\
\hline & н & PACFIC POWER \\
\hline & H & PACFIC POWER \\
\hline & $\mathrm{H}$ & 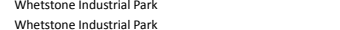 \\
\hline & M & Woodine Productions \\
\hline & H & Mustard Press \\
\hline & m & 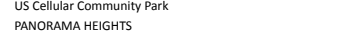 \\
\hline & н & 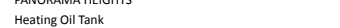 \\
\hline & m & CRATER LAKE ESTATES \\
\hline & H & Hawk Oil Co \\
\hline 658 & H & $\begin{array}{l}\text { Hawk oil Co } \\
\text { LEDOPBD }\end{array}$ \\
\hline & L & 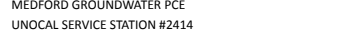 \\
\hline & เ & 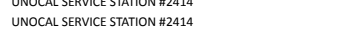 \\
\hline & m & 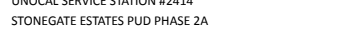 \\
\hline & M & 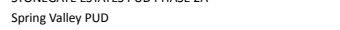 \\
\hline & M & PEARWOOD SUBDIVISION \\
\hline & th & Grififin Creek School \\
\hline & L & 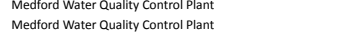 \\
\hline & м & Medford Water Quality Control Plant \\
\hline & M & $\begin{array}{l}\text { Griffin creek school } \\
\text { Stape stop store }\end{array}$ \\
\hline & L' & $\begin{array}{l}\text { Stage S Sopj sorer } \\
\text { Stage Stop Store }\end{array}$ \\
\hline & L & $\begin{array}{l}\text { Stage Stop store } \\
\text { Stope }\end{array}$ \\
\hline & M & $\begin{array}{l}\text { Lolod Concretet cutting } \\
\text { Aboveground Strogeg Tank }\end{array}$ \\
\hline & M & $\begin{array}{l}\text { BOSC VEEW EAST SUBDDIVISION } \\
\text { THEHEGTS }\end{array}$ \\
\hline & ${ }_{\mathrm{H}}^{\mathrm{H}}$ & 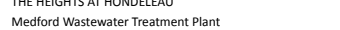 \\
\hline
\end{tabular}

PCSType
Sewer Lines- Close Proximity to pus

cigation Canal/Pond

Camprounds/RV Parks
Housing-High Densty- 1 Hous/0.5 Acres
Mining Activities - Gravel Mines/Gravel Pits

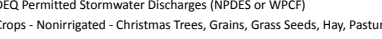

Dry Cleaners
Auto- Repari Shops

UST - Confirmed Leaking but listed as NFA - DEQ LUST Lis

Cops - Irrigated - Berries, Hops, Mint, Ochards, Vineyards $M$

Housing- - High Density $\rightarrow 1$ House/.5.5 Acres

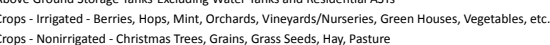

Grang Animals 5 S L Large Animals or Equivalent/Acre

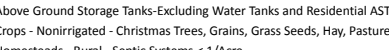

iv/Ministorage
Sewer Lines -closento

Won- Nonirigated - Christmas Trees, Grains, Grass Seeds, Hay, Pasture

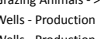

wells-Product

Lhoons/Liquid Wastes - Municipal//ndustrial Wastewater Treatment

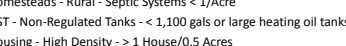

Irrigation Canal/Pond

Lawn Care - Highly Maintained Areas

Cops - Irrigated - Berries, Hops, Mint, OCh hards, Vineyardd/Nurseries, Green Howess, vegetables, etc.

Cops - Irigiated - Beries, Hops, Mint, Orchards, Vineyards/Nurseries, Green Houses, vegetables, etc DEQ Permitted Stormwater Discharges (NPDES or WPCF)

Known Contamination Sitess/Plumes/SPilis $s$ ECS

ousing - High Density $\rightarrow 1$ House/0.5 Acres
ousing - High Density $\rightarrow 1$ House/0.5 Acres

Housing - High Density $>1$ House/.5.5 Acres

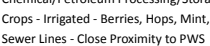

UST- Confirmed Leaking but listed as NFA - DEQQ LUST Lis

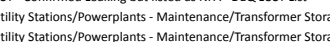

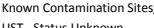

Grniture/Lumber//Parts stores

Photo Processing/Printing
Other Injection/Ory Wells, Sumps - Class V vics

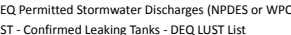

DEQ Permitited Stormwater Discharges N NDEES or WPCC

Chemical/Petroleum Processing//5
UST - Decommissioned - Inactive

Known Contaminination Siteses/Plumes/Spills EESSI)

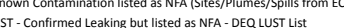

UST - Confirmed Leaking but listed as NAA- DEQ LUST Lis
DEQ Permitted Stormwater Discharges (NPDES Or WPCCFI

Construction/Demolition Area
DEQ Permitted Stormwater Discharges (NPDES or WPCF)

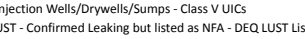

UST - Upgraded and/or Registered- Active

Wastew

Atto- Gas Stations

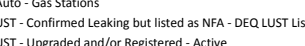

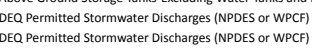




\begin{tabular}{|c|c|c|c|}
\hline & & & \\
\hline $\begin{array}{l}\text { Address } \\
1100 \text { Kirtand Road }\end{array}$ & City & County & RET_DATE \\
\hline 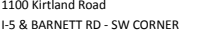 & $\begin{array}{l}\text { White city } \\
\text { Medford }\end{array}$ & $\begin{array}{l}\text { Jackson } \\
\text { JaCKSON }\end{array}$ & $\begin{array}{l}2005 \\
10 / 31 / 2018\end{array}$ \\
\hline 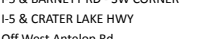 & Medford & JACKSON & $10 / 31 / 2018$ \\
\hline $\begin{array}{l}\text { Off West Antelop Rd } \\
\text { Lars Say }\end{array}$ & $\begin{array}{l}\text { White City } \\
\text { Medford }\end{array}$ & $\begin{array}{l}\text { Jackson } \\
\text { Jackson }\end{array}$ & $\begin{array}{l}2005 \\
2005\end{array}$ \\
\hline $\begin{array}{l}\text { Lars Way } \\
\text { Lars Way }\end{array}$ & $\begin{array}{l}\text { Metorod } \\
\text { Medford }\end{array}$ & $\begin{array}{l}\text { Jaccson } \\
\text { Jackson }\end{array}$ & \\
\hline Lars Way & $\begin{array}{l}\text { Menturod } \\
\text { Mentor }\end{array}$ & $\begin{array}{l}\text { Sackon } \\
\text { Jactson }\end{array}$ & 2005 \\
\hline $\begin{array}{l}\text { Downing } \\
\text { Down }\end{array}$ & Central Point & Jackson & 2005 \\
\hline Lars Way & Medford & Jackson & 2005 \\
\hline $\begin{array}{l}\text { Lars Way } \\
\text { Off }\end{array}$ & $\begin{array}{l}\text { Medford } \\
\text { Centrapits }\end{array}$ & Jackson & 2005 \\
\hline $\begin{array}{l}\text { Off Table Rock Road } \\
\text { Off Table Rock Road }\end{array}$ & $\begin{array}{l}\text { Centrata Point } \\
\text { Central Point }\end{array}$ & Jackson & $\begin{array}{l}2005 \\
2005\end{array}$ \\
\hline $\begin{array}{l}\text { Off Table Rock Roadd } \\
\text { Lars Way }\end{array}$ & $\begin{array}{l}\text { Centrata Point } \\
\text { Medford }\end{array}$ & $\begin{array}{l}\text { Jackson } \\
\text { Jackson }\end{array}$ & $\begin{array}{l}2005 \\
2005\end{array}$ \\
\hline Lars Way & Mettord & $\begin{array}{l}\text { Sackon } \\
\text { Jactson }\end{array}$ & 20005 \\
\hline Lars Way & Medford & Jackson & 2005 \\
\hline Downing & Central Point & Jackson & 2005 \\
\hline Downing & Central Point & Jackson & \\
\hline $\begin{array}{l}\text { LAT T22.3335, LONG G } 122.28385 \\
\text { LONE OAK DR \& SUNEAF }\end{array}$ & $\begin{array}{l}\text { Meforord } \\
\text { Mefford }\end{array}$ & $\begin{array}{l}\text { JaCCSON } \\
\text { JACKSON }\end{array}$ & $\begin{array}{l}10 / 1 / 1 / 2018 \\
1 / 131 / 2018\end{array}$ \\
\hline 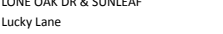 & Metford & $\begin{array}{l}\text { Jackson } \\
\text { Jackson }\end{array}$ & \\
\hline & Central Point & $\begin{array}{l}\text { Jackson } \\
\text { Jacton }\end{array}$ & 2005 \\
\hline Maple Park Dr. & Medford & Jackson & 2005 \\
\hline Maple Park Dr. & Mefford & Jackson & 2005 \\
\hline $\begin{array}{l}\text { Throughout DWPA } \\
\text { mCANDRES AND RIVERSIDE }\end{array}$ & $\begin{array}{l}\text { Central lonit } \\
\text { Medford }\end{array}$ & $\begin{array}{l}\text { Jactson } \\
\text { JacksoN }\end{array}$ & $\begin{array}{l}2005 \\
10 / 31 / 2018\end{array}$ \\
\hline McAndrews Rd. & Medford & Jackson & 2005 \\
\hline Mcandrews Rd. & $\begin{array}{l}\text { Mefford } \\
\text { Mefford }\end{array}$ & Jackson & \\
\hline 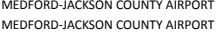 & $\begin{array}{l}\text { Melford } \\
\text { Medford }\end{array}$ & $\begin{array}{l}\text { JaCASSON } \\
\text { JACKSON }\end{array}$ & $\begin{array}{l}10 / 3 / 21 / 2018 \\
10 / 31 / 2018\end{array}$ \\
\hline Downing & Central Point & Jackson & 2005 \\
\hline Downing & $\begin{array}{l}\text { Central Point } \\
\text { Medford }\end{array}$ & $\begin{array}{l}\text { Jackson } \\
\text { accsoos }\end{array}$ & $\begin{array}{l}2005 \\
105 / 2018\end{array}$ \\
\hline 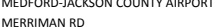 & $\begin{array}{l}\text { Meliterd } \\
\text { Medford }\end{array}$ & $\begin{array}{l}\text { JaCCSON } \\
\text { ACCSSON }\end{array}$ & $\begin{array}{l}10 / 3 / 1 / 2018 \\
10 / 13 / 2018\end{array}$ \\
\hline 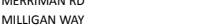 & $\begin{array}{l}\text { Mearora } \\
\text { Mefford }\end{array}$ & 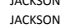 & $\begin{array}{l}101 / 3 / 21 / 2018 \\
10 / 131 / 2018\end{array}$ \\
\hline MOBILL SOURCE - JACKSON COUNTY & Mefford & 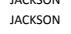 & $10 / 31 / 2018$ \\
\hline North of Well 2 & Central Point & Jackson & 2005 \\
\hline MT. ASHLAND RADAR STIE & Medford & JACKSON & $10 / 31 / 2018$ \\
\hline 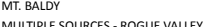 & $\begin{array}{l}\text { Mefford } \\
\text { Mefford }\end{array}$ & JACKSON & $\begin{array}{l}10 / 31 / 2018 \\
1013 / 2018\end{array}$ \\
\hline 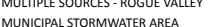 & $\begin{array}{l}\text { Meforord } \\
\text { Mefford }\end{array}$ & $\begin{array}{l}\text { JaCCSONO } \\
\text { ACCSSON }\end{array}$ & $\begin{array}{l}10 / 31 / 2018 \\
1013 / 2018\end{array}$ \\
\hline N 42-17-46 W 122-49 -38 & $\begin{array}{l}\text { MEDFORD } \\
\text { MEOTO }\end{array}$ & 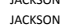 & $\begin{array}{l}10131 / 2018 \\
09 / 29 / 2008\end{array}$ \\
\hline N42-17-55 W122-44-57 & MEDFORD & Jackson & $\begin{array}{ll}09 / 29 / 2008 \\
0\end{array}$ \\
\hline N 42-18-52 W $122-51-48$ & MEDFORD & JaCKSON & 09/29/2008 \\
\hline 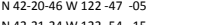 & MEOFORD & JaCKSON & $09 / 29 / 2008$ \\
\hline 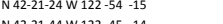 & МEFFRD & JACKSON & 09/29/2008 \\
\hline 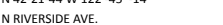 & $\begin{array}{l}\text { MEEForoD } \\
\text { Mefford }\end{array}$ & $\begin{array}{l}\text { JaCGSON } \\
\text { JACKSON }\end{array}$ & $\begin{array}{l}09 / 29 / 2008 \\
10 / 11 / 2018\end{array}$ \\
\hline N. Paccific Hwy & Medtord & $\begin{array}{l}\text { Jackson } \\
\text { Jacton }\end{array}$ & \\
\hline N. Pacific Hwy & Medtord & Jackson & 2005 \\
\hline N. Ross Ln. and W McAndrews & Medtord & Jackson & 2005 \\
\hline N. Ross Ln. and W Wcendrews & Medford & Jackson & 2005 \\
\hline 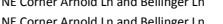 & $\begin{array}{l}\text { Meerorar } \\
\text { Metract }\end{array}$ & $\begin{array}{l}\text { Jackson } \\
\text { ackson }\end{array}$ & 2005 \\
\hline 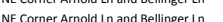 & 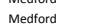 & $\begin{array}{l}\text { Sackon } \\
\text { Jactson }\end{array}$ & 2005 \\
\hline North of Well & Medford & Jackson & 2005 \\
\hline North of Well & Medford & Jackson & 2005 \\
\hline Not Applicable & MEFFORD & Jackson & 2013 \\
\hline Not Applicable & MEFFRO & Jackson & 2013 \\
\hline Not Applicable & $\begin{array}{ll}2 & -1\end{array}$ & $\begin{array}{l}\text { sacsson } \\
\text { backson }\end{array}$ & 2013 \\
\hline Not Applicable & $\begin{array}{l}\text { MEFFRD } \\
\text { MEFo }\end{array}$ & 急 & 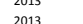 \\
\hline Not Applicable & МЕEFRO & Jackson & ${ }_{2013}$ \\
\hline Table Rock Rd. over Rogue River & White city & Jackson & 2005 \\
\hline $\begin{array}{l}\text { Not Applicable } \\
\text { Notentichbe }\end{array}$ & $\begin{array}{l}\text { MEDFORD } \\
\text { MEFOODD }\end{array}$ & $\begin{array}{l}\text { Jackson } \\
\text { Jackson }\end{array}$ & 2013 \\
\hline $\begin{array}{l}\text { Not Appolicable } \\
\text { Not palicable }\end{array}$ & $\begin{array}{l}\text { MEFEROD } \\
\text { MEDFRD }\end{array}$ & $\begin{array}{l}\text { Jackson } \\
\text { Jackson }\end{array}$ & 2 \\
\hline Non Appridarer & Whate Caty & 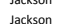 & 2005 \\
\hline 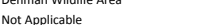 & MEFFOR & 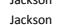 & 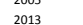 \\
\hline Not Applicable & MEDFORD & Jackson & 2013 \\
\hline Not Applicable & MEFFORD & Jackson & 2013 \\
\hline Off Table Rock Rd & White City & Jackson & 2005 \\
\hline Not Applicable & $\begin{array}{l}\text { MEEFERD } \\
\text { MEERO }\end{array}$ & Jackson & 2013 \\
\hline 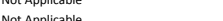 & MEFORD & 势 & 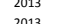 \\
\hline $\begin{array}{l}\text { Not Applicable } \\
\text { Not }\end{array}$ & $\begin{array}{lll} & 0\end{array}$ & $\begin{array}{l}\text { Sacson } \\
\text { Jactson }\end{array}$ & 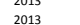 \\
\hline Throughout DWPA & White city & Jackson & 2005 \\
\hline able Rock Rd & White city & Jackson & 2005 \\
\hline able Rock Rd & White City & Jackson & 2005 \\
\hline Not Applicable & $\begin{array}{l}\text { MEEFROB } \\
\text { MEERO }\end{array}$ & Jackson & 2013 \\
\hline Nat & $\begin{array}{l}\text { MEEFBOB } \\
\text { MEERD }\end{array}$ & Jactson & 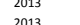 \\
\hline policable & MEEFORD & son & 2013 \\
\hline East of intake & White city & Jackson & 2005 \\
\hline actific Rd & White city & Jackson & 2005 \\
\hline 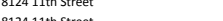 & White city & Jackson & 2005 \\
\hline
\end{tabular}

Oata. Sourc
OR Dept. of Environmental Quality ynd OR H Heath Authority Source Water Assessment database (DEQ/OHA SWA 2000 - 2005) OR Dept. of Environmental Quality L Leaking Underground Storage Tank database (DEQQIUST-NFEA

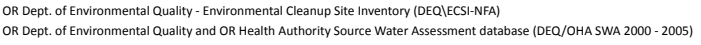

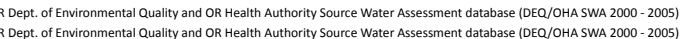

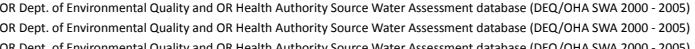
OR Dept. of Environmental Q Quality and OR Heath Authortity Source Water A Asessment database (DER/OHA SWA 2000- 2005 )

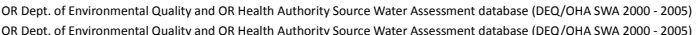

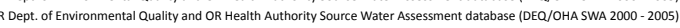

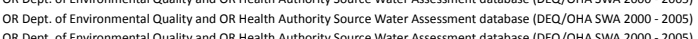

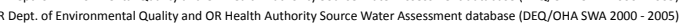

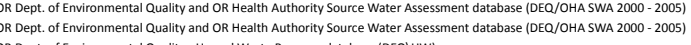

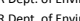

OR Dept. of Environmental Q Quality and OR Heath Authority Source Water Assessment database (DEQ//HA SWA 2000- 2005)

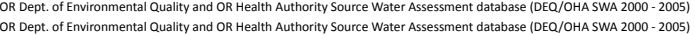

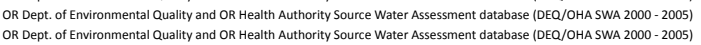

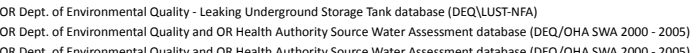

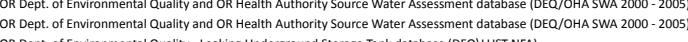

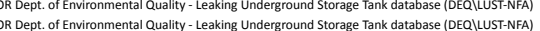

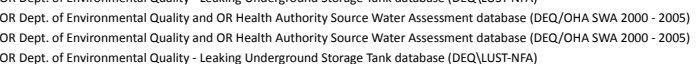

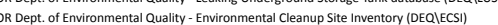

OR Dept. of Environmental Quality - Water Quality IIS database

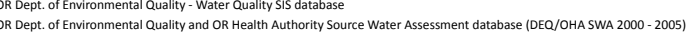

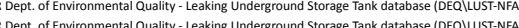

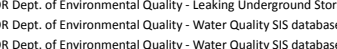

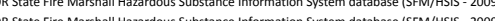

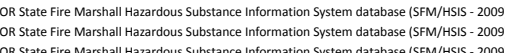

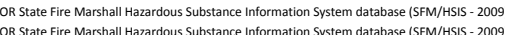

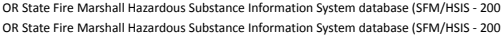

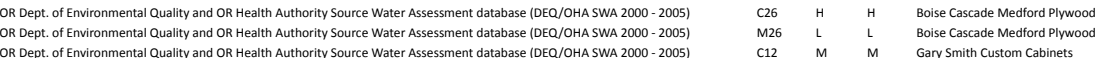

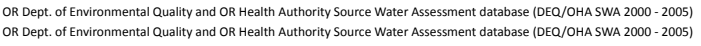

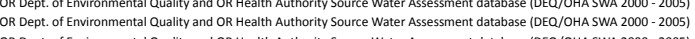
(2) Oregon Dept. of Transportation, Technical Services Branch, Brideges Section (ODOO)

Oregon Dept. of Transportation, Technicial Services Branch, Bridges Section (OODO)

Oregon Dept. of Transportation, Technical Services Branch, Bridges Section (OODT)
Oregon Dept. of Transportation, Technical Sevices reanch, Bridges Section (ODOT)

DEQ/OHA SWA 2000- 2005)

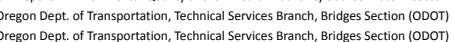

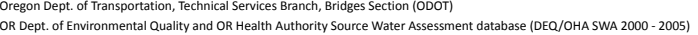
Oregon Dept. of Transportation, Technical Services Branch, Bridges Section (1000

Oregon Dept. of Transportation, Technical Sevices Branch, Bridges Section (looor)

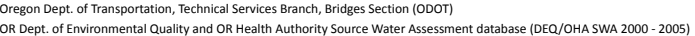
Oregon Dept. of Fransportation, Technical Services Branch, Bridges Section (1000)

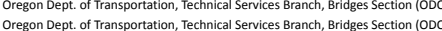

Oregon Dept. of Trans sortation, Technical Services Branch, Bridges Section (00007

(D)

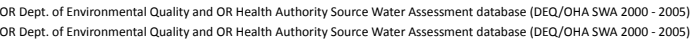

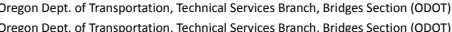

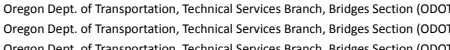

Oregon Dept. of Transportation, Technical Services Branch, Brideses Section (ODO)

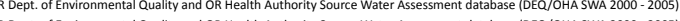

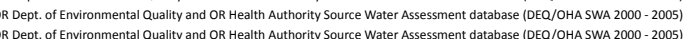
OR Dept. of Environmental Quality and OR Heath Authority Source Water Assessment datababse (DER/OHA SWA 2000- 2005)

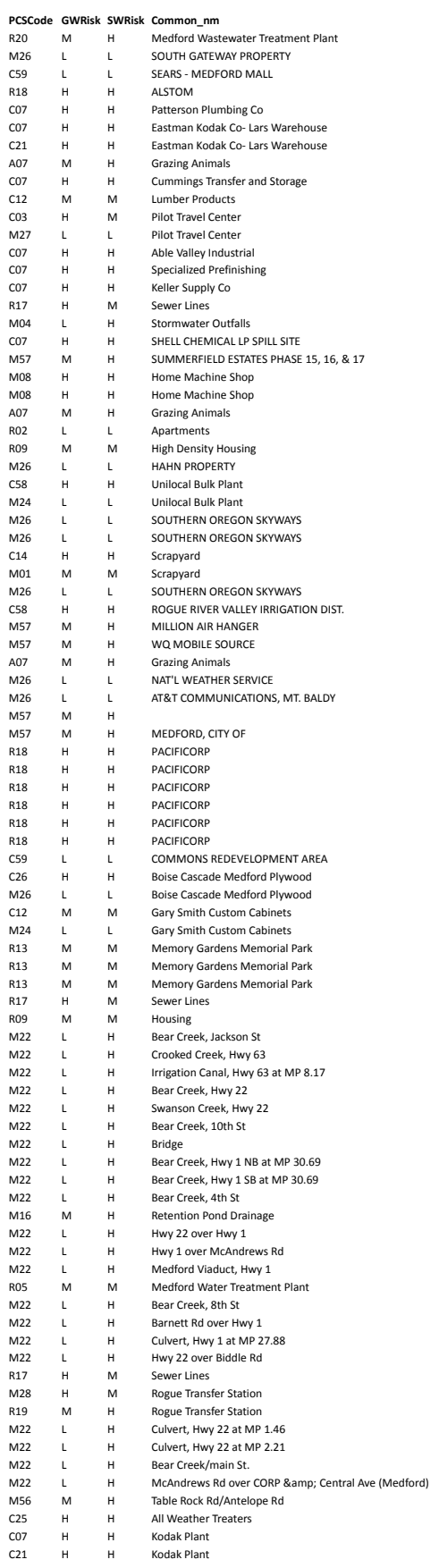

PCSType

Wastewater r Treatrent Plants/Collection Stations

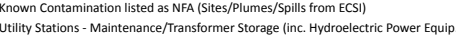

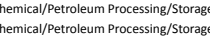

Photo Processing//rinting

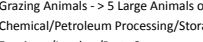

Furniture/lumber/Parts Stores
Auto - Gas Stations

UST- Upgraded and/or Registered-Active

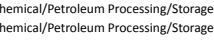

Sewer lines-close Proximity to PWS

Stormwater Outfall

DEQ Permitted Stormwater Discharges (NPDES or WPCA

Homesteads - Rural- Machine Shops/Equip Maintenance

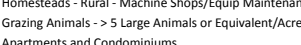

Housing - High Density $\rightarrow$ - 1 House/0.5 Acres

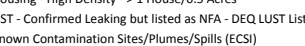

UST - Decommissioned - Inacture

WST - Confirmed Leaking but listed as NFA- DEQ LUST Lis
UST - Confirmed Leaking but isted a S NFA - DEQ UST

Junk/scrap//alaveg Yards

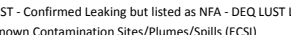

DEQ Permitted Stormwater Discharges (NPDES or WPCA

DEQR Permited Stormwater Discharges (NPDEE or WPCC
Grazing Animals $\rightarrow 5$ L Large Animals or Equivalent/Acre

UST - confirmed Leaking but listed as NFA- DEQ L UST List

UST - Confirmed Leaking but listed as NAA- DER LUST LAT

DEQ Perritted Stormwater Discharges (NPDES or WPC

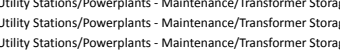

Utillty Stations/Powerplants - Maintenance//Transformer storage

Utility Stations/Powerplants - Maintenance//Transformer Storage

Known Contamination listed as NFA (Sities/Plumes/Spills from ECSI)

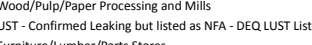

UST - Decommisisioned - Inac

Parks
Parks
Parks

Sewer Lines - Close Proximity to PWS

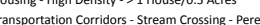

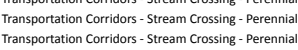

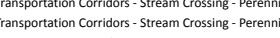

Transportation Corridiors - Straeac crossing - Perential

Transportation Corridors- S-Stream Crossing - Perennial

Coridors - Streamm Crossing - Peren

rransportation Corridors- Stream Crossing - Perenn

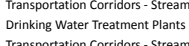

Transportation Corrididrs-Stream Crossing- - Perennial

Transportation Corrididors - Strtream Crossising - Perennial

Sewer Lines - Close Proximity to

Waste Transfer/Recycling Stations

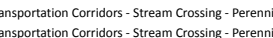

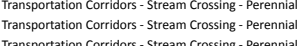

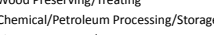




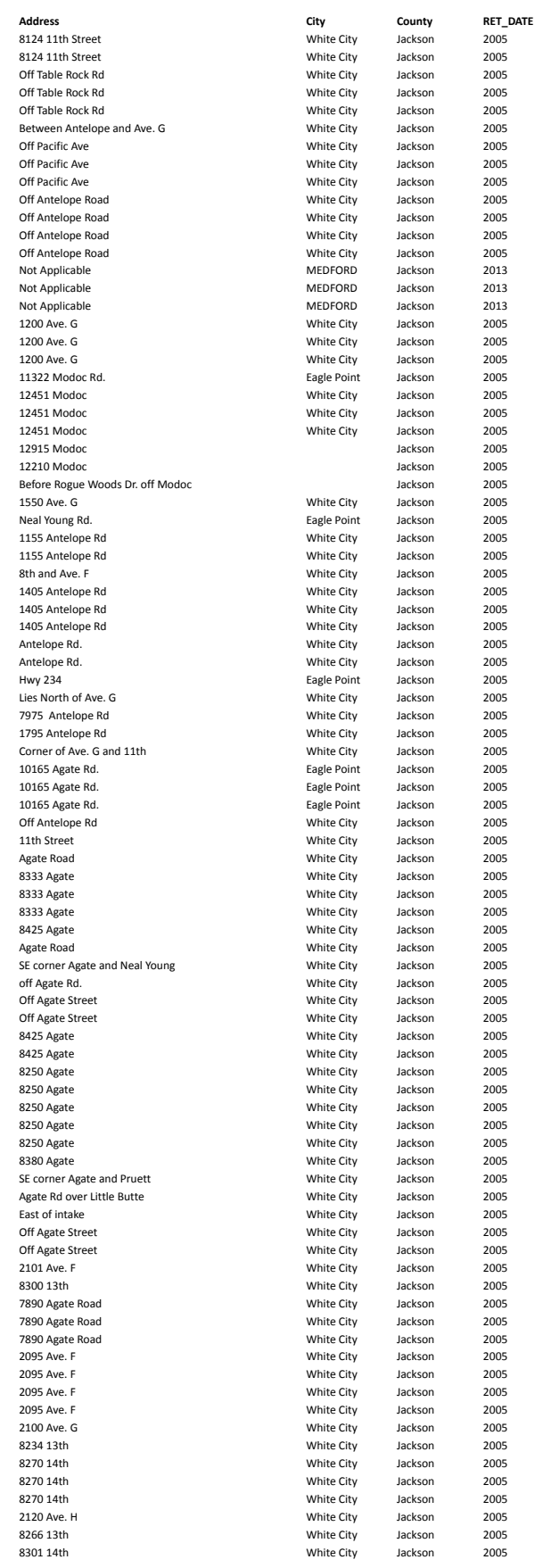

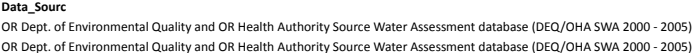

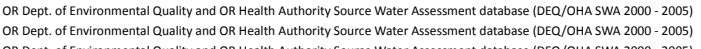

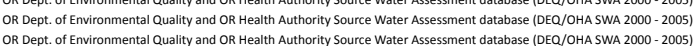

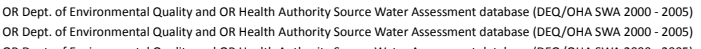

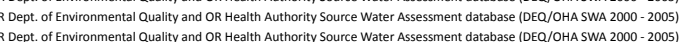

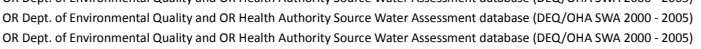

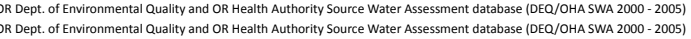

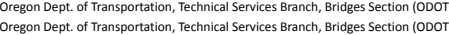

OR Dept. of Environmental Q Quality and OR Heatth Authortity Source Water Asesssment database (DEE/OHA SWA 2000 - 2005) $20005)$

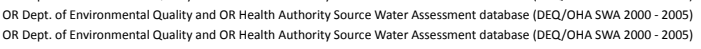

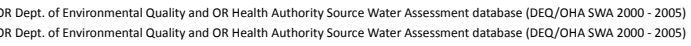
OR Dept. of Environmental Quality and OR Heath Authority Source Water ASSessment databse (DEO/OHA SWA 2000- 2005)

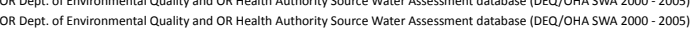
OR Dept. of Environmental Q Quality and OR Heath Authority Source Water A Assessment database (DEQ/OHA SWA 2000- 2005)

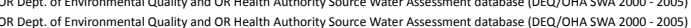
OR Dept. of Environmental Q Quality and OR Heath Authority Source Water Assessment database (DEQ/OHA SWA 2000- 2005)

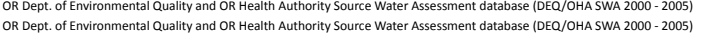

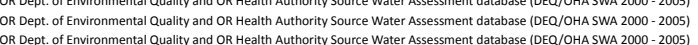

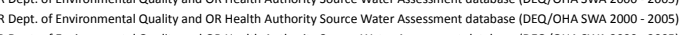
OR Dept. of Envirommental Q Quality and OR Health Authority SOurce Water Assessment databasese (DER/OOHA SWA 2000 - 2005)

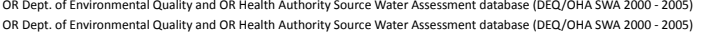

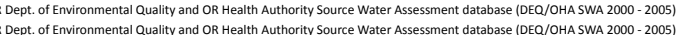
OR Dept. of Environmental Quality and OR Heath Authority Source Water Assessment datababse (DEC/OHA SWA $2000-2005)$ OR Dept. of Environmental (uality and OR Heath Authority Surce Water Assessment database (DEG/OHA SWA 2000 - 2005)

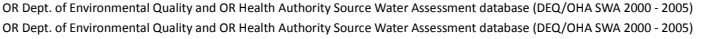

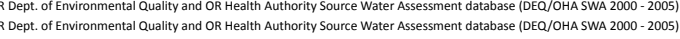

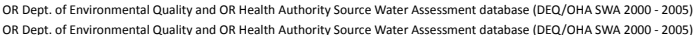
OR Dept. of Environmental Quality and OR Health Authority Source Water Assessment database (DEQ/OHA SWA 2000- 2005)

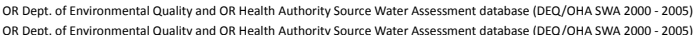

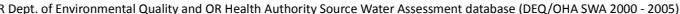

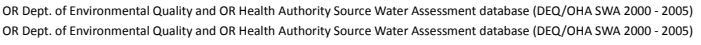

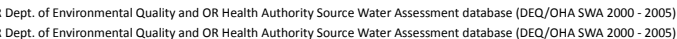

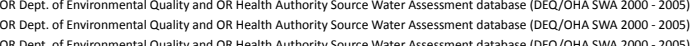

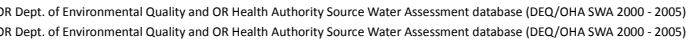
(D) Dase (OHA SWA 2000- 2005)

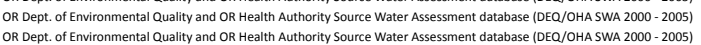

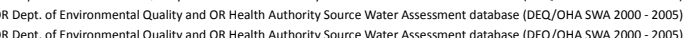
OR Dept. of Environmental l Quality and OR Health Authority S Surce Water A Asesssment database (DE//OHA SWA 2000-2005) OR Dept. of Environmental Q Quality and OR Heath Authority Source Water A A SSessment databbase (DEO/OHA SWA 2000 - 2005) OR Dept. of Environmental Q Quality and OR Heath Authority Source Water A Assessment database (DEQ/OHA SWA 2000- 2005)

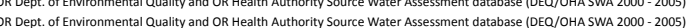
OR Dept. of Environmental Q Quality and OR Heath Authority Source Water ASSessment database (DEQ/OHA SWA 2000- 2005)

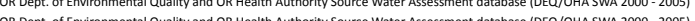
OR Dept. of Envirionmentat Q Quality and OR Heath Authority Source Water ASsessment database (DEQ//OHA SWA 2000 - 2005)

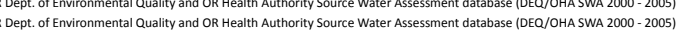

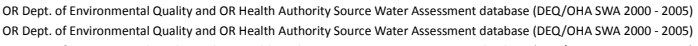

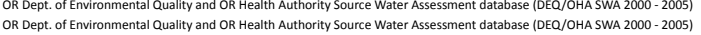

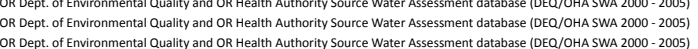

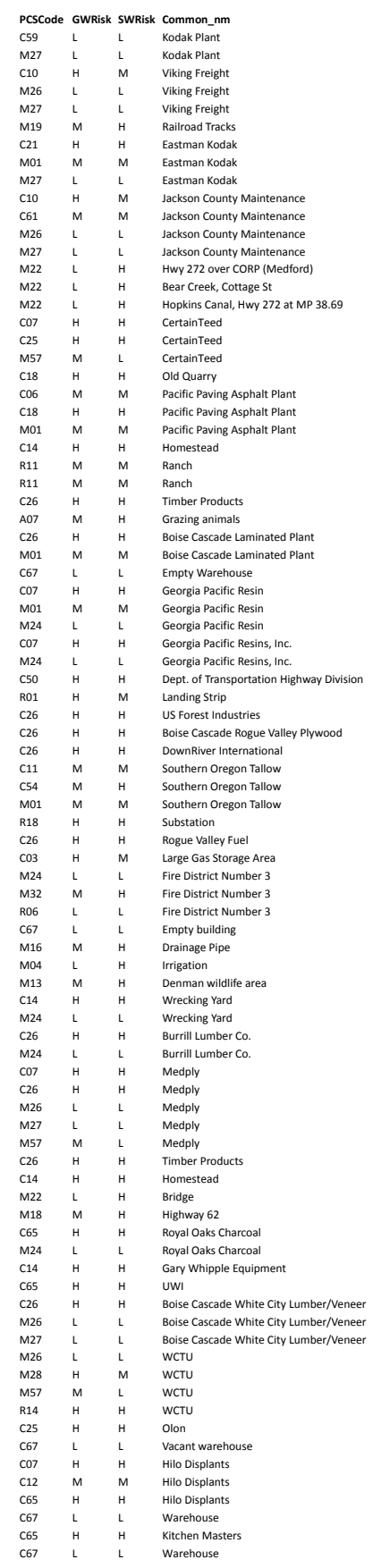

icstype

- Upgraded and/or Registered-Active

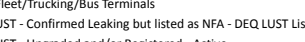
rransportation Corridors - Railroads Photo Processinip// Printing
Above Ground Strage Tank

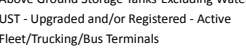
UST - Upgraded and/or Registered -Active Fransportation Corridoros- Stream crossing- Perenn Iransportation Corridors-Stream Crossing - Perenne Chemical/Petroleum Processing//Storage DEQ Permitted Stormwater Discharges (NDDES or WPCE)

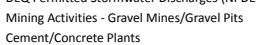

Cement/Concetete Plants
Mining Activities - Gravel Mines/Gravel Pits above Ground Storage Tanks-Excluding Water Tanks and Residential AST Lawn Care - Highly Maintained Areas
Lawn Care- - Hightly Maintaned

Wood/Pul//Paper Processing and Mills

Wood/Pulp/Paper Processing and Mills

Warehouses
Chemical Petroleum Processing/Storage

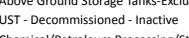

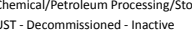

others (list)

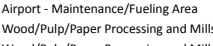

Wood/Pulp/Paper Processing and Mills
Wood/Pulp/Paper Processing and Mills
Wood/Pulp/Paper Processing and Mills

FEod Processing

Tank and Residential asts

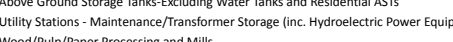
Auto- Gas Stations
UST-Decommissioned- - Inactive UST- Decommissined - Inactive
Construction/Demolition Area UST- Decom
Construction
Fire Station
Warehouses Warehouses
Stormwater Retention Basis Stormwater outtalls
Random Dumpsites

Junk/Scrap/Salvage Yards

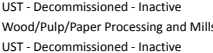
Chemical/Petroloum Processing / Stororage

UST- Confirmed Leaking but listed as NFA - DEQQ LUST List UST - Upgraded and/or Registered - Active

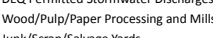

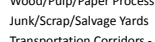

Transportation Corridors - Stream Crossing - Perennnal

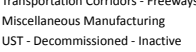
Junk/Scrap/salvage Yards
Miscelaneous Manuracturing

Wood/Pup/Paper Processing and Mills
UST - Confirmed Leaking but listed as N NA - DEQ LUST Lis

UST - Upgraded and/or Registered-Active
UST - Confirmed Leaking but listed as NFA- DEQ LUST Lis

UST-Status Unknown
DEQ Permitted Stormwater Discharges INPDES or WPCF

DEQ Permitted Stormwater Discharges (NPD
Railroad Vards/Maintenance/fuling Areas
Wood Preserving /Treating

Wood Preserving/Treating
Warehouses

Chemical/Petroleum Processing//s
furniture/Lumber/Part stores

Furniture/Lumber/Parts Stores
Miscellaneous Manufacturing

Warehouses
Miscellaneous Manufacturing 


\begin{tabular}{|c|c|c|}
\hline $\begin{array}{l}\text { Address } \\
\text { S }\end{array}$ & city & County \\
\hline $\begin{array}{l}\text { 8399 14th St. } \\
\text { Comer of 14thand ave } G\end{array}$ & $\begin{array}{l}\text { Whitit City } \\
\text { white city }\end{array}$ & Jackson \\
\hline $\begin{array}{l}\text { Cormer of } 1 \text { 14th and ava. } 6 \\
2250 \text { Ave. Hand } 83999 \text { 14th }\end{array}$ & $\begin{array}{l}\text { White City } \\
\text { White city }\end{array}$ & $\begin{array}{l}\text { Jackson } \\
\text { Jackson }\end{array}$ \\
\hline 2250 Ave. Hand 8399 14th & White city & Jackson \\
\hline 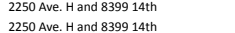 & $\begin{array}{l}\text { Whitit City } \\
\text { White city }\end{array}$ & $\begin{array}{l}\text { Jackson } \\
\text { Jackon }\end{array}$ \\
\hline 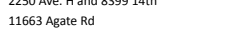 & $\begin{array}{l}\text { White city } \\
\text { Eagle Point }\end{array}$ & $\begin{array}{l}\text { Jackson } \\
\text { Jackson }\end{array}$ \\
\hline 11663 Agate Rd & $\begin{array}{l}\text { Eagle Pont } \\
\text { Eagle Point }\end{array}$ & $\begin{array}{l}\text { Jackson } \\
\text { Jackson }\end{array}$ \\
\hline 8400 14th & White city & Jackson \\
\hline 8399 14th St. & White city & Jackson \\
\hline $\begin{array}{l}23001 \text { 15th } \\
22211 \text { th }\end{array}$ & $\begin{array}{l}\text { White city } \\
\text { White city }\end{array}$ & Jackson \\
\hline 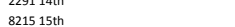 & $\begin{array}{l}\text { Whitit C City } \\
\text { White city }\end{array}$ & $\begin{array}{l}\text { Jacason } \\
\text { Jackson }\end{array}$ \\
\hline $\begin{array}{l}82115151 \mathrm{sin} \\
821515 \mathrm{sh}\end{array}$ & $\begin{array}{l}\text { White city } \\
\text { White city }\end{array}$ & $\begin{array}{l}\text { Jaccosson } \\
\text { Jackson }\end{array}$ \\
\hline 2091 Ave H & white city & Jackson \\
\hline 2050 Antelope Road & White city & Jackson \\
\hline 2050 Antelope Road & White city & Jackson \\
\hline 2050 Antelope Road & $\begin{array}{l}\text { Whitit City } \\
\text { white city }\end{array}$ & Jackson \\
\hline 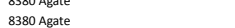 & $\begin{array}{l}\text { Whitit city } \\
\text { White city }\end{array}$ & $\begin{array}{l}\text { Jacasson } \\
\text { Jackson }\end{array}$ \\
\hline 15th Street & $\begin{array}{l}\text { White city } \\
\text { White city }\end{array}$ & $\begin{array}{l}\text { Jackson } \\
\text { Jackson }\end{array}$ \\
\hline 8333155 th & $\begin{array}{l}\text { White city } \\
\text { why }\end{array}$ & $\begin{array}{l}\text { Jackson } \\
\text { Jackson }\end{array}$ \\
\hline 2350 ave. F & White city & Jackson \\
\hline 2350 Ave. F & 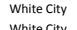 & Jackson \\
\hline $\begin{array}{ll}2355 \text { Ave.F } \\
2355 \text { Ave F F }\end{array}$ & $\begin{array}{l}\text { Whitit City } \\
\text { white city }\end{array}$ & $\begin{array}{l}\text { Jacksson } \\
\text { Jackson }\end{array}$ \\
\hline 2 & $\begin{array}{l}\text { White city } \\
\text { White city }\end{array}$ & $\begin{array}{l}\text { Jackson } \\
\text { Jackson }\end{array}$ \\
\hline 2301 Ave G & White city & $\begin{array}{l}\text { Jacksoun } \\
\text { Jackon }\end{array}$ \\
\hline 2350 Ave. 6 & White city & Jackson \\
\hline 2350 ave. $G$ & White city & Jackson \\
\hline 2390 Ave G & $\begin{array}{l}\text { White City } \\
\text { Eagle point }\end{array}$ & $\begin{array}{l}\text { Jackson } \\
\text { Jackson }\end{array}$ \\
\hline 2510 Ave G & $\begin{array}{l}\text { Egele oint } \\
\text { White city }\end{array}$ & $\begin{array}{l}\text { Jacsson } \\
\text { Jackson }\end{array}$ \\
\hline Rogue River Dr & & Jackson \\
\hline $\begin{array}{l}2630 \text { Ane G } \\
2630 \text { Ave G }\end{array}$ & White City & Jackson \\
\hline $\begin{array}{l}2630 \text { A Ave } G \\
64 \text { Rogue River Drive }\end{array}$ & $\begin{array}{l}\text { Ehtite City } \\
\text { Eagle Point }\end{array}$ & $\begin{array}{l}\text { Jacasson } \\
\text { Jackson }\end{array}$ \\
\hline 2650 Ave. G & $\begin{array}{l}\text { Whele pint } \\
\text { White city }\end{array}$ & $\begin{array}{l}\text { Jackson } \\
\text { Jackson }\end{array}$ \\
\hline 2625 Ave. $G$ & White city & $\begin{array}{l}\text { sackson } \\
\text { Jackon }\end{array}$ \\
\hline 2625 Ave. $G$ & White city & Jackson \\
\hline 2625 Ave. 6 & White city & Jackson \\
\hline 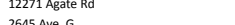 & $\begin{array}{l}\text { Eagle Point } \\
\text { skite }\end{array}$ & Jackson \\
\hline Net & $\begin{array}{l}\text { Eagle Point } \\
\text { Elate }\end{array}$ & 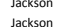 \\
\hline 12075 Agate Road & $\begin{array}{l}\text { Eagle P Pint } \\
\text { E. }\end{array}$ & $\begin{array}{l}\text { Jacksoun } \\
\text { Jackon }\end{array}$ \\
\hline 2655 ave. 6 & White city & Jackson \\
\hline Rogue River Dr & Eagle Point & Jackson \\
\hline Rogue River and Sam's Valley Hwy 234 & Eagle Point & Jackson \\
\hline Rogue River and Sam's Valley HWy 234 & $\begin{array}{l}\text { Eagle Point } \\
\text { White city }\end{array}$ & $\begin{array}{l}\text { Jacksson } \\
\text { Jackson }\end{array}$ \\
\hline $\begin{array}{l}82020 \text { Crater Lake Highway } \\
82 \text { Crater Lake Highway }\end{array}$ & $\begin{array}{l}\text { White city } \\
\text { White city }\end{array}$ & $\begin{array}{l}\text { Jackson } \\
\text { Jackson }\end{array}$ \\
\hline 8205 Crater Lake Highway & White city & Jackson \\
\hline 2727 Ave. $G$ & White city & Jackson \\
\hline 2727 Ave. $G$ & White city & Jackson \\
\hline $\begin{array}{l}2727 \text { Ave. } \\
1355 \text { Aate Road }\end{array}$ & $\begin{array}{l}\text { Whitite City } \\
\text { Eagle Point }\end{array}$ & $\begin{array}{l}\text { Jacksson } \\
\text { Jackson }\end{array}$ \\
\hline $\begin{array}{l}13555 \text { Agate torad } \\
1355 \text { Agte Raad }\end{array}$ & $\begin{array}{l}\text { Eagle Point } \\
\text { Eagle Point }\end{array}$ & $\begin{array}{l}\text { Jackson } \\
\text { Jackson }\end{array}$ \\
\hline 1331 old Hwy 234 & Eagle Point & Jackson \\
\hline 2841 Ave. $G$ & 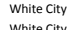 & Jackson \\
\hline $\begin{array}{l}550 \text { B Dutton } \\
\text { Crater lake Hiphway }\end{array}$ & $\begin{array}{l}\text { Whitit City } \\
\text { white city }\end{array}$ & 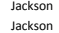 \\
\hline $\begin{array}{l}\text { Crater rateke Higwway } \\
\text { Crater Lake Highway }\end{array}$ & $\begin{array}{l}\text { Whitit City } \\
\text { White city }\end{array}$ & $\begin{array}{l}\text { Jackson } \\
\text { Jackson }\end{array}$ \\
\hline Crater Lake Highway & White city & Jackson \\
\hline Crater Lake Highway & White city & Jackson \\
\hline $\begin{array}{l}\text { Crater Lake Highway } \\
\text { p.e Box 258 Dutton }\end{array}$ & $\begin{array}{l}\text { Whitit City } \\
\text { white city }\end{array}$ & 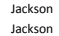 \\
\hline 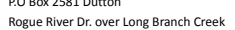 & $\begin{array}{l}\text { White city } \\
\text { Shady Cove }\end{array}$ & $\begin{array}{l}\text { Jacsson } \\
\text { Jackson }\end{array}$ \\
\hline 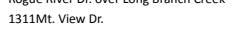 & Eagle Point & Jackson \\
\hline 740 Greenleaf Or & Eagle Point & Jackson \\
\hline $\begin{array}{l}\text { South Pacific Highway } 99 \\
\text { Sent }\end{array}$ & $\begin{array}{l}\text { Phoenix } \\
\text { Phoenix }\end{array}$ & $\begin{array}{l}\text { Jacksson } \\
\text { acckson }\end{array}$ \\
\hline 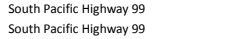 & $\begin{array}{l}\text { P. Phoenix } \\
\text { Phoenix }\end{array}$ & $\begin{array}{l}\text { Jackson } \\
\text { Jackson }\end{array}$ \\
\hline South Pacticich ighwway 99 & Phoenix & \\
\hline South Pacficic Highway 99 & Phoenix & Jackson \\
\hline $\begin{array}{l}\text { Birch } \\
\text { Sulth Pacifictiohway } 99\end{array}$ & $\begin{array}{l}\text { Phoenix } \\
\text { Phoenix }\end{array}$ & $\begin{array}{l}\text { Jackson } \\
\text { ackscon }\end{array}$ \\
\hline $\begin{array}{l}\text { th Pacifici ilihhway } 99 \\
\text { th Pacifici }\end{array}$ & $\begin{array}{l}\text { Phoenix } \\
\text { Phoenix }\end{array}$ & $\begin{array}{l}\text { Jackson } \\
\text { Jackson }\end{array}$ \\
\hline 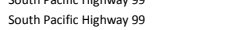 & $\begin{array}{l}\text { Pronix } \\
\text { Phoenix }\end{array}$ & $\begin{array}{l}\text { Jackson } \\
\text { Jackson }\end{array}$ \\
\hline Northwest of well & Shady Cove & $\begin{array}{l}\text { Jackson } \\
\text { Jackon }\end{array}$ \\
\hline 3525 Rogue River Drive & Shady Cove & Jackson \\
\hline in Paccific Highwway 99 & oenix & Jackson \\
\hline River Drive & $\begin{array}{l}\text { Shady Cove } \\
\text { Phhenix }\end{array}$ & $\begin{array}{l}\text { Jackson } \\
\text { accacon }\end{array}$ \\
\hline $\begin{array}{l}\text { h Pacacfic Cighway } 99 \\
\text { h Pacific Cighway } 99\end{array}$ & $\begin{array}{l}\text { Phonenix } \\
\text { Phoenix }\end{array}$ & $\begin{array}{l}\text { Jackson } \\
\text { Jackson }\end{array}$ \\
\hline ughout DWPA & $\begin{array}{l}\text { ofich } \\
\text { aby cove }\end{array}$ & $\begin{array}{l}\text { Jackson } \\
\text { Jackson }\end{array}$ \\
\hline ughout DWPA & Shady Cove & Jackson \\
\hline
\end{tabular}

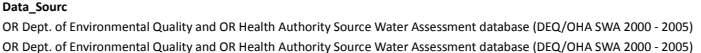

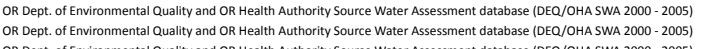

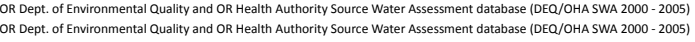

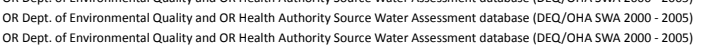

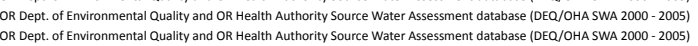
OR. Dept. of Environmental Quality ynd

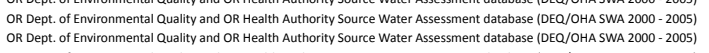

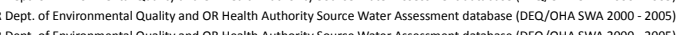

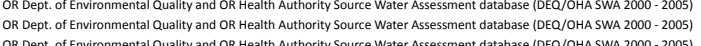

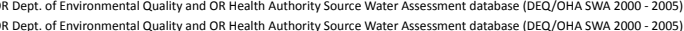

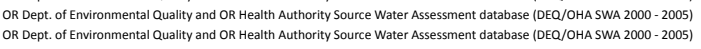

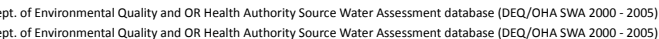

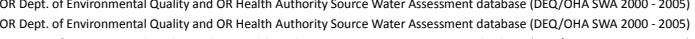

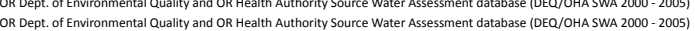
OR Dept. of Environmental Q Quality and OR Heath Authority Source Water Assessment database (DEQ/OHA SWA 2000- 2005)

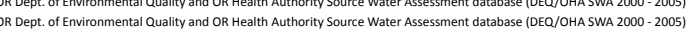
OR Dept. of Environmental Q Quality and OR Heath Authority Source Water Assessment database (DEQ/OHA SWA 2000- 2005) OR Dept. of Environmental Q Quality ynd OR Heath Authority Source Water ASSessment database (DEQ//OHA SWA 2000- 2005)

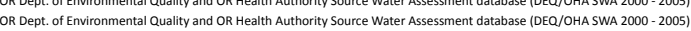

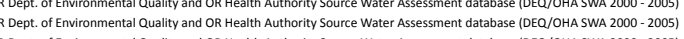
OR Dept. of Environmental Quality and OR Heath Authority S Surce Water A Asessment datababse (DER//OHA SWA 2000- 2005)

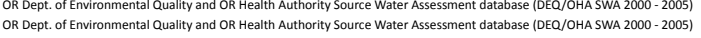

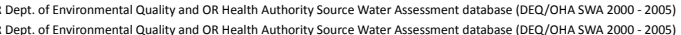

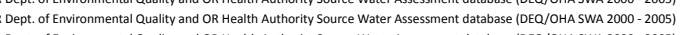

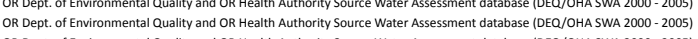
OR Dept. of Environmental Q Quality and OR Heath Authority Source Water AsSessment database (DEE//OHA SWA 2000-2005) OR Dept. of Environmental Q Quality and OR Heath Authority Source Water Assessment database (DER//OHA SWA 2000- 2005)

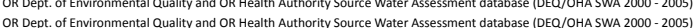
OR Dept. of Environmental Quality and OR Health Authority Source Water Assessment database (DEQ/OHA SWA 2000- 2005)

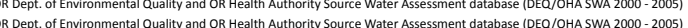

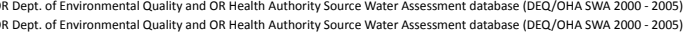

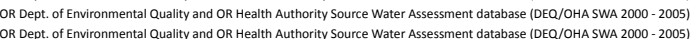

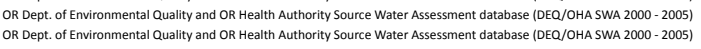

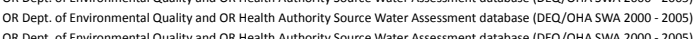
(2)

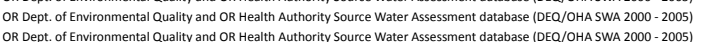

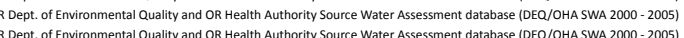
OR Dept. of Environmental Q Quality and OR Heathth Authority Source Water A Asessment database (DER/OHA SWA 2000 - 2005) OR Dept. of Environmental Q Quality and OR Health Authority S Surce Water Assessment database (DEQ/OHA SWA $2000-2005)$ OR Dept. of Environmental Q Quality and OR Heath Authority Source Water A Assessment database (DEQ/OHA SWA 2000- 2005)

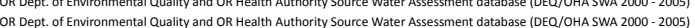
OR Dept. of Environmental Q Quality and OR Heath Authority Source Water Assessment database (DEQ/OHA SWA 2000- 2005)

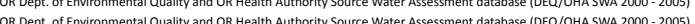

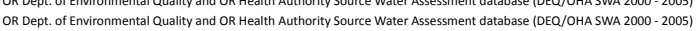

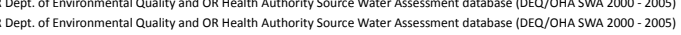

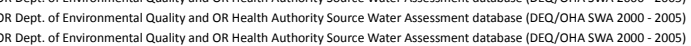

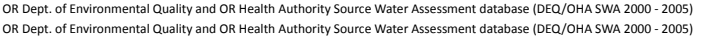

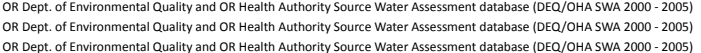

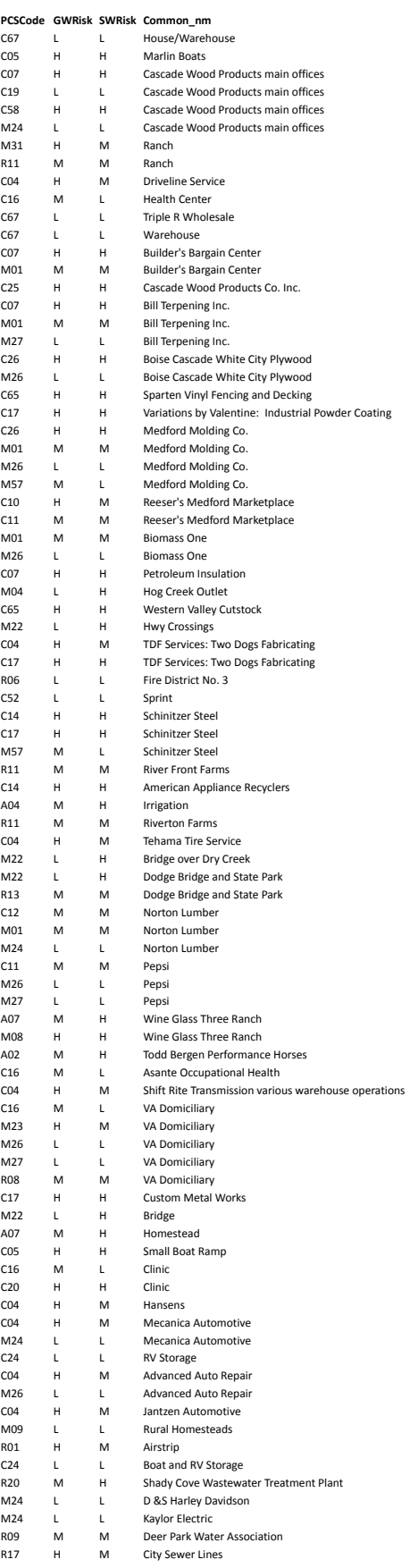

pCSType

oat Services/Repair/Refinishing office Buildings/Complexes UST-Decommissioned - Inactive
large Capacity Septic Systems - Class v VuC (serves 220

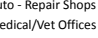

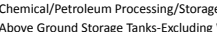
Above Ground Storage Tanks: Excll
Wood Presering/Treating

Chemical/Petroleum Processing/storage Above Ground Storage Tanks-Excluding Water Tanks and Residential ASS Wood/Pulp/Paper Processing and Mills UST - Confirmed Leaking but listed as N NA - DEQ L UST List Miscellaneous Manutacturing Wood/Pul//Paper Processing and Mills

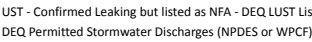
Fleet/Trucking//us Terminals

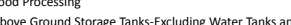
Water Tanks and Residential ASTs
NA - DEQ LuST List UST-Confirmed Leaking but isted as NFA- Did Stormwater Outfalls Processin rransportation Corridorts - Stream Crossing - Perennia Auto- Repair Shops
Metal lating/finishing/Fabrication Fire Station
Communications office
Sech Unk//crap/Salvage Yards DEQ Permitted Stormwater Discharges (NDDES or WPCF) Lawn Care - Highly Maintained Areas
Junk/Scrap/Salvage Yards Crops - Irrigated-Berries, Hops, Min Lawn Care - Highth Maintained Areas

Transportation Corridors- Stream Crossing - Perennia
Transportation Corridors - Stream Crossing- Perennia Parks
Funniture/Lumber/Parts Stores Above Ground Storage Tanks-Ex
UST - Decommissioned Inactive

UST - Confirmed Leaking but listed as NFA - DEQ L LST Lis

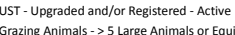

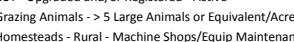

Boarding stables
Medical Net offices
Net

Nedical/Net offices

UST-Confirmed Leaking Tanks- DEQ UST List UST - Upgraded and/or Registered - Active
Golf Courses teal Patatin//inishing/Fabrication Janspon ation Coriduors-Stream Crosing - Perennial
Grazing Animals $\rightarrow 5$ Large Animals or Equivalent/Acre Boat Serices/Repair/Refinishing
Medicalvet offices

Parking Lots/Malls $\rightarrow 50$ spaces Auto - Repair Shops
Auto - Repair Shops UST - Decommissioned
Rv/Mini totrage
Auto - Repair Shops

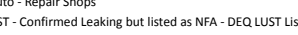
Homesteads - Rural- Septic Systems < 1 /Acre Airport- Maintenance//Uueling Area
RV/Mini Storage

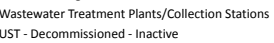
UST- Decommissioned - Inactive
UST - Decommissioned- Inactive

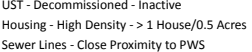




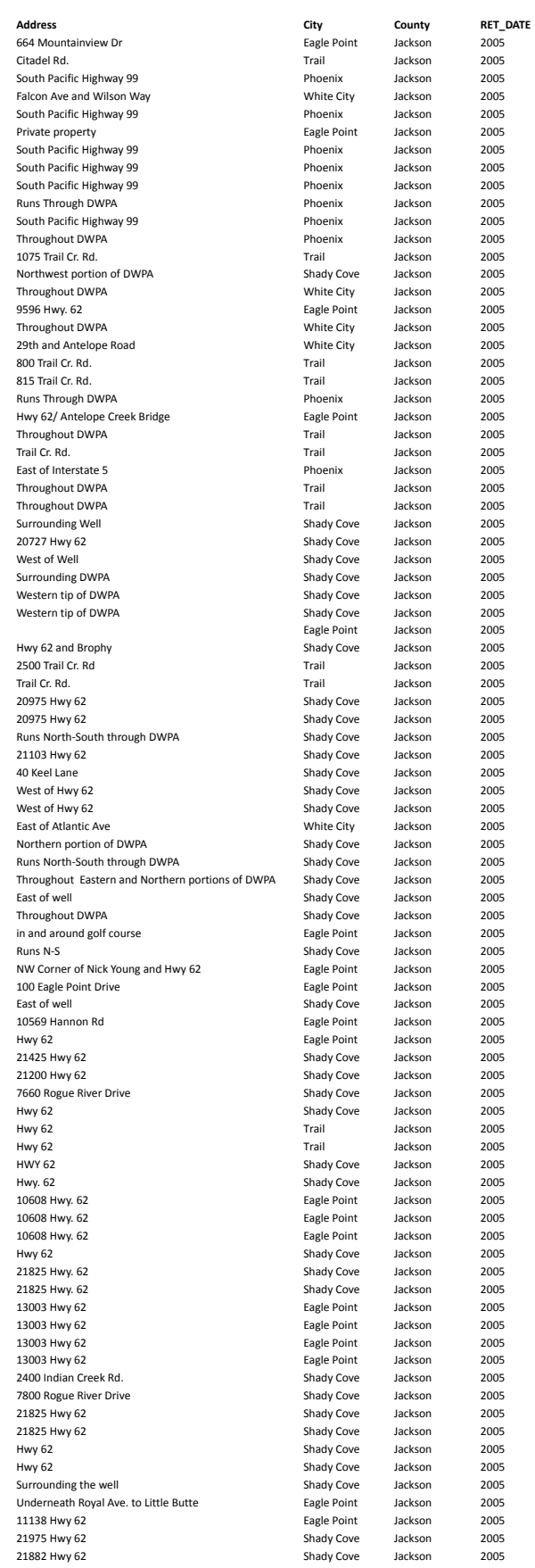

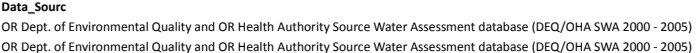

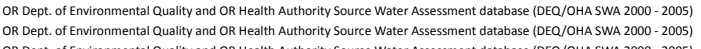

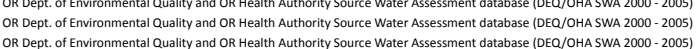

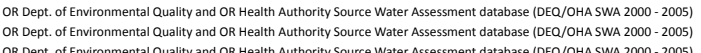
(2)

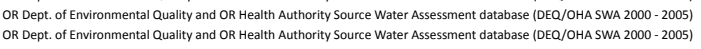

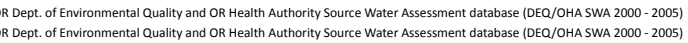

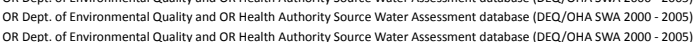

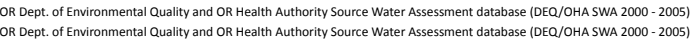

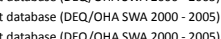

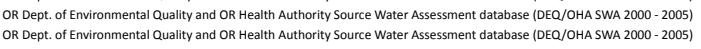

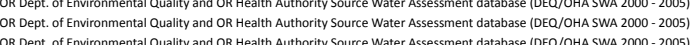

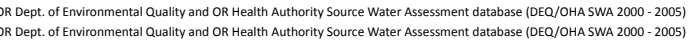

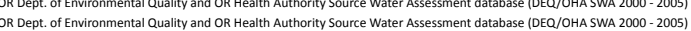
OR Dept. of Environmental Q Quality and OR Heath Authority Source Water A Asesssment database (DEQ/OHA SWA 2000- 2005)

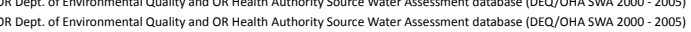
OR Dept. of Environmental Quality and OR Health Authority SOurce Water Assessment database (DEQ/OHA SWA 2000- 2005) OR Dept. of Environmental Q Quality and OR Heath Authority Source Water ASSessment database (DEE//OHA SWA $2000-2005)$

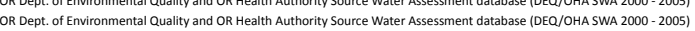

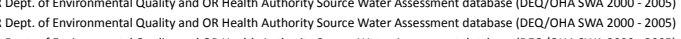
OR Dept. of Envirommental Quality and OR Heath Authority Source Water Asesssment datatabse (DEG//OOAA SWA 2000- 2005) OR Dept. of Environmental Quality and OR Health Authority SOurce Water Assessment database (DEQ//OHA SWA 20000 - 2005)

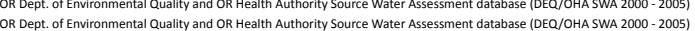

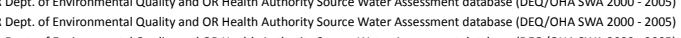

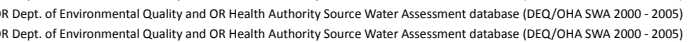
OR Dept. of Environmentatal Quality and OR Heath Authority Source Water Assessment database (DEE//OHA SWA 2000- 2005) OR Dept. of Environmental l Quallity nand OR Heath Authority Source Water Assessment database (DEE/OHA SWA 2000 - 2005)

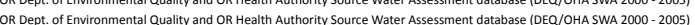

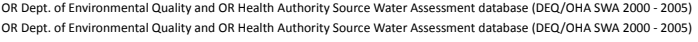

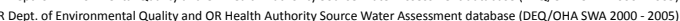

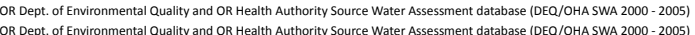

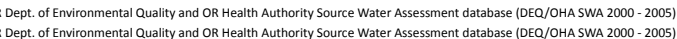

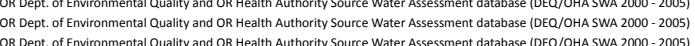
OR Dept. of Environmental Quality and OR Heath Authority Source Water A Ases Sment database (DEC/OHA SWA 2000-2005)

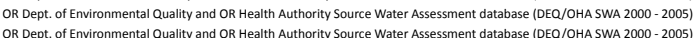

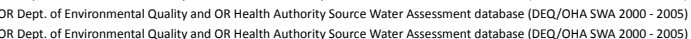
$\begin{array}{ll}2 & \end{array}$ OR Dept. of Environmental Quality and OR Health Authority SOurce Water Assessment database (DEQ/OHA SWA 2000- 2005) OR Dept. of Environmental Q Quality and OR Heath Authority Source Water ASSEssment database (DEQ/OHA SWA 2000-2005) OR Dept. of Environmental Q Quality and OR Heath Authority Source Water A Assessment databasese (DEQ/OHA SWA 2000 - 2005) OR Dept. of Environmental Q Quality and OR Heath Authority Source Water A Assessment database (DEQ/OHA SWA 2000- 2005)

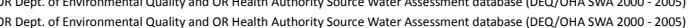
OR Dept. of Environmental Q Quality and OR Heath Authority Source Water Assessment database (DEQ/OHA SWA 2000- 2005)

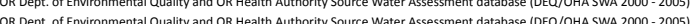
OR Dept. of Environmental Q Quality and OR Health Authority Source Water Assessment database (DEQ/OHA SWA 2000- 2005)

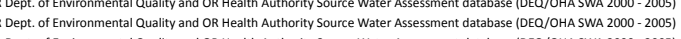

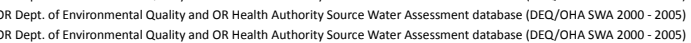

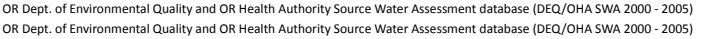

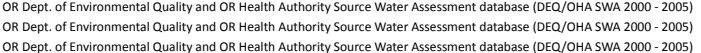

\begin{tabular}{|c|c|c|c|}
\hline & & & \\
\hline $\begin{array}{l}\text { Pcscod } \\
\text { c18 }\end{array}$ & & k swRis & $\begin{array}{l}\text { commonnm } \\
\text { Dirt/fravel }\end{array}$ \\
\hline A07 & M & & Grazing Animals \\
\hline $\mathrm{C}_{24}^{24}$ & t & t & 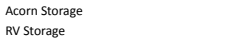 \\
\hline c16 & M & & $\begin{array}{l}\text { Phoenix Animal Hospital } \\
\text { Pon }\end{array}$ \\
\hline${ }_{2024}$ & ${ }_{L}^{M}$ & & $\begin{array}{l}\text { The whole aree around Voung Hill } \\
\text { Unkkonn o Oeretaion }\end{array}$ \\
\hline & m & & $\begin{array}{l}\text { Unknownw operation } \\
\text { Unknown Operation }\end{array}$ \\
\hline ס18 & H & & Jactson RV P Parts and Service \\
\hline & ${ }_{L}^{M}$ & & $\begin{array}{l}\text { South Pacific Aighway } 99 \\
\text { Vallay }\end{array}$ \\
\hline 09 & м & m & $\begin{array}{l}\text { Valey yotrallil } \\
\text { High Density Housing }\end{array}$ \\
\hline $\begin{array}{l}\text { noo } \\
\text { no9 }\end{array}$ & ${ }_{L}^{H}$ & & $\begin{array}{l}\text { Homestead } \\
\text { Rural Homesteads }\end{array}$ \\
\hline во9 & м & M & $\begin{array}{l}\text { Rurar Homesteads } \\
\text { High Density Housing }\end{array}$ \\
\hline c04 & H & M & $\begin{array}{l}\text { Watts Repair \& Welding } \\
\text { Wain }\end{array}$ \\
\hline 14 & ${ }_{H}^{H}$ & ${ }_{H}^{M}$ & $\begin{array}{l}\text { Sewer ines } \\
\text { scrapyard }\end{array}$ \\
\hline$c_{13}$ & H & & $\begin{array}{l}\text { Homestead } \\
\text { Host }\end{array}$ \\
\hline M18 & $\begin{array}{l}\mathrm{H} \\
\mathrm{M}\end{array}$ & ${ }_{H}^{H}$ & $\begin{array}{l}\text { Homestead } \\
\text { Intrestate } 5\end{array}$ \\
\hline M22 & L & & Hwy $62 /$ Antelope Creek Bridge \\
\hline $\begin{array}{ccc}\text { Mog } \\
\text { con }\end{array}$ & $\mathrm{L}_{\mathrm{H}}$ & L & $\begin{array}{l}\text { Rural Homes } \\
\text { RockPiles }\end{array}$ \\
\hline A04 & M & & $\begin{array}{l}\text { Rock Piles } \\
\text { IIritated Crops }\end{array}$ \\
\hline Mo9 & L & i & $\begin{array}{l}\text { Iritrated Crops } \\
\text { Rural Homes }\end{array}$ \\
\hline $\begin{array}{l}\text { R. } \\
\text { Ro9 }\end{array}$ & $M$ & M & $\begin{array}{l}\text { Rural Homes } \\
\text {. }\end{array}$ \\
\hline & & & $\begin{array}{l}\text { Leisure Day Mobile Home Park } \\
\text { Shayd Acres striel Park }\end{array}$ \\
\hline & m & M & $\begin{array}{l}\text { Shady Acres Sraler Park } \\
\text { Cabinitry Shop }\end{array}$ \\
\hline s & m & m & Shady Acres Trailer Park \\
\hline & н & & city Sewer Lines \\
\hline & H & M & city Sewer Lines \\
\hline & M & $\begin{array}{l}H \\
M\end{array}$ & $\begin{array}{l}\text { Irrigation Canal } \\
\text { Paradise Mobile Home Community }\end{array}$ \\
\hline & M & M & $\begin{array}{l}\text { Wilding Creations furniture } \\
\text { Whention }\end{array}$ \\
\hline $\begin{array}{c}\text { Mo8 } \\
022\end{array}$ & $\stackrel{H}{M}$ & H & $\begin{array}{l}\text { Homestead } \\
-\end{array}$ \\
\hline & L & t & 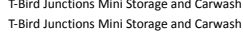 \\
\hline & M & ${ }_{H}^{H}$ & Highway 62 \\
\hline co4 & ${ }_{H}^{\mathrm{H}}$ & ${ }_{M}^{H}$ & $\begin{array}{l}\text { U-save Rentat EGuipment and Surf } \\
\text { David Y Young General Repair }\end{array}$ \\
\hline M61 & H & H & High Density Housing \\
\hline & $c^{M}$ & & $\begin{array}{l}\text { High Density Housing } \\
\text { Homes }\end{array}$ \\
\hline & ᄂ & ᄂ & Rural Homesteads \\
\hline & M & ${ }^{\mathrm{H}}$ & Highway 62 20 \\
\hline & & & $\begin{array}{l}\text { Homes } \\
\text { Hemal Homesteads }\end{array}$ \\
\hline 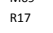 & H & M & $\begin{array}{l}\text { Rurar Homestseads } \\
\text { Sewer Lines }\end{array}$ \\
\hline 10 & m & m & $\begin{array}{l}\text { Eagle Point residential neighborhood } \\
\text { Eate }\end{array}$ \\
\hline M18 & M & н & Huy 62 \\
\hline Ro8 & $H$ & $\mathrm{M}$ & $\begin{array}{l}\text { Repair shop } \\
\text { Eaple Point Gels couse. }\end{array}$ \\
\hline Mog & $\mathrm{L}$ & & $\begin{array}{l}\text { Eagle Point Goif Course } \\
\text { Rural Homesteads }\end{array}$ \\
\hline & M & H & 3M Goat Farm \\
\hline $\begin{array}{c}\text { M18 } \\
004\end{array}$ & ${ }_{H}^{M}$ & $\stackrel{H}{M}$ & 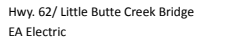 \\
\hline RO6 & & L & Jackson County Fire District No.4 \\
\hline & M & m & Shady Cove Park \\
\hline M31 & $\mathrm{H}$ & M & $\begin{array}{l}\text { Shadd Cove Minis itorage } \\
\text { Country View MH Estates }\end{array}$ \\
\hline R09 & M & M & Country View MH Estates \\
\hline 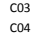 & ${ }_{H}^{H}$ & $\mathrm{M}$ & 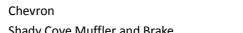 \\
\hline${ }^{03}$ & $\mathrm{H}$ & M & $\begin{array}{l}\text { hharyy Cove Muttrer and brake } \\
\text { ARCO Gas Station }\end{array}$ \\
\hline$M_{24}$ & L & L & ARCO Gas Station \\
\hline$C 24$ & & & $\begin{array}{l}\text { ARCO Gas Station } \\
\text { Fyy Caster's PV Park }\end{array}$ \\
\hline c04 & H & M & Shady Cove Automotive \\
\hline & H & & Shady Cove Automotive \\
\hline & & 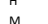 & $\begin{array}{l}\text { Willamette Egg Farm } \\
\text { willametre fer farm }\end{array}$ \\
\hline & 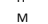 & 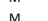 & $\begin{array}{l}\text { Willamette Egg Farm } \\
\text { Whillmette Ego Farm }\end{array}$ \\
\hline & & & $\begin{array}{l}\text { Willametete Egg Farm } \\
\text { Werm }\end{array}$ \\
\hline & & & Indian Creek Mini Storag \\
\hline & 4 & n & Edgewater Inn \\
\hline & $\mathrm{H}$ & 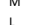 & $\begin{array}{l}\text { Texaco } \\
\text { Texaco }\end{array}$ \\
\hline c02 & M & M & $\begin{array}{l}\text { Texaco Wash } \\
\text { The Car Wash }\end{array}$ \\
\hline & H & H & Speed Queen Coin Laundry \\
\hline & M & ${ }_{\mathrm{H}}^{\mathrm{M}}$ & $\begin{array}{l}\text { Shad Cove MHP } \\
\text { Canal dumps into intle Butte Creek }\end{array}$ \\
\hline & M & ᄂ & Bear Creek Clinic Family Practice Me \\
\hline & ${ }_{\mathrm{H}}^{\mathrm{H}}$ & M & $\begin{array}{l}\text { Jerry Shale pit } \\
76 \text { Gas station }\end{array}$ \\
\hline
\end{tabular}

PCSType

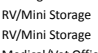
Housing - High Density $\rightarrow 1$ House//.5 Acres usT- Decommisisioned - Inach

Auto- Repair Shops

.

Housing - High Density $>1$ House/.5. A Acres

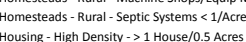

Auto- Repair Shops

Sewer Lines - Close Proximity to PUS

Home Manufacturing

Homesteads- Rural - Machine Shops/Equip Maintenance

ther heary use roads

Homesteads- Rural- Septic Systems $<1$ /Acre

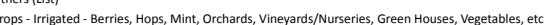

Homesteads - Rural- Septic Systems < 1/Acre

Aboverround Storage Tanks - Residential
Housing - High Density $\rightarrow 1$ House/0.5 Acres

furniture/Lumber/Parts Stores

Housing- High Density $\rightarrow 1$ House/ 0.5 . Acres
Sewer Lines - Close Proximity to PWs

Higation Canal/Pond
Housing - High Density $>1$ House/0.5 Acts

(a)

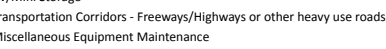

Auto- Repair Shops
Wells - Abandoned

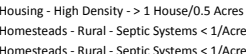

Fansportation Corridors- Freeways/Highways

Homesteads- Rural - Septic Systems $<1 /$ Acre
Homesteads - Rural - Septic Systems $<1 /$ Acre

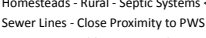

Lawn Care-Highty Maintained Areas Transportation Corrd
Auto- Repair Shoops

Solf Courses
Homesteads - Rural - Septic Systems $<1$ 1/Acre

Gazing An- als $>5$ L arge Animals or Equivalent/Acre

Auto- Repair Shops
Fire Station

Fire Station
Parks

Ni/Minis

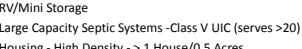

Auto Gas Stations
Auto - Repair Shops

Uuto - Gas stations
UST Decommissioned - Inactive
UST - Upgraded and/or Regitered - Active

RVyMini storage

Auto- Repari Shops
UST- Confirmed Leaking but listed as NFA- DEQ LUST List

Confined Animal Feeding Operations (CAFOS)

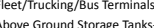

bove Ground Storage Tanks-Excluding Water Tanks and Residential ASts

RV/Mini Storage

Marking Lots/Malls $\rightarrow 50$ Spaces
atto- Gas Stations

UST - Upgraded and/or Registered - Active

Auto - Car Washes
Others (list)

Husing - - High Density > $>1$ House 0.5 Acres

Irrigation Canal/Pond
Medical/vet offices

Medicall(Net offices
others (ust) 


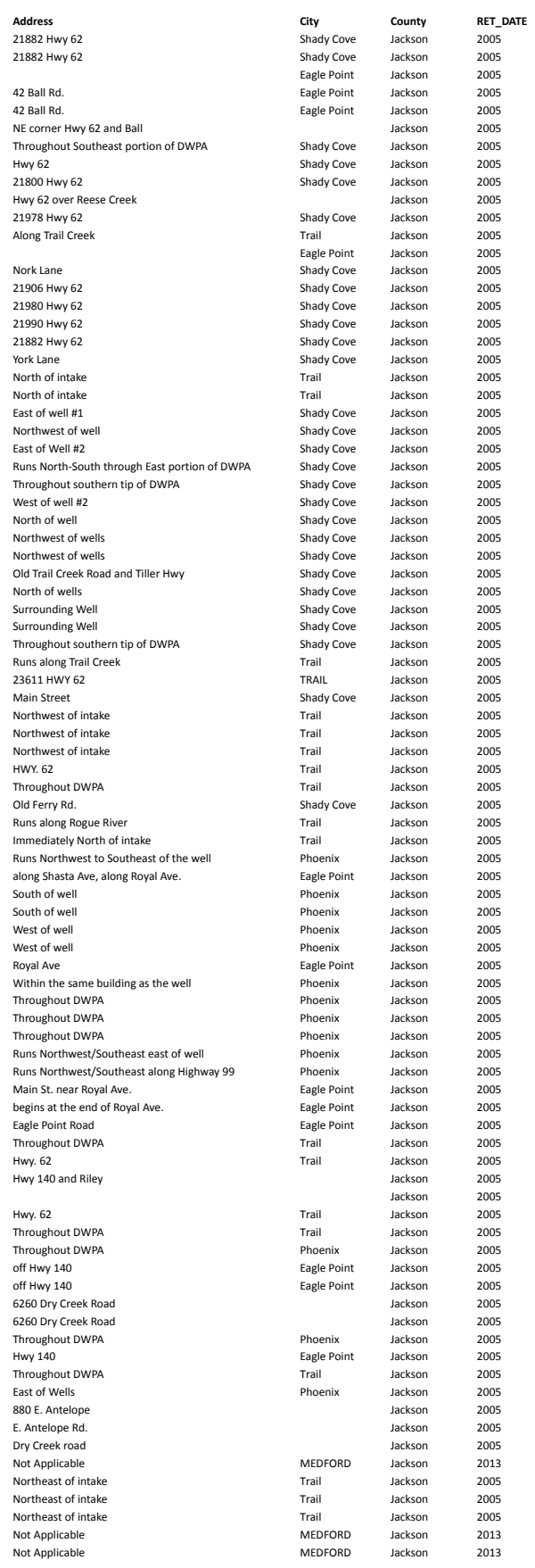

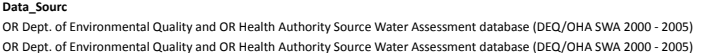

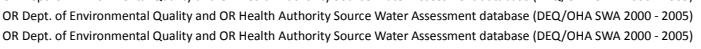

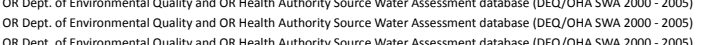

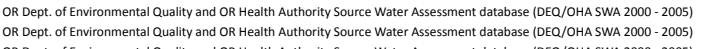

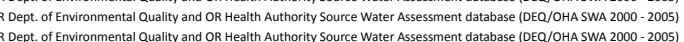

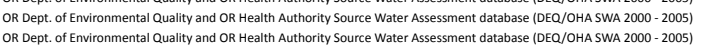

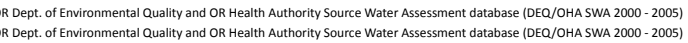

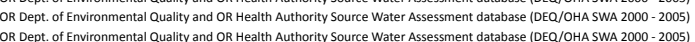

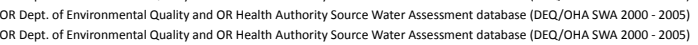
(D)

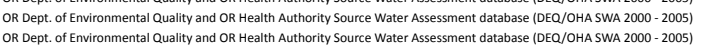

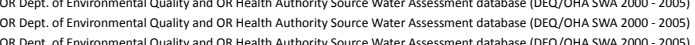

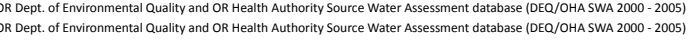

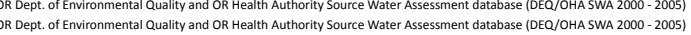
OR Dept. of Environmental Q Quality and OR Heath Authority Source Water ASSessment database (DEQ/OHA SWA 2000- 2005)

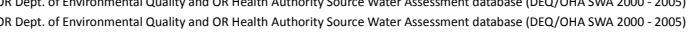
OR Dept. of Environmental Q Quality and OR Heath Authority Source Water Assessment database (DEQ/OHA SWA 2000- 2005) OR Dept. of Environmental Q Quality ynd OR Heath Authority Source Water ASSessment database (DEQ//OHA SWA 2000- 2005)

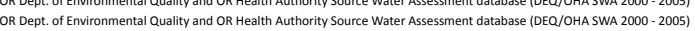

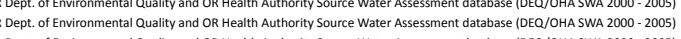
OR Dept. of Envirommental Quality and OR Heath Authority Source Water Asesssment datatabse (DEG//OOAA SWA 2000- 2005)

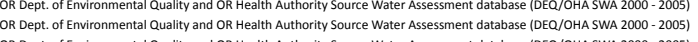

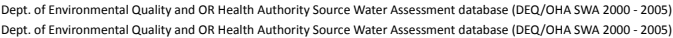

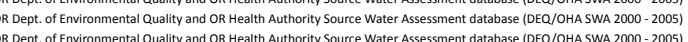
OR Dept. of Environmental a luality and OR Health Authority S Surce Water A Asessment database (DEQ/OHA SWA 2000 - 2005) OR Dept. of Environmental Q Quality and OR Heath Authority Source Water Assessment database (DEE//OHA SWA 2000 - 2005) OR Dept. of Environmental a uality and OR Heath Authority Source Water Assessment database (DEC//OHA SWA 2000-2005)

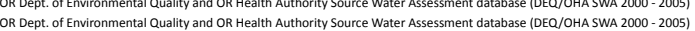

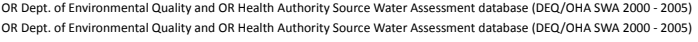

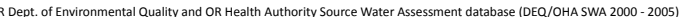

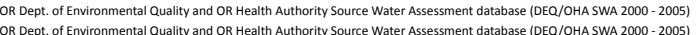

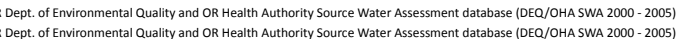

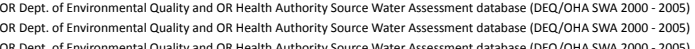

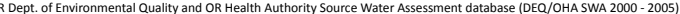

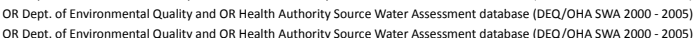

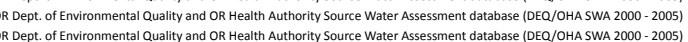

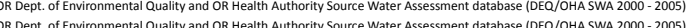
OR Dept. of Environmental Q Quality and OR Heath Authority S Surce Water Assessment database (DEQ/OHA SWA 2000- 2005) OR Dept. of Environmental Q Quality ynd OR Heath Authority Source Water ASSessment database (DEQ//OHA SWA $2000-2005)$

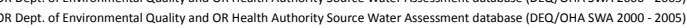
OR Dept. of Environmental Q Quality and OR Heath Authority Source Water Assessment database (DEQ/OHA SWA 2000- 2005)

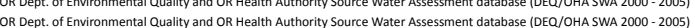
OR Dept. of Environmental Q Quality and OR Heath Authority Source Water Assessment database (DEQ/OHA SWA 2000- 2005)

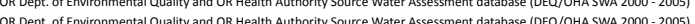

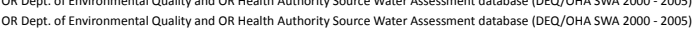

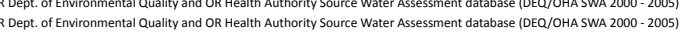

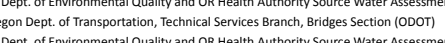

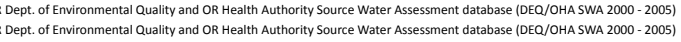
D.
D.

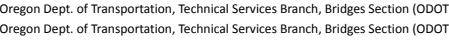

\begin{tabular}{|c|c|c|c|}
\hline $\begin{array}{l}\text { PScodi } \\
\text { M24 }\end{array}$ & e GWRisk & sweisk & $\begin{array}{l}\text { commonn } n \text { m } \\
76 \text { Gas Station }\end{array}$ \\
\hline M27 & i & i & 76 Gas station \\
\hline $\begin{array}{l}\text { C12 } \\
\text { M58 }\end{array}$ & ${ }_{M}^{M}$ & $M_{L}^{M}$ & $\begin{array}{l}\text { Oregon West Lumber Sales } \\
\text { Southerm Oregon Sanatition, Inc. }\end{array}$ \\
\hline R19 & & & Southern Oregon Sanitation, Inc. \\
\hline c24 & L & L & Ball Rd. Mini Storage \\
\hline 1222 & ${ }_{\mathrm{H}}^{\mathrm{H}}$ & ${ }_{\mathrm{H}}^{\mathrm{M}}$ & $\begin{array}{l}\text { Citis Sewer Lines } \\
\text { Shady Cove Bridge }\end{array}$ \\
\hline c24 & L & ᄂ & Rogue River RV Park \\
\hline $\begin{array}{c}\text { M22 } \\
\mathrm{C}_{16}\end{array}$ & L & & $\begin{array}{l}\text { Bridge } \\
\text { Family Dentistry }\end{array}$ \\
\hline c14 & & H & Junk/Scrap/Salvage \\
\hline M22 & L & & $\begin{array}{l}\text { Hog creek bridge } \\
\text { wis Site }\end{array}$ \\
\hline c20 & H & H & Royal Coachman Motel \\
\hline 16 & M & & Upper Rogue Eve Care \\
\hline & M & & $\begin{array}{l}\text { Shady Cove Medical Center } \\
\text { Paxifiperide Compercil }\end{array}$ \\
\hline & & & $\begin{array}{l}\begin{array}{l}\text { Pacific } \\
\text { Lustide Commercial fueling }\end{array} \\
\text { tuste }\end{array}$ \\
\hline קمe9 & M & & $\begin{array}{l}\text { LUTigh Site } \\
\text { High Density Housing }\end{array}$ \\
\hline R16 & H & & High Density Housing \\
\hline $\mathrm{c} 12$ & M & M $>2>>$ & Shady Cove Hardware \\
\hline c16 & M & L & $\begin{array}{l}\text { Shady Cove Medical Group } \\
\text { CaWose }\end{array}$ \\
\hline Co2 & M & & $\begin{array}{l}C_{\text {ar Wash }} \\
\text { Highway } 62\end{array}$ \\
\hline & & F & \\
\hline & M & & Shady Cove Meticial Center \\
\hline & & & 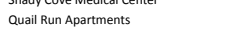 \\
\hline M24 & ᄂ & L & Shady Cove Middle School \\
\hline R15 & ᄂ & & Shady Cove Middle School \\
\hline $\begin{array}{l}\text { No2 } \\
\text { R02 }\end{array}$ & t & $H$ & $\begin{array}{l}\text { Bridge } \\
\text { Ouyian Run Apartments }\end{array}$ \\
\hline & L & & $\begin{array}{l}\text { Quallilun Appratments } \\
\text { Shady Cove Middle School }\end{array}$ \\
\hline R15 & 亡 & $M$ & 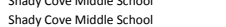 \\
\hline Ro9 & M & m & 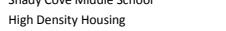 \\
\hline & $\mathrm{m}$ & H & Highway 227 \\
\hline R20 & & & COUNTRY VEWW MOBLIE HOME ESTATES \\
\hline 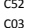 & $\mathrm{L}^{H}$ & $M$ & $\begin{array}{l}\text { Sprint Communications } \\
\text { John's Trailis Marate }\end{array}$ \\
\hline M24 & i & in & $\begin{array}{l}\text { John's Trails Market } \\
\text { John's Trails Market }\end{array}$ \\
\hline & t & t. & John's Trails Market \\
\hline M22 & i & H & Trail Creek Bridge \\
\hline мо9 & 2 & in $>2+2$ & Rural Homesteads \\
\hline Ro9 & M & M & $\begin{array}{l}\text { High Density Housing } \\
\text { Hilahway } 62\end{array}$ \\
\hline 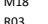 & (n) & ti & 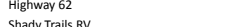 \\
\hline 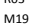 & $M$ & $\mathrm{H}$ & $\begin{array}{l}\text { Shady Iralls } \mathrm{s} \text { V } \\
\text { Railroad Tracks }\end{array}$ \\
\hline R11 & $m$ & M $>>>2$ & 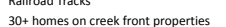 \\
\hline A05 & L & & 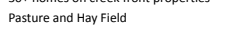 \\
\hline & M & & Pasture and Hay Field \\
\hline $\begin{array}{l}\text { A } 252 \\
\text { M01 }\end{array}$ & $\mathrm{M}$ & H & Irrigation Pond \\
\hline R13 & M & M & 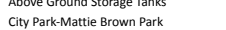 \\
\hline & & M & Rising Sun Farms \\
\hline M09 & 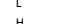 & L & Homestead-Rural \\
\hline & 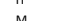 & & Homestead-Rural \\
\hline${ }_{M 18}$ & M & H & $\begin{array}{l}\text { Vineyards } \\
\text { Hiphway } 99\end{array}$ \\
\hline R17 & H & M & $\begin{array}{l}\text { Sewer Lines } \\
\text { Sers }\end{array}$ \\
\hline M22 & L & & Main St./Little Butte Creek Bridge \\
\hline & M & H & 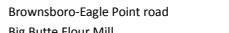 \\
\hline 1922 & ${ }_{L}^{M}$ & $\begin{array}{c}M \\
H\end{array}$ & 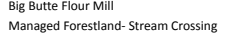 \\
\hline M22 & 2 & H & Lewis Creek Bridge \\
\hline (18 & H & H & 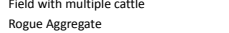 \\
\hline & L & & Bush Creek Bridge \\
\hline MOS & & L & 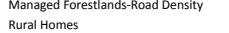 \\
\hline & m & m & Stone Ridge Golf Course \\
\hline R08 & M & M $>2>>$ & Stone Ridge Golf Course \\
\hline R10 & $\mathrm{H}$ & & $\begin{array}{l}\text { Dry Creek Landfill } \\
\text { Dry Creek tandfill }\end{array}$ \\
\hline & M & 1 & 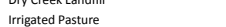 \\
\hline & m & & Boarding Faclities \\
\hline A11 & m & & Clear Cuts \\
\hline & $\mathrm{M}$ & & Irrigation Ditch \\
\hline R06 & & 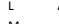 & Agate Lake Fire Station District No. 3 \\
\hline & & & $\begin{array}{l}\text { Agate lake } \\
\text { Bridepe } 531\end{array}$ \\
\hline & & & Hopkins Canal, Hwy 272 at MP \\
\hline & & $x_{1}$ & 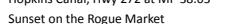 \\
\hline M24 & & & Sunset on the Rogue Market \\
\hline M27 & & L & Sunset on the Rogue Market \\
\hline$n_{122}$ & & & 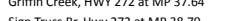 \\
\hline
\end{tabular}

PCSType

UST-Uopenissisoned- Inactive

Furniture/lumber/Parts Stores
DEQ Permitted Discharges - Domestic On-Site

Sewer Lines - Cosose Proximity to PWS
Transportation Corridors - Stream Crossing - Perennia

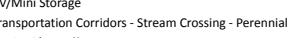

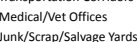

Transportation Corridors- Stream crossing- Perennial
UST - Confirmed Leaking but Isted as NAA - DEQ L LST Lis arking Lots/Malls $\rightarrow 50$ Spaces

MedicalNet offices
Meeticalvet offices

UST - Confirmed Leabis but listed as NFA- DEQ Q UST L

Housing- - High Density $\rightarrow 1$ House/0.5 Acres
Septic Systems - High Density $\rightarrow 1 /$ Acre

Medical/Net Offices
Auto - Car Wastes

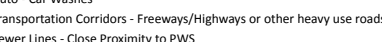

Sewer lines- closese
Medicalvet offices

Apartments and Condominiums
UST- Decommissioned- Inactive
schools

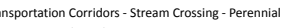

Apartments and Condominiums
UST - Decommissioned- Inactive

Schools
Housing- High Density- $>1$ House/0.5 Acres

rransportation Corrididrs - Freeways/Highways or other heavy use raads

Communications officic
Auto- Gas Stations

Auto- Gas Stations
UST- Decommissioned - Inactive

UST - Upgraded and/or Registered - Active

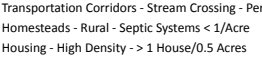

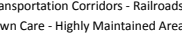

Cops - Noniririzated - Christmas as rees, Grains, Grass Seeds, Hay, Pasture

ririgation Canal/Pond
above Ground Storage Tanks-Excluding Water Tanks and Residential AS

Food Procosesing
Homesteass - Rural - Septic Systems $<1 /$ Acre

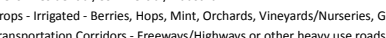

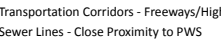

ransportation Corridorms Strteam Crossing - Perennial
reansportation Corridors - High Use Roads/Streets

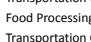

rensportatation Corridors - Stream Crossing - Perennial

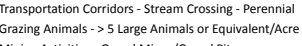

rransportation Corridors - stream Crossing - Perennia

Maraged forest tands- Road Density - 2 milsa mi

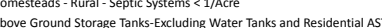

Solf Courses
DEQ Permitted

Crops - Irigigated- Berries, Hops, Mint, Orchards, Vineyards/Nursereres, Green Houses, Vegetables, etc.

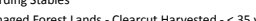

Managed forest Lands.
Irrigation Canal/Pond
Fire Station

Transportation Corridors-Stream Crossing- Perennial
Transportation Corridors - Stream Crossing - Perennial

Atto - Gas Stations
WT - Decommissioned- Inactive

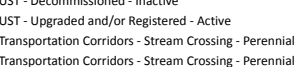




\begin{tabular}{|c|c|c|}
\hline $\begin{array}{l}\text { Address } \\
\text { Not Applicable }\end{array}$ & City & County \\
\hline $\begin{array}{l}\text { Not Appolicable } \\
\text { Not Applicable }\end{array}$ & $\begin{array}{l}\text { MEFFRD } \\
\text { MEFFRD }\end{array}$ & accksonon \\
\hline Talent Ave & Ashland & Jackson \\
\hline $\begin{array}{l}\text { Not Applicable } \\
\text { HWY. }\end{array}$ & $\begin{array}{l}\text { MEEFORD } \\
\text { Trail }\end{array}$ & ackckson \\
\hline Rogue Elk & Trail & Jackson \\
\hline Not Applicable & MEDFORD & Jackson \\
\hline 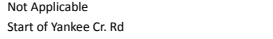 & MEFFORD & arcksonon \\
\hline $\begin{array}{l}\text { South of railroad tracks } \\
\text { westof weld to }\end{array}$ & Ashland & Jackson \\
\hline $\begin{array}{l}\text { West of well } \\
\text { Runs shrough DWPA }\end{array}$ & Ashland & Jackson \\
\hline 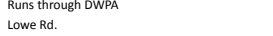 & $\begin{array}{l}\text { A Ashland } \\
\text { Ashland }\end{array}$ & $\begin{array}{l}\text { arckckon } \\
\text { acsson }\end{array}$ \\
\hline Runs through DWPA & Ashland & Iackson \\
\hline $\begin{array}{l}\text { Southwest of well } \\
\text { Corral ane }\end{array}$ & 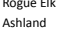 & ackckon \\
\hline $\begin{array}{l}\text { West of well } \\
\text { Whent }\end{array}$ & 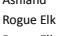 & acckson \\
\hline Northwest of well & $\begin{array}{l}\text { Sogue Elk } \\
\text { Rogut }\end{array}$ & sackson \\
\hline W. Butler Ln. & Ashland & Jackson \\
\hline $\begin{array}{l}\text { W. Butter In. } \\
\text { Trhoughout }\end{array}$ & 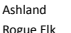 & Jackson \\
\hline $\begin{array}{l}\text { Throrghout twPA } \\
\text { Throughout DWPA }\end{array}$ & $\begin{array}{l}\text { Rogue Elk } \\
\text { Rogue Ekik }\end{array}$ & $\begin{array}{l}\text { Jackconon } \\
\text { Jackon }\end{array}$ \\
\hline southwest of well & Rogue Elk & Jackson \\
\hline & & Jackson \\
\hline $\begin{array}{l}\text { Runs west to south of the well } \\
\text { West of well }\end{array}$ & $\begin{array}{l}\text { Rogue Elk } \\
\text { Rogue Elk }\end{array}$ & $\begin{array}{l}\text { alckctson } \\
\text { aackon }\end{array}$ \\
\hline West of well & Rogue Elk & Jackson \\
\hline $\begin{array}{l}\text { North of W. Butter } L \text {. } \\
\text { Throushov Dwo }\end{array}$ & Asthand & Jackson \\
\hline $\begin{array}{l}\text { Throughout DWPA } \\
\text { Russ through PWPA }\end{array}$ & $\begin{array}{l}\text { Ashland } \\
\text { Ashland }\end{array}$ & $\begin{array}{l}\text { Jackconon } \\
\text { Jackon }\end{array}$ \\
\hline Hwy $99 \mathrm{~N}$ & Ashland & $\begin{array}{l}\text { Jackctson } \\
\text { Jackon }\end{array}$ \\
\hline South of RR Tracks & Ashland & Jackson \\
\hline South and East of well & Ashland & Jackson \\
\hline NW and SE of well & Ashland & Jackson \\
\hline $\begin{array}{l}\text { Lowe Rd. } \\
\text { Lowe Rd. }\end{array}$ & Ashland & Jackson \\
\hline $\begin{array}{l}\text { Lowe Rd. } \\
\text { Lowe Rd. }\end{array}$ & $\begin{array}{l}\text { A Ashand } \\
\text { Ashland }\end{array}$ & $\begin{array}{l}\text { Jackctson } \\
\text { Jackon }\end{array}$ \\
\hline Western edge of Ashland Country Estates & Ashland & Jackson \\
\hline South of Lowe Rd. & Ashland & Jackson \\
\hline W. Butler Lane & Ashland & Jackson \\
\hline 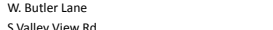 & Ashland & Jackson \\
\hline 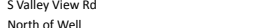 & $\begin{array}{l}\text { Ashland } \\
\text { Ahshand }\end{array}$ & Jackson \\
\hline $\begin{array}{l}\text { North or well } \\
\text { Lowe Rd. }\end{array}$ & $\begin{array}{l}\text { Asshand } \\
\text { Ashland }\end{array}$ & $\begin{array}{l}\text { Jackctson } \\
\text { Jackon }\end{array}$ \\
\hline Hwy 99N & $\begin{array}{l}\text { Ashina } \\
\text { Ashland }\end{array}$ & Jackson \\
\hline South of Well & Ashland & Jackson \\
\hline $\begin{array}{l}\text { Throughout DWPA } \\
\text { Hww y }\end{array}$ & $\begin{array}{l}\text { Ashland } \\
\text { Ashland }\end{array}$ & $\begin{array}{l}\text { Jackson } \\
\text { aackon }\end{array}$ \\
\hline $\begin{array}{l}\text { Hwy 99N } \\
\text { Rus through DWPA }\end{array}$ & $\begin{array}{l}\text { Asslalan } \\
\text { Ashland }\end{array}$ & Jackson \\
\hline 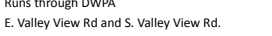 & $\begin{array}{l}\text { A Ashiand } \\
\text { Ashland }\end{array}$ & $\begin{array}{l}\text { alckctson } \\
\text { aackon }\end{array}$ \\
\hline E. Valley View Rd ands. Valley View Rd. & Ashland & Jackson \\
\hline 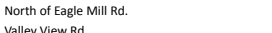 & Ashland & Jackson \\
\hline $\begin{array}{l}\text { Valley View Rd. } \\
\text { valey view Rd. }\end{array}$ & 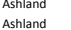 & $\begin{array}{l}\text { Jackoson } \\
\text { aackon }\end{array}$ \\
\hline $\begin{array}{l}\text { Valleyvew Kad } \\
\text { valley View Rd. }\end{array}$ & $\begin{array}{l}\text { A Ashina } \\
\text { Ashland }\end{array}$ & $\begin{array}{l}\text { Jackctson } \\
\text { Jackon }\end{array}$ \\
\hline Valley View Rd. & Ashland & Jackson \\
\hline NE Corner of East tand South Valley View Roads & Ashland & Jackson \\
\hline $\begin{array}{l}\text { Navley View Rd } \\
\text { Valley View Rd. }\end{array}$ & $\begin{array}{l}\text { Ashlalad } \\
\text { Ashland }\end{array}$ & $\begin{array}{l}\text { Jackson } \\
\text { aackon }\end{array}$ \\
\hline $\begin{array}{l}\text { Valley vivew Rd. } \\
\text { valey View Rd. }\end{array}$ & $\begin{array}{l}\text { A Ashland } \\
\text { Ashland }\end{array}$ & $\begin{array}{l}\text { alckctson } \\
\text { aackon }\end{array}$ \\
\hline Valley View Rd. & Ashland & Jackson \\
\hline $\begin{array}{l}\text { Valley View Rd. } \\
\text { Hyy 9 9N }\end{array}$ & $\begin{array}{l}\text { Ashlalad } \\
\text { Ashland }\end{array}$ & Jackson \\
\hline North of Huy $99 \mathrm{~N}$ & Ashland & Jackson \\
\hline $\begin{array}{l}\text { Hwy } \\
\text { North of Hwy } 99 \mathrm{~N}\end{array}$ & $\begin{array}{l}\text { Ashlalad } \\
\text { Ashland }\end{array}$ & $\begin{array}{l}\text { Jackson } \\
\text { azckon }\end{array}$ \\
\hline $\begin{array}{l}\text { North of HWy } 99 \mathrm{NO} \\
\text { Hwy } 999 \mathrm{~N}\end{array}$ & $\begin{array}{l}\text { A Ashiand } \\
\text { Ashland }\end{array}$ & $\begin{array}{l}\text { Jackocson } \\
\text { Jackon }\end{array}$ \\
\hline Hwy $99 \mathrm{~N}$ & Ashland & Jackson \\
\hline $\begin{array}{l}\text { Jackono Rd } \\
\text { HWy } 99 \mathrm{~N}\end{array}$ & $\begin{array}{l}\text { A Ashland } \\
\text { Ashland }\end{array}$ & $\begin{array}{l}\text { Jackson } \\
\text { Jackon }\end{array}$ \\
\hline Off Elk Creek Road & Trail & Jackson \\
\hline Off Elk Creek Road & Trail & Jackson \\
\hline Jackson Road & Ashland & Jackson \\
\hline Hwy $990 \mathrm{~N}$ & Ashland & Jackson \\
\hline $\begin{array}{l}\text { Jackson nd. } \\
\text { Hivy } 9 \mathrm{~N}\end{array}$ & $\begin{array}{l}\text { Ashlalan } \\
\text { Ashland }\end{array}$ & $\begin{array}{l}\text { Jackson } \\
\text { azckonon }\end{array}$ \\
\hline & $\begin{array}{l}\text { A Ashind } \\
\text { Ashland }\end{array}$ & $\begin{array}{l}\text { Jackonson } \\
\text { Jackon }\end{array}$ \\
\hline twy 140 & Eagle Point & Jackson \\
\hline Hwy 140 & Eagle Point & Jackson \\
\hline Jackson Rd. & Ashland & Jackson \\
\hline Hwy 140 & White City & Jackson \\
\hline & 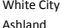 & k.son \\
\hline & & son \\
\hline & & \\
\hline ughout DWPA & Ashland & \\
\hline
\end{tabular}

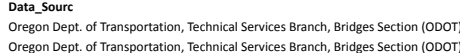

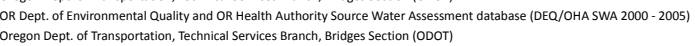

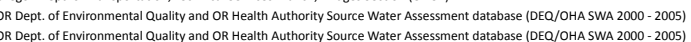
OR Dept. of Environmentata Qualitity and OR R Reath h Authority Source Water Assessm
Oregon Dept. of Transportation, Technical Services rranch, Bridges Section (ODOT)

database (DEQ/OHA SWA 2000- 2005)

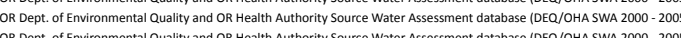

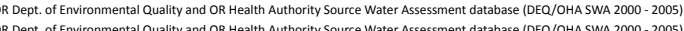

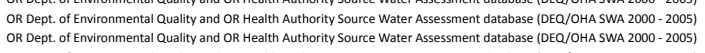
QR Dept. of Environmental Quality and OR Heath Authority Source Water Assessment database (DER/OOHA SWA 2000 - 20005

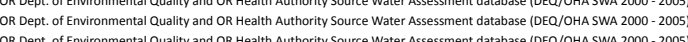
$\begin{array}{ll}2 & \end{array}$

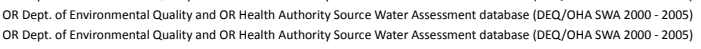

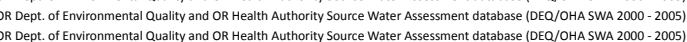

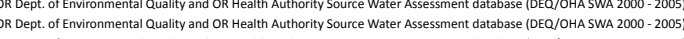

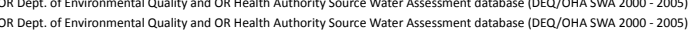
OR Dept. of Environmental Q Quality and OR Heath Authority Source Water AsSessment database (DEC/OHA SWA 2000- 2005)

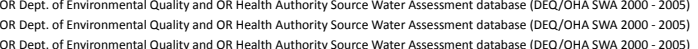
OR Dept. of Environmental Quality and OR Health Authority Source Water Assessment database (DEE//OHA SWA 2000 - 2005)

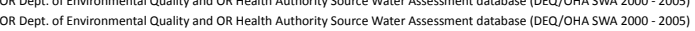

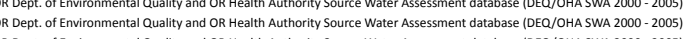
OR Dept. of Environmental Quality and OR Heath Authority Source Water Assessment tatatabse (DEQ/OOAA SWA 2000- 2005)

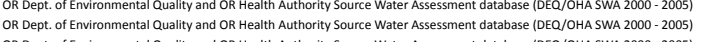

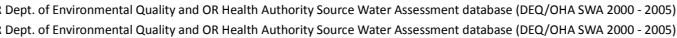

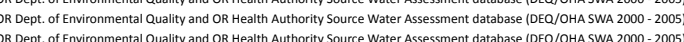

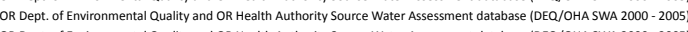

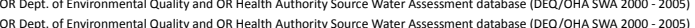
OR Dept. of Environmental Q Quality and OR Heath Authority Source Water Assessment database (DEQ//OHA SWA 2000- 2005)

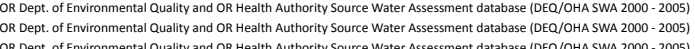

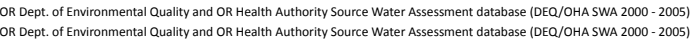

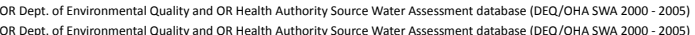

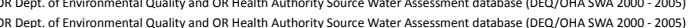

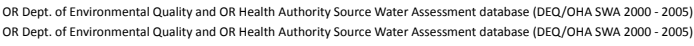

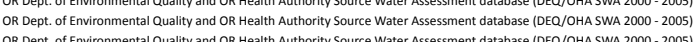

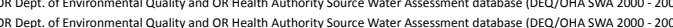
OR Dept. of Environmental auality and OR Heath Authority Source Water Assessment database (DEO/OHA SWA 2000 - 2005)

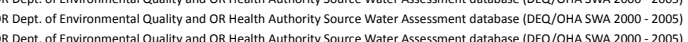
OR Dept. of Environmental l Quality and OR Health Authority S Surce Water A Asesssment database (DE//OHA SWA 2000- 2005) OR Dept. of Environmental Q Quality and OR Heath Authority Source Water A A SSessment database (DEE/OHA SWA 2000 - 2005) OR Dept. of Environmental Q Quality and OR Heath Authority Source Water Assessment database (DEQ/OHA SWA 2000- 2005)

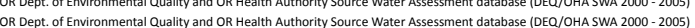
OR Dept. of Environmental Q Quality and OR Heath Authority Source Water Assessment database (DEQ/OHA SWA 2000- 2005)

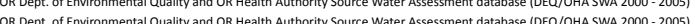

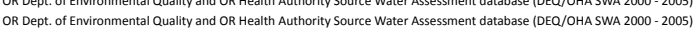

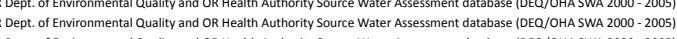

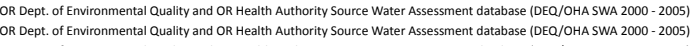

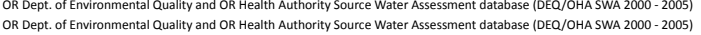

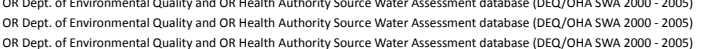

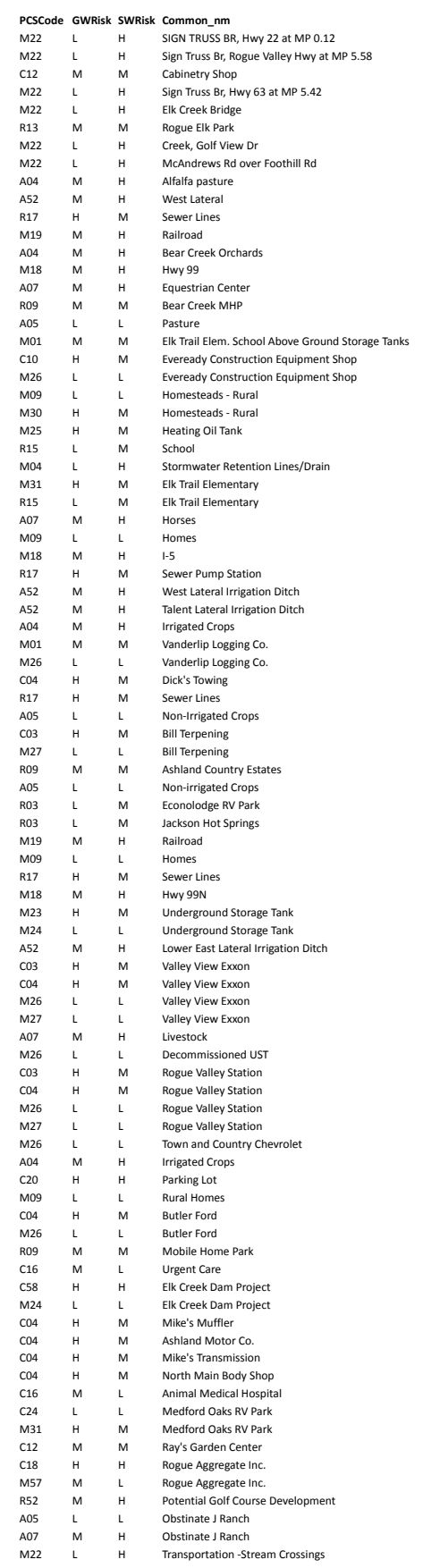

cSType

Corridors - Stream Crossing - Perennial

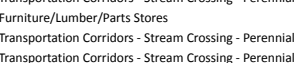
Parks
Transportation Corridors - Stream Crossing - Perennial
S Cops - Irigiated - Berries, Hops, Mint, Irizaztion Canal/Pond
Sewer Lines - Close Proximitity to PWs Crops-Irigated- Berries, Hops, Mint, Orchards, Vineyards/Nursereses, Green Houses, vegetables, etc.

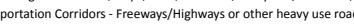

Housing - High Density $\rightarrow 1$ House/.5. A Acres

Crops - Noniririgated - Christmas Trees, Grains, Grass Seeds, Hay, Pasture
Above Ground Storage Tanks- Excluding Water Tanks and Residential ASTs Heet/Trucking//Bus Terminals

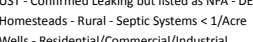

UST - Non-Regulated Tanks $<1,1,10$ gals or large heating oi t tanks schools
Stormwater Outfalls

Storrmwater Outfalls
Large Capacity Septic Systems-Class V VIC (serves 202$)$
Schools

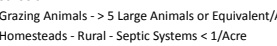
Tansportation Corridors - Freeways/Highways or other heavy use road Sewer Lines - Close Proximity to Pus Irrigation Canalpond
Irrigation Canal/Pond

Hops, Mint, Octhards, Vneyards/Wursenes, Green Houses, Vegetables, etc.

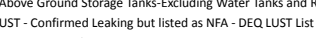

Auto- Repair Shops

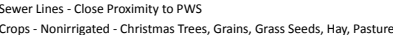
Auto - Gas Stations UST - Upgraded and/or Registered-Active

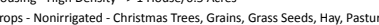

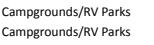

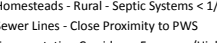

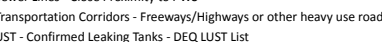
UST - Decommissioned- Inactive UST - Decommissioned
Irrigation Canal/Pond
Auto

auto- Gas Stations
unto - Repair Shoos

UST- Confirmed Leaking but listed as NFA - DEQ LUST List

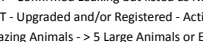

UST-Confirmed Leaking but Iisted as NFA - DEQ L USST LIS Auto- - Gas Stations
Auto - Reapir shoos

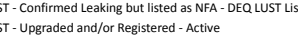

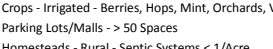

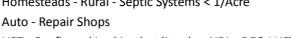
UST - Confirmed Leaking but listed as NFA - DEQ L UST L Housing- - - igh Density $\rightarrow 1$ House/0.5 Acres
Medical vet offices

Thown Contamination Sites/Plumes/Spills (ECSI)
UST - Decommissioned I Inactive

UST-Decommission
Auto - Repair hhops
Auto- Repair Shops

Auto- Repair Shops
Auto Repair shops
Auto - Repair Shops

Medicialvet offices

large Capacity Septic Systems - Class V UIC (Serves 20)

Mining Activities G Gravel Mines/Gravel Pits DEQ Permitted Stormwater Discharges (NPDES or WPCA

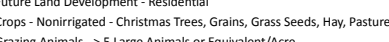

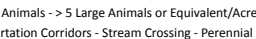




\begin{tabular}{|c|c|c|}
\hline Address & City & County \\
\hline $\begin{array}{l}\text { Throughout DWPA } \\
\text { Hwy } 4100\end{array}$ & Ashland & $\begin{array}{l}\text { Jackson } \\
\text { Jackson }\end{array}$ \\
\hline $\begin{array}{l}\text { Why } 140 \text { ughout DWPA } \\
\text { Throg }\end{array}$ & Ashland & $\begin{array}{l}\text { Jackson } \\
\text { Jackson }\end{array}$ \\
\hline $\begin{array}{l}\text { Throughout the D. WPA } \\
121206 \text { Yankee C.Rd }\end{array}$ & Assland & $\begin{array}{l}\text { Jackson } \\
\text { Jackono }\end{array}$ \\
\hline Southern most point in DWPA on Mt. Assland & Ashland & Jackson \\
\hline Hwy 140 & & Jackson \\
\hline 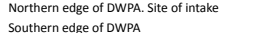 & $\begin{array}{l}\text { Astland } \\
\text { Asthend }\end{array}$ & $\begin{array}{l}\text { Jackson } \\
\text { Jatkson }\end{array}$ \\
\hline $\begin{array}{l}\text { Southere edge of ofWPA } \\
\text { Southern edge of DWPA }\end{array}$ & $\begin{array}{l}\text { Assland } \\
\text { Ashland } \\
\text { S }\end{array}$ & $\begin{array}{l}\text { Jackson } \\
\text { Jackon }\end{array}$ \\
\hline $\begin{array}{l}\text { Soutrem egae or owPA } \\
\text { Souther edge of DWPA }\end{array}$ & $\begin{array}{l}\text { Asslano } \\
\text { Ashland }\end{array}$ & $\begin{array}{l}\text { Jackson } \\
\text { Jackson }\end{array}$ \\
\hline Throughout DWPA & Ashland & $\begin{array}{l}\text { Jackson } \\
\text { Jackson }\end{array}$ \\
\hline 1/4 M I NW OF NEVADA ST \& OAK ST & ASHLAND & Jackson \\
\hline Hwy 140 & & $\begin{array}{l}\text { Jackson } \\
\text { Jackson }\end{array}$ \\
\hline Throughout OWPA & Trail & Jackson \\
\hline Northeast of intake. Lost Creek Lake & Trail & Jackson \\
\hline Northeast of intake. Lost Creek Lake & & Jackson \\
\hline $\begin{array}{l}\text { Runs through DWPA } \\
\text { N.Mountain Ave }\end{array}$ & $\begin{array}{l}\text { Asthand } \\
\text { Asshand }\end{array}$ & $\begin{array}{l}\text { Jackson } \\
\text { Jackonon }\end{array}$ \\
\hline $\begin{array}{l}\text { N. Mountain Ave } \\
\text { Throughout DWPA }\end{array}$ & 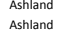 & $\begin{array}{l}\text { Jackson } \\
\text { Jackon }\end{array}$ \\
\hline $\begin{array}{l}\text { Throunhouot tWWPA } \\
\text { East of N Mountain Ave. }\end{array}$ & 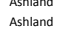 & $\begin{array}{l}\text { Jackson } \\
\text { Jackson }\end{array}$ \\
\hline East of Well & Ashland & Jackson \\
\hline Throughout DWPA & Ashland & Jackson \\
\hline East of N. Mountain R. & Ashland & $\begin{array}{l}\text { Jackson } \\
\text { Jackon }\end{array}$ \\
\hline $\begin{array}{l}\text { Hyy } 6 \text { 6 and Roguve River before Lost Creek Lake } \\
\text { Throghout OWPA }\end{array}$ & $\begin{array}{l}\text { Trail } \\
\text { Astland } \\
\end{array}$ & $\begin{array}{l}\text { Jackson } \\
\text { Jackon }\end{array}$ \\
\hline $\begin{array}{l}\text { Throunhouot UWWA } \\
\text { Crowfoot Rd over Big Butte Creek }\end{array}$ & $\begin{array}{l}\text { Assland } \\
\text { Trail }\end{array}$ & $\begin{array}{l}\text { Jackson } \\
\text { Jackson }\end{array}$ \\
\hline \multirow{4}{*}{$\begin{array}{l}\text { Crowwot tdd over Big Butte Creek } \\
\text { Takelma Drive }\end{array}$} & Trail & $\begin{array}{l}\text { Jacsonon } \\
\text { Jackson }\end{array}$ \\
\hline & Trail & Jackson \\
\hline & Trail & \\
\hline & Trail & \\
\hline Northeast of fintake. Located near dam. & 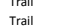 & $\begin{array}{l}\text { Jackson } \\
\text { Jackson }\end{array}$ \\
\hline 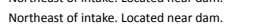 & Trail & 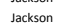 \\
\hline \multirow{3}{*}{ 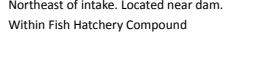 } & Trail & $\begin{array}{l}\text { Jackson } \\
\text { Jackson }\end{array}$ \\
\hline & Trail & Jackson \\
\hline & & \\
\hline Rogue River Dr. & Trail & Jackson \\
\hline $\begin{array}{l}\text { Nortreast of intake } \\
\text { Northeast of intake }\end{array}$ & 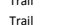 & $\begin{array}{l}\text { Jackson } \\
\text { Jackon }\end{array}$ \\
\hline Northeast of intiake & Trail & $\begin{array}{l}\text { Jackson } \\
\text { Jackson }\end{array}$ \\
\hline Northeast of intake & Trail & $\begin{array}{l}\text { Jackson } \\
\text { Jas }\end{array}$ \\
\hline Hwy 140 & Trail & Jackson \\
\hline Hwy 140 & Trail & Jackson \\
\hline $\begin{array}{l}\text { South Fork Litile Butte Creek Rd } \\
\text { Runs Through DWPA }\end{array}$ & & $\begin{array}{l}\text { Jackson } \\
\text { Jackon }\end{array}$ \\
\hline $\begin{array}{l}\text { Runs Through DWPA } \\
\text { R W }\end{array}$ & 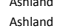 & $\begin{array}{l}\text { Jackson } \\
\text { Jackson }\end{array}$ \\
\hline Throughout DWPA & Ashland & $\begin{array}{l}\text { Jacsson } \\
\text { Jackon }\end{array}$ \\
\hline Mobile Dr: & Ashland & Jackson \\
\hline Reiten Dr. & Ashland & Jackson \\
\hline SW of Well & Assland & Jackson \\
\hline $\begin{array}{l}\text { Notrtheast of intake } \\
\text { Northeast of intake }\end{array}$ & Trail & $\begin{array}{l}\text { Jackson } \\
\text { Jackon }\end{array}$ \\
\hline 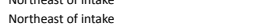 & $\begin{array}{l}\text { Trail } \\
\text { Trail }\end{array}$ & $\begin{array}{l}\text { Jackson } \\
\text { Jackson }\end{array}$ \\
\hline Northeast of intake & Trail & $\begin{array}{l}\text { Jacksonon } \\
\text { Jackon }\end{array}$ \\
\hline Northeast of intake & Trail & Jackson \\
\hline Throughout DWPA & Ashland & Jackson \\
\hline Moweta Dr. & Ashland & Jackson \\
\hline $\begin{array}{l}\text { Within Josepph State Park } \\
\text { Within os oseph State Park }\end{array}$ & $\begin{array}{l}\text { Trail } \\
\text { Trail }\end{array}$ & $\begin{array}{l}\text { Jackson } \\
\text { Jackon }\end{array}$ \\
\hline $\begin{array}{l}\text { Lake creek Loop } \\
\text { Lef }\end{array}$ & $\begin{array}{l}\text { Trail } \\
\text { Trail }\end{array}$ & $\begin{array}{l}\text { Jackson } \\
\text { Jackson }\end{array}$ \\
\hline Lake Creek Rd & Trail & $\begin{array}{l}\text { Jacksoun } \\
\text { Jackon }\end{array}$ \\
\hline Lake Creek Road & Trail & Jackson \\
\hline Lake Creek Road & Trail & Jackson \\
\hline Hwy 62 and lost creek Lake & Trail & Jackson \\
\hline $\begin{array}{l}\text { Southeasst of intake } \\
\text { Southeast of intake }\end{array}$ & $\begin{array}{l}\text { Butte a fals } \\
\text { Bunte ansts }\end{array}$ & Jackson \\
\hline $\begin{array}{l}\text { Throughout OWNA } \\
\text { The }\end{array}$ & 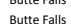 & $\begin{array}{l}\text { Jackson } \\
\text { Jackson }\end{array}$ \\
\hline $\begin{array}{l}\text { Throughout OWFA } \\
\text { The }\end{array}$ & 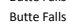 & 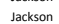 \\
\hline Northern Tip of DWPA & Butte falls & Jackson \\
\hline 120 Mill reek orvive & Prospect & Jackson \\
\hline 120 Mili reeek brive & Prospect & Jackson \\
\hline 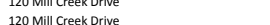 & $\begin{array}{l}\text { Prospect } \\
\text { prosect }\end{array}$ & $\begin{array}{l}\text { Jackson } \\
\text { ackson }\end{array}$ \\
\hline Mill creek Drive & Prospect & $\begin{array}{l}\text { Jackson } \\
\text { Jackson }\end{array}$ \\
\hline Mill creek Drive & Prospect & $\begin{array}{l}\text { Jackson } \\
\text { Jat }\end{array}$ \\
\hline rreek orive & Prospect & Jackson \\
\hline eek Drive & Prospect & Jackson \\
\hline & Prospect & \\
\hline 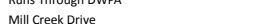 & $\begin{array}{l}\text { Porpsect } \\
\text { Prosect }\end{array}$ & \\
\hline $\begin{array}{l}\text { Creetex orve } \\
\text { crive }\end{array}$ & $\begin{array}{l}\text { Porspect } \\
\text { Prospect }\end{array}$ & \\
\hline rreek orive & & \\
\hline Creek Drive & Prosspect & \\
\hline creek Drive & Prospeet & Jackson \\
\hline
\end{tabular}

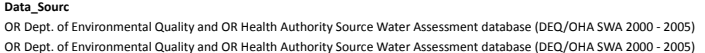

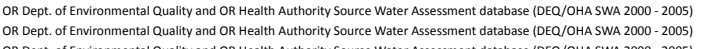

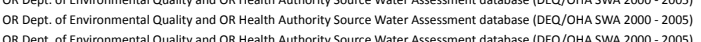

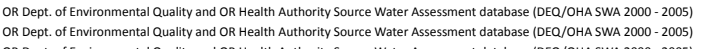
$\begin{array}{ll} & \\ 2 & \end{array}$

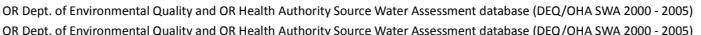

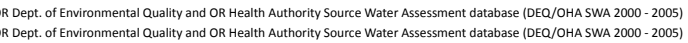

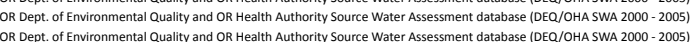

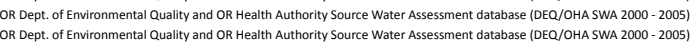

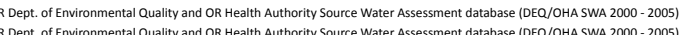

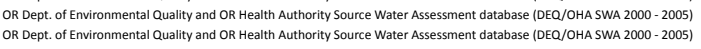

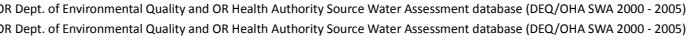
OR Dept. of Environmental Quality and OR Heath Authority Source Water A ASessment database (DEQ/OHA SWA 2000- 2005)

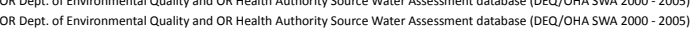
OR Dept. of Environmental Q Quality and OR Heath Authority Source Water A ASsessment database (DEQ/OHA SWA 2000- 2005)

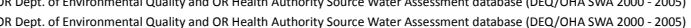
OR Dept. of Environmental Q Quality and OR Heath Authority Source Water Assessment database (DEQ/OHA SWA 2000- 2005) OR Dept. of Environmental Q Quality nnd OR Heath Authortity Source Water Assessment database (DEQ/JHA SWA $2000-2005)$

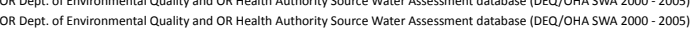

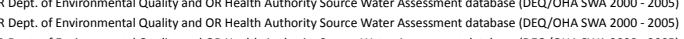
OR Dept. of Environmental Q Quality and OR Health Authority Source Water Assessment database (DER/OHA SWA 2000- 2005) OR Dept. of Environmental Q Quality ynd OR Heath Authority Source Water ASSessment database (DEQ//OHA SWA 2000- 2005)

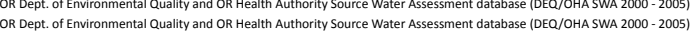

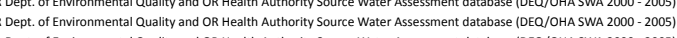

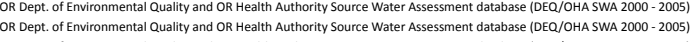
OR Dept. of Environmental Q Quality and OR Heath Authority Source Water Assessment database (DEE//OHA SWA 2000-2005) OR Dept. of Environmental Q Quality ynd OR Heath Authority Source Water Assessment database (DER//OHA SWA 2000- 2005)

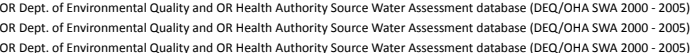

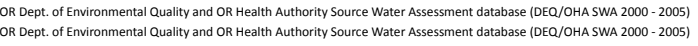

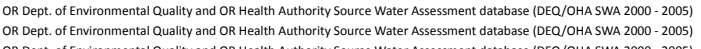

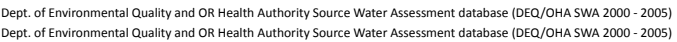

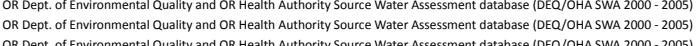

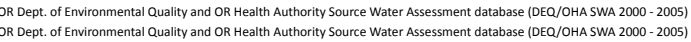

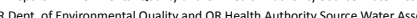

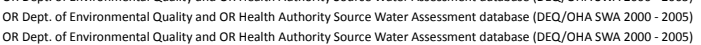

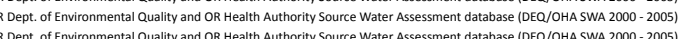
OR Dept. of Environmental Q vallity and OR Health Authority Source Water Assessment database (DEE/OHA SWA 2000- 2005) OR Dept. of Environmental Q Quality and OR Health Authority S Surce Water Assessment database (DEQ/OHA SWA $2000-2005)$ OR Dept. of Environmental Q Quality and OR Heath Authority Source Water Assessment database (DEQ/OHA SWA 2000- 2005)

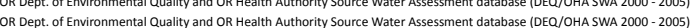
OR Dept. of Environmental Q Quality and OR Heath Authority Source Water Assessment database (DEQ/OHA SWA 2000- 2005)

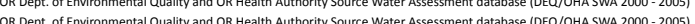

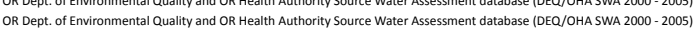

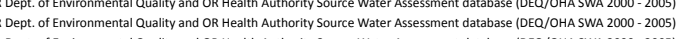

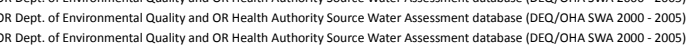

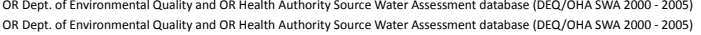

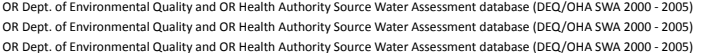

\begin{tabular}{|c|c|c|c|}
\hline & & & \\
\hline & ${ }_{L}^{M}$ & ${ }_{\mathrm{H}}^{\mathrm{H}}$ & $\begin{array}{l}\text { Transportation S-Stream Crossings } \\
\text { Little Butte Cr. Bridge }\end{array}$ \\
\hline & H & H & \\
\hline & m & M & 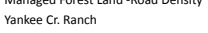 \\
\hline & L & L & TV Tower - Underground Storage Tan \\
\hline & t & $M$ & 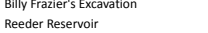 \\
\hline & ᄂ & L & Mount Ashland Ski Area \\
\hline & $H_{H}^{H}$ & ${ }_{H}^{M}$ & $\begin{array}{l}\text { Mount Assland Ski rarea } \\
\text { Mount Ashland Ski Area }\end{array}$ \\
\hline & H & & $\begin{array}{l}\text { Mount Assland Ski Area } \\
\text { Forest Fire Hazard }\end{array}$ \\
\hline & m & H & ASHLAND STP \\
\hline & M & & $\begin{array}{l}\text { High Cascade Emus } \\
\text { Panther Crushing }\end{array}$ \\
\hline & L & $\mathrm{H}$ & \\
\hline & & ${ }_{M}^{M}$ & 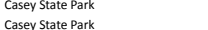 \\
\hline & M & H & \\
\hline & & & High Density Housing \\
\hline & M & H & 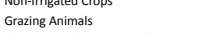 \\
\hline & a & & $\begin{array}{l}\text { Abovergound Storage Tanks } \\
\text { Rurar Hormes }\end{array}$ \\
\hline & M & H & $\begin{array}{l}\text { Rurar homes } \\
\text { Irrigated Crops }\end{array}$ \\
\hline & L & H & \\
\hline & t & H & $\begin{array}{l}\text { Nonirigigated Crops } \\
\text { Bridge }\end{array}$ \\
\hline & ᄂ & L & OOF Wildand Fire Station \\
\hline & & & McGregor Park \\
\hline & i & t & 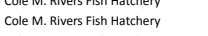 \\
\hline & t & L & 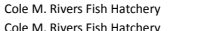 \\
\hline & m & $\begin{array}{l}H \\
M \\
M\end{array}$ & 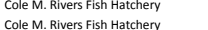 \\
\hline & $\mathrm{L}$ & L & 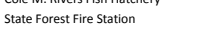 \\
\hline & L & $\begin{array}{c}M \\
M\end{array}$ & $\begin{array}{l}\text { Lost Creek Lake } \\
\text { Lost Creek Lake }\end{array}$ \\
\hline & ᄂ & m & $\begin{array}{l}\text { Losstreer Lake } \\
\text { Takelma Park }\end{array}$ \\
\hline & ᄂ & L & William L. Jess Dam \\
\hline & H & H & $\begin{array}{l}\text { William L. Jess Dam } \\
\text { William L. Jes Dam }\end{array}$ \\
\hline & L & M & Lost Creek Reservoir \\
\hline & & H & Bridge \\
\hline & 2 & 4 & Bridge \\
\hline & ${ }_{M}^{M}$ & 型 & Livestock \\
\hline & $m$ & H & Railroad \\
\hline & & & Non-Irrigated Crops \\
\hline & H & & $\begin{array}{l}\text { High Density Septic Systems } \\
\text { Decompisions }\end{array}$ \\
\hline & M & н & Irrigated Crops \\
\hline & ᄂ & M & Joseph Stewart State Park \\
\hline & & & 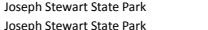 \\
\hline & H & $m$ & Joseph Stewart tstate Park \\
\hline & m & м & Joseph Stewart State Park \\
\hline & t & 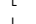 & Rural Homes \\
\hline & ᄂ & & $\begin{array}{l}\text { Decommisisioned UST } \\
\text { Lost Creek Marina }\end{array}$ \\
\hline & & & Lost Creek Marina \\
\hline & $\mathrm{m}$ & & Cascade Ranch \\
\hline & & & Lake Creek Rural Fire Station Dist. 1 \\
\hline & L & 4 & Bridge 235 \\
\hline & $\mathrm{L}$ & H & Bridge \\
\hline & $M$ & & city of Butte Falls \\
\hline & 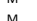 & & City of Butte Falls \\
\hline & m & & $\begin{array}{l}\text { Manageded forest lands } \\
\text { High Road Density }\end{array}$ \\
\hline & $m$ & n & Orinking Water Treatment Plant \\
\hline & m & & ОDणT Maintenance Station \\
\hline & & & ОоОтT Maintenanace Station \\
\hline & H & $\mathrm{m}$ & 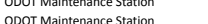 \\
\hline & н & м & Auto Service and Gas Station \\
\hline & H & & Auto Service and Gas Station \\
\hline & & & Auto Service and Gas station \\
\hline & $\mathrm{m}$ & $\mathrm{n}$ & 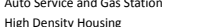 \\
\hline & m $>2>>$ & & Hwy 62 \\
\hline & M & $\mathrm{m}$ & School Greenhouse Heating oil Tank \\
\hline & н & $\mathrm{M}$ & 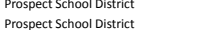 \\
\hline & H & $\mathrm{H}$ & Prospect School District \\
\hline & & & \\
\hline
\end{tabular}

PCSType

fation Corridors - High use Roads/streets

Others (lit)
Managed forest Lands - Road Density $\rightarrow>2 \mathrm{~m} / \mathrm{sq} \mathrm{mi}$ UST - Upgradedd and/or Registered-Active
Homesteads - Rural- Machine Shops/Equip Maintenance

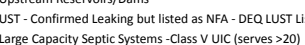

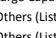

Wastewater Treatment Plants/Collection Stations

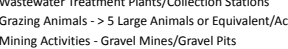
Transmission Lines- - Righto-f-Ways
River Recreation - Heary Use linc. Campgrounds Transportation Corridors - Freeways Highway crops - Noniririgated- Christsmas Trees, Grains, Grass Seeds, Hay, Pasture

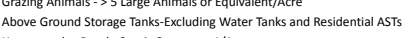

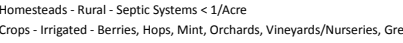
Transportation Corrididrs - Stream Crossing - Perennial Crops - Noniririgated - Christmas Trees, Grains, Grass Se Transport
Fire Station

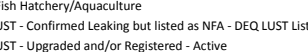
Excluding Water Tanks and Residential ASS Rir Station
River Recreation- Heary Use (inc. Campgrounds)

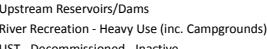

Utility Stations - Maintenance/rransformer Storage (inc. Hydroelectric Power Equip.) Transportation Corridors - Stream Crossing - Perennt

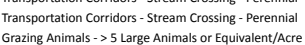
Transportation Corriders - Ralitrads
Crops - Nonirigated - Christmas rees, Grains, Grass Seeds, Hay, Pasture Crops - Nonirirgated - Christmas Tres,
Septic Systems - High Density $\rightarrow 1$ Acre

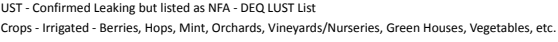

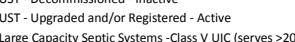
Large Capacity Septic Systems - Cass Wic (serves 200 )

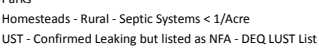
UST - Decommissioned - Inactive
Grazing Animals $\rightarrow 5$ Large Animals or Equivalent/Acte

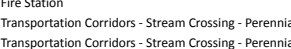

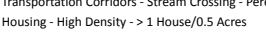
Wastewater Treatrent P Plants/Collection Stations Managed forest Lands - Clearcut Harvested $<35$ rs
Managed forest tands - Road Density $\rightarrow 2$ mi/sq $\mathrm{mi}$

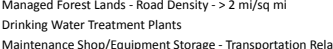

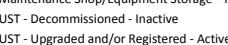
Large Capacity Septic Systems - Class V UIC (serves 20) Auto - Gas Stations

UST - Confirmed Leaking but listed as NFA- DEQ USST Lis UST - Upgraded and/or Registered- Active
Housing- $\rightarrow$ High Density $\rightarrow$ 1 House/0.5 Acres Housing - High Density $\rightarrow 1$ House/ 0.5 Acres
Transportation Corridors - Freeways/Highways or other heavy use roads

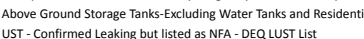

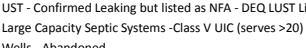




\begin{tabular}{|c|c|c|c|}
\hline Address & City & County & RET_DATE \\
\hline $\begin{array}{l}\text { North of WWll } \\
\text { Mill Creek Orive }\end{array}$ & $\begin{array}{l}\text { Prospect } \\
\text { Prospect }\end{array}$ & Jackson & $\begin{array}{l}2005 \\
2005\end{array}$ \\
\hline $\begin{array}{l}\text { Ninc ceek ruve } \\
\text { Throughout DWPA }\end{array}$ & $\begin{array}{l}\text { Prospect } \\
\text { Prospect }\end{array}$ & $\begin{array}{l}\text { Jackson } \\
\text { Jackson }\end{array}$ & 2005 \\
\hline Highway 62 & Prospect & Jackson & 2005 \\
\hline $\begin{array}{l}\text { Highway } 62 \\
\text { Highway } 62\end{array}$ & $\begin{array}{l}\text { Prospect } \\
\text { Prospect }\end{array}$ & $\begin{array}{l}\text { Jackson } \\
\text { Jackson }\end{array}$ & $\begin{array}{l}2005 \\
2005\end{array}$ \\
\hline & $\begin{array}{l}\text { Prospect } \\
\text { Prossect }\end{array}$ & $\begin{array}{l}\text { Jackson } \\
\text { Jackson }\end{array}$ & $\begin{array}{l}2005 \\
2005\end{array}$ \\
\hline Mill creek Drive & $\begin{array}{l}\text { Prospect } \\
\text { Prospect }\end{array}$ & $\begin{array}{l}\text { Jacsson } \\
\text { Jackson }\end{array}$ & 2005 \\
\hline Mill creek Drive & Prospect & Jackson & \\
\hline Mill Creek Drive & Prospect & Jackson & 2005 \\
\hline $\begin{array}{l}\text { Mill creek Drive } \\
\text { Millcree Dirive }\end{array}$ & Prospect & Jackson & 2005 \\
\hline $\begin{array}{l}\text { Mill creek Bive } \\
\text { Mill Creek Drive }\end{array}$ & $\begin{array}{l}\text { Prospect } \\
\text { Prospect }\end{array}$ & $\begin{array}{l}\text { Jackson } \\
\text { Jackson }\end{array}$ & $\begin{array}{l}2005 \\
2005\end{array}$ \\
\hline $\begin{array}{l}\text { Mill reeex urrve } \\
\text { Mill creek Drive }\end{array}$ & $\begin{array}{l}\text { Prospect } \\
\text { Prossect }\end{array}$ & $\begin{array}{l}\text { Jackson } \\
\text { Jackson }\end{array}$ & $\begin{array}{l}2005 \\
2005\end{array}$ \\
\hline Mill creek orive & $\begin{array}{l}\text { Prospect } \\
\text { Prospect }\end{array}$ & $\begin{array}{l}\text { Jackson } \\
\text { Jackson }\end{array}$ & 2005 \\
\hline Mill creek Drive & Prospect & Jackson & 2005 \\
\hline First Street & Prospect & Jackson & 2005 \\
\hline & Prospect & Jackson & 2005 \\
\hline $\begin{array}{l}\text { Throunhout DDPA } \\
\text { Mill rreek Drive }\end{array}$ & $\begin{array}{l}\text { Prospect } \\
\text { Prospect }\end{array}$ & $\begin{array}{l}\text { Jackson } \\
\text { Jackson }\end{array}$ & $\begin{array}{l}2005 \\
2005\end{array}$ \\
\hline 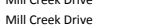 & $\begin{array}{l}\text { Prospect } \\
\text { Prossect }\end{array}$ & $\begin{array}{l}\text { Jacksson } \\
\text { Jackson }\end{array}$ & $\begin{array}{l}2005 \\
2005\end{array}$ \\
\hline First Street & $\begin{array}{l}\text { Prospect } \\
\text { Prospect }\end{array}$ & $\begin{array}{l}\text { Jackson } \\
\text { Jackson }\end{array}$ & 2005 \\
\hline East of Highway 62 & Prospect & Jackson & 2005 \\
\hline Runs Through DWPA & Prospect & Jackson & 2005 \\
\hline $\begin{array}{l}\text { Mili creee ovire } \\
\text { Mill reek Drive }\end{array}$ & $\begin{array}{l}\text { Prospect } \\
\text { Prospect }\end{array}$ & $\begin{array}{l}\text { Jackson } \\
\text { Jackson }\end{array}$ & $\begin{array}{l}2005 \\
2005\end{array}$ \\
\hline $\begin{array}{l}\text { Mill creee bivive } \\
\text { Crater Lake }\end{array}$ & $\begin{array}{l}\text { Prospect } \\
\text { Crater lake }\end{array}$ & $\begin{array}{l}\text { Jackson } \\
\text { Kamath }\end{array}$ & $\begin{array}{l}2005 \\
2005\end{array}$ \\
\hline 160 Mill Creek Dr & Prospect & $\begin{array}{l}\text { Kalamain } \\
\text { JacksoN }\end{array}$ & $\begin{array}{l}2005 / 02 / 2015 \\
0015\end{array}$ \\
\hline Not Applicable & MEFFORD & Jackson & \\
\hline Not Applicable & MEEFROR & Jackson & 2013 \\
\hline Not Applicable & MEDFORD & Jackson & 2013 \\
\hline $\begin{array}{l}\text { Not Applicable } \\
\text { Notiche }\end{array}$ & $\begin{array}{l}\text { MEFFORD } \\
\text { MEFFRD }\end{array}$ & $\begin{array}{l}\text { Jackson } \\
\text { Jackson }\end{array}$ & 2013 \\
\hline $\begin{array}{l}\text { Not Appocicable } \\
\text { Not Applicable }\end{array}$ & $\begin{array}{l}\text { MEEFRORD } \\
\text { MEFFRD }\end{array}$ & $\begin{array}{l}\text { Jackson } \\
\text { Jackson }\end{array}$ & $\begin{array}{l}2013 \\
2013\end{array}$ \\
\hline Not Applicable & $\begin{array}{l}\text { MEEFRO } \\
\text { MEFFRD }\end{array}$ & $\begin{array}{l}\text { Jackson } \\
\text { Jackson }\end{array}$ & $\begin{array}{l}2013 \\
2013\end{array}$ \\
\hline Not Applicable & MEFFRD & Jackson & 2013 \\
\hline 450 S 4th St & Central Point & JaCKSON & $06 / 02 / 2015$ \\
\hline 1955 Scenic Ave & $\begin{array}{l}\text { Central Point } \\
\text { Centrapeint }\end{array}$ & Jackson & $06 / 102 / 2015$ \\
\hline $\begin{array}{l}142351 \text { Mable Ronita Rd } \\
100 \text { St }\end{array}$ & $\begin{array}{l}\text { Central lonit } \\
\text { Central Point }\end{array}$ & $\begin{array}{l}\text { JaCCSSON } \\
\text { ACCSON }\end{array}$ & $\begin{array}{l}06 / 102 / 2015 \\
06602 / 2015\end{array}$ \\
\hline $200 \mathrm{~W}$ Pine St & $\begin{array}{l}\text { Central Pont } \\
\text { Centra Point }\end{array}$ & $\begin{array}{l}\text { JaCSSON } \\
\text { JACCSON }\end{array}$ & $\begin{array}{l}066202 / 2015 \\
06 / 202 / 215\end{array}$ \\
\hline Not Applicable & MEEFORD & Jackson & $\begin{array}{l}2013 \\
2012 / 215 \\
\end{array}$ \\
\hline 550 Wilson Way & $\begin{array}{l}\text { White city } \\
\text { Wherot }\end{array}$ & JaCKSON & $06 / 102 / 2015$ \\
\hline $\begin{array}{l}\text { Not Applicable } \\
\text { Notenticable }\end{array}$ & & Jackson & $\begin{array}{l}2013 \\
2013\end{array}$ \\
\hline $\begin{array}{l}\text { Not At Applcicale } \\
\text { Not Aplicable }\end{array}$ & $\begin{array}{l}\text { MEFFRO } \\
\text { MEFFORD }\end{array}$ & & 2013 \\
\hline Not Applicable & $\begin{array}{l}\text { MEFFRO } \\
\text { MEFO }\end{array}$ & $\begin{array}{l}\text { Jackson } \\
\text { Jackson }\end{array}$ & 2013 \\
\hline Not Applicable & MEFFORD & Jackson & 2013 \\
\hline Not Applicable & MEOFORD & Jackson & ${ }_{2013}$ \\
\hline Not Applicable & MEFFORD & Jackson & 2013 \\
\hline Not Applicable & MEEFORD & Jackson & 2013 \\
\hline 20135 Mountain $A \mathrm{~V}$ & Ashland & & 06/02/2015 \\
\hline 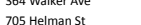 & $\begin{array}{l}\text { Assinga } \\
\text { Aschand }\end{array}$ & $\begin{array}{l}\text { JaCCSSON } \\
\text { ACCCSON }\end{array}$ & $\begin{array}{l}06 / 02 / 2015 \\
0.6202 / 2015\end{array}$ \\
\hline 100 Walker ave & 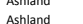 & 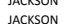 & $\begin{array}{l}06602 / 21 / 2015 \\
0.6202 / 215\end{array}$ \\
\hline 1070 Tolman Creek Rd & Ashland & JacksoN & . \\
\hline 625 fir st & Butte falls & JaCKSON & $06 / 102 / 2015$ \\
\hline 1280 araton Rd & Eagle Point & JACKSON & $06 / 102 / 2015$ \\
\hline 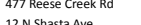 & $\begin{array}{l}\text { Eagle Point } \\
\text { Eante }\end{array}$ & JaCKSON & 06/102/2015 \\
\hline D. & 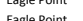 & $\begin{array}{l}\text { JaCCSON } \\
\text { ACCSSON }\end{array}$ & $\begin{array}{l}06 / 102 / 2015 \\
06502 / 2015\end{array}$ \\
\hline 806 6t have & Gold till & 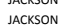 & $\begin{array}{l}066 / 2 / 212015 \\
06 / 202 / 2015\end{array}$ \\
\hline 15002 nd Ave & Gold Hill & JaCKSON & $\begin{array}{l}06 / 02 / 2015 \\
0015\end{array}$ \\
\hline 655 Hueners Ln & Jacksonvile & JaCKSON & $06 / 02 / 2015$ \\
\hline 215 N Rose St & Phoenix & JACKSON & $06 / 102 / 2015$ \\
\hline T. & $\begin{array}{l}\text { Phoenix } \\
\text { Tolent }\end{array}$ & JACCSON & $\begin{array}{l}06 / 102 / 2015 \\
06502 / 2015\end{array}$ \\
\hline 307 WW Wanper & $\begin{array}{l}\text { Talant } \\
\text { Talent } \\
\text { Thent }\end{array}$ & 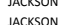 & $\begin{array}{l}06 / 20 / 21 / 2015 \\
0.6202 / 2015\end{array}$ \\
\hline 37 chooblhouse lane & Shand Cove & $\begin{array}{l}\text { ACCKSON } \\
\text { A. }\end{array}$ & $\begin{array}{l}0.06 / 2 / 2015 \\
06 / 2015\end{array}$ \\
\hline 655 N 3rd St & Central Point & Jackson & $06 / 02 / 2015$ \\
\hline 655 N 3rd St & Central Point & JaCKSON & 06/02/2015 \\
\hline Not Applicable & MEFFRD & Jackson & 2013 \\
\hline Not Appocicabe & MEEFOROD & Jackson & 2013 \\
\hline NotAplicable & MEFRD & 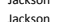 & 2013 \\
\hline 3070 Ross Ln & Central Point & JaCKSON & $\begin{array}{l}206 / 02 / 2015 \\
0\end{array}$ \\
\hline 300 Ash St & Central Point & JaCKSON & $006 / 02 / 2015$ \\
\hline 100 Walker Ave & Ashland & JACKSON & 06/02/2015 \\
\hline 885 Siskivou Blud & Ashland & JACKSON & 06/02/2015 \\
\hline 129N Patt & 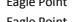 & The & $\begin{array}{l}06 / 202 / 2015 \\
0.6202 / 2015\end{array}$ \\
\hline & 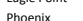 & IAcsson & $\begin{array}{l}06 / 202 / 2015 \\
0.6202 / 2015\end{array}$ \\
\hline $401 \mathrm{~W}$ 4th St & Phoenix & JaCKSON & $\begin{array}{l}0.06 / 2 / 2015 \\
06 / 2015\end{array}$ \\
\hline 160 Mill Creek or & Prospect & JaCKSON & $06 / 02 / 2015$ \\
\hline 2830 Maple ct & White City & & 06/02/2015 \\
\hline 720 Laurel Ave & Butte falls & JACKSON & 06/02/2015 \\
\hline
\end{tabular}

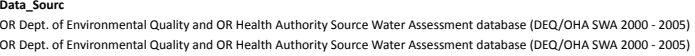

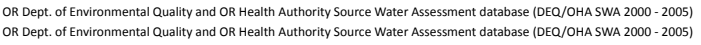

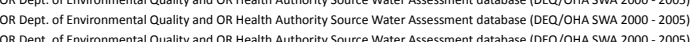

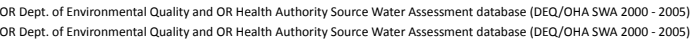

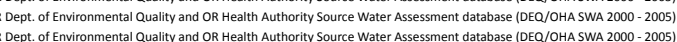

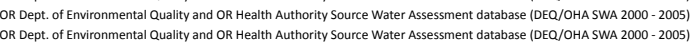

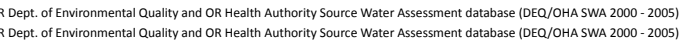

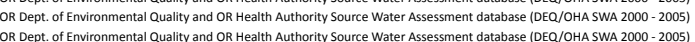
OR Dept. of Environmental Quality and O O Heath Authority Source Water A SSessment database (DEC/OHA SWA 2000-2005) OR Dept. of Environmental Q Quality and OR Heath Authority Source Water Assessment database (DEQ/OHA SWA 2000 - 2005)

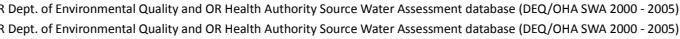

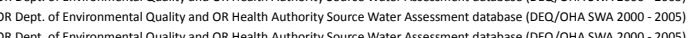

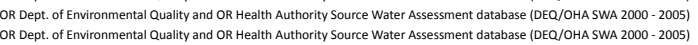

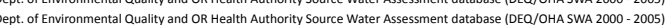
OR Dept. of Environmental Quality and OR Health Authority Source Water Assessment database (DEQ/OHA SWA 2000-2005)

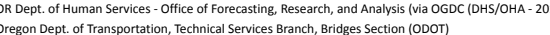

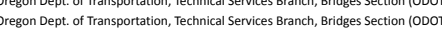

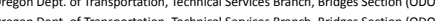
regon Dept of Transportation, Technical Services Branch, Brideses Section

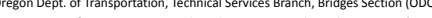

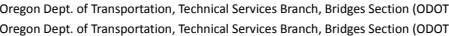
OR Dept. of Human Services- Office of Forecasting, Research, and Analysis Wva O6OC (DHS/OHA - 2015)

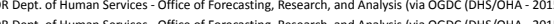

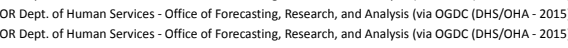

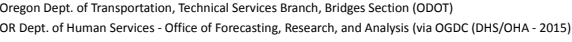

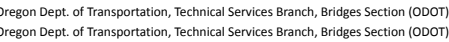

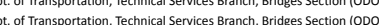
$\begin{array}{ll}0 & \end{array}$ Oregon Dept. of Transportation, Technical Services Branch, Bridges Section (1000) Oregon Dept. of fransportatation, Technical Services rranch, Bridges Section (0007)

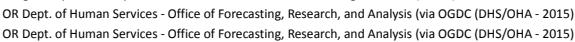

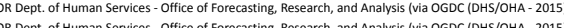

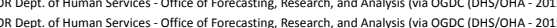
OR Dept. of Human Services- - Office of foreceasting, Research, and Analysis (via OGDC (DHS/OHA- 2015

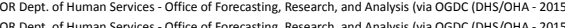

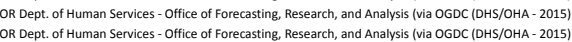

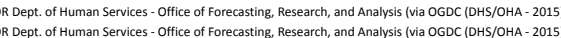
roper. $O R$ Dept. of Human Services - Office of Forecasting, Research, and Analysis (via OGDCC (OHS/OHA - 2015

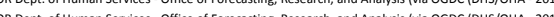

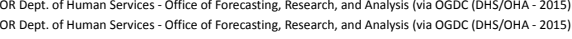
Oregon Dept. of Transportation, Technical Services Branch, Bridges Section (000)

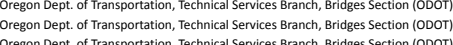

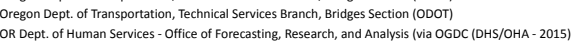

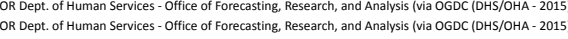

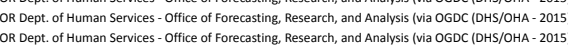

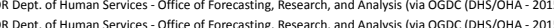
OR Dept. of Human Services - Office of Forecasting, Research, and Analysis Wia OGDC (DHS/OHA- 2015

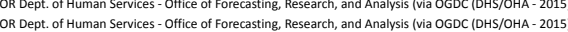

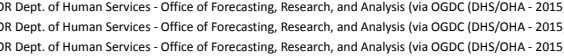

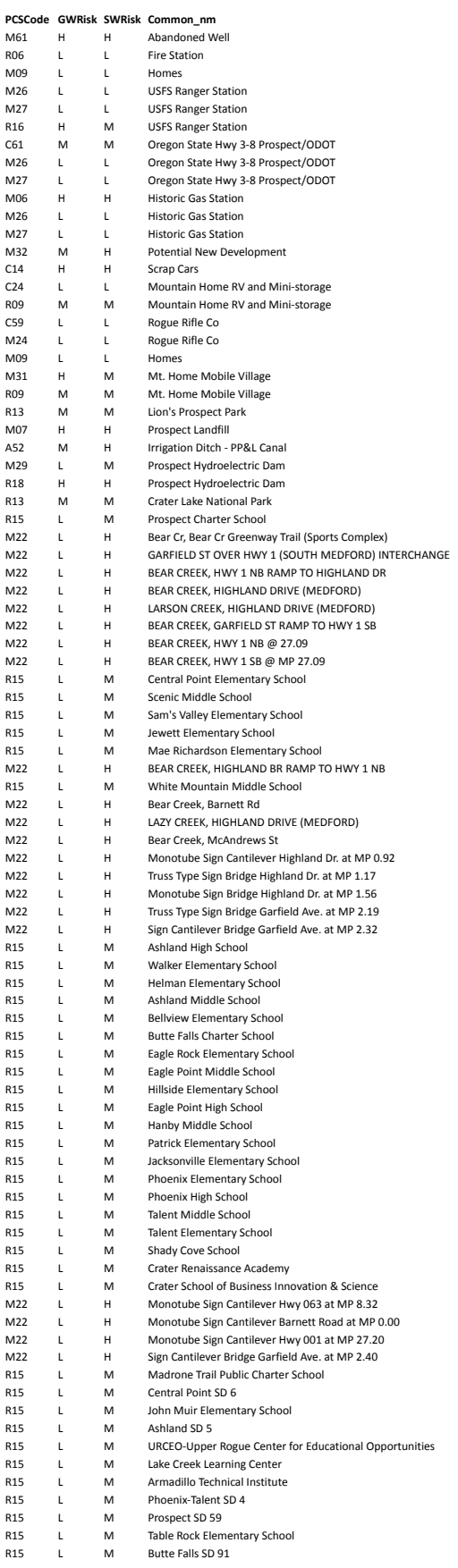

PCSType

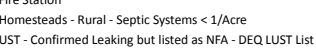

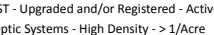

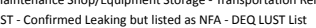

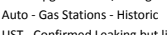

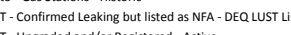

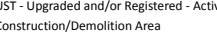
Junk/Scrap/Salvage Yards
RV/Mini Storage

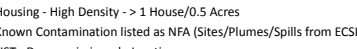

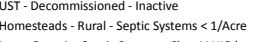

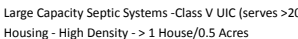
Housing - High Density $\rightarrow 1$ House/ $/ .5$

Historic Waste Dumps/Landfills

Irrization Canal/Pond
Upstream Reservoirs 0 ams

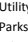

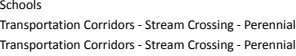

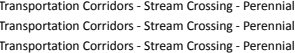

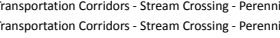

Transportat
Schools
Schools
Schools
Schols

Schools
Transpor
Schools

Iransportation Corridors - Stream Crossing - Perenn
Schools

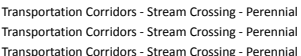
rransportatitin Corrididrs - Stream Crossing - Perennial

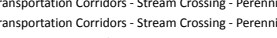

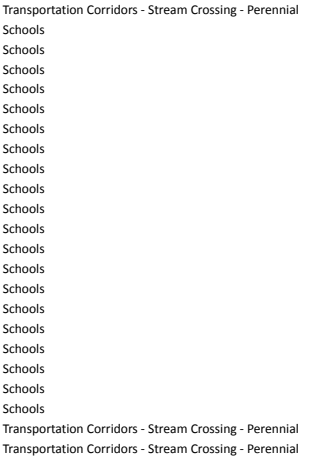

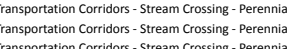
scheos

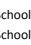

Schools
Schools
Schools
Schools 


\begin{tabular}{|c|c|c|c|}
\hline $\begin{array}{l}\text { Address } \\
\text { A1 N Royal Ave }\end{array}$ & $\begin{array}{l}\text { City } \\
\text { Eagle Point }\end{array}$ & $\begin{array}{l}\text { County } \\
\text { JACKSON }\end{array}$ & 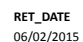 \\
\hline 1997 East Main St & $\begin{array}{l}\text { Ashland } \\
\text { And }\end{array}$ & JACKSON & $\begin{array}{l}06602 / 2015 \\
06 / 202 / 2015\end{array}$ \\
\hline $\begin{array}{l}\text { Not Applicable } \\
4411 \text { wanstst }\end{array}$ & MEDFORD & $\begin{array}{l}\text { Jackson } \\
\text { accscos }\end{array}$ & \\
\hline $\begin{array}{l}\text { Not W 2nd Splicatle } \\
\text { Not }\end{array}$ & 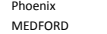 & $\begin{array}{l}\text { Jackson } \\
\text { Jackson }\end{array}$ & $\begin{array}{l}060 / 2 / 2015 \\
2013\end{array}$ \\
\hline 695 Mistleteo Rd & Ashland & $\begin{array}{l}\text { IACCSSON } \\
\text { Iats }\end{array}$ & $\begin{array}{l}2013 / 2 / 2 \\
00 / 02 / 2\end{array}$ \\
\hline Not applicable & MEDFORD & JACKSON & 2009 \\
\hline Not applicable & MEDFORD & JACKSON & 2009 \\
\hline Not applicable & MEDFORD & JACKSON & \\
\hline $\begin{array}{l}\text { Not applicable } \\
14611 \text { Hww } 62\end{array}$ & $\begin{array}{l}\text { Medford } \\
\text { Gavelepint }\end{array}$ & & \\
\hline $\begin{array}{l}114611 \text { Hww } 62 \\
631 \text { Clays }\end{array}$ & $\begin{array}{l}\text { Eagle Point } \\
\text { Ashland }\end{array}$ & $\begin{array}{l}\text { JACKSON } \\
\text { IACCSON }\end{array}$ & $\begin{array}{l}06 / 02 / 2015 \\
06 / 202 / 2015\end{array}$ \\
\hline $\begin{array}{l}\text { off Biddle Road } \\
\text { of Rat }\end{array}$ & $\begin{array}{l}\text { Assland } \\
\text { Mefford }\end{array}$ & $\begin{array}{l}\text { Jackson } \\
\text { Jackson }\end{array}$ & $\begin{array}{l}060202 / 2015 \\
2005\end{array}$ \\
\hline 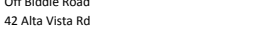 & $\begin{array}{l}\text { Meloror } \\
\text { Eagle Point }\end{array}$ & $\begin{array}{l}\text { JacSBOn } \\
\text { JACKSON }\end{array}$ & .06/02/2015 \\
\hline & $\begin{array}{l}\text { Cave Junction } \\
\text { Cons ent }\end{array}$ & JOSEPHINE & $006 / 02 / 2015$ \\
\hline 4300 Holland Loop Rd & Cave Junction & JOSEPHINE & $06 / 02 / 2015$ \\
\hline $\begin{array}{l}\text { Off Biddle Road } \\
\text { Off Bidtle Ragat }\end{array}$ & Medford & Jackson & \\
\hline $\begin{array}{l}\text { Off Biddle Read } \\
\text { 507 SOUTH FRONT }\end{array}$ & $\begin{array}{l}\text { Mediofd } \\
\text { CNETRAL POINT }\end{array}$ & $\begin{array}{l}\text { Jackson } \\
\text { Jacsson }\end{array}$ & \\
\hline 6061 C RAATER L LAKE HIIGHWAY & $\begin{array}{l}\text { CEETRARLOLONT } \\
\text { CENTRA POINT }\end{array}$ & $\begin{array}{l}\text { JaCSSON } \\
\text { JaCSSON }\end{array}$ & $\begin{array}{l}06 / 2 / 2 / 2015 \\
06 / 202 / 2015\end{array}$ \\
\hline 5588 CRATER LAKE HWY & 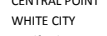 & 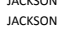 & $\begin{array}{l}06 / 02 / 2015 \\
0615\end{array}$ \\
\hline off Hammrick Road & Medford & Jackson & \\
\hline $\begin{array}{l}\text { Off Hammrick Road } \\
\text { off ftamprick Rapad }\end{array}$ & Medford & Jackson & 2005 \\
\hline $\begin{array}{l}\text { of Hammrick Road } \\
\text { Off Roguev Valley Blvd }\end{array}$ & $\begin{array}{l}\text { Medford } \\
\text { Meeford }\end{array}$ & Jackson & 2005 \\
\hline 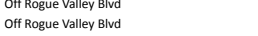 & $\begin{array}{l}\text { Medoford } \\
\text { Medford }\end{array}$ & $\begin{array}{l}\text { Jacksson } \\
\text { Jackson }\end{array}$ & $\begin{array}{l}2005 \\
2005\end{array}$ \\
\hline 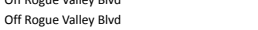 & $\begin{array}{l}\text { Mearora } \\
\text { Medford }\end{array}$ & $\begin{array}{l}\text { Jacksson } \\
\text { Jackson }\end{array}$ & $\begin{array}{l}2005 \\
2005\end{array}$ \\
\hline 401 WILIAMSSON WAY & $\begin{array}{l}\text { ATEarora } \\
\text { ASHLAND }\end{array}$ & $\begin{array}{l}\text { Jactson } \\
\text { Jacson }\end{array}$ & . \\
\hline 280 E. HERSEY ST & ASHLAND & JaCksoN & $06 / 02 / 2015$ \\
\hline 2425 SISKKYYu BLVD & ASHLAND & JaCkSON & $06 / 102 / 2015$ \\
\hline $\begin{array}{l}\text { Off Rogue Valley Blvd } \\
\text { Off Sage Roadd }\end{array}$ & $\begin{array}{l}\text { Medford } \\
\text { Meeford }\end{array}$ & Jackson & \\
\hline $\begin{array}{l}\text { Off Sage Road } \\
7800 \text { Pacific Ave }\end{array}$ & 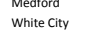 & $\begin{array}{l}\text { Jactson } \\
\text { JaCKSON }\end{array}$ & $\begin{array}{l}2005 \\
00 / 1 / 2 / 2015\end{array}$ \\
\hline 1250 Siskiyou Blvd & & 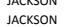 & $\begin{array}{l}06602 / 212015 \\
06 / 202 / 2015\end{array}$ \\
\hline $655 \mathrm{~N} 3 \mathrm{rd}$ St & $\begin{array}{l}\text { Astinat } \\
\text { Central point }\end{array}$ & 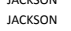 & o6/02/2015 \\
\hline 140 E Main Street & Eagle Point & JaCksoN & $06 / 02 / 2015$ \\
\hline SEC 20, T32S, R3E & N/A & JaCKSON & $10 / 31 / 2018$ \\
\hline 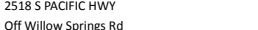 & N/A & JACKSON & $10 / 31 / 2018$ \\
\hline 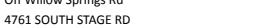 & $\begin{array}{l}\text { Metiord } \\
\text { N/A }\end{array}$ & $\begin{array}{l}\text { Jacason } \\
\text { IACKSON }\end{array}$ & $\begin{array}{l}2005 \\
1031 / 2018\end{array}$ \\
\hline 535 INDUSTRAAL CIRCLE & N/A & 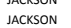 & $\begin{array}{l}101 / 3 / 21 / 2018 \\
10 / 131 / 2018\end{array}$ \\
\hline 2685 N PACIFIC HWY & N/A & JACKSON & $10 / 13 / 2018$ \\
\hline 3000 N. VALLEV VEWW RD. & N/A & JaCkSON & $10 / 31 / 2018$ \\
\hline 30000 N. VALLF VIEW RD. & N/A & JaCKSON & $10 / 31 / 2018$ \\
\hline $\begin{array}{l}8425 \text { AGAAE RRAD } \\
6250 \text { DRY CREEK RD }\end{array}$ & N/A & JACKSON & $10 / 13 / 2018$ \\
\hline $\begin{array}{l}62600 \text { DRY CREER RD } \\
6260 \text { DRY REE RD }\end{array}$ & $\begin{array}{l}\text { N/A } \\
N / A\end{array}$ & $\begin{array}{l}\text { JaCSSON } \\
\text { IACSSON }\end{array}$ & $\begin{array}{l}101 / 3 / 21 / 2018 \\
10 / 13 / 2018\end{array}$ \\
\hline 5205 N ROGUE RIVER RD & Rogue River & JACKSON & $\begin{array}{l}10 / 31 / 2018 \\
10 / 218\end{array}$ \\
\hline 5205 N ROGUE RIVER RD & Rogue River & JACKSON & $10 / 11 / 2018$ \\
\hline 8001 AABLE ROCKKD RD & & JACKSON & 10/31/2018 \\
\hline $\begin{array}{l}\text { Off Willow Springs Rd } \\
\text { PSOBSS PBK }\end{array}$ & Medford & Jackson & 2005 \\
\hline $\begin{array}{l}1 \text { 1PSOOII PAPAK } \\
4480 \text { ROYACREST ROAD }\end{array}$ & N/A & JACKSON & $10 / 13 / 2018$ \\
\hline 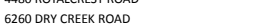 & $\begin{array}{l}N / A \\
N / A\end{array}$ & $\begin{array}{l}\text { JaCSSON } \\
\text { IACSSON }\end{array}$ & $\begin{array}{l}101 / 1 / 21 / 2018 \\
10 / 131 / 2018\end{array}$ \\
\hline 6260 DRY CREEK ROAD & N/A & $\begin{array}{l}\text { JaCKSON } \\
\text { Jack }\end{array}$ & $\begin{array}{l}10 / 131 / 218 \\
10 / 31 / 2018\end{array}$ \\
\hline 18460 HIGHWAY 62 & $\mathrm{N} / \mathrm{A}$ & JaCKSON & 10/31/2018 \\
\hline 18460 HIIGHWAV 62 & N/A & JACKSON & $10 / 31 / 2018$ \\
\hline SEC11,735S,RO2E & N/A & JaCKSON & $10 / 31 / 2018$ \\
\hline 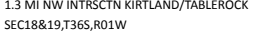 & N/A & JACCSON & $\begin{array}{l}10 / 31 / 2018 \\
1013 / 2018\end{array}$ \\
\hline 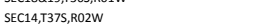 & N/A & 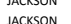 & $\begin{array}{l}101 / 1 / 2018 \\
10 / 13 / 2018\end{array}$ \\
\hline 9934 MODOC ROAD & N/A & JACKSON & $\begin{array}{l}10171 / 21 / 218 \\
10 / 31 / 2018\end{array}$ \\
\hline 9934 MODOC ROAD & $\mathrm{N} / \mathrm{A}$ & JaCKSON & $10 / 11 / 2018$ \\
\hline Ро вох 2803 & N/A & JACKSON & 10/31/2018 \\
\hline $\begin{array}{l}\text { A1E DUUTTNN RD } \\
\text { NEAR BUCK POINT ROAD }\end{array}$ & N/A & JACSKON & $\begin{array}{l}10 / 1 / 1 / 2018 \\
10131 / 2018\end{array}$ \\
\hline Off Willow Springs Rd & Medford & Jackson & 2005 \\
\hline 8087 BLACKWELL RD & $\mathrm{N} / \mathrm{A}$ & JaCKSON & $10 / 31 / 2018$ \\
\hline $\begin{array}{l}88077 \text { B BAAKWWELL LD } \\
3880 \text { KIRTLAND ROAD }\end{array}$ & N/A & JACSSON & $\begin{array}{l}10 / 31 / 2018 \\
103 / 212018\end{array}$ \\
\hline $\begin{array}{l}3033 \text { KRTTLAND RD } \\
30 \text { RD }\end{array}$ & N/A & $\begin{array}{l}\text { JACSSON } \\
\text { IACSSON }\end{array}$ & $\begin{array}{l}10 / 31 / 2018 \\
101312018\end{array}$ \\
\hline $\begin{array}{l}\text { D. } \\
\text { 2645 AVENUEG G }\end{array}$ & N/A & 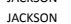 & $\begin{array}{l}10131 / 21 / 2018 \\
10131 / 2018\end{array}$ \\
\hline WEST MAIN ST. TO STEWART AVE & $\mathrm{N} / \mathrm{A}$ & JaCKSON & $10 / 31 / 2018$ \\
\hline 528 LINN ROAD & N/A & JACKSON & 10/31/2018 \\
\hline 8400 AGATE ROAD & N/A & JACKSON & 10/31/2018 \\
\hline $\begin{array}{l}1071 \text { West Jackson Road } \\
300 \text { W Vallev Vieiw Rd }\end{array}$ & $\begin{array}{l}\text { Astland, } \\
\text { Talent }\end{array}$ & Jackson & $\begin{array}{l}10 / 31 / 2018 \\
1013 / 2018\end{array}$ \\
\hline 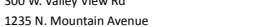 & $\begin{array}{l}\text { Talant } \\
\text { Ashland }\end{array}$ & 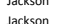 & $\begin{array}{l}10 / 31 / 2018 \\
101312018\end{array}$ \\
\hline Off Willow Springs Rd & Metford & Jackson & 2005 \\
\hline IE of Medford & Medford & Jackson & 10/31/2018 \\
\hline viver Hwy & Gold till & Jackson & $10 / 31 / 2018$ \\
\hline 190000 Caves Shighway & Caveresu & Josephine & $10 / 31 / 2018$ \\
\hline & $\begin{array}{l}\text { Eage font } \\
\text { Eaple point }\end{array}$ & & $\begin{array}{l}10 / 13 / 21 / 218 \\
1 / 1312018\end{array}$ \\
\hline & Phoenix & $\begin{array}{l}\text { Jackson } \\
\text { Jacton }\end{array}$ & $\begin{array}{l}10313218 \\
1018\end{array}$ \\
\hline 11663 Agate Road & Eagle Point & Jackson & $10 / 31 / 2018$ \\
\hline
\end{tabular}

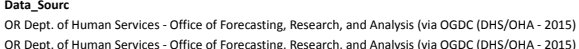

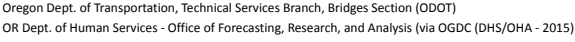
$\begin{array}{ll} & \\ & \end{array}$

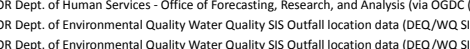
政

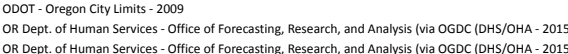
OR Dept. of Environmental Q uallity and OR Health Authority Source Water ASsessment database (DEQ/OHA SWA 2000 - 2005)

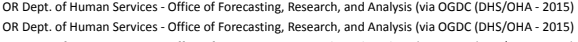

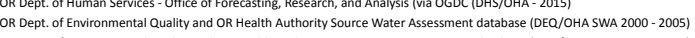

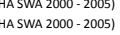

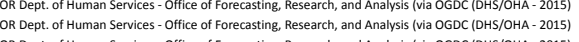

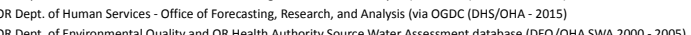

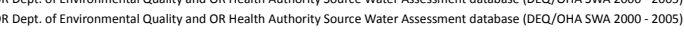
OR Dept. of Environmental Q Quality and OR Heath Authority SOurce Water Assessment databasese (DEE/OHA SWA 2000-2005)

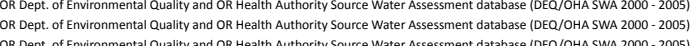
OR Dept. of Environmental Q Quality and OR Health Authority Source Water Assessment database (DEQ/OHA SWA 2000-2005)

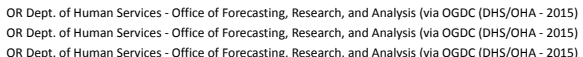

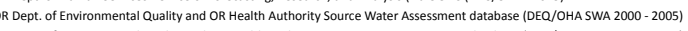

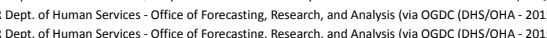

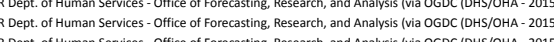
Dept. of Environmental Q Quality- Solid Waster Management System (DEQ|SWMS)

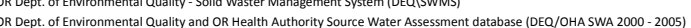

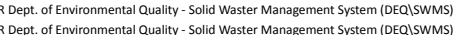

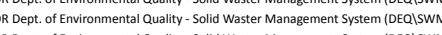

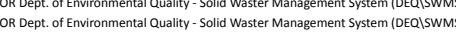
OR Dept. of Environmentat Quality - Solid Waster Management System (DEQ STSWMS

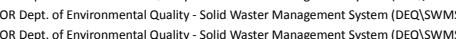

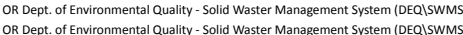
ment databse (DEQ/OHA SWA 2000 - 2005)

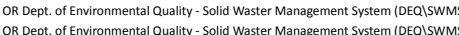

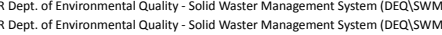

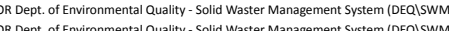

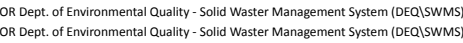

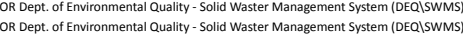

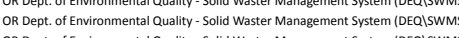

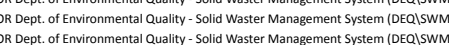
oR Dep. G f

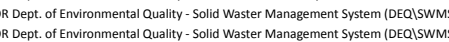

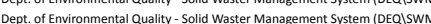

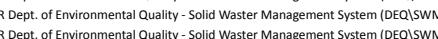

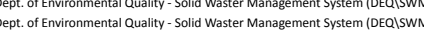

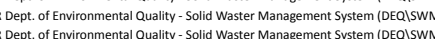

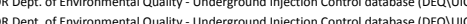

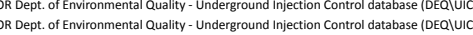

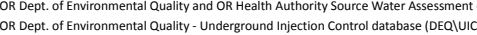

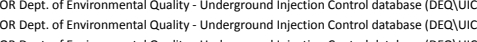
OR Dept. of Environmental Quality - Underground Injection Control databases (DEQQVivic

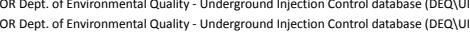

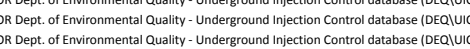

\begin{tabular}{|c|c|c|c|}
\hline \multirow{2}{*}{\multicolumn{4}{|c|}{ CScode GWRi }} \\
\hline Eagle Point so 9 & & & \\
\hline R15 & ᄂ & m & Willow Wind Community Learning Center \\
\hline 1215 & & M & $\begin{array}{l}\text { Monotube Sign nantilever Hwy } 065 \text { at MP } 8.73 \\
\text { Discover Correr }\end{array}$ \\
\hline M22 & ᄂ & & 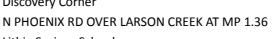 \\
\hline & M & & 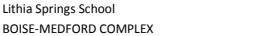 \\
\hline & M & & BOISE-MEFPROR COMPLEX \\
\hline M50 & $\begin{array}{l}M \\
M\end{array}$ & & $\begin{array}{l}\text { BOISE-MEDFORD COMPLEX } \\
\text { Medford }\end{array}$ \\
\hline 15 & & M & $\begin{array}{l}\text { Medtord In SDA School } \\
\text { Shady Point S A }\end{array}$ \\
\hline & t & & 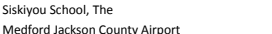 \\
\hline 125 & t & M & $\begin{array}{l}\text { Mediford a ackono County A Arp } \\
\text { St. John Lutheran School }\end{array}$ \\
\hline & t & & Dome School \\
\hline M27 & t & & $\begin{array}{l}\text { Madrone AAventist lemenentary chthool } \\
\text { Medford ackson County Airport }\end{array}$ \\
\hline & H & M & Medford J ackson County Airport \\
\hline R15 & & & $\begin{array}{l}\text { H\& R B Block Income Tax School, Central Point } \\
\text { IITR Truck School }\end{array}$ \\
\hline 215 & & & $\begin{array}{l}\text { IIRR Truck Kchool } \\
\text { Liberty Tax School White City - \#49224 }\end{array}$ \\
\hline & M & M & 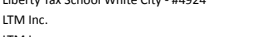 \\
\hline 127 & & & $\begin{array}{l}\text { LTM IIIC. } \\
\text { LTM Inc. }\end{array}$ \\
\hline & H & H & $\begin{array}{l}\text { Farwest steel } \\
\text { Fear }\end{array}$ \\
\hline & & & $\begin{array}{l}\text { Farwests Steel } \\
\text { Siera } \\
\text { Sine Medite Division }\end{array}$ \\
\hline R15 & i & $\mathrm{M}$ & 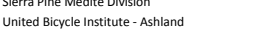 \\
\hline 215 & t & & $\begin{array}{l}\text { Ashland Institute of Massage } \\
\text { Imani nnstute of Cosmetology }\end{array}$ \\
\hline n28 & H & & $\begin{array}{l}\text { Imanal Institute of Cosmetology } \\
\text { Sierra Pine Medite Division }\end{array}$ \\
\hline & H & H & Industrial Park \\
\hline & & $M$ & Rogue Community College \\
\hline & & & $\begin{array}{l}\text { Southern norgen Unviversity } \\
\text { Crater Acadery of Heasth \& Public Services }\end{array}$ \\
\hline & & & $\begin{array}{l}\text { Crater Aadere Charter Academy } \\
\text { Cric Services }\end{array}$ \\
\hline & & & PROSPECT LANOFLL \\
\hline & m & & ASSOCIATED FRUIT CO. \\
\hline & M & & 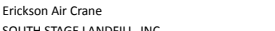 \\
\hline & & & $\begin{array}{l}\text { SOUTH HTSGE LANFFLL, INC. } \\
\text { MATT GARIS WASTE OL RECOVERY, INC. }\end{array}$ \\
\hline R10 & & & $\begin{array}{l}\text { MEDITE-MEDFORD DIVISION } \\
\text { MER, INC. }\end{array}$ \\
\hline & H & & VALLEY VIEW LANDFILL, INC. \\
\hline & & & $\begin{array}{l}\text { VALLY VEEW AADFFLL, INC. } \\
\text { BURRILL LUMBER LANDFILL }\end{array}$ \\
\hline & m & & 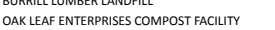 \\
\hline & m & & OAK LEAF ENTERPRISES COMPOST FACLITT \\
\hline & & & LOUIIANA-PACIFIC CORPORATION \\
\hline & H & H & LOUISIANA-PACLFC CORPORATION \\
\hline & & M & REOCUE TRAASFER \& RECYCLING, LC \\
\hline & H & H & 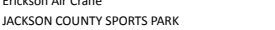 \\
\hline & M & H & BEAR CREEK ORCHARDS, INC. \\
\hline & & & DRY CREER LANOFLL, INC. \\
\hline & M & H & DRY CREE LANOFLL, INC. \\
\hline & m & ${ }_{H}^{H}$ & ROGUE WONDER FARM \\
\hline & M & H & $\begin{array}{l}\text { ROGUEE WNNDER FARM } \\
\text { BUTTE FALS LANDFIL }\end{array}$ \\
\hline R10 & H & H & ROTO-ROOTER SEPTAGE TRANSFER STATION \\
\hline & & & KEN DENMAN WILDLIFE MGMT. AREA \\
\hline & m & & $\begin{array}{l}\text { MOOOC ORCAARD } \\
\text { MOISPOSAL SIII }\end{array}$ \\
\hline & & H & MODOC ORCHARD \\
\hline & & H & BURNS TIRE RECYCLING, INC. \\
\hline & H & H & RICHARD R. WILSON TRUCKING \\
\hline & & H & JACKSON CREEK LSS STITE \\
\hline Mos & m & H & 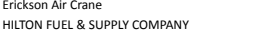 \\
\hline Mos & M & H & HILTON FUEL \& SUPPIY COMPANY \\
\hline 10 & & & BLACKWELL ROAD SITE \\
\hline & & H & WHITE CITY RECYCLING \\
\hline & & & LOZZER LANE IMPROVEMENT \\
\hline & & $H$ & TERBAMAI \\
\hline & & $\mathrm{L}$ & Village at Valley View Memory Care \\
\hline & & & Former Wal-Mart Store 12069 \\
\hline & & & 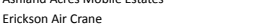 \\
\hline & M & M & OPRR - Casey State Recreatic \\
\hline & & m & Cypress Grove RV Park \\
\hline & H & $\begin{array}{l}M \\
M\end{array}$ & 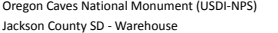 \\
\hline & & m & 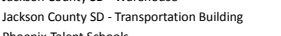 \\
\hline & H & M & $\begin{array}{l}\text { Phoenix-Talann Schools } \\
\text { Pheasant Brook at Eagle Point }\end{array}$ \\
\hline
\end{tabular}

PCSType
Schools

Thssontation Corridors- Stream Crossing - Perennial - Stream Crossing- Perenn Whols
Wasteter Treatment Plants/Collection Stations
Wastevater Treatment Plants/Collection stations

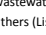

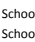

Schools
UST - Decommisioned - Inactive

STT-Decor
Schools
Schools
Schools

UST- Upgraded and/or Registered -Active

Schools
Schools

UST - Confirmed Leaking but listed as NFA- DEQ LUST Lis UST - Upgraded and/or Registered - Active
Metal Plating/Finishing/fabriction

UST-Confirmed Leaking but isted as N NA - DEQ WUST List

Sodools
Schools
Schools

Schools
Schools
ust- Status Unknown
Miscellaneous Manufacturing

Schools
Schools

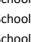

Municipal//ndustrial Land

Composting Facilities Maintenance Shop/Equipment Storage- Not Transportation Related

ustrial landfill

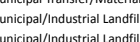

Municipal Transfer/Matern

Municipal/nnustrial landfil
Composting Facilities

Compossting facilitites

Municipal//ndustrtrat Landfil

Icover//kxporter

Municipal/ndustrial Landfil
Composting Facilities

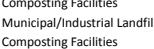

Composting Facilities
Composting Facilities

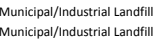

Municipal//ndustrial land

Composting Facilities
Compstion

Waste tire carrier/storage
Waste tire carrier/storage

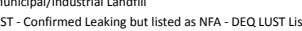

Composting Facilities
Composting facilities

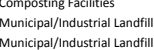

Waste tire carrier/storage
Muncicial//ndustrial landfill

Municipal/ndustrial landfif
Municipal//ndustrial landfil

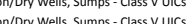

UST-Upgraded and/or Registered - Active (Serves 220)

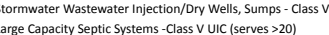

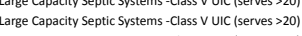

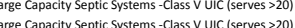

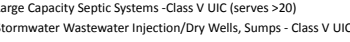




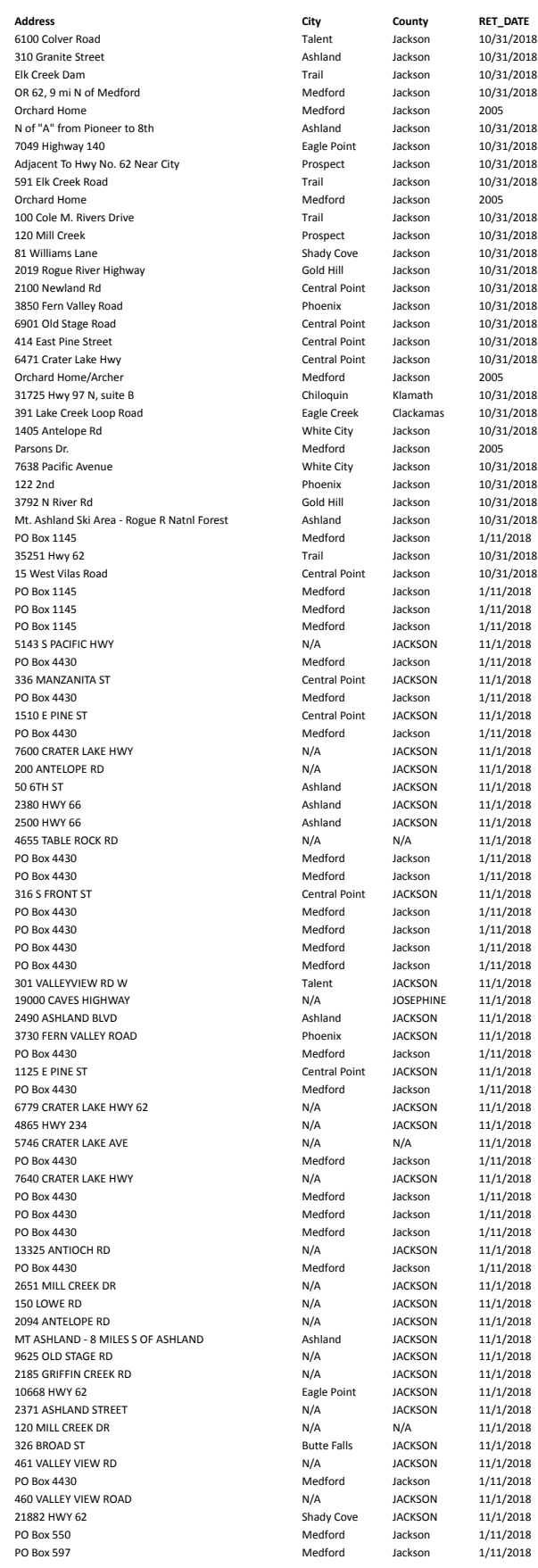

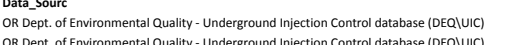

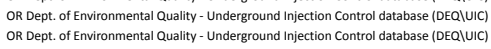

tabase (DEQ/OHA SWA 2000 - 2005)

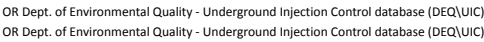

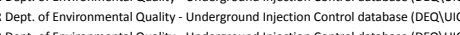

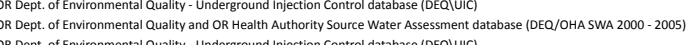

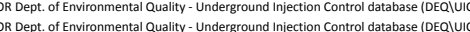

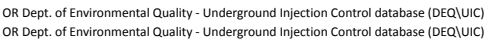

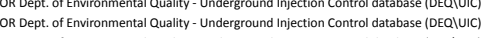

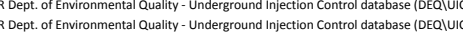

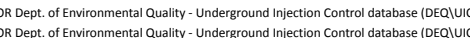

OR Dept. of Environmental Q Quality and OR Heath Authority Source Water Assessment database (DEQ/OHA SWA 2000- 2005)

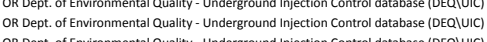

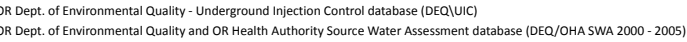

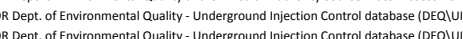

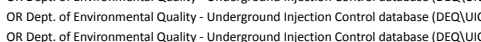

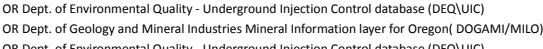

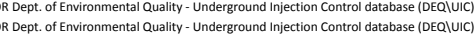

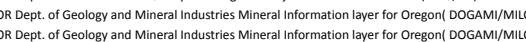

OR Dept. of Geology and Mineral industries Mineral hiformation layer for Oregon( DOGAM/MILO

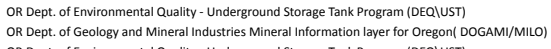

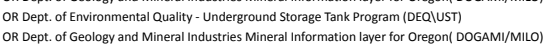

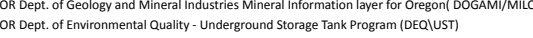

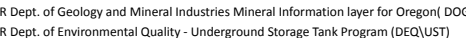

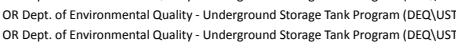

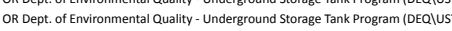

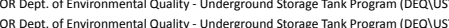

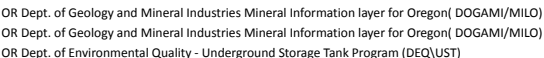

OR Dept. of Geology ynd Mineral Industries Mineral Information layer for Oregonn(DOGAM/MLILO

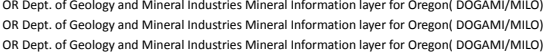

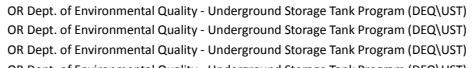

OR Dept. of Geelogy and Mineral Industries Mineral Information layer for Oregon( DOGAM/MILO

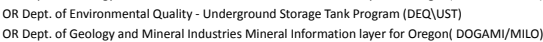

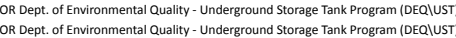

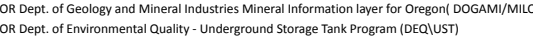

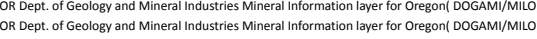

OR Dept. of Geology and Mineral Industries Mineral Information layer for Oregont ( D OGAMI/MILO)

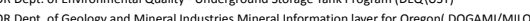

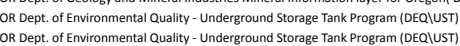

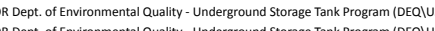

OR Dept. of Environmental Q uality - Underground Storage Tank Frogram (IEEQluST)

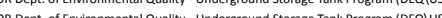

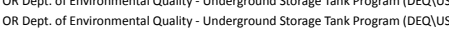

OR Dept. of Environmentat Q Quality - Undererground Storage Tank Program (DEQQUUST)

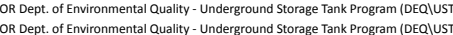

OR Dept. of Geology and Mineral Industries Mineral Information layer for Oregon( DOGAM/MIOO

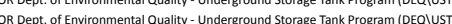

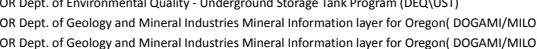

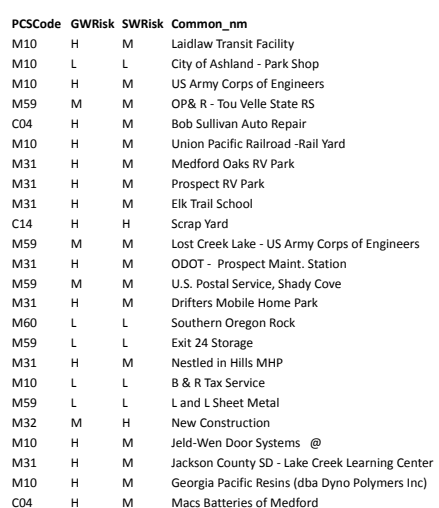

Jackson County SD- Lake Creek Learning Center
Georgia Pacific Resins (dba oyno Polymers

Vickers
city of phoent

city of hhoenix
OPBR - valley of the Rogue State Part

Ski Ashland
Table Rockstite

OPRR- Stewart State Park
former White's Midway Service

Former White'
Gebhard Pit
Hensen Site

Gebhard Pit
Hansen Stite
Kendal Bar

Kendall bar
Us west communications, inc

US WEST COMMUNICATIONS

CHERRON USA INC S 5983

CHEVRoN U.S.A.

JACKSON COUNTY RRADS AND PARKS SVS

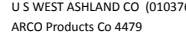

CHEVRON USA ANC

Modoc Road

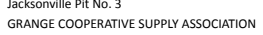

Whestsone Pit

ARCO PRODOCTS CO FAC 5959

USDOI; NATIONAL PARK SERVVC

PEETRO:UQEE 224

Black Rock Pit

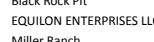

COUNN OIL (MEEFORO HE)

RANEY, MICHAELL.
HAYS OL CARDOOCK \#1

Jacksonville Pit N

PANoCo, INC.
Marr Daring Pit

Old dideal Quarry
Hi Banks Bock Produts

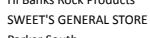

Parker South
CASCADE GORGE STORE

BILT TERENANG INC-ASHLAND
BILT TERENING, INC-WHTIE CII

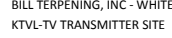

DARDANELL SERVICE
STAGE STOP STORE

EAGLE POINT AM/PM

OR STATE HWY 3-8 PROSPECT
BUTTE FALLS SERVICE STATION

BUTTE FALLS SERVICE STATIO
ROGUE VALLY STATION

Boise Cascade Pit
VAlLY VIEW EXXoN
PCSType

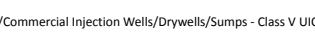

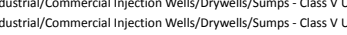

hidustria//Commercial lijection Wells/Orrwwells/Sumps - Classs VVics

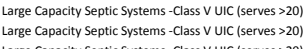

arge Capacity Septic Systems -Class V UIC (serves 20)

Sormwater Wastewater Injection/Ory Wells, Sumps - Class V Vics

Stormwater Wastewater Injection/Dry Wells, Sumps - Class V VICS

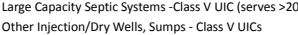

Stormwater Wastewater Ijjection/orr Wells, Sumps- Class V Vilcs

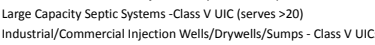

stormwater Wastewater rijection/or Wells, sumps - Class v Vics

dustrial/Commercial lnjection Wells/orywells/sumps - Class V Vics

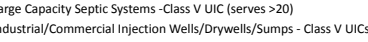

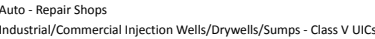

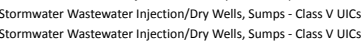

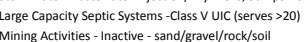

large Capacity Septic Systems - Class V UIC (Serves 20)

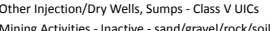

Mining Activities - Inactive - sand//ravel/rock/soil
Mining Activities - Inactive - sand/gravel/rock/soil

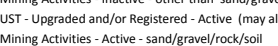

UST - Upgraded and/or Registered - Active (may als

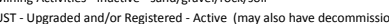
hactive - sand/grave//rock/soil

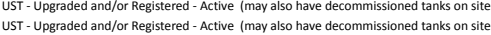

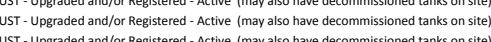

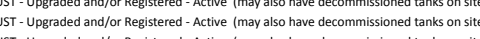

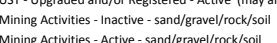

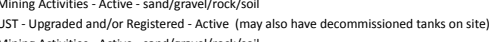

Mining Activities - Inactive - Sand/Farvel/rock/soil

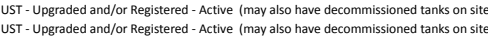

UST - Upgraded and/or Reeistered- Active me may dof

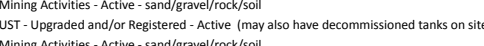

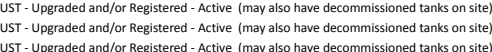

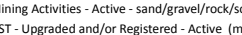

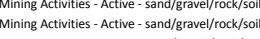

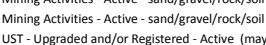

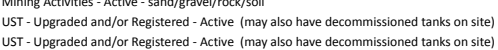

UST - Upgraded and/or Registered - Active (may a also have decommisisioned tanks on Situ

UST - Upgraded and/or Registered - Active (may also have decommissioned tanks on site

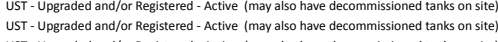

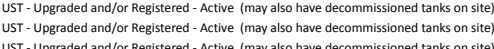

UT - Upgraded and/or Registered-Active (may also have decommissioned tanks on 5 il

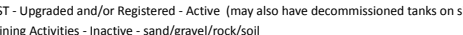

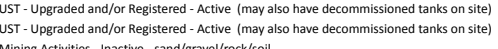

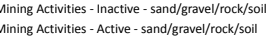




\begin{tabular}{|c|c|c|c|}
\hline $\begin{array}{l}\text { Address } \\
\text { PO B B } 8863\end{array}$ & City & $\begin{array}{c}\text { County } \\
\text { Jackson }\end{array}$ & RET_DATE \\
\hline $\begin{array}{l}\text { PO Box } 8363 \\
1515 \text { SISKYY BIV BIV }\end{array}$ & $\begin{array}{l}\text { Metiford } \\
\text { Ashland }\end{array}$ & $\begin{array}{l}\text { Jacason } \\
\text { JaCKSON }\end{array}$ & $\begin{array}{l}1 / 1112018 \\
1 / 1 / 2018\end{array}$ \\
\hline 16 NRONT ST & Central Point & N/A & $11 / 1 / 2018$ \\
\hline 60 SHAMROCK LANE & N/A & Jackson & 11/1/2018 \\
\hline $\begin{array}{l}24231 \text { HWV Y } 22 \\
\text { 21 TALENT AVE }\end{array}$ & $\begin{array}{l}\text { N/A } \\
\text { Talent } \\
\text { S }\end{array}$ & $\begin{array}{l}\text { N/A } \\
\text { Nacsoon }\end{array}$ & 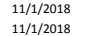 \\
\hline $\begin{array}{l}\text { 21 TAAENT A AE } \\
\text { PO BOX 999 }\end{array}$ & $\begin{array}{l}\text { Talant } \\
\text { Mefford }\end{array}$ & Jackson & 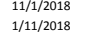 \\
\hline $\begin{array}{l}\text { Posse Ln } \\
\text { Pon }\end{array}$ & Medford & $\begin{array}{l}\text { Jacksoun } \\
\text { Jackson }\end{array}$ & $\begin{array}{l}1 / 1 / 12018 \\
2005\end{array}$ \\
\hline 945 N STH ST & 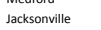 & N/A & 11/1/2018 \\
\hline Posse Ln. & Medford & Jackson & \\
\hline 5600 BUTTE FALL HWY & N/A & N/A & $11 / 1 / 2018$ \\
\hline $\begin{array}{l}90 \text { N MOONTAIN AVE } \\
20876 \text { HWY } 62\end{array}$ & N/A & $\begin{array}{l}\text { JACKSON } \\
\text { IACSSON }\end{array}$ & 的11/1/2018 \\
\hline $\begin{array}{l}26876 \text { HWW } 62 \\
21825 \text { HWY } 62\end{array}$ & $\begin{array}{l}N / A \\
\text { Shady Cove }\end{array}$ & JACSSON & 11/1/2018 $11 / 12018$ \\
\hline 540 MLL CREEK & & JACKSON & $\begin{array}{l}111 / 12018 \\
11 / 12018\end{array}$ \\
\hline Posse Ln. & & Jackson & 2005 \\
\hline RIVERSIDE \& MCANDREWS - SE CORNER & Medford & IACCSON & $10 / 31 / 2018$ \\
\hline Ross Ln and Stonefield Way & $\begin{array}{l}\text { Medford } \\
\text { Methat }\end{array}$ & Jackson & 2005 \\
\hline $\begin{array}{l}\text { 4M99 EAMAIN ST } \\
\text { Rossante }\end{array}$ & $\begin{array}{l}\text { Ashland } \\
\text { Mefford }\end{array}$ & Jackson & $\begin{array}{l}111 / 12018 \\
2005\end{array}$ \\
\hline $\begin{array}{l}\text { Rossanley } \\
\text { Rossanley and Ross Ln. }\end{array}$ & $\begin{array}{l}\text { Metotord } \\
\text { Metford }\end{array}$ & $\begin{array}{l}\text { Jacksson } \\
\text { Jackson }\end{array}$ & \\
\hline $\begin{array}{l}\text { Rossalley and Ross In. } \\
\text { 1065 E PINE ST }\end{array}$ & $\begin{array}{l}\text { Meadord } \\
\text { Central Point }\end{array}$ & $\begin{array}{l}\text { Jackson } \\
\text { JackSON }\end{array}$ & $\begin{array}{l}2005 \\
11 / 1 / 2018\end{array}$ \\
\hline Rossanley Dr. & Medford & Jackson & \\
\hline $\begin{array}{l}\text { Rossanley Dr. } \\
\text { DSogur }\end{array}$ & Medford & Jackson & \\
\hline $5595 \mathrm{SPACIFCCHWY}$ & N/A & Jackson & $\begin{array}{l}11 / 1 / 2018 \\
2005\end{array}$ \\
\hline 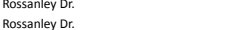 & $\begin{array}{l}\text { Mefored } \\
\text { Medford }\end{array}$ & $\begin{array}{l}\text { Jacksson } \\
\text { Jackson }\end{array}$ & \\
\hline $\begin{array}{l}\text { Kossantey r. } \\
\text { 404 2ND AVE }\end{array}$ & 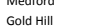 & $\begin{array}{l}\text { Jackson } \\
N / A\end{array}$ & $\begin{array}{l}2005 \\
11 / 12018\end{array}$ \\
\hline 2495 Ashland Street & Ashland & Jackson & $\begin{array}{l}111 / 1018 \\
11 / 1 / 2018\end{array}$ \\
\hline 21222 HWr 62 & Shady Cove & Jackson & $11 / 1 / 2018$ \\
\hline 1590 E PINE ST & Central Point & Jackson & 11/1/2018 \\
\hline 3082 SAMMIKE DR & N/A & Jackson & $\begin{array}{l}11 / 1 / 2018 \\
2005\end{array}$ \\
\hline 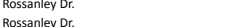 & $\begin{array}{l}\text { Medford } \\
\text { Mefford }\end{array}$ & $\begin{array}{l}\text { Jackson } \\
\text { Jackson }\end{array}$ & $\begin{array}{l}2005 \\
2005\end{array}$ \\
\hline 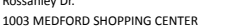 & $\begin{array}{l}\text { Mefiford } \\
N / A\end{array}$ & $\begin{array}{l}\text { Jackson } \\
\text { JackSON }\end{array}$ & $\begin{array}{l}2005 \\
11 / 1 / 2018\end{array}$ \\
\hline 2402 West Main Street & $\mathrm{N} / \mathrm{A}$ & Jackson & $\begin{array}{l}111 / 12018 \\
11 / 1 / 2018\end{array}$ \\
\hline 280 MAPLE STREET & Ashland & Jackson & 11/1/2018 \\
\hline 382 Wightman Street & Ashland & JaCKSON & $11 / 1 / 2018$ \\
\hline 105 North oregon Street & Jacksonville & Jackson & $\begin{array}{l}11 / 1 / 2018 \\
2005\end{array}$ \\
\hline $\begin{array}{l}\text { Rossanley D. } \\
\text { RT. 1, MISSION HWY (NEAR DELMARTER'S) }\end{array}$ & $\begin{array}{l}\text { Meford } \\
\text { Medford }\end{array}$ & $\begin{array}{l}\text { Jackson } \\
\text { Jackon }\end{array}$ & $\begin{array}{l}2005 \\
10 / 31 / 2018\end{array}$ \\
\hline Runs through Drinking Water Source Area & Medford & Jackson & $1 / 20 / 20178: 0$ \\
\hline Runs Through OWPA & Medford & & \\
\hline 730 EAST MAIN ST. & Phoenix & JaCKSON & $11 / 1 / 2018$ \\
\hline 501 E MAIN ST & Rogue River & JACKSON & 11/1/2018 \\
\hline $\begin{array}{l}1115 \text { ANAHONN WAY } \\
3075 \text { HAMRICK RD }\end{array}$ & $\begin{array}{l}N \text { NA } \\
\text { Central Point }\end{array}$ & $\begin{array}{l}\text { JaCGSON } \\
\text { ACCSSON }\end{array}$ & 11/1/2018 $11 / 1218$ \\
\hline 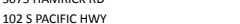 & $\begin{array}{l}\text { Talent } \\
\text { Talm }\end{array}$ & 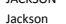 & 11/1/12018 11218 \\
\hline $\begin{array}{l}\text { Not applicable } \\
\text { Pate }\end{array}$ & SHADY COVE & JACKSON & $\begin{array}{l}1 / 1 / 1 / 2018 \\
2009\end{array}$ \\
\hline Not applicable & CENTRAL POINT & JaCKSON & 2009 \\
\hline Not applicable & CENTRAL POINT & JACKSON & 2009 \\
\hline 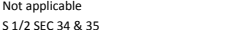 & $\begin{array}{l}\text { ButTef Falls } \\
\text { Medford }\end{array}$ & JACKSON & 2009 200912018 \\
\hline 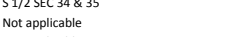 & ASHLAND & JACKSON & \\
\hline Not applicable & TrAal & JACKSON & 2009 \\
\hline $\begin{array}{l}\text { Not applicale } \\
\text { Notapicable }\end{array}$ & $\begin{array}{l}\text { ASHLLAND } \\
\text { ASHLND }\end{array}$ & JACKSON & 2009 \\
\hline 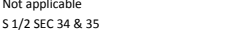 & $\begin{array}{l}\text { ASLLAND } \\
\text { Medford }\end{array}$ & $\begin{array}{l}\text { A AACSSON } \\
\text { JACSON }\end{array}$ & $\begin{array}{l}2009 / 2018 \\
10 / 31 / 21\end{array}$ \\
\hline Sage Rd. & Medford & Jackson & 2005 \\
\hline $\begin{array}{l}\text { Not applicable } \\
\text { sw HHuUSTON LAKE RD. }\end{array}$ & $\begin{array}{l}\text { Golo HILL } \\
\text { Portland }\end{array}$ & JaCKSON & 2009 $201 / 12018$ \\
\hline & $\begin{array}{l}\text { Portland } \\
\text { Mefford }\end{array}$ & $\begin{array}{l}\text { Crook } \\
\text { Jackson }\end{array}$ & $\begin{array}{l}100131 / 2018 \\
2005\end{array}$ \\
\hline 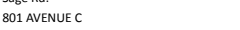 & $\mathrm{N} / \mathrm{A}$ & JACKSON & 10/31/2018 \\
\hline 8399 14TH ST & N/A & JACKSON & 10/31/2018 \\
\hline 1977 WWY Y9 N N & N/A & JACKSON & 10/31/2018 \\
\hline 2872 S PACIFIC HWY & N/A & JACKSON & 10/31/2018 \\
\hline 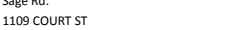 & $\begin{array}{l}\begin{array}{l}\text { Meford } \\
N / A\end{array} \\
\text { S }\end{array}$ & $\begin{array}{l}\text { Jackson } \\
\text { JaCKSON }\end{array}$ & 101/31/2018 \\
\hline & $\mathrm{N} / \mathrm{A}$ & JACKSON & 10/31/2018 \\
\hline 2518 S PACIFIC HWY & $\mathrm{N} / \mathrm{A}$ & JACKSON & 10/31/2018 \\
\hline 2518 S PACIFIC HWY & N/A & JACKSON & 10/31/2018 \\
\hline $\begin{array}{l}3959 \text { HAMRICK RD } \\
7797511 \text { ST ST }\end{array}$ & N/A & JACKSON & 10/31/2018 1018 \\
\hline $\begin{array}{l}7975111 \text { 11H ST } \\
1155 \text { ANTELOPE RD }\end{array}$ & $\begin{array}{l}N / A \\
N / A\end{array}$ & $\begin{array}{l}\text { J IACCSON } \\
\text { JACKSON }\end{array}$ & $\begin{array}{l}10 / 11 / 21 / 218 \\
10 / 31 / 2018\end{array}$ \\
\hline 25 E. McAndrews & $\mathrm{N} / \mathrm{A}$ & JACSON & 10/31/2018 \\
\hline 25 E. McAndrews & N/A & & 10/31/2018 \\
\hline 51 MODOC RD & N/A & JACKSON & 10/31/2018 \\
\hline 12451 MODOC RD & N/A & JACKSON & 10/31/2018 \\
\hline 2350 AVE G & N/A & JACKSON & 10/31/2018 \\
\hline 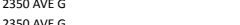 & N/A & JaCKSON & 10/31/2018 \\
\hline 1795 ANTELOPE RD & N/A & 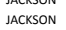 & $\begin{array}{l}10 / 3172018 \\
10 / 31 / 2018\end{array}$ \\
\hline 8124 PACIFIC AVE & N/A & 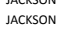 & $\begin{array}{lll}10 / 3112018 \\
10 / 1 / 2018\end{array}$ \\
\hline 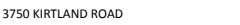 & & JaCKSON & 10/31/2018 \\
\hline Sage Rd. & Mefford & Jackson & 2005 \\
\hline 930 ANTELOPE RD & & JACKSON & 10/31/2018 \\
\hline
\end{tabular}

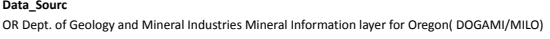

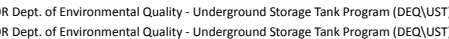

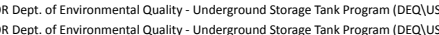

OR Dept. of Environmental Quality - Underground Storage Tank Program (DEQQUST)

Deept. of Gealogy and Mineral indastes

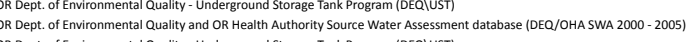

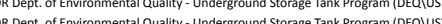

OR Dept. of Enironmental Q Quality - Underground Storogee Tank Program (DEQQUST)

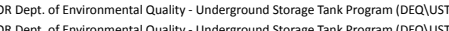

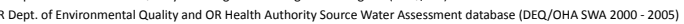

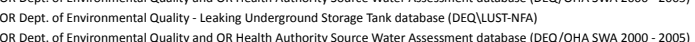
OR Dept. of Environmental Q uallity - Underground Storage Tank Program (DEQQUST)

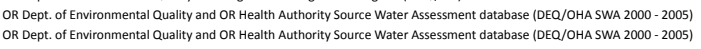

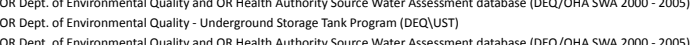

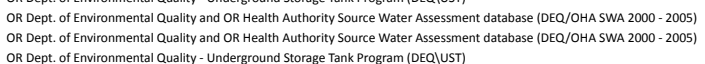

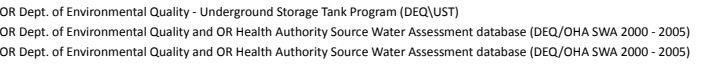

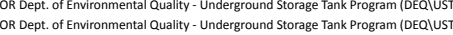

OR Dept. of Environmental Q Qually - Underground Storage Tank Rrogram (IEQQQUST)

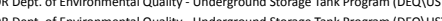

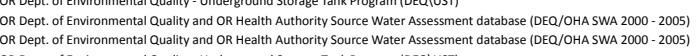

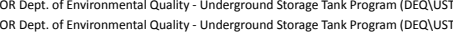

OR Dept. of Environmental Q Quality - Underground Storage Tank Frogram (DEQQU)ST)

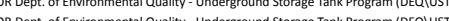

OR Dept. ff Environmental Q Quality and OR Heath Authority Source Water A Asessment database (DEG/OHA SWA 2000- 2005)

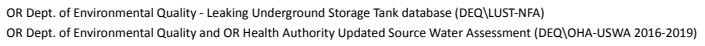

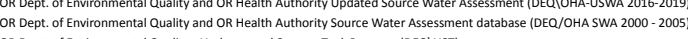

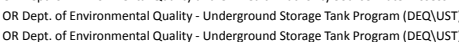

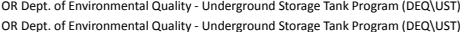

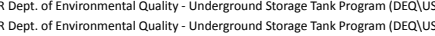

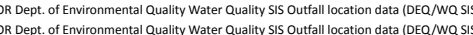

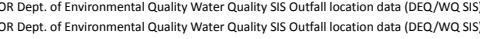

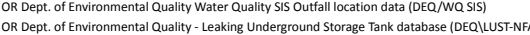

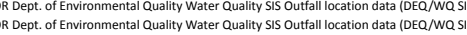

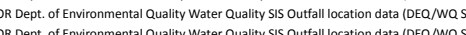

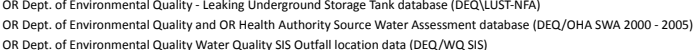

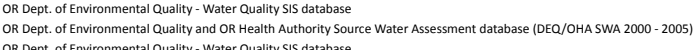

OR Dept. of Environmental Q Quality - Water Quality IS databass

OR Dept. of Environmental a uality and OR Heath h Authority Sor

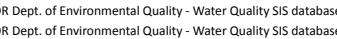

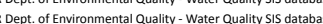

OR Dept. of Environmental Quality - Water Quality SIS database
OR Dept. of Environmental Quality - Water Quality SIS databas

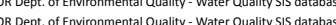

OR Dept. of Environmental Quality - Water Quality IS database

OR Dept. of Environmental Quality - Water quality IIS database

OR Dept. of Environmental Quality - Water Quality SIS datatabse

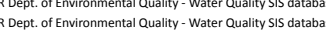

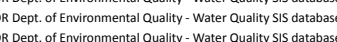

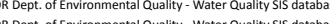

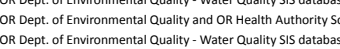

\begin{tabular}{|c|c|c|}
\hline & & \\
\hline 699 & M & 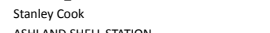 \\
\hline M27 & $t_{2}^{2}$ & $\begin{array}{l}\text { ASHLLND SHEL SATTION } \\
\text { B-MOR STATIONS \# }\end{array}$ \\
\hline M27 & $\mathrm{i}$ & \\
\hline M272 & L & $\begin{array}{l}\text { TRALL MAARET } \\
\text { TTLENGSA GES H9400 }\end{array}$ \\
\hline & M & Charter first Mortgage, Inc. \\
\hline${ }_{127}^{c 10}$ & ${ }^{\mathrm{M}}$ & All-star Towing \\
\hline & $\mathrm{L}_{\mathrm{H}}$ & $\begin{array}{l}\text { JAAKSONVILE TEXACO } \\
\text { Profile Grinding }\end{array}$ \\
\hline & L & $\begin{array}{l}\text { MIOWAY COUSTRYY STORE } \\
\text { MGAY }\end{array}$ \\
\hline 2027 & L & 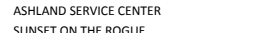 \\
\hline & L & $\begin{array}{l}\text { SUNSET TN THE RGGUE } \\
\text { SHAO COVE 7 } 6 \text { INC. }\end{array}$ \\
\hline & ᄂ & PROSPECT VILAGE SERVICE CENTER \\
\hline M26 & H & $\begin{array}{l}\text { Proflil Grinding } \\
\text { CIrT OF MEDFORD - RIVERSID/MCANDREWS }\end{array}$ \\
\hline & H & NW Meadows Subdivision \\
\hline $\mathrm{c} 17$ & L & \\
\hline м322 & M & $\begin{array}{l}\text { Lectra Coat } \\
\text { Hampton Place and Candlewood PUD }\end{array}$ \\
\hline M22 & & CENTRAL POINT BP \\
\hline 221 & н & $\begin{array}{l}\text { Decommissioned UST } \\
\text { Minuteman Press }\end{array}$ \\
\hline & L & JACCSON CNTY DPW, PHOENIX TALENT SHOP \\
\hline & ${ }_{H}^{H}$ & $\begin{array}{l}\text { TRA Supply Co } \\
\text { Paficichemtech }\end{array}$ \\
\hline M27 & $\mathrm{L}$ & GOLD HILL TEXACO \\
\hline & L & ASHLAND TEXACO \\
\hline & ᄂ & SHADY COVE CHEVRON \\
\hline & & PLLOT TRAVEL CENTERS LC \\
\hline & M & ARCO AM/PM \\
\hline & M & $\begin{array}{l}\text { Daviv Finisis Products } \\
\text { Ekerson Roofing }\end{array}$ \\
\hline 127 & i & $\begin{array}{l}\text { SAFEWAY GASOLLINE } \\
\text { SARE }\end{array}$ \\
\hline M27 & L & ASHLAND COMMUNITY HOSPTTAL \\
\hline & เ & SOUTHERN OREGON UNIVERSITY \\
\hline & ᄂ & QWEST - JACKSONVILEE CO (R00289) \\
\hline M26 & L & $\begin{array}{l}\text { Morton nillilg } \\
\text { PARRCIA LLTCERER }\end{array}$ \\
\hline M52 & i & Crooked Creek \\
\hline M27 & $L^{M}$ & $\begin{array}{l}\text { IIriration Canal } \\
\text { CIRCLE KAS GAS }\end{array}$ \\
\hline M27 & t & LIL \\
\hline & & WHTE CITY CARDLOCK \\
\hline & 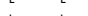 & COSTCO GASOLINE \# 1287 \\
\hline & & UNDEFNED \\
\hline & M & 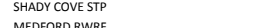 \\
\hline & m & $\begin{array}{l}\text { MEEFROR OWRF } \\
\text { MEDFOR RWRE }\end{array}$ \\
\hline & m & $\begin{array}{l}\text { BUTTE FALLS STP } \\
\text { S }\end{array}$ \\
\hline & ᄂ & MEDFRO VORTAC \\
\hline & $m$ & ASHLANO STP \\
\hline & $m$ & COUNTRY VEЕW MOB̈LE HOME ESTATES \\
\hline & M & VaLLEY VIEW LANDFFLL \\
\hline M26 & th & $\begin{array}{l}\text { VALLYY VIEL LANDFFLL } \\
\text { MEDOOD YORTAC }\end{array}$ \\
\hline M28 & M & Bigelow \\
\hline & $\begin{array}{c}M \\
M \\
M\end{array}$ & 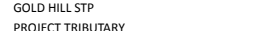 \\
\hline c17 & H & A \\
\hline & H & CROMAN CORPORATION LOGGING DIVISION \\
\hline & H & CASCADE WOOO PRoDUCTS, INC. \\
\hline & $m$ & $\begin{array}{l}\text { BUTLER FORD INC } \\
\text { TUUKRE SOC.CA CORPOBATION }\end{array}$ \\
\hline & H & $\begin{array}{l}\text { TUCKER SNOC-CAT CORPOOAATION } \\
\text { Timber Products Plywood }\end{array}$ \\
\hline & $m$ & $\begin{array}{l}\text { CSC, INC. } \\
\text { CSucts Plywood }\end{array}$ \\
\hline M62 2 & H & ALLWEATHER WOOD TREATERS \\
\hline & M & BEAR CREEK OPERATIONS INC \\
\hline & t & $\begin{array}{l}\text { BAER C CEEEE OPREATIONS INC } \\
\text { ITM, INCORPORATED }\end{array}$ \\
\hline 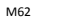 & & U.S. FOREST INDUSTRRES, INC. \\
\hline & m & BOISE CASCADE CORPOORATION \\
\hline & H & TIMBER PRODUCTS CO \\
\hline M5 & M & TIMBER PRODUCTS CO \\
\hline M62 & H & $\begin{array}{l}\text { PACFIII PAVIVG, NNC. } \\
\text { PAEIC PAIIG , }\end{array}$ \\
\hline & H & $\begin{array}{l}\text { PACFLC PAVIVE, INC. } \\
\text { BOMAS ONE }\end{array}$ \\
\hline & H & BIOMASS ONE LP \\
\hline & L & BIOMASS ONE LP \\
\hline 457 & $\mathrm{H}$ & EASTMAN KODAK COMPANY \\
\hline & ${ }_{H}^{H}$ & ROGUE AGGREGATES, INC. \\
\hline & $\mathrm{H}$ & 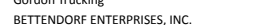 \\
\hline
\end{tabular}

Mining Activities- - Inactive - sand/fravel/rockssoil

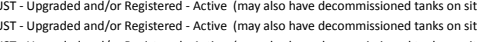

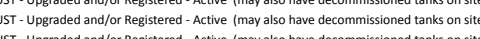
UST - Upgraded and/or Registered - Active (may also have deccommisisioned tanks on stite Mining Activities- Inactive-sand/gravel/rock/sol

UST - Upgrade
Machine Shop UST - Uppgraded and/or Registered - Active (may also have decommisisioned tanks on site) UST- Dppraded and/or Registered - Active (may also have decommisisioned tanks on Sil Metal Plating//Finshing//Fabrication
UST- Confirmed Leaking but isted as NFA - DEQ LusT Lis

UST - Uperaded and/or Reeis Construction/Demolition Area

UST - Upgraded and/or Registered - Active (may also hav
UST - Confirmed Leaking but isted as NFA - DEQ LUST LiS

toto Processing//rinting

政

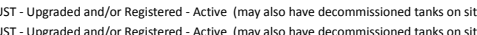
UST- Upgraded and/or Registered- -Active (may also have decommissioned tanks on 5 (t)

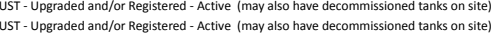
UST - Uprraded and/or Registered A A
Furniture/Lumber/Parts Stores

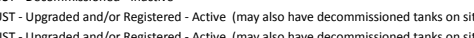
UST - Upgraded and/or Registered - Active (may also have decommissioned tanks on 5

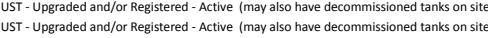
UST- Decommissioned - Inactive UST- Conffrmed leaking bist
Ponds/streass Wetrands

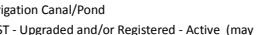
UST - Upgeraded and/or Registered - Active (may also have decommissioned tanks on Sit UST- Upgraded and/or Reisitered- - Active (may also have decommisisioned tanks on site UST - Uppraded andor Registered- -Active (may also have decommisisioned tanks on stete Wastewater Treatment Plants Collection Stations
Wastewater Treatment Plants//Colection Stations

Wastewater Treatment Plants/Collection Stations Wastewater Treatment Plants/Collection Stations Wastewater Treatment Plants/Collection Stations
Wastewater Treatment Plants//Collection Stations UST - Confirmed Leaking but listed as NFA - DEQ LUST List ust- Status snknowe Wastewater Treatment Plants/Collection Stations
DEQ Permited Stormwater Discharges NPDEES or WPCA Metal Plating//inishing//Fabrication

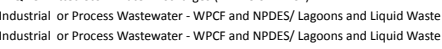
Wood Preserving/Treating DEQ Permitted Stormwater Discharges (NPDES or WPCF Industrial or Process Wastewater - WPCF and NPDES/ Lagons and Liquid Waste Industrial or Process Wastewater - WPCF and NPEES/ Lagoons snd Liquidid Waste

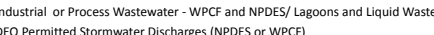
dustrial or Process Wastewater - WPCF and NPDES/L

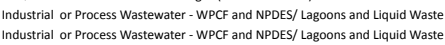
Industrial or Process Wastewater- - WPCC and NPDES/ Lagoons and Liquid Waste EeQ Permitited Stormwater Discharges (NPDEE or or WPCF) DEQ Permitted Stormwater Discharges (NPDES or WPCA Q Permitted Stormwater Discharges (NPDES or WPCF (S) Lageons and Liquid waste OEQ Permited Stormwater Discharges (NPDES or WPCF) 


\begin{tabular}{|c|c|c|}
\hline & & \\
\hline 1761 SOUTH STAGE RD & N/A & Jackson \\
\hline 5422 BLACKWELL RD & N/A & JACKSON \\
\hline $\begin{array}{l}1200 \text { AVENEUE G } \\
200 \text { COEE M RIVERS DR }\end{array}$ & NAA & $\begin{array}{l}\text { JACCSSON } \\
\text { JACKSON }\end{array}$ \\
\hline $\begin{array}{l}2000 \text { COEL M RVVERS DR } \\
3000 \text { N VALEY VEW RD. }\end{array}$ & N/A & JaCcson \\
\hline \multirow{3}{*}{$\begin{array}{l}72806 \text { BLCKWEEL RO. } \\
\text { 4865 HWV 234 }\end{array}$} & N/A & JACKSON \\
\hline & N/A & $\begin{array}{l}\text { IJCCSSON } \\
\text { ACKSON }\end{array}$ \\
\hline & $\mathrm{N} / \mathrm{A}$ & \\
\hline 800 HAMMEL RD. & & JACKSON \\
\hline $\begin{array}{l}\text { MT TSSHLNAD SKI AREA, ROGUE R NATL FOREST } \\
1550 \text { AVE G }\end{array}$ & N/A & $\begin{array}{l}\text { JACCSON } \\
\text { ACCSSON }\end{array}$ \\
\hline $\begin{array}{l}1350 \text { AVVE G } \\
300 \text { WEST ANTELOPE ROAD }\end{array}$ & N/A & $\begin{array}{l}\text { I ICCCSON } \\
\text { ACKSON }\end{array}$ \\
\hline PAC HWY/.3 M NE 1-5/MT ASHLAND INTCH & N/A & JACKSON \\
\hline 8001 TABLL ROCK ROAD & N/A & JACKSON \\
\hline 8301 TABLE ROCK ROAD & N/A & JACKSON \\
\hline 14745 HWY 234 & Gold Hill & JACKSON \\
\hline $\begin{array}{l}2505 \text { N R RGUUR RVVRR RD } \\
205 \text { N ROGUE RIVER RD }\end{array}$ & $\begin{array}{l}\text { Rogue River } \\
\text { Rogue River }\end{array}$ & $\begin{array}{l}\text { I ICCCSON } \\
\text { ACKSON }\end{array}$ \\
\hline $\begin{array}{l}24005 \text { BROPHY ROANED RD } \\
2\end{array}$ & & $\begin{array}{l}\text { A ACCSSON } \\
\text { JACKSON }\end{array}$ \\
\hline PROSFECT & N/A & $\begin{array}{l}\text { JACCSON } \\
\text { ACCSSON }\end{array}$ \\
\hline & N/A & $\begin{array}{l}\text { JaCCSSON } \\
\text { ACCSON }\end{array}$ \\
\hline 5505 Hwy 66 & N/A & 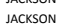 \\
\hline 2 MILES SOUTH OF CITY & N/A & JACKSON \\
\hline KIRTLAND RD & N/A & JACCSON \\
\hline 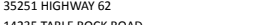 & N/A & JACKSON \\
\hline 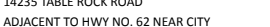 & $\begin{array}{l}\text { N/A } \\
\text { N/A }\end{array}$ & $\begin{array}{l}\text { A ACCCSSON } \\
\text { JACKON }\end{array}$ \\
\hline 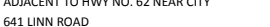 & Eagle Point & $\begin{array}{l}\text { APCCSSON } \\
\text { AACKSON }\end{array}$ \\
\hline 12665 HIGHWAY 62 & Eagle Point & JACKSON \\
\hline 403 DEAD INDIAN MEMORIAL RD & $\mathrm{N} / \mathrm{A}$ & JACKSON \\
\hline OFF -5 AT EXIT 45A & $\mathrm{N} / \mathrm{A}$ & Jackson \\
\hline 5630 TABLE ROCK RD & N/A & IACCSSON \\
\hline 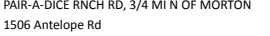 & N/A & $\begin{array}{l}\text { Jackckon } \\
\text { Jackson }\end{array}$ \\
\hline 2325 MERRY LANE & $\mathrm{N} / \mathrm{A}$ & \\
\hline $\begin{array}{l}2325 \text { MERRY LANE } \\
\text { Saeg Rd }\end{array}$ & N/A & Jaccson \\
\hline 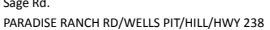 & $\begin{array}{l}\text { Medoford } \\
\text { N/A }\end{array}$ & $\begin{array}{l}\text { Jackson } \\
\text { AacsSon }\end{array}$ \\
\hline 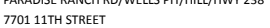 & $\begin{array}{l}N / A \\
N / A\end{array}$ & $\begin{array}{l}\text { APCCSSON } \\
\text { AACKSON }\end{array}$ \\
\hline $1235 \mathrm{~N}$. MOUNTAIN AVENUE & N/A & Son \\
\hline NEAR TRALl, OREGON & N/A & JACKSON \\
\hline & N/A & JACKSON \\
\hline $\begin{array}{l}501 \text { MIIL CRREE KRRVE } \\
2019 \text { ROGUE RIVER HWY }\end{array}$ & $\begin{array}{l}N / A \\
N / A\end{array}$ & KSON \\
\hline 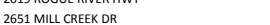 & N/A & $\begin{array}{l}\text { A ACCCSSON } \\
\text { JACKON }\end{array}$ \\
\hline Sage Rd. & Medford & Jackson \\
\hline Sage Rd. & Medford & \\
\hline 24904 HIGHWAY 62 & N/A & JACKSON \\
\hline WLLOW CREEK OFF OLD STAGE RD. & N/A & IACCSSON \\
\hline 18881 HIGHWAY 62 & Mettord & Jaccson \\
\hline 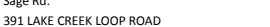 & $\begin{array}{l}\text { Nedortar } \\
\text { N/A }\end{array}$ & $\begin{array}{l}\text { JackCon } \\
\text { JackSON }\end{array}$ \\
\hline 260 DRY CREE ROAD & N/A & son \\
\hline 2625 A AEANUE G & N/A & \\
\hline 14484 AVALLN ROAD & N/A & JACKSON \\
\hline COLVER RD/HARTLY RO/1 MILES OF PHOENIX & N/A & \\
\hline 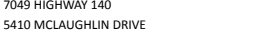 & 政 & $\begin{array}{l}\text { KSON } \\
\text { KSON }\end{array}$ \\
\hline & N/A & KSON \\
\hline 531: & N/A & \\
\hline TTE FaLS & N/A & son \\
\hline A2 BALL RD. & N/A & Son \\
\hline & N & \\
\hline & 醇 & \\
\hline 12285 & N/A & \\
\hline 5500 HWV 140 & N/A & \\
\hline ARTLET ST AND E 4TH ST & Mettord & Jackson \\
\hline 1100 & N/A & \\
\hline RSDIDE \&MCANDREWS & Mentord & \\
\hline $58 \mathrm{~N}$ & $\begin{array}{l}\text { N/A } \\
N / A\end{array}$ & \\
\hline o & $\mathrm{N} / \mathrm{A}$ & \\
\hline 7896 & N/A & \\
\hline 7890 & N/A & Son \\
\hline A & $\begin{array}{l}\text { Mestord } \\
\text { Ashhand }\end{array}$ & \\
\hline & $\begin{array}{l}\text { Asslan } \\
\text { N/A }\end{array}$ & \\
\hline $\begin{array}{l}\text { N PACFIC HWY } \\
\text { N HW }\end{array}$ & N N/A & \\
\hline $2309 \mathrm{SAG}$ & N/A & \\
\hline & & \\
\hline 960 2ND AVENUEN & Gold Hill & ACKSON \\
\hline 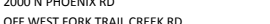 & 政 & 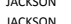 \\
\hline
\end{tabular}

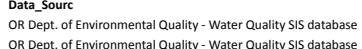
OR Dept. of Environmental Qualitiv- Water Quality IS d database
OR Dept. of Environmental luality- Water Quality SIS database

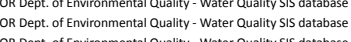
OR Dept. of Environmental Q Quality - Water Quality II database
OR Dept. of Environmental quality - Water Quality SIS database OR Dept. of E Evirionmental Q uality - Water Quality SIS databasese

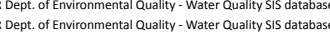

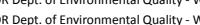
OR Dept. of Environmental Quality - Water Quality SIS datababse OR Dept. of fnvironmental Q Quality- Water Quality IIS databasese

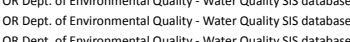

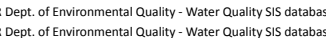
OR Rept. of Evirionmental Q Quality- Water Quality SIS datababe

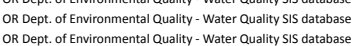

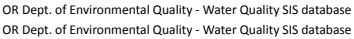

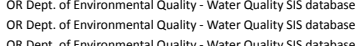

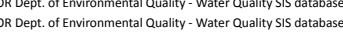
OR Dept. of Ef nvirionmentental Qualitity - Wateter Quaulity yIS dis databasase OR Dept. of Environmental Quality - Water Quality SIS datababse OR Dept. of Environmental Quality- Water Quality SIS database
OR Dept. of Environmental Quality - Water auality IIS databsese

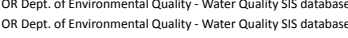

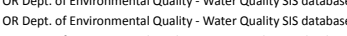
OR Dept. of Evironmental Quality - Water Q Quality SIS databass (DE/OHA SWA 2000 - 2005)

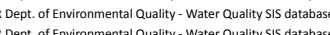

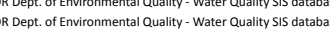

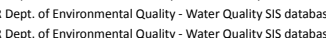
OR Dept. of Environmental Quality - Water Quality SIS database (D) R Dept. of Environmenta Q Quality and OR Heath Authority Source Water A Asses OR Dept. of Environmental Quality - Water auality SIS databasse OR Dept. of Environmental Quality - Water Quality SIS databasese
OR Dept of Environmental Quality ynd OR Helty

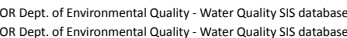
OR Dept. of Environmental Quality - Water Quality SIS database
OR Dept. of Environmental Quality - Water Quality IIS databasese $Q R$ Dept. of Environmental Q Quality - Water Quality SIS database OR Dept. of Environmental Q Quality - Water Quality SIS database

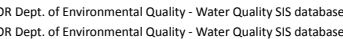

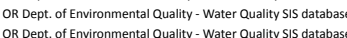

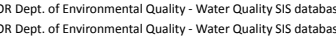
OR Dept. of Environmental Quality- Water Qualiti IS IS database
OR Dept. of nnironmental Quality - Water Q Quality IS datababse OR Dept. of Environmental Quality - Water Quality SIS databasse

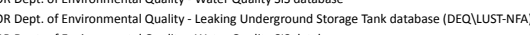
OR Dept. of Environme tha

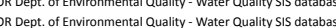
OR Dept. of Environmental Quality - Water Quality SS database OR Dept. of Envirionmentat quality - Water Quality Sis datababe OR Dept. of Environmental Quality and OR Health Authority SOurce Water Assessment database (DEQ/OHA SWA 2000 - 2005)

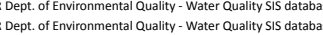

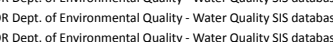

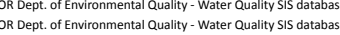

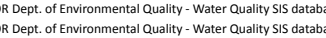

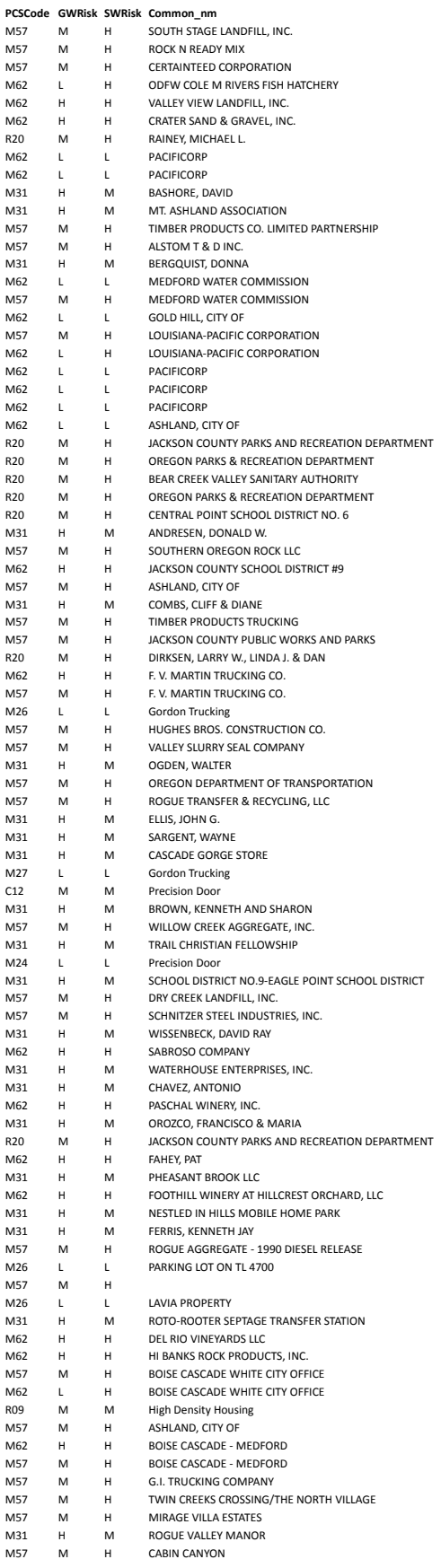

DEQQ Reermited Stormwater Discharges (NPDES or WPCC EQ Perminited Stormwater Discharges (NPDES or WPCC (a) Industrial or Process Wastewater- -WPCF and NPDES/ Lagoons and Liquid Waste

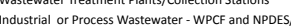
Domessic S Sevage Treatment with On-Site System/Large Capacity Septic Systems Serving $>20$

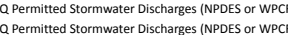
a Industrial or Process Wastewater - WPCF and NPDES/ Lagoons and Liquid Waste industrial or Procoess Wastewater - WPCF and N NDES/ Lagoons and Liquid Waste

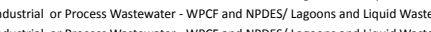
Industrial or Process Wastewater - WPCF and NPDES/ Lagoons and Liquid Waste

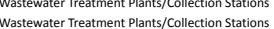

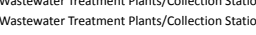

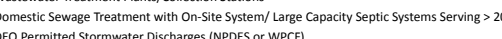
dustrial or Process Wastewater - WPCF and NPDES/ L DPDES/Lagoons and Liquid Waste omestic Sewaze Treatment with On-Site System/Large Capactity Septic Systems Serving $>20$

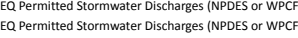
Industrial or Process Wastewater - WPCF and NPDES/ Lagons and Liquid Waste DEQ Permitted Stormwater Discharges NNDEE or WPCF

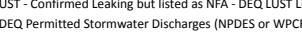
EEQ Permitted Stormwater Discharges (NPDES or WPC) Domestic Sewage Treatment with on-site System/ Large DEQ Permitted Stormwater D Discharges (NPDES or WPCF)

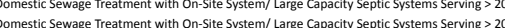
UST - Upgraded and/or Registered - Active

Destic Sewaze Treatment with On-Site System/Large Capacity Septic Systems Serving > 20

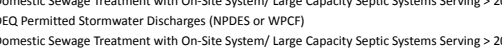

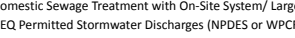
Domestic Sevage Treattennt with on-site System/Large Capacity Septic Systems Serving $>20$ Industrial or Process Wastewater - WPCF and NDEES/ Lagons and Liquid Waste Domestic Sewage Treatment with On-Site System/Large Capactity Septic SSystems Seving $>20$

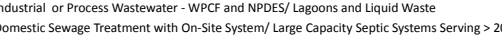
Idustrial or Proceess Wastewater- -WPCF and NPDES/ Lagoons and Liquid Waste Domestic Sevage Treatment with on-Site System/ Large Capacity Septic Systems Serving $>20$

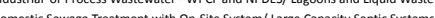

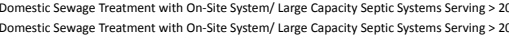
DEQ Permitited Stormwater Discharges (NPDEE or WPCF

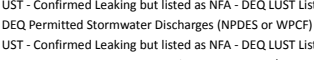
Industrial or Pr Process Wastevater- - WPCF and NPDES/ Lagonons and liquidid Waste

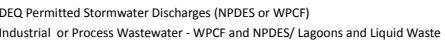
Housing - High Density $\rightarrow 1$ House/0.5 Acres DEQ Permitted Stormwater Discharges (NPDES or WPCH

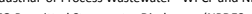
DEQ Permitted Stormwater Discharges (NPDES or WPC DEQ Permited Stormwater Dischareses (NDDES Sor WPCC De Permitted Storrmwater Discharges $($ NNe

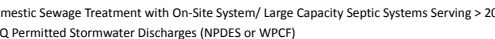




\begin{tabular}{|c|c|c|c|}
\hline $\begin{array}{l}\text { Address } \\
\text { SSNDEF WAY }\end{array}$ & city & County & RET_DATE \\
\hline $\begin{array}{l}\text { SIINEY WAY } \\
\text { MUNIIPAL STORMWATER AREA }\end{array}$ & $\begin{array}{l}\begin{array}{l}\text { Medeford } \\
\text { Phoenix }\end{array} \\
\text { (a) }\end{array}$ & $\begin{array}{l}\text { JACKSON } \\
\text { ICCCSON }\end{array}$ & $\begin{array}{l}10 / 31 / 2018 \\
103 / 212018\end{array}$ \\
\hline 24TH ST/ AVENUEC C & N/A A n & $\begin{array}{l}\text { JACKSON } \\
\text { JAON }\end{array}$ & $\begin{array}{l}10 / 3171 / 2018 \\
1018\end{array}$ \\
\hline $\begin{array}{l}\text { South East of fintake } \\
2005 \text { Ragef RIVR HWYy }\end{array}$ & $\begin{array}{l}\text { Medford } \\
N / \mathrm{A}\end{array}$ & $\begin{array}{l}\text { Jackson } \\
\text { JACKSON }\end{array}$ & \\
\hline $\begin{array}{l}20055 \text { ROGGUE RVVR HWYI } \\
\text { 217 R RSS LANE }\end{array}$ & $\begin{array}{l}N / A \\
N / A\end{array}$ & $\begin{array}{l}\text { JACSSON } \\
\text { IACKSON }\end{array}$ & $\begin{array}{l}10 / 3 / 1 / 1028 \\
10131 / 2018\end{array}$ \\
\hline 7130 C CAATER LAKE HWY & N/A & $\begin{array}{l}\text { JACSSON } \\
\text { IACKSON }\end{array}$ & $\begin{array}{l}10 / 3 / 1 / 2018 \\
10 / 1 / 2018 \\
\end{array}$ \\
\hline INT. OF GARFELL RD AND CENTER DRVIE & $\mathrm{N} / \mathrm{A}$ & JACKSON & $\begin{array}{l}10 / 31 / 2018 \\
1018\end{array}$ \\
\hline 1930 PARRA-ADCE RANCH ROAD & N/A & IACKSON & 10/31/2018 \\
\hline 246 RIDGE RD & 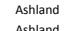 & JACKSON & $10 / 31 / 2018$ \\
\hline 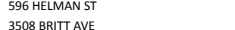 & $\begin{array}{l}\text { Ashland } \\
N / A\end{array}$ & IACKSON & 10/31/2018 \\
\hline $\begin{array}{l}3008 \text { BRRIII AVE } \\
1014 \text { OLD STAGE ROAD }\end{array}$ & $\begin{array}{l}N / A \\
N / A\end{array}$ & $\begin{array}{l}\text { JACSSON } \\
\text { IACKSON }\end{array}$ & $\begin{array}{l}10 / 3 / 1 / 1028 \\
10131 / 2018\end{array}$ \\
\hline 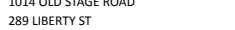 & & $\begin{array}{l}\text { JaCSSON } \\
\text { IACCSON }\end{array}$ & $\begin{array}{l}10 / 3 / 2018 \\
10 / 1 / 2018\end{array}$ \\
\hline 195 SHERMAN ST & $\begin{array}{l}\text { Asthand } \\
\text { Ashand }\end{array}$ & 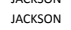 & $\begin{array}{l}10 / 31 / 2018 \\
1018\end{array}$ \\
\hline 117 HELMAN ST & Astland & IACKSON & 10/31/2018 \\
\hline 798 OAK ST & Ashland & JACKSON & $10 / 31 / 2018$ \\
\hline 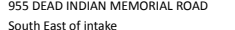 & $\begin{array}{l}N / A \\
\text { Nefterd }\end{array}$ & $\begin{array}{l}\text { Jackson } \\
\text { Jackson }\end{array}$ & $\begin{array}{l}10 / 31 / 2018 \\
2005\end{array}$ \\
\hline 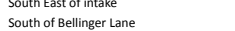 & $\begin{array}{l}\text { Mefiford } \\
\text { Mefford }\end{array}$ & $\begin{array}{l}\text { Jackson } \\
\text { Jackson }\end{array}$ & $\begin{array}{l}2005 \\
2005\end{array}$ \\
\hline 2546 W MAIN ST & $\begin{array}{l}\text { Metard } \\
N / A\end{array}$ & $\begin{array}{l}\text { Jackson } \\
\text { IAcKSoN }\end{array}$ & $\begin{array}{l}2005 \\
10 / 31 / 2018\end{array}$ \\
\hline 743 sкoоkum LN & $\mathrm{N} / \mathrm{A}$ & JACKSON & $\begin{array}{l}10 / 31 / 2018 \\
1\end{array}$ \\
\hline 1391 EBERGREEN LANE & Ashland & JACKSON & $10 / 31 / 2018$ \\
\hline South of Well & Medford & Jackson & \\
\hline $\begin{array}{l}\text { South Stage Roadd } \\
\text { Southwest of well }\end{array}$ & $\begin{array}{l}\text { Mefford } \\
\text { Mefford }\end{array}$ & $\begin{array}{l}\text { Jackson } \\
\text { Jackson }\end{array}$ & 2005 \\
\hline 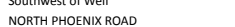 & 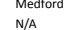 & $\begin{array}{l}\text { Jactson } \\
\text { IAcKSON }\end{array}$ & $\begin{array}{l}2005 \\
10 / 1 / 2018\end{array}$ \\
\hline 3919 AVE. H/3757 AVE. H & $\begin{array}{l}N / A \\
N / A\end{array}$ & $\begin{array}{l}\text { JACSSON } \\
\text { IACSSON }\end{array}$ & $\begin{array}{l}10 / 31 / 2018 \\
10 / 21 / 2018\end{array}$ \\
\hline SPRINGBROOK ROAD & Medford & JACKSON & 10/31/2018 \\
\hline 3791 ROGUE RIVER HWY & N/A & JACKSON & 10/31/2018 \\
\hline 1730 THOMAS ROAD & N/A & IACKSON & 10/31/2018 \\
\hline $\begin{array}{l}\text { FRODD DOVIVE \& CHETTENHAM WAY } \\
813 \text { LONARD ST }\end{array}$ & $\begin{array}{l}\text { NA } \\
\text { Ashland }\end{array}$ & $\begin{array}{l}\text { JACKSON } \\
\text { JACKSON }\end{array}$ & $\begin{array}{l}10 / 3 / 1 / 2018 \\
10131 / 2018\end{array}$ \\
\hline 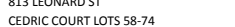 & $\begin{array}{l}\text { Asthand } \\
\text { N/A }\end{array}$ & $\begin{array}{l}\text { JaCKSON } \\
\text { IACKSON }\end{array}$ & $\begin{array}{l}10 / 3 / 1 / 2018 \\
10 / 31 / 2018\end{array}$ \\
\hline MAPLE PARK DR \& KATTLN LANE & $N / A$ & JACKSON & $\begin{array}{l}10 / 3 / 1 / 1018 \\
10 / 2018\end{array}$ \\
\hline 131 TERRACE ST & Ashland & Jackson & 10/31/2018 \\
\hline 699 ASHLAND CREEK DR & Ashland & JaCksoN & 10/31/2018 \\
\hline STEWART AND COLUMBUS & Mediford & IACKSON & 10/31/2018 \\
\hline $\begin{array}{l}\text { STEWART AVENUE } \\
\text { Stowe ANe }\end{array}$ & $\begin{array}{l}\text { Medford } \\
\text { Mefford }\end{array}$ & $\begin{array}{l}\text { Jackson } \\
\text { Jackson }\end{array}$ & $\begin{array}{l}10 / 31 / 2018 \\
2005\end{array}$ \\
\hline $\begin{array}{l}\text { Stowe Ave } \\
\text { CAMPBEL RD }\end{array}$ & 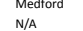 & $\begin{array}{l}\text { Jactson } \\
\text { JacksoN }\end{array}$ & $\begin{array}{l}2005 \\
10 / 31 / 2018\end{array}$ \\
\hline 2763 BLLEY ROAD & N/A & 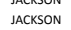 & $\begin{array}{l}10 / 31 / 12018 \\
1018\end{array}$ \\
\hline RLLEY ROAD & Eagle Point & JACKSON & 10/31/2018 \\
\hline WEST MAII ST. TO STEWART AVE & $\mathrm{N} / \mathrm{A}$ & JACKSON & $10 / 31 / 2018$ \\
\hline $\begin{array}{l}\text { Stowe Ave. } \\
\text { W NEVAAD ST \& HEMMEN ST. }\end{array}$ & $\begin{array}{l}\text { Metorord } \\
N / A\end{array}$ & $\begin{array}{l}\text { Jactson } \\
\text { Iacsson }\end{array}$ & $\begin{array}{l}{ }_{103}^{2005} \\
103 / 2018\end{array}$ \\
\hline 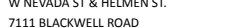 & $\begin{array}{l}N / A \\
N / A\end{array}$ & $\begin{array}{l}\text { JACSSON } \\
\text { IACKSON }\end{array}$ & $\begin{array}{l}10 / 3 / 1 / 2018 \\
10 / 13 / 2018\end{array}$ \\
\hline SUNLEAF- LONE OAK DR & Metorord & 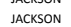 & $\begin{array}{l}10131 / 2018 \\
10 / 31 / 2018\end{array}$ \\
\hline Sunset Drive & Medtord & Jactoon & 2005 \\
\hline Sunset Drive & Medtord & Jackson & 2005 \\
\hline Sunset Drive & Medford & $\begin{array}{l}\text { Jackson } \\
\text { Accscos }\end{array}$ & 2005 \\
\hline 3075 HAMRRCK ROAD & $\begin{array}{l}\text { Central Poin } \\
N / A\end{array}$ & $\begin{array}{l}\text { JACKSON } \\
\text { IACSSON }\end{array}$ & $\begin{array}{l}10 / 31 / 2018 \\
1013 / 2018\end{array}$ \\
\hline $\begin{array}{l}5422 \text { SWCLOUGLLNN DRRV } \\
\text { SW ORNER OF GTH \& RIVERSIDE }\end{array}$ & $\begin{array}{l}\text { N/A } \\
\text { Meftord }\end{array}$ & $\begin{array}{l}\text { JaCSSON } \\
\text { IACCSON }\end{array}$ & $\begin{array}{l}10 / 3 / 1 / 2018 \\
10 / 31 / 2018\end{array}$ \\
\hline $\begin{array}{l}1900 \text { N PACIFIC HIGHWAY } \\
\text { lat }\end{array}$ & N/A & 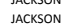 & $\begin{array}{l}10131 / 2018 \\
10 / 31 / 2018\end{array}$ \\
\hline Syringa Rd. & Meftord & Jackson & 2005 \\
\hline 10175 WHEELER ROAD & $\mathrm{N} / \mathrm{A}$ & JACKSON & $10 / 31 / 2018$ \\
\hline T275, RR, SEC G, TL25000 & Medford & JACKSON & $10 / 31 / 2018$ \\
\hline 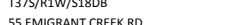 & Metorord & $\begin{array}{l}\text { IACKSON } \\
\text { ACGSOONH }\end{array}$ & $10 / 31 / 2018$ \\
\hline KINGS SIGHAY WA & NA & $\begin{array}{l}\text { JACSKON } \\
\text { IACKSON }\end{array}$ & $\begin{array}{l}10 / 3 / 1 / 2018 \\
1013 / 2018\end{array}$ \\
\hline STEVENS RD & Eagle Point & 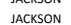 & $\begin{array}{l}1013 / 1 / 2018 \\
10 / 31 / 2018\end{array}$ \\
\hline DIAMOND STREET \& ULLAN STREET & & JACKSON & $10 / 31 / 2018$ \\
\hline TAX LOT 38-1-W5-600 (NACANT) & Medford & JACKSON & 10/31/2018 \\
\hline $\begin{array}{l}\text { GOOD ETRRAAE D DIVE } \\
1193 \text { ASHLAND MIN RD }\end{array}$ & $\begin{array}{l}\text { Jacksonvile } \\
\text { Asshand }\end{array}$ & $\begin{array}{l}\text { JACKSON } \\
\text { JACKSON }\end{array}$ & $\begin{array}{l}10 / 3 / 1 / 2018 \\
10 / 13 / 2018\end{array}$ \\
\hline 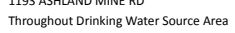 & Medford & Jackson & 1 1/20/20178 \\
\hline Throughout DWPA & Medford & $\begin{array}{l}\text { Jackson } \\
\text { Jospewer }\end{array}$ & 2005 \\
\hline $\begin{array}{l}19000 \text { CAVES SIIGHWAY } \\
\text { 654 KENDA RD. }\end{array}$ & N/A & $\begin{array}{l}\text { JoSEPHNIN } \\
\text { ISSPPNNEE }\end{array}$ & $\begin{array}{l}10 / 31 / 2018 \\
101312018\end{array}$ \\
\hline $\begin{array}{l}\text { 6544 KENDAARLD. } \\
\text { TRS - } 395 \text { 7W } 36 \text { L LT } 500\end{array}$ & N/A & $\begin{array}{l}\text { JSSERANE } \\
\text { JOSPPHNE }\end{array}$ & $\begin{array}{l}10 / 3 / 1 / 2018 \\
10 / 31 / 2018\end{array}$ \\
\hline $\begin{array}{l}4210 \text { HOLAND LOOP RD } \\
4\end{array}$ & N/A & JOSEPHINE & $\begin{array}{l}10131 / 2018 \\
10 / 31 / 2018\end{array}$ \\
\hline Throughout DWPA & Medford & Jackson & 2005 \\
\hline HARRISON STREET AND $3 R D$ STREET & Cartton & Vambill & $10 / 31 / 2018$ \\
\hline 1195 oak St. & Astland & JACKSON & $\begin{array}{l}11 / 1 / 2018 \\
111 / 2018\end{array}$ \\
\hline 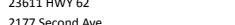 & 列 & & $\begin{array}{l}11 / 1 / 2018 \\
111 / 20218\end{array}$ \\
\hline 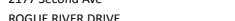 & 作 & 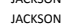 & $\begin{array}{l}111 / 1 / 21218 \\
11 / 12018\end{array}$ \\
\hline FALS RD & Butte falls & IACKSON & $\begin{array}{l}111 / 12018 \\
11 / 1 / 2018\end{array}$ \\
\hline 1100 KIRTLAND RD & & JaCKSON & 11/1/2018 \\
\hline Throughout DWPA & Medford & Jackson & 2005 \\
\hline Throughout DWPA & Metford & Jackson & 2005 \\
\hline 1654 Asthanand St & & & \\
\hline $\begin{array}{l}\text { unghout DWPA } \\
\text { unhout DWPA }\end{array}$ & Mentford & 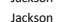 & \\
\hline Antelope Rd & White City & Jackson & $8 / 27 / 2018$ \\
\hline
\end{tabular}

Data_Sourc
OR Dept. of Environmental Q Quality - Water Quality IS d database
OR Dept. of Environmental Quality- Water Quality IIS database

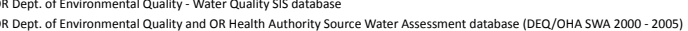

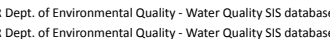

OR Dept. of Environmental Quality - Water Quality SIS datababse

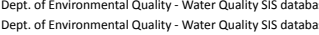

OR Dept. of Environmental quality - Water Quality SIS datababse

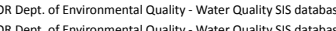

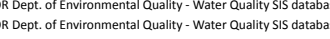

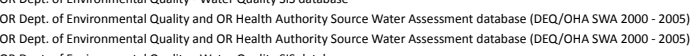

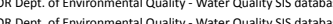

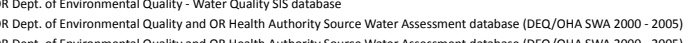

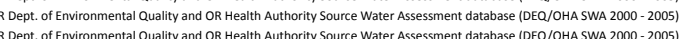

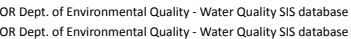

OR Dept. of Environmentat Qualiti - Water Quality SIS datababse
OR Dept. of Envirironmental a uality - Water Q Quality SIS database

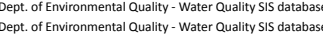

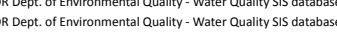

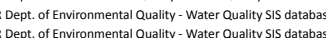

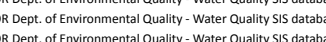

OR Dept. of fnvirommental Quality - Water Quality SS databasese

(a)

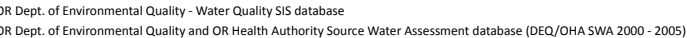

OR Dept. of Environmental Quality - Water Quality SIS database

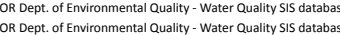

OR Dept. of Envirommental Quality - Water Quality SIS database

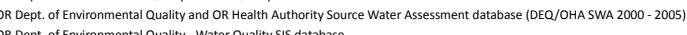

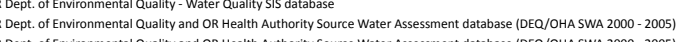

OR Dept. of Environmental Quality and OR Heath Authority SOurce Water Assessment database (DEQ/OHA SWA 2000- 2005)

OR Dept. of Environmental Quality - Water Quality SII databass

und Storage Tank database (DEQQULST-NFA)

OR Dept. of Environmental Quality - Water Quality IS databass

(DEO/OHA SWA 2000-2005)

tal Quality- Water Ouality sis databasc

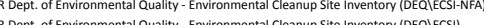

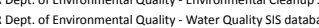

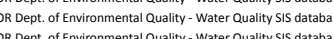

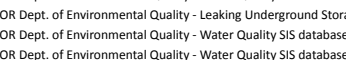

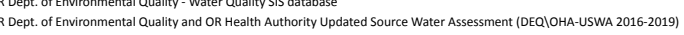

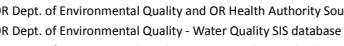

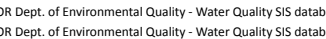

OR Dept. of Environmental Q Quality - Water Quality SIS database

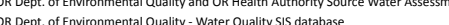

OR Dept. of Environmental Q uality - Waste Water Treatment Plants (DEQQIWWTP-SIS)

OR Dept. of Environmental Quality - Waste Water Treatment Plants (DEQQIWWTP-SIS)

OR Deet of Environmental Q Quality- Waste Water Treatment Plans (DESOWWTPSSIS

OR Dept. of Environmental Quality - Waste Water Treatment Plants (DEQ QIWWTP-SIS)

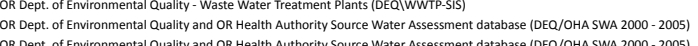
OR Dept. of Environmental Q Quality and OR Heath Authority Source Water Assessment database (DEQ/OHA SWA 2000- 2005)

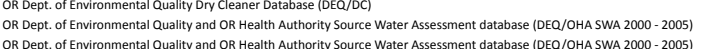

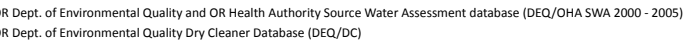

\begin{tabular}{|c|c|c|c|}
\hline M57 & M & ${ }_{\mathrm{H}}^{\mathrm{H}}$ & 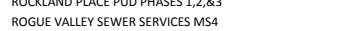 \\
\hline M57 & M & H & AUBURN ESTATES SUBDOVISION \\
\hline & ${ }_{H}^{H}$ & M & 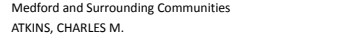 \\
\hline M57 & & $\mathrm{H}$ & $\begin{array}{l}\text { ARNAS, CHARLSM. } \\
\text { SCHATZRROPERT- HAC }\end{array}$ \\
\hline & $\mathrm{M}$ & ${ }_{H}^{H}$ & WHITE CITY METALL \& SUPPLY \\
\hline & $\mathrm{M}$ & ${ }_{H}^{H}$ & $\begin{array}{l}\text { ROCGH GRADING TAX LOT } 100 \\
\text { JACSSONVIIF PI NO } 1\end{array}$ \\
\hline & ${ }_{M}^{M}$ & M & $\begin{array}{l}\text { JAAKSOVVILE PII NO.1 } \\
\text { DENISON LEE }\end{array}$ \\
\hline M50 & M & M & JUUE NORMAN \\
\hline M62 & H & H & VAUGHN'S FARM \& ORCHARD \\
\hline $\begin{array}{c}m 50 \\
\text { Mso }\end{array}$ & M & M & $\begin{array}{l}\text { HAGEN, BRADLEY } \\
\text { SAWWIITECE }\end{array}$ \\
\hline $\begin{array}{c}M_{350} \\
M 50\end{array}$ & ${ }_{M}^{M}$ & $M_{M}^{M}$ & $\begin{array}{l}\text { SSM WHITIIDGE } \\
\text { PHYUI NORRIS }\end{array}$ \\
\hline M50 & M & m & RISA E BUCK \\
\hline & $H$ & $\stackrel{H}{M}$ & $\begin{array}{l}\text { BELEFFORE WINERY, LCC } \\
\text { Medford and Survonidiog Communities }\end{array}$ \\
\hline & & ${ }_{\mathrm{H}}^{\mathrm{M}}$ & $\begin{array}{l}\text { Medford and Surrounding Communities } \\
\text { Irrigitade Crops }\end{array}$ \\
\hline & M & H & 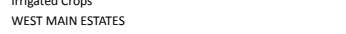 \\
\hline & m & m & BRIAN WILKERSON \\
\hline & M & M & DEBBA SWARZMAN \\
\hline & M & $\mathrm{H}$ & Irrigated Crops \\
\hline & M & ${ }_{\mathrm{H}}^{\mathrm{H}}$ & 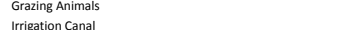 \\
\hline & M & H & $\begin{array}{l}\text { Irrigation Canal } \\
\text { STONEGTE ESTATES PHASE } 5\end{array}$ \\
\hline & & H & \\
\hline & M & $\mathrm{H}$ & KENNEDY PARK \\
\hline & & H & WATNE BUMA \\
\hline & ${ }_{M}^{M}$ & ${ }_{\mathrm{H}}^{\mathrm{H}}$ & $\begin{array}{l}\text { ANOU UEWW SUBBDISION } \\
\text { DEEIA ESTASS PHASE } 1\end{array}$ \\
\hline Mso & м & M & $\begin{array}{l}\text { DELTA SEATES PPASEE } 1 \\
\text { TABTHA VINER }\end{array}$ \\
\hline & M & H & SIENNA HILIS PHASE 2 \\
\hline 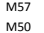 & ${ }_{M}^{M}$ & $\stackrel{H}{M}$ & $\begin{array}{l}\text { ROSSS LAN SUBDDVISIN } \\
\text { KARE GROVE \& IAY ACH }\end{array}$ \\
\hline Mso & M & M & KATHERINE MORSE \\
\hline & L & $\mathrm{L}_{\mathrm{H}}$ & $\begin{array}{l}\text { THOMAAP PKRPATARCK } \\
\text { MOUNATIN VISA }\end{array}$ \\
\hline $\mathrm{C}_{12}$ & M & M & Fosters Wholessale IWP \\
\hline & M & H & 2HAWK WINERY, PRoceEsSinG FACLUTY \\
\hline 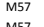 & $\mathrm{M}$ & ${ }^{H}$ & EAGLE POIIT NATIONAL CEMEETER, EXPANSION 603 CMM3012 \\
\hline $\begin{array}{l}\text { MS7 } \\
\text { M57 }\end{array}$ & ${ }_{M}^{M}$ & ${ }_{H}^{\mathrm{H}}$ & $\begin{array}{l}\text { EAALEC CEEST OORELOOKK } \\
\text { LOZER LANE IMPROVEMENTS }\end{array}$ \\
\hline & H & $\mathrm{H}$ & Valley Web Printing \\
\hline & & & VERDE VILAGE \\
\hline M57 & M & н & COMBINED TANSPORT LOGISTICS GROUP, INC. \\
\hline & & ${ }^{H}$ & SUMMERFELL SUBDIVISION PHASES 9 \& 10 \\
\hline R1 & m & M & $\begin{array}{l}\text { Sub Station } \\
\text { Aboveround Storage Tank }\end{array}$ \\
\hline & & H & 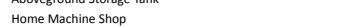 \\
\hline & m & H & 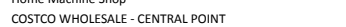 \\
\hline M5 & m $>2>>2$ & н & EAGLE POINT SOLAR FARM \\
\hline M26 & L & ᄂ & PARKING GARAGE PROJECT \\
\hline & $\mathrm{M}$ & $\mathrm{H}$ & NORTHGATE OFFICE PARK \\
\hline Mo & M & H & $\begin{array}{l}\text { Home Machine Shop } \\
\text { DOGS FOR THE DEAF }\end{array}$ \\
\hline c59 & L & L & MEDCO ROAD FORMER ORCHARD \\
\hline c58 & H & ${ }_{H}^{H}$ & $\begin{array}{l}\text { NORTHMONT UUBDIVISION } \\
\text { WETTAAND MTTGGTON }\end{array}$ \\
\hline & $m$ & H & $\begin{array}{l}\text { WELTRAND MITICATION } \\
\text { FRANLIN PLACE }\end{array}$ \\
\hline & м & H & IDLEWOOD SUBDVIISION \\
\hline M57 7 & m $>2>>>2$ & н & KING ARTHUR VILAGE, PHASES 1 AND 2 \\
\hline M26 & ᄂ & L & WESTERN BANK ORCHARD \\
\hline & $M$ & $\mathrm{H}$ & GOLD TERRACE HEIGHTS \\
\hline $\begin{array}{l}\text { M50 } \\
\text { Mog }\end{array}$ & ${ }^{M}$ & $M^{m}$ & $\begin{array}{l}\text { COOK, ALAN } \\
\text { Buran home with sentic cystems }\end{array}$ \\
\hline & $m$ & H & \\
\hline мз & н & m & USDOI; NATIONAL PARK SERVICE \\
\hline$m$ & н & н & GERBER, TED \& MERIDETH \\
\hline & $\mathrm{m}$ & $\mathrm{H}$ & CARLONS GRAVEL PIT \\
\hline & H & H & BRIDGEVIEW VINEYARDS, INC. \\
\hline M5: & M & ${ }_{H}^{M}$ & $\begin{array}{l}\text { Sewer Lines } \\
\text { ADELAADE SUBDIVISION }\end{array}$ \\
\hline 220 & M & $\mathrm{H}$ & ASHLAND, CITY OF \\
\hline & & ${ }_{H}^{H}$ & $\begin{array}{l}\text { EATINGER, AL } \\
\text { GOD HII, ITY OF }\end{array}$ \\
\hline R20 & M & $\mathrm{H}$ & SHADY COVE, CITY OF \\
\hline & M & $\mathrm{H}$ & BUTTE FALLS STP \\
\hline мо9 & L & L & Rural Homes \\
\hline & & & Rural Homes \\
\hline & & $\mathrm{m}$ & Ashland Southide Cleaner \\
\hline & & & $\begin{array}{l}\text { Rurat inomes } \\
\text { Rural Homes }\end{array}$ \\
\hline & H & M & \\
\hline
\end{tabular}

a Permitted Storrmwater Discharges (NDDES or WPCA

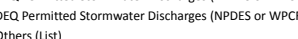

Desstic Sewage Treatment with On-Site System/Large Capacity Septic Systems Serving > 20

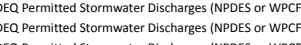

DEQ Permitted Stormwater Discharges NDDES or WPCA

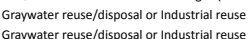

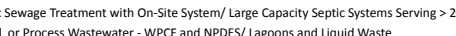
Craywater reuse/lisposal or Industrial reuse

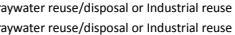

(n)

Crops - Irigigated- Berries, Hops, Mint, Orchards, Vineyards/Nurseries, Green Houses, Vegetables, etc.

Graywater reuse/disposal or Industrial reuse

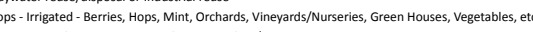

Degation Canal/Pond

ter Discharges (NPDES or WPCA

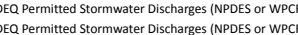

Wastewater Treatment Plants//ollection Stations

DEQ Permitted Stormwater Discharges SNDES or WPCC

Graywater reuse/disposal or Industrial reuse

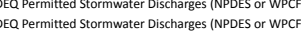

UTT- Confirmed Leaking but listed as N NA - DEQ WST L

Uurniture/Lumber/Parts stores

DEQ Permitted Stormwater Discharges (NPDES or WPCh

DEQ Permitted Stormwater Discharges $N$ wites of

DEQ Permitited Stormwater Discharges N NDDES or or WPCC

Photo Processing/Printing
DEQ P Pernited Stormwater Discharges (NPDES or WPCF)

DQ Perminited Stormwater D Discharges

(a)

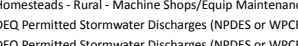

DEQ Permitted Stormwater Discharges N NPEES or WPCC

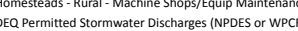

Known Contamination listed as NFA (Sites/Plumes/Spills from ECSS)

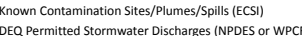

DEQ Permitted Stormwater Discharges (NPDES or WPCA)

DEQ Permitted Stormwater Discharges (NPEES or WPCh

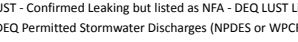

Comesteads - Rural - Septic Systems $<1 /$ Accere

Crops - Irrigated - Berries, Hops, Mint, Orchards, Vineyards/Nurseries, Green Houses, Vegetables, etc.

EQ Permitted Stormwater Dischareses (NDDES or WPCF)

SEe Perritted Stormwater Discharges (NPDES or WPCA)

Sewage Treatment Plants
Sewage Treatment Plants

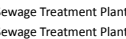

Sewage Treatment Plants

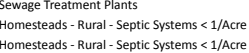

Ory Cleaners
Homesteads- Rural - Septic Systems $<1 /$ Acre 


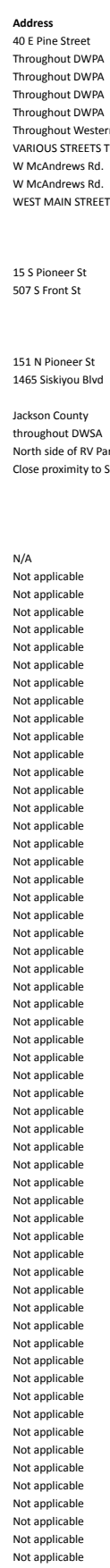

\begin{tabular}{|c|c|c|c|}
\hline & $\begin{array}{c}\text { County } \\
\text { Jackeon }\end{array}$ & RET_DATE & 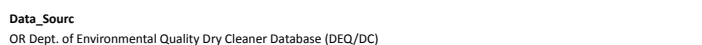 \\
\hline $\begin{array}{l}\text { Central lo oint } \\
\text { Medford }\end{array}$ & $\begin{array}{l}\text { Jackson } \\
\text { Jackson }\end{array}$ & & 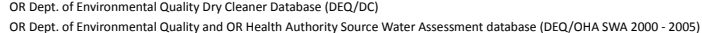 \\
\hline Medford & Jackson & & 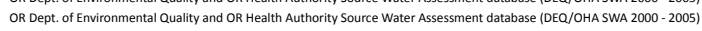 \\
\hline & & & 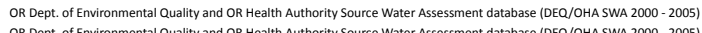 \\
\hline & & & 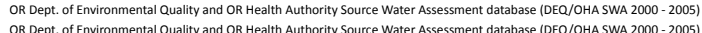 \\
\hline & $\begin{array}{l}\text { Jackson } \\
\text { Jacsson }\end{array}$ & 然/13/2018 & 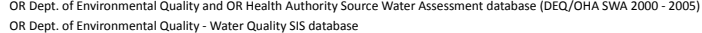 \\
\hline & & & 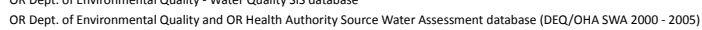 \\
\hline & & & 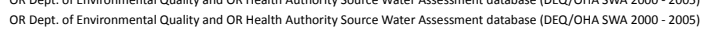 \\
\hline Medford & CKSON & 10/31/2018 & 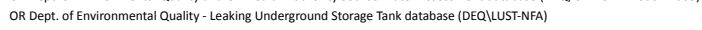 \\
\hline & & & olegy and Mineral Industries Mineral Information layer for reregon( DOGAMM/MLLO) \\
\hline & & & and Mineral Industries Mineral hiformation \\
\hline $\begin{array}{l}\text { Metorora } \\
\text { Ashland }\end{array}$ & & $8 / 27 / 2$ & 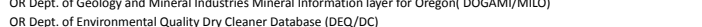 \\
\hline Central Poin & & $8 / 27 / 2$ & st. of Environmental C \\
\hline & & & d Mineral Industries Mineral Information layer for $\mathrm{C}$ \\
\hline dford & & & d Mineral Industries Mineral Information layer for $\mathrm{O}$ \\
\hline & & & averfo \\
\hline & & & 然: of \\
\hline Shady Cove & Jackson & 5/31/20177:00:00 & tal Quality and OR Heath Authority Updated Source Weter Assessment t1 \\
\hline & & 5/31/20177: & tal Quality and OR Heath Authority Y Dodated Source Water Assessment to \\
\hline & & 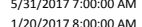 & $\begin{array}{l}\text { OR Dept. of Environ } \\
\text { OR Dept. of Evviron }\end{array}$ \\
\hline cove & & $1 / 20 / 20178$ & 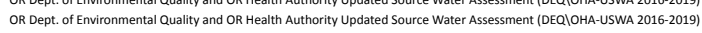 \\
\hline & & 1/11/2018 & \\
\hline & & & \\
\hline & & & \\
\hline & & & \\
\hline
\end{tabular}

PCSCode GWRisk SWRisk Common nm
Co8 H M Central Point Cleaners

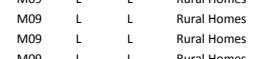

ALDV A REPLACEMEN PROAECT
Irongate Ornamental Ironuorts

Rogue Pacific Lumber
MEDEOBD HOTEL

MEEFRR HОТТ

Tru-Mix Construction Company
Tru-Mix Construction Company

Oregon Shakespeare Festivi

Tru-Mix Construction Company
Tru-MMix construction Company
Tru-Mix Construction Company

Towne \& Country Cleaners \& Laundry
Weldon's cleaners

Rural homes with septic systems in Dws

Septic systems

AST tor propane
Tu-Mix Construction Company

Sever Lines
city of Metiord Dept. of Public Works

YMCA Park Fields
Vineyard
SOS

South Stage L.F.

Bear Creek
Bowen Creek
Clark creek

OR Dept. of Envirionmentatal Quality Water Quality Assessment (DER/W/W

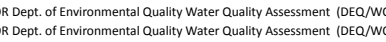

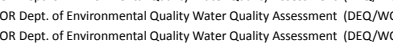

OR Dept. of Environmental Quality Water Quality Assessment (DEQ/WO

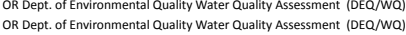

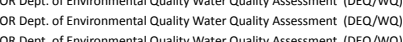

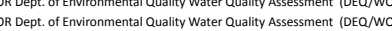

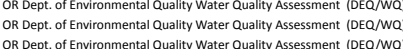

OR Dept. of Environmental Q uality Water Quality Assessment (DEQ/NGD

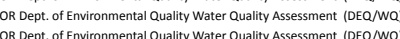

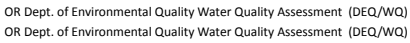

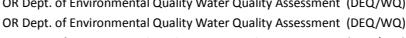

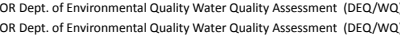

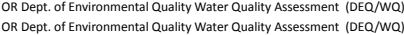

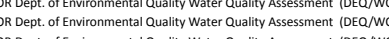

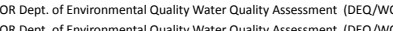

OR Dept. of Environmental Q Quality Water Quality Assessment (DEC/WO

OR Dept. of Environmental Quality Water Quality Assessment (DEQ/WD

OR Dept. of Environmental Q Qually Water Quality Assessment (DEC/WO

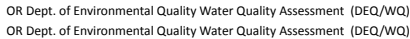

OR Dept. of Environmental Quality Water Quality Assessment (DEQ/WQI
OR Dept. of Environmental Quality Water Quality Assessment (DEQ/Wa)

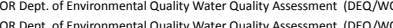

OR Dept. of Environmental Quality Water Quality Assessment (DEQ/WO

OR Dept. of Environmental Quality Water Quality Assessment (DEQ/WOS

OR Dept. of Environmentantal Quality Water Quality Assessment (IDEO/WO)

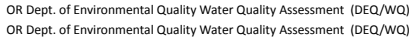

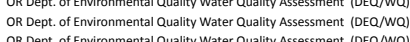

OR Dept. of Environmental l Quality Water Quality Assessment (DEG/WG

OR Dept. of Environmental Q Quality Water quality Assessment (DEO/Wa)
OR Dept. of Environmental Luality Water Quality Assessment (DER/Wa)

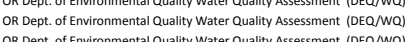

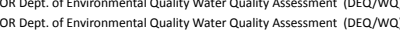

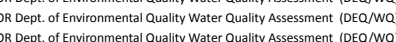

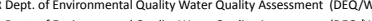

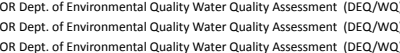

Clark Creek
Dry Creek

East Ferk Ashland creek
Elder Creek

Grayback Creel
kane creek

Kane Creek
Lake reeek
Lick creek

Lost Creek
Soda creek
Succer Creek

Sucker Creek
Trail Creek
West Branch Elk Cr

West Branch Elk Creek
West fork Ashland creek

Bear Creek

Bowen creek
Buter creek

Clark creek
Dry Creek
East Fork Ashland creek

East fork kshlara
Elder creek
Grazback creek

Grayback Creek
Hopkins Canal

Horn Creek
Hukill Creek

Kane Creek
Kenutchen Creek

Kenutchenctreed
Lake Crek
Larson Creek

Larson Creek
Lick Creek

Lick Creek
Lost creek
Merer creek

Payne cree,
Sodat creek

South Fork Big Butte creek

Sucker creek
Trail creek

Trail Creek
Wagner reek
West Branch Elk cree

West Fork Ashland creek

Dry Creek
Dry rreek

Emigrant Creek
Griffin Creek

Sardine Creek
Walker creek

Dry creek
Dry creek

Dry creek
Sout Stage L.

Dry creek
Sount fork Big Butte creek

South Stage L.F.
Bowen Creek
PCSType
Dry Cleaners

Homesteads - Rural - Septic Systems $<1 /$ Acre
Homesteads - Rural- Septic Systems $<1 /$ Acre
Homesteads

Homesteads - Rural - Sepptic Systems $<1 /$ Acre
Homesteads - Rural - Septic systems $<1$ /Acre

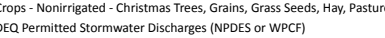

Metal Plating//Finshing//Fabrication

UST-Confirmed Leaking but listed as NFA- DEQ LUST List

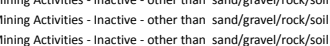

Dry cleaners

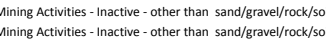

vycleanes

Homesteads - Rural - Septit Systems $\leq 1 /$ Acre

Funture Land Development- - Residentials

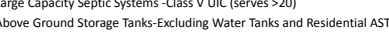

Mining Activities - Inactive- other than sand/fravel/rock/sol

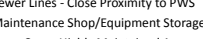

Surface Water on $303 \mathrm{~d}$ list

Surface Water on $303 \mathrm{~d}$ list
Surface Water on $303 \mathrm{~d}$ ist

Surface Water on $303 \mathrm{~d} d$ ist

Surface Water on $303 \mathrm{~d}$ list
Surface Water on $303 \mathrm{~d}$ ist

Surface Water on $303 \mathrm{~d}$ list

Surface Water on 3030 dist
Surface Water on $303 \mathrm{~d}$ ist
Surfece water

Surface Water on $303 d$ list

Surface Water on 3030 dis
Surface Water on 033 lis.

Surface Water on $303 d$ list

Surface Water on 3030 dist

Surface WWeter on $300 \mathrm{~d} d$ ilit
Surface Water on $303 \mathrm{~d}$ list

Surface Water on $303 \mathrm{~d}$ i ist
Surface Water on $303 \mathrm{~d}$ ist

Surface Water on 3030 dis
Surface Water on 3038 dis
Sustrec water

Surface Water on 3030 dist
Surface Water on 303 dist

Surface Water on 303 di his

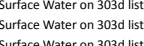

surface Water on $303 d$ lis

Surface Water on $3030 \mathrm{~d}$ list
Surface Water on $303 \mathrm{~d}$ list

Surface Water on $303 d$ list
Surface Water on $303 \mathrm{~d}$ list

Surface Water on $303 \mathrm{~d} d$ ist

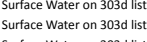

Surface Water on $303 d$ list

Surface Water on $303 \mathrm{~d} d$ lis

Surface Water on 3030 dist

Surface Weter on $n 303 d$ ilst
Surface Water on 3030 list

Surrace Watere wanter on 3030 did ist

Surface Water on 3030 lis
Surface Water on 030 dist

Surface Water on $303 \mathrm{~d} d \mathrm{dis}$

Surface Water on 3030 dist
Surface Watete on $303 \mathrm{dil}$
Sust

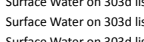

surface Water on $303 d$ list 
PCSType
Surface Wor

Surface Water on $303 d$ list
surface Water on 303 d ist Surface Water on 3030 di:s

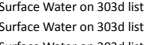
Surface Water on 3030 dis
Surface Water on 0303 lis surface Water Surface Water on 3030 disis Surface Water on 3030 dili: Surface Water on 303 dilis
Surface Wateter on 303 dilis Surface Water on $303 \mathrm{~d}$ lis
Surface Water on $303 \mathrm{~d}$ lis Surface Water on $303 \mathrm{~d}$ lis
Surface Water on $303 \mathrm{din}$ Surface Water on 3030 dist
Surface Water on $303 d$ ist Surface Water on $303 \mathrm{da}$
Surface Water on $0303 \mathrm{~d}$ Surface Water on $303 \mathrm{~d}$ lis

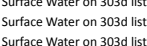
Surface Water on 3030 dis Surface Water on $303 d$ lis
Surface Water on $303 \mathrm{~d}$ lis Surface Water on $303 \mathrm{~d}$ lis

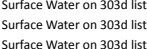
Surface Water on 303 dilis
Surface Water on 303 lis Surface Water on 3033 lis
Surface Water on $303 \mathrm{~d}$ lis
S Surface Water on 303 lis
Surface Water on 303 lis
Suster Sufface Water on 300 dilis
Surface Water on 3030 disis Surface Water on $303 \mathrm{~d}$ lis:
Surface Water on $303 \mathrm{~d}$ lis: Surface Water on 3030 dist
Surface Water on 3030 dist Surface Water on 0303 dist
Surface Water on 303 dist

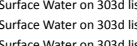
Surface Water on $303 \mathrm{~d} d$ list
Surface Water on $303 \mathrm{~d}$ list Surface Water on $0330 \mathrm{~d}$ dis
surface Water on 303 dias Surface Water on 3033 dis
Surface Water on $303 \mathrm{~d}$ lis surface Water on $303 \mathrm{~d}$ lis

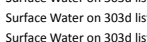
Surface Water on 303d dis
Surface Water on $303 \mathrm{~d}$ lise

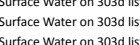
Surface Water on 303d lis. Surface Water on $303 \mathrm{~d} d$ lis Surface Water on $303 \mathrm{~d}$ lis Surface Water on 303d lis
Surface Water on $303 d$ lis Surface Water on $303 \mathrm{~d}$ is Surface Water on 3030 dis
Surface Water on $303 \mathrm{~d}$ lis Surface Water on 3033 lis
Surtace Water Surface Water on 3030 d list
Surface Water on $303 d$ ist Surface Water on $303 d \mathrm{~d}$
Surface Water on $303 \mathrm{~d}$

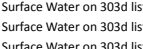




\begin{tabular}{|c|c|c|c|}
\hline & County & RET_DATE & Data_sourc \\
\hline $\begin{array}{l}\text { Not applicable } \\
\text { Not applicable }\end{array}$ & $\begin{array}{l}\text { Not applicible } \\
\text { Not aplicable }\end{array}$ & $\begin{array}{l}1 / 0131 / 2014 \\
10 / 11 / 2014\end{array}$ & 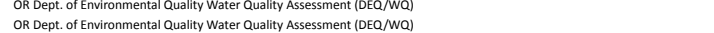 \\
\hline Not applicable & $\begin{array}{l}\text { Not applicule } \\
\text { Not }\end{array}$ & $10 / 31 / 2014$ & 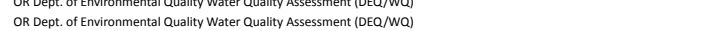 \\
\hline Not applicable & & $10 / 31 / 2014$ & OR Dept. of Environmental Quality Water Quality Assessment ( $(\mathrm{EE} / \mathrm{WO})$ \\
\hline Not applicable & Not applicable & 10/31/2014 & OR Dept. of Environmental Quality Water Quality Assessment (DEQ/Wa) \\
\hline $\begin{array}{l}\text { Not applicable } \\
\text { Notaplicable. }\end{array}$ & $\begin{array}{l}\text { Not applicable } \\
\text { Notaznicible }\end{array}$ & $10 / 31 / 2014$ & 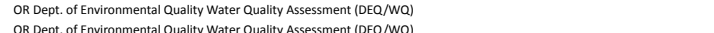 \\
\hline $\begin{array}{l}\text { Not applicable } \\
\text { Not applicable }\end{array}$ & $\begin{array}{l}\text { Not applicible } \\
\text { Not applicale }\end{array}$ & $\begin{array}{l}10 / 131 / 2014 \\
10 / 31 / 2014\end{array}$ & 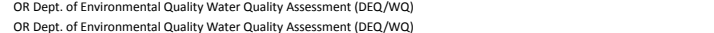 \\
\hline $\begin{array}{l}\text { Not appicable } \\
\text { Not applicable }\end{array}$ & $\begin{array}{l}\text { Not applicable } \\
\text { Not applicable }\end{array}$ & $\begin{array}{l}10 / 111 / 2014 \\
10 / 31 / 2014\end{array}$ & 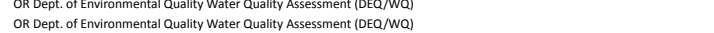 \\
\hline Not applicable & Not applicable & $10 / 31 / 2014$ & 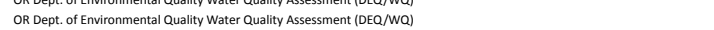 \\
\hline Not applicable & $\begin{array}{l}\text { Not applicable } \\
\text { Nots }\end{array}$ & 10/31/2014 & OR Dept. of Environmental Quality Water Quality Assessment ( $(\mathrm{EE} / \mathrm{W}$ ) \\
\hline Not applicable & Not applicable & 10/31/2014 & OR Dept. of Environmental Quality Water Quality Assessment (DEa/Wa) \\
\hline Not applicable & Notapplicable & 10/31/2014 & OR Dept. of Environmental Q Quality Water Q Quality Assessment (DEG/Wa) \\
\hline $\begin{array}{l}\text { Not applicable } \\
\text { Not applicable }\end{array}$ & & $\begin{array}{l}10 / 31 / 2014 \\
03 / 14 / 2016\end{array}$ & 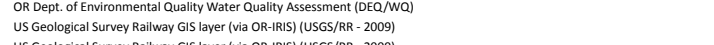 \\
\hline $\begin{array}{l}\text { Not applicable } \\
\text { Nota tolicable }\end{array}$ & & & US Geological Survey Railway GIS IVyer (Via OR-RIS) (USSS/RR - 2009) \\
\hline $\begin{array}{l}\text { Not applicable } \\
\text { Not applicable }\end{array}$ & $\begin{array}{l}\text { Not A Appicicable } \\
\text { Not policable }\end{array}$ & $\begin{array}{l}2008 \\
2008\end{array}$ & 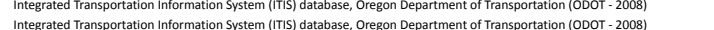 \\
\hline $\begin{array}{l}\text { Not appicable } \\
\text { Not applicable }\end{array}$ & $\begin{array}{l}\text { Not Appincabbe } \\
\text { Not Applicable }\end{array}$ & ${ }_{2008}^{2008}$ & 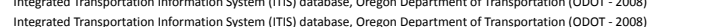 \\
\hline Not applicable & Not Applicable & & 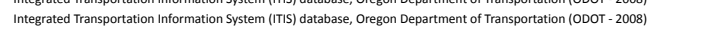 \\
\hline Not applicable & & & Integrated Transportation Information System (ITIS) databasse, Oregon Department of Transportation (ODOT- 2008) \\
\hline Not applicable & Not Applicable & & Integrated Transportation Information System (ITIS) database, Oregon Department of Transportation (ODOT- 2008) \\
\hline $\begin{array}{l}\text { Not applicable } \\
\text { Notaplicable. }\end{array}$ & $\begin{array}{l}\text { Not Applicable } \\
\text { Notentichele }\end{array}$ & ${ }_{2008}^{2008}$ & 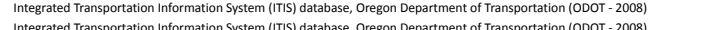 \\
\hline $\begin{array}{l}\text { Not appicable } \\
\text { Not applicable }\end{array}$ & $\begin{array}{l}\text { Not A A pplicable } \\
\text { Not pplicale }\end{array}$ & ${ }_{2008}^{2008}$ & 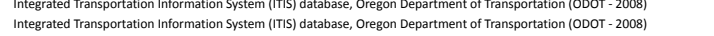 \\
\hline Not applicable & Not Applicable & & Integr rated Transportation Information System (ITIS) database, Oregon Department of Transportation (ODOT- 2008) \\
\hline $\begin{array}{l}\text { Not appliable } \\
\text { Notapolicable }\end{array}$ & $\begin{array}{l}\text { Not Applicable } \\
\text { Not palicable }\end{array}$ & ${ }_{2008}^{2008}$ & 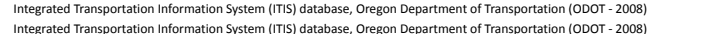 \\
\hline $\begin{array}{l}\text { Not appicable } \\
\text { Not applicable }\end{array}$ & $\begin{array}{l}\text { Not pappicicable } \\
\text { Not Applicale }\end{array}$ & $\begin{array}{l}2008 \\
2008\end{array}$ & 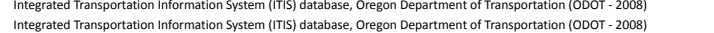 \\
\hline Not applicable & $\begin{array}{l}\text { Not Atppicicabe } \\
\text { Not Applicale }\end{array}$ & & 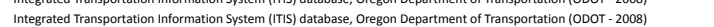 \\
\hline Not applicable & $\begin{array}{l}\text { Not applicable } \\
\text { Note }\end{array}$ & 10/31/2014 & OR Dept of Envirom mental Quality Water Quality Assessment (DEa/Wa) \\
\hline Not applicable & Not applicable & 10/31/2014 & OR Dept. of Environmental Quality Water Quality Assessment (DEQ/Wa) \\
\hline Not applicable & $\begin{array}{l}\text { Not applicable } \\
\text { Notazniche }\end{array}$ & 10/31/2014 & OR Dept. of Environmental Quality Water Quality Assessment (DEQ/Wa) \\
\hline $\begin{array}{l}\text { Not applicable } \\
\text { Not appicale }\end{array}$ & $\begin{array}{l}\text { Nota applicable } \\
\text { Not aplicable }\end{array}$ & $\begin{array}{l}10 / 131 / 2014 \\
10 / 11 / 2014\end{array}$ & 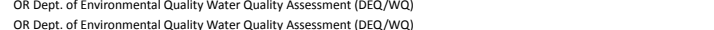 \\
\hline $\begin{array}{l}\text { Not appicable } \\
\text { Not applicable }\end{array}$ & $\begin{array}{l}\text { Noto papplacble } \\
\text { Not applicable }\end{array}$ & $\begin{array}{l}10 / 313 / 2014 \\
10 / 31 / 2014\end{array}$ & 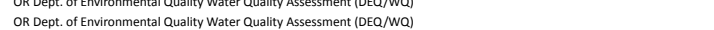 \\
\hline Not applicable & Not applicable & $10 / 31 / 2014$ & 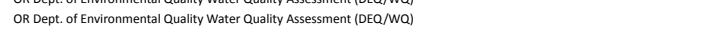 \\
\hline Not applicable & Not applicable & $10 / 31 / 2014$ & OR Dept. of Environmental Quality Water Quality Assessment ( $(\mathrm{EEO} / \mathrm{WQ})$ \\
\hline Not applicable & Not applicable & 10/31/2014 & OR Dept. of Environmental Quality Water Quality Assessment ( $(\mathrm{DEQ} / \mathrm{Wa})$ \\
\hline Not applicable & $\begin{array}{l}\text { Not applicable } \\
\text { Notal }\end{array}$ & $10 / 31 / 2014$ & OR Dept. of Environmentala Quality Water Quality Assessment (DEQ/Wa) \\
\hline $\begin{array}{l}\text { Not applicable } \\
\text { Not applicable }\end{array}$ & $\begin{array}{l}\text { Notapplicable } \\
\text { Not applicable }\end{array}$ & $\begin{array}{l}10 / 131 / 2014 \\
10 / 11 / 2014\end{array}$ & 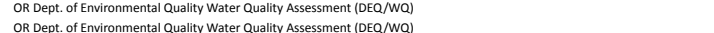 \\
\hline $\begin{array}{l}\text { Not appicable } \\
\text { Not applicable }\end{array}$ & $\begin{array}{l}\text { Not appinable } \\
\text { Not applicable }\end{array}$ & $\begin{array}{l}10 / 313 / 2014 \\
10 / 31 / 2014\end{array}$ & 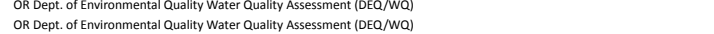 \\
\hline Not appicable & Not applicable & $10 / 31 / 2014$ & 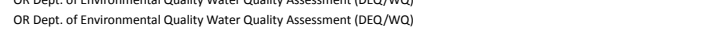 \\
\hline Not applicable & Not applicable & 10/31/2014 & OR Dept. of Environmental Quality Water Quality Assessment ( $(\mathrm{EE} / \mathrm{WO})$ \\
\hline Not applicable & Not applicable & 10/13/2014 & OR Dept. of Environmental Q Quality Water Quality Assessment (DEQ/Wa) \\
\hline $\begin{array}{l}\text { Not applicable } \\
\text { Notaplicable }\end{array}$ & $\begin{array}{l}\text { Not applicable } \\
\text { Notaplicable }\end{array}$ & $\begin{array}{l}10 / 31 / 2014 \\
10 / 131 / 2014\end{array}$ & 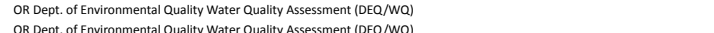 \\
\hline $\begin{array}{l}\text { Not applicable } \\
\text { Not applicable }\end{array}$ & $\begin{array}{l}\text { Notappliable } \\
\text { Not applicable }\end{array}$ & $\begin{array}{l}10 / 11 / 2014 \\
10 / 31 / 2014\end{array}$ & 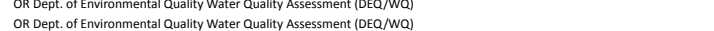 \\
\hline $\begin{array}{l}\text { Not appinabie } \\
\text { Not applicable }\end{array}$ & $\begin{array}{l}\text { Notapplicable } \\
\text { Not applicable }\end{array}$ & $\begin{array}{l}10 / 11 / 2014 \\
10 / 31 / 2014\end{array}$ & 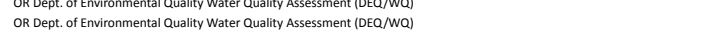 \\
\hline Not applicable & Not appicable & ${ }_{10131 / 2014}$ & OR Dept. of Environmental Qualitit Water Quality Assessment (DEG/Wa) \\
\hline Not applicable & Not applicable & 10/31/2014 & OR Dept. of Environmental Quality Water Quality Assessment ( $(\mathrm{EE} / \mathrm{W}$ WO) \\
\hline Not applicable & Not applicable & 10/31/2014 & OR Dept. of Environmental Quality Water Quality Assessment (DEQ/Wa) \\
\hline Not applicable & $\begin{array}{l}\text { Not applicable } \\
\text { Jackson }\end{array}$ & $\begin{array}{l}10 / 31 / 2014 \\
1 / 20201780.00 .\end{array}$ & OR Dept. of Environmental Quality Water quality Assessment (DEQ/WO) \\
\hline 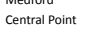 & $\begin{array}{l}\text { Jackson } \\
\text { Not applicable }\end{array}$ & $\begin{array}{l}\text { 1/20/20178:00:00 AM } \\
\text { 2009 }\end{array}$ & 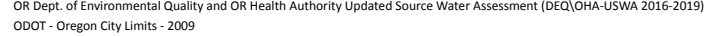 \\
\hline Asshand & Not applicable & 2009 & $\begin{array}{l}\text { ODOT - Oregon City Limits - } 2009 \\
\text { O. }\end{array}$ \\
\hline Shady Cove & Not tapplicable & 2009 & ODOT - Oregon City Limits - 2009 \\
\hline $\begin{array}{l}\text { Cave Junction } \\
\text { Talent }\end{array}$ & $\begin{array}{l}\text { Not applicable } \\
\text { Notanlicable }\end{array}$ & 2009 & ODOT- Oregon City Limits - 2009 \\
\hline $\begin{array}{l}\text { Talent } \\
\text { Eagle point }\end{array}$ & $\begin{array}{l}\text { Not applicable } \\
\text { Notaplicable }\end{array}$ & ${ }_{2009}^{2009}$ & 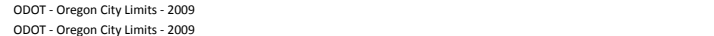 \\
\hline Phoenix & Not applicable & 2009 & $\begin{array}{l}\text { ODOO- OOegon Citit Limitit- }-2009 \\
\text { ODOT - Oregon City Lims - } 2009\end{array}$ \\
\hline Jacksonville & Not applicable & & ODOT- Oregon City Limits - 2009 \\
\hline ue River & Not applicable & 2009 & ODOT- Oregon City Limits - 2009 \\
\hline 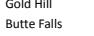 & $\begin{array}{l}\begin{array}{l}\text { Nota taplicable } \\
\text { Not applicable }\end{array} \\
\text {. }\end{array}$ & & 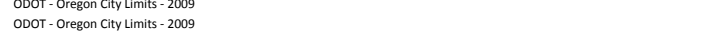 \\
\hline applicable & $\begin{array}{l}\text { Jackson } \\
\text { Jas }\end{array}$ & & Oregon Heath Authority Y HAB Advisories \\
\hline applicable & Jackson & 12/26/18 & Oregon Heath Authority Y HAB Advisorories \\
\hline Not applicable & Jackson & 12/26/18 & Oregon Health Authority $、$ HAB Advisories \\
\hline applica & Jackson & 12/26/18 & Oregon Health Authority \ HAB Advisories \\
\hline No No $_{\text {No }}$ & Jacksonon & 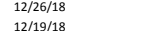 & Oregon Heath Authority HAB Advisories \\
\hline $\begin{array}{llll} & \end{array}$ & 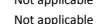 & $\begin{array}{l}12 / 191 / 18 \\
12 / 1918\end{array}$ & 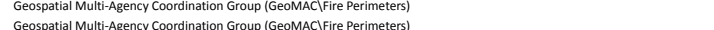 \\
\hline tapplicable & Not appicable & ${ }_{12 / 191 / 18}$ & 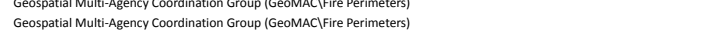 \\
\hline bicable & Not appicable & 12/19/18 & Geospatial Mult-ABency Coordination Group (GeoMAClFire Perimeters) \\
\hline & Not appicable & 12/19/18 & Geospatail Multi-Bgency Coordination Group (Geod \\
\hline & Not tapplicable & 12/19/18 & Geospatial Multi-ABency Coordination Group Geeon \\
\hline 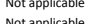 & $\begin{array}{l}\text { Not appicicable } \\
\text { votie }\end{array}$ & $\begin{array}{l}12 / 191 / 18 \\
12 / 1918\end{array}$ & Geospatial Mutti-ABency Coordination Group Geeo \\
\hline & 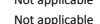 & 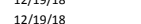 & 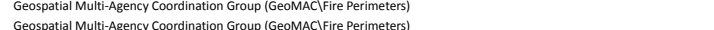 \\
\hline & Not appicable & ${ }_{12 / 19 / 18}$ & 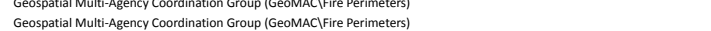 \\
\hline & Not applicable & 12/19/18 & Geospatial Multi-Agency Coordination Group \\
\hline & Not appicable & 12/19/18 & Geospatial Multi-Agency Coordination Grou \\
\hline picable & $\begin{array}{l}\text { Not applicable } \\
\text {. }\end{array}$ & $\begin{array}{l}12 / 19 / 18 \\
12 / 18918\end{array}$ & Geospatial Multi-Agency Coordination Grou \\
\hline & $\begin{array}{l}\text { Not atppicable } \\
\text { Not applicable }\end{array}$ & $\begin{array}{l}12719 / 8 \\
12 / 19 / 18\end{array}$ & 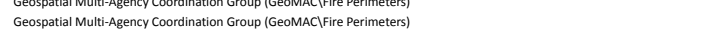 \\
\hline
\end{tabular}

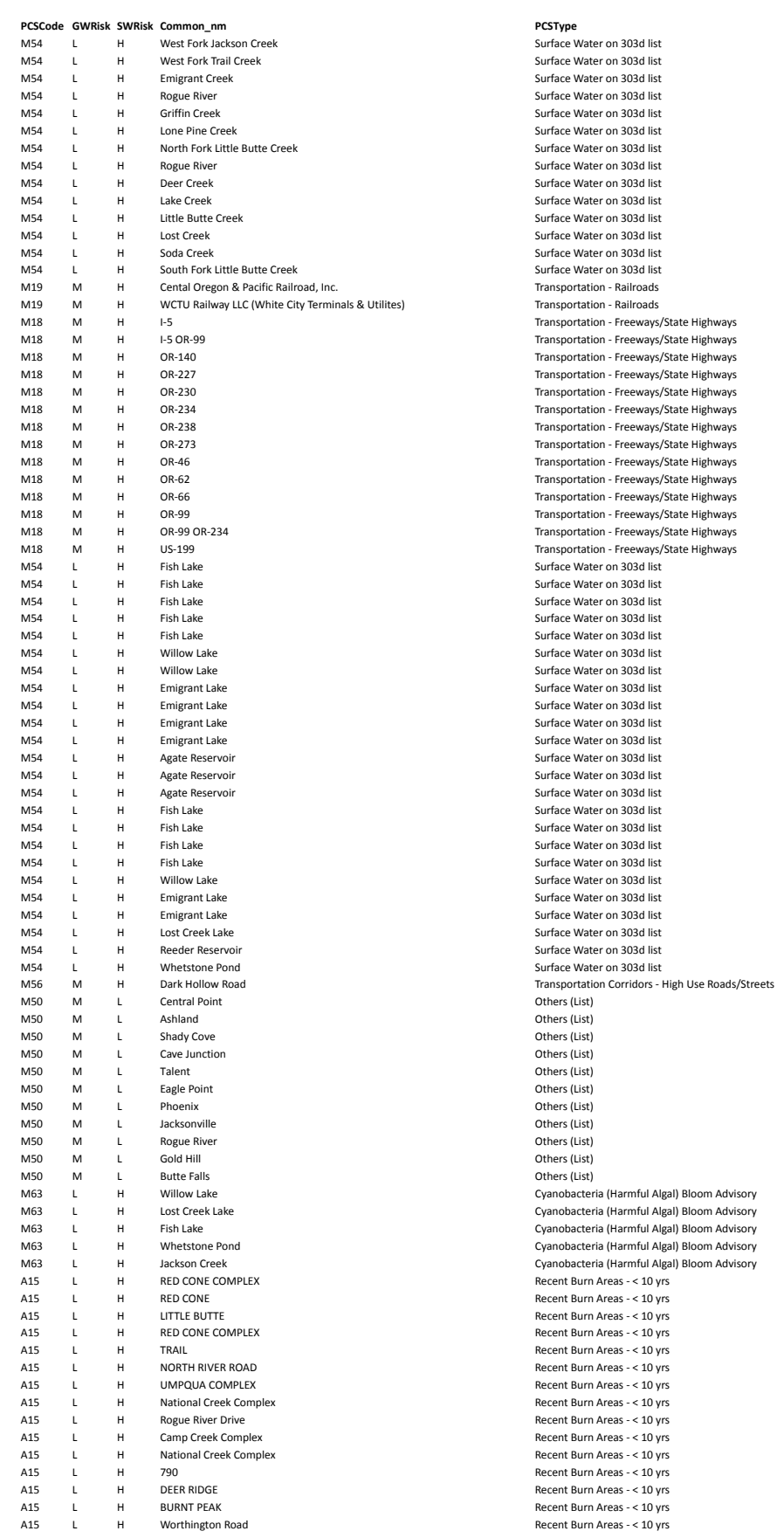


Recent Burn Areas $<10$ yrs

Recent Burn Areas $<10 \mathrm{ys}$
Recent Burn Areas -10 yrs

Recent Burn Areas $<10$ yrs
Recent Burn Areas $<10$ yrs

Recent Burn Areas $<10$ yrs
Recent Burn Areas $<10$ yrs

Recent Burn Areas $<10$ yrs

Recent Burn Areas $<10$ yrs
Recent Burn Areas $<10$ yrs

Recent burn Areas $<10$ yrs
Recent turn Areas $<10$ yrs

Recent Burn Areas $<10$ vis
Recent Burn Areas $<10$ ys 
APPENDIX I. Windshield Survey Database 


\begin{tabular}{|c|c|c|c|c|c|c|c|c|c|c|c|}
\hline Facility Name & Address & Phone Number & Receiving Waterbody & Inspector & Date & Time & Activity at Site & Visual Sheen & Spilled Materials & Erosion & Bulk Storage \\
\hline \begin{tabular}{|l|} 
Automania Car Sales (Moved) \\
\end{tabular} & 1455 Rogue River Highway/895 SE Gladiola & \begin{tabular}{|l}
$(541) 479-8888$ \\
\end{tabular} & \begin{tabular}{|l|} 
Fruitdale Creek \\
\end{tabular} & AS & $12 / 17 / 2018$ & 12:00 & \begin{tabular}{|l|} 
Residential \\
\end{tabular} & N/A & N/A & No & Yes \\
\hline Big Sam's Auto Repair (Closed) & Highway 99 & N/A & Fruitdale Creek & AS & $12 / 17 / 2018$ & 13:05 & Could Not Verify & N/A & N/A & $\mathrm{N} / \mathrm{A}$ & N/A \\
\hline Brakes Plus & 1499 Rogue River Highway & (541) 474-0307 & Fruitdale Creek & AS & $12 / 17 / 2018$ & 12:06 & Auto Repair & No & No & No & Yes \\
\hline Caveman RV & 1182/1190 Rogue River Highway & (541) 476-1253 & Fruitdale Creek & AS & $12 / 17 / 2018$ & $13: 24$ & RV Sales/Service & $\mathrm{N} / \mathrm{A}$ & N/A & No & Yes \\
\hline Clovercrest Service Center (now NAPA Auto) & 2600 Cloverlawn Drive & (541) 955-9541 & Fruitdale Creek & AS & $12 / 17 / 2018$ & $12: 32$ & Auto Repair & No & No & No & Yes \\
\hline Fruitdale Elementary School & 1560 Bill Baker Way & (541) 476-2276 & Fruitdale Creek & AS & $12 / 17 / 2018$ & $12: 22$ & School & No & No & Yes & Yes \\
\hline Grants Pass Irrigation District (GPID) Easement & 2202 Haviland Drive & (541) 476-2582 & Fruitdale Creek & AS & $12 / 17 / 2018$ & 12:44 & Irrigation Canal & No & No & Yes & No \\
\hline Heating Oil Tank & 1560 Hamilton Lane & N/A & Fruitdale Creek & AS & $12 / 17 / 2018$ & 12:17 & Could Not Verify & N/A & N/A & N/A & N/A \\
\hline Heating oil Tank & 1980 Rogue River Highway & N/A & Fruitdale Creek & AS & $12 / 17 / 2018$ & 3:10 & Could Not Verify & N/A & N/A & N/A & $\mathrm{N} / \mathrm{A}$ \\
\hline High Density Housing - Developed & 1560 Hamilton Lane & N/A & Fruitdale Creek & AS & $12 / 17 / 2018$ & $12: 15$ & Fully Developed & N/A & N/A & N/A & $\mathrm{N} / \mathrm{A}$ \\
\hline High Density Housing - Developed & SE Mystic Drive & N/A & Fruitdale Creek & AS & $12 / 17 / 2018$ & $13: 20$ & Fully Developed & N/A & N/A & N/A & N/A \\
\hline Jerry's Appliance and Repair (Closed) & 1077 Rogue River Highway & N/A & Fruitdale Creek & AS & $12 / 17 / 2018$ & $11: 47$ & Could Not Verify & N/A & N/A & Yes & Yes \\
\hline Muffler King (Closed)/A1 Exterminators (Moved) & 1492 Rogue River Highway & |(541) 472-1094 & Fruitdale Creek & AS & $12 / 17 / 2018$ & $13: 15$ & Could Not Verify & N/A & N/A & N/A & $\mathrm{N} / \mathrm{A}$ \\
\hline New Construction Site & 2263 Haviland Drive & N/A & Fruitdale Creek & AS & $12 / 17 / 2018$ & 12:51 & Active Construction Site & No & No & Yes & Yes \\
\hline Panoramic Estates - Developed & Panoramic Loop & N/A & Fruitdale Creek & AS & $12 / 17 / 2018$ & $12: 38$ & Fully Developed & N/A & N/A & N/A & N/A \\
\hline Parkdale Subdivision - Developed & South end of Parkdale Drive & IN/A & Fruitdale Creek & AS & $12 / 17 / 2018$ & $11: 42$ & Fully Developed & IN/A & N/A & N/A & N/A \\
\hline Powell Distributing (now Timber Mountain Construction, Inc.) & 1919/1935 Rogue River Highway & |(541) $956-8700$ & Fruitdale Creek & AS & $12 / 17 / 2018$ & 13:02 & Storage & N/A & N/A & Yes & Yes \\
\hline Swiss Metric & 1610 Parkdale Drive & |(541) 476-1771 & Fruitdale Creek & AS & $12 / 17 / 2018$ & $11: 37$ & Warehouse & No & No & No & No \\
\hline Willow Development - Developed & 2221 Haviland Drive & N/A & Fruitdale Creek & AS & $12 / 17 / 2018$ & $12: 40$ & Fully Developed & N/A & N/A & N/A & N/A \\
\hline Cabinet Crafters & 1584 SE N Street & (541) 474-5063 & Skunk Creek & AS & $12 / 28 / 2018$ & 12:11 & Could Not Verify & N/A & N/A & $\mathrm{N} / \mathrm{A}$ & N/A \\
\hline Clearwater Co-op (Closed?) & 1130 SE Gladiola Drive & N/A & Skunk Creek & AS & $12 / 28 / 2018$ & $12: 32$ & Could Not Verify & No & No & No & Yes \\
\hline Edgewater Christian Fellowship - Developed & 101 Assembly Circle & (541) 474-7172 & Jones Creek & AS & $12 / 28 / 2018$ & $13: 15$ & Fully Developed & N/A & N/A & N/A & N/A \\
\hline Ferguson Fabrication (now Warehouse Rebuilders) & 959/961 SE Gladiola Drive & (541) 476-1593 & Skunk Creek & AS & $12 / 28 / 2018$ & 12:19 & Storage & N/A & N/A & Yes & Yes \\
\hline Gary Peterson & 910 SE M Street & (541) 479-2981 & Skunk Creek & AS & $12 / 28 / 2018$ & $11: 30$ & Office/Warehouse/storage & N/A & N/A & Yes & Yes \\
\hline Gladiola Campus & 1137 SE Gladiola Drive & (541) 474-5790 & Skunk Creek & AS & $12 / 28 / 2018$ & $12: 35$ & School & No & No & No & No \\
\hline Grants Pass Equipment Rentals (now Southern Oregon Tire) & 851 SE Milbank Road & (541) 955-4444 & Skunk Creek & AS & $12 / 28 / 2018$ & $11: 41$ & Auto Repair & No & No & Yes & Yes \\
\hline Grants Pass School District 7 & 1053 SE Gladiola Drive & (541) 474-5785 & Skunk Creek & AS & $12 / 28 / 2018$ & $12: 25$ & Bus Yard & No & No & No & Yes \\
\hline Indian Creek Auto Dismantlers & 1098 SE M Street & (541) 479-7203 & Skunk Creek & AS & $12 / 28 / 2018$ & 12:06 & Salvage Yard & N/A & N/A & N/A & Yes \\
\hline Oregon Department of Transportation (ODOT) & 345 NE Agness Avenue & (541) 474-3149 & Skunk Creek & AS & $12 / 28 / 2018$ & $13: 12$ & Warehouse & N/A & N/A & No & Yes \\
\hline Oregon Swiss Precision (now Caveman Fence) & 1080 SE M Street & (541) 474-0302 & Skunk Creek & AS & $12 / 28 / 2018$ & $11: 48$ & Office/Warehouse/storage & No & No & Yes & Yes \\
\hline Riverside Elementary School & 1200 SE Harvey Drive & (541) 474-5780 & Skunk Creek & AS & $12 / 28 / 2018$ & 12:43 & School & No & No & No & No \\
\hline Roger Hansen (Closed) & 450 Ament Road & N/A & Jones Creek & AS & $12 / 28 / 2018$ & $12: 57$ & Could Not Verify & N/A & N/A & N/A & N/A \\
\hline RV Marble/Castle Marble and Granite, LLC & 2375 Foothill Boulevard & (541) 476-3343 & Jones Creek & AS & $12 / 28 / 2018$ & 13:01 & Warehouse & No & No & No & Yes \\
\hline Schnitzer Steel/Edmond Corporation (Moved) & 605 NE Agness Avenue/2625 Avenue G, White City, OR 97503 & (541) 826-5758 & Skunk Creek & AS & $12 / 28 / 2018$ & $13: 20$ & Could Not Verify & N/A & N/A & N/A & N/A \\
\hline Spalding and Son & 2345 SE N Street/2251 NE Spalding Avenue & (541) 476-4448 & Jones Creek & AS & $12 / 28 / 2018$ & $12: 55$ & Warehouse/storage & N/A & N/A & N/A & Yes \\
\hline Timber Products Company & 1090 SE M Street & (541) 479-6822 & Skunk Creek & AS & $12 / 28 / 2018$ & $11: 56$ & Factory/Warehouse/Storage & Yes & No & Yes & Yes \\
\hline Umpqua Dairy Products Company & 1686 SE N Street & (541) 479-6832 & Skunk Creek & AS & $12 / 28 / 2018$ & $12: 15$ & Warehouse & N/A & N/A & No & N/A \\
\hline Warehouse Rebuilders (Moved) & |961 SE N Street/961 SE Gladiola Drive & |(541) $476-1593$ & |skunk Creek & AS & |12/28/2018 & 31:35 & Could Not Verify & N/A & N/A & $\mathrm{N} / \mathrm{A}$ & $\mathrm{N} / \mathrm{A}$ \\
\hline
\end{tabular}




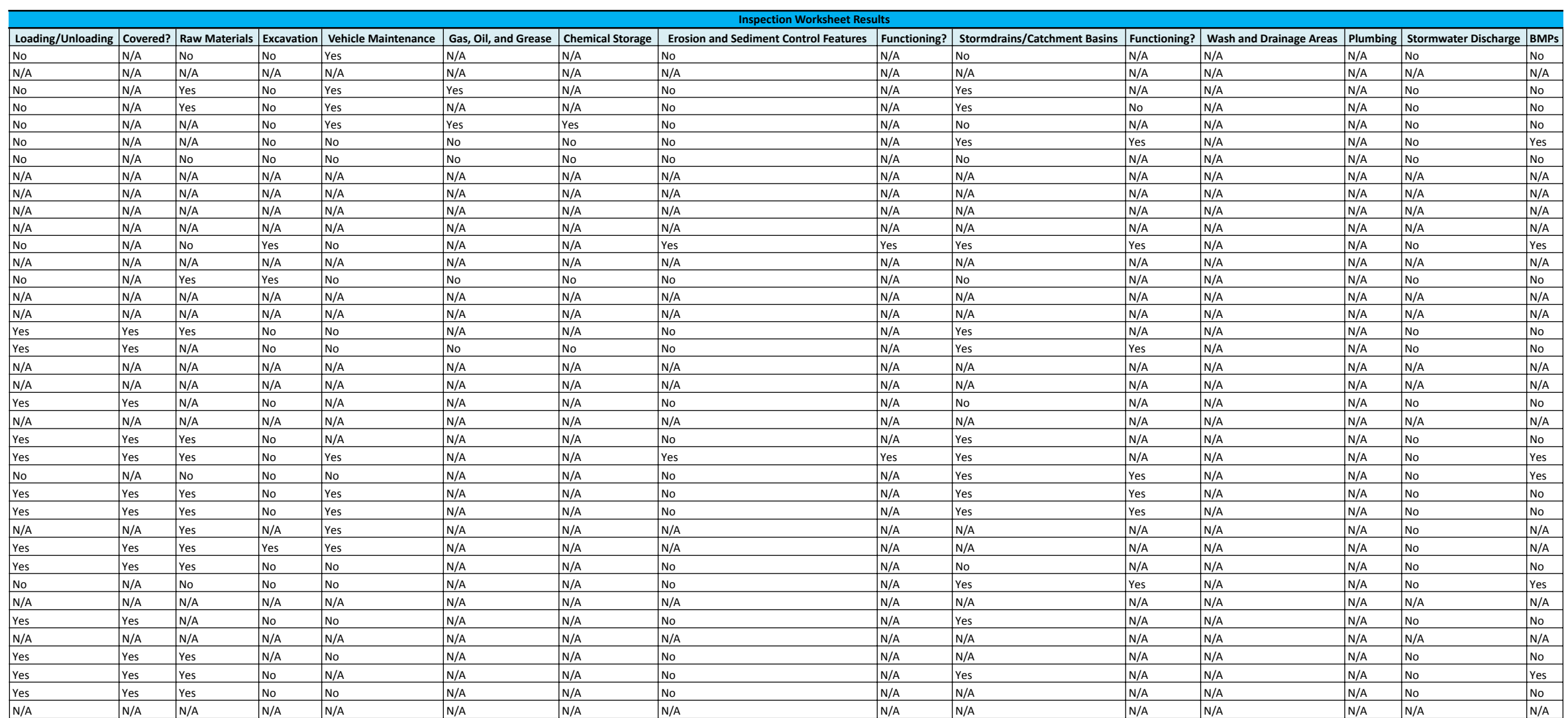




\begin{tabular}{|c|c|c|c|c|c|c|c|}
\hline Functioning? & Stormwater Controls Observed & SWPCP & Spill Prevention and Response Plan & Employees Aware? & Photo\# & PCS code & PCS Recommendation \\
\hline N/A & ( & N/A & N/A & N/A & 4488 & & - \\
\hline N/A & N/A & N/A & N/A & N/A & N/A & - & \\
\hline $\mathrm{N} / \mathrm{A}$ & Catch Basin and Dumpster/Equipment Cover & N/A & N/A & N/A & 4489,4490 & c04, 007 & BMP12, 13, 20, 22, 23, 30-34, 36-41, 49-51. \\
\hline N/A & N/A & N/A & N/A & N/A & $4505,4506,4507$ & $\mathrm{C} 04, \mathrm{C} 10, \mathrm{C} 12$ & BMP12, 13, 20, 22, 23, 30-41, 49, 51. \\
\hline N/A & Dumpster/Equipment Cover and Drum/Container Containment & N/A & N/A & N/A & 4492,4493 & C04 & BMP13, 20, 22, 23, 30-34, 36, 38, 39, 40, 49, 51. \\
\hline Yes & Oil/Water Separator, Dumpster/Equipment Cover, and Vegetated Buffer Zone & N/A & N/A & N/A & 4491 & $\mathrm{C} 20, \mathrm{M} 28, \mathrm{R} 15$ & BMP3, $9,12,13,17,20,22,23,26-29,31,36,37,40,41,46-49,51-53$. \\
\hline N/A & Vegetated Buffer Zone & N/A & N/A & N/A & $4494,4495,4497$ & C07 & BMP12, 13, 22, 23, 30-32, 34, 36, 37, 40, 41, 49-51. \\
\hline N/A & N/A & N/A & N/A & N/A & N/A & - & 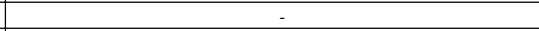 \\
\hline N/A & N/A & N/A & N/A & $\mathrm{N} / \mathrm{A}$ & N/A & - & - \\
\hline N/A & N/A & N/A & N/A & N/A & N/A & - & - \\
\hline N/A & N/A & N/A & N/A & N/A & N/A & - & \\
\hline Yes & Catch Basin, On-Site Bio-Swale, and Vegetated Buffer Zone & N/A & N/A & N/A & $\mid 4483,4484,4485,4486,4487$ & c15 & BMP12, 22, 23, 31, 33, 34, 36, 37, 40, 41, 49-51. \\
\hline N/A & $\mathrm{N} / \mathrm{A}$ & N/A & N/A & $\mathrm{N} / \mathrm{A}$ & N/A & - & \\
\hline N/A & N/A & N/A & N/A & N/A & 4498,4499 & - & - \\
\hline N/A & N/A & N/A & N/A & N/A & N/A & - & - \\
\hline N/A & N/A & N/A & N/A & N/A & N/A & - & - \\
\hline N/A & N/A & N/A & N/A & N/A & $4500,4501,4502$ & M23, M62 & BMP2, 13, 24, 31, 49. \\
\hline N/A & Catch Basin & N/A & N/A & N/A & 4482 & c07 & BMP12, 13, 22, 23, 30-32, 34, 36, 37, 40, 41, 49-51. \\
\hline N/A & N/A & N/A & N/A & N/A & N/A & - & $x_{-1}^{2}$ \\
\hline N/A & N/A & N/A & N/A & N/A & N/A & - & \\
\hline N/A & Vegetated Buffer Zone & N/A & N/A & N/A & 4527 & C14, R10 & BMP12, 13, 17-20, 22-24, 28, 30-34, 37, 39-41, 49-51. \\
\hline N/A & N/A & N/A & N/A & N/A & N/A & - & \\
\hline N/A & Catch Basin & N/A & N/A & N/A & 4524,4525 & $c 07,, 17$ & BMP12, 13, 22, 23, 30-34, 36, 37, 40, 41, 49-51. \\
\hline Yes & Catch Basin and Dumpster/Equipment Cover & N/A & N/A & N/A & $4509,4510,4511,4513$ & $\mathrm{Co6}, \mathrm{C07}$ & BMP11-13, 20, 22, 23, 30-32, 34, 36, 37, 40, 41, 49-51. \\
\hline Yes & Dumpster/Equipment Cover and Vegetated Buffer Zone & N/A & N/A & N/A & 4528,4529 & R15 & BMP3, 12, 13, 17, 20, 22, 23, 26-29, 36, 37, 40, 41, 46-48, 51-53. \\
\hline N/A & Catch Basin, Dumpster/Equipment Cover, and Vegetated Buffer Zone & N/A & N/A & N/A & 4514 & C04, C61 (for SO Tire) & BMP12, 13, 20, 22, 23, 30-34, 36-41, 49, 51. \\
\hline N/A & Dumpster/Equipment Cover and Vegetated Buffer Zone & N/A & N/A & N/A & 4526 & $\mathrm{CO}, \mathrm{M} 27, \mathrm{R} 15$ & BMP3, 12, 13, 17, 20, 22, 23, 26-32, 34, 36, 37, 40, 41, 46-48, 49-53. \\
\hline N/A & N/A & N/A & N/A & N/A & 4522 & C61 & BMP12, 13, 20, 22, 23, 30-34, 36-41, 49, 51. \\
\hline N/A & N/A & N/A & N/A & N/A & 4537 & $\mathrm{C} 07, \mathrm{M} 26$ & BMP12, 13, 22, 23, 30-32, 34, 36, 37, 40, 41, 49-51. \\
\hline N/A & Dumpster/Equipment Cover & N/A & N/A & N/A & $4515,4516,4517,4518$ & C12, C67 (for Caveman Fence) & BMP12, 13, 22, 23, 36, 40, 49, 51 \\
\hline Yes & Catch Basin, Oil/Water Separator, and Vegetated Buffer Zone & N/A & N/A & N/A & 4530,4531 & M26, R15 & BMP3, 12, 13, 17, 20, 22, 23, 26-29, 31, 36, 37, 40, 41, 46-49, 51-53. \\
\hline N/A & N/A & N/A & N/A & N/A & N/A & - & 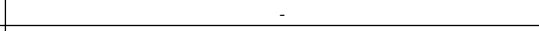 \\
\hline N/A & Drum and Container Containment & N/A & N/A & N/A & 4536 & $\mathrm{C} 65$ & BMP9, 10, 12, 13, 22-24, 27, 28, 30-37, 39, 41, 49-51. \\
\hline N/A & N/A & N/A & N/A & N/A & N/A & - & \\
\hline N/A & Dumpster/Equipment Cover & N/A & N/A & N/A & $4532,4533,4534,4535$ & $\mathrm{C} 07, \mathrm{C} 26, \mathrm{C} 58, \mathrm{C} 60, \mathrm{M} 26, \mathrm{M} 28$ & BMP2, 9, 10, 12, 13, 20, 22-24, 27, 30-32, 34, 36, 37, 39-41, 49-51. \\
\hline N/A & Catch Basin and Oil/Water Separator & N/A & N/A & N/A & $4519,4520,4521$ & $\mathrm{C} 07, \mathrm{C} 26$ & BMP9, 10, 12, 13, 22, 23, 27, 30-32, 34, 36, 37, 40, 41, 49-51. \\
\hline N/A & N/A & N/A & N/A & $\mathrm{N} / \mathrm{A}$ & 4523 & c007, c67 & BMP12, 13, 22, 23, 30-32, 34, 36, 37, 40, 41, 49-51. \\
\hline N/A & N/A & N/A & N/A & $\mathrm{N} / \mathrm{A}$ & N/A & - & \\
\hline
\end{tabular}

